\title{
Topics in Differential Geometry
}

Peter W. Michor

Fakultät für Mathematik der Universität Wien, Nordbergstrasse 15, A-1090 Wien, Austria.

Erwin Schrödinger Institut für Mathematische Physik, Boltzmanngasse 9, A-1090 Wien, Austria.

peter.michor@univie.ac.at 

To the ladies of my life, Elli, Franziska, and Johanna 



\section{Contents}

Preface ix

CHAPTER I. Manifolds and Vector Fields 1

1. Differentiable Manifolds 1

2. Submersions and Immersions 16

3. Vector Fields and Flows 21

CHAPTER II. Lie Groups and Group Actions 43

4. Lie Groups I 43

5. Lie Groups II. Lie Subgroups and Homogeneous Spaces 62

6. Transformation Groups and G-Manifolds 68

7. Polvnomial and Smooth Invariant Theory 87

CHAPTER III. Differential Forms and de Rham Cohomology 101

$\begin{array}{ll}\text { 8. Vector Bundles } & 101\end{array}$

9. Differential Forms 115

10. Integration on Manifolds 124

11. De Rham Cohomology 131

12. Cohomology with Compact Supports and Poincaré Duality 141

13. De Rham Cohomology of Compact Manifolds 153

14. Lie Groups III. Analysis on Lie Groups 160

15. Extensions of Lie Algebras and Lie Groups 171

CHAPTER IV. Bundles and Connections 193

16. Derivations on the Algebra of Differential Forms 193 
17. Fiber Bundles and Connections 202

18. Principal Fiber Bundles and $G$-Bundles 212

19. Principal and Induced Connections 231

20. Characteristic Classes 253

21. Jets 268

CHAPTER V. Riemann Manifolds 275

22. Pseudo-Riemann Metrics and Covariant Derivatives 275

23. Geometry of Geodesics 291

24. Parallel Transport and Curvature 300

25. Computing with Adapted Frames and Examples 312

26. Riemann Immersions and Submersions 329

$\begin{array}{ll}27 . \text { Jacobi Fields } & 347\end{array}$

CHAPTER VI. Isometric Group Actions or Riemann G-Manifolds 365

28. Isometries, Homogeneous Manifolds, and Symmetric Spaces 365

29. Riemann G-Manifolds 373

30. Polar Actions 387

CHAPTER VII. Symplectic and Poisson Geometry 413

31. Symplectic Geometry and Classical Mechanics 413

32. Completely Integrable Hamiltonian Systems 435

33. Poisson Manifolds 441

34. Hamiltonian Group Actions and Momentum Mappings 453

\begin{tabular}{ll}
\hline List of Symbols & 479
\end{tabular}

\begin{tabular}{ll}
\hline Bibliography & 481
\end{tabular}

Index 493 


\section{Preface}

This book is an introduction to the fundamentals of differential geometry (manifolds, flows, Lie groups and their actions, invariant theory, differential forms and de Rham cohomology, bundles and connections, Riemann manifolds, isometric actions, symplectic geometry) which stresses naturality and functoriality from the beginning and is as coordinate-free as possible. The material presented in the beginning is standard - but some parts are not so easily found in text books: Among these are initial submanifolds (2.13) and the extension of the Frobenius theorem for distributions of nonconstant rank (the Stefan-Sussman theory) in (3.21)- (3.28). A quick proof of the Campbell-Baker-Hausdorff formula for Lie groups is in (4.29), Lie group actions are studied in detail: Palais' results that an infinitesimal action of a finite-dimensional Lie algebra on a manifold integrates to a local action of a Lie group and that proper actions admit slices are presented with full proofs in sections (5) and (6). The basics of invariant theory are given in section (7): The Hilbert-Nagata theorem is proved, and Schwarz's theorem on smooth invariant functions is discussed, but not proved.

In the section on vector bundles, the Lie derivative is treated for natural vector bundles, i.e., functors which associate vector bundles to manifolds and vector bundle homomorphisms to local diffeomorphisms. A formula for the Lie derivative is given in the form of a commutator, but it involves the tangent bundle of the vector bundle. So also a careful treatment of tangent bundles of vector bundles is given. Then follows a standard presentation of differential forms and de Rham cohomoloy including the theorems of de Rham and Poincaré duality. This is used to compute the cohomology of compact Lie groups, and a section on extensions of Lie algebras and Lie groups follows. 
The chapter on bundles and connections starts with a thorough treatment of the Frölicher-Nijenhuis bracket via the study of all graded derivations of the algebra of differential forms. This bracket is a natural extension of the Lie bracket from vector fields to tangent bundle valued differential forms; it is one of the basic structures of differential geometry. We begin our treatment of connections in the general setting of fiber bundles (without structure group). A connection on a fiber bundle is just a projection onto the vertical bundle. Curvature and the Bianchi identity are expressed with the help of the Frölicher-Nijenhuis bracket. The parallel transport for such a general connection is not defined along the whole of the curve in the base in general - if this is the case, the connection is called complete. We show that every fiber bundle admits complete connections. For complete connections we treat holonomy groups and the holonomy Lie algebra, a subalgebra of the Lie algebra of all vector fields on the standard fiber. Then we present principal bundles and associated bundles in detail together with the most important examples. Finally we investigate principal connections by requiring equivariance under the structure group. It is remarkable how fast the usual structure equations can be derived from the basic properties of the Frölicher-Nijenhuis bracket. Induced connections are investigated thoroughly — we describe tools to recognize induced connections among general ones. If the holonomy Lie algebra of a connection on a fiber bundle with compact standard fiber turns out to be finite-dimensional, we are able to show that in fact the fiber bundle is associated to a principal bundle and the connection is an induced one. I think that the treatment of connections presented here offers some didactical advantages: The geometric content of a connection is treated first, and the additional requirement of equivariance under a structure group is seen to be additional and can be dealt with later - so the student is not required to grasp all the structures at the same time. Besides that it gives new results and new insights. This treatment is taken from [147.

The chapter on Riemann geometry contains a careful treatment of connections to geodesic structures to sprays to connectors and back to connections considering also the roles of the second and third tangent bundles in this. Most standard results are proved. Isometric immersions and Riemann submersions are treated in analogy to each other. A unusual feature is the Jacobi flow on the second tangent bundle. The chapter on isometric actions starts off with homogeneous Riemann manifolds and the beginnings of symmetric space theory; then Riemann $G$-manifolds and polar actions are treated.

The final chapter on symplectic and Poisson geometry puts some emphasis on group actions, momentum mappings and reductions. 
There are some glaring omissions: The Laplace-Beltrami operator is treated only summarily, there is no spectral theory, and the structure theory of Lie algebras is not treated and used. Thus the finer theory of symmetric spaces is outside of the scope of this book.

The exposition is not always linear. Sometimes concepts treated in detail in later sections are used or pointed out earlier on when they appear in a natural way. Text cross-references to sections, subsections, theorems, numbered equations, items in a list, etc., appear in parantheses, for example, section (1), subsection (1.1), theorem (3.16), equation (3.16.3) which will be called (3) within (3.16) and its proof, property (3.22.1).

This book grew out of lectures which I have given during the last three decades on advanced differential geometry, Lie groups and their actions, Riemann geometry, and symplectic geometry. I have benefited a lot from the advise of colleagues and remarks by readers and students. In particular I want to thank Konstanze Rietsch whose write-up of my lecture course on isometric group actions was very helpful in the preparation of this book and Simon Hochgerner who helped with the last section.

Support by the Austrian FWF-projects P 4661, P 7724-PHY, P 10037MAT, P 14195-MAT, and P 17108-N04 during the years $1983-2007$ is acknowledged.

Kritzendorf, April 2008 



\section{CHAPTER I. Manifolds and Vector Fields}

\section{Differentiable Manifolds}

1.1. Manifolds. A topological manifold is a separable metrizable space $M$ which is locally homeomorphic to $\mathbb{R}^{n}$. So for any $x \in M$ there is some homeomorphism $u: U \rightarrow u(U) \subseteq \mathbb{R}^{n}$, where $U$ is an open neighborhood of $x$ in $M$ and $u(U)$ is an open subset in $\mathbb{R}^{n}$. The pair $(U, u)$ is called a chart on $M$.

One of the basic results of algebraic topology, called 'invariance of domain', conjectured by Dedekind and proved by Brouwer in 1911, says that the number $n$ is locally constant on $M$; if $n$ is constant, $M$ is sometimes called a pure manifold. We will only consider pure manifolds and consequently we will omit the prefix pure.

A family $\left(U_{\alpha}, u_{\alpha}\right)_{\alpha \in A}$ of charts on $M$ such that the $U_{\alpha}$ form a cover of $M$ is called an atlas. The mappings

$$
u_{\alpha \beta}:=u_{\alpha} \circ u_{\beta}^{-1}: u_{\beta}\left(U_{\alpha \beta}\right) \rightarrow u_{\alpha}\left(U_{\alpha \beta}\right)
$$

are called the chart changings for the atlas $\left(U_{\alpha}\right)$, where we use the notation $U_{\alpha \beta}:=U_{\alpha} \cap U_{\beta}$.

An atlas $\left(U_{\alpha}, u_{\alpha}\right)_{\alpha \in A}$ for a manifold $M$ is said to be a $C^{k}$-atlas, if all chart changings $u_{\alpha \beta}: u_{\beta}\left(U_{\alpha \beta}\right) \rightarrow u_{\alpha}\left(U_{\alpha \beta}\right)$ are differentiable of class $C^{k}$. Two $C^{k}$-atlases are called $C^{k}$-equivalent if their union is again a $C^{k}$-atlas for $M$. An equivalence class of $C^{k}$-atlases is called a $C^{k}$-structure on $M$. 
From differential topology we know that if $M$ has a $C^{1}$-structure, then it also has a $C^{1}$-equivalent $C^{\infty}$-structure and even a $C^{1}$-equivalent $C^{\omega}$-structure, where $C^{\omega}$ is shorthand for real analytic; see $[\mathbf{8 4}$.

By a $C^{k}$-manifold $M$ we mean a topological manifold together with a $C^{k}$ structure and a chart on $M$ will be a chart belonging to some atlas of the $C^{k}$-structure.

But there are topological manifolds which do not admit differentiable structures. For example, every 4-dimensional manifold is smooth off some point, but there are such which are not smooth; see [196, 62. There are also topological manifolds which admit several inequivalent smooth structures. The spheres from dimension 7 on have finitely many; see [157]. But the most surprising result is that on $\mathbb{R}^{4}$ there are uncountably many pairwise inequivalent (exotic) differentiable structures. This follows from the results of $[42$ and 62 ; see $[78$ ] for an overview.

Note that for a Hausdorff $C^{\infty}$-manifold in a more general sense the following properties are equivalent:

(1) It is paracompact.

(2) It is metrizable.

(3) It admits a Riemann metric.

(4) Each connected component is separable.

In this book a manifold will usually mean a $C^{\infty}$-manifold, and smooth is used synonymously for $C^{\infty}$ - it will be Hausdorff, separable, finite-dimensional, to state it precisely.

Note finally that any manifold $M$ admits a finite atlas consisting of $\operatorname{dim} M+$ 1 (not connected) charts. This is a consequence of topological dimension theory [169]; a proof for manifolds may be found in $[\mathbf{8 0}, \mathrm{I}]$.

1.2. Example: Spheres. We consider the space $\mathbb{R}^{n+1}$, equipped with the standard inner product $\langle x, y\rangle=\sum x^{i} y^{i}$. The $n$-sphere $S^{n}$ is then the subset $\left\{x \in \mathbb{R}^{n+1}:\langle x, x\rangle=1\right\}$. Since $f(x)=\langle x, x\rangle, f: \mathbb{R}^{n+1} \rightarrow \mathbb{R}$, satisfies $d f(x) y=2\langle x, y\rangle$, it is of rank 1 off 0 and by (1.12) the sphere $S^{n}$ is a submanifold of $\mathbb{R}^{n+1}$.

In order to get some feeling for the sphere, we will describe an explicit atlas for $S^{n}$, the stereographic atlas. Choose $a \in S^{n}$ ('south pole'). Let

$$
\begin{array}{lll}
U_{+}:=S^{n} \backslash\{a\}, & u_{+}: U_{+} \rightarrow\{a\}^{\perp}, & u_{+}(x)=\frac{x-\langle x, a\rangle a}{1-\langle x, a\rangle}, \\
U_{-}:=S^{n} \backslash\{-a\}, & u_{-}: U_{-} \rightarrow\{a\}^{\perp}, & u_{-}(x)=\frac{x-\langle x, a\rangle a}{1+\langle x, a\rangle} .
\end{array}
$$


From the following drawing in the 2-plane through $0, x$, and $a$ it is easily seen that $u_{+}$is the usual stereographic projection. We also get

$$
u_{+}^{-1}(y)=\frac{|y|^{2}-1}{|y|^{2}+1} a+\frac{2}{|y|^{2}+1} y \quad \text { for } y \in\{a\}^{\perp} \backslash\{0\}
$$

and $\left(u_{-} \circ u_{+}^{-1}\right)(y)=\frac{y}{|y|^{2}}$. The latter equation can directly be seen from the drawing using the intercept theorem.

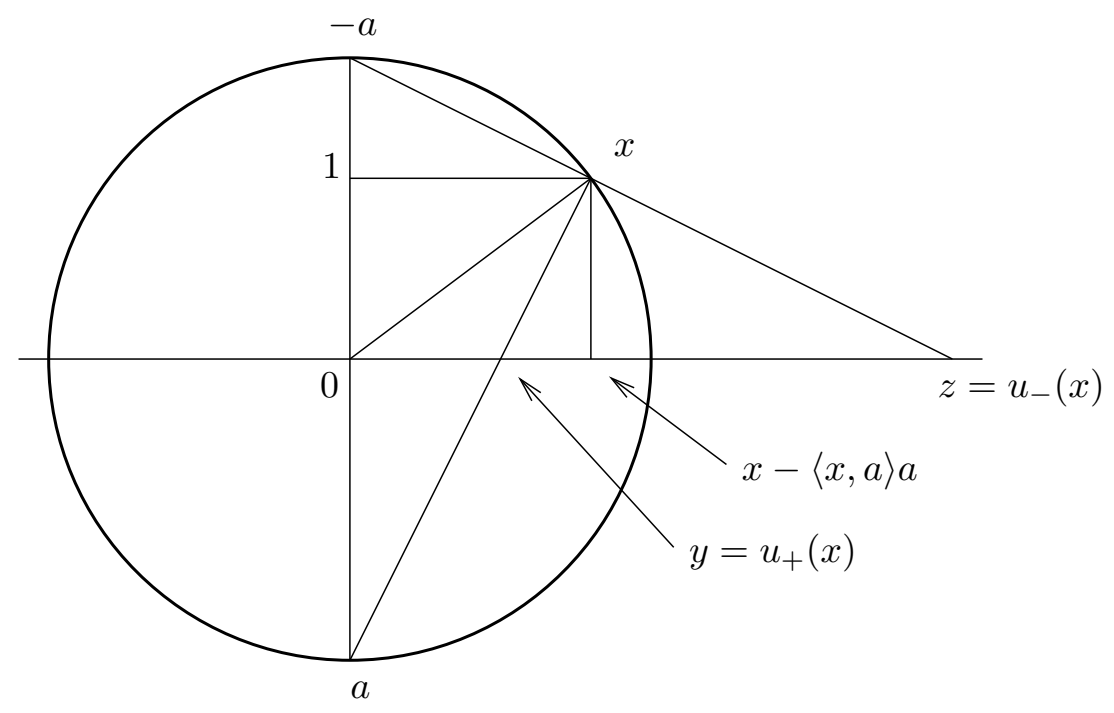

1.3. Smooth mappings. A mapping $f: M \rightarrow N$ between manifolds is said to be $C^{k}$ if for each $x \in M$ and one (equivalently: any) chart $(V, v)$ on $N$ with $f(x) \in V$ there is a chart $(U, u)$ on $M$ with $x \in U, f(U) \subseteq V$, and $v \circ f \circ u^{-1}$ is $C^{k}$. We will denote by $C^{k}(M, N)$ the space of all $C^{k}$-mappings from $M$ to $N$.

A $C^{k}$-mapping $f: M \rightarrow N$ is called a $C^{k}$-diffeomorphism if $f^{-1}: N \rightarrow M$ exists and is also $C^{k}$. Two manifolds are called diffeomorphic if there exists a diffeomorphism between them. From differential topology (see [84]) we know that if there is a $C^{1}$-diffeomorphism between $M$ and $N$, then there is also a $C^{\infty}$-diffeomorphism.

There are manifolds which are homeomorphic but not diffeomorphic: On $\mathbb{R}^{4}$ there are uncountably many pairwise nondiffeomorphic differentiable structures; on every other $\mathbb{R}^{n}$ the differentiable structure is unique. There are finitely many different differentiable structures on the spheres $S^{n}$ for $n \geq 7$. A mapping $f: M \rightarrow N$ between manifolds of the same dimension is called a local diffeomorphism if each $x \in M$ has an open neighborhood $U$ such that $f \mid U: U \rightarrow f(U) \subset N$ is a diffeomorphism. Note that a local diffeomorphism need not be surjective. 
1.4. Smooth functions. The set of smooth real valued functions on a manifold $M$ will be denoted by $C^{\infty}(M)$, in order to distinguish it clearly from spaces of sections which will appear later. The space $C^{\infty}(M)$ is a real commutative algebra.

The support of a smooth function $f$ is the closure of the set where it does not vanish, $\operatorname{supp}(f)=\overline{\{x \in M: f(x) \neq 0\}}$. The zero set of $f$ is the set where $f$ vanishes, $Z(f)=\{x \in M: f(x)=0\}$.

1.5. Theorem. Any (separable, metrizable, smooth) manifold admits smooth partitions of unity: Let $\left(U_{\alpha}\right)_{\alpha \in A}$ be an open cover of $M$.

Then there is a family $\left(\varphi_{\alpha}\right)_{\alpha \in A}$ of smooth functions on $M$, such that:

(1) $\varphi_{\alpha}(x) \geq 0$ for all $x \in M$ and all $\alpha \in A$.

(2) $\operatorname{supp}\left(\varphi_{\alpha}\right) \subset U_{\alpha}$ for all $\alpha \in A$.

(3) $\left(\operatorname{supp}\left(\varphi_{\alpha}\right)\right)_{\alpha \in A}$ is a locally finite family (so each $x \in M$ has an open neighborhood which meets only finitely many $\left.\operatorname{supp}\left(\varphi_{\alpha}\right)\right)$.

(4) $\sum_{\alpha} \varphi_{\alpha}=1$ (locally this is a finite sum).

Proof. Any (separable, metrizable) manifold is a 'Lindelöf space', i.e., each open cover admits a countable subcover. This can be seen as follows:

Let $\mathcal{U}$ be an open cover of $M$. Since $M$ is separable, there is a countable dense subset $S$ in $M$. Choose a metric on $M$. For each $U \in \mathcal{U}$ and each $x \in U$ there is a $y \in S$ and $n \in \mathbb{N}$ such that the ball $B_{1 / n}(y)$ with respect to that metric with center $y$ and radius $\frac{1}{n}$ contains $x$ and is contained in $U$. But there are only countably many of these balls; for each of them we choose an open set $U \in \mathcal{U}$ containing it. This is then a countable subcover of $\mathcal{U}$.

Now let $\left(U_{\alpha}\right)_{\alpha \in A}$ be the given cover. Let us fix first $\alpha$ and $x \in U_{\alpha}$. We choose a chart $(U, u)$ centered at $x$ (i.e., $u(x)=0$ ) and $\varepsilon>0$ such that $\varepsilon \mathbb{D}^{n} \subset u\left(U \cap U_{\alpha}\right)$, where $\mathbb{D}^{n}=\left\{y \in \mathbb{R}^{n}:|y| \leq 1\right\}$ is the closed unit ball. Let

$$
h(t):= \begin{cases}e^{-1 / t} & \text { for } t>0, \\ 0 & \text { for } t \leq 0,\end{cases}
$$

a smooth function on $\mathbb{R}$. Then

$$
f_{\alpha, x}(z):= \begin{cases}h\left(\varepsilon^{2}-|u(z)|^{2}\right) & \text { for } z \in U, \\ 0 & \text { for } z \notin U\end{cases}
$$

is a nonnegative smooth function on $M$ with support in $U_{\alpha}$ which is positive at $x$.

We choose such a function $f_{\alpha, x}$ for each $\alpha$ and $x \in U_{\alpha}$. The interiors of the supports of these smooth functions form an open cover of $M$ which refines 
$\left(U_{\alpha}\right)$, so by the argument at the beginning of the proof there is a countable subcover with corresponding functions $f_{1}, f_{2}, \ldots$ Let

$$
W_{n}=\left\{x \in M: f_{n}(x)>0 \text { and } f_{i}(x)<\frac{1}{n} \text { for } 1 \leq i<n\right\},
$$

and denote by $\bar{W}_{n}$ the closure. Then $\left(W_{n}\right)_{n}$ is an open cover. We claim that $\left(\bar{W}_{n}\right)_{n}$ is locally finite: Let $x \in M$. Then there is a smallest $n$ such that $x \in W_{n}$. Let $V:=\left\{y \in M: f_{n}(y)>\frac{1}{2} f_{n}(x)\right\}$. If $y \in V \cap \bar{W}_{k}$, then we have $f_{n}(y)>\frac{1}{2} f_{n}(x)$ and $f_{i}(y) \leq \frac{1}{k}$ for $i<k$, which is possible for finitely many $k$ only.

Consider the nonnegative smooth function

$$
g_{n}(x)=h\left(f_{n}(x)\right) h\left(\frac{1}{n}-f_{1}(x)\right) \ldots h\left(\frac{1}{n}-f_{n-1}(x)\right), \quad n \in \mathbb{N} .
$$

Then obviously $\operatorname{supp}\left(g_{n}\right)=\bar{W}_{n}$. So $g:=\sum_{n} g_{n}$ is smooth, since it is locally only a finite sum, and everywhere positive; thus $\left(g_{n} / g\right)_{n \in \mathbb{N}}$ is a smooth partition of unity on $M$. Since $\operatorname{supp}\left(g_{n}\right)=\bar{W}_{n}$ is contained in some $U_{\alpha(n)}$, we may put $\varphi_{\alpha}=\sum_{\{n: \alpha(n)=\alpha\}} \frac{g_{n}}{g}$ to get the required partition of unity which is subordinated to $\left(U_{\alpha}\right)_{\alpha \in A}$.

1.6. Germs. Let $M$ and $N$ be manifolds and $x \in M$. We consider all smooth mappings $f: U_{f} \rightarrow N$, where $U_{f}$ is some open neighborhood of $x$ in $M$, and we put $f \sim_{x} g$ if there is some open neighborhood $V$ of $x$ with $f|V=g| V$. This is an equivalence relation on the set of mappings considered. The equivalence class of a mapping $f$ is called the germ of $f$ at $x$, sometimes denoted by $\operatorname{germ}_{x} f$. The set of all these germs is denoted by $C_{x}^{\infty}(M, N)$.

Note that for a germs at $x$ of a smooth mapping only the value at $x$ is defined. We may also consider composition of germs: $\operatorname{germ}_{f(x)} g \circ \operatorname{germ}_{x} f:=$ $\operatorname{germ}_{x}(g \circ f)$.

If $N=\mathbb{R}$, we may add and multiply germs of smooth functions, so we get the real commutative algebra $C_{x}^{\infty}(M, \mathbb{R})$ of germs of smooth functions at $x$. This construction works also for other types of functions like real analytic or holomorphic ones if $M$ has a real analytic or complex structure.

Using smooth partitions of unity (1.4) it is easily seen that each germ of a smooth function has a representative which is defined on the whole of $M$. For germs of real analytic or holomorphic functions this is not true. So $C_{x}^{\infty}(M, \mathbb{R})$ is the quotient of the algebra $C^{\infty}(M)$ by the ideal of all smooth functions $f: M \rightarrow \mathbb{R}$ which vanish on some neighborhood (depending on $f$ ) of $x$.

1.7. The tangent space of $\mathbb{R}^{n}$. Let $a \in \mathbb{R}^{n}$. A tangent vector with foot point $a$ is simply a pair $(a, X)$ with $X \in \mathbb{R}^{n}$, also denoted by $X_{a}$. It induces a derivation $X_{a}: C^{\infty}\left(\mathbb{R}^{n}\right) \rightarrow \mathbb{R}$ by $X_{a}(f)=d f(a)\left(X_{a}\right)$. The value depends 
only on the germ of $f$ at $a$ and we have $X_{a}(f \cdot g)=X_{a}(f) \cdot g(a)+f(a) \cdot X_{a}(g)$ (the derivation property).

If conversely $D: C^{\infty}\left(\mathbb{R}^{n}\right) \rightarrow \mathbb{R}$ is linear and satisfies

$$
D(f \cdot g)=D(f) \cdot g(a)+f(a) \cdot D(g)
$$

(a derivation at $a$ ), then $D$ is given by the action of a tangent vector with foot point $a$. This can be seen as follows. For $f \in C^{\infty}\left(\mathbb{R}^{n}\right)$ we have

$$
\begin{aligned}
f(x) & =f(a)+\int_{0}^{1} \frac{d}{d t} f(a+t(x-a)) d t \\
& =f(a)+\sum_{i=1}^{n} \int_{0}^{1} \frac{\partial f}{\partial x^{i}}(a+t(x-a)) d t\left(x^{i}-a^{i}\right) \\
& =f(a)+\sum_{i=1}^{n} h_{i}(x)\left(x^{i}-a^{i}\right) .
\end{aligned}
$$

On the constant function 1 the derivation gives $D(1)=D(1 \cdot 1)=2 D(1)$, so $D($ constant $)=0$. Therefore,

$$
\begin{aligned}
D(f) & =D\left(f(a)+\sum_{i=1}^{n} h_{i}\left(x^{i}-a^{i}\right)\right) \\
& =0+\sum_{i=1}^{n} D\left(h_{i}\right)\left(a^{i}-a^{i}\right)+\sum_{i=1}^{n} h_{i}(a)\left(D\left(x^{i}\right)-0\right) \\
& =\sum_{i=1}^{n} \frac{\partial f}{\partial x^{i}}(a) D\left(x^{i}\right),
\end{aligned}
$$

where $x^{i}$ is the $i$-th coordinate function on $\mathbb{R}^{n}$. So we have

$$
D(f)=\left.\sum_{i=1}^{n} D\left(x^{i}\right) \frac{\partial}{\partial x^{i}}\right|_{a}(f), \quad D=\left.\sum_{i=1}^{n} D\left(x^{i}\right) \frac{\partial}{\partial x^{i}}\right|_{a} .
$$

Thus $D$ is induced by the tangent vector $\left(a, \sum_{i=1}^{n} D\left(x^{i}\right) e_{i}\right)$, where $\left(e_{i}\right)$ is the standard basis of $\mathbb{R}^{n}$.

1.8. The tangent space of a manifold. Let $M$ be a manifold and let $x \in M$ and $\operatorname{dim} M=n$. Let $T_{x} M$ be the vector space of all derivations at $x$ of $C_{x}^{\infty}(M, \mathbb{R})$, the algebra of germs of smooth functions on $M$ at $x$. Using $(1.5)$, it may easily be seen that a derivation of $C^{\infty}(M)$ at $x$ factors to a derivation of $C_{x}^{\infty}(M, \mathbb{R})$.

So $T_{x} M$ consists of all linear mappings $X_{x}: C^{\infty}(M) \rightarrow \mathbb{R}$ with the property $X_{x}(f \cdot g)=X_{x}(f) \cdot g(x)+f(x) \cdot X_{x}(g)$. The space $T_{x} M$ is called the tangent space of $M$ at $x$. 
If $(U, u)$ is a chart on $M$ with $x \in U$, then $u^{*}: f \mapsto f \circ u$ induces an isomorphism of algebras $C_{u(x)}^{\infty}\left(\mathbb{R}^{n}, \mathbb{R}\right) \cong C_{x}^{\infty}(M, \mathbb{R})$, and thus also an isomorphism $T_{x} u: T_{x} M \rightarrow T_{u(x)} \mathbb{R}^{n}$, given by $\left(T_{x} u . X_{x}\right)(f)=X_{x}(f \circ u)$. So $T_{x} M$ is an $n$-dimensional vector space.

We will use the following notation: $u=\left(u^{1}, \ldots, u^{n}\right)$, so $u^{i}$ denotes the $i$-th coordinate function on $U$, and

$$
\left.\frac{\partial}{\partial u^{i}}\right|_{x}:=\left(T_{x} u\right)^{-1}\left(\left.\frac{\partial}{\partial x^{i}}\right|_{u(x)}\right)=\left(T_{x} u\right)^{-1}\left(u(x), e_{i}\right) .
$$

So $\left.\frac{\partial}{\partial u^{i}}\right|_{x} \in T_{x} M$ is the derivation given by

$$
\left.\frac{\partial}{\partial u^{i}}\right|_{x}(f)=\frac{\partial\left(f \circ u^{-1}\right)}{\partial x^{i}}(u(x)) .
$$

From (1.7) we have now

$$
\begin{aligned}
T_{x} u \cdot X_{x} & =\left.\sum_{i=1}^{n}\left(T_{x} u \cdot X_{x}\right)\left(x^{i}\right) \frac{\partial}{\partial x^{i}}\right|_{u(x)}=\left.\sum_{i=1}^{n} X_{x}\left(x^{i} \circ u\right) \frac{\partial}{\partial x^{i}}\right|_{u(x)} \\
& =\left.\sum_{i=1}^{n} X_{x}\left(u^{i}\right) \frac{\partial}{\partial x^{i}}\right|_{u(x)}, \\
X_{x} & =\left(T_{x} u\right)^{-1} \cdot T_{x} u \cdot X_{x}=\left.\sum_{i=1}^{n} X_{x}\left(u^{i}\right) \frac{\partial}{\partial u^{i}}\right|_{x} .
\end{aligned}
$$

1.9. The tangent bundle. For a manifold $M$ of dimension $n$ we put $T M:=\bigsqcup_{x \in M} T_{x} M$, the disjoint union of all tangent spaces. This is a family of vector spaces parameterized by $M$, with projection $\pi_{M}: T M \rightarrow M$ given by $\pi_{M}\left(T_{x} M\right)=x$.

For any chart $\left(U_{\alpha}, u_{\alpha}\right)$ of $M$ consider the chart $\left(\pi_{M}^{-1}\left(U_{\alpha}\right), T u_{\alpha}\right)$ on $T M$, where $T u_{\alpha}: \pi_{M}^{-1}\left(U_{\alpha}\right) \rightarrow u_{\alpha}\left(U_{\alpha}\right) \times \mathbb{R}^{n}$ is given by

$$
T u_{\alpha} \cdot X=\left(u_{\alpha}\left(\pi_{M}(X)\right), T_{\pi_{M}(X)} u_{\alpha} \cdot X\right) .
$$

Then the chart changings look as follows:

$$
\begin{aligned}
T u_{\beta} \circ\left(T u_{\alpha}\right)^{-1}: & T u_{\alpha}\left(\pi_{M}^{-1}\left(U_{\alpha \beta}\right)\right)=u_{\alpha}\left(U_{\alpha \beta}\right) \times \mathbb{R}^{n} \rightarrow \\
& \rightarrow u_{\beta}\left(U_{\alpha \beta}\right) \times \mathbb{R}^{n}=T u_{\beta}\left(\pi_{M}^{-1}\left(U_{\alpha \beta}\right)\right), \\
\left(\left(T u_{\beta} \circ\left(T u_{\alpha}\right)^{-1}\right)(y, Y)\right)(f) & =\left(\left(T u_{\alpha}\right)^{-1}(y, Y)\right)\left(f \circ u_{\beta}\right) \\
& =(y, Y)\left(f \circ u_{\beta} \circ u_{\alpha}^{-1}\right)=d\left(f \circ u_{\beta} \circ u_{\alpha}^{-1}\right)(y) . Y \\
& =d f\left(u_{\beta} \circ u_{\alpha}^{-1}(y)\right) \cdot d\left(u_{\beta} \circ u_{\alpha}^{-1}\right)(y) . Y \\
& =\left(u_{\beta} \circ u_{\alpha}^{-1}(y), d\left(u_{\beta} \circ u_{\alpha}^{-1}\right)(y) . Y\right)(f) .
\end{aligned}
$$

So the chart changings are smooth. We choose the topology on $T M$ in such a way that all $T u_{\alpha}$ become homeomorphisms. This is a Hausdorff topology, since $X, Y \in T M$ may be separated in $M$ if $\pi(X) \neq \pi(Y)$; and they may be 
separated in one chart if $\pi(X)=\pi(Y)$. So $T M$ is again a smooth manifold in a canonical way; the triple $\left(T M, \pi_{M}, M\right)$ is called the tangent bundle of the manifold $M$.

1.10. Kinematic definition of the tangent space. Let $C_{0}^{\infty}(\mathbb{R}, M)$ denote the space of germs at 0 of smooth curves $\mathbb{R} \rightarrow M$. We put the following equivalence relation on $C_{0}^{\infty}(\mathbb{R}, M)$ : the germ of $c$ is equivalent to the germ of $e$ if and only if $c(0)=e(0)$ and in one (equivalently: each) chart $(U, u)$ with $c(0)=e(0) \in U$ we have $\left.\frac{d}{d t}\right|_{0}(u \circ c)(t)=\left.\frac{d}{d t}\right|_{0}(u \circ e)(t)$. The equivalence classes are also called velocity vectors of curves in $M$. We have the following diagram of mappings where $\alpha(c)\left(\operatorname{germ}_{c(0)} f\right)=\left.\frac{d}{d t}\right|_{0} f(c(t))$ and $\beta: T M \rightarrow C_{0}^{\infty}(\mathbb{R}, M)$ is given by: $\beta\left((T u)^{-1}(y, Y)\right)$ is the germ at 0 of $t \mapsto u^{-1}(y+t Y)$. So $T M$ is canonically identified with the set of all possible velocity vectors of curves in $M$ :

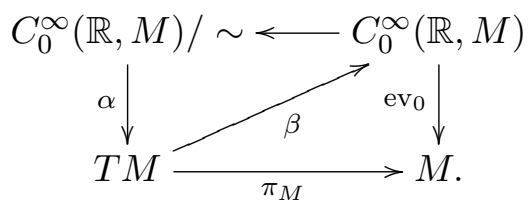

1.11. Tangent mappings. Let $f: M \rightarrow N$ be a smooth mapping between manifolds. Then $f$ induces a linear mapping $T_{x} f: T_{x} M \rightarrow T_{f(x)} N$ for each $x \in M$ by $\left(T_{x} f . X_{x}\right)(h)=X_{x}(h \circ f)$ for $h \in C_{f(x)}^{\infty}(N, \mathbb{R})$. This mapping is well defined and linear since $f^{*}: C_{f(x)}^{\infty}(N, \mathbb{R}) \rightarrow C_{x}^{\infty}(M, \mathbb{R})$, given by $h \mapsto h \circ f$, is linear and an algebra homomorphism, and $T_{x} f$ is its adjoint, restricted to the subspace of derivations.

If $(U, u)$ is a chart around $x$ and $(V, v)$ is one around $f(x)$, then

$$
\begin{aligned}
\left(\left.T_{x} f \cdot \frac{\partial}{\partial u^{i}}\right|_{x}\right)\left(v^{j}\right) & =\left.\frac{\partial}{\partial u^{i}}\right|_{x}\left(v^{j} \circ f\right)=\frac{\partial}{\partial x^{i}}\left(v^{j} \circ f \circ u^{-1}\right)(u(x)), \\
\left.T_{x} f \cdot \frac{\partial}{\partial u^{i}}\right|_{x} & =\left.\sum_{j}\left(\left.T_{x} f \cdot \frac{\partial}{\partial u^{i}}\right|_{x}\right)\left(v^{j}\right) \frac{\partial}{\partial v^{j}}\right|_{f(x)} \quad \text { by }(1.8) \\
& =\left.\sum_{j} \frac{\partial\left(v^{j} \circ f \circ u^{-1}\right)}{\partial x^{i}}(u(x)) \frac{\partial}{\partial v^{j}}\right|_{f(x)} .
\end{aligned}
$$

So the matrix of $T_{x} f: T_{x} M \rightarrow T_{f(x)} N$ in the bases $\left(\left.\frac{\partial}{\partial u^{i}}\right|_{x}\right)$ and $\left(\left.\frac{\partial}{\partial v^{j}}\right|_{f(x)}\right)$ is just the Jacobi matrix $d\left(v \circ f \circ u^{-1}\right)(u(x))$ of the mapping $v \circ f \circ u^{-1}$ at $u(x)$, so $T_{f(x)} v \circ T_{x} f \circ\left(T_{x} u\right)^{-1}=d\left(v \circ f \circ u^{-1}\right)(u(x))$.

Let us denote by $T f: T M \rightarrow T N$ the total mapping which is given by $T f \mid T_{x} M:=T_{x} f$. Then the composition

$$
\begin{gathered}
T v \circ T f \circ(T u)^{-1}: u(U) \times \mathbb{R}^{m} \rightarrow v(V) \times \mathbb{R}^{n}, \\
(y, Y) \mapsto\left(\left(v \circ f \circ u^{-1}\right)(y), d\left(v \circ f \circ u^{-1}\right)(y) Y\right),
\end{gathered}
$$

is smooth; thus $T f: T M \rightarrow T N$ is again smooth. 
If $f: M \rightarrow N$ and $g: N \rightarrow P$ are smooth, then we have $T(g \circ f)=T g \circ T f$. This is a direct consequence of $(g \circ f)^{*}=f^{*} \circ g^{*}$, and it is the global version of the chain rule. Furthermore we have $T\left(I d_{M}\right)=I d_{T M}$.

If $f \in C^{\infty}(M)$, then $T f: T M \rightarrow T \mathbb{R}=\mathbb{R} \times \mathbb{R}$. We define the differential of $f$ by $d f:=\operatorname{pr}_{2} \circ T f: T M \rightarrow \mathbb{R}$. Let $t$ denote the identity function on $\mathbb{R}$. Then $\left(T f . X_{x}\right)(t)=X_{x}(t \circ f)=X_{x}(f)$, so we have $d f\left(X_{x}\right)=X_{x}(f)$.

1.12. Submanifolds. A subset $N$ of a manifold $M$ is called a submanifold if for each $x \in N$ there is a chart $(U, u)$ of $M$ such that $u(U \cap N)=$ $u(U) \cap\left(\mathbb{R}^{k} \times 0\right)$, where $\mathbb{R}^{k} \times 0 \hookrightarrow \mathbb{R}^{k} \times \mathbb{R}^{n-k}=\mathbb{R}^{n}$. Then clearly $N$ is itself a manifold with $(U \cap N, u \mid(U \cap N))$ as charts, where $(U, u)$ runs through all submanifold charts as above.

1.13. Let $f: \mathbb{R}^{n} \rightarrow \mathbb{R}^{q}$ be smooth. A point $x \in \mathbb{R}^{q}$ is called a regular value of $f$ if the rank of $f$ (more exactly: the rank of its derivative) is $q$ at each point $y$ of $f^{-1}(x)$. In this case, $f^{-1}(x)$ is a submanifold of $\mathbb{R}^{n}$ of dimension $n-q$ (or empty). This is an immediate consequence of the implicit function theorem, as follows: Let $x=0 \in \mathbb{R}^{q}$. Permute the coordinates $\left(x^{1}, \ldots, x^{n}\right)$ on $\mathbb{R}^{n}$ such that the Jacobi matrix

$$
d f(y)=\left(\left(\frac{\partial f^{i}}{\partial x^{j}}(y)\right)_{1 \leq j \leq q}^{1 \leq i \leq q} \mid\left(\frac{\partial f^{i}}{\partial x^{j}}(y)\right)_{q+1 \leq j \leq n}^{1 \leq i \leq q}\right)
$$

has the left hand part invertible. Then $u:=\left(f, \operatorname{pr}_{n-q}\right): \mathbb{R}^{n} \rightarrow \mathbb{R}^{q} \times \mathbb{R}^{n-q}$ has invertible differential at $y$, so $(U, u)$ is a chart at any $y \in f^{-1}(0)$, and we have $f \circ u^{-1}\left(z^{1}, \ldots, z^{n}\right)=\left(z^{1}, \ldots, z^{q}\right)$, so $u\left(f^{-1}(0)\right)=u(U) \cap\left(0 \times \mathbb{R}^{n-q}\right)$ as required.

Constant rank theorem ([41, I 10.3.1]). Let $f: W \rightarrow \mathbb{R}^{q}$ be a smooth mapping, where $W$ is an open subset of $\mathbb{R}^{n}$. If the derivative $d f(x)$ has constant rank $k$ for each $x \in W$, then for each $a \in W$ there are charts $(U, u)$ of $W$ centered at a and $(V, v)$ of $\mathbb{R}^{q}$ centered at $f(a)$ such that $v \circ f \circ u^{-1}$ : $u(U) \rightarrow v(V)$ has the following form:

$$
\left(x_{1}, \ldots, x_{n}\right) \mapsto\left(x_{1}, \ldots, x_{k}, 0, \ldots, 0\right) .
$$

So $f^{-1}(b)$ is a submanifold of $W$ of dimension $n-k$ for each $b \in f(W)$.

Proof. We will use the inverse function theorem several times. The derivative $d f(a)$ has rank $k \leq n, q$; without loss we may assume that the upper left $(k \times k)$-submatrix of $d f(a)$ is invertible. Moreover, let $a=0$ and $f(a)=0$.

We consider $u: W \rightarrow \mathbb{R}^{n}, u\left(x^{1}, \ldots, x^{n}\right):=\left(f^{1}(x), \ldots, f^{k}(x), x^{k+1}, \ldots, x^{n}\right)$. Then

$$
d u=\left(\begin{array}{cc}
\left(\frac{\partial f^{i}}{\partial z^{j}}\right)_{1 \leq j \leq k}^{1 \leq i \leq k} & \left(\frac{\partial f^{i}}{\partial z^{j}}\right)_{k+1 \leq j \leq n}^{1 \leq i \leq k} \\
0 & \mathbb{I}_{\mathbb{R}^{n-k}}
\end{array}\right)
$$


is invertible, so $u$ is a diffeomorphism $U_{1} \rightarrow U_{2}$ for suitable open neighborhoods of 0 in $\mathbb{R}^{n}$. Consider $g=f \circ u^{-1}: U_{2} \rightarrow \mathbb{R}^{q}$. Then we have

$$
\begin{aligned}
& g\left(z_{1}, \ldots, z_{n}\right)=\left(z_{1}, \ldots, z_{k}, g_{k+1}(z), \ldots, g_{q}(z)\right), \\
& d g(z)=\left(\begin{array}{cc}
\mathbb{I}_{\mathbb{R}^{k}} & 0 \\
* & \left(\frac{\partial g^{i}}{\partial z^{j}}\right)_{k+1 \leq j \leq n}^{k+1 \leq i \leq q}
\end{array}\right), \\
& \operatorname{rank}(d g(z))=\operatorname{rank}\left(d\left(f \circ u^{-1}\right)(z)\right) \\
& =\operatorname{rank}\left(d f\left(u^{-1}(z)\right) \cdot d u^{-1}(z)\right)=\operatorname{rank}(d f(z))=k .
\end{aligned}
$$

Therefore, $\quad \frac{\partial g^{i}}{\partial z^{j}}(z)=0 \quad$ for $k+1 \leq i \leq q$ and $k+1 \leq j \leq n$;

$$
g^{i}\left(z^{1}, \ldots, z^{n}\right)=g^{i}\left(z^{1}, \ldots, z^{k}, 0, \ldots, 0\right) \quad \text { for } k+1 \leq i \leq q .
$$

Let $v: U_{3} \rightarrow \mathbb{R}^{q}$, where $U_{3}=\left\{y \in \mathbb{R}^{q}:\left(y^{1}, \ldots, y^{k}, 0, \ldots, 0\right) \in U_{2} \subset \mathbb{R}^{n}\right\}$, be given by

$$
v\left(\begin{array}{c}
y^{1} \\
\vdots \\
y^{q}
\end{array}\right)=\left(\begin{array}{c}
y^{1} \\
\vdots \\
y^{k} \\
y^{k+1}-g^{k+1}\left(y^{1}, \ldots, y^{k}, 0, \ldots, 0\right) \\
\vdots \\
y^{q}-g^{q}\left(y^{1}, \ldots, y^{k}, 0, \ldots, 0\right)
\end{array}\right)=\left(\begin{array}{c}
y^{1} \\
\vdots \\
y^{k} \\
y^{k+1}-g^{k+1}(\bar{y}) \\
\vdots \\
y^{q}-g^{q}(\bar{y})
\end{array}\right)
$$

where $\bar{y}=\left(y^{1}, \ldots, y^{q}, 0, \ldots, 0\right) \in \mathbb{R}^{n}$ if $q<n$ and $\bar{y}=\left(y^{1}, \ldots, y^{n}\right)$ if $q \geq n$. We have $v(0)=0$, and

$$
d v=\left(\begin{array}{cc}
\mathbb{I}_{\mathbb{R}^{k}} & 0 \\
* & \mathbb{I}_{\mathbb{R}^{q}-k}
\end{array}\right)
$$

is invertible; thus $v: V \rightarrow \mathbb{R}^{q}$ is a chart for a suitable neighborhood of 0 . Now let $U:=f^{-1}(V) \cup U_{1}$. Then $v \circ f \circ u^{-1}=v \circ g: \mathbb{R}^{n} \supseteq u(U) \rightarrow v(V) \subseteq \mathbb{R}^{q}$ looks as follows:

$$
\left(\begin{array}{c}
x^{1} \\
\vdots \\
x^{n}
\end{array}\right) \stackrel{g}{\longrightarrow}\left(\begin{array}{c}
x^{1} \\
\vdots \\
x^{k} \\
g^{k+1}(x) \\
\vdots \\
g^{q}(x)
\end{array}\right) \stackrel{v}{\longrightarrow}\left(\begin{array}{c}
x^{1} \\
\vdots \\
x^{k} \\
g^{k+1}(x)-g^{k+1}(x) \\
\vdots \\
g^{q}(x)-g^{q}(x)
\end{array}\right)=\left(\begin{array}{c}
x^{1} \\
\vdots \\
x^{k} \\
0 \\
\vdots \\
0
\end{array}\right) .
$$

Corollary. Let $f: M \rightarrow N$ be $C^{\infty}$ with $T_{x} f$ of constant rank $k$ for all $x \in M$.

Then for each $b \in f(M)$ the set $f^{-1}(b) \subset M$ is a submanifold of $M$ of dimension $\operatorname{dim} M-k$. 
1.14. Products. Let $M$ and $N$ be smooth manifolds described by smooth atlases $\left(U_{\alpha}, u_{\alpha}\right)_{\alpha \in A}$ and $\left(V_{\beta}, v_{\beta}\right)_{\beta \in B}$, respectively. Then the family $\left(U_{\alpha} \times\right.$ $\left.V_{\beta}, u_{\alpha} \times v_{\beta}: U_{\alpha} \times V_{\beta} \rightarrow \mathbb{R}^{m} \times \mathbb{R}^{n}\right)_{(\alpha, \beta) \in A \times B}$ is a smooth atlas for the cartesian product $M \times N$. Clearly the projections

$$
M \stackrel{\mathrm{pr}_{1}}{\longleftarrow} M \times N \stackrel{\mathrm{pr}_{2}}{\longrightarrow} N
$$

are also smooth. The product $\left(M \times N, \mathrm{pr}_{1}, \mathrm{pr}_{2}\right)$ has the following universal property:

For any smooth manifold $P$ and smooth mappings $f: P \rightarrow M$ and $g: P \rightarrow N$ the mapping

$$
(f, g): P \rightarrow M \times N, \quad(f, g)(x)=(f(x), g(x)),
$$

is the unique smooth mapping with $\operatorname{pr}_{1} \circ(f, g)=f$ and $\operatorname{pr}_{2} \circ(f, g)=g$.

From the construction of the tangent bundle in (1.9) it is immediately clear that

$$
T M \stackrel{T\left(\mathrm{pr}_{1}\right)}{\longleftarrow} T(M \times N) \stackrel{T\left(\mathrm{pr}_{2}\right)}{\longrightarrow} T N
$$

is again a product, so that $T(M \times N)=T M \times T N$ in a canonical way.

Clearly we can form products of finitely many manifolds.

1.15. Theorem. Let $M$ be a connected manifold and suppose that $f: M \rightarrow$ $M$ is smooth with $f \circ f=f$. Then the image $f(M)$ of $f$ is a submanifold of $M$.

This result can also be expressed as: 'smooth retracts' of manifolds are manifolds. If we do not suppose that $M$ is connected, then $f(M)$ will not be a pure manifold in general; it will have different dimensions in different connected components.

Proof. We claim that there is an open neighborhood $U$ of $f(M)$ in $M$ such that the rank of $T_{y} f$ is constant for $y \in U$. Then by theorem (1.13) the result follows.

For $x \in f(M)$ we have $T_{x} f \circ T_{x} f=T_{x} f$; thus $\operatorname{im} T_{x} f=\operatorname{ker}\left(I d-T_{x} f\right)$ and $\operatorname{rank} T_{x} f+\operatorname{rank}\left(I d-T_{x} f\right)=\operatorname{dim} M$. Since $\operatorname{rank} T_{x} f$ and $\operatorname{rank}\left(I d-T_{x} f\right)$ cannot fall locally, $\operatorname{rank} T_{x} f$ is locally constant for $x \in f(M)$, and since $f(M)$ is connected, $\operatorname{rank} T_{x} f=r$ for all $x \in f(M)$.

But then for each $x \in f(M)$ there is an open neighborhood $U_{x}$ in $M$ with $\operatorname{rank} T_{y} f \geq r$ for all $y \in U_{x}$. On the other hand

$$
\operatorname{rank} T_{y} f=\operatorname{rank} T_{y}(f \circ f)=\operatorname{rank} T_{f(y)} f \circ T_{y} f \leq \operatorname{rank} T_{f(y)} f=r
$$

since $f(y) \in f(M)$.

So the neighborhood we need is given by $U=\bigcup_{x \in f(M)} U_{x}$. 
1.16. Corollary. (1) The (separable) connected smooth manifolds are exactly the smooth retracts of connected open subsets of $\mathbb{R}^{n}$ 's.

(2) A smooth mapping $f: M \rightarrow N$ is an embedding of a submanifold if and only if there is an open neighborhood $U$ of $f(M)$ in $N$ and a smooth mapping $r: U \rightarrow M$ with $r \circ f=I d_{M}$.

Proof. Any manifold $M$ may be embedded into some $\mathbb{R}^{n}$; see (1.19) below. Then there exists a tubular neighborhood of $M$ in $R^{n}$ (see later or [84, pp. 109-118]), and $M$ is clearly a retract of such a tubular neighborhood. The converse follows from (1.15).

For the second assertion we repeat the argument for $N$ instead of $\mathbb{R}^{n}$.

1.17. Sets of Lebesque measure 0 in manifolds. An $m$-cube of width $w>0$ in $\mathbb{R}^{m}$ is a set of the form $C=\left[x_{1}, x_{1}+w\right] \times \ldots \times\left[x_{m}, x_{m}+w\right]$. The measure $\mu(C)$ is then $\mu(C)=w^{n}$. A subset $S \subset \mathbb{R}^{m}$ is called a set of (Lebesque) measure 0 if for each $\varepsilon>0$ these are at most countably many $m$-cubes $C_{i}$ with $S \subset \bigcup_{i=0}^{\infty} C_{i}$ and $\sum_{i=0}^{\infty} \mu\left(C_{i}\right)<\varepsilon$. Obviously, a countable union of sets of Lebesque measure 0 is again of measure 0 .

Lemma. Let $U \subset \mathbb{R}^{m}$ be open and let $f: U \rightarrow \mathbb{R}^{m}$ be $C^{1}$. If $S \subset U$ is of measure 0 , then also $f(S) \subset \mathbb{R}^{m}$ is of measure 0 .

Proof. Every point of $S$ belongs to an open ball $B \subset U$ such that the operator norm $\|d f(x)\| \leq K_{B}$ for all $x \in B$. Then $|f(x)-f(y)| \leq K_{B}|x-y|$ for all $x, y \in B$. So if $C \subset B$ is an $m$-cube of width $w$, then $f(C)$ is contained in an $m$-cube $C^{\prime}$ of width $\sqrt{m} K_{B} w$ and measure $\mu\left(C^{\prime}\right) \leq m^{m / 2} K_{B}^{m} \mu(C)$. Now let $S=\bigcup_{j=1}^{\infty} S_{j}$ where each $S_{j}$ is a subset of a ball $B_{j}$ as above. It suffices to show that each $f\left(S_{j}\right)$ is of measure 0 .

For each $\varepsilon>0$ there are $m$-cubes $C_{i}$ in $B_{j}$ with $S_{j} \subset \bigcup_{i} C_{i}$ and $\sum_{i} \mu\left(C_{i}\right)<\varepsilon$. As we saw above, then $f\left(S_{j}\right) \subset \bigcup_{i} C_{i}^{\prime}$ with $\sum_{i} \mu\left(C_{i}^{\prime}\right)<m^{m / 2} K_{B_{j}}^{m} \varepsilon$.

Let $M$ be a smooth (separable) manifold. A subset $S \subset M$ is called a set of (Lebesque) measure 0 if for each chart $(U, u)$ of $M$ the set $u(S \cap U)$ is of measure 0 in $\mathbb{R}^{m}$. By the lemma it suffices that there is some atlas whose charts have this property. Obviously, a countable union of sets of measure 0 in a manifold is again of measure 0 .

An $m$-cube is not of measure 0 . Thus a subset of $\mathbb{R}^{m}$ of measure 0 does not contain any $m$-cube; hence its interior is empty. Thus a closed set of measure 0 in a manifold is nowhere dense. More generally, let $S$ be a subset of a manifold which is of measure 0 and $\sigma$-compact, i.e., a countable union of compact subsets. Then each of the latter is nowhere dense, so $S$ is nowhere dense by the Baire category theorem. The complement of $S$ is residual, i.e., it contains the intersection of a countable family of open dense subsets. 
The Baire theorem says that a residual subset of a complete metric space is dense.

1.18. Regular values. Let $f: M \rightarrow N$ be a smooth mapping between manifolds.

(1) A point $x \in M$ is called a singular point of $f$ if $T_{x} f$ is not surjective, and it is called a regular point of $f$ if $T_{x} f$ is surjective.

(2) A point $y \in N$ is called a regular value of $f$ if $T_{x} f$ is surjective for all $x \in f^{-1}(y)$. If not, $y$ is called a singular value. Note that any $y \in N \backslash f(M)$ is a regular value.

Theorem ([167], 197]). The set of all singular values of a $C^{k}$ mapping $f: M \rightarrow N$ is of Lebesgue measure 0 in $N$ if $k>\max \{0, \operatorname{dim}(M)-\operatorname{dim}(N)\}$.

So any smooth mapping has regular values.

Proof. We prove this only for smooth mappings. It is sufficient to prove this locally. Thus we consider a smooth mapping $f: U \rightarrow \mathbb{R}^{n}$ where $U \subset \mathbb{R}^{m}$ is open. If $n>m$, then the result follows from lemma (1.17) above (consider the set $U \times 0 \subset \mathbb{R}^{m} \times \mathbb{R}^{n-m}$ of measure 0$)$. Thus let $m \geq n$.

Let $\Sigma(f) \subset U$ denote the set of singular points of $f$. Let $f=\left(f^{1}, \ldots, f^{n}\right)$, and let $\Sigma(f)=\Sigma_{1} \cup \Sigma_{2} \cup \Sigma_{3}$ where:

$\Sigma_{1}$ is the set of singular points $x$ such that $\operatorname{Pf}(x)=0$ for all linear differential operators $P$ of order $\leq \frac{m}{n}$.

$\Sigma_{2}$ is the set of singular points $x$ such that $\operatorname{Pf}(x) \neq 0$ for some differential operator $P$ of order $\geq 2$.

$\Sigma_{3}$ is the set of singular points $x$ such that $\frac{\partial f^{i}}{x^{j}}(x)=0$ for some $i, j$.

We first show that $f\left(\Sigma_{1}\right)$ has measure 0 . Let $\nu=\left\lceil\frac{m}{n}+1\right\rceil$ be the smallest integer $>m / n$. Then each point of $\Sigma_{1}$ has an open neighborhood $W \subset U$ such that $|f(x)-f(y)| \leq K|x-y|^{\nu}$ for all $x \in \Sigma_{1} \cap W$ and $y \in W$ and for some $K>0$, by Taylor expansion. We take $W$ to be a cube, of width $w$. It suffices to prove that $f\left(\Sigma_{1} \cap W\right)$ has measure 0 . We divide $W$ into $p^{m}$ cubes of width $\frac{w}{p}$; those which meet $\Sigma_{1}$ will be denoted by $C_{1}, \ldots, C_{q}$ for $q \leq p^{m}$. Each $C_{k}$ is contained in a ball of radius $\frac{w}{p} \sqrt{m}$ centered at a point of $\Sigma_{1} \cap W$. The set $f\left(C_{k}\right)$ is contained in a cube $C_{k}^{\prime} \subset \mathbb{R}^{n}$ of width $2 K\left(\frac{w}{p} \sqrt{m}\right)^{\nu}$. Then

$$
\sum_{k} \mu^{n}\left(C_{k}^{\prime}\right) \leq p^{m}(2 K)^{n}\left(\frac{w}{p} \sqrt{m}\right)^{\nu n}=p^{m-\nu n}(2 K)^{n} w^{\nu n} \rightarrow 0 \text { for } p \rightarrow \infty,
$$

since $m-\nu n<0$.

Note that $\Sigma(f)=\Sigma_{1}$ if $n=m=1$. So the theorem is proved in this case. We proceed by induction on $m$. So let $m>1$ and assume that the 
theorem is true for each smooth map between manifolds $M^{\prime} \rightarrow N^{\prime}$ where $\operatorname{dim}\left(M^{\prime}\right)<m$.

We prove that $f\left(\Sigma_{2} \backslash \Sigma_{3}\right)$ has measure 0. For each $x \in \Sigma_{2} \backslash \Sigma_{3}$ there is a linear differential operator $P$ such that $\operatorname{Pf}(x)=0$ and $\frac{\partial f^{i}}{\partial x^{j}}(x) \neq 0$ for some $i, j$. Let $W$ be the set of all such points, for fixed $P, i, j$. It suffices to show that $f(W)$ has measure 0 . By assumption, $0 \in \mathbb{R}$ is a regular value for the function $P f^{i}: W \rightarrow \mathbb{R}$. Therefore $W$ is a smooth submanifold of dimension $m-1$ in $\mathbb{R}^{m}$. Clearly, $\Sigma(f) \cap W$ is contained in the set of all singular points of $f \mid W: W \rightarrow \mathbb{R}^{n}$, and by induction we get that $f\left(\left(\Sigma_{2} \backslash \Sigma_{3}\right) \cap W\right) \subset f(\Sigma(f) \cap W) \subset f(\Sigma(f \mid W))$ has measure 0 .

It remains to prove that $f\left(\Sigma_{3}\right)$ has measure 0 . Every point of $\Sigma_{3}$ has an open neighborhood $W \subset U$ on which $\frac{\partial f^{i}}{\partial x^{j}} \neq 0$ for some $i, j$. By shrinking $W$ if necessary and applying diffeomorphisms, we may assume that

$$
\mathbb{R}^{m-1} \times \mathbb{R} \supseteq W_{1} \times W_{2}=W \stackrel{f}{\longrightarrow} \mathbb{R}^{n-1} \times \mathbb{R}, \quad(y, t) \mapsto(g(y, t), t) .
$$

Clearly, $(y, t)$ is a critical point for $f$ if and only if $y$ is a critical point for $g(, t)$. Thus $\Sigma(f) \cap W=\bigcup_{t \in W_{2}}(\Sigma(g(, t)) \times\{t\})$. Since $\operatorname{dim}\left(W_{1}\right)=m-1$, by induction we get that $\mu^{n-1}(g(\Sigma(g(, t), t)))=0$, where $\mu^{n-1}$ is the Lebesque measure in $\mathbb{R}^{n-1}$. By Fubini's theorem we get

$$
\begin{aligned}
\mu^{n}\left(\bigcup_{t \in W_{2}}(\Sigma(g(\quad, t)) \times\{t\})\right) & =\int_{W_{2}} \mu^{n-1}(g(\Sigma(g(\quad, t), t))) d t \\
& =\int_{W_{2}} 0 d t=0 . \quad \square
\end{aligned}
$$

1.19. Embeddings into $\mathbb{R}^{n}$ 's. Let $M$ be a smooth manifold of dimension $m$. Then $M$ can be embedded into $\mathbb{R}^{n}$ if

(1) $n=2 m+1$ (this is due to [229]; see also [84, p. 55] or [26, p. 73]).

(2) $n=2 m$ (see 229 ).

(3) Conjecture (still unproved): The minimal $n$ is $n=2 m-\alpha(m)+1$, where $\alpha(m)$ is the number of 1's in the dyadic expansion of $m$.

There exists an immersion (see section (2) $M \rightarrow \mathbb{R}^{n}$ if

(4) $n=2 m($ see $[\mathbf{8 4}])$.

(5) $n=2 m-1$ (see [229]).

(6) Conjecture: The minimal $n$ is $n=2 m-\alpha(m)$. The article [34] claims to have proven this. The proof is believed to be incomplete. 


\section{Examples and Exercises}

1.20. Discuss the following submanifolds of $\mathbb{R}^{n}$; in particular make drawings of them:

The unit sphere $S^{n-1}=\left\{x \in \mathbb{R}^{n}:\langle x, x\rangle=1\right\} \subset \mathbb{R}^{n}$.

The ellipsoid $\left\{x \in \mathbb{R}^{n}: f(x):=\sum_{i=1}^{n} \frac{x_{i}^{2}}{a_{i}^{2}}=1\right\}, a_{i} \neq 0$, with principal axis $a_{1}, \ldots, a_{n}$.

The hyperboloid $\left\{x \in \mathbb{R}^{n}: f(x):=\sum_{i=1}^{n} \varepsilon_{i} \frac{x_{i}^{2}}{a_{i}^{2}}=1\right\}, \varepsilon_{i}= \pm 1, a_{i} \neq 0$, with principal axis $a_{i}$ and index $=\sum \varepsilon_{i}$.

The saddle $\left\{x \in \mathbb{R}^{3}: x_{3}=x_{1} x_{2}\right\}$.

The torus: the rotation surface generated by rotation of $(y-R)^{2}+z^{2}=r^{2}$, $0<r<R$, with center the $z$-axis, i.e.,

$$
\left\{(x, y, z):\left(\sqrt{x^{2}+y^{2}}-R\right)^{2}+z^{2}=r^{2}\right\} .
$$

1.21. A compact surface of genus $g$. Let $f(x):=x(x-1)^{2}(x-$ $2)^{2} \ldots(x-(g-1))^{2}(x-g)$. For small $r>0$ the set $\left\{(x, y, z):\left(y^{2}+\right.\right.$ $\left.f(x))^{2}+z^{2}=r^{2}\right\}$ describes a surface of genus $g$ (topologically a sphere with $g$ handles) in $\mathbb{R}^{3}$. Visualize this:

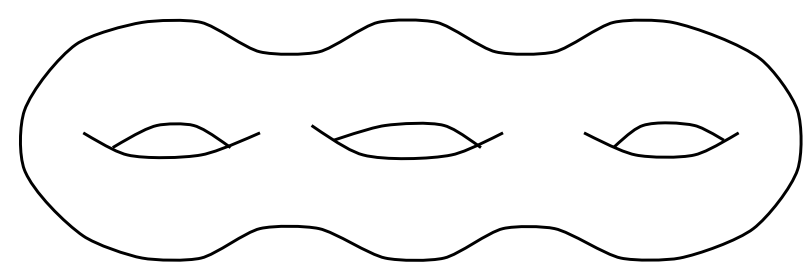

1.22. The Moebius strip. It is not the set of zeros of a regular function on an open neighborhood of $\mathbb{R}^{n}$. Why not? But it may be represented by the following parameterization:

$$
\begin{aligned}
f(r, \varphi) & :=\left(\begin{array}{c}
\cos \varphi(R+r \cos (\varphi / 2)) \\
\sin \varphi(R+r \cos (\varphi / 2)) \\
r \sin (\varphi / 2)
\end{array}\right), \\
(r, \varphi) & =(-1,1) \times[0,2 \pi),
\end{aligned}
$$

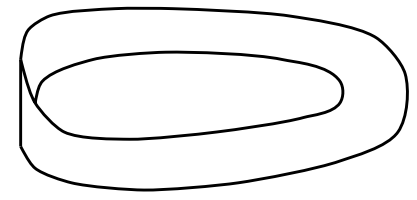

where $R$ is quite big.

1.23. Describe an atlas for the real projective plane which consists of three charts (homogeneous coordinates) and compute the chart changings.

Then describe an atlas for the $n$-dimensional real projective space $P^{n}(\mathbb{R})$ and compute the chart changes. 
1.24. Let $f: L\left(\mathbb{R}^{n}, \mathbb{R}^{n}\right) \rightarrow L\left(\mathbb{R}^{n}, \mathbb{R}^{n}\right)$ be given by $f(A):=A^{\top} A$. Where is $f$ of constant rank? What is $f^{-1}\left(\mathbb{I}_{n}\right)$ ?

1.25. Let $f: L\left(\mathbb{R}^{n}, \mathbb{R}^{m}\right) \rightarrow L\left(\mathbb{R}^{n}, \mathbb{R}^{n}\right), n<m$, be given by $f(A):=A^{\top} A$. Where is $f$ of constant rank? What is $f^{-1}\left(I d_{\mathbb{R}^{n}}\right)$ ?

1.26. Let $S$ be a symmetric matrix, i.e., $S(x, y):=x^{\top} S y$ is a symmetric bilinear form on $\mathbb{R}^{n}$. Let $f: L\left(\mathbb{R}^{n}, \mathbb{R}^{n}\right) \rightarrow L\left(\mathbb{R}^{n}, \mathbb{R}^{n}\right)$ be given by $f(A):=$ $A^{\top} S A$. Where is $f$ of constant rank? What is $f^{-1}(S)$ ?

1.27. Describe $T S^{2} \subset \mathbb{R}^{6}$.

\section{Submersions and Immersions}

2.1. Definition. A mapping $f: M \rightarrow N$ between manifolds is called a submersion at $x \in M$ if the rank of $T_{x} f: T_{x} M \rightarrow T_{f(x)} N$ equals $\operatorname{dim} N$. Since the rank cannot fall locally (the determinant of a submatrix of the Jacobi matrix is not 0$), f$ is then a submersion in a whole neighborhood of $x$. The mapping $f$ is said to be a submersion if it is a submersion at each $x \in M$.

2.2. Lemma. If $f: M \rightarrow N$ is a submersion at $x \in M$, then for any chart $(V, v)$ centered at $f(x)$ on $N$ there is chart $(U, u)$ centered at $x$ on $M$ such that $v \circ f \circ u^{-1}$ looks as follows:

$$
\left(y^{1}, \ldots, y^{n}, y^{n+1}, \ldots, y^{m}\right) \mapsto\left(y^{1}, \ldots, y^{n}\right)
$$

Proof. Use the inverse function theorem once: Apply the argument from the beginning of (1.13) to $v \circ f \circ u_{1}^{-1}$ for some chart $\left(U_{1}, u_{1}\right)$ centered at the point $x$.

2.3. Corollary. Any submersion $f: M \rightarrow N$ is open: For each open $U \subset M$ the set $f(U)$ is open in $N$.

2.4. Definition. A triple $(M, p, N)$, where $p: M \rightarrow N$ is a surjective submersion, is called a fibered manifold. The manifold $M$ is called the total space and $N$ is called the base.

A fibered manifold admits local sections: For each $x \in M$ there is an open neighborhood $U$ of $p(x)$ in $N$ and a smooth mapping $s: U \rightarrow M$ with $p \circ s=I d_{U}$ and $s(p(x))=x$. 
The existence of local sections in turn implies the following universal property:

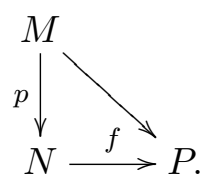

If $(M, p, N)$ is a fibered manifold and $f: N \rightarrow P$ is a mapping into some further manifold such that $f \circ p: M \rightarrow P$ is smooth, then $f$ is smooth.

2.5. Definition. A smooth mapping $f: M \rightarrow N$ is called an immersion at $x \in M$ if the rank of $T_{x} f: T_{x} M \rightarrow T_{f(x)} N$ equals $\operatorname{dim} M$. Since the rank is maximal at $x$ and cannot fall locally, $f$ is an immersion on a whole neighborhood of $x$. The mapping $f$ is called an immersion if it is so at every $x \in M$.

2.6. Lemma. If $f: M \rightarrow N$ is an immersion, then for any chart $(U, u)$ centered at $x \in M$ there is a chart $(V, v)$ centered at $f(x)$ on $N$ such that $v \circ f \circ u^{-1}$ has the form

$$
\left(y^{1}, \ldots, y^{m}\right) \mapsto\left(y^{1}, \ldots, y^{m}, 0, \ldots, 0\right) .
$$

Proof. Use the inverse function theorem.

2.7. Corollary. If $f: M \rightarrow N$ is an immersion, then for any $x \in M$ there is an open neighborhood $U$ of $x \in M$ such that $f(U)$ is a submanifold of $N$ and $f \mid U: U \rightarrow f(U)$ is a diffeomorphism.

2.8. Corollary. If an injective immersion $i: M \rightarrow N$ is a homeomorphism onto its image, then $i(M)$ is a submanifold of $N$.

Proof. Use (2.7).

2.9. Definition. If $i: M \rightarrow N$ is an injective immersion, then $(M, i)$ is called an immersed submanifold of $N$.

A submanifold is an immersed submanifold, but the converse is wrong in general. The structure of an immersed submanifold $(M, i)$ is in general not determined by the subset $i(M) \subset N$. All this is illustrated by the following example. Consider the curve $\gamma(t)=\left(\sin ^{3} t, \sin t \cdot \cos t\right)$ in $\mathbb{R}^{2}$. Then $((-\pi, \pi), \gamma \mid(-\pi, \pi))$ and $((0,2 \pi), \gamma \mid(0,2 \pi))$ are two different immersed submanifolds, but the image of the embedding is in both cases just the figure eight. 
2.10. Let $M$ be a submanifold of $N$. Then the embedding $i: M \rightarrow N$ is an injective immersion with the following property:

(1) For any manifold $Z$ a mapping $f: Z \rightarrow M$ is smooth if and only if $i \circ f: Z \rightarrow N$ is smooth.

There are injective immersions without property (1); see (2.9).

We want to determine all injective immersions $i: M \rightarrow N$ with property (11). To require that $i$ is a homeomorphism onto its image is too strong as (2.11) below shows. To look for all smooth mappings $i: M \rightarrow N$ with property (2.10.1) (initial mappings in categorical terms) is too difficult as remark (2.12) below shows.

2.11. Example. We consider the 2-dimensional torus $\mathbb{T}^{2}=\mathbb{R}^{2} / \mathbb{Z}^{2}$. Then the quotient mapping $\pi: \mathbb{R}^{2} \rightarrow \mathbb{T}^{2}$ is a covering map, so locally a diffeomorphism. Let us also consider the mapping $f: \mathbb{R} \rightarrow \mathbb{R}^{2}, f(t)=(t, \alpha . t)$, where $\alpha$ is irrational. Then $\pi \circ f: \mathbb{R} \rightarrow \mathbb{T}^{2}$ is an injective immersion with dense image, and it is obviously not a homeomorphism onto its image. But $\pi \circ f$ has property (2.10.1), which follows from the fact that $\pi$ is a covering map.

2.12. Remark. If $f: \mathbb{R} \rightarrow \mathbb{R}$ is a function such that the powers $f^{p}$ and $f^{q}$ are smooth for some $p, q$ which are relatively prime in $\mathbb{N}$, then $f$ itself turns out to be smooth; see [97. So the mapping $i: t \mapsto\left(\begin{array}{c}t^{p} \\ t^{q}\end{array}\right), \mathbb{R} \rightarrow \mathbb{R}^{2}$, has property (2.10.1), but $i$ is not an immersion at 0 .

In [98] all germs of mappings at 0 with property (2.10.1) are characterized as in the following way: Let $g:(\mathbb{R}, 0) \rightarrow\left(\mathbb{R}^{n}, 0\right)$ be a germ of a $C^{\infty}$-curve, $g(t)=\left(g_{1}(t), \ldots, g_{n}(t)\right)$. Without loss we may suppose that $g$ is not infinitely flat at 0 , so that $g_{1}(t)=t^{r}$ for $r \in \mathbb{N}$ after a suitable change of coordinates. Then $g$ has property (2.10.1) near 0 if and only if the Taylor series of $g$ is not contained in any $\mathbb{R}^{n}\left[\left[t^{s}\right]\right]$ for $s \geq 2$.

2.13. Definition. For an arbitrary subset $A$ of a manifold $N$ and $x_{0} \in A$ let $C_{x_{0}}(A)$ denote the set of all $x \in A$ which can be joined to $x_{0}$ by a smooth curve in $M$ lying in $A$.

A subset $M$ in a manifold $N$ is called an initial submanifold of dimension $m$ if the following property is true:

(1) For each $x \in M$ there exists a chart $(U, u)$ centered at $x$ on $N$ such that $u\left(C_{x}(U \cap M)\right)=u(U) \cap\left(\mathbb{R}^{m} \times 0\right)$.

The following three lemmas explain the name initial submanifold.

2.14. Lemma. Let $f: M \rightarrow N$ be an injective immersion between manifolds with the universal property $(2.10 .1)$. Then $f(M)$ is an initial submanifold of $N$. 
Proof. Let $x \in M$. By (2.6) we may choose a chart $(V, v)$ centered at $f(x)$ on $N$ and another chart $(W, w)$ centered at $x$ on $M$ such that

$$
\left(v \circ f \circ w^{-1}\right)\left(y^{1}, \ldots, y^{m}\right)=\left(y^{1}, \ldots, y^{m}, 0, \ldots, 0\right) .
$$

Let $r>0$ be small enough such that $\left\{y \in \mathbb{R}^{m}:|y|<2 r\right\} \subset w(W)$ and also $\left\{z \in \mathbb{R}^{n}:|z|<2 r\right\} \subset v(V)$. Put

$$
\begin{gathered}
U:=v^{-1}\left(\left\{z \in \mathbb{R}^{n}:|z|<r\right\}\right) \subset N, \\
W_{1}:=w^{-1}\left(\left\{y \in \mathbb{R}^{m}:|y|<r\right\}\right) \subset M .
\end{gathered}
$$

We claim that $(U, u=v \mid U)$ satisfies the condition of (2.13.1).

$$
\begin{aligned}
& u^{-1}\left(u(U) \cap\left(\mathbb{R}^{m} \times 0\right)\right)=u^{-1}\left(\left\{\left(y^{1}, \ldots, y^{m}, 0 \ldots, 0\right):|y|<r\right\}\right) \\
& \quad=f \circ w^{-1} \circ\left(u \circ f \circ w^{-1}\right)^{-1}\left(\left\{\left(y^{1}, \ldots, y^{m}, 0 \ldots, 0\right):|y|<r\right\}\right) \\
& \quad=f \circ w^{-1}\left(\left\{y \in \mathbb{R}^{m}:|y|<r\right\}\right)=f\left(W_{1}\right) \subseteq C_{f(x)}(U \cap f(M)),
\end{aligned}
$$

since $f\left(W_{1}\right) \subseteq U \cap f(M)$ and $f\left(W_{1}\right)$ is $C^{\infty}$-contractible.

Now let conversely $z \in C_{f(x)}(U \cap f(M))$. By definition there is a smooth curve $c:[0,1] \rightarrow N$ with $c(0)=f(x), c(1)=z$, and $c([0,1]) \subseteq U \cap f(M)$. By property (2.10.1) the unique curve $\bar{c}:[0,1] \rightarrow M$ with $f \circ \bar{c}=c$ is smooth.

We claim that $\bar{c}([0,1]) \subseteq W_{1}$. If not, then there is some $t \in[0,1]$ with $\bar{c}(t) \in w^{-1}\left(\left\{y \in \mathbb{R}^{m}: r \leq|y|<2 r\right\}\right)$ since $\bar{c}$ is smooth and thus continuous. But then we have

$$
\begin{aligned}
(v \circ f)(\bar{c}(t)) & \in\left(v \circ f \circ w^{-1}\right)\left(\left\{y \in \mathbb{R}^{m}: r \leq|y|<2 r\right\}\right) \\
= & \left\{(y, 0) \in \mathbb{R}^{m} \times 0: r \leq|y|<2 r\right\} \subseteq\left\{z \in \mathbb{R}^{n}: r \leq|z|<2 r\right\} .
\end{aligned}
$$

This means $(v \circ f \circ \bar{c})(t)=(v \circ c)(t) \in\left\{z \in \mathbb{R}^{n}: r \leq|z|<2 r\right\}$, so $c(t) \notin U$, a contradiction.

So $\bar{c}([0,1]) \subseteq W_{1}$; thus $\bar{c}(1)=f^{-1}(z) \in W_{1}$ and $z \in f\left(W_{1}\right)$. Consequently we have $C_{f(x)}(U \cap f(M))=f\left(W_{1}\right)$ and finally $f\left(W_{1}\right)=u^{-1}\left(u(U) \cap\left(\mathbb{R}^{m} \times 0\right)\right)$ by the first part of the proof.

2.15. Lemma. Let $M$ be an initial submanifold of a manifold $N$. Then there is a unique $C^{\infty}$-manifold structure on $M$ such that the injection $i$ : $M \rightarrow N$ is an injective immersion with property (2.10.1):

(1) For any manifold $Z$ a mapping $f: Z \rightarrow M$ is smooth if and only if $i \circ f: Z \rightarrow N$ is smooth.

The connected components of $M$ are separable (but there may be uncountably many of them).

Proof. We use the sets $C_{x}\left(U_{x} \cap M\right)$ as charts for $M$, where $x \in M$ and $\left(U_{x}, u_{x}\right)$ is a chart for $N$ centered at $x$ with the property required in (2.13.1). Then the chart changings are smooth since they are just restrictions of the 
chart changings on $N$. But the sets $C_{x}\left(U_{x} \cap M\right)$ are not open in the induced topology on $M$ in general. So the identification topology with respect to the charts $\left(C_{x}\left(U_{x} \cap M\right), u_{x}\right)_{x \in M}$ yields a topology on $M$ which is finer than the induced topology, so it is Hausdorff. Clearly $i: M \rightarrow N$ is then an injective immersion. Uniqueness of the smooth structure follows from the universal property (1) which we prove now: For $z \in Z$ we choose a chart $(U, u)$ on $N$, centered at $f(z)$, such that $u\left(C_{f(z)}(U \cap M)\right)=u(U) \cap\left(\mathbb{R}^{m} \times 0\right)$. Then $f^{-1}(U)$ is open in $Z$ and contains a chart $(V, v)$ centered at $z$ on $Z$ with $v(V)$ a ball. Then $f(V)$ is $C^{\infty}$-contractible in $U \cap M$, so $f(V) \subseteq C_{f(z)}(U \cap M)$, and $\left(u \mid C_{f(z)}(U \cap M)\right) \circ f \circ v^{-1}=u \circ f \circ v^{-1}$ is smooth.

Finally note that $N$ admits a Riemann metric (22.1) which induces one on $M$, so each connected component of $M$ is separable, by (1.1.4).

2.16. Transversal mappings. Let $M_{1}, M_{2}$, and $N$ be manifolds and let $f_{i}: M_{i} \rightarrow N$ be smooth mappings for $i=1,2$. We say that $f_{1}$ and $f_{2}$ are transversal at $y \in N$ if

$$
\operatorname{im} T_{x_{1}} f_{1}+\operatorname{im} T_{x_{2}} f_{2}=T_{y} N \quad \text { whenever } \quad f_{1}\left(x_{1}\right)=f_{2}\left(x_{2}\right)=y .
$$

Note that they are transversal at any $y$ which is not in $f_{1}\left(M_{1}\right)$ or not in $f_{2}\left(M_{2}\right)$. The mappings $f_{1}$ and $f_{2}$ are simply said to be transversal if they are transversal at every $y \in N$.

If $P$ is an initial submanifold of $N$ with embedding $i: P \rightarrow N$, then a mapping $f: M \rightarrow N$ is said to be transversal to $P$ if $i$ and $f$ are transversal.

Lemma. In this case $f^{-1}(P)$ is an initial submanifold of $M$ with the same codimension in $M$ as $P$ has in $N$; or $f^{-1}(P)$ is the empty set. If $P$ is a submanifold, then also $f^{-1}(P)$ is a submanifold.

Proof. Let $x \in f^{-1}(P)$ and let $(U, u)$ be an initial submanifold chart for $P$ centered at $f(x)$ on $N$, i.e., $u\left(C_{f(x)}(U \cap P)\right)=u(U) \cap\left(\mathbb{R}^{p} \times 0\right)$. Then the mapping

$$
M \supseteq f^{-1}(U) \stackrel{f}{\longrightarrow} U \stackrel{u}{\longrightarrow} u(U) \subseteq \mathbb{R}^{p} \times \mathbb{R}^{n-p} \stackrel{\mathrm{pr}_{2}}{\longrightarrow} \mathbb{R}^{n-p}
$$

is a submersion at $x$ since $f$ is transversal to $P$. So by lemma (2.2) there is a chart $(V, v)$ on $M$ centered at $x$ such that we have

$$
\left(\operatorname{pr}_{2} \circ u \circ f \circ v^{-1}\right)\left(y^{1}, \ldots, y^{n-p}, \ldots, y^{m}\right)=\left(y^{1}, \ldots, y^{n-p}\right) .
$$

But then $z \in C_{x}\left(f^{-1}(P) \cap V\right)$ if and only if $v(z) \in v(V) \cap\left(0 \times \mathbb{R}^{m-n+p}\right)$, so $v\left(C_{x}\left(f^{-1}(P) \cap V\right)\right)=v(V) \cap\left(0 \times \mathbb{R}^{m-n+p}\right)$. 
2.17. Corollary. If $f_{1}: M_{1} \rightarrow N$ and $f_{2}: M_{2} \rightarrow N$ are smooth and transversal, then the topological pullback

$$
M_{1} \underset{\left(f_{1}, N, f_{2}\right)}{\times} M_{2}=M_{1} \times_{N} M_{2}:=\left\{\left(x_{1}, x_{2}\right) \in M_{1} \times M_{2}: f_{1}\left(x_{1}\right)=f_{2}\left(x_{2}\right)\right\}
$$

is a submanifold of $M_{1} \times M_{2}$, and it has the following universal property:

For any smooth mappings $g_{1}: P \rightarrow M_{1}$ and $g_{2}: P \rightarrow M_{2}$ with $f_{1} \circ g_{1}=$ $f_{2} \circ g_{2}$ there is a unique smooth mapping $\left(g_{1}, g_{2}\right): P \rightarrow M_{1} \times_{N} M_{2}$ with $\operatorname{pr}_{1} \circ\left(g_{1}, g_{2}\right)=g_{1}$ and $\operatorname{pr}_{2} \circ\left(g_{1}, g_{2}\right)=g_{2}$.

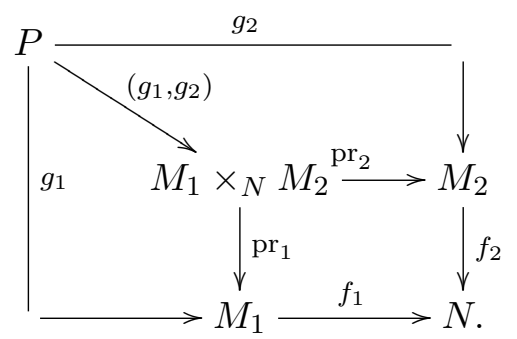

This is also called the pullback property in the category $\mathcal{M f}$ of smooth manifolds and smooth mappings. So one may say that transversal pullbacks exist in the category $\mathcal{M} f$. But there also exist pullbacks which are not transversal.

Proof. $M_{1} \times{ }_{N} M_{2}=\left(f_{1} \times f_{2}\right)^{-1}(\Delta)$, where $f_{1} \times f_{2}: M_{1} \times M_{2} \rightarrow N \times N$ and where $\Delta$ is the diagonal of $N \times N$, and $f_{1} \times f_{2}$ is transversal to $\Delta$ if and only if $f_{1}$ and $f_{2}$ are transversal.

\section{Vector Fields and Flows}

3.1. Definition. A vector field $X$ on a manifold $M$ is a smooth section of the tangent bundle; so $X: M \rightarrow T M$ is smooth and $\pi_{M} \circ X=I d_{M}$. A local vector field is a smooth section which is defined on an open subset only. We denote the set of all vector fields by $\mathfrak{X}(M)$. With pointwise addition and scalar multiplication $\mathfrak{X}(M)$ becomes a vector space.

Example. Let $(U, u)$ be a chart on $M$. Then the $\frac{\partial}{\partial u^{i}}: U \rightarrow T M \mid U, x \mapsto$ $\left.\frac{\partial}{\partial u^{i}}\right|_{x}$, described in (1.8), are local vector fields defined on $U$.

Lemma. If $X$ is a vector field on $M$ and $(U, u)$ is a chart on $M$ and $x \in U$, then we have $X(x)=\left.\sum_{i=1}^{m} X(x)\left(u^{i}\right) \frac{\partial}{\partial u^{i}}\right|_{x}$. We write $X \mid U=$ $\sum_{i=1}^{m} X\left(u^{i}\right) \frac{\partial}{\partial u^{i}}$. 
3.2. The vector fields $\left(\frac{\partial}{\partial u^{i}}\right)_{i=1}^{m}$ on $U$, where $(U, u)$ is a chart on $M$, form a holonomic frame field. By a frame field on some open set $V \subset M$ we mean $m=\operatorname{dim} M$ vector fields $s_{i} \in \mathfrak{X}(U)$ such that $s_{1}(x), \ldots, s_{m}(x)$ is a linear basis of $T_{x} M$ for each $x \in V$. A frame field is said to be holonomic if $s_{i}=\frac{\partial}{\partial v^{i}}$ for some chart $(V, v)$. If no such chart may be found locally, the frame field is called anholonomic.

With the help of partitions of unity and holonomic frame fields one may construct 'many' vector fields on $M$. In particular the values of a vector field can be arbitrarily preassigned on a discrete set $\left\{x_{i}\right\} \subset M$.

3.3. Lemma. The space $\mathfrak{X}(M)$ of vector fields on $M$ coincides canonically with the space of all derivations of the algebra $C^{\infty}(M)$ of smooth functions, i.e., those $\mathbb{R}$-linear operators $D: C^{\infty}(M) \rightarrow C^{\infty}(M)$ with

$$
D(f g)=D(f) g+f D(g) .
$$

Proof. Clearly each vector field $X \in \mathfrak{X}(M)$ defines a derivation (again called $X$; later sometimes called $\mathcal{L}_{X}$ ) of the algebra $C^{\infty}(M)$ by stipulating $X(f)(x):=X(x)(f)=d f(X(x))$.

If conversely a derivation $D$ of $C^{\infty}(M)$ is given, for any $x \in M$ we consider $D_{x}: C^{\infty}(M) \rightarrow \mathbb{R}, D_{x}(f)=D(f)(x)$. Then $D_{x}$ is a derivation at $x$ of $C^{\infty}(M)$ in the sense of (1.7), so $D_{x}=X_{x}$ for some $X_{x} \in T_{x} M$. In this way we get a section $X: M \rightarrow T M$. If $(U, u)$ is a chart on $M$, we have $D_{x}=\left.\sum_{i=1}^{m} X(x)\left(u^{i}\right) \frac{\partial}{\partial u^{i}}\right|_{x}$ by (1.7), Choose $V$ open in $M, V \subset \bar{V} \subset U$, and $\varphi \in C^{\infty}(M, \mathbb{R})$ such that $\operatorname{supp}(\varphi) \subset U$ and $\varphi \mid V=1$. Then $\varphi \cdot u^{i} \in C^{\infty}(M)$ and $\left(\varphi u^{i}\right)\left|V=u^{i}\right| V$. So $D\left(\varphi u^{i}\right)(x)=X(x)\left(\varphi u^{i}\right)=X(x)\left(u^{i}\right)$ and $X \mid V=$ $\sum_{i=1}^{m} D\left(\varphi u^{i}\right)\left|V \cdot \frac{\partial}{\partial u^{i}}\right| V$ is smooth.

3.4. The Lie bracket. By lemma (3.3) we can identify $\mathfrak{X}(M)$ with the vector space of all derivations of the algebra $C^{\infty}(M)$, which we will do without any notational change in the following.

If $X, Y$ are two vector fields on $M$, then the mapping $f \mapsto X(Y(f))-$ $Y(X(f))$ is again a derivation of $C^{\infty}(M)$, as a simple computation shows. Thus there is a unique vector field $[X, Y] \in \mathfrak{X}(M)$ such that $[X, Y](f)=$ $X(Y(f))-Y(X(f))$ holds for all $f \in C^{\infty}(M)$.

In a local chart $(U, u)$ on $M$ one easily checks that for $X \mid U=\sum X^{i} \frac{\partial}{\partial u^{i}}$ and $Y \mid U=\sum Y^{i} \frac{\partial}{\partial u^{i}}$ we have

$$
\begin{aligned}
{\left[\sum_{i} X^{i} \frac{\partial}{\partial u^{i}}, \sum_{j} Y^{j} \frac{\partial}{\partial u^{j}}\right] } & =\sum_{i, j}\left(X^{i}\left(\frac{\partial}{\partial u^{i}} Y^{j}\right)-Y^{i}\left(\frac{\partial}{\partial u^{i}} X^{j}\right)\right) \frac{\partial}{\partial u^{j}} \\
& =\sum_{j}\left(X\left(Y^{j}\right)-Y\left(X^{j}\right)\right) \frac{\partial}{\partial u^{j}}
\end{aligned}
$$


since second partial derivatives commute. The $\mathbb{R}$-bilinear mapping

$$
[\quad, \quad]: \mathfrak{X}(M) \times \mathfrak{X}(M) \rightarrow \mathfrak{X}(M)
$$

is called the Lie bracket. Note also that $\mathfrak{X}(M)$ is a module over the algebra $C^{\infty}(M)$ by pointwise multiplication $(f, X) \mapsto f X$.

Theorem. The Lie bracket [ , ] : $\mathfrak{X}(M) \times \mathfrak{X}(M) \rightarrow \mathfrak{X}(M)$ has the following properties:

$$
\begin{aligned}
& {[X, Y]=-[Y, X]} \\
& {[X,[Y, Z]]=[[X, Y], Z]+[Y,[X, Z]], \quad \text { the Jacobi identity, }} \\
& {[f X, Y]=f[X, Y]-(Y f) X} \\
& {[X, f Y]=f[X, Y]+(X f) Y .}
\end{aligned}
$$

The form of the Jacobi identity we have chosen says that $\operatorname{ad}(X)=[X, \quad]$ is a derivation for the Lie algebra $(\mathfrak{X}(M),[]$,$) . The pair (\mathfrak{X}(M),[]$, is the prototype of a Lie algebra. The concept of a Lie algebra is one of the most important notions of modern mathematics.

Proof. All these properties are checked easily for the commutator $[X, Y]=$ $X \circ Y-Y \circ X$ in the space of derivations of the algebra $C^{\infty}(M)$.

3.5. Integral curves. Let $c: J \rightarrow M$ be a smooth curve in a manifold $M$ defined on an interval $J$. We will use the following notations: $c^{\prime}(t)=\dot{c}(t)=$ $\frac{d}{d t} c(t):=T_{t} c .1$. Clearly $c^{\prime}: J \rightarrow T M$ is smooth. We call $c^{\prime}$ a vector field along $c$ since we have $\pi_{M} \circ c^{\prime}=c$ :

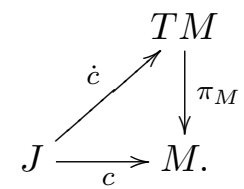

A smooth curve $c: J \rightarrow M$ will be called an integral curve or flow line of a vector field $X \in \mathfrak{X}(M)$ if $c^{\prime}(t)=X(c(t))$ holds for all $t \in J$.

3.6. Lemma. Let $X$ be a vector field on $M$. Then for any $x \in M$ there is an open interval $J_{x}$ containing 0 and an integral curve $c_{x}: J_{x} \rightarrow M$ for $X$ (i.e., $c_{x}^{\prime}=X \circ c_{x}$ ) with $c_{x}(0)=x$. If $J_{x}$ is maximal, then $c_{x}$ is unique.

Proof. In a chart $(U, u)$ on $M$ with $x \in U$ the equation $c^{\prime}(t)=X(c(t))$ is a system ordinary differential equations with initial condition $c(0)=x$. Since $X$ is smooth, there is a unique local solution which even depends smoothly on the initial values, by the theorem of Picard-Lindelöf, 41, 10.7.4]. So on $M$ there are always local integral curves. If $J_{x}=(a, b)$ and $\lim _{t \rightarrow b-} c_{x}(t)=$ : $c_{x}(b)$ exists in $M$, there is a unique local solution $c_{1}$ defined in an open interval containing $b$ with $c_{1}(b)=c_{x}(b)$. By uniqueness of the solution on 
the intersection of the two intervals, $c_{1}$ prolongs $c_{x}$ to a larger interval. This may be repeated (also on the left hand side of $J_{x}$ ) as long as the limit exists. So if we suppose $J_{x}$ to be maximal, $J_{x}$ either equals $\mathbb{R}$ or the integral curve leaves the manifold in finite (parameter-)time in the past or future or both.

3.7. The flow of a vector field. Let $X \in \mathfrak{X}(M)$ be a vector field. Let us write $\mathrm{Fl}_{t}^{X}(x)=\mathrm{Fl}^{X}(t, x):=c_{x}(t)$, where $c_{x}: J_{x} \rightarrow M$ is the maximally defined integral curve of $X$ with $c_{x}(0)=x$, constructed in lemma (3.6).

Theorem. For each vector field $X$ on $M$, the mapping $\mathrm{Fl}^{X}: \mathcal{D}(X) \rightarrow M$ is smooth, where $\mathcal{D}(X)=\bigcup_{x \in M} J_{x} \times\{x\}$ is an open neighborhood of $0 \times M$ in $\mathbb{R} \times M$. We have

$$
\mathrm{Fl}^{X}(t+s, x)=\mathrm{Fl}^{X}\left(t, \mathrm{Fl}^{X}(s, x)\right)
$$

in the following sense. If the right hand side exists, then the left hand side exists and we have equality. If both $t, s \geq 0$ or both are $\leq 0$, and if the left hand side exists, then also the right hand side exists and we have equality.

Proof. As mentioned in the proof of $(3.6), \mathrm{Fl}^{X}(t, x)$ is smooth in $(t, x)$ for small $t$, and if it is defined for $(t, x)$, then it is also defined for $(s, y)$ nearby. These are local properties which follow from the theory of ordinary differential equations.

Now let us treat the equation $\mathrm{Fl}^{X}(t+s, x)=\mathrm{Fl}^{X}\left(t, \mathrm{Fl}^{X}(s, x)\right)$. If the right hand side exists, then we consider the equation

$$
\left\{\begin{array}{l}
\frac{d}{d t} \mathrm{Fl}^{X}(t+s, x)=\left.\frac{d}{d u} \mathrm{Fl}^{X}(u, x)\right|_{u=t+s}=X\left(\mathrm{Fl}^{X}(t+s, x)\right), \\
\left.\mathrm{Fl}^{X}(t+s, x)\right|_{t=0}=\mathrm{Fl}^{X}(s, x) .
\end{array}\right.
$$

But the unique solution of this is $\mathrm{Fl}^{X}\left(t, \mathrm{Fl}^{X}(s, x)\right)$. So the left hand side exists and equals the right hand side.

If the left hand side exists, let us suppose that $t, s \geq 0$. We put

$$
c_{x}(u)= \begin{cases}\mathrm{Fl}^{X}(u, x) & \text { if } u \leq s, \\ \mathrm{Fl}^{X}\left(u-s, \mathrm{Fl}^{X}(s, x)\right) & \text { if } u \geq s .\end{cases}
$$

Then we have

$$
\begin{aligned}
\frac{d}{d u} c_{x}(u) & =\left\{\begin{array}{l}
\frac{d}{d u} \mathrm{Fl}^{X}(u, x)=X\left(\mathrm{Fl}^{X}(u, x)\right) \text { for } u \leq s, \\
\frac{d}{d u} \mathrm{Fl}^{X}\left(u-s, \mathrm{Fl}^{X}(s, x)\right)=X\left(\mathrm{Fl}^{X}\left(u-s, \mathrm{Fl}^{X}(s, x)\right)\right)
\end{array}\right. \\
& =X\left(c_{x}(u)\right) \text { for } 0 \leq u \leq t+s .
\end{aligned}
$$

Also $c_{x}(0)=x$ and on the overlap both definitions coincide by the first part of the proof; thus we conclude that $c_{x}(u)=\mathrm{Fl}^{X}(u, x)$ for $0 \leq u \leq t+s$ and we have $\mathrm{Fl}^{X}\left(t, \mathrm{Fl}^{X}(s, x)\right)=c_{x}(t+s)=\mathrm{Fl}^{X}(t+s, x)$. 
Now we show that $\mathcal{D}(X)$ is open and $\mathrm{Fl}^{X}$ is smooth on $\mathcal{D}(X)$. We know already that $\mathcal{D}(X)$ is a neighborhood of $0 \times M$ in $\mathbb{R} \times M$ and that $\mathrm{Fl}^{X}$ is smooth near $0 \times M$.

For $x \in M$ let $J_{x}^{\prime}$ be the set of all $t \in \mathbb{R}$ such that $\mathrm{Fl}^{X}$ is defined and smooth on an open neighborhood of $[0, t] \times\{x\}$ (respectively on $[t, 0] \times\{x\}$ for $t<0$ ) in $\mathbb{R} \times M$. We claim that $J_{x}^{\prime}=J_{x}$, which finishes the proof. It suffices to show that $J_{x}^{\prime}$ is not empty, open and closed in $J_{x}$. It is open by construction, and not empty, since $0 \in J_{x}^{\prime}$. If $J_{x}^{\prime}$ is not closed in $J_{x}$, let $t_{0} \in J_{x} \cap\left(\overline{J_{x}^{\prime}} \backslash J_{x}^{\prime}\right)$ and suppose that $t_{0}>0$, say. By the local existence and smoothness $\mathrm{Fl}^{X}$ exists and is smooth near $[-\varepsilon, \varepsilon] \times\left\{y:=\mathrm{Fl}^{X}\left(t_{0}, x\right)\right\}$ in $\mathbb{R} \times M$ for some $\varepsilon>0$, and by construction $\mathrm{Fl}^{X}$ exists and is smooth near $\left[0, t_{0}-\varepsilon\right] \times\{x\}$. Since $\mathrm{Fl}^{X}(-\varepsilon, y)=\mathrm{Fl}^{X}\left(t_{0}-\varepsilon, x\right)$, we conclude for $t$ near $\left[0, t_{0}-\varepsilon\right], x^{\prime}$ near $x$, and $t^{\prime}$ near $[-\varepsilon, \varepsilon]$ that $\mathrm{Fl}^{X}\left(t+t^{\prime}, x^{\prime}\right)=\mathrm{Fl}^{X}\left(t^{\prime}, \mathrm{Fl}^{X}\left(t, x^{\prime}\right)\right)$ exists and is smooth. So $t_{0} \in J_{x}^{\prime}$, a contradiction.

3.8. Let $X \in \mathfrak{X}(M)$ be a vector field. Its flow $\mathrm{Fl}^{X}$ is called global or complete if its domain of definition $\mathcal{D}(X)$ equals $\mathbb{R} \times M$. Then the vector field $X$ itself will be called a complete vector field. In this case $\mathrm{Fl}_{t}^{X}$ is also sometimes called $\exp t X$; it is a diffeomorphism of $M$. The support $\operatorname{supp}(X)$ of a vector field $X$ is the closure of the set $\{x \in M: X(x) \neq 0\}$.

Lemma. A vector field with compact support on $M$ is complete.

Proof. Let $K=\operatorname{supp}(X)$ be compact. Then the compact set $0 \times K$ has positive distance to the disjoint closed set $(\mathbb{R} \times M) \backslash \mathcal{D}(X)$ (if it is not empty), so $[-\varepsilon, \varepsilon] \times K \subset \mathcal{D}(X)$ for some $\varepsilon>0$. If $x \notin K$, then $X(x)=0$, so $\mathrm{Fl}^{X}(t, x)=x$ for all $t$ and $\mathbb{R} \times\{x\} \subset \mathcal{D}(X)$. So we have $[-\varepsilon, \varepsilon] \times M \subset \mathcal{D}(X)$. Since $\mathrm{Fl}^{X}(t+\varepsilon, x)=\mathrm{Fl}^{X}\left(t, \mathrm{Fl}^{X}(\varepsilon, x)\right)$ exists for $|t| \leq \varepsilon$ by theorem $(3.7)$, we have $[-2 \varepsilon, 2 \varepsilon] \times M \subset \mathcal{D}(X)$ and by repeating this argument we get $\mathbb{R} \times M=\mathcal{D}(X)$.

So on a compact manifold $M$ each vector field is complete. If $M$ is not compact and of dimension $\geq 2$, then in general the set of complete vector fields on $M$ is neither a vector space nor is it closed under the Lie bracket, as the following example on $\mathbb{R}^{2}$ shows: $X=y \frac{\partial}{\partial x}$ and $Y=\frac{x^{2}}{2} \frac{\partial}{\partial y}$ are complete, but neither $X+Y$ nor $[X, Y]$ is complete. In general one may embed $\mathbb{R}^{2}$ as a closed submanifold into $M$ and extend the vector fields $X$ and $Y$.

3.9. $f$-related vector fields. If $f: M \rightarrow M$ is a diffeomorphism, then for any vector field $X \in \mathfrak{X}(M)$ the mapping $T f^{-1} \circ X \circ f$ is also a vector field, which we will denote by $f^{*} X$. We also put $f_{*} X:=T f \circ X \circ f^{-1}=\left(f^{-1}\right)^{*} X$. But if $f: M \rightarrow N$ is a smooth mapping and $Y \in \mathfrak{X}(N)$ is a vector field, there may or may not exist a vector field $X \in \mathfrak{X}(M)$ such that the following 
diagram commutes:

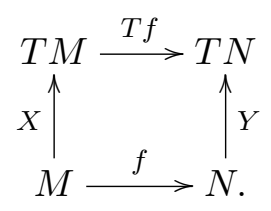

Definition. Let $f: M \rightarrow N$ be a smooth mapping. Two vector fields $X \in \mathfrak{X}(M)$ and $Y \in \mathfrak{X}(N)$ are called $f$-related if $T f \circ X=Y \circ f$ holds, i.e., if diagram (11) commutes.

Example. If $X \in \mathfrak{X}(M)$ and $Y \in \mathfrak{X}(N)$ and if $X \times Y \in \mathfrak{X}(M \times N)$ is given by $(X \times Y)(x, y)=(X(x), Y(y))$, then we have:

(2) $X \times Y$ and $X$ are $\mathrm{pr}_{1}$-related.

(3) $X \times Y$ and $Y$ are $\operatorname{pr}_{2}$-related.

(4) $X$ and $X \times Y$ are ins(y)-related if and only if $Y(y)=0$, where the mapping $\operatorname{ins}(y): M \rightarrow M \times N$ is given by $\operatorname{ins}(y)(x)=(x, y)$.

3.10. Lemma. Consider vector fields $X_{i} \in \mathfrak{X}(M)$ and $Y_{i} \in \mathfrak{X}(N)$ for $i=1,2$, and a smooth mapping $f: M \rightarrow N$. If $X_{i}$ and $Y_{i}$ are $f$-related for $i=1,2$, then also $\lambda_{1} X_{1}+\lambda_{2} X_{2}$ and $\lambda_{1} Y_{1}+\lambda_{2} Y_{2}$ are $f$-related, and also $\left[X_{1}, X_{2}\right]$ and $\left[Y_{1}, Y_{2}\right]$ are $f$-related.

Proof. The first assertion is immediate. To prove the second, we choose $h \in C^{\infty}(N)$. Then by assumption we have $T f \circ X_{i}=Y_{i} \circ f$; thus:

$$
\begin{aligned}
& \left(X_{i}(h \circ f)\right)(x)=X_{i}(x)(h \circ f)=\left(T_{x} f \cdot X_{i}(x)\right)(h) \\
& \quad=\left(T f \circ X_{i}\right)(x)(h)=\left(Y_{i} \circ f\right)(x)(h)=Y_{i}(f(x))(h)=\left(Y_{i}(h)\right)(f(x)),
\end{aligned}
$$

so $X_{i}(h \circ f)=\left(Y_{i}(h)\right) \circ f$, and we may continue:

$$
\begin{aligned}
{\left[X_{1}, X_{2}\right](h \circ f) } & =X_{1}\left(X_{2}(h \circ f)\right)-X_{2}\left(X_{1}(h \circ f)\right) \\
& =X_{1}\left(Y_{2}(h) \circ f\right)-X_{2}\left(Y_{1}(h) \circ f\right) \\
& =Y_{1}\left(Y_{2}(h)\right) \circ f-Y_{2}\left(Y_{1}(h)\right) \circ f=\left[Y_{1}, Y_{2}\right](h) \circ f .
\end{aligned}
$$

But this means $T f \circ\left[X_{1}, X_{2}\right]=\left[Y_{1}, Y_{2}\right] \circ f$.

3.11. Corollary. If $f: M \rightarrow N$ is a local diffeomorphism $\left(s o\left(T_{x} f\right)^{-1}\right.$ makes sense for each $x \in M)$, then for $Y \in \mathfrak{X}(N)$ a vector field $f^{*} Y \in$ $\mathfrak{X}(M)$ is defined by $\left(f^{*} Y\right)(x)=\left(T_{x} f\right)^{-1} . Y(f(x))$. The linear mapping $f^{*}$ : $\mathfrak{X}(N) \rightarrow \mathfrak{X}(M)$ is then a Lie algebra homomorphism, i.e.,

$$
f^{*}\left[Y_{1}, Y_{2}\right]=\left[f^{*} Y_{1}, f^{*} Y_{2}\right] .
$$


3.12. The Lie derivative of functions. For a vector field $X \in \mathfrak{X}(M)$ and $f \in C^{\infty}(M)$ we define $\mathcal{L}_{X} f \in C^{\infty}(M)$ by

$$
\begin{aligned}
\mathcal{L}_{X} f(x) & :=\left.\frac{d}{d t}\right|_{0} f\left(\mathrm{Fl}^{X}(t, x)\right) \quad \text { or } \\
\mathcal{L}_{X} f & :=\left.\frac{d}{d t}\right|_{0}\left(\mathrm{Fl}_{t}^{X}\right)^{*} f=\left.\frac{d}{d t}\right|_{0}\left(f \circ \mathrm{Fl}_{t}^{X}\right) .
\end{aligned}
$$

Since $\mathrm{Fl}^{X}(t, x)$ is defined for small $t$, for any $x \in M$, the expressions above make sense.

Lemma. We have

$$
\frac{d}{d t}\left(\mathrm{Fl}_{t}^{X}\right)^{*} f=\left(\mathrm{Fl}_{t}^{X}\right)^{*} X(f)=X\left(\left(\mathrm{Fl}_{t}^{X}\right)^{*} f\right)
$$

in particular for $t=0$ we have $\mathcal{L}_{X} f=X(f)=d f(X)$.

Proof. We have

$$
\frac{d}{d t}\left(\mathrm{Fl}_{t}^{X}\right)^{*} f(x)=d f\left(\frac{d}{d t} \mathrm{Fl}^{X}(t, x)\right)=d f\left(X\left(\mathrm{Fl}^{X}(t, x)\right)\right)=\left(\mathrm{Fl}_{t}^{X}\right)^{*}(X f)(x) .
$$

From this we get $\mathcal{L}_{X} f=X(f)=d f(X)$ and then in turn

$$
\frac{d}{d t}\left(\mathrm{Fl}_{t}^{X}\right)^{*} f=\left.\frac{d}{d s}\right|_{0}\left(\mathrm{Fl}_{t}^{X} \circ \mathrm{Fl}_{s}^{X}\right)^{*} f=\left.\frac{d}{d s}\right|_{0}\left(\mathrm{Fl}_{s}^{X}\right)^{*}\left(\mathrm{Fl}_{t}^{X}\right)^{*} f=X\left(\left(\mathrm{Fl}_{t}^{X}\right)^{*} f\right) .
$$

3.13. The Lie derivative for vector fields. For $X, Y \in \mathfrak{X}(M)$ we define $\mathcal{L}_{X} Y \in \mathfrak{X}(M)$ by

$$
\mathcal{L}_{X} Y:=\left.\frac{d}{d t}\right|_{0}\left(\mathrm{Fl}_{t}^{X}\right)^{*} Y=\left.\frac{d}{d t}\right|_{0}\left(T\left(\mathrm{Fl}_{-t}^{X}\right) \circ Y \circ \mathrm{Fl}_{t}^{X}\right),
$$

and call it the Lie derivative of $Y$ along $X$.

Lemma. We have

$$
\begin{gathered}
\mathcal{L}_{X} Y=[X, Y] \\
\frac{d}{d t}\left(\mathrm{Fl}_{t}^{X}\right)^{*} Y=\left(\mathrm{Fl}_{t}^{X}\right)^{*} \mathcal{L}_{X} Y=\left(\mathrm{Fl}_{t}^{X}\right)^{*}[X, Y]=\mathcal{L}_{X}\left(\mathrm{Fl}_{t}^{X}\right)^{*} Y=\left[X,\left(\mathrm{Fl}_{t}^{X}\right)^{*} Y\right]
\end{gathered}
$$

Proof. For $f \in C^{\infty}(M)$ consider the mapping $\alpha(t, s):=Y\left(\mathrm{Fl}^{X}(t, x)\right)(f \circ$ $\mathrm{Fl}_{s}^{X}$ ), which is locally defined near 0 . It satisfies

$$
\begin{aligned}
\alpha(t, 0) & =Y\left(\mathrm{Fl}^{X}(t, x)\right)(f), \\
\alpha(0, s) & =Y(x)\left(f \circ \mathrm{Fl}_{s}^{X}\right), \\
\frac{\partial}{\partial t} \alpha(0,0) & =\left.\partial\right|_{0} Y\left(\mathrm{Fl}^{X}(t, x)\right)(f)=\left.\partial\right|_{0}(Y f)\left(\mathrm{Fl}^{X}(t, x)\right)=X(x)(Y f), \\
\frac{\partial}{\partial s} \alpha(0,0) & =\left.\frac{\partial}{\partial s}\right|_{0} Y(x)\left(f \circ \mathrm{Fl}_{s}^{X}\right)=\left.Y(x) \frac{\partial}{\partial s}\right|_{0}\left(f \circ \mathrm{Fl}_{s}^{X}\right)=Y(x)(X f) .
\end{aligned}
$$

But on the other hand we have

$$
\begin{aligned}
\left.\frac{\partial}{\partial u}\right|_{0} \alpha(u,-u) & =\left.\frac{\partial}{\partial u}\right|_{0} Y\left(\mathrm{Fl}^{X}(u, x)\right)\left(f \circ \mathrm{Fl}_{-u}^{X}\right) \\
& =\left.\frac{\partial}{\partial u}\right|_{0}\left(T\left(\mathrm{Fl}_{-u}^{X}\right) \circ Y \circ \mathrm{Fl}_{u}^{X}\right)_{x}(f)=\left(\mathcal{L}_{X} Y\right)_{x}(f),
\end{aligned}
$$


so the first assertion follows. For the second claim we compute as follows:

$$
\begin{aligned}
\frac{\partial}{\partial t}\left(\mathrm{Fl}_{t}^{X}\right)^{*} Y & =\left.\frac{\partial}{\partial s}\right|_{0}\left(T\left(\mathrm{Fl}_{-t}^{X}\right) \circ T\left(\mathrm{Fl}_{-s}^{X}\right) \circ Y \circ \mathrm{Fl}_{s}^{X} \circ \mathrm{Fl}_{t}^{X}\right) \\
& =\left.T\left(\mathrm{Fl}_{-t}^{X}\right) \circ \frac{\partial}{\partial s}\right|_{0}\left(T\left(\mathrm{Fl}_{-s}^{X}\right) \circ Y \circ \mathrm{Fl}_{s}^{X}\right) \circ \mathrm{Fl}_{t}^{X} \\
& =T\left(\mathrm{Fl}_{-t}^{X}\right) \circ[X, Y] \circ \mathrm{Fl}_{t}^{X}=\left(\mathrm{Fl}_{t}^{X}\right)^{*}[X, Y] \\
\frac{\partial}{\partial t}\left(\mathrm{Fl}_{t}^{X}\right)^{*} Y & =\left.\frac{\partial}{\partial s}\right|_{0}\left(\mathrm{Fl}_{s}^{X}\right)^{*}\left(\mathrm{Fl}_{t}^{X}\right)^{*} Y=\mathcal{L}_{X}\left(\mathrm{Fl}_{t}^{X}\right)^{*} Y .
\end{aligned}
$$

3.14. Lemma. Let $X \in \mathfrak{X}(M)$ and $Y \in \mathfrak{X}(N)$ be $f$-related vector fields for a smooth mapping $f: M \rightarrow N$. Then we have $\left(f \circ \mathrm{Fl}_{t}^{X}\right)(x)=\left(\mathrm{Fl}_{t}^{Y} \circ f\right)(x)$ whenever $\mathrm{Fl}_{t}^{X}(x)$ is defined. In particular, if $f$ is a diffeomorphism, we have $\mathrm{Fl}_{t}^{f^{*} Y}=f^{-1} \circ \mathrm{Fl}_{t}^{Y} \circ f$.

Proof. We have $\frac{d}{d t}\left(f \circ \mathrm{Fl}_{t}^{X}\right)(x)=\left(T f \circ \frac{d}{d t} \mathrm{Fl}_{t}^{X}\right)(x)=(T f \circ X)\left(\mathrm{Fl}^{X}(t, x)\right)=$ $\left(Y \circ f \circ \mathrm{Fl}_{t}^{X}\right)(x)$ and $f\left(\mathrm{Fl}^{X}(0, x)\right)=f(x)$. So $t \mapsto f\left(\mathrm{Fl}^{X}(t, x)\right)$ is an integral curve of the vector field $Y$ on $N$ with initial value $f(x)$, so we have $f\left(\mathrm{Fl}^{X}(t, x)\right)=\mathrm{Fl}^{Y}(t, f(x))$ or $f \circ \mathrm{Fl}_{t}^{X}=\mathrm{Fl}_{t}^{Y} \circ f$.

3.15. Corollary. Let $X, Y \in \mathfrak{X}(M)$. Then the following assertions are equivalent:

(1) $\mathcal{L}_{X} Y=[X, Y]=0$.

(2) $\left(\mathrm{Fl}_{t}^{X}\right)^{*} Y=Y$ wherever the felt hand side is defined.

(3) $\left(\mathrm{Fl}_{t}^{X} \circ \mathrm{Fl}_{s}^{Y}\right)(x)=\left(\mathrm{Fl}_{s}^{Y} \circ \mathrm{Fl}_{t}^{X}\right)(x)$ for all $(t, s, x)$ such that one side is defined even along $[0, t] \times[0, s]$ for $t, s>0$, similarly for other cases.

The open condition in (3) on $(t, s, x)$ is necessary; see [121, 9.19]: On $\mathbb{R}^{3} \backslash$ $\{z$-axis $\}$ the vector fields $X=\partial_{x}-\frac{y}{x^{2}+y^{2}} \partial_{z}$ and $Y=\partial_{y}+\frac{x}{x^{2}+y^{2}} \partial_{z}$ commute but their flows do not satisfy (3) for all $(t, s, p)$.

Proof. (1) $\Leftrightarrow(2)$ is immediate from lemma (3.13). To see (2) $\Leftrightarrow(3)$, we note that, locally under the open condition on $(t, s, x), \mathrm{Fl}_{t}^{X} \circ \mathrm{Fl}_{s}^{Y}=\mathrm{Fl}_{s}^{Y} \circ \mathrm{Fl}_{t}^{X}$ if and only if $\mathrm{Fl}_{s}^{Y}=\mathrm{Fl}_{-t}^{X} \circ \mathrm{Fl}_{s}^{Y} \circ \mathrm{Fl}_{t}^{X}=\mathrm{Fl}_{s}^{\left(\mathrm{Fl}_{t}^{X}\right)^{*} Y}$ by lemma (3.14) which is applicable since the integral curves exist; and this in turn is equivalent to $Y=\left(\mathrm{Fl}_{t}^{X}\right)^{*} Y$.

3.16. Theorem. Let $M$ be a manifold, let $\varphi^{i}: \mathbb{R} \times M \supset U_{\varphi^{i}} \rightarrow M$ be smooth mappings for $i=1, \ldots, k$ where each $U_{\varphi^{i}}$ is an open neighborhood of $\{0\} \times M$ in $\mathbb{R} \times M$, such that each $\varphi_{t}^{i}$ is a diffeomorphism on its domain, $\varphi_{0}^{i}=I d_{M}$, and $\left.\partial\right|_{0} \varphi_{t}^{i}=X_{i} \in \mathfrak{X}(M)$. We put $\left[\varphi^{i}, \varphi^{j}\right]_{t}=\left[\varphi_{t}^{i}, \varphi_{t}^{j}\right]:=\left(\varphi_{t}^{j}\right)^{-1} \circ$ $\left(\varphi_{t}^{i}\right)^{-1} \circ \varphi_{t}^{j} \circ \varphi_{t}^{i}$. Then for each formal bracket expression $P$ of length $k$ we have

$$
0=\left.\frac{\partial^{\ell}}{\partial t^{\ell}}\right|_{0} P\left(\varphi_{t}^{1}, \ldots, \varphi_{t}^{k}\right) \quad \text { for } 1 \leq \ell<k
$$




$$
P\left(X_{1}, \ldots, X_{k}\right)=\left.\frac{1}{k !} \frac{\partial^{k}}{\partial t^{k}}\right|_{0} P\left(\varphi_{t}^{1}, \ldots, \varphi_{t}^{k}\right) \in \mathfrak{X}(M)
$$

in the sense explained in step 2 of the proof. In particular we have for vector fields $X, Y \in \mathfrak{X}(M)$

$$
\begin{aligned}
0 & =\left.\partial\right|_{0}\left(\mathrm{Fl}_{-t}^{Y} \circ \mathrm{Fl}_{-t}^{X} \circ \mathrm{Fl}_{t}^{Y} \circ \mathrm{Fl}_{t}^{X}\right), \\
{[X, Y] } & =\left.\frac{1}{2} \frac{\partial^{2}}{\partial t^{2}}\right|_{0}\left(\mathrm{Fl}_{-t}^{Y} \circ \mathrm{Fl}_{-t}^{X} \circ \mathrm{Fl}_{t}^{Y} \circ \mathrm{Fl}_{t}^{X}\right) .
\end{aligned}
$$

Proof. Step 1. Let $c: \mathbb{R} \rightarrow M$ be a smooth curve. If $c(0)=x \in M$, $c^{\prime}(0)=0, \ldots, c^{(k-1)}(0)=0$, then $c^{(k)}(0)$ is a well defined tangent vector in $T_{x} M$ which is given by the derivation $f \mapsto(f \circ c)^{(k)}(0)$ at $x$. Namely, we have

$$
\begin{aligned}
((f . g) \circ c)^{(k)}(0) & =((f \circ c) \cdot(g \circ c))^{(k)}(0)=\sum_{j=0}^{k}\left(\begin{array}{l}
k \\
j
\end{array}\right)(f \circ c)^{(j)}(0)(g \circ c)^{(k-j)}(0) \\
& =(f \circ c)^{(k)}(0) g(x)+f(x)(g \circ c)^{(k)}(0),
\end{aligned}
$$

since all other summands vanish: $(f \circ c)^{(j)}(0)=0$ for $1 \leq j<k$.

Step 2. Let $\varphi: \mathbb{R} \times M \supset U_{\varphi} \rightarrow M$ be a smooth mapping where $U_{\varphi}$ is an open neighborhood of $\{0\} \times M$ in $\mathbb{R} \times M$, such that each $\varphi_{t}$ is a diffeomorphism on its domain and $\varphi_{0}=I d_{M}$. We say that $\varphi_{t}$ is a curve of local diffeomorphisms through $I d_{M}$.

From step 11 we see that if $\left.\frac{\partial^{j}}{\partial t^{j}}\right|_{0} \varphi_{t}=0$ for all $1 \leq j<k$, then $X:=\left.\frac{1}{k !} \frac{\partial^{k}}{\partial t^{k}}\right|_{0} \varphi_{t}$ is a well defined vector field on $M$. We say that $X$ is the first nonvanishing derivative at 0 of the curve $\varphi_{t}$ of local diffeomorphisms. We may paraphrase this as $\left(\left.\partial_{t}^{k}\right|_{0} \varphi_{t}^{*}\right) f=k ! \mathcal{L}_{X} f$.

Claim 3. Let $\varphi_{t}, \psi_{t}$ be curves of local diffeomorphisms through $I d_{M}$ and let $f \in C^{\infty}(M)$. Then we have

$$
\left.\partial_{t}^{k}\right|_{0}\left(\varphi_{t} \circ \psi_{t}\right)^{*} f=\left.\partial_{t}^{k}\right|_{0}\left(\psi_{t}^{*} \circ \varphi_{t}^{*}\right) f=\sum_{j=0}^{k}\left(\begin{array}{c}
k \\
j
\end{array}\right)\left(\left.\partial_{t}^{j}\right|_{0} \psi_{t}^{*}\right)\left(\left.\partial_{t}^{k-j}\right|_{0} \varphi_{t}^{*}\right) f .
$$

Also the multinomial version of this formula holds:

$$
\left.\partial_{t}^{k}\right|_{0}\left(\varphi_{t}^{1} \circ \ldots \circ \varphi_{t}^{\ell}\right)^{*} f=\sum_{j_{1}+\cdots+j_{\ell}=k} \frac{k !}{j_{1} ! \ldots j_{\ell} !}\left(\left.\partial_{t}^{j_{\ell}}\right|_{0}\left(\varphi_{t}^{\ell}\right)^{*}\right) \ldots\left(\left.\partial_{t}^{j_{1}}\right|_{0}\left(\varphi_{t}^{1}\right)^{*}\right) f .
$$

We only show the binomial version. For a function $h(t, s)$ of two variables we have

$$
\partial_{t}^{k} h(t, t)=\left.\sum_{j=0}^{k}\left(\begin{array}{c}
k \\
j
\end{array}\right) \partial_{t}^{j} \partial_{s}^{k-j} h(t, s)\right|_{s=t}
$$

since for $h(t, s)=f(t) g(s)$ this is just a consequence of the Leibniz rule, and linear combinations of such decomposable tensors are dense in the space of all 
functions of two variables in the compact $C^{\infty}$-topology, so that by continuity the formula holds for all functions. In the following form it implies the claim:

$$
\left.\partial_{t}^{k}\right|_{0} f(\varphi(t, \psi(t, x)))=\left.\sum_{j=0}^{k}\left(\begin{array}{l}
k \\
j
\end{array}\right) \partial_{t}^{j} \partial_{s}^{k-j} f(\varphi(t, \psi(s, x)))\right|_{t=s=0} .
$$

Claim 4. Let $\varphi_{t}$ be a curve of local diffeomorphisms through $I d_{M}$ with first nonvanishing derivative $k ! X=\left.\partial_{t}^{k}\right|_{0} \varphi_{t}$. Then the inverse curve of local diffeomorphisms $\varphi_{t}^{-1}$ has first nonvanishing derivative $-k ! X=\left.\partial_{t}^{k}\right|_{0} \varphi_{t}^{-1}$, for we have $\varphi_{t}^{-1} \circ \varphi_{t}=I d$, so by claim 3 we get for $1 \leq j \leq k$

$$
\begin{aligned}
0 & =\left.\partial_{t}^{j}\right|_{0}\left(\varphi_{t}^{-1} \circ \varphi_{t}\right)^{*} f=\sum_{i=0}^{j}\left(\begin{array}{c}
j \\
i
\end{array}\right)\left(\left.\partial_{t}^{i}\right|_{0} \varphi_{t}^{*}\right)\left(\partial_{t}^{j-i}\left(\varphi_{t}^{-1}\right)^{*}\right) f \\
& =\left.\partial_{t}^{j}\right|_{0} \varphi_{t}^{*}\left(\varphi_{0}^{-1}\right)^{*} f+\left.\varphi_{0}^{*} \partial_{t}^{j}\right|_{0}\left(\varphi_{t}^{-1}\right)^{*} f,
\end{aligned}
$$

i.e., $\left.\partial_{t}^{j}\right|_{0} \varphi_{t}^{*} f=-\left.\partial_{t}^{j}\right|_{0}\left(\varphi_{t}^{-1}\right)^{*} f$ as required.

Claim 5. Let $\varphi_{t}$ be a curve of local diffeomorphisms through $I d_{M}$ with first nonvanishing derivative $m ! X=\left.\partial_{t}^{m}\right|_{0} \varphi_{t}$, and let $\psi_{t}$ be a curve of local diffeomorphisms through $I d_{M}$ with first nonvanishing derivative $n ! Y=\left.\partial_{t}^{n}\right|_{0} \psi_{t}$.

Then the curve of local diffeomorphisms $\left[\varphi_{t}, \psi_{t}\right]=\psi_{t}^{-1} \circ \varphi_{t}^{-1} \circ \psi_{t} \circ \varphi_{t}$ has first nonvanishing derivative

$$
(m+n) ![X, Y]=\left.\partial_{t}^{m+n}\right|_{0}\left[\varphi_{t}, \psi_{t}\right] .
$$

From this claim the theorem follows.

By the multinomial version of claim 3 we have

$$
\begin{aligned}
A_{N} f: & =\left.\partial_{t}^{N}\right|_{0}\left(\psi_{t}^{-1} \circ \varphi_{t}^{-1} \circ \psi_{t} \circ \varphi_{t}\right)^{*} f \\
& =\sum_{i+j+k+\ell=N} \frac{N !}{i ! j ! k ! \ell !}\left(\left.\partial_{t}^{i}\right|_{0} \varphi_{t}^{*}\right)\left(\left.\partial_{t}^{j}\right|_{0} \psi_{t}^{*}\right)\left(\left.\partial_{t}^{k}\right|_{0}\left(\varphi_{t}^{-1}\right)^{*}\right)\left(\left.\partial_{t}^{\ell}\right|_{0}\left(\psi_{t}^{-1}\right)^{*}\right) f .
\end{aligned}
$$

Let us suppose that $1 \leq n \leq m$; the case $m \leq n$ is similar. If $N<n$, all summands are 0 . If $N=n$, we have by claim 4

$$
A_{N} f=\left(\left.\partial_{t}^{n}\right|_{0} \varphi_{t}^{*}\right) f+\left(\left.\partial_{t}^{n}\right|_{0} \psi_{t}^{*}\right) f+\left(\left.\partial_{t}^{n}\right|_{0}\left(\varphi_{t}^{-1}\right)^{*}\right) f+\left(\left.\partial_{t}^{n}\right|_{0}\left(\psi_{t}^{-1}\right)^{*}\right) f=0 .
$$

If $n<N \leq m$, we have, using again claim 4 :

$$
\begin{aligned}
A_{N} f & =\sum_{j+\ell=N} \frac{N !}{j ! \ell !}\left(\left.\partial_{t}^{j}\right|_{0} \psi_{t}^{*}\right)\left(\left.\partial_{t}^{\ell}\right|_{0}\left(\psi_{t}^{-1}\right)^{*}\right) f+\delta_{N}^{m}\left(\left(\left.\partial_{t}^{m}\right|_{0} \varphi_{t}^{*}\right) f+\left(\left.\partial_{t}^{m}\right|_{0}\left(\varphi_{t}^{-1}\right)^{*}\right) f\right) \\
& =\left(\left.\partial_{t}^{N}\right|_{0}\left(\psi_{t}^{-1} \circ \psi_{t}\right)^{*}\right) f+0=0 .
\end{aligned}
$$

Now we come to the difficult case $m, n<N \leq m+n$.

$$
A_{N} f=\left.\partial_{t}^{N}\right|_{0}\left(\psi_{t}^{-1} \circ \varphi_{t}^{-1} \circ \psi_{t}\right)^{*} f+\left(\begin{array}{l}
N \\
m
\end{array}\right)\left(\left.\partial_{t}^{m}\right|_{0} \varphi_{t}^{*}\right)\left(\left.\partial_{t}^{N-m}\right|_{0}\left(\psi_{t}^{-1} \circ \varphi_{t}^{-1} \circ \psi_{t}\right)^{*}\right) f
$$




$$
+\left(\left.\partial_{t}^{N}\right|_{0} \varphi_{t}^{*}\right) f
$$

by claim 3 , since all other terms vanish; see (8) below. By claim 3 again we get:

$$
\begin{aligned}
\left.\partial_{t}^{N}\right|_{0}( & \left.\psi_{t}^{-1} \circ \varphi_{t}^{-1} \circ \psi_{t}\right)^{*} f \\
= & \sum_{j+k+\ell=N} \frac{N !}{j ! k ! \ell !}\left(\left.\partial_{t}^{j}\right|_{0} \psi_{t}^{*}\right)\left(\left.\partial_{t}^{k}\right|_{0}\left(\varphi_{t}^{-1}\right)^{*}\right)\left(\left.\partial_{t}^{\ell}\right|_{0}\left(\psi_{t}^{-1}\right)^{*}\right) f \\
= & \sum_{j+\ell=N}\left(\begin{array}{c}
N \\
j
\end{array}\right)\left(\left.\partial_{t}^{j}\right|_{0} \psi_{t}^{*}\right)\left(\left.\partial_{t}^{\ell}\right|_{0}\left(\psi_{t}^{-1}\right)^{*}\right) f \\
& +\left(\begin{array}{c}
N \\
m
\end{array}\right)\left(\left.\partial_{t}^{N-m}\right|_{0} \psi_{t}^{*}\right)\left(\left.\partial_{t}^{m}\right|_{0}\left(\varphi_{t}^{-1}\right)^{*}\right) f \\
& +\left(\begin{array}{c}
N \\
m
\end{array}\right)\left(\left.\partial_{t}^{m}\right|_{0}\left(\varphi_{t}^{-1}\right)^{*}\right)\left(\left.\partial_{t}^{N-m}\right|_{0}\left(\psi_{t}^{-1}\right)^{*}\right) f+\left.\partial_{t}^{N}\right|_{0}\left(\varphi_{t}^{-1}\right)^{*} f \\
= & 0+\left(\begin{array}{l}
N \\
m
\end{array}\right)\left(\left.\partial_{t}^{N-m}\right|_{0} \psi_{t}^{*}\right) m ! \mathcal{L}_{-X} f+\left(\begin{array}{c}
N \\
m
\end{array}\right) m ! \mathcal{L}_{-X}\left(\left.\partial_{t}^{N-m}\right|_{0}\left(\psi_{t}^{-1}\right)^{*}\right) f \\
& +\left.\partial_{t}^{N}\right|_{0}\left(\varphi_{t}^{-1}\right)^{*} f \\
= & \delta_{m+n}^{N}(m+n) !\left(\mathcal{L}_{X} \mathcal{L}_{Y}-\mathcal{L}_{Y} \mathcal{L}_{X}\right) f+\left.\partial_{t}^{N}\right|_{0}\left(\varphi_{t}^{-1}\right)^{*} f \\
= & \delta_{m+n}^{N}(m+n) ! \mathcal{L}_{[X, Y]} f+\left.\partial_{t}^{N}\right|_{0}\left(\varphi_{t}^{-1}\right)^{*} f .
\end{aligned}
$$

From the second expression in (77) one can also read off that

$$
\left.\partial_{t}^{N-m}\right|_{0}\left(\psi_{t}^{-1} \circ \varphi_{t}^{-1} \circ \psi_{t}\right)^{*} f=\left.\partial_{t}^{N-m}\right|_{0}\left(\varphi_{t}^{-1}\right)^{*} f .
$$

If we put (7) and (8) into (6), we get, using claims 3 and 4 again, the final result which proves claim 5 and the theorem:

$$
\begin{aligned}
A_{N} f= & \delta_{m+n}^{N}(m+n) ! \mathcal{L}_{[X, Y]} f+\left.\partial_{t}^{N}\right|_{0}\left(\varphi_{t}^{-1}\right)^{*} f \\
& +\left(\begin{array}{c}
N \\
m
\end{array}\right)\left(\left.\partial_{t}^{m}\right|_{0} \varphi_{t}^{*}\right)\left(\left.\partial_{t}^{N-m}\right|_{0}\left(\varphi_{t}^{-1}\right)^{*}\right) f+\left(\left.\partial_{t}^{N}\right|_{0} \varphi_{t}^{*}\right) f \\
= & \delta_{m+n}^{N}(m+n) ! \mathcal{L}_{[X, Y]} f+\left.\partial_{t}^{N}\right|_{0}\left(\varphi_{t}^{-1} \circ \varphi_{t}\right)^{*} f \\
= & \delta_{m+n}^{N}(m+n) ! \mathcal{L}_{[X, Y]} f+0 . \quad \square
\end{aligned}
$$

3.17. Theorem. Let $X_{1}, \ldots, X_{m}$ be vector fields on $M$ defined in a neighborhood of a point $x \in M$ such that $X_{1}(x), \ldots, X_{m}(x)$ are a basis for $T_{x} M$ and $\left[X_{i}, X_{j}\right]=0$ for all $i, j$.

Then there is a chart $(U, u)$ of $M$ centered at $x$ such that $X_{i} \mid U=\frac{\partial}{\partial u^{2}}$.

Proof. For small $t=\left(t^{1}, \ldots, t^{m}\right) \in \mathbb{R}^{m}$ we put

$$
f\left(t^{1}, \ldots, t^{m}\right)=\left(\mathrm{Fl}_{t^{1}}^{X_{1}} \circ \cdots \circ \mathrm{Fl}_{t^{m}}^{X_{m}}\right)(x) .
$$

By (3.15) we may interchange the order of the flows arbitrarily. Therefore

$$
\frac{\partial}{\partial t^{i}} f\left(t^{1}, \ldots, t^{m}\right)=\frac{\partial}{\partial t^{i}}\left(\mathrm{Fl}_{t^{i}}^{X_{i}} \circ \mathrm{Fl}_{t^{1}}^{X_{1}} \circ \cdots\right)(x)=X_{i}\left(\left(\mathrm{Fl}_{t^{1}}^{x_{1}} \circ \cdots\right)(x)\right) .
$$


So $T_{0} f$ is invertible, $f$ is a local diffeomorphism, and its inverse gives a chart with the desired properties.

3.18. The theorem of Frobenius. The next three subsections will be devoted to the theorem of Frobenius for distributions of constant rank. We will give a powerful generalization for distributions of nonconstant rank below in (3.21)-(3.28),

Let $M$ be a manifold. By a vector subbundle $E$ of $T M$ of fiber dimension $k$ we mean a subset $E \subset T M$ such that each $E_{x}:=E \cap T_{x} M$ is a linear subspace of dimension $k$ and such that for each $x \operatorname{im} M$ there are $k$ vector fields defined on an open neighborhood of $M$ with values in $E$ and spanning $E$, called a local frame for $E$. Such an $E$ is also called a smooth distribution of constant rank $k$. See section (8) for a thorough discussion of the notion of vector bundles. The space of all vector fields with values in $E$ will be called $\Gamma(E)$.

The vector subbundle $E$ of $T M$ is called integrable or involutive, if for all $X, Y \in \Gamma(E)$ we have $[X, Y] \in \Gamma(E)$.

Local version of Frobenius's theorem. Let $E \subset T M$ be an integrable vector subbundle of fiber dimension $k$ of $T M$.

Then for each $x \in M$ there exists a chart $(U, u)$ of $M$ centered at $x$ with $u(U)=V \times W \subset \mathbb{R}^{k} \times \mathbb{R}^{m-k}$, such that $T\left(u^{-1}(V \times\{y\})\right)=E \mid\left(u^{-1}(V \times\{y\})\right)$ for each $y \in W$.

Proof. Let $x \in M$. We choose a chart $(U, u)$ of $M$ centered at $x$ such that there exist $k$ vector fields $X_{1}, \ldots, X_{k} \in \Gamma(E)$ which form a frame of $E \mid U$. Then we have $X_{i}=\sum_{j=1}^{m} f_{i}^{j} \frac{\partial}{\partial u^{j}}$ for $f_{i}^{j} \in C^{\infty}(U)$. Then $f=\left(f_{i}^{j}\right)$ is a $(k \times m)$-matrix valued smooth function on $U$ which has rank $k$ on $U$. So some $(k \times k)$-submatrix, say the top one, is invertible at $x$ and thus we may take $U$ so small that this top $(k \times k)$-submatrix is invertible everywhere on $U$. Let $g=\left(g_{i}^{j}\right)$ be the inverse of this submatrix, so that the $(k \times m)$-matrix $f . g$ is given by

$$
f . g=\left(\begin{array}{c}
\mathbb{I}_{k} \\
*
\end{array}\right)
$$

We put

$$
Y_{i}:=\sum_{j=1}^{k} g_{i}^{j} X_{j}=\sum_{j=1}^{k} \sum_{l=1}^{m} g_{i}^{j} f_{j}^{l} \frac{\partial}{\partial u^{l}}=\frac{\partial}{\partial u^{i}}+\sum_{p \geq k+1} h_{i}^{p} \frac{\partial}{\partial u^{p}} .
$$

We claim that $\left[Y_{i}, Y_{j}\right]=0$ for all $1 \leq i, j \leq k$. Since $E$ is integrable, we have $\left[Y_{i}, Y_{j}\right]=\sum_{l=1}^{k} c_{i j}^{l} Y_{l}$. But from (11) we conclude (using the coordinate 
formula in (3.4) that $\left[Y_{i}, Y_{j}\right]=\sum_{p \geq k+1} a^{p} \frac{\partial}{\partial u^{p}}$. Again by (10) this implies that $c_{i j}^{l}=0$ for all $l$, and the claim follows.

Now we consider an $(m-k)$-dimensional linear subspace $W_{1}$ in $\mathbb{R}^{m}$ which is transversal to the $k$ vectors $T_{x} u . Y_{i}(x) \in T_{0} \mathbb{R}^{m}$ spanning $\mathbb{R}^{k}$, and we define $f: V \times W \rightarrow U$ by

$$
f\left(t^{1}, \ldots, t^{k}, y\right):=\left(\mathrm{Fl}_{t^{1}}^{Y_{1}} \circ \mathrm{Fl}_{t^{2}}^{Y_{2}} \circ \ldots \circ \mathrm{Fl}_{t^{k}}^{Y_{k}}\right)\left(u^{-1}(y)\right),
$$

where $t=\left(t^{1}, \ldots, t^{k}\right) \in V$, a small neighborhood of 0 in $\mathbb{R}^{k}$, and where $y \in W$, a small neighborhood of 0 in $W_{1}$. By (3.15) we may interchange the order of the flows in the definition of $f$ arbitrarily. Thus

$$
\begin{aligned}
\frac{\partial}{\partial t^{i}} f(t, y) & =\frac{\partial}{\partial t^{i}}\left(\mathrm{Fl}_{t^{i}}^{Y_{i}} \circ \mathrm{Fl}_{t^{1}}^{Y_{1}} \circ \ldots\right)\left(u^{-1}(y)\right)=Y_{i}(f(t, y)), \\
\frac{\partial}{\partial y^{k}} f(0, y) & =\frac{\partial}{\partial y^{k}}\left(u^{-1}\right)(y),
\end{aligned}
$$

and so $T_{0} f$ is invertible and the inverse of $f$ on a suitable neighborhood of $x$ gives us the required chart.

3.19. Remark. Any charts $\left(U, u: U \rightarrow V \times W \subset \mathbb{R}^{k} \times \mathbb{R}^{m-k}\right)$ as constructed in theorem (3.18) with $V$ and $W$ open balls is called a distinguished chart for $E$. The submanifolds $u^{-1}(V \times\{y\})$ are called plaques. Two plaques of different distinguished charts intersect in open subsets in both plaques or not at all: This follows immediately by flowing a point in the intersection into both plaques with the same construction as in the proof of (3.18). Thus an atlas of distinguished charts on $M$ has chart change mappings which respect the submersion $\mathbb{R}^{k} \times \mathbb{R}^{m-k} \rightarrow \mathbb{R}^{m-k}$ (the plaque structure on $M$ ). Such an atlas (or the equivalence class of such atlases) is called the foliation corresponding to the integrable vector subbundle $E \subset T M$.

3.20. Global version of Frobenius's theorem. Let $E \subsetneq T M$ be an integrable vector subbundle of $T M$. Then, using the restrictions of distinguished charts to plaques as charts, we get a new structure of a smooth manifold on $M$, which we denote by $M_{E}$. If $E \neq T M$, the topology of $M_{E}$ is finer than that of $M, M_{E}$ has uncountably many connected components called the leaves of the foliation, and the identity induces a bijective immersion $M_{E} \rightarrow M$. Each leaf $L$ is a second countable initial submanifold of $M$, and it is a maximal integrable submanifold of $M$ for $E$ in the sense that $T_{x} L=E_{x}$ for each $x \in L$.

Proof. Let $\left(U_{\alpha}, u_{\alpha}: U_{\alpha} \rightarrow V_{\alpha} \times W_{\alpha} \subseteq \mathbb{R}^{k} \times \mathbb{R}^{m-k}\right)$ be an atlas of distinguished charts corresponding to the integrable vector subbundle $E \subset T M$, as given by theorem (3.18). Let us now use for each plaque the homeomorphisms $\operatorname{pr}_{1} \circ u_{\alpha} \mid\left(u_{\alpha}^{-1}\left(V_{\alpha} \times\{y\}\right)\right): u_{\alpha}^{-1}\left(V_{\alpha} \times\{y\}\right) \rightarrow V_{\alpha} \subset \mathbb{R}^{m-k}$ as charts; 
then we describe on $M$ a new smooth manifold structure $M_{E}$ with finer topology which however has uncountably many connected components, and the identity on $M$ induces a bijective immersion $M_{E} \rightarrow M$. The connected components of $M_{E}$ are called the leaves of the foliation.

In order to check the rest of the assertions made in the theorem, let us construct the unique leaf $L$ through an arbitrary point $x \in M$ : choose a plaque containing $x$ and take the union with any plaque meeting the first one, and keep going. Now choose $y \in L$ and a curve $c:[0,1] \rightarrow L$ with $c(0)=x$ and $c(1)=y$. Then there are finitely many distinguished charts $\left(U_{1}, u_{1}\right), \ldots,\left(U_{n}, u_{n}\right)$ and $a_{1}, \ldots, a_{n} \in \mathbb{R}^{m-k}$ such that $x \in u_{1}^{-1}\left(V_{1} \times\left\{a_{1}\right\}\right)$, $y \in u_{n}^{-1}\left(V_{n} \times\left\{a_{n}\right\}\right)$ and such that for each $i$

$$
u_{i}^{-1}\left(V_{i} \times\left\{a_{i}\right\}\right) \cap u_{i+1}^{-1}\left(V_{i+1} \times\left\{a_{i+1}\right\}\right) \neq \emptyset .
$$

Given $u_{i}, u_{i+1}$, and $a_{i}$, there are only countably many points $a_{i+1}$ such that (11) holds: If not, then we get a cover of the the separable submanifold $u_{i}^{-1}\left(V_{i} \times\left\{a_{i}\right\}\right) \cap U_{i+1}$ by uncountably many pairwise disjoint open sets of the form given in (1), which contradicts separability.

Finally, since (each component of) $M$ is a Lindelöf space, any distinguished atlas contains a countable subatlas. So each leaf is the union of at most countably many plaques. The rest is clear.

3.21. Singular distributions. Let $M$ be a manifold. Suppose that for each $x \in M$ we are given a vector subspace $E_{x}$ of $T_{x} M$. The disjoint union $E=\bigsqcup_{x \in M} E_{x}$ is called a (singular) distribution on $M$. We do not suppose that the dimension of $E_{x}$ is locally constant in $x$.

Let $\mathfrak{X}_{l o c}(M)$ denote the set of all locally defined smooth vector fields on $M$, i.e., $\mathfrak{X}_{\text {loc }}(M)=\bigcup \mathfrak{X}(U)$, where $U$ runs through all open sets in $M$. Furthermore let $\mathfrak{X}_{E}$ denote the set of all local vector fields $X \in \mathfrak{X}_{l o c}(M)$ with $X(x) \in E_{x}$ whenever defined. We say that a subset $\mathcal{V} \subset \mathfrak{X}_{E}$ spans $E$ if for each $x \in M$ the vector space $E_{x}$ is the linear hull of the set $\{X(x): X \in \mathcal{V}\}$. We say that $E$ is a smooth distribution if $\mathfrak{X}_{E}$ spans $E$. Note that every subset $\mathcal{W} \subset \mathfrak{X}_{\text {loc }}(M)$ spans a distribution denoted by $E(\mathcal{W})$, which is obviously smooth (the linear span of the empty set is the vector space 0). From now on we will consider only smooth distributions.

An integral manifold of a smooth distribution $E$ is a connected immersed submanifold $(N, i)$ (see $(2.9)$ such that $T_{x} i\left(T_{x} N\right)=E_{i(x)}$ for all $x \in N$. We will see in theorem (3.25) below that any integral manifold is in fact an initial submanifold of $M$ (see (2.13), so that we need not specify the injective immersion $i$. An integral manifold of $E$ is called maximal if it is not contained in any strictly larger integral manifold of $E$.

3.22. Lemma. Let $E$ be a smooth distribution on $M$. Then we have: 
(1) If $(N, i)$ is an integral manifold of $E$ and $X \in \mathfrak{X}_{E}$, then $i^{*} X$ makes sense and is an element of $\mathfrak{X}_{\text {loc }}(N)$, which is $i \mid i^{-1}\left(U_{X}\right)$-related to $X$, where $U_{X} \subset M$ is the open domain of $X$.

(2) If $\left(N_{j}, i_{j}\right)$ are integral manifolds of $E$ for $j=1,2$, then $i_{1}^{-1}\left(i_{1}\left(N_{1}\right) \cap\right.$ $\left.i_{2}\left(N_{2}\right)\right)$ and $i_{2}^{-1}\left(i_{1}\left(N_{1}\right) \cap i_{2}\left(N_{2}\right)\right)$ are open subsets in $N_{1}$ and $N_{2}$, respectively; furthermore $i_{2}^{-1} \circ i_{1}$ is a diffeomorphism between them.

(3) If $x \in M$ is contained in some integral submanifold of $E$, then it is contained in a unique maximal one.

Proof. (11) Let $U_{X}$ be the open domain of $X \in \mathfrak{X}_{E}$. If $i(x) \in U_{X}$ for $x \in N$, we have $X(i(x)) \in E_{i(x)}=T_{x} i\left(T_{x} N\right)$, so $i^{*} X(x):=\left(\left(T_{x} i\right)^{-1} \circ X \circ i\right)(x)$ makes sense. The vector field $i^{*} X$ is clearly defined on an open subset of $N$ and is smooth.

(2) Let $X \in \mathfrak{X}_{E}$. Then $i_{j}^{*} X \in \mathfrak{X}_{l o c}\left(N_{j}\right)$ and is $i_{j}$-related to $X$. So by lemma (3.14) for $j=1,2$ we have

$$
i_{j} \circ \mathrm{Fl}_{t}^{i_{j}^{*} X}=F l_{t}^{X} \circ i_{j} .
$$

Now choose $x_{j} \in N_{j}$ such that $i_{1}\left(x_{1}\right)=i_{2}\left(x_{2}\right)=x_{0} \in M$ and choose vector fields $X_{1}, \ldots, X_{n} \in \mathfrak{X}_{E}$ such that $\left(X_{1}\left(x_{0}\right), \ldots, X_{n}\left(x_{0}\right)\right)$ is a basis of $E_{x_{0}}$. Then

$$
f_{j}\left(t^{1}, \ldots, t^{n}\right):=\left(\mathrm{Fl}_{t^{1}}^{i_{j}^{*} X_{1}} \circ \cdots \circ \mathrm{Fl}_{t^{n}}^{i_{j}^{*} X_{n}}\right)\left(x_{j}\right)
$$

is a smooth local mapping $\mathbb{R}^{n} \rightarrow N_{j}$ defined near zero. Since obviously $\left.\frac{\partial}{\partial t^{k}}\right|_{0} f_{j}=i_{j}^{*} X_{k}\left(x_{j}\right)$ for $j=1,2$, we see that $f_{j}$ is a diffeomorphism near 0 . Finally we have

$$
\begin{aligned}
\left(i_{2}^{-1} \circ i_{1} \circ f_{1}\right)\left(t^{1}, \ldots, t^{n}\right) & =\left(i_{2}^{-1} \circ i_{1} \circ \mathrm{Fl}_{t^{1}}^{i_{1}^{*} X_{1}} \circ \ldots \circ \mathrm{Fl}_{t^{n}}^{i_{1}^{*} X_{n}}\right)\left(x_{1}\right) \\
& =\left(i_{2}^{-1} \circ \mathrm{Fl}_{t^{1}}^{X_{1}} \circ \cdots \circ \mathrm{Fl}_{t^{n}}^{X_{n}} \circ i_{1}\right)\left(x_{1}\right) \\
& =\left(\mathrm{Fl}_{t^{1}}^{i_{2}^{*} X_{1}} \circ \cdots \circ \mathrm{Fl}_{t^{n}}^{i_{2}^{*} X_{n}} \circ i_{2}^{-1} \circ i_{1}\right)\left(x_{1}\right) \\
& =f_{2}\left(t^{1}, \ldots, t^{n}\right) .
\end{aligned}
$$

So $i_{2}^{-1} \circ i_{1}$ is a diffeomorphism, as required.

(3) Let $N$ be the union of all integral manifolds containing $x$. Choose the union of all the atlases of these integral manifolds as atlas for $N$, which is a smooth atlas for $N$ by (2). Note that a connected immersed submanifold of a separable manifold is automatically separable (since it carries a Riemann metric).

3.23. Integrable singular distributions and singular foliations. A smooth singular distribution $E$ on a manifold $M$ is called integrable if each point of $M$ is contained in some integral manifold of $E$. By (3.22.3) each 
point is then contained in a unique maximal integral manifold, so the maximal integral manifolds form a partition of $M$. This partition is called the (singular) foliation of $M$ induced by the integrable (singular) distribution $E$, and each maximal integral manifold is called a leaf of this foliation. If $X \in \mathfrak{X}_{E}$, then by (3.22.1) the integral curve $t \mapsto \mathrm{Fl}^{X}(t, x)$ of $X$ through $x \in M$ stays in the leaf through $x$.

Let us now consider an arbitrary subset $\mathcal{V} \subset \mathfrak{X}_{\text {loc }}(M)$. We say that $\mathcal{V}$ is stable if for all $X, Y \in \mathcal{V}$ and for all $t$ for which it is defined the local vector field $\left(\mathrm{Fl}_{t}^{X}\right)^{*} Y$ is again an element of $\mathcal{V}$.

If $\mathcal{W} \subset \mathfrak{X}_{l o c}(M)$ is an arbitrary subset, we call $\mathcal{S}(\mathcal{W})$ the set of all local vector fields of the form $\left(\mathrm{Fl}_{t_{1}}^{X_{1}} \circ \cdots \circ \mathrm{Fl}_{t_{k}}^{X_{k}}\right)^{*} Y$ for $X_{i}, Y \in \mathcal{W}$. By lemma (3.14) the flow of this vector field is

$$
\operatorname{Fl}\left(\left(\mathrm{Fl}_{t_{1}}^{X_{1}} \circ \cdots \circ \mathrm{Fl}_{t_{k}}^{X_{k}}\right)^{*} Y, t\right)=\mathrm{Fl}_{-t_{k}}^{X_{k}} \circ \cdots \circ \mathrm{Fl}_{-t_{1}}^{X_{1}} \circ \mathrm{Fl}_{t}^{Y} \circ \mathrm{Fl}_{t_{1}}^{X_{1}} \circ \cdots \circ \mathrm{Fl}_{t_{k}}^{X_{k}},
$$

so $\mathcal{S}(\mathcal{W})$ is the minimal stable set of local vector fields which contains $\mathcal{W}$.

Now let $F$ be an arbitrary distribution. A local vector field $X \in \mathfrak{X}_{\text {loc }}(M)$ is called an infinitesimal automorphism of $F$ if $T_{x}\left(\mathrm{Fl}_{t}^{X}\right)\left(F_{x}\right) \subset F_{\mathrm{Fl}^{X}(t, x)}$ whenever defined. We denote by $\operatorname{aut}(F)$ the set of all infinitesimal automorphisms of $F$. By arguments given just above, $\operatorname{aut}(F)$ is stable.

3.24. Lemma. Let $E$ be a smooth distribution on a manifold $M$. Then the following conditions are equivalent:

(1) E is integrable.

(2) $\mathfrak{X}_{E}$ is stable.

(3) There exists a subset $\mathcal{W} \subset \mathfrak{X}_{\text {loc }}(M)$ such that $\mathcal{S}(\mathcal{W})$ spans $E$.

(4) $\operatorname{aut}(E) \cap \mathfrak{X}_{E}$ spans $E$.

Proof. (11) $\Longrightarrow$ (2) Let $X \in \mathfrak{X}_{E}$ and let $L$ be the leaf through $x \in M$, with $i: L \rightarrow M$ the inclusion. Then $\mathrm{Fl}_{-t}^{X} \circ i=i \circ \mathrm{Fl}_{-t}^{i^{*} X}$ by lemma (3.14), so we have

$$
\begin{aligned}
& T_{x}\left(\mathrm{Fl}_{-t}^{X}\right)\left(E_{x}\right)=T\left(\mathrm{Fl}_{-t}^{X}\right) \cdot T_{x} i \cdot T_{x} L=T\left(\mathrm{Fl}_{-t}^{X} \circ i\right) \cdot T_{x} L \\
&=T i \cdot T_{x}\left(\mathrm{Fl}_{-t}^{i^{*} X}\right) \cdot T_{x} L \\
&=T i \cdot T_{F l^{*} X}(-t, x) \\
& L=E_{F l^{X}}(-t, x)
\end{aligned}
$$

This implies that $\left(\mathrm{Fl}_{t}^{X}\right)^{*} Y \in \mathfrak{X}_{E}$ for any $Y \in \mathfrak{X}_{E}$.

(21) $\Longrightarrow$ (4) In fact (2) says that $\mathfrak{X}_{E} \subset \operatorname{aut}(E)$.

(41) $\Longrightarrow$ (3) We can choose $\mathcal{W}=\operatorname{aut}(E) \cap \mathfrak{X}_{E}$ : For $X, Y \in \mathcal{W}$ we have $\left(\mathrm{Fl}_{t}^{X}\right)^{*} Y \in \mathfrak{X}_{E} ;$ so $\mathcal{W} \subset \mathcal{S}(\mathcal{W}) \subset \mathfrak{X}_{E}$ and $E$ is spanned by $\mathcal{W}$.

(3) $\Longrightarrow$ (1) We have to show that each point $x \in M$ is contained in some integral submanifold for the distribution $E$. Since $\mathcal{S}(\mathcal{W})$ spans $E$ and is 
stable, we have

$$
T\left(\mathrm{Fl}_{t}^{X}\right) \cdot E_{x}=E_{\mathrm{Fl}^{X}(t, x)}
$$

for each $X \in \mathcal{S}(\mathcal{W})$. Let $\operatorname{dim} E_{x}=n$. There are $X_{1}, \ldots, X_{n} \in \mathcal{S}(\mathcal{W})$ such that $X_{1}(x), \ldots, X_{n}(x)$ is a basis of $E_{x}$, since $E$ is smooth. As in the proof of (3.22.2) we consider the mapping

$$
f\left(t^{1}, \ldots, t^{n}\right):=\left(\mathrm{Fl}_{t^{1}}^{X_{1}} \circ \cdots \circ \mathrm{Fl}_{t^{n}}^{X_{n}}\right)(x),
$$

defined and smooth near 0 in $\mathbb{R}^{n}$. Since the rank of $f$ at 0 is $n$, the image under $f$ of a small open neighborhood of 0 is a submanifold $N$ of $M$. We claim that $N$ is an integral manifold of $E$. The tangent space $T_{f\left(t^{1}, \ldots, t^{n}\right)} N$ is linearly generated by

$$
\begin{aligned}
\frac{\partial}{\partial t^{k}}\left(\mathrm{Fl}_{t^{1}}^{X_{1}} \circ \cdots \circ \mathrm{Fl}_{t^{n}}^{X_{n}}\right)(x) & =T\left(\mathrm{Fl}_{t^{1}}^{X_{1}} \circ \cdots \circ \mathrm{Fl}_{t^{k-1}}^{X_{k-1}}\right) X_{k}\left(\left(\mathrm{Fl}_{t^{k}}^{X_{k}} \circ \cdots \circ \mathrm{Fl}_{t^{n}}^{X_{n}}\right)(x)\right) \\
& =\left(\left(\mathrm{Fl}_{-t^{1}}^{X_{1}}\right)^{*} \cdots\left(\mathrm{Fl}_{-t^{k-1}}^{X_{k-1}}\right)^{*} X_{k}\right)\left(f\left(t^{1}, \ldots, t^{n}\right)\right) .
\end{aligned}
$$

Since $\mathcal{S}(\mathcal{W})$ is stable, these vectors lie in $E_{f(t)}$. From the form of $f$ and from (5) we see that $\operatorname{dim} E_{f(t)}=\operatorname{dim} E_{x}$, so these vectors even span $E_{f(t)}$ and we have $T_{f(t)} N=E_{f(t)}$ as required.

3.25. Theorem (Local structure of singular foliations). Let $E$ be an integrable (singular) distribution of a manifold $M$. Then for each $x \in M$ there exist a chart $(U, u)$ with $u(U)=\left\{y \in \mathbb{R}^{m}:\left|y^{i}\right|<\varepsilon\right.$ for all $\left.i\right\}$ for some $\varepsilon>0$ and a countable subset $A \subset \mathbb{R}^{m-n}$, such that for the leaf $L$ through $x$ we have

$$
u(U \cap L)=\left\{y \in u(U):\left(y^{n+1}, \ldots, y^{m}\right) \in A\right\} .
$$

Each leaf is an initial submanifold.

If furthermore the distribution $E$ has locally constant rank, this property holds for each leaf meeting $U$ with the same $n$.

This chart $(U, u)$ is called a distinguished chart for the (singular) distribution or the (singular) foliation. A connected component of $U \cap L$ is called a plaque.

Proof. Let $L$ be the leaf through $x, \operatorname{dim} L=n$. Let $X_{1}, \ldots, X_{n} \in \mathfrak{X}_{E}$ be local vector fields such that $X_{1}(x), \ldots, X_{n}(x)$ is a basis of $E_{x}$. We choose a chart $(V, v)$ centered at $x$ on $M$ such that the vectors

$$
X_{1}(x), \ldots, X_{n}(x),\left.\frac{\partial}{\partial v^{n+1}}\right|_{x}, \ldots,\left.\frac{\partial}{\partial v^{m}}\right|_{x}
$$

form a basis of $T_{x} M$. Then

$$
f\left(t^{1}, \ldots, t^{m}\right)=\left(\mathrm{Fl}_{t^{1}}^{X_{1}} \circ \cdots \circ \mathrm{Fl}_{t^{n}}^{X_{n}}\right)\left(v^{-1}\left(0, \ldots, 0, t^{n+1}, \ldots, t^{m}\right)\right)
$$

is a diffeomorphism from a neighborhood of 0 in $\mathbb{R}^{m}$ onto a neighborhood of $x$ in $M$. Let $(U, u)$ be the chart given by $f^{-1}$, suitably restricted. We have

$$
y \in L \Longleftrightarrow\left(\mathrm{Fl}_{t^{1}}^{X_{1}} \circ \ldots \circ \mathrm{Fl}_{t^{n}}^{X_{n}}\right)(y) \in L
$$


for all $y$ and all $t^{1}, \ldots, t^{n}$ for which both expressions make sense. So we have

$$
f\left(t^{1}, \ldots, t^{m}\right) \in L \Longleftrightarrow f\left(0, \ldots, 0, t^{n+1}, \ldots, t^{m}\right) \in L,
$$

and consequently $L \cap U$ is the disjoint union of connected sets of the form $\left\{y \in U:\left(u^{n+1}(y), \ldots, u^{m}(y)\right)=\right.$ constant $\}$. Since $L$ is a connected immersive submanifold of $M$, it is second countable and only a countable set of constants can appear in the description of $u(L \cap U)$ given above. From this description it is clear that $L$ is an initial submanifold (2.13) since $u\left(C_{x}(L \cap U)\right)=u(U) \cap\left(\mathbb{R}^{n} \times 0\right)$.

The argument given above is valid for any leaf of dimension $n$ meeting $U$, so also the assertion for an integrable distribution of constant rank follows.

3.26. Involutive singular distributions. A subset $\mathcal{V} \subset \mathfrak{X}_{l o c}(M)$ is called involutive if $[X, Y] \in \mathcal{V}$ for all $X, Y \in \mathcal{V}$. Here $[X, Y]$ is defined on the intersection of the domains of $X$ and $Y$.

A smooth distribution $E$ on $M$ is called involutive if there exists an involutive subset $\mathcal{V} \subset \mathfrak{X}_{l o c}(M)$ spanning $E$.

For an arbitrary subset $\mathcal{W} \subset \mathfrak{X}_{\text {loc }}(M)$ let $\mathcal{L}(\mathcal{W})$ be the set consisting of all local vector fields on $M$ which can be written as finite expressions using Lie brackets and starting from elements of $\mathcal{W}$. Clearly $\mathcal{L}(\mathcal{W})$ is the smallest involutive subset of $\mathfrak{X}_{l o c}(M)$ which contains $\mathcal{W}$.

3.27. Lemma. For each subset $\mathcal{W} \subset \mathfrak{X}_{\text {loc }}(M)$ we have

$$
E(\mathcal{W}) \subset E(\mathcal{L}(\mathcal{W})) \subset E(\mathcal{S}(\mathcal{W}))
$$

In particular we have $E(\mathcal{S}(\mathcal{W}))=E(\mathcal{L}(\mathcal{S}(\mathcal{W})))$.

Proof. We will show that for $X, Y \in \mathcal{W}$ we have $[X, Y] \in \mathfrak{X}_{E(\mathcal{S}(\mathcal{W}))}$, for then by induction we get $\mathcal{L}(\mathcal{W}) \subset \mathfrak{X}_{E(\mathcal{S}(\mathcal{W}))}$ and $E(\mathcal{L}(\mathcal{W})) \subset E(\mathcal{S}(\mathcal{W}))$.

Let $x \in M$; since by $(3.24) E(\mathcal{S}(\mathcal{W}))$ is integrable, we can choose the leaf $L$ through $x$, with the inclusion $i$. Then $i^{*} X$ is $i$-related to $X$ and $i^{*} Y$ is $i$-related to $Y$; thus by $(3.10)$ the local vector field $\left[i^{*} X, i^{*} Y\right] \in \mathfrak{X}_{l o c}(L)$ is $i$-related to $[X, Y]$, and $[X, Y](x) \in E(\mathcal{S}(\mathcal{W}))_{x}$, as required.

3.28. Theorem. Let $\mathcal{V} \subset \mathfrak{X}_{\text {loc }}(M)$ be an involutive subset. Then the distribution $E(\mathcal{V})$ spanned by $\mathcal{V}$ is integrable under each of the following conditions.

(1) $M$ is real analytic and $\mathcal{V}$ consists of real analytic vector fields.

(2) The dimension of $E(\mathcal{V})$ is constant along flow lines of vector fields in $\mathcal{V}$.

Proof. (11) For $X, Y \in \mathcal{V}$ we have $\frac{d}{d t}\left(\mathrm{Fl}_{t}^{X}\right)^{*} Y=\left(\mathrm{Fl}_{t}^{X}\right)^{*} \mathcal{L}_{X} Y$; consequently $\frac{d^{k}}{d t^{k}}\left(\mathrm{Fl}_{t}^{X}\right)^{*} Y=\left(\mathrm{Fl}_{t}^{X}\right)^{*}\left(\mathcal{L}_{X}\right)^{k} Y$, and since everything is real analytic, we get 
for $x \in M$ and small $t$

$$
\left(\mathrm{Fl}_{t}^{X}\right)^{*} Y(x)=\left.\sum_{k \geq 0} \frac{t^{k}}{k !} \frac{d^{k}}{d t^{k}}\right|_{0}\left(\mathrm{Fl}_{t}^{X}\right)^{*} Y(x)=\sum_{k \geq 0} \frac{t^{k}}{k !}\left(\mathcal{L}_{X}\right)^{k} Y(x) .
$$

Since $\mathcal{V}$ is involutive, all $\left(\mathcal{L}_{X}\right)^{k} Y \in \mathcal{V}$. Therefore we get $\left(\mathrm{Fl}_{t}^{X}\right)^{*} Y(x) \in$ $E(\mathcal{V})_{x}$ for small $t$. By the flow property of $\mathrm{Fl}^{X}$ the set of all $t$ satisfying $\left(\mathrm{Fl}_{t}^{X}\right)^{*} Y(x) \in E(\mathcal{V})_{x}$ is open and closed, so it follows that (3.24.2) is satisfied and thus $E(\mathcal{V})$ is integrable.

(22) We choose $X_{1}, \ldots, X_{n} \in \mathcal{V}$ such that $X_{1}(x), \ldots, X_{n}(x)$ is a basis of $E(\mathcal{V})_{x}$. For any $X \in \mathcal{V}$, by hypothesis, $E(\mathcal{V})_{\mathrm{Fl}^{X}(t, x)}$ has also dimension $n$ and admits the vectors $X_{1}\left(\mathrm{Fl}^{X}(t, x)\right), \ldots, X_{n}\left(\mathrm{Fl}^{X}(t, x)\right)$ as basis, for small $t$. So there are smooth functions $f_{i j}(t)$ such that

$$
\left[X, X_{i}\right]\left(\mathrm{Fl}^{X}(t, x)\right)=\sum_{j=1}^{n} f_{i j}(t) X_{j}\left(\mathrm{Fl}^{X}(t, x)\right) .
$$

Therefore,

$$
\begin{aligned}
\frac{d}{d t} T\left(\mathrm{Fl}_{-t}^{X}\right) \cdot X_{i}\left(\mathrm{Fl}^{X}(t, x)\right) & =T\left(\mathrm{Fl}_{-t}^{X}\right) \cdot\left[X, X_{i}\right]\left(\mathrm{Fl}^{X}(t, x)\right) \\
& =\sum_{j=1}^{n} f_{i j}(t) T\left(\mathrm{Fl}_{-t}^{X}\right) \cdot X_{j}\left(\mathrm{Fl}^{X}(t, x)\right) .
\end{aligned}
$$

So the $T_{x} M$-valued functions $g_{i}(t)=T\left(\mathrm{Fl}_{-t}^{X}\right) \cdot X_{i}\left(\mathrm{Fl}^{X}(t, x)\right)$ satisfy the linear ordinary differential equation $\frac{d}{d t} g_{i}(t)=\sum_{j=1}^{n} f_{i j}(t) g_{j}(t)$ and have initial values in the linear subspace $E(\mathcal{V})_{x}$, so they have values in it for all small $t$. Therefore $T\left(\mathrm{Fl}_{-t}^{X}\right) E(\mathcal{V})_{\mathrm{Fl}^{X}{ }_{(t, x)}} \subset E(\mathcal{V})_{x}$ for small $t$. Using compact time intervals and the flow property, one sees that condition (3.24.2) is satisfied and $E(\mathcal{V})$ is integrable.

3.29. Examples. (1) The singular distribution spanned by $\mathcal{W} \subset \mathfrak{X}_{\text {loc }}\left(\mathbb{R}^{2}\right)$ is involutive, but not integrable, where $\mathcal{W}$ consists of all global vector fields with support in $\mathbb{R}^{2} \backslash\{0\}$ and the field $\frac{\partial}{\partial x^{1}}$; the leaf through 0 should have dimension 1 at 0 and dimension 2 elsewhere.

(2) Let $f: \mathbb{R} \rightarrow \mathbb{R}$ be a smooth function with $f\left(x^{1}\right)=0$ for $x^{1} \leq 0$ and $f\left(x^{1}\right)>0$ for $x^{1}>0$. Then the singular distribution on $\mathbb{R}^{2}$ spanned by the two vector fields $X\left(x^{1}, x^{2}\right)=\frac{\partial}{\partial x^{1}}$ and $Y\left(x^{1}, x^{2}\right)=f\left(x^{1}\right) \frac{\partial}{\partial x^{2}}$ is involutive, but not integrable. Any leaf should pass $\left(0, x^{2}\right)$ tangentially to $\frac{\partial}{\partial x^{1}}$, should have dimension 1 for $x^{1} \leq 0$ and should have dimension 2 for $x^{1}>0$.

3.30. By a time dependent vector field on a manifold $M$ we mean a smooth mapping $X: J \times M \rightarrow T M$ with $\pi_{M} \circ X=\operatorname{pr}_{2}$, where $J$ is an open interval. An integral curve of $X$ is a smooth curve $c: I \rightarrow M$ with $\dot{c}(t)=X(t, c(t))$ for all $t \in I$, where $I$ is a subinterval of $J$. 
There is an associated vector field $\bar{X} \in \mathfrak{X}(J \times M)$, given by $\bar{X}(t, x)=$ $\left(\frac{\partial}{\partial t}, X(t, x)\right) \in T_{t} \mathbb{R} \times T_{x} M$.

By the evolution operator of $X$ we mean the mapping $\Phi^{X}: J \times J \times M \rightarrow$ $M$, defined in a maximal open neighborhood of $\Delta_{J} \times M$ (where $\Delta_{J}$ is the diagonal of $J$ ) and satisfying the differential equation

$$
\left\{\begin{array}{l}
\frac{d}{d t} \Phi^{X}(t, s, x)=X\left(t, \Phi^{X}(t, s, x)\right) \\
\Phi^{X}(s, s, x)=x .
\end{array}\right.
$$

It is easily seen that $\left(t, \Phi^{X}(t, s, x)\right)=\mathrm{Fl}^{\bar{X}}(t-s,(s, x))$, so the maximally defined evolution operator exists and is unique, and it satisfies

$$
\Phi_{t, s}^{X}=\Phi_{t, r}^{X} \circ \Phi_{r, s}^{X}, \quad \text { where } \Phi_{t, s}^{X}(x)=\Phi(t, s, x),
$$

whenever one side makes sense (with the restrictions of (3.7)].

\section{Examples and Exercises}

3.31. Compute the flow of the vector field $\xi_{1}(x, y):=y \frac{\partial}{\partial x}$ in $\mathbb{R}^{2}$. Is it a global flow? Answer the same questions for $\xi_{2}(x, y):=\frac{x^{2}}{2} \frac{\partial}{\partial y}$. Now compute $\left[\xi_{1}, \xi_{2}\right]$ and investigate its flow. This time it is not global! In fact, $F l_{t}^{\left[\xi_{1}, \xi_{2}\right]}(x, y)=\left(\frac{2 x}{2+x t}, \frac{y}{4}(t x+2)^{2}\right)$. Investigate the flow of $\xi_{1}+\xi_{2}$. It is not global either! Thus the set of complete vector fields on $\mathbb{R}^{2}$ is neither a vector space nor closed under the Lie bracket.

3.32. Driving a car. The phase space consists of all $(x, y, \vartheta, \varphi) \in \mathbb{R}^{2} \times$ $S^{1} \times(-\pi / 4, \pi / 4)$, where

$$
\begin{array}{ll}
(x, y) & \text { is the position of the midpoint of the rear axle, } \\
\vartheta & \text { is the direction of the car axle, } \\
\phi & \text { is the steering angle of the front wheels. }
\end{array}
$$

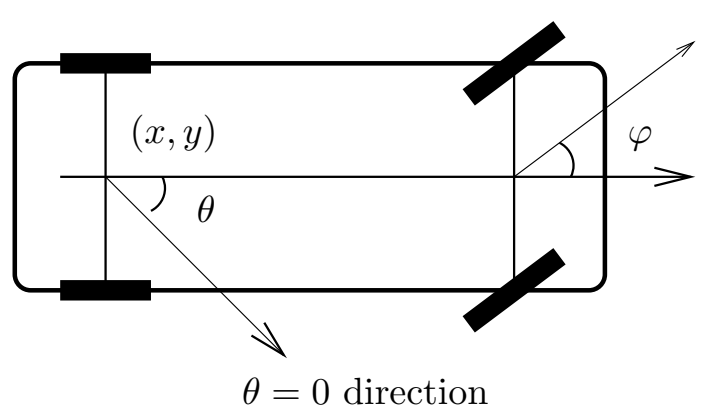

There are two 'control' vector fields:

$$
\text { steer }=\frac{\partial}{\partial \phi} \text {, }
$$




$$
\text { drive }=\cos (\vartheta) \frac{\partial}{\partial x}+\sin (\vartheta) \frac{\partial}{\partial y}+\tan (\phi) \frac{1}{l} \frac{\partial}{\partial \vartheta} \text { (why?). }
$$

Compute [steer, drive] =: park (why?) and [drive,park], and interpret the results. Is it not convenient that the two control vector fields do not span an integrable distribution?

3.33. Describe the Lie algebra of all vector fields on $S^{1}$ in terms of Fourier expansion. This is nearly (up to a central extension) the Virasoro algebra of theoretical physics. 



\section{CHAPTER II. Lie Groups and Group Actions}

\section{Lie Groups I}

4.1. Definition. A Lie group $G$ is a smooth manifold and a group such that the multiplication $\mu: G \times G \rightarrow G$ is smooth. We shall see in a moment that then also the inversion $\nu: G \rightarrow G$ turns out to be smooth.

We shall use the following notation:

$$
\begin{aligned}
& \mu: G \times G \rightarrow G, \text { multiplication, } \mu(x, y)=x . y . \\
& \mu_{a}: G \rightarrow G, \text { left translation, } \mu_{a}(x)=a . x . \\
& \mu^{a}: G \rightarrow G, \text { right translation, } \mu^{a}(x)=x . a . \\
& \nu: G \rightarrow G, \text { inversion, } \nu(x)=x^{-1} . \\
& e \in G, \text { the unit element. }
\end{aligned}
$$

Then we have

$$
\begin{aligned}
& \mu_{a} \circ \mu_{b}=\mu_{a . b}, \quad \quad \mu^{a} \circ \mu^{b}=\mu^{b . a}, \quad \quad \mu^{a} \circ \mu_{b}=\mu_{b} \circ \mu^{a}, \\
& \mu_{a}^{-1}=\mu_{a^{-1}}, \quad\left(\mu^{a}\right)^{-1}=\mu^{a^{-1}} .
\end{aligned}
$$

If $\varphi: G \rightarrow H$ is a smooth homomorphism between Lie groups, then we have $\varphi \circ \mu_{a}=\mu_{\varphi(a)} \circ \varphi, \varphi \circ \mu^{a}=\mu^{\varphi(a)} \circ \varphi$ and thus also $T \varphi \cdot T \mu_{a}=T \mu_{\varphi(a)} . T \varphi$, etc. So $T_{e} \varphi$ is injective (surjective) if and only if $T_{a} \varphi$ is injective (surjective) for all $a \in G$. 
4.2. Lemma. The tangent mapping $T_{(a, b)} \mu: T_{a} G \times T_{b} G \rightarrow T_{a b} G$ of the multiplication $\mu$ is given by

$$
T_{(a, b)} \mu \cdot\left(X_{a}, Y_{b}\right)=T_{a}\left(\mu^{b}\right) \cdot X_{a}+T_{b}\left(\mu_{a}\right) \cdot Y_{b} .
$$

Proof. Let $r i_{a}: G \rightarrow G \times G, r i_{a}(x)=(a, x)$ be the right insertion and let $l i_{b}: G \rightarrow G \times G, l i_{b}(x)=(x, b)$ be the left insertion. Then we have

$$
\begin{aligned}
& T_{(a, b)} \mu \cdot\left(X_{a}, Y_{b}\right)=T_{(a, b)} \mu \cdot\left(T_{a}\left(l i_{b}\right) \cdot X_{a}+T_{b}\left(r i_{a}\right) \cdot Y_{b}\right) \\
& \quad=T_{a}\left(\mu \circ l i_{b}\right) \cdot X_{a}+T_{b}\left(\mu \circ r i_{a}\right) \cdot Y_{b}=T_{a}\left(\mu^{b}\right) \cdot X_{a}+T_{b}\left(\mu_{a}\right) \cdot Y_{b} .
\end{aligned}
$$

4.3. Corollary. The inversion $\nu: G \rightarrow G$ is smooth and

$$
T_{a} \nu=-T_{e}\left(\mu^{a^{-1}}\right) \cdot T_{a}\left(\mu_{a^{-1}}\right)=-T_{e}\left(\mu_{a^{-1}}\right) \cdot T_{a}\left(\mu^{a^{-1}}\right) .
$$

Proof. The equation $\mu(x, \nu(x))=e$ determines $\nu$ implicitly. The mapping $\nu$ is smooth in a neighborhood of $e$ by the implicit function theorem since $T_{e}(\mu(e, \quad))=T_{e}\left(\mu_{e}\right)=I d$. From $\left(\nu \circ \mu_{a}\right)(x)=x^{-1} \cdot a^{-1}=\left(\mu^{a^{-1}} \circ \nu\right)(x)$ we may conclude that $\nu$ is everywhere smooth. Now we differentiate the equation $\mu(a, \nu(a))=e$; this gives in turn

$$
\begin{gathered}
0_{e}=T_{\left(a, a^{-1}\right)} \mu \cdot\left(X_{a}, T_{a} \nu \cdot X_{a}\right)=T_{a}\left(\mu^{a^{-1}}\right) \cdot X_{a}+T_{a^{-1}}\left(\mu_{a}\right) \cdot T_{a} \nu \cdot X_{a} \\
T_{a} \nu \cdot X_{a}=-T_{e}\left(\mu_{a}\right)^{-1} \cdot T_{a}\left(\mu^{a^{-1}}\right) \cdot X_{a}=-T_{e}\left(\mu_{a^{-1}}\right) \cdot T_{a}\left(\mu^{a^{-1}}\right) \cdot X_{a} .
\end{gathered}
$$

4.4. Example. The general linear group $G L(n, \mathbb{R})$ is the group of all invertible real $n \times n$-matrices. It is an open subset of $L\left(\mathbb{R}^{n}, \mathbb{R}^{n}\right)$, given by det $\neq 0$ and a Lie group.

Similarly $G L(n, \mathbb{C})$, the group of invertible complex $n \times n$-matrices, is a Lie group; also $G L(n, \mathbb{H})$, the group of all invertible quaternionic $n \times n$-matrices, is a Lie group, since it is open in the real Banach algebra $L_{\mathbb{H}}\left(\mathbb{H}^{n}, \mathbb{H}^{n}\right)$ as a glance at the von Neumann series shows; but the quaternionic determinant is a more subtle instrument here.

4.5. Example. The orthogonal group $O(n, \mathbb{R})$ is the group of all linear isometries of $\left(\mathbb{R}^{n},\langle, \quad\rangle\right)$, where $\langle, \quad\rangle$ is the standard positive definite inner product on $\mathbb{R}^{n}$. The special orthogonal group $S O(n, \mathbb{R}):=\{A \in O(n, \mathbb{R})$ : $\operatorname{det} A=1\}$ is open in $O(n, \mathbb{R})$, since we have the disjoint union

$$
O(n, \mathbb{R})=S O(n, \mathbb{R}) \sqcup\left(\begin{array}{cc}
-1 & 0 \\
0 & \mathbb{I}_{n-1}
\end{array}\right) S O(n, \mathbb{R}),
$$

where $\mathbb{I}_{k}$ is short for the identity matrix $I d_{\mathbb{R}^{k}}$. We claim that $O(n, \mathbb{R})$ and $S O(n, \mathbb{R})$ are submanifolds of $L\left(\mathbb{R}^{n}, \mathbb{R}^{n}\right)$. For that we consider the mapping $f: L\left(\mathbb{R}^{n}, \mathbb{R}^{n}\right) \rightarrow L\left(\mathbb{R}^{n}, \mathbb{R}^{n}\right)$, given by $f(A)=A^{\top} \cdot A$. Then $O(n, \mathbb{R})=$ $f^{-1}\left(\mathbb{I}_{n}\right)$; so $O(n, \mathbb{R})$ is closed. Since it is also bounded, $O(n, \mathbb{R})$ is compact. 
We have $d f(A) \cdot X=X^{\top} \cdot A+A^{\top} \cdot X$, so ker $d f\left(\mathbb{I}_{n}\right)=\left\{X: X^{\top}+X=0\right\}$ is the space $\mathfrak{o}(n, \mathbb{R})$ of all skew-symmetric $n \times n$-matrices. Note that $\operatorname{dim} \mathfrak{o}(n, \mathbb{R})=$ $\frac{1}{2}(n-1) n$. If $A$ is invertible, we get

$$
\begin{aligned}
\operatorname{ker} d f(A) & =\left\{Y: Y^{\top} \cdot A+A^{\top} . Y=0\right\}=\left\{Y: A^{\top} . Y \in \mathfrak{o}(n, \mathbb{R})\right\} \\
& =\left(A^{-1}\right)^{\top} \cdot \mathfrak{o}(n, \mathbb{R}) .
\end{aligned}
$$

The mapping $f$ takes values in $L_{\text {sym }}\left(\mathbb{R}^{n}, \mathbb{R}^{n}\right)$, the space of all symmetric $n \times n$-matrices, and $\operatorname{dim} \operatorname{ker} d f(A)+\operatorname{dim} L_{\text {sym }}\left(\mathbb{R}^{n}, \mathbb{R}^{n}\right)=\frac{1}{2}(n-1) n+\frac{1}{2} n(n+$ $1)=n^{2}=\operatorname{dim} L\left(\mathbb{R}^{n}, \mathbb{R}^{n}\right)$, so $f: G L(n, \mathbb{R}) \rightarrow L_{\text {sym }}\left(\mathbb{R}^{n}, \mathbb{R}^{n}\right)$ is a submersion. Since obviously $f^{-1}\left(\mathbb{I}_{n}\right) \subset G L(n, \mathbb{R})$, we conclude from $(1.12)$ that $O(n, \mathbb{R})$ is a submanifold of $G L(n, \mathbb{R})$. It is also a Lie group, since the group operations are smooth as the restrictions of the ones from $G L(n, \mathbb{R})$.

4.6. Example. The special linear group $S L(n, \mathbb{R})$ is the group of all $n \times n$ matrices of determinant 1 . The function det $: L\left(\mathbb{R}^{n}, \mathbb{R}^{n}\right) \rightarrow \mathbb{R}$ is smooth and $d \operatorname{det}(A) X=\operatorname{Trace}(C(A) \cdot X)$, where $C(A)_{j}^{i}$, the cofactor of $A_{i}^{j}$, is the determinant of the matrix, which results from putting 1 instead of $A_{i}^{j}$ into $A$ and 0 in the rest of the $j$-th row and the $i$-th column of $A$; see (4.33), We recall Cramer's rule $C(A) \cdot A=A \cdot C(A)=\operatorname{det}(A) \cdot \mathbb{I}_{n}$. So if $C(A) \neq 0$ (i.e., $\operatorname{rank}(A) \geq n-1)$, then the linear functional $d f(A)$ is nonzero. So det $: G L(n, \mathbb{R}) \rightarrow \mathbb{R}$ is a submersion and $S L(n, \mathbb{R})=(\text { det })^{-1}(1)$ is a manifold and a Lie group of dimension $n^{2}-1$. Note finally that $T_{\mathbb{I}_{n}} S L(n, \mathbb{R})=$ $\operatorname{ker} d \operatorname{det}\left(\mathbb{I}_{n}\right)=\{X: \operatorname{Trace}(X)=0\}$. This space of traceless matrices is usually called $\mathfrak{s l}(n, \mathbb{R})$.

4.7. Example. The symplectic group $S p(n, \mathbb{R})$ is the group of all $2 n \times 2 n$ matrices $A$ such that $\omega(A x, A y)=\omega(x, y)$ for all $x, y \in \mathbb{R}^{2 n}$, where $\omega$ is a (the standard) nondegenerate skew-symmetric bilinear form on $\mathbb{R}^{2 n}$.

Such a form exists on a vector space if and only if the dimension is even, and on $\mathbb{R}^{n} \times\left(\mathbb{R}^{n}\right)^{*}$ the form $\omega\left(\left(x, x^{*}\right),\left(y, y^{*}\right)\right)=\left\langle x, y^{*}\right\rangle-\left\langle y, x^{*}\right\rangle$ (where we use the duality pairing), in coordinates $\omega\left(\left(x^{i}\right)_{i=1}^{2 n},\left(y^{j}\right)_{j=1}^{2 n}\right)=\sum_{i=1}^{n}\left(x^{i} y^{n+i}-x^{n+i} y^{i}\right)$, is such a form. Any symplectic form on $\mathbb{R}^{2 n}$ looks like that after choosing a suitable basis; see $(31.2)$ and $(31.4)$. Let $\left(e_{i}\right)_{i=1}^{2 n}$ be the standard basis in $\mathbb{R}^{2 n}$. Then we have

$$
\left(\omega\left(e_{i}, e_{j}\right)_{j}^{i}\right)=\left(\begin{array}{cc}
0 & \mathbb{I}_{n} \\
-\mathbb{I}_{n} & 0
\end{array}\right)=: J
$$

and the matrix $J$ satisfies $J^{\top}=-J, J^{2}=-\mathbb{I}_{2 n}, J\left(\begin{array}{l}x \\ y\end{array}\right)=\left(\begin{array}{c}y \\ -x\end{array}\right)$ in $\mathbb{R}^{n} \times \mathbb{R}^{n}$, and $\omega(x, y)=\langle x, J y\rangle$ in terms of the standard inner product on $\mathbb{R}^{2 n}$.

For $A \in L\left(\mathbb{R}^{2 n}, \mathbb{R}^{2 n}\right)$ we have $\omega(A x, A y)=\langle A x, J A y\rangle=\left\langle x, A^{\top} J A y\right\rangle$. Thus $A \in S p(n, \mathbb{R})$ if and only if $A^{\top} J A=J$. 
We consider now the mapping $f: L\left(\mathbb{R}^{2 n}, \mathbb{R}^{2 n}\right) \rightarrow L\left(\mathbb{R}^{2 n}, \mathbb{R}^{2 n}\right)$ given by $f(A)=A^{\top} J A$. Then $f(A)^{\top}=\left(A^{\top} J A\right)^{\top}=-A^{\top} J A=-f(A)$, so $f$ takes values in the space $\mathfrak{o}(2 n, \mathbb{R})$ of skew-symmetric matrices. We have $d f(A) X=$ $X^{\top} J A+A^{\top} J X$, and therefore

$$
\begin{aligned}
\operatorname{ker} d f\left(\mathbb{I}_{2 n}\right) & =\left\{X \in L\left(\mathbb{R}^{2 n}, \mathbb{R}^{2 n}\right): X^{\top} J+J X=0\right\} \\
& =\{X: J X \text { is symmetric }\}=: \mathfrak{s p}(n, \mathbb{R}) .
\end{aligned}
$$

We see that $\operatorname{dim} \mathfrak{s p}(n, \mathbb{R})=\frac{2 n(2 n+1)}{2}=\left(\begin{array}{c}2 n+1 \\ 2\end{array}\right)$. Furthermore ker $d f(A)=$ $\left\{X: X^{\top} J A+A^{\top} J X=0\right\}$ and the mapping $X \mapsto A^{\top} J X$ is an isomorphism ker $d f(A) \rightarrow L_{\text {sym }}\left(\mathbb{R}^{2 n}, \mathbb{R}^{2 n}\right)$ if $A$ is invertible. Thus dim ker $d f(A)=\left(\begin{array}{c}2 n+1 \\ 2\end{array}\right)$ for all $A \in G L(2 n, \mathbb{R})$. If $f(A)=J$, then $A^{\top} J A=J$, so $A$ has rank $2 n$ and is invertible, and we have $\operatorname{dim} \operatorname{ker} d f(A)+\operatorname{dim} \mathfrak{o}(2 n, \mathbb{R})=\left(\begin{array}{c}2 n+1 \\ 2\end{array}\right)+\frac{2 n(2 n-1)}{2}=$ $4 n^{2}=\operatorname{dim} L\left(\mathbb{R}^{2 n}, \mathbb{R}^{2 n}\right)$. So $f: G L(2 n, \mathbb{R}) \rightarrow \mathfrak{o}(2 n, \mathbb{R})$ is a submersion and $f^{-1}(J)=S p(n, \mathbb{R})$ is a manifold and a Lie group. It is the symmetry group of 'classical mechanics'.

4.8. Example. The complex general linear group $G L(n, \mathbb{C})$ of all invertible complex $n \times n$-matrices is open in $L_{\mathbb{C}}\left(\mathbb{C}^{n}, \mathbb{C}^{n}\right)$, so it is a real Lie group of real dimension $2 n^{2}$; it is also a complex Lie group of complex dimension $n^{2}$. The complex special linear group $S L(n, \mathbb{C})$ of all matrices of determinant 1 is a submanifold of $G L(n, \mathbb{C}$ ) of complex codimension 1 (or real codimension $2)$.

The complex orthogonal group $O(n, \mathbb{C})$ is the set

$$
\left\{A \in L\left(\mathbb{C}^{n}, \mathbb{C}^{n}\right): g(A z, A w)=g(z, w) \text { for all } z, w\right\},
$$

where $g(z, w)=\sum_{i=1}^{n} z^{i} w^{i}$. This is a complex Lie group of complex dimension $\frac{(n-1) n}{2}$, and it is not compact. Since $O(n, \mathbb{C})=\left\{A: A^{\top} A=\mathbb{I}_{n}\right\}$, we have $1=\operatorname{det}_{\mathbb{C}}\left(\mathbb{I}_{n}\right)=\operatorname{det}_{\mathbb{C}}\left(A^{\top} A\right)=\operatorname{det}_{\mathbb{C}}(A)^{2}$, so $\operatorname{det}_{\mathbb{C}}(A)= \pm 1$. Thus $S O(n, \mathbb{C}):=\left\{A \in O(n, \mathbb{C}): \operatorname{det}_{\mathbb{C}}(A)=1\right\}$ is an open subgroup of index 2 in $O(n, \mathbb{C})$.

The group $S p(n, \mathbb{C})=\left\{A \in L_{\mathbb{C}}\left(\mathbb{C}^{2 n}, \mathbb{C}^{2 n}\right): A^{\top} J A=J\right\}$ is also a complex Lie group of complex dimension $n(2 n+1)$.

The groups treated here are the classical complex Lie groups. The groups $S L(n, \mathbb{C})$ for $n \geq 2, S O(n, \mathbb{C})$ for $n \geq 3, S p(n, \mathbb{C})$ for $n \geq 4$, and five more exceptional groups exhaust all simple complex Lie groups up to coverings.

4.9. Example. Let $\mathbb{C}^{n}$ be equipped with the standard Hermitian inner product $(z, w)=\sum_{i=1}^{n} \bar{z}^{i} w^{i}$. The unitary group $U(n)$ consists of all complex $n \times n$-matrices $A$ such that $(A z, A w)=(z, w)$ for all $z, w$ holds, or equivalently $U(n)=\left\{A: A^{*} A=\mathbb{I}_{n}\right\}$, where $A^{*}=\bar{A}^{\top}$. 
We consider the mapping $f: L_{\mathbb{C}}\left(\mathbb{C}^{n}, \mathbb{C}^{n}\right) \rightarrow L_{\mathbb{C}}\left(\mathbb{C}^{n}, \mathbb{C}^{n}\right)$, given by $f(A)=$ $A^{*} A$. Then $f$ is smooth but not holomorphic. Its derivative is $d f(A) X=$ $X^{*} A+A^{*} X$, so $\operatorname{ker} d f\left(\mathbb{I}_{n}\right)=\left\{X: X^{*}+X=0\right\}=: \mathfrak{u}(n)$, the space of all skew-Hermitian matrices. We have $\operatorname{dim}_{\mathbb{R}} \mathfrak{u}(n)=n^{2}$. As above we may check that $f: G L(n, \mathbb{C}) \rightarrow L_{\text {herm }}\left(\mathbb{C}^{n}, \mathbb{C}^{n}\right)$ is a submersion, so $U(n)=f^{-1}\left(\mathbb{I}_{n}\right)$ is a compact real Lie group of dimension $n^{2}$.

The special unitary group is $S U(n)=U(n) \cap S L(n, \mathbb{C})$. For $A \in U(n)$ we have $\left|\operatorname{det}_{\mathbb{C}}(A)\right|=1$; thus $\operatorname{dim}_{\mathbb{R}} S U(n)=n^{2}-1$.

4.10. Example. The group $S p(n)$. Let $\mathbb{H}$ be the division algebra of quaternions. We will use the following description of quaternions: Let $\left(\mathbb{R}^{3},\langle\rangle,, \Delta\right)$ be the oriented Euclidean space of dimension 3 , where $\Delta$ is a determinant function with value 1 on a positive oriented orthonormal basis. The vector product on $R^{3}$ is then given by $\langle X \times Y, Z\rangle=\Delta(X, Y, Z)$. Now we let $\mathbb{H}:=\mathbb{R}^{3} \times \mathbb{R}$, equipped with the following product:

$$
(X, s)(Y, t):=(X \times Y+s Y+t X, s t-\langle X, Y\rangle) .
$$

Now we take a positively oriented orthonormal basis of $\mathbb{R}^{3}$, call it $(i, j, k)$, and identify $(0,1)$ with 1 . Then the last formula implies visibly the usual product rules for the basis $(1, i, j, k)$ of the quaternions.

The group $S p(1):=S^{3} \subset \mathbb{H} \cong \mathbb{R}^{4}$ is then the group of unit quaternions, obviously a Lie group.

Now let $V$ be a right vector space over $\mathbb{H}$. Since $\mathbb{H}$ is not commutative, we have to distinguish between left and right vector spaces and we choose right ones as basic, so that matrices can multiply from the left. By choosing a basis, we get $V=\mathbb{R}^{n} \otimes_{\mathbb{R}} \mathbb{H}=\mathbb{H}^{n}$. For $u=\left(u^{i}\right), v=\left(v^{i}\right) \in \mathbb{H}^{n}$ we put $\langle u, v\rangle:=\sum_{i=1}^{n} \bar{u}^{i} v^{i}$. Then $\langle\quad, \quad\rangle$ is $\mathbb{R}$-bilinear and $\langle u a, v b\rangle=\bar{a}\langle u, v\rangle b$ for $a, b \in \mathbb{H}$.

An $\mathbb{R}$ linear mapping $A: V \rightarrow V$ is called $\mathbb{H}$-linear or quaternionically linear if $A(u a)=A(u) a$ holds. The space of all such mappings shall be denoted by $L_{\mathbb{H}}(V, V)$. It is real isomorphic to the space of all quaternionic $n \times n$ matrices with the usual multiplication, since for the standard basis $\left(e_{i}\right)_{i=1}^{n}$ in $V=\mathbb{H}^{n}$ we have $A(u)=A\left(\sum_{i} e_{i} u^{i}\right)=\sum_{i} A\left(e_{i}\right) u^{i}=\sum_{i, j} e_{j} A_{i}^{j} u^{i}$. If $V$ is a right quaternionic vector space, then $L_{\mathbb{H}}(V, V)$ is only a real vector space - any further structure must come from a second (left) quaternionic vector space structure on $V$.

The group $G L(n, \mathbb{H})$ of invertible $\mathbb{H}$-linear mappings of $\mathbb{H}^{n}$, is a Lie group, because it is $G L(4 n, \mathbb{R}) \cap L_{\mathbb{H}}\left(\mathbb{H}^{n}, \mathbb{H}^{n}\right)$ which is open in $L_{\mathbb{H}}\left(\mathbb{H}^{n}, \mathbb{H}^{n}\right)$.

A quaternionically linear mapping $A$ is called isometric or quaternionically unitary if $\langle A(u), A(v)\rangle=\langle u, v\rangle$ for all $u, v \in \mathbb{H}^{n}$. We denote by $S p(n)$ the group of all quaternionic isometries of $\mathbb{H}^{n}$, the quaternionic unitary group. 
The reason for its name is that $S p(n)=S p(n, \mathbb{C}) \cap U(2 n)$, since we can decompose the quaternionic Hermitian form $\langle\quad, \quad\rangle$ into a complex Hermitian one and a complex symplectic one. Also we have $S p(n) \subset O(4 n, \mathbb{R})$, since the real part of $\langle$,$\rangle is a positive definite real inner product. For$ $A \in L_{\mathbb{H}}\left(\mathbb{H}^{n}, \mathbb{H}^{n}\right)$ we put $A^{*}:=\bar{A}^{\top}$. Then we have $\langle u, A(v)\rangle=\left\langle A^{*}(u), v\right\rangle$, so $\langle A(u), A(v)\rangle=\left\langle A^{*} A(u), v\right\rangle$. Thus $A \in S p(n)$ if and only if $A^{*} A=I d$.

Again $f: L_{\mathbb{H}}\left(\mathbb{H}^{n}, \mathbb{H}^{n}\right) \rightarrow L_{\mathbb{H}, \text { herm }}\left(\mathbb{H}^{n}, \mathbb{H}^{n}\right)=\left\{A: A^{*}=A\right\}$, given by $f(A)=A^{*} A$, is a smooth mapping with $d f(A) X=X^{*} A+A^{*} X$. So we have ker $d f(I d)=\left\{X: X^{*}=-X\right\}=: \mathfrak{s p}(n)$, the space of quaternionic skew-Hermitian matrices. The usual proof shows that $f$ has maximal rank on $G L(n, \mathbb{H})$, so $S p(n)=f^{-1}(I d)$ is a compact real Lie group of dimension $2 n(n-1)+3 n$.

The groups $S O(n, \mathbb{R})$ for $n \geq 3, S U(n)$ for $n \geq 2, S p(n)$ for $n \geq 2$ and the real forms of the five exceptional complex Lie groups exhaust all simple compact Lie groups up to coverings.

4.11. Invariant vector fields and Lie algebras. Let $G$ be a (real) Lie group. A vector field $\xi$ on $G$ is called left invariant if $\mu_{a}^{*} \xi=\xi$ for all $a \in G$, where $\mu_{a}^{*} \xi=T\left(\mu_{a^{-1}}\right) \circ \xi \circ \mu_{a}$ as in section (3). Since by (3.11) we have $\mu_{a}^{*}[\xi, \eta]=\left[\mu_{a}^{*} \xi, \mu_{a}^{*} \eta\right]$, the space $\mathfrak{X}_{L}(G)$ of all left invariant vector fields on $G$ is closed under the Lie bracket, so it is a Lie subalgebra of $\mathfrak{X}(G)$. Any left invariant vector field $\xi$ is uniquely determined by $\xi(e) \in T_{e} G$, since $\xi(a)=T_{e}\left(\mu_{a}\right) \cdot \xi(e)$. Thus the Lie algebra $\mathfrak{X}_{L}(G)$ of left invariant vector fields is linearly isomorphic to $T_{e} G$, and on $T_{e} G$ the Lie bracket on $\mathfrak{X}_{L}(G)$ induces a Lie algebra structure, whose bracket is again denoted by [ , ]. This Lie algebra will be denoted as usual by $\mathfrak{g}$, sometimes by $\operatorname{Lie}(G)$.

We will also give a name to the isomorphism with the space of left invariant vector fields: $L: \mathfrak{g} \rightarrow \mathfrak{X}_{L}(G), X \mapsto L_{X}$, where $L_{X}(a)=T_{e} \mu_{a} . X$. Thus $[X, Y]=\left[L_{X}, L_{Y}\right](e)$.

A vector field $\eta$ on $G$ is called right invariant if $\left(\mu^{a}\right)^{*} \eta=\eta$ for all $a \in G$. If $\xi$ is left invariant, then $\nu^{*} \xi$ is right invariant, since $\nu \circ \mu^{a}=\mu_{a^{-1}} \circ \nu$ implies that $\left(\mu^{a}\right)^{*} \nu^{*} \xi=\left(\nu \circ \mu^{a}\right)^{*} \xi=\left(\mu_{a^{-1}} \circ \nu\right)^{*} \xi=\nu^{*}\left(\mu_{a^{-1}}\right)^{*} \xi=\nu^{*} \xi$. The right invariant vector fields form a Lie subalgebra $\mathfrak{X}_{R}(G)$ of $\mathfrak{X}(G)$, which is again linearly isomorphic to $T_{e} G$ and induces also a Lie algebra structure on $T_{e} G$. Since $\nu^{*}: \mathfrak{X}_{L}(G) \rightarrow \mathfrak{X}_{R}(G)$ is an isomorphism of Lie algebras by (3.11). $T_{e} \nu=-I d: T_{e} G \rightarrow T_{e} G$ is an isomorphism between the two Lie algebra structures. We will denote by $R: \mathfrak{g}=T_{e} G \rightarrow \mathfrak{X}_{R}(G)$ the isomorphism discussed, which is given by $R_{X}(a)=T_{e}\left(\mu^{a}\right) . X$.

4.12. Lemma. If $L_{X}$ is a left invariant vector field and $R_{Y}$ is a right invariant one, then $\left[L_{X}, R_{Y}\right]=0$. Thus the flows of $L_{X}$ and $R_{Y}$ commute. 
Proof. We consider the vector field $0 \times L_{X} \in \mathfrak{X}(G \times G)$, given by $(0 \times$ $\left.L_{X}\right)(a, b)=\left(0_{a}, L_{X}(b)\right)$. Then $T_{(a, b)} \mu \cdot\left(0_{a}, L_{X}(b)\right)=T_{a} \mu^{b} \cdot 0_{a}+T_{b} \mu_{a} \cdot L_{X}(b)=$ $L_{X}(a b)$, so $0 \times L_{X}$ is $\mu$-related to $L_{X}$. Likewise $R_{Y} \times 0$ is $\mu$-related to $R_{Y}$. But then $0=\left[0 \times L_{X}, R_{Y} \times 0\right]$ is $\mu$-related to $\left[L_{X}, R_{Y}\right]$ by (3.10). Since $\mu$ is surjective, $\left[L_{X}, R_{Y}\right]=0$ follows.

4.13. Lemma. Let $\varphi: G \rightarrow H$ be a smooth homomorphism of Lie groups. Then $\varphi^{\prime}:=T_{e} \varphi: \mathfrak{g}=T_{e} G \rightarrow \mathfrak{h}=T_{e} H$ is a Lie algebra homomorphism.

Later, in (4.21), we shall see that any continuous homomorphism between Lie groups is automatically smooth.

Proof. For $X \in \mathfrak{g}$ and $x \in G$ we have

$$
\begin{aligned}
T_{x} \varphi \cdot L_{X}(x) & =T_{x} \varphi \cdot T_{e} \mu_{x} \cdot X=T_{e}\left(\varphi \circ \mu_{x}\right) \cdot X \\
& =T_{e}\left(\mu_{\varphi(x)} \circ \varphi\right) \cdot X=T_{e}\left(\mu_{\varphi(x)}\right) \cdot T_{e} \varphi \cdot X=L_{\varphi^{\prime}(X)}(\varphi(x)) .
\end{aligned}
$$

So $L_{X}$ is $\varphi$-related to $L_{\varphi^{\prime}(X)}$. By (3.10) the field $\left[L_{X}, L_{Y}\right]=L_{[X, Y]}$ is $\varphi$-related to $\left[L_{\varphi^{\prime}(X)}, L_{\varphi^{\prime}(Y)}\right]=L_{\left[\varphi^{\prime}(X), \varphi^{\prime}(Y)\right]}$. So we have $T \varphi \circ L_{[X, Y]}=$ $L_{\left[\varphi^{\prime}(X), \varphi^{\prime}(Y)\right]} \circ \varphi$. If we evaluate this at $e$, the result follows.

Now we will determine the Lie algebras of all the examples given above.

4.14. For the Lie group $G L(n, \mathbb{R})$ we have $T_{e} G L(n, \mathbb{R})=L\left(\mathbb{R}^{n}, \mathbb{R}^{n}\right)=$ : $\mathfrak{g l}(n, \mathbb{R})$ and $T G L(n, \mathbb{R})=G L(n, \mathbb{R}) \times L\left(\mathbb{R}^{n}, \mathbb{R}^{n}\right)$ by the affine structure of the surrounding vector space. For $A \in G L(n, \mathbb{R})$ we have $\mu_{A}(B)=$ $A$. $B$, so $\mu_{A}$ extends to a linear isomorphism of $L\left(\mathbb{R}^{n}, \mathbb{R}^{n}\right)$, and for $(B, X) \in$ $T G L(n, \mathbb{R})$ we get $T_{B}\left(\mu_{A}\right) \cdot(B, X)=(A . B, A . X)$. So the left invariant vector field $L_{X} \in \mathfrak{X}_{L}(G L(n, \mathbb{R}))$ is given by $L_{X}(A)=T_{e}\left(\mu_{A}\right) . X=(A, A . X)$.

Let $f: G L(n, \mathbb{R}) \rightarrow \mathbb{R}$ be the restriction of a linear functional on $L\left(\mathbb{R}^{n}, \mathbb{R}^{n}\right)$. Then we have $L_{X}(f)(A)=d f(A)\left(L_{X}(A)\right)=d f(A)(A . X)=f(A . X)$, which we may write as $L_{X}(f)=f(. X)$. Therefore

$$
\begin{aligned}
L_{[X, Y]}(f) & =\left[L_{X}, L_{Y}\right](f)=L_{X}\left(L_{Y}(f)\right)-L_{Y}\left(L_{X}(f)\right) \\
& =L_{X}(f(. Y))-L_{Y}(f(. X))=f(. X . Y)-f(\quad . Y . X) \\
& =f(.(X Y-Y X))=L_{X Y-Y X}(f) .
\end{aligned}
$$

So the Lie bracket on $\mathfrak{g l}(n, \mathbb{R})=L\left(\mathbb{R}^{n}, \mathbb{R}^{n}\right)$ is given by $[X, Y]=X Y-Y X$, the usual commutator.

4.15. Example. Let $V$ be a vector space. Then $(V,+)$ is a Lie group, $T_{0} V=V$ is its Lie algebra, $T V=V \times V$, left translation is $\mu_{v}(w)=v+w$, $T_{w}\left(\mu_{v}\right) \cdot(w, X)=(v+w, X)$. So $L_{X}(v)=(v, X)$, a constant vector field. Thus the Lie bracket is 0 . 
4.16. Example. The special linear group is $S L(n, \mathbb{R})=\operatorname{det}^{-1}(1)$ and its Lie algebra is given by $T_{e} S L(n, \mathbb{R})=\operatorname{ker} d \operatorname{det}(\mathbb{I})=\left\{X \in L\left(\mathbb{R}^{n}, \mathbb{R}^{n}\right)\right.$ : Trace $X=0\}=\mathfrak{s l}(n, \mathbb{R})$ by (4.6), The injection $i: S L(n, \mathbb{R}) \rightarrow G L(n, \mathbb{R})$ is a smooth homomorphism of Lie groups, so $T_{e} i=i^{\prime}: \mathfrak{s l}(n, \mathbb{R}) \rightarrow \mathfrak{g l}(n, \mathbb{R})$ is an injective homomorphism of Lie algebras. Thus the Lie bracket is given by $[X, Y]=X Y-Y X$.

The same argument gives the commutator as the Lie bracket in all other examples we have treated. We have already determined the Lie algebras as $T_{e} G$.

4.17. 1-parameter subgroups. Let $G$ be a Lie group with Lie algebra $\mathfrak{g}$. A 1-parameter subgroup of $G$ is a Lie group homomorphism $\alpha:(\mathbb{R},+) \rightarrow G$, i.e., a smooth curve $\alpha$ in $G$ with $\alpha(s+t)=\alpha(s) \cdot \alpha(t)$, and hence $\alpha(0)=e$.

Lemma. Let $\alpha: \mathbb{R} \rightarrow G$ be a smooth curve with $\alpha(0)=e$. Let $X \in \mathfrak{g}$. Then the following assertions are equivalent.

(1) $\alpha$ is a 1-parameter subgroup with $X=\left.\partial\right|_{0} \alpha(t)$.

(2) $\alpha(t)=\mathrm{Fl}^{L_{X}}(t, e)$ for all $t$.

(3) $\alpha(t)=\mathrm{Fl}^{R_{X}}(t, e)$ for all $t$.

(4) $x . \alpha(t)=\mathrm{Fl}^{L_{X}}(t, x)$, or $\mathrm{Fl}_{t}^{L_{X}}=\mu^{\alpha(t)}$, for all $t$.

(5) $\alpha(t) . x=\mathrm{Fl}^{R_{X}}(t, x)$, or $\mathrm{Fl}_{t}^{R_{X}}=\mu_{\alpha(t)}$, for all $t$.

Proof. (11) $\Longrightarrow$ (44) We have $\frac{d}{d t} x \cdot \alpha(t)=\left.\frac{d}{d s}\right|_{0} x \cdot \alpha(t+s)=\left.\frac{d}{d s}\right|_{0} x \cdot \alpha(t) \cdot \alpha(s)=$ $\left.\frac{d}{d s}\right|_{0} \mu_{x . \alpha(t)} \alpha(s)=\left.T_{e}\left(\mu_{x . \alpha(t)}\right) \cdot \frac{d}{d s}\right|_{0} \alpha(s)=T_{e}\left(\mu_{x . \alpha(t)}\right) \cdot X=L_{X}(x . \alpha(t))$. Вy uniqueness of solutions we get $x \cdot \alpha(t)=\mathrm{Fl}^{L_{X}}(t, x)$.

(41) $\Longrightarrow$ (2) This is clear.

(2) $\Longrightarrow$ (1) We have

$$
\begin{aligned}
\frac{d}{d s} \alpha(t) \alpha(s) & =\frac{d}{d s}\left(\mu_{\alpha(t)} \alpha(s)\right)=T\left(\mu_{\alpha(t)}\right) \frac{d}{d s} \alpha(s) \\
& =T\left(\mu_{\alpha(t)}\right) L_{X}(\alpha(s))=L_{X}(\alpha(t) \alpha(s))
\end{aligned}
$$

and $\alpha(t) \alpha(0)=\alpha(t)$. So we get $\alpha(t) \alpha(s)=\mathrm{Fl}^{L_{X}}(s, \alpha(t))=\mathrm{Fl}_{s}^{L_{X}} \mathrm{Fl}_{t}^{L_{X}}(e)=$ $\mathrm{Fl}^{L_{X}}(t+s, e)=\alpha(t+s)$.

(44) $\Longleftrightarrow$ (5) We have $\mathrm{Fl}_{t}^{\nu^{*} \xi}=\nu^{-1} \circ \mathrm{Fl}_{t}^{\xi} \circ \nu$ by (3.14), Therefore we have by $(4.11)$

$$
\begin{aligned}
\left(\mathrm{Fl}_{t}^{R_{X}}\left(x^{-1}\right)\right)^{-1} & =\left(\nu \circ \mathrm{Fl}_{t}^{R_{X}} \circ \nu\right)(x)=\mathrm{Fl}_{t}^{\nu^{*} R_{X}}(x) \\
& =\mathrm{Fl}_{-t}^{L_{X}}(x)=x \cdot \alpha(-t) .
\end{aligned}
$$

So $\mathrm{Fl}_{t}^{R_{X}}\left(x^{-1}\right)=\alpha(t) \cdot x^{-1}$, and $\mathrm{Fl}_{t}^{R_{X}}(y)=\alpha(t) \cdot y$.

(51) $\Longrightarrow$ (3) $\Longrightarrow$ (11) can be shown in a similar way. 
An immediate consequence of the foregoing lemma is that left invariant and right invariant vector fields on a Lie group are always complete, so they have global flows, because a locally defined 1-parameter group can always be extended to a globally defined one by multiplying it up: $\alpha(n t)=\alpha(t)^{n}$.

4.18. Definition. The exponential mapping $\exp : \mathfrak{g} \rightarrow G$ of a Lie group is defined by

$$
\exp X=\mathrm{Fl}^{L_{X}}(1, e)=\mathrm{Fl}^{R_{X}}(1, e)=\alpha_{X}(1),
$$

where $\alpha_{X}$ is the 1-parameter subgroup of $G$ with $\dot{\alpha}_{X}(0)=X$.

\section{Theorem.}

(1) $\exp : \mathfrak{g} \rightarrow G$ is smooth.

(2) $\exp (t X)=\mathrm{Fl}^{L_{X}}(t, e)$.

(3) $\mathrm{Fl}^{L_{X}}(t, x)=x \cdot \exp (t X)$.

(4) $\mathrm{Fl}^{R_{X}}(t, x)=\exp (t X) \cdot x$.

(5) $\exp (0)=e$ and $T_{0} \exp =I d: T_{0} \mathfrak{g}=\mathfrak{g} \rightarrow T_{e} G=\mathfrak{g}$; thus $\exp$ is a diffeomorphism from a neighborhood of 0 in $\mathfrak{g}$ onto a neighborhood of e in $G$.

Proof. (1) Let $0 \times L \in \mathfrak{X}(\mathfrak{g} \times G)$ be given by $(0 \times L)(X, x)=\left(0_{X}, L_{X}(x)\right)$. Then $\operatorname{pr}_{2} \mathrm{Fl}^{0 \times L}(t,(X, e))=\alpha_{X}(t)$ is smooth in $(t, X)$.

(2) $\exp (t X)=\mathrm{Fl}^{t \cdot L_{X}}(1, e)=\mathrm{Fl}^{L_{X}}(t, e)=\alpha_{X}(t)$.

(3) and (4) follow from lemma (4.17).

(5) $T_{0} \exp \cdot X=\left.\frac{d}{d t}\right|_{0} \exp (0+t \cdot X)=\left.\frac{d}{d t}\right|_{0} \mathrm{Fl}^{L_{X}}(t, e)=X$.

4.19. Remark. If $G$ is connected and $U \subset \mathfrak{g}$ is open with $0 \in U$, then the group generated by $\exp (U)$ equals $G$.

Namely, this group is a subgroup of $G$ containing some open neighborhood of $e$, so it is open. The complement in $G$ is also open (as union of the other cosets), so this subgroup is open and closed. Since $G$ is connected, it coincides with $G$.

If $G$ is not connected, then the subgroup generated by $\exp (U)$ is the connected component $G_{0}$ of $e$ in $G$, an open connected normal subgroup.

4.20. Remark. Let $\varphi: G \rightarrow H$ be a smooth homomorphism of Lie groups. Then the diagram

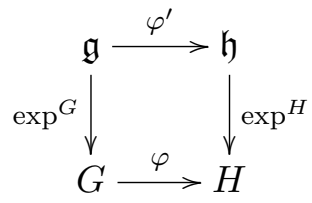


commutes, since $t \mapsto \varphi\left(\exp ^{G}(t X)\right)$ is a 1-parameter subgroup of $H$ which satisfies $\left.\frac{d}{d t}\right|_{0} \varphi\left(\exp ^{G} t X\right)=\varphi^{\prime}(X)$, so $\varphi\left(\exp ^{G} t X\right)=\exp ^{H}\left(t \varphi^{\prime}(X)\right)$.

If $G$ is connected and $\varphi, \psi: G \rightarrow H$ are homomorphisms of Lie groups with $\varphi^{\prime}=\psi^{\prime}: \mathfrak{g} \rightarrow \mathfrak{h}$, then $\varphi=\psi$. Namely, $\varphi=\psi$ on the subgroup generated by $\exp ^{G} \mathfrak{g}$ which equals $G$ by $(4.19)$

4.21. Theorem. A continuous homomorphism $\varphi: G \rightarrow H$ between Lie groups is smooth. In particular a topological group can carry at most one compatible Lie group structure.

Proof. Let first $\varphi=\alpha:(\mathbb{R},+) \rightarrow G$ be a continuous 1-parameter subgroup. Then $\alpha(-\varepsilon, \varepsilon) \subset \exp (U)$, where $U$ is an open ball with center 0 in $\mathfrak{g}$ such that $\exp \lceil 2 U$ is a diffeomorphism, for some $\varepsilon>0$. Put

$$
\beta:=\left(\exp \lceil 2 U)^{-1} \circ \alpha:(-\varepsilon, \varepsilon) \rightarrow \mathfrak{g} .\right.
$$

Then for $|t|<\frac{\varepsilon}{2}$ we have

$$
\exp (2 \beta(t))=\exp (\beta(t))^{2}=\alpha(t)^{2}=\alpha(2 t)=\exp (\beta(2 t)),
$$

so $2 \beta(t)=\beta(2 t)$; thus $\beta\left(\frac{s}{2}\right)=\frac{1}{2} \beta(s)$ for $|s|<\varepsilon$. Applying exp we have $\alpha\left(\frac{s}{2}\right)=\exp \left(\beta\left(\frac{s}{2}\right)\right)=\exp \left(\frac{1}{2} \beta(s)\right)$ for all $|s|<\varepsilon$ and by recursion we get $\alpha\left(\frac{s}{2^{n}}\right)=\exp \left(\frac{1}{2^{n}} \beta(s)\right)$ for $n \in \mathbb{N}$ and in turn

$$
\alpha\left(\frac{k}{2^{n}} s\right)=\alpha\left(\frac{s}{2^{n}}\right)^{k}=\exp \left(\frac{1}{2^{n}} \beta(s)\right)^{k}=\exp \left(\frac{k}{2^{n}} \beta(s)\right)
$$

for $k \in \mathbb{Z}$. Since the $\frac{k}{2^{n}}$ for $k \in \mathbb{Z}$ and $n \in \mathbb{N}$ are dense in $\mathbb{R}$ and since $\alpha$ is continuous, we get $\alpha(t s)=\exp (t \beta(s))$ for all $t \in \mathbb{R}$. So $\alpha$ is smooth.

Now let $\varphi: G \rightarrow H$ be a continuous homomorphism. Let $X_{1}, \ldots, X_{n}$ be a linear basis of $\mathfrak{g}$. We define $\psi: \mathbb{R}^{n} \rightarrow G$ by

$$
\psi\left(t^{1}, \ldots, t^{n}\right)=\exp \left(t^{1} X_{1}\right) \cdots \exp \left(t^{n} X_{n}\right) .
$$

Then $T_{0} \psi$ is invertible, so $\psi$ is a diffeomorphism near 0 . Sometimes $\psi^{-1}$ is called a coordinate system of the second kind. The curve $t \mapsto \varphi\left(\exp ^{G} t X_{i}\right)$ is a continuous 1-parameter subgroup of $H$, so it is smooth by the first part of the proof.

We have $(\varphi \circ \psi)\left(t^{1}, \ldots, t^{n}\right)=\left(\varphi \exp \left(t^{1} X_{1}\right)\right) \cdots\left(\varphi \exp \left(t^{n} X_{n}\right)\right)$, so $\varphi \circ \psi$ is smooth. Thus $\varphi$ is smooth near $e \in G$ and so everywhere on $G$.

4.22. Theorem. Let $G$ and $H$ be Lie groups ( $G$ separable is essential here), and let $\varphi: G \rightarrow H$ be a continuous bijective homomorphism. Then $\varphi$ is a diffeomorphism.

Proof. Our first aim is to show that $\varphi$ is a homeomorphism. Let $V$ be an open $e$-neighborhood in $G$, and let $K$ be a compact $e$-neighborhood in $G$ such that $K . K^{-1} \subset V$. Since $G$ is separable, there is a sequence $\left(a_{i}\right)_{i \in \mathbb{N}}$ in $G$ such that $G=\bigcup_{i=1}^{\infty} a_{i} . K$. Since $H$ is locally compact, it is a Baire space (i.e., $V_{i}$ 
open and dense for $i \in \mathbb{N}$ implies $\bigcap V_{i}$ dense). The set $\varphi\left(a_{i}\right) \varphi(K)$ is compact, thus closed. Since $H=\bigcup_{i} \varphi\left(a_{i}\right) \cdot \varphi(K)$, there is some $i$ such that $\varphi\left(a_{i}\right) \varphi(K)$ has nonempty interior, so $\varphi(K)$ has nonempty interior. Choose $b \in G$ such that $\varphi(b)$ is an interior point of $\varphi(K)$ in $H$. Then $e_{H}=\varphi(b) \varphi\left(b^{-1}\right)$ is an interior point of $\varphi(K) \varphi\left(K^{-1}\right) \subset \varphi(V)$. So if $U$ is open in $G$ and $a \in U$, then $e_{H}$ is an interior point of $\varphi\left(a^{-1} U\right)$, so $\varphi(a)$ is in the interior of $\varphi(U)$. Thus $\varphi(U)$ is open in $H$, and $\varphi$ is a homeomorphism.

Now by (4.21) $\varphi$ and $\varphi^{-1}$ are smooth.

4.23. Examples. We first describe the exponential mapping of the general linear group $G L(n, \mathbb{R})$. Let $X \in \mathfrak{g l}(n, \mathbb{R})=L\left(\mathbb{R}^{n}, \mathbb{R}^{n}\right)$; then the left invariant vector field is given by $L_{X}(A)=(A, A . X) \in G L(n, \mathbb{R}) \times \mathfrak{g l}(n, \mathbb{R})$ and the 1-parameter group $\alpha_{X}(t)=\mathrm{Fl}^{L_{X}}\left(t, \mathbb{I}_{n}\right)$ is given by the differential equation $\frac{d}{d t} \alpha_{X}(t)=L_{X}\left(\alpha_{X}(t)\right)=\alpha_{X}(t) . X$, with initial condition $\alpha_{X}(0)=\mathbb{I}_{n}$. But the unique solution of this equation is $\alpha_{X}(t)=e^{t X}=\sum_{k=0}^{\infty} \frac{t^{k}}{k !} X^{k}$. So

$$
\exp ^{G L(n, \mathbb{R})}(X)=e^{X}=\sum_{k=0}^{\infty} \frac{1}{k !} X^{k} .
$$

If $n=1$, we get the usual exponential mapping of one real variable. For all Lie subgroups of $G L(n, \mathbb{R})$, the exponential mapping is given by the same formula $\exp (X)=e^{X}$; this follows from (4.20).

4.24. The adjoint representation. A representation of a Lie group $G$ on a finite-dimensional vector space $V$ (real or complex) is a homomorphism $\rho: G \rightarrow G L(V)$ of Lie groups. Its derivative $\rho^{\prime}: \mathfrak{g} \rightarrow \mathfrak{g l}(V)=L(V, V)$ is a Lie algebra homomorphism by (4.13).

For $a \in G$ we define $\operatorname{conj}_{a}: G \rightarrow G$ by $\operatorname{conj}_{a}(x)=a x a^{-1}$. It is called the conjugation or the inner automorphism by $a \in G$. We have $\operatorname{conj}_{a}(x y)=$ $\operatorname{conj}_{a}(x) \operatorname{conj}_{a}(y), \operatorname{conj}_{a b}=\operatorname{conj}_{a} \circ \operatorname{conj}_{b}$, and conj is smooth in all variables. Next we define for $a \in G$ the mapping $\operatorname{Ad}(a)=\left(\operatorname{conj}_{a}\right)^{\prime}=T_{e}\left(\operatorname{conj}_{a}\right): \mathfrak{g} \rightarrow \mathfrak{g}$. By (4.13) the linear map $\operatorname{Ad}(a)$ is a Lie algebra homomorphism, so we have $\operatorname{Ad}(a)[X, Y]=[\operatorname{Ad}(a) X, \operatorname{Ad}(a) Y]$. Furthermore $\operatorname{Ad}: G \rightarrow G L(\mathfrak{g})$ is a representation, called the adjoint representation of $G$, since

$$
\begin{aligned}
\operatorname{Ad}(a b) & =T_{e}\left(\operatorname{conj}_{a b}\right)=T_{e}\left(\operatorname{conj}_{a} \circ \operatorname{conj}_{b}\right) \\
& =T_{e}\left(\operatorname{conj}_{a}\right) \circ T_{e}\left(\operatorname{conj}_{b}\right)=\operatorname{Ad}(a) \circ \operatorname{Ad}(b) .
\end{aligned}
$$

The relations $\operatorname{Ad}(a)=T_{e}\left(\operatorname{conj}_{a}\right)=T_{a}\left(\mu^{a^{-1}}\right) \cdot T_{e}\left(\mu_{a}\right)=T_{a^{-1}}\left(\mu_{a}\right) \cdot T_{e}\left(\mu^{a^{-1}}\right)$ will be used later.

Now we define the (lower case) adjoint representation of the Lie algebra $\mathfrak{g}$,

$$
\operatorname{ad}: \mathfrak{g} \rightarrow \mathfrak{g l}(\mathfrak{g})=L(\mathfrak{g}, \mathfrak{g}), \quad \text { ad }:=\mathrm{Ad}^{\prime}=T_{e} \mathrm{Ad} .
$$




\section{Lemma.}

(1) $L_{X}(a)=R_{\operatorname{Ad}(a) X}(a)$ for $X \in \mathfrak{g}$ and $a \in G$.

(2) $\operatorname{ad}(X) Y=[X, Y]$ for $X, Y \in \mathfrak{g}$.

Proof. (11) $L_{X}(a)=T_{e}\left(\mu_{a}\right) \cdot X=T_{e}\left(\mu^{a}\right) \cdot T_{e}\left(\mu^{a^{-1}} \circ \mu_{a}\right) \cdot X=R_{\operatorname{Ad}(a) X}(a)$. (21) Let $X_{1}, \ldots, X_{n}$ be a linear basis of $\mathfrak{g}$ and fix $X \in \mathfrak{g}$. Then $\operatorname{Ad}(x) X=$ $\sum_{i=1}^{n} f_{i}(x) \cdot X_{i}$ for $f_{i} \in C^{\infty}(G, \mathbb{R})$ and we have in turn:

$$
\begin{aligned}
\operatorname{Ad}^{\prime}(Y) X & =T_{e}(\operatorname{Ad}(\quad) X) Y=\left.d(\operatorname{Ad}(\quad) X)\right|_{e} Y=\left.d\left(\sum f_{i} X_{i}\right)\right|_{e} Y \\
& =\left.\sum d f_{i}\right|_{e}(Y) X_{i}=\sum L_{Y}\left(f_{i}\right)(e) \cdot X_{i} . \\
L_{X}(x) & =R_{\operatorname{Ad}(x) X}(x)=R\left(\sum f_{i}(x) X_{i}\right)(x)=\sum f_{i}(x) \cdot R_{X_{i}}(x) \text { by (11). } \\
{\left[L_{Y}, L_{X}\right] } & =\left[L_{Y}, \sum f_{i} \cdot R_{X_{i}}\right]=0+\sum L_{Y}\left(f_{i}\right) \cdot R_{X_{i}} \text { by }(3.4) \text { and }(4.12) . \\
{[Y, X] } & =\left[L_{Y}, L_{X}\right](e)=\sum L_{Y}\left(f_{i}\right)(e) \cdot R_{X_{i}}(e)=\operatorname{Ad}^{\prime}(Y) X=\operatorname{ad}(Y) X .
\end{aligned}
$$

4.25. Corollary. From (4.20) and (4.23) we have

$$
\begin{aligned}
\operatorname{Ad} \circ \exp ^{G} & =\exp ^{G L(\mathfrak{g})} \circ \mathrm{ad}, \\
\operatorname{Ad}\left(\exp ^{G} X\right) Y & =\sum_{k=0}^{\infty} \frac{1}{k !}(\operatorname{ad} X)^{k} Y=e^{\operatorname{ad} X} Y \\
& =Y+[X, Y]+\frac{1}{2 !}[X,[X, Y]]+\frac{1}{3 !}[X,[X,[X, Y]]]+\cdots
\end{aligned}
$$

so that also $\operatorname{ad}(X)=\left.\partial\right|_{0} \operatorname{Ad}(\exp (t X))$.

4.26. The right logarithmic derivative. Let $M$ be a manifold and let $f: M \rightarrow G$ be a smooth mapping into a Lie group $G$ with Lie algebra $\mathfrak{g}$. We define the mapping $\delta f: T M \rightarrow \mathfrak{g}$ by the formula $\delta f\left(\xi_{x}\right):=$ $T_{f(x)}\left(\mu^{f(x)^{-1}}\right) \cdot T_{x} f \cdot \xi_{x}$. Then $\delta f$ is a $\mathfrak{g}$-valued 1 -form on $M, \delta f \in \Omega^{1}(M, \mathfrak{g})$, as we will write later. We call $\delta f$ the right logarithmic derivative of $f$, since for $f: \mathbb{R} \rightarrow\left(\mathbb{R}^{+}, \cdot\right)$ we have $\delta f(x) .1=\frac{f^{\prime}(x)}{f(x)}=(\log \circ f)^{\prime}(x)$.

Lemma. Let $f, g: M \rightarrow G$ be smooth. Then we have

$$
\delta(f . g)(x)=\delta f(x)+\operatorname{Ad}(f(x)) \cdot \delta g(x) .
$$

Proof. We compute as follows:

$$
\begin{aligned}
\delta(f \cdot g)(x) & =T\left(\mu^{g(x)^{-1} \cdot f(x)^{-1}}\right) \cdot T_{x}(f \cdot g) \\
& =T\left(\mu^{f(x)^{-1}}\right) \cdot T\left(\mu^{g(x)^{-1}}\right) \cdot T_{(f(x), g(x))} \mu \cdot\left(T_{x} f, T_{x} g\right) \\
& =T\left(\mu^{f(x)^{-1}}\right) \cdot T\left(\mu^{g(x)^{-1}}\right) \cdot\left(T\left(\mu^{g(x)}\right) \cdot T_{x} f+T\left(\mu_{f(x)}\right) \cdot T_{x} g\right) \\
& =\delta f(x)+\operatorname{Ad}(f(x)) \cdot \delta g(x) . \quad \square
\end{aligned}
$$


Remark. The left logarithmic derivative $\delta^{\text {left }} f \in \Omega^{1}(M, \mathfrak{g})$ of a smooth mapping $f: M \rightarrow G$ is given by $\delta^{\text {left }} f \cdot \xi_{x}=T_{f(x)}\left(\mu_{f(x)^{-1}}\right) \cdot T_{x} f \cdot \xi_{x}$. The corresponding Leibniz rule for it is uglier than that for the right logarithmic derivative:

$$
\delta^{\text {left }}(f g)(x)=\delta^{\text {left }} g(x)+\operatorname{Ad}\left(g(x)^{-1}\right) \delta^{\text {left }} f(x) .
$$

The form $\delta^{\text {left }}\left(I_{G}\right) \in \Omega^{1}(G, \mathfrak{g})$ is also called the Maurer-Cartan form of the Lie group $G$.

4.27. Lemma. For $\exp : \mathfrak{g} \rightarrow G$ and for $g(z):=\frac{e^{z}-1}{z}$ we have

$$
\delta(\exp )(X)=T\left(\mu^{\exp (-X)}\right) \cdot T_{X} \exp =\sum_{p=0}^{\infty} \frac{1}{(p+1) !}(\operatorname{ad} X)^{p}=g(\operatorname{ad} X)
$$

Proof. We put $M(X)=\delta(\exp )(X): \mathfrak{g} \rightarrow \mathfrak{g}$. Then

$$
\begin{aligned}
(s+t) & M((s+t) X)=(s+t) \delta(\exp )((s+t) X) \\
& =\delta(\exp ((s+t) \quad)) X \quad \text { by the chain rule } \\
& =\delta(\exp (s \quad) \cdot \exp (t \quad)) \cdot X \\
& =\delta(\exp (s \quad)) \cdot X+\operatorname{Ad}(\exp (s X)) \cdot \delta(\exp (t \quad)) \cdot X \quad \text { by }(4.26) \\
& =s \cdot \delta(\exp )(s X)+\operatorname{Ad}(\exp (s X)) \cdot t \cdot \delta(\exp )(t X) \\
& =s \cdot M(s X)+\operatorname{Ad}(\exp (s X)) \cdot t \cdot M(t X) .
\end{aligned}
$$

Next we put

$$
\begin{aligned}
N(t): & =t \cdot M(t X) \in L(\mathfrak{g}, \mathfrak{g}) ; \\
N(s+t) & =N(s)+\operatorname{Ad}(\exp (s X)) \cdot N(t) .
\end{aligned}
$$

We fix $t$, apply $\left.\frac{d}{d s}\right|_{0}$, and get

$$
\begin{aligned}
& N^{\prime}(t)=N^{\prime}(0)+\operatorname{ad}(X) \cdot N(t), \\
& N^{\prime}(0)=M(0)+0=\delta(\exp )(0)=I d_{\mathfrak{g}} .
\end{aligned}
$$

So we have the differential equation

$$
N^{\prime}(t)=I d_{\mathfrak{g}}+\operatorname{ad}(X) \cdot N(t)
$$

in $L(\mathfrak{g}, \mathfrak{g})$ with initial condition $N(0)=0$. The unique solution is

$$
\begin{gathered}
N(s)=\sum_{p=0}^{\infty} \frac{1}{(p+1) !} \operatorname{ad}(X)^{p} \cdot s^{p+1}, \quad \text { and so } \\
\delta(\exp )(X)=M(X)=N(1)=\sum_{p=0}^{\infty} \frac{1}{(p+1) !} \operatorname{ad}(X)^{p} .
\end{gathered}
$$


4.28. Corollary. The tangent mapping $T_{X} \exp$ is bijective if and only if no eigenvalue of $\operatorname{ad}(X): \mathfrak{g} \rightarrow \mathfrak{g}$ is of the form $\sqrt{-1} 2 k \pi$ for $k \in \mathbb{Z} \backslash\{0\}$.

Proof. The zeros of $g(z)=\frac{e^{z}-1}{z}$ are $z=2 k \pi \sqrt{-1}$ for $k \in \mathbb{Z} \backslash\{0\}$. The linear mapping $T_{X}$ exp is bijective if and only if no eigenvalue of

$$
g(\operatorname{ad}(X))=T\left(\mu^{\exp (-X)}\right) \cdot T_{X} \exp
$$

is 0 . But the eigenvalues of $g(\operatorname{ad}(X))$ are the images under $g$ of the eigenvalues of $\operatorname{ad}(X)$.

\subsection{Theorem. The Baker-Campbell-Hausdorff formula.}

Let $G$ be a Lie group with Lie algebra $\mathfrak{g}$. For complex $z$ near 1 we consider the function $f(z):=\frac{\log (z)}{z-1}=\sum_{n \geq 0} \frac{(-1)^{n}}{n+1}(z-1)^{n}$.

Then for $X, Y$ near 0 in $\mathfrak{g}$ we have $\exp X$. $\exp Y=\exp C(X, Y)$, where

$$
\begin{aligned}
& C(X, Y)=Y+\int_{0}^{1} f\left(e^{t \cdot \operatorname{ad} X} \cdot e^{\operatorname{ad} Y}\right) \cdot X d t \\
& =X+Y+\sum_{n \geq 1} \frac{(-1)^{n}}{n+1} \int_{0}^{1}\left(\sum_{\substack{k, \ell \geq 0 \\
k+\ell \geq 1}} \frac{t^{k}}{k ! \ell !}(\operatorname{ad} X)^{k}(\operatorname{ad} Y)^{\ell}\right)^{n} X d t \\
& =X+Y+\sum_{n \geq 1} \frac{(-1)^{n}}{n+1} \sum_{\begin{array}{c}
k_{1}, \ldots, k_{n} \geq 0 \\
\ell_{1}, \ldots, \ell_{n} \geq 0 \\
k_{i}+\ell_{i} \geq 1
\end{array}} \frac{(\operatorname{ad} X)^{k_{1}}(\operatorname{ad} Y)^{\ell_{1}} \ldots(\operatorname{ad} X)^{k_{n}}(\operatorname{ad} Y)^{\ell_{n}}}{\left(k_{1}+\cdots+k_{n}+1\right) k_{1} ! \ldots k_{n} ! \ell_{1} ! \ldots \ell_{n} !} X \\
& =X+Y+\frac{1}{2}[X, Y]+\frac{1}{12}([X,[X, Y]]+[Y,[Y, X]])+\cdots \text {. }
\end{aligned}
$$

Proof. Let $C(X, Y):=\exp ^{-1}(\exp X \cdot \exp Y)$ for $X, Y$ near 0 in $\mathfrak{g}$, and let $C(t):=C(t X, Y)$. Then by (4.27) we have

$$
\begin{aligned}
T\left(\mu^{\exp (-C(t))}\right) \frac{d}{d t}(\exp C(t)) & =\delta(\exp \circ C)(t) \cdot 1=\delta \exp (C(t)) \cdot \dot{C}(t) \\
& =\sum_{k \geq 0} \frac{1}{(k+1) !}(\operatorname{ad} C(t))^{k} \dot{C}(t) \\
& =g(\operatorname{ad} C(t)) \cdot \dot{C}(t),
\end{aligned}
$$

where $g(z):=\frac{e^{z}-1}{z}=\sum_{k \geq 0} \frac{z^{k}}{(k+1) !}$. We have $\exp C(t)=\exp (t X) \exp Y$ and

$$
\exp (-C(t))=\exp (C(t))^{-1}=\exp (-Y) \exp (-t X) ;
$$

therefore

$$
\begin{aligned}
& T\left(\mu^{\exp (-C(t))}\right) \frac{d}{d t}(\exp C(t))=T\left(\mu^{\exp (-Y) \exp (-t X)}\right) \frac{d}{d t}(\exp (t X) \exp Y) \\
&=T\left(\mu^{\exp (-t X)}\right) T\left(\mu^{\exp (-Y)}\right) T\left(\mu^{\exp Y}\right) \frac{d}{d t} \exp (t X) \\
&=T\left(\mu^{\exp (-t X)}\right) \cdot R_{X}(\exp (t X))=X \quad \text { by }(4.18 .4) \text { and }(4.11) . \\
& X=g(\operatorname{ad} C(t)) \cdot \dot{C}(t) .
\end{aligned}
$$




$$
\begin{aligned}
e^{\operatorname{ad} C(t)} & =\operatorname{Ad}(\exp C(t)) \quad \text { by }(4.25) \\
& =\operatorname{Ad}(\exp (t X) \exp Y)=\operatorname{Ad}(\exp (t X)) \cdot \operatorname{Ad}(\exp Y) \\
& =e^{\operatorname{ad}(t X)} \cdot e^{\operatorname{ad} Y}=e^{t \cdot \operatorname{ad} X} \cdot e^{\operatorname{ad} Y} .
\end{aligned}
$$

If $X, Y$, and $t$ are small enough, we get ad $C(t)=\log \left(e^{t \cdot \operatorname{ad} X} \cdot e^{\text {ad } Y}\right)$, where $\log (z)=\sum_{n \geq 1} \frac{(-1)^{n+1}}{n}(z-1)^{n} ;$ thus we have

$$
X=g(\operatorname{ad} C(t)) \cdot \dot{C}(t)=g\left(\log \left(e^{t \cdot \operatorname{ad} X} \cdot e^{\operatorname{ad} Y}\right)\right) \cdot \dot{C}(t) .
$$

For $z$ near 1 we put $f(z):=\frac{\log (z)}{z-1}=\sum_{n \geq 0} \frac{(-1)^{n}}{n+1}(z-1)^{n}$. This function satisfies $g(\log (z)) \cdot f(z)=1$. So we have

$$
\begin{aligned}
& X=g\left(\log \left(e^{t \cdot \operatorname{ad} X} \cdot e^{\operatorname{ad} Y}\right)\right) \cdot \dot{C}(t)=f\left(e^{t \cdot \operatorname{ad} X} \cdot e^{\operatorname{ad} Y}\right)^{-1} \cdot \dot{C}(t), \\
& \left\{\begin{array}{l}
\dot{C}(t)=f\left(e^{t \cdot \operatorname{ad} X} \cdot e^{\operatorname{ad} Y}\right) \cdot X \\
C(0)=Y .
\end{array}\right.
\end{aligned}
$$

Passing to the definite integral, we get the desired formula

$$
\begin{aligned}
& C(X, Y)=C(1)=C(0)+\int_{0}^{1} \dot{C}(t) d t \\
& =Y+\int_{0}^{1} f\left(e^{t \cdot \operatorname{ad} X} \cdot e^{\operatorname{ad} Y}\right) \cdot X d t \\
& =X+Y+\sum_{n \geq 1} \frac{(-1)^{n}}{n+1} \int_{0}^{1}\left(\sum_{\substack{k, \ell \geq 0 \\
k+\ell \geq 1}} \frac{t^{k}}{k ! \ell !}(\operatorname{ad} X)^{k}(\operatorname{ad} Y)^{\ell}\right)^{n} X d t \\
& =X+Y+\sum_{n \geq 1} \frac{(-1)^{n}}{n+1} \sum_{\begin{array}{c}
k_{1}, \ldots, k_{n} \geq 0 \\
\ell_{1}, \ldots, \ell_{n} \geq 0 \\
k_{i}+\ell_{i} \geq 1
\end{array}} \frac{(\operatorname{ad} X)^{k_{1}}(\operatorname{ad} Y)^{\ell_{1}} \ldots(\operatorname{ad} X)^{k_{n}}(\operatorname{ad} Y)^{\ell_{n}}}{\left(k_{1}+\cdots+k_{n}+1\right) k_{1} ! \ldots k_{n} ! \ell_{1} ! \ldots \ell_{n} !} X \\
& =X+Y+\frac{1}{2}[X, Y]+\frac{1}{12}([X,[X, Y]]+[Y,[Y, X]])+\cdots \text {. }
\end{aligned}
$$

4.30. Remarks. (1) If $G$ is a Lie group of differentiability class $C^{2}$, then we may define $T G$ and the Lie bracket of vector fields. The proof above then makes sense and the theorem shows that in the chart given by $\exp ^{-1}$ the multiplication $\mu: G \times G \rightarrow G$ is $C^{\omega}$ near $e$, hence everywhere. So in this case $G$ is a real analytic Lie group. See also remark (5.7) below.

(2) Let $G$ be a Lie groups with Lie algebra $\mathfrak{g}$. Then Trotter's formula holds:

For $X, Y \in \mathfrak{g}$ we have, by (4.29).

$$
\begin{aligned}
\left(\exp \left(\frac{1}{n} X\right) \exp \left(\frac{1}{n}\right)\right)^{n} & =\exp \left(n \cdot C\left(\frac{1}{n} X, \frac{1}{n} Y\right)\right) \\
& =\exp \left(X+Y+\frac{1}{n} \cdot(\text { bounded })\right) \underset{n \rightarrow \infty}{\longrightarrow} \exp (X+Y) .
\end{aligned}
$$


(3) Similarly, by (4.29),

$$
\begin{aligned}
& \left(\exp \left(\frac{1}{n} X\right) \exp \left(\frac{1}{n} Y\right) \exp \left(\frac{-1}{n} X\right) \exp \left(\frac{-1}{n} Y\right)\right)^{n^{2}} \\
& =\exp \left(n^{2} C\left(C\left(\frac{1}{n} X, \frac{1}{n} Y\right), C\left(\frac{-1}{n} X, \frac{-1}{n} Y\right)\right)\right) \\
& \quad=\exp \left([X, Y]+\frac{1}{n}(\text { bounded })\right) \underset{n \rightarrow \infty}{\longrightarrow} \exp ([X, Y]) .
\end{aligned}
$$

(4) Let $P$ be a formal bracket expression of length $k$ as in (3.16). On $G$ we use $[g, h]=g h g^{-1} h^{-1}$ as commutator. We consider smooth curves $g_{i}: \mathbb{R} \rightarrow G$ with $g_{i}(0)=e$ and $g_{i}^{\prime}(0)=X_{i} \in \mathfrak{g}$. Then $\varphi_{i}(t, h)=h . g_{i}(t)=\mu^{g_{i}(t)}(h)$ are global curves of diffeomorphisms on $G$ with $\left.\partial_{t}\right|_{0} \varphi_{i}(t, h)=L_{X_{i}}(h)$. Evaluating (3.16) at $e$, we then get

$$
\begin{aligned}
0 & =\left.\frac{\partial^{\ell}}{\partial t^{\ell}}\right|_{0} P\left(g_{t}^{1}, \ldots, g_{t}^{k}\right) \quad \text { for } 1 \leq \ell<k, \\
P\left(X_{1}, \ldots, X_{k}\right) & =\left.\frac{1}{k !} \frac{\partial^{k}}{\partial t^{k}}\right|_{0} P\left(g_{t}^{1}, \ldots, g_{t}^{k}\right) \in \mathfrak{X}(M) .
\end{aligned}
$$

A special case of this is: For $X_{i} \in \mathfrak{g}$ we have

$$
\begin{aligned}
0 & =\left.\frac{\partial^{\ell}}{\partial t^{\ell}}\right|_{0} P\left(\exp \left(t . X_{1}\right), \ldots, \exp \left(t . X_{k}\right)\right) \quad \text { for } 1 \leq \ell<k, \\
P\left(X_{1}, \ldots, X_{k}\right) & =\left.\frac{1}{k !} \frac{\partial^{k}}{\partial t^{k}}\right|_{0} P\left(\exp \left(t . X_{1}\right), \ldots, \exp \left(t . X_{k}\right)\right) \in \mathfrak{g} .
\end{aligned}
$$

4.31. Example. The group $S O(3, \mathbb{R})$. From (4.5) and (4.16) we know that the Lie algebra $\mathfrak{o}(3, \mathbb{R})$ of $S O(3, \mathbb{R})$ is the space $L_{\text {skew }}\left(\mathbb{R}^{3}, \mathbb{R}^{3}\right)$ of all linear mappings which are skew-symmetric with respect to the inner product, with the commutator as Lie bracket.

The group $S p(1)=S^{3}$ of unit quaternions has as Lie algebra $T_{1} S^{3}=1^{\perp}$, the space of imaginary quaternions, with the commutator of the quaternion multiplications as bracket. From (4.10) we see that this is $[X, Y]=2 X \times Y$. Then we observe that the mapping

$$
\alpha: \mathfrak{s p}(1) \rightarrow \mathfrak{o}(3, \mathbb{R})=L_{\text {skew }}\left(\mathbb{R}^{3}, \mathbb{R}^{3}\right), \quad \alpha(X) Y=2 X \times Y,
$$

is a linear isomorphism between two 3 -dimensional vector spaces and is also an isomorphism of Lie algebras because $[\alpha(X), \alpha(Y)] Z=4(X \times(Y \times Z)-$ $Y \times(X \times Z))=4(X \times(Y \times Z)+Y \times(Z \times X))=-4(Z \times(Y \times X))=$ $2(2 X \times Y) \times Z=\alpha([X, Y]) Z$. Since $S^{3}$ is simply connected, we may conclude from (5.4) below that $S p(1)$ is the universal cover of $S O(3)$.

We can also see this directly as follows: Consider the mapping $\tau: S^{3} \subset \mathbb{H} \rightarrow$ $S O(3, \mathbb{R})$ which is given by $\tau(P) X=P X \bar{P}$, where $X \in \mathbb{R}^{3} \times\{0\} \subset \mathbb{H}$ is an imaginary quaternion. It is clearly a homomorphism $\tau: S^{3} \rightarrow G L(3, \mathbb{R})$, and since $|\tau(P) X|=|P X \bar{P}|=|X|$ and $S^{3}$ is connected, it has values in $S O(3, \mathbb{R})$. The tangent mapping of $\tau$ is computed as $\left(T_{1} \tau . X\right) Y=X Y 1+$ $1 Y(-X)=2(X \times Y)=\alpha(X) Y$, so it is an isomorphism. Thus $\tau$ is a local diffeomorphism, the image of $\tau$ is an open and compact (since $S^{3}$ is compact) 
subgroup of $S O(3, \mathbb{R})$, so $\tau$ is surjective since $S O(3, \mathbb{R})$ is connected. The kernel of $\tau$ is the set of all $P \in S^{3}$ with $P X \bar{P}=X$ for all $X \in \mathbb{R}^{3}$, i.e., the intersection of the center of $\mathbb{H}$ with $S^{3}$, the set $\{1,-1\}$. So $\tau$ is a two sheeted covering mapping.

So the universal cover of $S O(3, \mathbb{R})$ is the group $S^{3}=S p(1)=S U(2)=$ $\operatorname{Spin}(3)$. Here $\operatorname{Spin}(n)$ is just a name for the universal cover of $S O(n)$, and the isomorphism $S p(1)=S U(2)$ is just given by the fact that the quaternions can also be described as the set of all complex matrices

$$
\left(\begin{array}{cc}
a & b \\
-\bar{b} & \bar{a}
\end{array}\right) \sim a 1+b j
$$

The fundamental group $\pi_{1}(S O(3, \mathbb{R}))=\mathbb{Z}_{2}=\mathbb{Z} / 2 \mathbb{Z}$.

4.32. Example. The group $S O(4, \mathbb{R})$. We consider the smooth homomorphism $\rho: S^{3} \times S^{3} \rightarrow S O(4, \mathbb{R})$ given by $\rho(P, Q) Z:=P Z \bar{Q}$ in terms of multiplications of quaternions. The derived mapping is $\rho^{\prime}(X, Y) Z=$ $\left(T_{(1,1)} \rho .(X, Y)\right) Z=X Z 1+1 Z(-Y)=X Z-Z Y$, and its kernel consists of all pairs of imaginary quaternions $(X, Y)$ with $X Z=Z Y$ for all $Z \in \mathbb{H}$. If we put $Z=1$, we get $X=Y$; then $X$ is in the center of $\mathbb{H}$ which intersects $\mathfrak{s p}(1)$ at 0 only. So $\rho^{\prime}$ is a Lie algebra isomorphism since the dimensions are equal, and $\rho$ is a local diffeomorphism. Its image is open and closed in $S O(4, \mathbb{R})$, so $\rho$ is surjective, a covering mapping. The kernel of $\rho$ is easily seen to be $\{(1,1),(-1,-1)\} \subset S^{3} \times S^{3}$. So the universal cover of $S O(4, \mathbb{R})$ is $S^{3} \times S^{3}=S p(1) \times S p(1)=\operatorname{Spin}(4)$, and the fundamental group $\pi_{1}(S O(4, \mathbb{R}))=\mathbb{Z}_{2}$ again.

\section{Examples and Exercises}

4.33. Let $A \in L\left(\mathbb{R}^{n}, \mathbb{R}^{n}\right)$ be an $(n \times n)$-matrix. Let $C(A)$ be the matrix of the signed algebraic complements of $A$, i.e.,

$$
C(A)_{j}^{i}:=\operatorname{det}\left(\begin{array}{ccccccc}
A_{1}^{1} & \ldots & A_{i-1}^{1} & 0 & A_{i+1}^{1} & \ldots & A_{n}^{1} \\
\vdots & & \vdots & \vdots & \vdots & & \vdots \\
A_{1}^{j-1} & \ldots & A_{i-1}^{j-1} & 0 & A_{i+1}^{j-1} & \ldots & A_{n}^{j-1} \\
0 & \ldots & 0 & 1 & 0 & \ldots & 0 \\
A_{1}^{j+1} & \ldots & A_{i-1}^{j+1} & 0 & A_{i+1}^{j+1} & \ldots & A_{n}^{j+1} \\
\vdots & & \vdots & \vdots & \vdots & & \vdots \\
A_{1}^{n} & \ldots & A_{i-1}^{n} & 0 & A_{i+1}^{n} & \ldots & A_{n}^{n}
\end{array}\right) .
$$

Prove that $C(A) A=A C(A)=\operatorname{det}(A) \cdot \mathbb{I}_{n}$ (Cramer's rule)! This can be done by remembering the expansion formula for the determinant while multiplying it out. 
Prove that $d(\operatorname{det})(A) X=\operatorname{Trace}(C(A) X)$ ! There are two ways to do this. The first one is to check that the standard inner product on $L\left(\mathbb{R}^{n}, \mathbb{R}^{n}\right)$ is given by $\langle A, X\rangle=\operatorname{Trace}\left(A^{\top} X\right)$ and by computing the gradient of det at $A$. The second way uses (14.19). $\operatorname{det}\left(A+t \mathbb{I}_{n}\right)=t^{n}+t^{n-1} \operatorname{Trace}(A)+t^{n-2} c_{2}^{n}(A)+\cdots+t c_{n-1}^{n}(A)+\operatorname{det}(A)$.

Assume that $A$ is invertible. Then:

$$
\begin{aligned}
\operatorname{det}(A+t X) & =t^{n} \operatorname{det}\left(t^{-1} A+X\right)=t^{n} \operatorname{det}\left(A\left(A^{-1} X+t^{-1} \mathbb{I}_{n}\right)\right) \\
& =t^{n} \operatorname{det}(A) \operatorname{det}\left(A^{-1} X+t^{-1} \mathbb{I}_{n}\right) \\
& =t^{n} \operatorname{det}(A)\left(t^{-n}+t^{1-n} \operatorname{Trace}\left(A^{-1} X\right)+\cdots+\operatorname{det}\left(A^{-1} X\right)\right) \\
& =\operatorname{det}(A)\left(1+t \operatorname{Trace}\left(A^{-1} X\right)+O\left(t^{2}\right)\right), \\
d \operatorname{det}(A) X & =\left.\partial\right|_{0} \operatorname{det}(A+t X)=\left.\partial\right|_{0} \operatorname{det}(A)\left(1+t \operatorname{Trace}\left(A^{-1} X\right)+O\left(t^{2}\right)\right) \\
& =\operatorname{det}(A) \operatorname{Trace}\left(A^{-1} X\right)=\operatorname{Trace}\left(\operatorname{det}(A) A^{-1} X\right) \\
& =\operatorname{Trace}(C(A) X) .
\end{aligned}
$$

Since invertible matrices are dense, the formula follows by continuity. What about $\operatorname{det}_{\mathbb{C}}: L_{\mathbb{C}}\left(\mathbb{C}^{n}, \mathbb{C}^{n}\right) \rightarrow \mathbb{C}$ ?

4.34. For a matrix $A \in L\left(\mathbb{R}^{n}, \mathbb{R}^{n}\right)$ let $e^{A}:=\sum_{k \geq 0} \frac{1}{k !} A^{k}$. Prove that $e^{A}$ converges everywhere, that $\operatorname{det}\left(e^{A}\right)=e^{\operatorname{Trace}(A)}$, and thus $e^{A} \in G L(n, \mathbb{R})$ for all $A \in L\left(\mathbb{R}^{n}, \mathbb{R}^{n}\right)$.

4.35. We can insert matrices into real analytic functions in one variable:

$$
f(A):=f(0) \cdot \mathbb{I}_{n}+\sum_{k \geq 0} \frac{f^{(k)}(0)}{k !} A^{k}, \quad \text { if the norm }|A| \leq \rho,
$$

where $\rho$ is the radius of convergence of $f$ at 0 . Develop some theory about that (pay attention to constants): $(f \cdot g)(A)=f(A) \cdot g(A),(f \circ g)(A)=$ $f(g(A)), d f(A) X=f^{\prime}(A) X$ if $[A, X]=0$. What about $d f(A) X$ in the general case?

4.36. Quaternions. Let $\langle$,$\rangle denote the standard inner product on$ oriented $\mathbb{R}^{4}$. Put $1:=(0,0,0,1) \in \mathbb{R}^{4}$ and $\mathbb{R}^{3} \cong \mathbb{R}^{3} \times\{0\}=1^{\perp} \subset \mathbb{R}^{4}$. The vector product on $\mathbb{R}^{3}$ is then given by $\langle x \times y, z\rangle:=\operatorname{det}(x, y, z)$. We define a multiplication on $\mathbb{R}^{4}$ by $(X, s)(Y, t):=(X \times Y+s Y+t X$, st $-\langle X, Y\rangle)$. Prove that we get the skew-field of quaternions $\mathbb{H}$, and derive all properties: associativity, $|p \cdot q|=|p| \cdot|q|, p \cdot \bar{p}=|p|^{2} \cdot 1, p^{-1}=|p|^{-2} \cdot p, \bar{p} \cdot \bar{q}=\bar{q} \cdot \bar{p}$. How many representations of the form $x=x_{0} 1+x_{1} i+x_{2} j+x_{3} k$ can we find? Show that $\mathbb{H}$ is isomorphic to the algebra of all complex $(2 \times 2)$-matrices of the form

$$
\left(\begin{array}{cc}
u & v \\
-\bar{v} & \bar{u}
\end{array}\right), \quad u, v \in \mathbb{C}
$$


4.37. The exponential mapping for self-adjoint operators. Let $V$ be a Euclidean vector space with positive definite inner product $(\mid)$ (or a Hermitian vector space over $\mathbb{C}$ ). Let $S(V)$ be the vector space of all symmetric (or self-adjoint) linear operators on $V$. Let $S^{+}(V)$ be the open subset of all positive definite symmetric operators $A$, so that $(A v \mid v)>0$ for $v \neq 0$. Then the exponential mapping exp : $A \mapsto e^{A}=\sum_{k=0}^{\infty} \frac{1}{k !} A^{k}$ maps $S(V)$ into $S^{+}(V)$.

Lemma. The exponential mapping exp $: S(V) \rightarrow S^{+}(V)$ is a diffeomorphism.

Proof. We start with a complex Hermitian vector space $V$. Let $\mathbb{C}^{+}:=\{\lambda \in$ $\mathbb{C}: \operatorname{Re}(\lambda)>0\}$, and let $\log : \mathbb{C}^{+} \rightarrow \mathbb{C}$ be given by $\log (\lambda)=\int_{[1, \lambda]} z^{-1} d z$, where $[1, \lambda]$ denotes the line segment from 1 to $\lambda$.

Let $B \in S^{+}(V)$. Then all eigenvalues of $B$ are real and positive. We chose a (positively oriented) circle $\gamma \subset \mathbb{C}^{+}$such that all eigenvalues of $B$ are contained in the interior of $\gamma$. We consider $\lambda \mapsto \log (\lambda)\left(\lambda \operatorname{Id}_{V}-B\right)^{-1}$ as a meromorphic function in $\mathbb{C}^{+}$with values in the complex vector space $\mathbb{C} \otimes S(V)$, and we define

$$
\log (B):=\frac{1}{2 \pi \sqrt{-1}} \int_{\gamma} \log (\lambda)\left(\lambda \operatorname{Id}_{V}-B\right)^{-1} d \lambda, \quad B \in S^{+}(V) .
$$

We shall see that this does not depend on the choice of $\gamma$. We may use the same choice of the curve $\gamma$ for all $B$ in an open neighborhood in $S^{+}(V)$; thus $\log (B)$ is real analytic in $B$.

We claim that $\log =\exp ^{-1}$. If $B \in S^{+}(V)$, then $B$ has eigenvalues $\lambda_{i}>0$ with eigenvectors $v_{i}$ forming an orthonormal basis of $V$, so that $B v_{i}=\lambda_{i} v_{i}$. Thus $\left(\lambda \operatorname{Id}_{V}-B\right)^{-1} v_{i}=\frac{1}{\lambda-\lambda_{i}} v_{i}$ for $\lambda \neq \lambda_{i}$, and

$$
(\log B) v_{i}=\left(\frac{1}{2 \pi \sqrt{-1}} \int_{\gamma} \frac{\log \lambda}{\lambda-\lambda_{i}} d \lambda\right) v_{i}=\log \left(\lambda_{i}\right) v_{i}
$$

by Cauchy's integral formula. Thus $\log (B)$ does not depend on the choice of $\gamma$ and $\exp (\log (B)) v_{i}=e^{\log \left(\lambda_{i}\right)} v_{i}=\lambda_{i} v_{i}=B v_{i}$ for all $i$. Thus exp $\circ \log =$ $\operatorname{Id}_{S^{+}(V)}$. Similarly one sees that $\log \circ \exp =\operatorname{Id}_{S(V)}$.

Now let $V$ be a real Euclidean vector space. Let $V^{\mathbb{C}}=\mathbb{C} \otimes V$ be the complexified Hermitian vector space. If $B: V \rightarrow V$ is symmetric, then $j(B):=B^{\mathbb{C}}=\operatorname{Id}_{\mathbb{C}} \otimes B: V^{\mathbb{C}} \rightarrow V^{\mathbb{C}}$ is self-adjoint. Thus we have an embedding of real vector spaces $j: S(V) \rightarrow S\left(V^{\mathbb{C}}\right)$. The eigenvalues of $j(B)$ are the same as the eigenvalues of $B$; thus $j$ restricts to an embedding $j: S^{+}(V) \rightarrow S^{+}\left(V^{\mathbb{C}}\right)$. By definition the left hand diagram below commutes 
and thus also the right hand one:
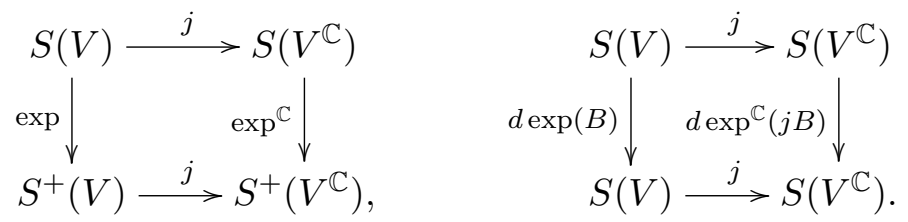

Thus $d \exp (B): S(V) \rightarrow S(V)$ is injective for each $B$, thus a linear isomorphism, and by the inverse function theorem exp $: S(V) \rightarrow S^{+}(V)$ is locally a diffeomorphism and is injective by the diagram. It is also surjective: for $B \in S^{+}(V)$ we have $B v_{i}=\lambda_{i} v_{i}$ for an orthonormal basis $v_{i}$, where $\lambda_{i}>0$. Let $A \in S(V)$ be given by $A v_{i}=\log \left(\lambda_{i}\right) v_{i}$; then $\exp (A)=B$.

4.38. Polar decomposition. Let $(V, g)$ be a Euclidean real vector space (positive definite). Then we have a real analytic diffeomorphism

$$
G L(V) \cong S^{+}(V, g) \times O(V, g) ;
$$

thus each $A \in G L(V)$ decomposes uniquely and real analytically as $A=B . U$ where $B$ is $g$-symmetric and $g$-positive definite and $U \in O(V, g)$.

Proof. The decomposition $A=B U$, if it exists, must satisfy $A A^{\top}=$ $B U U^{\top} B^{\top}=B^{2}$. By (4.37) the exponential mapping $X \mapsto e^{X}$ is a real analytic diffeomorphism exp $: S(V, g) \rightarrow S^{+}(V, g)$ from the real vector space of $g$-symmetric operators in $V$ onto the submanifold of $g$-symmetric positive definite operators in $G L(V)$, with inverse $B \mapsto \log (B)$. The operator $A A^{\top}$ is $g$-symmetric and positive definite. Thus we may put $B:=\sqrt{A A^{\top}}=$ $\exp \left(\frac{1}{2} \log \left(A A^{\top}\right)\right) \in S^{+}(V, g)$. Moreover, $B$ commutes with $A A^{\top}$. Let $U:=$ $B^{-1} A$. Then $U U^{\top}=B^{-1} A A^{\top}\left(B^{-1}\right)^{\top}=\operatorname{Id}_{V}$, so $U \in O(V, g)$.

\section{Lie Groups II. Lie Subgroups and Homogeneous Spaces}

5.1. Definition. Let $G$ be a Lie group. A subgroup $H$ of $G$ is called a Lie subgroup if $H$ is itself a Lie group (so it is separable) and the inclusion $i: H \rightarrow G$ is smooth.

In this case the inclusion is even an immersion. It suffices to check that $T_{e} i$ is injective: If $X \in \mathfrak{h}$ is in the kernel of $T_{e} i$, then $i \circ \exp ^{H}(t X)=$ $\exp ^{G}\left(t . T_{e} i . X\right)=e$. Since $i$ is injective, $X=0$.

From the next result it follows that $H \subset G$ is then an initial submanifold in the sense of (2.13) If $H_{0}$ is the connected component of $H$, then $i\left(H_{0}\right)$ is the Lie subgroup of $G$ generated by $i^{\prime}(\mathfrak{h}) \subset \mathfrak{g}$, which is an initial submanifold, and this is true for all components of $H$. 
5.2. Theorem. Let $G$ be a Lie group with Lie algebra $\mathfrak{g}$. If $\mathfrak{h} \subset \mathfrak{g}$ is a Lie subalgebra, then there is a unique connected Lie subgroup $H$ of $G$ with Lie algebra $\mathfrak{h}$. Moreover, $H$ is an initial submanifold of $G$.

Proof. Put $E_{x}:=\left\{T_{e}\left(\mu_{x}\right) \cdot X: X \in \mathfrak{h}\right\} \subset T_{x} G$. Then $E:=\bigsqcup_{x \in G} E_{x}$ is a distribution of constant rank on $G$. It is spanned by the involutive set $\left\{L_{X}, X \in \mathfrak{h}\right\}$ of vector fields. So by theorem (3.20) the distribution $E$ is integrable and the leaf $H$ through $e$ is an initial submanifold. It is even a subgroup, since for $x \in H$ the initial submanifold $\mu_{x} H$ is again a leaf (since $E$ is left invariant) and intersects $H$ at $x$, so $\mu_{x}(H)=H$. Thus $H . H=H$ and consequently $H^{-1}=H$. The multiplication $\mu: H \times H \rightarrow G$ is smooth by restriction and smooth as a mapping $H \times H \rightarrow H$, since $H$ is an initial submanifold, by lemma (2.15).

5.3. Theorem. Let $\mathfrak{g}$ be a finite-dimensional real Lie algebra. Then there exists a connected Lie group $G$ whose Lie algebra is $\mathfrak{g}$.

Sketch of Proof. By the theorem of Ado (see [96] or [224, p. 237]) $\mathfrak{g}$ has a faithful (i.e., injective) representation on a finite-dimensional vector space $V$, i.e., $\mathfrak{g}$ can be viewed as a Lie subalgebra of $\mathfrak{g l}(V)=L(V, V)$. By theorem $(5.2)$ above there is a Lie subgroup $G$ of $G L(V)$ with $\mathfrak{g}$ as its Lie algebra.

This is a rather involved proof, since the theorem of Ado needs the structure theory of Lie algebras for its proof. There are simpler proofs available, starting from a neighborhood of $e$ in $G$ (a neighborhood of 0 in $\mathfrak{g}$ with the Baker-Campbell-Hausdorff formula (4.29) as multiplication) and extending the Lie group structure.

5.4. Theorem. Let $G$ and $H$ be Lie groups with Lie algebras $\mathfrak{g}$ and $\mathfrak{h}$, respectively. Let $f: \mathfrak{g} \rightarrow \mathfrak{h}$ be a homomorphism of Lie algebras. Then there is a Lie group homomorphism $\varphi$, locally defined near e, from $G$ to $H$, such that $\varphi^{\prime}=T_{e} \varphi=f$. If $G$ is simply connected, then there is a globally defined homomorphism of Lie groups $\varphi: G \rightarrow H$ with this property.

Proof. Let $\mathfrak{k}:=\operatorname{graph}(f) \subset \mathfrak{g} \times \mathfrak{h}$. Then $\mathfrak{k}$ is a Lie subalgebra of $\mathfrak{g} \times \mathfrak{h}$, since $f$ is a homomorphism of Lie algebras. The product $\mathfrak{g} \times \mathfrak{h}$ is the Lie algebra of $G \times H$, so by theorem (5.2) there is a connected Lie subgroup $K \subset G \times H$ with algebra $\mathfrak{k}$. We consider the homomorphism $g:=\operatorname{pr}_{1} \circ$ incl $: K \rightarrow G \times H \rightarrow G$, whose tangent mapping satisfies

$$
T_{e} g(X, f(X))=T_{(e, e)} \operatorname{pr}_{1} \cdot T_{e} \text { incl. }(X, f(X))=X
$$

so it is invertible. Thus $g$ is a local diffeomorphism, so $g: K \rightarrow G_{0}$ is a covering of the connected component $G_{0}$ of $e$ in $G$. If $G$ is simply connected, $g$ is an isomorphism. Now we consider the homomorphism $\psi:=\operatorname{pr}_{2} \circ$ incl : $K \rightarrow G \times H \rightarrow H$, whose tangent mapping satisfies $T_{e} \psi \cdot(X, f(X))=f(X)$. 
We see that $\varphi:=\psi \circ\left(g\lceil U)^{-1}: G \supset U \rightarrow H\right.$ solves the problem, where $U$ is an $e$-neighborhood in $K$ such that $g \uparrow U$ is a diffeomorphism. If $G$ is simply connected, $\varphi=\psi \circ g^{-1}$ is the global solution.

5.5. Theorem. Let $H$ be a closed subgroup of a Lie group $G$. Then $H$ is a Lie subgroup and a submanifold of $G$.

Proof. Let $\mathfrak{g}$ be the Lie algebra of $G$. We consider the subset $\mathfrak{h}:=\left\{c^{\prime}(0)\right.$ : $\left.c \in C^{\infty}(\mathbb{R}, G), c(\mathbb{R}) \subset H, c(0)=e\right\}$.

Claim 1. $\mathfrak{h}$ is a linear subspace.

If $c_{i}^{\prime}(0) \in \mathfrak{h}$ and $t_{i} \in \mathbb{R}$, we define $c(t):=c_{1}\left(t_{1} \cdot t\right) \cdot c_{2}\left(t_{2} . t\right)$. Then we have $c^{\prime}(0)=T_{(e, e)} \mu \cdot\left(t_{1} \cdot c_{1}^{\prime}(0), t_{2} \cdot c_{2}^{\prime}(0)\right)=t_{1} \cdot c_{1}^{\prime}(0)+t_{2} \cdot c_{2}^{\prime}(0) \in \mathfrak{h}$.

Claim 2. $\mathfrak{h}=\{X \in \mathfrak{g}: \exp (t X) \in H$ for all $t \in \mathbb{R}\}$.

Clearly we have ' $\supseteq$ '. To check the other inclusion, let $X=c^{\prime}(0) \in \mathfrak{h}$ and consider $v(t):=\left(\exp ^{G}\right)^{-1} c(t)$ for small $t$. Then we have $X=c^{\prime}(0)=$ $\left.\frac{d}{d t}\right|_{0} \exp (v(t))=v^{\prime}(0)=\lim _{n \rightarrow \infty} n . v\left(\frac{1}{n}\right)$. We put $t_{n}:=\frac{1}{n}$ and $X_{n}:=n . v\left(\frac{1}{n}\right)$, so that $\exp \left(t_{n} \cdot X_{n}\right)=\exp \left(v\left(\frac{1}{n}\right)\right)=c\left(\frac{1}{n}\right) \in H$. By claim 3 below we then get $\exp (t X) \in H$ for all $t$.

Claim 3. Let $X_{n} \rightarrow X$ in $\mathfrak{g}, 0<t_{n} \rightarrow 0$ in $\mathbb{R}$ with $\exp \left(t_{n} X_{n}\right) \in H$. Then $\exp (t X) \in H$ for all $t \in \mathbb{R}$.

Let $t \in \mathbb{R}$ and take $m_{n} \in\left(\frac{t}{t_{n}}-1, \frac{t}{t_{n}}\right] \cap \mathbb{Z}$ for large $n$. Then $t_{n} . m_{n} \rightarrow t$ and $m_{n} . t_{n} . X_{n} \rightarrow t X$, and since $H$ is closed, we may conclude that

$$
\exp (t X)=\lim _{n} \exp \left(m_{n} \cdot t_{n} \cdot X_{n}\right)=\lim _{n} \exp \left(t_{n} \cdot X_{n}\right)^{m_{n}} \in H .
$$

Claim 4. Let $\mathfrak{k}$ be a complementary linear subspace for $\mathfrak{h}$ in $\mathfrak{g}$. Then there is an open 0-neighborhood $W$ in $\mathfrak{k}$ such that $\exp (W) \cap H=\{e\}$.

If not, there are $0 \neq Y_{k} \in \mathfrak{k}$ with $Y_{k} \rightarrow 0$ such that $\exp \left(Y_{k}\right) \in H$. Choose a norm $\mid \quad$ on $\mathfrak{g}$ and let $X_{n}=Y_{n} /\left|Y_{n}\right|$. Passing to a subsequence, we may assume that $X_{n} \rightarrow X$ in $\mathfrak{k}$; then $|X|=1$. But $\exp \left(\left|Y_{n}\right| . X_{n}\right)=\exp \left(Y_{n}\right) \in H$ and $0<\left|Y_{n}\right| \rightarrow 0$, so by claim 3 we have $\exp (t X) \in H$ for all $t \in \mathbb{R}$. By claim 2 we get $X \in \mathfrak{h}$, a contradiction.

Claim 5. Put $\varphi: \mathfrak{h} \times \mathfrak{k} \rightarrow G, \varphi(X, Y)=\exp X$. $\exp Y$. Then there are 0-neighborhoods $V$ in $\mathfrak{h}, W$ in $\mathfrak{k}$, and an $e$-neighborhood $U$ in $G$ such that $\varphi: V \times W \rightarrow U$ is a diffeomorphism and $U \cap H=\exp (V)$.

Choose $V, W$, and $U$ so small that $\varphi$ becomes a diffeomorphism. By claim 4 the set $W$ may be chosen so small that $\exp (W) \cap H=\{e\}$. By claim 2 we have $\exp (V) \subseteq H \cap U$. Let $x \in H \cap U$. Since $x \in U$, we have $x=\exp X \cdot \exp Y$ for unique $(X, Y) \in V \times W$. Then $x$ and $\exp X \in H$, so $\exp Y \in H \cap \exp (W)=\{e\}$; thus $Y=0$. So $x=\exp X \in \exp (V)$.

Claim 6. $H$ is a submanifold and a Lie subgroup.

The pair $\left(U,(\varphi \uparrow V \times W)^{-1}=: u\right)$ is a submanifold chart for $H$ centered at $e$ 
by claim 5. For $x \in H$ the pair $\left(\mu_{x}(U), u \circ \mu_{x^{-1}}\right)$ is a submanifold chart for $H$ centered at $x$. So $H$ is a closed submanifold of $G$, and the multiplication is smooth since it is a restriction.

5.6. Theorem. Let $H$ be a subgroup of a Lie group $G$ which is $C^{\infty}$-pathwise connected (see (2.13)). Then $H$ is a connected Lie group and an initial Lie subgroup of $G$.

Proof. Let us call any smooth curve $c: \mathbb{R} \rightarrow G$ with $c(0)=e$ and $c(\mathbb{R}) \subseteq H$ an $H$-curve in $G$. As in the proof of $(5.5)$ let $\mathfrak{h}$ be the set of $c^{\prime}(0)$ for all $H$-curves $c$ in $G$. Claim 1 in the proof of (5.5) shows that $\mathfrak{h}$ is a linear subspace of $\mathfrak{g}$. For $H$-curves $c_{i}$ in $G$ we use $(4.30 .3)$ to see that $\left[c_{1}^{\prime}(0), c_{2}^{\prime}(0)\right]=$ $\left.\frac{1}{2} \partial_{t}^{2}\right|_{0} g_{1}(t) g_{2}(t) g_{1}(t)^{-1} g_{2}(t)^{-1}$ is again in $\mathfrak{h}$; so $\mathfrak{h}$ is a Lie subalgebra of $\mathfrak{g}$.

Let $H_{1}$ be the connected initial Lie subgroup of $G$ corresponding to $\mathfrak{h}$ which is the leaf through $e$ of the foliation given by the left invariant distribution of constant rank generated by $\mathfrak{h}$; see $(5.2)$. For any $H$-curve $c$ in $G$ we

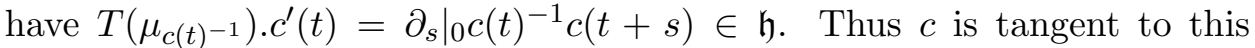
distribution and thus lies in the leaf $H_{1}$ through $e$. By assumption, any point in $H$ is connected to $e$ with such a curve. Thus $H \subseteq H_{1}$.

To prove that $H_{1} \subseteq H$, we choose a basis $X_{1}, \ldots, X_{k}$ of $\mathfrak{h}$ and $H$-curves $c_{i}$ in $G$ with $c_{i}^{\prime}(0)=X_{i}$. We consider the mapping $f: \mathbb{R}^{k} \rightarrow H$ and $H_{1}$ which is given by $f\left(t_{1}, \ldots, t_{k}\right):=c_{1}\left(t_{1}\right) \ldots c_{k}\left(t_{k}\right)$. Since $T_{0} f$ is invertible $\mathbb{R}^{k} \rightarrow \mathfrak{h}$, the mapping $f$ is a local diffeomorphism near 0 onto an open $e$ neighborhood in $H_{1}$. This shows that an open $e$-neighborhood of $H_{1}$ is in $H$; thus $H_{1} \subset H$.

5.7. Remarks. The following stronger results on subgroups and the relation between topological groups and Lie groups in general are available.

Any $C^{0}$-pathwise connected subgroup of a Lie group is a connected Lie subgroup, 231. Theorem (5.6) is a weaker version of this, fitting the spirit of (2.13). The proof of (5.6) works also for $C^{1}$-pathwise connected subgroups, without any changes.

Let $G$ be a separable locally compact topological group. If it has an $e$ neighborhood which does not contain a proper subgroup, then $G$ is a Lie group. This is the solution of the 5-th problem of Hilbert; see [163, p. 107]. Any subgroup $H$ of a Lie group $G$ has a coarsest Lie group structure, but it might be nonseparable. To indicate a proof of this statement, consider all continuous curves $c: \mathbb{R} \rightarrow G$ with $c(\mathbb{R}) \subset H$, and equip $H$ with the final topology with respect to them. Then apply the Yamabe theorem cited above to the component of the identity. Or consider all smooth $H$-curves in $G$ (as in the proof of (5.6) and put the final topology with respect to these on $H$, and apply (5.6) to the connected component. 
5.8. Let $\mathfrak{g}$ be a Lie algebra. An ideal $\mathfrak{k}$ in $\mathfrak{g}$ is a linear subspace $\mathfrak{k}$ such that $[\mathfrak{k}, \mathfrak{g}] \subset \mathfrak{k}$. Then the quotient space $\mathfrak{g} / \mathfrak{k}$ carries a unique Lie algebra structure such that $\mathfrak{g} \rightarrow \mathfrak{g} / \mathfrak{k}$ is a Lie algebra homomorphism.

Lemma. A connected Lie subgroup $H$ of a connected Lie group $G$ is a normal subgroup if and only if its Lie algebra $\mathfrak{h}$ is an ideal in $\mathfrak{g}$.

Proof. $H$ normal in $G$ means $x H x^{-1}=\operatorname{conj}_{x}(H) \subset H$ for all $x \in G$. By remark (4.20) this is equivalent to $T_{e}\left(\operatorname{conj}_{x}\right)(\mathfrak{h}) \subset \mathfrak{h}$, i.e., $\operatorname{Ad}(x) \mathfrak{h} \subset \mathfrak{h}$, for all $x \in G$. But this in turn is equivalent to $\operatorname{ad}(X) \mathfrak{h} \subset \mathfrak{h}$ for all $X \in \mathfrak{g}$, so to the fact that $\mathfrak{h}$ is an ideal in $\mathfrak{g}$.

5.9. Let $G$ be a connected Lie group. If $A \subset G$ is an arbitrary subset, the centralizer of $A$ in $G$ is the closed subgroup $Z_{G}(A):=\{x \in G: x a=$ $a x$ for all $a \in A$ \}, which by (5.5) is a Lie subgroup.

The Lie algebra $\mathfrak{z}_{\mathfrak{g}}(A)$ of $Z_{G}(A)$ consists of all $X \in \mathfrak{g}$ with $a \cdot \exp (t X) \cdot a^{-1}=$ $\exp (t X)$ for all $a \in A$, i.e., $\mathfrak{z}_{\mathfrak{g}}(A)=\{X \in \mathfrak{g}: \operatorname{Ad}(a) X=X$ for all $a \in A\}$.

If $A$ is itself a connected Lie subgroup of $G$ with Lie algebra $\mathfrak{a}$, then $\mathfrak{z}_{\mathfrak{g}}(A)=$ $\{X \in \mathfrak{g}: \operatorname{ad}(Y) X=0$ for all $Y \in \mathfrak{a}\}$. This set is also called the centralizer of $\mathfrak{a}$ in $\mathfrak{g}$. If $A=G$ is connected, then $Z_{G}=Z_{G}(G)$ is called the center of $G$ and $\mathfrak{z}_{\mathfrak{g}}(G)=\mathfrak{z}_{\mathfrak{g}}=\{X \in \mathfrak{g}:[X, Y]=0$ for all $Y \in \mathfrak{g}\}$ is then the center of the Lie algebra $\mathfrak{g}$.

5.10. The normalizer of a subset $A$ of a connected Lie group $G$ is the subgroup

$$
N_{G}(A)=\left\{x \in G: \mu_{x}(A)=\mu^{x}(A)\right\}=\left\{x \in G: \operatorname{conj}_{x}(A)=A\right\} .
$$

If $A$ is closed, then $N_{G}(A)$ is also closed.

If $A$ is a connected Lie subgroup of $G$, then $N_{G}(A)=\{x \in G: \operatorname{Ad}(x) \mathfrak{a} \subset \mathfrak{a}\}$. Its Lie algebra

$$
\mathfrak{n}_{G}(A)=\{X \in \mathfrak{g}: \operatorname{ad}(X) \mathfrak{a} \subset \mathfrak{a}\}=\mathfrak{n}_{\mathfrak{g}}(\mathfrak{a})
$$

is then the normalizer or idealizer of $\mathfrak{a}$ in $\mathfrak{g}$.

5.11. Homogeneous spaces. Let $G$ be a Lie group and let $H \subset G$ be a closed subgroup. By theorem (5.5), $H$ is a Lie subgroup of $G$. We denote by $G / H$ the space of all right cosets of $G$, i.e., $G / H=\{g H: g \in G\}$. Let $p: G \rightarrow G / H$ be the projection. We equip $G / H$ with the quotient topology, i.e., $U \subset G / H$ is open if and only if $p^{-1}(U)$ is open in $G$. Since $H$ is closed, $G / H$ is a Hausdorff space.

The quotient space $G / H$ is called a homogeneous space of $G$. We have a left action of $G$ on $G / H$, which is induced by the left translation and is given by $\bar{\mu}_{g}\left(g_{1} H\right)=g g_{1} H$. 
Theorem. If $H$ is a closed subgroup of $G$, then there exists a unique structure of a smooth manifold on $G / H$ such that $p: G \rightarrow G / H$ is a submersion. Thus $\operatorname{dim} G / H=\operatorname{dim} G-\operatorname{dim} H$.

Proof. Surjective submersions have the universal property (2.4) thus the manifold structure on $G / H$ is unique, if it exists. Let $\mathfrak{h}$ be the Lie algebra of the Lie subgroup $H$. We choose a complementary linear subspace $\mathfrak{k}$ such that $\mathfrak{g}=\mathfrak{h} \oplus \mathfrak{k}$.

Claim 1. We consider the mapping $f: \mathfrak{k} \times H \rightarrow G$, given by $f(X, h):=$ $\exp X . h$. Then there is an open 0-neighborhood $W$ in $\mathfrak{k}$ and an open $e$-neighborhood $U$ in $G$ such that $f: W \times H \rightarrow U$ is a diffeomorphism.

By claim 5 in the proof of theorem (5.5) there are open 0-neighborhoods $V$ in $\mathfrak{h}, W^{\prime}$ in $\mathfrak{k}$, and an open $e$-neighborhood $U^{\prime}$ in $G$ such that $\varphi: W^{\prime} \times V \rightarrow U^{\prime}$ is a diffeomorphism, where $\varphi(X, Y)=\exp X$. $\exp Y$, and such that $U^{\prime} \cap H=$ $\exp V$. Now we choose $W$ in $W^{\prime} \subset \mathfrak{k}$ so small that $\exp (W)^{-1} \cdot \exp (W) \subset U^{\prime}$. We will check that this $W$ satisfies claim 1 .

Claim 2. $f \uparrow W \times H$ is injective.

The equality $f\left(X_{1}, h_{1}\right)=f\left(X_{2}, h_{2}\right)$ means $\exp X_{1} \cdot h_{1}=\exp X_{2} \cdot h_{2}$; thus $h_{2} h_{1}^{-1}=\left(\exp X_{2}\right)^{-1} \exp X_{1} \in \exp (W)^{-1} \exp (W) \cap H \subset U^{\prime} \cap H=\exp V$. So there is a unique $Y \in V$ with $h_{2} h_{1}^{-1}=\exp Y$. But then $\varphi\left(X_{1}, 0\right)=\exp X_{1}=$ $\exp X_{2} \cdot h_{2} \cdot h_{1}^{-1}=\exp X_{2} \cdot \exp Y=\varphi\left(X_{2}, Y\right)$. Since $\varphi$ is injective, $X_{1}=X_{2}$ and $Y=0$, so $h_{1}=h_{2}$.

Claim 3. $f \uparrow W \times H$ is a local diffeomorphism.

The diagram

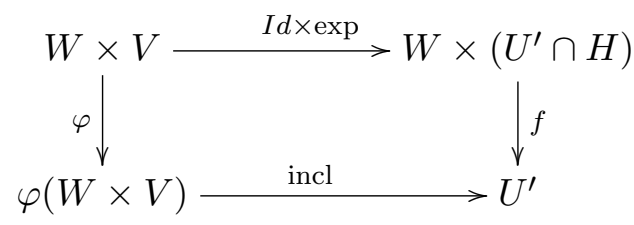

commutes, and $I d_{W} \times \exp$ and $\varphi$ are diffeomorphisms. So $f \uparrow W \times\left(U^{\prime} \cap H\right)$ is a diffeomorphism. Since $f(X, h)=f(X, e) . h$, we conclude that $f \uparrow W \times H$ is everywhere a local diffeomorphism. So finally claim 1 follows, where $U=f(W \times H)$.

Now we put $g:=p \circ(\exp \uparrow W): \mathfrak{k} \supset W \rightarrow G / H$. Then the following diagram commutes:

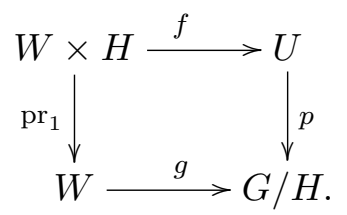

Claim 4. $g$ is a homeomorphism onto $p(U)=: \bar{U} \subset G / H$.

Clearly $g$ is continuous, and $g$ is open, since $p$ is open. If $g\left(X_{1}\right)=g\left(X_{2}\right)$, 
then $\exp X_{1}=\exp X_{2} . h$ for some $h \in H$, so $f\left(X_{1}, e\right)=f\left(X_{2}, h\right)$. By claim 1 we get $X_{1}=X_{2}$, so $\mathrm{g}$ is injective. Finally $g(W)=\bar{U}$, so claim 4 follows.

For $a \in G$ we consider $\bar{U}_{a}=\bar{\mu}_{a}(\bar{U})=a \cdot \bar{U}$ and the mapping

$$
u_{a}:=g^{-1} \circ \bar{\mu}_{a^{-1}}: \bar{U}_{a} \rightarrow W \subset \mathfrak{k} .
$$

Claim 5. $\left(\bar{U}_{a}, u_{a}=g^{-1} \circ \bar{\mu}_{a^{-1}}: \bar{U}_{a} \rightarrow W\right)_{a \in G}$ is a smooth atlas for $G / H$. Let $a, b \in G$ such that $\bar{U}_{a} \cap \bar{U}_{b} \neq \emptyset$. Then

$$
\begin{aligned}
u_{a} \circ u_{b}^{-1} & =g^{-1} \circ \bar{\mu}_{a^{-1}} \circ \bar{\mu}_{b} \circ g: u_{b}\left(\bar{U}_{a} \cap \bar{U}_{b}\right) \rightarrow u_{a}\left(\bar{U}_{a} \cap \bar{U}_{b}\right) \\
& =g^{-1} \circ \bar{\mu}_{a^{-1} b} \circ p \circ(\exp \uparrow W) \\
& =g^{-1} \circ p \circ \mu_{a^{-1} b} \circ(\exp \uparrow W) \\
& =\operatorname{pr}_{1} \circ f^{-1} \circ \mu_{a^{-1} b} \circ(\exp \lceil W) \text { is smooth. } \square
\end{aligned}
$$

\section{Transformation Groups and $G$-Manifolds}

6.1. Group actions. A left action of a Lie group $G$ on a manifold $M$ is a smooth mapping $\ell: G \times M \rightarrow M$ such that $\ell_{g} \circ \ell_{h}=\ell_{g h}$ and $\ell_{e}=I d_{M}$, where $\ell_{g}(z)=\ell(g, z)$.

A right action of a Lie group $G$ on a manifold $M$ is a smooth mapping $r: M \times G \rightarrow M$ such that $r^{g} \circ r^{h}=r^{h g}$ and $r^{e}=I d_{M}$, where $r^{g}(z)=r(z, g)$.

A $G$-space or a $G$-manifold is a manifold $M$ together with a right or left action of $G$ on $M$.

We will describe the following notions only for a left action of $G$ on $M$. They make sense also for right actions.

The orbit through $z \in M$ is the set $G . z=\ell(G, z) \subset M$.

The action is called:

- Transitive if $M$ is one orbit, i.e., for all $z, w \in M$ there is some $g \in G$ with $g . z=w$.

- Free if $g_{1} . z=g_{2} . z$ for some $z \in M$ implies already $g_{1}=g_{2}$.

- Effective if $\ell_{g}=\ell_{h}$ implies $g=h$, i.e., if $\ell: G \rightarrow \operatorname{Diff}(M)$ is injective where $\operatorname{Diff}(M)$ denotes the group of all diffeomorphisms of $M$.

- Infinitesimally free if $T_{e}\left(\ell^{x}\right): \mathfrak{g} \rightarrow T_{x} M$ is injective for each $x \in M$.

- Infinitesimally transitive if $T_{e}\left(\ell^{x}\right): \mathfrak{g} \rightarrow T_{x} M$ is surjective for each $x \in M$.

- Linear if $M$ is a vector space and the action defines a representation.

- Affine if $M$ is an affine space, and every $\ell_{g}: M \rightarrow M$ is an affine map.

- Orthogonal if $(M, \gamma)$ is a Euclidean vector space and $\ell_{g} \in O(M, \gamma)$ for all $g \in G$. 
- Isometric if $(M, \gamma)$ is a Riemann manifold and $\ell_{g}$ is an isometry for all $g \in G$; see section $(22)$.

- Symplectic if $(M, \omega)$ is a symplectic manifold and $\ell_{g}$ is a symplectomorphism for all $g \in G$; see section (31).

- Principal fiber bundle action if it is free and if the projection onto the orbit space $\pi: M \rightarrow M / G$ is a principal fiber bundle; see section (17).

More generally, a continuous transformation group of a topological space $M$ is a pair $(G, M)$ where $G$ is a topological group and where to each element $g \in G$ there is given a homeomorphism $\ell_{g}$ of $M$ such that $\ell: G \times M \rightarrow M$ is continuous and $\ell_{g} \circ \ell_{h}=\ell_{g h}$. The continuity is an obvious geometrical requirement, but in accordance with the general observation that group properties often force more regularity than explicitly postulated (cf. (5.7)), differentiability follows in many situations. So, if $G$ is locally compact, $M$ is a smooth or real analytic manifold, all $\ell_{g}$ are smooth or real analytic homeomorphisms and the action is effective, then $G$ is a Lie group and $\ell$ is smooth or real analytic, respectively; see [163, p. 212].

6.2. Let $\ell: G \times M \rightarrow M$ be a left action. Then we have partial mappings $\ell_{a}: M \rightarrow M$ and $\ell^{x}: G \rightarrow M$, given by $\ell_{a}(x)=\ell^{x}(a)=\ell(a, x)=a . x$, where $a \in G$ and $x \in M$.

For any $X \in \mathfrak{g}$ we define the fundamental vector field $\zeta_{X}=\zeta_{X}^{M} \in \mathfrak{X}(M)$ by

$$
\zeta_{X}(x)=T_{e}\left(\ell^{x}\right) \cdot X=T_{(e, x)} \ell \cdot\left(X, 0_{x}\right) .
$$

Lemma. In this situation the following assertions hold:

(1) $\zeta: \mathfrak{g} \rightarrow \mathfrak{X}(M)$ is a linear mapping.

(2) $T_{x}\left(\ell_{a}\right) \cdot \zeta_{X}(x)=\zeta_{\operatorname{Ad}(a) X}(a . x)$.

(3) $R_{X} \times 0_{M} \in \mathfrak{X}(G \times M)$ is $\ell$-related to $\zeta_{X} \in \mathfrak{X}(M)$.

(4) $\left[\zeta_{X}, \zeta_{Y}\right]=-\zeta_{[X, Y]}$

Proof. (1) is clear.

(2) We have $\ell_{a} \ell^{x}(b)=a b x=a b a^{-1} a x=\ell^{a x} \operatorname{conj}_{a}(b)$, so

$$
\begin{aligned}
T_{x}\left(\ell_{a}\right) \cdot \zeta_{X}(x) & =T_{x}\left(\ell_{a}\right) \cdot T_{e}\left(\ell^{x}\right) \cdot X=T_{e}\left(\ell_{a} \circ \ell^{x}\right) \cdot X \\
& =T_{e}\left(\ell^{a x}\right) \cdot \operatorname{Ad}(a) \cdot X=\zeta_{\operatorname{Ad}(a) X}(a x) .
\end{aligned}
$$

(3) We have $\ell \circ\left(I d \times \ell_{a}\right)=\ell \circ\left(\mu^{a} \times I d\right): G \times M \rightarrow M$, so

$$
\begin{aligned}
\zeta_{X}(\ell(a, x)) & =T_{(e, a x)} \ell .\left(X, 0_{a x}\right)=T \ell \cdot\left(I d \times T\left(\ell_{a}\right)\right) \cdot\left(X, 0_{x}\right) \\
& =T \ell \cdot\left(T\left(\mu^{a}\right) \times I d\right) .\left(X, 0_{x}\right)=T \ell .\left(R_{X} \times 0_{M}\right)(a, x) .
\end{aligned}
$$


(4) $\left[R_{X} \times 0_{M}, R_{Y} \times 0_{M}\right]=\left[R_{X}, R_{Y}\right] \times 0_{M}=-R_{[X, Y]} \times 0_{M}$ is $\ell$-related to $\left[\zeta_{X}, \zeta_{Y}\right]$ by (3) and by $(3.10)$. On the other hand $-R_{[X, Y]} \times 0_{M}$ is $\ell$-related to $-\zeta_{[X, Y]}$ by (3) again. Since $\ell$ is surjective, we get $\left[\zeta_{X}, \zeta_{Y}\right]=-\zeta_{[X, Y]}$.

6.3. Let $r: M \times G \rightarrow M$ be a right action, so $\check{r}: G \rightarrow \operatorname{Diff}(M)$ is a group antihomomorphism. We will use the following notation: $r^{a}: M \rightarrow M$ and $r_{x}: G \rightarrow M$, given by $r_{x}(a)=r^{a}(x)=r(x, a)=x . a$.

For any $X \in \mathfrak{g}$ we define the fundamental vector field $\zeta_{X}=\zeta_{X}^{M} \in \mathfrak{X}(M)$ by $\zeta_{X}(x)=T_{e}\left(r_{x}\right) \cdot X=T_{(x, e)} r \cdot\left(0_{x}, X\right)$.

Lemma. In this situation the following assertions hold:

(1) $\zeta: \mathfrak{g} \rightarrow \mathfrak{X}(M)$ is a linear mapping.

(2) $T_{x}\left(r^{a}\right) \cdot \zeta_{X}(x)=\zeta_{\operatorname{Ad}\left(a^{-1}\right) X}(x . a)$.

(3) $0_{M} \times L_{X} \in \mathfrak{X}(M \times G)$ is r-related to $\zeta_{X} \in \mathfrak{X}(M)$.

(4) $\left[\zeta_{X}, \zeta_{Y}\right]=\zeta_{[X, Y]}$.

6.4. Theorem. Let $\ell: G \times M \rightarrow M$ be a smooth left action. For $x \in M$ let $G_{x}=\{a \in G: a x=x\}$ be the isotropy subgroup or fixpoint group of $x$ in $G$, a closed subgroup of $G$. Then $\ell^{x}: G \rightarrow M$ factors over $p: G \rightarrow G / G_{x}$ to an injective immersion $i^{x}: G / G_{x} \rightarrow M$, which is G-equivariant, i.e., $\ell_{a} \circ i^{x}=i^{x} \circ \bar{\mu}_{a}$ for all $a \in G$. The image of $i^{x}$ is the orbit through $x$.

The fundamental vector fields span an integrable distribution on $M$ in the sense of (3.23). Its leaves are the connected components of the orbits, and each orbit is an initial submanifold. Thus $i^{x}: G / G_{x} \rightarrow M$ is an initial immersion.

Proof. Clearly $\ell^{x}$ factors over $p$ to an injective mapping $i^{x}: G / G_{x} \rightarrow M$; by the universal property of surjective submersions $i^{x}$ is smooth, and obviously it is equivariant. Thus $T_{p(a)}\left(i^{x}\right) \cdot T_{p(e)}\left(\bar{\mu}_{a}\right)=T_{p(e)}\left(i^{x} \circ \bar{\mu}_{a}\right)=T_{p(e)}\left(\ell_{a} \circ i^{x}\right)=$ $T_{x}\left(\ell_{a}\right) \cdot T_{p(e)}\left(i^{x}\right)$ for all $a \in G$ and it suffices to show that $T_{p(e)}\left(i^{x}\right)$ is injective. Let $X \in \mathfrak{g}$ and consider its fundamental vector field $\zeta_{X} \in \mathfrak{X}(M)$. By (3.14) and (6.2.3) we have

$$
\ell(\exp (t X), x)=\ell\left(\mathrm{Fl}_{t}^{R_{X} \times 0_{M}}(e, x)\right)=\mathrm{Fl}_{t}^{\zeta X}(\ell(e, x))=\mathrm{Fl}_{t}^{\zeta X}(x) .
$$

So $\exp (t X) \in G_{x}$, i.e., $X \in \mathfrak{g}_{x}$, if and only if $\zeta_{X}(x)=0_{x}$. In other words, $0_{x}=\zeta_{X}(x)=T_{e}\left(\ell^{x}\right) \cdot X=T_{p(e)}\left(i^{x}\right) \cdot T_{e} p \cdot X$ if and only if $T_{e} p \cdot X=0_{p(e)}$. Thus $i^{x}$ is an immersion.

Since the connected components of the orbits are integral manifolds, the fundamental vector fields span an integrable distribution in the sense of $(3.23)$ but also the condition (3.28.2) is satisfied. So by theorem (3.25) each orbit is an initial submanifold in the sense of (2.13). By uniqueness 
of the manifold structure on an initial submanifold, the mapping $i^{x}$ is an initial immersion.

6.5. Theorem ([186]). Let $M$ be a smooth manifold and let $\zeta: \mathfrak{g} \rightarrow \mathfrak{X}(M)$ be a homomorphism from a finite-dimensional Lie algebra $\mathfrak{g}$ into the Lie algebra of vector fields on $M$. Let $G$ be a simply connected Lie group with Lie algebra $\mathfrak{g}$.

Then there exists a local left action $G \times M \supset U \stackrel{l}{\longrightarrow} M$, where $U$ is an open neighborhood of $\{e\} \times M$ in $G \times M$ whose fundamental vector field mapping equals $-\zeta$. Here $U$ is an open neighborhood of $\{e\} \times M$ in $G \times M$ and $l(g, l(h, x))=l(g h, x)$ whenever both sides are defined.

Suppose moreover that each element $\zeta_{X}$ in the image of $\zeta$ is a complete vector field. Then there exists a left action $\ell: G \times M \rightarrow M$ of the Lie group $G$ on the manifold $M$ whose fundamental vector field mapping equals $-\zeta$.

The domain $U$ of the local action cannot be chosen maximal in general: When trying to do so, one glues together open pieces of covering spaces of subsets of $G$ and gets sets spread over $G$ but not contained in $G$. See [102] for more information and examples.

Proof. On the product manifold $G \times M$ we consider the vector subbundle $E=\left\{\left(L_{X}(g), \zeta_{X}(x)\right):(g, x) \in G \times M, X \in \mathfrak{g}\right\} \subset T G \times T M$ where $L_{X} \in \mathfrak{X}(G)$ is the left invariant vector field generated by $X \in \mathfrak{g}$. We have $\operatorname{dim} E_{g, x}=\operatorname{dim} \mathfrak{g}$. The subbundle $E$ is integrable since $\left[L_{X} \times \zeta_{X}, L_{Y} \times \zeta_{Y}\right]=$ $\left[L_{X}, L_{Y}\right] \times\left[\zeta_{X}, \zeta_{Y}\right]=L_{[X, Y]} \times \zeta_{[X, Y]}$. Thus by theorem (3.20) (or (3.28) the bundle $E$ induces a foliation on $G \times M$. Let us denote the leaf through $(g, x) \in G \times M$ by $L(g, x)$. Note that by (4.18.3) for the flow we have

$$
\mathrm{Fl}_{t}^{L_{X} \times \zeta_{X}}(g, x)=\left(g \cdot \exp (t X), \mathrm{Fl}_{t}^{\zeta_{X}}(x)\right) .
$$

This flow line lies in the leaf $L(g, x)$ since it is tangent to it.

Thus for $\zeta_{X}$ a complete vector field we have $\left(\mu^{\exp X} \times \mathrm{Fl}_{1}^{\zeta X}\right): L(g, x) \rightarrow$ $L(g, x)$. In particular,

$$
L\left(g \cdot \exp (X), \mathrm{Fl}_{1}^{\zeta X}(x)\right)=L(g, x), \quad L(g \cdot \exp (X), y)=L\left(g, \mathrm{Fl}_{-1}^{\zeta_{X}}(y)\right) .
$$

If $\zeta_{X}$ is not complete, then (2) holds only whenever both sides (of any equation) are defined.

We have $T\left(\mu_{g} \times \operatorname{Id}_{M}\right)\left(L_{X}(h), \zeta_{X}(x)\right)=\left(L_{X}(g h), \zeta_{X}(x)\right)$ for $X \in \mathfrak{g}, g, h \in G$, and $x \in M$. Thus $\left(\mu_{g} \times \operatorname{Id}_{M}\right)$ maps leaves to leaves:

$$
L(g, x)=\{(g h, y):(h, y) \in L(e, x)\}=\left(\mu_{g} \times \operatorname{Id}_{M}\right)(L(e, x)) .
$$

We suppose now that each vector field $\zeta_{X}$ for $X \in \mathfrak{g}$ is complete.

(4) Claim. Then for any leaf $L \subset G \times M$, the restriction $\operatorname{pr}_{1} \mid L: L \rightarrow G$ is a covering map. 
For $(g, x) \in L$ we have $T_{(g, x)}\left(\operatorname{pr}_{1}\right)\left(L_{X}(g), \zeta_{X}(x)\right)=L_{X}(g)$; thus $\operatorname{pr}_{1} \mid L$ is locally a diffeomorphism. For any $g_{1} \in G$ we can find a piecewise smooth curve $c$ in $G$ connecting $g$ with $g_{1}$ consisting of pieces of the form $t \mapsto$ $g_{i} \cdot \exp \left(t X_{i}\right)$. Starting from $(g, x) \in L$, we can fit together corresponding pieces of the form $\mathrm{Fl}_{t}^{L_{X_{i}} \times \zeta_{X_{i}}}$ to obtain a curve $\tilde{c}$ in $L$ with $\operatorname{pr}_{1} \circ \tilde{c}=c$ which connects $(g, x)$ with $\left(g_{1}, x_{1}\right) \in L$ for some $x_{1} \in M$. Thus $\operatorname{pr}_{1}: L \rightarrow G$ is surjective. Next we consider some absolutely convex ball $B \subset \mathfrak{g}$ such that $\exp : \mathfrak{g} \supset B \rightarrow U \subset G$ is a diffeomorphism onto an open neighborhood $U$ of $e$ in $G$. We consider the inverse image $\left(\operatorname{pr}_{1} \mid L\right)^{-1}(g . U) \subset L$ and decompose it into its connected components, $\left(\operatorname{pr}_{1} \mid L\right)^{-1}(g . U)=\bigsqcup V_{i} \subset L$. Choose $x_{i}$ such that $\left(g, x_{i}\right) \in V_{i}$. Any point in $g . U$ is of the form $g \cdot \exp (X)$ for a unique $X \in B$, with unique inverse image $\mathrm{Fl}_{t}^{L_{X} \times \zeta_{X}}\left(g, x_{i}\right) \in V_{i}$ under $\operatorname{pr}_{1} \mid V_{i}$. Since $\left\{\mathrm{Fl}_{t}^{L_{X} \times \zeta_{X}}\left(g, x_{i}\right): X \in B\right\}$ is open and closed in $\left(\mathrm{pr}_{1} \mid L\right)^{-1}(g . U)$, it equals $V_{i}$, which is diffeomorphic to $g . U$ via $\operatorname{pr}_{1} \mid V_{i}$, and the claim follows.

Since $G$ is simply connected, we conclude that for each leaf $L$ the mapping $\operatorname{pr}_{1} \mid L: L \rightarrow G$ is a diffeomorphism. We now define the action as follows: For $g \in G$ and $x \in M$ consider the leaf $L(e, x)$ through $(e, x)$ and put

$$
\ell(g, x)=g \cdot x=\operatorname{pr}_{2}\left(\left(\operatorname{pr}_{1} \mid L(e, x)\right)^{-1}\left(g^{-1}\right)\right) \in M .
$$

Obviously, $\ell$ is smooth.

Let us now pass to the general case, where some $\zeta_{X}$ may be incomplete. Then claim (4) is wrong in general. Consider the following diagram, where $W_{x} \subset G$ is the image of the leaf $L(e, x)$ in $G$ :

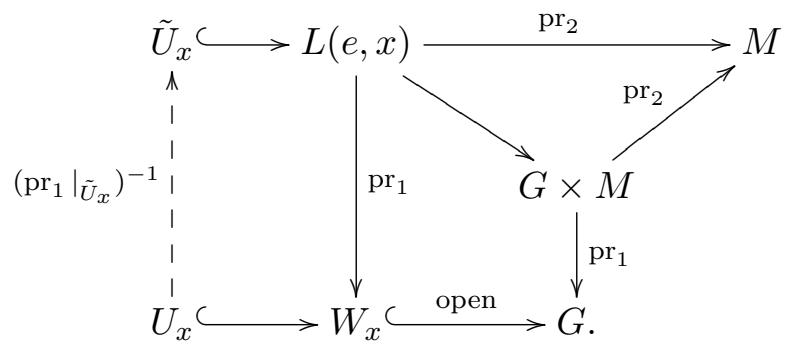

To describe $U_{x}$, we consider the vector field $\zeta \in \mathfrak{X}(\mathfrak{g} \times M)$ given by $\zeta(X, x)=$ $\left(0_{X}, \zeta_{X}(x)\right)$ with flow $\mathrm{Fl}_{t}^{\zeta}(X, x)=\left(X, \mathrm{Fl}_{t}^{\zeta X}(x)\right)$ defined as $\mathrm{Fl}^{\zeta}: \mathcal{D}(\zeta) \rightarrow M$ where the (maximal) domain of definition $\mathcal{D}(\zeta)$ is an open neighborhood of $\{0\} \times \mathfrak{g} \times M$ in $\mathbb{R} \times \mathfrak{g} \times M$ by (3.7), Let $U^{\prime}=\{(X, x) \in \mathfrak{g} \times M$ : $[-1,1] \times\{X\} \times\{x\} \subset \mathcal{D}(\zeta)\}$. Since $[-1,1]$ is compact, $U^{\prime}$ is open. Now we consider an open ball $B \subset \mathfrak{g}$ centered at 0 such that $\exp : B \rightarrow \exp (B) \subset G$ is a diffeomorphism. Then we let $U=\left(\exp \times \operatorname{Id}_{M}\right)\left(U^{\prime} \cap(B \times M)\right) \stackrel{\text { open }}{\longrightarrow} G \times M$ and we denote $U_{x}:=\operatorname{pr}_{1}(U \cap(G \times\{x\}))$ which is open in $\exp (B)$ inside $G$ and which is also simply connected, since $(t, X, x) \in \mathcal{D}(\zeta) \Longleftrightarrow\left(1, \frac{1}{t} X, x\right) \in$ $\mathcal{D}(\zeta)$. By construction, $U_{x} \subset W_{x}$, and there is a branch $\tilde{U}_{x} \subset L(e, x)$ of 
$\operatorname{pr}_{1}: L(e, x) \rightarrow W_{x}$ over $U_{x}$ such that $\operatorname{pr}_{1} \mid \tilde{U}_{x}: \tilde{U}_{x} \rightarrow U_{x}$ is a diffeomorphism. So all entries of diagram (6) have now been explained. We can define the local action for $(g, x) \in U$ by

$$
G \times M \stackrel{\text { open }}{\longleftarrow} U \stackrel{\ell}{\longrightarrow} M, \quad \ell(g, x)=g \cdot x:=\operatorname{pr}_{2}\left(\left(\left.\operatorname{pr}_{1}\right|_{\tilde{U}_{x}}\right)^{-1}\left(g^{-1}\right)\right) .
$$

Note that (7) is the local version of (5). Again $\ell$ is smooth.

It remains to show that $\ell$ is a global or local action. Both definitions say: $g \cdot x=y \Longleftrightarrow\left(g^{-1}, y\right) \in L(e, x) \Longleftrightarrow L\left(g^{-1}, g \cdot x\right)=L\left(g^{-1}, y\right)=L(e, x)$ (for $(g, x) \in U$ in the noncomplete case). So $L(e, h . z)=L(h, z)$ determines $h . z$ uniquely; compare with (3). Applying $\mu_{g} \times \operatorname{Id}_{M}$ and (3), we get $L(g, h . z)=$ $L(g . h, z)$ for all $g, h \in G$ and $z \in M$. Thus $\ell: G \times M \rightarrow M$ is a (local) action, since $L(e, g .(h . x))=L(g, h . x)=L(g . h, z)=L(e,(g . h) . z)$. From the considerations in the proof of the claim (4) and from (11) and (7) it follows that for $X \in \mathfrak{g}$ we also have (for $X$ small in the noncomplete case)

$$
\ell(\exp (X), x)=\exp (X) \cdot x=\mathrm{Fl}_{-1}^{\zeta_{X}}(x) \in M .
$$

So the fundamental vector field mapping of $\ell$ is $-\zeta$.

6.6. Semidirect products of Lie groups. Let $H$ and $K$ be two Lie groups and let $\ell: H \times K \rightarrow K$ be a smooth left action of $H$ in $K$ such that each $\ell_{h}: K \rightarrow K$ is a group automorphism. So the associated mapping $\check{\ell}: H \rightarrow \operatorname{Aut}(K)$ is a smooth homomorphism into the automorphism group of $K$. Then we can introduce the following multiplication and inversion on $K \times H$ :

$$
(k, h)\left(k^{\prime}, h^{\prime}\right):=\left(k \ell_{h}\left(k^{\prime}\right), h h^{\prime}\right), \quad(k, h)^{-1}:=\left(\ell_{h^{-1}}\left(k^{-1}\right), h^{-1}\right) .
$$

It is easy to see that this defines a Lie group $G=K \rtimes_{\ell} H$ called the semidirect product of $H$ and $K$ with respect to $\ell$. If the action $\ell$ is clear from the context, we write $G=K \rtimes H$ only. The second projection $\operatorname{pr}_{2}: K \rtimes H \rightarrow H$ is a surjective smooth homomorphism with kernel $K \times\{e\}$, and the insertion ins $_{e}: H \rightarrow K \rtimes H$ ins $_{e}(h)=(e, h)$ is a smooth group homomorphism with $\mathrm{pr}_{2} \circ$ ins $_{e}=I d_{H}$.

Conversely we consider an exact sequence of Lie groups and homomorphisms

$$
\{e\} \rightarrow K \stackrel{j}{\longrightarrow} G \stackrel{p}{\longrightarrow} H \rightarrow\{e\} .
$$

So $j$ is injective, $p$ is surjective, and the kernel of $p$ equals the image of $j$. We suppose furthermore that the sequence splits, so that there is a smooth homomorphism $s: H \rightarrow G$ with $p \circ s=I d_{H}$. Then the rule $\ell_{h}(k)=s(h) k s\left(h^{-1}\right)$ (where we suppress $j$ ) defines a left action of $H$ on $K$ by automorphisms. It is easily seen that the mapping $K \rtimes_{\ell} H \rightarrow G$ given by $(k, h) \mapsto k . s(h)$ is an isomorphism of Lie groups with inverse $g \mapsto$ (g.sp $\left.(g)^{-1}, s p(g)\right)$. Note that $g \mapsto g \cdot s p(g)^{-1}$ is not a homomorphism of 
groups but only of $H$-modules $G \rightarrow K$. So we see that semidirect products of Lie groups correspond exactly to splitting short exact sequences.

6.7. The tangent group of a Lie group. Let $G$ be a Lie group with Lie algebra $\mathfrak{g}$. We will use the notation from (4.1). First note that $T G$ is also a Lie group with multiplication $T \mu$ and inversion $T \nu$, given by (see (4.2)) $T_{(a, b)} \mu \cdot\left(\xi_{a}, \eta_{b}\right)=T_{a}\left(\mu^{b}\right) \cdot \xi_{a}+T_{b}\left(\mu_{a}\right) \cdot \eta_{b}$ and $T_{a} \nu \cdot \xi_{a}=-T_{e}\left(\mu_{a^{-1}}\right) \cdot T_{a}\left(\mu^{a^{-1}}\right) \cdot \xi_{a}$.

Lemma. Via the isomorphism given by the right trivialization $\mathfrak{g} \times G \rightarrow T G$, $(X, g) \mapsto T_{e}\left(\mu^{g}\right) . X$, the group structure on $T G$ looks as follows:

$$
(X, a) \cdot(Y, b)=(X+\operatorname{Ad}(a) Y, a \cdot b) \quad \text { and } \quad(X, a)^{-1}=\left(-\operatorname{Ad}\left(a^{-1}\right) X, a^{-1}\right) .
$$

So $T G$ is isomorphic to the semidirect product $\mathfrak{g} \rtimes G$.

Proof. We compute:

$$
\begin{aligned}
T_{(a, b)} \mu \cdot\left(T \mu^{a} \cdot X, T \mu^{b} \cdot Y\right) & =T \mu^{b} \cdot T \mu^{a} \cdot X+T \mu_{a} \cdot T \mu^{b} \cdot Y \\
& =T \mu^{a b} \cdot X+T \mu^{b} \cdot T \mu^{a} \cdot T \mu^{a^{-1}} \cdot T \mu_{a} \cdot Y=T \mu^{a b}(X+\operatorname{Ad}(a) Y), \\
T_{a} \nu \cdot T \mu^{a} \cdot X & =-T \mu^{a^{-1}} \cdot T \mu_{a^{-1}} \cdot T \mu^{a} \cdot X=-T \mu^{a^{-1}} \cdot \operatorname{Ad}\left(a^{-1}\right) X . \quad \square
\end{aligned}
$$

Remark. In the left trivialization $G \times \mathfrak{g} \rightarrow T G,(g, X) \mapsto T_{e}\left(\mu_{g}\right) . X$, the semidirect product structure looks awkward:

$(a, X) \cdot(b, Y)=\left(a b, \operatorname{Ad}\left(b^{-1}\right) X+Y\right)$ and $(a, X)^{-1}=\left(a^{-1},-\operatorname{Ad}(a) X\right)$.

6.8. $G$-actions and their orbit spaces. If $M$ is a left $G$-manifold, then $M / G$, the space of all $G$-orbits endowed with the quotient topology, is called the orbit space. We consider some examples:

The standard action of $O(n)$ on $\mathbb{R}^{n}$. It is orthogonal. The orbits are the concentric spheres around the fixed point 0 and 0 itself. The orbit space is $\mathbb{R}^{n} / O(n) \cong[0, \infty)$.

Every Lie group $G$ acts on itself by conjugation conj : $G \times G \rightarrow G$ which is defined by $(g, h) \mapsto \operatorname{conj}_{g}(h):=g . h . g^{-1}$ and which is a smooth left action of $G$ on itself.

The adjoint action Ad : $G \rightarrow G L(\mathfrak{g})$ of a Lie group $G$ on its Lie algebra $\mathfrak{g}$ from (4.24), In particular, the orthogonal group acts orthogonally on $\mathfrak{o}(n)$, the Lie algebra of all skew-symmetric $n \times n$-matrices.

The $O(n)$-action on $S(n)$ treated in $(7.1)$. Similarly, $S U(n)$ acts unitarily on the Hermitian $(n \times n)$ matrices by conjugation. 
6.9. Isotropy groups. Let $M$ be a $G$-manifold; then the closed subgroup $G_{x}=\{g \in G: g \cdot x=x\}$ of $G$ is called the isotropy subgroup of $x$.

The map $i^{x}: G / G_{x} \longrightarrow M$ defined by $i^{x}: g \cdot G_{x} \mapsto g \cdot x \in M$ is a $G$ equivariant initial immersion with image $G . x$, by (6.4).

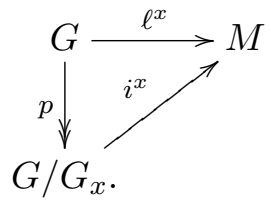

6.10. Lemma. Let $M$ be a $G$-manifold and $x, y \in M$; then

(1) $G_{g x}=g \cdot G_{x} \cdot g^{-1}$,

(2) If $G . x \cap G . y \neq \emptyset$, then $G . x=G . y$,

(3) $T_{x}(G \cdot x)=T_{e}\left(\ell^{x}\right) \cdot \mathfrak{g}$.

Proof. (11) $a \in G_{g x}$ means ag.x $=g \cdot x$ or $g^{-1} a g \cdot x=x$ and again $g^{-1} a g \in G_{x}$ which in turn is equivalent to $a \in g G_{x} g^{-1}$.

(2) If $z \in G . x \cap G . y$, then $z=g_{1} . x=g_{2} . y$ for some $g_{1}, g_{2} \in G$. So $x=$ $g_{1}^{-1} \cdot g_{2} \cdot y$; therefore $G \cdot x=G \cdot\left(g_{1}^{-1} \cdot g_{2} \cdot y\right)=G \cdot y$.

(3) $X \in T_{x}(G . x) \Leftrightarrow X=\left.\partial_{t}\right|_{0} c(t)$ for some smooth curve $c(t)=g(t) . x \in G \cdot x$ with $g(0)=e$. So we have $X=\left.\partial_{t}\right|_{0} \ell^{x}\left(g_{t}\right) \in T_{e}\left(\ell^{x}\right) \cdot \mathfrak{g}$.

6.11. Conjugacy classes. The closed subgroups of $G$ can be partitioned into equivalence classes by writing

$$
H \sim H^{\prime} \text { if there exists } g \in G \text { for which } H=g H^{\prime} g^{-1} .
$$

The equivalence class of $H$ is denoted by $(H)$.

Using lemma (6.10.1) we have as a first consequence: The conjugacy class of an isotropy subgroup is invariant under the action of $G:\left(G_{x}\right)=\left(G_{g x}\right)$. Therefore we can assign to each orbit $G . x$ the conjugacy class $\left(G_{x}\right)$. We will call $\left(G_{x}\right)$ the isotropy type or the orbit type of the orbit through $x$.

If $G$ is compact, we can define a partial ordering on the conjugacy classes simply by transferring the usual partial ordering " $\subseteq$ " on the subgroups to the classes:

$$
\begin{aligned}
(H) \leq\left(H^{\prime}\right) \quad: & \Longleftrightarrow \quad \exists K \in(H), K^{\prime} \in\left(H^{\prime}\right): K \subseteq K^{\prime} \\
& \Longleftrightarrow \quad \exists g \in G: H \subseteq g H^{\prime} g^{-1} .
\end{aligned}
$$

If $G$ is not compact, this relation may not be reflexive. For compact $G$ the reflexivity of this relation is a consequence of the following: 
6.12. Lemma. Let $G$ be a compact Lie group and $H$ a closed subgroup of $G$; then

$$
g H g^{-1} \subseteq H \quad \Longrightarrow \quad g H g^{-1}=H
$$

Proof. By iteration, $g H g^{-1} \subseteq H$ implies $g^{n} H g^{-n} \subseteq H$ for all $n \in \mathbb{N}$. Now let us study the set $A:=\left\{g^{n}: n \in \mathbb{Z}_{\geq 0}\right\}$. We will show that $g^{-1}$ is contained in its closure $\bar{A}$.

Suppose that $e$ is discrete in $\bar{A}$. So there is an $e$-neighborhood $U$ in $G$ such that $U \cap \bar{A}=\{e\}$. Then $\left.g^{n} U \cap \overline{\left\{g^{k}: k \geq n\right.}\right\}=\left\{g^{n}\right\}$, so by induction $\bar{A}$ is discrete. Since $G$ is compact, $\bar{A}=A$ is finite. Therefore $g^{n}=e$ for some $n>0$, and $g^{n-1}=g^{-1} \in A$.

Suppose now that $e$ is an accumulation point of $A$. Then for any neighborhood $U$ of $e$ there is a $g^{n} \in U$ where $n>0$. This implies $g^{n-1} \in g^{-1} U \cap A$. Since the sets $g^{-1} U$ form a neighborhood basis of $g^{-1}$, we see that $g^{-1}$ is an accumulation point of $A$ as well. That is, $g^{-1} \in \bar{A}$.

Since conj : $G \times G \rightarrow G$ is continuous and $H$ is closed, we have $\operatorname{conj}(\bar{A}, H) \subseteq$ $H$. In particular, $g^{-1} H g \subseteq H$ which together with our premise implies that $g H g^{-1}=H$.

6.13. Principal orbits. Let $M$ be a $G$-manifold. The orbit $G$. $x$ is called a principal orbit if there is an invariant open neighborhood $U$ of $x$ in $M$ and for all $y \in U$ an equivariant map

$$
f: G . x \rightarrow G . y .
$$

Note that $f$ is automatically surjective: Namely, let $f(x)=: a . y$. For an arbitrary $z=g . y \in G . y$ this gives us

$$
z=g \cdot y=g a^{-1} a \cdot y=g a^{-1} f(x)=f\left(g a^{-1} \cdot x\right) .
$$

The existence of $f$ in the above definition is equivalent to the condition: $G_{x} \subseteq a G_{y} a^{-1}$ for some $a \in G$ :

If $f$ exists, then for $g \in G_{x}$ we have $g . x=x$ and thus $g . f(x)=f(g . x)=f(x)$. For $f(x)=: a . y$ we get $g a . y=a . y$; thus $g \in G_{a y}=a G_{y} a^{-1}$ by (6.10.1).

To show the converse, we define $f: G . x \rightarrow G . y$ explicitly by $f(g . x):=g a . y$. We have to check: If $g_{1} . x=g_{2} . x$, i.e., $g:=g_{2}^{-1} g_{1} \in G_{x}$, then $g_{1} a . y=g_{2} a . y$ or $g \in G_{a y}=a G_{y} a^{-1}$. This is guaranteed by our assumption.

We call $x \in M$ a regular point if $G$. $x$ is a principal orbit. Otherwise, $x$ is called singular. The subset of all regular points in $M$ is denoted by $M_{\text {reg, }}$, and $M_{\text {sing }}$ denotes the subset of all singular points. 
6.14. Slices. Let $M$ be a $G$-manifold and $x \in M$, then a subset $S \subseteq M$ is called a slice at $x$ if there is a $G$-invariant open neighborhood $U$ of $G . x$ and a smooth equivariant retraction $r: U \rightarrow G . x$ such that $S=r^{-1}(x)$. Since $r$ is equivariant, it is a submersion onto G.x.

6.15. Proposition. If $M$ is a $G$-manifold and $S=r^{-1}(x)$ a slice at $x \in M$, where $r: U \rightarrow G . x$ is the corresponding retraction, then:

(1) $x \in S$ and $G_{x} . S \subseteq S$.

(2) If $g . S \cap S \neq \emptyset$, then $g \in G_{x}$.

(3) $G . S=\{g . s: g \in G, s \in S\}=U$.

Proof. (1) We have $x \in S$ since $S=r^{-1}(x)$ and $r(x)=x$. To show that $G_{x} . S \subseteq S$, take an $s \in S$ and $g \in G_{x}$. Then $r(g . s)=g \cdot r(s)=g \cdot x=x$, and therefore $g . s \in r^{-1}(x)=S$.

(2) If $g . S \cap S \neq \emptyset$, then $g . s \in S$ for some $s \in S$. So we get $x=r(g . s)=$ $g . r(s)=g . x ;$ thus $g \in G_{x}$.

(3) We have $G \cdot S=G \cdot r^{-1}(x)=r^{-1}(G \cdot x)=U$.

6.16. Corollary. If $M$ is a $G$-manifold and $S$ a slice at $x \in M$, then:

(1) $S$ is a $G_{x}$-manifold.

(2) $G_{s} \subseteq G_{x}$ for all $s \in S$.

(3) If $G . x$ is a principal orbit and $G_{x}$ compact, then $G_{y}=G_{x}$ for all $y \in S$ if the slice $S$ at $x$ is chosen small enough. In other words, all orbits near G.x are principal as well.

(4) If two $G_{x}$-orbits $G_{x} . s_{1}, G_{x} . s_{2}$ in $S$ have the same orbit type as $G_{x}$-orbits in $S$, then $G . s_{1}$ and $G . s_{2}$ have the same orbit type as $G$-orbits in $M$.

(5) $S / G_{x} \cong G . S / G$ is an open neighborhood of $G . x$ in the orbit space $M / G$.

Proof. (11) This is clear from (6.15.1),

(2) If $g \in G_{y}$ then $g . y=y \in S$; thus $g \in G_{x}$ by (6.15.2).

(3) By (2) we have $G_{y} \subseteq G_{x}$, so $G_{y}$ is compact as well. Because $G$.x is principal it follows that for $y \in S$ close to $x, G_{x}$ is conjugate to a subgroup of $G_{y}, G_{y} \subseteq G_{x} \subseteq g . G_{y} g^{-1}$. Since $G_{y}$ is compact, $G_{y} \subseteq g . G_{y} g^{-1}$ implies $G_{y}=g \cdot G_{y} g^{-1}$ by (6.12), Therefore $G_{y}=G_{x}$, and $G . y$ is also a principal orbit.

(44) For any $s \in S$ we have $\left(G_{x}\right)_{s}=G_{s}$, since $\left(G_{x}\right)_{s} \subseteq G_{s}$. Conversely, by (2), $G_{s} \subseteq G_{x}$; therefore $G_{s} \subseteq\left(G_{x}\right)_{s}$. So $\left(G_{x}\right)_{s_{1}}=g\left(G_{x}\right)_{s_{2}} g^{-1}$ implies $G_{s_{1}}=g G_{s_{2}} g^{-1}$ and the $G$-orbits have the same orbit type. 
(5) The isomorphism $S / G_{x} \cong G . S / G$ is given by the map $G_{x} . s \mapsto G . s$ which is an injection by (6.15.2), Since $G . S=U$ is an open $G$-invariant neighborhood of $G . x$ in $M$ by (6.15.3), we have $G . S / G$ is an open neighborhood of $G . x$ in $M / G$.

6.17. Remark. The converse to (6.16.4) is generally false. If the two $G$ orbits G.s $s_{1}, G . s_{2}$ are of the same type, then the isotropy groups $G_{s_{1}}$ and $G_{s_{2}}$ are conjugate in $G$. They need not be conjugate in $G_{x}$. For example, consider the following semidirect product, the compact Lie group $G:=\left(S^{1} \times\right.$ $\left.S^{1}\right) \rtimes \mathbb{Z}_{2}$ with multiplication defined as follows. Let $\varphi_{1}, \varphi_{2}, \psi_{1}, \psi_{2} \in S^{1}$ and $\alpha, \beta \in \mathbb{Z}_{2}$. Take on $S^{1} \times S^{1}$ the usual multiplication by components, and as $\mathbb{Z}_{2}$-action:

$$
\mathbb{Z}_{2} \ni \overline{0} \mapsto i_{0}:=\operatorname{Id}_{S^{1} \times S^{1}}, \quad \overline{1} \mapsto\left(i_{1}:\left(\varphi_{1}, \varphi_{2}\right) \mapsto\left(\varphi_{2}, \varphi_{1}\right)\right) .
$$

Then we consider the semidirect product structure:

$$
\left(\varphi_{1}, \varphi_{2}, \alpha\right) \cdot\left(\psi_{1}, \psi_{2}, \beta\right):=\left(\left(\varphi_{1}, \varphi_{2}\right) \cdot i_{\alpha}\left(\psi_{1}, \psi_{2}\right), \alpha+\beta\right) .
$$

Now we let $G$ act on $M:=V \sqcup W$ where $V=W=\mathbb{C} \times \mathbb{C}$. For any element in $M$ we will indicate its connected component by the index $(x, y)_{V}$ or $(x, y)_{W}$. The action shall be the following:

$$
\begin{aligned}
& \left(\varphi_{1}, \varphi_{2}, \overline{0}\right) \cdot(x, y)_{V}:=\left(\varphi_{1} \cdot x, \varphi_{2} \cdot y\right)_{V} \\
& \left(\varphi_{1}, \varphi_{2}, \overline{1}\right) \cdot(x, y)_{V}:=\left(\varphi_{1} \cdot y, \varphi_{2} \cdot x\right)_{W} .
\end{aligned}
$$

The action on $W$ is simply given by interchanging the $V$ 's and $W$ 's in the above formulae. This defines an action. Denote by $H$ the abelian subgroup $S^{1} \times S^{1} \times\{\overline{0}\}$. Then $H$ is the isotropy subgroup of $(0,0)_{V}$, and $V$ is a slice at $(0,0)_{V}$. Now consider $s_{1}:=\left(0, v^{1}\right)_{V}$ and $s_{2}:=\left(v^{2}, 0\right)_{V}$, both not equal to zero. Then let

$$
\begin{aligned}
& H_{1}:=G_{s_{1}}=S^{1} \times\{1\} \times\{\overline{0}\}, \\
& H_{2}:=G_{s_{2}}=\{1\} \times S^{1} \times\{\overline{0}\} .
\end{aligned}
$$

The subgroups $H_{1}$ and $H_{2}$ are conjugate in $G$ by $(1,1, \overline{1})$. Yet they are clearly not conjugate in $H$ since $H$ is abelian. So $H . s_{1}$ and $H . s_{2}$ have different orbit types in $H$ while $G . s_{1}$ and $G . s_{2}$ are of the same $G$-orbit type.

6.18. Proposition. Let $M$ be a $G$-manifold and $S$ a slice at $x$; then there is a $G$-equivariant diffeomorphism of the associated bundle $G[S]$ onto $G . S$,

$$
f: G[S]:=G \times_{G_{x}} S \rightarrow G . S
$$

which maps the zero section $G \times{ }_{G_{x}}\{x\}$ onto G.x. See (18.7) below for more information on associated bundles. 
Proof. Since $\ell\left(g h, h^{-1} . s\right)=g . s=\ell(g, s)$ for all $h \in G_{x}$, there is an $f$ : $G[S] \rightarrow G . S$ such that the following diagram commutes:

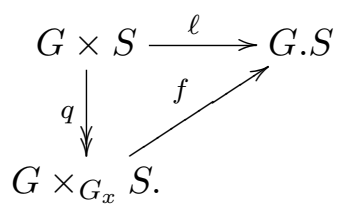

The map $f$ is smooth because $f \circ q=\ell$ is smooth and $q$ is a submersion. It is equivariant since $\ell$ and $q$ are equivariant. Also, $f$ maps the zero section $G \times{ }_{G_{x}}\{x\}$ onto G.x. The map $f$ is bijective: If $g_{1} . s_{1}=g_{2} . s_{2}$, then $s_{1}=$ $g_{1}^{-1} g_{2} . s_{2}$; thus $h=g_{1}^{-1} g_{2} \in G_{x}$ by (6.15.2). But then $g_{1}=g_{2} h$ and $s_{1}=h . s_{2}$. This is equivalent to $q\left(g_{1}, s_{1}\right)=q\left(g_{2}, s_{2}\right)$.

To see that $f$ is a diffeomorphism, let us prove that the rank of $f$ equals the dimension of $M$. First of all, note that $\operatorname{rank}\left(\ell_{g}\right)=\operatorname{dim}(g \cdot S)=\operatorname{dim} S$ and $\operatorname{rank}\left(\ell^{x}\right)=\operatorname{dim}(G . x)$. Since $S=r^{-1}(x)$ and $r: G . S \rightarrow G . x$ is a submersion, it follows that $\operatorname{dim}(G \cdot x)=\operatorname{codim} S$. Therefore,

$$
\begin{array}{r}
\operatorname{rank}(f)=\operatorname{rank}(\ell)=\operatorname{rank}\left(\ell_{g}\right)+\operatorname{rank}\left(\ell^{x}\right)=\operatorname{dim} S+\operatorname{dim}(G \cdot x) \\
=\operatorname{dim} S+\operatorname{codim} S=\operatorname{dim} M .
\end{array}
$$

6.19. Remark. The converse also holds. If $\bar{f}: G \times_{G_{x}} S \rightarrow G . S$ is a $G$-equivariant diffeomorphism, then for some $\bar{g} \in G$ and $\bar{s} \in S$ whe have $\bar{f}[\bar{g}, \bar{s}]=x$. So $f[g, s]:=\bar{f}[g \bar{g}, s]$ defines a $G$-equivariant diffeomorphism with the additional property that $x=f[e, \bar{s}]$ :

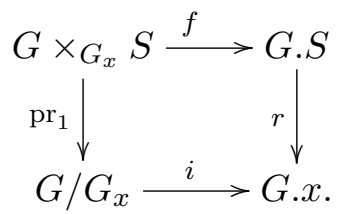

If we define $r:=i \circ \mathrm{pr}_{1} \circ f^{-1}: G . S \rightarrow G . x$, then $r$ is again a smooth $G$ equivariant map, and it is a retraction onto G.x since

$$
x \stackrel{f^{-1}}{\longrightarrow}[e, \bar{s}] \stackrel{\mathrm{pr}_{1}}{\longrightarrow} e . G_{x} \stackrel{i}{\longrightarrow} e . x .
$$

Furthermore, $r^{-1}(x)=S$, making $S$ a slice.

6.20. Proper actions. Recall that a continuous mapping between topological spaces is called proper if compact subsets have compact inverse images. A smooth action $\ell: G \times M \rightarrow M$ is called proper if it satisfies one of the following three equivalent conditions:

(1) $\left(\ell, \mathrm{pr}_{2}\right): G \times M \rightarrow M \times M,(g, x) \mapsto(g . x, x)$, is a proper mapping 
(2) $g_{n} \cdot x_{n} \rightarrow y$ and $x_{n} \rightarrow x$ in $M$, for some $g_{n} \in G$ and $x_{n}, x, y \in M$, implies that these $g_{n}$ have a convergent subsequence in $G$.

(3) $K$ and $L$ compact in $M$ implies that $\{g \in G: g \cdot K \cap L \neq \emptyset\}$ is compact as well.

Proof. (11) $\Rightarrow$ (2) This is a direct consequence of the definitions.

(2) $\Rightarrow$ (3) Let $g_{n}$ be a sequence in $\{g \in G: g . K \cap L \neq \emptyset\}$ and $x_{n} \in K$ such that $g_{n} . x_{n} \in L$. Since $K$ is compact, we can choose a convergent subsequence $x_{n_{k}} \rightarrow x \in K$ of $x_{n}$. Since $L$ is compact, we can do the same for $g_{n_{k}} \cdot x_{n_{k}}$ there. Now (2) tells us that in such a case $g_{n}$ must have a convergent subsequence; therefore $\{g \in G: g . K \cap L \neq \emptyset\}$ is compact.

(3) $\Rightarrow$ (11) Let $R$ be a compact subset of $M \times M$. Then $L:=\operatorname{pr}_{1}(R)$ and $K:=\operatorname{pr}_{2}(R)$ are compact, and $\left(\ell, \operatorname{pr}_{2}\right)^{-1}(R) \subseteq\{g \in G: g \cdot K \cap L \neq \emptyset\} \times K$. By (3), $\{g \in G: g . K \cap L \neq \emptyset\}$ is compact. Therefore $\left(\ell, \operatorname{pr}_{2}\right)^{-1}(R)$ is compact, and $\left(\ell, \mathrm{pr}_{2}\right)$ is proper.

6.21. Remark. If $G$ is compact, then every $G$-action is proper. If $\ell: G \times$ $M \rightarrow M$ is a proper action and $G$ is not compact, then for any noncompact $H \subseteq G$ and $x \in M$ the set $H . x$ is noncompact in $M$. Furthermore, all isotropy groups are compact (most easily seen from (6.20.3) by setting $K=$ $L=\{x\})$.

6.22. Lemma. A continuous, proper map $f: X \rightarrow Y$ between two topological spaces is closed.

Proof (For metric spaces). Consider a closed subset $A \subseteq X$, and take a point $y$ in the closure of $f(A)$. Let $f\left(a_{n}\right) \in f(A)$ converge to $y\left(a_{n} \in A\right)$. Then the $f\left(a_{n}\right)$ are contained in a compact subset $K \subseteq Y$. Therefore $a_{n} \subseteq f^{-1}(K) \cap A$ which is now, since $f$ is proper, a compact subset of $A$. Consequently, $\left(a_{n}\right)$ has a convergent subsequence with limit $a \in A$, and by continuity of $f$, it gives a convergent subsequence of $f\left(a_{n}\right)$ with limit $f(a) \in f(A)$. Since $f\left(a_{n}\right)$ converges to $y$, we have $y=f(a) \in f(A)$.

6.23. Proposition. The orbits of a proper action $\ell: G \times M \rightarrow M$ are closed submanifolds.

Proof. By the preceding lemma, $\left(\ell, \mathrm{pr}_{2}\right)$ is closed. Therefore $\left(\ell, \mathrm{pr}_{2}\right)(G, x)=$ $G . x \times\{x\}$, and with it $G . x$ is closed.

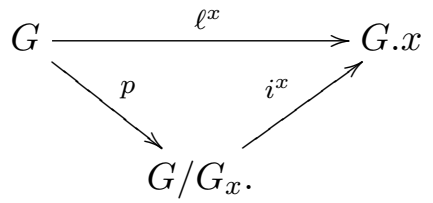


As a maximal integral manifold of the involutive distribution of (in general) nonconstant rank spanned by all fundamental vector fields, $G . x$ is an initial submanifold, and $i^{x}$ is an initial immersion by (6.4). Thus $i^{x}: G / G_{x} \rightarrow G . x$ is open.

6.24 Examples of nonproper actions. The standard action of $S L(2, \mathbb{R})$ on $\mathbb{R}^{2}$ has two orbits, 0 and $\mathbb{R}^{2} \backslash 0$ which is not closed. By (6.23) this action is not proper.

The action of $G L(n, \mathbb{R})$ on the space $L_{\mathrm{sym}}\left(\mathbb{R}^{n}, \mathbb{R}^{n}\right)$ of symmetric matrices given by $(g, A) \mapsto g^{\top} . A . g$ is not proper, since the isotropy group of the symmetric bilinear form with signature $p, n-p$ is the group $O(p, n-p, \mathbb{R})$ which is not compact for $0<p<n$.

6.25. Lemma. Let $(M, \gamma)$ be a Riemann manifold and $\ell: G \times M \rightarrow M$ an effective isometric action (i.e., g. $x=x$ for all $x \in M \Rightarrow g=e$ ), such that $\ell(G) \subseteq \operatorname{Isom}(M, \gamma)$ is closed in the compact open topology. Then $\ell$ is proper.

Proof. Assume without loss that $M$ is connected. Let $g_{n} \in G$ and $x_{n}, x, y \in$ $M$ such that $g_{n} . x_{n} \rightarrow y$ and $x_{n} \rightarrow x$; then we have to show that $g_{n}$ has a convergent subsequence which is the same as proving that $\left\{g_{n}: n \in \mathbb{N}\right\}$ is relatively compact, since $\ell(G) \subseteq \operatorname{Isom}(M, \gamma)$ is closed.

Let us choose a compact neighborhood $K$ of $x$ in $M$. Put a metric on $M$ (e.g., the Riemann distance function). Note first $\left.\operatorname{Isom}(M) \ni \varphi \mapsto \varphi\right|_{K} \in$ $C^{0}(K, M)$ is an injective embedding, where we put the uniform metric on $C^{0}(K, M)$. Then, since the $g_{n}$ act isometrically, we can find a compact neighborhood $L \subseteq M$ of $y$ such that $\bigcup_{n=1}^{\infty} g_{n} . K$ is contained in $L$. So $\left\{g_{n}\right\}$ is bounded in $C^{0}(K, M)$. Furthermore, the set of all $g_{n}$ is equicontinuous as subset of $\operatorname{Isom}(M)$. Therefore, by the theorem of Ascoli-Arzela, $\left\{g_{n}: n \in \mathbb{N}\right\}$ is relatively compact in $\operatorname{Isom}(M)$.

6.26. Theorem (Existence of slices, [187]. Let $M$ be a $G$-space and let $x \in M$ be a point with compact isotropy group $G_{x}$. If for all open neighborhoods $W$ of $G_{x}$ in $G$ there is a neighborhood $V$ of $x$ in $M$ such that $\{g \in G: g . V \cap V \neq \emptyset\} \subseteq W$, then there exists a slice at $x$.

Proof. Let $\tilde{\gamma}$ be any Riemann metric on $M$. Since $G_{x}$ is compact, we can get a $G_{x}$-invariant Riemann metric on $M$ by integrating over the Haar measure for the action of $G_{x}$; see (14.4).

$$
\gamma(X, Y):=\int_{G_{x}}\left(\ell_{a}^{*} \tilde{\gamma}\right)(X, Y) d a=\int_{G_{x}} \tilde{\gamma}\left(T \ell_{a} X, T \ell_{a} Y\right) d a
$$


We choose $\varepsilon>0$ small enough for $T_{x} M \supseteq B_{0_{x}}(\varepsilon) \stackrel{\exp _{x}^{\gamma}}{\longrightarrow} M$ to be a diffeomorphism onto its image and we define:

$$
\tilde{S}:=\exp _{x}^{\gamma}\left(T_{x}(G \cdot x)^{\perp} \cap B_{0_{x}}(\varepsilon)\right) \subseteq M .
$$

Then $\tilde{S}$ is a submanifold of $M$ and the first step towards obtaining a real slice. Let us show that $\tilde{S}$ is $G_{x}$-invariant. Since $G_{x}$ leaves $\gamma$ unchanged and $T_{x}(G . x)$ is invariant under $T_{x} \ell_{g}$ (for $g \in G_{x}$ ), $T_{x} \ell_{g}$ is an isometry and leaves $T_{x}(G . x)^{\perp} \cap B_{0_{x}}(\varepsilon)$ invariant. Therefore:

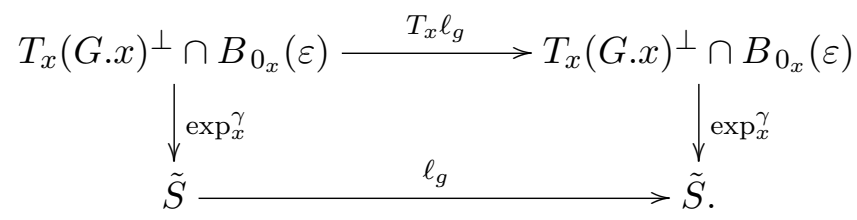

We have to shrink $\tilde{S}$ to an open subset $S$ such that for $g \in G$ with $g . S \cap S \neq \emptyset$ we have $g \in G_{x}$. This property is necessary for a slice. At this point, we shall need the condition that for every open neighborhood $W$ of $G_{x}$ in $G$, there is a neighborhood $V$ of $x$ in $M$ such that $\{g \in G: g . V \cap V \neq \emptyset\} \subseteq W$.

First we must construct a $W$ fitting our purposes. Choose an open neighborhood $U \subseteq G / G_{x}$ of $e . G_{x}$ such that there is a smooth section $\chi: U \rightarrow G$ of $\pi: G \rightarrow G / G_{x}$ with $\chi\left(e . G_{x}\right)=e$. Also, let $U$ and possibly $\tilde{S}$ be small enough to get an embedding

$$
f: U \times \tilde{S} \rightarrow M:(u, s) \mapsto \chi(u) . s .
$$

Our neighborhood of $G_{x}$ will be $W:=\pi^{-1}(U)$. Now by our assumption, there is a neighborhood $V$ of $x$ in $M$ such that $\{g \in G: g . V \cap V \neq \emptyset\} \subseteq W$.

Next we will prove that $V$ can be chosen $G_{x}$-invariant. Suppose we can choose an open neighborhood $\tilde{W}$ of $G_{x}$ in $G$ such that $G_{x} \cdot \tilde{W} \subseteq W$ (we will prove this below). Let $V^{\prime}$ be the neighborhood of $x$ in $M$ satisfying $\left\{g \in G: g . V^{\prime} \cap V^{\prime} \neq \emptyset\right\} \subseteq \tilde{W}$. Now $V:=G_{x} . V^{\prime}$ has the desired property, since:

$$
\begin{aligned}
\{g \in G & \left.: g \cdot G_{x} \cdot V^{\prime} \cap G_{x} \cdot V^{\prime} \neq \emptyset\right\}=\bigcup_{g_{1}, g_{2} \in G_{x}}\left\{g \in G: g \cdot g_{1} \cdot V^{\prime} \cap g_{2} \cdot V^{\prime} \neq \emptyset\right\} \\
& =\bigcup_{g_{1}, g_{2} \in G_{x}}\left\{g \in G: g_{2}^{-1} g g_{1} \cdot V^{\prime} \cap V^{\prime} \neq \emptyset\right\} \\
& =\bigcup_{g_{1}, g_{2} \in G_{x}} g_{2}\left\{g \in G: g \cdot V^{\prime} \cap V^{\prime} \neq \emptyset\right\} g_{1}^{-1} \\
& =G_{x} \cdot\left\{g \in G: g \cdot V^{\prime} \cap V^{\prime} \neq \emptyset\right\} \cdot G_{x} \subseteq G_{x} \cdot \tilde{W} \cdot G_{x} \subseteq W \cdot G_{x}=W .
\end{aligned}
$$


To complete the above argumentation, we have only to prove the

Claim. To any open neighborhood $W$ of $G_{x}$ in $G$ there is an open neighborhood $\tilde{W}$ of $G_{x}$ such that $G_{x} . \tilde{W} \subseteq W$.

The proof of this claim relies on the compactness of $G_{x}$. For all $(a, b) \in G_{x} \times$ $G_{x}$ we choose neighborhoods $A_{a, b}$ of $a$ and $B_{a, b}$ of $b$, such that $A_{a, b} . B_{a, b} \subseteq W$. This is possible by continuity, since $G_{x} \cdot G_{x}=G_{x} .\left\{B_{a, b}: b \in G_{x}\right\}$ is an open cover of $G_{x}$. Then there is a finite subcover $\bigcup_{j=1}^{N} B_{a, b_{j}}:=B_{a} \supseteq G_{x}$. Since $A_{a, b_{j}} \cdot B_{a, b_{j}} \subseteq W$, we must choose $A_{a}:=\bigcap_{j=1}^{N} A_{a, b_{j}}$, to get $A_{a} . B_{a} \subseteq W$. Now since $A_{a}$ is a neighborhood of $a$ in $G_{x}$, the $A_{a}$ cover $G_{x}$ again. Consider a finite subcovering $A:=\bigcup_{j=1}^{n} A_{a_{j}} \supseteq G_{x}$, and as before define $B:=\bigcap_{j=1}^{n} B_{a_{j}}$, so that $A . B \subseteq W$. In particular, this gives us $G_{x} . B \subseteq W$, so $\tilde{W}:=B$ is an open neighborhood of $G_{x}$ with the desired property.

So we have a $G_{x}$-invariant neighborhood $V$ of $x$ with $\{g \in G: g V \cap V \neq \emptyset\}$ contained in $W$. Now we define $S:=\tilde{S} \cap V$ and hope for the best. The $S$ is an open subset of $\tilde{S}$, and it is again invariant under $G_{x}$. Let us check whether we have the converse: $\{g \in G: g . S \cap S \neq \emptyset\} \subseteq G_{x}$. If $g . s_{1}=s_{2}$ for some $s_{1}, s_{2} \in S$, then $g \in W=\pi^{-1}(U)$ by the above effort. Therefore $\pi(g) \in U$. Choose $h=g^{-1} \chi(\pi(g)) \in G_{x}$. Then

$$
f\left(\pi(g), h^{-1} s_{1}\right)=\chi(\pi(g)) h^{-1} s_{1}=g \cdot s_{1}=s_{2}=f\left(\pi(e), s_{2}\right) .
$$

Since $f$ is a diffeomorphism onto its image, we have shown that $\pi(g)=\pi(e)$, that is, $g \in G_{x}$.

Now, it is easy to see that $F: G \times{ }_{G_{x}} S \rightarrow G . S:[g, s] \mapsto g . s$ is well defined, $G$-equivariant and smooth. We have the diagram

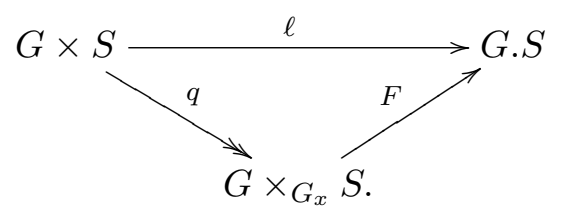

To finish the proof, we have to show that $F$ is a diffeomorphism, according to (6.19), Firstly, $F$ is injective because:

$$
\begin{aligned}
& F[g, s]=F\left[g^{\prime}, s^{\prime}\right] \Rightarrow g . s=g^{\prime} . s^{\prime} \Rightarrow g^{-1} g^{\prime} . s^{\prime}=s \\
& \Rightarrow g^{-1} g^{\prime} \in G_{x} \Rightarrow[g, s]=\left[g, g^{-1} g^{\prime} . s^{\prime}\right]=\left[g^{\prime}, s^{\prime}\right] .
\end{aligned}
$$

Next, we notice that $\ell(W, S)=W . S=f(U, S)$ is open in $M$ since $f$ : $U \times \tilde{S} \rightarrow M$ is an embedding with an open image. Consequently, G.S = $\ell(G, W . S)$ is open, since $\ell$ is open, and thus $F$ is a diffeomorphism.

6.27. Theorem ([187]). If $M$ is a proper $G$-manifold, then for all $x \in M$ the conditions of the previous theorem are satisfied, so each $x$ has slices. 
Proof. We have already shown that each isotropy group $G_{x}$ is compact (6.21). Now for every neighborhood $U$ of $G_{x}$ in $G$, for every $x \in M$, it remains to find a neighborhood $V$ of $x$ in $M$ such that

$$
\{g \in G: g \cdot V \cap V \neq \emptyset\} \subseteq U .
$$

Claim. $U$ contains an open neighborhood $\tilde{U}$ with $G_{x} \cdot \tilde{U}=\tilde{U}$; so we will be able to assume $G_{x} . U=U$ without loss of generality.

The claim in the proof of theorem (6.26) shows the existence of a neighborhood $B$ of $G_{x}$ such that $G_{x} . B \subseteq U$, using only the compactness of $G_{x}$. So $\tilde{U}:=G_{x} \cdot B=\bigcup_{g \in G_{x}} g . B$ is again an open neighborhood of $G_{x}$, and it has the desired properties.

Now we can suppose $U=G_{x} . U$. Next, we have to construct an open neighborhood $V \subseteq M$ of $x$, such that $\{g \in G: g . V \cap V \neq \emptyset\} \subseteq U$. This is the same as saying $(G \backslash U) . V \cap V$ should be empty. So we have to look for $V$ in the complement of $(G \backslash U) . x$.

We see that $M \backslash((G \backslash U) . x)$ is open, or rather that $(G \backslash U) . x$ is closed. This is because $(G \backslash U) \cdot x \times\{x\}=\left(\ell, \operatorname{pr}_{2}\right)((G \backslash U) \times\{x\})$ is the image of a closed set under $\left(\ell, \mathrm{pr}_{2}\right)$ which is a closed mapping by lemma (6.22).

Now let us choose a compact neighborhood $W$ of $x$ in $M \backslash((G \backslash U) . x)$. Then since $G$ acts properly, it follows that $\{g \in G: g . W \cap W \neq \emptyset\}$ is compact; in particular $K:=\{g \in G \backslash U: g . W \cap W \neq \emptyset\}$ is compact. But what we need is for $\{g \in G \backslash U: g . V \cap V \neq \emptyset\}$ to be empty. An $x$-neighborhood $V$ contained in $W$ fulfills this if $K . V \subseteq M \backslash W$. Let us find such a neighborhood.

Our choice of $W$ guarantees $K . x \subseteq M \backslash W$. But $M \backslash W$ is open; therefore for each $k \in K$ we can choose a neighborhood $Q_{k}$ of $k$ in $G$ and $V_{k}$ of $x$ in $W$, such that $Q_{k} . V_{k} \subseteq M \backslash W$. The neighborhoods $Q_{k}$ cover $K$, and we can choose a finite subcovering $\bigcup_{j=1}^{m} Q_{k_{j}}$. Then $V:=\bigcap_{j=1}^{m} V_{k_{j}}$ has the desired property: $K . V \subseteq M \backslash W$.

6.28. Lemma. Let $M$ be a proper $G$-manifold, $V$ a linear $G$-space and $f: M \rightarrow V$ smooth with compact support. Then

$$
\tilde{f}: x \mapsto \int_{G} g^{-1} f(g \cdot x) d_{R} g
$$

is a $G$-equivariant $C^{\infty}$-map with $\tilde{f}(x)=0$ for $x \notin G$. supp $f$ (where $d_{R}$ stands for a right Haar measure on $G$ ).

Proof. Since $G$ acts properly, $\{g \in G: g . x \in \operatorname{supp} f\}$ is compact. Therefore the map $g \mapsto g^{-1} f(g \cdot x)$ has compact support, and $\tilde{f}$ is well defined. To see that $\tilde{f}$ is smooth, let $x_{0}$ be in $M$, and let $U$ be a compact neighborhood of $x_{0}$. Then the set $\{g \in G: g . U \cap \operatorname{supp} f \neq \emptyset\}$ is compact. Therefore, 
$\tilde{f}$ restricted to $U$ is smooth; in particular $\tilde{f}$ is smooth in $x_{0}$. Also, $\tilde{f}$ is $G$-equivariant, since

$$
\begin{aligned}
\tilde{f}(h . x) & =\int_{G} g^{-1} f(g h . x) d_{R} g=\int_{G} h(g h)^{-1} f(g h . x) d_{R} g \\
& =h . \int_{G} g^{-1} f(g . x) d_{R} g=h \tilde{f}(x) .
\end{aligned}
$$

Furthermore, if $x \notin G$. $\operatorname{supp} f$, then $f(g \cdot x)=0$ for all $g \in G$; thus $\tilde{f}(x)=$ 0 .

6.29. Corollary. If $M$ is a proper $G$-manifold, then $M / G$ is completely regular.

Proof. Choose $F \subseteq M / G$ closed and $\bar{x}_{0}=\pi\left(x_{0}\right) \notin F$. Now let $U$ be a compact neighborhood of $x_{0}$ in $M$ fulfilling $U \cap \pi^{-1}(F)=\emptyset$, and choose $f \in C^{\infty}(M,[0, \infty))$ with support in $U$ such that $f\left(x_{0}\right)>0$. If we take the trivial representation of $G$ on $\mathbb{R}$, then from lemma (6.28) it follows that

$$
\tilde{f}(x)=\int_{G} f(g \cdot x) d_{R} g
$$

defines a smooth $G$-invariant function. Here $d_{R} g$ denote the right Harr measure on $G$; see $(14.4)$. Moreover, $\tilde{f}\left(x_{0}\right)>0$. Since $\operatorname{supp}(\tilde{f}) \subseteq G \cdot \operatorname{supp}(f) \subseteq$ $G . U$, we have $\operatorname{supp}(f) \cap \pi^{-1}(F)=\emptyset$. Since $\tilde{f} \in C^{\infty}(M,[0, \infty))^{G}$ is invariant, $f$ factors over $\pi$ to a map $\bar{f} \in C^{0}(M / G,[0, \infty))$, with $\bar{f}\left(\bar{x}_{0}\right)>0$ and $\left.\bar{f}\right|_{F}=0$.

6.30. Theorem. If $M$ is a proper $G$-manifold, then there is a $G$-invariant Riemann metric on $M$.

Proof. By (6.27) there is a slice $S_{x}$ at $x$ for all $x \in M$. Let $\pi: M \rightarrow M / G$ be the quotient map. Notice first that $M / G$ is Hausdorff by (6.29),

For each $x$ choose $f_{x} \in C^{\infty}(M,[0, \infty))$ with $f_{x}(x>0)$ and $\operatorname{supp}\left(f_{x}\right) \subseteq G . S_{x}$ compact; then by (6.28)

$$
\bar{f}_{x}(y):=\int_{G} f_{x}(g \cdot y) d_{R} g \in C^{\infty}(M,[0, \infty))^{G}
$$

is $G$-invariant, positive on $G . x$, and has $\operatorname{supp}\left(\bar{f}_{n}\right) \subseteq G \cdot S_{n}$. Moreover, $\pi\left(\operatorname{supp} \bar{f}_{x}\right)$ is a compact neighborhood of $\pi(x)$, so $M / G$ is locally compact. The interiors of the supports of the smooth functions $\bar{f}_{x}$ form an open cover of $M$. Since $M$ is a Lindelöf-space (1.6), there is a countable subcover with corresponding functions $\bar{f}_{x_{1}}, \bar{f}_{x_{2}}, \ldots$ We write $\bar{f}_{n}:=\bar{f}_{x_{n}}$ and $S_{N}:=S_{x_{n}}$. Let

$$
W_{n}=\left\{x \in M: \bar{f}_{n}(x)>0 \text { and } \bar{f}_{i}(x)<\frac{1}{n} \text { for } 1 \leq i<n\right\} \subseteq G . S_{n}
$$


and denote by $\bar{W}_{n}$ the closure. Then $\left\{W_{n}\right\}$ is a $G$-invariant open cover. We claim that $\left\{\bar{W}_{n}: n \in \mathbb{N}\right\}$ is locally finite: Let $x \in M$. Then there is a smallest $n$ such that $x \in W_{n}$. Let $V:=\left\{y \in M: \bar{f}_{n}(y)>\frac{1}{2} \bar{f}_{n}(x)\right\}$. If $y \in V \cap \bar{W}_{k}$, then we have $\bar{f}_{n}(y)>\frac{1}{2} \bar{f}_{n}(x)$ and $\bar{f}_{i}(y) \leq \frac{1}{k}$ for $i<k$, which is possible for finitely many $k$ only. Let $h(t)=e^{-1 / t}$ for $t>0$ and $h(t)=0$ for $t \leq 0$. Consider the nonnegative smooth function

$$
f_{n}(x):=h\left(\bar{f}_{n}(x)\right) h\left(\frac{1}{n}-f_{1}(x)\right) \ldots h\left(\frac{1}{n}-f_{n-1}(x)\right)
$$

for each $n$. Then obviously $\operatorname{supp}\left(f_{n}\right)=\bar{W}_{n} \subseteq G \cdot S_{n}$.

The action of the compact group $G_{x_{n}}$ on $\left.T M\right|_{S_{n}}$ is fiber linear, so there is a $G_{x_{n}}$-invariant Riemann metric $\gamma^{(n)}$ on the vector bundle $\left.T M\right|_{S_{x_{n}}}$ by integration over the compact group $G_{x_{n}}$. To get a Riemann metric on $\left.T M\right|_{G . S_{n}}$ invariant under the whole group $G$, consider the following diagram:

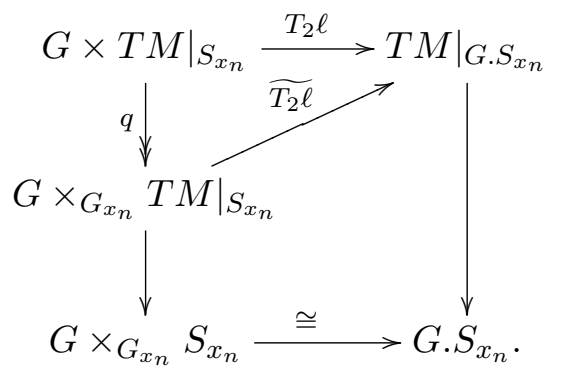

The map $T_{2} \ell:\left(g, X_{s}\right) \mapsto T_{s} \ell_{g} \cdot X_{s}$ factors over $q$ to a map $\widetilde{T_{2} \ell}$ which is injective, since if $T_{2} \ell\left(g, X_{s}\right)=T_{2} \ell\left(g^{\prime}, X_{s^{\prime}}\right)$, then on the one side $\ell(g . s)=$ $\ell\left(g^{\prime} . s^{\prime}\right)$ so $g^{-1} g^{\prime} . s^{\prime}=s$ and $g^{-1} g^{\prime} \in G_{x}$. On the other side, $T_{s} \ell_{g} . X_{s}=$ $T_{s^{\prime}} \ell_{g^{\prime}} \cdot X_{s^{\prime}}$. So

$$
\left(g^{\prime}, X_{s^{\prime}}\right)=\left\{g\left(g^{-1} g^{\prime}\right), T_{s^{\prime}} \ell_{g^{\prime-1}} T_{s} \ell_{g} \cdot X_{s}\right\}
$$

thus $q\left(g^{\prime}, X_{s^{\prime}}\right)=q\left(g, X_{s}\right)$.

The Riemann metric $\gamma^{(n)}$ induces a $G$-invariant vector bundle metric on $G \times\left. T M\right|_{S_{n}} \rightarrow G \times S_{n}$ by

$$
\gamma_{n}\left(\left(g, X_{s}\right),\left(g, Y_{s}\right)\right):=\gamma^{(n)}\left(X_{s}, Y_{s}\right) .
$$

It is also invariant under the right $G_{x_{n}}$-action $\left(g, X_{s}\right) \cdot h=\left(g h, T \ell_{h^{-1}} \cdot X_{s}\right)$ and, therefore, induces a Riemann metric $\tilde{\gamma}_{n}$ on $G \times\left._{G_{x}} T M\right|_{S_{n}}$. This metric is again $G$-invariant, since the actions of $G$ and $G_{x}$ commute. Now $\left(\widetilde{T_{2} \ell}\right)_{*} \tilde{\gamma}_{n}=$ : $\bar{\gamma}_{n}$ is a $G$-invariant Riemann metric on $\left.T M\right|_{G . S_{n}}$, and $\gamma:=\sum_{n=1}^{\infty} f_{n} \bar{\gamma}_{n}$ is a $G$-invariant Riemann metric on $M$.

6.31. Result ([187]). Let $G$ be a matrix group, that is, a Lie group with a faithful finite-dimensional representation, and let $M$ be a proper $G$-space with only a finite number of orbit types. Then there is a G-equivariant embedding $f: M \rightarrow V$ into a linear $G$-space $V$. 


\section{Polynomial and Smooth Invariant Theory}

7.1. A motivating example. Let $S(n)$ denote the space of symmetric $n \times n$ matrices with entries in $\mathbb{R}$ and $O(n)$ the orthogonal group. Consider the action:

$$
\ell: O(n) \times S(n) \rightarrow S(n), \quad(A, B) \mapsto A B A^{-1}=A B A^{T} .
$$

If $\Sigma$ is the space of all real diagonal matrices and $\mathcal{S}_{n}$ is the symmetric group on $n$ letters, then we have the following:

\section{Theorem.}

(1) This is an orthogonal $O(n)$-action on $S(n)$ for the inner product given by $\langle A, B\rangle=\operatorname{Trace}\left(A B^{T}\right)=\operatorname{Trace}(A B)$.

(2) $\Sigma$ meets every $O(n)$-orbit.

(3) If $B \in \Sigma$, then $\ell(O(n), B) \cap \Sigma$, the intersection of the $O(n)$-orbit through $B$ with $\Sigma$, equals the $\mathcal{S}_{n}$-orbit through $B$, where $\mathcal{S}_{n}$ acts on $B \in \Sigma$ by permuting the eigenvalues.

(4) $\Sigma$ intersects each orbit orthogonally with respect to the inner product $\langle A, B\rangle=\operatorname{Trace}\left(A B^{T}\right)=\operatorname{Trace}(A B)$ on $S(n)$.

(5) $\mathbb{R}[S(n)]^{O(n)}$, the space of all $O(n)$-invariant polynomials in $S(n)$ is isomorphic to $\mathbb{R}[\Sigma]^{\mathcal{S}_{n}}$, the symmetric polynomials in $\Sigma$ (by restriction).

(6) The space $C^{\infty}(S(n))^{O(n)}$ of $O(n)$-invariant $C^{\infty}$-functions is isomorphic to $C^{\infty}(\Sigma)^{\mathcal{S}_{n}}$, the space of all symmetric $C^{\infty}$-functions in $\Sigma$ (again by restriction), and these again are isomorphic to the $C^{\infty}$-functions in the elementary symmetric polynomials.

(7) The space of all $O(n)$-invariant horizontal $p$-forms on $S(n)$, the space of all $O(n)$-invariant $p$-forms $\omega$ with the property $i_{X} \omega=0$ for all $X \in$ $T_{A}(O(n) . A)$, is isomorphic to the space of $\mathcal{S}_{n}$-invariant $p$-forms on $\Sigma$ :

$$
\Omega_{h o r}^{p}(S(n))^{O(n)} \cong \Omega^{p}(\Sigma)^{\mathcal{S}_{n}} .
$$

Proof. (11) Let $A \in O(n)$ act on $H_{1}, H_{2} \in S(n)$; then

$$
\begin{aligned}
& \operatorname{Trace}\left(A H_{2} A^{-1}\left(A H_{1} A^{-1}\right)^{T}\right)=\operatorname{Trace}\left(A H_{2} A^{-1}\left(A^{-1}\right)^{T} H_{1}^{T} A^{T}\right) \\
& \quad=\operatorname{Trace}\left(A H_{2} A^{-1} A H_{1}^{T} A^{-1}\right)=\operatorname{Trace}\left(A H_{2} H_{1}^{T} A^{-1}\right)=\operatorname{Trace}\left(H_{2} H_{1}^{T}\right) .
\end{aligned}
$$

(21) Clear from linear algebra.

(3) The transformation of a symmetric matrix into normal form is unique except for the order in which the eigenvalues appear.

(44) Take an $A$ in $\Sigma$. For any $X \in \mathfrak{o}(n)$, that is, for any skew-symmetric $X$, let $\zeta_{X}$ denote the corresponding fundamental vector field on $S(n)$. Then we 
have

$$
\begin{gathered}
\zeta_{X}(A)=\left.\frac{d}{d t}\right|_{t=0} \exp _{e}(t X) A \exp _{e}\left(t X^{T}\right) \\
=X A i d+i d A X^{T}=X A-A X
\end{gathered}
$$

Now the inner product with $\eta \in T_{A} \Sigma \cong \Sigma$ computes to

$$
\begin{gathered}
\left\langle\zeta_{X}(A), \eta\right\rangle=\operatorname{Trace}\left(\zeta_{X}(A) \eta\right)=\operatorname{Trace}((X A-A X) \eta) \\
=\operatorname{Trace}(\underbrace{X A \eta}_{=X \eta A})-\operatorname{Trace}(A X \eta)=\operatorname{Trace}(X \eta A)-\operatorname{Trace}(X \eta A)=0 .
\end{gathered}
$$

(5) If $p \in \mathbb{R}[S(n)]^{O(n)}$, then clearly $\tilde{p}:=\left.p\right|_{\Sigma} \in \mathbb{R}[\Sigma]^{\mathcal{S}_{n}}$. To construct $p$ from $\tilde{p}$, we use the result from algebra that $\mathbb{R}\left[\mathbb{R}^{n}\right]^{\mathcal{S}_{n}}$ is just the ring of all polynomials in the elementary symmetric functions. So if we use the isomorphism

$$
A:=\left(\begin{array}{cccc}
a_{1} & 0 & \ldots & 0 \\
0 & a_{2} & \ldots & \\
\vdots & \vdots & \ddots & \\
0 & 0 & \ldots & a_{n}
\end{array}\right) \mapsto\left(a_{1}, a_{2}, \ldots, a_{n}\right)=: a
$$

to replace $\mathbb{R}^{n}$ by $\Sigma$, we find that each symmetric polynomial $\tilde{p}$ on $\Sigma$ is of the form

$$
\tilde{p}(A)=\bar{p}\left(\sigma_{1}(A), \sigma_{2}(A), \ldots, \sigma_{n}(A)\right) .
$$

It can be expressed as a polynomial $\bar{p}$ in the elementary symmetric functions

$$
\begin{aligned}
\sigma_{1} & =-x^{1}-x^{2}-\cdots-x^{n} \\
\sigma_{2} & =x^{1} x^{2}+x^{1} x^{3}+\ldots \\
& \ldots \\
\sigma_{k} & =(-1)^{k} \sum_{j_{1}<\cdots<j_{k}} x^{j_{1}} \ldots x^{j_{k}}, \\
& \ldots \\
\sigma_{n} & =(-1)^{n} X^{1} \ldots x^{n} .
\end{aligned}
$$

Let us consider the characteristic polynomial of the diagonal matrix $X$ with eigenvalues $x^{1}, \ldots, x^{n}$ :

$$
\begin{aligned}
\prod_{i=1}^{n}\left(t-x^{i}\right) & =t^{n}+\sigma_{1} \cdot t^{n-1}+\cdots+\sigma_{n-1} \cdot t+\sigma_{n} \\
& =\operatorname{det}(t \cdot I d-X) \\
& =\sum_{i=0}^{n}(-1)^{n-i} t^{i} c_{n-i}(X), \quad \text { where } \\
c_{k}(Y) & =\operatorname{Trace}\left(\bigwedge^{k} Y: \bigwedge^{k} \mathbb{R}^{n} \rightarrow \bigwedge^{k} \mathbb{R}^{n}\right)
\end{aligned}
$$


is the $k$-th characteristic coefficient of a matrix $Y$; see (14.9), So the $\sigma_{i}$ extend to $O(n)$-invariant polynomials $c_{i}$ on $S(n)$. We can now extend $\tilde{p}$ to a polynomial on $S(n)$ by

$$
\tilde{p}(H):=\bar{p}\left(c_{1}(H), c_{2}(H), \ldots, c_{n}(H)\right) \quad \text { for all } H \in S(n) .
$$

Therefore, $\tilde{p}$ is an $O(n)$-invariant polynomial on $S(n)$ and is unique as such due to (11).

(6) Again we have that $f \in C^{\infty}(S(n))^{O(n)}$ implies $\tilde{f}:=\left.f\right|_{\Sigma} \in C^{\infty}(\Sigma)^{\mathcal{S}_{n}}$. Finding an inverse map $\tilde{f} \mapsto f$ as above is possible due to the following theorem by Gerald Schwarz; see (7.13) below:

Let $G$ be a compact Lie group with a finite-dimensional representation $G \rightarrow$ $G L(V)$ and let $\rho_{1}, \rho_{2}, \ldots, \rho_{k}$ be generators for the algebra $\mathbb{R}[V]^{G}$ of $G$-invariant polynomials on $V$. It is finitely generated as an algebra due to Hilbert; see (7.2). Then, for any smooth function $h \in C^{\infty}(V)^{G}$, there is a function $\bar{h} \in C^{\infty}\left(\mathbb{R}^{k}\right)$ such that $h(v)=\bar{h}\left(\rho_{1}(v), \ldots, \rho_{k}(v)\right)$.

Now we can prove the assertion as in (5) above. Again we take the symmetric polynomials $\sigma_{1}, \ldots, \sigma_{n}$ as generators of $\mathbb{R}[\Sigma]^{\mathcal{S}_{n}}$. By Schwarz's theorem $(7.13)$, any $\tilde{f} \in C^{\infty}(\Sigma)^{\mathcal{S}_{n}}$ can be written as a smooth function in $\sigma_{1}, \ldots, \sigma_{n}$. So we have an $\bar{f} \in C^{\infty}\left(\mathbb{R}^{n}\right)$ such that

$$
\tilde{f}(A)=\bar{f}\left(\sigma_{1}(A), \ldots \sigma_{n}(A)\right) \quad \text { for all } A \in \Sigma .
$$

If we extend the $\sigma_{i}$ onto $S(n)$ as in (4), we can define

$$
f(H):=\bar{f}\left(c_{1}(H), c_{2}(H), \ldots, c_{n}(H)\right) \quad \text { for } H \in S(n) .
$$

Then $f$ is again a smooth function and it is the unique $O(n)$-invariant extension of $\tilde{f}$.

(17) Consider $\sigma=\left(\sigma_{1}, \ldots, \sigma_{n}\right): \Sigma \rightarrow \mathbb{R}^{n}$ and put $J(x):=\operatorname{det}(d \sigma(x))$. For each $\alpha \in \mathcal{S}_{n}$ we have

$$
\begin{aligned}
J . d x^{1} \wedge \cdots \wedge d x^{n} & =d \sigma_{1} \wedge \cdots \wedge d \sigma_{n} \\
& =\alpha^{*}\left(d \sigma_{1} \wedge \cdots \wedge d \sigma_{n}\right) \\
& =(J \circ \alpha) \cdot \alpha^{*}\left(d x^{1} \wedge \cdots \wedge d x^{n}\right) \\
& =(J \circ \alpha) \cdot \operatorname{det}(\alpha) \cdot d x^{1} \wedge \cdots \wedge d x^{n}, \\
J \circ \alpha=\operatorname{det}\left(\alpha^{-1}\right) . J . &
\end{aligned}
$$

From this we see firstly that $J$ is a homogeneous polynomial of degree

$$
0+1+\cdots+(n-1)=\frac{n(n-1)}{2}=\left(\begin{array}{l}
n \\
2
\end{array}\right) .
$$

The mapping $\sigma$ is a local diffeomorphism on the open set $U=\Sigma \backslash J^{-1}(0)$; thus $d \sigma_{1}, \ldots, d \sigma_{n}$ is a coframe on $U$, i.e., a basis of the cotangent bundle 
everywhere on $U$. Let $(i j)$ be the transpositions in $\mathcal{S}_{n}$, and let

$$
H_{(i j)}:=\left\{x \in \Sigma: x^{i}-x^{j}=0\right\}
$$

be the reflection hyperplanes of the $(i j)$. If $x \in H_{(i j)}$, then by (8) we have $J(x)=J((i j) x)=-J(x)$, so $J(X)=0$. Thus $J \mid H_{(i j)}=0$, so the polynomial $J$ is divisible by the linear form $x^{i}-x^{j}$, for each $i<j$. By comparing degrees, we see that

$$
J(x)=c . \prod_{i<j}\left(x^{i}-x^{j}\right), \quad \text { where } 0 \neq c \in \mathbb{R} .
$$

By the same argument we see that:

(10) If $g \in C^{\infty}(\Sigma)$ satisfies $g \circ \alpha=\operatorname{det}\left(\alpha^{-1}\right) . g$ for all $\alpha \in \mathcal{S}_{n}$, then $g=J . h$ for $h \in C^{\infty}(\Sigma)^{\mathcal{S}_{n}}$.

(11) Claim. Let $\omega \in \Omega^{p}(\Sigma)^{\mathcal{S}_{n}}$. Then we have

$$
\omega=\sum_{j_{1}<j_{2}<\cdots<j_{p}} \omega_{j_{1}, \ldots, j_{p}} d \sigma_{j_{1}} \wedge \cdots \wedge d \sigma_{j_{p}}
$$

on $\Sigma$, for $\omega_{j_{1}, \ldots, j_{p}} \in C^{\infty}(\Sigma)^{\mathcal{S}_{n}}$.

To prove claim (11) recall that $d \sigma_{1}, \ldots, d \sigma_{n}$ is an $\mathcal{S}_{n}$-invariant coframe on the $\mathcal{S}_{n}$-invariant open set $U$. Thus

$$
\begin{aligned}
\omega \mid U & =\sum_{j_{1}<j_{2}<\cdots<j_{p}} \underbrace{g_{j_{1}, \ldots, j_{p}}}_{\in C^{\infty}(U)} d \sigma_{j_{1}} \wedge \cdots \wedge d \sigma_{j_{p}} \\
& =\sum_{j_{1}<j_{2}<\cdots<j_{p}} \underbrace{\left(\frac{1}{n !} \sum_{\alpha \in \mathcal{S}_{n}} \alpha^{*} g_{j_{1}, \ldots, j_{p}}\right)}_{h_{j_{1}, \ldots, j_{p}} \in C^{\infty}(U)^{\mathcal{S}_{n}}} d \sigma_{j_{1}} \wedge \cdots \wedge d \sigma_{j_{p}} .
\end{aligned}
$$

Now choose $I=\left\{i_{1}<\cdots<i_{p}\right\} \subseteq\{1, \ldots, n\}$ and let $\bar{I}=\{1, \ldots, n\} \backslash I=$ $\left\{i_{p+1}<\cdots<i_{n}\right\}$. Then we have for a sign $\varepsilon= \pm 1$

$$
\begin{aligned}
\omega \mid U \wedge \underbrace{d \sigma_{i_{p+1}} \wedge \cdots \wedge d \sigma_{i_{n}}}_{d \sigma^{\bar{I}}} & =\varepsilon \cdot h_{I} \cdot d \sigma_{1} \wedge \cdots \wedge d \sigma_{n} \\
& =\varepsilon \cdot h_{I} . J . d x^{1} \wedge \cdots \wedge d x^{n} .
\end{aligned}
$$

On the whole of $\Sigma$ we have

$$
\omega \wedge d \sigma^{\bar{I}}=\varepsilon \cdot k_{I} \cdot d x^{1} \wedge \cdots \wedge d x^{n}
$$

for suitable $k_{I} \in C^{\infty}(\Sigma)$. By comparing the two expressions on $U$, we see from (8) that $k_{I} \circ \alpha=\operatorname{det}\left(\alpha^{-1}\right) \cdot k_{I}$ since $U$ is dense in $\Sigma$. So from (10) we may conclude that $k_{I}=J \cdot \omega_{I}$ for $\omega_{I} \in C^{\infty}(\Sigma)^{\mathcal{S}_{n}}$, but then $h_{I}=\omega_{I} \mid U$ and $\omega=\sum_{I} \omega_{I} d \sigma^{I}$ as asserted in claim (11). 
Now we may finish the proof. By the theorem of G. Schwarz (7.13) there exist $f_{I} \in C^{\infty}\left(\mathbb{R}^{n}\right)$ with $\omega_{I}=f_{I}\left(\sigma_{1}, \ldots, \sigma_{n}\right)$. Recall now the characteristic coefficients $c_{i} \in \mathbb{R}[S(n)]$ from the proof of (5) which satisfy $c_{i} \mid \Sigma=\sigma_{i}$. If we put now

$$
\tilde{\omega}:=\sum_{i_{1}<\cdots<i_{p}} f_{i_{1}, \ldots, i_{p}}\left(c_{1}, \ldots, c_{n}\right) d c_{i_{1}} \wedge \cdots \wedge d c_{i_{p}} \in \Omega_{\mathrm{hor}}^{p}(S(n))^{O(n)},
$$

then the pullback of $\tilde{\omega}$ to $\Sigma$ equals $\omega$.

7.2. Theorem of Hilbert and Nagata. Let $G$ be a Lie group with a finite-dimensional representation $G \rightarrow G L(V)$ and let one of the following conditions be fulfilled:

(1) $G$ is semisimple and has only a finite number of connected components.

(2) $V$ and $\langle G . f\rangle_{\mathbb{R}}$ are completely reducible for all $f \in \mathbb{R}[V]$; see (7.8).

Then $\mathbb{R}[V]^{G}$ is finitely generated as an algebra, or equivalently, there is a finite set of polynomials $\rho_{1}, \ldots, \rho_{k} \in \mathbb{R}[V]^{G}$, such that the map $\rho:=$ $\left(\rho_{1}, \ldots, \rho_{k}\right): V \rightarrow R^{k}$ induces a surjection

$$
\mathbb{R}\left[\mathbb{R}^{k}\right] \stackrel{\rho^{*}}{\longrightarrow} \mathbb{R}[V]^{G} .
$$

Remark. The first condition is stronger than the second since for a connected, semisimple Lie group, or for one with a finite number of connected components, every finite-dimensional representation is completely reducible. To prove the theorem, we will only need to know complete reducibility for the finite-dimensional representations $V$ and $\langle G . f\rangle_{\mathbb{R}}$ though as in (2).

7.3. Lemma. Let $A=\bigoplus_{i>0} A_{i}$ be a graded $\mathbb{R}$-algebra with $A_{0}=\mathbb{R}$. If $A_{+}:=\bigoplus_{i>0} A_{i}$ is finitely generated as an $A$-module, then $A$ is finitely generated as an $\mathbb{R}$-algebra.

Proof. Let $a_{1}, \ldots, a_{n} \in A_{+}$be generators of $A_{+}$as an $A$-module. Since they can be chosen homogeneous, we assume $a_{i} \in A_{d_{i}}$ for positive integers $d_{i}$.

Claim. The $a_{i}$ generate $A$ as an $\mathbb{R}$-algebra: $A=\mathbb{R}\left[a_{1}, \ldots, a_{n}\right]$.

We will show by induction that $A_{i} \subseteq \mathbb{R}\left[a_{1}, \ldots, a_{n}\right]$ for all $i$. For $i=0$ the assertion is clearly true, since $A_{0}=\mathbb{R}$. Now suppose $A_{i} \subseteq \mathbb{R}\left[a_{1}, \ldots, a_{n}\right]$ for all $i<N$. Then we have to show that

$$
A_{N} \subseteq \mathbb{R}\left[a_{1}, \ldots, a_{n}\right]
$$

as well. Take any $a \in A_{N}$. Then $a$ can be expressed as

$$
a=\sum_{i, j} c_{j}^{i} a_{i}, \quad c_{j}^{i} \in A_{j} .
$$


Since $a$ is homogeneous of degree $N$, we can discard all $c_{j}^{i} a_{i}$ with total degree $j+d_{i} \neq N$ from the right hand side of the equation. If we set $c_{N-d_{i}}^{i}=: c^{i}$, we get

$$
a=\sum_{i} c^{i} a_{i}
$$

In this equation all terms are homogeneous of degree $N$. In particular, any occurring $a_{i}$ have degree $d_{i} \leq N$. Consider first the $a_{i}$ of degree $d_{i}=N$. The corresponding $c^{i}$ then automatically lie in $A_{0}=\mathbb{R}$, so $c^{i} a_{i} \in \mathbb{R}\left[a_{1}, \ldots, a_{n}\right]$. To handle the remaining $a_{i}$, we use the induction hypothesis. Since $a_{i}$ and $c^{i}$ are of degree $<N$, they are both contained in $\mathbb{R}\left[a_{1}, \ldots, a_{n}\right]$. Therefore, $c^{i} a_{i}$ lies in $\mathbb{R}\left[a_{1}, \ldots, a_{n}\right]$ as well. So $a=\sum c^{i} a_{i} \in \mathbb{R}\left[a_{1}, \ldots, a_{n}\right]$, which completes the proof.

Remark. If we apply this lemma for $A=\mathbb{R}[V]^{G}$, we see that to prove (7.2) we only have to show that $\mathbb{R}[V]_{+}^{G}$, the algebra of all invariant polynomials of strictly positive degree, is finitely generated as a module over $[V]^{G}$. The first step in this direction will be to prove the weaker statement:

$$
B:=\left\langle\mathbb{R}[V]_{+}^{G}\right\rangle_{\mathbb{R}[V]}=\mathbb{R}[V] \cdot \mathbb{R}[V]_{+}^{G} \text { is finitely generated as an ideal. }
$$

This is a consequence of a well known theorem by Hilbert:

7.4. Theorem (Hilbert's ideal basis theorem). If $A$ is a commutative Noetherian ring, then the polynomial ring $A[x]$ is Noetherian as well.

A ring is Noetherian if every strictly ascending sequence of left ideals $I_{0} \subset$ $I_{1} \subset I_{2} \subset \ldots$ is finite, or equivalently, if every left ideal is finitely generated. If we choose $A=\mathbb{R}$, the theorem states that $\mathbb{R}[x]$ is again Noetherian. Now consider $A=\mathbb{R}[x]$; then $\mathbb{R}[x][y]=\mathbb{R}[x, y]$ is Noetherian, and so on. By induction, we see that $\mathbb{R}[V]$ is Noetherian. Therefore, any left ideal in $\mathbb{R}[V]$, in particular $B$, is finitely generated.

Proof of (7.4). Take any ideal $I \subseteq A[x]$ and denote by $A_{i}$ the set of leading coefficients of all $i$-th degree polynomials in $I$. Then $A_{i}$ is an ideal in $A$, and we have a sequence of ideals

$$
A_{0} \subseteq A_{1} \subseteq A_{2} \subseteq \cdots \subseteq A
$$

Since $A$ is Noetherian, this sequence stabilizes after a certain index $r$, i.e., $A_{r}=A_{r+1}=\cdots$. Let $\left\{a_{i 1}, \ldots, a_{i n_{i}}\right\}$ be a set of generators for $A_{i}(i=$ $1, \ldots, r)$, and let $p_{i j}$ be a polynomial of degree $i$ in $I$ with leading coefficient $a_{i j}$.

Claim. These polynomials generate $I$.

Let $\mathcal{P}=\left\langle p_{i j}\right\rangle_{A[x]} \subseteq A[x]$ be the ideal generated by the $p_{i j}$. Then $\mathcal{P}$ clearly contains all constants in $I\left(A_{0} \subseteq I\right)$. Let us show by induction that it contains all polynomials in $I$ of degree $d>0$ as well. Take any polynomial 
$p$ of degree $d$. We distinguish between two cases.

(1) Suppose $d \leq r$. Then we can find coefficients $c_{1}, \ldots, c_{n_{d}} \in A$ such that

$$
\tilde{p}:=p-c_{1} p_{d 1}-c_{2} p_{d 2}-\ldots-c_{n_{d}} p_{d n_{d}}
$$

has degree $<d$.

(2) Suppose $d>r$. Then the leading coefficients of $x^{d-r} p_{r 1}, \ldots, x^{d-r} p_{r n_{r}} \in I$ generate $A_{d}$. So we can find coefficients $c_{1}, \ldots, c_{n_{r}} \in A$ such that

$$
\tilde{p}:=p-c_{1} x^{d-r} p_{r 1}-c_{2} x^{d-r} p_{r 2}-\ldots-c_{n_{r}} x^{d-r} p_{r n_{r}}
$$

has degree $<d$.

In both cases we have $p \in \tilde{p}+\mathcal{P}$ and $\operatorname{deg} \tilde{p}<d$. Therefore by the induction hypothesis $\tilde{p}$, and with it $p$, lies in $\mathcal{P}$.

To prove theorem (7.2), it remains only to show the following:

7.5. Lemma. Let $G$ be a Lie group acting on $V$ such that the same conditions as in Hilbert and Nagata's theorem are satisfied. Then for $f_{1}, \ldots, f_{k} \in$ $\mathbb{R}[V]^{G}$ :

$$
\mathbb{R}[V]^{G} \cap\left\langle f_{1}, \ldots, f_{k}\right\rangle_{\mathbb{R}[V]}=\left\langle f_{1}, \ldots, f_{k}\right\rangle_{\mathbb{R}[V]^{G}}
$$

where the brackets denote the generated ideal (module) in the specified space.

7.6. Remark. In our case, if we take $f_{i}=\rho_{i} \in \mathbb{R}[V]_{+}^{G}$ to be the finite system of generators of $B$ as an ideal in $\mathbb{R}[V]$, we get:

$$
\mathbb{R}[V]_{+}^{G}=\mathbb{R}[V]^{G} \cap B=\left\langle\rho_{1}, \ldots, \rho_{k}\right\rangle_{\mathbb{R}[V]^{G}}
$$

That is, the $\rho_{i}$ generate $\mathbb{R}[V]_{+}^{G}$ as an $\mathbb{R}[V]^{G}$-module. With lemma (7.3), Hilbert and Nagata's theorem (7.2) follows immediately.

7.7. Remark. The inclusion $(\supseteq)$ in lemma (7.5) is trivial. If $G$ is compact, then the opposite inclusion

$$
\mathbb{R}[V]^{G} \cap\left\langle f_{1}, \ldots, f_{k}\right\rangle_{\mathbb{R}[V]} \subseteq\left\langle f_{1}, \ldots, f_{k}\right\rangle_{\mathbb{R}[V]^{G}}
$$

is easily seen as well. Take any $f \in \mathbb{R}[V]^{G} \cap\left\langle f_{1}, \ldots, f_{k}\right\rangle_{\mathbb{R}[V]}$. Then $f$ can be written as

$$
f=\sum p_{i} f_{i}, \quad p_{i} \in \mathbb{R}[V]
$$

Since $G$ is compact, we can integrate both sides over $G$ using the Haar measure $d g$; see (14.4).

$$
f(x)=\int_{G} f(g \cdot x) d g=\sum_{i} \int_{G} p_{i}(g \cdot x) f_{i}(g \cdot x) d g=\sum_{i} \underbrace{\left(\int_{G} p_{i}(g \cdot x) d g\right)}_{=: p_{i}^{*}(x)} f_{i}(x) .
$$

The $p_{i}^{*}$ are $G$-invariant polynomials; therefore $f$ is in $\left\langle f_{1}, \ldots, f_{k}\right\rangle_{\mathbb{R}[V]^{G}}$.

To show the lemma in its general form, we will need to find a replacement for the integral. This is done in the following central lemma. 
7.8. Lemma ([170]). Under the same conditions as theorem (7.2), for any $f \in \mathbb{R}[V]$ there exists an $f^{*} \in \mathbb{R}[V]^{G} \cap\langle G . f\rangle_{\mathbb{R}}$ such that

$$
f-f^{*} \in\langle G f-G f\rangle_{\mathbb{R}}
$$

Proof. Take $f \in \mathbb{R}[V]$. Clearly, $f$ is contained in $M_{f}:=\langle G . f\rangle_{\mathbb{R}}$, where $f^{*}$ is supposed to lie as well. The vector space $M_{f}$ is a finite-dimensional subspace of $\mathbb{R}[V]$ since it is contained in

$$
M_{f} \subseteq \bigoplus_{i \leq \operatorname{deg} f} \mathbb{R}[V]_{i} .
$$

In addition we have that

$$
\langle G . f-G . f\rangle_{\mathbb{R}}=: N_{f} \subseteq M_{f}
$$

is an invariant subspace. So we can restrict all our considerations to the finite-dimensional $G$-space $M_{f}$ which is completely reducible by our assumption.

If $f \in N_{f}$, then we can set $f^{*}=0$ and we are done. Suppose $f \notin N_{f}$. Then the $f^{*}$ we are looking for must also lie in $M_{f} \backslash N_{f}$. From the identity

$$
g . f=f+\underbrace{(g . f-f)}_{\in N_{f}} \quad \text { for all } g \in G
$$

it follows that

$$
M_{f}=N_{f} \oplus \mathbb{R} . f .
$$

In particular, $N_{f}$ has codimension 1 in $M_{f}$.

Since we require $f^{*}$ to be $G$-invariant, $\mathbb{R}$. $f^{*}$ will be a 1 -dimensional $G$ invariant subspace of $M_{f}$ which is not contained in $N_{f}$. As we just saw, $N_{f}$ has codimension 1 in $M_{f}$; therefore $\mathbb{R} . f^{*}$ will be a complementary subspace to $N_{f}$.

If we now write $M_{f}$ as the direct sum

$$
M_{f}=N_{f} \oplus P
$$

where $P$ is the invariant subspace complementary to $N_{f}$ guaranteed by the complete irreducibility of $M_{f}$, then $P$ is a good place to look for $f^{*}$.

Now $P \cong M_{f} / N_{f}$ as a $G$-module, so let us take a look at the action of $G$ on $M_{f} / N_{f}$. Every element of $M_{f} / N_{f}$ has a representative in $\mathbb{R}$. $f$, so we need only consider elements of the form $\lambda f+N_{f}(\lambda \in \mathbb{R})$. For arbitrary $g \in G$ we have:

$$
g \cdot\left(\lambda f+N_{f}\right)=\lambda g . f+N_{f}=\lambda f+\underbrace{(\lambda g \cdot f-\lambda f)}_{\in N_{f}}+N_{f}=\lambda f+N_{f} .
$$


So $G$ acts trivially on $M_{f} / N_{f}$ and therefore on $P$. This is good news, since now every $f^{\prime} \in P$ is $G$-invariant and we only have to project $f$ onto $P$ (along $N_{f}$ ) to get the desired $f^{*} \in \mathbb{R}[V]^{G} \cap M_{f}$.

Proof of lemma (7.5), Recall that for arbitrary $f_{1}, \ldots, f_{k}$ we have to show

$$
\mathbb{R}[V]^{G} \cap\left\langle f_{1}, \ldots, f_{k}\right\rangle_{\mathbb{R}[V]} \subseteq\left\langle f_{1}, \ldots, f_{k}\right\rangle_{\mathbb{R}[V]^{G}}
$$

We will do so by induction on $k$. For $k=0$ the assertion is trivial.

Suppose the lemma is valid for $k=r-1$. Consider $f_{1}, \ldots, f_{r} \in \mathbb{R}[V]^{G}$ and $f \in \mathbb{R}[V]^{G} \cap\left\langle f_{1}, \ldots, f_{r}\right\rangle_{\mathbb{R}[V]}$. Then

$$
f=\sum_{i=1}^{r} p_{i} f_{i}, \quad p_{i} \in \mathbb{R}[V] .
$$

By Nagata's lemma (7.8), we can approximate $p_{i}$ up to $\left\langle G . p_{i}-G \cdot p_{i}\right\rangle_{\mathbb{R}}$ by a $p_{i}^{*} \in \mathbb{R}[V]^{G}$. So for some finite subset $F \subset G \times G$ we have

$$
p_{i}=p_{i}^{*}+\sum_{s, t \in F} \lambda_{s, t}^{i}\left(s \cdot p_{i}-t \cdot p_{i}\right), \quad \lambda_{s, t}^{i} \in \mathbb{R} .
$$

Therefore we have

$$
f-\sum_{i=1}^{r} p_{i}^{*} f_{i}=\sum_{i=1}^{r} \sum_{s, t \in F} \lambda_{s, t}^{i}\left(s . p_{i}-t . p_{i}\right) f_{i} \in \mathbb{R}[V]^{G} .
$$

It remains to show that the right hand side of this lies in $\left\langle f_{1}, \ldots, f_{r}\right\rangle_{\mathbb{R}[V]^{G}}$. Notice that by the $G$-invariance of $f$ :

$$
\sum_{i=1}^{r}\left(s p_{i}-t p_{i}\right) f_{i}=s . \sum_{i=1}^{r} p_{i} f_{i}-t . \sum_{i=1}^{r} p_{i} f_{i}=s . f-t . f=0
$$

for all $s, t \in G$. Therefore

$$
\sum_{i=1}^{r-1}\left(s \cdot p_{i}-t . p_{i}\right) f_{i}=\left(t \cdot p_{r}-s \cdot p_{r}\right) f_{r} .
$$

Now we can use the induction hypothesis on

$$
\begin{aligned}
& \sum_{i=1}^{r} \sum_{s, t \in F} \lambda_{s, t}^{i}\left(s . p_{i}-t . p_{i}\right) f_{i} \\
& \quad=\sum_{i=1}^{r-1} \sum_{s, t \in F}\left(\lambda_{s, t}^{i}-\lambda_{s, t}^{r}\right)\left(s . p_{i}-t . p_{i}\right) f_{i} \in \mathbb{R}[V]^{G} \cap\left\langle f_{1}, \ldots, f_{r-1}\right\rangle_{\mathbb{R}[V]}
\end{aligned}
$$

to complete the proof. 
7.9. Remark. With lemma (7.5), Hilbert and Nagata's theorem is proved as well. So in the setting of (7.2) we now have an exact sequence

$$
0 \longrightarrow \rho^{*} \longrightarrow \mathbb{R}\left[\mathbb{R}^{k}\right] \stackrel{\rho^{*}}{\longrightarrow} \mathbb{R}[V]^{G} \longrightarrow 0
$$

where ker $\rho^{*}=\left\{R \in \mathbb{R}\left[\mathbb{R}^{k}\right]: R\left(\rho_{1}, \ldots, \rho_{k}\right)=0\right\}$ is just the finitely generated ideal consisting of all relations between the $\rho_{i}$.

Since the action of $G$ respects the grading of $\mathbb{R}[V]=\bigoplus_{k} \mathbb{R}[V]_{k}$, it induces an action on the space of all power series, $\mathbb{R}[[V]]=\Pi_{k=1}^{\infty} \mathbb{R}[V]_{k}$, and we have the following:

7.10. Theorem. Let $G$ be a Lie group with a finite-dimensional representation $G \rightarrow G L(V)$ satisfying the conditions of Hilbert and Nagata's theorem $(7.2)$. Let $\rho_{1}, \ldots, \rho_{k} \in \mathbb{R}[V]^{G}$ be generators for the algebra $\mathbb{R}[V]^{G}$ which exist by (7.2), Then the map

$$
\rho:=\left(\rho_{1}, \ldots, \rho_{k}\right): V \rightarrow R^{k}
$$

induces a surjection

$$
\mathbb{R}\left[\left[\mathbb{R}^{k}\right]\right] \stackrel{\rho^{*}}{\longrightarrow} \mathbb{R}[[V]]^{G}
$$

between the spaces of formal power series.

Proof. Write the formal power series $f \in \mathbb{R}[[V]]^{G}$ as the sum of its homogeneous parts:

$$
f(x)=f_{0}+f_{1}(x)+f_{2}(x)+\ldots
$$

Then to each $f_{i}(x) \in \mathbb{R}[V]_{i}^{G}$ there is a $g_{i}(y) \in \mathbb{R}\left[\mathbb{R}^{k}\right]$ such that $f_{i}(x)=$ $g_{i}\left(\rho_{1}(x), \ldots, \rho_{k}(x)\right)$. Before we can set

$$
g(y)=g_{0}+g_{1}(y)+g_{2}(y)+\ldots
$$

to finish the proof, we have to check whether this expression is finite in each degree. This is the case, since the lowest degree $\lambda_{i}$ that can appear in $g_{i}$ goes to infinity with $i$ :

Write explicitly $g_{i}=\sum_{|\alpha| \leq i} A_{i, \alpha} y^{\alpha}$ and take an $A_{i, \alpha} \neq 0$. Then $\operatorname{deg} f_{i}=i=$ $\alpha_{1} d_{1}+\cdots+\alpha_{k} d_{k}$ where $\bar{d}_{i}=\operatorname{deg} \rho_{i}$ and

$$
\lambda_{i}=\inf \left\{|\alpha|: i=\sum \alpha_{j} d_{j}\right\} \rightarrow \infty \quad(i \rightarrow \infty) .
$$

7.11. The orbit space of a representation. If $G$ is a Lie group acting smoothly on a manifold $M$, then the orbit space $M / G$ is not generally again a smooth manifold. Yet, it still has a functional structure induced by the smooth structure on $M$ simply by calling a function $f: M / G \rightarrow \mathbb{R}$ smooth if and only if $f \circ \pi: M \rightarrow \mathbb{R}$ is smooth (where $\pi: M \rightarrow M / G$ is the quotient map). 
In the following, let $G$ be a compact Lie group, $\ell: G \rightarrow L(V)$ a representation on $V=\mathbb{R}^{n}$. Let $\rho_{1}, \ldots, \rho_{k} \in \mathbb{R}[V]^{G}$ denote a finite system of generators for the algebra $\mathbb{R}[V]^{G}$, and let $\rho$ denote the polynomial mapping:

$$
\rho:=\left(\rho_{1}, \ldots, \rho_{k}\right): V \rightarrow \mathbb{R}^{k} .
$$

Lemma. Let $G$ be a compact Lie group. Then we have

(1) $\rho$ is proper (6.20) so $\rho^{-1}$ (compact) is compact.

(2) $\rho$ separates the orbits of $G$.

(3) There is a map $\bar{\rho}: V / G \rightarrow R^{k}$ such that the diagram

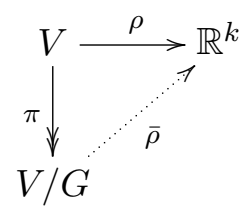

commutes and $\bar{\rho}$ is a homeomorphism onto its image.

Proof. (1) Let $r(x)=|x|^{2}=\langle x, x\rangle$, for an invariant inner product on $V$. Then $r \in \mathbb{R}[V]^{G}$. By (7.2) there is a polynomial $p \in \mathbb{R}\left[\mathbb{R}^{k}\right]$ such that $r(x)=$ $p(\rho(x))$. If $\left(x_{n}\right) \in V$ is an unbounded sequence, then $r\left(x_{n}\right)$ is unbounded. Therefore $p\left(\rho\left(x_{n}\right)\right)$ is unbounded, and, since $p$ is a polynomial, $\rho\left(x_{n}\right)$ is also unbounded. For compact $K \subset \mathbb{R}^{k}$ then $\rho^{-1}(K)$ is closed and bounded, thus compact. So $\rho$ is proper.

(2) Choose two different orbits $G . x \neq G . y(x, y \in V)$ and consider the map:

$$
f: G . x \cup G . y \rightarrow \mathbb{R}, \quad f(v):= \begin{cases}0 & \text { for } v \in G . x, \\ 1 & \text { for } v \in G . y .\end{cases}
$$

Both orbits are compact and $f$ is continuous. Therefore, by the Weierstrass approximation theorem, there is a polynomial $p \in \mathbb{R}[V]$ such that

$$
\|p-f\|_{G . x \cup G . y}=\sup \{|p(z)-f(z)|: z \in G . x \cup G . y\}<\frac{1}{10} .
$$

Now we can average $p$ over the group using the Haar measure $d g$ on $G$ from (14.4) to get a $G$-invariant function

$$
q(v):=\int_{G} p(g \cdot v) d g
$$

Note that since the action of $G$ is linear, $q$ is again a polynomial. For $v \in G . x \cup G . y$, we have

$$
|\underbrace{\int_{G} f(g \cdot v) d g}_{=f(v)}-\int_{G} p(g \cdot v) d g .| \leq \int_{G}|f(g \cdot v)-p(g \cdot v)| d g \leq \frac{1}{10} \underbrace{\int_{G} d g}_{=1} .
$$


Recalling how $f$ was defined, we get

$$
\begin{aligned}
|q(v)| & \leq \frac{1}{10} & & \text { for } v \in G . x, \\
|1-q(v)| & \leq \frac{1}{10} & & \text { for } v \in G . y .
\end{aligned}
$$

Therefore $q(G . x) \neq q(G . y)$, and since $q$ can be expressed in the Hilbert generators, we can conclude that $\rho(G . x) \neq \rho(G . y)$.

(3) Clearly, $\bar{\rho}$ is well defined and continuous for the quotient topology on $V / G$. By (2) the mapping $\bar{\rho}$ is injective, and by (11) it is proper, thus closed by (6.22). So it is a homeomorphism onto its image.

7.12. Remark. (1) If $f: V \rightarrow \mathbb{R}$ is in $C^{0}(V)^{G}$, then $f$ factors over $\pi$ to a continuous map $\tilde{f}: V / G \rightarrow \mathbb{R}$. By (7.11.3) there is a continuous map $\bar{f}: \rho(V) \rightarrow R$ given by $\bar{f}=\tilde{f} \circ \bar{\rho}^{-1}$. It has the property $f=\bar{f} \circ \rho$. Since $\rho(V)$ is closed, $\bar{f}$ extends to a continuous function $\bar{f} \in C^{0}\left(\mathbb{R}^{k}\right)$ (Tietze-Urysohn). So for continuous functions we have the assertion that

$$
\rho^{*}: C^{0}\left(\mathbb{R}^{k}\right) \rightarrow C^{0}(V)^{G} \quad \text { is surjective. }
$$

(2) The subset $\rho(V) \subset \mathbb{R}^{k}$ is a real semialgebraic variety, i.e., it is described by a finite number of polynomial equations and inequalities. In the complex case, the image of an algebraic variety under a polynomial map is again an algebraic variety, meaning it is described by polynomial equations only. In the real case this is already disproved by the simple polynomial map: $x \mapsto x^{2}$.

7.13. Result. $C^{\infty}$-Invariant Theorem. Let $G$ be a compact Lie group, $\ell$ : $G \rightarrow O(V)$ a finite-dimensional representation, and $\rho_{1}, \rho_{2}, \ldots, \rho_{k}$ generators for the algebra $\mathbb{R}[V]^{G}$ of $G$-invariant polynomials on $V$ (this space is finitely generated as an algebra by (7.2). If

$$
\rho:=\left(\rho_{1}, \ldots, \rho_{k}\right): V \rightarrow \mathbb{R}^{k},
$$

then

$$
\rho^{*}: C^{\infty}\left(\mathbb{R}^{k}\right) \rightarrow C^{\infty}(V)^{G}
$$

is surjective with a continuous linear section.

This theorem is due to Schwarz [204], who showed surjectivity. The article [138] extended the result to split surjective (existence of a continuous section). Later, [18] and [19] generalized this to 'semiproper real analytic mappings' $\rho$. For the action of $G=\{ \pm 1\}$ on $\mathbb{R}^{1}$ the result is due to $[\mathbf{2 2 8}$. If $G=\mathcal{S}_{n}$ acting on $\mathbb{R}^{n}$ by the standard representation, it was shown by [75. It is easy to see that $\rho^{*} C^{\infty}\left(\mathbb{R}^{k}\right)$ is dense in $C^{\infty}(V)^{G}$ in the compact $C^{\infty}$-topology. Therefore, Schwarz's theorem is equivalent to: $\rho^{*} C^{\infty}\left(\mathbb{R}^{k}\right)$ is closed in $C^{\infty}(V)^{G}$. Further results in this direction were obtained by Luna who, among other things, generalized the theorem of Schwarz to reductive 
Lie groups losing only the property of the Hilbert generators separating the orbits.

7.14. Result (Luna's Theorem [126]). Consider a representation of a reductive Lie group $G$ on $\mathbb{K}^{m}$ (where $\mathbb{K}=\mathbb{C}, \mathbb{R}$ ), and let $\sigma=\left(\sigma_{1}, \ldots, \sigma_{n}\right)$ : $\mathbb{K}^{m} \rightarrow \mathbb{K}^{n}$, where $\sigma_{1}, \ldots, \sigma_{n}$ generate the algebra $\mathbb{K}\left[\mathbb{K}^{m}\right]^{G}$. Then the following assertions hold:

(1) If $\mathbb{K}=\mathbb{C}$, then $\sigma^{*}: \mathcal{O}\left(\mathbb{C}^{n}\right) \rightarrow \mathcal{O}\left(\mathbb{C}^{m}\right)^{G}$ is surjective.

(2) If $\mathbb{K}=\mathbb{R}$, then $\sigma^{*}: C^{\omega}\left(\mathbb{R}^{n}\right) \rightarrow C^{\omega}\left(\mathbb{R}^{m}\right)^{G}$ is surjective.

(3) If $\mathbb{K}=\mathbb{R}$, then also

$\sigma^{*}: C^{\infty}\left(\mathbb{R}^{n}\right) \longrightarrow\left\{f \in C^{\infty}\left(\mathbb{R}^{m}\right)^{G}: f\right.$ constant on $\left.\sigma^{-1}(y) \forall y \in \mathbb{R}^{n}\right\}$

is surjective. 



\section{CHAPTER III. Differential Forms and de Rham Cohomology}

\section{Vector Bundles}

8.1. Vector bundles. Let $p: E \rightarrow M$ be a smooth mapping between manifolds. By a vector bundle chart on $(E, p, M)$ we mean a pair $(U, \psi)$, where $U$ is an open subset in $M$ and where $\psi$ is a fiber respecting diffeomorphism as in the following diagram:

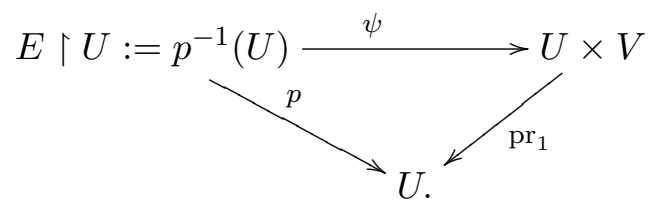

Here $V$ is a fixed finite-dimensional vector space, called the standard fiber or the typical fiber, real for the moment.

Two vector bundle charts $\left(U_{1}, \psi_{1}\right)$ and $\left(U_{2}, \psi_{2}\right)$ are said to be compatible if $\psi_{1} \circ \psi_{2}^{-1}$ is a fiber linear isomorphism, i.e.,

$$
\left(\psi_{1} \circ \psi_{2}^{-1}\right)(x, v)=\left(x, \psi_{1,2}(x) v\right)
$$

for some smooth mapping $\psi_{1,2}: U_{1,2}:=U_{1} \cap U_{2} \rightarrow G L(V)$. The mapping $\psi_{1,2}$ is then unique and smooth, and it is called the transition function between the two vector bundle charts.

A vector bundle atlas $\left(U_{\alpha}, \psi_{\alpha}\right)_{\alpha \in A}$ for $(E, p, M)$ is a set of pairwise compatible vector bundle charts $\left(U_{\alpha}, \psi_{\alpha}\right)$ such that $\left(U_{\alpha}\right)_{\alpha \in A}$ is an open cover of 
$M$. Two vector bundle atlases are called equivalent if their union is again a vector bundle atlas.

A vector bundle $(E, p, M)$ consists of manifolds $E$ (the total space), $M$ (the base), and a smooth mapping $p: E \rightarrow M$ (the projection) together with an equivalence class of vector bundle atlases: So we must know at least one vector bundle atlas. The projection $p$ turns out to be a surjective submersion.

8.2. Let us fix a vector bundle $(E, p, M)$ for the moment. On each fiber $E_{x}:=p^{-1}(x)$ (for $x \in M$ ) there is a unique structure of a real vector space, induced from any vector bundle chart $\left(U_{\alpha}, \psi_{\alpha}\right)$ with $x \in U_{\alpha}$. So $0_{x} \in E_{x}$ is a special element and $0: M \rightarrow E, 0(x)=0_{x}$, is a smooth mapping which is called the zero section.

A section $u$ of $(E, p, M)$ is a smooth mapping $u: M \rightarrow E$ with $p \circ u=I d_{M}$. The support of the section $u$ is the closure of the set $\left\{x \in M: u(x) \neq 0_{x}\right\}$ in $M$. The space of all smooth sections of the bundle $(E, p, M)$ will be denoted by either $\Gamma(E)=\Gamma(E, p, M)=\Gamma(E \rightarrow M)$. Clearly it is a vector space with fiberwise addition and scalar multiplication.

If $\left(U_{\alpha}, \psi_{\alpha}\right)_{\alpha \in A}$ is a vector bundle atlas for $(E, p, M)$, then any smooth mapping $f_{\alpha}: U_{\alpha} \rightarrow V$ (where $V$ is the standard fiber) defines a local section $x \mapsto \psi_{\alpha}^{-1}\left(x, f_{\alpha}(x)\right)$ on $U_{\alpha}$. If $\left(g_{\alpha}\right)_{\alpha \in A}$ is a partition of unity subordinated to $\left(U_{\alpha}\right)$, then a global section can be formed by $x \mapsto \sum_{\alpha} g_{\alpha}(x) \cdot \psi_{\alpha}^{-1}\left(x, f_{\alpha}(x)\right)$. So a smooth vector bundle has 'many' smooth sections.

8.3. We will now give a formal description of the set of equivalence classes of vector bundles with fixed base $M$ and fixed standard fiber $V$.

Let us first fix an open cover $\left(U_{\alpha}\right)_{\alpha \in A}$ of $M$. If $(E, p, M)$ is a vector bundle which admits a vector bundle atlas $\left(U_{\alpha}, \psi_{\alpha}\right)$ with the given open cover, then we have $\psi_{\alpha} \circ \psi_{\beta}^{-1}(x, v)=\left(x, \psi_{\alpha \beta}(x) v\right)$ for transition functions $\psi_{\alpha \beta}: U_{\alpha \beta}=$ $U_{\alpha} \cap U_{\beta} \rightarrow G L(V)$, which are smooth. This family of transition functions satisfies

$$
\begin{cases}\psi_{\alpha \beta}(x) \cdot \psi_{\beta \gamma}(x)=\psi_{\alpha \gamma}(x) & \text { for each } x \in U_{\alpha \beta \gamma}=U_{\alpha} \cap U_{\beta} \cap U_{\gamma}, \\ \psi_{\alpha \alpha}(x)=e & \text { for all } x \in U_{\alpha} .\end{cases}
$$

Condition (11) is called the cocycle condition and thus we call the family $\left(\psi_{\alpha \beta}\right)$ the cocycle of transition functions for the vector bundle atlas $\left(U_{\alpha}, \psi_{\alpha}\right)$.

Let us suppose now that the same vector bundle $(E, p, M)$ is described by an equivalent vector bundle atlas $\left(U_{\alpha}, \varphi_{\alpha}\right)$ with the same open cover $\left(U_{\alpha}\right)$. Then the vector bundle charts $\left(U_{\alpha}, \psi_{\alpha}\right)$ and $\left(U_{\alpha}, \varphi_{\alpha}\right)$ are compatible for each $\alpha$, so

$$
\varphi_{\alpha} \circ \psi_{\alpha}^{-1}(x, v)=\left(x, \tau_{\alpha}(x) v\right)
$$


for some smooth mapping $\tau_{\alpha}: U_{\alpha} \rightarrow G L(V)$. But then we have

$$
\begin{aligned}
\left(x, \tau_{\alpha}(x) \psi_{\alpha \beta}(x) v\right) & =\left(\varphi_{\alpha} \circ \psi_{\alpha}^{-1}\right)\left(x, \psi_{\alpha \beta}(x) v\right) \\
& =\left(\varphi_{\alpha} \circ \psi_{\alpha}^{-1} \circ \psi_{\alpha} \circ \psi_{\beta}^{-1}\right)(x, v)=\left(\varphi_{\alpha} \circ \psi_{\beta}^{-1}\right)(x, v) \\
& =\left(\varphi_{\alpha} \circ \varphi_{\beta}^{-1} \circ \varphi_{\beta} \circ \psi_{\beta}^{-1}\right)(x, v)=\left(x, \varphi_{\alpha \beta}(x) \tau_{\beta}(x) v\right) .
\end{aligned}
$$

So we get

$$
\tau_{\alpha}(x) \psi_{\alpha \beta}(x)=\varphi_{\alpha \beta}(x) \tau_{\beta}(x) \text { for all } x \in U_{\alpha \beta} .
$$

We say that the two cocycles $\left(\psi_{\alpha \beta}\right)$ and $\left(\varphi_{\alpha \beta}\right)$ of transition functions over the cover $\left(U_{\alpha}\right)$ are cohomologous. The cohomology classes of cocycles $\left(\psi_{\alpha \beta}\right)$ over the open cover $\left(U_{\alpha}\right)$ (where we identify cohomologous ones) form a set $\check{H}^{1}\left(\left(U_{\alpha}\right), \underline{G L}(V)\right)$, the first $\check{C} e c h$ cohomology set of the open cover $\left(U_{\alpha}\right)$ with values in the sheaf $C^{\infty}(, G L(V))=: \underline{G L}(V)$.

Now let $\left(W_{i}\right)_{i \in I}$ be an open cover of $M$ that refines $\left(U_{\alpha}\right)$ with $W_{i} \subset U_{\varepsilon(i)}$, where $\varepsilon: I \rightarrow A$ is some refinement mapping; then for any cocycle $\left(\psi_{\alpha \beta}\right)$ over $\left(U_{\alpha}\right)$ we define the cocycle $\varepsilon^{*}\left(\psi_{\alpha \beta}\right)=$ : $\left(\varphi_{i j}\right)$ by the prescription $\varphi_{i j}:=$ $\psi_{\varepsilon(i), \varepsilon(j)} \uparrow W_{i j}$. The mapping $\varepsilon^{*}$ respects the cohomology relations and induces therefore a mapping $\varepsilon^{\sharp}: \check{H}^{1}\left(\left(U_{\alpha}\right), \underline{G L}(V)\right) \rightarrow \check{H}^{1}\left(\left(W_{i}\right), \underline{G L}(V)\right)$. One can show that the mapping $\varepsilon^{*}$ depends on the choice of the refinement mapping $\varepsilon$ only up to cohomology (use $\tau_{i}=\psi_{\varepsilon(i), \eta(i)} \uparrow W_{i}$ if $\varepsilon$ and $\eta$ are two refinement mappings), so we may form the inductive limit $\underset{\lim }{\longrightarrow} \breve{H}^{1}(\mathcal{U}, \underline{G L}(V))=$ : $\check{H}^{1}(M, \underline{G L}(V))$ over all open covers of $M$ directed by refinement.

Theorem. There is a bijective correspondence between the (nonabelian if $\operatorname{dim}(V)>1)$ cohomology space $\check{H}^{1}(M, \underline{G L}(V))$ and the set of isomorphism classes of vector bundles over $M$ with typical fiber $V$.

Proof. Let $\left(\psi_{\alpha \beta}\right)$ be a cocycle of transition functions $\psi_{\alpha \beta}: U_{\alpha \beta} \rightarrow G L(V)$ over some open cover $\left(U_{\alpha}\right)$ of $M$. We consider the disjoint union $\bigsqcup_{\alpha \in A}\{\alpha\} \times$ $U_{\alpha} \times V$ and the following relation on it: $(\alpha, x, v) \sim(\beta, y, w)$ if and only if $x=y$ and $\psi_{\beta \alpha}(x) v=w$.

By the cocycle property (11) of $\left(\psi_{\alpha \beta}\right)$ this is an equivalence relation. The space of all equivalence classes is denoted by $E=V B\left(\psi_{\alpha \beta}\right)$ and it is equipped with the quotient topology. We put $p: E \rightarrow M, p[(\alpha, x, v)]=x$, and we define the vector bundle charts $\left(U_{\alpha}, \psi_{\alpha}\right)$ by $\psi_{\alpha}[(\alpha, x, v)]=(x, v)$, $\psi_{\alpha}: p^{-1}\left(U_{\alpha}\right)=: E \uparrow U_{\alpha} \rightarrow U_{\alpha} \times V$. Then the mapping $\psi_{\alpha} \circ \psi_{\beta}^{-1}(x, v)=$ $\psi_{\alpha}[(\beta, x, v)]=\psi_{\alpha}\left[\left(\alpha, x, \psi_{\alpha \beta}(x) v\right)\right]=\left(x, \psi_{\alpha \beta}(x) v\right)$ is smooth; so $E$ becomes a smooth manifold which is is Hausdorff: Let $u \neq v$ in $E$; if $p(u) \neq p(v)$, we can separate them in $M$ and take the inverse image under $p$; if $p(u)=p(v)$, we can separate them in one chart. So $(E, p, M)$ is a vector bundle.

Now suppose that we have two cocycles, $\left(\psi_{\alpha \beta}\right)$ over $\left(U_{\alpha}\right)$ and $\left(\varphi_{i j}\right)$ over $\left(V_{i}\right)$. Then there is a common refinement $\left(W_{\gamma}\right)$ for the two covers $\left(U_{\alpha}\right)$ and $\left(V_{i}\right)$. 
The construction described a moment ago gives isomorphic vector bundles if we restrict the cocycle to a finer open cover. So we may assume that $\left(\psi_{\alpha \beta}\right)$ and $\left(\varphi_{\alpha \beta}\right)$ are cocycles over the same open cover $\left(U_{\alpha}\right)$. If the two cocycles are cohomologous, so $\tau_{\alpha} \cdot \psi_{\alpha \beta}=\varphi_{\alpha \beta} \cdot \tau_{\beta}$ on $U_{\alpha \beta}$, then a fiber linear diffeomorphism $\tau: V B\left(\psi_{\alpha \beta}\right) \rightarrow V B\left(\varphi_{\alpha \beta}\right)$ is given by $\varphi_{\alpha} \tau[(\alpha, x, v)]=\left(x, \tau_{\alpha}(x) v\right)$. By relation (21) this is well defined, so the vector bundles $V B\left(\psi_{\alpha \beta}\right)$ and $V B\left(\varphi_{\alpha \beta}\right)$ are isomorphic.

Most of the converse direction was already shown in the discussion before the theorem, and the argument can be easily refined to show also that isomorphic bundles give cohomologous cocycles.

8.4. Remark. If $G L(V)$ is an abelian group (only if $V$ is of real or complex dimension 1), then $\check{H}^{1}(M, \underline{G L}(V))$ is a usual cohomology group with coefficients in the sheaf $G L(V)$ and it can be computed with the methods of algebraic topology. We will treat the two situations in a moment. If $G L(V)$ is not abelian, then the situation is rather mysterious: There is no clear definition for $\check{H}^{2}(M, \underline{G L}(V))$ for example. So $\check{H}^{1}(M, \underline{G L}(V))$ is more a notation than a mathematical concept.

A coarser relation on vector bundles (stable isomorphism) leads to the concept of topological K-theory, which can be handled much better, but is only a quotient of the real situation.

Example: Real line bundles. As an example we want to determine here the set of all real line bundles on a smooth manifold $M$. Let us first consider the following exact sequence of abelian Lie groups:

$$
0 \rightarrow(\mathbb{R},+) \stackrel{\exp }{\longrightarrow} G L(1, \mathbb{R})=(\mathbb{R} \backslash 0, \cdot) \stackrel{p}{\longrightarrow} \mathbb{Z}_{2} \rightarrow 0 . \rightarrow 0
$$

where $\mathbb{Z}_{2}:=\mathbb{Z} / 2 \mathbb{Z}$ is the two element group. This gives rise to an exact sequence of sheafs with values in abelian groups:

$$
0 \rightarrow C^{\infty}(\quad, \mathbb{R}) \stackrel{\exp _{*}}{\longrightarrow} C^{\infty}(\quad, G L(1, \mathbb{R})) \stackrel{p_{*}}{\longrightarrow} \mathbb{Z}_{2} \rightarrow 0
$$

where in the end we find the constant sheaf. This induces the following long exact sequence in cohomology (the Bockstein sequence):

$$
\begin{aligned}
\cdots \rightarrow 0=\check{H}^{1}\left(M, C^{\infty}(, \mathbb{R})\right) \stackrel{\exp _{*}}{\longrightarrow} \check{H}^{1}\left(M, C^{\infty}(\quad, G L(1, \mathbb{R}))\right) \\
\stackrel{p_{*}}{\longrightarrow} H^{1}\left(M, \mathbb{Z}_{2}\right) \stackrel{\delta}{\longrightarrow} \check{H}^{2}\left(M, C^{\infty}(, \mathbb{R})\right)=0 \rightarrow \ldots
\end{aligned}
$$

Here the sheaf $C^{\infty}(, \mathbb{R})$ has 0 cohomology in dimensions $\geq 1$ since this is a fine sheaf, i.e., it admits partitions of unity; see for example [77. Thus the pullback $p_{*}: \check{H}^{1}\left(M, C^{\infty}(\quad, G L(1, \mathbb{R}))\right) \rightarrow H^{1}\left(M, \mathbb{Z}_{2}\right)$ is an isomorphism, and by theorem (8.3) a real line bundle $E$ over $M$ is uniquely determined by 
a certain cohomology class in $H^{1}\left(M, \mathbb{Z}_{2}\right)$, namely the first Stiefel-Whitney class $w_{1}(E)$ of this line bundle.

Example: Complex line bundles. As another example we want to determine here the set of all smooth complex line bundles on a smooth manifold $M$. Again we first consider the following exact sequence of abelian Lie groups:

$$
0 \rightarrow \mathbb{Z} \stackrel{2 \pi \sqrt{-1}}{\longrightarrow}(\mathbb{C},+) \stackrel{\exp }{\longrightarrow} G L(1, \mathbb{C})=(\mathbb{C} \backslash 0, \cdot) \rightarrow 0 .
$$

This gives rise to the following exact sequence of sheafs with values in abelian groups:

$$
0 \rightarrow \mathbb{Z} \rightarrow C^{\infty}(\quad, \mathbb{C}) \stackrel{\exp _{*}}{\longrightarrow} C^{\infty}(\quad, G L(1, \mathbb{C})) \rightarrow 0
$$

where in the beginning we find the constant sheaf. This induces the following long exact sequence in cohomology (the Bockstein sequence):

$$
\begin{aligned}
\cdots \rightarrow 0=\check{H}^{1}\left(M, C^{\infty}(, \mathbb{C})\right) \stackrel{\exp _{*}}{\longrightarrow} \check{H}^{1}\left(M, C^{\infty}(\quad, G L(1, \mathbb{C}))\right) \\
\stackrel{\delta}{\longrightarrow} H^{2}(M, \mathbb{Z}) \stackrel{2 \pi \sqrt{-1}}{\longrightarrow} \check{H}^{2}\left(M, C^{\infty}(\quad, \mathbb{C})\right)=0 \rightarrow \ldots .
\end{aligned}
$$

Again the sheaf $C^{\infty}(, \mathbb{R})$ has 0 cohomology in dimensions $\geq 1$ since it is a fine sheaf. Thus $\delta: \breve{H}^{1}\left(M, C^{\infty}(, G L(1, \mathbb{C}))\right) \rightarrow H^{2}(M, \mathbb{Z})$ is an isomorphism, and by theorem (8.3) a complex smooth line bundle $E$ over $M$ is uniquely determined by a certain cohomology class in $H^{2}(M, \mathbb{Z})$, namely the first Chern class $c_{1}(E)$ of this line bundle.

8.5. Let $\left(U_{\alpha}, \psi_{\alpha}\right)$ be a vector bundle atlas for a vector bundle $(E, p, M)$. Let $\left(e_{j}\right)_{j=1}^{k}$ be a basis of the standard fiber $V$. We consider the section $s_{j}(x):=\psi_{\alpha}^{-1}\left(x, e_{j}\right)$ for $x \in U_{\alpha}$. Then the $s_{j}: U_{\alpha} \rightarrow E$ are local sections of $E$ such that $\left(s_{j}(x)\right)_{j=1}^{k}$ is a basis of $E_{x}$ for each $x \in U_{\alpha}$ : We say that

$$
s=\left(s_{1}, \ldots, s_{k}\right)
$$

is a local frame field for $E$ over $U_{\alpha}$.

Now let conversely $U \subset M$ be an open set and let $s_{j}: U \rightarrow E$ be local sections of $E$ such that $s=\left(s_{1}, \ldots, s_{k}\right)$ is a local frame field of $E$ over $U$. Then $s$ determines a unique vector bundle chart $(U, \psi)$ of $E$ such that $s_{j}(x)=\psi^{-1}\left(x, e_{j}\right)$, in the following way. We define $f: U \times \mathbb{R}^{k} \rightarrow E \uparrow U$ by $f\left(x, v^{1}, \ldots, v^{k}\right):=\sum_{j=1}^{k} v^{j} s_{j}(x)$. Then $f$ is smooth, invertible, and a fiber linear isomorphism, so $\left(U, \psi=f^{-1}\right)$ is the vector bundle chart promised above. 
8.6. Let $(E, p, M)$ and $(F, q, N)$ be vector bundles. A vector bundle homomorphism $\varphi: E \rightarrow F$ is a fiber respecting, fiber linear smooth mapping

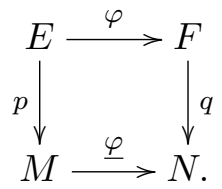

So we require that $\varphi_{x}: E_{x} \rightarrow F_{\underline{\varphi}(x)}$ is linear. We say that $\varphi$ covers $\underline{\varphi}$. If $\varphi$ is invertible, it is called a vector bundle isomorphism.

8.7. A vector subbundle $(F, p, M)$ of a vector bundle $(E, p, M)$ is a vector bundle and a vector bundle homomorphism $\tau: F \rightarrow E$, which covers $I d_{M}$, such that $\tau_{x}: F_{x} \rightarrow E_{x}$ is a linear embedding for each $x \in M$.

Lemma. Let $\varphi:(E, p, M) \rightarrow\left(E^{\prime}, q, N\right)$ be a vector bundle homomorphism such that $\operatorname{rank}\left(\varphi_{x}: E_{x} \rightarrow E_{\varphi(x)}^{\prime}\right)$ is locally constant in $x \in M$. Then $\operatorname{ker} \varphi$, given by $(\operatorname{ker} \varphi)_{x}=\operatorname{ker}\left(\varphi_{x}\right)$, is a vector subbundle of $(E, p, M)$.

Proof. This is a local question, so we may assume that both bundles are trivial: Let $E=M \times \mathbb{R}^{p}$ and let $F=N \times \mathbb{R}^{q}$; then $\varphi(x, v)=(\varphi(x), \bar{\varphi}(x) \cdot v)$, where $\bar{\varphi}: M \rightarrow L\left(\mathbb{R}^{p}, \mathbb{R}^{q}\right)$. The matrix $\bar{\varphi}(x)$ has rank $k$, so by the elimination procedure we can find $p-k$ linearly independent solutions $v_{i}(x)$ of the equation $\bar{\varphi}(x) \cdot v=0$. The elimination procedure (with the same lines) gives solutions $v_{i}(y)$ for $y$ near $x$ which are smooth in $y$, so near $x$ we get a local frame field $v=\left(v_{1}, \ldots, v_{p-k}\right)$ for $\operatorname{ker} \varphi \cdot \operatorname{By}(8.5)$, $\operatorname{ker} \varphi$ is then a vector subbundle.

8.8. Constructions with vector bundles. Let $\mathcal{F}$ be a covariant functor from the category of finite-dimensional vector spaces and linear mappings into itself, such that $\mathcal{F}: L(V, W) \rightarrow L(\mathcal{F}(V), \mathcal{F}(W))$ is smooth. Then $\mathcal{F}$ will be called a smooth functor for shortness sake. Well known examples of smooth functors are $\mathcal{F}(V)=\bigwedge^{k}(V)$ (the $k$-th exterior power), or $\mathcal{F}(V)=$ $\otimes^{k} V$, and the like.

If $(E, p, M)$ is a vector bundle, described by a vector bundle atlas with cocycle of transition functions $\varphi_{\alpha \beta}: U_{\alpha \beta} \rightarrow G L(V)$, where $\left(U_{\alpha}\right)$ is an open cover of $M$, then we may consider the smooth functions $\mathcal{F}\left(\varphi_{\alpha \beta}\right): x \mapsto \mathcal{F}\left(\varphi_{\alpha \beta}(x)\right)$, $U_{\alpha \beta} \rightarrow G L(\mathcal{F}(V))$. Since $\mathcal{F}$ is a covariant functor, $\mathcal{F}\left(\varphi_{\alpha \beta}\right)$ satisfies again the cocycle condition (8.3.1), and cohomology of cocycles (8.3.2) is respected, so there exists a unique vector bundle $\left(\mathcal{F}(E):=V B\left(\mathcal{F}\left(\varphi_{\alpha \beta}\right)\right), p, M\right)$, the value at the vector bundle $(E, p, M)$ of the canonical extension of the functor $\mathcal{F}$ to the category of vector bundles and their homomorphisms.

If $\mathcal{F}$ is a contravariant smooth functor like the duality functor $\mathcal{F}(V)=V^{*}$, then we have to consider the new cocycle $\mathcal{F}\left(\varphi_{\alpha \beta}^{-1}\right)$ instead of $\mathcal{F}\left(\varphi_{\alpha \beta}\right)$. 
If $\mathcal{F}$ is a contra-covariant smooth bifunctor like $L(V, W)$, then the construction $\mathcal{F}\left(V B\left(\psi_{\alpha \beta}\right), V B\left(\varphi_{\alpha \beta}\right)\right):=V B\left(\mathcal{F}\left(\psi_{\alpha \beta}^{-1}, \varphi_{\alpha \beta}\right)\right)$ describes the induced canonical vector bundle construction, and similarly in other constructions.

So for vector bundles $(E, p, M)$ and $(F, q, M)$ we have the following vector bundles with base $M: \bigwedge^{k} E, E \oplus F, E^{*}, \bigwedge E=\bigoplus_{k \geq 0} \wedge^{k} E, E \otimes F$, $L(E, F) \cong E^{*} \otimes F$, and so on.

8.9. Pullbacks of vector bundles. Let $(E, p, M)$ be a vector bundle and let $f: N \rightarrow M$ be smooth. Then the pullback vector bundle $\left(f^{*} E, f^{*} p, N\right)$ with the same typical fiber and a vector bundle homomorphism

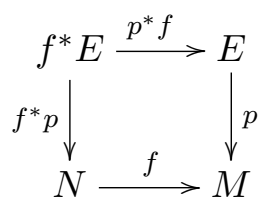

is defined as follows. Let $E$ be described by a cocycle $\left(\psi_{\alpha \beta}\right)$ of transition functions over an open cover $\left(U_{\alpha}\right)$ of $M, E=V B\left(\psi_{\alpha \beta}\right)$. Then $\left(\psi_{\alpha \beta} \circ f\right)$ is a cocycle of transition functions over the open cover $\left(f^{-1}\left(U_{\alpha}\right)\right)$ of $N$ and the bundle is given by $f^{*} E:=V B\left(\psi_{\alpha \beta} \circ f\right)$. As a manifold we have $f^{*} E=N \times_{(f, M, p)} E$ in the sense of (2.17).

The vector bundle $f^{*} E$ has the following universal property: For any vector bundle $(F, q, P)$, vector bundle homomorphism $\varphi: F \rightarrow E$ and smooth $g$ : $P \rightarrow N$ such that $f \circ g=\underline{\varphi}$, there is a unique vector bundle homomorphism $\psi: F \rightarrow f^{*} E$ with $\underline{\psi}=g$ and $p^{*} f \circ \psi=\varphi$ :

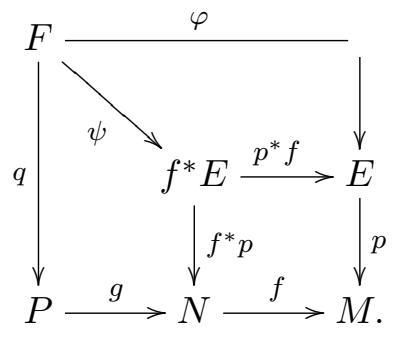

8.10. Theorem. Any vector bundle admits a finite vector bundle atlas.

Proof. Let $(E, p, M)$ be the vector bundle in question, where $\operatorname{dim} M=m$. Let $\left(U_{\alpha}, \psi_{\alpha}\right)_{\alpha \in A}$ be a vector bundle atlas. By topological dimension theory, since $M$ is separable, there exists a refinement of the open cover $\left(U_{\alpha}\right)_{\alpha \in A}$ of the form $\left(V_{i j}\right)_{i=1, \ldots, m+1 ; j \in \mathbb{N}}$, such that $V_{i j} \cap V_{i k}=\emptyset$ for $j \neq k$; see the remarks at the end of (1.1), We define the set $W_{i}:=\bigsqcup_{j \in \mathbb{N}} V_{i j}$ (a disjoint union) and $\psi_{i} \uparrow V_{i j}=\psi_{\alpha(i, j)}$, where $\alpha:\{1, \ldots, m+1\} \times \mathbb{N} \rightarrow A$ is a refining map. Then $\left(W_{i}, \psi_{i}\right)_{i=1, \ldots, m+1}$ is a finite vector bundle atlas of $E$. 
8.11. Theorem. For any vector bundle $(E, p, M)$ there is a second vector bundle $(F, p, M)$ such that $(E \oplus F, p, M)$ is a trivial vector bundle, i.e., isomorphic to $M \times \mathbb{R}^{N}$ for some $N \in \mathbb{N}$.

Proof. Let $\left(U_{i}, \psi_{i}\right)_{i=1}^{n}$ be a finite vector bundle atlas for $(E, p, M)$. Let $\left(g_{i}\right)$ be a smooth partition of unity subordinated to the open cover $\left(U_{i}\right)$. Let $\ell_{i}: \mathbb{R}^{k} \rightarrow\left(\mathbb{R}^{k}\right)^{n}=\mathbb{R}^{k} \times \cdots \times \mathbb{R}^{k}$ be the embedding on the $i$-th factor, where $\mathbb{R}^{k}$ is the typical fiber of $E$. Let us define $\psi: E \rightarrow M \times \mathbb{R}^{n k}$ by

$$
\psi(u)=\left(p(u), \sum_{i=1}^{n} g_{i}(p(u))\left(\ell_{i} \circ \mathrm{pr}_{2} \circ \psi_{i}\right)(u)\right) ;
$$

then $\psi$ is smooth, fiber linear, and an embedding on each fiber, so $E$ is a vector subbundle of $M \times \mathbb{R}^{n k}$ via $\psi$. Now we define $F_{x}=E_{x}^{\perp}$ in $\{x\} \times \mathbb{R}^{n k}$ with respect to the standard inner product on $\mathbb{R}^{n k}$. Then $F \rightarrow M$ is a vector bundle and $E \oplus F \cong M \times \mathbb{R}^{n k}$.

8.12. The tangent bundle of a vector bundle. Let $(E, p, M)$ be a vector bundle with fiber addition $+_{E}: E \times_{M} E \rightarrow E$ and fiber scalar multiplication $m_{t}^{E}: E \rightarrow E$. Then $\left(T E, \pi_{E}, E\right)$, the tangent bundle of the manifold $E$, is itself a vector bundle, with fiber addition denoted by $+_{T E}$ and scalar multiplication denoted by $m_{t}^{T E}$.

If $\left(U_{\alpha}, \psi_{\alpha}: E\left\lceil U_{\alpha} \rightarrow U_{\alpha} \times V\right)_{\alpha \in A}\right.$ is a vector bundle atlas for $E$, such that $\left(U_{\alpha}, u_{\alpha}\right)$ is also a manifold atlas for $M$, then $\left(E \uparrow U_{\alpha}, \psi_{\alpha}^{\prime}\right)_{\alpha \in A}$ is an atlas for the manifold $E$, where

$$
\psi_{\alpha}^{\prime}:=\left(u_{\alpha} \times I d_{V}\right) \circ \psi_{\alpha}: E \uparrow U_{\alpha} \rightarrow U_{\alpha} \times V \rightarrow u_{\alpha}\left(U_{\alpha}\right) \times V \subset \mathbb{R}^{m} \times V .
$$

Hence the family $\left(T\left(E \uparrow U_{\alpha}\right), T \psi_{\alpha}^{\prime}: T\left(E \uparrow U_{\alpha}\right) \rightarrow T\left(u_{\alpha}\left(U_{\alpha}\right) \times V\right)=\right.$ $\left.u_{\alpha}\left(U_{\alpha}\right) \times V \times \mathbb{R}^{m} \times V\right)_{\alpha \in A}$ is the atlas describing the canonical vector bundle structure of $\left(T E, \pi_{E}, E\right)$. The transition functions are in turn:

$$
\begin{aligned}
\left(\psi_{\alpha} \circ \psi_{\beta}^{-1}\right)(x, v)= & \left(x, \psi_{\alpha \beta}(x) v\right) \quad \text { for } x \in U_{\alpha \beta}, \\
\left(u_{\alpha} \circ u_{\beta}^{-1}\right)(y)= & u_{\alpha \beta}(y) \quad \text { for } y \in u_{\beta}\left(U_{\alpha \beta}\right), \\
\left(\psi_{\alpha}^{\prime} \circ\left(\psi_{\beta}^{\prime}\right)^{-1}\right)(y, v)= & \left(u_{\alpha \beta}(y), \psi_{\alpha \beta}\left(u_{\beta}^{-1}(y)\right) v\right), \\
\left(T \psi_{\alpha}^{\prime} \circ T\left(\psi_{\beta}^{\prime}\right)^{-1}\right)(y, v ; \xi, w)= & \left(u_{\alpha \beta}(y), \psi_{\alpha \beta}\left(u_{\beta}^{-1}(y)\right) v ; d\left(u_{\alpha \beta}\right)(y) \xi,\right. \\
& \left.\left(d\left(\psi_{\alpha \beta} \circ u_{\beta}^{-1}\right)(y) \xi\right) v+\psi_{\alpha \beta}\left(u_{\beta}^{-1}(y)\right) w\right) .
\end{aligned}
$$

So we see that for fixed $(y, v)$ the transition functions are linear in $(\xi, w) \in$ $\mathbb{R}^{m} \times V$. This describes the vector bundle structure of the tangent bundle $\left(T E, \pi_{E}, E\right)$.

For fixed $(y, \xi)$ the transition functions of $T E$ are also linear in $(v, w) \in$ $V \times V$. This gives a vector bundle structure on $(T E, T p, T M)$. Its fiber addition will be denoted by $T\left(+_{E}\right): T\left(E \times_{M} E\right)=T E \times_{T M} T E \rightarrow T E$, 
since it is the tangent mapping of $+_{E}$. Likewise its scalar multiplication will be denoted by $T\left(m_{t}^{E}\right)$. One may say that the second vector bundle structure on $T E$, that one over $T M$, is the derivative of the original one on $E$.

The space $\{\Xi \in T E: T p . \Xi=0$ in $T M\}=(T p)^{-1}(0)$ is denoted by $V E$ and is called the vertical bundle over $E$. The local form of a vertical vector $\Xi$ is $T \psi_{\alpha}^{\prime} . \Xi=(y, v ; 0, w)$, so the transition function looks like

$$
\left(T \psi_{\alpha}^{\prime} \circ T\left(\psi_{\beta}^{\prime}\right)^{-1}\right)(y, v ; 0, w)=\left(u_{\alpha \beta}(y), \psi_{\alpha \beta}\left(u_{\beta}^{-1}(y)\right) v ; 0, \psi_{\alpha \beta}\left(u_{\beta}^{-1}(y)\right) w\right) .
$$

They are linear in $(v, w) \in V \times V$ for fixed $y$, so $V E$ is a vector bundle over $M$. It coincides with $0_{M}^{*}(T E, T p, T M)$, the pullback of the bundle $T E \rightarrow T M$ over the zero section. We have a canonical isomorphism $\mathrm{vl}_{E}$ : $E \times{ }_{M} E \rightarrow V E$, called the vertical lift, given by vl $\mathrm{v}_{E}\left(u_{x}, v_{x}\right):=\left.\frac{d}{d t}\right|_{0}\left(u_{x}+t v_{x}\right)$, which is fiber linear over $M$. The local representation of the vertical lift is $\left(T \psi_{\alpha}^{\prime} \circ \mathrm{vl}_{E} \circ\left(\psi_{\alpha}^{\prime} \times \psi_{\alpha}^{\prime}\right)^{-1}\right)((y, u),(y, v))=(y, u ; 0, v)$.

If (and only if) $\varphi:(E, p, M) \rightarrow(F, q, N)$ is a vector bundle homomorphism, then we have $\operatorname{vl}_{F} \circ\left(\varphi \times_{M} \varphi\right)=T \varphi \circ v_{E}: E \times_{M} E \rightarrow V F \subset T F$. So vl is a natural transformation between certain functors on the category of vector bundles and their homomorphisms.

The mapping $\mathrm{vpr}_{E}:=\mathrm{pr}_{2} \circ \mathrm{vl}_{E}^{-1}: V E \rightarrow E$ is called the vertical projection. Note also the relation $\mathrm{pr}_{1} \circ \mathrm{vl}_{E}^{-1}=\pi_{E} \uparrow V E$.

8.13. The second tangent bundle of a manifold. All of (8.12) is valid for the second tangent bundle $T^{2} M=T T M$ of a manifold, but here we have one more natural structure at our disposal. The canonical flip or involution $\kappa_{M}: T^{2} M \rightarrow T^{2} M$ is defined locally by

$$
\left(T^{2} u \circ \kappa_{M} \circ T^{2} u^{-1}\right)(x, \xi ; \eta, \zeta)=(x, \eta ; \xi, \zeta),
$$

where $(U, u)$ is a chart on $M$. Clearly this definition is invariant under changes of charts.

The flip $\kappa_{M}$ has the following properties:

(1) $\kappa_{N} \circ T^{2} f=T^{2} f \circ \kappa_{M}$ for each $f \in C^{\infty}(M, N)$.

(2) $T\left(\pi_{M}\right) \circ \kappa_{M}=\pi_{T M}$.

(3) $\pi_{T M} \circ \kappa_{M}=T\left(\pi_{M}\right)$.

(4) $\kappa_{M}^{-1}=\kappa_{M}$.

(5) $\kappa_{M}$ is a linear isomorphism from the bundle $\left(T T M, T\left(\pi_{M}\right), T M\right)$ to the bundle $\left(T T M, \pi_{T M}, T M\right)$, so it interchanges the two vector bundle structures on $T T M$.

(6) It is the unique smooth mapping TTM $\rightarrow T T M$ which satisfies the equation $\frac{\partial}{\partial t} \frac{\partial}{\partial s} c(t, s)=\kappa_{M} \frac{\partial}{\partial s} \frac{\partial}{\partial t} c(t, s)$ for each $c: \mathbb{R}^{2} \rightarrow M$. 
All this follows from the local formula given above.

8.14. Lemma. For vector fields $X, Y \in \mathfrak{X}(M)$ we have

$$
\begin{aligned}
& {[X, Y]=\operatorname{vpr}_{T M} \circ\left(T Y \circ X-\kappa_{M} \circ T X \circ Y\right),} \\
& T Y \circ X-\kappa_{M} \circ T X \circ Y=\operatorname{vl}_{T M}(Y,[X, Y]) .
\end{aligned}
$$

We will give global proofs of this result later on: the first one is (8.19).

Proof. We prove this locally, so we may assume that $M$ is open in $\mathbb{R}^{m}$, $X(x)=(x, \bar{X}(x))$, and $Y(x)=(x, \bar{Y}(x))$. Then by (3.4) we have

$$
[X, Y](x)=(x, d \bar{Y}(x) \cdot \bar{X}(x)-d \bar{X}(x) \cdot \bar{Y}(x)),
$$

and thus:

$$
\begin{aligned}
& \left(T Y \circ X-\kappa_{M} \circ T X \circ Y\right)(x)=T Y \cdot(x, \bar{X}(x))-\kappa_{M} \circ T X \cdot(x, \bar{Y}(x)) \\
& \quad=(x, \bar{Y}(x) ; \bar{X}(x), d \bar{Y}(x) \cdot \bar{X}(x))-\kappa_{M}(x, \bar{X}(x) ; \bar{Y}(x), d \bar{X}(x) \cdot \bar{Y}(x)) \\
& \quad=(x, \bar{Y}(x) ; 0, d \bar{Y}(x) \cdot \bar{X}(x)-d \bar{X}(x) \cdot \bar{Y}(x)), \\
& \operatorname{vpr}_{T M} \circ\left(T Y \circ X-\kappa_{M} \circ T X \circ Y\right)(x)=(x, d \bar{Y}(x) \cdot \bar{X}(x)-d \bar{X}(x) \cdot \bar{Y}(x)) .
\end{aligned}
$$

8.15. Natural vector bundles or vector bundle functors. Let $\mathcal{M} f_{m}$ denote the category of all $m$-dimensional smooth manifolds and local diffeomorphisms (i.e., immersions) between them. A vector bundle functor or natural vector bundle is a functor $F$ which associates a vector bundle $\left(F(M), p_{M}, M\right)$ to each $m$-manifold $M$ and a vector bundle homomorphism

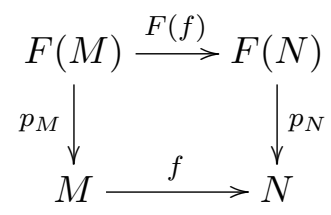

to each $f: M \rightarrow N$ in $\mathcal{M} f_{m}$, which covers $f$ and is fiberwise a linear isomorphism. We also require that for smooth $f: \mathbb{R} \times M \rightarrow N$ the mapping $(t, x) \mapsto F\left(f_{t}\right)(x)$ is smooth $\mathbb{R} \times F(M) \rightarrow F(N)$. We will say that $F$ maps smoothly parametrized families to smoothly parametrized families. See [108] for more information on naturality in differential geometry.

Examples. (1) $T M$, the tangent bundle. This is even a functor on the category $\mathcal{M} f$ of all manifolds and all smooth mappings, not only local diffeomorphisms.

(2) $T^{*} M$, the cotangent bundle, where by (8.8) the action on morphisms is given by $\left(T^{*} f\right)_{x}:=\left(\left(T_{x} f\right)^{-1}\right)^{*}: T_{x}^{*} M \rightarrow T_{f(x)}^{*} N$. This functor is defined on $\mathcal{M} f_{m}$ only.

(3) $\bigwedge^{k} T^{*} M, \wedge T^{*} M=\bigoplus_{k \geq 0} \wedge^{k} T^{*} M$. 
(4) $\otimes^{k} T^{*} M \otimes \bigotimes^{\ell} T M=T^{*} M \otimes \cdots \otimes T^{*} M \otimes T M \otimes \cdots \otimes T M$, where the action on morphisms involves $T f^{-1}$ in the $T^{*} M$-parts and $T f$ in the $T M$-parts.

(5) $\mathcal{F}(T M)$, where $\mathcal{F}$ is any smooth functor on the category of finitedimensional vector spaces and linear mappings, as in (8.8).

(6) All examples discussed till now are of the following form: For a manifold of dimension $m$, consider the linear frame bundle $G L\left(\mathbb{R}^{m}, T M\right)=$ inv $J_{0}^{1}\left(\mathbb{R}^{m}, M\right)$ (see (18.11) and (21.6) $)$ and a representation of the structure group $\rho: G L(m, \mathbb{R}) \rightarrow G L(V)$ on some vector space $V$. Then the associated bundle $G L\left(\mathbb{R}^{m}, T M\right) \times_{G L(m, \mathbb{R})} V$ is a natural bundle. This can be generalized to frame bundles of higher order, which is described in (21.6).

8.16. Lie derivative. Let $F$ be a vector bundle functor on $\mathcal{M} f_{m}$ as described in (8.15), Let $M$ be a manifold and let $X \in \mathfrak{X}(M)$ be a vector field on $M$. Then the flow $\mathrm{Fl}_{t}^{X}$, for fixed $t$, is a diffeomorphism defined on an open subset of $M$, which we do not specify. The mapping

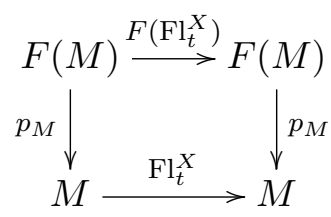

is then a vector bundle isomorphism, defined over an open subset of $M$.

We consider a section $s \in \Gamma(F(M))$ of the vector bundle $\left(F(M), p_{M}, M\right)$ and we define for $t \in \mathbb{R}$

$$
\left(\mathrm{Fl}_{t}^{X}\right)^{*} s:=F\left(\mathrm{Fl}_{-t}^{X}\right) \circ s \circ \mathrm{Fl}_{t}^{X},
$$

a local section of the bundle $F(M)$. For each $x \in M$ the value $\left(\left(\mathrm{Fl}_{t}^{X}\right)^{*} s\right)(x) \in$ $F(M)_{x}$ is defined, if $t$ is small enough (depending on $x$ ). So in the vector space $F(M)_{x}$ the expression $\left.\frac{d}{d t}\right|_{0}\left(\left(\mathrm{Fl}_{t}^{X}\right)^{*} s\right)(x)$ makes sense and therefore the section

$$
\mathcal{L}_{X} s:=\left.\frac{d}{d t}\right|_{0}\left(\mathrm{Fl}_{t}^{X}\right)^{*} s
$$

is globally defined and is an element of $\Gamma(F(M))$. It is called the Lie derivative of $s$ along $X$.

Lemma. In this situation we have

(1) $\left(\mathrm{Fl}_{t}^{X}\right)^{*}\left(\mathrm{Fl}_{r}^{X}\right)^{*} s=\left(\mathrm{Fl}_{t+r}^{X}\right)^{*} s$, wherever defined.

(2) $\frac{d}{d t}\left(\mathrm{Fl}_{t}^{X}\right)^{*} s=\left(\mathrm{Fl}_{t}^{X}\right)^{*} \mathcal{L}_{X} s=\mathcal{L}_{X}\left(\mathrm{Fl}_{t}^{X}\right)^{*} s$, so $\left[\mathcal{L}_{X},\left(\mathrm{Fl}_{t}^{X}\right)^{*}\right]:=\mathcal{L}_{X} \circ\left(\mathrm{Fl}_{t}^{X}\right)^{*}-\left(\mathrm{Fl}_{t}^{X}\right)^{*} \circ \mathcal{L}_{X}=0$, whenever defined.

(3) $\left(\mathrm{Fl}_{t}^{X}\right)^{*} s=s$ for all relevant $t$ if and only if $\mathcal{L}_{X} s=0$. 
Proof. (11) is clear. (2) is seen by the following computations:

$$
\begin{aligned}
\frac{d}{d t}\left(\mathrm{Fl}_{t}^{X}\right)^{*} s & =\left.\frac{d}{d r}\right|_{0}\left(\mathrm{Fl}_{r}^{X}\right)^{*}\left(\mathrm{Fl}_{t}^{X}\right)^{*} s=\mathcal{L}_{X}\left(\mathrm{Fl}_{t}^{X}\right)^{*} s \\
\frac{d}{d t}\left(\left(\mathrm{Fl}_{t}^{X}\right)^{*} s\right)(x) & =\left.\frac{d}{d r}\right|_{0}\left(\left(\mathrm{Fl}_{t}^{X}\right)^{*}\left(\mathrm{Fl}_{r}^{X}\right)^{*} s\right)(x) \\
& =\left.\frac{d}{d r}\right|_{0} F\left(\mathrm{Fl}_{-t}^{X}\right)\left(F\left(\mathrm{Fl}_{-r}^{X}\right) \circ s \circ \mathrm{Fl}_{r}^{X}\right)\left(\mathrm{Fl}_{t}^{X}(x)\right) \\
& =\left.F\left(\mathrm{Fl}_{-t}^{X}\right) \frac{d}{d r}\right|_{0}\left(F\left(\mathrm{Fl}_{-r}^{X}\right) \circ s \circ \mathrm{Fl}_{r}^{X}\right)\left(\mathrm{Fl}_{t}^{X}(x)\right) \\
& =\left(\left(\mathrm{Fl}_{t}^{X}\right)^{*} \mathcal{L}_{X} s\right)(x),
\end{aligned}
$$

since $F\left(\mathrm{Fl}_{-t}^{X}\right): F(M)_{\mathrm{Fl}_{t}^{X}(x)} \rightarrow F(M)_{x}$ is linear.

(3) follows from (2).

8.17. Let $F_{1}, F_{2}$ be two vector bundle functors on $\mathcal{M} f_{m}$. Then the (fiberwise) tensor product $\left(F_{1} \otimes F_{2}\right)(M):=F_{1}(M) \otimes F_{2}(M)$ is again a vector bundle functor and for $s_{i} \in \Gamma\left(F_{i}(M)\right)$ there is a section $s_{1} \otimes s_{2} \in$ $\Gamma\left(\left(F_{1} \otimes F_{2}\right)(M)\right)$, given by the pointwise tensor product.

Lemma. In this situation, for $X \in \mathfrak{X}(M)$ we have

$$
\mathcal{L}_{X}\left(s_{1} \otimes s_{2}\right)=\mathcal{L}_{X} s_{1} \otimes s_{2}+s_{1} \otimes \mathcal{L}_{X} s_{2} .
$$

In particular, for $f \in C^{\infty}(M)$ we have $\mathcal{L}_{X}(f s)=d f(X) s+f \mathcal{L}_{X} s$.

Proof. Using bilinearity of the tensor product, we have

$$
\begin{aligned}
\mathcal{L}_{X}\left(s_{1} \otimes s_{2}\right) & =\left.\frac{d}{d t}\right|_{0}\left(\mathrm{Fl}_{t}^{X}\right)^{*}\left(s_{1} \otimes s_{2}\right) \\
& =\left.\frac{d}{d t}\right|_{0}\left(\left(\mathrm{Fl}_{t}^{X}\right)^{*} s_{1} \otimes\left(\mathrm{Fl}_{t}^{X}\right)^{*} s_{2}\right) \\
& =\left.\frac{d}{d t}\right|_{0}\left(\mathrm{Fl}_{t}^{X}\right)^{*} s_{1} \otimes s_{2}+\left.s_{1} \otimes \frac{d}{d t}\right|_{0}\left(\mathrm{Fl}_{t}^{X}\right)^{*} s_{2} \\
& =\mathcal{L}_{X} s_{1} \otimes s_{2}+s_{1} \otimes \mathcal{L}_{X} s_{2} .
\end{aligned}
$$

8.18. Let $\varphi: F_{1} \rightarrow F_{2}$ be a linear natural transformation between vector bundle functors on $\mathcal{M} f_{m}$. So for each $M \in \mathcal{M} f_{m}$ we have a vector bundle homomorphism $\varphi_{M}: F_{1}(M) \rightarrow F_{2}(M)$ covering the identity on $M$, such that $F_{2}(f) \circ \varphi_{M}=\varphi_{N} \circ F_{1}(f)$ holds for any $f: M \rightarrow N$ in $\mathcal{M} f_{m}$.

Example. A tensor field of type $\left(\begin{array}{l}p \\ q\end{array}\right)$ is a smooth section of the natural bundle $\bigotimes^{q} T^{*} M \otimes \bigotimes^{p} T M$. For such tensor fields, by (8.16) the Lie derivative along any vector field is defined and by (8.17) it is a derivation with respect to the tensor product. For functions and vector fields the Lie derivative was already defined in section (3). This natural bundle admits many natural transformations: Any 'contraction' like the trace $T^{*} M \otimes T M=L(T M, T M) \rightarrow M \times \mathbb{R}$, but applied just to one specified factor $T^{*} M$ and another one of type $T M$, is a natural transformation. Also, any 'permutation of the same kind of factors' is a natural transformation. 
Lemma. In this situation, for $s \in \Gamma\left(F_{1}(M)\right)$ and $X \in \mathfrak{X}(M)$ we have $\mathcal{L}_{X}\left(\varphi_{M} s\right)=\varphi_{M}\left(\mathcal{L}_{X} s\right)$,

Proof. Since $\varphi_{M}$ is fiber linear and natural, we can compute as follows:

$$
\begin{aligned}
\mathcal{L}_{X}\left(\varphi_{M} s\right)(x) & =\left.\frac{d}{d t}\right|_{0}\left(\left(\mathrm{Fl}_{t}^{X}\right)^{*}\left(\varphi_{M} s\right)\right)(x)=\left.\frac{d}{d t}\right|_{0}\left(F_{2}\left(\mathrm{Fl}_{-t}^{X}\right) \circ \varphi_{M} \circ s \circ \mathrm{Fl}_{t}^{X}\right)(x) \\
& =\left.\varphi_{M} \circ \frac{d}{d t}\right|_{0}\left(F_{1}\left(\mathrm{Fl}_{-t}^{X}\right) \circ s \circ \mathrm{Fl}_{t}^{X}\right)(x)=\left(\varphi_{M} \mathcal{L}_{X} s\right)(x) . \quad \square
\end{aligned}
$$

Thus the Lie derivative on tensor fields commutes with any kind of 'contraction' or 'permutation of the indices'.

8.19. Let $F$ be a vector bundle functor on $\mathcal{M} f_{m}$ and let $X \in \mathfrak{X}(M)$ be a vector field. We consider the local vector bundle homomorphism $F\left(\mathrm{Fl}_{t}^{X}\right)$ on $F(M)$. Since $F\left(\mathrm{Fl}_{t}^{X}\right) \circ F\left(\mathrm{Fl}_{s}^{X}\right)=F\left(\mathrm{Fl}_{t+s}^{X}\right)$ and $F\left(\mathrm{Fl}_{0}^{X}\right)=I d_{F(M)}$, we have $\frac{d}{d t} F\left(\mathrm{Fl}_{t}^{X}\right)=\left.\frac{d}{d s}\right|_{0} F\left(\mathrm{Fl}_{s}^{X}\right) \circ F\left(\mathrm{Fl}_{t}^{X}\right)=X^{F} \circ F\left(\mathrm{Fl}_{t}^{X}\right)$, so we get $F\left(\mathrm{Fl}_{t}^{X}\right)=\mathrm{Fl}_{t}^{X^{F}}$, where $X^{F}=\left.\frac{d}{d s}\right|_{0} F\left(\mathrm{Fl}_{s}^{X}\right) \in \mathfrak{X}(F(M))$ is a vector field on $F(M)$, which is called the flow prolongation or the natural lift of $X$ to $F(M)$.

\section{Lemma.}

(1) $X^{T}=\kappa_{M} \circ T X$.

(2) $[X, Y]^{F}=\left[X^{F}, Y^{F}\right]$.

(3) $X^{F}:\left(F(M), p_{M}, M\right) \rightarrow\left(T F(M), T\left(p_{M}\right), T M\right)$ is a vector bundle homomorphism for the $T(+)$-structure.

(4) For $s \in \Gamma(F(M))$ and $X \in \mathfrak{X}(M)$ we have

$$
\mathcal{L}_{X} s=\operatorname{vpr}_{F(M)} \circ\left(T s \circ X-X^{F} \circ s\right) .
$$

(5) $\mathcal{L}_{X} s$ is linear in $X$ and $s$.

Proof. (11) is an easy computation. The mapping $F\left(\mathrm{Fl}_{t}^{X}\right)$ is fiber linear and this implies (3).

(4) is seen as follows:

$$
\begin{aligned}
\left(\mathcal{L}_{X} s\right)(x) & =\left.\frac{d}{d t}\right|_{0}\left(F\left(\mathrm{Fl}_{-t}^{X}\right) \circ s \circ \mathrm{Fl}_{t}^{X}\right)(x) \quad \text { in } F(M)_{x} \\
& =\operatorname{vpr}_{F(M)}\left(\left.\frac{d}{d t}\right|_{0}\left(F\left(\mathrm{Fl}_{-t}^{X}\right) \circ s \circ \mathrm{Fl}_{t}^{X}\right)(x) \text { in } V F(M)\right) \\
& =\operatorname{vpr}_{F(M)}\left(-X^{F} \circ s \circ \mathrm{Fl}_{0}^{X}(x)+T\left(F\left(\mathrm{Fl}_{0}^{X}\right)\right) \circ T s \circ X(x)\right) \\
& =\operatorname{vpr}_{F(M)}\left(T s \circ X-X^{F} \circ s\right)(x) .
\end{aligned}
$$

(5) $\mathcal{L}_{X} s$ is homogeneous of degree 1 in $X$ by formula (44), and it is smooth as a mapping $\mathfrak{X}(M) \rightarrow \Gamma(F(M))$, so it is linear. See [64] or [113 for the convenient calculus in infinite dimensions.

(2) Note first that $F$ induces a smooth mapping between appropriate spaces of local diffeomorphisms which are infinite-dimensional manifolds; see [113. 
By $(3.16)$ we have

$$
\begin{aligned}
0 & =\left.\partial\right|_{0}\left(\mathrm{Fl}_{-t}^{Y} \circ \mathrm{Fl}_{-t}^{X} \circ \mathrm{Fl}_{t}^{Y} \circ \mathrm{Fl}_{t}^{X}\right), \\
{[X, Y] } & =\left.\frac{1}{2} \frac{\partial^{2}}{\partial t^{2}}\right|_{0}\left(\mathrm{Fl}_{-t}^{Y} \circ \mathrm{Fl}_{-t}^{X} \circ \mathrm{Fl}_{t}^{Y} \circ \mathrm{Fl}_{t}^{X}\right) \\
& =\left.\partial\right|_{0} \mathrm{Fl}_{t}^{[X, Y]} .
\end{aligned}
$$

Applying $F$ to these curves of local diffeomorphisms, we get

$$
\begin{aligned}
0 & =\left.\partial\right|_{0}\left(\mathrm{Fl}_{-t}^{Y^{F}} \circ \mathrm{Fl}_{-t}^{X^{F}} \circ \mathrm{Fl}_{t}^{Y^{F}} \circ \mathrm{Fl}_{t}^{X^{F}}\right), \\
{\left[X^{F}, Y^{F}\right] } & =\left.\frac{1}{2} \frac{\partial^{2}}{\partial t^{2}}\right|_{0}\left(\mathrm{Fl}_{-t}^{Y^{F}} \circ \mathrm{Fl}_{-t}^{X^{F}} \circ \mathrm{Fl}_{t}^{Y^{F}} \circ \mathrm{Fl}_{t}^{X^{F}}\right) \\
& =\left.\frac{1}{2} \frac{\partial^{2}}{\partial t^{2}}\right|_{0} F\left(\mathrm{Fl}_{-t}^{Y} \circ \mathrm{Fl}_{-t}^{X} \circ \mathrm{Fl}_{t}^{Y} \circ \mathrm{Fl}_{t}^{X}\right) \\
& =\left.\partial\right|_{0} F\left(\mathrm{Fl}_{t}^{[X, Y]}\right)=[X, Y]^{F} .
\end{aligned}
$$

8.20. Theorem. For any vector bundle functor $F$ on $\mathcal{M} f_{m}$ and $X, Y \in$ $\mathfrak{X}(M)$ we have

$$
\left[\mathcal{L}_{X}, \mathcal{L}_{Y}\right]:=\mathcal{L}_{X} \circ \mathcal{L}_{Y}-\mathcal{L}_{Y} \circ \mathcal{L}_{X}=\mathcal{L}_{[X, Y]}: \Gamma(F(M)) \rightarrow \Gamma(F(M)) .
$$

So $\mathcal{L}: \mathfrak{X}(M) \rightarrow$ End $\Gamma(F(M))$ is a Lie algebra homomorphism.

Proof. We need some preparation.

$$
\begin{aligned}
X^{F} \circ \operatorname{vpr}_{F(M)}=\left.\frac{d}{d t}\right|_{0} F\left(\mathrm{Fl}_{t}^{X}\right) \circ \operatorname{vpr}_{F(M)} \\
\quad=\left.\frac{d}{d t}\right|_{0} \operatorname{vpr}_{F(M)} \circ T F\left(\mathrm{Fl}_{t}^{X}\right) \uparrow V F(M) \\
\quad=\left.T\left(\operatorname{vpr}_{F(M)}\right) \circ \frac{d}{d t}\right|_{0} T F\left(\mathrm{Fl}_{t}^{X}\right) \uparrow V F(M) \\
\quad=T\left(\operatorname{vpr}_{F(M)}\right) \circ \kappa_{F(M)} \circ T\left(\left.\frac{d}{d t}\right|_{0} F\left(\mathrm{Fl}_{t}^{X}\right)\right) \uparrow V F(M) \\
\quad=T\left(\operatorname{vpr}_{F(M)}\right) \circ \kappa_{F(M)} \circ T\left(X^{F}\right) \uparrow V F(M) .
\end{aligned}
$$

(2) Sublemma. For any vector bundle $(E, p, M)$ we have

$\operatorname{vpr}_{E} \circ T\left(\operatorname{vpr}_{E}\right) \circ \kappa_{E}=\operatorname{vpr}_{E} \circ T\left(\operatorname{vpr}_{E}\right)=\operatorname{vpr}_{E} \circ \operatorname{vpr}_{T E}: V T E \cap T V E \rightarrow E$, and this is linear for all three vector bundle structures on TTE.

The assertion of this sublemma is local over $M$, so one may assume that $(E, p, M)$ is trivial. Then one may carefully write out the action of the three mappings on a typical element $\left(x, v ; 0, w ; ; 0,0 ; 0, w^{\prime}\right) \in V T E \cap T V E$ and get the result.

Now we can start the actual proof.

$$
\begin{aligned}
& \mathcal{L}_{[X, Y]} s=\operatorname{vpr}_{F(M)}\left(T s \circ[X, Y]-[X, Y]^{F} \circ s\right) \quad \text { by (8.19) } \\
&=\operatorname{vpr}_{F(M)} \circ\left(T s \circ \operatorname{vpr}_{T M} \circ\left(T Y \circ X-\kappa_{M} \circ T X \circ Y\right)\right. \\
&\left.-\operatorname{vpr}_{T F(M)} \circ\left(T Y^{F} \circ X^{F}-\kappa_{F(M)} \circ T X^{F} \circ Y^{F}\right) \circ s\right)
\end{aligned}
$$




$$
\begin{aligned}
& =\operatorname{vpr}_{F(M)} \circ \operatorname{vpr}_{T F(M)} \circ\left(T^{2} s \circ T Y \circ X-\kappa_{F(M)} \circ T^{2} s \circ T X \circ Y\right. \\
& \left.-T Y^{F} \circ X^{F} \circ s-\kappa_{F(M)} \circ T X^{F} \circ Y^{F} \circ s\right), \\
& \mathcal{L}_{X} \mathcal{L}_{Y} s=\mathcal{L}_{X}\left(\operatorname{vpr}_{F(M)} \circ\left(T s \circ Y-Y^{F} \circ s\right)\right) \\
& =\operatorname{vpr}_{F(M)} \circ\left(T\left(\operatorname{vpr}_{F(M)}\right) \circ\left(T^{2} s \circ T Y T(-) T\left(Y^{F}\right) \circ T s\right) \circ X\right. \\
& \left.-X^{F} \circ \operatorname{vpr}_{F(M)} \circ\left(T s \circ Y-Y^{F} \circ s\right)\right) \\
& =\operatorname{vpr}_{F(M)} \circ T\left(\operatorname{vpr}_{F(M)}\right) \circ\left(T^{2} s \circ T Y \circ X T(-) T\left(Y^{F}\right) \circ T s \circ X\right) \\
& -\operatorname{vpr}_{F(M)} \circ T\left(\operatorname{vpr}_{F(M)}\right) \circ \kappa_{F(M)} \circ T\left(X^{F}\right) \circ\left(T s \circ Y-Y^{F} \circ s\right) \\
& =\operatorname{vpr}_{F(M)} \circ \operatorname{vpr}_{T F(M)} \circ\left(T^{2} s \circ T Y \circ X-T\left(Y^{F}\right) \circ T s \circ X\right. \\
& \left.-\kappa_{F(M)} \circ T\left(X^{F}\right) \circ T s \circ Y+\kappa_{F(M)} \circ T\left(X^{F}\right) \circ Y^{F} \circ s\right) .
\end{aligned}
$$

Finally we have

$$
\begin{aligned}
& {\left[\mathcal{L}_{X}, \mathcal{L}_{Y}\right] s=\mathcal{L}_{X} \mathcal{L}_{Y} s-\mathcal{L}_{Y} \mathcal{L}_{X} s} \\
& =\operatorname{vpr}_{F(M)} \circ \operatorname{vpr}_{T F(M)} \circ\left(T^{2} s \circ T Y \circ X-T\left(Y^{F}\right) \circ T s \circ X\right. \\
& \left.\quad-\kappa_{F(M)} \circ T\left(X^{F}\right) \circ T s \circ Y+\kappa_{F(M)} \circ T\left(X^{F}\right) \circ Y^{F} \circ s\right) \\
& -\operatorname{vpr}_{F(M)} \circ \operatorname{vpr}_{T F(M)} \circ \kappa_{F(M)} \circ\left(T^{2} s \circ T Y \circ X T(-) T\left(Y^{F}\right) \circ T s \circ X\right. \\
& \left.T(-) \kappa_{F(M)} \circ T\left(X^{F}\right) \circ T s \circ Y T(+) \kappa_{F(M)} \circ T\left(X^{F}\right) \circ Y^{F} \circ s\right) \\
& =\mathcal{L}_{[X, Y]} s .
\end{aligned}
$$

\section{Differential Forms}

9.1. The cotangent bundle of a manifold $M$ is the vector bundle $T^{*} M:=$ $(T M)^{*}$, the (real) dual of the tangent bundle.

If $(U, u)$ is a chart on $M$, then $\left(\frac{\partial}{\partial u^{1}}, \ldots, \frac{\partial}{\partial u^{m}}\right)$ is the associated frame field over $U$ of $T M$. Since $\left.\frac{\partial}{\partial u^{i}}\right|_{x}\left(u^{j}\right)=d u^{j}\left(\left.\frac{\partial}{\partial u^{i}}\right|_{x}\right)=\delta_{i}^{j}$, we see that $\left(d u^{1}, \ldots, d u^{m}\right)$ is the dual frame field on $T^{*} M$ over $U$. It is also called a holonomic frame field. A section of $T^{*} M$ is also called a 1-form.

9.2. According to (8.18) a tensor field of type $\left(\begin{array}{l}p \\ q\end{array}\right)$ on a manifold $M$ is a smooth section of the vector bundle

$$
\bigotimes^{p} T M \otimes \bigotimes^{q} T^{*} M=\overbrace{T M \otimes \cdots \otimes T M}^{p \text { times }} \otimes \overbrace{T^{*} M \otimes \cdots \otimes T^{*} M}^{q \text { times }} .
$$

The position of $p$ (up) and $q$ (down) can be explained as follows: If $(U, u)$ is a chart on $M$, we have the holonomous frame field

$$
\left(\frac{\partial}{\partial u^{i_{1}}} \otimes \frac{\partial}{\partial u^{i_{2}}} \otimes \cdots \otimes \frac{\partial}{\partial u^{i_{p}}} \otimes d u^{j_{1}} \otimes \cdots \otimes d u^{j_{q}}\right)_{i \in\{1, \ldots, m\}^{p}, j \in\{1, \ldots, m\}^{q}}
$$


over $U$ of this tensor bundle, and for any $\left(\begin{array}{l}p \\ q\end{array}\right)$-tensor field $A$ we have

$$
A \mid U=\sum_{i, j} A_{j_{1} \ldots j_{q}}^{i_{1} \ldots i_{p}} \frac{\partial}{\partial u^{i} i_{1}} \otimes \cdots \otimes \frac{\partial}{\partial u^{i_{p}}} \otimes d u^{j_{1}} \otimes \cdots \otimes d u^{j_{q}} .
$$

The coefficients have $p$ indices up and $q$ indices down, and they are smooth functions on $U$.

From a categorical point of view one should look where the indices of the frame field are, but this convention here has a long tradition.

9.3. Lemma. Let

$$
\Phi: \mathfrak{X}(M) \times \cdots \times \mathfrak{X}(M)=\mathfrak{X}(M)^{k} \rightarrow \Gamma\left(\bigotimes^{l} T M\right)
$$

be a mapping which is $k$-linear over $C^{\infty}(M)$. Then $\Phi$ is given by the action of a $\left(\begin{array}{l}l \\ k\end{array}\right)$-tensor field.

Proof. For simplicity's sake we put $k=1, \ell=0$, so $\Phi: \mathfrak{X}(M) \rightarrow C^{\infty}(M)$ is a $C^{\infty}(M)$-linear mapping: $\Phi(f . X)=f . \Phi(X)$. In the general case we subject each entry to the treatment described below.

Claim 1. If $X \mid U=0$ for some open subset $U \subset M$, then we have $\Phi(X) \mid U=0$.

Let $x \in U$. We choose $f \in C^{\infty}(M)$ with $f(x)=0$ and $f \mid M \backslash U=1$. Then $f . X=X$, so $\Phi(X)(x)=\Phi(f . X)(x)=f(x) . \Phi(X)(x)=0$.

Claim 2. If $X(x)=0$, then also $\Phi(X)(x)=0$.

Let $(U, u)$ be a chart centered at $x$, and let $V$ be open with $x \in V \subset \bar{V} \subset U$. Then

$$
X \mid U=\sum X^{i} \frac{\partial}{\partial u^{i}} \quad \text { and } \quad X^{i}(x)=0 .
$$

We choose $g \in C^{\infty}(M)$ with $g \mid V \equiv 1$ and with support contained in $U$. Then $\left(g^{2} . X\right)|V=X| V$ and by claim 1 the restriction $\Phi(X) \mid V$ depends only on $X \mid V$; thus $g^{2} \cdot X=\sum_{i}\left(g \cdot X^{i}\right)\left(g \cdot \frac{\partial}{\partial u^{i}}\right)$ is a decomposition which is globally defined on $M$. Therefore we have

$$
\begin{aligned}
\Phi(X)(x) & =\Phi\left(g^{2} \cdot X\right)(x)=\Phi\left(\sum_{i}\left(g \cdot X^{i}\right)\left(g \cdot \frac{\partial}{\partial u^{i}}\right)\right)(x) \\
& =\sum\left(g \cdot X^{i}\right)(x) \cdot \Phi\left(g \cdot \frac{\partial}{\partial u^{i}}\right)(x)=0 .
\end{aligned}
$$

So we see that for a general vector field $X$ the value $\Phi(X)(x)$ depends only on the value $X(x)$, for each $x \in M$. So there is a linear map $\varphi_{x}: T_{x} M \rightarrow \mathbb{R}$ for each $x \in M$ with $\Phi(X)(x)=\varphi_{x}(X(x))$. Then $\varphi: M \rightarrow T^{*} M$ is smooth since $\varphi \mid V=\sum_{i} \Phi\left(g \cdot \frac{\partial}{\partial u^{i}}\right) d u^{i}$ in the setting of claim 2 . 
9.4. Definition. A differential form of degree $k$, or a $k$-form for short, is a section of the (natural) vector bundle $\wedge^{k} T^{*} M$. The space of all $k$ forms will be denoted by $\Omega^{k}(M)$. It may also be viewed as the space of all skew-symmetric $\left(\begin{array}{l}0 \\ k\end{array}\right)$-tensor fields, i.e., (by (9.3) the space of all mappings

$$
\varphi: \mathfrak{X}(M) \times \cdots \times \mathfrak{X}(M)=\mathfrak{X}(M)^{k} \rightarrow C^{\infty}(M),
$$

which are $k$-linear over $C^{\infty}(M)$ and are skew-symmetric:

$$
\varphi\left(X_{\sigma 1}, \ldots, X_{\sigma k}\right)=\operatorname{sign} \sigma \cdot \varphi\left(X_{1}, \ldots, X_{k}\right)
$$

for each permutation $\sigma \in \mathcal{S}_{k}$.

We put $\Omega^{0}(M):=C^{\infty}(M)$. Then the space

$$
\Omega(M):=\bigoplus_{k=0}^{\operatorname{dim} M} \Omega^{k}(M)
$$

is an algebra with the following product, called the wedge product. For $\varphi \in \Omega^{k}(M)$ and $\psi \in \Omega^{\ell}(M)$ and for $X_{i}$ in $\mathfrak{X}(M)$ (or in $T_{x} M$ ) we put

$$
\begin{aligned}
& (\varphi \wedge \psi)\left(X_{1}, \ldots, X_{k+\ell}\right) \\
& =\frac{1}{k ! \ell !} \sum_{\sigma \in \mathcal{S}_{k+\ell}} \operatorname{sign} \sigma \cdot \varphi\left(X_{\sigma 1}, \ldots, X_{\sigma k}\right) \cdot \psi\left(X_{\sigma(k+1)}, \ldots, X_{\sigma(k+\ell)}\right) .
\end{aligned}
$$

This product is defined fiberwise, i.e., $(\varphi \wedge \psi)_{x}=\varphi_{x} \wedge \psi_{x}$ for each $x \in M$. It is also associative, i.e., $(\varphi \wedge \psi) \wedge \tau=\varphi \wedge(\psi \wedge \tau)$, and graded commutative, i.e., $\varphi \wedge \psi=(-1)^{k \ell} \psi \wedge \varphi$. There are differing conventions for the factor in the definition of the wedge product: in [192] the factor $\frac{1}{(k+\ell) !}$ is used. But then the insertion operator of (9.7) is no longer a graded derivation.

9.5. If $f: N \rightarrow M$ is a smooth mapping and $\varphi \in \Omega^{k}(M)$, then the pullback $f^{*} \varphi \in \Omega^{k}(N)$ is defined for $X_{i} \in T_{x} N$ by

$$
\left(f^{*} \varphi\right)_{x}\left(X_{1}, \ldots, X_{k}\right):=\varphi_{f(x)}\left(T_{x} f \cdot X_{1}, \ldots, T_{x} f \cdot X_{k}\right) .
$$

Then we have $f^{*}(\varphi \wedge \psi)=f^{*} \varphi \wedge f^{*} \psi$, so $f^{*}: \Omega(M) \rightarrow \Omega(N)$ is an algebra homomorphism. Moreover we have $(g \circ f)^{*}=f^{*} \circ g^{*}: \Omega(P) \rightarrow \Omega(N)$ if $g: M \rightarrow P$, and $\left(I d_{M}\right)^{*}=I d_{\Omega(M)}$.

So $M \mapsto \Omega(M)=\Gamma\left(\bigwedge T^{*} M\right)$ is a contravariant functor from the category $\mathcal{M} f$ of all manifolds and all smooth mappings into the category of real graded commutative algebras, whereas $M \mapsto \wedge T^{*} M$ is a covariant vector bundle functor defined only on $\mathcal{M} f_{m}$, the category of $m$-dimensional manifolds and local diffeomorphisms, for each $m$ separately. 
9.6. The Lie derivative of differential forms. Since $M \mapsto \bigwedge^{k} T^{*} M$ is a vector bundle functor on $\mathcal{M} f_{m}$, by (8.16) for $X \in \mathfrak{X}(M)$ the Lie derivative of a $k$-form $\varphi$ along $X$ is defined by

$$
\mathcal{L}_{X} \varphi=\left.\frac{d}{d t}\right|_{0}\left(\mathrm{Fl}_{t}^{X}\right)^{*} \varphi .
$$

Lemma. The Lie derivative has the following properties.

(1) $\mathcal{L}_{X}(\varphi \wedge \psi)=\mathcal{L}_{X} \varphi \wedge \psi+\varphi \wedge \mathcal{L}_{X} \psi$, so $\mathcal{L}_{X}$ is a derivation.

(2) For $Y_{i} \in \mathfrak{X}(M)$ we have

$$
\left(\mathcal{L}_{X} \varphi\right)\left(Y_{1}, \ldots, Y_{k}\right)=X\left(\varphi\left(Y_{1}, \ldots, Y_{k}\right)\right)-\sum_{i=1}^{k} \varphi\left(Y_{1}, \ldots,\left[X, Y_{i}\right], \ldots, Y_{k}\right)
$$

(3) $\left[\mathcal{L}_{X}, \mathcal{L}_{Y}\right] \varphi=\mathcal{L}_{[X, Y]} \varphi$

(4) $\frac{\partial}{\partial t}\left(\mathrm{Fl}_{t}^{X}\right)^{*} \varphi=\left(\mathrm{Fl}_{t}^{X}\right)^{*} \mathcal{L}_{X} \varphi=\mathcal{L}_{X}\left(\left(\mathrm{Fl}_{t}^{X}\right)^{*} \varphi\right)$.

Proof. (11) The mapping Alt: $\bigotimes^{k} T^{*} M \rightarrow \wedge^{k} T^{*} M$, given by

$$
(\operatorname{Alt} A)\left(Y_{1}, \ldots, Y_{k}\right):=\frac{1}{k !} \sum_{\sigma} \operatorname{sign}(\sigma) A\left(Y_{\sigma 1}, \ldots, Y_{\sigma k}\right),
$$

is a linear natural transformation in the sense of (8.18) and induces an algebra homomorphism from $\bigoplus_{k \geq 0} \Gamma\left(\otimes^{k} T^{*} M\right)$ onto $\Omega(M)$. So (1) follows from (8.17) and (8.18).

Second, direct proof, using the definition and (9.5):

$$
\begin{aligned}
\mathcal{L}_{X}(\varphi \wedge \psi) & =\left.\frac{d}{d t}\right|_{0}\left(\mathrm{Fl}_{t}^{X}\right)^{*}(\varphi \wedge \psi)=\left.\frac{d}{d t}\right|_{0}\left(\left(\mathrm{Fl}_{t}^{X}\right)^{*} \varphi \wedge\left(\mathrm{Fl}_{t}^{X}\right)^{*} \psi\right) \\
& =\left.\frac{d}{d t}\right|_{0}\left(\mathrm{Fl}_{t}^{X}\right)^{*} \varphi \wedge\left(\mathrm{Fl}_{0}^{X}\right)^{*} \psi+\left.\left(\mathrm{Fl}_{0}^{X}\right)^{*} \varphi \wedge \frac{d}{d t}\right|_{0}\left(\mathrm{Fl}_{t}^{X}\right)^{*} \psi \\
& =\mathcal{L}_{X} \varphi \wedge \psi+\varphi \wedge \mathcal{L}_{X} \psi
\end{aligned}
$$

(2) Again by (8.17) and (8.18) we may compute as follows, where Trace is the full evaluation of the form on all vector fields:

$$
\begin{aligned}
& X\left(\varphi\left(Y_{1}, \ldots, Y_{k}\right)\right)=\mathcal{L}_{X} \circ \operatorname{Trace}\left(\varphi \otimes Y_{1} \otimes \cdots \otimes Y_{k}\right) \\
& \quad=\operatorname{Trace} \circ \mathcal{L}_{X}\left(\varphi \otimes Y_{1} \otimes \cdots \otimes Y_{k}\right) \\
& \quad=\operatorname{Trace}\left(\mathcal{L}_{X} \varphi \otimes\left(Y_{1} \otimes \cdots \otimes Y_{k}\right)+\varphi \otimes\left(\sum_{i} Y_{1} \otimes \cdots \otimes \mathcal{L}_{X} Y_{i} \otimes \cdots \otimes Y_{k}\right)\right) .
\end{aligned}
$$

Now we use $\mathcal{L}_{X} Y_{i}=\left[X, Y_{i}\right]$ from $(3.13)$,

Second, independent proof:

$$
\begin{aligned}
X\left(\varphi\left(Y_{1}, \ldots, Y_{k}\right)\right) & =\left.\frac{d}{d t}\right|_{0}\left(\mathrm{Fl}_{t}^{X}\right)^{*}\left(\varphi\left(Y_{1}, \ldots, Y_{k}\right)\right) \\
& \left.=\left.\frac{d}{d t}\right|_{0}\left(\left(\mathrm{Fl}_{t}^{X}\right)^{*} \varphi\right)\left(\left(\mathrm{Fl}_{t}^{X}\right)^{*} Y_{1}, \ldots,\left(\mathrm{Fl}_{t}^{X}\right)^{*} Y_{k}\right)\right) \\
& =\left(\mathcal{L}_{X} \varphi\right)\left(Y_{1}, \ldots, Y_{k}\right)+\sum_{i=1}^{k} \varphi\left(Y_{1}, \ldots, \mathcal{L}_{X} Y_{i}, \ldots, Y_{k}\right) .
\end{aligned}
$$


(3) is a special case of (8.20), See (9.9.7) below for another proof.

$$
\text { (44) } \begin{aligned}
\frac{\partial}{\partial t}\left(\mathrm{Fl}_{t}^{X}\right)^{*} \varphi & =\left.\frac{\partial}{\partial s}\right|_{0}\left(\bigwedge^{k} T\left(\mathrm{Fl}_{-t}^{X}\right) \circ T\left(\mathrm{Fl}_{-s}^{X}\right)^{*} \circ \varphi \circ \mathrm{Fl}_{s}^{X} \circ \mathrm{Fl}_{t}^{X}\right) \\
& =\left.\bigwedge_{k}^{k} T\left(\mathrm{Fl}_{-t}^{X}\right)^{*} \circ \frac{\partial}{\partial s}\right|_{0}\left(\bigwedge^{k} T\left(\mathrm{Fl}_{-s}^{X}\right)^{*} \circ \varphi \circ \mathrm{Fl}_{s}^{X}\right) \circ \mathrm{Fl}_{t}^{X} \\
& =\bigwedge^{k} T\left(\mathrm{Fl}_{-t}^{X}\right)^{*} \circ \mathcal{L}_{X} \varphi \circ \mathrm{Fl}_{t}^{X}=\left(\mathrm{Fl}_{t}^{X}\right)^{*} \mathcal{L}_{X} \varphi \\
\frac{\partial}{\partial t}\left(\mathrm{Fl}_{t}^{X}\right)^{*} Y & =\left.\frac{\partial}{\partial s}\right|_{0}\left(\mathrm{Fl}_{s}^{X}\right)^{*}\left(\mathrm{Fl}_{t}^{X}\right)^{*} Y=\mathcal{L}_{X}\left(\mathrm{Fl}_{t}^{X}\right)^{*} \varphi
\end{aligned}
$$

9.7. The insertion operator. For a vector field $X \in \mathfrak{X}(M)$ we define the insertion operator $i_{X}=i(X): \Omega^{k}(M) \rightarrow \Omega^{k-1}(M)$ by

$$
\left(i_{X} \varphi\right)\left(Y_{1}, \ldots, Y_{k-1}\right):=\varphi\left(X, Y_{1}, \ldots, Y_{k-1}\right) .
$$

\section{Lemma.}

(1) $i_{X}$ is a graded derivation of degree -1 of the graded algebra $\Omega(M)$, so we have $i_{X}(\varphi \wedge \psi)=i_{X} \varphi \wedge \psi+(-1)^{-\operatorname{deg} \varphi} \varphi \wedge i_{X} \psi$.

(2) $i_{X} \circ i_{Y}+i_{Y} \circ i_{X}=0$.

(3) $\left[\mathcal{L}_{X}, i_{Y}\right]:=\mathcal{L}_{X} \circ i_{Y}-i_{Y} \circ \mathcal{L}_{X}=i_{[X, Y]} \cdot$

Proof. (11) For $\varphi \in \Omega^{k}(M)$ and $\psi \in \Omega^{\ell}(M)$ we have

$$
\begin{aligned}
& \left(i_{X_{1}}(\varphi \wedge \psi)\right)\left(X_{2}, \ldots, X_{k+\ell}\right)=(\varphi \wedge \psi)\left(X_{1}, \ldots, X_{k+\ell}\right) \\
& \quad=\frac{1}{k ! \ell !} \sum_{\sigma} \operatorname{sign}(\sigma) \varphi\left(X_{\sigma 1}, \ldots, X_{\sigma k}\right) \psi\left(X_{\sigma(k+1)}, \ldots, X_{\sigma(k+\ell)}\right), \\
& \left(i_{X_{1}} \varphi \wedge \psi+(-1)^{k} \varphi \wedge i_{X_{1}} \psi\right)\left(X_{2}, \ldots, X_{k+\ell}\right) \\
& =\frac{1}{(k-1) ! \ell !} \sum_{\sigma} \operatorname{sign}(\sigma) \varphi\left(X_{1}, X_{\sigma 2}, \ldots, X_{\sigma k}\right) \psi\left(X_{\sigma(k+1)}, \ldots, X_{\sigma(k+\ell)}\right) \\
& \quad+\frac{(-1)^{k}}{k !(\ell-1) !} \sum_{\sigma} \operatorname{sign}(\sigma) \varphi\left(X_{\sigma 2}, \ldots, X_{\sigma(k+1)}\right) \psi\left(X_{1}, X_{\sigma(k+2)}, \ldots\right) .
\end{aligned}
$$

Using the skew-symmetry of $\varphi$ and $\psi$, we may distribute $X_{1}$ to each position by adding an appropriate sign. There are $k+\ell$ summands. Since

$$
\frac{1}{(k-1) ! \ell !}+\frac{1}{k !(\ell-1) !}=\frac{k+\ell}{k ! \ell !},
$$

and since we can generate each permutation in $\mathcal{S}_{k+\ell}$ in this way, the result follows. 
(2) $\left(i_{X} i_{Y} \varphi\right)\left(Z_{1}, \ldots, Z_{k-2}\right)=\varphi\left(Y, X, Z_{1}, \ldots, Z_{n}\right)$

$$
=-\varphi\left(X, Y, Z_{1}, \ldots, Z_{n}\right)=-\left(i_{Y} i_{X} \varphi\right)\left(Z_{1}, \ldots, Z_{k-2}\right) .
$$

(3) By (8.17) and (8.18) we have:

$$
\begin{aligned}
\mathcal{L}_{X} i_{Y} \varphi & =\mathcal{L}_{X} \operatorname{Trace}_{1}(Y \otimes \varphi)=\operatorname{Trace}_{1} \mathcal{L}_{X}(Y \otimes \varphi) \\
& =\operatorname{Trace}_{1}\left(\mathcal{L}_{X} Y \otimes \varphi+Y \otimes \mathcal{L}_{X} \varphi\right)=i_{[X, Y]} \varphi+i_{Y} \mathcal{L}_{X} \varphi .
\end{aligned}
$$

See (9.9.6) below for another proof.

9.8. The exterior differential. We want to construct a differential operator $\Omega^{k}(M) \rightarrow \Omega^{k+1}(M)$ which is natural. We will show that the simplest choice will work and (later) that it is essentially unique.

Let $U$ be open in $\mathbb{R}^{n}$, and let $\varphi \in \Omega^{k}(U)=C^{\infty}\left(U, L_{a l t}^{k}\left(\mathbb{R}^{n}, \mathbb{R}\right)\right)$. We consider the derivative $D \varphi \in C^{\infty}\left(U, L\left(\mathbb{R}^{n}, L_{a l t}^{k}\left(\mathbb{R}^{n}, \mathbb{R}\right)\right)\right)$, and we take its canonical image in $C^{\infty}\left(U, L_{\text {alt }}^{k+1}\left(\mathbb{R}^{n}, \mathbb{R}\right)\right)$. Here we write $D$ for the derivative in order to distinguish it from the exterior differential, which we define as

$$
d \varphi:=(k+1) \text { Alt } D \varphi,
$$

more explicitly as

$$
\begin{aligned}
(d \varphi)_{x}\left(X_{0}, \ldots, X_{k}\right) & =\frac{1}{k !} \sum_{\sigma} \operatorname{sign}(\sigma) D \varphi(x)\left(X_{\sigma 0}\right)\left(X_{\sigma 1}, \ldots, X_{\sigma k}\right) \\
& =\sum_{i=0}^{k}(-1)^{i} D \varphi(x)\left(X_{i}\right)\left(X_{0}, \ldots, \widehat{X}_{i}, \ldots, X_{k}\right),
\end{aligned}
$$

where the hat over a symbol means that this is to be omitted and where $X_{i} \in \mathbb{R}^{n}$.

Now we pass to an arbitrary manifold $M$. For a $k$-form $\varphi \in \Omega^{k}(M)$ and vector fields $X_{i} \in \mathfrak{X}(M)$ we try to replace $D \varphi(x)\left(X_{i}\right)\left(X_{0}, \ldots\right)$ in formula (11) by Lie derivatives. We differentiate

$$
\begin{aligned}
& X_{i}\left(\varphi(x)\left(X_{0}, \ldots\right)\right) \\
& \quad=D \varphi(x)\left(X_{i}\right)\left(X_{0}, \ldots\right)+\sum_{0 \leq j \leq k, j \neq i} \varphi(x)\left(X_{0}, \ldots, D X_{j}(x) X_{i}, \ldots\right)
\end{aligned}
$$

and insert this expression into formula (1) in order to get (cf. (3.4) our working definition

$$
\begin{aligned}
d \varphi\left(X_{0}, \ldots, X_{k}\right) & :=\sum_{i=0}^{k}(-1)^{i} X_{i}\left(\varphi\left(X_{0}, \ldots, \widehat{X}_{i}, \ldots, X_{k}\right)\right) \\
& +\sum_{i<j}(-1)^{i+j} \varphi\left(\left[X_{i}, X_{j}\right], X_{0}, \ldots, \widehat{X}_{i}, \ldots, \widehat{X}_{j}, \ldots, X_{k}\right) .
\end{aligned}
$$


This formula gives $d \varphi$ as a $(k+1)$-linear mapping over $C^{\infty}(M)$, as a short computation involving (3.4) shows. It is obviously skew-symmetric, so $d \varphi$ is a $(k+1)$-form by (9.3), and the operator $d: \Omega^{k}(M) \rightarrow \Omega^{k+1}(M)$ is called the exterior derivative.

If $(U, u)$ is a chart on $M$, then we have

$$
\varphi \uparrow U=\sum_{i_{1}<\cdots<i_{k}} \varphi_{i_{1}, \ldots, i_{k}} d u^{i_{1}} \wedge \cdots \wedge d u^{i_{k}}
$$

where

$$
\varphi_{i_{1}, \ldots, i_{k}}=\varphi\left(\frac{\partial}{\partial u^{i_{1}}}, \ldots, \frac{\partial}{\partial u^{i_{k}}}\right) .
$$

An easy computation shows that (2) leads to

$$
d \varphi \uparrow U=\sum_{i_{1}<\cdots<i_{k}} d \varphi_{i_{1}, \ldots, i_{k}} \wedge d u^{i_{1}} \wedge \cdots \wedge d u^{i_{k}},
$$

so that formulas (11) and (2) really define the same operator.

9.9. Theorem. The exterior derivative $d: \Omega^{k}(M) \rightarrow \Omega^{k+1}(M)$ has the following properties:

(1) $d(\varphi \wedge \psi)=d \varphi \wedge \psi+(-1)^{\operatorname{deg} \varphi} \varphi \wedge d \psi$, so $d$ is a graded derivation of degree 1 .

(2) $\mathcal{L}_{X}=i_{X} \circ d+d \circ i_{X}$ for any vector field $X$.

(3) $d^{2}=d \circ d=0$.

(4) $f^{*} \circ d=d \circ f^{*}$ for any smooth $f: N \rightarrow M$.

(5) $\mathcal{L}_{X} \circ d=d \circ \mathcal{L}_{X}$ for any vector field $X$.

(6) $\left[\mathcal{L}_{X}, i_{Y}\right]:=\mathcal{L}_{X} \circ i_{Y}-i_{Y} \circ \mathcal{L}_{X}=i_{[X, Y]}$. See also (9.7.3).

(7) $\left[\mathcal{L}_{X}, \mathcal{L}_{Y}\right]=\mathcal{L}_{[X, Y]}$ for any two vector fields $X, Y$.

Remark. In terms of the graded commutator

$$
\left[D_{1}, D_{2}\right]:=D_{1} \circ D_{2}-(-1)^{\operatorname{deg}\left(D_{1}\right) \operatorname{deg}\left(D_{2}\right)} D_{2} \circ D_{1}
$$

for graded homomorphisms and graded derivations (see (16.1) the assertions of this theorem take the following form:
(2) $\mathcal{L}_{X}=\left[i_{X}, d\right]$.
(3) $\frac{1}{2}[d, d]=0$.
(44) $\left[f^{*}, d\right]=0$.
(5) $\left[\mathcal{L}_{X}, d\right]=0$.

This point of view will be developed in section (16) below. The equation (17) is a special case of (8.20). 
Proof. (2) For $\varphi \in \Omega^{k}(M)$ and $X_{i} \in \mathfrak{X}(M)$ we have

$$
\begin{aligned}
& \left(\mathcal{L}_{X_{0}} \varphi\right)\left(X_{1}, \ldots, X_{k}\right)=X_{0}\left(\varphi\left(X_{1}, \ldots, X_{k}\right)\right) \\
& +\sum_{j=1}^{k}(-1)^{0+j} \varphi\left(\left[X_{0}, X_{j}\right], X_{1}, \ldots, \widehat{X_{j}}, \ldots, X_{k}\right) \text { by }(9.6 .2) \\
& \left(i_{X_{0}} d \varphi\right)\left(X_{1}, \ldots, X_{k}\right)=d \varphi\left(X_{0}, \ldots, X_{k}\right) \\
& =\sum_{i=0}^{k}(-1)^{i} X_{i}\left(\varphi\left(X_{0}, \ldots, \widehat{X}_{i}, \ldots, X_{k}\right)\right) \\
& +\sum_{0 \leq i<j}(-1)^{i+j} \varphi\left(\left[X_{i}, X_{j}\right], X_{0}, \ldots, \widehat{X}_{i}, \ldots, \widehat{X}_{j}, \ldots, X_{k}\right), \\
& \left(d i_{X_{0}} \varphi\right)\left(X_{1}, \ldots, X_{k}\right)=\sum_{i=1}^{k}(-1)^{i-1} X_{i}\left(\left(i_{X_{0}} \varphi\right)\left(X_{1}, \ldots, \widehat{X}_{i}, \ldots, X_{k}\right)\right) \\
& +\sum_{1 \leq i<j}(-1)^{i+j-2}\left(i_{X_{0}} \varphi\right)\left(\left[X_{i}, X_{j}\right], X_{1}, \ldots, \widehat{X_{i}}, \ldots, \widehat{X_{j}}, \ldots, X_{k}\right) \\
& =-\sum_{i=1}^{k}(-1)^{i} X_{i}\left(\varphi\left(X_{0}, X_{1}, \ldots, \widehat{X}_{i}, \ldots, X_{k}\right)\right) \\
& -\sum_{1 \leq i<j}(-1)^{i+j} \varphi\left(\left[X_{i}, X_{j}\right], X_{0}, X_{1}, \ldots, \widehat{X_{i}}, \ldots, \widehat{X_{j}}, \ldots, X_{k}\right) .
\end{aligned}
$$

By summing up, the result follows.

(11) Let $\varphi \in \Omega^{p}(M)$ and $\psi \in \Omega^{q}(M)$. We prove the result by induction on $p+q$.

$p+q=0: d(f \cdot g)=d f \cdot g+f \cdot d g$.

Suppose that (1) is true for $p+q<k$. Then for $X \in \mathfrak{X}(M)$ we have by part (2) and (9.6), (9.7) and by induction

$$
\begin{aligned}
i_{X} d(\varphi \wedge \psi)= & \mathcal{L}_{X}(\varphi \wedge \psi)-d i_{X}(\varphi \wedge \psi) \\
= & \mathcal{L}_{X} \varphi \wedge \psi+\varphi \wedge \mathcal{L}_{X} \psi-d\left(i_{X} \varphi \wedge \psi+(-1)^{p} \varphi \wedge i_{X} \psi\right) \\
= & i_{X} d \varphi \wedge \psi+d i_{X} \varphi \wedge \psi+\varphi \wedge i_{X} d \psi+\varphi \wedge d i_{X} \psi-d i_{X} \varphi \wedge \psi \\
& \quad-(-1)^{p-1} i_{X} \varphi \wedge d \psi-(-1)^{p} d \varphi \wedge i_{X} \psi-\varphi \wedge d i_{X} \psi \\
= & i_{X}\left(d \varphi \wedge \psi+(-1)^{p} \varphi \wedge d \psi\right) .
\end{aligned}
$$

Since $X$ is arbitrary, (11) follows.

(3) By (11) the operator $d$ is a graded derivation of degree 1 , so $d^{2}=\frac{1}{2}[d, d]$ is a graded derivation of degree 2 ; see (16.1), It is obviously local: $d^{2}(\varphi \wedge \psi)=$ $d^{2}(\varphi) \wedge \psi+\varphi \wedge d(\psi)$. Since $\Omega(M)$ is locally generated as an algebra by $C^{\infty}(M)$ and $\left\{d f: f \in C^{\infty}(M)\right\}$, it suffices to show that $d^{2} f=0$ for each 
$f \in C^{\infty}(M)\left(d^{3} f=0\right.$ is a consequence). But this is easy:

$d^{2} f(X, Y)=X d f(Y)-Y d f(X)-d f([X, Y])=X Y f-Y X f-[X, Y] f=0$.

(44) $f^{*}: \Omega(M) \rightarrow \Omega(N)$ is an algebra homomorphism by (9.6), so $f^{*} \circ d$ and $d \circ f^{*}$ are both graded derivations over $f^{*}$ of degree 1 . So if $f^{*} \circ d$ and $d \circ f^{*}$ agree on $\varphi$ and on $\psi$, then they also agree on $\varphi \wedge \psi$. By the same argument as in the proof of (3) above it suffices to show that they agree on $g$ and $d g$ for all $g \in C^{\infty}(M)$. We have

$$
\left(f^{*} d g\right)_{y}(Y)=(d g)_{f(y)}\left(T_{y} f . Y\right)=\left(T_{y} f . Y\right)(g)=Y(g \circ f)(y)=\left(d f^{*} g\right)_{y}(Y) ;
$$

thus also $d f^{*} d g=d d f^{*} g=0$, and $f^{*} d d g=0$.

(5) $d \mathcal{L}_{X}=d i_{X} d+d d i_{X}=d i_{X} d+i_{X} d d=\mathcal{L}_{X} d$.

(6) We use the graded commutator alluded to in the remarks. Both $\mathcal{L}_{X}$ and $i_{Y}$ are graded derivations; thus the graded commutator $\left[L_{X}, i_{Y}\right]$ is also a graded derivation as is $i_{[X, Y]}$. Thus it suffices to show that they agree on 0 -forms $g \in C^{\infty}(M)$ and on exact 1-forms $d g$. We have

$$
\begin{aligned}
{\left[\mathcal{L}_{X}, i_{Y}\right] g } & =\mathcal{L}_{X} i_{Y} g-i_{Y} \mathcal{L}_{X} g=\mathcal{L}_{X} 0-i_{Y}(d g(X))=0=i_{[X, Y]} g \\
{\left[\mathcal{L}_{X}, i_{Y}\right] d g } & =\mathcal{L}_{X} i_{Y} d g-i_{Y} \mathcal{L}_{X} d g=\mathcal{L}_{X} \mathcal{L}_{Y} g-i_{Y} d \mathcal{L}_{X} g \\
& =(X Y-Y X) g=[X, Y] g=i_{[X, Y]} d g .
\end{aligned}
$$

(17) By the (graded) Jacobi identity and by (6) (or lemma (9.7.3) we have

$$
\begin{aligned}
{\left[\mathcal{L}_{X}, \mathcal{L}_{Y}\right] } & =\left[\mathcal{L}_{X},\left[i_{Y}, d\right]\right]=\left[\left[\mathcal{L}_{X}, i_{Y}\right], d\right]+\left[i_{Y},\left[\mathcal{L}_{X}, d\right]\right] \\
& =\left[i_{[X, Y]}, d\right]+0=\mathcal{L}_{[X, Y]} .
\end{aligned}
$$

9.10. A differential form $\omega \in \Omega^{k}(M)$ is called closed if $d \omega=0$, and it is called exact if $\omega=d \varphi$ for some $\varphi \in \Omega^{k-1}(M)$. Since $d^{2}=0$, any exact form is closed. The quotient space

$$
H^{k}(M):=\frac{\operatorname{ker}\left(d: \Omega^{k}(M) \rightarrow \Omega^{k+1}(M)\right)}{\operatorname{im}\left(d: \Omega^{k-1}(M) \rightarrow \Omega^{k}(M)\right)}
$$

is called the $k$-th de Rham cohomology space of $M$. As a preparation for our treatment of cohomology we finish with the

Lemma of Poincaré. A closed differential form of degree $k \geq 1$ is locally exact. More precisely: let $\omega \in \Omega^{k}(M)$ with $d \omega=0$. Then for any $x \in M$ there is an open neighborhood $U$ of $x$ in $M$ and $a \varphi \in \Omega^{k-1}(U)$ with $d \varphi=$ $\omega \uparrow U$.

Proof. Let $(U, u)$ be a chart on $M$ centered at $x$ such that $u(U)=\mathbb{R}^{m}$. So we may just assume that $M=\mathbb{R}^{m}$. 
We consider $\alpha: \mathbb{R} \times \mathbb{R}^{m} \rightarrow \mathbb{R}^{m}$, given by $\alpha(t, x)=\alpha_{t}(x)=t x$. Let $I \in$ $\mathfrak{X}\left(\mathbb{R}^{m}\right)$ be the vector field $I(x)=x$; then $\alpha\left(e^{t}, x\right)=\mathrm{Fl}_{t}^{I}(x)$. So for $t>0$ we have

$$
\begin{aligned}
\frac{d}{d t} \alpha_{t}^{*} \omega & =\frac{d}{d t}\left(\mathrm{Fl}_{\log t}^{I}\right)^{*} \omega=\frac{1}{t}\left(\mathrm{Fl}_{\log t}^{I}\right)^{*} \mathcal{L}_{I} \omega \\
& =\frac{1}{t} \alpha_{t}^{*}\left(i_{I} d \omega+d i_{I} \omega\right)=\frac{1}{t} d \alpha_{t}^{*} i_{I} \omega .
\end{aligned}
$$

Note that $T_{x}\left(\alpha_{t}\right)=t . I d$. Therefore

$$
\begin{aligned}
\left(\frac{1}{t} \alpha_{t}^{*} i_{I} \omega\right)_{x}\left(X_{2}, \ldots, X_{k}\right) & =\frac{1}{t}\left(i_{I} \omega\right)_{t x}\left(t X_{2}, \ldots, t X_{k}\right) \\
& =\frac{1}{t} \omega_{t x}\left(t x, t X_{2}, \ldots, t X_{k}\right)=\omega_{t x}\left(x, t X_{2}, \ldots, t X_{k}\right) .
\end{aligned}
$$

So if $k \geq 1$, the $(k-1)$-form $\frac{1}{t} \alpha_{t}^{*} i_{I} \omega$ is defined and smooth in $(t, x)$ for all $t \in \mathbb{R}$. Clearly $\alpha_{1}^{*} \omega=\omega$ and $\alpha_{0}^{*} \omega=0$; thus

$$
\begin{aligned}
\omega & =\alpha_{1}^{*} \omega-\alpha_{0}^{*} \omega=\int_{0}^{1} \frac{d}{d t} \alpha_{t}^{*} \omega d t \\
& =\int_{0}^{1} d\left(\frac{1}{t} \alpha_{t}^{*} i_{I} \omega\right) d t=d\left(\int_{0}^{1} \frac{1}{t} \alpha_{t}^{*} i_{I} \omega d t\right)=d \varphi .
\end{aligned}
$$

\section{Integration on Manifolds}

10.1. Let $U \subset \mathbb{R}^{n}$ be an open subset, let $d x$ denote Lebesque measure on $\mathbb{R}^{n}$ (which depends on the Euclidean structure), let $g: U \rightarrow g(U)$ be a diffeomorphism onto some other open subset in $\mathbb{R}^{n}$, and let $f: g(U) \rightarrow \mathbb{R}$ be an integrable continuous function. Then the transformation formula for multiple integrals reads

$$
\int_{g(U)} f(y) d y=\int_{U} f(g(x))|\operatorname{det} d g(x)| d x .
$$

This suggests that the suitable objects for integration on a manifold are sections of a 1-dimensional vector bundle whose cocycle of transition functions is given by the absolute value of the Jacobi matrix of the chart changes. They will be called densities below.

10.2. The volume bundle. Let $M$ be a manifold and let $\left(U_{\alpha}, u_{\alpha}\right)$ be a smooth atlas for it. The volume bundle $\left(\operatorname{Vol}(M), \pi_{M}, M\right)$ of $M$ is the 1-dimensional vector bundle (line bundle) which is given by the following cocycle of transition functions; see (8.3);

$$
\begin{gathered}
\psi_{\alpha \beta}: U_{\alpha \beta}=U_{\alpha} \cap U_{\beta} \rightarrow \mathbb{R} \backslash\{0\}=G L(1, \mathbb{R}), \\
\psi_{\alpha \beta}(x)=\left|\operatorname{det} d\left(u_{\beta} \circ u_{\alpha}^{-1}\right)\left(u_{\alpha}(x)\right)\right|=\frac{1}{\left|\operatorname{det} d\left(u_{\alpha} \circ u_{\beta}^{-1}\right)\left(u_{\beta}(x)\right)\right|} .
\end{gathered}
$$

Lemma. $\operatorname{Vol}(\mathrm{M})$ is a trivial line bundle over $M$. 
But there is no natural trivialization.

Proof. We choose a positive local section over each $U_{\alpha}$ and we glue them with a partition of unity. Since positivity is invariant under the transitions, the resulting global section $\mu$ is nowhere 0 . By (8.5), $\mu$ is a global frame field and trivializes $\operatorname{Vol}(M)$.

Definition. Sections of the line bundle $\operatorname{Vol}(M)$ are called densities.

10.3. Integral of a density. Let $\mu \in \Gamma(\operatorname{Vol}(M))$ be a density with compact support on the manifold $M$. We define the integral of the density $\mu$ as follows:

Let $\left(U_{\alpha}, u_{\alpha}\right)$ be an atlas on $M$, and let $f_{\alpha}$ be a partition of unity with $\operatorname{supp}\left(f_{\alpha}\right) \subset U_{\alpha}$. Then we put

$$
\int_{M} \mu=\sum_{\alpha} \int_{U_{\alpha}} f_{\alpha} \mu:=\sum_{\alpha} \int_{u_{\alpha}\left(U_{\alpha}\right)} f_{\alpha}\left(u_{\alpha}^{-1}(y)\right) \cdot \psi_{\alpha}\left(\mu\left(u_{\alpha}^{-1}(y)\right)\right) d y .
$$

If $\mu$ does not have compact support, we require that $\sum \int_{U_{\alpha}} f_{\alpha}|\mu|<\infty$. The series is then absolutely convergent.

Lemma. $\int_{M} \mu$ is well defined.

Proof. Let $\left(V_{\beta}, v_{\beta}\right)$ be another atlas on $M$, and let $\left(g_{\beta}\right)$ be a partition of unity with $\operatorname{supp}\left(g_{\beta}\right) \subset V_{\beta}$. Let $\left(U_{\alpha}, \psi_{\alpha}\right)$ be the vector bundle atlas of $\operatorname{Vol}(M)$ induced by the atlas $\left(U_{\alpha}, u_{\alpha}\right)$, and let $\left(V_{\beta}, \varphi_{\beta}\right)$ be the one induced by $\left(V_{\beta}, v_{\beta}\right)$. By the transformation formula of integrals for the diffeomorphisms $u_{\alpha} \circ v_{\beta}^{-1}: v_{\beta}\left(U_{\alpha} \cap V_{\beta}\right) \rightarrow u_{\alpha}\left(U_{\alpha} \cap V_{\beta}\right)$ we have:

$$
\begin{aligned}
\sum_{\alpha} \int_{U_{\alpha}} f_{\alpha} \mu & =\sum_{\alpha} \int_{u_{\alpha}\left(U_{\alpha}\right)}\left(f_{\alpha} \circ u_{\alpha}^{-1}\right)(y) \psi_{\alpha}\left(\mu\left(u_{\alpha}^{-1}(y)\right)\right) d y \\
& =\sum_{\alpha} \int_{u_{\alpha}\left(U_{\alpha}\right)} \sum_{\beta}\left(g_{\beta} \circ u_{\alpha}^{-1}\right)(y)\left(f_{\alpha} \circ u_{\alpha}^{-1}\right)(y) \psi_{\alpha}\left(\mu\left(u_{\alpha}^{-1}(y)\right)\right) d y \\
& =\sum_{\alpha \beta} \int_{u_{\alpha}\left(U_{\alpha} \cap V_{\beta}\right)}\left(g_{\beta} \circ u_{\alpha}^{-1}\right)(y)\left(f_{\alpha} \circ u_{\alpha}^{-1}\right)(y) \psi_{\alpha}\left(\mu\left(u_{\alpha}^{-1}(y)\right)\right) d y \\
& =\sum_{\alpha \beta} \int_{v_{\beta}\left(U_{\alpha} \cap V_{\beta}\right)}\left(g_{\beta} \circ v_{\beta}^{-1}\right)(x)\left(f_{\alpha} \circ v_{\beta}^{-1}\right)(x) . \\
& =\sum_{\alpha \beta} \int_{v_{\beta}\left(U_{\alpha} \cap V_{\beta}\right)}\left(g_{\beta} \circ v_{\beta}^{-1}\right)(x)\left(f_{\alpha} \circ v_{\beta}^{-1}\right)(x) \varphi_{\beta}\left(\mu\left(v_{\beta}^{-1}(x)\right)\right) d x \\
& =\sum_{\beta} \int_{V_{\beta}} g_{\beta} \mu . \quad \square
\end{aligned}
$$


Remark. If $\mu \in \Gamma(\operatorname{Vol}(M))$ is an arbitrary section and $f \in C_{c}^{\infty}(M)$ is a function with compact support, then we may define the integral of $f$ with respect to $\mu$ by $\int_{M} f \mu$, since $f \mu$ is a density with compact support. In this way $\mu$ defines a Radon measure on $M$.

For the converse we note first that ( $C^{1}$ suffices) diffeomorphisms between open subsets on $\mathbb{R}^{m}$ map sets of Lebesque measure zero to sets of Lebesque measure zero. Thus on a manifold we have a well defined notion of sets of Lebesque measure zero - but no measure. If $\nu$ is a Radon measure on $M$ which is absolutely continuous, i.e., the $|\nu|$-measure of a set of Lebesque measure zero is zero, then it is given by a uniquely determined measurable section of the line bundle Vol. Here a section is called measurable if in any line bundle chart it is given by a measurable function.

10.4. $p$-densities. For $0 \leq p \leq 1$ let $\operatorname{Vol}^{p}(M)$ be the line bundle defined by the cocycle of transition functions

$$
\begin{gathered}
\psi_{\alpha \beta}^{p}: U_{\alpha \beta} \rightarrow \mathbb{R} \backslash\{0\}, \\
\psi_{\alpha \beta}^{p}(x)=\left|\operatorname{det} d\left(u_{\alpha} \circ u_{\beta}^{-1}\right)\left(u_{\beta}(x)\right)\right|^{-p} .
\end{gathered}
$$

This is also a trivial line bundle. Its sections are called $p$-densities. Note that 1-densities are just densities and that 0-densities are functions. If $\mu$ is a $p$-density and $\nu$ is a $q$-density with $p+q \leq 1$, then $\mu . \nu:=\mu \otimes \nu$ is a $p+q$-density, i.e., $\operatorname{Vol}^{p}(M) \otimes \operatorname{Vol}^{q}(M)=\mathrm{Vol}^{p+q}(M)$. Thus the product of two $\frac{1}{2}$-densities with compact support can be integrated, so $\Gamma_{c}\left(\operatorname{Vol}^{1 / 2}(M)\right)$ is a pre-Hilbert space in a natural way.

Distributions on $M$ (in the sense of generalized functions) are elements of the dual space of the space $\Gamma_{c}(\operatorname{Vol}(M))$ of densities with compact support equipped with the inductive limit topology — so they contain functions.

10.5. Example. The density of a Riemann metric. Let $g$ be a Riemann metric on a manifold $M$; see section (22) below. So $g$ is a symmetric $\left(\begin{array}{l}0 \\ 2\end{array}\right)$-tensor field such that $g_{x}$ is a positive definite inner product on $T_{x} M$ for each $x \in M$. If $(U, u)$ is a chart on $M$, then we have

$$
g \mid U=\sum_{i, j=1}^{m} g_{i j}^{u} d u^{i} \otimes d u^{j}
$$

where the functions $g_{i j}^{u}=g\left(\frac{\partial}{\partial u^{i}}, \frac{\partial}{\partial u^{j}}\right)$ form a positive definite symmetric matrix. So $\operatorname{det}\left(g_{i j}^{u}\right)=\operatorname{det}\left(\left(g\left(\frac{\partial}{\partial u^{i}}, \frac{\partial}{\partial u^{j}}\right)\right)_{i, j=1}^{m}\right)>0$. We put

$$
\operatorname{vol}(g)^{u}:=\sqrt{\operatorname{det}\left(\left(g\left(\frac{\partial}{\partial u^{i}}, \frac{\partial}{\partial u^{j}}\right)\right)_{i, j=1}^{m}\right)} .
$$


If $(V, v)$ is another chart, we have

$$
\begin{aligned}
\operatorname{vol}(g)^{u} & =\sqrt{\operatorname{det}\left(\left(g\left(\frac{\partial}{\partial u^{i}}, \frac{\partial}{\partial u^{j}}\right)\right)_{i, j=1}^{m}\right)} \\
& =\sqrt{\operatorname{det}\left(\left(g\left(\sum_{k} \frac{\partial v^{k}}{\partial u^{i}} \frac{\partial}{\partial v^{k}}, \sum_{\ell} \frac{\partial v^{\ell}}{\partial u^{j}} \frac{\partial}{\partial v^{\ell}}\right)\right)_{i, j=1}^{m}\right)} \\
& =\sqrt{\operatorname{det}\left(\left(\frac{\partial v^{k}}{\partial u^{i}}\right)_{k, i}\right)^{2} \operatorname{det}\left(\left(g\left(\frac{\partial}{\partial v^{\ell}}, \frac{\partial}{\partial v^{j}}\right)\right)_{\ell, j}\right)} \\
& =\left|\operatorname{det} d\left(v \circ u^{-1}\right)\right| \operatorname{vol}(g)^{v},
\end{aligned}
$$

so these local representatives determine a section $\operatorname{vol}(g) \in \Gamma(\operatorname{Vol}(M))$, which is called the density or volume of the Riemann metric $\mathrm{g}$. If $M$ is compact, then $\int_{M} \operatorname{vol}(g)$ is called the volume of the Riemann manifold $(M, g)$.

10.6. The orientation bundle. For a manifold $M$ with $\operatorname{dim} M=m$ and an atlas $\left(U_{\alpha}, u_{\alpha}\right)$ for $M$ the line bundle $\bigwedge^{m} T^{*} M$ is given by the cocycle of transition functions

$$
\varphi_{\alpha \beta}(x)=\operatorname{det} d\left(u_{\beta} \circ u_{\alpha}^{-1}\right)\left(u_{\alpha}(x)\right)=\bigwedge^{m} d\left(u_{\beta} \circ u_{\alpha}^{-1}\right)\left(u_{\alpha}(x)\right) .
$$

We consider the line bundle $\operatorname{Or}(M)$ which is given by the cocycle of transition functions

$$
\tau_{\alpha \beta}(x)=\operatorname{sign} \varphi_{\alpha \beta}(x)=\operatorname{sign} \operatorname{det} d\left(u_{\beta} \circ u_{\alpha}^{-1}\right)\left(u_{\alpha}(x)\right) .
$$

Since $\tau_{\alpha \beta}(x) \varphi_{\alpha \beta}(x)=\psi_{\alpha \beta}(x)$, the cocycle of the volume bundle of (10.2), we have

$$
\begin{aligned}
& \operatorname{Vol}(M)=\operatorname{Or}(M) \otimes \bigwedge^{m} T^{*} M, \\
& \bigwedge^{m} T^{*} M=\operatorname{Or}(M) \otimes \operatorname{Vol}(M) .
\end{aligned}
$$

10.7. Definition. A manifold $M$ is called orientable if the orientation bundle $\operatorname{Or}(M)$ is trivial. Obviously this is the case if and only if there exists an atlas $\left(U_{\alpha}, u_{\alpha}\right)$ for the smooth structure of $M$ such that $\operatorname{det} d\left(u_{\alpha} \circ\right.$ $\left.u_{\beta}^{-1}\right)\left(u_{\beta}(x)\right)>0$ for all $x \in U_{\alpha \beta}$.

Since the transition functions of $\operatorname{Or}(M)$ take only the values +1 and -1 , there is a well defined notion of a fiberwise absolute value on $\operatorname{Or}(M)$, given by $|s(x)|:=p r_{2} \tau_{\alpha}(s(x))$, where $\left(U_{\alpha}, \tau_{\alpha}\right)$ is a vector bundle chart of $\operatorname{Or}(M)$ induced by an atlas for $M$. If $M$ is orientable, there are two distinguished global frames for the orientation bundle $\operatorname{Or}(M)$, namely those with absolute value $|s(x)|=1$.

The two normed frames $s_{1}$ and $s_{2}$ of $\operatorname{Or}(M)$ will be called the two possible orientations of the orientable manifold $M$. We call $M$ an oriented manifold if one of these two normed frames of $\operatorname{Or}(M)$ is specified: We call it $\mathfrak{o}_{M}$. 
If $M$ is oriented, then $\operatorname{Or}(M) \cong M \times \mathbb{R}$ with the help of the orientation, so we have also

$$
\bigwedge^{m} T^{*} M=\operatorname{Or}(M) \otimes \operatorname{Vol}(M)=(M \times \mathbb{R}) \otimes \operatorname{Vol}(M)=\operatorname{Vol}(M) .
$$

So an orientation gives us a canonical identification of $m$-forms and densities. Thus for any $m$-form $\omega \in \Omega^{m}(M)$ the integral $\int_{M} \omega$ is defined by the isomorphism above as the integral of the associated density; see (10.3). If $\left(U_{\alpha}, u_{\alpha}\right)$ is an oriented atlas (i.e., in each induced vector bundle chart $\left(U_{\alpha}, \tau_{\alpha}\right)$ for $\operatorname{Or}(M)$ we have $\left.\tau_{\alpha}\left(\mathfrak{o}_{M}\right)=1\right)$, then the integral of the $m$-form $\omega$ is given by

$$
\begin{aligned}
\int_{M} \omega & =\sum_{\alpha} \int_{U_{\alpha}} f_{\alpha} \omega:=\sum_{\alpha} \int_{U_{\alpha}} f_{\alpha} \cdot \omega^{\alpha} d u^{1} \wedge \cdots \wedge d u^{m} \\
& :=\sum_{\alpha} \int_{u_{\alpha}\left(U_{\alpha}\right)} f_{\alpha}\left(u_{\alpha}^{-1}(y)\right) \cdot \omega^{\alpha}\left(u_{\alpha}^{-1}(y)\right) d y^{1} \wedge \cdots \wedge d y^{m},
\end{aligned}
$$

where the last integral has to be interpreted as an oriented integral on an open subset in $\mathbb{R}^{m}$.

10.8. Manifolds with boundary. A manifold with boundary $M$ is a second countable metrizable topological space together with an equivalence class of smooth atlases $\left(U_{\alpha}, u_{\alpha}\right)$ which consist of charts with boundary: So $u_{\alpha}: U_{\alpha} \rightarrow u_{\alpha}\left(U_{\alpha}\right)$ is a homeomorphism from $U_{\alpha}$ onto an open subset of a half-space

$$
(-\infty, 0] \times \mathbb{R}^{m-1}=\left\{\left(x_{1}, \ldots, x_{m}\right): x_{1} \leq 0\right\},
$$

and all chart changes $u_{\alpha \beta}: u_{\beta}\left(U_{\alpha} \cap U_{\beta}\right) \rightarrow u_{\alpha}\left(U_{\alpha} \cap U_{\beta}\right)$ are smooth in the sense that they are restrictions of smooth mappings defined on open (in $\mathbb{R}^{m}$ ) neighborhoods of the respective domains. There is a more intrinsic treatment of this notion of smoothness by means of Whitney jets, [227, [221, and for the case of half-spaces and quadrants as here, 205.

We have $u_{\alpha \beta}\left(u_{\beta}\left(U_{\alpha} \cap U_{\beta}\right) \cap\left(0 \times \mathbb{R}^{m-1}\right)\right)=u_{\alpha}\left(U_{\alpha} \cap U_{\beta}\right) \cap\left(0 \times \mathbb{R}^{m-1}\right)$ since interior points (with respect to $\mathbb{R}^{m}$ ) are mapped to interior points by the inverse function theorem.

Thus the boundary of $M$, denoted by $\partial M$, is uniquely given as the set of all points $x \in M$ such that $u_{\alpha}(x) \in 0 \times \mathbb{R}^{m-1}$ for one (equivalently any) chart $\left(U_{\alpha}, u_{\alpha}\right)$ of $M$. Obviously the boundary $\partial M$ is itself a smooth manifold of dimension $m-1$.

A simple example: The closed unit ball $B^{m}=\left\{x \in \mathbb{R}^{m}:|x| \leq 1\right\}$ is a manifold with boundary; its boundary is $\partial B^{m}=S^{m-1}$.

The notions of smooth functions, smooth mappings, tangent bundle (use the approach (1.9) without any change in notation) are analogous to the usual 
ones. If $x \in \partial M$, we may distinguish in $T_{x} M$ tangent vectors pointing into the interior, pointing into the exterior, and those in $T_{x}(\partial M)$.

10.9. Lemma. Let $M$ be a manifold with boundary of dimension $m$. Then $M$ is a submanifold with boundary of an $m$-dimensional manifold $\tilde{M}$ without boundary.

Proof. Using partitions of unity, we construct a vector field $X$ on $M$ which points strictly into the interior of $M$. We may multiply $X$ by a strictly positive function so that the flow $\mathrm{Fl}_{t}^{X}$ exists for all $0 \leq t<2 \varepsilon$ for some $\varepsilon>0$. Then $\mathrm{Fl}_{\varepsilon}^{X}: M \rightarrow M \backslash \partial M$ is a diffeomorphism onto its image which embeds $M$ as a submanifold with boundary of $M \backslash \partial M$.

10.10. Lemma. Let $M$ be an oriented manifold with boundary. Then there is a canonical induced orientation on the boundary $\partial M$.

Proof. Let $\left(U_{\alpha}, u_{\alpha}\right)$ be an oriented atlas for $M$. Then the chart changes respect the boundary,

$$
u_{\alpha \beta}: u_{\beta}\left(U_{\alpha \beta} \cap \partial M\right) \rightarrow u_{\alpha}\left(U_{\alpha \beta} \cap \partial M\right) .
$$

Thus for $x \in u_{\beta}\left(U_{\alpha \beta} \cap \partial M\right)$ we have $d u_{\alpha \beta}(x): 0 \times \mathbb{R}^{m-1} \rightarrow 0 \times \mathbb{R}^{m-1}$,

$$
d u_{\alpha \beta}(x)=\left(\begin{array}{cccc}
\lambda & 0 & \ldots & 0 \\
* & & * &
\end{array}\right),
$$

where $\lambda>0$ since $d u_{\alpha \beta}(x)\left(-e_{1}\right)$ is again pointing downwards. So

$$
\operatorname{det} d u_{\alpha \beta}(x)=\lambda \operatorname{det}\left(d u_{\alpha \beta}(x) \mid 0 \times \mathbb{R}^{m-1}\right)>0 ;
$$

consequently $\operatorname{det}\left(d u_{\alpha \beta}(x) \mid 0 \times \mathbb{R}^{m-1}\right)>0$ and the restriction of the atlas $\left(U_{\alpha}, u_{\alpha}\right)$ is an oriented atlas for $\partial M$.

10.11. Theorem of Stokes. Let $M$ be an m-dimensional oriented manifold with boundary $\partial M$. Then for any $(m-1)$-form $\omega \in \Omega_{c}^{m-1}(M)$ with compact support on $M$ we have

$$
\int_{M} d \omega=\int_{\partial M} i^{*} \omega=\int_{\partial M} \omega
$$

where $i: \partial M \rightarrow M$ is the embedding.

Proof. Clearly $d \omega$ again has compact support. Let $\left(U_{\alpha}, u_{\alpha}\right)$ be an oriented smooth atlas for $M$ and let $\left(f_{\alpha}\right)$ be a smooth partition of unity with $\operatorname{supp}\left(f_{\alpha}\right) \subset U_{\alpha}$. Then we have $\sum_{\alpha} f_{\alpha} \omega=\omega$ and $\sum_{\alpha} d\left(f_{\alpha} \omega\right)=d \omega$. Consequently

$$
\int_{M} d \omega=\sum_{\alpha} \int_{U_{\alpha}} d\left(f_{\alpha} \omega\right) \quad \text { and } \quad \int_{\partial M} \omega=\sum_{\alpha} \int_{\partial U_{\alpha}} f_{\alpha} \omega .
$$


It suffices to show that for each $\alpha$ we have

$$
\int_{U_{\alpha}} d\left(f_{\alpha} \omega\right)=\int_{\partial U_{\alpha}} f_{\alpha} \omega
$$

For simplicity's sake we now omit the index $\alpha$. The form $f \omega$ has compact support in $U$ and we have in turn

$$
\begin{aligned}
f \omega & =\sum_{k=1}^{m} \omega_{k} d u^{1} \wedge \cdots \wedge \widehat{d u^{k}} \cdots \wedge d u^{m} \\
d(f \omega) & =\sum_{k=1}^{m} \frac{\partial \omega_{k}}{\partial u^{k}} d u^{k} \wedge d u^{1} \wedge \cdots \wedge \widehat{d u^{k}} \cdots \wedge d u^{m} \\
& =\sum_{k=1}^{m}(-1)^{k-1} \frac{\partial \omega_{k}}{\partial u^{k}} d u^{1} \wedge \cdots \wedge d u^{m} .
\end{aligned}
$$

Since $i^{*} d u^{1}=0$, we have $f \omega \mid \partial U=i^{*}(f \omega)=\omega_{1} d u^{2} \wedge \cdots \wedge d u^{m}$, where $i: \partial U \rightarrow U$ is the embedding. Finally we get

$$
\begin{aligned}
\int_{U} d(f \omega)= & \int_{U} \sum_{k=1}^{m}(-1)^{k-1} \frac{\partial \omega_{k}}{\partial u^{k}} d u^{1} \wedge \ldots \wedge d u^{m} \\
= & \sum_{k=1}^{m}(-1)^{k-1} \int_{U} \frac{\partial \omega_{k}}{\partial u^{k}} d u^{1} \wedge \ldots \wedge d u^{m} \\
= & \sum_{k=1}^{m}(-1)^{k-1} \int_{u(U)} \frac{\partial \omega_{k}}{\partial x^{k}} d x^{1} \wedge \ldots \wedge d x^{m} \\
= & \int_{\mathbb{R}^{m-1}}\left(\int_{-\infty}^{0} \frac{\partial \omega_{1}}{\partial x^{1}} d x^{1}\right) d x^{2} \ldots d x^{m} \\
& +\sum_{k=2}^{m}(-1)^{k-1} \int_{(-\infty, 0] \times \mathbb{R}^{m-2}}\left(\int_{-\infty}^{\infty} \frac{\partial \omega_{k}}{\partial x^{k}} d x^{k}\right) d x^{1} \ldots \widehat{d x^{k}} \ldots d x^{m} \\
= & \int_{\mathbb{R}^{m-1}}\left(\omega_{1}\left(0, x^{2}, \ldots, x^{m}\right)-0\right) d x^{2} \ldots d x^{m} \\
= & \int_{\partial U}\left(\omega_{1} \mid \partial U\right) d u^{2} \ldots d u^{m}=\int_{\partial U} f \omega .
\end{aligned}
$$

We used the fundamental theorem of calculus twice,

$$
\int_{-\infty}^{0} \frac{\partial \omega_{1}}{\partial x^{1}} d x^{1}=\omega_{1}\left(0, x^{2}, \ldots, x^{m}\right)-0, \quad \int_{-\infty}^{\infty} \frac{\partial \omega_{k}}{\partial x^{k}} d x^{k}=0
$$

which holds since $f \omega$ has compact support in $U$. 


\section{De Rham Cohomology}

11.1. De Rham cohomology. Let $M$ be a smooth manifold which may have boundary. We consider the graded algebra $\Omega(M)=\bigoplus_{k=0}^{\operatorname{dim} M} \Omega^{k}(M)$ of all differential forms on $M$. The space $Z(M):=\{\omega \in \Omega(M): d \omega=0\}$ of closed forms is a graded subalgebra of $\Omega$, i.e., it is a subalgebra and satisfies $Z(M)=\bigoplus_{k=0}^{\operatorname{dim} M}\left(\Omega^{k}(M) \cap Z(M)\right)=\bigoplus_{k=0}^{\operatorname{dim} M} Z^{k}(M)$. The space $B(M):=\{d \varphi: \varphi \in \Omega(M)\}$ of exact forms is a graded ideal in $Z(M)$ : $B(M) \wedge Z(M) \subset B(M)$. This follows directly from the derivation property $d(\varphi \wedge \psi)=d \varphi \wedge \psi+(-1)^{\operatorname{deg} \varphi} \varphi \wedge d \psi$ of the exterior derivative.

Definition. The algebra

$$
H^{*}(M):=\frac{Z(M)}{B(M)}=\frac{\{\omega \in \Omega(M): d \omega=0\}}{\{d \varphi: \varphi \in \Omega(M)\}}
$$

is called the de Rham cohomology algebra of the manifold $M$. It is graded by

$$
H^{*}(M)=\bigoplus_{k=0}^{\operatorname{dim} M} H^{k}(M)=\bigoplus_{k=0}^{\operatorname{dim} M} \frac{\operatorname{ker}\left(d: \Omega^{k}(M) \rightarrow \Omega^{k+1}(M)\right)}{\operatorname{im} d: \Omega^{k-1}(M) \rightarrow \Omega^{k}(M)} .
$$

If $f: M \rightarrow N$ is a smooth mapping between manifolds, then $f^{*}: \Omega(N) \rightarrow$ $\Omega(M)$ is a homomorphism of graded algebras by (9.5) which satisfies $d \circ f^{*}=$ $f^{*} \circ d$ by (9.9). Thus $f^{*}$ induces an algebra homomorphism which we again call $f^{*}: H^{*}(N) \rightarrow H^{*}(M)$.

11.2. Remark. Since $\Omega^{k}(M)=0$ for $k>\operatorname{dim} M=: m$, we have

$$
\begin{aligned}
H^{m}(M) & =\frac{\Omega^{m}(M)}{\left\{d \varphi: \varphi \in \Omega^{m-1}(M)\right\}}, \\
H^{k}(M) & =0 \quad \text { for } k>m, \\
H^{0}(M) & =\frac{\left\{f \in \Omega^{0}(M)=C^{\infty}(M): d f=0\right\}}{0} \\
& =\text { the space of locally constant functions on } M \\
& =\mathbb{R}^{b_{0}(M)},
\end{aligned}
$$

where $b_{0}(M)$ is the number of pathwise connected components of $M$. We put $b_{k}(M):=\operatorname{dim}_{\mathbb{R}} H^{k}(M)$ and call it the $k$-th Betti number of $M$. If $b_{k}(M)<\infty$ for all $k$, we put

$$
f_{M}(t):=\sum_{k=0}^{m} b_{k}(M) t^{k}
$$


and call it the Poincaré polynomial of $M$. The number

$$
\chi_{M}:=\sum_{k=0}^{m} b_{k}(M)(-1)^{k}=f_{M}(-1)
$$

is called the Euler-Poincaré characteristic of $M$; see also (13.7) below.

11.3. Examples. We have $H^{0}\left(\mathbb{R}^{m}\right)=\mathbb{R}$ since it has only one connected component. We have $H^{k}\left(\mathbb{R}^{m}\right)=0$ for $k>0$ by the proof of the lemma of Poincaré (9.10),

For the 1-dimensional sphere we have $H^{0}\left(S^{1}\right)=\mathbb{R}$ since it is connected, and clearly $H^{k}\left(S^{1}\right)=0$ for $k>1$ by reasons of dimension. Also, we have

$$
\begin{aligned}
H^{1}\left(S^{1}\right) & =\frac{\left\{\omega \in \Omega^{1}\left(S^{1}\right): d \omega=0\right\}}{\left\{d \varphi: \varphi \in \Omega^{0}\left(S^{1}\right)\right\}} \\
& =\frac{\Omega^{1}\left(S^{1}\right)}{\left\{d f: f \in C^{\infty}\left(S^{1}\right)\right\}}, \\
\Omega^{1}\left(S^{1}\right) & =\left\{f d \vartheta: f \in C^{\infty}\left(S^{1}\right)\right\} \\
& \cong\left\{f \in C^{\infty}(\mathbb{R}): f \text { is periodic with period } 2 \pi\right\},
\end{aligned}
$$

where $d \vartheta$ denotes the global coframe of $T^{*} S^{1}$. If $f \in C^{\infty}(\mathbb{R})$ is periodic with period $2 \pi$, then $f d t$ is exact if and only if $\int f d t$ is also $2 \pi$ periodic, i.e., $\int_{0}^{2 \pi} f(t) d t=0$. So we have

$$
H^{1}\left(S^{1}\right)=\frac{\left\{f \in C^{\infty}(\mathbb{R}): f \text { is periodic with period } 2 \pi\right\}}{\left\{f \in C^{\infty}(\mathbb{R}): f \text { is periodic with period } 2 \pi, \int_{0}^{2 \pi} f d t=0\right\}}=\mathbb{R},
$$

where $f \mapsto \int_{0}^{2 \pi} f d t$ factors to the isomorphism.

11.4. Lemma. Let $f, g: M \rightarrow N$ be smooth mappings between manifolds which are $C^{\infty}$-homotopic: There exists $h \in C^{\infty}(\mathbb{R} \times M, N)$ with $h(0, x)=f(x)$ and $h(1, x)=g(x)$. Then $f$ and $g$ induce the same mapping in cohomology:

$$
f^{*}=g^{*}: H(N) \rightarrow H(M) .
$$

Remark. $f, g \in C^{\infty}(M, N)$ are called homotopic if there exists a continuous mapping $h:[0,1] \times M \rightarrow N$ with $h(0, x)=f(x)$ and $h(1, x)=g(x)$. This seemingly looser relation in fact coincides with the relation of $C^{\infty}$-homotopy. We sketch a proof of this statement: Let $\varphi: \mathbb{R} \rightarrow[0,1]$ be a smooth function with $\varphi((-\infty, 1 / 4])=0$ and $\varphi([3 / 4, \infty))=1$, and with $\varphi$ monotone in between. Then consider $\bar{h}: \mathbb{R} \times M \rightarrow N$, given by $\bar{h}(t, x)=h(\varphi(t), x)$. Now we may approximate $\bar{h}$ by smooth functions $\tilde{h}: \mathbb{R} \times M \rightarrow N$ without changing it on $(-\infty, 1 / 8) \times M$ where it equals $f$ and on $(7 / 8, \infty) \times M$ where it equals $g$. This is done chartwise by convolution with a smooth function with small 
support on $\mathbb{R}^{m}$. See [26] for a careful presentation of the approximation. So we will use the equivalent concept of homotopic mappings below.

Proof. For $\omega \in \Omega^{k}(N)$ we have $h^{*} \omega \in \Omega^{k}(\mathbb{R} \times M)$. We consider the insertion operator ins $t: M \rightarrow \mathbb{R} \times M$, given by $\operatorname{ins}_{t}(x)=(t, x)$. For $\varphi \in \Omega^{k}(\mathbb{R} \times M)$ we then have a smooth curve $t \mapsto \operatorname{ins}_{t}^{*} h^{*} \varphi$ in $\Omega^{k}(M)$ (this can be made precise with the help of the calculus in infinite dimensions of [64]). We define the integral operator $I_{0}^{1}: \Omega^{k}(\mathbb{R} \times M) \rightarrow \Omega^{k}(M)$ by $I_{0}^{1}(\varphi):=\int_{0}^{1} \operatorname{ins}_{t}^{*} \varphi d t$. Looking at this locally on $M$, one sees that it is well defined, even without infinite-dimensional calculus. Let $T:=\frac{\partial}{\partial t} \in \mathfrak{X}(\mathbb{R} \times M)$ be the unit vector field in direction $\mathbb{R}$.

We have $\operatorname{ins}_{t+s}=\mathrm{Fl}_{t}^{T} \circ$ ins $_{s}$ for $s, t \in \mathbb{R}$, so

$$
\begin{aligned}
\frac{\partial}{\partial s} \operatorname{ins}_{s}^{*} \varphi & =\left.\partial\right|_{0}\left(\mathrm{Fl}_{t}^{T} \circ \operatorname{ins}_{s}\right)^{*} \varphi=\left.\partial\right|_{0} \operatorname{ins}_{s}^{*}\left(\mathrm{Fl}_{t}^{T}\right)^{*} \varphi \\
& =\left.\operatorname{ins}_{s}^{*} \partial\right|_{0}\left(\mathrm{Fl}_{t}^{T}\right)^{*} \varphi=\left(\mathrm{ins}_{s}\right)^{*} \mathcal{L}_{T} \varphi \quad \text { by }(9.6) .
\end{aligned}
$$

We have used that $\left(\text { ins }_{s}\right)^{*}: \Omega^{k}(\mathbb{R} \times M) \rightarrow \Omega^{k}(M)$ is linear and continuous and so one may differentiate through it by the chain rule. This can also be checked by evaluating at $x \in M$. Then we have in turn

$$
\begin{aligned}
d I_{0}^{1} \varphi & =d \int_{0}^{1} \operatorname{ins}_{t}^{*} \varphi d t=\int_{0}^{1} d \operatorname{ins}_{t}^{*} \varphi d t \\
& =\int_{0}^{1} \operatorname{ins}_{t}^{*} d \varphi d t=I_{0}^{1} d \varphi \quad \text { by (9.9.4), } \\
\left(\mathrm{ins}_{1}^{*}-\operatorname{ins}_{0}^{*}\right) \varphi & =\int_{0}^{1} \frac{\partial}{\partial t} \operatorname{ins}_{t}^{*} \varphi d t=\int_{0}^{1} \operatorname{ins}_{t}^{*} \mathcal{L}_{T} \varphi d t \\
& =I_{0}^{1} \mathcal{L}_{T} \varphi=I_{0}^{1}\left(d i_{T}+i_{T} d\right) \varphi \quad \text { by }(9.9)
\end{aligned}
$$

Now we define the homotopy operator $\bar{h}:=I_{0}^{1} \circ i_{T} \circ h^{*}: \Omega^{k}(N) \rightarrow \Omega^{k-1}(M)$. Then we get

$$
\begin{aligned}
g^{*}-f^{*} & =\left(h \circ \operatorname{ins}_{1}\right)^{*}-\left(h \circ \operatorname{ins}_{0}\right)^{*}=\left(\operatorname{ins}_{1}^{*}-\operatorname{ins}_{0}^{*}\right) \circ h^{*} \\
& =\left(d \circ I_{0}^{1} \circ i_{T}+I_{0}^{1} \circ i_{T} \circ d\right) \circ h^{*}=d \circ \bar{h}-\bar{h} \circ d,
\end{aligned}
$$

which implies the desired result since for $\omega \in \Omega^{k}(M)$ with $d \omega=0$ we have $g^{*} \omega-f^{*} \omega=\bar{h} d \omega+d \bar{h} \omega=d \bar{h} \omega$.

11.5. Lemma. If a manifold is decomposed into a disjoint union $M=$ $\bigsqcup_{\alpha} M_{\alpha}$ of open submanifolds, then $H^{k}(M)=\prod_{\alpha} H^{k}\left(M_{\alpha}\right)$ for all $k$.

Proof. $\Omega^{k}(M)$ is isomorphic to $\prod_{\alpha} \Omega^{k}\left(M_{\alpha}\right)$ via $\varphi \mapsto\left(\varphi \mid M_{\alpha}\right)_{\alpha}$. This isomorphism commutes with exterior differential $d$ and induces the result. 
11.6. The setting for the Mayer-Vietoris sequence. Let $M$ be a smooth manifold, and let $U, V \subset M$ be open subsets such that $M=U \cup V$. We consider the following embeddings:

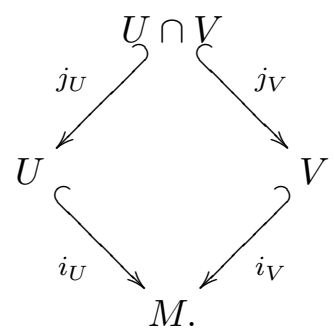

Lemma. In this situation the sequence

$$
0 \rightarrow \Omega(M) \stackrel{\alpha}{\longrightarrow} \Omega(U) \oplus \Omega(V) \stackrel{\beta}{\longrightarrow} \Omega(U \cap V) \rightarrow 0
$$

is exact, where $\alpha(\omega):=\left(i_{U}^{*} \omega, i_{V}^{*} \omega\right)$ and $\beta(\varphi, \psi)=j_{U}^{*} \varphi-j_{V}^{*} \psi$. We also have $(d \oplus d) \circ \alpha=\alpha \circ d$ and $d \circ \beta=\beta \circ(d \oplus d)$.

Proof. We have to show that $\alpha$ is injective, $\operatorname{ker} \beta=\operatorname{im} \alpha$, and that $\beta$ is surjective. The first two assertions are obvious and for the last one we let $\left\{f_{U}, f_{V}\right\}$ be a partition of unity with supp $f_{U} \subset U$ and $\operatorname{supp} f_{V} \subset V$. For $\varphi \in \Omega(U \cap V)$ we consider $f_{V} \varphi \in \Omega(U \cap V)$; note that $\operatorname{supp}\left(f_{V} \varphi\right)$ is closed in the set $U \cap V$ which is open in $U$, so we may extend $f_{V} \varphi$ by 0 to $\varphi_{U} \in \Omega(U)$. Likewise we extend $-f_{U} \varphi$ by 0 to $\varphi_{V} \in \Omega(V)$. Then we have $\beta\left(\varphi_{U}, \varphi_{V}\right)=\left(f_{U}+f_{V}\right) \varphi=\varphi$.

Now we are in the situation where we may apply the main theorem of homological algebra, (11.8). So we deviate now to develop the basics of homological algebra.

11.7. The essentials of homological algebra. A graded differential space $(\mathrm{GDS}) K=(K, d)$ is a sequence

$$
\cdots \rightarrow K^{n-1} \stackrel{d^{n-1}}{\longrightarrow} K^{n} \stackrel{d^{n}}{\longrightarrow} K^{n+1} \rightarrow \cdots
$$

of abelian groups $K^{n}$ and group homomorphisms $d^{n}: K^{n} \rightarrow K^{n+1}$ such that $d^{n+1} \circ d^{n}=0$. In our case these are the vector spaces $K^{n}=\Omega^{n}(M)$ and the exterior derivative. The group

$$
H^{n}(K):=\frac{\operatorname{ker}\left(d^{n}: K^{n} \rightarrow K^{n+1}\right)}{\operatorname{im}\left(d^{n-1}: K^{n-1} \rightarrow K^{n}\right)}
$$

is called the $n$-th cohomology group of the GDS $K$. We consider also the direct sum

$$
H^{*}(K):=\bigoplus_{n=-\infty}^{\infty} H^{n}(K)
$$


as a graded group. A homomorphism $f: K \rightarrow L$ of graded differential spaces is a sequence of homomorphisms $f^{n}: K^{n} \rightarrow L^{n}$ such that $d^{n} \circ f^{n}=f^{n+1} \circ d^{n}$. It induces a homomorphism

$$
f_{*}=H^{*}(f): H^{*}(K) \rightarrow H^{*}(L)
$$

and $H^{*}$ has clearly the properties of a functor from the category of graded differential spaces into the category of graded groups:

$$
\begin{aligned}
H^{*}\left(I d_{K}\right) & =I d_{H^{*}(K)}, \\
H^{*}(f \circ g) & =H^{*}(f) \circ H^{*}(g) .
\end{aligned}
$$

A graded differential space $(K, d)$ is called a graded differential algebra if $\bigoplus_{n} K^{n}$ is an associative algebra which is graded (so $K^{n} \cdot K^{m} \subset K^{n+m}$ ), such that the differential $d$ is a graded derivation: $d(x . y)=d x \cdot y+(-1)^{\operatorname{deg} x} x \cdot d y$. The cohomology group $H^{*}(K, d)$ of a graded differential algebra is a graded algebra; see (11.1).

By a short exact sequence of graded differential spaces we mean a sequence

$$
0 \rightarrow K \stackrel{i}{\longrightarrow} L \stackrel{p}{\longrightarrow} M \rightarrow 0
$$

of homomorphisms of graded differential spaces which is degreewise exact: For each $n$ the sequence $0 \rightarrow K^{n} \rightarrow L^{n} \rightarrow M^{n} \rightarrow 0$ is exact.

11.8. Theorem. Mayer-Vietoris sequence. Let

$$
0 \rightarrow K \stackrel{i}{\longrightarrow} L \stackrel{p}{\longrightarrow} M \rightarrow 0
$$

be an exact sequence of graded differential spaces. Then there exists a graded homomorphism $\delta=\left(\delta^{n}: H^{n}(M) \rightarrow H^{n+1}(K)\right)_{n \in \mathbb{Z}}$ called the 'connecting homomorphism' such that the following is an exact sequence of abelian groups:

$$
\cdots \rightarrow H^{n-1}(M) \stackrel{\delta}{\longrightarrow} H^{n}(K) \stackrel{i_{*}}{\longrightarrow} H^{n}(L) \stackrel{p_{*}}{\longrightarrow} H^{n}(M) \stackrel{\delta}{\longrightarrow} H^{n+1}(K) \rightarrow \cdots
$$

It is called the 'long exact sequence in cohomology'. Here $\delta$ is a natural transformation in the following sense: Let

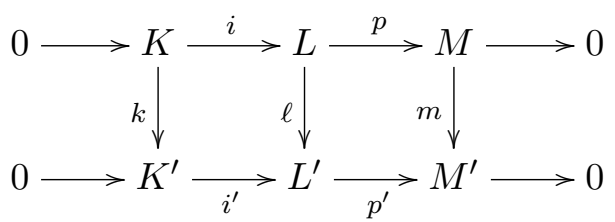


be a commutative diagram of homomorphisms of graded differential spaces with exact lines. Then also the following diagram is commutative:

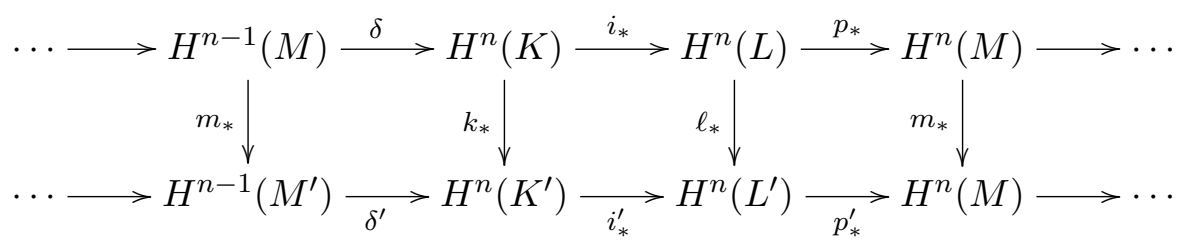

The long exact sequence in cohomology can also be written in the following way:

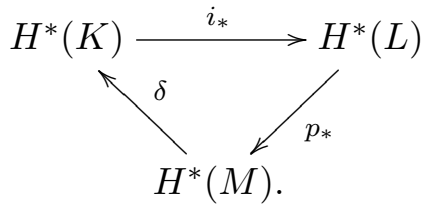

Definition of $\delta$. The connecting homomorphism is defined by ' $\delta=i^{-1}$ 。 $d \circ p^{-1}$ or $\delta[p \ell]=\left[i^{-1} d \ell\right]$. This is meant as follows:

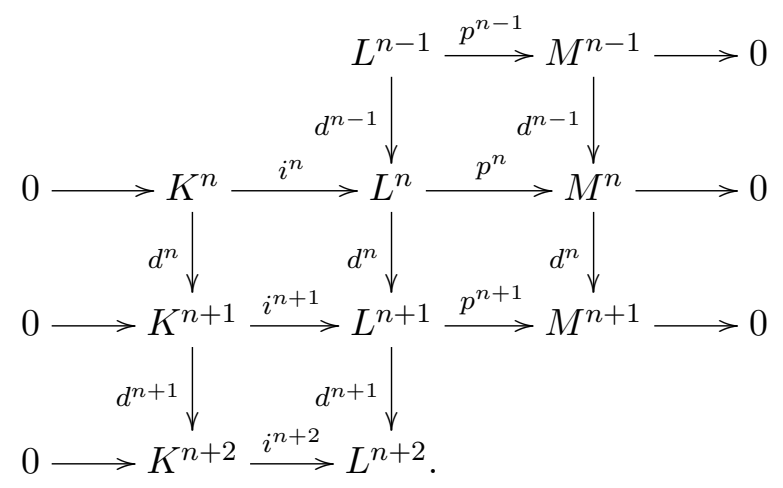

The following argument is called a diagram chase. Let $[m] \in H^{n}(M)$. Then $m \in M^{n}$ with $d m=0$. Since $p$ is surjective, there is $\ell \in L^{n}$ with $p \ell=m$. We consider $d \ell \in L^{n+1}$ for which we have $p d \ell=d p \ell=d m=0$, so $d \ell \in$ $\operatorname{ker} p=\operatorname{im} i$; thus there is an element $k \in K^{n+1}$ with $i k=d \ell$. We have $i d k=d i k=d d \ell=0$. Since $i$ is injective, we have $d k=0$, so $[k] \in H^{n+1}(K)$. Now we put $\delta[m]:=[k]$ or $\delta[p \ell]=\left[i^{-1} d \ell\right]$.

This method of diagram chasing can be used for the whole proof of the theorem. The reader is advised to do it at least once in his life with fingers on the diagram above. For the naturality imagine two copies of the diagram lying above each other with homomorphisms going up. 


\subsection{Five-lemma. Let}

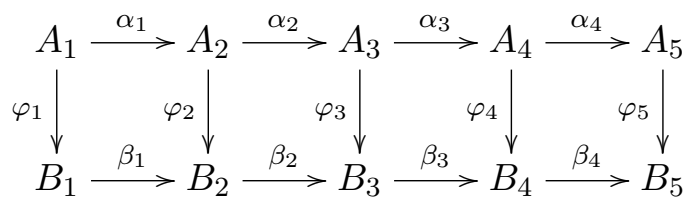

be a commutative diagram of abelian groups with exact lines. If $\varphi_{1}, \varphi_{2}, \varphi_{4}$, and $\varphi_{5}$ are isomorphisms, then also the middle $\varphi_{3}$ is an isomorphism.

Proof. Diagram chasing in this diagram leads to the result. The chase becomes simpler if one first replaces the diagram by the following equivalent one with exact lines:

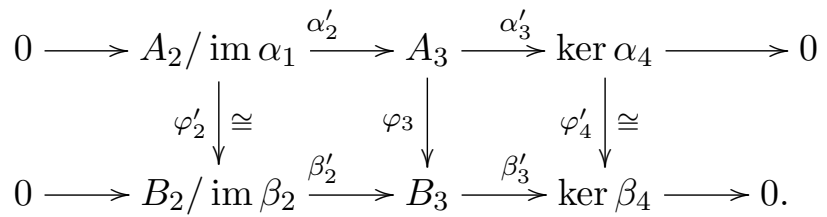

11.10. Theorem. Mayer-Vietoris sequence. Let $U$ and $V$ be open subsets in a manifold $M$ such that $M=U \cup V$. Then there is an exact sequence

$\cdots \rightarrow H^{k}(M) \stackrel{\alpha_{*}}{\longrightarrow} H^{k}(U) \oplus H^{k}(V) \stackrel{\beta_{*}}{\longrightarrow} H^{k}(U \cap V) \stackrel{\delta}{\longrightarrow} H^{k+1}(M) \rightarrow \cdots$

which is natural in the triple $(M, U, V)$ in the sense explained in (11.8). The homomorphisms $\alpha_{*}$ and $\beta_{*}$ are algebra homomorphisms, but $\delta$ is not.

Proof. This follows from (11.6) and theorem (11.8).

Since we shall need it later, we will give now a detailed description of the connecting homomorphism $\delta$. Let $\left\{f_{U}, f_{V}\right\}$ be a partition of unity with $\operatorname{supp} f_{U} \subset U$ and $\operatorname{supp} f_{V} \subset V$. Let $\omega \in \Omega^{k}(U \cap V)$ with $d \omega=0$ so that $[\omega] \in H^{k}(U \cap V)$. Then $\left(f_{V} \cdot \omega,-f_{U} \cdot \omega\right) \in \Omega^{k}(U) \oplus \Omega^{k}(V)$ is mapped to $\omega$ by $\beta$ and so we have by the description of $\delta$ in (11.8)

$$
\begin{aligned}
\delta[\omega] & =\left[\alpha^{-1} d\left(f_{V} \cdot \omega,-f_{U} \cdot \omega\right)\right]=\left[\alpha^{-1}\left(d f_{V} \wedge \omega,-d f_{U} \wedge \omega\right)\right] \\
& =\left[d f_{V} \wedge \omega\right]=-\left[d f_{U} \wedge \omega\right],
\end{aligned}
$$

where we have used the following fact: $f_{U}+f_{V}=1$ implies that on $U \cap V$ we have $d f_{V}=-d f_{U}$; thus $d f_{V} \wedge \omega=-d f_{U} \wedge \omega$ and off $U \cap V$ both are 0 .

11.11. Axioms for cohomology. The de Rham cohomology is uniquely determined by the following properties which we have already verified: 
(1) $H^{*}(\quad)$ is a contravariant functor from the category of smooth manifolds and smooth mappings into the category of $\mathbb{Z}$-graded groups and graded homomorphisms.

(2) $H^{k}$ (point) $=\mathbb{R}$ for $k=0$ and $H^{k}$ (point) $=0$ for $k \neq 0$.

(3) If $f$ and $g$ are $C^{\infty}$-homotopic, then $H^{*}(f)=H^{*}(g)$.

(4) If $M=\bigsqcup_{\alpha} M_{\alpha}$ is a disjoint union of open subsets, then $H^{*}(M)=\prod_{\alpha} H^{*}\left(M_{\alpha}\right)$.

(5) If $U$ and $V$ are open in $M$, then there exists a connecting homomorphism $\delta: H^{k}(U \cap V) \rightarrow H^{k+1}(U \cup V)$ which is natural in the triple $(U \cup V, U, V)$ such that the following sequence is exact:

$$
\cdots \rightarrow H^{k}(U \cup V) \rightarrow H^{k}(U) \oplus H^{k}(V) \rightarrow H^{k}(U \cap V) \stackrel{\delta}{\longrightarrow} H^{k+1}(U \cup V) \rightarrow \cdots
$$

There are many other cohomology theories for topological spaces, like singular cohomology, Čech cohomology, simplicial cohomology, Alexander-Spanier cohomology, etc., which satisfy the above axioms for smooth manifolds when defined with real coefficients, so they all coincide with the de Rham cohomology on manifolds. See books on algebraic topology or sheaf theory for all this and look for the abstract theorem of de Rham in sheaf cohomology.

11.12. Example. If $M$ is contractible (which is equivalent to the seemingly stronger concept of $C^{\infty}$-contractibility; see the remark in (11.4), then $H^{0}(M)=\mathbb{R}$ since $M$ is connected, and $H^{k}(M)=0$ for $k \neq 0$, because the constant mapping $c: M \rightarrow$ point $\rightarrow M$ onto some fixed point of $M$ is homotopic to $I d_{M}$, so $H^{*}(c)=H^{*}\left(I d_{M}\right)=I d_{H^{*}(M)}$ by (11.4), But we have

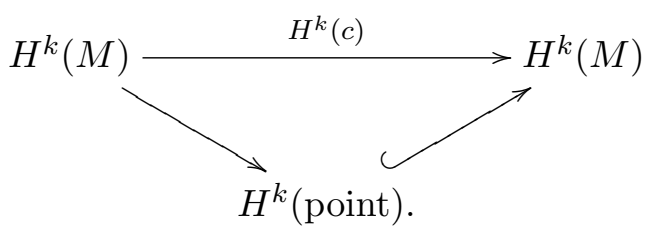

More generally, two manifolds $M$ and $N$ are called smoothly homotopy equivalent if there exist smooth mappings $f: M \rightarrow N$ and $g: N \rightarrow M$ such that $g \circ f$ is homotopic to $I d_{M}$ and $f \circ g$ is homotopic to $I d_{N}$. If this is the case, both $H^{*}(f)$ and $H^{*}(g)$ are isomorphisms, since

$$
H^{*}(g) \circ H^{*}(f)=I d_{H^{*}(M)} \quad \text { and } \quad H^{*}(f) \circ H^{*}(g)=I d_{H^{*}(N)} .
$$

As an example consider a vector bundle $(E, p, M)$ with zero section $0_{E}$ : $M \rightarrow E$. Then $p \circ 0_{E}=I d_{M}$ whereas $0_{E} \circ p$ is homotopic to $I d_{E}$ via $(t, u) \mapsto t$.u. Thus $H^{*}(E)$ is isomorphic to $H^{*}(M)$. 
11.13. Example. The cohomology of spheres. For $n \geq 1$ we have

$$
H^{k}\left(S^{n}\right)=\left\{\begin{array}{ll}
\mathbb{R} & \text { for } k=0, \\
0 & \text { for } 1 \leq k \leq n-1, \\
\mathbb{R} & \text { for } k=n, \\
0 & \text { for } k>n,
\end{array} \quad H^{k}\left(S^{0}\right)= \begin{cases}\mathbb{R}^{2} & \text { for } k=0, \\
0 & \text { for } k>0 .\end{cases}\right.
$$

We may say: The cohomology of $S^{n}$ has two generators as a graded vector space, one in dimension 0 and one in dimension n. The Poincaré polynomial is given by $f_{S^{n}}(t)=1+t^{n}$.

Proof. The assertion for $S^{0}$ is obvious, and for $S^{1}$ it was proved in (11.3) so let $n \geq 2$. Then $H^{0}\left(S^{n}\right)=\mathbb{R}$ since it is connected, so let $k>0$. Now fix a north pole $a \in S^{n}, 0<\varepsilon<1$, and let

$$
\begin{aligned}
S^{n} & =\left\{x \in \mathbb{R}^{n+1}:|x|^{2}=\langle x, x\rangle=1\right\}, \\
U & =\left\{x \in S^{n}:\langle x, a\rangle>-\varepsilon\right\}, \\
V & =\left\{x \in S^{n}:\langle x, a\rangle<\varepsilon\right\},
\end{aligned}
$$

so $U$ and $V$ are overlapping northern and southern hemispheres, respectively, which are diffeomorphic to an open ball and thus smoothly contractible. Their cohomology is thus described in (11.12). Clearly $U \cup V=S^{n}$ and $U \cap V \cong S^{n-1} \times(-\varepsilon, \varepsilon)$ which is obviously (smoothly) homotopy equivalent to $S^{n-1}$. By theorem (11.10) we have the following part of the MayerVietoris sequence

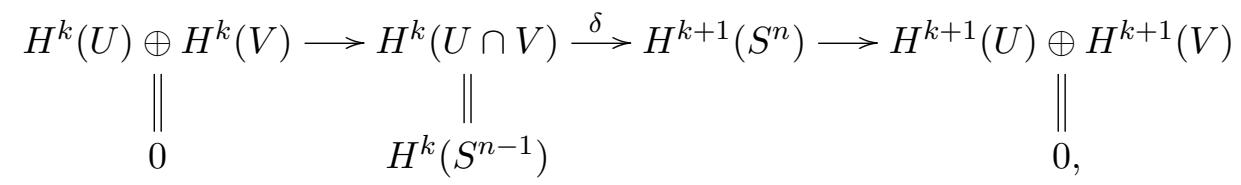

where the vertical isomorphisms are from (11.12). Thus $H^{k}\left(S^{n-1}\right)$ is isomorphic to $H^{k+1}\left(S^{n}\right)$ for $k>0$ and $n \geq 2$.

Next we look at the initial segment of the Mayer-Vietoris sequence:

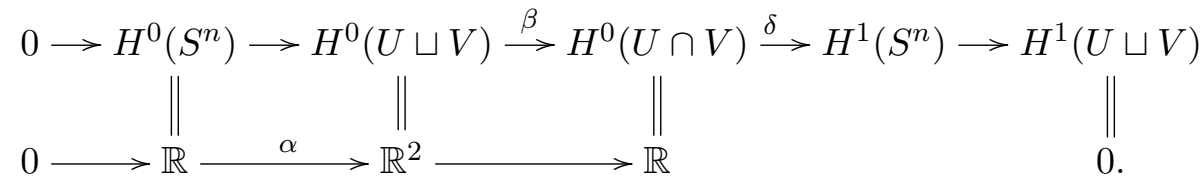

From exactness we have: In the lower line $\alpha$ is injective, so $\operatorname{dim}(\operatorname{ker} \beta)=1$, so $\beta$ is surjective and thus $\delta=0$. This implies that $H^{1}\left(S^{n}\right)=0$ for $n \geq 2$. Starting from $H^{k}\left(S^{1}\right)$ for $k>0$ the result now follows by induction on $n$.

By looking more closely on the initial segment of the Mayer-Vietoris sequence for $n=1$ and taking into account the form of $\delta: H^{0}\left(S^{0}\right) \rightarrow H^{1}\left(S^{1}\right)$, 
we could even derive the result for $S^{1}$ without using (11.3), The reader is advised to try this.

11.14. Example. The Stiefel manifold $V(k, n ; \mathbb{R})$ of oriented orthonormal $k$-frames in $\mathbb{R}^{n}$ (see (18.5) has the following Poincaré polynomial:

\begin{tabular}{|ll}
\hline For: & $f_{V(k, n)}=$ \\
$n=2 m, k=2 l+1, l \geq 0:$ & $\left(1+t^{2 m-1}\right) \prod_{i=1}^{l}\left(1+t^{4 m-4 i-1}\right)$ \\
$n=2 m+1, k=2 l, l \geq 1:$ & $\prod_{i=1}^{l}\left(1+t^{4 m-4 i+3}\right)$ \\
$n=2 m, k=2 l, m>l \geq 1:$ & $\left(1+t^{2 m-2 l}\right)\left(1+t^{2 m-1}\right) \prod_{i=1}^{l-1}\left(1+t^{4 m-4 i-1}\right)$ \\
$n=2 m+1, k=2 l+1$, & $\left(1+t^{2 m-2 l}\right) \prod_{i=1}^{l-1}\left(1+t^{4 m-4 i+3}\right)$ \\
$m>l \geq 0:$ &
\end{tabular}

Since $V(n-1, n ; \mathbb{R})=S O(n ; \mathbb{R})$, we get

$$
\begin{aligned}
& f_{S O(2 m ; \mathbb{R})}(t)=\left(1+t^{2 m-1}\right) \prod_{i=1}^{m-1}\left(1+t^{4 i-1}\right), \\
& f_{S O(2 m+1, \mathbb{R})}(t)=\prod_{i=1}^{m}\left(1+t^{4 i-1}\right) .
\end{aligned}
$$

So the cohomology can be quite complicated. For a proof of these formulas using the Gysin sequence for sphere bundles, see [80, II].

11.15. Relative de Rham cohomology. Let $N \subset M$ be a closed submanifold and let

$$
\Omega^{k}(M, N):=\left\{\omega \in \Omega^{k}(M): i^{*} \omega=0\right\},
$$

where $i: N \rightarrow M$ is the embedding. Since $i^{*} \circ d=d \circ i^{*}$, we get a graded differential subalgebra $\left(\Omega^{*}(M, N), d\right)$ of $\left(\Omega^{*}(M), d\right)$. Its cohomology, denoted by $H^{*}(M, N)$, is called the relative de Rham cohomology of the manifold pair $(M, N)$.

11.16. Lemma. In the setting of (11.15),

$$
0 \rightarrow \Omega^{*}(M, N) \hookrightarrow \Omega^{*}(M) \stackrel{i^{*}}{\longrightarrow} \Omega^{*}(N) \rightarrow 0
$$


is an exact sequence of differential graded algebras. Thus by (11.8) we have the long exact sequence in cohomology

$$
\cdots \rightarrow H^{k}(M, N) \rightarrow H^{k}(M) \rightarrow H^{k}(N) \stackrel{\delta}{\longrightarrow} H^{k+1}(M, N) \rightarrow \ldots
$$

which is natural in the manifold pair $(M, N)$. It is called the long exact cohomology sequence of the pair $(M, N)$.

Proof. We only have to show that $i^{*}: \Omega^{*}(M) \rightarrow \Omega^{*}(N)$ is surjective. So we have to extend each $\omega \in \Omega^{k}(N)$ to the whole of $M$. We cover $N$ by submanifold charts of $M$ with respect to $N$. These and $M \backslash N$ cover $M$. On each of the submanifold charts one can easily extend the restriction of $\omega$ and one can glue all these extensions by a partition of unity which is subordinated to the cover of $M$.

\section{Cohomology with Compact Supports and Poincaré Duality}

12.1. Cohomology with compact supports. Let $\Omega_{c}^{k}(M)$ denote the space of all $k$-forms with compact support on the manifold $M$. Since $\operatorname{supp}(d \omega) \subset \operatorname{supp}(\omega), \operatorname{supp}\left(\mathcal{L}_{X} \omega\right) \subset \operatorname{supp}(X) \cap \operatorname{supp}(\omega)$, and $\operatorname{supp}\left(i_{X} \omega\right) \subset$ $\operatorname{supp}(X) \cap \operatorname{supp}(\omega)$, all formulas of section $(9)$ are also valid in $\Omega_{c}^{*}(M)=$ $\bigoplus_{k=0}^{\operatorname{dim} M} \Omega_{c}^{k}(M)$. So $\Omega_{c}^{*}(M)$ is an ideal and a differential graded subalgebra of $\Omega^{*}(M)$. The cohomology of $\Omega_{c}^{*}(M)$

$$
\begin{aligned}
& H_{c}^{k}(M):=\frac{\operatorname{ker}\left(d: \Omega_{c}^{k}(M) \rightarrow \Omega_{c}^{k+1}(M)\right)}{\operatorname{im} d: \Omega_{c}^{k-1}(M) \rightarrow \Omega_{c}^{k}(M)}, \\
& H_{c}^{*}(M):=\bigoplus_{k=0}^{\operatorname{dim} M} H_{c}^{k}(M)
\end{aligned}
$$

is called the de Rham cohomology algebra with compact supports of the manifold $M$. It has no unit if $M$ is not compact.

12.2. Mappings. If $f: M \rightarrow N$ is a smooth mapping between manifolds and if $\omega \in \Omega_{c}^{k}(N)$ is a form with compact support, then $f^{*} \omega$ is a $k$-form on $M$, in general with noncompact support. So $\Omega_{c}^{*}$ is not a functor on the category of all smooth manifolds and all smooth mappings. But if we restrict the morphisms suitably, then $\Omega_{c}^{*}$ becomes a functor. There are two ways to do this:

(1) $\Omega_{c}^{*}$ is a contravariant functor on the category of all smooth manifolds and proper smooth mappings ( $f$ is called proper if $f^{-1}$ (compact set) is a compact set) by the usual pullback operation. 
(2) $\Omega_{c}^{*}$ is a covariant functor on the category of all smooth manifolds and embeddings of open submanifolds: For $i: U \hookrightarrow M$ and $\omega \in \Omega_{c}^{k}(U)$ just extend $\omega$ by 0 off $U$ to get $i_{*} \omega \in \Omega_{c}^{k}(M)$. Clearly $i_{*} \circ d=d \circ i_{*}$.

12.3. Remarks. (1) If a manifold $M$ is a disjoint union, $M=\bigsqcup_{\alpha} M_{\alpha}$, then we have obviously $H_{c}^{k}(M)=\bigoplus_{\alpha} H_{c}^{k}\left(M_{\alpha}\right)$.

(2) $H_{c}^{0}(M)$ is a direct sum of copies of $\mathbb{R}$, one for each compact connected component of $M$.

(3) If $M$ is compact, then $H_{c}^{k}(M)=H^{k}(M)$.

12.4. The Mayer-Vietoris sequence with compact supports. Let $M$ be a smooth manifold, and let $U, V \subset M$ be open subsets such that $M=U \cup V$. We consider the following embeddings:

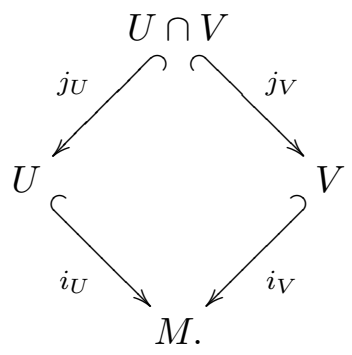

Theorem. The following sequence of graded differential algebras is exact:

$$
0 \rightarrow \Omega_{c}^{*}(U \cap V) \stackrel{\beta_{c}}{\longrightarrow} \Omega_{c}^{*}(U) \oplus \Omega_{c}^{*}(V) \stackrel{\alpha_{c}}{\longrightarrow} \Omega_{c}^{*}(M) \rightarrow 0,
$$

where $\beta_{c}(\omega):=\left(\left(j_{U}\right)_{*} \omega,\left(j_{V}\right)_{*} \omega\right)$ and $\alpha_{c}(\varphi, \psi)=\left(i_{U}\right)_{*} \varphi-\left(i_{V}\right)_{*} \psi$. So by (11.8) we have the long exact sequence

$$
\begin{aligned}
\cdots \rightarrow H_{c}^{k-1}(M) \stackrel{\delta_{c}}{\longrightarrow} H_{c}^{k}(U \cap V) & \rightarrow H_{c}^{k}(U) \oplus H_{c}^{k}(V) \rightarrow \\
& \rightarrow H_{c}^{k}(M) \stackrel{\delta_{c}}{\longrightarrow} H_{c}^{k+1}(U \cap V) \rightarrow \ldots
\end{aligned}
$$

which is natural in the triple $(M, U, V)$. It is called the Mayer Vietoris sequence with compact supports.

The connecting homomorphism $\delta_{c}: H_{c}^{k}(M) \rightarrow H_{c}^{k+1}(U \cap V)$ is given by

$$
\begin{aligned}
\delta_{c}[\varphi] & =\left[\beta_{c}^{-1} d \alpha_{c}^{-1}(\varphi)\right]=\left[\beta_{c}^{-1} d\left(f_{U} \varphi,-f_{V} \varphi\right)\right] \\
& =\left[d f_{U} \wedge \varphi\lceil U \cap V]=-\left[d f_{V} \wedge \varphi \uparrow U \cap V\right] .\right.
\end{aligned}
$$

Proof. The only part that is not completely obvious is that $\alpha_{c}$ is surjective. Let $\left\{f_{U}, f_{V}\right\}$ be a partition of unity with $\operatorname{supp}\left(f_{U}\right) \subset U$ and $\operatorname{supp}\left(f_{V}\right) \subset$ $V$, and let $\varphi \in \Omega_{c}^{k}(M)$. Then $f_{U} \varphi \in \Omega_{c}^{k}(U)$ and $-f_{V} \varphi \in \Omega_{c}^{k}(V)$ satisfy $\alpha_{c}\left(f_{U} \varphi,-f_{V} \varphi\right)=\left(f_{U}+f_{V}\right) \varphi=\varphi$. 
12.5. Proper homotopies. A smooth mapping $h: \mathbb{R} \times M \rightarrow N$ is called a proper homotopy if $h^{-1}$ (compact set) $\cap([0,1] \times M)$ is compact. A continuous homotopy $h:[0,1] \times M \rightarrow N$ is a proper homotopy if and only if it is a proper mapping.

Lemma. Let $f, g: M \rightarrow N$ be proper and proper homotopic. Then $f^{*}=$ $g^{*}: H_{c}^{k}(N) \rightarrow H_{c}^{k}(M)$ for all $k$.

Proof. Recall the proof of lemma (11.4).

Claim. In the proof of (11.4) we have furthermore $\bar{h}: \Omega_{c}^{k}(N) \rightarrow \Omega_{c}^{k-1}(M)$. Let $\omega \in \Omega_{c}^{k}(N)$ and let $K_{1}:=\operatorname{supp}(\omega)$, a compact set in $N$. Then $K_{2}:=$ $h^{-1}\left(K_{1}\right) \cap([0,1] \times M)$ is compact in $\mathbb{R} \times M$, and finally $K_{3}:=p r_{2}\left(K_{2}\right)$ is compact in $M$. If $x \notin K_{3}$, then we have

$$
(\bar{h} \omega)_{x}=\left(\left(I_{0}^{1} \circ i_{T} \circ h^{*}\right) \omega\right)_{x}=\int_{0}^{1}\left(\operatorname{ins}_{t}^{*}\left(i_{T} h^{*} \omega\right)\right)_{x} d t=0 .
$$

The rest of the proof is then again as in (11.4).

\subsection{Lemma.}

$$
H_{c}^{k}\left(\mathbb{R}^{n}\right)=\left\{\begin{array}{lc}
\mathbb{R} & \text { for } k=n \\
0 & \text { otherwise }
\end{array}\right.
$$

Proof. We embed $\mathbb{R}^{n}$ into its one point compactification $\mathbb{R}^{n} \cup\{\infty\}$ which is diffeomorphic to $S^{n}$; see (1.2), The embedding induces the exact sequence of complexes

$$
0 \rightarrow \Omega_{c}\left(\mathbb{R}^{n}\right) \rightarrow \Omega\left(S^{n}\right) \rightarrow \Omega\left(S^{n}\right)_{\infty} \rightarrow 0,
$$

where $\Omega\left(S^{n}\right)_{\infty}$ denotes the space of germs at the point $\infty \in S^{n}$. For germs at a point the lemma of Poincaré (9.10) is valid, so we have $H^{0}\left(\Omega\left(S^{n}\right)_{\infty}\right)=\mathbb{R}$ and $H^{k}\left(\Omega\left(S^{n}\right)_{\infty}\right)=0$ for $k>0$. By theorem (11.8) there is a long exact sequence in cohomology whose beginning is:

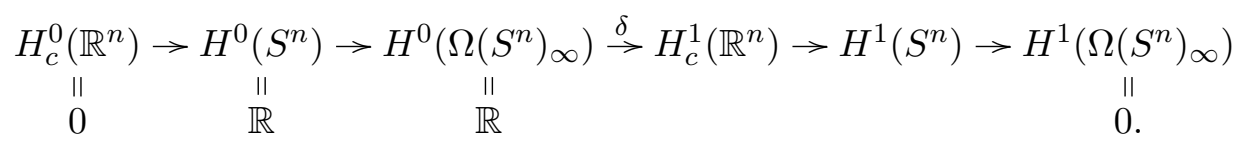

From this we see that $\delta=0$ and consequently $H_{c}^{1}\left(\mathbb{R}^{n}\right) \cong H^{1}\left(S^{n}\right)$. Another part of this sequence for $k \geq 2$ is:

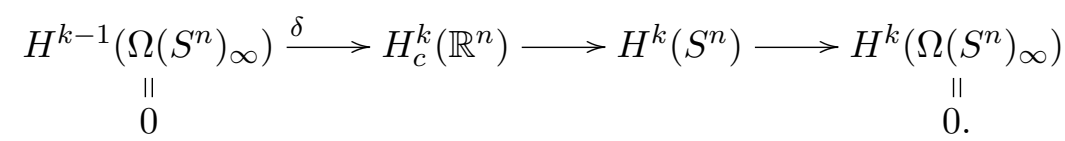

It implies $H_{c}^{k}\left(\mathbb{R}^{n}\right) \cong H^{k}\left(S^{n}\right)$ for all $k$. 
12.7. Fiber integration. Let $M$ be a manifold, and let $\operatorname{pr}_{1}: M \times \mathbb{R} \rightarrow M$. We define an operator called the fiber integration

$$
\int_{\text {fiber }}: \Omega_{c}^{k}(M \times \mathbb{R}) \rightarrow \Omega_{c}^{k-1}(M)
$$

as follows. Let $t$ be the coordinate function on $\mathbb{R}$. A differential form with compact support on $M \times \mathbb{R}$ is a finite linear combination of two types of forms:

(1) $\operatorname{pr}_{1}^{*} \varphi \cdot f(x, t)$, or for short $\varphi \cdot f$,

(2) $\operatorname{pr}_{1}^{*} \varphi \wedge f(x, t) d t$, or for short $\varphi \wedge f d t$,

where $\varphi \in \Omega(M)$ and $f \in C_{c}^{\infty}(M \times \mathbb{R}, \mathbb{R})$. We then put

(11) $\int_{\text {fiber }} \operatorname{pr}_{1}^{*} \varphi f:=0$,

(2) $\int_{\text {fiber }} \operatorname{pr}_{1}^{*} \varphi \wedge f d t:=\varphi \int_{-\infty}^{\infty} f(, t) d t$.

This is well defined since the only relation which we have to satisfy is $\operatorname{pr}_{1}^{*}(\varphi g) \wedge f(x, t) d t=\operatorname{pr}_{1}^{*} \varphi g(x) \wedge f(x, t) d t$.

Lemma. We have $d \circ \int_{\text {fiber }}=\int_{\text {fiber }} \circ d$. Thus $\int_{\text {fiber }}$ induces a linear mapping in cohomology

$$
\left(\int_{\text {fiber }}\right)_{*}: H_{c}^{k}(M \times \mathbb{R}) \rightarrow H_{c}^{k-1}(M),
$$

which however is not an algebra homomorphism.

Proof. In case (1) we have

$$
\begin{aligned}
\int_{\text {fiber }} d(\varphi \cdot f) & =\int_{\text {fiber }} d \varphi \cdot f+(-1)^{k} \int_{\text {fiber }} \varphi \cdot d_{M} f+(-1)^{k} \int_{\text {fiber }} \varphi \cdot \frac{\partial f}{\partial t} d t \\
& =(-1)^{k} \varphi \int_{-\infty}^{\infty} \frac{\partial f}{\partial t} d t=0 \quad \text { since } f \text { has compact support } \\
& =d \int_{\text {fiber }} \varphi \cdot f .
\end{aligned}
$$

In case (2) we get

$$
\begin{aligned}
\int_{\text {fiber }} d(\varphi \wedge f d t) & =\int_{\text {fiber }} d \varphi \wedge f d t+(-1)^{k} \int_{\text {fiber }} \varphi \wedge d_{M} f \wedge d t \\
& =d \varphi \int_{-\infty}^{\infty} f(, t) d t+(-1)^{k} \varphi \int_{-\infty}^{\infty} d_{M} f(, t) d t \\
& =d\left(\varphi \int_{-\infty}^{\infty} f(, t) d t\right)=d \int_{\text {fiber }} \varphi \wedge f d t .
\end{aligned}
$$

In order to find a mapping in the converse direction, we let $e=e(t) d t$ be a compactly supported 1 -form on $\mathbb{R}$ with $\int_{-\infty}^{\infty} e(t) d t=1$. We define 
$e_{*}: \Omega_{c}^{k}(M) \rightarrow \Omega_{c}^{k+1}(M \times \mathbb{R})$ by $e_{*}(\varphi)=\varphi \wedge e$. Then

$$
d e_{*}(\varphi)=d(\varphi \wedge e)=d \varphi \wedge e+0=e_{*}(d \varphi),
$$

so we have an induced mapping in cohomology $e_{*}: H_{c}^{k}(M) \rightarrow H_{c}^{k+1}(M \times \mathbb{R})$. We have $\int_{\text {fiber }} \circ e_{*}=I d_{\Omega_{c}^{k}(M)}$, since

$$
\int_{\text {fiber }} e_{*}(\varphi)=\int_{\text {fiber }} \varphi \wedge e(\quad) d t=\varphi \int_{-\infty}^{\infty} e(t) d t=\varphi
$$

Next we define $K: \Omega_{c}^{k}(M \times \mathbb{R}) \rightarrow \Omega_{c}^{k-1}(M \times \mathbb{R})$ by

(11) $K(\varphi \cdot f):=0$,

(21) $K(\varphi \wedge f d t)=\varphi \int_{-\infty}^{t} f d t-\varphi \cdot A(t) \int_{-\infty}^{\infty} f d t$, where $A(t):=\int_{-\infty}^{t} e(t) d t$.

Lemma. Then we have

$$
I d_{\Omega_{c}^{k}(M \times \mathbb{R})}-e_{*} \circ \int_{\text {fiber }}=(-1)^{k-1}(d \circ K-K \circ d) .
$$

Proof. We have to check the two cases. In case (11) we have

$$
\begin{aligned}
\left(I d-e_{*} \circ \int_{\text {fiber }}\right)(\varphi \cdot f) & =\varphi \cdot f-0, \\
(d \circ K-K \circ d)(\varphi \cdot f) & =0-K\left(d \varphi \cdot f+(-1)^{k} \varphi \wedge d_{1} f+(-1)^{k} \varphi \wedge \frac{\partial f}{\partial t} d t\right) \\
& =-(-1)^{k}\left(\varphi \int_{-\infty}^{t} \frac{\partial f}{\partial t} d t-\varphi \cdot A(t) \int_{-\infty}^{\infty} \frac{\partial f}{\partial t} d t\right) \\
& =(-1)^{k-1} \varphi \cdot f+0 .
\end{aligned}
$$

In case (2) we get

$$
\begin{aligned}
\left(I d-e_{*} \circ \int_{\text {fiber }}\right)(\varphi \wedge f d t)= & \varphi \wedge f d t-\varphi \int_{-\infty}^{\infty} f d t \wedge e \\
(d \circ K-K \circ d)(\varphi \wedge f d t)= & d\left(\varphi \int_{-\infty}^{t} f d t-\varphi \cdot A(t) \int_{-\infty}^{\infty} f d t\right) \\
& -K\left(d \varphi \wedge f d t+(-1)^{k-1} \varphi \wedge d_{1} f \wedge d t\right) \\
= & (-1)^{k-1}\left(\varphi \wedge f d t-\varphi \wedge e \int_{-\infty}^{\infty} f d t\right) .
\end{aligned}
$$

Corollary. The induced mappings $\left(\int_{\text {fiber }}\right)_{*}$ and $e_{*}$ are inverse to each other and thus isomorphisms between $H_{c}^{k}(M \times \mathbb{R})$ and $H_{c}^{k-1}(M)$.

Proof. This is clear from the chain homotopy (3). 
12.8. Second proof of (12.6). For $k \leq n$ we have

$$
\begin{aligned}
H_{c}^{k}\left(\mathbb{R}^{n}\right) & \cong H_{c}^{k-1}\left(\mathbb{R}^{n-1}\right) \cong \ldots \cong H_{c}^{0}\left(\mathbb{R}^{n-k}\right) \\
& = \begin{cases}0 & \text { for } k<n, \\
H_{c}^{0}\left(\mathbb{R}^{0}\right)=\mathbb{R} & \text { for } k=n .\end{cases}
\end{aligned}
$$

Note that the isomorphism $H_{c}^{n}\left(\mathbb{R}^{n}\right) \cong \mathbb{R}$ is given by integrating the differential form with compact support with respect to the standard orientation. This is well defined since by Stokes's theorem (10.11) we have

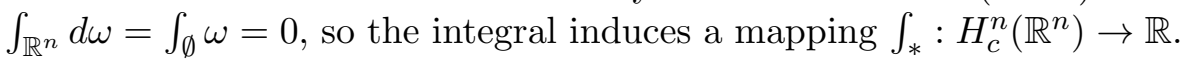

12.9. Example. We consider the open Möbius strip $M$ in $\mathbb{R}^{3}$; see $(1.22)$, Open means without boundary. Then $M$ is contractible onto $S^{1}$; in fact $M$ is the total space of a real line bundle over $S^{1}$. So from (11.12) we see that $H^{k}(M) \cong H^{k}\left(S^{1}\right)=\mathbb{R}$ for $k=0,1$ and $=0$ for $k>1$.

Now we claim that $H_{c}^{k}(M)=0$ for all $k$. For that we cut the Möbius strip in two pieces which are glued at the end with one turn,

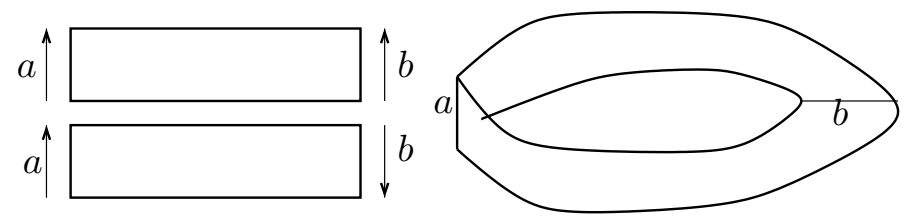

so that $M=U \cup V$ where $U \cong \mathbb{R}^{2}, V \cong \mathbb{R}^{2}$, and $U \cap V \cong \mathbb{R}^{2} \sqcup \mathbb{R}^{2}$, the disjoint union. We also know that $H_{c}^{0}(M)=0$ since $M$ is not compact and connected. Then the Mayer-Vietoris sequence (see (12.4) is given by

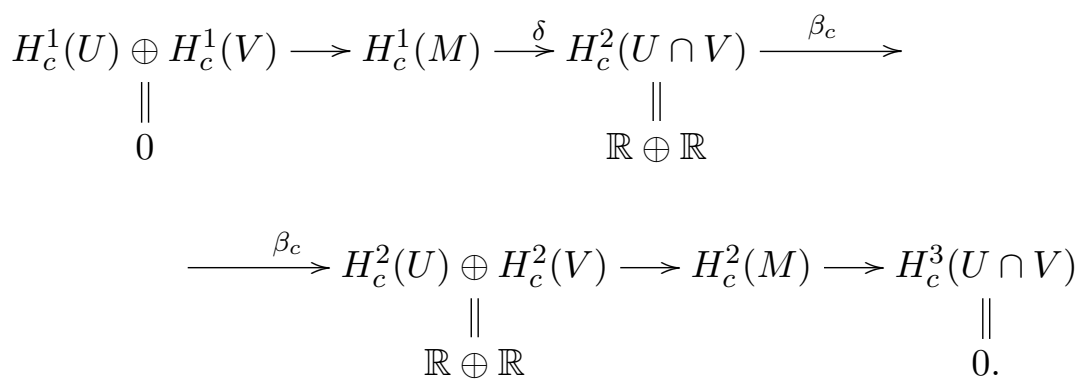

We shall show that the linear mapping $\beta_{c}$ has rank 2 . So we read from the sequence that $H_{c}^{1}(M)=0$ and $H_{c}^{2}(M)=0$. By reasons of dimension $H^{k}(M)=0$ for $k>2$.

Let $\varphi, \psi \in \Omega_{c}^{2}(U \cap V)$ be two forms, supported in the two connected components, respectively, with integral 1 in the orientation induced from one on $U$. Then $\int_{U} \varphi=1, \int_{U} \psi=1$, but for some orientation on $V$ we have 
$\int_{V} \varphi=1$ and $\int_{V} \psi=-1$. So the matrix of the mapping $\beta_{c}$ in these bases is $\left(\begin{array}{cc}1 & 1 \\ 1 & -1\end{array}\right)$, which has rank 2 .

12.10. Mapping degree for proper mappings. Let $f: \mathbb{R}^{n} \rightarrow \mathbb{R}^{n}$ be a smooth proper mapping; then $f^{*}: \Omega_{c}^{k}\left(\mathbb{R}^{n}\right) \rightarrow \Omega_{c}^{k}\left(\mathbb{R}^{n}\right)$ is defined and is an algebra homomorphism. So also the induced mapping in cohomology with compact supports makes sense and by

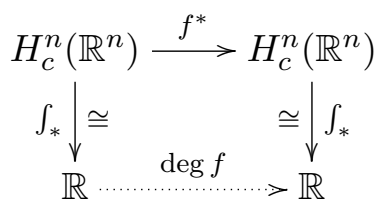

a linear mapping $\mathbb{R} \rightarrow \mathbb{R}$, i.e., multiplication by a real number, is defined. This number $\operatorname{deg} f$ is called the mapping degree of $f$.

12.11. Lemma. The mapping degree of proper mappings has the following properties:

(1) If $f, g: \mathbb{R}^{n} \rightarrow \mathbb{R}^{n}$ are proper, then $\operatorname{deg}(f \circ g)=\operatorname{deg}(f) \cdot \operatorname{deg}(g)$.

(2) If $f$ and $g: \mathbb{R}^{n} \rightarrow \mathbb{R}^{n}$ are proper homotopic (see (12.5)), then $\operatorname{deg}(f)=$ $\operatorname{deg}(g)$.

(3) $\operatorname{deg}\left(I d_{\mathbb{R}^{n}}\right)=1$.

(4) If $f: \mathbb{R}^{n} \rightarrow \mathbb{R}^{n}$ is proper and not surjective, then $\operatorname{deg}(f)=0$.

Proof. Only statement (4) needs a proof. Since $f$ is proper, $f\left(\mathbb{R}^{n}\right)$ is closed in $\mathbb{R}^{n}$ : For $K$ compact in $\mathbb{R}^{n}$ the inverse image $K_{1}=f^{-1}(K)$ is compact, so $f\left(K_{1}\right)=f\left(\mathbb{R}^{n}\right) \cap K$ is compact, thus closed. By local compactness $f\left(\mathbb{R}^{n}\right)$ is closed.

Suppose that there exists $x \in \mathbb{R}^{n} \backslash f\left(\mathbb{R}^{n}\right)$; then there is an open neighborhood $U \subset \mathbb{R}^{n} \backslash f\left(\mathbb{R}^{n}\right)$. We choose a bump $n$-form $\alpha$ on $\mathbb{R}^{n}$ with support in $U$ and $\int \alpha=1$. Then $f^{*} \alpha=0$, so $\operatorname{deg}(f)=0$ since $[\alpha]$ is a generator of $H_{c}^{n}\left(\mathbb{R}^{n}\right)$.

12.12. Lemma. For a proper smooth mapping $f: \mathbb{R}^{n} \rightarrow \mathbb{R}^{n}$ the mapping degree is an integer; in fact for any regular value $y$ of $f$ we have

$$
\operatorname{deg}(f)=\sum_{x \in f^{-1}(y)} \operatorname{sign}(\operatorname{det}(d f(x))) \in \mathbb{Z} .
$$

Proof. By the Morse-Sard theorem, see (1.18), there exists a regular value $y$ of $f$. If $f^{-1}(y)=\emptyset$, then $f$ is not surjective, so $\operatorname{deg}(f)=0$ by $(12.11 .4)$ and the formula holds. If $f^{-1}(y) \neq \emptyset$, then for all $x \in f^{-1}(y)$ the tangent mapping $T_{x} f$ is surjective, thus an isomorphism. By the inverse mapping 
theorem $f$ is locally a diffeomorphism from an open neighborhood of $x$ onto a neighborhood of $y$. Thus $f^{-1}(y)$ is a discrete and compact set, say $f^{-1}(y)=$ $\left\{x_{1}, \ldots, x_{k}\right\} \subset \mathbb{R}^{n}$.

Now we choose pairwise disjoint open neighborhoods $U_{i}$ of $x_{i}$ and an open neighborhood $V$ of $y$ such that $f: U_{i} \rightarrow V$ is a diffeomorphism for each $i$. We choose an $n$-form $\alpha$ on $\mathbb{R}^{n}$ with support in $V$ and $\int \alpha=1$. So $f^{*} \alpha=\sum_{i}\left(f \mid U_{i}\right)^{*} \alpha$ and moreover

$$
\begin{aligned}
\int_{U_{i}}\left(f \mid U_{i}\right)^{*} \alpha & =\operatorname{sign}\left(\operatorname{det}\left(d f\left(x_{i}\right)\right)\right) \int_{V} \alpha=\operatorname{sign}\left(\operatorname{det}\left(d f\left(x_{i}\right)\right)\right), \\
\operatorname{deg}(f) & =\int_{\mathbb{R}^{n}} f^{*} \alpha=\sum_{i} \int_{U_{i}}\left(f \mid U_{i}\right)^{*} \alpha=\sum_{i}^{k} \operatorname{sign}\left(\operatorname{det}\left(d f\left(x_{i}\right)\right)\right) \in \mathbb{Z} .
\end{aligned}
$$

12.13. Example. The last result for a proper smooth mapping $f: \mathbb{R} \rightarrow \mathbb{R}$ can be interpreted as follows: Think of $f$ as parametrizing the path of a car on an (infinite) street. A regular value of $f$ is then a position on the street where the car never stops. Wait there and count the directions of the passes of the car: The sum is the mapping degree, the number of journeys from $-\infty$ to $\infty$. In dimension 1 it can be only $-1,0$, or +1 (why?).

12.14. Poincaré duality. Let $M$ be an oriented smooth manifold of dimension $m$ without boundary. By Stokes's theorem (10.11) the integral operator $\int: \Omega_{c}^{m}(M) \rightarrow \mathbb{R}$ vanishes on exact forms and induces the cohomological integral

$$
\int_{*}: H_{c}^{m}(M) \rightarrow \mathbb{R}
$$

It is surjective (use a bump $m$-form with small support). The Poincaré product is the bilinear form

$$
\begin{gathered}
P_{M}^{k}: H^{k}(M) \times H_{c}^{m-k}(M) \rightarrow \mathbb{R}, \\
P_{M}^{k}([\alpha],[\beta])=\int_{*}[\alpha] \wedge[\beta]=\int_{M} \alpha \wedge \beta .
\end{gathered}
$$

It is well defined since for $\beta$ closed $d \gamma \wedge \beta=d(\gamma \wedge \beta)$, etc. If $j: U \rightarrow M$ is an orientation preserving embedding of an open submanifold, then for $[\alpha] \in H^{k}(M)$ and for $[\beta] \in H_{c}^{m-k}(U)$ we may compute as follows:

$$
\begin{aligned}
P_{U}^{k}\left(j^{*}[\alpha],[\beta]\right) & =\int_{*}\left(j^{*}[\alpha]\right) \wedge[\beta]=\int_{U} j^{*} \alpha \wedge \beta \\
& =\int_{U} j^{*}\left(\alpha \wedge j_{*} \beta\right)=\int_{j(U)} \alpha \wedge j_{*} \beta \\
& =\int_{M} \alpha \wedge j_{*} \beta=P_{M}^{k}\left([\alpha], j_{*}[\beta]\right) .
\end{aligned}
$$


Now we define the Poincaré duality operator

$$
\begin{aligned}
D_{M}^{k}: H^{k}(M) & \rightarrow\left(H_{c}^{m-k}(M)\right)^{*}, \\
\left\langle[\beta], D_{M}^{k}[\alpha]\right\rangle & =P_{M}^{k}([\alpha],[\beta]) .
\end{aligned}
$$

For example, we have

$$
D_{\mathbb{R}^{n}}^{0}(1)=\left(\int_{\mathbb{R}^{n}}\right)_{*} \in\left(H_{c}^{n}\left(\mathbb{R}^{n}\right)\right)^{*} .
$$

Let $M=U \cup V$ with $U, V$ open in $M$; then we have the two Mayer-Vietoris sequences from (11.10) and from (12.4)

$$
\begin{aligned}
& \cdots \rightarrow H^{k}(M) \stackrel{\alpha_{*}}{\longrightarrow} H^{k}(U) \oplus H^{k}(V) \stackrel{\beta_{*}}{\longrightarrow} H^{k}(U \cap V) \stackrel{\delta}{\longrightarrow} H^{k+1}(M) \rightarrow \cdots \\
& H_{c}^{m-k}(M) \leftarrow H_{c}^{m-k}(U) \oplus H_{c}^{m-k}(V) \leftarrow H_{c}^{m-k}(U \cap V) \stackrel{\delta_{c}}{\longleftarrow} H_{c}^{m-(k+1)}(M) .
\end{aligned}
$$

We take dual spaces and dual mappings in the second sequence and we replace $\delta$ in the first sequence by $(-1)^{k-1} \delta$ and get the following diagram which is commutative as we will see in a moment:

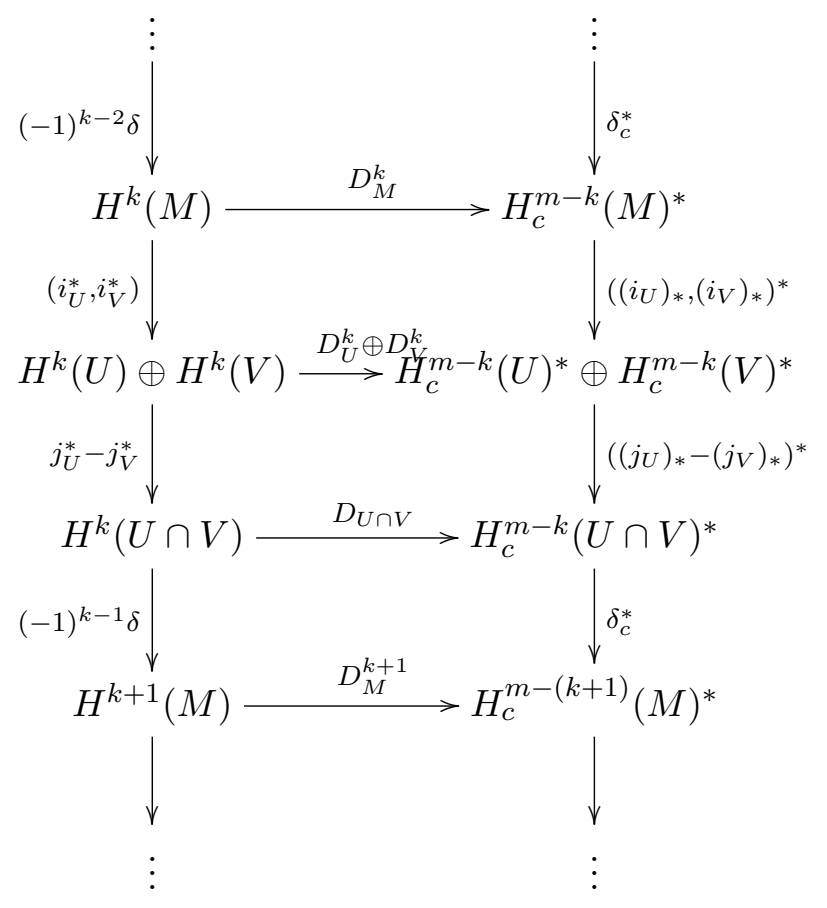

12.15. Lemma. Diagram (12.14.5) commutes.

Proof. The first and the second square from the top commute by (12.14.3). So we have to check that the bottom one commutes. Let $[\alpha] \in H^{k}(U \cap V)$ 
and $[\beta] \in H_{c}^{m-(k+1)}(M)$, and let $\left(f_{U}, f_{V}\right)$ be a partition of unity which is subordinated to the open cover $(U, V)$ of $M$. Then we have

$$
\begin{aligned}
\left\langle[\beta], D_{M}^{k+1}(-1)^{k-1} \delta[\alpha]\right\rangle=P_{M}^{k+1}\left((-1)^{k-1} \delta[\alpha],[\beta]\right) & \\
& =P_{M}^{k+1}\left((-1)^{k-1}\left[d f_{V} \wedge \alpha\right],[\beta]\right) \quad \text { by }[11.10) \\
& =(-1)^{k-1} \int_{M} d f_{V} \wedge \alpha \wedge \beta, \\
\left\langle[\beta], \delta_{c}^{*} D_{U \cap V}^{k}[\alpha]\right\rangle & =\left\langle\delta_{c}[\beta], D_{U \cap V}^{k}[\alpha]\right\rangle=P_{U \cap V}^{k}\left([\alpha], \delta_{c}[\beta]\right) \\
& =P_{U \cap V}^{k}\left([\alpha],\left[d f_{U} \wedge \beta\right]=-\left[d f_{V} \wedge \beta\right]\right) \quad \text { by }(12.4) \\
& =-\int_{U \cap V} \alpha \wedge d f_{V} \wedge \beta=-(-1)^{k} \int_{M} d f_{V} \wedge \alpha \wedge \beta .
\end{aligned}
$$

12.16. Theorem. Poincaré duality. If $M$ is an oriented manifold of dimension $m$ without boundary, then the Poincaré duality mapping

$$
D_{M}^{k}: H^{k}(M) \rightarrow H_{c}^{m-k}(M)^{*}
$$

is a linear isomorphism for each $k$.

Proof. Step 1. Let $\mathcal{O}$ be an $i$-base for the open sets of $M$, i.e., $\mathcal{O}$ is a basis containing all finite intersections of sets in $\mathcal{O}$. Let $\mathcal{O}_{f}$ be the set of all open sets in $M$ which are finite unions of sets in $\mathcal{O}$. Let $\mathcal{O}_{s}$ be the set of all open sets in $M$ which are at most countable disjoint unions of sets in $\mathcal{O}$. Then obviously $\mathcal{O}_{f}$ and $\mathcal{O}_{s}$ are again $i$-bases.

Step 2. Let $\mathcal{O}$ be an $i$-base for $M$. If $D_{O}: H(O) \rightarrow H_{c}(O)^{*}$ is an isomorphism for all $O \in \mathcal{O}$, then it is so also for all $O \in \mathcal{O}_{f}$.

Let $U \in \mathcal{O}_{f}, U=O_{1} \cup \cdots \cup O_{k}$ for $O_{i} \in \mathcal{O}$. We consider $O_{1}$ and $V=$ $O_{2} \cup \cdots \cup O_{k}$. Then $O_{1} \cap V=\left(O_{1} \cap O_{2}\right) \cup \cdots \cup\left(O_{1} \cap O_{k}\right)$ is again a union of elements of $\mathcal{O}$ since it is an $i$-base. Now we prove the claim by induction on $k$. The case $k=1$ is trivial. By induction $D_{O_{1}}, D_{V}$, and $D_{O_{1} \cap V}$ are isomorphisms, so $D_{U}$ is also an isomorphism by the five-lemma (11.9) applied to the diagram (12.14.5).

Step 3. If $\mathcal{O}$ is a basis of open sets in $M$ such that $D_{O}$ is an isomorphism for all $O \in \mathcal{O}$, then it is so also for all $O \in \mathcal{O}_{s}$.

If $U \in \mathcal{O}_{s}$, we have $U=O_{1} \sqcup O_{2} \sqcup \ldots=\bigsqcup_{i=1}^{\infty} O_{i}$ for $O_{i} \in \mathcal{O}$. But then the diagram

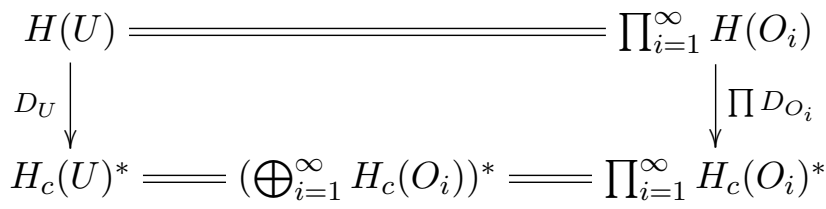

commutes and implies that $D_{U}$ is an isomorphism. 
Step 4. If $D_{O}$ is an isomorphism for each $O \in \mathcal{O}$ where $\mathcal{O}$ is an $i$-base for the open sets of $M$, then $D_{U}$ is an isomorphism for each open set $U \subset M$.

Namely, $\left(\left(\mathcal{O}_{f}\right)_{s}\right)_{f}$ contains all open sets of $M$; then the result follows from steps 2 and 3. Indeed, given an open $U \subset M$, choose compact sets $K_{i} \subset M$ with $K_{i} \subset K_{i+1}$ and $U=\bigcup_{i=1}^{\infty} K_{i}$. Then we choose open sets $O_{i} \in \mathcal{O}_{f}$ for $i=1,2, \ldots$ of $U$ such that $\overline{O_{i}}$ is compact, $\bigcup_{i=1}^{k} O_{i} \supset K_{k}$ so that the $O_{i}$ also cover $U, \bigcup_{i=1}^{k} O_{i} \supset \bigcup_{i=1}^{k-1} O_{i}$, and $O_{i} \cap O_{j}=\emptyset$ unless $j=i-1$ or $j=i+1$. Then let $V_{1}=\bigcup_{i \geq 0} O_{2 i+1}$ and $V_{2}=\bigcup_{i \geq 1} O_{2 i}$ which are elements of $\left(\mathcal{O}_{f}\right)_{s}$. Hence $U=V_{1} \cup V_{2}$ is in $\left(\left(\mathcal{O}_{f}\right)_{s}\right)_{f}$.

Step 5. $D_{\mathbb{R}^{m}}: H\left(\mathbb{R}^{m}\right) \rightarrow H_{c}\left(\mathbb{R}^{m}\right)^{*}$ is an isomorphism.

We have

$$
H^{k}\left(\mathbb{R}^{m}\right)=\left\{\begin{array}{ll}
\mathbb{R} & \text { for } k=0, \\
0 & \text { for } k>0,
\end{array} \quad H_{c}^{k}\left(\mathbb{R}^{m}\right)= \begin{cases}\mathbb{R} & \text { for } k=m, \\
0 & \text { for } k \neq m .\end{cases}\right.
$$

The class [1] is a generator for $H^{0}\left(\mathbb{R}^{m}\right)$, and $[\alpha]$ is a generator for $H_{c}^{m}\left(\mathbb{R}^{m}\right)$ where $\alpha$ is any $m$-form with compact support and $\int_{M} \alpha=1$. But then $P_{\mathbb{R}^{m}}^{0}([1],[\alpha])=\int_{\mathbb{R}^{m}} 1 . \alpha=1$.

Step 6. For each open subset $U \subset \mathbb{R}^{m}$ the mapping $D_{U}$ is an isomorphism. The set $\left\{\left\{x \in \mathbb{R}^{m}: a^{i}<x^{i}<b^{i}\right.\right.$ for all $\left.\left.i\right\}: a^{i}<b^{i}\right\}$ is an $i$-base of $\mathbb{R}^{m}$. Each element $O$ in it is diffeomorphic (with orientation preserved) to $\mathbb{R}^{m}$, so $D_{O}$ is an isomorphism by step 5. From step 4 the result follows.

Step 7. $D_{M}$ is an isomorphism for each oriented manifold $M$.

Let $\mathcal{O}$ be the set of all open subsets of $M$ which are diffeomorphic to an open subset of $\mathbb{R}^{m}$, i.e., all charts of a maximal atlas. Then $\mathcal{O}$ is an $i$-base for $M$, and $D_{O}$ is an isomorphism for each $O \in \mathcal{O}$. By step 4 the operator $D_{U}$ is an isomorphism for each open $U$ in $M$; thus also $D_{M}$ is an isomorphism.

12.17. Corollary. For each oriented manifold $M$ without boundary the bilinear pairings

$$
\begin{gathered}
P_{M}: H^{*}(M) \times H_{c}^{*}(M) \rightarrow \mathbb{R}, \\
P_{M}^{k}: H^{k}(M) \times H_{c}^{m-k}(M) \rightarrow \mathbb{R}
\end{gathered}
$$

are not degenerate.

12.18. Corollary. Let $j: U \rightarrow M$ be the embedding of an open submanifold of an oriented manifold $M$ of dimension $m$ without boundary. Then of the following two mappings one is an isomorphism if and only if the other one is:

$$
\begin{aligned}
j^{*}: H^{k}(U) & \leftarrow H^{k}(M), \\
j_{*}: H_{c}^{m-k}(U) & \rightarrow H_{c}^{m-k}(M) .
\end{aligned}
$$


Proof. Use (12.14.3), $P_{U}^{k}\left(j^{*}[\alpha],[\beta]\right)=P_{M}^{k}\left([\alpha], j_{*}[\beta]\right)$.

12.19. Theorem. Let $M$ be an oriented connected manifold of dimension $m$ without boundary. Then the integral

$$
\int_{*}: H_{c}^{m}(M) \rightarrow \mathbb{R}
$$

is an isomorphism. So $\operatorname{ker} \int_{M}=d\left(\Omega_{c}^{m-1}(M)\right) \subset \Omega_{c}^{m}(M)$.

Proof. Considering $m$-forms with small support shows that the integral is surjective. By Poincaré duality (12.16), $\operatorname{dim}_{\mathbb{R}} H_{c}^{m}(M)^{*}=\operatorname{dim}_{\mathbb{R}} H^{0}(M)=1$ since $M$ is connected.

Definition. The uniquely defined cohomology class $\omega_{M} \in H_{c}^{m}(M)$ with integral $\int_{M} \omega_{M}=1$ is called the orientation class of the manifold $M$.

12.20. Relative cohomology with compact supports. Let $M$ be a smooth manifold and let $N$ be a closed submanifold. Then the injection $i: N \rightarrow M$ is a proper smooth mapping. We consider the spaces

$$
\Omega_{c}^{k}(M, N):=\left\{\omega \in \Omega_{c}^{k}(M): \omega \mid N=i^{*} \omega=0\right\}
$$

whose direct $\operatorname{sum}\left(\Omega_{c}^{*}(M, N), d\right)$ is a graded differential subalgebra of the graded differential algebra $\left(\Omega_{c}^{*}(M), d\right)$. Its cohomology, $H_{c}^{*}(M, N)$, is called the relative de Rham cohomology with compact supports of the manifold pair $(M, N)$. The sequence of graded differential algebras

$$
0 \rightarrow \Omega_{c}^{*}(M, N) \hookrightarrow \Omega_{c}^{*}(M) \stackrel{i^{*}}{\longrightarrow} \Omega_{c}^{*}(N) \rightarrow 0
$$

is exact. This is seen by the same proof as of (11.16) with some obvious changes. Thus by (11.8) we have the long exact sequence in cohomology

$$
\cdots \rightarrow H_{c}^{k}(M, N) \rightarrow H_{c}^{k}(M) \rightarrow H_{c}^{k}(N) \stackrel{\delta}{\longrightarrow} H_{c}^{k+1}(M, N) \rightarrow \ldots
$$

which is natural in the manifold pair $(M, N)$. It is called the long exact cohomology sequence with compact supports of the pair $(M, N)$.

12.21. Now let $M$ be an oriented smooth manifold of dimension $m$ with boundary $\partial M$. Then $\partial M$ is a closed submanifold of $M$. Since for $\omega \in$ $\Omega_{c}^{m-1}(M, \partial M)$ we have $\int_{M} d \omega=\int_{\partial M} \omega=\int_{\partial M} 0=0$, the integral of $m$ forms factors as

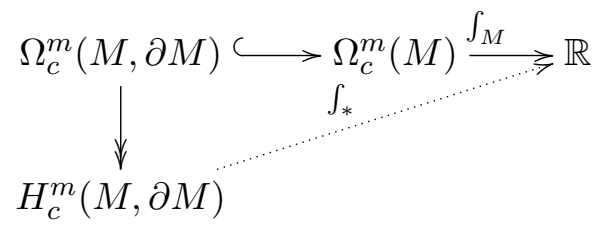

to the cohomological integral $\int_{*}: H_{c}^{m}(M, \partial M) \rightarrow \mathbb{R}$. 
Example. Let $I=[a, b]$ be a compact interval; then $\partial I=\{a, b\}$. We have $H^{1}(I)=0$ since $f d t=d \int_{a}^{t} f(s) d s$. The long exact sequence in cohomology of the pair $(I, \partial I)$ is

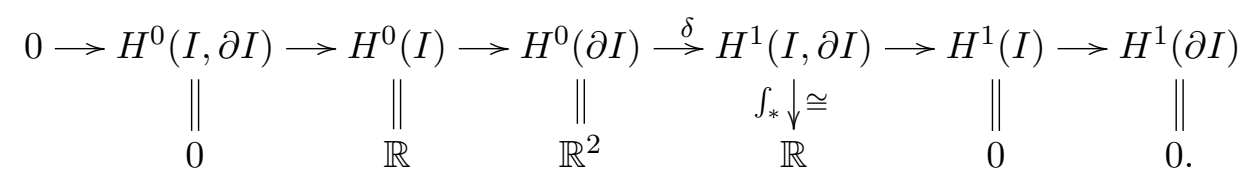

The connecting homomorphism $\delta: H^{0}(\partial I) \rightarrow H^{1}(I, \partial I)$ is given by the following procedure: Let $(f(a), f(b)) \in H^{0}(\partial I)$, where $f \in C^{\infty}(I)$. Then

$$
\delta(f(a), f(b))=[d f]=\int_{*}[d f]=\int_{a}^{b} d f=\int_{a}^{b} f^{\prime}(t) d t=f(b)-f(a) .
$$

So the fundamental theorem of calculus can be interpreted as the connecting homomorphism for the long exact sequence of the relative cohomology for the pair $(I, \partial I)$.

The general situation. Let $M$ be an oriented smooth manifold with boundary $\partial M$. We consider the following piece of the long exact sequence in cohomology with compact supports of the pair $(M, \partial M)$ :

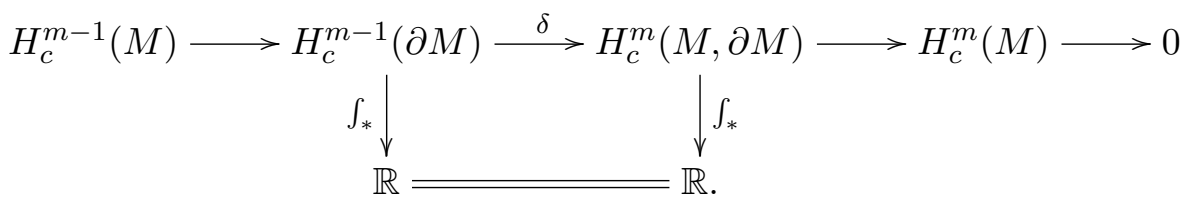

The connecting homomorphism is given by

$$
\delta[\omega \mid \partial M]=[d \omega]_{H_{c}^{m}(M, \partial M)}, \quad \omega \in \Omega_{c}^{m-1}(M),
$$

so commutation of the diagram above is equivalent to the validity of Stokes's theorem.

\section{De Rham Cohomology of Compact Manifolds}

13.1. The oriented double cover. Let $M$ be a manifold. We consider the orientation bundle $\operatorname{Or}(M)$ of $M$ which we discussed in (10.6), and we consider the subset $\operatorname{or}(M):=\{v \in \operatorname{Or}(M):|v|=1\}$; see (10.7) for the modulus. We shall see shortly that it is a submanifold of the total space $\operatorname{Or}(M)$, that it is orientable, and that $\pi_{M}: \operatorname{or}(M) \rightarrow M$ is a double cover of $M$. The manifold or $(M)$ is called the orientable double cover of $M$.

We first check that the total space $\operatorname{Or}(M)$ of the orientation bundle is orientable. Let $\left(U_{\alpha}, u_{\alpha}\right)$ be an atlas for $M$. Then the orientation bundle is given by the cocycle of transition functions

$$
\tau_{\alpha \beta}(x)=\operatorname{sign} \varphi_{\alpha \beta}(x)=\operatorname{sign} \operatorname{det} d\left(u_{\beta} \circ u_{\alpha}^{-1}\right)\left(u_{\alpha}(x)\right) .
$$


Let $\left(U_{\alpha}, \tau_{\alpha}\right)$ be the induced vector bundle atlas for $\operatorname{Or}(M)$; see (8.3). We consider the mappings

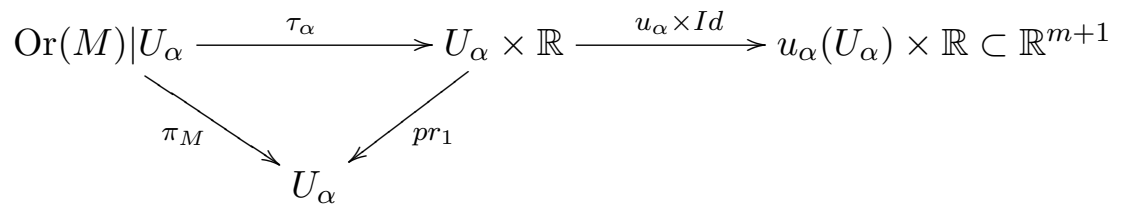

and we use them as charts for $\operatorname{Or}(M)$. The chart changes $u_{\beta}\left(U_{\alpha \beta}\right) \times \mathbb{R} \rightarrow$ $u_{\alpha}\left(U_{\alpha \beta}\right) \times \mathbb{R}$ are then given by

$$
\begin{aligned}
(y, t) & \mapsto\left(u_{\alpha} \circ u_{\beta}^{-1}(y), \tau_{\alpha \beta}\left(u_{\beta}^{-1}(y)\right) t\right) \\
& =\left(u_{\alpha} \circ u_{\beta}^{-1}(y), \operatorname{sign} \operatorname{det} d\left(u_{\beta} \circ u_{\alpha}^{-1}\right)\left(\left(u_{\alpha} \circ u_{\beta}^{-1}\right)(y)\right) t\right) \\
& =\left(u_{\alpha} \circ u_{\beta}^{-1}(y), \operatorname{sign} \operatorname{det} d\left(u_{\alpha} \circ u_{\beta}^{-1}\right)(y) t\right) .
\end{aligned}
$$

The Jacobi matrix of this mapping is

$$
\left(\begin{array}{cc}
d\left(u_{\alpha} \circ u_{\beta}^{-1}\right)(y) & * \\
0 & \operatorname{sign} \operatorname{det} d\left(u_{\alpha} \circ u_{\beta}^{-1}\right)(y)
\end{array}\right)
$$

which has positive determinant.

Now we let $Z:=\{v \in \operatorname{Or}(M):|v| \leq 1\}$ which is a submanifold with boundary in $\operatorname{Or}(M)$ of the same dimension and thus orientable. Its boundary $\partial Z$ coincides with or $(M)$, which is thus orientable.

Next we consider the diffeomorphism $\varphi: \operatorname{or}(M) \rightarrow \operatorname{or}(M)$ which is induced by the multiplication with -1 in $\operatorname{Or}(M)$. We have $\varphi \circ \varphi=I d$ and $\pi_{M}^{-1}(x)=$ $\{z, \varphi(z)\}$ for $z \in$ or $(M)$ and $\pi_{M}(z)=x$.

Suppose that the manifold $M$ is connected. Then the oriented double cover $\operatorname{or}(M)$ has at most two connected components, since $\pi_{M}$ is a two sheeted covering map. If or $(M)$ has two components, then $\varphi$ restricts to a diffeomorphism between them. The projection $\pi_{M}$, if restricted to one of the components, becomes invertible, so $\operatorname{Or}(M)$ admits a section which vanishes nowhere; thus $M$ is orientable. So we see that $\operatorname{or}(M)$ is connected if and only if $M$ is not orientable.

The pullback mapping $\varphi^{*}: \Omega(\operatorname{or}(M)) \rightarrow \Omega(\operatorname{or}(M))$ also satisfies $\varphi^{*} \circ \varphi^{*}=I d$. We put

$$
\begin{aligned}
& \Omega_{+}(\operatorname{or}(M)):=\left\{\omega \in \Omega(\operatorname{or}(M)): \varphi^{*} \omega=\omega\right\}, \\
& \Omega_{-}(\operatorname{or}(M)):=\left\{\omega \in \Omega(\operatorname{or}(M)): \varphi^{*} \omega=-\omega\right\} .
\end{aligned}
$$

For each $\omega \in \Omega(\operatorname{or}(M))$ we have $\omega=\frac{1}{2}\left(\omega+\varphi^{*} \omega\right)+\frac{1}{2}\left(\omega-\varphi^{*} \omega\right) \in \Omega_{+}(\operatorname{or}(M)) \oplus$ $\Omega_{-}(\operatorname{or}(M))$, so $\Omega(\operatorname{or}(M))=\Omega_{+}(\operatorname{or}(M)) \oplus \Omega_{-}(\operatorname{or}(M))$. Since $d \circ \varphi^{*}=\varphi^{*} \circ d$, these two subspaces are invariant under $d$; thus we conclude that

$$
H^{k}(\operatorname{or}(M))=H^{k}\left(\Omega_{+}(\operatorname{or}(M))\right) \oplus H^{k}\left(\Omega_{-}(\operatorname{or}(M))\right) .
$$


Since $\pi_{M}^{*}: \Omega(M) \rightarrow \Omega(\operatorname{or}(M))$ is an embedding with image $\Omega_{+}(\operatorname{or}(M))$, we see that the induced mapping $\pi_{M}^{*}: H^{k}(M) \rightarrow H^{k}(\operatorname{or}(M))$ is also an embedding with image $H^{k}\left(\Omega_{+}(\operatorname{or}(M))\right)$.

13.2. Theorem. For a compact manifold $M$ we have $\operatorname{dim}_{\mathbb{R}} H^{*}(M)<\infty$.

Proof. Step 1. If $M$ is orientable, we have by Poincaré duality (12.16)

$$
H^{k}(M) \stackrel{D_{M}^{k}}{\cong}\left(H_{c}^{m-k}(M)\right)^{*}=\left(H^{m-k}(M)\right)^{*} \underset{\cong}{\stackrel{\left(D_{M}^{m-k}\right)^{*}}{\cong}}\left(H_{c}^{k}(M)\right)^{* *},
$$

so $H^{k}(M)$ is finite-dimensional since otherwise $\operatorname{dim}\left(H^{k}(M)\right)^{*}>\operatorname{dim} H^{k}(M)$.

Step 2. Let $M$ be not orientable. Then from (13.1) we see that the oriented double cover or $(M)$ of $M$ is compact, oriented, and connected, and we have $\operatorname{dim} H^{k}(M)=\operatorname{dim} H^{k}\left(\Omega_{+}(\operatorname{or}(M))\right) \leq \operatorname{dim} H^{k}(\operatorname{or}(M))<\infty$.

13.3. Theorem. Let $M$ be a connected manifold of dimension $m$. Then

$$
H^{m}(M) \cong \begin{cases}\mathbb{R} & \text { if } M \text { is compact and orientable } \\ 0 & \text { otherwise }\end{cases}
$$

Proof. If $M$ is compact and orientable, the integral $\int_{*}: H^{m}(M) \rightarrow \mathbb{R}$ is an isomorphism, by (12.19).

Next let $M$ be compact but not orientable. Then the oriented double cover $\operatorname{or}(M)$ is connected, compact and oriented. Let $\omega \in \Omega^{m}(\operatorname{or}(M))$ be an $m$ form which vanishes nowhere. Then also $\varphi^{*} \omega$ is nowhere zero where $\varphi$ : $\operatorname{or}(M) \rightarrow \operatorname{or}(M)$ is the covering transformation from (13.1). So $\varphi^{*} \omega=f \omega$ for a function $f \in C^{\infty}(\operatorname{or}(M))$ which vanishes nowhere. So $f>0$ or $f<0$. If $f>0$, then $\alpha:=\omega+\varphi^{*} \omega=(1+f) \omega$ is again nowhere 0 and $\varphi^{*} \alpha=\alpha$, so $\alpha=\pi_{M}^{*} \beta$ for an $m$-form $\beta$ on $M$ without zeros. So $M$ is orientable, a contradiction. Thus $f<0$ and $\varphi$ changes the orientation.

The $m$-form $\gamma:=\omega-\varphi^{*} \omega=(1-f) \omega$ has no zeros, so $\int_{\operatorname{or}(M)} \gamma>0$ if we orient $\operatorname{or}(M)$ using $\omega$; thus the cohomology class $[\gamma] \in H^{m}(\operatorname{or}(M))$ is not zero. But $\varphi^{*} \gamma=-\gamma$ so $\gamma \in \Omega_{-}(\operatorname{or}(M))$; thus $H^{m}\left(\Omega_{-}(\operatorname{or}(M))\right) \neq 0$. By the first part of the proof we have $H^{m}(\operatorname{or}(M))=\mathbb{R}$ and from (13.1) we get $H^{m}(\operatorname{or}(M))=H^{m}\left(\Omega_{-}(\operatorname{or}(M))\right)$, so $H^{m}(M)=H^{m}\left(\Omega_{+}(\operatorname{or}(M))\right)=0$.

Finally let us suppose that $M$ is not compact. If $M$ is orientable, we have by Poincaré duality (12.16) and by (12.3.1) that $H^{m}(M) \cong H_{c}^{0}(M)^{*}=0$.

If $M$ is not orientable, then or $(M)$ is connected by (13.1) and not compact, so $H^{m}(M)=H^{m}\left(\Omega_{+}(\operatorname{or}(M))\right) \subset H^{m}(\operatorname{or}(M))=0$. 
13.4. Corollary. Let $M$ be a connected manifold which is not orientable. Then $\operatorname{or}(M)$ is orientable and the Poincaré duality pairing of $\operatorname{or}(M)$ satisfies

$$
\begin{aligned}
& P_{\operatorname{or}(M)}^{k}\left(H_{+}^{k}(\operatorname{or}(M)),\left(H_{c}^{m-k}\right)_{+}(\operatorname{or}(M))\right)=0, \\
& P_{\operatorname{or}(M)}^{k}\left(H_{-}^{k}(\operatorname{or}(M)),\left(H_{c}^{m-k}\right)_{-}(\operatorname{or}(M))\right)=0, \\
& H_{+}^{k}(\operatorname{or}(M)) \cong\left(H_{c}^{m-k}\right)_{-}(\operatorname{or}(M))^{*}, \\
& H_{-}^{k}(\operatorname{or}(M)) \cong\left(H_{c}^{m-k}\right)_{+}(\operatorname{or}(M))^{*} .
\end{aligned}
$$

Proof. From (13.1) we know that or $(M)$ is connected and orientable. So $\mathbb{R}=H^{0}(\operatorname{or}(M)) \cong H_{c}^{m}(\operatorname{or}(M))^{*}$.

Now we orient or $(M)$ and choose a nonnegative bump $m$-form $\omega$ with compact support on $\operatorname{or}(M)$ so that $\int_{\operatorname{or}(M)} \omega>0$. From the proof of (13.3) we know that the covering transformation $\varphi: \operatorname{or}(M) \rightarrow \operatorname{or}(M)$ changes the orientation, so $\varphi^{*} \omega$ is negatively oriented, i.e., $\int_{\operatorname{or}(M)} \varphi^{*} \omega<0$. Then $\omega-\varphi^{*} \omega \in \Omega_{-}^{m}(\operatorname{or}(M))$ and $\int_{\operatorname{or}(M)}\left(\omega-\varphi^{*} \omega\right)>0$, so $\left(H_{c}^{m}\right)_{-}(\operatorname{or}(M))=\mathbb{R}$ and $\left(H_{c}^{m}\right)_{+}(\operatorname{or}(M))=0$.

Since $\varphi^{*}$ is an algebra homomorphism, we have

$$
\begin{aligned}
& \Omega_{+}^{k}(\operatorname{or}(M)) \wedge\left(\Omega_{c}^{m-k}\right)_{+}(\operatorname{or}(M)) \subset\left(\Omega_{c}^{m}\right)_{+}(\operatorname{or}(M)), \\
& \Omega_{-}^{k}(\operatorname{or}(M)) \wedge\left(\Omega_{c}^{m-k}\right)_{-}(\operatorname{or}(M)) \subset\left(\Omega_{c}^{m}\right)_{+}(\operatorname{or}(M)) .
\end{aligned}
$$

From $\left(H_{c}^{m}\right)_{+}(\operatorname{or}(M))=0$ the first two results follows. The last two assertions then follow from this and $H^{k}(\operatorname{or}(M))=H_{+}^{k}(\operatorname{or}(M)) \oplus H_{-}^{k}(\operatorname{or}(M))$ and the analogous decomposition of $H_{c}^{k}(\operatorname{or}(M))$.

13.5. Theorem. For the real projective spaces we have

$$
\begin{aligned}
& H^{0}\left(\mathbb{R} \mathbb{P}^{n}\right)=\mathbb{R}, \\
& H^{k}\left(\mathbb{R P}^{n}\right)=0 \quad \text { for } 1 \leq k<n, \\
& H^{n}\left(\mathbb{R P}^{n}\right)= \begin{cases}\mathbb{R} & \text { for odd } n, \\
0 & \text { for even } n .\end{cases}
\end{aligned}
$$

Proof. The projection $\pi: S^{n} \rightarrow \mathbb{R} \mathbb{P}^{n}$ is a smooth covering mapping with two sheets; the covering transformation is the antipodal mapping $A: S^{n} \rightarrow$ $S^{n}, x \mapsto-x$. We put $\Omega_{+}\left(S^{n}\right)=\left\{\omega \in \Omega\left(S^{n}\right): A^{*} \omega=\omega\right\}$ and $\Omega_{-}\left(S^{n}\right)=$ $\left\{\omega \in \Omega\left(S^{n}\right): A^{*} \omega=-\omega\right\}$. The pullback $\pi^{*}: \Omega\left(\mathbb{R} \mathbb{P}^{n}\right) \rightarrow \Omega\left(S^{n}\right)$ is an embedding onto $\Omega_{+}\left(S^{n}\right)$.

Let $\Delta$ be the determinant function on the oriented Euclidean space $\mathbb{R}^{n+1}$. We identify $T_{x} S^{n}$ with $\{x\}^{\perp}$ in $\mathbb{R}^{n+1}$ and we consider the $n$-form $\omega_{S^{n}} \in$ 
$\Omega^{n}\left(S^{n}\right)$ which is given by $\left(\omega_{S^{n}}\right)_{x}\left(X_{1}, \ldots, X_{n}\right)=\Delta\left(x, X_{1}, \ldots, X_{n}\right)$. Then we have

$$
\begin{aligned}
\left(A^{*} \omega_{S^{n}}\right)_{x}\left(X_{1}, \ldots, X_{n}\right) & =\left(\omega_{S^{n}}\right)_{A(x)}\left(T_{x} A \cdot X_{1}, \ldots, T_{x} A \cdot X_{n}\right) \\
& =\left(\omega_{S^{n}}\right)_{-x}\left(-X_{1}, \ldots,-X_{n}\right) \\
& =\Delta\left(-x,-X_{1}, \ldots,-X_{n}\right) \\
& =(-1)^{n+1} \Delta\left(x, X_{1}, \ldots, X_{n}\right) \\
& =(-1)^{n+1}\left(\omega_{S^{n}}\right)_{x}\left(X_{1}, \ldots, X_{n}\right) .
\end{aligned}
$$

Since $\omega_{S^{n}}$ is invariant under the action of the group $S O(n+1, \mathbb{R})$, it must be the Riemann volume form, so

$$
\int_{S^{n}} \omega_{S^{n}}=\operatorname{vol}\left(S^{n}\right)=\frac{(n+1) \pi^{\frac{n+1}{2}}}{\Gamma\left(\frac{n+3}{2}\right)}= \begin{cases}\frac{2 \pi^{k}}{(k-1) !} & \text { for } n=2 k-1, \\ \frac{2^{k} \pi^{k-1}}{1 \cdot 3 \cdot 5 \ldots(2 k-3)} & \text { for } n=2 k-2 .\end{cases}
$$

Thus $\left[\omega_{S^{n}}\right] \in H^{n}\left(S^{n}\right)$ is a generator for the cohomology. We have $A^{*} \omega_{S^{n}}=$ $(-1)^{n+1} \omega_{S^{n}}$, so

$$
\omega_{S^{n}} \in \begin{cases}\Omega_{+}^{n}\left(S^{n}\right) & \text { for odd } n, \\ \Omega_{-}^{n}\left(S^{n}\right) & \text { for even } n .\end{cases}
$$

Thus $H^{n}\left(\mathbb{R} \mathbb{P}^{n}\right)=H^{n}\left(\Omega_{+}\left(S^{n}\right)\right)$ equals $H^{n}\left(S^{n}\right)=\mathbb{R}$ for odd $n$ and equals 0 for even $n$.

Since $\mathbb{R} \mathbb{P}^{n}$ is connected, we have $H^{0}\left(\mathbb{R P}^{n}\right)=\mathbb{R}$. For $1 \leq k<n$ we have $H^{k}\left(\mathbb{R P}^{n}\right)=H^{k}\left(\Omega_{+}\left(S^{n}\right)\right) \subset H^{k}\left(S^{n}\right)=0$.

13.6. Corollary. Let $M$ be a compact manifold. Then for all Betti numbers, we have $b_{k}(M):=\operatorname{dim}_{\mathbb{R}} H^{k}(M)<\infty$. If $M$ is compact and orientable of dimension $m$, we have $b_{k}(M)=b_{m-k}(M)$.

Proof. This follows from (13.2) and from Poincaré duality (12.16).

13.7. Euler-Poincaré characteristic. If $M$ is compact, then all Betti numbers are finite, so the Euler-Poincaré characteristic (see also (11.2)

$$
\chi_{M}=\sum_{k=0}^{\operatorname{dim} M}(-1)^{k} b_{k}(M)=f_{M}(-1)
$$

is defined.

Theorem. Let $M$ be a compact and orientable manifold of dimension $m$. Then we have:

(1) If $m$ is odd, then $\chi_{M}=0$.

(2) If $m=2 n$ for odd $n$, then $\chi_{M} \equiv b_{n}(M) \equiv 0 \bmod (2)$.

(3) If $m=4 k$, then $\chi_{M} \equiv b_{2 k}(M) \equiv \operatorname{signature}\left(P_{M}^{2 k}\right) \bmod (2)$. 
Proof. From (13.6) we have $b_{q}(M)=b_{m-q}(M)$. Thus the Euler-Poincaré characteristic is $\chi_{M}=\sum_{q=0}^{m}(-1)^{q} b_{q}=\sum_{q=0}^{m}(-1)^{q} b_{m-q}=(-1)^{m} \chi_{M}$ which implies (1).

If $m=2 n$, we have $\chi_{M}=\sum_{q=0}^{2 n}(-1)^{q} b_{q}=2 \sum_{q=0}^{n-1}(-1)^{q} b_{q}+(-1)^{n} b_{n}$, so $\chi_{M} \equiv b_{n} \bmod (2)$. In general we have for a compact oriented manifold

$P_{M}^{q}([\alpha],[\beta])=\int_{M} \alpha \wedge \beta=(-1)^{q(m-q)} \int_{M} \beta \wedge \alpha=(-1)^{q(m-q)} P_{M}^{m-q}([\beta],[\alpha])$.

For odd $n$ and $m=2 n$ we see that $P_{M}^{n}$ is a skew-symmetric nondegenerate bilinear form on $H^{n}(M)$, so $b_{n}$ must be even (see (4.7) or (31.4) below) which implies (2).

(3) If $m=4 k$, then $P_{M}^{2 k}$ is a nondegenerate symmetric bilinear form on $H^{2 k}(M)$, an inner product. By the signature of a nondegenerate symmetric inner product one means the number of positive eigenvalues minus the number of negative eigenvalues, so the number $\operatorname{dim} H^{2 k}(M)_{+}-\operatorname{dim} H^{2 k}(M)_{-}=$: $a_{+}-a_{-}$, but since $H^{2 k}(M)_{+} \oplus H^{2 k}(M)_{-}=H^{2 k}(M)$, we have $a_{+}+a_{-}=b_{2 k}$, so $a_{+}-a_{-}=b_{2 k}-2 a_{-} \equiv b_{2 k} \bmod (2)$.

13.8. The mapping degree. Let $M$ and $N$ be smooth compact oriented manifolds, both of the same dimension $m$. Then for any smooth mapping $f: M \rightarrow N$ there is a real number $\operatorname{deg} f$, called the degree of $f$, which is given in the bottom row of the diagram

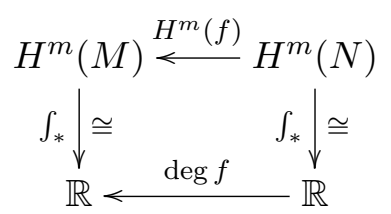

where the vertical arrows are isomorphisms by (12.19) and where $\operatorname{deg} f$ is the linear mapping given by multiplication with that number. So we also have the defining relation

$$
\int_{M} f^{*} \omega=\operatorname{deg} f \int_{N} \omega \quad \text { for all } \omega \in \Omega^{m}(N) .
$$

13.9. Lemma. The mapping degree deg has the following properties:

(1) $\operatorname{deg}(f \circ g)=\operatorname{deg} f \cdot \operatorname{deg} g$, and $\operatorname{deg}\left(I d_{M}\right)=1$.

(2) If $f, g: M \rightarrow N$ are (smoothly) homotopic, then $\operatorname{deg} f=\operatorname{deg} g$.

(3) If $\operatorname{deg} f \neq 0$, then $f$ is surjective.

(4) If $f: M \rightarrow M$ is a diffeomorphism, then $\operatorname{deg} f=1$ if $f$ respects the orientation and $\operatorname{deg} f=-1$ if $f$ reverses the orientation. 
Proof. (11) and (2) are clear. (3) If $f(M) \neq N$, we choose a bump $m$-form $\omega$ on $N$ with support in the open set $N \backslash f(M)$. Then $f^{*} \omega=0$ so we have $0=\int_{M} f^{*} \omega=\operatorname{deg} f \int_{N} \omega$. Since $\int_{N} \omega \neq 0$, we get $\operatorname{deg} f=0$.

(44) follows either directly from the definition of the integral (10.7) or from (13.11) below.

13.10. Examples on spheres. Let $f \in O(n+1, \mathbb{R})$ and restrict it to a mapping $f: S^{n} \rightarrow S^{n}$. Then $\operatorname{deg} f=\operatorname{det} f$. This follows from the description of the volume form on $S^{n}$ given in the proof of (13.5).

Let $f, g: S^{n} \rightarrow S^{n}$ be smooth mappings. If $f(x) \neq-g(x)$ for all $x \in S^{n}$, then the mappings $f$ and $g$ are smoothly homotopic: The homotopy moves $f(x)$ along the shorter arc of the geodesic (big circle) to $g(x)$. So $\operatorname{deg} f=$ $\operatorname{deg} g$.

If $f(x) \neq-x$ for all $x \in S^{n}$, then $f$ is homotopic to $I d_{S^{n}}$, so $\operatorname{deg} f=1$.

If $f(x) \neq x$ for all $x \in S^{n}$, then $f$ is homotopic to $-I d_{S^{n}}$, so $\operatorname{deg} f=(-1)^{n+1}$.

The hairy ball theorem says that on $S^{n}$ for even $n$ each vector field vanishes somewhere. This can be seen as follows. The tangent bundle of the sphere is

$$
T S^{n}=\left\{(x, y) \in \mathbb{R}^{n+1} \times \mathbb{R}^{n+1}:|x|^{2}=1,\langle x, y\rangle=0\right\},
$$

so a vector field without zeros is a mapping $x \mapsto(x, g(x))$ with $g(x) \perp x$; then $f(x):=g(x) /|g(x)|$ defines a smooth mapping $f: S^{n} \rightarrow S^{n}$ with $f(x) \perp x$ for all $x$. So $f(x) \neq x$ for all $x$; thus $\operatorname{deg} f=(-1)^{n+1}=-1$. But also $f(x) \neq-x$ for all $x$, so $\operatorname{deg} f=1$, a contradiction.

Finally we consider the unit circle $S^{1} \stackrel{i}{\longrightarrow} \mathbb{C}=\mathbb{R}^{2}$. Its volume form is given by $\omega:=i^{*}(x d y-y d x)=i^{*} \frac{x d y-y d x}{x^{2}+y^{2}}$; obviously we have $\int_{S^{1}} x d y-y d x=2 \pi$. Now let $f: S^{1} \rightarrow S^{1}$ be smooth, $f(t)=(x(t), y(t))$ for $0 \leq t \leq 2 \pi$. Then

$$
\operatorname{deg} f=\frac{1}{2 \pi} \int_{S^{1}} f^{*}(x d y-y d x)
$$

is the winding number about 0 from complex analysis.

13.11. The mapping degree is an integer. Let $f: M \rightarrow N$ be a smooth mapping between compact oriented manifolds of dimension $m$. Let $b \in N$ be a regular value for $f$ which exists by Sard's theorem; see (1.18). Then for each $x \in f^{-1}(b)$ the tangent mapping $T_{x} f$ is invertible, so $f$ is a diffeomorphism near $x$. Thus $f^{-1}(b)$ is a finite set, since $M$ is compact. We define the mapping $\varepsilon: M \rightarrow\{-1,0,1\}$ by

$$
\varepsilon(x)= \begin{cases}0 & \text { if } T_{x} f \text { is not invertible } \\ 1 & \text { if } T_{x} f \text { is invertible and respects orientations } \\ -1 & \text { if } T_{x} f \text { is invertible and changes orientations }\end{cases}
$$


13.12. Theorem. In the setting of (13.11), if $b \in N$ is a regular value for $f$, then

$$
\operatorname{deg} f=\sum_{x \in f^{-1}(b)} \varepsilon(x) .
$$

In particular $\operatorname{deg} f$ is always an integer.

Proof. The proof is the same as for lemma (12.12) with obvious changes.

\section{Lie Groups III. Analysis on Lie Groups}

\section{Invariant Integration on Lie Groups}

14.1. Invariant differential forms on Lie groups. Let $G$ be a real Lie group of dimension $n$ with Lie algebra $\mathfrak{g}$. Then the tangent bundle of $G$ is a trivial vector bundle, see (6.7), so $G$ is orientable. Recall from section (4) the notation:

$$
\begin{aligned}
& \mu: G \times G \rightarrow G, \text { multiplication, } \mu(x, y)=x . y . \\
& \mu_{a}: G \rightarrow G, \text { left translation, } \mu_{a}(x)=a . x . \\
& \mu^{a}: G \rightarrow G, \text { right translation, } \mu^{a}(x)=x . a . \\
& \nu: G \rightarrow G, \text { inversion, } \nu(x)=x^{-1} . \\
& e \in G, \text { the unit element. }
\end{aligned}
$$

A differential form $\omega \in \Omega^{n}(G)$ is called left invariant if

$$
\mu_{x}^{*} \omega=\omega \quad \text { for all } \quad x \in G .
$$

Then $\omega$ is uniquely determined by its value

$$
\omega_{e} \in \bigwedge^{n} T^{*} G=\bigwedge^{n} \mathfrak{g}^{*} .
$$

For each determinant function $\Delta$ on $\mathfrak{g}$ there is a unique left invariant $n$-form $L_{\Delta}$ on $G$ which is given by

$$
\begin{gathered}
\left(L_{\Delta}\right)_{x}\left(X_{1}, \ldots, X_{n}\right):=\Delta\left(T_{x}\left(\mu_{x^{-1}}\right) \cdot X_{1}, \ldots, T_{x}\left(\mu_{x^{-1}}\right) \cdot X_{n}\right), \\
\left(L_{\Delta}\right)_{x}=T_{x}\left(\mu_{x^{-1}}\right)^{*} \Delta .
\end{gathered}
$$

Likewise there is a unique right invariant $n$-form $R_{\Delta}$ which is given by

$$
\left(R_{\Delta}\right)_{x}\left(X_{1}, \ldots, X_{n}\right):=\Delta\left(T_{x}\left(\mu^{x^{-1}}\right) . X_{1}, \ldots, T_{x}\left(\mu^{x^{-1}}\right) \cdot X_{n}\right) .
$$

14.2. Lemma. We have for all $a \in G$

$$
\begin{aligned}
\left(\mu^{a}\right)^{*} L_{\Delta} & =\operatorname{det}\left(\operatorname{Ad}\left(a^{-1}\right)\right) L_{\Delta}, \\
\left(\mu_{a}\right)^{*} R_{\Delta} & =\operatorname{det}(\operatorname{Ad}(a)) R_{\Delta}, \\
\left(R_{\Delta}\right)_{a} & =\operatorname{det}(\operatorname{Ad}(a))\left(L_{\Delta}\right)_{a} .
\end{aligned}
$$


Proof. We compute as follows:

$$
\begin{aligned}
& \left(\left(\mu^{a}\right)^{*} L_{\Delta}\right)_{x}\left(X_{1}, \ldots, X_{n}\right)=\left(L_{\Delta}\right)_{x a}\left(T_{x}\left(\mu^{a}\right) \cdot X_{1}, \ldots, T_{x}\left(\mu^{a}\right) \cdot X_{n}\right) \\
& =\Delta\left(T_{x a}\left(\mu_{(x a)^{-1}}\right) \cdot T_{x}\left(\mu^{a}\right) \cdot X_{1}, \ldots, T_{x a}\left(\mu_{(x a)^{-1}}\right) \cdot T_{x}\left(\mu^{a}\right) \cdot X_{n}\right) \\
& =\Delta\left(T_{a}\left(\mu_{a^{-1}}\right) \cdot T_{x a}\left(\mu_{x^{-1}}\right) \cdot T_{x}\left(\mu^{a}\right) \cdot X_{1}, \ldots, T_{a}\left(\mu_{a^{-1}}\right) \cdot T_{x a}\left(\mu_{x^{-1}}\right) \cdot T_{x}\left(\mu^{a}\right) \cdot X_{n}\right) \\
& =\Delta\left(T_{a}\left(\mu_{a^{-1}}\right) \cdot T_{e}\left(\mu^{a}\right) \cdot T_{x}\left(\mu_{x^{-1}}\right) \cdot X_{1}, \ldots, T_{a}\left(\mu_{a^{-1}}\right) \cdot T_{e}\left(\mu^{a}\right) \cdot T_{x}\left(\mu_{x^{-1}}\right) \cdot X_{n}\right) \\
& =\Delta\left(\operatorname{Ad}\left(a^{-1}\right) \cdot T_{x}\left(\mu_{x^{-1}}\right) \cdot X_{1}, \ldots, \operatorname{Ad}\left(a^{-1}\right) \cdot T_{x}\left(\mu_{x^{-1}}\right) \cdot X_{n}\right) \\
& =\operatorname{det}\left(\operatorname{Ad}\left(a^{-1}\right)\right) \Delta\left(T_{x}\left(\mu_{x^{-1}}\right) \cdot X_{1}, \ldots, T_{x}\left(\mu_{x^{-1}}\right) \cdot X_{n}\right) \\
& =\operatorname{det}\left(\operatorname{Ad}\left(a^{-1}\right)\right)\left(L_{\Delta}\right)_{x}\left(X_{1}, \ldots, X_{n}\right) \text {, } \\
& \left(\left(\mu_{a}\right)^{*} R_{\Delta}\right)_{x}\left(X_{1}, \ldots, X_{n}\right)=\left(R_{\Delta}\right)_{a x}\left(T_{x}\left(\mu_{a}\right) \cdot X_{1}, \ldots, T_{x}\left(\mu_{a}\right) \cdot X_{n}\right) \\
& =\Delta\left(T_{a x}\left(\mu^{(a x)^{-1}}\right) \cdot T_{x}\left(\mu_{a}\right) \cdot X_{1}, \ldots, T_{a x}\left(\mu^{(a x)^{-1}}\right) \cdot T_{x}\left(\mu_{a}\right) \cdot X_{n}\right) \\
& =\Delta\left(T_{a}\left(\mu^{a^{-1}}\right) \cdot T_{a x}\left(\mu^{x^{-1}}\right) \cdot T_{x}\left(\mu_{a}\right) \cdot X_{1}, \ldots, T_{a}\left(\mu^{a^{-1}}\right) \cdot T_{a x}\left(\mu^{x^{-1}}\right) \cdot T_{x}\left(\mu_{a}\right) \cdot X_{n}\right) \\
& =\Delta\left(T_{a}\left(\mu^{a^{-1}}\right) \cdot T_{e}\left(\mu_{a}\right) \cdot T_{x}\left(\mu^{x^{-1}}\right) \cdot X_{1}, \ldots, T_{a}\left(\mu^{a^{-1}}\right) \cdot T_{e}\left(\mu_{a}\right) \cdot T_{x}\left(\mu^{x^{-1}}\right) \cdot X_{n}\right) \\
& =\Delta\left(\operatorname{Ad}(a) \cdot T_{x}\left(\mu^{x^{-1}}\right) \cdot X_{1}, \ldots, \operatorname{Ad}(a) \cdot T_{x}\left(\mu^{x^{-1}}\right) \cdot X_{n}\right) \\
& =\operatorname{det}(\operatorname{Ad}(a)) \Delta\left(T_{x}\left(\mu^{x^{-1}}\right) \cdot X_{1}, \ldots, T_{x}\left(\mu^{x^{-1}}\right) \cdot X_{n}\right) \\
& =\operatorname{det}(\operatorname{Ad}(a))\left(R_{\Delta}\right)_{x}\left(X_{1}, \ldots, X_{n}\right), \\
& \operatorname{det}(\operatorname{Ad}(a))\left(L_{\Delta}\right)_{a}\left(X_{1}, \ldots, X_{n}\right) \\
& =\operatorname{det}(\operatorname{Ad}(a)) \Delta\left(T_{a}\left(\mu_{a^{-1}}\right) \cdot X_{1}, \ldots, T_{a}\left(\mu_{a^{-1}}\right) \cdot X_{n}\right) \\
& =\Delta\left(\operatorname{Ad}(a) \cdot T_{a}\left(\mu_{a^{-1}}\right) \cdot X_{1}, \ldots, \operatorname{Ad}(a) \cdot T_{a}\left(\mu_{a^{-1}}\right) \cdot X_{n}\right) \\
& =\Delta\left(T_{a}\left(\mu^{a^{-1}}\right) \cdot T_{e}\left(\mu_{a}\right) \cdot T_{a}\left(\mu_{a^{-1}}\right) \cdot X_{1}, \ldots, T_{a}\left(\mu^{a^{-1}}\right) \cdot T_{e}\left(\mu_{a}\right) \cdot T_{a}\left(\mu_{a^{-1}}\right) \cdot X_{n}\right) \\
& =\Delta\left(T_{a}\left(\mu^{a^{-1}}\right) \cdot X_{1}, \ldots, T_{a}\left(\mu^{a^{-1}}\right) \cdot X_{n}\right)=\left(R_{\Delta}\right)_{a}\left(X_{1}, \ldots, X_{n}\right) .
\end{aligned}
$$

14.3. Corollary and Definition. The Lie group $G$ admits a bi-invariant (i.e., left and right invariant) $n$-form if and only if $\operatorname{det}(\operatorname{Ad}(a))=1$ for all $a \in G$.

The Lie group $G$ is called unimodular if $|\operatorname{det}(\operatorname{Ad}(a))|=1$ for all $a \in G$.

Note that $\operatorname{det}(\operatorname{Ad}(a))>0$ if $G$ is connected.

Proof. This is obvious from lemma (14.2).

14.4. Haar measure. We orient the Lie group $G$ by a left invariant $n$-form $L_{\Delta}$ where $n=\operatorname{dim}(G)$. If $f \in C_{c}^{\infty}(G, \mathbb{R})$ is a smooth function with compact support on $G$, then the integral $\int_{G} f L_{\Delta}$ is defined and we have

$$
\int_{G}\left(\mu_{a}^{*} f\right) L_{\Delta}=\int_{G} \mu_{a}^{*}\left(f L_{\Delta}\right)=\int_{G} f L_{\Delta}
$$


because $\mu_{a}: G \rightarrow G$ is an orientation preserving diffeomorphism of $G$. Thus $f \mapsto \int_{G} f L_{\Delta}$ is a left invariant integration on $G$, which is also denoted by $\int_{G} f(x) d_{L} x$ and which gives rise to a left invariant measure on $G$, the socalled left Haar measure. It is unique up to a multiplicative constant, since $\operatorname{dim}\left(\bigwedge^{n} \mathfrak{g}^{*}\right)=1$. In the other notation the left invariance looks like

$$
\int_{G} f(a x) d_{L} x=\int_{G} f(x) d_{L} x \text { for all } f \in C_{c}^{\infty}(G, \mathbb{R}), a \in G .
$$

From lemma (14.2.1) we have

$$
\int_{G}\left(\left(\mu^{a}\right)^{*} f\right) L_{\Delta}=\operatorname{det}(\operatorname{Ad}(a)) \int_{G}\left(\mu^{a}\right)^{*}\left(f L_{\Delta}\right)=|\operatorname{det}(\operatorname{Ad}(a))| \int_{G} f L_{\Delta},
$$

since the mapping $\mu^{a}$ is orientation preserving if and only if $\operatorname{det}(\operatorname{Ad}(a))>0$. So a left invariant Haar measure is also a right invariant one if and only if the Lie group $G$ is unimodular.

14.5. Lemma. Each compact Lie group is unimodular.

Proof. The mapping det $\circ \mathrm{Ad}: G \rightarrow G L(1, \mathbb{R})$ is a homomorphism of Lie groups, so its image is a compact subgroup of $G L(1, \mathbb{R})$. Thus $\operatorname{det}(\operatorname{Ad}(G))$ equals $\{1\}$ or $\{1,-1\}$. In both cases we have $|\operatorname{det}(\operatorname{Ad}(a))|=1$ for all $a \in G$.

\section{Analysis for Mappings between Lie Groups}

14.6. Definition. Let $G$ and $H$ be Lie groups with Lie algebras $\mathfrak{g}$ and $\mathfrak{h}$, respectively, and let $f: G \rightarrow H$ be a smooth mapping. Then we define the mapping $D f: G \rightarrow L(\mathfrak{g}, \mathfrak{h})$ by

$$
D f(x):=T_{f(x)}\left(\left(\mu^{f(x)}\right)^{-1}\right) \cdot T_{x} f \cdot T_{e}\left(\mu^{x}\right)=\delta f(x) \cdot T_{e}\left(\mu^{x}\right),
$$

and we call it the right trivialized derivative of $f$.

14.7. Lemma. The chain rule: For smooth $g: K \rightarrow G$ and $f: G \rightarrow H$ we have

$$
D(f \circ g)(x)=D f(g(x)) \circ D g(x) .
$$

The product rule: For $f, h \in C^{\infty}(G, H)$ we have

$$
D(f h)(x)=D f(x)+\operatorname{Ad}(f(x)) D h(x) .
$$

Proof. We compute as follows:

$$
\begin{aligned}
& D(f \circ g)(x)=T\left(\mu^{f(g(x))^{-1}}\right) \cdot T_{x}(f \circ g) \cdot T_{e}\left(\mu^{x}\right) \\
& \quad=T\left(\mu^{f(g(x))^{-1}}\right) \cdot T_{g(x)}(f) \cdot T_{e}\left(\mu^{g(x)}\right) \cdot T\left(\mu^{g(x)^{-1}}\right) \cdot T_{x}(g) \cdot T_{e}\left(\mu^{x}\right) \\
& \quad=D f(g(x)) \cdot D g(x),
\end{aligned}
$$




$$
\begin{aligned}
& =T\left(\mu^{f(x)^{-1}}\right) \cdot T\left(\mu^{h(x)^{-1}}\right) \cdot T_{f(x), h(x)} \mu \cdot\left(T_{x} f \cdot T_{e}\left(\mu^{x}\right), T_{x} h \cdot T_{e}\left(\mu^{x}\right)\right) \\
& =T\left(\mu^{f(x)^{-1}}\right) \cdot T\left(\mu^{h(x)^{-1}}\right) \cdot\left(T\left(\mu^{h(x)}\right) \cdot T_{x} f \cdot T_{e}\left(\mu^{x}\right)+T\left(\mu_{f(x)}\right) \cdot T_{x} h \cdot T_{e}\left(\mu^{x}\right)\right) \\
& =T\left(\mu^{f(x)^{-1}}\right) \cdot T_{x} f \cdot T_{e}\left(\mu^{x}\right)+T\left(\mu^{f(x)^{-1}}\right) \cdot T\left(\mu_{f(x)}\right) \cdot T\left(\mu^{h(x)^{-1}}\right) \cdot T_{x} h \cdot T_{e}\left(\mu^{x}\right) \\
& =D f(x)+\operatorname{Ad}(f(x)) \cdot D h(x) .
\end{aligned}
$$

14.8. Inverse function theorem. Let $f: G \rightarrow H$ be smooth and for some $x \in G$ let $D f(x): \mathfrak{g} \rightarrow \mathfrak{h}$ be invertible. Then $f$ is a diffeomorphism from a suitable neighborhood of $x$ in $G$ onto a neighborhood of $f(x)$ in $H$, and for the derivative we have $D\left(f^{-1}\right)(f(x))=(D f(x))^{-1}$.

Proof. This follows from the usual inverse function theorem.

14.9. Lemma. Let $f \in C^{\infty}(G, G)$ and let $\Delta \in \bigwedge^{\operatorname{dim} G} \mathfrak{g}^{*}$ be a determinant function on $\mathfrak{g}$. Then we have for all $x \in G$,

$$
\left(f^{*} R_{\Delta}\right)_{x}=\operatorname{det}(D f(x))\left(R_{\Delta}\right)_{x}
$$

Proof. Let $\operatorname{dim} G=n$. We compute as follows:

$$
\begin{aligned}
\left(f^{*}\right. & \left.R_{\Delta}\right)_{x}\left(X_{1}, \ldots, X_{n}\right)=\left(R_{\Delta}\right)_{f(x)}\left(T_{x} f \cdot X_{1}, \ldots, T_{x} f \cdot X_{n}\right) \\
& =\Delta\left(T\left(\mu^{f(x)^{-1}}\right) \cdot T_{x} f \cdot X_{1}, \ldots\right) \\
& =\Delta\left(T\left(\mu^{f(x)^{-1}}\right) \cdot T_{x} f \cdot T\left(\mu^{x}\right) \cdot T\left(\mu^{x^{-1}}\right) \cdot X_{1}, \ldots\right) \\
& =\Delta\left(D f(x) \cdot T\left(\mu^{x^{-1}}\right) \cdot X_{1}, \ldots\right) \\
& =\operatorname{det}(D f(x)) \Delta\left(T\left(\mu^{x^{-1}}\right) \cdot X_{1}, \ldots\right) \\
& =\operatorname{det}(D f(x))\left(R_{\Delta}\right)_{x}\left(X_{1}, \ldots, X_{n}\right) .
\end{aligned}
$$

14.10. Theorem. Transformation formula for multiple integrals. Let $f: G \rightarrow G$ be a diffeomorphism, and let $\Delta \in \bigwedge^{\operatorname{dim} G} \mathfrak{g}^{*}$. Then for any $g \in C_{c}^{\infty}(G, \mathbb{R})$ we have

$$
\int_{G} g(f(x))|\operatorname{det}(D f(x))| d_{R} x=\int_{G} g(y) d_{R} y,
$$

where $d_{R} x$ is the right Haar measure, given by $R_{\Delta}$.

Proof. We consider the locally constant function $\varepsilon(x)=\operatorname{sign} \operatorname{det}(D f(x))$ which is 1 on those connected components where $f$ respects the orientation and is -1 on the other components. Then the integral is the sum of all integrals over the connected components and we may investigate each one separately, so let us restrict attention to the component $G_{0}$ of the identity. By a right translation (which does not change the integrals) we may assume 
that $f\left(G_{0}\right)=G_{0}$. So finally let us assume without loss of generality that $G$ is connected, so that $\varepsilon$ is constant. Then by lemma (14.9) we have

$$
\begin{aligned}
\int_{G} g R_{\Delta} & =\varepsilon \int_{G} f^{*}\left(g R_{\Delta}\right)=\varepsilon \int_{G} f^{*}(g) f^{*}\left(R_{\Delta}\right) \\
& =\int_{G}(g \circ f) \varepsilon \operatorname{det}(D f) R_{\Delta}=\int_{G}(g \circ f)|\operatorname{det}(D f)| R_{\Delta} .
\end{aligned}
$$

14.11. Theorem. Let $G$ be a compact and connected Lie group, and let $f \in C^{\infty}(G, G)$ and $\Delta \in \bigwedge^{\operatorname{dim} G} \mathfrak{g}^{*}$. Then we have for $g \in C^{\infty}(G)$,

$$
\begin{gathered}
\operatorname{deg} f \int_{G} g R_{\Delta}=\int_{G}(g \circ f) \operatorname{det}(D f) R_{\Delta}, \text { or } \\
\operatorname{deg} f \int_{G} g(y) d_{R} y=\int_{G} g(f(x)) \operatorname{det}(D f(x)) d_{R} x .
\end{gathered}
$$

Here $\operatorname{deg} f$, the mapping degree of $f$, see (13.8), is an integer.

Proof. From lemma (14.9) we have $f^{*} R_{\Delta}=\operatorname{det}(D f) R_{\Delta}$. Using this and the defining relation from $(13.8)$ for $\operatorname{deg} f$, we may compute as follows:

$$
\begin{aligned}
\operatorname{deg} f \int_{G} g R_{\Delta} & =\int_{G} f^{*}\left(g R_{\Delta}\right)=\int_{G} f^{*}(g) f^{*}\left(R_{\Delta}\right) \\
& =\int_{G}(g \circ f) \operatorname{det}(D f) R_{\Delta} \cdot \square
\end{aligned}
$$

14.12. Examples. Let $G$ be a compact connected Lie group.

(1) If $f=\mu^{a}: G \rightarrow G$, then $D\left(\mu^{a}\right)(x)=I d_{\mathfrak{g}}$. From theorem (14.11) we get $\int_{G} g R_{\Delta}=\int_{G}\left(g \circ \mu^{a}\right) R_{\Delta}$, the right invariance of the right Haar measure.

(2) If $f=\mu_{a}: G \rightarrow G$, then $D\left(\mu_{a}\right)(x)=T\left(\mu^{(a x)^{-1}}\right) \cdot T_{x}\left(\mu_{a}\right) \cdot T_{e}\left(\mu^{x}\right)=\operatorname{Ad}(a)$. So the last two results give $\int_{G} g R_{\Delta}=\int_{G}\left(g \circ \mu_{a}\right)|\operatorname{det} \operatorname{Ad}(a)| R_{\Delta}$ which we already know from (14.4).

(3) If $f(x)=x^{2}=\mu(x, x)$, we have

$$
\begin{aligned}
D f(x) & =T_{x^{2}}\left(\mu^{x^{-2}}\right) \cdot T_{(x, x)} \mu \cdot\left(T_{e}\left(\mu^{x}\right), T_{e}\left(\mu^{x}\right)\right) \\
& =T_{x}\left(\mu^{x^{-1}}\right) \cdot T_{x^{2}}\left(\mu^{x^{-1}}\right)\left(T_{x}\left(\mu_{x}\right) \cdot T_{e}\left(\mu^{x}\right)+T_{x}\left(\mu^{x}\right) \cdot T_{e}\left(\mu^{x}\right)\right) \\
& =\operatorname{Ad}(x)+I d_{\mathfrak{g}} .
\end{aligned}
$$

Let us now suppose that $\int_{G} R_{\Delta}=1$; then we get

$$
\begin{gathered}
\operatorname{deg}\left((\quad)^{2}\right)=\operatorname{deg}\left((\quad)^{2}\right) \int_{G} R_{\Delta}=\int_{G} \operatorname{det}\left(I d_{\mathfrak{g}}+\operatorname{Ad}(x)\right) d_{R} x \\
\int_{G} g\left(x^{2}\right) \operatorname{det}\left(I d_{\mathfrak{g}}+\operatorname{Ad}(x)\right) d_{R} x=\int_{G} \operatorname{det}\left(I d_{\mathfrak{g}}+\operatorname{Ad}(x)\right) d_{R} x \int_{G} g(x) d_{R} x .
\end{gathered}
$$


(4) Let $f(x)=x^{k}$ for $k \in \mathbb{N}$, and suppose that $\int_{G} d_{R} x=1$. Then we claim that

$$
D\left((\quad)^{k}\right)(x)=\sum_{i=0}^{k-1} \operatorname{Ad}\left(x^{i}\right) .
$$

This follows from induction, starting from example (3) above, since

$$
\begin{aligned}
D\left((\quad)^{k}\right)(x) & =D\left(I d_{G} \cdot(\quad)^{k-1}\right)(x) \\
& =D\left(I d_{G}\right)(x)+\operatorname{Ad}(x) \cdot D\left((\quad)^{k-1}\right)(x) \quad \text { by (14.7) } \\
& =I d_{\mathfrak{g}}+\operatorname{Ad}(x)\left(\sum_{i=0}^{k-2} \operatorname{Ad}\left(x^{i}\right)\right)=\sum_{i=0}^{k-1} \operatorname{Ad}\left(x^{i}\right) .
\end{aligned}
$$

We conclude that

$$
\operatorname{deg}(\quad)^{k}=\int_{G} \operatorname{det}\left(\sum_{i=0}^{k-1} \operatorname{Ad}\left(x^{i}\right)\right) d_{R} x .
$$

If $G$ is abelian, we have $\operatorname{deg}(\quad)^{k}=k$ since then $\operatorname{Ad}(x)=I d_{\mathfrak{g}}$.

(5) Let $f(x)=\nu(x)=x^{-1}$. Then we have $D \nu(x)=T \mu^{\nu(x)^{-1}} \cdot T_{x} \nu \cdot T_{e} \mu^{x}=$ $-\operatorname{Ad}\left(x^{-1}\right)$. Using this, we see that the result in (4) holds also for negative $k$ if the summation is interpreted in the right way:

$$
D\left((\quad)^{-k}\right)(x)=\sum_{i=-k+1}^{0} \operatorname{Ad}\left(x^{i}\right)=-\sum_{i=0}^{k-1} \operatorname{Ad}\left(x^{-i}\right) .
$$

\section{Cohomology of Compact Connected Lie Groups}

14.13. Let $G$ be a connected Lie group with Lie algebra $\mathfrak{g}$. The de Rham cohomology of $G$ is the cohomology of the graded differential algebra $(\Omega(G), d)$. We will investigate now what is contributed by the subcomplex of the left invariant differential forms.

Definition. A differential form $\omega \in \Omega(G)$ is called left invariant if $\mu_{a}^{*} \omega=\omega$ for all $a \in G$. We denote by $\Omega_{L}(G)$ the subspace of all left invariant forms. Clearly the mapping

$$
\begin{aligned}
L & : \bigwedge \mathfrak{g}^{*} \rightarrow \Omega_{L}(G), \\
\left(L_{\omega}\right)_{x}\left(X_{1}, \ldots, X_{k}\right) & =\omega\left(T\left(\mu_{x^{-1}}\right) \cdot X_{1}, \ldots, T\left(\mu_{x^{-1}}\right) \cdot X_{k}\right),
\end{aligned}
$$

is a linear isomorphism. Since $\mu_{a}^{*} \circ d=d \circ \mu_{a}^{*}$, the space $\left(\Omega_{L}(G), d\right)$ is a graded differential subalgebra of $(\Omega(G), d)$.

We shall also need the representation $\widetilde{\mathrm{Ad}}: G \rightarrow G L\left(\bigwedge \mathfrak{g}^{*}\right)$ which is given by $\widetilde{\operatorname{Ad}}(a)=\bigwedge\left(\operatorname{Ad}\left(a^{-1}\right)^{*}\right)$ or

$$
(\widetilde{\operatorname{Ad}}(a) \omega)\left(X_{1}, \ldots, X_{k}\right)=\omega\left(\operatorname{Ad}\left(a^{-1}\right) \cdot X_{1}, \ldots, \operatorname{Ad}\left(a^{-1}\right) \cdot X_{k}\right) \text {. }
$$


14.14. Lemma. (1) Via the isomorphism $L: \bigwedge \mathfrak{g}^{*} \rightarrow \Omega_{L}(G)$ the exterior differential $d$ has the following form on $\wedge \mathfrak{g}^{*}$ :

$$
d \omega\left(X_{0}, \ldots, X_{k}\right)=\sum_{0 \leq i<j \leq k}(-1)^{i+j} \omega\left(\left[X_{i}, X_{j}\right], X_{0}, \ldots, \widehat{X}_{i}, \ldots \widehat{X}_{j}, \ldots, X_{k}\right)
$$

where $\omega \in \bigwedge^{k} \mathfrak{g}^{*}$ and $X_{i} \in \mathfrak{g}$.

(2) For $X \in \mathfrak{g}$ we have $i\left(L_{X}\right) \Omega_{L}(G) \subset \Omega_{L}(G)$ and $\mathcal{L}_{L_{X}} \Omega_{L}(G) \subset \Omega_{L}(G)$. Thus we have induced mappings

$$
\begin{aligned}
& i_{X}: \bigwedge^{k} \mathfrak{g}^{*} \rightarrow \bigwedge^{k-1} \mathfrak{g}^{*} \\
&\left(i_{X} \omega\right)\left(X_{1}, \ldots, X_{k-1}\right)=\omega\left(X, X_{1}, \ldots, X_{k-1}\right) \\
& \mathcal{L}_{X}: \bigwedge^{k} \mathfrak{g}^{*} \rightarrow \bigwedge^{k} \mathfrak{g}^{*} \\
&\left(\mathcal{L}_{X} \omega\right)\left(X_{1}, \ldots, X_{k}\right)=\sum_{i=1}^{k}(-1)^{i} \omega\left(\left[X, X_{i}\right], X_{1}, \ldots, \widehat{X}_{i}, \ldots X_{k}\right) .
\end{aligned}
$$

(3) These mappings satisfy all the properties from section (9), in particular

$$
\begin{array}{ll}
\mathcal{L}_{X}=i_{X} \circ d+d \circ i_{X}, & \text { see }(9.9 .2), \\
\mathcal{L}_{X} \circ d=d \circ \mathcal{L}_{X}, & \text { see }(9.9 .5), \\
{\left[\mathcal{L}_{X}, \mathcal{L}_{Y}\right]=\mathcal{L}_{[X, Y]},} & \text { see }(9.6 .3) . \\
{\left[\mathcal{L}_{X}, i_{Y}\right]=i_{[X, Y]},} & \text { see }(9.7 .3) .
\end{array}
$$

(4) The representation $\widetilde{\mathrm{Ad}}: G \rightarrow G L\left(\bigwedge \mathfrak{g}^{*}\right)$ has derivative $T_{e} \widetilde{\mathrm{Ad}} . X=\mathcal{L}_{X}$.

Proof. For $\omega \in \bigwedge^{k} \mathfrak{g}^{*}$ and $X_{i} \in \mathfrak{g}$ the function

$$
\begin{aligned}
\left(L_{\omega}\right)_{x}\left(L_{X_{1}}(x), \ldots, L_{X_{k}}(x)\right) & =\omega\left(T\left(\mu_{x^{-1}}\right) \cdot L_{X_{1}}(x), \ldots\right) \\
& =\omega\left(T\left(\mu_{x^{-1}}\right) \cdot T\left(\mu_{x}\right) \cdot X_{1}, \ldots\right) \\
& =\omega\left(X_{1}, \ldots, X_{k}\right)
\end{aligned}
$$

is constant in $x$. This implies already that $i\left(L_{X}\right) \Omega_{L}(G) \subset \Omega_{L}(G)$ and the form of $i_{X}$ in (2). Then by (9.8.2) we have

$$
\begin{aligned}
& (d \omega)\left(X_{0}, \ldots, X_{k}\right)=\left(d L_{\omega}\right)\left(L_{X_{0}}, \ldots, L_{X_{k}}\right)(e) \\
& =\sum_{i=0}^{k}(-1)^{i} L_{X_{i}}(e)\left(\omega\left(X_{0}, \ldots \widehat{X}_{i}, \ldots X_{k}\right)\right) \\
& \quad+\sum_{0 \leq i<j \leq k}(-1)^{i+j} \omega\left(\left[X_{i}, X_{j}\right], X_{0}, \ldots, \widehat{X}_{i}, \ldots, \widehat{X}_{j}, \ldots X_{k}\right),
\end{aligned}
$$

from which assertion (1) follows since the first summand is 0. Similarly we have 


$$
\begin{aligned}
& \left(\mathcal{L}_{X} \omega\right)\left(X_{1}, \ldots, X_{k}\right)=\left(\mathcal{L}_{L_{X}} L_{\omega}\right)\left(L_{X_{1}}, \ldots, L_{X_{k}}\right)(e) \\
& =L_{X}(e)\left(\omega\left(X_{1}, \ldots, X_{k}\right)\right)+\sum_{i=1}^{k}(-1)^{i} \omega\left(\left[X, X_{i}\right], X_{1}, \ldots, \widehat{X}_{i}, \ldots X_{k}\right) .
\end{aligned}
$$

Again the first summand is 0 and the second result of (2) follows.

(3) This is obvious.

(41) For $X$ and $X_{i} \in \mathfrak{g}$ and for $\omega \in \bigwedge^{k} \mathfrak{g}^{*}$ we have

$$
\begin{aligned}
& \left(\left(T_{e} \widetilde{\operatorname{Ad}} \cdot X\right) \omega\right)\left(X_{1}, \ldots, X_{k}\right)=\left.\partial\right|_{0}(\widetilde{\operatorname{Ad}}(\exp (t X)) \omega)\left(X_{1}, \ldots, X_{k}\right) \\
& \quad=\left.\partial\right|_{0} \omega\left(\operatorname{Ad}(\exp (-t X)) \cdot X_{1}, \ldots, \operatorname{Ad}(\exp (-t X)) \cdot X_{k}\right) \\
& \quad=\sum_{i=1}^{k} \omega\left(X_{1}, \ldots, X_{i-1},-\operatorname{ad}(X) X_{i}, X_{i+1}, \ldots X_{k}\right) \\
& \quad=\sum_{i=1}^{k}(-1)^{i} \omega\left(\left[X, X_{i}\right], X_{1}, \ldots, \widehat{X}_{i}, \ldots X_{k}\right) \\
& \quad=\left(\mathcal{L}_{X} \omega\right)\left(X_{1}, \ldots, X_{k}\right) .
\end{aligned}
$$

14.15. Lemma of Maschke. Let $G$ be a compact Lie group, and let

$$
(0 \rightarrow) V_{1} \stackrel{i}{\longrightarrow} V_{2} \stackrel{p}{\longrightarrow} V_{3} \rightarrow 0
$$

be an exact sequence of $G$-modules and module homomorphisms such that each $V_{i}$ is a complete locally convex vector space, $i$ and $p$ are continuous, and the representation of $G$ on each $V_{i}$ consists of continuous linear mappings with $g \mapsto g . v$ continuous $G \rightarrow V_{i}$ for each $v \in V_{i}$. Then also the sequence

$$
(0 \rightarrow) V_{1}^{G} \stackrel{i}{\longrightarrow} V_{2}^{G} \stackrel{p^{G}}{\longrightarrow} V_{3}^{G} \rightarrow 0
$$

is exact, where $V_{i}^{G}:=\left\{v \in V_{i}: g . v=v\right.$ for all $\left.g \in G\right\}$.

Convenient vector spaces are sufficient for this lemma; see[113.

Proof. We prove first that $p^{G}$ is surjective. Let $v_{3} \in V_{3}^{G} \subset V_{3}$. Since $p: V_{2} \rightarrow V_{3}$ is surjective, there is a $v_{2} \in V_{2}$ with $p\left(v_{2}\right)=v_{3}$. We consider the element $\tilde{v}_{2}:=\int_{G} x \cdot v_{2} d_{L} x$; the integral makes sense since $x \mapsto x \cdot v_{2}$ is a continuous mapping $G \rightarrow V_{2}, G$ is compact, and Riemann sums converge in the locally convex topology of $V_{2}$. We assume that $\int_{G} d_{L} x=1$. Then we have

$$
a \cdot \tilde{v}_{2}=a \cdot \int_{G} x \cdot v_{2} d_{L} x=\int_{G}(a x) \cdot v_{2} d_{L} x=\int_{G} x \cdot v_{2} d_{L} x=\tilde{v}_{2}
$$

by the left invariance of the integral, see (14.4), where one uses continuous linear functionals to reduce to the scalar valued case. So $\tilde{v}_{2} \in V_{2}^{G}$ and since 
$p$ is a $G$-homomorphism, we get

$$
\begin{aligned}
p^{G}\left(\tilde{v}_{2}\right) & =p\left(\tilde{v}_{2}\right)=p\left(\int_{G} x \cdot v_{2} d_{L} x\right) \\
& =\int_{G} p\left(x \cdot v_{2}\right) d_{L} x=\int_{G} x \cdot p\left(v_{2}\right) d_{L} x \\
& =\int x \cdot v_{3} d_{L} x=\int_{G} v_{3} d_{L} x=v_{3} .
\end{aligned}
$$

So $p^{G}$ is surjective.

Now we prove that the sequence is exact at $V_{2}^{G}$. Clearly $p^{G} \circ i^{G}=(p \circ i) \mid V_{1}^{G}=$ 0 . Suppose conversely that $v_{2} \in V_{2}^{G}$ with $p^{G}\left(v_{2}\right)=p\left(v_{2}\right)=0$. Then there is a $v_{1} \in V_{1}$ with $i\left(v_{1}\right)=v_{2}$. Consider $\tilde{v}_{1}:=\int_{G} x \cdot v_{1} d_{L} x$. As above we see that $\tilde{v}_{1} \in V_{1}^{G}$ and that $i^{G}\left(\tilde{v}_{1}\right)=v_{2}$.

14.16. Theorem (Chevalley, Eilenberg). Let $G$ be a compact connected Lie group with Lie algebra $\mathfrak{g}$. Then we have:

(1) $H^{*}(G)=H^{*}\left(\bigwedge \mathfrak{g}^{*}, d\right)=: H^{*}(\mathfrak{g})$.

(2) $H^{*}(\mathfrak{g})=H^{*}\left(\bigwedge \mathfrak{g}^{*}, d\right)=\left(\bigwedge \mathfrak{g}^{*}\right)^{\mathfrak{g}}=\left\{\omega \in \bigwedge \mathfrak{g}^{*}: \mathcal{L}_{X} \omega=0\right.$ for all $X \in$ $\mathfrak{g}\}$, the space of all $\mathfrak{g}$-invariant forms on $\mathfrak{g}$.

The algebra $H^{*}(\mathfrak{g})=H\left(\bigwedge \mathfrak{g}^{*}, d\right)$ is called the Chevalley cohomology of the Lie algebra $\mathfrak{g}$. For the proof we follow [194].

Proof of (11). Let $Z^{k}(G)=\operatorname{ker}\left(d: \Omega^{k}(G) \rightarrow \Omega^{k+1}(G)\right)$, and let us consider the following exact sequence of vector spaces:

$$
\Omega^{k-1}(G) \stackrel{d}{\longrightarrow} Z^{k}(G) \rightarrow H^{k}(G) \rightarrow 0 .
$$

The group $G$ acts on $\Omega(G)$ by $a \mapsto \mu_{a^{-1}}^{*}$; this action commutes with $d$ and induces thus an action of $G$ on $Z^{k}(G)$ and also on $H^{k}(G)$. On the space $\Omega(G)$ we may consider the compact $C^{\infty}$-topology (uniform convergence on the compact $G$, in all derivatives separately, in a fixed set of charts). In this topology $d$ is continuous, $Z^{k}(G)$ is closed, and the action of $G$ is pointwise continuous. So the assumptions of the lemma of Maschke (14.15) are satisfied and we conclude that the following sequence is also exact:

$$
\Omega_{L}^{p-1}(G) \stackrel{d}{\longrightarrow} Z^{k}(G)^{G} \rightarrow H^{k}(G)^{G} \rightarrow 0 .
$$

Since $G$ is connected, for each $a \in G$ we may find a smooth curve $c:[0,1] \rightarrow$ $G$ with $c(0)=e$ and $c(1)=a$. Then $(t, x) \mapsto \mu_{c(t)^{-1}}(x)=c(t)^{-1} x$ is a smooth homotopy between $I d_{G}$ and $\mu_{a^{-1}}$, so by (11.4) the two mappings induce the same mapping in homology; we have

$$
\mu_{a^{-1}}^{*}=I d: H^{k}(G) \rightarrow H^{k}(G) \quad \text { for each } \quad a \in G .
$$


Thus $H^{k}(G)^{G}=H^{k}(G)$. Moreover $Z^{k}(G)^{G}=\operatorname{ker}\left(d: \Omega_{L}^{k}(G) \rightarrow \Omega_{L}^{k+1}(G)\right)$, so from the exact sequence (4) we may conclude that

$$
H^{k}(G)=H^{k}(G)^{G}=\frac{\operatorname{ker}\left(d: \Omega_{L}^{k}(G) \rightarrow \Omega_{L}^{k+1}(G)\right)}{\operatorname{im}\left(d: \Omega_{L}^{k-1}(G) \rightarrow \Omega_{L}^{k}(G)\right)}=H^{k}\left(\bigwedge \mathfrak{g}^{*}, d\right) .
$$

Proof of (2). From (14.14.3) we have $\mathcal{L}_{X} \circ d=d \circ \mathcal{L}_{X}$, so by (14.14.4) we conclude that $\widetilde{\operatorname{Ad}}(a) \circ d=d \circ \widetilde{\operatorname{Ad}}(a): \wedge \mathfrak{g}^{*} \rightarrow \wedge \mathfrak{g}^{*}$ since $G$ is connected. Thus the sequence

$$
\bigwedge^{k-1} \mathfrak{g}^{*} \stackrel{d}{\longrightarrow} Z^{k}\left(\mathfrak{g}^{*}\right) \rightarrow H^{k}\left(\bigwedge \mathfrak{g}^{*}, d\right) \rightarrow 0
$$

is an exact sequence of $G$-modules and $G$-homomorphisms, where $Z^{k}\left(\mathfrak{g}^{*}\right)=$ $\operatorname{ker}\left(d: \bigwedge^{k} \mathfrak{g}^{*} \rightarrow \bigwedge^{k+1} \mathfrak{g}^{*}\right)$. All spaces are finite-dimensional, so the lemma of Maschke (14.15) is applicable and we may conclude that also the following sequence is exact:

$$
\left(\bigwedge^{k-1} \mathfrak{g}^{*}\right)^{G} \stackrel{d}{\longrightarrow} Z^{k}\left(\mathfrak{g}^{*}\right)^{G} \rightarrow H^{k}\left(\bigwedge \mathfrak{g}^{*}, d\right)^{G} \rightarrow 0 .
$$

The space $H^{k}\left(\bigwedge \mathfrak{g}^{*}, d\right)^{G}$ consists of all cohomology classes $\alpha$ with $\widetilde{\operatorname{Ad}}(a) \alpha=$ $\alpha$ for all $a \in G$. Since $G$ is connected, by (14.14.4) these are exactly the $\alpha$ with $\mathcal{L}_{X} \alpha=0$ for all $X \in \mathfrak{g}$. For $\omega \in \wedge \mathfrak{g}^{*}$ with $d \omega=0$ we have by (14.14.3) that $\mathcal{L}_{X} \omega=i_{X} d \omega+d i_{X} \omega=d i_{X} \omega$, so that $\mathcal{L}_{X} \alpha=0$ for all $\alpha \in H^{k}\left(\bigwedge \mathfrak{g}^{*}, d\right)$. Thus we get $H^{k}\left(\bigwedge \mathfrak{g}^{*}, d\right)=H^{k}\left(\bigwedge \mathfrak{g}^{*}, d\right)^{G}$. Also we have $\left(\bigwedge \mathfrak{g}^{*}\right)^{G}=\left(\bigwedge \mathfrak{g}^{*}\right)^{\mathfrak{g}}$ so that the exact sequence (6) translates to

$$
H^{k}(\mathfrak{g})=H^{k}\left(\bigwedge \mathfrak{g}^{*}, d\right)=H^{k}\left(\left(\bigwedge \mathfrak{g}^{*}\right)^{\mathfrak{g}}, d\right) .
$$

Now let $\omega \in\left(\bigwedge^{k} \mathfrak{g}^{*}\right)^{\mathfrak{g}}=\left\{\varphi: \mathcal{L}_{X} \varphi=0\right.$ for all $\left.X \in \mathfrak{g}\right\}$ and consider the inversion $\nu: G \rightarrow G$. Then we have for $\omega \in \bigwedge^{k} \mathfrak{g}^{*}$ and $X_{i} \in \mathfrak{g}$ :

$$
\begin{aligned}
\left(\nu^{*} L_{\omega}\right)_{a}\left(T_{e}\left(\mu_{a}\right) \cdot X_{1}, \ldots, T_{e}\left(\mu_{a}\right) \cdot X_{k}\right) \\
\quad=\left(L_{\omega}\right)_{a^{-1}}\left(T_{a} \nu \cdot T_{e}\left(\mu_{a}\right) \cdot X_{1}, \ldots, T_{a} \nu \cdot T_{e}\left(\mu_{a}\right) \cdot X_{k}\right) \\
\quad=\left(L_{\omega}\right)_{a^{-1}}\left(-T\left(\mu^{a^{-1}}\right) \cdot T\left(\mu_{a^{-1}}\right) \cdot T_{e}\left(\mu_{a}\right) \cdot X_{1}, \ldots\right) \\
\quad=\left(L_{\omega}\right)_{a^{-1}}\left(-T_{e}\left(\mu^{a^{-1}}\right) \cdot X_{1}, \ldots,-T_{e}\left(\mu^{a^{-1}}\right) \cdot X_{k}\right) \\
\quad=(-1)^{k} \omega\left(T \mu_{a} \cdot T \mu^{a^{-1}} \cdot X_{1}, \ldots, T \mu_{a} \cdot T \mu^{a^{-1}} \cdot X_{k}\right) \\
\quad=(-1)^{k} \omega\left(\operatorname{Ad}(a) \cdot X_{1}, \ldots, \operatorname{Ad}(a) \cdot X_{k}\right) \\
=(-1)^{k}\left(\widetilde{\operatorname{Ad}}\left(a^{-1}\right) \omega\right)\left(X_{1}, \ldots, X_{k}\right) \\
\quad=(-1)^{k} \omega\left(X_{1}, \ldots, X_{k}\right) \quad \text { since } \omega \in\left(\bigwedge \mathfrak{g}^{*}\right)^{\mathfrak{g}} \\
=(-1)^{k}\left(L_{\omega}\right)_{a}\left(T_{e}\left(\mu_{a}\right) \cdot X_{1}, \ldots, T_{e}\left(\mu_{a}\right) \cdot X_{k}\right) .
\end{aligned}
$$


So for $\omega \in\left(\bigwedge^{k} \mathfrak{g}^{*}\right)^{\mathfrak{g}}$ we have $\nu^{*} L_{\omega}=(-1)^{k} L_{\omega}$ and thus also $(-1)^{k+1} L_{d \omega}=$ $\nu^{*} d L_{\omega}=d \nu^{*} L_{\omega}=(-1)^{k} d L_{\omega}=(-1)^{k} L_{d \omega}$ which implies $d \omega=0$. Hence we have $d \mid\left(\bigwedge \mathfrak{g}^{*}\right)^{\mathfrak{g}}=0$.

From (7) we now get $H^{k}(\mathfrak{g})=H^{k}\left(\left(\bigwedge \mathfrak{g}^{*}\right)^{\mathfrak{g}}, 0\right)=\left(\bigwedge^{k} \mathfrak{g}^{*}\right)^{\mathfrak{g}}$ as required.

14.17. Corollary. Let $G$ be a compact connected Lie group. Then its Poincaré polynomial is given by

$$
f_{G}(t)=\int_{G} \operatorname{det}\left(\operatorname{Ad}(x)+t I d_{\mathfrak{g}}\right) d_{L} x .
$$

Proof. Let $\operatorname{dim} G=n$. By (11.2) and (13.6) we have

$$
f_{G}(t)=\sum_{k=0}^{n} b_{k}(G) t^{k}=\sum_{k=0}^{n} b_{k}(G) t^{n-k}=\sum_{k=0}^{n} \operatorname{dim}_{\mathbb{R}} H^{k}(G) t^{n-k} .
$$

On the other hand we have

$$
\begin{aligned}
\int_{G} \operatorname{det}\left(\operatorname{Ad}(x)+t I d_{\mathfrak{g}}\right) d_{L} x=\int_{G} \operatorname{det}\left(\operatorname{Ad}\left(x^{-1}\right)^{*}+t I d_{\mathfrak{g}^{*}}\right) d_{L} x \\
=\int_{G} \sum_{k=0}^{n} \operatorname{Trace}\left(\bigwedge \operatorname{Ad}\left(x^{-1}\right)^{*}\right) t^{n-k} d_{L} x \quad \text { by (14.19) below } \\
=\sum_{k=0}^{n} \int_{G} \operatorname{Trace}\left(\widetilde{\operatorname{Ad}}(x) \mid \bigwedge^{k} \mathfrak{g}^{*}\right) d_{L} x t^{n-k} .
\end{aligned}
$$

If $\rho: G \rightarrow G L(V)$ is a finite-dimensional representation of $G$, then the operator $\int_{G} \rho(x) d_{L} x: V \rightarrow V$ is just a projection onto $V^{G}$, the space of fixed points of the representation; see the proof of the lemma of Maschke (14.15), The trace of a projection is the dimension of the image. So

$$
\begin{aligned}
\int_{G} \operatorname{Trace}\left(\widetilde{\operatorname{Ad}}(a) \mid \bigwedge^{k} \mathfrak{g}^{*}\right) d_{L} x & =\operatorname{Trace}\left(\int_{G}\left(\widetilde{\operatorname{Ad}}(a) \mid \bigwedge^{k} \mathfrak{g}^{*}\right) d_{L} x\right) \\
& =\operatorname{dim}\left(\bigwedge^{k} \mathfrak{g}^{*}\right)^{G}=\operatorname{dim} H^{k}(G) .
\end{aligned}
$$

14.18. Let $\mathbb{T}^{n}=\left(S^{1}\right)^{n}$ be the $n$-dimensional torus, and let $\mathfrak{t}^{n}$ be its Lie algebra. The Lie bracket is zero since the torus is an abelian group. From theorem (14.16) we have then that $H^{*}\left(\mathbb{T}^{n}\right)=\left(\bigwedge\left(\mathfrak{t}^{n}\right)^{*}\right)^{\mathfrak{t}^{n}}=\bigwedge\left(\mathfrak{t}^{n}\right)^{*}$, so the Poincaré polynomial is $f_{\mathbb{T}^{n}}(t)=(1+t)^{n}$. 
14.19. Lemma. Let $V$ be an $n$-dimensional vector space and let $A: V \rightarrow V$ be a linear mapping. Then we have

$$
\operatorname{det}\left(A+t I d_{V}\right)=\sum_{k=0}^{n} t^{n-k} \operatorname{Trace}\left(\bigwedge^{k} A\right)
$$

Proof. By $\wedge^{k} A: \bigwedge^{k} V \rightarrow \bigwedge^{k} V$ we mean the mapping $v_{1} \wedge \cdots \wedge v_{k} \mapsto$ $A v_{1} \wedge \cdots \wedge A v_{k}$. Let $e_{1}, \ldots, e_{n}$ be a basis of $V$. By the definition of the determinant we have

$$
\begin{gathered}
\operatorname{det}\left(A+t I d_{V}\right)\left(e_{1} \wedge \cdots \wedge e_{n}\right)=\left(A e_{1}+t e_{1}\right) \wedge \cdots \wedge\left(A e_{n}+t e_{n}\right) \\
=\sum_{k=0}^{n} t^{n-k} \sum_{i_{1}<\cdots<i_{k}} e_{1} \wedge \cdots \wedge A e_{i_{1}} \wedge \cdots \wedge A e_{i_{k}} \wedge \cdots \wedge e_{n} .
\end{gathered}
$$

The multivectors $\left(e_{i_{1}} \wedge \cdots \wedge e_{i_{k}}\right)_{i_{1}<\cdots<i_{k}}$ are a basis of $\bigwedge^{k} V$ and we can thus write

$$
\left(\bigwedge^{k} A\right)\left(e_{i_{1}} \wedge \cdots \wedge e_{i_{k}}\right)=A e_{i_{1}} \wedge \cdots \wedge A e_{i_{k}}=\sum_{j_{1}<\cdots<j_{k}} A_{i_{1} \ldots i_{k}}^{j_{1} \ldots j_{k}} e_{j_{1}} \wedge \cdots \wedge e_{j_{k}}
$$

where $\left(A_{i_{1} \ldots i_{k}}^{j_{1} \ldots j_{k}}\right)$ is the matrix of $\bigwedge^{k} A$ in this basis. We see that

$$
e_{1} \wedge \cdots \wedge A e_{i_{1}} \wedge \cdots \wedge A e_{i_{k}} \wedge \cdots \wedge e_{n}=A_{i_{1} \ldots i_{k}}^{i_{1} \ldots i_{k}} e_{1} \wedge \cdots \wedge e_{n} .
$$

Consequently we have

$$
\begin{aligned}
\operatorname{det}( & \left.A+t I d_{V}\right) e_{1} \wedge \cdots \wedge e_{n}=\sum_{k=0}^{n} t^{n-k} \sum_{i_{1}<\cdots<i_{k}} A_{i_{1} \ldots i_{k}}^{i_{1} \ldots i_{k}} e_{1} \wedge \cdots \wedge e_{n} \\
= & \sum_{k=0}^{n} t^{n-k} \operatorname{Trace}(\bigwedge \mathrm{k}) e_{1} \wedge \cdots \wedge e_{n},
\end{aligned}
$$

which implies the result.

\section{Extensions of Lie Algebras and Lie Groups \\ Extension of Lie Algebras}

In this section we describe first the theory of semidirect products and central extensions of Lie algebras, later the more involved theory of general extensions with noncommutative kernels. For the latter we follow the presentation from [6], with special emphasis on relations with the (algebraic) theory of covariant exterior derivatives, curvature and the Bianchi identity in differential geometry (see section (15.3)]. The results are due to [89, [164, [209], and generalizations for Lie algebroids are in [127]. The analogous result for Lie super-algebras are available in [7]. 
15.1. Extensions. An extension of a Lie algebra $\mathfrak{g}$ with kernel $\mathfrak{h}$ is an exact sequence of homomorphisms of Lie algebras:

$$
0 \rightarrow \mathfrak{h} \stackrel{i}{\longrightarrow} \mathfrak{e} \stackrel{p}{\longrightarrow} \mathfrak{g} \rightarrow 0 .
$$

(1) This extension is called a semidirect product if we can find a section $s$ : $\mathfrak{g} \rightarrow \mathfrak{e}$ which is a Lie algebra homomorphism. Then we have a representation of the Lie algebra $\alpha: \mathfrak{g} \rightarrow L(\mathfrak{h}, \mathfrak{h})$ which is given by $\alpha_{X}(H)=[s(X), H]$ where we suppress the injection $i$. It is a representation since $\alpha_{[X, Y]} H=$ $[s([X, Y]), H]=[[s(X), s(Y)], H]=[s(X),[s(Y), H]]-[s(Y),[s(X, H)]]=$ $\left(\alpha_{X} \alpha_{Y}-\alpha_{Y} \alpha_{X}\right) H$. This representation takes values in the Lie algebra $\operatorname{der}(\mathfrak{h})$ of derivations of $\mathfrak{h}$, so $\alpha: \mathfrak{g} \rightarrow \operatorname{der}(\mathfrak{h})$. From the data $\alpha, s$ we can reconstruct the extension $\mathfrak{e}$ since on $\mathfrak{h} \times \mathfrak{g}$ we have $\left[H+s(X), H^{\prime}+s\left(X^{\prime}\right)\right]=\left[H, H^{\prime}\right]+$ $\left[s(X), H^{\prime}\right]-\left[s\left(X^{\prime}\right), H\right]+\left[X, X^{\prime}\right]=\left[H, H^{\prime}\right]+\alpha_{X}\left(H^{\prime}\right)-\alpha_{X^{\prime}}(H)+\left[X, X^{\prime}\right]$.

(2) The extension is called a central extension if $\mathfrak{h}$ or rather $i(\mathfrak{h})$ is in the center of $\mathfrak{e}$.

15.2. Describing extensions. Consider any exact sequence of homomorphisms of Lie algebras:

$$
0 \rightarrow \mathfrak{h} \stackrel{i}{\longrightarrow} \mathfrak{e} \stackrel{p}{\longrightarrow} \mathfrak{g} \rightarrow 0
$$

Consider a linear mapping $s: \mathfrak{g} \rightarrow \mathfrak{e}$ with $p \circ s=\operatorname{Id}_{\mathfrak{g}}$. Then $s$ induces mappings

$$
\begin{aligned}
\alpha: \mathfrak{g} \rightarrow \operatorname{der}(\mathfrak{h}), & \alpha_{X}(H)=[s(X), H], \\
\rho: \bigwedge \mathfrak{g} \rightarrow \mathfrak{h}, & \rho(X, Y)=[s(X), s(Y)]-s([X, Y]),
\end{aligned}
$$

which are easily seen to satisfy

$$
\begin{gathered}
{\left[\alpha_{X}, \alpha_{Y}\right]-\alpha_{[X, Y]}=\operatorname{ad}_{\rho(X, Y)},} \\
\sum_{\text {cyclic }\{X, Y, Z\}}\left(\alpha_{X} \rho(Y, Z)-\rho([X, Y], Z)\right)=0 .
\end{gathered}
$$

We can completely describe the Lie algebra structure on $\mathfrak{e}=\mathfrak{h} \oplus s(\mathfrak{g})$ in terms of $\alpha$ and $\rho$ :

$$
\begin{aligned}
& {\left[H_{1}+s\left(X_{1}\right), H_{2}+s\left(X_{2}\right)\right]} \\
& \quad=\left(\left[H_{1}, H_{2}\right]+\alpha_{X_{1}} H_{2}-\alpha_{X_{2}} H_{1}+\rho\left(X_{1}, X_{2}\right)\right)+s\left[X_{1}, X_{2}\right]
\end{aligned}
$$

and one can check that formula (5) gives a Lie algebra structure on $\mathfrak{h} \oplus s(\mathfrak{g})$ if $\alpha: \mathfrak{g} \rightarrow \operatorname{der}(\mathfrak{h})$ and $\rho: \bigwedge^{2} \mathfrak{g} \rightarrow \mathfrak{h}$ satisfy (3) and (4). 
15.3. Motivation: Lie algebra extensions associated to a principal bundle. Let $\pi: P \rightarrow M=P / K$ be a principal bundle with structure group $K$; see section (18): $P$ is a manifold with a free right action of a Lie group $K$ and $\pi$ is the projection on the orbit space $M=P / K$. Denote by $\mathfrak{g}=\mathfrak{X}(M)$ the Lie algebra of the vector fields on $M$, by $\mathfrak{e}=\mathfrak{X}(P)^{K}$ the Lie algebra of $K$-invariant vector fields on $P$ and by $\mathfrak{h}=\mathfrak{X}_{\text {vert }}(P)^{K}$ the ideal of the $K$-invariant vertical vector fields of $\mathfrak{e}$. Geometrically, $\mathfrak{e}$ is the Lie algebra of infinitesimal automorphisms of the principal bundle $P$ and $\mathfrak{h}$ is the ideal of infinitesimal automorphisms acting trivially on $M$, i.e., the Lie algebra of infinitesimal gauge transformations. We have a natural homomorphism $\pi_{*}: \mathfrak{e} \rightarrow \mathfrak{g}$ with the kernel $\mathfrak{h}$, i.e., $\mathfrak{e}$ is an extension of $\mathfrak{g}$ by $\mathfrak{h}$.

Note that we have additional structures of $C^{\infty}(M)$-modules on $\mathfrak{g}, \mathfrak{h}, \mathfrak{e}$, such that $[X, f Y]=f[X, Y]+\left(\pi_{*} X\right) f Y$, where $X, Y \in \mathfrak{e}, f \in C^{\infty}(M)$. In particular, $\mathfrak{h}$ is a Lie algebra over $C^{\infty}(M)$. The extension

$$
0 \rightarrow \mathfrak{h} \rightarrow \mathfrak{e} \rightarrow \mathfrak{g} \rightarrow 0
$$

is also an extension of $C^{\infty}(M)$-modules.

Assume now that the section $s: \mathfrak{g} \rightarrow \mathfrak{e}$ is a homomorphism of $C^{\infty}(M)$ modules. Then it can be considered as a connection in the principal bundle $\pi$, see section (19), and the $\mathfrak{h}$-valued 2 -form $\rho$ as its curvature. In this sense we interpret the constructions from section (15.1) as follows in (15.4) below. The analogy with differential geometry has also been noticed by [117] and 118.

15.4. Geometric interpretation. Note that (15.2.2) is similar to the Maurer-Cartan formula for the curvature on principal bundles of differential geometry (19.2.3)

$$
\rho=d s+\frac{1}{2}[s, s]_{\wedge},
$$

where for an arbitrary vector space $V$ the usual Chevalley differential, see $(14.14 .2)$, is given by

$$
\begin{gathered}
d: L_{\text {skew }}^{p}(\mathfrak{g} ; V) \rightarrow L_{\text {skew }}^{p+1}(\mathfrak{g} ; V), \\
d \varphi\left(X_{0}, \ldots, X_{p}\right)=\sum_{i<j}(-1)^{i+j} \varphi\left(\left[X_{i}, X_{j}\right], X_{0}, \ldots, \widehat{X_{i}}, \ldots, \widehat{X_{j}}, \ldots, X_{p}\right)
\end{gathered}
$$

and where for a vector space $W$ and a Lie algebra $\mathfrak{f}$ the $\mathbb{N}_{0}$-graded Lie bracket $[, \quad] \wedge$ on $L_{\text {skew }}^{*}(W, \mathfrak{f})$, see $(19.2)$, is given by

$[\varphi, \psi]_{\wedge}\left(X_{1}, \ldots, X_{p+q}\right)=\frac{1}{p ! q !} \sum_{\sigma} \operatorname{sign}(\sigma)\left[\varphi\left(X_{\sigma 1}, \ldots, X_{\sigma p}\right), \psi\left(X_{\sigma(p+1)}, \ldots\right)\right]_{\mathfrak{f}}$.

Similarly formula (15.2.3) reads as

$$
\operatorname{ad}_{\rho}=d \alpha+\frac{1}{2}[\alpha, \alpha]_{\wedge}
$$


Thus we view $s$ as a connection in the sense of a horizontal lift of vector fields on the base of a bundle and $\alpha$ as an induced connection; see (19.8). Namely, for every $\operatorname{der}(\mathfrak{h})$-module $V$ we put

$$
\begin{gathered}
\alpha_{\wedge}: L_{\text {skew }}^{p}(\mathfrak{g} ; V) \rightarrow L_{\text {skew }}^{p+1}(\mathfrak{g} ; V), \\
\alpha_{\wedge} \varphi\left(X_{0}, \ldots, X_{p}\right)=\sum_{i=0}^{p}(-1)^{i} \alpha_{X_{i}}\left(\varphi\left(X_{0}, \ldots, \widehat{X}_{i}, \ldots, X_{p}\right)\right) .
\end{gathered}
$$

Then we have the covariant exterior differential (on the sections of an associated vector bundle; see (19.12)

$$
\delta_{\alpha}: L_{\text {skew }}^{p}(\mathfrak{g} ; V) \rightarrow L_{\text {skew }}^{p+1}(\mathfrak{g} ; V), \quad \delta_{\alpha} \varphi=\alpha_{\wedge} \varphi+d \varphi,
$$

for which formula (15.2.4) looks like the Bianchi identity, see (19.5.6), $\delta_{\alpha} \rho=$ 0 . Moreover one can prove by direct evaluation that another well known result from differential geometry holds, namely (19.5.9), i.e.,

$$
\delta_{\alpha} \delta_{\alpha}(\varphi)=[\rho, \varphi]_{\wedge}, \quad \varphi \in L_{\text {skew }}^{p}(\mathfrak{g} ; \mathfrak{h}) .
$$

If we change the linear section $s$ to $s^{\prime}=s+b$ for linear $b: \mathfrak{g} \rightarrow \mathfrak{h}$, then we get

$$
\begin{aligned}
\alpha_{X}^{\prime} & =\alpha_{X}+\operatorname{ad}_{b(X)}^{\mathfrak{h}}, \\
\rho^{\prime}(X, Y) & =\rho(X, Y)+\alpha_{X} b(Y)-\alpha_{Y} b(X)-b([X, Y])+[b X, b Y] \\
& =\rho(X, Y)+\left(\delta_{\alpha} b\right)(X, Y)+[b X, b Y], \\
\rho^{\prime} & =\rho+\delta_{\alpha} b+\frac{1}{2}[b, b]_{\wedge} .
\end{aligned}
$$

15.5. Theorem. Let $\mathfrak{h}$ and $\mathfrak{g}$ be Lie algebras.

Then isomorphism classes of extensions of $\mathfrak{g}$ over $\mathfrak{h}$, i.e., short exact sequences of Lie algebras $0 \rightarrow \mathfrak{h} \rightarrow \mathfrak{e} \rightarrow \mathfrak{g} \rightarrow 0$, modulo the equivalence described by the commutative diagram of Lie algebra homomorphisms

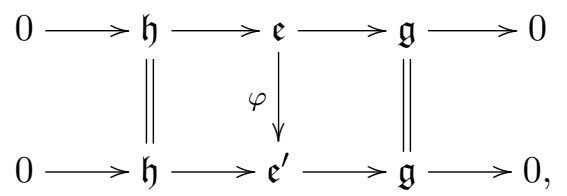

correspond bijectively to equivalence classes of data of the following form:

a linear mapping $\alpha: \mathfrak{g} \rightarrow \operatorname{der}(\mathfrak{h})$,

such that

$$
\begin{aligned}
& {\left[\alpha_{X}, \alpha_{Y}\right]-\alpha_{[X, Y]}=\operatorname{ad}_{\rho(X, Y)},} \\
& \sum_{\text {cyclic }}\left(\alpha_{X} \rho(Y, Z)-\rho([X, Y], Z)\right)=0, \quad \text { equivalently, } \quad \delta_{\alpha} \rho=0 .
\end{aligned}
$$


On the vector space $\mathfrak{e}:=\mathfrak{h} \oplus \mathfrak{g}$ a Lie algebra structure is given by

$$
\begin{aligned}
& {\left[H_{1}+X_{1}, H_{2}+X_{2}\right]_{\mathfrak{e}}} \\
& \quad=\left[H_{1}, H_{2}\right]_{\mathfrak{h}}+\alpha_{X_{1}} H_{2}-\alpha_{X_{2}} H_{1}+\rho\left(X_{1}, X_{2}\right)+\left[X_{1}, X_{2}\right]_{\mathfrak{g}},
\end{aligned}
$$

and the associated exact sequence is

$$
0 \rightarrow \mathfrak{h} \stackrel{i_{1}}{\longrightarrow} \mathfrak{h} \oplus \mathfrak{g}=\mathfrak{e} \stackrel{\mathrm{pr}_{2}}{\longrightarrow} \mathfrak{g} \rightarrow 0 .
$$

Two data $(\alpha, \rho)$ and $\left(\alpha^{\prime}, \rho^{\prime}\right)$ are equivalent if there exists a linear mapping $b: \mathfrak{g} \rightarrow \mathfrak{h}$ such that

$$
\begin{aligned}
\alpha_{X}^{\prime} & =\alpha_{X}+\operatorname{ad}_{b(X)}^{\mathfrak{h}}, \\
\rho^{\prime}(X, Y) & =\rho(X, Y)+\alpha_{X} b(Y)-\alpha_{Y} b(X)-b([X, Y])+[b(X), b(Y)], \\
\rho^{\prime} & =\rho+\delta_{\alpha} b+\frac{1}{2}[b, b]_{\wedge},
\end{aligned}
$$

the corresponding isomorphism being

$$
\mathfrak{e}=\mathfrak{h} \oplus \mathfrak{g} \rightarrow \mathfrak{h} \oplus \mathfrak{g}=\mathfrak{e}^{\prime}, \quad H+X \mapsto H-b(X)+X .
$$

Moreover, a datum $(\alpha, \rho)$ corresponds to a split extension (a semidirect prod$u c t)$ if and only if $(\alpha, \rho)$ is equivalent to a datum of the form $\left(\alpha^{\prime}, 0\right)$ (then $\alpha^{\prime}$ is a homomorphism). This is the case if and only if there exists a mapping $b: \mathfrak{g} \rightarrow \mathfrak{h}$ such that

$$
\rho=-\delta_{\alpha} b-\frac{1}{2}[b, b]_{\wedge}
$$

Proof. Straightforward computations.

15.6. Corollary ([120]). Let $\mathfrak{g}$ and $\mathfrak{h}$ be Lie algebras such that $\mathfrak{h}$ has no center. Then isomorphism classes of extensions of $\mathfrak{g}$ over $\mathfrak{h}$ correspond bijectively to Lie homomorphisms

$$
\bar{\alpha}: \mathfrak{g} \rightarrow \operatorname{out}(\mathfrak{h})=\operatorname{der}(\mathfrak{h}) / \operatorname{ad}(\mathfrak{h}) .
$$

Proof. If $(\alpha, \rho)$ is a datum, then the map $\bar{\alpha}: \mathfrak{g} \rightarrow \operatorname{der}(\mathfrak{h}) / \operatorname{ad}(\mathfrak{h})$ is a Lie algebra homomorphism by (15.5.3). Conversely, let $\bar{\alpha}$ be given. Choose a linear lift $\alpha: \mathfrak{g} \rightarrow \operatorname{der}(\mathfrak{h})$ of $\bar{\alpha}$. Since $\bar{\alpha}$ is a Lie algebra homomorphism and $\mathfrak{h}$ has no center, there is a uniquely defined skew-symmetric linear mapping $\rho: \mathfrak{g} \times \mathfrak{g} \rightarrow \mathfrak{h}$ such that $\left[\alpha_{X}, \alpha_{Y}\right]-\alpha_{[X, Y]}=\operatorname{ad}_{\rho(X, Y)}$. Condition (15.5.4) is then automatically satisfied. For later use also, we record the simple proof:

$$
\begin{aligned}
& \sum_{\text {cyclic } X, Y, Z}\left[\alpha_{X} \rho(Y, Z)-\rho([X, Y], Z), H\right] \\
= & \sum_{\text {cyclic } X, Y, Z}\left(\alpha_{X}[\rho(Y, Z), H]-\left[\rho(Y, Z), \alpha_{X} H\right]-[\rho([X, Y], Z), H]\right)
\end{aligned}
$$




$$
\begin{array}{r}
=\sum_{\text {cyclic } X, Y, Z}\left(\alpha_{X}\left[\alpha_{Y}, \alpha_{Z}\right]-\alpha_{X} \alpha_{[Y, Z]}-\left[\alpha_{Y}, \alpha_{Z}\right] \alpha_{X}+\alpha_{[Y, Z]} \alpha_{X}\right. \\
\left.-\left[\alpha_{[X, Y]}, \alpha_{Z}\right]+\alpha_{[[X, Y] Z]}\right) H \\
=\sum_{\text {cyclic } X, Y, Z}\left(\left[\alpha_{X},\left[\alpha_{Y}, \alpha_{Z}\right]\right]-\left[\alpha_{X}, \alpha_{[Y, Z]}\right]-\left[\alpha_{[X, Y]}, \alpha_{Z}\right]+\alpha_{[[X, Y] Z]}\right) H=0 .
\end{array}
$$

Thus $(\alpha, \rho)$ describes an extension by theorem (15.5). The rest is clear.

15.7. Remarks. If $\mathfrak{h}$ has no center and $\bar{\alpha}: \mathfrak{g} \rightarrow \operatorname{out}(\mathfrak{h})=\operatorname{der}(\mathfrak{h}) / \operatorname{ad}(\mathfrak{h})$ is a given homomorphism, the extension corresponding to $\bar{\alpha}$ can be constructed in the following easy way: It is given by the pullback diagram

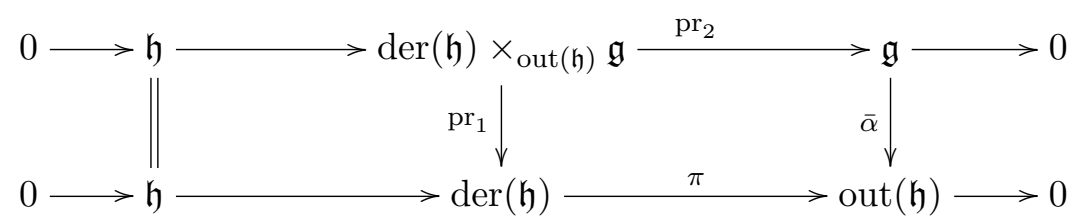

where $\operatorname{der}(\mathfrak{h}) \times_{\text {out }(\mathfrak{h})} \mathfrak{g}$ is the Lie subalgebra

$$
\operatorname{der}(\mathfrak{h}) \times{ }_{\text {out }(\mathfrak{h})} \mathfrak{g}:=\{(D, X) \in \operatorname{der}(\mathfrak{h}) \times \mathfrak{g}: \pi(D)=\bar{\alpha}(X)\} \subset \operatorname{der}(\mathfrak{h}) \times \mathfrak{g} .
$$

We owe this remark to E. Vinberg.

If $\mathfrak{h}$ has no center and satisfies $\operatorname{der}(\mathfrak{h})=\mathfrak{h}$ and if $\mathfrak{h}$ is normal in a Lie algebra $\mathfrak{e}$, then $\mathfrak{e} \cong \mathfrak{h} \oplus \mathfrak{e} / \mathfrak{h}$, since $\operatorname{Out}(\mathfrak{h})=0$.

15.8. Theorem. Let $\mathfrak{g}$ and $\mathfrak{h}$ be Lie algebras and let

$$
\bar{\alpha}: \mathfrak{g} \rightarrow \operatorname{out}(\mathfrak{h})=\operatorname{der}(\mathfrak{h}) / \operatorname{ad}(\mathfrak{h})
$$

be a Lie algebra homomorphism. For a linear lift $\alpha: \mathfrak{g} \rightarrow \operatorname{der}(\mathfrak{h})$ of $\bar{\alpha}$ choose $\rho: \bigwedge^{2} \mathfrak{g} \rightarrow \mathfrak{h}$ satisfying $\left(\left[\alpha_{X}, \alpha_{Y}\right]-\alpha_{[X, Y]}\right)=\operatorname{ad}_{\rho(X, Y)}$. Then $\lambda=\lambda(\alpha, \rho):=$ $\delta_{\alpha} \rho: \bigwedge^{3} \mathfrak{g} \rightarrow Z(\mathfrak{h})$ is a cocycle for the cochain complex

$$
\delta_{\bar{\alpha}}: L_{\text {skew }}^{k}(\mathfrak{g} ; Z(\mathfrak{h})) \rightarrow L_{\text {skew }}^{k+1}(\mathfrak{g} ; Z(\mathfrak{h})), \quad \delta_{\bar{\alpha}} \circ \delta_{\bar{\alpha}}=0 .
$$

The cohomology class $[\lambda] \in H^{3}(\mathfrak{g} ; Z(\mathfrak{h}))$ depends only on $\bar{\alpha}$ and not on the choices of $\alpha$ and $\rho$. Then the following are equivalent:

(1) The $\delta_{\bar{\alpha}}$-cohomology class of $\lambda$ vanishes: $[\lambda]=0 \in H^{3}(\mathfrak{g} ; Z(\mathfrak{h}))$.

(2) There exists an extension $0 \rightarrow \mathfrak{h} \rightarrow \mathfrak{e} \rightarrow \mathfrak{g} \rightarrow 0$ inducing the homomorphism $\bar{\alpha}$.

If this is the case, then all extensions $0 \rightarrow \mathfrak{h} \rightarrow \mathfrak{e} \rightarrow \mathfrak{g} \rightarrow 0$ inducing the homomorphism $\bar{\alpha}$ are parameterized by $H^{2}(\mathfrak{g},(Z(\mathfrak{h}), \bar{\alpha}))$, the second Chevalley cohomology space of $\mathfrak{g}$ with values in the center $Z(\mathfrak{h})$, considered as $\mathfrak{g}$-module via $\bar{\alpha}$. 
Proof. Using once more the computation in the proof of corollary (15.6), we see that $\operatorname{ad}(\lambda(X, Y, Z))=\operatorname{ad}\left(\delta_{\alpha} \rho(X, Y, Z)\right)=0$ so that $\lambda(X, Y, Z) \in Z(\mathfrak{h})$. The Lie algebra out $(\mathfrak{h})=\operatorname{der}(\mathfrak{h}) / \operatorname{ad}(\mathfrak{h})$ acts on the center $Z(\mathfrak{h})$; thus $Z(\mathfrak{h})$ is a $\mathfrak{g}$-module via $\bar{\alpha}$, and $\delta_{\bar{\alpha}}$ is the differential of the Chevalley cohomology. Using (15.4.2), we see that

$$
\delta_{\bar{\alpha}} \lambda=\delta_{\alpha} \delta_{\alpha} \rho=[\rho, \rho]_{\wedge}=-(-1)^{2 \cdot 2}[\rho, \rho]_{\wedge}=0,
$$

so that $[\lambda] \in H^{3}(\mathfrak{g} ; Z(\mathfrak{h}))$.

Let us check next that the cohomology class $[\lambda]$ does not depend on the choices we made. If we are given a pair $(\alpha, \rho)$ as above and we take another linear lift $\alpha^{\prime}: \mathfrak{g} \rightarrow \operatorname{der}(\mathfrak{h})$, then $\alpha_{X}^{\prime}=\alpha_{X}+\operatorname{ad}_{b(X)}$ for some linear $b: \mathfrak{g} \rightarrow \mathfrak{h}$. We consider

$$
\rho^{\prime}: \bigwedge^{2} \mathfrak{g} \rightarrow \mathfrak{h}, \quad \rho^{\prime}(X, Y)=\rho(X, Y)+\left(\delta_{\alpha} b\right)(X, Y)+[b(X), b(Y)] .
$$

Computations involving only the definitions and the Jacobi identity show that

$$
\left[\alpha_{X}^{\prime}, \alpha_{Y}^{\prime}\right]-\alpha_{[X, Y]}^{\prime}=\operatorname{ad}_{\rho^{\prime}(X, Y)}, \quad \lambda(\alpha, \rho)=\delta_{\alpha} \rho=\delta_{\alpha^{\prime}} \rho^{\prime}=\lambda\left(\alpha^{\prime}, \rho^{\prime}\right),
$$

so that even the cochain did not change. So let us consider for fixed $\alpha$ two linear mappings

$$
\rho, \rho^{\prime}: \bigwedge^{2} \mathfrak{g} \rightarrow \mathfrak{h}, \quad\left[\alpha_{X}, \alpha_{Y}\right]-\alpha_{[X, Y]}=\operatorname{ad}_{\rho(X, Y)}=\operatorname{ad}_{\rho^{\prime}(X, Y)} .
$$

Then $\rho-\rho^{\prime}=: \mu: \bigwedge^{2} \mathfrak{g} \rightarrow Z(\mathfrak{h})$ and $\lambda(\alpha, \rho)-\lambda\left(\alpha, \rho^{\prime}\right)=\delta_{\alpha} \rho-\delta_{\alpha} \rho^{\prime}=\delta_{\bar{\alpha}} \mu$.

If there exists an extension inducing $\bar{\alpha}$, then for any lift $\alpha$ we may find $\rho$ as in (15.5) such that $\lambda(\alpha, \rho)=0$. On the other hand, given a pair $(\alpha, \rho)$ as in (1) such that $[\lambda(\alpha, \rho)]=0 \in H^{3}(\mathfrak{g},(Z(\mathfrak{h}), \bar{\alpha}))$, there exists $\mu: \bigwedge^{2} \mathfrak{g} \rightarrow Z(\mathfrak{h})$ such that $\delta_{\bar{\alpha}} \mu=\lambda$. But then

$$
\operatorname{ad}_{(\rho-\mu)(X, Y)}=\operatorname{ad}_{\rho(X, Y)}, \quad \delta_{\alpha}(\rho-\mu)=0,
$$

so that $(\alpha, \rho-\mu)$ satisfies the conditions of (15.5) and thus defines an extension which induces $\bar{\alpha}$.

Finally, suppose that (1) is satisfied, and let us determine how many extensions there exist which induce $\bar{\alpha}$. By (15.5) we have to determine all equivalence classes of data $(\alpha, \rho)$ as in (15.5) . We may fix the linear lift $\alpha$ and one mapping $\rho: \bigwedge^{2} \mathfrak{g} \rightarrow \mathfrak{h}$ which satisfies (15.5.3) and (15.5.4), and we have to find all $\rho^{\prime}$ with this property. But then $\rho-\rho^{\prime}=\mu: \bigwedge^{2} \mathfrak{g} \rightarrow Z(\mathfrak{h})$ and

$$
\delta_{\bar{\alpha}} \mu=\delta_{\alpha} \rho-\delta_{\alpha} \rho^{\prime}=0-0=0
$$

so that $\mu$ is a 2 -cocycle. We may still pass to equivalent data in the sense of (15.5) using some $b: \mathfrak{g} \rightarrow \mathfrak{h}$ which does not change $\alpha$, i.e., $b: \mathfrak{g} \rightarrow Z(\mathfrak{h})$. 
The corresponding $\rho^{\prime}$ is, by (15.5.7), $\rho^{\prime}=\rho+\delta_{\alpha} b+\frac{1}{2}[b, b]_{\wedge}=\rho+\delta_{\bar{\alpha}} b$. Thus only the cohomology class of $\mu$ matters.

15.9. Corollary. Let $\mathfrak{g}$ and $\mathfrak{h}$ be Lie algebras such that $\mathfrak{h}$ is abelian. Then isomorphism classes of extensions of $\mathfrak{g}$ over $\mathfrak{h}$ correspond bijectively to the set of all pairs $(\alpha,[\rho])$, where $\alpha: \mathfrak{g} \rightarrow \mathfrak{g l}(\mathfrak{h})=\operatorname{der}(\mathfrak{h})$ is a homomorphism of Lie algebras and $[\rho] \in H^{2}(\mathfrak{g}, \mathfrak{h})$ is a Chevalley cohomology class with coefficients in the $\mathfrak{g}$-module $\mathfrak{h}$ given by $\alpha$.

Isomorphism classes of central extensions correspond bijectively to elements $[\rho] \in H^{2}(\mathfrak{g}, \mathbb{R}) \otimes \mathfrak{h}$ (0 action of $\mathfrak{g}$ on $\left.\mathfrak{h}\right)$.

Proof. This is obvious from theorem (15.8).

15.10. An interpretation of the class $\lambda$. Let $\mathfrak{h}$ and $\mathfrak{g}$ be Lie algebras and let a homomorphism $\bar{\alpha}: \mathfrak{g} \rightarrow \operatorname{der}(\mathfrak{h}) / \operatorname{ad}(\mathfrak{h})$ be given. We consider the extension

$$
0 \rightarrow \operatorname{ad}(\mathfrak{h}) \rightarrow \operatorname{der}(\mathfrak{h}) \rightarrow \operatorname{der}(\mathfrak{h}) / \operatorname{ad}(\mathfrak{h}) \rightarrow 0
$$

and the following diagram, where the bottom right hand square is a pullback (compare with remark (15.7)):

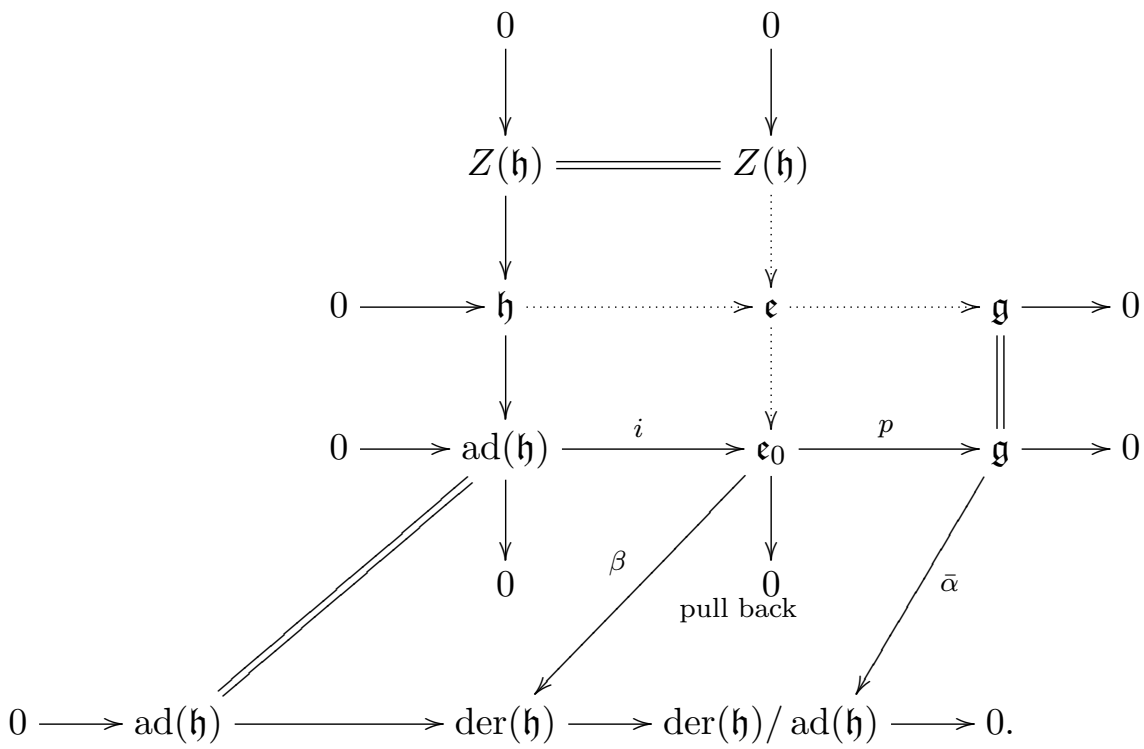

The left hand vertical column describes $\mathfrak{h}$ as a central extension of $\operatorname{ad}(\mathfrak{h})$ with abelian kernel $Z(\mathfrak{h})$ which is moreover killed under the action of $\mathfrak{g}$ via $\bar{\alpha}$; it is given by a cohomology class $[\nu] \in H^{2}(\operatorname{ad}(\mathfrak{h}) ; Z(\mathfrak{h}))^{\mathfrak{g}}$. In order to get an extension $\mathfrak{e}$ of $\mathfrak{g}$ with kernel $\mathfrak{h}$ as in the third row, we have to check that the cohomology class $[\nu]$ is in the image of $i^{*}: H^{2}\left(\mathfrak{e}_{0} ; Z(\mathfrak{h})\right) \rightarrow H^{2}(\operatorname{ad}(\mathfrak{h}) ; Z(\mathfrak{h}))^{\mathfrak{g}}$. It would be interesting to express this in terms of the Hochschild-Serre exact sequence; see [92]. 


\section{Extensions of Groups and Lie Groups}

In this section we present a discussion and variants of cohomology results going back to O. Schreier [201, 202], R. Baer [15], S. Eilenberg and S. MacLane, [56], G. Hochschild [88, 89], and G. Hochschild and J.-P Serre [91. A convenient source for group cohomology is [74. We have to be careful when taking sections; see (15.12) for a discussion of this.

15.11. Describing extensions. Let $G$ and $N$ be Lie groups. An extension of $G$ over $N$ is an exact sequence of homomorphism of groups:

$$
e \rightarrow N \stackrel{i}{\longrightarrow} E \stackrel{p}{\longrightarrow} G \rightarrow e .
$$

Two extensions are defined to be equivalent if there exists a homomorphism $\varphi$ fitting commutatively into the diagram

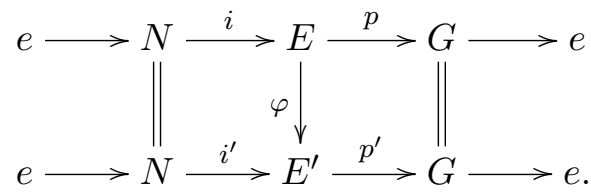

Note that if such a morphism $\varphi$ exists, then it is an isomorphism.

For a given extension let us choose a section $s: G \rightarrow E$ of $p$ with $s(e)=e$. We may assume that $s$ is smooth on an open $e$-neighborhood $U$ in $G$. Then $s$ defines mappings

$$
\begin{aligned}
\alpha: G \rightarrow \operatorname{Aut}(N), & \alpha_{x}(h) & =s(x) h s(x)^{-1}, \\
f: G \times G \rightarrow N, & f(x, y) & =s(x) s(y) s(x y)^{-1},
\end{aligned}
$$

which are smooth near $e$ in $G$ and, by the definition of $\alpha$ and by associativity, have the following properties:

$$
\begin{aligned}
\alpha_{x} \circ \alpha_{y} & =\operatorname{conj}_{f(x, y)} \circ \alpha_{x y}, \\
\alpha_{x}(f(y, z)) f(x, y z) & =f(x, y) f(x y, z), \\
f(e, e) & =f(x, e)=f(e, x)=e,
\end{aligned}
$$

where $\operatorname{conj}_{h}(n)=h n h^{-1}$ is conjugation by $H$, an inner automorphism. We shall denote by $\operatorname{Int}(N) \subseteq \operatorname{Aut}(N)$ the normal subgroup of all inner automorphisms in the group of all automorphisms. If we choose another section $s^{\prime}: G \rightarrow E$ which is smooth near $e$, then $s^{\prime}(x)=b(x) s(x)$ for a mapping $b: G \rightarrow N$ which is smooth near $e$ in $G$. We have

$$
\begin{aligned}
\alpha_{x}^{\prime} & =\operatorname{conj}_{b(x)} \circ \alpha_{x} \\
f^{\prime}(x, y) & =b(x) \alpha_{x}(b(y)) f(x, y) b(x y)^{-1} .
\end{aligned}
$$


The group multiplication on $E$ is then described in terms of $\alpha$ and $f$ by

$$
\begin{aligned}
m s(x) . n s(y) & =m s(x) n s(x)^{-1} s(x) s(y)=m \alpha_{x}(n) f(x, y) . s(x y), \\
(m s(x))^{-1} & =\alpha_{x^{-1}}\left(f\left(x, x^{-1}\right)^{-1} m^{-1}\right) \cdot s\left(x^{-1}\right) \\
& =\left(\alpha_{x}\right)^{-1}\left(m^{-1} f\left(x, x^{-1}\right)^{-1}\right) s\left(x^{-1}\right) \\
& =f\left(x^{-1}, x\right)^{-1} \alpha_{x^{-1}}\left(m^{-1}\right) \cdot s\left(x^{-1}\right) .
\end{aligned}
$$

See (15.12) below for the reconstruction of the smooth structure.

\subsection{Choosing sections smoothly or reconstructing the smooth manifold structure. Let}

$$
e \rightarrow N \stackrel{i}{\longrightarrow} E \stackrel{p}{\longrightarrow} G \rightarrow e
$$

be an exact sequence of smooth homomorphisms of Lie groups. In particular, $E$ is a principal fiber bundle over $G$ with structure group $N$. If we are able to choose a smooth section $s: G \rightarrow E$ of $p$ as in (15.11), then this is a trivial fiber bundle, so $E \cong N \times G$ as a smooth manifold, and we can use all constructions of (15.13) (15.27) below to describe Lie group extensions of $G$ over $N$ which are topologically trivial.

Let us look at the long exact sequence in homotopy:

$$
\cdots \rightarrow \pi_{2}(G) \rightarrow \pi_{1}(N) \rightarrow \pi_{1}(E) \rightarrow \pi_{1}(G) \rightarrow \pi_{0}(N) \rightarrow \ldots
$$

We always have $\pi_{2}(N)=0$. So if $N$ is connected and $E$ is simply connected, then both $N$ and $G$ are simply connected. Using structure theory of Lie algebras and Lie groups, one can prove the following (see [90]): If $E$ is simply connected and $N$ is connected, then there is a closed submanifold $M$ of $E$ meeting $N$ only in $\{e\}$ transversally, such that $E \cong N \times M$. Thus there exists a global smooth section $s: G \rightarrow E$.

For the topologically nontrivial case, we can find a global section $s$ which is smooth only on a neighborhood $U$ of $e$ in $G$ which also satisfies $U^{-1}=U$.

Lemma. Then we can reconstruct the Lie group structure on E from the extension data (which are all smooth near e on $G$ ) and the smooth manifold structure on $N \times U \cong \tilde{U}:=p^{-1}(U) \subset E$.

Proof. Choose $e \in V \subset U$ open with $V^{-1}=V$ and $V \cdot V \subset U$, and let $\tilde{V}:=p^{-1}(V)$. In the setting of (15.11) we then have: $\alpha: U \rightarrow \operatorname{Aut}(N)$ and $f: V \times V \rightarrow N$ are smooth and the group multiplication (15.13.4) is smooth on $\tilde{V} \times \tilde{V} \rightarrow \tilde{U}$. We then use $\left(x \cdot \tilde{V}, \mu_{x^{-1}}: x \cdot \tilde{V} \rightarrow \tilde{V}\right)_{x \in E}$ as atlas for $E$. The chart changes are $\mu_{y^{-1}} \circ \mu_{x}=\mu_{y^{-1} \cdot x}: x^{-1} \cdot(x \cdot \tilde{V} \cap y \cdot \tilde{V})=\tilde{V} \cap\left(x^{-1} \cdot y \cdot \tilde{V}\right) \rightarrow$ $\left(y^{-1} \cdot x \cdot \tilde{V}\right) \cap \tilde{V}$, so they are smooth. The resulting smooth manifold structure on $E$ has the property that $p: E \rightarrow G$ and $i: N \rightarrow E$ are smooth, and the group structure maps $\mu$ and $\nu$ are smooth also. Moreover $E$ is Hausdorff: 
Either $p(x)=p(y)$ and then we can separate them already in one chart $x \cdot \tilde{V}=p^{-1}(p(x) \cdot V)$, or we can separate them with open sets of the form $p^{-1}\left(U_{1}\right)$ and $p^{-1}\left(U_{2}\right)$.

We shall use this lemma in all constructions below without mentioning it. Note that a homomorphism between Lie groups which is smooth near $e$ is smooth everywhere.

15.13. Proposition ([201, 202]). Let $G$ and $N$ be Lie groups. We consider pairs $(\alpha, f)$ of mappings which are smooth near $e$ :

$$
\alpha: G \rightarrow \operatorname{Aut}(N) \quad \text { and } \quad f: G \times G \rightarrow N
$$

with the properties

$$
\begin{aligned}
\alpha_{x} \circ \alpha_{y} & =\operatorname{conj}_{f(x, y)} \circ \alpha_{x y}, \\
f(e, e) & =f(x, e)=f(e, y)=e, \\
e & =\alpha_{x}(f(y, z)) f(x, y z) f(x y, z)^{-1} f(x, y)^{-1} .
\end{aligned}
$$

Then the following assertions hold:

(4) Every such pair $(\alpha, f)$ defines a Lie group extension of $G$ over $N$, given by the set $E=N \times G$, with the group structure

$$
\begin{aligned}
(m, x) \cdot(n, y) & =\left(m \alpha_{x}(n) f(x, y), x y\right), \\
(n, x)^{-1} & =\left(f\left(x^{-1}, x\right)^{-1} \alpha_{x^{-1}}\left(n^{-1}\right), x^{-1}\right) .
\end{aligned}
$$

Up to isomorphism, every extension of $G$ over $N$ can be so obtained.

(5) Two data $(\alpha, f)$ and $\left(\alpha^{\prime}, f^{\prime}\right)$ define equivalent extensions if there exists a mapping $b: G \rightarrow N$ (smooth near e) such that

$$
\begin{aligned}
\alpha_{x}^{\prime} & =\operatorname{conj}_{b(x)} \circ \alpha_{x}, \\
f^{\prime}(x, y) & =b(x) \alpha_{x}(b(y)) f(x, y) b(x y)^{-1} .
\end{aligned}
$$

The induced smooth isomorphism $E \rightarrow E^{\prime}$ between the extensions defined by $(\alpha, f)$ and $\left(\alpha^{\prime}, f^{\prime}\right)$ is given by $(n, x) \mapsto\left(n b(x)^{-1}, x\right)$.

(6) A datum $(\alpha, f)$ describes a splitting extension (a semidirect product) if and only if it is equivalent to a datum $\left(\alpha^{\prime}, f^{\prime}\right)$, where $f^{\prime}$ is constant $=e$. This is the case if and only if there exists a map $b: G \rightarrow N$ (smooth near e) with

$$
f(x, y)=b(x) \alpha_{x}(b(y)) b(x y)^{-1} .
$$

Note that for such a pair $\left(\alpha^{\prime}, f^{\prime}=e\right)$ the map $\alpha^{\prime}$ must be a homomorphism and thus is smooth everywhere.

Proof. (15.11) and routine calculations. 
15.14. Remarks. (1) The center $Z(N)$ of $N$ is preserved by all automorphisms of $N$ and pointwise fixed by all inner automorphisms, so the Lie group $\operatorname{Aut}(N) / \operatorname{Int}(N)$ acts by automorphisms on $Z(N)$. Every homomorphism $\bar{\alpha}: G \rightarrow \operatorname{Aut}(N) / \operatorname{Int}(N)$ naturally induces a homomorphism $G \rightarrow$ $\operatorname{Aut}(Z(N))$ and therefore turns $Z(N)$ into a smooth $G$-module $(Z(N), \bar{\alpha})$. Condition (15.13.1) implies that every extension of $G$ over $N$ induces a smooth homomorphism $\bar{\alpha}: G \rightarrow \operatorname{Aut}(N) / \operatorname{Int}(N)$, hence defines a $G$-module structure on $Z(N)$. Thus we have the following commutative diagram with exact rows:

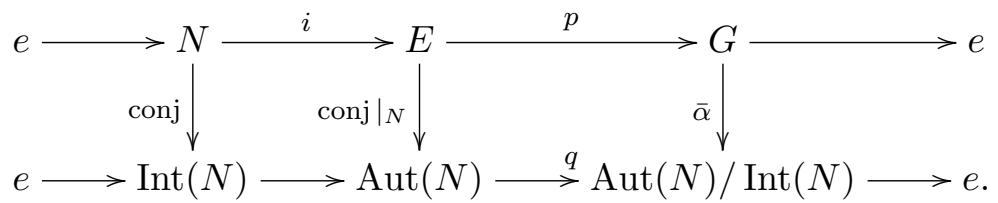

Note that the commutativity of this diagram yields a surjective homomorphism $E \rightarrow \Gamma$, where $\Gamma$ is the pullback object of the morphisms $q$ and $\bar{\alpha}$. We shall exploit this fact later.

(2) Note that if $(\alpha, f)$ is the data of an extension then every lift $\alpha^{\prime}: G \rightarrow N$ (smooth near $e$ ) of $\bar{\alpha}$ shows up in a data pair $\left(\alpha^{\prime}, f^{\prime}\right)$ equivalent to $(\alpha, f)$. This is a consequence of (15.13.5).

(3) In 15 and 56 a triplet $(N, G, \bar{\alpha})$, where $N$ and $G$ are groups and $\bar{\alpha}$ is a homomorphism $G \rightarrow \operatorname{Aut}(N) / \operatorname{Int}(N)$, is usually called an abstract kernel or kernel for short. The kernel $(N, G, \bar{\alpha})$ is said to be extendible if it can be derived from an extension of $G$ over $N$.

In the following we want to characterize those smooth homomorphisms $\bar{\alpha}$ for which $(N, G, \bar{\alpha})$ is an extendible kernel.

15.15. Notation. Let us fix a smooth homomorphism of Lie groups $\bar{\alpha}$ : $G \rightarrow \operatorname{Aut}(N) / \operatorname{Int}(N)$ and consider all pairs $(\alpha, f)$ consisting of a lift $\alpha$ : $G \rightarrow \operatorname{Aut}(N), x \mapsto \alpha_{x}$, of $\bar{\alpha}$, and of $f: G \times G \rightarrow N$ which are smooth near $e$ and satisfy conditions (15.13.1) and (15.13.2).

$$
\begin{aligned}
\alpha_{x} \circ \alpha_{y} & =\operatorname{conj}_{f(x, y)} \circ \alpha_{x y} \\
f(e, e) & =f(x, e)=f(e, x)=e, \quad \alpha_{e}=\operatorname{Id}_{N} .
\end{aligned}
$$

For the sake of brevity, we call such a pair $(\alpha, f)$ an $\bar{\alpha}$-pair. We write

$$
\lambda(x, y, z)=\alpha_{x}(f(y, z)) f(x, y z) f(x y, z)^{-1} f(x, y)^{-1}
$$

for the right side of equation (15.13.3), To avoid taking inverses it will be often convenient to write (3) in the equivalent form

$$
\lambda(x, y, z) f(x, y) f(x y, z)=\alpha_{x}(f(y, z)) f(x, y z) .
$$


Note that the normalization condition (15.13.2) implies that $\lambda$ is also normalized, i.e.,

$$
\lambda(e, y, z)=\lambda(x, e, z)=\lambda(x, y, e)=e \quad \text { for all } x, y, z \in G .
$$

Two $\bar{\alpha}$-pairs $(\alpha, f)$ and $\left(\alpha^{\prime}, f^{\prime}\right)$ are said to be equivalent if there exists a mapping $b: G \rightarrow N$ such that

$$
\begin{aligned}
\alpha_{x}^{\prime} & =\operatorname{conj}_{b(x)} \circ \alpha_{x} \\
f^{\prime}(x, y) & =b(x) \alpha_{x}(b(y)) f(x, y) b(x y)^{-1} .
\end{aligned}
$$

Following [93, the function $f$ is traditionally called a factor set, and $\lambda$ is called the obstruction of $(\alpha, f)$ to form an extension. We shall not use this terminology here.

15.16. Nonabelian cohomology. Let $(Z, \beta)$ be a smooth $G$-module, i.e., an abelian Lie group with a smooth $G$-action. The boundary operator of group cohomology with values in $(Z, \beta)$ is given by

$$
\begin{aligned}
& \delta_{\beta}: \operatorname{Map}_{e}\left(G^{k}, Z\right) \rightarrow \operatorname{Map}_{e}\left(G^{k+1}, Z\right), \\
& \left(\delta_{\beta} f\right)\left(x_{0}, x_{1}, \ldots, x_{k}\right)=\beta_{x_{0}}\left(f\left(x_{1}, \ldots, x_{k}\right)\right) . f\left(x_{0} x_{1}, x_{2}, \ldots, x_{k}\right)^{-1} \\
& \quad . f\left(x_{0}, x_{1} x_{2}, x_{3}, \ldots, x_{k}\right) \ldots f\left(x_{0}, \ldots, x_{k-1} x_{k}\right)^{(-1)^{k}} \cdot f\left(x_{0}, \ldots, x_{k-1}\right)^{(-1)^{k+1}}
\end{aligned}
$$

where $\mathrm{Map}_{e}$ denotes the space of mappings which are smooth near $e$. This gives rise to abelian group cohomology; here $Z$ is abelian!

Now we discuss a nonabelian version. Inspired by condition (15.13.3) or by (15.15.3), for every map $\alpha: G \rightarrow \operatorname{Aut}(N)$ and $f: G \times G \rightarrow N$ which are smooth near $e$ we consider

$$
\begin{gathered}
\delta_{\alpha} f: G \times G \times G \rightarrow N, \\
\left(\delta_{\alpha} f\right)(x, y, z)=\alpha_{x}(f(y, z)) f(x, y z) f(x y, z)^{-1} f(x, y)^{-1} .
\end{gathered}
$$

Then $\delta_{\alpha}$ looks like the nonabelian version of a coboundary - except that

(a) $\alpha$ is not a homomorphism, and that

(b) in comparison with the above traditional definition the order of the two middle terms of the expression for $\delta_{\alpha} f$ is reversed.

Likewise assertion (15.13.6) suggests to consider for $b: G \rightarrow N$ (smooth near $e$ ) the 'nonabelian coboundary'

$$
\delta_{\alpha} b: G \times G \rightarrow N, \quad\left(\delta_{\alpha} b\right)(x, y)=b(x) \alpha_{x}(b(y)) b(x y)^{-1} .
$$

Also in this case the terms in the expression on the right hand side do not follow the traditional order. 
A straightforward computation shows that

$$
\begin{aligned}
& \delta_{\alpha} \delta_{\alpha} b(x, y, z) \\
& =\alpha_{x}\left(b(y) \alpha_{y}(b(z)) b(y z)^{-1}\right) b(x) \alpha_{x}(b(y z)) \alpha_{x y}(b(z))^{-1} \alpha_{x}(b(y))^{-1} b(x)^{-1} .
\end{aligned}
$$

If the image of $b$ is central in $N$, then this reduces to

$$
\delta_{\alpha} \delta_{\alpha} b(x, y, z)=\alpha_{x} \circ \alpha_{y}(b(z)) \alpha_{x y}(b(z))^{-1} .
$$

Thus we cannot expect $\delta_{\alpha} \delta_{\alpha} b=e$ in general.

15.17. Remarks. By (15.13) an $\bar{\alpha}$-pair $(\alpha, f)$ is the data of an extension if and only if the associated map $\lambda=\delta_{\alpha} f$ is identically $=e$.

If $\alpha^{\prime}: G \rightarrow \operatorname{Aut}(N)$ is another lift (smooth near $e$ ) of $\bar{\alpha}$, then there exists a map $f^{\prime}: G \times G \rightarrow N$ (smooth near $e$ ) such that $\left(\alpha^{\prime}, f^{\prime}\right)$ is equivalent to $(\alpha, f)$.

For fixed $\alpha$ the $\bar{\alpha}$-pairs $(\alpha, f)$ and $\left(\alpha, f^{\prime}\right)$ are equivalent if and only if there exists a map $b: G \rightarrow Z(N)$ which is smooth near $e$ such that

$$
f^{\prime}(x, y)=b(x) \alpha_{x}(b(y)) f(x, y) b(x y)^{-1},
$$

that is, the maps $f^{\prime}$ and $f$ differ only by the coboundary $\delta_{\bar{\alpha}} b$ with respect to cohomology with values in the $G$-module $(Z(N), \bar{\alpha})$. Since $\alpha=\alpha^{\prime}$, the equation $\alpha_{x}=\operatorname{conj}_{b(x)} \circ \alpha_{x}^{\prime}$ implies conj $_{b(x)}=i d$, so $b(x)$ must be central.

\subsection{Lemma.}

(1) For any $\bar{\alpha}$-pair $(\alpha, f)$ the associated $\lambda=\delta_{\alpha} f$ takes values in the center of $N$.

(2) If the pairs $(\alpha, f)$ and $\left(\alpha^{\prime}, f^{\prime}\right)$ are equivalent, then the associated maps $\lambda$ and $\lambda^{\prime}$ coincide. In particular, if $(\alpha, f)$ is the data of an extension, then so is every equivalent pair $\left(\alpha^{\prime}, f^{\prime}\right)$.

Proof of (1). Applying condition (15.13.1), we find

$$
\begin{aligned}
\operatorname{conj}_{\lambda(x, y, z)} & =\operatorname{conj}_{\alpha_{x}(f(y, z))} \operatorname{conj}_{f(x, y z)} \operatorname{conj}_{f(x y, z)^{-1}} \operatorname{conj}_{f(x, y)^{-1}} \\
& =\alpha_{x} \alpha_{y} \alpha_{z} \alpha_{y z}^{-1} \alpha_{x}^{-1} \alpha_{x} \alpha_{y z} \alpha_{x y z}^{-1} \alpha_{x y z} \alpha_{z}^{-1} \alpha_{x y}^{-1} \alpha_{x y} \alpha_{y}^{-1} \alpha_{x}^{-1} \\
& =\operatorname{Id}_{N}
\end{aligned}
$$

which means that $\lambda(x, y, z)$ must lie in the center of $N$.

Proof of (2) . Let $\left(\alpha^{\prime}, f^{\prime}\right)$ be equivalent to $(\alpha, f)$. Then there exists a map $b: G \rightarrow N$ with

$$
\alpha_{x}^{\prime}=\operatorname{conj}_{b(x)} \circ \alpha_{x}, \quad f^{\prime}(x, y)=b(x) \alpha_{x}(b(y)) f(x, y) b(x y)^{-1} .
$$

By definition we have

$$
\lambda^{\prime}(x, y, z) f^{\prime}(x, y) f^{\prime}(x y, z)=\alpha_{x}^{\prime}\left(f^{\prime}(y, z)\right) f^{\prime}(x, y z) .
$$


Inserting the identities (3), the left side of this equation reads

$$
\begin{aligned}
& \lambda^{\prime}(x, y, z) \cdot f^{\prime}(x, y) \cdot f^{\prime}(x y, z) \\
& =\lambda^{\prime}(x, y, z) \cdot b(x) \alpha_{x}(b(y)) f(x, y) b(x y)^{-1} \cdot b(x y) \alpha_{x y}(b(z)) f(x y, z) b(x y z)^{-1} \\
& =\lambda^{\prime}(x, y, z) \cdot b(x) \alpha_{x}(b(y)) f(x, y) \alpha_{x y}(b(z)) f(x y, z) b(x y z)^{-1} .
\end{aligned}
$$

Since $\operatorname{conj}_{f(x, y)} \circ \alpha_{x y}=\alpha_{x} \circ \alpha_{y}$, we have $f(x, y) \alpha_{x y}(b(z))=\alpha_{x} \alpha_{y}(b(z)) f(x, y)$ and therefore (using also that $\lambda^{\prime}(x, y, z)$ is central in $\left.N\right)$ :

$$
\begin{aligned}
\lambda^{\prime} & (x, y, z) \cdot f^{\prime}(x, y) \cdot f^{\prime}(x y, z) \\
\quad & =\lambda^{\prime}(x, y, z) \cdot b(x) \alpha_{x}(b(y)) \alpha_{x} \alpha_{y}(b(z)) f(x, y) f(x y, z) b(x y z)^{-1} \\
& =\lambda^{\prime}(x, y, z) \cdot b(x) \alpha_{x}\left(b(y) \alpha_{y}(b(z))\right) f(x, y) f(x y, z) b(x y z)^{-1} \\
& =b(x) \alpha_{x}\left(b(y) \alpha_{y}(b(z))\right) \lambda^{\prime}(x, y, z) f(x, y) f(x y, z) b(x y z)^{-1} .
\end{aligned}
$$

Similarly, the right side can be transformed into

$$
\begin{aligned}
& \alpha_{x}^{\prime}\left(f^{\prime}(y, z)\right) \cdot f^{\prime}(x, y z) \\
& =\operatorname{conj}_{b(x)} \alpha_{x}\left(b(y) \alpha_{y}(b(z)) f(y, z) b(y z)^{-1}\right) \cdot b(x) \alpha_{x}(b(y z)) f(x, y z) b(x y z)^{-1} \\
& =b(x) \alpha_{x}\left(b(y) \alpha_{y}(b(z)) f(y, z) b(y z)^{-1}\right) b(x)^{-1} b(x) \alpha_{x}(b(y z)) f(x, y z) b(x y z)^{-1} \\
& =b(x) \alpha_{x}\left(b(y) \alpha_{y}(b(z))\right) \alpha_{x}(f(y, z)) f(x, y z) b(x y z)^{-1} .
\end{aligned}
$$

Canceling the term $b(x) \alpha_{x}\left(b(y) \alpha_{y}(b(z))\right)$ on the right and the term $b(x y z)^{-1}$ on the left, we see that $\lambda^{\prime}(x, y, z)$ satisfies

$$
\lambda^{\prime}(x, y, z) f(x, y) f(x y, z)=\alpha_{x}(f(y, z)) f(x, y z),
$$

the defining equation for $\lambda(x, y, z)$. Thus $\lambda=\lambda^{\prime}$.

15.19. Lemma. Let $(\alpha, f)$ be an $\bar{\alpha}$-pair and let $\lambda=\delta_{\alpha} f$.

(1) The map

$$
\lambda: G \times G \times G \rightarrow Z(N), \quad(x, y, z) \mapsto \lambda(x, y, z),
$$

is a normalized 3-cocycle with respect to $\delta_{\bar{\alpha}}$ cohomology with values in the $G$-module $(Z(N), \bar{\alpha})$ and is smooth near e.

(2) The cocycles (smooth near e) in the $\delta_{\bar{\alpha}}$ cohomology class $[\lambda]$ of $\lambda$ are exactly the maps $\lambda^{\prime}=\delta_{\alpha} f^{\prime}$ which are induced by an $\bar{\alpha}$-pair of the form $\left(\alpha, f^{\prime}\right)$.

(3) An $\bar{\alpha}$-pair $\left(\alpha, f^{\prime}\right)$ induces the same cocycle $\lambda \in[\lambda]$ as $(\alpha, f)$ if and only if $f=f^{\prime} \cdot c$, where $c: G \times G \rightarrow Z(N)$, is a 2-cocycle with respect to $\delta_{\bar{\alpha}}$ cohomology, normalized by the condition $c(x, e)=c(e, y)=e$, and is smooth near e. 
Proof. (11) In order to show that $\lambda$ is a 3 -cocycle, we have to prove that, for any quadruplet $(x, y, z, u)$ of elements in $G$,

$$
\begin{aligned}
& \left(\delta_{\bar{\alpha}} \lambda\right)(x, y, z, u) \\
& \quad=\alpha_{x}(\lambda(y, z, u)) \lambda(x y, z, u)^{-1} \lambda(x, y z, u) \lambda(x, y, z u)^{-1} \lambda(x, y, z)=e,
\end{aligned}
$$

or, equivalently, that

$$
\alpha_{x}(\lambda(y, z, u)) \lambda(x, y z, u) \lambda(x, y, z)=\lambda(x y, z, u) \lambda(x, y, z u) .
$$

By the definition of $\lambda$ and the centrality of the $\lambda$ 's, we have for the right side $R$ of this equation:

$$
\begin{aligned}
R & =\alpha_{x y}(f(z, u)) f(x y, z u) f(x y z, u)^{-1} f(x y, z)^{-1} \cdot \lambda(x, y, z u) \\
& =\alpha_{x y}(f(z, u)) \cdot \lambda(x, y, z u) \cdot f(x y, z u) f(x y z, u)^{-1} f(x y, z)^{-1} .
\end{aligned}
$$

Applying the equation $\alpha_{x y}=\operatorname{conj}_{f(x, y)^{-1}} \circ \alpha_{x} \circ \alpha_{y}$, we conclude

$$
R f(x y, z) f(x y z, u)=f(x, y)^{-1} \alpha_{x} \alpha_{y}(f(z, u)) f(x, y) \lambda(x, y, z u) f(x y, z u),
$$

and, by the centrality of $R$ and the $\lambda$ 's,

$$
\begin{aligned}
& R f(x, y) f(x y, z) f(x y z, u)=\alpha_{x} \alpha_{y}(f(z, u)) \cdot \lambda(x, y, z u) f(x, y) f(x y, z u) \\
& =\alpha_{x} \alpha_{y}(f(z, u)) \alpha_{x}(f(y, z u)) f(x, y z u) f(x y, z u)^{-1} f(x, y)^{-1} f(x, y) f(x y, z u) \\
& =\alpha_{x} \alpha_{y}(f(z, u)) \alpha_{x}(f(y, z u)) f(x, y z u) .
\end{aligned}
$$

For the left side $L=\alpha_{x}(\lambda(y, z, u)) \lambda(x, y z, u) \lambda(x, y, z)$ we see

$$
\begin{aligned}
L= & \alpha_{x}(\lambda(y, z, u)) \cdot \lambda(x, y z, u) \cdot \lambda(x, y, z) \\
= & \alpha_{x}(\lambda(y, z, u)) \cdot \lambda(x, y z, u) \cdot \alpha_{x}(f(y, z)) f(x, y z) f(x y, z)^{-1} f(x, y)^{-1} \\
= & \alpha_{x}(\lambda(y, z, u) \cdot f(y, z)) \cdot \lambda(x, y z, u) f(x, y z) f(x y, z)^{-1} f(x, y)^{-1} \\
= & \alpha_{x}(\lambda(y, z, u) \cdot f(y, z)) \cdot \alpha_{x}(f(y z, u)) f(x, y z u) f(x y z, u)^{-1} f(x, y z)^{-1} \\
& \quad \cdot f(x, y z) f(x y, z)^{-1} f(x, y)^{-1} \\
= & \alpha_{x}\left(\alpha_{y}(f(z, u)) f(y, z u) f(y z, u)^{-1} f(y, z)^{-1} f(y, z) f(y z, u)\right) \\
& \quad \cdot f(x, y z u) f(x y z, u)^{-1} f(x y, z)^{-1} f(x, y)^{-1} \\
= & \alpha_{x}\left(\alpha_{y}(f(z, u)) f(y, z u)\right) f(x, y z u) f(x y z, u)^{-1} f(x y, z)^{-1} f(x, y)^{-1} .
\end{aligned}
$$

Thus we conclude that

$$
\begin{aligned}
L f(x, y) f(x y, z) f(x y z, u) & =\alpha_{x}\left(\alpha_{y}(f(z, u)) f(y, z u)\right) f(x, y z u) \\
& =R f(x, y) f(x y, z) f(x y z, u)
\end{aligned}
$$

and, upon cancellation, $L=R$. This finishes the proof of (1).

(2) Consider any mapping $f^{\prime}: G \times G \rightarrow N$ such that $\left(\alpha, f^{\prime}\right)$ is an $\bar{\alpha}$-pair. Then

$$
\operatorname{conj}_{f(x, y)}=\operatorname{conj}_{f^{\prime}(x, y)}=\left(\alpha_{x y}\right)^{-1} \alpha_{x}^{-1} \alpha_{y},
$$


and therefore the element $c(x, y)=f(x, y)^{-1} f^{\prime}(x, y)$ lies in the center $Z(N)$ of $N$. Now

$$
\begin{aligned}
& \lambda^{\prime}(x, y, z) \lambda(x, y, z)^{-1} \\
& =\alpha_{x}(f(y, z) c(y, z)) f(x, y z) c(x, y z) c(x y, z)^{-1} f(x y, z)^{-1} c(x, y)^{-1} f(x, y)^{-1} \\
& \cdot f(x, y) f(x y, z) f(x, y z)^{-1} \alpha_{x}\left(f(y, z)^{-1}\right) \\
& =\alpha_{x}(c(y, z)) c(x, y z) c(x y, z)^{-1} c(x, y)^{-1}=\left(\delta_{\bar{\alpha}} c\right)(x, y, z)
\end{aligned}
$$

Reading the above calculations backwards, we see that, conversely, every cochain $\lambda^{\prime}$ lying in the cohomology class of $\lambda$ is induced by some pair $\left(\alpha, f^{\prime}\right)$. (3) We have seen in the proof of (2) that the cochains $\lambda=\delta_{\alpha} f, \lambda^{\prime}=\delta_{\alpha} f^{\prime}$ induced, respectively, by the $\bar{\alpha}$-pairs $(\alpha, f)$ and $\left(\alpha, f^{\prime}\right)$ differ by the cocycle $\delta_{\bar{\alpha}} c$, where $c(x, y)=f(x, y)^{-1} f^{\prime}(x, y)$. Thus $(\alpha, f)$ and $\left(\alpha, f^{\prime}\right)$ induce the same cocycle $\lambda$ if and only if $\delta_{\bar{\alpha}} c$ vanishes. This implies that the cocycles in the cohomology class $[\lambda]$ are in 1-1 correspondence to the 2 -cocycles with respect to $\delta_{\bar{\alpha}}$-cohomology. This finishes the proof.

15.20. Corollary. The cohomology class of $\lambda=\delta_{\alpha} f$ depends only on $\bar{\alpha}$, not on the particular choice of the $\bar{\alpha}$-pair $(\alpha, f)$.

Proof. Suppose that $\left(\alpha^{\prime}, f^{\prime}\right)$ is another $\bar{\alpha}$-pair and let $\lambda^{\prime}=\delta_{\alpha^{\prime}} f^{\prime}$. By $(15.17)$, the pair $\left(\alpha^{\prime}, f^{\prime}\right)$ is equivalent to some pair $\left(\alpha, f^{\prime \prime}\right)$. Since by lemma (15.18.2) equivalent pairs produce the same $\lambda$, we have $\lambda^{\prime}=\delta_{\alpha} f^{\prime \prime}$. By lemma $(15.19 .2), \delta_{\alpha} f$ and $\delta_{\alpha} f^{\prime \prime}$ are in the same cohomology class. This proves the assertion.

Notation. For given $\bar{\alpha}$ we henceforth write $\lambda^{\bar{\alpha}}$ for the cohomology class $[\lambda] \in H^{3}(G ;(Z(N), \bar{\alpha})$ ) (smooth near $e$ ). By the corollary above this notation is unambiguous.

15.21. Theorem ([56]). Let $G$ and $N$ be Lie groups and consider a smooth homomorphism $\bar{\alpha}: G \rightarrow \operatorname{Aut}(N) / \operatorname{Int}(N)$. Then the following assertions hold:

(1) The homomorphism $\bar{\alpha}$ is induced by a Lie group extension if and only if the corresponding cohomology class $\lambda^{\bar{\alpha}} \in H^{3}(G, Z(N))$ vanishes.

(2) If $\bar{\alpha}$ is induced by an extension, then all extensions inducing $\bar{\alpha}$ are parameterized by $H^{2}(G, Z(N))$.

(3) The homomorphism $\bar{\alpha}$ is induced by a splitting extension if and only if it can be lifted to a (smooth) homomorphism $\alpha: G \rightarrow \operatorname{Aut}(N)$.

Here $\left.H^{k}(G, Z(N))\right)$ denotes the group cohomology (smooth near e) of $G$ with values in the $G$-module $(Z(N), \bar{\alpha})$. 
Proof. (1) We know already from (15.13) that if $(\alpha, f)$ is the data associated with an extension, then $\lambda^{\bar{\alpha}}=e$.

Conversely, if $\lambda^{\bar{\alpha}}$ is trivial, then by (15.19) for any lift $\alpha: G \rightarrow \operatorname{Aut}(N)$ of $\bar{\alpha}$ we can find a map $f: G \times G \rightarrow N$ such that $(\alpha, f)$ is a pair with $\delta_{\alpha} f=e$; by (15.13) this pair $(\alpha, f)$ defines an extension inducing $\bar{\alpha}$.

(2) $\mathrm{By}(15.13)$ an $\bar{\alpha}$-pair $(\alpha, f)$ is the data of an extension if and only if $\delta_{\alpha} f=e$. By (15.19.3) we know that if $\delta_{\alpha} f=\delta_{\alpha} f^{\prime}$, then $f=f^{\prime} \cdot c$, where $c: G \times G \rightarrow Z(N)$ is a 2-cocycle. Furthermore, by (15.15.2) two such $\bar{\alpha}$ pairs are equivalent - and thus define equivalent extensions - if and only if $f=f^{\prime} \cdot c$ where $c$ is the coboundary $c=\delta_{\bar{\alpha}} b$ for $b: G \rightarrow Z(N)$. Thus we see that the extensions inducing $\bar{\alpha}$ are in $1-1$ correspondence with the elements of $H^{2}(G ;(Z(N), \bar{\alpha}))$.

(3) By (15.13.6) we know that if $(\alpha, f)$ is an $\bar{\alpha}$-pair inducing a splitting extension, then there exists a map $b: G \rightarrow N$ such that the map $\alpha^{\prime}: G \rightarrow N$, $x \mapsto \operatorname{conj}_{b(x)} \circ \alpha$ is a homomorphism, so $\bar{\alpha}$ has a homomorphic lift. The converse is obvious.

15.22. Corollary. Let $G$ and $N$ be Lie groups, $N$ abelian. Then isomorphism classes of Lie group extensions of $G$ over $N$ correspond bijectively to the set of pairs $(\alpha,[f])$ where $\alpha: G \rightarrow \operatorname{Aut}(N)$ is a smooth homomorphism and $[f] \in H^{2}(G ;(N, \alpha))$ is an element in the second group cohomology (smooth near e) of $G$ with values in the $G$-module $(N, \alpha)$.

Proof. Since $N$ is abelian, $\operatorname{Int}(N)=e$ and therefore $\bar{\alpha}$ can be considered as a homomorphism $\alpha: G \rightarrow \operatorname{Aut}(N)$. Thus we can form the semidirect product $N \rtimes_{\alpha} G$, so extensions inducing $\bar{\alpha}$ exist. Now theorem (15.21) applies and yields the assertion.

15.23. Corollary ([15]). Let $G$ and $N$ be Lie groups, $N$ without center. Then isomorphism classes of Lie group extensions correspond bijectively to smooth group homomorphisms $\bar{\alpha}: G \rightarrow \operatorname{Aut}(N) / \operatorname{Int}(N)$.

Proof. Since $Z(N)=e$, the cohomologies $H^{3}(G ; Z(N))$ and $H^{2}(G ; Z(N))$ obviously vanish; hence by theorem (15.21) every homomorphism $\bar{\alpha}$ induces a unique extension.

Conversely, every extension induces some $\bar{\alpha}: G \rightarrow \operatorname{Aut}(N) / \operatorname{Int}(N)$ by the construction in (15.11).

Alternative proof of (15.23). For a given $\bar{\alpha}$ consider the group

$$
\Gamma=\{(g, \varphi) \in G \times \operatorname{Aut}(N) \mid \varphi \in \bar{\alpha}(g)\}
$$


which is the pullback object of the diagram

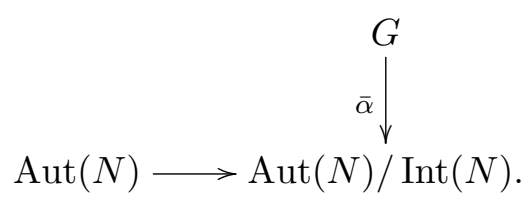

By assumption, $N$ has no center. Therefore the map $N \rightarrow \operatorname{Aut}(N), h \mapsto$ $\operatorname{conj}_{h}$ is injective and hence the map $N \rightarrow \Gamma, h \mapsto\left(e, \operatorname{conj}_{h}\right)$ is a homomorphic injection. Moreover, its image $N$ is the kernel of the quotient map $\Gamma \rightarrow G,(g, \varphi) \mapsto g$. Thus we have an extension

$$
e \rightarrow N \rightarrow \Gamma \longrightarrow G \rightarrow e
$$

of $G$ by $N$ which induces $\bar{\alpha}$. Conversely, let

$$
e \rightarrow N \stackrel{i}{\longrightarrow} E \stackrel{p}{\longrightarrow} G \rightarrow e
$$

be an extension inducing $\bar{\alpha}$. Then the map $\vartheta: E \rightarrow \Gamma, x \mapsto(p(x), q(x))$, where $q(x)$ denotes the automorphism of $N$ induced by $\operatorname{conj}_{x}$, is a homomorphism. Thus (1) and (2) are equivalent extensions.

15.24. In the general case this construction runs as follows: Define $\Gamma$ and $\vartheta$ as above. Then every extension

$$
e \rightarrow N \stackrel{i}{\longrightarrow} E \stackrel{p}{\longrightarrow} G \rightarrow e
$$

gives rise to an extension of $\Gamma$ over the center $Z(N)$ of $N$ :

$$
e \rightarrow Z(N) \stackrel{i \mid Z(N)}{\longrightarrow} E \stackrel{\theta}{\longrightarrow} \Gamma \rightarrow e,
$$

where $\Gamma$ operates on $Z(N)$ via $z \cdot(g, \varphi)=\varphi(z)$. These two extensions fit into the commutative diagram

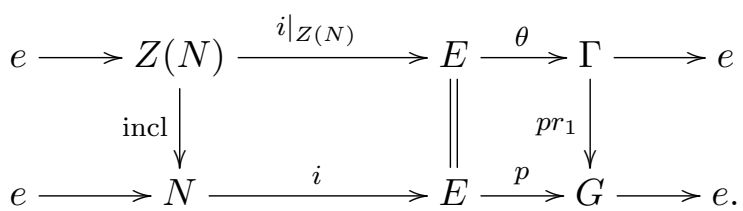

Roughly speaking, $E$ can be regarded both as an extension of $G$ over $N$ and as an extension of $\Gamma$ over $Z(N)$. It can be shown that if $\bar{\alpha}$ admits an extension, then every extension inducing $\bar{\alpha}$ is obtained in this way.

Note that for a given abstract kernel $(N, G, \bar{\alpha})$ there is always an extension of $\Gamma$ over $Z(N)$, but if $\left[\lambda^{\bar{\alpha}}\right] \in H^{3}(G, Z(N))$ is nonzero, then the inclusion $Z(N) \rightarrow E$ does not extend to an inclusion $N \rightarrow E$. 
15.25. In [56] a pair $(K, \psi)$, with $\psi: G \rightarrow \operatorname{Aut}(K) / \operatorname{Int}(K)$ a homomorphism ( $G$ being fixed), is called a kernel. As we have seen above, $\psi$ induces a homomorphism $\psi_{0}: G \rightarrow \operatorname{Aut}(Z(K))$. Consider all kernels $(K, \psi)$, with fixed center $C=Z(K)$ and fixed restriction $\psi_{0}$. Two such kernels are said to be similar if they differ only by a kernel coming from a homomorphism. One of the results in [56] is that the similarity classes of kernels form a group under a multiplication which is defined using the amalgamated direct product of two kernels with $C$ as amalgamating subgroup and that this group can be naturally identified with the third cohomology group $H^{3}\left(G,\left(C, \psi_{0}\right)\right)$. In the following we outline the arguments.

15.26. Proposition. Suppose that we are given an abelian group $Z, a$ homomorphism $\alpha^{0}: G \rightarrow \operatorname{Aut}(Z)$ and a normalized 3-cocycle $\lambda: G \times G \times$ $G \rightarrow Z$. Then there exists a group $N$ containing $Z$ as its center and a homomorphism $\bar{\alpha}: G \rightarrow \operatorname{Aut}(N) / \operatorname{Int}(N)$ inducing both $\alpha^{0}$ and $\lambda$.

Proof. Let $S$ be the free group generated by the symbols $[x, y]$ with $e \neq$ $x \in G$ and $e \neq y \in G$. For convenience the identity $e$ of $F$ is identified with all symbols $[x, y]$ such that either $x=e$ or $y=e$. The group $F$ is centerfree except in the case where $G$ is cyclic of order two. For the moment we set aside the exceptional case.

We define $N$ to be the direct product $F \times Z$ and, for every $g \in G$, we define a homomorphism $\alpha_{g}: N \rightarrow N$ by the formula

$$
\alpha_{g}([x, y], z)=\left([g, x][g x, y][g, x y]^{-1}, \lambda(g, x, y) \alpha_{g}^{0}(z)\right) .
$$

Since $\lambda$ is normalized, we see that $\alpha_{e}$ is the identity. We claim that

$$
\alpha_{x} \alpha_{y}=\operatorname{conj}_{([x, y], e)} \circ \alpha_{x y} .
$$

To see this, we apply the left side of this equation to an element $([u, v], z)$ :

$$
\begin{gathered}
\alpha_{x} \alpha_{y}([u, v], z)=\alpha_{x}\left([y, u][y u, v][y, u v]^{-1}, \lambda(y, u, v) \alpha_{y}^{0}(z)\right) \\
=\left([x, y][x y, u][x, y u]^{-1} \cdot[x, y u][x y u, v][x, y u v]^{-1}\right. \\
\cdot[x, y u v][x y, u v]^{-1}[x, y]^{-1}, \\
\left.\lambda(x, y, u) \lambda(x, y u, v) \lambda(x, y, u v)^{-1} \alpha_{x}^{0}(\lambda(y, u, v)) \alpha_{x y}^{0}(z)\right) \\
=\operatorname{conj}_{([x, y], e)}\left([x y, u][x y u, v][x y, u v]^{-1}[x, y]^{-1},\right. \\
\left.\lambda(x, y, u) \lambda(x, y u, v) \lambda(x, y, u v)^{-1} \alpha_{x}^{0}(\lambda(y, u, v)) \alpha_{x y}^{0}(z)\right) .
\end{gathered}
$$

Since $\lambda$ is a cocycle, we have

$$
\begin{aligned}
e & =\delta_{\alpha^{0}} \lambda(x, y, u, v) \\
& =\alpha_{x}^{0}(\lambda(y, u, v)) \lambda(x y, u, v)^{-1} \lambda(x, y u, v) \lambda(x, y, u v)^{-1} \lambda(x, y, u)
\end{aligned}
$$


and therefore

$$
\alpha_{x}^{0}(\lambda(y, u, v)) \lambda(x, y u, v) \lambda(x, y, u v)^{-1} \lambda(x, y, u)=\lambda(x y, u, v) .
$$

Thus we find

$$
\begin{aligned}
& \alpha_{x} \alpha_{y}([u, v], z) \\
& \quad=\operatorname{conj}_{([x, y], e)}\left([x y, u][x y u, v][x y, u v]^{-1}[x, y]^{-1}, \lambda(x y, u v) \alpha_{x y}^{0}(z)\right) \\
& \quad=\operatorname{conj}_{([x, y], e)}\left(\alpha_{x y}([u, v])\right)
\end{aligned}
$$

which establishes our claim (1).

By (1) we have the equations $\alpha_{x^{-1}} \circ \alpha_{x}=\operatorname{conj}_{\left[x^{-1}, x\right]}$ and $\alpha_{x} \circ \alpha_{x^{-1}}=$ $\operatorname{conj}_{\left[x, x^{-1}\right]}$, so every homomorphism $\alpha_{x}, x \in G$, is injective as well as surjective, hence an automorphism.

If we assume that $G$ is not cyclic of order two, then $e \times Z$ is exactly the center of $N$ and equation (1) defines a homomorphism $\bar{\alpha}: G \rightarrow \operatorname{Aut}(N) / \operatorname{Int}(N)$ which, by construction, induces both $\lambda$ and $\alpha^{0}$.

15.27. An interpretation of the class $\lambda$. Let $N$ and $G$ be Lie groups and let a homomorphism $\alpha: G \rightarrow \operatorname{Aut}(N) / \operatorname{Int}(N)$ be given. We consider the extension

$$
e \rightarrow \operatorname{Int}(N) \rightarrow \operatorname{Aut}(N) \rightarrow \operatorname{Aut}(N) / \operatorname{Int}(N) \rightarrow e
$$

and the following diagram, where the bottom right hand square is a pullback (compare with the alternative proof of (15.23)):

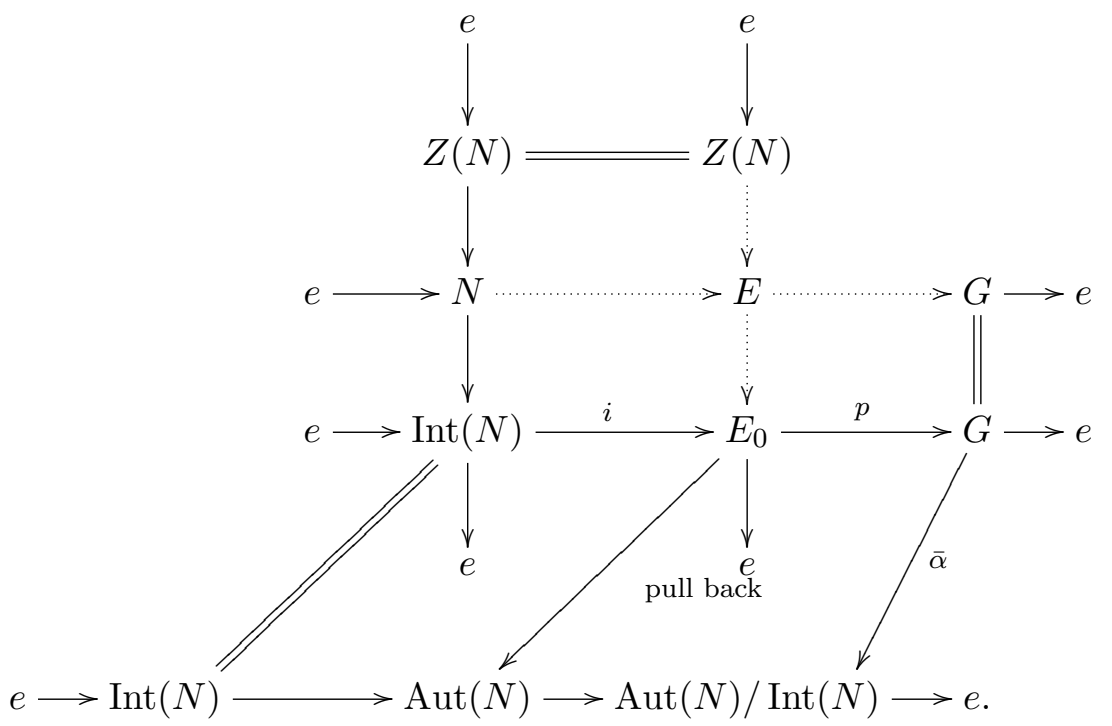

The left hand vertical column describes $N$ as a central extension of $\operatorname{Int}(N)$ with abelian kernel $Z(N)$ which is moreover invariant under the action of $G$ via $\bar{\alpha}$; it is given by a cohomology class $[\nu] \in H^{2}(N ; Z(N))^{G}$. In order 
to get an extension $E$ of $G$ with kernel $N$ as in the third row, we have to check that the cohomology class $[\nu]$ is in the image of $i^{*}: H^{2}\left(E_{0} ; Z(N)\right) \rightarrow$ $H^{2}(N ; Z(N))^{G}$. 


\section{CHAPTER IV. Bundles and Connections}

\section{Derivations on the Algebra of Differential Forms}

16.1. Derivations. In this section let $M$ be a smooth manifold. We consider the graded commutative algebra

$$
\Omega(M)=\bigoplus_{k=0}^{\operatorname{dim} M} \Omega^{k}(M)=\bigoplus_{k=-\infty}^{\infty} \Omega^{k}(M)
$$

of differential forms on $M$, where we put $\Omega^{k}(M)=0$ for $k<0$ and $k>$ $\operatorname{dim} M$. We denote by $\operatorname{Der}_{k} \Omega(M)$ the space of all (graded) derivations of degree $k$, i.e., all linear mappings $D: \Omega(M) \rightarrow \Omega(M)$ with $D\left(\Omega^{\ell}(M)\right) \subset$ $\Omega^{k+\ell}(M)$ and $D(\varphi \wedge \psi)=D(\varphi) \wedge \psi+(-1)^{k \ell} \varphi \wedge D(\psi)$ for $\varphi \in \Omega^{\ell}(M)$.

Lemma. Then the space $\operatorname{Der} \Omega(M)=\bigoplus_{k} \operatorname{Der}_{k} \Omega(M)$ is a graded Lie algebra with the graded commutator $\left[D_{1}, D_{2}\right]:=D_{1} \circ D_{2}-(-1)^{k_{1} k_{2}} D_{2} \circ D_{1}$ as bracket. This means that the bracket is graded anticommutative and satisfies the graded Jacobi identity

$$
\begin{gathered}
{\left[D_{1}, D_{2}\right]=-(-1)^{k_{1} k_{2}}\left[D_{2}, D_{1}\right]} \\
{\left[D_{1},\left[D_{2}, D_{3}\right]\right]=\left[\left[D_{1}, D_{2}\right], D_{3}\right]+(-1)^{k_{1} k_{2}}\left[D_{2},\left[D_{1}, D_{3}\right]\right]}
\end{gathered}
$$

(so that $\operatorname{ad}\left(D_{1}\right)=\left[D_{1}, \quad\right]$ is itself a derivation of degree $\left.k_{1}\right)$.

Proof. Plug in the definition of the graded commutator and compute. 
In section (9) we have already met some graded derivations: For a vector field $X$ on $M$ the derivation $i_{X}$ is of degree $-1, \mathcal{L}_{X}$ is of degree 0 , and $d$ is of degree 1 . Note also that the important formula $\mathcal{L}_{X}=d i_{X}+i_{X} d$ translates to $\mathcal{L}_{X}=\left[i_{X}, d\right]$.

16.2. Algebraic derivations. A derivation $D \in \operatorname{Der}_{k} \Omega(M)$ is called algebraic if $D \mid \Omega^{0}(M)=0$. Then $D(f . \omega)=f . D(\omega)$ for $f \in C^{\infty}(M)$, so $D$ is of tensorial character by (9.3). So $D$ induces a derivation $D_{x} \in \operatorname{Der}_{k} \wedge T_{x}^{*} M$ for each $x \in M$. It is uniquely determined by its restriction to 1 -forms $D_{x} \mid T_{x}^{*} M: T_{x}^{*} M \rightarrow \bigwedge^{k+1} T^{*} M$ which we may view as an element $K_{x} \in$ $\bigwedge^{k+1} T_{x}^{*} M \otimes T_{x} M$ depending smoothly on $x \in M$. To express this dependence, we write $D=i_{K}=i(K)$, where $K \in \Gamma\left(\bigwedge^{k+1} T^{*} M \otimes T M\right)=$ : $\Omega^{k+1}(M ; T M)$. Note the defining equation: $i_{K}(\omega)=\omega \circ K$ for $\omega \in \Omega^{1}(M)$. We call $\Omega(M, T M)=\bigoplus_{k=0}^{\operatorname{dim} M} \Omega^{k}(M, T M)$ the space of all vector valued differential forms.

Theorem. (1) For $K \in \Omega^{k+1}(M, T M)$ the formula

$$
\begin{aligned}
& \left(i_{K} \omega\right)\left(X_{1}, \ldots, X_{k+\ell}\right) \\
& \quad=\frac{1}{(k+1) !(\ell-1) !} \sum_{\sigma \in \mathcal{S}_{k+\ell}} \operatorname{sign} \sigma \cdot \omega\left(K\left(X_{\sigma 1}, \ldots, X_{\sigma(k+1)}\right), X_{\sigma(k+2)}, \ldots\right)
\end{aligned}
$$

for $\omega \in \Omega^{\ell}(M), X_{i} \in \mathfrak{X}(M)$ (or $\left.T_{x} M\right)$ defines an algebraic graded derivation $i_{K} \in \operatorname{Der}_{k} \Omega(M)$ and any algebraic derivation is of this form.

(2) By $i\left([K, L]^{\wedge}\right):=\left[i_{K}, i_{L}\right]$ we get a bracket $[,]^{\wedge}$ on $\Omega^{*+1}(M, T M)$ which defines a graded Lie algebra structure with the grading as indicated, and for $K \in \Omega^{k+1}(M, T M), L \in \Omega^{\ell+1}(M, T M)$ we have

$$
[K, L]^{\wedge}=i_{K} L-(-1)^{k \ell} i_{L} K
$$

where $i_{K}(\omega \otimes X):=i_{K}(\omega) \otimes X$.

The bracket $[\quad, \quad]^{\wedge}$ is called the algebraic bracket or the Nijenhuis-Richardson bracket; see $\mathbf{1 7 8}$.

Proof. Since $\wedge T_{x}^{*} M$ is the free graded commutative algebra generated by the vector space $T_{x}^{*} M$, any $K \in \Omega^{k+1}(M, T M)$ extends to a graded derivation. By applying it to an exterior product of 1-forms, one can derive the formula in (11). The graded commutator of two algebraic derivations is again algebraic, so the injection $i: \Omega^{*+1}(M, T M) \rightarrow \operatorname{Der}_{*}(\Omega(M))$ induces a graded Lie bracket on $\Omega^{*+1}(M, T M)$ whose form can be seen by applying it to a 1-form. 
16.3. Lie derivations. The exterior derivative $d$ lies in $\operatorname{Der}_{1} \Omega(M)$. In view of the formula $\mathcal{L}_{X}=\left[i_{X}, d\right]=i_{X} d+d i_{X}$ for vector fields $X$, we define for $K \in \Omega^{k}(M ; T M)$ the Lie derivative $\mathcal{L}_{K}=\mathcal{L}(K) \in \operatorname{Der}_{k} \Omega(M)$ by $\mathcal{L}_{K}:=\left[i_{K}, d\right]=i_{K} d-(-1)^{k-1} d i_{K}$.

Then the mapping $\mathcal{L}: \Omega(M, T M) \rightarrow \operatorname{Der} \Omega(M)$ is injective, since $\mathcal{L}_{K} f=$ $i_{K} d f=d f \circ K$ for $f \in C^{\infty}(M)$.

Theorem. For any graded derivation $D \in \operatorname{Der}_{k} \Omega(M)$ there are unique $K \in \Omega^{k}(M ; T M)$ and $L \in \Omega^{k+1}(M ; T M)$ such that

$$
D=\mathcal{L}_{K}+i_{L} .
$$

We have $L=0$ if and only if $[D, d]=0$. The derivation $D$ is algebraic if and only if $K=0$.

Proof. Let $X_{i} \in \mathfrak{X}(M)$ be vector fields. Then $f \mapsto(D f)\left(X_{1}, \ldots, X_{k}\right)$ is a derivation $C^{\infty}(M) \rightarrow C^{\infty}(M)$, so there exists a vector field $K\left(X_{1}, \ldots, X_{k}\right) \in$ $\mathfrak{X}(M)$ by $(3.3)$ such that

$$
(D f)\left(X_{1}, \ldots, X_{k}\right)=K\left(X_{1}, \ldots, X_{k}\right) f=d f\left(K\left(X_{1}, \ldots, X_{k}\right)\right) .
$$

Clearly $K\left(X_{1}, \ldots, X_{k}\right)$ is $C^{\infty}(M)$-linear in each $X_{i}$ and alternating, so $K$ is tensorial by (9.3), $K \in \Omega^{k}(M ; T M)$.

The defining equation for $K$ is $D f=d f \circ K=i_{K} d f=\mathcal{L}_{K} f$ for $f \in C^{\infty}(M)$. Thus $D-\mathcal{L}_{K}$ is an algebraic derivation, so $D-\mathcal{L}_{K}=i_{L}$ by (16.2) for unique $L \in \Omega^{k+1}(M ; T M)$.

Since we have $[d, d]=2 d^{2}=0$, by the graded Jacobi identity, we obtain $0=\left[i_{K},[d, d]\right]=\left[\left[i_{K}, d\right], d\right]+(-1)^{k-1}\left[d,\left[i_{K}, d\right]\right]=2\left[\mathcal{L}_{K}, d\right]$. The mapping $K \mapsto\left[i_{K}, d\right]=\mathcal{L}_{K}$ is injective, so the last assertions follow.

16.4. Applying $i\left(I d_{T M}\right)$ on a $k$-fold exterior product of 1 -forms, we get $i\left(I d_{T M}\right) \omega=k \omega$ for $\omega \in \Omega^{k}(M)$. Thus we have $\mathcal{L}\left(I d_{T M}\right) \omega=i\left(I d_{T M}\right) d \omega-$ $d i\left(I d_{T M}\right) \omega=(k+1) d \omega-k d \omega=d \omega$. Thus $\mathcal{L}\left(I d_{T M}\right)=d$.

16.5. Let $K \in \Omega^{k}(M ; T M)$ and $L \in \Omega^{\ell}(M ; T M)$. Then $\left[\left[\mathcal{L}_{K}, \mathcal{L}_{L}\right], d\right]=0$, so we have

$$
[\mathcal{L}(K), \mathcal{L}(L)]=\mathcal{L}([K, L])
$$

for a uniquely defined $[K, L] \in \Omega^{k+\ell}(M ; T M)$. This vector valued form $[K, L]$ is called the Frölicher-Nijenhuis bracket of $K$ and $L$.

Theorem. The space $\Omega(M ; T M)=\bigoplus_{k=0}^{\operatorname{dim} M} \Omega^{k}(M ; T M)$ with its usual grading is a graded Lie algebra for the Frölicher-Nijenhuis bracket. So we have

$$
\begin{gathered}
{[K, L]=-(-1)^{k \ell}[L, K],} \\
{\left[K_{1},\left[K_{2}, K_{3}\right]\right]=\left[\left[K_{1}, K_{2}\right], K_{3}\right]+(-1)^{k_{1} k_{2}}\left[K_{2},\left[K_{1}, K_{3}\right]\right] .}
\end{gathered}
$$


The 1-form $\operatorname{Id}_{T M} \in \Omega^{1}(M ; T M)$ is in the center, i.e., $\left[K, I d_{T M}\right]=0$ for all $K$. The operator $\mathcal{L}:(\Omega(M ; T M),[, \quad]) \rightarrow \operatorname{Der} \Omega(M)$ is an injective homomorphism of graded Lie algebras. For vector fields the Frölicher-Nijenhuis bracket coincides with the Lie bracket.

Proof. $d f \circ[X, Y]=\mathcal{L}([X, Y]) f=\left[\mathcal{L}_{X}, \mathcal{L}_{Y}\right] f$. The rest is clear.

16.6. Lemma. For $K \in \Omega^{k}(M ; T M)$ and $L \in \Omega^{\ell+1}(M ; T M)$ we have

$$
\begin{aligned}
& {\left[\mathcal{L}_{K}, i_{L}\right]=i([K, L])-(-1)^{k \ell} \mathcal{L}\left(i_{L} K\right), \text { or }} \\
& {\left[i_{L}, \mathcal{L}_{K}\right]=\mathcal{L}\left(i_{L} K\right)-(-1)^{k} i([L, K]) .}
\end{aligned}
$$

This generalizes (9.7.3)

Proof. For $f \in C^{\infty}(M)$ we have $\left[i_{L}, \mathcal{L}_{K}\right] f=i_{L} i_{K} d f-0=i_{L}(d f \circ K)=$ $d f \circ\left(i_{L} K\right)=\mathcal{L}\left(i_{L} K\right) f$. So $\left[i_{L}, \mathcal{L}_{K}\right]-\mathcal{L}\left(i_{L} K\right)$ is an algebraic derivation.

$$
\begin{aligned}
{\left[\left[i_{L}, \mathcal{L}_{K}\right], d\right]=\left[i_{L},\left[\mathcal{L}_{K}, d\right]\right]-} & (-1)^{k \ell}\left[\mathcal{L}_{K},\left[i_{L}, d\right]\right] \\
& =0-(-1)^{k \ell} \mathcal{L}([K, L])=(-1)^{k}[i([L, K]), d] .
\end{aligned}
$$

Since $[, d]$ kills the ' $\mathcal{L}$ 's' and is injective on the ' $i$ 's', the algebraic part of $\left[i_{L}, \mathcal{L}_{K}\right]$ is $(-1)^{k} i([L, K])$.

16.7. Module structure. The space $\operatorname{Der} \Omega(M)$ is a graded module over the graded algebra $\Omega(M)$ with the action $(\omega \wedge D) \varphi=\omega \wedge D(\varphi)$, because $\Omega(M)$ is graded commutative.

Theorem. Let the degree of $\omega$ be $q$, of $\varphi$ be $k$, and of $\psi$ be $\ell$. Let the other degrees be as indicated. Then we have:

$$
\begin{aligned}
{\left[\omega \wedge D_{1}, D_{2}\right]=} & \omega \wedge\left[D_{1}, D_{2}\right]-(-1)^{\left(q+k_{1}\right) k_{2}} D_{2}(\omega) \wedge D_{1} . \\
i(\omega \wedge L)= & \omega \wedge i(L) . \\
\omega \wedge \mathcal{L}_{K}= & \mathcal{L}(\omega \wedge K)+(-1)^{q+k-1} i(d \omega \wedge K) . \\
{\left[\omega \wedge L_{1}, L_{2}\right]^{\wedge}=} & \omega \wedge\left[L_{1}, L_{2}\right]^{\wedge}-(-1)^{\left(q+\ell_{1}-1\right)\left(\ell_{2}-1\right)} i\left(L_{2}\right) \omega \wedge L_{1} . \\
{\left[\omega \wedge K_{1}, K_{2}\right]=} & \omega \wedge\left[K_{1}, K_{2}\right]-(-1)^{\left(q+k_{1}\right) k_{2}} \mathcal{L}\left(K_{2}\right) \omega \wedge K_{1} \\
& +(-1)^{q+k_{1}} d \omega \wedge i\left(K_{1}\right) K_{2} . \\
{[\varphi \otimes X, \psi \otimes Y]=} & \varphi \wedge \psi \otimes[X, Y] \\
& -\left(i_{Y} d \varphi \wedge \psi \otimes X-(-1)^{k \ell} i_{X} d \psi \wedge \varphi \otimes Y\right) \\
& -\left(d\left(i_{Y} \varphi \wedge \psi\right) \otimes X-(-1)^{k \ell} d\left(i_{X} \psi \wedge \varphi\right) \otimes Y\right) \\
= & \varphi \wedge \psi \otimes[X, Y]+\varphi \wedge \mathcal{L}_{X} \psi \otimes Y-\mathcal{L}_{Y} \varphi \wedge \psi \otimes X \\
& +(-1)^{k}\left(d \varphi \wedge i_{X} \psi \otimes Y+i_{Y} \varphi \wedge d \psi \otimes X\right) .
\end{aligned}
$$


Proof. For (11), (2), (3) write out the definitions. For (44) compute $i([\omega \wedge$ $\left.\left.L_{1}, L_{2}\right]^{\wedge}\right)$. For (5) compute $\mathcal{L}\left(\left[\omega \wedge K_{1}, K_{2}\right]\right)$. For (66) use (5) .

16.8. Theorem. For $K \in \Omega^{k}(M ; T M)$ and $\omega \in \Omega^{\ell}(M)$ the Lie derivative of $\omega$ along $K$ is given by the following formula, where the $X_{i}$ are vector fields on $M$ :

$$
\begin{aligned}
\left(\mathcal{L}_{K} \omega\right)( & \left.X_{1}, \ldots, X_{k+\ell}\right) \\
= & \frac{1}{k ! \ell !} \sum_{\sigma} \operatorname{sign} \sigma \mathcal{L}\left(K\left(X_{\sigma 1}, \ldots, X_{\sigma k}\right)\right)\left(\omega\left(X_{\sigma(k+1)}, \ldots, X_{\sigma(k+\ell)}\right)\right) \\
& +\frac{-1}{k !(\ell-1) !} \sum_{\sigma} \operatorname{sign} \sigma \omega\left(\left[K\left(X_{\sigma 1}, \ldots, X_{\sigma k}\right), X_{\sigma(k+1)}\right], X_{\sigma(k+2)}, \ldots\right) \\
& +\frac{(-1)^{k-1}}{(k-1) !(\ell-1) ! 2 !} \sum_{\sigma} \operatorname{sign} \sigma \omega\left(K\left(\left[X_{\sigma 1}, X_{\sigma 2}\right], X_{\sigma 3}, \ldots\right), X_{\sigma(k+2)}, \ldots\right) .
\end{aligned}
$$

Proof. It suffices to consider $K=\varphi \otimes X$. Then by (16.7.3) we have $\mathcal{L}(\varphi \otimes X)=\varphi \wedge \mathcal{L}_{X}-(-1)^{k-1} d \varphi \wedge i_{X}$. Now use the global formulas of section $(9)$ to expand this.

16.9. Theorem. For $K \in \Omega^{k}(M ; T M)$ and $L \in \Omega^{\ell}(M ; T M)$ we have for the Frölicher-Nijenhuis bracket $[K, L]$ the following formula, where the $X_{i}$ are vector fields on $M$ :

$$
\begin{aligned}
{[K, L](} & \left.X_{1}, \ldots, X_{k+\ell}\right) \\
= & \frac{1}{k ! \ell !} \sum_{\sigma} \operatorname{sign} \sigma\left[K\left(X_{\sigma 1}, \ldots, X_{\sigma k}\right), L\left(X_{\sigma(k+1)}, \ldots, X_{\sigma(k+\ell)}\right)\right] \\
& +\frac{-1}{k !(\ell-1) !} \sum_{\sigma} \operatorname{sign} \sigma L\left(\left[K\left(X_{\sigma 1}, \ldots, X_{\sigma k}\right), X_{\sigma(k+1)}\right], X_{\sigma(k+2)}, \ldots\right) \\
& +\frac{(-1)^{k \ell}}{(k-1) ! \ell !} \sum_{\sigma} \operatorname{sign} \sigma K\left(\left[L\left(X_{\sigma 1}, \ldots, X_{\sigma \ell}\right), X_{\sigma(\ell+1)}\right], X_{\sigma(\ell+2)}, \ldots\right) \\
& +\frac{(-1)^{k-1}}{(k-1) !(\ell-1) ! 2 !} \sum_{\sigma} \operatorname{sign} \sigma L\left(K\left(\left[X_{\sigma 1}, X_{\sigma 2}\right], X_{\sigma 3}, \ldots\right), X_{\sigma(k+2)}, \ldots\right) \\
& +\frac{(-1)^{(k-1) \ell}}{(k-1) !(\ell-1) ! 2 !} \sum_{\sigma} \operatorname{sign} \sigma K\left(L\left(\left[X_{\sigma 1}, X_{\sigma 2}\right], X_{\sigma 3}, \ldots\right), X_{\sigma(\ell+2)}, \ldots\right) .
\end{aligned}
$$

Proof. It suffices to consider $K=\varphi \otimes X$ and $L=\psi \otimes Y$; then for $[\varphi \otimes$ $X, \psi \otimes Y]$ we may use (16.7.6) and evaluate that at $\left(X_{1}, \ldots, X_{k+\ell}\right)$. After some combinatorial computation we get the right hand side of the above formula for $K=\varphi \otimes X$ and $L=\psi \otimes Y$.

There are more illuminating ways to prove this formula; see [147]. 
16.10. Local formulas. In a local chart $(U, u)$ on the manifold $M$ we put $K\left|U=\sum K_{\alpha}^{i} d^{\alpha} \otimes \partial_{i}, L\right| U=\sum L_{\beta}^{j} d^{\beta} \otimes \partial_{j}$, and $\omega \mid U=\sum \omega_{\gamma} d^{\gamma}$, where $\alpha=\left(1 \leq \alpha_{1}<\alpha_{2}<\cdots<\alpha_{k} \leq \operatorname{dim} M\right)$ is a form index, $d^{\alpha}=$ $d u^{\alpha_{1}} \wedge \ldots \wedge d u^{\alpha_{k}}, \partial_{i}=\frac{\partial}{\partial u^{i}}$ and so on.

Plugging $X_{j}=\partial_{i_{j}}$ into the global formulas (16.2), (16.8), and (16.9), we get the following local formulas:

$$
\begin{aligned}
& i_{K} \omega \mid U=\sum K_{\alpha_{1} \ldots \alpha_{k}}^{i} \omega_{i \alpha_{k+1} \ldots \alpha_{k+\ell-1}} d^{\alpha} \\
& {[K, L]^{\wedge} \mid U }=\sum\left(K_{\alpha_{1} \ldots \alpha_{k}}^{i} L_{i \alpha_{k+1} \ldots \alpha_{k+\ell}}^{j}\right. \\
&\left.-(-1)^{(k-1)(\ell-1)} L_{\alpha_{1} \ldots \alpha_{\ell}}^{i} K_{i \alpha_{\ell+1} \ldots \alpha_{k+\ell}}^{j}\right) d^{\alpha} \otimes \partial_{j}, \\
& \mathcal{L}_{K} \omega \mid U=\sum\left(K_{\alpha_{1} \ldots \alpha_{k}}^{i} \partial_{i} \omega_{\alpha_{k+1} \ldots \alpha_{k+\ell}}\right. \\
&\left.+(-1)^{k}\left(\partial_{\alpha_{1}} K_{\alpha_{2} \ldots \alpha_{k+1}}^{i}\right) \omega_{i \alpha_{k+2} \ldots \alpha_{k+\ell}}\right) d^{\alpha}, \\
& {[K, L] \mid U=\sum\left(\begin{array}{l}
K_{\alpha_{1} \ldots \alpha_{k}}^{i} \partial_{i} L_{\alpha_{k+1} \ldots \alpha_{k+\ell}}^{j} \\
-(-1)^{k \ell} L_{\alpha_{1} \ldots \alpha_{\ell}}^{i} \partial_{i} K_{\alpha_{\ell+1} \ldots \alpha_{k+\ell}}^{j} \\
-k K_{\alpha_{1} \ldots \alpha_{k-1} i}^{j} \partial_{\alpha_{k}} L_{\alpha_{k+1} \ldots \alpha_{k+\ell}}^{i} \\
\left.+(-1)^{k \ell} \ell L_{\alpha_{1} \ldots \alpha_{\ell-1} i}^{j} \partial_{\alpha_{\ell}} K_{\alpha_{\ell+1} \ldots \alpha_{k+\ell}}^{i}\right) d^{\alpha} \otimes \partial_{j} .
\end{array}\right.}
\end{aligned}
$$

16.11. Theorem. For $K_{i} \in \Omega^{k_{i}}(M ; T M)$ and $L_{i} \in \Omega^{k_{i}+1}(M ; T M)$ we have

$$
\begin{aligned}
{\left[\mathcal{L}_{K_{1}}+i_{L_{1}}, \mathcal{L}_{K_{2}}+i_{L_{2}}\right] } & =\mathcal{L}\left(\left[K_{1}, K_{2}\right]+i_{L_{1}} K_{2}-(-1)^{k_{1} k_{2}} i_{L_{2}} K_{1}\right) \\
& +i\left(\left[L_{1}, L_{2}\right]^{\wedge}+\left[K_{1}, L_{2}\right]-(-1)^{k_{1} k_{2}}\left[K_{2}, L_{1}\right]\right) .
\end{aligned}
$$

Each summand of this formula looks like a semidirect product of graded Lie algebras, but the mappings

$$
\begin{aligned}
i: \Omega(M ; T M) & \rightarrow \operatorname{End}(\Omega(M ; T M),[\quad, \quad]), \\
\operatorname{ad}: \Omega(M ; T M) & \rightarrow \operatorname{End}\left(\Omega(M ; T M),[\quad, \quad]^{\wedge}\right)
\end{aligned}
$$

do not take values in the subspaces of graded derivations. We have instead for $K \in \Omega^{k}(M ; T M)$ and $L \in \Omega^{\ell+1}(M ; T M)$ the following relations:

$$
\begin{gathered}
i_{L}\left[K_{1}, K_{2}\right]=\left[i_{L} K_{1}, K_{2}\right]+(-1)^{k_{1} \ell}\left[K_{1}, i_{L} K_{2}\right], \\
-\left((-1)^{k_{1} \ell} i\left(\left[K_{1}, L\right]\right) K_{2}-(-1)^{\left(k_{1}+\ell\right) k_{2}} i\left(\left[K_{2}, L\right]\right) K_{1}\right) \\
{\left[K,\left[L_{1}, L_{2}\right]^{\wedge}\right]=\left[\left[K, L_{1}\right], L_{2}\right]^{\wedge}+(-1)^{k k_{1}}\left[L_{1},\left[K, L_{2}\right]\right]^{\wedge}} \\
\quad-\left((-1)^{k k_{1}}\left[i\left(L_{1}\right) K, L_{2}\right]-(-1)^{\left(k+k_{1}\right) k_{2}}\left[i\left(L_{2}\right) K, L_{1}\right]\right) .
\end{gathered}
$$


The algebraic meaning of the relations of this theorem and its consequences in group theory have been investigated in $\mathbf{1 5 0}$. The corresponding product of groups is well known to algebraists under the name Zappa-Szep product.

Proof. Equation (11) is an immediate consequence of (16.6), Equations (21) and (3) follow from (11) by writing out the graded Jacobi identity or as follows: Consider $\mathcal{L}\left(i_{L}\left[K_{1}, K_{2}\right]\right)$ and use (16.6) repeatedly to obtain $\mathcal{L}$ of the right hand side of (2). Then consider $i\left(\left[K,\left[L_{1}, L_{2}\right]^{\wedge}\right]\right)$ and use again (16.6) several times to obtain $i$ of the right hand side of (3) .

16.12. Corollary (of (16.9)]. For $K, L \in \Omega^{1}(M ; T M)$ we have

$$
\begin{aligned}
{[K, L](X, Y)=[K X, L Y] } & -[K Y, L X]-L([K X, Y]-[K Y, X]) \\
& -K([L X, Y]-[L Y, X])+(L K+K L)[X, Y] .
\end{aligned}
$$

16.13. Curvature. Let $P \in \Omega^{1}(M ; T M)$ be a fiber projection, i.e., $P \circ P=$ $P$. This is the most general case of a (first order) connection. We may call ker $P$ the horizontal space and im $P$ the vertical space of the connection. If $P$ is of constant rank, then both are vector subbundles of $T M$. If $\operatorname{im} P$ is some primarily fixed vector subbundle or (tangent bundle of) a foliation, $P$ can be called a connection for it. Special cases of this will be treated extensively later on. The following result is immediate from (16.12).

Lemma. We have

$$
[P, P]=2 R+2 \bar{R}
$$

where $R, \bar{R} \in \Omega^{2}(M ; T M)$ are given by $R(X, Y)=P[(I d-P) X,(I d-P) Y]$ and $\bar{R}(X, Y)=(I d-P)[P X, P Y]$.

If $P$ has constant rank, then $R$ is the obstruction against integrability of the horizontal bundle ker $P$, and $\bar{R}$ is the obstruction against integrability of the vertical bundle $\operatorname{im} P$. Thus we call $R$ the curvature and $\bar{R}$ the cocurvature of the connection $P$. We will see later that for a principal fiber bundle $R$ is just the negative of the usual curvature.

16.14. Lemma (Bianchi identity). If $P \in \Omega^{1}(M ; T M)$ is a connection (fiber projection) with curvature $R$ and cocurvature $\bar{R}$, then we have

$$
\begin{aligned}
& {[P, R+\bar{R}]=0,} \\
& {[R, P]=i_{R} \bar{R}+i_{\bar{R}} R .}
\end{aligned}
$$

Proof. We have $[P, P]=2 R+2 \bar{R}$ by $(16.13)$ and $[P,[P, P]]=0$ by the graded Jacobi identity. So the first formula follows. We have $2 R=P$ 。 $[P, P]=i_{[P, P]} P$. By (16.11.2) we get $i_{[P, P]}[P, P]=2\left[i_{[P, P]} P, P\right]-0=4[R, P]$. 
Therefore $[R, P]=\frac{1}{4} i_{[P, P]}[P, P]=i(R+\bar{R})(R+\bar{R})=i_{R} \bar{R}+i_{\bar{R}} R$ since $R$ has vertical values and kills vertical vectors, so $i_{R} R=0$; likewise for $\bar{R}$.

16.15. Naturality of the Frölicher-Nijenhuis bracket. Let $f: M \rightarrow$ $N$ be a smooth mapping between manifolds. Two vector valued forms $K \in$ $\Omega^{k}(M ; T M)$ and $K^{\prime} \in \Omega^{k}(N ; T N)$ are called $f$-related or $f$-dependent if for all $X_{i} \in T_{x} M$ we have

$$
K_{f(x)}^{\prime}\left(T_{x} f \cdot X_{1}, \ldots, T_{x} f \cdot X_{k}\right)=T_{x} f \cdot K_{x}\left(X_{1}, \ldots, X_{k}\right) .
$$

\section{Theorem.}

(2) If $K$ and $K^{\prime}$ as above are $f$-related, then $i_{K} \circ f^{*}=f^{*} \circ i_{K^{\prime}}: \Omega(N) \rightarrow$ $\Omega(M)$.

(3) If $i_{K} \circ f^{*}\left|B^{1}(N)=f^{*} \circ i_{K^{\prime}}\right| B^{1}(N)$, then $K$ and $K^{\prime}$ are $f$-related, where $B^{1}$ denotes the space of exact 1 -forms.

(4) If $K_{j}$ and $K_{j}^{\prime}$ are $f$-related for $j=1,2$, then $i_{K_{1}} K_{2}$ and $i_{K_{1}^{\prime}} K_{2}^{\prime}$ are $f$-related, and also $\left[K_{1}, K_{2}\right]^{\wedge}$ and $\left[K_{1}^{\prime}, K_{2}^{\prime}\right]^{\wedge}$ are $f$-related.

(5) If $K$ and $K^{\prime}$ are $f$-related, then $\mathcal{L}_{K} \circ f^{*}=f^{*} \circ \mathcal{L}_{K^{\prime}}: \Omega(N) \rightarrow \Omega(M)$.

(6) If $\mathcal{L}_{K} \circ f^{*}\left|\Omega^{0}(N)=f^{*} \circ \mathcal{L}_{K^{\prime}}\right| \Omega^{0}(N)$, then $K$ and $K^{\prime}$ are $f$-related.

(7) If $K_{j}$ and $K_{j}^{\prime}$ are $f$-related for $j=1,2$, then their Frölicher-Nijenhuis brackets $\left[K_{1}, K_{2}\right]$ and $\left[K_{1}^{\prime}, K_{2}^{\prime}\right]$ are also $f$-related.

Proof. (2) By (16.2) we have for $\omega \in \Omega^{q}(N)$ and $X_{i} \in T_{x} M$ :

$$
\begin{aligned}
\left(i_{K} f^{*} \omega\right)_{x}\left(X_{1}, \ldots, X_{q+k-1}\right) & \\
& =\frac{1}{k !(q-1) !} \sum_{\sigma} \operatorname{sign} \sigma\left(f^{*} \omega\right)_{x}\left(K_{x}\left(X_{\sigma 1}, \ldots, X_{\sigma k}\right), X_{\sigma(k+1)}, \ldots\right) \\
& =\frac{1}{k !(q-1) !} \sum_{\sigma} \operatorname{sign} \sigma \omega_{f(x)}\left(T_{x} f \cdot K_{x}\left(X_{\sigma 1}, \ldots\right), T_{x} f \cdot X_{\sigma(k+1)}, \ldots\right) \\
& =\frac{1}{k !(q-1) !} \sum_{\sigma} \operatorname{sign} \sigma \omega_{f(x)}\left(K_{f(x)}^{\prime}\left(T_{x} f \cdot X_{\sigma 1}, \ldots\right), T_{x} f \cdot X_{\sigma(k+1)}, \ldots\right) \\
& =\left(f^{*} i_{K^{\prime}} \omega\right)_{x}\left(X_{1}, \ldots, X_{q+k-1}\right) .
\end{aligned}
$$

(3) follows from this computation, since the $d f, f \in C^{\infty}(M)$, separate points. (41) follows from the same computation for $K_{2}$ instead of $\omega$; the result for the bracket then follows from (16.2.2),

(5) By (2) the algebra homomorphism $f^{*}$ intertwines the operators $i_{K}$ and $i_{K^{\prime}}$, and $f^{*}$ commutes with the exterior derivative $d$. Thus $f^{*}$ intertwines the commutators $\left[i_{K}, d\right]=\mathcal{L}_{K}$ and $\left[i_{K^{\prime}}, d\right]=\mathcal{L}_{K^{\prime}}$.

(6) For $g \in \Omega^{0}(N)$ we have $\mathcal{L}_{K} f^{*} g=i_{K} d f^{*} g=i_{K} f^{*} d g$ and $f^{*} \mathcal{L}_{K^{\prime}} g=$ $f^{*} i_{K^{\prime}} d g$. By (3) the result follows. 
(17) The algebra homomorphism $f^{*}$ intertwines $\mathcal{L}_{K_{j}}$ and $\mathcal{L}_{K_{j}^{\prime}}$, so also their graded commutators which equal $\mathcal{L}\left(\left[K_{1}, K_{2}\right]\right)$ and $\mathcal{L}\left(\left[K_{1}^{\prime}, K_{2}^{\prime}\right]\right)$, respectively. Now use (6).

16.16. Let $f: M \rightarrow N$ be a local diffeomorphism. Then we can consider the pullback operator $f^{*}: \Omega(N ; T N) \rightarrow \Omega(M ; T M)$, given by

$$
\left(f^{*} K\right)_{x}\left(X_{1}, \ldots, X_{k}\right)=\left(T_{x} f\right)^{-1} K_{f(x)}\left(T_{x} f \cdot X_{1}, \ldots, T_{x} f \cdot X_{k}\right) .
$$

Note that this is a special case of the pullback operator for sections of natural vector bundles in (8.16). Clearly $K$ and $f^{*} K$ are then $f$-related.

Theorem. In this situation we have:

(2) $f^{*}[K, L]=\left[f^{*} K, f^{*} L\right]$.

(3) $f^{*} i_{K} L=i_{f^{*} K} f^{*} L$.

(4) $f^{*}[K, L]^{\wedge}=\left[f^{*} K, f^{*} L\right]^{\wedge}$.

(5) For a vector field $X \in \mathfrak{X}(M)$ and $K \in \Omega(M ; T M)$ by (8.16) the Lie derivative $\mathcal{L}_{X} K=\left.\partial\right|_{0}\left(\mathrm{Fl}_{t}^{X}\right)^{*} K$ is defined. Then we have $\mathcal{L}_{X} K=[X, K]$, the Frölicher-Nijenhuis bracket.

We may say that the Frölicher-Nijenhuis bracket, [ , $]^{\wedge}$, etc., are natural bilinear mappings.

Proof. (2) - (4) are obvious from (16.15) (5) Obviously $\mathcal{L}_{X}$ is $\mathbb{R}$-linear, so it suffices to check this formula for $K=\psi \otimes Y, \psi \in \Omega(M)$ and $Y \in \mathfrak{X}(M)$. But then

$$
\begin{aligned}
\mathcal{L}_{X}(\psi \otimes Y) & =\mathcal{L}_{X} \psi \otimes Y+\psi \otimes \mathcal{L}_{X} Y \quad \text { by }(8.17) \\
& =\mathcal{L}_{X} \psi \otimes Y+\psi \otimes[X, Y] \\
& =[X, \psi \otimes Y] \quad \text { by }(16.7 .6), \quad \square
\end{aligned}
$$

16.17. Remark. At last we mention the best known application of the Frölicher-Nijenhuis bracket, which also led to its discovery. A vector valued 1form $J \in \Omega^{1}(M ; T M)$ with $J \circ J=-I d$ is called an almost complex structure; if it exists, $\operatorname{dim} M$ is even and $J$ can be viewed as a fiber multiplication with $\sqrt{-1}$ on $T M$. By (16.12) we have

$$
[J, J](X, Y)=2([J X, J Y]-[X, Y]-J[X, J Y]-J[J X, Y]) .
$$

The vector valued form $\frac{1}{2}[J, J]$ is also called the Nijenhuis tensor of $J$. For it the following result is true:

A manifold $M$ with an almost complex structure $J$ is a complex manifold (i.e., there exists an atlas for $M$ with holomorphic chartchange mappings) if and only if $[J, J]=0$. See [173]. 


\section{Fiber Bundles and Connections}

17.1. Definition. A (fiber) bundle $(E, p, M, S)$ consists of manifolds $E$, $M, S$, and a smooth mapping $p: E \rightarrow M$; furthermore each $x \in M$ has an open neighborhood $U$ such that $E \mid U:=p^{-1}(U)$ is diffeomorphic to $U \times S$ via a fiber respecting diffeomorphism:

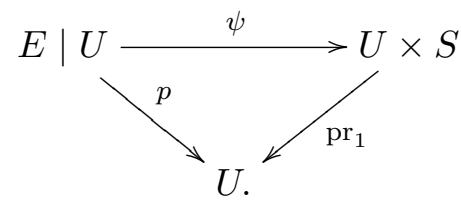

The manifold $E$ is called the total space, $M$ is called the base space or basis, $p$ is a surjective submersion, called the projection, $S$ is called standard fiber, and $(U, \psi)$ as above is called a fiber chart.

A collection of fiber charts $\left(U_{\alpha}, \psi_{\alpha}\right)$, such that $\left(U_{\alpha}\right)$ is an open cover of $M$, is called a fiber bundle atlas. If we fix such an atlas, then $\psi_{\alpha} \circ \psi_{\beta}{ }^{-1}(x, s)=$ $\left(x, \psi_{\alpha \beta}(x, s)\right)$, where $\psi_{\alpha \beta}:\left(U_{\alpha} \cap U_{\beta}\right) \times S \rightarrow S$ is smooth and $\psi_{\alpha \beta}(x$,$) is a$ diffeomorphism of $S$ for each $x \in U_{\alpha \beta}:=U_{\alpha} \cap U_{\beta}$. We may thus consider the mappings $\psi_{\alpha \beta}: U_{\alpha \beta} \rightarrow \operatorname{Diff}(S)$ with values in the group $\operatorname{Diff}(S)$ of all diffeomorphisms of $S$; their differentiability is a subtle question, which will not be discussed in this book, but see [148. In either form these mappings $\psi_{\alpha \beta}$ are called the transition functions of the bundle. They satisfy the cocycle condition: $\psi_{\alpha \beta}(x) \circ \psi_{\beta \gamma}(x)=\psi_{\alpha \gamma}(x)$ for $x \in U_{\alpha \beta \gamma}$ and $\psi_{\alpha \alpha}(x)=I d_{S}$ for $x \in U_{\alpha}$. Therefore the collection $\left(\psi_{\alpha \beta}\right)$ is called a cocycle of transition functions.

Given an open cover $\left(U_{\alpha}\right)$ of a manifold $M$ and a cocycle of transition functions $\left(\psi_{\alpha \beta}\right)$, we may construct a fiber bundle $(E, p, M, S)$ in a similar way as in (8.3).

17.2. Lemma. Let $p: N \rightarrow M$ be a surjective submersion (a fibered manifold) which is proper, so that $p^{-1}(K)$ is compact in $N$ for each compact $K \subset M$, and let $M$ be connected. Then $(N, p, M)$ is a fiber bundle.

Proof. We have to produce a fiber chart at each $x_{0} \in M$. So let $(U, u)$ be a chart centered at $x_{0}$ on $M$ such that $u(U) \cong \mathbb{R}^{m}$. For each $x \in U$ let $\xi_{x}(y):=\left(T_{y} u\right)^{-1} \cdot u(x)$; then we have $\xi_{x} \in \mathfrak{X}(U)$ which depends smoothly on $x \in U$, such that $u\left(\mathrm{Fl}_{t}^{\xi_{x}} u^{-1}(z)\right)=z+t . u(x)$. Thus each $\xi_{x}$ is a complete vector field on $U$. Since $p$ is a submersion, with the help of a partition of unity on $p^{-1}(U)$ we may construct vector fields $\eta_{x} \in \mathfrak{X}\left(p^{-1}(U)\right)$ which depend smoothly on $x \in U$ and are $p$-related to $\xi_{x}: T p \cdot \eta_{x}=\xi_{x} \circ p$. Thus $p \circ \mathrm{Fl}_{t}^{\eta_{x}}=\mathrm{Fl}_{t}^{\xi_{x}} \circ p$ by (3.14), so $\mathrm{Fl}_{t}^{\eta_{x}}$ is fiber respecting, and since $p$ is proper and $\xi_{x}$ is complete, $\eta_{x}$ has a global flow too. Denote $p^{-1}\left(x_{0}\right)$ by $S$. Then $\varphi: U \times S \rightarrow p^{-1}(U)$, defined by $\varphi(x, y)=\mathrm{Fl}_{1}^{\eta_{x}}(y)$, is a diffeomorphism and 
is fiber respecting, so $\left(U, \varphi^{-1}\right)$ is a fiber chart. Since $M$ is connected, the fibers $p^{-1}(x)$ are all diffeomorphic.

17.3. Let $(E, p, M, S)$ be a fiber bundle; we consider the fiber linear tangent mapping $T p: T E \rightarrow T M$ and its kernel ker $T p=: V E$ which is called the vertical bundle of $E$. The following is a special case of (16.13)

Definition. A connection on the fiber bundle $(E, p, M, S)$ is a vector valued 1-form $\Phi \in \Omega^{1}(E ; V E)$ with values in the vertical bundle $V E$ such that $\Phi \circ \Phi=\Phi$ and $\operatorname{Im} \Phi=V E$; so $\Phi$ is just a projection $T E \rightarrow V E$.

Then $\operatorname{ker} \Phi$ is of constant rank, so $\operatorname{ker} \Phi$ is a vector subbundle of $T E$ by (8.7), it is called the space of horizontal vectors or the horizontal bundle and it is denoted by $H E=\operatorname{ker} \Phi$. Clearly $T E=H E \oplus V E$ and $T_{u} E=H_{u} E \oplus V_{u} E$ for $u \in E$.

Now we consider the mapping $\left(T p, \pi_{E}\right): T E \rightarrow T M \times_{M} E$. Then by definition $\left(T p, \pi_{E}\right)^{-1}\left(0_{p(u)}, u\right)=V_{u} E$, so $\left(T p, \pi_{E}\right) \mid H E: H E \rightarrow T M \times_{M} E$ is fiber linear over $E$ and injective, so by reason of dimensions it is a fiber linear isomorphism: Its inverse is denoted by

$$
C:=\left(\left(T p, \pi_{E}\right) \mid H E\right)^{-1}: T M \times_{M} E \rightarrow H E \hookrightarrow T E .
$$

So $C: T M \times_{M} E \rightarrow T E$ is fiber linear over $E$ and is a right inverse for $\left(T p, \pi_{E}\right)$. The mapping $C$ is called the horizontal lift associated to the connection $\Phi$.

Note the formula $\Phi\left(\xi_{u}\right)=\xi_{u}-C\left(T p \cdot \xi_{u}, u\right)$ for $\xi_{u} \in T_{u} E$. So we can equally well describe a connection $\Phi$ by specifying $C$. Then we call $\Phi$ the vertical projection (no confusion with (8.12) will arise) and $\chi:=\operatorname{id}_{T E}-\Phi=C$ 。 $\left(T p, \pi_{E}\right)$ will be called the horizontal projection.

17.4. Curvature. If $\Phi: T E \rightarrow V E$ is a connection on the bundle $(E, p, M, S)$, then as in (16.13) the curvature $R$ of $\Phi$ is given by

$$
2 R=[\Phi, \Phi]=[I d-\Phi, I d-\Phi]=[\chi, \chi] \in \Omega^{2}(E ; V E) .
$$

The cocurvature $\bar{R}$ vanishes since the vertical bundle $V E$ is integrable. We have

$$
R(X, Y)=\frac{1}{2}[\Phi, \Phi](X, Y)=\Phi[\chi X, \chi Y],
$$

so $R$ is an obstruction against integrability of the horizontal subbundle. Note that for vector fields $\xi, \eta \in \mathfrak{X}(M)$ and their horizontal lifts $C \xi, C \eta \in \mathfrak{X}(E)$ we have

$$
R(C \xi, C \eta)=[C \xi, C \eta]-C([\xi, \eta]) .
$$

Since the vertical bundle $V E$ is integrable, by (16.14) we have the Bianchi identity $[\Phi, R]=0$. 
17.5. Pullback. Let $(E, p, M, S)$ be a fiber bundle and consider a smooth mapping $f: N \rightarrow M$. Since $p$ is a submersion, $f$ and $p$ are transversal in the sense of (2.16) and thus the pullback $N \times_{(f, M, p)} E$ exists. It will be called the pullback of the fiber bundle $E$ by $f$ and we will denote it by $f^{*} E$. The following diagram sets up some further notation for it:

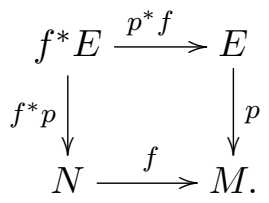

Proposition. In the situation above we have:

(1) $\left(f^{*} E, f^{*} p, N, S\right)$ is again a fiber bundle, and $p^{*} f$ is a fiberwise diffeomorphism.

(2) If $\Phi \in \Omega^{1}(E ; V E) \subset \Omega^{1}(E ; T E)$ is a connection on $E$, then the vector valued form $f^{*} \Phi$, given by $\left(f^{*} \Phi\right)_{u}(X):=V_{u}\left(p^{*} f\right)^{-1} \cdot \Phi \cdot T_{u}\left(p^{*} f\right) . X$ for $X \in T_{u} E$, is a connection on the bundle $f^{*} E$. The forms $f^{*} \Phi$ and $\Phi$ are $p^{*} f$-related in the sense of (16.15).

(3) The curvatures of $f^{*} \Phi$ and $\Phi$ are also $p^{*} f$-related.

Proof. (11) If $\left(U_{\alpha}, \psi_{\alpha}\right)$ is a fiber bundle atlas of $(E, p, M, S)$ in the sense of (17.1), then $\left(f^{-1}\left(U_{\alpha}\right),\left(f^{*} p, \operatorname{pr}_{2} \circ \psi_{\alpha} \circ p^{*} f\right)\right)$ is a fiber bundle atlas for $\left(f^{*} E, f^{*} p, N, S\right)$, by the formal universal properties of a pullback (2.17). Part (22) is obvious. Part (3) follows from (2) and (16.15.7).

17.6. Let us suppose that a connection $\Phi$ on the bundle $(E, p, M, S)$ has zero curvature. Then by (17.4) the horizontal bundle is integrable and gives rise to the horizontal foliation by (3.28.2). Each point $u \in E$ lies on a unique leaf $L(u)$ such that $T_{v} L(u)=H_{v} E$ for each $v \in L(u)$. The restriction $p \mid L(u)$ is locally a diffeomorphism, but in general it is neither surjective nor is it a covering onto its image. This is seen by devising suitable horizontal foliations on the trivial bundle $\mathrm{pr}_{2}: \mathbb{R} \times S^{1} \rightarrow S^{1}$, or $\mathrm{pr}_{2} \mathbb{R} \times \mathbb{R} \rightarrow \mathbb{R}$, like $L(0, t)=\{(\tan (s-t), s): s \in \mathbb{R}\}$.

17.7. Local description. Let $\Phi$ be a connection on $(E, p, M, S)$. Let us fix a fiber bundle atlas $\left(U_{\alpha}\right)$ with transition functions $\left(\psi_{\alpha \beta}\right)$, and let us consider the connection $\left(\left(\psi_{\alpha}\right)^{-1}\right)^{*} \Phi \in \Omega^{1}\left(U_{\alpha} \times S ; U_{\alpha} \times T S\right)$, which may be written in the form

$$
\left(\left(\left(\psi_{\alpha}\right)^{-1}\right)^{*} \Phi\right)\left(\xi_{x}, \eta_{y}\right)=:-\Gamma^{\alpha}\left(\xi_{x}, y\right)+\eta_{y} \text { for } \xi_{x} \in T_{x} U_{\alpha} \text { and } \eta_{y} \in T_{y} S,
$$

since it reproduces vertical vectors. The $\Gamma^{\alpha}$ are given by

$$
\left(0_{x}, \Gamma^{\alpha}\left(\xi_{x}, y\right)\right):=-T\left(\psi_{\alpha}\right) . \Phi \cdot T\left(\psi_{\alpha}\right)^{-1} \cdot\left(\xi_{x}, 0_{y}\right) .
$$


We consider $\Gamma^{\alpha}$ as an element of the space $\Omega^{1}\left(U_{\alpha} ; \mathfrak{X}(S)\right)$, a 1-form on $U^{\alpha}$ with values in the infinite-dimensional Lie algebra $\mathfrak{X}(S)$ of all vector fields on the standard fiber. The $\Gamma^{\alpha}$ are called the Christoffel forms of the connection $\Phi$ with respect to the bundle atlas $\left(U_{\alpha}, \psi_{\alpha}\right)$.

Lemma. The transformation law for the Christoffel forms is

$$
T_{y}\left(\psi_{\alpha \beta}(x, \quad)\right) \cdot \Gamma^{\beta}\left(\xi_{x}, y\right)=\Gamma^{\alpha}\left(\xi_{x}, \psi_{\alpha \beta}(x, y)\right)-T_{x}\left(\psi_{\alpha \beta}(\quad, y)\right) . \xi_{x} .
$$

The curvature $R$ of $\Phi$ satisfies

$$
\left(\psi_{\alpha}^{-1}\right)^{*} R=d \Gamma^{\alpha}+\left[\Gamma^{\alpha}, \Gamma^{\alpha}\right]_{\mathfrak{X}(S)} .
$$

Here $d \Gamma^{\alpha}$ is the exterior derivative of the 1 -form $\Gamma^{\alpha} \in \Omega^{1}\left(U_{\alpha} ; \mathfrak{X}(S)\right)$ with values in the complete locally convex space $\mathfrak{X}(S)$. We will later also use the Lie derivative of it and the usual formulas apply: Consult [113 for calculus in infinite-dimensional spaces.

The formula for the curvature is the Maurer-Cartan formula which in this general setting appears only in the level of local description.

Proof. From $\left(\psi_{\alpha} \circ\left(\psi_{\beta}\right)^{-1}\right)(x, y)=\left(x, \psi_{\alpha \beta}(x, y)\right)$ we get that

$$
T\left(\psi_{\alpha} \circ\left(\psi_{\beta}\right)^{-1}\right) \cdot\left(\xi_{x}, \eta_{y}\right)=\left(\xi_{x}, T_{(x, y)}\left(\psi_{\alpha \beta}\right) \cdot\left(\xi_{x}, \eta_{y}\right)\right)
$$

and thus:

$$
\begin{aligned}
& T\left(\psi_{\beta}^{-1}\right) \cdot\left(0_{x}, \Gamma^{\beta}\left(\xi_{x}, y\right)\right)=-\Phi\left(T\left(\psi_{\beta}^{-1}\right)\left(\xi_{x}, 0_{y}\right)\right) \\
& =-\Phi\left(T\left(\psi_{\alpha}^{-1}\right) \cdot T\left(\psi_{\alpha} \circ \psi_{\beta}^{-1}\right) \cdot\left(\xi_{x}, 0_{y}\right)\right) \\
& =-\Phi\left(T\left(\psi_{\alpha}^{-1}\right)\left(\xi_{x}, T_{(x, y)}\left(\psi_{\alpha \beta}\right)\left(\xi_{x}, 0_{y}\right)\right)\right) \\
& =-\Phi\left(T\left(\psi_{\alpha}^{-1}\right)\left(\xi_{x}, 0_{\psi_{\alpha \beta}(x, y)}\right)\right)-\Phi\left(T\left(\psi_{\alpha}^{-1}\right)\left(0_{x}, T_{(x, y)} \psi_{\alpha \beta}\left(\xi_{x}, 0_{y}\right)\right)\right. \\
& =T\left(\psi_{\alpha}^{-1}\right) \cdot\left(0_{x}, \Gamma^{\alpha}\left(\xi_{x}, \psi_{\alpha \beta}(x, y)\right)\right)-T\left(\psi_{\alpha}^{-1}\right)\left(0_{x}, T_{x}\left(\psi_{\alpha \beta}(\quad, y)\right) \cdot \xi_{x}\right) .
\end{aligned}
$$

This implies the transformation law.

For the curvature $R$ of $\Phi$ we have by (17.4) and (17.5.3)

$$
\begin{aligned}
& \left(\psi_{\alpha}^{-1}\right)^{*} R\left(\left(\xi^{1}, \eta^{1}\right),\left(\xi^{2}, \eta^{2}\right)\right) \\
& \quad=\left(\psi_{\alpha}^{-1}\right)^{*} \Phi\left[\left(I d-\left(\psi_{\alpha}^{-1}\right)^{*} \Phi\right)\left(\xi^{1}, \eta^{1}\right),\left(I d-\left(\psi_{\alpha}^{-1}\right)^{*} \Phi\right)\left(\xi^{2}, \eta^{2}\right)\right] \\
& \quad=\left(\psi_{\alpha}^{-1}\right)^{*} \Phi\left[\left(\xi^{1}, \Gamma^{\alpha}\left(\xi^{1}\right)\right),\left(\xi^{2}, \Gamma^{\alpha}\left(\xi^{2}\right)\right)\right] \\
& \quad=\left(\psi_{\alpha}^{-1}\right)^{*} \Phi\left(\left[\xi^{1}, \xi^{2}\right], \xi^{1} \Gamma^{\alpha}\left(\xi^{2}\right)-\xi^{2} \Gamma^{\alpha}\left(\xi^{1}\right)+\left[\Gamma^{\alpha}\left(\xi^{1}\right), \Gamma^{\alpha}\left(\xi^{2}\right)\right]\right) \\
& \quad=-\Gamma^{\alpha}\left(\left[\xi^{1}, \xi^{2}\right]\right)+\xi^{1} \Gamma^{\alpha}\left(\xi^{2}\right)-\xi^{2} \Gamma^{\alpha}\left(\xi^{1}\right)+\left[\Gamma^{\alpha}\left(\xi^{1}\right), \Gamma^{\alpha}\left(\xi^{2}\right)\right] \\
& \quad=d \Gamma^{\alpha}\left(\xi^{1}, \xi^{2}\right)+\left[\Gamma^{\alpha}\left(\xi^{1}\right), \Gamma^{\alpha}\left(\xi^{2}\right)\right] \mathfrak{X}(S) .
\end{aligned}
$$


17.8. Theorem (Parallel transport). Let $\Phi$ be a connection on a bundle $(E, p, M, S)$ and let $c:(a, b) \rightarrow M$ be a smooth curve with $0 \in(a, b)$, $c(0)=x$.

Then there is a neighborhood $U$ of $E_{x} \times\{0\}$ in $E_{x} \times(a, b)$ and a smooth mapping $\mathrm{Pt}_{c}: U \rightarrow E$ such that:

(1) $p\left(\operatorname{Pt}\left(c, u_{x}, t\right)\right)=c(t)$ if defined, and $\operatorname{Pt}\left(c, u_{x}, 0\right)=u_{x}$.

(2) $\Phi\left(\frac{d}{d t} \operatorname{Pt}\left(c, u_{x}, t\right)\right)=0$ if defined.

(3) Reparameterization invariance: If $f:\left(a^{\prime}, b^{\prime}\right) \rightarrow(a, b)$ is smooth with $0 \in\left(a^{\prime}, b^{\prime}\right)$, then $\operatorname{Pt}\left(c, u_{x}, f(t)\right)=\operatorname{Pt}\left(c \circ f, \operatorname{Pt}\left(c, u_{x}, f(0)\right), t\right)$ if defined.

(4) $U$ is maximal for properties (11) and (2).

(5) In a certain sense Pt depends smoothly also on $c$.

First proof. In local bundle coordinates $\Phi\left(\frac{d}{d t} \operatorname{Pt}\left(c, u_{x}, t\right)\right)=0$ is an ordinary differential equation of first order, nonlinear, with initial condition $\operatorname{Pt}\left(c, u_{x}, 0\right)=u_{x}$. So there is a maximally defined local solution curve which is unique. All further properties are consequences of uniqueness.

Second proof. Consider the pullback bundle $\left(c^{*} E, c^{*} p,(a, b), S\right)$ and the pullback connection $c^{*} \Phi$ on it. It has zero curvature, since the horizontal bundle is 1-dimensional. By (17.6) the horizontal foliation exists and the parallel transport just follows a leaf and we may map it back to $E$, in detail: $\operatorname{Pt}\left(c, u_{x}, t\right)=p^{*} c\left(\left(c^{*} p \mid L\left(u_{x}\right)\right)^{-1}(t)\right)$.

Third proof. Consider a fiber bundle atlas $\left(U_{\alpha}, \psi_{\alpha}\right)$ as in (17.7), Then we have $\psi_{\alpha}\left(\operatorname{Pt}\left(c, \psi_{\alpha}^{-1}(x, y), t\right)\right)=(c(t), \gamma(y, t))$, where

$$
0=\left(\left(\psi_{\alpha}^{-1}\right)^{*} \Phi\right)\left(\frac{d}{d t} c(t), \frac{d}{d t} \gamma(y, t)\right)=-\Gamma^{\alpha}\left(\frac{d}{d t} c(t), \gamma(y, t)\right)+\frac{d}{d t} \gamma(y, t),
$$

so $\gamma(y, t)$ is the integral curve (evolution line) through $y \in S$ of the time dependent vector field $\Gamma^{\alpha}\left(\frac{d}{d t} c(t)\right)$ on $S$. This vector field visibly depends smoothly on $c$. Clearly local solutions exist and all properties follow, even (5). For more detailed information on (5) we refer to [143] or [113.

17.9. A connection $\Phi$ on $(E, p, M, S)$ is called a complete connection if the parallel transport $\mathrm{Pt}_{c}$ along any smooth curve $c:(a, b) \rightarrow M$ is defined on the whole of $E_{c(0)} \times(a, b)$. The third proof of theorem (17.8) shows that on a fiber bundle with compact standard fiber any connection is complete.

The following is a sufficient condition for a connection $\Phi$ to be complete:

There exists a fiber bundle atlas $\left(U_{\alpha}, \psi_{\alpha}\right)$ and complete Riemann metrics $g_{\alpha}$ on the standard fiber $S$ such that each Christoffel form $\Gamma^{\alpha} \in \Omega^{1}\left(U_{\alpha}, \mathfrak{X}(S)\right)$ takes values in the linear subspace of $g_{\alpha}$-bounded vector fields on $S$. 
This is true because in the third proof of theorem (17.8) above the time dependent vector field $\Gamma^{\alpha}\left(\frac{d}{d t} c(t)\right)$ on $S$ is $g_{\alpha}$-bounded for compact time intervals. By (23.9) this vector field is complete. So by continuation the solution exists globally.

A complete connection is called an Ehresmann connection in [80, I, p. 314], where the following result is given as an exercise.

Theorem. Each fiber bundle admits complete connections.

Proof. Let $\operatorname{dim} M=m$. Let $\left(U_{\alpha}, \psi_{\alpha}\right)$ be a fiber bundle atlas as in (17.1), By topological dimension theory 169 the open cover $\left(U_{\alpha}\right)$ of $M$ admits a refinement such that any $m+2$ members have empty intersection; see also (1.1). Let $\left(U_{\alpha}\right)$ itself have this property. Choose a smooth partition of unity $\left(f_{\alpha}\right)$ subordinated to $\left(U_{\alpha}\right)$. Then the sets $V_{\alpha}:=\left\{x: f_{\alpha}(x)>\frac{1}{m+2}\right\} \subset U_{\alpha}$ still form an open cover of $M$ since $\sum f_{\alpha}(x)=1$ and at most $m+1$ of the $f_{\alpha}(x)$ can be nonzero. By renaming, assume that each $V_{\alpha}$ is connected. Then we choose an open cover $\left(W_{\alpha}\right)$ of $M$ such that $\overline{W_{\alpha}} \subset V_{\alpha}$.

Now let $g_{1}$ and $g_{2}$ be complete Riemann metrics on $M$ and $S$, respectively (see (23.8)]. For not connected Riemann manifolds complete means that each connected component is complete. Then $g_{1} \mid U_{\alpha} \times g_{2}$ is a Riemann metric on $U_{\alpha} \times S$ and we consider the metric $g:=\sum f_{\alpha} \psi_{\alpha}^{*}\left(g_{1} \mid U_{\alpha} \times g_{2}\right)$ on $E$. Obviously $p: E \rightarrow M$ is a Riemann submersion for the metrics $g$ and $g_{1}$ : This means that $T_{u} p:\left(T_{u}\left(E_{p(u)}\right)^{\perp}, g_{u}\right) \rightarrow\left(T_{p(u)} M,\left(g_{1}\right)_{p(u)}\right)$ is an isometry for each $u \in E$. We choose now the connection $\Phi: T E \rightarrow V E$ as the orthonormal projection with respect to the Riemann metric $g$.

Claim. $\Phi$ is a complete connection on $E$.

Let $c:[0,1] \rightarrow M$ be a smooth curve. We choose a partition $0=t_{0}<$ $t_{1}<\cdots<t_{k}=1$ such that $c\left(\left[t_{i}, t_{i+1}\right]\right) \subset V_{\alpha_{i}}$ for suitable $\alpha_{i}$. It suffices to show that $\operatorname{Pt}\left(c\left(t_{i}+\right), u_{c\left(t_{i}\right)}, t\right)$ exists for all $0 \leq t \leq t_{i+1}-t_{i}$ and all $u_{c\left(t_{i}\right)}$, for all $i$, since then we may piece them together. So we may assume that $c:[0,1] \rightarrow V_{\alpha}$ for some $\alpha$. Let us now assume that for $x=c(0)$ and some $y \in S$ the parallel transport $\operatorname{Pt}\left(c, \psi_{\alpha}(x, y), t\right)$ is defined only for $t \in\left[0, t^{\prime}\right)$ for some $0<t^{\prime}<1$. By the third proof of theorem (17.8) we have

$$
\operatorname{Pt}\left(c, \psi_{\alpha}^{-1}(x, y), t\right)=\psi_{\alpha}^{-1}(c(t), \gamma(t))
$$

where $\gamma:\left[0, t^{\prime}\right) \rightarrow S$ is the maximally defined integral curve through $y \in S$ of the time dependent vector field $\Gamma^{\alpha}\left(\frac{d}{d t} c(t), \quad\right)$ on $S$. We put $g_{\alpha}:=\left(\psi_{\alpha}^{-1}\right)^{*} g$; then

$$
\left(g_{\alpha}\right)_{(x, y)}=\left(g_{1}\right)_{x} \times\left(\sum_{\beta} f_{\beta}(x) \psi_{\beta \alpha}(x, \quad)^{*} g_{2}\right)_{y} .
$$

Since $\operatorname{pr}_{1}:\left(V_{\alpha} \times S, g_{\alpha}\right) \rightarrow\left(V_{\alpha}, g_{1} \mid V_{\alpha}\right)$ is a Riemann submersion and since the connection $\left(\psi_{\alpha}^{-1}\right)^{*} \Phi$ is also given by orthonormal projection onto the 
vertical bundle, we get

$$
\begin{gathered}
\infty>g_{1} \text {-length } t_{0}^{t^{\prime}}(c)=g_{\alpha} \text {-length }(c, \gamma)=\int_{0}^{t^{\prime}}\left|\left(c^{\prime}(t), \frac{d}{d t} \gamma(t)\right)\right|_{g_{\alpha}} d t \\
=\int_{0}^{t^{\prime}} \sqrt{\left|c^{\prime}(t)\right|_{g_{1}}^{2}+\sum_{\beta} f_{\beta}(c(t))\left(\psi_{\alpha \beta}(c(t),-)^{*} g_{2}\right)\left(\frac{d}{d t} \gamma(t), \frac{d}{d t} \gamma(t)\right)} d t \\
\geq \int_{0}^{t^{\prime}} \sqrt{f_{\alpha}(c(t))}\left|\frac{d}{d t} \gamma(t)\right|_{g_{2}} d t \geq \frac{1}{\sqrt{m+2}} \int_{0}^{t^{\prime}}\left|\frac{d}{d t} \gamma(t)\right|_{g_{2}} d t .
\end{gathered}
$$

So $g_{2}$-length $(\gamma)$ is finite and since the Riemann metric $g_{2}$ on $S$ is complete, the limit $\lim _{t \rightarrow t^{\prime}} \gamma(t)=: \gamma\left(t^{\prime}\right)$ exists in $S$ and the integral curve $\gamma$ can be continued.

17.10. Holonomy groups and Lie algebras. Let $(E, p, M, S)$ be a fiber bundle with a complete connection $\Phi$, and let us assume that $M$ is connected. We choose a fixed base point $x_{0} \in M$ and we identify $E_{x_{0}}$ with the standard fiber $S$. For each closed piecewise smooth curve $c:[0,1] \rightarrow M$ through $x_{0}$ the parallel transport $\operatorname{Pt}(c,, 1)=: \operatorname{Pt}(c, 1)$ (pieced together over the smooth parts of $c$ ) is a diffeomorphism of $S$. All these diffeomorphisms form together the group $\operatorname{Hol}\left(\Phi, x_{0}\right)$, the holonomy group of $\Phi$ at $x_{0}$, a subgroup of the diffeomorphism group $\operatorname{Diff}(S)$. If we consider only those piecewise smooth curves which are homotopic to zero, we get a subgroup $\operatorname{Hol}_{0}\left(\Phi, x_{0}\right)$, called the restricted holonomy group of the connection $\Phi$ at $x_{0}$.

Now let $C: T M \times_{M} E \rightarrow T E$ be the horizontal lifting as in (17.3), and let $R$ be the curvature (see (17.4) of the connection $\Phi$. For any $x \in M$ and $X_{x} \in T_{x} M$ the horizontal lift $C\left(X_{x}\right):=C\left(X_{x}, \quad\right): E_{x} \rightarrow T E$ is a vector field along $E_{x}$. For $X_{x}$ and $Y_{x} \in T_{x} M$ we consider $R\left(C X_{x}, C Y_{x}\right) \in$ $\mathfrak{X}\left(E_{x}\right)$. Now we choose any piecewise smooth curve $c$ from $x_{0}$ to $x$ and consider the diffeomorphism $\operatorname{Pt}(c, t): S=E_{x_{0}} \rightarrow E_{x}$ and the pullback $\operatorname{Pt}(c, 1)^{*} R\left(C X_{x}, C Y_{x}\right) \in \mathfrak{X}(S)$. Let us denote by hol $\left(\Phi, x_{0}\right)$ the closed linear subspace, generated by all these vector fields (for all $x \in M, X_{x}, Y_{x} \in T_{x} M$ and curves $c$ from $x_{0}$ to $\left.x\right)$ in $\mathfrak{X}(S)$ with respect to the compact $C^{\infty}$-topology, and let us call it the holonomy Lie algebra of $\Phi$ at $x_{0}$.

Lemma. $\operatorname{hol}\left(\Phi, x_{0}\right)$ is a Lie subalgebra of $\mathfrak{X}(S)$.

Proof. For $X \in \mathfrak{X}(M)$ we consider the local flow $\mathrm{Fl}_{t}^{C X}$ of the horizontal lift of $X$. It restricts to parallel transport along any of the flow lines of $X$ in $M$. Then for vector fields on $M$ the expression

$$
\begin{aligned}
& \left.\frac{d}{d t}\right|_{0}\left(\mathrm{Fl}_{s}^{C X}\right)^{*}\left(\mathrm{Fl}_{t}^{C Y}\right)^{*}\left(\mathrm{Fl}_{-s}^{C X}\right)^{*}\left(\mathrm{Fl}_{z}^{C Z}\right)^{*} R(C U, C V) \uparrow E_{x_{0}} \\
& \quad=\left(\mathrm{Fl}_{s}^{C X}\right)^{*}\left[C Y,\left(\mathrm{Fl}_{-s}^{C X}\right)^{*}\left(\mathrm{Fl}_{z}^{C Z}\right)^{*} R(C U, C V)\right] \uparrow E_{x_{0}} \\
& \quad=\left[\left(\mathrm{Fl}_{s}^{C X}\right)^{*} C Y,\left(\mathrm{Fl}_{z}^{C Z}\right)^{*} R(C U, C V)\right] \uparrow E_{x_{0}}
\end{aligned}
$$


is in $\operatorname{hol}\left(\Phi, x_{0}\right)$, since it is closed in the compact $C^{\infty}$-topology and the derivative can be written as a limit. Thus

$$
\left[\left(\mathrm{Fl}_{s}^{C X}\right)^{*}\left[C Y_{1}, C Y_{2}\right],\left(\mathrm{Fl}_{z}^{C Z}\right)^{*} R(C U, C V)\right] \uparrow E_{x_{0}} \in \operatorname{hol}\left(\Phi, x_{0}\right)
$$

by the Jacobi identity and

$$
\left[\left(\mathrm{Fl}_{s}^{C X}\right)^{*} C\left[Y_{1}, Y_{2}\right],\left(\mathrm{Fl}_{z}^{C Z}\right)^{*} R(C U, C V)\right] \uparrow E_{x_{0}} \in \operatorname{hol}\left(\Phi, x_{0}\right),
$$

so also their difference

$$
\left[\left(\mathrm{Fl}_{s}^{C X}\right)^{*} R\left(C Y_{1}, C Y_{2}\right),\left(\mathrm{Fl}_{z}^{C Z}\right)^{*} R(C U, C V)\right] \uparrow E_{x_{0}}
$$

is in $\operatorname{hol}\left(\Phi, x_{0}\right)$.

17.11. The following theorem is a generalization of the theorem of $\mathbf{1 7 4}$, 175. and 9] on principal connections. The reader who does not know principal connections is advised to read parts of sections (18) and (19) first. We include this result here in order not to disturb the development in section (19) later.

Theorem. Let $\Phi$ be a complete connection on the fiber bundle $(E, p, M, S)$ and let $M$ be connected. Suppose that for some (hence any) $x_{0} \in M$ the holonomy Lie algebra $\operatorname{hol}\left(\Phi, x_{0}\right)$ is finite-dimensional and consists of complete vector fields on the fiber $E_{x_{0}}$.

Then there is a principal bundle $(P, p, M, G)$ with finite-dimensional structure group $G$, a connection $\omega$ on it and a smooth action of $G$ on $S$ such that the Lie algebra $\mathfrak{g}$ of $G$ equals the holonomy Lie algebra $\operatorname{hol}\left(\Phi, x_{0}\right)$, the fiber bundle $E$ is isomorphic to the associated bundle $P[S]$, and $\Phi$ is the connection induced by $\omega$. The structure group $G$ equals the holonomy group $\operatorname{Hol}\left(\Phi, x_{0}\right)$. The principle bundle $P$ and its connection $\omega$ are unique up to isomorphism.

By a theorem of [186] a finite-dimensional Lie subalgebra of $\mathfrak{X}\left(E_{x_{0}}\right)$ like $\operatorname{hol}\left(\Phi, x_{0}\right)$ consists of complete vector fields if and only if it is generated by complete vector fields as a Lie algebra.

Proof. Let us again identify $E_{x_{0}}$ and $S$. Then $\mathfrak{g}:=\operatorname{hol}\left(\Phi, x_{0}\right)$ is a finitedimensional Lie subalgebra of $\mathfrak{X}(S)$, and since each vector field in it is complete, there is a finite-dimensional connected Lie group $G_{0}$ of diffeomorphisms of $S$ with Lie algebra $\mathfrak{g}$, by theorem (6.5).

Claim 1. $G_{0}$ contains $\operatorname{Hol}_{0}\left(\Phi, x_{0}\right)$, the restricted holonomy group.

Let $f \in \operatorname{Hol}_{0}\left(\Phi, x_{0}\right)$; then $f=\operatorname{Pt}(c, 1)$ for a piecewise smooth closed curve $c$ through $x_{0}$, which is nullhomotopic. Since the parallel transport is essentially invariant under reparametrization, (17.8), we can replace $c$ by $c \circ g$, where $g$ is smooth and flat at each corner of $c$. So we may assume that $c$ itself is smooth. Since $c$ is homotopic to zero, by approximation we may 
assume that there is a smooth homotopy $H: \mathbb{R}^{2} \rightarrow M$ with $H_{1} \mid[0,1]=c$ and $H_{0} \mid[0,1]=x_{0}$. Then $f_{t}:=\operatorname{Pt}\left(H_{t}, 1\right)$ is a curve in $\operatorname{Hol}_{0}\left(\Phi, x_{0}\right)$ which is smooth as a mapping $\mathbb{R} \times S \rightarrow S$; this can be seen by using the proof of claim 2 below or as in the proof of (19.7.4). We will continue the proof of claim 1 below.

Claim 2. $\left(\frac{d}{d t} f_{t}\right) \circ f_{t}^{-1}=: Z_{t}$ is in $\mathfrak{g}$ for all $t$.

To prove claim 2 , we consider the pullback bundle $H^{*} E \rightarrow \mathbb{R}^{2}$ with the induced connection $H^{*} \Phi$. It is sufficient to prove claim 2 there. Let $X=\frac{d}{d s}$ and $Y=\frac{d}{d t}$ be constant vector fields on $\mathbb{R}^{2}$, so $[X, Y]=0$. Then $\operatorname{Pt}(c, s)=$ $\mathrm{Fl}_{s}^{C X} \mid S$ and so on. We put

$$
f_{t, s}=\mathrm{Fl}_{-s}^{C X} \circ \mathrm{Fl}_{-t}^{C Y} \circ \mathrm{Fl}_{s}^{C X} \circ \mathrm{Fl}_{t}^{C Y}: S \rightarrow S,
$$

so $f_{t, 1}=f_{t}$. Then we have in the vector space $\mathfrak{X}(S)$

$$
\begin{aligned}
& \left(\frac{d}{d t} f_{t, s}\right) \circ f_{t, s}^{-1}=-\left(\mathrm{Fl}_{s}^{C X}\right)^{*} C Y+\left(\mathrm{Fl}_{s}^{C X}\right)^{*}\left(\mathrm{Fl}_{t}^{C Y}\right)^{*}\left(\mathrm{Fl}_{-s}^{C X}\right)^{*} C Y, \\
& \left(\frac{d}{d t} f_{t, 1}\right) \circ f_{t, 1}^{-1}=\int_{0}^{1} \frac{d}{d s}\left(\left(\frac{d}{d t} f_{t, s}\right) \circ f_{t, s}^{-1}\right) d s \\
& =\int_{0}^{1}\left(-\left(\mathrm{Fl}_{s}^{C X}\right)^{*}[C X, C Y]+\left(\mathrm{Fl}_{s}^{C X}\right)^{*}\left[C X,\left(\mathrm{Fl}_{t}^{C Y}\right)^{*}\left(\mathrm{Fl}_{-s}^{C X}\right)^{*} C Y\right]\right. \\
& \left.-\left(\mathrm{Fl}_{s}^{C X}\right)^{*}\left(\mathrm{Fl}_{t}^{C Y}\right)^{*}\left(\mathrm{Fl}_{-s}^{C X}\right)^{*}[C X, C Y]\right) d s .
\end{aligned}
$$

Since $[X, Y]=0$, we have $[C X, C Y]=\Phi[C X, C Y]=R(C X, C Y)$ and $\left(\mathrm{Fl}_{t}^{X}\right)^{*} Y=Y$; thus

$$
\begin{aligned}
& \left(\mathrm{Fl}_{t}^{C X}\right)^{*} C Y=C\left(\left(\mathrm{Fl}_{t}^{X}\right)^{*} Y\right)+\Phi\left(\left(\mathrm{Fl}_{t}^{C X}\right)^{*} C Y\right) \\
& =C Y+\int_{0}^{t} \frac{d}{d t} \Phi\left(\mathrm{Fl}_{t}^{C X}\right)^{*} C Y d t=C Y+\int_{0}^{t} \Phi\left(\mathrm{Fl}_{t}^{C X}\right)^{*}[C X, C Y] d t \\
& =C Y+\int_{0}^{t} \Phi\left(\mathrm{Fl}_{t}^{C X}\right)^{*} R(C X, C Y) d t=C Y+\int_{0}^{t}\left(\mathrm{Fl}_{t}^{C X}\right)^{*} R(C X, C Y) d t .
\end{aligned}
$$

The flows $\left(\mathrm{Fl}_{s}^{C X}\right)^{*}$ and their derivatives $\mathcal{L}_{C X}=[C X, \quad]$ do not lead out of $\mathfrak{g}$; thus all parts of the integrand above are in $\mathfrak{g}$ and so $\left(\frac{d}{d t} f_{t, 1}\right) \circ f_{t, 1}^{-1}$ is in $\mathfrak{g}$ for all $t$ and claim 2 follows.

Now claim 1 can be shown as follows. There is a unique smooth curve $g(t)$ in $G_{0}$ satisfying $T_{e}\left(\mu^{g(t)}\right) Z_{t}=Z_{t} . g(t)=\frac{d}{d t} g(t)$ and $g(0)=e$; via the action of $G_{0}$ on $S$ the curve $g(t)$ is a curve of diffeomorphisms on $S$, generated by the time dependent vector field $Z_{t}$, so $g(t)=f_{t}$ and $f=f_{1}$ is in $G_{0}$. So we get $\operatorname{Hol}_{0}\left(\Phi, x_{0}\right) \subseteq G_{0}$.

Claim 3. $\operatorname{Hol}_{0}\left(\Phi, x_{0}\right)$ equals $G_{0}$.

In the proof of claim 1 we have seen that $\operatorname{Hol}_{0}\left(\Phi, x_{0}\right)$ is a smoothly arcwise connected subgroup of $G_{0}$, so it is a connected Lie subgroup by the theorem (5.6). It suffices thus to show that the Lie algebra $\mathfrak{g}$ of $G_{0}$ is contained 
in the Lie algebra of $\operatorname{Hol}_{0}\left(\Phi, x_{0}\right)$, and for that it is enough to show that for each $\xi$ in a linearly spanning subset of $\mathfrak{g}$ there is a smooth mapping $f:[-1,1] \times S \rightarrow S$ such that the associated curve $\check{f}$ lies in $\operatorname{Hol}_{0}\left(\Phi, x_{0}\right)$ with $\check{f}^{\prime}(0)=0$ and $\check{f}^{\prime \prime}(0)=\xi$.

By definition we may assume $\xi=\operatorname{Pt}(c, 1)^{*} R\left(C X_{x}, C Y_{x}\right)$ for $X_{x}, Y_{x} \in T_{x} M$ and a smooth curve $c$ in $M$ from $x_{0}$ to $x$. We extend $X_{x}$ and $Y_{x}$ to vector fields $X$ and $Y \in \mathfrak{X}(M)$ with $[X, Y]=0$ near $x$. We may also suppose that $Z \in \mathfrak{X}(M)$ is a vector field which extends $c^{\prime}(t)$ along $c(t)$ : If $c$ is simple, we approximate it by an embedding and can consequently extend $c^{\prime}(t)$ to such a vector field. If $c$ is not simple, we do this for each simple piece of $c$, and then have several vector fields $Z$ instead of one below. So we have

$$
\begin{aligned}
\xi & =\left(\mathrm{Fl}_{1}^{C Z}\right)^{*} R(C X, C Y)=\left(\mathrm{Fl}_{1}^{C Z}\right)^{*}[C X, C Y] \quad \text { since }[X, Y](x)=0 \\
& =\left.\left(\mathrm{Fl}_{1}^{C Z}\right)^{*} \frac{1}{2} \frac{d^{2}}{d t^{2}}\right|_{t=0}\left(\mathrm{Fl}_{-t}^{C Y} \circ \mathrm{Fl}_{-t}^{C X} \circ \mathrm{Fl}_{t}^{C Y} \circ \mathrm{Fl}_{t}^{C X}\right) \quad \text { by }(3.16) \\
& =\left.\frac{1}{2} \frac{d^{2}}{d t^{2}}\right|_{t=0}\left(\mathrm{Fl}_{-1}^{C Z} \circ \mathrm{Fl}_{-t}^{C Y} \circ \mathrm{Fl}_{-t}^{C X} \circ \mathrm{Fl}_{t}^{C Y} \circ \mathrm{Fl}_{t}^{C X} \circ \mathrm{Fl}_{1}^{C Z}\right),
\end{aligned}
$$

where the parallel transport in the last equation first follows $c$ from $x_{0}$ to $x$, then follows a small closed parallelogram near $x$ in $M$ (since $[X, Y]=0$ near $x$ ) and then follows $c$ back to $x_{0}$. This curve is clearly nullhomotopic.

Step 4. Now we make $\operatorname{Hol}\left(\Phi, x_{0}\right)$ into a Lie group which we call $G$, by taking $\operatorname{Hol}_{0}\left(\Phi, x_{0}\right)=G_{0}$ as its connected component of the identity. Then the quotient $\operatorname{Hol}\left(\Phi, x_{0}\right) / \operatorname{Hol}_{0}\left(\Phi, x_{0}\right)$ is a countable group, since the fundamental group $\pi_{1}(M)$ is countable (by Morse theory $M$ is homotopy equivalent to a countable CW-complex).

Step 5. Construction of a cocycle of transition functions with values in $G$. Let $\left(U_{\alpha}, u_{\alpha}: U_{\alpha} \rightarrow \mathbb{R}^{m}\right)$ be a locally finite smooth atlas for $M$ such that each $u_{\alpha}: U_{\alpha} \rightarrow \mathbb{R}^{m}$ is surjective. Put $x_{\alpha}:=u_{\alpha}^{-1}(0)$ and choose smooth curves $c_{\alpha}:[0,1] \rightarrow M$ with $c_{\alpha}(0)=x_{0}$ and $c_{\alpha}(1)=x_{\alpha}$. For each $x \in U_{\alpha}$ let $c_{\alpha}^{x}:[0,1] \rightarrow M$ be the smooth curve $t \mapsto u_{\alpha}^{-1}\left(t . u_{\alpha}(x)\right)$; then $c_{\alpha}^{x}$ connects $x_{\alpha}$ and $x$ and the mapping $(x, t) \mapsto c_{\alpha}^{x}(t)$ is smooth $U_{\alpha} \times[0,1] \rightarrow M$. Now we define a fiber bundle atlas $\left(U_{\alpha}, \psi_{\alpha}: E \mid U_{\alpha} \rightarrow U_{\alpha} \times S\right)$ by $\psi_{\alpha}^{-1}(x, s)=$ $\operatorname{Pt}\left(c_{\alpha}^{x}, 1\right) \operatorname{Pt}\left(c_{\alpha}, 1\right) s$. Then $\psi_{\alpha}$ is smooth since $\operatorname{Pt}\left(c_{\alpha}^{x}, 1\right)=\mathrm{Fl}_{1}^{C X_{x}}$ for a local vector field $X_{x}$ depending smoothly on $x$. Let us investigate the transition functions:

$$
\begin{aligned}
\psi_{\alpha} \psi_{\beta}^{-1}(x, s) & =\left(x, \operatorname{Pt}\left(c_{\alpha}, 1\right)^{-1} \operatorname{Pt}\left(c_{\alpha}^{x}, 1\right)^{-1} \operatorname{Pt}\left(c_{\beta}^{x}, 1\right) \operatorname{Pt}\left(c_{\beta}, 1\right) s\right) \\
& =\left(x, \operatorname{Pt}\left(c_{\beta} \cdot c_{\beta}^{x} \cdot\left(c_{\alpha}^{x}\right)^{-1} \cdot\left(c_{\alpha}\right)^{-1}, 4\right) s\right) \\
& =:\left(x, \psi_{\alpha \beta}(x) s\right), \text { where } \psi_{\alpha \beta}: U_{\alpha \beta} \rightarrow G .
\end{aligned}
$$

Clearly $\psi_{\beta \alpha}: U_{\beta \alpha} \times S \rightarrow S$ is smooth, which implies that $\psi_{\beta \alpha}: U_{\beta \alpha} \rightarrow G$ is also smooth. $\left(\psi_{\alpha \beta}\right)$ is a cocycle of transition functions and we use it to glue a principal bundle with structure group $G$ over $M$ which we call $(P, p, M, G)$. 
From its construction it is clear that the associated bundle $P[S]=P \times_{G} S$ equals $(E, p, M, S)$.

Step 6. Lifting the connection $\Phi$ to $P$.

For this we have to compute the Christoffel symbols of $\Phi$ with respect to the atlas of step 5. To do this directly is quite difficult since we have to differentiate the parallel transport with respect to the curve. Fortunately there is another way. Let $c:[0,1] \rightarrow U_{\alpha}$ be a smooth curve. Then we have

$$
\begin{aligned}
\psi_{\alpha} & \left(\operatorname{Pt}(c, t) \psi_{\alpha}^{-1}(c(0), s)\right) \\
& =\left(c(t), \operatorname{Pt}\left(\left(c_{\alpha}\right)^{-1}, 1\right) \operatorname{Pt}\left(\left(c_{\alpha}^{c(0)}\right)^{-1}, 1\right) \operatorname{Pt}(c, t) \operatorname{Pt}\left(c_{\alpha}^{c(0)}, 1\right) \operatorname{Pt}\left(c_{\alpha}, 1\right) s\right) \\
& =(c(t), \gamma(t) . s),
\end{aligned}
$$

where $\gamma$ is a smooth curve in the holonomy group $G$. Let $\Gamma^{\alpha} \in \Omega^{1}\left(U_{\alpha}, \mathfrak{X}(S)\right)$ be the Christoffel symbol of the connection $\Phi$ with respect to the chart $\left(U_{\alpha}, \psi_{\alpha}\right)$. From the third proof of theorem (17.8) we have

$$
\psi_{\alpha}\left(\operatorname{Pt}(c, t) \psi_{\alpha}^{-1}(c(0), s)\right)=(c(t), \bar{\gamma}(t, s)),
$$

where $\bar{\gamma}(t, s)$ is the integral curve through $s$ of the time dependent vector field $\Gamma^{\alpha}\left(\frac{d}{d t} c(t)\right)$ on $S$. But then we get

$$
\begin{aligned}
\Gamma^{\alpha}\left(\frac{d}{d t} c(t)\right)(\bar{\gamma}(t, s)) & =\frac{d}{d t} \bar{\gamma}(t, s)=\frac{d}{d t}(\gamma(t) . s)=\left(\frac{d}{d t} \gamma(t)\right) . s, \\
\Gamma^{\alpha}\left(\frac{d}{d t} c(t)\right) & =\left(\frac{d}{d t} \gamma(t)\right) \circ \gamma(t)^{-1} \in \mathfrak{g} .
\end{aligned}
$$

So $\Gamma^{\alpha}$ takes values in the Lie subalgebra of fundamental vector fields for the action of $G$ on $S$. By theorem (19.9) below the connection $\Phi$ is thus induced by a principal connection $\omega$ on $P$. Since by (19.8) the principal connection $\omega$ has the 'same' holonomy group as $\Phi$ and since this is also the structure group of $P$, the principal connection $\omega$ is irreducible; see (19.7).

\section{Principal Fiber Bundles and $G$-Bundles}

18.1. Definition. Let $G$ be a Lie group and let $(E, p, M, S)$ be a fiber bundle as in (17.1). A $G$-bundle structure on the fiber bundle consists of the following data:

(1) a left action $\ell: G \times S \rightarrow S$ of the Lie group on the standard fiber,

(2) a fiber bundle atlas $\left(U_{\alpha}, \psi_{\alpha}\right)$ whose transition functions $\left(\psi_{\alpha \beta}\right)$ act on $S$ via the $G$-action: There is a family of smooth mappings $\left(\varphi_{\alpha \beta}: U_{\alpha \beta} \rightarrow\right.$ $G)$ which satisfies the cocycle condition $\varphi_{\alpha \beta}(x) \varphi_{\beta \gamma}(x)=\varphi_{\alpha \gamma}(x)$ for $x \in U_{\alpha \beta \gamma}$ and $\varphi_{\alpha \alpha}(x)=e$, the unit in the group, such that $\psi_{\alpha \beta}(x, s)=$ $\ell\left(\varphi_{\alpha \beta}(x), s\right)=\varphi_{\alpha \beta}(x) . s$. 
A fiber bundle with a $G$-bundle structure is called a $G$-bundle. A fiber bundle atlas as in (2) is called a $G$-atlas and the family $\left(\varphi_{\alpha \beta}\right)$ is also called a cocycle of transition functions, but now for the $G$-bundle.

To be more precise, two $G$-atlases are said to be equivalent (to describe the same $G$-bundle) if their union is also a $G$-atlas. This translates as follows to the two cocycles of transition functions, where we assume that the two coverings of $M$ are the same (by passing to the common refinement, if necessary): $\left(\varphi_{\alpha \beta}\right)$ and $\left(\varphi_{\alpha \beta}^{\prime}\right)$ are called cohomologous if there is a family $\left(\tau_{\alpha}: U_{\alpha} \rightarrow G\right)$ such that $\varphi_{\alpha \beta}(x)=\tau_{\alpha}(x)^{-1} \cdot \varphi_{\alpha \beta}^{\prime}(x) \cdot \tau_{\beta}(x)$ holds for all $x \in$ $U_{\alpha \beta}$; compare with (8.3).

In (2) one should specify only an equivalence class of $G$-bundle structures or only a cohomology class of cocycles of $G$-valued transition functions. The proof of (8.3) now shows that from any open cover $\left(U_{\alpha}\right)$ of $M$, some cocycle of transition functions $\left(\varphi_{\alpha \beta}: U_{\alpha \beta} \rightarrow G\right)$ for it, and a left $G$-action on a manifold $S$, we may construct a $G$-bundle, which depends only on the cohomology class of the cocycle. By some abuse of notation we write $(E, p, M, S, G)$ for a fiber bundle with specified $G$-bundle structure.

Examples. The tangent bundle of a manifold $M$ is a fiber bundle with structure group $G L(m)$. More generally, a vector bundle $(E, p, M, V)$ as in (8.1) is a fiber bundle with standard fiber the vector space $V$ and with $G L(V)$-structure.

18.2. Definition. A principal (fiber) bundle $(P, p, M, G)$ is a $G$-bundle with typical fiber a Lie group $G$, where the left action of $G$ on $G$ is just the left translation.

So by (18.1) we are given a bundle atlas $\left(U_{\alpha}, \varphi_{\alpha}: P \mid U_{\alpha} \rightarrow U_{\alpha} \times G\right)$ such that we have $\varphi_{\alpha} \varphi_{\beta}^{-1}(x, a)=\left(x, \varphi_{\alpha \beta}(x) . a\right)$ for the cocycle of transition functions $\left(\varphi_{\alpha \beta}: U_{\alpha \beta} \rightarrow G\right)$. This is now called a principal bundle atlas. Clearly the principal bundle is uniquely specified by the cohomology class of its cocycle of transition functions.

Each principal bundle admits a unique right action $r: P \times G \rightarrow P$, called the principal right action, given by $\varphi_{\alpha}\left(r\left(\varphi_{\alpha}^{-1}(x, a), g\right)\right)=(x, a g)$. Since left and right translation on $G$ commute, this is well defined. As in (6.1) we write $r(u, g)=u . g$ when the meaning is clear. The principal right action is visibly free and for any $u_{x} \in P_{x}$ the partial mapping $r_{u_{x}}=r\left(u_{x}, \quad\right): G \rightarrow P_{x}$ is a diffeomorphism onto the fiber through $u_{x}$, whose inverse is denoted by $\tau_{u_{x}}$ : $P_{x} \rightarrow G$. These inverses together give a smooth mapping $\tau: P \times_{M} P \rightarrow G$, whose local expression is $\tau\left(\varphi_{\alpha}^{-1}(x, a), \varphi_{\alpha}^{-1}(x, b)\right)=a^{-1} . b$. This mapping is also uniquely determined by the implicit equation $r\left(u_{x}, \tau\left(u_{x}, v_{x}\right)\right)=v_{x}$; thus we also have $\tau\left(u_{x} . g, u_{x}^{\prime} . g^{\prime}\right)=g^{-1} \cdot \tau\left(u_{x}, u_{x}^{\prime}\right) \cdot g^{\prime}$ and $\tau\left(u_{x}, u_{x}\right)=e$. 
When considering principal bundles, the reader should think of frame bundles as the foremost examples for this book. They will be treated in (18.11) below.

18.3. Lemma. Let $p: P \rightarrow M$ be a surjective submersion (a fibered manifold), and let $G$ be a Lie group which acts freely on $P$ such that the orbits of the action are exactly the fibers $p^{-1}(x)$ of $p$. Then $(P, p, M, G)$ is a principal fiber bundle.

Proof. Let the action be a right one by using the group inversion if necessary. Let $s_{\alpha}: U_{\alpha} \rightarrow P$ be local sections (right inverses) for $p: P \rightarrow M$ such that $\left(U_{\alpha}\right)$ is an open cover of $M$. Let $\varphi_{\alpha}^{-1}: U_{\alpha} \times G \rightarrow P \mid U_{\alpha}$ be given by $\varphi_{\alpha}^{-1}(x, a)=s_{\alpha}(x) . a$, which is obviously injective with invertible tangent mapping, so its inverse $\varphi_{\alpha}: P \mid U_{\alpha} \rightarrow U_{\alpha} \times G$ is a fiber respecting diffeomorphism. So $\left(U_{\alpha}, \varphi_{\alpha}\right)$ is already a fiber bundle atlas. Let $\tau: P \times_{M} P \rightarrow G$ be given by the implicit equation $r\left(u_{x}, \tau\left(u_{x}, u_{x}^{\prime}\right)\right)=u_{x}^{\prime}$, where $r$ is the right $G$-action. The mapping $\tau$ is smooth by the implicit function theorem and clearly we have

$$
\tau\left(u_{x}, u_{x}^{\prime} \cdot g\right)=\tau\left(u_{x}, u_{x}^{\prime}\right) \cdot g \quad \text { and } \quad \varphi_{\alpha}\left(u_{x}\right)=\left(x, \tau\left(s_{\alpha}(x), u_{x}\right)\right) .
$$

Thus we have

$$
\begin{aligned}
\varphi_{\alpha} \varphi_{\beta}^{-1}(x, g) & =\varphi_{\alpha}\left(s_{\beta}(x) . g\right)=\left(x, \tau\left(s_{\alpha}(x), s_{\beta}(x) . g\right)\right) \\
& =\left(x, \tau\left(s_{\alpha}(x), s_{\beta}(x)\right) \cdot g\right)
\end{aligned}
$$

and $\left(U_{\alpha}, \varphi_{\alpha}\right)$ is a principal bundle atlas.

18.4. Remarks. In the proof of lemma (18.3) we have seen that a principal bundle atlas of a principal fiber bundle $(P, p, M, G)$ is already determined if we specify a family of smooth sections of $P$ whose domains of definition cover the base $M$.

Lemma (18.3) can serve as an equivalent definition for a principal bundle. But this is true only if an implicit function theorem is available, so in topology or in infinite-dimensional differential geometry one should stick to our original definition.

From lemma (18.3) itself it follows that the pullback $f^{*} P$ over a smooth mapping $f: M^{\prime} \rightarrow M$ is again a principal fiber bundle.

18.5. Homogeneous spaces. Let $G$ be a Lie group with Lie algebra $\mathfrak{g}$. Let $K$ be a closed subgroup of $G$; then by theorem (5.5), $K$ is a closed Lie subgroup whose Lie algebra will be denoted by k. By theorem (5.11) there is a unique structure of a smooth manifold on the quotient space $G / K$ such that the projection $p: G \rightarrow G / K$ is a submersion, so by the implicit function theorem $p$ admits local sections. 
Theorem. $(G, p, G / K, K)$ is a principal fiber bundle.

Proof. The group multiplication of $G$ restricts to a free right action $\mu$ : $G \times K \rightarrow G$ whose orbits are exactly the fibers of $p$. By lemma (18.3) the result follows.

For the convenience of the reader we discuss now the best known homogeneous spaces.

The group $S O(n)$ acts transitively on $S^{n-1} \subset \mathbb{R}^{n}$. The isotropy group of the 'north pole' $(1,0, \ldots, 0)$ is the subgroup

$$
\left(\begin{array}{cc}
1 & 0 \\
0 & S O(n-1)
\end{array}\right)
$$

which we identify with $S O(n-1)$. So

$$
S^{n-1}=S O(n) / S O(n-1)
$$

and we get a principal fiber bundle

$$
\left(S O(n), p, S^{n-1}, S O(n-1)\right) .
$$

Likewise the follwing are principal fiber bundles:

$$
\begin{aligned}
& \left(O(n), p, S^{n-1}, O(n-1)\right), \\
& \left(S U(n), p, S^{2 n-1}, S U(n-1)\right), \\
& \left(U(n), p, S^{2 n-1}, U(n-1)\right), \\
& \left(S p(n), p, S^{4 n-1}, S p(n-1)\right) .
\end{aligned}
$$

The Grassmann manifold $G(k, n ; \mathbb{R})$ is the space of all $k$-planes containing 0 in $\mathbb{R}^{n}$. The group $O(n)$ acts transitively on it and the isotropy group of the $k$-plane $\mathbb{R}^{k} \times\{0\}$ is the subgroup

$$
\left(\begin{array}{cc}
O(k) & 0 \\
0 & O(n-k)
\end{array}\right)
$$

therefore

$$
G(k, n ; \mathbb{R})=O(n) / O(k) \times O(n-k)
$$

is a compact manifold and we get the principal fiber bundle

$$
(O(n), p, G(k, n ; \mathbb{R}), O(k) \times O(n-k)) .
$$

Likewise the follwing are principal fiber bundles:

$$
\begin{aligned}
& (S O(n), p, G(k, n ; \mathbb{R}), S(O(k) \times O(n-k))), \\
& (S O(n), p, \tilde{G}(k, n ; \mathbb{R}), S O(k) \times S O(n-k)), \\
& (U(n), p, G(k, n ; \mathbb{C}), U(k) \times U(n-k)), \\
& (S p(n), p, G(k, n ; \mathbb{H}), S p(k) \times S p(n-k)) .
\end{aligned}
$$


The Stiefel manifold $V(k, n ; \mathbb{R})$ is the space of all orthonormal $k$-frames in $\mathbb{R}^{n}$. Clearly the group $O(n)$ acts transitively on $V(k, n ; \mathbb{R})$ and the isotropy subgroup of $\left(e_{1}, \ldots, e_{k}\right)$ is $\mathbb{I}_{k} \times O(n-k)$, so

$$
V(k, n ; \mathbb{R})=O(n) / O(n-k)
$$

is a compact manifold, and

$$
(O(n), p, V(k, n ; \mathbb{R}), O(n-k))
$$

is a principal fiber bundle. But $O(k)$ also acts from the right on $V(k, n ; \mathbb{R})$; its orbits are exactly the fibers of the projection $p: V(k, n ; \mathbb{R}) \rightarrow G(k, n ; \mathbb{R})$. So by lemma (18.3) we get a principal fiber bundle

$$
(V(k, n, \mathbb{R}), p, G(k, n ; \mathbb{R}), O(k)) .
$$

Indeed we have the following diagram where all arrows are projections of principal fiber bundles and where the respective structure groups are written on the arrows:

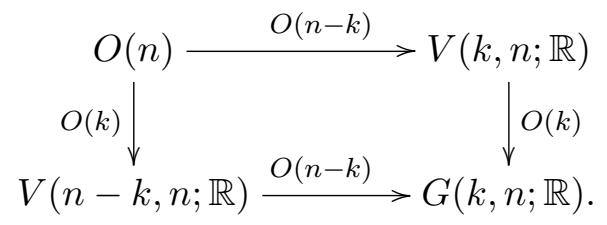

The Stiefel manifold $V(k, n ; \mathbb{R})$ is also diffeomorphic to the space $\{A \in$ $\left.L\left(\mathbb{R}^{k}, \mathbb{R}^{n}\right): A^{\top} \cdot A=\mathbb{I}_{k}\right\}$, i.e., the space of all linear isometries $\mathbb{R}^{k} \rightarrow \mathbb{R}^{n}$. There are furthermore complex and quaternionic versions of Stiefel manifolds and flag manifolds.

18.6. Homomorphisms. Let $\chi:(P, p, M, G) \rightarrow\left(P^{\prime}, p^{\prime}, M^{\prime}, G\right)$ be a principal fiber bundle homomorphism, i.e., a smooth $G$-equivariant mapping $\chi: P \rightarrow P^{\prime}$. Then obviously the diagram

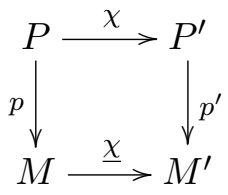

commutes for a uniquely determined smooth mapping $\underline{\chi}: M \rightarrow M^{\prime}$. For each $x \in M$ the mapping $\chi_{x}:=\chi \mid P_{x}: P_{x} \rightarrow P_{\bar{\chi}(x)}^{\prime}$ is $G$-equivariant and therefore a diffeomorphism, so diagram (1) is a pullback diagram.

But the most general notion of a homomorphism of principal bundles is the following. Let $\Phi: G \rightarrow G^{\prime}$ be a homomorphism of Lie groups. A mapping $\chi:(P, p, M, G) \rightarrow\left(P^{\prime}, p^{\prime}, M^{\prime}, G^{\prime}\right)$ is called a homomorphism over $\Phi$ of principal bundles if $\chi: P \rightarrow P^{\prime}$ is smooth and $\chi(u . g)=\chi(u) . \Phi(g)$ holds 
in general. Then $\chi$ is fiber respecting, so diagram (11) again makes sense, but it is no longer a pullback diagram in general.

If $\chi$ covers the identity on the base, it is called a reduction of the structure group $G^{\prime}$ to $G$ for the principal bundle $\left(P^{\prime}, p^{\prime}, M^{\prime}, G^{\prime}\right)$ - the name comes from the case when $\Phi$ is the embedding of a subgroup.

By the universal property of the pullback any general homomorphism $\chi$ of principal fiber bundles over a group homomorphism can be written as the composition of a reduction of structure groups and a pullback homomorphism as follows, where we also indicate the structure groups:

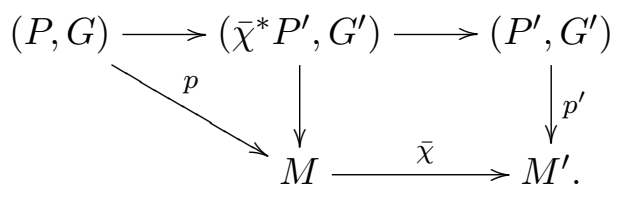

18.7. Associated bundles. Let $(P, p, M, G)$ be a principal bundle and let $\ell: G \times S \rightarrow S$ be a left action of the structure group $G$ on a manifold $S$. We consider the right action $R:(P \times S) \times G \rightarrow P \times S$, given by $R((u, s), g)=\left(u . g, g^{-1} . s\right)$.

Theorem. In this situation we have:

(1) The space $P \times_{G} S$ of orbits of the action $R$ carries a unique smooth manifold structure such that the quotient map $q: P \times S \rightarrow P \times{ }_{G} S$ is a submersion.

(2) $\left(P \times_{G} S, \bar{p}, M, S, G\right)$ is a $G$-bundle in a canonical way, where $\bar{p}: P \times_{G}$ $S \rightarrow M$ is given as in the following diagram, where $q_{u}:\{u\} \times S \rightarrow$ $\left(P \times_{G} S\right)_{p(u)}$ is a diffeomorphism for each $u \in P$ :

(a)

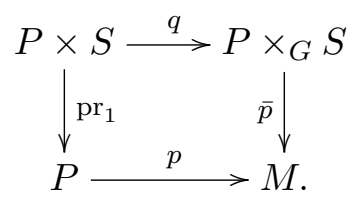

(3) $\left(P \times S, q, P \times_{G} S, G\right)$ is a principal fiber bundle with principal action $R$.

(4) If $\left(U_{\alpha}, \varphi_{\alpha}: P \mid U_{\alpha} \rightarrow U_{\alpha} \times G\right)$ is a principal bundle atlas with cocycle of transition functions $\left(\varphi_{\alpha \beta}: U_{\alpha \beta} \rightarrow G\right)$, then together with the left action $\ell: G \times S \rightarrow S$ this cocycle is also one for the $G$-bundle $\left(P \times_{G}\right.$ $S, \bar{p}, M, S, G)$.

Notation. $\left(P \times{ }_{G} S, \bar{p}, M, S, G\right)$ is called the associated bundle for the action $\ell: G \times S \rightarrow S$. We will also denote it by $P[S, \ell]$ or simply $P[S]$ and we will write $p$ for $\bar{p}$ if no confusion is possible. We also define the smooth 
mapping $\tau=\tau^{S}: P \times_{M} P[S, \ell] \rightarrow S$ by $\tau\left(u_{x}, v_{x}\right):=q_{u_{x}}^{-1}\left(v_{x}\right)$ which satisfies $\tau(u, q(u, s))=s, q\left(u_{x}, \tau\left(u_{x}, v_{x}\right)\right)=v_{x}$, and $\tau\left(u_{x} . g, v_{x}\right)=g^{-1} \cdot \tau\left(u_{x}, v_{x}\right)$. In the special situation where $S=G$ and the action is left translation, so that $P[G]=P$, this mapping coincides with $\tau$ considered in $(18.2)$.

Proof. In the setting of diagram (a) in (2) the mapping $p \circ \operatorname{pr}_{1}$ is constant on the $R$-orbits, so $\bar{p}$ exists as a mapping. Let $\left(U_{\alpha}, \varphi_{\alpha}: P \mid U_{\alpha} \rightarrow U_{\alpha} \times G\right)$ be a principal bundle atlas with transition functions $\left(\varphi_{\alpha \beta}: U_{\alpha \beta} \rightarrow G\right)$. We define $\psi_{\alpha}^{-1}: U_{\alpha} \times S \rightarrow \bar{p}^{-1}\left(U_{\alpha}\right) \subset P \times_{G} S$ by $\psi_{\alpha}^{-1}(x, s)=q\left(\varphi_{\alpha}^{-1}(x, e), s\right)$, which is fiber respecting. For each point in $\bar{p}^{-1}(x) \subset P \times{ }_{G} S$ there is exactly one $s \in S$ such that the orbit corresponding to this point passes through $\left(\varphi_{\alpha}^{-1}(x, e), s\right)$, namely $s=\tau^{G}\left(u_{x}, \varphi_{\alpha}^{-1}(x, e)\right)^{-1} \cdot s^{\prime}$ if $\left(u_{x}, s^{\prime}\right)$ is the orbit, since the principal right action is free. Thus $\psi_{\alpha}^{-1}(x, \quad): S \rightarrow \bar{p}^{-1}(x)$ is bijective.

Furthermore

$$
\begin{aligned}
\psi_{\beta}^{-1}(x, s) & =q\left(\varphi_{\beta}^{-1}(x, e), s\right) \\
& =q\left(\varphi_{\alpha}^{-1}\left(x, \varphi_{\alpha \beta}(x) \cdot e\right), s\right)=q\left(\varphi_{\alpha}^{-1}(x, e) \cdot \varphi_{\alpha \beta}(x), s\right) \\
& =q\left(\varphi_{\alpha}^{-1}(x, e), \varphi_{\alpha \beta}(x) . s\right)=\psi_{\alpha}^{-1}\left(x, \varphi_{\alpha \beta}(x) . s\right),
\end{aligned}
$$

so $\psi_{\alpha} \psi_{\beta}^{-1}(x, s)=\left(x, \varphi_{\alpha \beta}(x) . s\right)$. So $\left(U_{\alpha}, \psi_{\alpha}\right)$ is a $G$-atlas for $P \times_{G} S$ and makes it into a smooth manifold and a $G$-bundle. The defining equation for $\psi_{\alpha}$ shows that $q$ is smooth and a submersion and consequently the smooth structure on $P \times_{G} S$ is uniquely defined, and $\bar{p}$ is smooth by the universal properties of a submersion.

By the definition of $\psi_{\alpha}$ the diagram

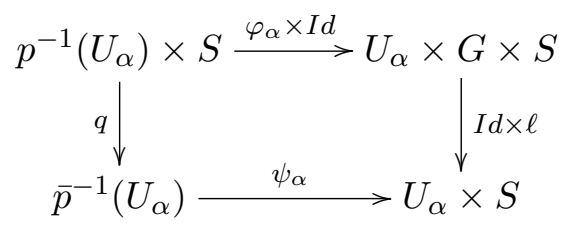

commutes; since its lines are diffeomorphisms, we conclude that $q_{u}:\{u\} \times$ $S \rightarrow \bar{p}^{-1}(p(u))$ is a diffeomorphism. So (1), (2), and (4) are checked.

(3) follows directly from lemma (18.3), We give below an explicit chart construction. We rewrite the last diagram in the following form:

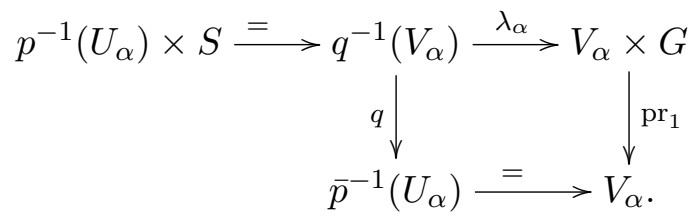


Here $V_{\alpha}:=\bar{p}^{-1}\left(U_{\alpha}\right) \subset P \times_{G} S$, and the diffeomorphism $\lambda_{\alpha}$ is given by the expression $\lambda_{\alpha}^{-1}\left(\psi_{\alpha}^{-1}(x, s), g\right):=\left(\varphi_{\alpha}^{-1}(x, g), g^{-1} . s\right)$. Then we have

$$
\begin{aligned}
\lambda_{\beta}^{-1}\left(\psi_{\alpha}^{-1}(x, s), g\right) & =\lambda_{\beta}^{-1}\left(\psi_{\beta}^{-1}\left(x, \varphi_{\beta \alpha}(x) . s\right), g\right) \\
& =\left(\varphi_{\beta}^{-1}(x, g), g^{-1} \cdot \varphi_{\beta \alpha}(x) \cdot s\right) \\
& =\left(\varphi_{\alpha}^{-1}\left(x, \varphi_{\alpha \beta}(x) \cdot g\right), g^{-1} \cdot \varphi_{\alpha \beta}(x)^{-1} \cdot s\right) \\
& =\lambda_{\alpha}^{-1}\left(\psi_{\alpha}^{-1}(x, s), \varphi_{\alpha \beta}(x) \cdot g\right),
\end{aligned}
$$

so $\lambda_{\alpha} \lambda_{\beta}^{-1}\left(\psi_{\alpha}^{-1}(x, s), g\right)=\left(\psi_{\alpha}^{-1}(x, s), \varphi_{\alpha \beta}(x) . g\right)$ and $\left(P \times S, q, P \times_{G} S, G\right)$ is a principal bundle with structure group $G$ and the same cocycle $\left(\varphi_{\alpha \beta}\right)$ we started with.

18.8. Corollary. Let $(E, p, M, S, G)$ be a $G$-bundle, specified by a cocycle of transition functions $\left(\varphi_{\alpha \beta}\right)$ with values in $G$ and a left action $\ell$ of $G$ on $S$. Then from the cocycle of transition functions we may glue a unique principal bundle $(P, p, M, G)$ such that $E=P[S, \ell]$.

This is the usual way a differential geometer thinks of an associated bundle. He is given a bundle $E$ and a principal bundle $P$, and the $G$-bundle structure then is described with the help of the mappings $\tau$ and $q$.

\subsection{Equivariant mappings and associated bundles.}

(1) Let $(P, p, M, G)$ be a principal fiber bundle and consider two left actions of $G, \ell: G \times S \rightarrow S$ and $\ell^{\prime}: G \times S^{\prime} \rightarrow S^{\prime}$. Let furthermore $f: S \rightarrow S^{\prime}$ be a $G$-equivariant smooth mapping, so $f(g . s)=g . f(s)$ or $f \circ \ell_{g}=\ell_{g}^{\prime} \circ f$. Then $I d_{P} \times f: P \times S \rightarrow P \times S^{\prime}$ is equivariant for the actions $R:(P \times S) \times G \rightarrow P \times S$ and $R^{\prime}:\left(P \times S^{\prime}\right) \times G \rightarrow P \times S^{\prime}$ and is thus a homomorphism of principal bundles, so there is an induced mapping

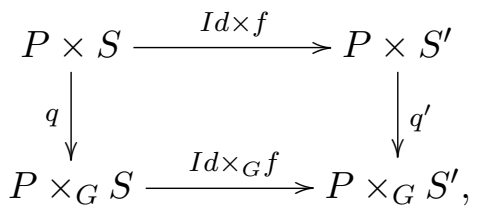

which is fiber respecting over $M$, and a homomorphism of $G$-bundles in the sense of the definition (18.10) below.

(3) Let $\chi:(P, p, M, G) \rightarrow\left(P^{\prime}, p^{\prime}, M^{\prime}, G\right)$ be a principal fiber bundle homomorphism as in (18.6). Furthermore we consider a smooth left action $\ell: G \times S \rightarrow S$. Then $\chi \times I d_{S}: P \times S \rightarrow P^{\prime} \times S$ is $G$-equivariant and induces a mapping $\chi \times_{G} I d_{S}: P \times_{G} S \rightarrow P^{\prime} \times_{G} S$, which is fiber respecting over $M$, fiberwise a diffeomorphism, and again a homomorphism of $G$-bundles in the sense of definition (18.10) below. 
(4) We consider the situation of (11) and (2) at the same time. Given two associated bundles $P[S, \ell]$ and $P^{\prime}\left[S^{\prime}, \ell^{\prime}\right]$, let

$$
\chi:(P, p, M, G) \rightarrow\left(P^{\prime}, p^{\prime}, M^{\prime}, G\right)
$$

be a principal fiber bundle homomorphism and let $f: S \rightarrow S^{\prime}$ be a $G$ equivariant mapping. Then $\chi \times f: P \times S \rightarrow P^{\prime} \times S^{\prime}$ is clearly $G$-equivariant and therefore induces a mapping

$$
\chi \times_{G} f: P[S, \ell] \rightarrow P^{\prime}\left[S^{\prime}, \ell^{\prime}\right]
$$

which again is a homomorphism of $G$-bundles.

(5) Let $S$ be a point. Then $P[S]=P \times_{G} S=M$. Furthermore let $y \in S^{\prime}$ be a fixed point of the action $\ell^{\prime}: G \times S^{\prime} \rightarrow S^{\prime}$; then the inclusion $i:\{y\} \hookrightarrow S^{\prime}$ is $G$-equivariant. Thus $I d_{P} \times i$ induces

$$
I d_{P} \times_{G} i: M=P[\{y\}] \rightarrow P\left[S^{\prime}\right],
$$

which is a global section of the associated bundle $P\left[S^{\prime}\right]$.

If the action of $G$ on $S$ is trivial, so $g . s=s$ for all $s \in S$, then the associated bundle is trivial: $P[S]=M \times S$. For a trivial principal fiber bundle any associated bundle is trivial.

18.10. Definition. In the situation of (18.9), a smooth fiber respecting mapping $\gamma: P[S, \ell] \rightarrow P^{\prime}\left[S^{\prime}, \ell^{\prime}\right]$ covering a smooth mapping $\bar{\gamma}: M \rightarrow$ $M^{\prime}$ of the bases is called a homomorphism of G-bundles if the following conditions are satisfied: $P$ is isomorphic to the pullback $\bar{\gamma}^{*} P^{\prime}$, and the local representations of $\gamma$ in pullback-related fiber bundle atlases belonging to the two $G$-bundles are fiberwise $G$-equivariant.

Let us describe this in more detail now. Let $\left(U_{\alpha}^{\prime}, \psi_{\alpha}^{\prime}\right)$ be a $G$-atlas for $P^{\prime}\left[S^{\prime}, \ell^{\prime}\right]$ with cocycle of transition functions $\left(\varphi_{\alpha \beta}^{\prime}\right)$, belonging to the principal fiber bundle atlas $\left(U_{\alpha}^{\prime}, \varphi_{\alpha}^{\prime}\right)$ of $\left(P^{\prime}, p^{\prime}, M^{\prime}, G\right)$. Then the pullback-related principal fiber bundle atlas $\left(U_{\alpha}=\bar{\gamma}^{-1}\left(U_{\alpha}^{\prime}\right), \varphi_{\alpha}\right)$ for $P=\bar{\gamma}^{*} P^{\prime}$ as described in the proof of (17.5) has the cocycle of transition functions $\left(\varphi_{\alpha \beta}=\varphi_{\alpha \beta}^{\prime} \circ \bar{\gamma}\right)$; it induces the $G$-atlas $\left(U_{\alpha}, \psi_{\alpha}\right)$ for $P[S, \ell]$. Then $\left(\psi_{\alpha}^{\prime} \circ \gamma \circ \psi_{\alpha}^{-1}\right)(x, s)=$ $\left(\bar{\gamma}(x), \gamma_{\alpha}(x, s)\right)$ and $\gamma_{\alpha}(x, \quad): S \rightarrow S^{\prime}$ is required to be $G$-equivariant for all $\alpha$ and all $x \in U_{\alpha}$.

Lemma. Let $\gamma: P[S, \ell] \rightarrow P^{\prime}\left[S^{\prime}, \ell^{\prime}\right]$ be a homomorphism of $G$-bundles as in (18.9), Then there is a homomorphism

$$
\chi:(P, p, M, G) \rightarrow\left(P^{\prime}, p^{\prime}, M^{\prime}, G\right)
$$

of principal bundles and a $G$-equivariant mapping $f: S \rightarrow S^{\prime}$ such that

$$
\gamma=\chi \times_{G} f: P[S, \ell] \rightarrow P^{\prime}\left[S^{\prime}, \ell^{\prime}\right] .
$$


Proof. The homomorphism $\chi:(P, p, M, G) \rightarrow\left(P^{\prime}, p^{\prime}, M^{\prime}, G\right)$ of principal fiber bundles is already determined by the requirement that $P=\bar{\gamma}^{*} P^{\prime}$, and we have $\bar{\gamma}=\bar{\chi}$. The $G$-equivariant mapping $f: S \rightarrow S^{\prime}$ can be read off the following diagram:

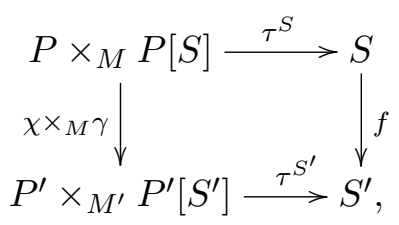

which by the assumptions is seen to be well defined in the right column.

So a homomorphism of $G$-bundles is described by the whole triple $(\chi: P \rightarrow$ $P^{\prime}, f: S \rightarrow S^{\prime}$ (G-equivariant), $\left.\gamma: P[S] \rightarrow P^{\prime}\left[S^{\prime}\right]\right)$, such that diagram (11) commutes.

18.11. Associated vector bundles. Let $(P, p, M, G)$ be a principal fiber bundle, and consider a representation $\rho: G \rightarrow G L(V)$ of $G$ on a finitedimensional vector space $V$. Then $P[V, \rho]$ is an associated fiber bundle with structure group $G$, but also with structure group $G L(V)$, for in the canonically associated fiber bundle atlas the transition functions also have values in $G L(V)$. So by section $[(8), P[V, \rho]$ is a vector bundle.

Now let $\mathcal{F}$ be a covariant smooth functor from the category of finite-dimensional vector spaces and linear mappings into itself, as considered in section (8.8). Then clearly $\mathcal{F} \circ \rho: G \rightarrow G L(V) \rightarrow G L(\mathcal{F}(V))$ is another representation of $G$ and the associated bundle $P[\mathcal{F}(V), \mathcal{F} \circ \rho]$ coincides with the vector bundle $\mathcal{F}(P[V, \rho])$ constructed with the method of $(8.8)$, but now it has an extra $G$-bundle structure. For contravariant functors $\mathcal{F}$ we have to consider the representation $\mathcal{F} \circ \rho \circ \nu$, where $\nu(g)=g^{-1}$. A similar choice works for bifunctors. In particular the bifunctor $L(V, W)$ may be applied to two different representations of two structure groups of two principal bundles over the same base $M$ to construct a vector bundle

$$
L\left(P[V, \rho], P^{\prime}\left[V^{\prime}, \rho^{\prime}\right]\right)=\left(P \times_{M} P^{\prime}\right)\left[L\left(V, V^{\prime}\right), L \circ\left((\rho \circ \nu) \times \rho^{\prime}\right)\right] .
$$

If $(E, p, M)$ is a vector bundle with $n$-dimensional fibers, we may consider the open subset $G L\left(\mathbb{R}^{n}, E\right) \subset L\left(M \times \mathbb{R}^{n}, E\right)$, a fiber bundle over the base $M$, whose fiber over $x \in M$ is the space $G L\left(\mathbb{R}^{n}, E_{x}\right)$ of all invertible linear mappings. Composition from the right by elements of $G L(n)$ gives a free right action on $G L\left(\mathbb{R}^{n}, E\right)$ whose orbits are exactly the fibers, so by lemma $(18.3)$ we have a principal fiber bundle $\left(G L\left(\mathbb{R}^{n}, E\right), p, M, G L(n)\right)$. The associated bundle $G L\left(\mathbb{R}^{n}, E\right)\left[\mathbb{R}^{n}\right]$ for the banal representation of $G L(n)$ on $\mathbb{R}^{n}$ is isomorphic to the vector bundle $(E, p, M)$ we started with, for the evaluation mapping $e v: G L\left(\mathbb{R}^{n}, E\right) \times \mathbb{R}^{n} \rightarrow E$ is invariant under the right 
action $R$ of $G L(n)$, and locally in the image there are smooth sections to it, so it factors to a fiber linear diffeomorphism

$$
G L\left(\mathbb{R}^{n}, E\right)\left[\mathbb{R}^{n}\right]=G L\left(\mathbb{R}^{n}, E\right) \times_{G L(n)} \mathbb{R}^{n} \rightarrow E .
$$

The principal bundle $G L\left(\mathbb{R}^{n}, E\right)$ is called the linear frame bundle of $E$. Note that local sections of $G L\left(\mathbb{R}^{n}, E\right)$ are exactly the local frame fields of the vector bundle $E$ as discussed in (8.5),

To illustrate the notion of reduction of structure groups, we consider now a vector bundle $\left(E, p, M, \mathbb{R}^{n}\right)$ equipped with a Riemann metric $g$, that is, a section $g \in C^{\infty}\left(S^{2} E^{*}\right)$ such that $g_{x}$ is a positive definite inner product on $E_{x}$ for each $x \in M$. Any vector bundle admits Riemann metrics: local existence is clear and we may glue with the help of a partition of unity on $M$, since the positive definite sections form an open convex subset. Now let

$$
s^{\prime}=\left(s_{1}^{\prime}, \ldots, s_{n}^{\prime}\right) \in C^{\infty}\left(G L\left(\mathbb{R}^{n}, E\right) \mid U\right)
$$

be a local frame field of the bundle $E$ over $U \subset M$. Now we may apply the Gram-Schmidt orthonormalization procedure to the basis $\left(s_{1}(x), \ldots, s_{n}(x)\right)$ of $E_{x}$ for each $x \in U$. Since this procedure is smooth (even real analytic), we obtain a frame field $s=\left(s_{1}, \ldots, s_{n}\right)$ of $E$ over $U$ which is orthonormal with respect to $g$. We call it an orthonormal frame field. Now let $\left(U_{\alpha}\right)$ be an open cover of $M$ with orthonormal frame fields $s^{\alpha}=\left(s_{1}^{\alpha}, \ldots, s_{n}^{\alpha}\right)$, where $s^{\alpha}$ is defined on $U_{\alpha}$. We consider the vector bundle charts

$$
\left(U_{\alpha}, \psi_{\alpha}: E \mid U_{\alpha} \rightarrow U_{\alpha} \times \mathbb{R}^{n}\right)
$$

given by the orthonormal frame fields:

$$
\psi_{\alpha}^{-1}\left(x, v^{1}, \ldots, v^{n}\right)=\sum s_{i}^{\alpha}(x) \cdot v^{i}=: s^{\alpha}(x) \cdot v .
$$

For $x \in U_{\alpha \beta}$ we have $s_{i}^{\alpha}(x)=\sum s_{j}^{\beta}(x) \cdot g_{\beta \alpha}{ }_{i}^{j}(x)$ for $C^{\infty}$-functions $g_{\alpha \beta}{ }_{i}^{j}$ : $U_{\alpha \beta} \rightarrow \mathbb{R}$. Since $s^{\alpha}(x)$ and $s^{\beta}(x)$ are both orthonormal bases of $E_{x}$, the matrix $g_{\alpha \beta}(x)=\left(g_{\alpha \beta}{ }_{i}^{j}(x)\right)$ is an element of $O(n, \mathbb{R})$. We write $s^{\alpha}=s^{\beta} \cdot g_{\beta \alpha}$ for short. Then we have

$$
\psi_{\beta}^{-1}(x, v)=s^{\beta}(x) \cdot v=s^{\alpha}(x) \cdot g_{\alpha \beta}(x) \cdot v=\psi_{\alpha}^{-1}\left(x, g_{\alpha \beta}(x) \cdot v\right)
$$

and consequently $\psi_{\alpha} \psi_{\beta}^{-1}(x, v)=\left(x, g_{\alpha \beta}(x) . v\right)$. So the $\left(g_{\alpha \beta}: U_{\alpha \beta} \rightarrow O(n, \mathbb{R})\right)$ are the cocycle of transition functions for the vector bundle atlas $\left(U_{\alpha}, \psi_{\alpha}\right)$. So we have constructed an $O(n, \mathbb{R})$-structure on $E$. The corresponding principal fiber bundle will be denoted by $O\left(\mathbb{R}^{n},(E, g)\right)$; it is usually called the orthonormal frame bundle of $E$. It is derived from the linear frame bundle $G L\left(\mathbb{R}^{n}, E\right)$ by reduction of the structure group from $G L(n)$ to $O(n)$. The phenomenon discussed here plays a prominent role in the theory of classifying spaces. 
18.12. Sections of associated bundles. Let $(P, p, M, G)$ be a principal fiber bundle and $\ell: G \times S \rightarrow S$ a left action. Let $C^{\infty}(P, S)^{G}$ denote the space of all smooth mappings $f: P \rightarrow S$ which are $G$-equivariant in the sense that $f(u . g)=g^{-1} . f(u)$ holds for $g \in G$ and $u \in P$.

Theorem. The sections of the associated bundle $P[S, \ell]$ correspond exactly to the $G$-equivariant mappings $P \rightarrow S$; we have a bijection

$$
C^{\infty}(P, S)^{G} \cong \Gamma(P[S])
$$

This result follows from (18.9) and (18.10). Since it is very important, we include a direct proof.

Proof. If $f \in C^{\infty}(P, S)^{G}$, we construct $s_{f} \in \Gamma(P[S])$ in the following way: The mapping graph $(f)=(I d, f): P \rightarrow P \times S$ is $G$-equivariant, since $(I d, f)(u . g)=(u . g, f(u . g))=\left(u . g, g^{-1} \cdot f(u)\right)=((I d, f)(u)) . g$. So it induces a smooth section $s_{f} \in \Gamma(P[S])$ as seen from (18.9) and the diagram:

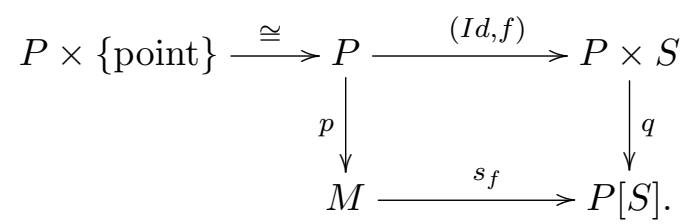

For $s \in \Gamma(P[S])$ we define $f_{s} \in C^{\infty}(P, S)^{G}$ by

$$
f_{s}:=\tau^{S} \circ\left(I d_{P} \times_{M} s\right): P=P \times_{M} M \rightarrow P \times_{M} P[S] \rightarrow S .
$$

This is $G$-equivariant since we have by (18.7)

$$
f_{s}\left(u_{x} \cdot g\right)=\tau^{S}\left(u_{x} . g, s(x)\right)=g^{-1} \cdot \tau^{S}\left(u_{x}, s(x)\right)=g^{-1} \cdot f_{s}\left(u_{x}\right) .
$$

These constructions are inverse to each other since we have

$$
\begin{gathered}
f_{s(f)}(u)=\tau^{S}\left(u, s_{f}(p(u))\right)=\tau^{S}(u, q(u, f(u)))=f(u), \\
s_{f(s)}(p(u))=q\left(u, f_{s}(u)\right)=q\left(u, \tau^{S}(u, s(p(u)))\right)=s(p(u)) .
\end{gathered}
$$

18.13. Induced representations. Let $K$ be a closed subgroup of a Lie group $G$. Let $\rho: K \rightarrow G L(V)$ be a representation in a vector space $V$, which we assume to be finite-dimensional to begin with. Then we consider the principal fiber bundle $(G, p, G / K, K)$ and the associated vector bundle $(G[V], p, G / K)$. The smooth (or even continuous) sections of $G[V]$ correspond exactly to the $K$-equivariant mappings $f: G \rightarrow V$, those satisfying $f(g k)=\rho\left(k^{-1}\right) f(g)$, by lemma (18.12), Each $g \in G$ acts as a principal 
bundle homomorphism by left translation:

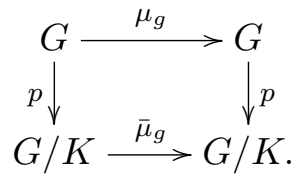

So by (18.9) we have an induced isomorphism of vector bundles

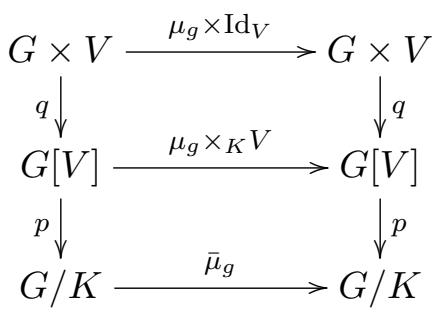

which gives rise to the representation $\widetilde{\operatorname{ind}}_{K}^{G} \rho$ of $G$ in the space $\Gamma(G[V])$, defined by

$$
\left.\widetilde{\operatorname{ind}}_{K}^{G} \rho\right)(g)(s):=\left(\mu_{g} \times_{K} V\right) \circ s \circ \bar{\mu}_{g^{-1}}=\left(\mu_{g} \times_{K} V\right)_{*}(s) .
$$

Now let us assume that the original representation $\rho$ is unitary, $\rho: K \rightarrow$ $U(V)$ for a complex vector space $V$ with inner product $\langle\quad, \quad\rangle_{V}$. Then $v \mapsto$ $\|v\|^{2}=\langle v, v\rangle$ is an invariant symmetric homogeneous polynomial $V \rightarrow \mathbb{R}$ of degree 2 , so it is equivariant where $K$ acts trivially on $\mathbb{R}$. By (18.9) again we get an induced mapping $G[V] \rightarrow G[\mathbb{R}]=G / K \times \mathbb{R}$, which we can polarize to a smooth fiberwise Hermitian form $\langle,\rangle_{G[V]}$ on the vector bundle $G[V]$. We may also express this by

$$
\begin{aligned}
\left\langle v_{x}, w_{x}\right\rangle_{G[V]} & =\left\langle\tau^{V}\left(u_{x}, v_{x}\right), \tau^{V}\left(u_{x}, w_{x}\right)\right\rangle_{V} \\
& =\left\langle k^{-1} \tau^{V}\left(u_{x}, v_{x}\right), k^{-1} \tau^{V}\left(u_{x}, w_{x}\right)\right\rangle_{V} \\
& =\left\langle\tau^{V}\left(u_{x} . k, v_{x}\right), \tau^{V}\left(u_{x} . k, w_{x}\right)\right\rangle_{V}
\end{aligned}
$$

for some $u_{x} \in G_{x}$, using the mapping $\tau^{V}: G \times_{G / M} G[V] \rightarrow V$ from (18.7) it does not depend on the choice of $u_{x}$. Still another way to describe the fiberwise Hermitian form is

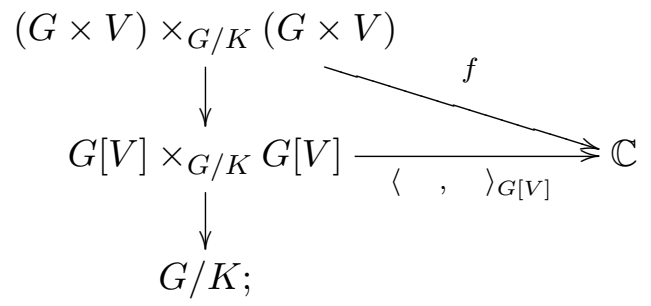

here $f\left(\left(g_{1}, v_{1}\right),\left(g_{2}, v_{2}\right)\right):=\left\langle v_{1}, \rho\left(\tau^{K}\left(g_{1}, g_{2}\right)\right) v_{2}\right\rangle_{V}$ where we use the mapping $\tau^{K}: G \times{ }_{G / K} G \rightarrow K$ given by $\tau^{K}\left(g_{1}, g_{2}\right)=g_{1}^{-1} g_{2}$ from (18.2). From this 
last description it is also clear that each $g \in G$ acts as an isometric vector bundle homomorphism.

Now we consider the natural line bundle $\operatorname{Vol}^{1 / 2}(G / K)$ of all $\frac{1}{2}$-densities on the manifold $G / K$ from $(10.4)$, Then for $\frac{1}{2}$-densities $\mu_{i} \in \Gamma\left(\mathrm{Vol}^{1 / 2}(G / M)\right)$ and any diffeomorphism $f: G / K \rightarrow G / K$ the pushforward $f_{*} \mu_{i}$ is defined and for those with compact support we have

$$
\int_{G / K}\left(f_{*} \mu_{1} \cdot f_{*} \mu_{2}\right)=\int_{G / K} f_{*}\left(\mu_{1} \cdot \mu_{2}\right)=\int_{G / K} \mu_{1} \cdot \mu_{2} .
$$

The Hermitian inner product on $G[V]$ now defines a fiberwise Hermitian mapping

$\left(G[V] \otimes \operatorname{Vol}^{1 / 2}(G / K)\right) \times_{G / K}\left(G[V] \otimes \operatorname{Vol}^{1 / 2}(G / K)\right) \stackrel{\langle\quad, \quad\rangle_{G[V]}}{\longrightarrow} \operatorname{Vol}^{1}(G / L)$

and on the space $C_{c}^{\infty}\left(G[V] \otimes \operatorname{Vol}^{1 / 2}(G / K)\right)$ of all smooth sections with compact support we have the following Hermitian inner product:

$$
\left\langle\sigma_{1}, \sigma_{2}\right\rangle:=\int_{G / K}\left\langle\sigma_{1}, \sigma_{2}\right\rangle_{G[V]} .
$$

For a decomposable section $\sigma_{i}=s_{i} \otimes \alpha_{i}$ (where $s_{i} \in \Gamma(G[V])$ and where $\alpha_{i} \in C_{c}^{\infty}\left(\operatorname{Vol}^{1 / 2}(G / K)\right)$ ) we may consider (using (18.12)) the equivariant lifts $f_{s_{i}}: G \rightarrow V$, their invariant inner product $\left\langle f_{s_{1}}, f_{s_{2}}\right\rangle_{V}: G \rightarrow \mathbb{C}$, and its factorization to $\left\langle f_{s_{1}}, f_{s_{2}}\right\rangle_{V}^{-}: G / K \rightarrow \mathbb{C}$. Then

$$
\left\langle\sigma_{1}, \sigma_{2}\right\rangle:=\int_{G / K}\left\langle f_{s_{1}}, f_{s_{2}}\right\rangle_{V}^{-} \alpha_{1} \alpha_{2}
$$

Obviously the resulting action of the group $G$ on $\Gamma\left(G[V] \otimes \operatorname{Vol}^{1 / 2}(G / K)\right)$ is unitary with respect to the Hermitian inner product, and it can be extended to the Hilbert space completion of this space of sections. The resulting unitary representation is called the induced representation and is denoted by $\operatorname{ind}_{K}^{G} \rho$.

If the original unitary representation $\rho: K \rightarrow U(V)$ is in an infinitedimensional Hilbert space $V$, one can first restrict the representation $\rho$ to the subspace of smooth vectors, on which it is differentiable, and repeat the above construction with some modifications. See [151] for more details on this infinite-dimensional construction.

18.14. Theorem. Consider a principal fiber bundle $(P, p, M, G)$ and a closed subgroup $K$ of $G$. Then the reductions of structure group from $G$ to $K$ correspond bijectively to the global sections of the associated bundle $P[G / K, \bar{\lambda}]$ in a canonical way, where $\bar{\lambda}: G \times G / K \rightarrow G / K$ is the left action on the homogeneous space from (5.11). 
Proof. By (18.12) the section $s \in \Gamma(P[G / K])$ corresponds to an equivariant mapping $f_{s} \in C^{\infty}(P, G / K)^{G}$, which is a surjective submersion since the action $\bar{\lambda}: G \times G / K \rightarrow G / K$ is transitive. Thus $P_{s}:=f_{s}^{-1}(\bar{e})$ is a submanifold of $P$ which is stable under the right action of $K$ on $P$. Furthermore the $K$ orbits are exactly the fibers of the mapping $p: P_{s} \rightarrow M$, so by lemma (18.3) we get a principal fiber bundle $\left(P_{s}, p, M, K\right)$. The embedding $P_{s} \hookrightarrow P$ is then a reduction of structure groups as required.

If conversely we have a principal fiber bundle $\left(P^{\prime}, p^{\prime}, M, K\right)$ and a reduction of structure groups $\chi: P^{\prime} \rightarrow P$, then $\chi$ is an embedding covering the identity of $M$ and is $K$-equivariant, so we may view $P^{\prime}$ as a fiber subbundle of $P$ which is stable under the right action of $K$. Now we consider the mapping $\tau: P \times_{M} P \rightarrow G$ from $(18.2)$ and restrict it to $P \times_{M} P^{\prime}$. Since we have $\tau\left(u_{x}, v_{x} . k\right)=\tau\left(u_{x}, v_{x}\right) . k$ for $k \in K$, this restriction induces $f: P \rightarrow G / K$ by

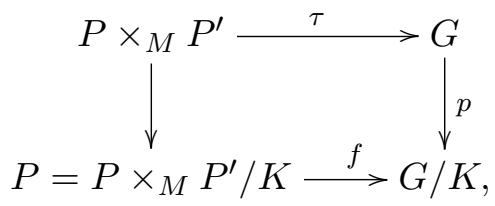

since $P^{\prime} / K=M$; and from $\tau\left(u_{x} . g, v_{x}\right)=g^{-1} \cdot \tau\left(u_{x}, v_{x}\right)$ it follows that $f$ is $G$ equivariant as required. Finally $f^{-1}(\bar{e})=\left\{u \in P: \tau\left(u, P_{p(u)}^{\prime}\right) \subseteq K\right\}=P^{\prime}$, so the two constructions are inverse to each other.

18.15. The bundle of gauges. If $(P, p, M, G)$ is a principal fiber bundle, we denote by $\operatorname{Aut}(P)$ the group of all $G$-equivariant diffeomorphisms $\chi$ : $P \rightarrow P$. Then $p \circ \chi=\bar{\chi} \circ p$ for a unique diffeomorphism $\bar{\chi}$ of $M$, so there is a group homomorphism from $\operatorname{Aut}(P)$ into the group $\operatorname{Diff}(M)$ of all diffeomorphisms of $M$. The kernel of this homomorphism is called $\operatorname{Gau}(P)$, the group of gauge transformations. So $\operatorname{Gau}(P)$ is the space of all $\chi: P \rightarrow P$ which satisfy $p \circ \chi=p$ and $\chi(u . g)=\chi(u) . g$. A vector field $\xi \in \mathfrak{X}(P)$ is an infinitesimal gauge transformation if its flow $\mathrm{Fl}_{t}^{\xi}$ consists of gauge transformations, i.e., if $\xi$ is vertical and $G$-invariant, $\left(r^{g}\right)^{*} \xi=\xi$.

Theorem. The group Gau $(P)$ of gauge transformations is equal to the space

$$
G a u(P) \cong C^{\infty}(P,(G, \text { conj }))^{G} \cong \Gamma(P[G, \text { conj }]) .
$$

The Lie algebra $\mathfrak{X}_{\text {vert }}(P)^{G}$ of infinitesimal gauge transformations is equal to the space

$$
\mathfrak{X}_{\text {vert }}(P)^{G} \cong C^{\infty}(P,(\mathfrak{g}, \mathrm{Ad}))^{G} \cong \Gamma(P[\mathfrak{g}, \mathrm{Ad}])
$$

Proof. We use again the mapping $\tau: P \times_{M} P \rightarrow G$ from (18.2). For $\chi \in \operatorname{Gau}(P)$ we define $f_{\chi} \in C^{\infty}(P,(G, \operatorname{conj}))^{G}$ by $f_{\chi}:=\tau \circ(I d, \chi)$. Then 
$f_{\chi}(u . g)=\tau(u . g, \chi(u . g))=g^{-1} \cdot \tau(u, \chi(u)) . g=\operatorname{conj}_{g^{-1}} f_{\chi}(u)$, so $f_{\chi}$ is indeed $G$-equivariant.

If conversely $f \in C^{\infty}(P,(G, \text { conj }))^{G}$ is given, we define $\chi_{f}: P \rightarrow P$ by $\chi_{f}(u):=u . f(u)$. It is easy to check that $\chi_{f}$ is indeed in $\operatorname{Gau}(P)$ and that the two constructions are inverse to each other, namely

$$
\begin{aligned}
& \chi_{f}(u g)=u g f(u g)=u g g^{-1} f(u) g=\chi_{f}(u) g, \\
& f_{\chi_{f}}(u)=\tau^{G}\left(u, \chi_{f}(u)\right)=\tau^{G}(u, u . f(u))=\tau^{G}(u, u) f(u)=f(u), \\
& \chi_{f_{\chi}}(u)=u f_{\chi}(u)=u \tau^{G}(u, \chi(u))=\chi(u) .
\end{aligned}
$$

The isomorphism $C^{\infty}(P,(G, \text { conj }))^{G} \cong \Gamma(P[G$, conj $])$ is a special case of theorem (18.12).

A vertical vector field $\xi \in \mathfrak{X}_{\text {vert }}(P)=\Gamma(V P)$ is given uniquely by a mapping $f_{\xi}: P \rightarrow \mathfrak{g}$ via $\xi(u)=T_{e}\left(r_{u}\right) \cdot f_{\xi}(u)$, and it is $G$-equivariant if and only if

$$
\begin{aligned}
T_{e}\left(r_{u}\right) \cdot f_{\xi}(u) & =\xi(u)=\left(\left(r^{g}\right)^{*} \xi\right)(u)=T\left(r^{g^{-1}}\right) \cdot \xi(u \cdot g) \\
& =T\left(r^{g^{-1}}\right) \cdot T_{e}\left(r_{u . g}\right) \cdot f_{\xi}(u \cdot g)=T_{e}\left(r^{g^{-1}} \circ r_{u . g}\right) \cdot f_{\xi}(u . g) \\
& =T_{e}\left(r_{u} \circ \operatorname{conj}_{g}\right) \cdot f_{\xi}(u . g)=T_{e}\left(r_{u}\right) \cdot \operatorname{Ad}_{g} \cdot f_{\xi}(u . g) .
\end{aligned}
$$

The isomorphism $C^{\infty}(P,(\mathfrak{g}, \mathrm{Ad}))^{G} \cong \Gamma(P[\mathfrak{g}, \mathrm{Ad}])$ is again a special case of theorem (18.12).

18.16. The tangent bundles of homogeneous spaces. Let $G$ be a Lie group and $K$ a closed subgroup, with Lie algebras $\mathfrak{g}$ and $\mathfrak{k}$, respectively. We recall the mapping $\operatorname{Ad}_{G}: G \rightarrow \operatorname{Aut}_{\text {Lie }}(\mathfrak{g})$ from (4.24) and put $\operatorname{Ad}_{G, K}:=\operatorname{Ad}_{G} \mid K: K \rightarrow \operatorname{Aut}_{\text {Lie }}(\mathfrak{g})$. For $X \in \mathfrak{k}$ and $k \in K$ we have $\operatorname{Ad}_{G, K}(k) X=\operatorname{Ad}_{G}(k) X=\operatorname{Ad}_{K}(k) X \in \mathfrak{k}$, so $\mathfrak{k}$ is an invariant subspace for the representation $\operatorname{Ad}_{G, K}$ of $K$ in $\mathfrak{g}$, and we have the factor representation $\mathrm{Ad}^{\perp}: K \rightarrow G L(\mathfrak{g} / \mathfrak{k})$. Then

$$
0 \rightarrow \mathfrak{k} \rightarrow \mathfrak{g} \rightarrow \mathfrak{g} / \mathfrak{k} \rightarrow 0
$$

is short exact and $K$-equivariant.

Now we consider the principal fiber bundle $(G, p, G / K, K)$ and the associated vector bundles $G\left[\mathfrak{g} / \mathfrak{k}, \mathrm{Ad}^{\perp}\right]$ and $G\left[\mathfrak{k}, \operatorname{Ad}_{K}\right]$.

Theorem. In these circumstances we have

$$
T(G / K)=G\left[\mathfrak{g} / \mathfrak{k}, \mathrm{Ad}^{\perp}\right]=\left(G \times_{K} \mathfrak{g} / \mathfrak{k}, p, G / K, \mathfrak{g} / \mathfrak{k}\right) .
$$

The left action $g \mapsto T\left(\bar{\mu}_{g}\right)$ of $G$ on $T(G / K)$ corresponds to the canonical left action of $G$ on $G \times_{K} \mathfrak{g} / \mathfrak{k}$. Furthermore $G\left[\mathfrak{g} / \mathfrak{k}, \mathrm{Ad}^{\perp}\right] \oplus G\left[\mathfrak{k}, \mathrm{Ad}_{K}\right]$ is a trivial vector bundle.

Proof. For $p: G \rightarrow G / K$ we consider the tangent mapping $T_{e} p: \mathfrak{g} \rightarrow$ $T_{\bar{e}}(G / K)$ which is linear and surjective and induces a linear isomorphism 
$\overline{T_{e} p}: \mathfrak{g} / \mathfrak{k} \rightarrow T_{\bar{e}}(G / K)$. For $k \in K$ we have $p \circ \operatorname{conj}_{k}=p \circ \mu_{k} \circ \mu^{k^{-1}}=\bar{\mu}_{k} \circ p$ and consequently $T_{e} p \circ \operatorname{Ad}_{G, K}(k)=T_{e} p \circ T_{e}\left(\operatorname{conj}_{k}\right)=T_{\bar{e}} \bar{\mu}_{k} \circ T_{e} p$. Thus the isomorphism $\overline{T_{e} p}: \mathfrak{g} / \mathfrak{k} \rightarrow T_{\bar{e}}(G / K)$ is $K$-equivariant for the representations $\operatorname{Ad}^{\perp}$ and $T_{\bar{e}} \bar{\lambda}: k \mapsto T_{\bar{e}} \bar{\mu}_{k}$, where, for the moment, we use the notation $\bar{\lambda}: G \times G / K \rightarrow G / K$ for the left action.

Let us now consider the associated vector bundle

$$
G\left[T_{\bar{e}}(G / K), T_{\bar{e}} \bar{\lambda}\right]=\left(G \times{ }_{K} T_{\bar{e}}(G / K), p, G / K, T_{\bar{e}}(G / K)\right),
$$

which is isomorphic to the vector bundle $G\left[\mathfrak{g} / \mathfrak{k}, \mathrm{Ad}^{\perp}\right]$, since the representation spaces are isomorphic. The mapping $T_{2} \bar{\lambda}: G \times T_{\bar{e}}(G / K) \rightarrow T(G / K)$ (where $T_{2}$ is the second partial tangent functor) is $K$-invariant, since

$$
T_{2} \bar{\lambda}((g, X) k)=T_{2} \bar{\lambda}\left(g k, T_{\bar{e}} \bar{\mu}_{k^{-1}} \cdot X\right)=T \bar{\mu}_{g k} \cdot T \bar{\mu}_{k^{-1}} \cdot X=T \bar{\mu}_{g} \cdot X
$$

Therefore it induces a mapping $\psi$ as in the following diagram:

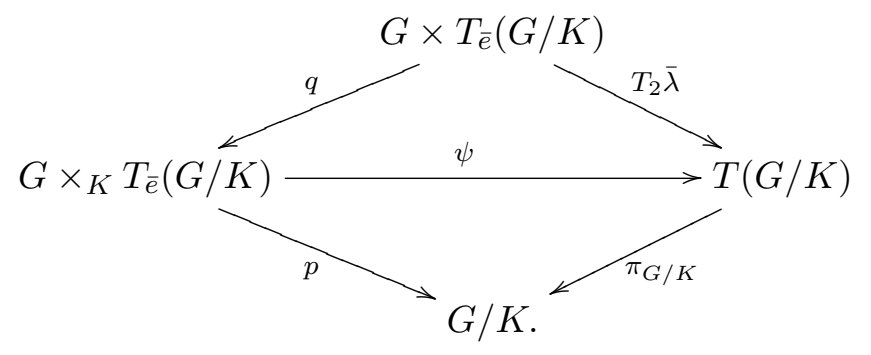

This mapping $\psi$ is an isomorphism of vector bundles.

It remains to show the last assertion. The short exact sequence (1) induces a sequence of vector bundles over $G / K$ :

$$
G / K \times 0 \rightarrow G\left[\mathfrak{k}, \operatorname{Ad}_{K}\right] \rightarrow G\left[\mathfrak{g}, \operatorname{Ad}_{G, K}\right] \rightarrow G\left[\mathfrak{g} / \mathfrak{k}, \operatorname{Ad}^{\perp}\right] \rightarrow G / K \times 0
$$

This sequence splits fiberwise thus also locally over $G / K$, so we get

$$
G\left[\mathfrak{g} / \mathfrak{k}, \operatorname{Ad}^{\perp}\right] \oplus G\left[\mathfrak{k}, \operatorname{Ad}_{K}\right] \cong G\left[\mathfrak{g}, \operatorname{Ad}_{G, K}\right]
$$

We have to show that $G\left[\mathfrak{g}, \operatorname{Ad}_{G, K}\right]$ is a trivial vector bundle. Let $\varphi: G \times \mathfrak{g} \rightarrow$ $G \times \mathfrak{g}$ be given by $\varphi(g, X)=\left(g, \operatorname{Ad}_{G}(g) X\right)$. Then for $k \in K$ we have

$$
\begin{aligned}
\varphi((g, X) . k) & =\varphi\left(g k, \operatorname{Ad}_{G, K}\left(k^{-1}\right) X\right) \\
& =\left(g k, \operatorname{Ad}_{G}\left(g . k \cdot k^{-1}\right) X\right)=\left(g k, \operatorname{Ad}_{G}(g) X\right) .
\end{aligned}
$$


So $\varphi$ is $K$-equivariant for the 'joint' $K$-action to the 'on the left' $K$-action and therefore induces a mapping $\bar{\varphi}$ as in the diagram:

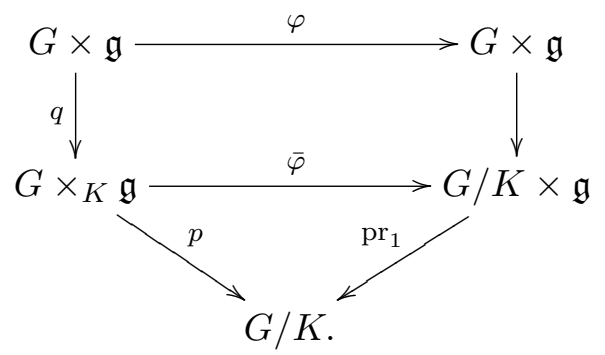

The map $\bar{\varphi}$ is a vector bundle isomorphism.

18.17. Tangent bundles of Grassmann manifolds. From (18.5) we know that $(V(k, n)=O(n) / O(n-k), p, G(k, n), O(k))$ is a principal fiber bundle. Using the standard representation of $O(k)$, we consider the associated vector bundle $\left(E_{k}:=V(k, n)\left[\mathbb{R}^{k}\right], p, G(k, n)\right)$. Recall from (18.5) the description of $V(k, n)$ as the space of all linear isometries $\mathbb{R}^{k} \rightarrow \mathbb{R}^{n}$; we get from it the evaluation mapping $e v: V(k, n) \times \mathbb{R}^{k} \rightarrow \mathbb{R}^{n}$. The mapping $(p, e v)$ in the diagram

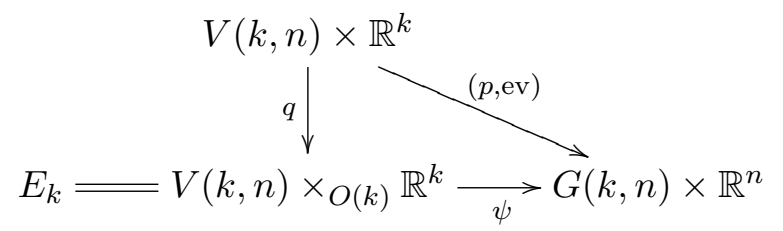

is $O(k)$-invariant for the action $R$ and factors therefore to an embedding of vector bundles $\psi: E_{k} \rightarrow G(k, n) \times \mathbb{R}^{n}$. So the fiber $\left(E_{k}\right)_{W}$ over the $k$-plane $W$ in $\mathbb{R}^{n}$ is just the linear subspace $W$. Note finally that the fiberwise orthogonal complement $E_{k}{ }^{\perp}$ of $E_{k}$ in the trivial vector bundle $G(k, n) \times \mathbb{R}^{n}$ with its standard Riemann metric is isomorphic to the universal vector bundle $E_{n-k}$ over $G(n-k, n)$, where the isomorphism covers the diffeomorphism $G(k, n) \rightarrow G(n-k, n)$ given also by the orthogonal complement mapping.

Corollary. The tangent bundle of the Grassmann manifold is

$$
T G(k, n) \cong L\left(E_{k}, E_{k}^{\perp}\right) .
$$

Proof. We have $G(k, n)=O(n) /(O(k) \times O(n-k))$, so by theorem (18.16) we get

$$
T G(k, n)=O(n) \underset{O(k) \times \stackrel{\times}{\times}(n-k)}{\times}(\mathfrak{s o}(n) /(\mathfrak{s o}(k) \times \mathfrak{s o}(n-k))) .
$$


On the other hand we have $V(k, n)=O(n) / O(n-k)$ and the right action of $O(k)$ commutes with the right action of $O(n-k)$ on $O(n)$; therefore

$$
V(k, n)\left[\mathbb{R}^{k}\right]=(O(n) / O(n-k)) \underset{O(k)}{\times} \mathbb{R}^{k}=O(n) \underset{O(k) \times O(n-k)}{\times} \mathbb{R}^{k},
$$

where $O(n-k)$ acts trivially on $\mathbb{R}^{k}$. We have

$$
\begin{aligned}
& L\left(E_{k}, E_{k}{ }^{\perp}\right)=L\left(O(n) \underset{O(k) \times O(n-k)}{\times} \mathbb{R}^{k}, O(n) \underset{O(k) \times O(n-k)}{\times} \underset{\mathbb{R}^{n-k}}{\times}\right) \\
& =O(n) \underset{O(k) \times O(n-k)}{\times} L\left(\mathbb{R}^{k}, \mathbb{R}^{n-k}\right),
\end{aligned}
$$

where $O(k) \times O(n-k)$ acts on $L\left(\mathbb{R}^{k}, \mathbb{R}^{n-k}\right)$ by $(A, B)(C)=$ B.C. $A^{-1}$. Finally, we have an $(O(k) \times O(n-k))$-equivariant linear isomorphism as follows:

$$
\begin{aligned}
L\left(\mathbb{R}^{k}, \mathbb{R}^{n-k}\right) & \rightarrow \mathfrak{s o}(n) /(\mathfrak{s o}(k) \times \mathfrak{s o}(n-k)), \\
\mathfrak{s o}(n) /(\mathfrak{s o}(k) \times \mathfrak{s o}(n-k)) & =(\text { skew }) /\left(\begin{array}{cc}
\text { skew } & 0 \\
0 & \text { skew }
\end{array}\right) \\
& =\left\{\left(\begin{array}{cc}
0 & -A^{\top} \\
A & 0
\end{array}\right): \quad A \in L\left(\mathbb{R}^{k}, \mathbb{R}^{n-k}\right)\right\} .
\end{aligned}
$$

18.18. Tangent bundles and vertical bundles. Let $(E, p, M, S)$ be a fiber bundle. The vector subbundle $V E=\{\xi \in T E: T p . \xi=0\}$ of $T E$ is called the vertical bundle and is denoted by $\left(V E, \pi_{E}, E\right)$.

Theorem. Let $(P, p, M, G)$ be a principal fiber bundle with principal right action $r: P \times G \rightarrow P$. Let $\ell: G \times S \rightarrow S$ be a left action. Then the following assertions hold:

(1) $(T P, T p, T M, T G)$ is again a principal fiber bundle with principal right action $T r: T P \times T G \rightarrow T P$, where the structure group $T G$ is the tangent group of $G$; see $(6.7)$.

(2) The vertical bundle $(V P, \pi, P, \mathfrak{g})$ of the principal bundle is trivial as a vector bundle over $P: V P \cong P \times \mathfrak{g}$.

(3) The vertical bundle of the principal bundle as bundle over $M$ is again a principal bundle: $(V P, p \circ \pi, M, T G)$.

(4) The tangent bundle of the associated bundle $P[S, \ell]$ is given by $T(P[S, \ell])=T P[T S, T \ell]$.

(5) The vertical bundle of the associated bundle $P[S, \ell]$ is given by $V(P[S, \ell])=P\left[T S, T_{2} \ell\right]=P \times_{G} T S$.

Proof. Let $\left(U_{\alpha}, \varphi_{\alpha}: P \mid U_{\alpha} \rightarrow U_{\alpha} \times G\right)$ be a principal fiber bundle atlas with cocycle of transition functions $\left(\varphi_{\alpha \beta}: U_{\alpha \beta} \rightarrow G\right)$. Since $T$ is a functor which 
respects products, $\left(T U_{\alpha}, T \varphi_{\alpha}: T P \mid T U_{\alpha} \rightarrow T U_{\alpha} \times T G\right)$ is again a principal fiber bundle atlas with cocycle of transition functions $\left(T \varphi_{\alpha \beta}: T U_{\alpha \beta} \rightarrow T G\right)$, describing the principal fiber bundle $(T P, T p, T M, T G)$. The assertion about the principal action is obvious. So (11) follows. For completeness sake we include here the transition formula for this atlas in the right trivialization of $T G$ :

$T\left(\varphi_{\alpha} \circ \varphi_{\beta}^{-1}\right)\left(\xi_{x}, T_{e}\left(\mu^{g}\right) \cdot X\right)=\left(\xi_{x}, T_{e}\left(\mu^{\varphi_{\alpha \beta}(x) \cdot g}\right) \cdot\left(\delta^{r} \varphi_{\alpha \beta}\left(\xi_{x}\right)+\operatorname{Ad}\left(\varphi_{\alpha \beta}(x)\right) X\right)\right)$,

where $\delta \varphi_{\alpha \beta} \in \Omega^{1}\left(U_{\alpha \beta} ; \mathfrak{g}\right)$ is the right logarithmic derivative of $\varphi_{\alpha \beta}$; see $(4.26)$

(2) The mapping $(u, X) \mapsto T_{e}\left(r_{u}\right) \cdot X=T_{(u, e)} r .\left(0_{u}, X\right)$ is a vector bundle isomorphism $P \times \mathfrak{g} \rightarrow V P$ over $P$.

(3) Obviously $\operatorname{Tr}: T P \times T G \rightarrow T P$ is a free right action which acts transitively on the fibers of $T p: T P \rightarrow T M$. Since $V P=(T p)^{-1}\left(0_{M}\right)$, the bundle $V P \rightarrow M$ is isomorphic to $T P \mid 0_{M}$ and $T r$ restricts to a free right action, which is transitive on the fibers, so by lemma (18.3) the result follows.

(41) The transition functions of the fiber bundle $P[S, \ell]$ are given by the expression $\ell \circ\left(\varphi_{\alpha \beta} \times I d_{S}\right): U_{\alpha \beta} \times S \rightarrow G \times S \rightarrow S$. Then the transition functions of $T(P[S, \ell])$ are $T\left(\ell \circ\left(\varphi_{\alpha \beta} \times I d_{S}\right)\right)=T \ell \circ\left(T \varphi_{\alpha \beta} \times I d_{T S}\right): T U_{\alpha \beta} \times$ $T S \rightarrow T G \times T S \rightarrow T S$, from which the result follows.

(5) Vertical vectors in $T(P[S, \ell])$ have local representations $\left(0_{x}, \eta_{s}\right) \in T U_{\alpha \beta} \times$ $T S$. Under the transition functions of $T(P[S, \ell])$ they transform as $T(\ell \circ$ $\left.\left(\varphi_{\alpha \beta} \times I d_{S}\right)\right) \cdot\left(0_{x}, \eta_{s}\right)=T \ell .\left(0_{\varphi_{\alpha \beta}(x)}, \eta_{s}\right)=T\left(\ell_{\varphi_{\alpha \beta}(x)}\right) \cdot \eta_{s}=T_{2} \ell \cdot\left(\varphi_{\alpha \beta}(x), \eta_{s}\right)$ and this implies the result

\section{Principal and Induced Connections}

19.1. Principal connections. Let $(P, p, M, G)$ be a principal fiber bundle. Recall from (17.3) that a (general) connection on $P$ is a fiber projection $\Phi: T P \rightarrow V P$, viewed as a 1 -form in $\Omega^{1}(P, T P)$. Such a connection $\Phi$ is called a principal connection if it is $G$-equivariant for the principal right action $r: P \times G \rightarrow P$, so that $T\left(r^{g}\right) . \Phi=\Phi . T\left(r^{g}\right)$ and $\Phi$ is $r^{g}$-related to itself, or $\left(r^{g}\right)^{*} \Phi=\Phi$ in the sense of (16.16), for all $g \in G$. By theorem $(16.15 .6)$ the curvature $R=\frac{1}{2} \cdot[\Phi, \Phi]$ is then also $r^{g}$-related to itself for all $g \in G$.

Recall from (18.18.2) that the vertical bundle of $P$ is trivialized as a vector bundle over $P$ by the principal action. So

$$
\omega\left(X_{u}\right):=T_{e}\left(r_{u}\right)^{-1} . \Phi\left(X_{u}\right) \in \mathfrak{g}
$$

and in this way we get a $\mathfrak{g}$-valued 1 -form $\omega \in \Omega^{1}(P, \mathfrak{g})$, which is called the (Lie algebra valued) connection form of the connection $\Phi$. Recall from 
(6.3) the fundamental vector field mapping $\zeta: \mathfrak{g} \rightarrow \mathfrak{X}(P)$ for the principal right action given by $\zeta_{X}(u)=T_{e}\left(r_{u}\right) X$ which satisfies $T_{u}\left(r^{g}\right) \zeta_{X}(u)=$ $\zeta_{\operatorname{Ad}\left(g^{-1}\right) X}(u . g)$. The defining equation for $\omega$ can be written also as $\Phi\left(X_{u}\right)=$ $\zeta_{\omega\left(X_{u}\right)}(u)$.

Lemma. If $\Phi \in \Omega^{1}(P, V P)$ is a principal connection on the principal fiber bundle $(P, p, M, G)$, then the connection form has the following two properties:

(2) $\omega$ reproduces the generators of fundamental vector fields:

$$
\omega\left(\zeta_{X}(u)\right)=X \quad \text { for all } \quad X \in \mathfrak{g} .
$$

(3) $\omega$ is G-equivariant, i.e.,

$$
\left(\left(r^{g}\right)^{*} \omega\right)\left(X_{u}\right)=\omega\left(T_{u}\left(r^{g}\right) \cdot X_{u}\right)=\operatorname{Ad}\left(g^{-1}\right) \cdot \omega\left(X_{u}\right)
$$

for all $g \in G$ and $X_{u} \in T_{u} P$. Consequently we have for the Lie derivative $\mathcal{L}_{\zeta_{X}} \omega=-\operatorname{ad}(X) . \omega$.

Conversely a 1-form $\omega \in \Omega^{1}(P, \mathfrak{g})$ satisfying (2) defines a connection $\Phi$ on $P$ by $\Phi\left(X_{u}\right)=T_{e}\left(r_{u}\right) \cdot \omega\left(X_{u}\right)$, which is a principal connection if and only if (3) is satisfied.

Proof. (2) $T_{e}\left(r_{u}\right) \cdot \omega\left(\zeta_{X}(u)\right)=\Phi\left(\zeta_{X}(u)\right)=\zeta_{X}(u)=T_{e}\left(r_{u}\right) \cdot X$. Since $T_{e}\left(r_{u}\right): \mathfrak{g} \rightarrow V_{u} P$ is an isomorphism, the result follows.

(3) Both directions follow from

$$
\begin{aligned}
T_{e}\left(r_{u g}\right) \cdot \omega\left(T_{u}\left(r^{g}\right) \cdot X_{u}\right) & =\zeta_{\omega\left(T_{u}\left(r^{g}\right) \cdot X_{u}\right)}(u g)=\Phi\left(T_{u}\left(r^{g}\right) \cdot X_{u}\right), \\
T_{e}\left(r_{u g}\right) \cdot \operatorname{Ad}\left(g^{-1}\right) \cdot \omega\left(X_{u}\right) & =\zeta_{\operatorname{Ad}\left(g^{-1}\right) \cdot \omega\left(X_{u}\right)}(u g)=T_{u}\left(r^{g}\right) \cdot \zeta_{\omega\left(X_{u}\right)}(u) \\
& =T_{u}\left(r^{g}\right) \cdot \Phi\left(X_{u}\right) .
\end{aligned}
$$

19.2. Curvature. Let $\Phi$ be a principal connection on the principal fiber bundle $(P, p, M, G)$ with connection form $\omega \in \Omega^{1}(P, \mathfrak{g})$. We already noted in $(19.1)$ that the curvature $R=\frac{1}{2}[\Phi, \Phi]$ is then also $G$-equivariant, $\left(r^{g}\right)^{*} R=R$ for all $g \in G$. Since $R$ has vertical values, we may again define a $\mathfrak{g}$-valued 2 -form

$$
\Omega \in \Omega^{2}(P, \mathfrak{g}), \quad \Omega\left(X_{u}, Y_{u}\right):=-T_{e}\left(r_{u}\right)^{-1} \cdot R\left(X_{u}, Y_{u}\right),
$$

which is called the (Lie algebra valued) curvature form of the connection. We also have

$$
R\left(X_{u}, Y_{u}\right)=-\zeta_{\Omega\left(X_{u}, Y_{u}\right)}(u)
$$

We take the negative sign to get the usual curvature form as in [107, I].

We equip the space $\Omega(P, \mathfrak{g})$ of all $\mathfrak{g}$-valued forms on $P$ in a canonical way with the structure of a graded Lie algebra by 


$$
\begin{aligned}
& {[\Psi, \Theta]_{\wedge}\left(X_{1}, \ldots, X_{p+q}\right)} \\
& \quad=\frac{1}{p ! q !} \sum_{\sigma} \operatorname{sign} \sigma\left[\Psi\left(X_{\sigma 1}, \ldots, X_{\sigma p}\right), \Theta\left(X_{\sigma(p+1)}, \ldots, X_{\sigma(p+q)}\right)\right]_{\mathfrak{g}}
\end{aligned}
$$

or equivalently by

$$
[\psi \otimes X, \vartheta \otimes Y]_{\wedge}:=\psi \wedge \vartheta \otimes[X, Y]_{\mathfrak{g}}
$$

From the latter description it is clear that

$$
d[\Psi, \Theta]_{\wedge}=[d \Psi, \Theta]_{\wedge}+(-1)^{\operatorname{deg} \Psi}[\Psi, d \Theta]_{\wedge} .
$$

In particular for $\omega \in \Omega^{1}(P, \mathfrak{g})$ we have

$$
[\omega, \omega]_{\wedge}(X, Y)=2[\omega(X), \omega(Y)]_{\mathfrak{g}}
$$

Theorem. The curvature form $\Omega$ of a principal connection with connection form $\omega$ has the following properties:

(1) $\Omega$ is horizontal, i.e., it kills vertical vectors.

(2) $\Omega$ is G-equivariant in the following sense: $\left(r^{g}\right)^{*} \Omega=\operatorname{Ad}\left(g^{-1}\right) . \Omega$. Consequently $\mathcal{L}_{\zeta_{X}} \Omega=-\operatorname{ad}(X) . \Omega$.

(3) The Maurer-Cartan formula holds: $\Omega=d \omega+\frac{1}{2}[\omega, \omega]_{\wedge}$.

Proof. (11) is true for $R$ by (17.4), For (2) we compute as follows:

$$
\begin{aligned}
T_{e}\left(r_{u g}\right) & \cdot\left(\left(r^{g}\right)^{*} \Omega\right)\left(X_{u}, Y_{u}\right)=T_{e}\left(r_{u g}\right) \cdot \Omega\left(T_{u}\left(r^{g}\right) \cdot X_{u}, T_{u}\left(r^{g}\right) \cdot Y_{u}\right) \\
& =-R_{u g}\left(T_{u}\left(r^{g}\right) \cdot X_{u}, T_{u}\left(r^{g}\right) \cdot Y_{u}\right)=-T_{u}\left(r^{g}\right) \cdot\left(\left(r^{g}\right)^{*} R\right)\left(X_{u}, Y_{u}\right) \\
& =-T_{u}\left(r^{g}\right) \cdot R\left(X_{u}, Y_{u}\right)=T_{u}\left(r^{g}\right) \cdot \zeta_{\Omega\left(X_{u}, Y_{u}\right)}(u) \\
& =\zeta_{\operatorname{Ad}\left(g^{-1}\right) \cdot \Omega\left(X_{u}, Y_{u}\right)}(u g)=T_{e}\left(r_{u g}\right) \cdot \operatorname{Ad}\left(g^{-1}\right) \cdot \Omega\left(X_{u}, Y_{u}\right), \quad \text { by }(6.3)
\end{aligned}
$$

(3) For $X \in \mathfrak{g}$ we have $i_{\zeta_{X}} R=0$ by (11), and using (19.1.2), we get

$$
\begin{aligned}
i_{\zeta_{X}}\left(d \omega+\frac{1}{2}[\omega, \omega]_{\wedge}\right) & =i_{\zeta_{X}} d \omega+\frac{1}{2}\left[i_{\zeta_{X}} \omega, \omega\right]_{\wedge}-\frac{1}{2}\left[\omega, i_{\zeta_{X}} \omega\right]_{\wedge} \\
& =\mathcal{L}_{\zeta_{X}} \omega+[X, \omega]_{\wedge}=-\operatorname{ad}(X) \omega+\operatorname{ad}(X) \omega=0 .
\end{aligned}
$$

So the formula holds for vertical vectors, and for horizontal vector fields $\xi, \eta \in \Gamma(H(P))$ we have

$$
\begin{aligned}
R(\xi, \eta) & =\Phi[\xi-\Phi \xi, \eta-\Phi \eta]=\Phi[\xi, \eta]=\zeta_{\omega([\xi, \eta])} \\
\left(d \omega+\frac{1}{2}[\omega, \omega]\right)(\xi, \eta) & =\xi \omega(\eta)-\eta \omega(\xi)-\omega([\xi, \eta])+0=-\omega([\xi, \eta]) .
\end{aligned}
$$

19.3. Lemma. Any principal fiber bundle $(P, p, M, G)$ (with paracompact basis) admits principal connections. 
Proof. Let $\left(U_{\alpha}, \varphi_{\alpha}: P \mid U_{\alpha} \rightarrow U_{\alpha} \times G\right)_{\alpha}$ be a principal fiber bundle atlas. Let us define $\gamma_{\alpha}\left(T \varphi_{\alpha}^{-1}\left(\xi_{x}, T_{e} \mu_{g} . X\right)\right):=X$ for $\xi_{x} \in T_{x} U_{\alpha}$ and $X \in \mathfrak{g}$. Using lemma (6.3), we get

$$
\begin{aligned}
\left(\left(r^{h}\right)^{*} \gamma_{\alpha}\right)\left(T \varphi _ { \alpha } ^ { - 1 } \left(\xi_{x},\right.\right. & \left.\left.T_{e} \mu_{g} \cdot X\right)\right)=\gamma_{\alpha}\left(T r^{h} \cdot T \varphi_{\alpha}^{-1}\left(\xi_{x}, T_{e} \mu_{g} \cdot X\right)\right) \\
& =\gamma_{\alpha}\left(T \varphi_{\alpha}^{-1}\left(\xi_{x}, T \mu^{h} \cdot T_{e} \mu_{g} \cdot X\right)\right) \\
& =\gamma_{\alpha}\left(T \varphi_{\alpha}^{-1}\left(\xi_{x}, T_{e} \mu_{g h} \cdot \operatorname{Ad}\left(h^{-1}\right) \cdot X\right)\right)=\operatorname{Ad}\left(h^{-1}\right) \cdot X,
\end{aligned}
$$

so that $\gamma_{\alpha} \in \Omega^{1}\left(P \mid U_{\alpha}, \mathfrak{g}\right)$ satisfies the requirements of lemma (19.1) and thus is a principal connection on $P \mid U_{\alpha}$. Now let $\left(f_{\alpha}\right)$ be a smooth partition of unity on $M$ which is subordinated to the open cover $\left(U_{\alpha}\right)$, and let $\omega:=$ $\sum_{\alpha}\left(f_{\alpha} \circ p\right) \gamma_{\alpha}$. Since both requirements of lemma (19.1) are invariant under convex linear combinations, $\omega$ is a principal connection on $P$.

19.4. Local descriptions of principal connections. We consider a principal fiber bundle $(P, p, M, G)$ with some principal fiber bundle atlas $\left(U_{\alpha}, \varphi_{\alpha}: P \mid U_{\alpha} \rightarrow U_{\alpha} \times G\right)$ and corresponding cocycle $\left(\varphi_{\alpha \beta}: U_{\alpha \beta} \rightarrow G\right)$ of transition functions. We consider the sections $s_{\alpha} \in \Gamma\left(P \mid U_{\alpha}\right)$ which are given by $\varphi_{\alpha}\left(s_{\alpha}(x)\right)=(x, e)$ and satisfy $s_{\alpha} \cdot \varphi_{\alpha \beta}=s_{\beta}$, since we have in turn:

$$
\begin{aligned}
\varphi_{\alpha}\left(s_{\beta}(x)\right) & =\varphi_{\alpha} \varphi_{\beta}^{-1}(x, e)=\left(x, \varphi_{\alpha \beta}(x)\right), \\
s_{\beta}(x) & =\varphi_{\alpha}^{-1}\left(x, e \cdot \varphi_{\alpha \beta}(e)\right)=\varphi_{\alpha}^{-1}(x, e) \varphi_{\alpha \beta}(x)=s_{\alpha}(x) \varphi_{\alpha \beta}(x) .
\end{aligned}
$$

(1) Let $\kappa^{l} \in \Omega^{1}(G, \mathfrak{g})$ be the left logarithmic derivative of the identity, also called the left Maurer-Cartan form, i.e., $\kappa^{l}\left(\eta_{g}\right):=T_{g}\left(\mu_{g^{-1}}\right) \cdot \eta_{g}$. We will use the forms $\kappa_{\alpha \beta}^{l}:=\varphi_{\alpha \beta}{ }^{*} \kappa^{l} \in \Omega^{1}\left(U_{\alpha \beta}, \mathfrak{g}\right)$.

Let $\Phi=\zeta \circ \omega \in \Omega^{1}(P, V P)$ be a principal connection with connection form $\omega \in \Omega^{1}(P, \mathfrak{g})$. We may associate the following local data to the connection:

(2) $\omega_{\alpha}:=s_{\alpha}{ }^{*} \omega \in \Omega^{1}\left(U_{\alpha}, \mathfrak{g}\right)$, the physicists' version or Cartan moving frame version of the connection,

(3) the Christoffel forms $\Gamma^{\alpha} \in \Omega^{1}\left(U_{\alpha}, \mathfrak{X}(G)\right)$ from (17.7), which are given by $\left(0_{x}, \Gamma^{\alpha}\left(\xi_{x}, g\right)\right)=-T\left(\varphi_{\alpha}\right) \cdot \Phi \cdot T\left(\varphi_{\alpha}\right)^{-1}\left(\xi_{x}, 0_{g}\right)$,

(4) $\gamma_{\alpha}:=\left(\varphi_{\alpha}^{-1}\right)^{*} \omega \in \Omega^{1}\left(U_{\alpha} \times G, \mathfrak{g}\right)$, the local expressions of $\omega$.

Lemma. These local data have the following properties and are related by the following formulas.

(5) The forms $\omega_{\alpha} \in \Omega^{1}\left(U_{\alpha}, \mathfrak{g}\right)$ satisfy the transition formulas

$$
\omega_{\alpha}=\operatorname{Ad}\left(\varphi_{\beta \alpha}^{-1}\right) \omega_{\beta}+\kappa_{\beta \alpha}^{l},
$$

and any set of forms like that with this transition behavior determines a unique principal connection.

(6) We have $\gamma_{\alpha}\left(\xi_{x}, T \mu_{g} \cdot X\right)=\gamma_{\alpha}\left(\xi_{x}, 0_{g}\right)+X=\operatorname{Ad}\left(g^{-1}\right) \omega_{\alpha}\left(\xi_{x}\right)+X$. 
(7) We have $\Gamma^{\alpha}\left(\xi_{x}\right)=-R_{\omega_{\alpha}\left(\xi_{x}\right)}$, a right invariant vector field, since

$$
\begin{aligned}
\Gamma^{\alpha}\left(\xi_{x}, g\right) & =-T_{e}\left(\mu_{g}\right) \cdot \gamma_{\alpha}\left(\xi_{x}, 0_{g}\right) \\
& =-T_{e}\left(\mu_{g}\right) \cdot \operatorname{Ad}\left(g^{-1}\right) \omega_{\alpha}\left(\xi_{x}\right)=-T\left(\mu^{g}\right) \omega_{\alpha}\left(\xi_{x}\right) .
\end{aligned}
$$

Proof. (7) From the definition of the Christoffel forms we have

$$
\begin{aligned}
\left(0_{x}, \Gamma^{\alpha}\left(\xi_{x}, g\right)\right) & =-T\left(\varphi_{\alpha}\right) \cdot \Phi \cdot T\left(\varphi_{\alpha}\right)^{-1}\left(\xi_{x}, 0_{g}\right) \\
& =-T\left(\varphi_{\alpha}\right) \cdot T_{e}\left(r_{\varphi_{\alpha}^{-1}(x, g)}\right) \cdot \omega \cdot T\left(\varphi_{\alpha}\right)^{-1}\left(\xi_{x}, 0_{g}\right) \quad \text { by }[19.1 \cdot 1) \\
& =-T_{e}\left(\varphi_{\alpha} \circ r_{\varphi_{\alpha}^{-1}(x, g)}\right) \omega \cdot T\left(\varphi_{\alpha}\right)^{-1}\left(\xi_{x}, 0_{g}\right) \\
& =-\left(0_{x}, T_{e}\left(\mu_{g}\right) \omega \cdot T\left(\varphi_{\alpha}\right)^{-1}\left(\xi_{x}, 0_{g}\right)\right) \\
& =-\left(0_{x}, T_{e}\left(\mu_{g}\right) \gamma_{\alpha}\left(\xi_{x}, 0_{g}\right)\right), \quad \text { by (4) },
\end{aligned}
$$

where we also used $\varphi_{\alpha}\left(r_{\varphi_{\alpha}^{-1}(x, g)} h\right)=\varphi_{\alpha}\left(\varphi_{\alpha}^{-1}(x, g) h\right)=\varphi_{\alpha}\left(\varphi_{\alpha}^{-1}(x, g h)\right)=$ $(x, g h)$. This is the first part of (7). The second part follows from (6).

(6)

$$
\begin{aligned}
\gamma_{\alpha}\left(\xi_{x}, T \mu_{g} . X\right) & =\gamma_{\alpha}\left(\xi_{x}, 0_{g}\right)+\gamma_{\alpha}\left(0_{x}, T \mu_{g} . X\right) \\
& =\gamma_{\alpha}\left(\xi_{x}, 0_{g}\right)+\omega\left(T\left(\varphi_{\alpha}\right)^{-1}\left(0_{x}, T \mu_{g} . X\right)\right) \\
& =\gamma_{\alpha}\left(\xi_{x}, 0_{g}\right)+\omega\left(\zeta_{X}\left(\varphi_{\alpha}^{-1}(x, g)\right)\right) \\
& =\gamma_{\alpha}\left(\xi_{x}, 0_{g}\right)+X .
\end{aligned}
$$

So the first part of (마) holds. The second part is seen from

$$
\begin{aligned}
\gamma_{\alpha}\left(\xi_{x}, 0_{g}\right) & =\gamma_{\alpha}\left(\xi_{x}, T_{e}\left(\mu^{g}\right) 0_{e}\right)=\left(\omega \circ T\left(\varphi_{\alpha}\right)^{-1} \circ T\left(I d_{X} \times \mu^{g}\right)\right)\left(\xi_{x}, 0_{e}\right) \\
& =\left(\omega \circ T\left(r^{g} \circ \varphi_{\alpha}^{-1}\right)\right)\left(\xi_{x}, 0_{e}\right)=\operatorname{Ad}\left(g^{-1}\right) \omega\left(T\left(\varphi_{\alpha}^{-1}\right)\left(\xi_{x}, 0_{e}\right)\right) \\
& =\operatorname{Ad}\left(g^{-1}\right)\left(s_{\alpha}{ }^{*} \omega\right)\left(\xi_{x}\right)=\operatorname{Ad}\left(g^{-1}\right) \omega_{\alpha}\left(\xi_{x}\right) .
\end{aligned}
$$

(5) Via (7) the transition formulas for the $\omega_{\alpha}$ are easily seen to be equivalent to the transition formulas for the Christoffel forms in lemma (17.7). A direct proof goes as follows: We have $s_{\alpha}(x)=s_{\beta}(x) \varphi_{\beta \alpha}(x)=r\left(s_{\beta}(x), \varphi_{\beta \alpha}(x)\right)$ and thus

$$
\begin{aligned}
\omega_{\alpha}\left(\xi_{x}\right)=\omega\left(T_{x}\left(s_{\alpha}\right) \cdot \xi_{x}\right) \\
=\left(\omega \circ T_{\left(s_{\beta}(x), \varphi_{\beta \alpha}(x)\right)} r\right)\left(\left(T_{x} s_{\beta} \cdot \xi_{x}, 0_{\varphi_{\beta \alpha}(x)}\right)+\left(0_{s_{\beta}}(x), T_{x} \varphi_{\beta \alpha} \cdot \xi_{x}\right)\right) \\
=\omega\left(T\left(r^{\varphi_{\beta \alpha}(x)}\right) \cdot T_{x}\left(s_{\beta}\right) \cdot \xi_{x}\right)+\omega\left(T_{\varphi_{\beta \alpha}(x)}\left(r_{s_{\beta}(x)}\right) \cdot T_{x}\left(\varphi_{\beta \alpha}\right) \cdot \xi_{x}\right) \\
=\operatorname{Ad}\left(\varphi_{\beta \alpha}(x)^{-1}\right) \omega\left(T_{x}\left(s_{\beta}\right) \cdot \xi_{x}\right) \\
\quad+\omega\left(T _ { \varphi _ { \beta \alpha } ( x ) } ( r _ { s _ { \beta } ( x ) } ) \cdot T \left(\mu_{\varphi_{\beta \alpha}(x)} \circ \mu_{\left.\left.\varphi_{\beta \alpha}(x)^{-1}\right) T_{x}\left(\varphi_{\beta \alpha}\right) \cdot \xi_{x}\right)}\right.\right. \\
=\operatorname{Ad}\left(\varphi_{\beta \alpha}(x)^{-1}\right) \omega_{\beta}\left(\xi_{x}\right) \\
\quad+\omega\left(T_{e}\left(r_{s_{\beta}(x) \varphi_{\beta \alpha}(x)}\right) \cdot \kappa_{\beta \alpha}^{l} \cdot \xi_{x}\right) \\
=\operatorname{Ad}\left(\varphi_{\beta \alpha}(x)^{-1}\right) \omega_{\beta}\left(\xi_{x}\right)+\kappa_{\beta \alpha}^{l}\left(\xi_{x}\right) .
\end{aligned}
$$


19.5. The covariant derivative. Let $(P, p, M, G)$ be a principal fiber bundle with principal connection $\Phi=\zeta \circ \omega$. We consider the horizontal projection $\chi=I d_{T P}-\Phi: T P \rightarrow H P$, cf. (17.3), which satisfies

$$
\chi \circ \chi=\chi, \quad \operatorname{im} \chi=H P, \quad \operatorname{ker} \chi=V P, \quad \chi \circ T\left(r^{g}\right)=T\left(r^{g}\right) \circ \chi
$$

for all $g \in G$.

If $W$ is a finite-dimensional vector space, we consider the mapping $\chi^{*}$ : $\Omega(P, W) \rightarrow \Omega(P, W)$ which is given by

$$
\left(\chi^{*} \varphi\right)_{u}\left(X_{1}, \ldots, X_{k}\right)=\varphi_{u}\left(\chi\left(X_{1}\right), \ldots, \chi\left(X_{k}\right)\right) .
$$

The mapping $\chi^{*}$ is a projection onto the subspace of horizontal differential forms, i.e., the space $\Omega_{h o r}(P, W):=\left\{\psi \in \Omega(P, W): i_{X} \psi=0\right.$ for $\left.X \in V P\right\}$. The notion of horizontal form is independent of the choice of a connection.

The projection $\chi^{*}$ has the following properties: $\chi^{*}(\varphi \wedge \psi)=\chi^{*} \varphi \wedge \chi^{*} \psi$ if one of the two forms has values in $\mathbb{R} ; \chi^{*} \circ \chi^{*}=\chi^{*} ; \chi^{*} \circ\left(r^{g}\right)^{*}=\left(r^{g}\right)^{*} \circ \chi^{*}$ for all $g \in G ; \chi^{*} \omega=0$; and $\chi^{*} \circ \mathcal{L}\left(\zeta_{X}\right)=\mathcal{L}\left(\zeta_{X}\right) \circ \chi^{*}$. They follow easily from the corresponding properties of $\chi$; the last property uses that $\mathrm{Fl}_{t}^{\zeta(X)}=r^{\exp t X}$. We define the covariant exterior derivative $d_{\omega}: \Omega^{k}(P, W) \rightarrow \Omega^{k+1}(P, W)$ by prescribing $d_{\omega}:=\chi^{*} \circ d$.

Theorem. The covariant exterior derivative $d_{\omega}$ has the following properties.

(1) $d_{\omega}(\varphi \wedge \psi)=d_{\omega}(\varphi) \wedge \chi^{*} \psi+(-1)^{\operatorname{deg} \varphi} \chi^{*} \varphi \wedge d_{\omega}(\psi)$ if $\varphi$ or $\psi$ is real valued.

(2) $\mathcal{L}\left(\zeta_{X}\right) \circ d_{\omega}=d_{\omega} \circ \mathcal{L}\left(\zeta_{X}\right)$ for each $X \in \mathfrak{g}$.

(3) $\left(r^{g}\right)^{*} \circ d_{\omega}=d_{\omega} \circ\left(r^{g}\right)^{*}$ for each $g \in G$.

(4) $d_{\omega} \circ p^{*}=d \circ p^{*}=p^{*} \circ d: \Omega(M, W) \rightarrow \Omega_{h o r}(P, W)$.

(5) $d_{\omega} \omega=\Omega$, the curvature form.

(6) $d_{\omega} \Omega=0$, the Bianchi identity.

(7) $d_{\omega} \circ \chi^{*}-d_{\omega}=\chi^{*} \circ i(R)$, where $R$ is the curvature.

(8) $d_{\omega} \circ d_{\omega}=\chi^{*} \circ i(R) \circ d$.

(9) Let $\Omega_{\text {hor }}(P, \mathfrak{g})^{G}$ be the algebra of all horizontal $G$-equivariant $\mathfrak{g}$-valued forms, i.e., $\left(r^{g}\right)^{*} \psi=\operatorname{Ad}\left(g^{-1}\right) \psi$. Then for any $\psi \in \Omega_{\text {hor }}(P, \mathfrak{g})^{G}$ we have $d_{\omega} \psi=d \psi+[\omega, \psi]_{\wedge}$.

(10) The mapping $\psi \mapsto \zeta_{\psi}$, where $\zeta_{\psi}\left(X_{1}, \ldots, X_{k}\right)(u)=\zeta_{\psi\left(X_{1}, \ldots, X_{k}\right)(u)}(u)$, is an isomorphism between $\Omega_{h o r}(P, \mathfrak{g})^{G}$ and the algebra $\Omega_{\text {hor }}(P, V P)^{G}$ of all horizontal $G$-equivariant forms with values in the vertical bundle $V P$. Then we have $\zeta_{d_{\omega} \psi}=-\left[\Phi, \zeta_{\psi}\right]$.

Proof. Parts (11) through (4) follow from the properties of $\chi^{*}$. 
(5) We have

$$
\begin{aligned}
\left(d_{\omega} \omega\right)(\xi, \eta) & =\left(\chi^{*} d \omega\right)(\xi, \eta)=d \omega(\chi \xi, \chi \eta) \\
& =(\chi \xi) \omega(\chi \eta)-(\chi \eta) \omega(\chi \xi)-\omega([\chi \xi, \chi \eta]) \\
& =-\omega([\chi \xi, \chi \eta]), \\
-\zeta(\Omega(\xi, \eta)) & =R(\xi, \eta)=\Phi[\chi \xi, \chi \eta]=\zeta_{\omega([\chi \xi, \chi \eta])} .
\end{aligned}
$$

(6) Using (19.2), we have

$$
\begin{aligned}
d_{\omega} \Omega & =d_{\omega}\left(d \omega+\frac{1}{2}[\omega, \omega]_{\wedge}\right) \\
& =\chi^{*} d d \omega+\frac{1}{2} \chi^{*} d[\omega, \omega]_{\wedge} \\
& =\frac{1}{2} \chi^{*}\left([d \omega, \omega]_{\wedge}-[\omega, d \omega]_{\wedge}\right)=\chi^{*}[d \omega, \omega]_{\wedge} \\
& =\left[\chi^{*} d \omega, \chi^{*} \omega\right]_{\wedge}=0, \text { since } \chi^{*} \omega=0 .
\end{aligned}
$$

(17) For $\varphi \in \Omega(P, W)$ we have

$$
\begin{aligned}
\left(d_{\omega} \chi^{*} \varphi\right)( & \left.X_{0}, \ldots, X_{k}\right)=\left(d \chi^{*} \varphi\right)\left(\chi\left(X_{0}\right), \ldots, \chi\left(X_{k}\right)\right) \\
= & \sum_{0 \leq i \leq k}(-1)^{i} \chi\left(X_{i}\right)\left(\left(\chi^{*} \varphi\right)\left(\chi\left(X_{0}\right), \ldots, \widehat{\chi\left(X_{i}\right)}, \ldots, \chi\left(X_{k}\right)\right)\right) \\
& +\sum_{i<j}(-1)^{i+j}\left(\chi^{*} \varphi\right)\left(\left[\chi\left(X_{i}\right), \chi\left(X_{j}\right)\right], \chi\left(X_{0}\right), \ldots\right. \\
& \left.\ldots, \widehat{\chi\left(X_{i}\right)}, \ldots, \widehat{\chi\left(X_{j}\right)}, \ldots\right) \\
= & \sum_{0 \leq i \leq k}(-1)^{i} \chi\left(X_{i}\right)\left(\varphi\left(\chi\left(X_{0}\right), \ldots, \widehat{\chi\left(X_{i}\right)}, \ldots, \chi\left(X_{k}\right)\right)\right) \\
& +\sum_{i<j}(-1)^{i+j} \varphi\left(\left[\chi\left(X_{i}\right), \chi\left(X_{j}\right)\right]-\Phi\left[\chi\left(X_{i}\right), \chi\left(X_{j}\right)\right], \chi\left(X_{0}\right), \ldots\right. \\
\ldots & \left.\ldots, \widehat{\chi\left(X_{i}\right)}, \ldots, \widehat{\chi\left(X_{j}\right)}, \ldots\right) \\
= & (d \varphi)\left(\chi\left(X_{0}\right), \ldots, \chi\left(X_{k}\right)\right)+\left(i_{R} \varphi\right)\left(\chi\left(X_{0}\right), \ldots, \chi\left(X_{k}\right)\right) \\
= & \left(d_{\omega}+\chi^{*} i_{R}\right)(\varphi)\left(X_{0}, \ldots, X_{k}\right) .
\end{aligned}
$$

(8) $d_{\omega} d_{\omega}=\chi^{*} d \chi^{*} d=\left(\chi^{*} i_{R}+\chi^{*} d\right) d=\chi^{*} i_{R} d$ holds by (7).

(9) If we insert one vertical vector field, say $\zeta_{X}$ for $X \in \mathfrak{g}$, into $d_{\omega} \psi$, we get 0 by definition. For the right hand side we use $i_{\zeta_{X}} \psi=0$ and $\mathcal{L}_{\zeta_{X}} \psi=$ $\left.\partial\right|_{0}\left(\mathrm{Fl}_{t}^{\zeta X}\right)^{*} \psi=\left.\partial\right|_{0}\left(r^{\exp t X}\right) * \psi=\left.\partial\right|_{0} \operatorname{Ad}(\exp (-t X)) \psi=-\operatorname{ad}(X) \psi$ to get

$$
\begin{aligned}
i_{\zeta_{X}}\left(d \psi+[\omega, \psi]_{\wedge}\right) & =i_{\zeta_{X}} d \psi+d i_{\zeta_{X}} \psi+\left[i_{\zeta_{X}} \omega, \psi\right]-\left[\omega, i_{\zeta_{X}} \psi\right] \\
& =\mathcal{L}_{\zeta_{X}} \psi+[X, \psi]=-\operatorname{ad}(X) \psi+[X, \psi]=0 .
\end{aligned}
$$

Now let all vector fields $\xi_{i}$ be horizontal; then we get

$$
\begin{gathered}
\left(d_{\omega} \psi\right)\left(\xi_{0}, \ldots, \xi_{k}\right)=\left(\chi^{*} d \psi\right)\left(\xi_{0}, \ldots, \xi_{k}\right)=d \psi\left(\xi_{0}, \ldots, \xi_{k}\right), \\
\left(d \psi+[\omega, \psi]_{\wedge}\right)\left(\xi_{0}, \ldots, \xi_{k}\right)=d \psi\left(\xi_{0}, \ldots, \xi_{k}\right) .
\end{gathered}
$$


So the first formula holds.

(10) We proceed in a similar manner. Let $\Psi$ be in the space $\Omega_{\text {hor }}^{\ell}(P, V P)^{G}$ of all horizontal $G$-equivariant forms with vertical values. Then for each $X \in \mathfrak{g}$ we have $i_{\zeta_{X}} \Psi=0$; furthermore the $G$-equivariance $\left(r^{g}\right)^{*} \Psi=\Psi$ implies that $\mathcal{L}_{\zeta_{X}} \Psi=\left[\zeta_{X}, \Psi\right]=0$ by (16.16.5), Using formula (16.11.2), we have

$$
\begin{aligned}
i_{\zeta_{X}}[\Phi, \Psi] & =\left[i_{\zeta_{X}} \Phi, \Psi\right]-\left[\Phi, i_{\zeta_{X}} \Psi\right]+i\left(\left[\Phi, \zeta_{X}\right]\right) \Psi+i\left(\left[\Psi, \zeta_{X}\right]\right) \Phi \\
& =\left[\zeta_{X}, \Psi\right]-0+0+0=0 .
\end{aligned}
$$

Now let all vector fields $\xi_{i}$ again be horizontal; then from the huge formula $(16.9)$ for the Frölicher-Nijenhuis bracket only the following terms in the third and fifth lines survive:

$$
\begin{aligned}
{[\Phi, \Psi]\left(\xi_{1}, \ldots, \xi_{\ell+1}\right) } & =\frac{(-1)^{\ell}}{\ell !} \sum_{\sigma} \operatorname{sign} \sigma \Phi\left(\left[\Psi\left(\xi_{\sigma 1}, \ldots, \xi_{\sigma \ell}\right), \xi_{\sigma(\ell+1)}\right]\right) \\
& +\frac{1}{(\ell-1) ! 2 !} \sum_{\sigma} \operatorname{sign} \sigma \Phi\left(\Psi\left(\left[\xi_{\sigma 1}, \xi_{\sigma 2}\right], \xi_{\sigma 3}, \ldots, \xi_{\sigma(\ell+1)}\right)\right) .
\end{aligned}
$$

For $f: P \rightarrow \mathfrak{g}$ and horizontal $\xi$ we have $\Phi\left[\xi, \zeta_{f}\right]=\zeta_{\xi(f)}=\zeta_{d f(\xi)}$ : It is $C^{\infty}(P)$-linear in $\xi$; or imagine it in local coordinates. So the last expression becomes

$$
-\zeta\left(d_{\omega} \psi\left(\xi_{0}, \ldots, \xi_{k}\right)\right)=-\zeta\left(d \psi\left(\xi_{0}, \ldots, \xi_{k}\right)\right)=-\zeta\left(\left(d \psi+[\omega, \psi]_{\wedge}\right)\left(\xi_{0}, \ldots, \xi_{k}\right)\right)
$$

as required.

19.6. Theorem. Let $(P, p, M, G)$ be a principal fiber bundle with principal connection $\omega$. Then the parallel transport for the principal connection is globally defined and G-equivariant.

In detail: For each smooth curve $c: \mathbb{R} \rightarrow M$ there is a smooth mapping $\mathrm{Pt}_{c}: \mathbb{R} \times P_{c(0)} \rightarrow P$ such that the following hold:

(1) $\operatorname{Pt}(c, t, u) \in P_{c(t)}, \operatorname{Pt}(c, 0)=I d_{P_{c(0)}}$, and $\omega\left(\frac{d}{d t} \operatorname{Pt}(c, t, u)\right)=0$.

(2) $\operatorname{Pt}(c, t): P_{c(0)} \rightarrow P_{c(t)}$ is G-equivariant, i.e., $\operatorname{Pt}(c, t, u . g)=\operatorname{Pt}(c, t, u) . g$ holds for all $g \in G$ and $u \in P$. Moreover we have $\operatorname{Pt}(c, t)^{*}\left(\zeta_{X} \mid P_{c(t)}\right)=$ $\zeta_{X} \mid P_{c(0)}$ for all $X \in \mathfrak{g}$.

(3) For any smooth function $f: \mathbb{R} \rightarrow \mathbb{R}$ we have

$\operatorname{Pt}(c, f(t), u)=\operatorname{Pt}(c \circ f, t, \operatorname{Pt}(c, f(0), u))$.

Proof. By (19.4) the Christoffel forms $\Gamma^{\alpha} \in \Omega^{1}\left(U_{\alpha}, \mathfrak{X}(G)\right)$ of the connection $\omega$ with respect to a principal fiber bundle atlas $\left(U_{\alpha}, \varphi_{\alpha}\right)$ are given by $\Gamma^{\alpha}\left(\xi_{x}\right)=R_{\omega_{\alpha}\left(\xi_{x}\right)}$, so they take values in the Lie subalgebra $\mathfrak{X}_{R}(G)$ of all right invariant vector fields on $G$, which are bounded with respect to any right invariant Riemann metric on $G$. Each right invariant metric on a Lie group is complete. So the connection is complete by proposition (23.9). 
Properties (11) and (3) follow from theorem (17.8), and (2) is seen as follows: We have $\omega\left(\frac{d}{d t} \operatorname{Pt}(c, t, u) \cdot g\right)=\operatorname{Ad}\left(g^{-1}\right) \omega\left(\frac{d}{d t} \operatorname{Pt}(c, t, u)\right)=0$, and this implies $\operatorname{Pt}(c, t, u) \cdot g=\operatorname{Pt}(c, t, u . g)$. For the second assertion we compute for $u \in$ $P_{c(0)}$ :

$$
\begin{aligned}
\operatorname{Pt}(c, t)^{*}\left(\zeta_{X} \mid P_{c(t)}\right)(u) & =T \operatorname{Pt}(c, t)^{-1} \zeta_{X}(\operatorname{Pt}(c, t, u)) \\
& =\left.T \operatorname{Pt}(c, t)^{-1} \frac{d}{d s}\right|_{0} \operatorname{Pt}(c, t, u) \cdot \exp (s X) \\
& =\left.T \operatorname{Pt}(c, t)^{-1} \frac{d}{d s}\right|_{0} \operatorname{Pt}(c, t, u \cdot \exp (s X)) \\
& =\left.\frac{d}{d s}\right|_{0} \operatorname{Pt}(c, t)^{-1} \operatorname{Pt}(c, t, u \cdot \exp (s X)) \\
& =\left.\frac{d}{d s}\right|_{0} u \cdot \exp (s X)=\zeta_{X}(u) . \quad \square
\end{aligned}
$$

19.7. Holonomy groups. Let $(P, p, M, G)$ be a principal fiber bundle with principal connection $\Phi=\zeta \circ \omega$. We assume that $M$ is connected and we fix $x_{0} \in M$.

In (17.10) we defined the holonomy group $\operatorname{Hol}\left(\Phi, x_{0}\right) \subset \operatorname{Diff}\left(P_{x_{0}}\right)$ as the group of all $\operatorname{Pt}(c, 1): P_{x_{0}} \rightarrow P_{x_{0}}$ for $c$ any piecewise smooth closed loop through $x_{0}$. (Reparameterizing $c$ by a function which is flat at each corner of $c$, we may assume that any $c$ is smooth.) If we consider only those curves $c$ which are nullhomotopic, we obtain the restricted holonomy group $\operatorname{Hol}_{0}\left(\Phi, x_{0}\right)$, a normal subgroup.

Now let us fix $u_{0} \in P_{x_{0}}$. The elements $\tau\left(u_{0}, \operatorname{Pt}\left(c, 1, u_{0}\right)\right) \in G$ (for $c$ all piecewise smooth closed loops through $x_{0}$ ) form a subgroup of the structure group $G$ which is isomorphic to $\operatorname{Hol}\left(\Phi, x_{0}\right)$; we denote it by $\operatorname{Hol}\left(\omega, u_{0}\right)$ and we call it again the holonomy group of the connection. Considering only nullhomotopic curves, we get the restricted holonomy group $\operatorname{Hol}_{0}\left(\omega, u_{0}\right)$, a normal subgroup of $\operatorname{Hol}\left(\omega, u_{0}\right)$.

Theorem. Let $(P, p, M, G)$ be a principal fiber bundle with principal connection $\Phi=\zeta \circ \omega$. We assume that $M$ is connected and we fix $x_{0} \in M$ and $u_{0} \in P_{x_{0}}$.

(1) We have an isomorphism $\operatorname{Hol}\left(\omega, u_{0}\right) \rightarrow \operatorname{Hol}\left(\Phi, x_{0}\right)$ given by $g \mapsto\left(u \mapsto f_{g}(u)=u_{0} . g \cdot \tau\left(u_{0}, u\right)\right)$ with inverse $g_{f}:=\tau\left(u_{0}, f\left(u_{0}\right)\right) \leftarrow f$.

(2) We have $\operatorname{Hol}\left(\omega, u_{0} . g\right)=\operatorname{conj}\left(g^{-1}\right) \operatorname{Hol}\left(\omega, u_{0}\right)$ and $\operatorname{Hol}_{0}\left(\omega, u_{0} . g\right)=\operatorname{conj}\left(g^{-1}\right) \operatorname{Hol}_{0}\left(\omega, u_{0}\right)$.

(3) For any curve $c$ with $c(0)=x_{0}$ we have $\operatorname{Hol}\left(\omega, \operatorname{Pt}\left(c, t, u_{0}\right)\right)=\operatorname{Hol}\left(\omega, u_{0}\right)$ and $\operatorname{Hol}_{0}\left(\omega, \operatorname{Pt}\left(c, t, u_{0}\right)\right)=\operatorname{Hol}_{0}\left(\omega, u_{0}\right)$.

(4) The restricted holonomy group $\mathrm{Hol}_{0}\left(\omega, u_{0}\right)$ is a connected Lie subgroup of $G$. The quotient group $\operatorname{Hol}\left(\omega, u_{0}\right) / \operatorname{Hol}_{0}\left(\omega, u_{0}\right)$ is at most countable, so $\operatorname{Hol}\left(\omega, u_{0}\right)$ is also a Lie subgroup of $G$. 
(5) The Lie algebra $\operatorname{hol}\left(\omega, u_{0}\right) \subset \mathfrak{g}$ of $\operatorname{Hol}\left(\omega, u_{0}\right)$ is generated by $\left\{\Omega\left(X_{u}, Y_{u}\right): X_{u}, Y_{u} \in T_{u} P, u=\operatorname{Pt}\left(c, 1, u_{0}\right), c:[0,1] \rightarrow M, c(0)=x_{0}\right\}$ as a vector space. It is isomorphic to the Lie algebra $\operatorname{hol}\left(\Phi, x_{0}\right)$ we considered in (17.10).

(6) For $u_{0} \in P_{x_{0}}$ let $P\left(\omega, u_{0}\right)$ be the set of all $\mathrm{Pt}\left(c, t, u_{0}\right)$ for $c$ any (piecewise) smooth curve in $M$ with $c(0)=x_{0}$ and for $t \in \mathbb{R}$. Then $P\left(\omega, u_{0}\right)$ is a fiber subbundle of $P$ which is invariant under the right action of $\operatorname{Hol}\left(\omega, u_{0}\right)$; so it is itself a principal fiber bundle over $M$ with structure group $\mathrm{Hol}\left(\omega, u_{0}\right)$ and we have a reduction of structure group; see (18.6) and (18.14). The pullback of $\omega$ to $P\left(\omega, u_{0}\right)$ is also a principal connection form $i^{*} \omega \in \Omega^{1}\left(P\left(\omega, u_{0}\right) ; \operatorname{hol}\left(\omega, u_{0}\right)\right)$.

(7) $P$ is foliated by the leaves $P(\omega, u), u \in P_{x_{0}}$.

(8) If the curvature $\Omega=0$, then $\operatorname{Hol}_{0}\left(\omega, u_{0}\right)=\{e\}$ and each $P(\omega, u)$ is a covering of $M$. The leaves $P(\omega, u)$ are all isomorphic and are associated to the universal covering of $M$, which is a principal fiber bundle with structure group the fundamental group $\pi_{1}(M)$.

In view of assertion (6) a principal connection $\omega$ is called irreducible if $\operatorname{Hol}\left(\omega, u_{0}\right)$ equals the structure group $G$ for some (equivalently: any) $u_{0} \in$ $P_{x_{0}}$.

Proof. (11) follows from the definition of $\operatorname{Hol}\left(\omega, u_{0}\right)$.

(2) This follows from the properties of the mapping $\tau$ from (18.2) and from the $G$-equivariance of the parallel transport:

$$
\tau\left(u_{0} . g, \operatorname{Pt}\left(c, 1, u_{0} . g\right)\right)=\tau\left(u_{0}, \operatorname{Pt}\left(c, 1, u_{0}\right) . g\right)=g^{-1} \cdot \tau\left(u_{0}, \operatorname{Pt}\left(c, 1, u_{0}\right)\right) . g .
$$

So via the diffeomorphism $\tau\left(u_{0}, \quad\right): P_{x_{0}} \rightarrow G$ the action of the holonomy group $\operatorname{Hol}\left(\Phi, u_{0}\right)$ on $P_{x_{0}}$ is conjugate to the left translation of $\operatorname{Hol}\left(\omega, u_{0}\right)$ on $G$.

(3) By reparameterizing the curve $c$, we may assume that $t=1$, and we put $\operatorname{Pt}\left(c, 1, u_{0}\right)=: u_{1}$. Then by definition, for an element $g \in G$ we have $g \in \operatorname{Hol}\left(\omega, u_{1}\right)$ if and only if $g=\tau\left(u_{1}, \operatorname{Pt}\left(e, 1, u_{1}\right)\right)$ for some closed smooth loop $e$ through $x_{1}:=c(1)=p\left(u_{1}\right)$, i.e.,

$$
\begin{aligned}
\operatorname{Pt}(c, 1)\left(u_{0} . g\right) & =\operatorname{Pt}(c, 1)\left(r^{g}\left(u_{0}\right)\right)=r^{g}\left(\operatorname{Pt}(c, 1)\left(u_{0}\right)\right)=u_{1} g \\
& =\operatorname{Pt}(e, 1)\left(\operatorname{Pt}(c, 1)\left(u_{0}\right)\right) \\
u_{0} . g & =\operatorname{Pt}(c, 1)^{-1} \operatorname{Pt}(e, 1) \operatorname{Pt}(c, 1)\left(u_{0}\right)=\operatorname{Pt}\left(c . e . c^{-1}, 3\right)\left(u_{0}\right),
\end{aligned}
$$

where c.e. $c^{-1}$ is the curve traveling along $c(t)$ for $0 \leq t \leq 1$, along $e(t-1)$ for $1 \leq t \leq 2$, and along $c(3-t)$ for $2 \leq t \leq 3$. This is equivalent to $g \in \operatorname{Hol}\left(\omega, u_{0}\right)$. Furthermore $e$ is nullhomotopic if and only if c.e. $c^{-1}$ is nullhomotopic, so we also have $\operatorname{Hol}_{0}\left(\omega, u_{1}\right)=\operatorname{Hol}_{0}\left(\omega, u_{0}\right)$. 
(44) Let $c:[0,1] \rightarrow M$ be a nullhomotopic curve through $x_{0}$ and let $h: \mathbb{R}^{2} \rightarrow$ $M$ be a smooth homotopy with $h_{1} \mid[0,1]=c$ and $h(0, s)=h(t, 0)=h(t, 1)=$ $x_{0}$. We consider the pullback bundle

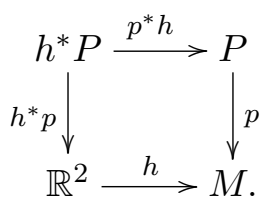

Then for the parallel transport $\mathrm{Pt}^{\Phi}$ on $P$ and for the parallel transport $\mathrm{Pt}^{h^{*}} \Phi$ of the pulled back connection we have

$$
\mathrm{Pt}^{\Phi}\left(h_{t}, 1, u_{0}\right)=\left(p^{*} h\right) \mathrm{Pt}^{h^{*} \Phi}\left((t, \quad), 1, u_{0}\right)=\left(p^{*} h\right) \mathrm{Fl}_{1}^{C^{h^{*} \Phi} \partial_{s}}\left(t, u_{0}\right) .
$$

So $t \mapsto \tau\left(u_{0}, \mathrm{Pt}^{\Phi}\left(h_{t}, 1, u_{0}\right)\right)$ is a smooth curve in the Lie group $G$ starting from $e$, so $\operatorname{Hol}_{0}\left(\omega, u_{0}\right)$ is a smoothly arcwise connected subgroup of $G$. By theorem (5.6) the subgroup $\operatorname{Hol}_{0}\left(\omega, u_{0}\right)$ is a Lie subgroup of $G$. The quotient group $\operatorname{Hol}\left(\omega, u_{0}\right) / \operatorname{Hol}_{0}\left(\omega, u_{0}\right)$ is a countable group, since by Morse theory $M$ is homotopy equivalent to a countable CW-complex, so the fundamental group $\pi_{1}(M)$ is countably generated, thus countable.

(51) Note first that for $g \in G$ and $X \in \mathfrak{X}(M)$ we have for the horizontal lift $\left(r^{g}\right)^{*} C X=C X$, since $\left(r^{g}\right)^{*} \Phi=\Phi$ implies $T_{u}\left(r^{g}\right) \cdot H_{u} P=H_{u . g} P$ and thus

$$
\begin{aligned}
T_{u}\left(r^{g}\right) \cdot C(X, u) & =T_{u}\left(r^{g}\right) \cdot\left(T_{u} p \mid H_{u} P\right)^{-1}(X(p(u))) \\
& =\left(T_{u . g} p \mid H_{u . g} P\right)^{-1}(X(p(u)))=C(X, u . g) .
\end{aligned}
$$

The vector space $\operatorname{hol}(\omega) \subset \mathfrak{g}$ is normalized by the subgroup $\operatorname{Hol}\left(\omega, u_{0}\right) \subseteq$ $G$ since for $g=\tau\left(u_{0}, \operatorname{Pt}\left(c, 1, u_{0}\right)\right)$ (where $c$ is a loop at $x_{0}$ ) and for $u=$ $\operatorname{Pt}\left(c_{1}, 1, u_{0}\right)$ (where $\left.c_{1}(0)=x_{0}\right)$ we have

$$
\begin{aligned}
\operatorname{Ad}\left(g^{-1}\right) \Omega(C(X, u), C(Y, u)) & =\Omega\left(T_{u}\left(r^{g}\right) \cdot C(X, u), T_{u}\left(r^{g}\right) \cdot C(Y, u)\right) \\
& =\Omega(C(X, u \cdot g), C(Y, u \cdot g)) \in \operatorname{hol}(\omega), \\
u . g=\operatorname{Pt}\left(c_{1}, 1, u_{0}\right) \cdot g & =\operatorname{Pt}\left(c_{1}, 1, u_{0} \cdot g\right)=\operatorname{Pt}\left(c_{1}, 1, \operatorname{Pt}\left(c, 1, u_{0}\right)\right) \\
& =\operatorname{Pt}\left(c_{1} \cdot c, 2, u_{0}\right) .
\end{aligned}
$$

We consider now the mapping

$$
\begin{gathered}
\xi^{u_{0}}: \operatorname{hol}(\omega) \rightarrow \mathfrak{X}\left(P_{x_{0}}\right), \\
\xi_{X}^{u_{0}}(u)=\zeta_{\operatorname{Ad}\left(\tau\left(u_{0}, u\right)^{-1}\right) X}(u) .
\end{gathered}
$$

It turns out that $\xi_{X}^{u_{0}}$ is related to the right invariant vector field $R_{X}$ on $G$ under the diffeomorphism $\tau\left(u_{0}, \quad\right)=\left(r_{u_{0}}\right)^{-1}: P_{x_{0}} \rightarrow G$, since we have

$$
\begin{aligned}
T_{g}\left(r_{u_{0}}\right) \cdot R_{X}(g) & =T_{g}\left(r_{u_{0}}\right) \cdot T_{e}\left(\mu^{g}\right) \cdot X=T_{u_{0}}\left(r^{g}\right) \cdot T_{e}\left(r_{u_{0}}\right) \cdot X \\
& =T_{u_{0}}\left(r^{g}\right) \zeta_{X}\left(u_{0}\right)=\zeta_{\operatorname{Ad}\left(g^{-1}\right) X}\left(u_{0} \cdot g\right)=\xi_{X}^{u_{0}}\left(u_{0} \cdot g\right) .
\end{aligned}
$$


Thus $\xi^{u_{0}}$ is the restriction to $\operatorname{hol}(\omega) \subseteq \mathfrak{g}$ of a Lie algebra antihomomorphism $\mathfrak{g} \rightarrow \mathfrak{X}\left(P_{x_{0}}\right)$, and each vector field $\xi_{X}^{u_{0}}$ on $P_{x_{0}}$ is complete. The dependence of $\xi^{u_{0}}$ on $u_{0}$ is explained by

$$
\begin{aligned}
\xi_{X}^{u_{0} g}(u) & =\zeta_{\operatorname{Ad}\left(\tau\left(u_{0} g, u\right)^{-1}\right) X}(u)=\zeta_{\operatorname{Ad}\left(\tau\left(u_{0}, u\right)^{-1}\right) \operatorname{Ad}(g) X}(u) \\
& =\xi_{\operatorname{Ad}(g) X}^{u_{0}}(u)
\end{aligned}
$$

Recall now that the holonomy Lie algebra hol $\left(\Phi, x_{0}\right)$ is the closed linear span of all vector fields of the form $\operatorname{Pt}(c, 1)^{*} R(C X, C Y)$, where $X, Y \in T_{x} M$ and $c$ is a curve from $x_{0}$ to $x$. Then we have for $u=\operatorname{Pt}\left(c, 1, u_{0}\right)$

$$
\begin{aligned}
R(C(X, u), C(Y, u)) & =\zeta_{\Omega(C(X, u), C(Y, u))}(u), \\
R(C X, C Y)(u g) & =T\left(r^{g}\right) R(C X, C Y)(u)=T\left(r^{g}\right) \zeta_{\Omega(C(X, u), C(Y, u))}(u) \\
& =\zeta_{\operatorname{Ad}\left(g^{-1}\right) \Omega(C(X, u), C(Y, u))}(u g)=\xi_{\Omega(C(X, u), C(Y, u))}^{u}(u g), \\
\left(\operatorname{Pt}(c, 1)^{*} R(C X, C Y)\right)\left(u_{0} . g\right)= & \\
& =T\left(\operatorname{Pt}(c, 1)^{-1}\right) \zeta_{\operatorname{Ad}\left(g^{-1}\right) \Omega(C(X, u), C(Y, u))}\left(\operatorname{Pt}\left(c, 1, u_{0} . g\right)\right) \\
& =\left(\operatorname{Pt}(c, 1)^{*} \zeta_{\operatorname{Ad}\left(g^{-1}\right) \Omega(C(X, u), C(Y, u))}\right)\left(u_{0} . g\right) \\
& =\zeta_{\operatorname{Ad}\left(g^{-1}\right) \Omega(C(X, u), C(Y, u))}\left(u_{0} . g\right) \quad \text { by }(19.6 .2) \\
& =\xi_{\Omega(C(X, u), C(Y, u))}^{u_{0}}\left(u_{0} . g\right) .
\end{aligned}
$$

So $\xi^{u_{0}}: \operatorname{hol}(\omega) \rightarrow \operatorname{hol}\left(\Phi, x_{0}\right)$ is a linear isomorphic. Since $\operatorname{hol}\left(\Phi, x_{0}\right)$ is a Lie subalgebra of $\mathfrak{X}\left(P_{x_{0}}\right)$ by (17.10) and $\xi^{u_{0}}: \mathfrak{g} \rightarrow \mathfrak{X}\left(P_{x_{0}}\right)$ is a Lie algebra antihomomorphism, $\operatorname{hol}(\omega)$ is a Lie subalgebra of $\mathfrak{g}$. Moreover $\operatorname{hol}\left(\Phi, x_{0}\right)$ consists of complete vector fields and we may apply theorem (17.11) (only claim 3) which tells us that the Lie algebra of the Lie group $\operatorname{Hol}\left(\Phi, x_{0}\right)$ is $\operatorname{hol}\left(\Phi, x_{0}\right)$. The diffeomorphism $\tau\left(u_{0}, \quad\right): P_{x_{0}} \rightarrow G$ intertwines the actions and the infinitesimal actions in the right way.

(66) We define the vector subbundle $E \subset T P$ by $E_{u}:=H_{u} P+T_{e}\left(r_{u}\right) \cdot \operatorname{hol}(\omega)$. From the proof of (4) it follows that $\xi_{X}^{u_{0}}$ are sections of $E$ for each $X \in \operatorname{hol}(\omega)$; thus $E$ is a vector bundle. Any vector field $\eta \in \mathfrak{X}(P)$ with values in $E$ is a linear combination with coefficients in $C^{\infty}(P)$ of horizontal vector fields $C X$ for $X \in \mathfrak{X}(M)$ and of $\zeta_{Z}$ for $Z \in \operatorname{hol}(\omega)$. Their Lie brackets are in turn

$$
\begin{aligned}
{[C X, C Y](u) } & =C[X, Y](u)+R(C X, C Y)(u) \\
& =C[X, Y](u)+\zeta_{\Omega(C(X, u), C(Y, u))}(u) \in \Gamma(E), \\
{\left[\zeta_{Z}, C X\right] } & =\mathcal{L}_{\zeta_{Z}} C X=\left.\frac{d}{d t}\right|_{0}\left(\mathrm{Fl}_{t}^{\zeta Z}\right)^{*} C X=0,
\end{aligned}
$$

since $\left(r^{g}\right)^{*} C X=C X$; see step (5) above. So $E$ is an integrable subbundle and induces a foliation by (3.28.2), Let $L\left(u_{0}\right)$ be the leaf of the foliation through $u_{0}$. Since for a curve $c$ in $M$ the parallel transport $\operatorname{Pt}\left(c, t, u_{0}\right)$ is tangent to the leaf, we have $P\left(\omega, u_{0}\right) \subseteq L\left(u_{0}\right)$. By definition the holonomy $\operatorname{group} \operatorname{Hol}\left(\Phi, x_{0}\right)$ acts transitively and freely on $P\left(\omega, u_{0}\right) \cap P_{x_{0}}$, and by (5) the 
restricted holonomy group $\operatorname{Hol}_{0}\left(\Phi, x_{0}\right)$ acts transitively on each connected component of $L\left(u_{0}\right) \cap P_{x_{0}}$, since the vertical part of $E$ is spanned by the generating vector fields of this action. This is true for any fiber since we may conjugate the holonomy groups by a suitable parallel transport to each fiber. Thus $P\left(\omega, u_{0}\right)=L\left(u_{0}\right)$ and by lemma (18.3) the fiber subbundle $P\left(\omega, x_{0}\right)$ is a principal fiber bundle with structure group $\operatorname{Hol}\left(\omega, u_{0}\right)$. Since all horizontal spaces $H_{u} P$ with $u \in P\left(\omega, x_{0}\right)$ are tangential to $P\left(\omega, x_{0}\right)$, the connection $\Phi$ restricts to a principal connection on $P\left(\omega, x_{0}\right)$ and we obtain the reduction we looked for of the structure group.

(77) This is obvious from the proof of (6) .

(8) If the curvature $\Omega$ is everywhere 0 , the holonomy Lie algebra is zero, so $P(\omega, u)$ is a principal fiber bundle with discrete structure group; thus $p \mid P(\omega, u): P(\omega, u) \rightarrow M$ is a local diffeomorphism, since $T_{u} P(\omega, u)=H_{u} P$ and $T p$ is invertible on it. By the right action of the structure group we may translate each local section of $p$ to any point of the fiber, so $p$ is a covering map. Parallel transport defines a group homomorphism $\varphi: \pi_{1}\left(M, x_{0}\right) \rightarrow$ $\operatorname{Hol}\left(\Phi, u_{0}\right) \cong \operatorname{Hol}\left(\omega, u_{0}\right)$ (see the proof of (4) $)$. Let $\tilde{M}$ be the universal covering space of $M$; then from topology one knows that $\tilde{M} \rightarrow M$ is a principal fiber bundle with discrete structure group $\pi_{1}\left(M, x_{0}\right)$. Let $\pi_{1}(M)$ act on $\operatorname{Hol}\left(\omega, u_{0}\right)$ by left translation via $\varphi$; then the mapping $f: \tilde{M} \times$ $\operatorname{Hol}\left(\omega, u_{0}\right) \rightarrow P\left(\omega, u_{0}\right)$ which is given by $f([c], g)=\operatorname{Pt}\left(c, 1, u_{0}\right) \cdot g$ is $\pi_{1}(M)$ invariant and thus factors to a mapping

$$
\tilde{M} \times_{\pi_{1}(M)} \operatorname{Hol}\left(\omega, u_{0}\right)=\tilde{M}\left[\operatorname{Hol}\left(\omega, u_{0}\right)\right] \rightarrow P\left(\omega, u_{0}\right)
$$

which is an isomorphism of $\operatorname{Hol}\left(\omega, u_{0}\right)$-bundles since the upper mapping admits local sections by the curve lifting property of the universal cover.

19.8. Inducing principal connections on associated bundles. We consider a principal bundle $(P, p, M, G)$ with principal right action $r: P \times$ $G \rightarrow P$ and let $\ell: G \times S \rightarrow S$ be a left action of the structure group $G$ on some manifold $S$. Then we consider the associated bundle $P[S]=P[S, \ell]=$ $P \times_{G} S$, constructed in (18.7), Recall from (18.18) that its tangent and vertical bundle are given by $T(P[S, \ell])=T P[T S, T \ell]=T P \times_{T G} T S$ and $V(P[S, \ell])=P\left[T S, T_{2} \ell\right]=P \times_{G} T S$.

Let $\Phi=\zeta \circ \omega \in \Omega^{1}(P, T P)$ be a principal connection on the principal bundle $P$. We construct the induced connection $\bar{\Phi} \in \Omega^{1}(P[S], T(P[S]))$ by factorizing as in the following diagram:

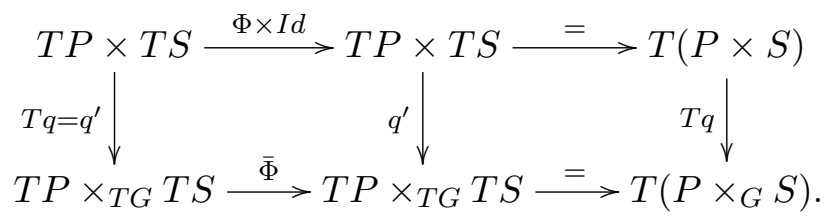


Let us first check that the top mapping $\Phi \times I d$ is $T G$-equivariant. For $g \in G$ and $X \in \mathfrak{g}$ the inverse of $T_{e}\left(\mu_{g}\right) X$ in the Lie group $T G$ is denoted by $\left(T_{e}\left(\mu_{g}\right) X\right)^{-1}$; see lemma (6.7), Furthermore by (6.3) we have

$$
\begin{aligned}
\operatorname{Tr}\left(\xi_{u}, T_{e}\left(\mu_{g}\right) X\right) & =T_{u}\left(r^{g}\right) \xi_{u}+\operatorname{Tr}\left(\left(0_{P} \times L_{X}\right)(u, g)\right) \\
& =T_{u}\left(r^{g}\right) \xi_{u}+T_{g}\left(r_{u}\right)\left(T_{e}\left(\mu_{g}\right) X\right) \\
& =T_{u}\left(r^{g}\right) \xi_{u}+\zeta_{X}(u g) .
\end{aligned}
$$

We may compute

$$
\begin{aligned}
(\Phi \times & I d)\left(\operatorname{Tr}\left(\xi_{u}, T_{e}\left(\mu_{g}\right) X\right), T \ell\left(\left(T_{e}\left(\mu_{g}\right) X\right)^{-1}, \eta_{s}\right)\right) \\
& =\left(\Phi\left(T_{u}\left(r^{g}\right) \xi_{u}+\zeta_{X}(u g)\right), T \ell\left(\left(T_{e}\left(\mu_{g}\right) X\right)^{-1}, \eta_{s}\right)\right) \\
& =\left(\Phi\left(T_{u}\left(r^{g}\right) \xi_{u}\right)+\Phi\left(\zeta_{X}(u g)\right), T \ell\left(\left(T_{e}\left(\mu_{g}\right) X\right)^{-1}, \eta_{s}\right)\right) \\
& =\left(\left(T_{u}\left(r^{g}\right) \Phi \xi_{u}\right)+\zeta_{X}(u g), T \ell\left(\left(T_{e}\left(\mu_{g}\right) X\right)^{-1}, \eta_{s}\right)\right) \\
& =\left(\operatorname{Tr}\left(\Phi\left(\xi_{u}\right), T_{e}\left(\mu_{g}\right) X\right), T \ell\left(\left(T_{e}\left(\mu_{g}\right) X\right)^{-1}, \eta_{s}\right)\right) .
\end{aligned}
$$

So the mapping $\Phi \times I d$ factors to $\bar{\Phi}$ as indicated in the diagram, and we have $\bar{\Phi} \circ \bar{\Phi}=\bar{\Phi}$ from $(\Phi \times I d) \circ(\Phi \times I d)=\Phi \times I d$. The mapping $\bar{\Phi}$ is fiberwise linear, since $\Phi \times I d$ and $q^{\prime}=T q$ are. The image of $\bar{\Phi}$ is

$$
\begin{aligned}
q^{\prime}(V P \times T S) & =q^{\prime}(\operatorname{ker}(T p: T P \times T S \rightarrow T M)) \\
& =\operatorname{ker}\left(T p: T P \times_{T G} T S \rightarrow T M\right)=V(P[S, \ell]) .
\end{aligned}
$$

Thus $\bar{\Phi}$ is a connection on the associated bundle $P[S]$. We call it the induced connection.

From the diagram it also follows that the vector valued forms

$$
\begin{aligned}
\Phi \times I d & \in \Omega^{1}(P \times S, T P \times T S) \\
\text { and } \quad \bar{\Phi} & \in \Omega^{1}(P[S], T(P[S]))
\end{aligned}
$$

are $(q: P \times S \rightarrow P[S])$-related. So by (16.15) we have for the curvatures

$$
\begin{aligned}
R_{\Phi \times I d} & =\frac{1}{2}[\Phi \times I d, \Phi \times I d]=\frac{1}{2}[\Phi, \Phi] \times 0 \\
& =R_{\Phi} \times 0 \\
R_{\bar{\Phi}} & =\frac{1}{2}[\bar{\Phi}, \bar{\Phi}]
\end{aligned}
$$

that they are also $q$-related, i.e., $T q \circ\left(R_{\Phi} \times 0\right)=R_{\bar{\Phi}} \circ\left(T q \times{ }_{M} T q\right)$.

By uniqueness of the solutions of the defining differential equation we also get that

$$
\operatorname{Pt}_{\bar{\Phi}}(c, t, q(u, s))=q\left(\mathrm{Pt}_{\Phi}(c, t, u), s\right)
$$


19.9. Recognizing induced connections. We consider again a principal fiber bundle $(P, p, M, G)$ and a left action $\ell: G \times S \rightarrow S$. Suppose that we have a connection $\Psi \in \Omega^{1}(P[S], T(P[S]))$ on the associated bundle $P[S]=P[S, \ell]$. Then the following question arises: When is the connection $\Psi$ induced from a principal connection on $P$ ? If this is the case, we say that $\Psi$ is compatible with the $G$-structure on $P[S]$. The answer is given in the following

Theorem. Let $\Psi$ be a (general) connection on the associated bundle $P[S]$. Let us suppose that the action $\ell$ is infinitesimally effective, i.e., the fundamental vector field mapping $\zeta: \mathfrak{g} \rightarrow \mathfrak{X}(S)$ is injective.

Then the connection $\Psi$ is induced from a principal connection $\omega$ on $P$ if and only if the following condition is satisfied:

- In some (equivalently: any) fiber bundle atlas $\left(U_{\alpha}, \psi_{\alpha}\right)$ of $P[S]$ belonging to the G-structure of the associated bundle the Christoffel forms $\Gamma^{\alpha} \in \Omega^{1}\left(U_{\alpha}, \mathfrak{X}(S)\right)$ have values in the Lie subalgebra $\mathfrak{X}_{\text {fund }}(S)$ of fundamental vector fields for the action $\ell$.

Proof. Let $\left(U_{\alpha}, \varphi_{\alpha}: P \mid U_{\alpha} \rightarrow U_{\alpha} \times G\right)$ be a principal fiber bundle atlas for $P$. Then by the proof of theorem (18.7) the induced fiber bundle atlas

$$
\left(U_{\alpha}, \psi_{\alpha}: P[S] \mid U_{\alpha} \rightarrow U_{\alpha} \times S\right)
$$

is given by

$$
\begin{gathered}
\psi_{\alpha}^{-1}(x, s)=q\left(\varphi_{\alpha}^{-1}(x, e), s\right), \\
\left(\psi_{\alpha} \circ q\right)\left(\varphi_{\alpha}^{-1}(x, g), s\right)=(x, g . s) .
\end{gathered}
$$

Let $\Phi=\zeta \circ \omega$ be a principal connection on $P$ and let $\bar{\Phi}$ be the induced connection on the associated bundle $P[S]$. By (17.7) its Christoffel symbols are given by

$$
\begin{array}{rlrl}
\left(0_{x}, \Gamma_{\bar{\Phi}}^{\alpha}\left(\xi_{x}, s\right)\right) & =-\left(T\left(\psi_{\alpha}\right) \circ \bar{\Phi} \circ T\left(\psi_{\alpha}^{-1}\right)\right)\left(\xi_{x}, 0_{s}\right) & \\
& =-\left(T\left(\psi_{\alpha}\right) \circ \bar{\Phi} \circ T q \circ\left(T\left(\varphi_{\alpha}^{-1}\right) \times I d\right)\right)\left(\xi_{x}, 0_{e}, 0_{s}\right) & & \text { by (1) } \\
& =-\left(T\left(\psi_{\alpha}\right) \circ T q \circ(\Phi \times I d)\right)\left(T\left(\varphi_{\alpha}^{-1}\right)\left(\xi_{x}, 0_{e}\right), 0_{s}\right) & & \text { by (19.8) } \\
& =-\left(T\left(\psi_{\alpha}\right) \circ T q\right)\left(\Phi\left(T\left(\varphi_{\alpha}^{-1}\right)\left(\xi_{x}, 0_{e}\right)\right), 0_{s}\right) & & \\
& =\left(T\left(\psi_{\alpha}\right) \circ T q\right)\left(T\left(\varphi_{\alpha}^{-1}\right)\left(0_{x}, \Gamma_{\Phi}^{\alpha}\left(\xi_{x}, e\right)\right), 0_{s}\right) & & \text { by (19.4.3) } \\
& =-T\left(\psi_{\alpha} \circ q \circ\left(\varphi_{\alpha}^{-1} \times I d\right)\right)\left(0_{x}, \omega_{\alpha}\left(\xi_{x}\right), 0_{s}\right) & & \text { by (19.4.7) } \\
& =-T_{e}\left(\ell^{s}\right) \omega_{\alpha}\left(\xi_{x}\right) & & \text { by (2) } \\
& =-\zeta_{\omega_{\alpha}\left(\xi_{x}\right)}(s) . & &
\end{array}
$$

So the condition is necessary. 
Now let us conversely suppose that a connection $\Psi$ on $P[S]$ is given such that the Christoffel forms $\Gamma_{\Psi}^{\alpha}$ with respect to a fiber bundle atlas of the $G$-structure have values in $\mathfrak{X}_{\text {fund }}(S)$. Then unique $\mathfrak{g}$-valued forms $\omega_{\alpha} \in$ $\Omega^{1}\left(U_{\alpha}, \mathfrak{g}\right)$ are given by the equation

$$
\Gamma_{\Psi}^{\alpha}\left(\xi_{x}\right)=-\zeta\left(\omega_{\alpha}\left(\xi_{x}\right)\right)
$$

since the action is infinitesimally effective. From the transition formulas (17.7) for the $\Gamma_{\Psi}^{\alpha}$ follow the transition formulas (19.4.5) for the $\omega^{\alpha}$, so that they give a unique principal connection on $P$, which by the first part of the proof induces the given connection $\Psi$ on $P[S]$.

19.10. Inducing principal connections on associated vector bundles. Let $(P, p, M, G)$ be a principal fiber bundle and let $\rho: G \rightarrow G L(W)$ be a representation of the structure group $G$ on a finite-dimensional vector space $W$. We consider the associated vector bundle $(E:=P[W, \rho], p, M, W)$, which was treated in some detail in (18.11).

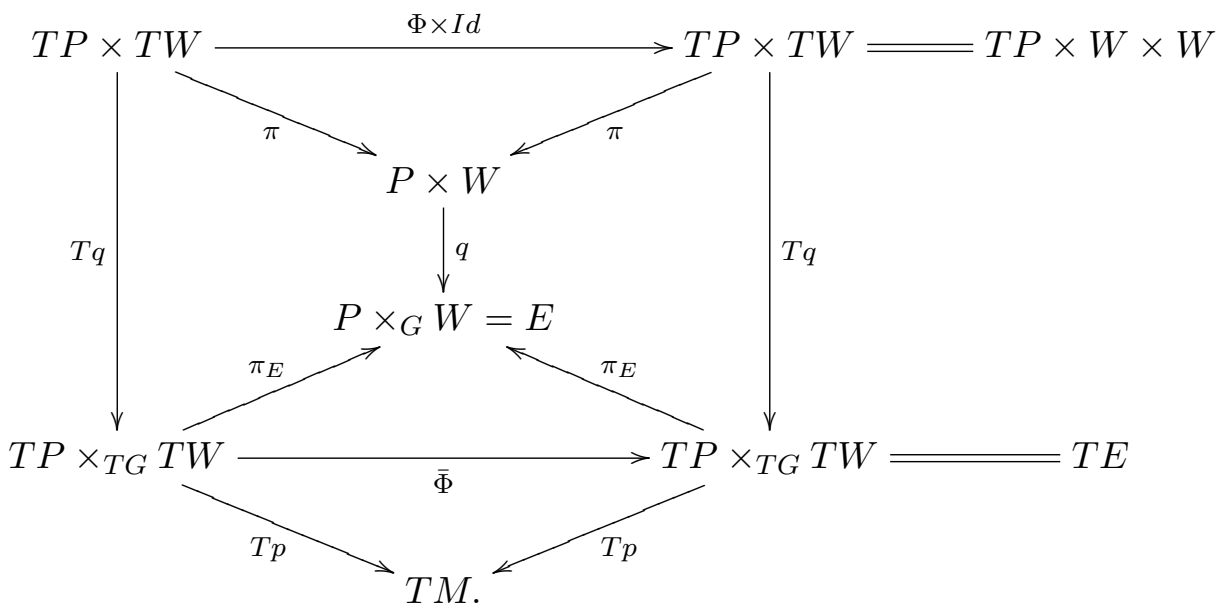

Recall from (8.12) that $T(E)=T P \times_{T G} T W$ has two vector bundle structures with the projections

$$
\begin{gathered}
\pi_{E}: T(E)=T P \times_{T G} T W \rightarrow P \times_{G} W=E, \\
T p \circ \operatorname{pr}_{1}: T(E)=T P \times_{T G} T W \rightarrow T M .
\end{gathered}
$$

Now let $\Phi=\zeta \circ \omega \in \Omega^{1}(P, T P)$ be a principal connection on $P$. We consider the induced connection $\bar{\Phi} \in \Omega^{1}(E, T(E))$ from (19.8).

A look at the diagram above shows that the induced connection is linear in both vector bundle structures. We say that it is a linear connection on the associated bundle.

Recall now from (8.12) the vertical lift $\mathrm{vl}_{E}: E \times_{M} E \rightarrow V E$, which is an isomorphism, $\mathrm{pr}_{1}-\pi_{E}$-fiberwise linear and also $\mathrm{pr}_{2}-T p$-fiberwise linear. 
Now we define the connector $K$ of the linear connection $\bar{\Phi}$ by

$$
K:=\operatorname{pr}_{2} \circ\left(\mathrm{vl}_{E}\right)^{-1} \circ \bar{\Phi}: T E \rightarrow V E \rightarrow E \times_{M} E \rightarrow E .
$$

Lemma. The connector $K: T E \rightarrow E$ is a vector bundle homomorphism for both vector bundle structures on $T E$ and satisfies

$$
K \circ \mathrm{vl}_{E}=\operatorname{pr}_{2}: E \times_{M} E \rightarrow T E \rightarrow E .
$$

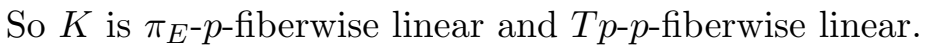

Proof. This follows from the fiberwise linearity of the components of $K$ and from its definition.

19.11. Linear connections. If $(E, p, M)$ is a vector bundle, a connection $\Psi \in \Omega^{1}(E, T E)$ such that $\Psi: T E \rightarrow V E \rightarrow T E$ is also $T p$-Tp-fiberwise linear is called a linear connection. An easy check with (19.9) or a direct construction shows that $\Psi$ is then induced from a unique principal connection on the linear frame bundle $G L\left(\mathbb{R}^{n}, E\right)$ of $E$ (where $n$ is the fiber dimension of $E$ ).

Equivalently, a linear connection may be specified by a connector

$$
K: T E \rightarrow E
$$

with the three properties of lemma (19.10), For then

$$
H E:=\left\{\xi_{u}: K\left(\xi_{u}\right)=0_{p(u)}\right\}
$$

is a complement to $V E$ in $T E$ which is $T p$-fiberwise linearly chosen.

19.12. Covariant derivative on vector bundles. Let $(E, p, M)$ be a vector bundle with a linear connection, given by a connector $K: T E \rightarrow E$ with the properties in lemma (19.10).

For any manifold $N$, smooth mapping $s: N \rightarrow E$, and vector field $X \in \mathfrak{X}(N)$ we define the covariant derivative of $s$ along $X$ by

$$
\nabla_{X} s:=K \circ T s \circ X: N \rightarrow T N \rightarrow T E \rightarrow E .
$$

If $f: N \rightarrow M$ is a fixed smooth mapping, let us denote by $C_{f}^{\infty}(N, E)$ the vector space of all smooth mappings $s: N \rightarrow E$ with $p \circ s=f$ - they are called sections of $E$ along $f$. From the universal property of the pullback it follows that the vector space $C_{f}^{\infty}(N, E)$ is canonically linearly isomorphic to the space $\Gamma\left(f^{*} E\right)$ of sections of the pullback bundle. Then the covariant derivative may be viewed as a bilinear mapping

$$
\nabla: \mathfrak{X}(N) \times C_{f}^{\infty}(N, E) \rightarrow C_{f}^{\infty}(N, E) .
$$

In particular for $f=I d_{M}$ we have

$$
\nabla: \mathfrak{X}(M) \times \Gamma(E) \rightarrow \Gamma(E) .
$$


Lemma. This covariant derivative has the following properties:

(3) $\nabla_{X} s$ is $C^{\infty}(N)$-linear in $X \in \mathfrak{X}(N)$. So for a tangent vector $X_{x} \in$ $T_{x} N$ the mapping $\nabla_{X_{x}}: C_{f}^{\infty}(N, E) \rightarrow E_{f(x)}$ makes sense and we have $\left(\nabla_{X} s\right)(x)=\nabla_{X(x)} s$.

(4) $\nabla_{X} s$ is $\mathbb{R}$-linear in $s \in C_{f}^{\infty}(N, E)$.

(5) $\nabla_{X}(h . s)=d h(X) . s+h . \nabla_{X} s$ for $h \in C^{\infty}(N)$, the derivation property of $\nabla_{X}$.

(6) For any manifold $Q$ and smooth mapping $g: Q \rightarrow N$ and $Y_{y} \in T_{y} Q$ we have $\nabla_{T g . Y_{y}} s=\nabla_{Y_{y}}(s \circ g)$. If $Y \in \mathfrak{X}(Q)$ and $X \in \mathfrak{X}(N)$ are g-related, then we have $\nabla_{Y}(s \circ g)=\left(\nabla_{X} s\right) \circ g$.

Proof. All these properties follow easily from the definition (1).

Property (6) is not well understood in some differential geometric literature.

For vector fields $X, Y \in \mathfrak{X}(M)$ and a section $s \in \Gamma(E)$ an easy computation shows that

$$
\begin{aligned}
R^{E}(X, Y) s: & =\nabla_{X} \nabla_{Y} s-\nabla_{Y} \nabla_{X} s-\nabla_{[X, Y]} s \\
& =\left(\left[\nabla_{X}, \nabla_{Y}\right]-\nabla_{[X, Y]}\right) s
\end{aligned}
$$

is $C^{\infty}(M)$-linear in $X, Y$, and $s$. By the method of (9.3) it follows that $R^{E}$ is a 2 -form on $M$ with values in the vector bundle $L(E, E)$, i.e., $R^{E} \in$ $\Omega^{2}(M, L(E, E))$. It is called the curvature of the covariant derivative. See (19.16) below for the relation to the principal curvature if $E$ is an associated bundle.

For $f: N \rightarrow M$, vector fields $X, Y \in \mathfrak{X}(N)$ and a section $s \in C_{f}^{\infty}(N, E)$ along $f$ one may prove that

$$
\nabla_{X} \nabla_{Y} s-\nabla_{Y} \nabla_{X} s-\nabla_{[X, Y]} s=\left(f^{*} R^{E}\right)(X, Y) s:=R^{E}(T f . X, T f . Y) s .
$$

19.13. Covariant exterior derivative. Let $(E, p, M)$ be a vector bundle with a linear connection, given by a connector $K: T E \rightarrow E$.

For a smooth mapping $f: N \rightarrow M$ let $\Omega\left(N, f^{*} E\right)$ be the vector space of all forms on $N$ with values in the vector bundle $f^{*} E$. We can also view them as forms on $N$ with values along $f$ in $E$, but we do not introduce an extra notation for this.

The graded space $\Omega\left(N, f^{*} E\right)$ is a graded $\Omega(N)$-module via

$$
\begin{aligned}
& (\varphi \wedge \Phi)\left(X_{1}, \ldots, X_{p+q}\right) \\
& \quad=\frac{1}{p ! q !} \sum_{\sigma} \operatorname{sign}(\sigma) \varphi\left(X_{\sigma 1}, \ldots, X_{\sigma p}\right) \Phi\left(X_{\sigma(p+1)}, \ldots, X_{\sigma(p+q)}\right) .
\end{aligned}
$$


The graded module homomorphisms $H: \Omega\left(N, f^{*} E\right) \rightarrow \Omega\left(N, f^{*} E\right)$ (so that $\left.H(\varphi \wedge \Phi)=(-1)^{\operatorname{deg} H \cdot \operatorname{deg} \varphi} \varphi \wedge H(\Phi)\right)$ are easily seen to coincide with the mappings $\mu(A)$ for $A \in \Omega^{p}\left(N, f^{*} L(E, E)\right)$, which are given by

$$
\begin{aligned}
& (\mu(A) \Phi)\left(X_{1}, \ldots, X_{p+q}\right) \\
& \quad=\frac{1}{p ! q !} \sum_{\sigma} \operatorname{sign}(\sigma) A\left(X_{\sigma 1}, \ldots, X_{\sigma p}\right)\left(\Phi\left(X_{\sigma(p+1)}, \ldots, X_{\sigma(p+q)}\right)\right) .
\end{aligned}
$$

The covariant exterior derivative $d_{\nabla}: \Omega^{p}\left(N, f^{*} E\right) \rightarrow \Omega^{p+1}\left(N, f^{*} E\right)$ is defined by (where the $X_{i}$ are vector fields on $N$ )

$$
\begin{aligned}
\left(d_{\nabla} \Phi\right)\left(X_{0}, \ldots,\right. & \left.X_{p}\right)=\sum_{i=0}^{p}(-1)^{i} \nabla_{X_{i}} \Phi\left(X_{0}, \ldots, \widehat{X}_{i}, \ldots, X_{p}\right) \\
& +\sum_{0 \leq i<j \leq p}(-1)^{i+j} \Phi\left(\left[X_{i}, X_{j}\right], X_{0}, \ldots, \widehat{X}_{i}, \ldots, \widehat{X}_{j}, \ldots, X_{p}\right) .
\end{aligned}
$$

Lemma. The covariant exterior derivative is well defined and has the following properties.

(1) For $s \in \Gamma\left(f^{*} E\right)=\Omega^{0}\left(N, f^{*} E\right)$ we have $\left(d_{\nabla} s\right)(X)=\nabla_{X} s$.

(2) $d_{\nabla}(\varphi \wedge \Phi)=d \varphi \wedge \Phi+(-1)^{\operatorname{deg} \varphi} \varphi \wedge d_{\nabla} \Phi$.

(3) For smooth $g: Q \rightarrow N$ and $\Phi \in \Omega\left(N, f^{*} E\right)$ we have $d_{\nabla}\left(g^{*} \Phi\right)=$ $g^{*}\left(d_{\nabla} \Phi\right)$.

(4) $d_{\nabla} d_{\nabla} \Phi=\mu\left(f^{*} R^{E}\right) \Phi$.

Proof. It suffices to investigate decomposable forms $\Phi=\varphi \otimes s$ where $\varphi \in \Omega^{p}(N)$ and $s \in \Gamma\left(f^{*} E\right)$. Then from the definition we have

$$
d_{\nabla}(\varphi \otimes s)=d \varphi \otimes s+(-1)^{p} \varphi \wedge d_{\nabla} s .
$$

Since $d_{\nabla} s \in \Omega^{1}\left(N, f^{*} E\right)$ by (19.12.3), the mapping $d_{\nabla}$ is well defined. This formula also implies (2) immediately. Part (3) follows from (19.12.6), Part (41) is checked as follows:

$$
\begin{aligned}
d_{\nabla} d_{\nabla}(\varphi \otimes s) & =d_{\nabla}\left(d \varphi \otimes s+(-1)^{p} \varphi \wedge d_{\nabla} s\right) \text { by (2) } \\
& =0+(-1)^{2 p} \varphi \wedge d_{\nabla} d_{\nabla} s \\
& =\varphi \wedge \mu\left(f^{*} R^{E}\right) s \text { by the definition of } R^{E} \\
& =\mu\left(f^{*} R^{E}\right)(\varphi \otimes s) . \quad \square
\end{aligned}
$$


19.14. Let $(P, p, M, G)$ be a principal fiber bundle and let $\rho: G \rightarrow G L(W)$ be a representation of the structure group $G$ on a finite-dimensional vector space $W$.

Theorem. There is a canonical isomorphism from the space of $P[W, \rho]$ valued differential forms on $M$ onto the space of horizontal $G$-equivariant $W$-valued differential forms on $P$ :

$$
\begin{aligned}
q^{\sharp}: \Omega(M, P[W, \rho]) \rightarrow & \Omega_{\text {hor }}(P, W)^{G}=\left\{\varphi \in \Omega(P, W): i_{X} \varphi=0\right. \\
& \text { for all } \left.X \in V P,\left(r^{g}\right)^{*} \varphi=\rho\left(g^{-1}\right) \circ \varphi \text { for all } g \in G\right\} .
\end{aligned}
$$

In particular for $W=\mathbb{R}$ with trivial representation we see that

$$
p^{*}: \Omega(M) \rightarrow \Omega_{h o r}(P)^{G}=\left\{\varphi \in \Omega_{h o r}(P):\left(r^{g}\right)^{*} \varphi=\varphi\right\}
$$

is also an isomorphism. The isomorphism

$$
q^{\sharp}: \Omega^{0}(M, P[W])=\Gamma(P[W]) \rightarrow \Omega_{h o r}^{0}(P, W)^{G}=C^{\infty}(P, W)^{G}
$$

is a special case of the one from (18.12).

Proof. Recall the smooth mapping $\tau^{G}: P \times_{M} P \rightarrow G$ from (18.2) with

$$
\begin{aligned}
r\left(u_{x}, \tau^{G}\left(u_{x}, v_{x}\right)\right) & =v_{x}, \\
\tau^{G}\left(u_{x} . g, u_{x}^{\prime} . g^{\prime}\right) & =g^{-1} \cdot \tau^{G}\left(u_{x}, u_{x}^{\prime}\right) \cdot g^{\prime}, \\
\tau^{G}\left(u_{x}, u_{x}\right) & =e .
\end{aligned}
$$

Let $\varphi \in \Omega_{h o r}^{k}(P, W)^{G}, X_{1}, \ldots, X_{k} \in T_{u} P$, and $X_{1}^{\prime}, \ldots, X_{k}^{\prime} \in T_{u^{\prime}} P$ such that $T_{u} p . X_{i}=T_{u^{\prime}} p . X_{i}^{\prime}$ for each $i$. For $g=\tau^{G}\left(u, u^{\prime}\right)$, so that $u g=u^{\prime}$, we then have:

$$
\begin{aligned}
q(u, & \left.\varphi_{u}\left(X_{1}, \ldots, X_{k}\right)\right)=q\left(u g, \rho\left(g^{-1}\right) \varphi_{u}\left(X_{1}, \ldots, X_{k}\right)\right) \\
& =q\left(u^{\prime},\left(\left(r^{g}\right)^{*} \varphi\right)_{u}\left(X_{1}, \ldots, X_{k}\right)\right) \\
& =q\left(u^{\prime}, \varphi_{u g}\left(T_{u}\left(r^{g}\right) \cdot X_{1}, \ldots, T_{u}\left(r^{g}\right) \cdot X_{k}\right)\right) \\
& =q\left(u^{\prime}, \varphi_{u^{\prime}}\left(X_{1}^{\prime}, \ldots, X_{k}^{\prime}\right)\right), \text { since } T_{u}\left(r^{g}\right) X_{i}-X_{i}^{\prime} \in V_{u^{\prime}} P .
\end{aligned}
$$

By this a vector bundle valued form $\Phi \in \Omega^{k}(M, P[W])$ is uniquely determined.

For the converse recall the smooth mapping $\tau^{W}: P \times_{M} P[W, \rho] \rightarrow W$ from (18.7), which satisfies

$$
\begin{aligned}
\tau^{W}(u, q(u, w)) & =w \\
q\left(u_{x}, \tau^{W}\left(u_{x}, v_{x}\right)\right) & =v_{x} \\
\tau^{W}\left(u_{x} g, v_{x}\right) & =\rho\left(g^{-1}\right) \tau^{W}\left(u_{x}, v_{x}\right) .
\end{aligned}
$$


For $\Phi \in \Omega^{k}(M, P[W])$ we define $q^{\sharp} \Phi \in \Omega^{k}(P, W)$ as follows. For $X_{i} \in T_{u} P$ we put

$$
\left(q^{\sharp} \Phi\right)_{u}\left(X_{1}, \ldots, X_{k}\right):=\tau^{W}\left(u, \Phi_{p(u)}\left(T_{u} p \cdot X_{1}, \ldots, T_{u} p \cdot X_{k}\right)\right) .
$$

Then $q^{\sharp} \Phi$ is smooth and horizontal. For $g \in G$ we have

$$
\begin{aligned}
& \left(\left(r^{g}\right)^{*}\left(q^{\sharp} \Phi\right)\right)_{u}\left(X_{1}, \ldots, X_{k}\right)=\left(q^{\sharp} \Phi\right)_{u g}\left(T_{u}\left(r^{g}\right) \cdot X_{1}, \ldots, T_{u}\left(r^{g}\right) \cdot X_{k}\right) \\
& \quad=\tau^{W}\left(u g, \Phi_{p(u g)}\left(T_{u g} p \cdot T_{u}\left(r^{g}\right) \cdot X_{1}, \ldots, T_{u g} p \cdot T_{u}\left(r^{g}\right) \cdot X_{k}\right)\right) \\
& \quad=\rho\left(g^{-1}\right) \tau^{W}\left(u, \Phi_{p(u)}\left(T_{u} p \cdot X_{1}, \ldots, T_{u} p \cdot X_{k}\right)\right) \\
& \quad=\rho\left(g^{-1}\right)\left(q^{\sharp} \Phi\right)_{u}\left(X_{1}, \ldots, X_{k}\right) .
\end{aligned}
$$

Clearly the two constructions are inverse to each other.

19.15. Let $(P, p, M, G)$ be a principal fiber bundle with a principal connection $\Phi=\zeta \circ \omega$, and let $\rho: G \rightarrow G L(W)$ be a representation of the structure group $G$ on a finite-dimensional vector space $W$. We consider the associated vector bundle $(E:=P[W, \rho], p, M, W)$, the induced connection $\bar{\Phi}$ on it and the corresponding covariant derivative.

Theorem. The covariant exterior derivative $d_{\omega}$ from (19.5) on $P$ and the covariant exterior derivative for $P[W]$-valued forms on $M$ are connected by the mapping $q^{\sharp}$ from (19.14), as follows:

$$
q^{\sharp} \circ d_{\nabla}=d_{\omega} \circ q^{\sharp}: \Omega(M, P[W]) \rightarrow \Omega_{h o r}(P, W)^{G} .
$$

Proof. Let us consider first $f \in \Omega_{h o r}^{0}(P, W)^{G}=C^{\infty}(P, W)^{G}$; then $f=q^{\sharp} s$ for $s \in \Gamma(P[W])$ and we have $f(u)=\tau^{W}(u, s(p(u)))$ and $s(p(u))=q(u, f(u))$ by (19.14) and (18.12). Therefore we have Ts.Tp. $X_{u}=T q\left(X_{u}, T f . X_{u}\right)$, where $T f . X_{u}=\left(f(u), d f\left(X_{u}\right)\right) \in T W=W \times W$. If $\chi: T P \rightarrow H P$ is the horizontal projection as in (19.5), we have Ts.Tp. $X_{u}=T$ s.Tp. $\chi \cdot X_{u}=$ $T q\left(\chi \cdot X_{u}, T f \cdot \chi \cdot X_{u}\right)$. So we get

$$
\begin{array}{ll}
\left(q^{\sharp} d_{\nabla} s\right)\left(X_{u}\right)=\tau^{W}\left(u,\left(d_{\nabla} s\right)\left(T p \cdot X_{u}\right)\right) & \\
=\tau^{W}\left(u, \nabla_{T p \cdot X_{u}} s\right) & \text { by }(19.13 \cdot 1) \\
=\tau^{W}\left(u, K \cdot T s \cdot T p \cdot X_{u}\right) & \text { by (19.12.1) } \\
=\tau^{W}\left(u, K \cdot T q\left(\chi \cdot X_{u}, T f \cdot \chi \cdot X_{u}\right)\right) & \text { from above } \\
=\tau^{W}\left(u, \mathrm{pr}_{2} \cdot \mathrm{vl}_{P[W]}^{-1} \cdot \bar{\Phi} \cdot T q\left(\chi \cdot X_{u}, T f \cdot \chi \cdot X_{u}\right)\right) & \text { by (19.10) } \\
=\tau^{W}\left(u, \mathrm{pr}_{2} \cdot \mathrm{vl}_{P[W]}^{-1} \cdot T q \cdot(\Phi \times I d)\left(\chi \cdot X_{u}, T f \cdot \chi \cdot X_{u}\right)\right) & \text { by (19.8) } \\
=\tau^{W}\left(u, \mathrm{pr}_{2} \cdot \mathrm{vl}_{P[W]}^{-1} \cdot T q\left(0_{u}, T f \cdot \chi \cdot X_{u}\right)\right) & \text { since } \Phi \cdot \chi=0 \\
=\tau^{W}\left(u, q \cdot \mathrm{pr}_{2} \cdot \mathrm{vl}_{P \times W}^{-1} \cdot\left(0_{u}, T f \cdot \chi \cdot X_{u}\right)\right) & \text { since } q \text { is fiber linear } \\
=\tau^{W}\left(u, q\left(u, d f \cdot \chi \cdot X_{u}\right)\right)=\left(\chi^{*} d f\right)\left(X_{u}\right)=\left(d_{\omega} q^{\sharp} s\right)\left(X_{u}\right) . &
\end{array}
$$


Now we turn to the general case. It suffices to check the formula for a decomposable $P[W]$-valued form $\Psi=\psi \otimes s \in \Omega^{k}(M, P[W])$, where $\psi \in$ $\Omega^{k}(M)$ and $s \in \Gamma(P[W])$. Then we have

$$
\begin{aligned}
& d_{\omega} q^{\sharp}(\psi \otimes s)=d_{\omega}\left(p^{*} \psi \cdot q^{\sharp} s\right) \\
& =d_{\omega}\left(p^{*} \psi\right) \cdot q^{\sharp} s+(-1)^{k} \chi^{*} p^{*} \psi \wedge d_{\omega} q^{\sharp} s \quad \text { by (19.5.1) } \\
& =\chi^{*} p^{*} d \psi \cdot q^{\sharp} s+(-1)^{k} p^{*} \psi \wedge q^{\sharp} d_{\nabla} s \quad \text { from above and (19.5.4) } \\
& =p^{*} d \psi \cdot q^{\sharp} s+(-1)^{k} p^{*} \psi \wedge q^{\sharp} d_{\nabla} s \\
& =q^{\sharp}\left(d \psi \otimes s+(-1)^{k} \psi \wedge d_{\nabla} s\right) \\
& =q^{\sharp} d_{\nabla}(\psi \otimes s) \text {. }
\end{aligned}
$$

19.16. Corollary. In the situation of theorem (19.15), the curvature $R^{P[W]} \in \Omega^{2}(M, L(P[W], P[W]))$ is related to the Lie algebra valued curvature form $\Omega \in \Omega_{\text {hor }}^{2}(P, \mathfrak{g})$ by

$$
q_{L(P[W], P[W])}^{\sharp} R^{P[W]}=\rho^{\prime} \circ \Omega,
$$

where $\rho^{\prime}=T_{e} \rho: \mathfrak{g} \rightarrow L(W, W)$ is the derivative of the representation $\rho$.

Proof. We use the notation of the proof of theorem (19.15), By this theorem we have for $X, Y \in T_{u} P$

$$
\begin{aligned}
\left(d_{\omega} d_{\omega} q_{P[W]}^{\sharp} s\right)_{u}(X, Y) & =\left(q^{\sharp} d_{\nabla} d_{\nabla} s\right)_{u}(X, Y) \\
& =\left(q^{\sharp} R^{P[W]} s\right)_{u}(X, Y) \\
& =\tau^{W}\left(u, R^{P[W]}\left(T_{u} p \cdot X, T_{u} p . Y\right) s(p(u))\right) \\
& =\left(q_{L(P[W], P[W]}^{\sharp} R^{P[W]}\right)_{u}(X, Y)\left(q_{P[W]}^{\sharp} s\right)(u) .
\end{aligned}
$$

On the other hand we have by theorem (19.5.8)

$$
\begin{aligned}
\left(d_{\omega} d_{\omega} q^{\sharp} s\right)_{u}(X, Y) & =\left(\chi^{*} i_{R} d q^{\sharp} s\right)_{u}(X, Y) \\
& =\left(d q^{\sharp} s\right)_{u}(R(X, Y)) \quad \text { since } R \text { is horizontal } \\
& =\left(d q^{\sharp} s\right)\left(-\zeta_{\Omega(X, Y)}(u)\right) \quad \text { by }(19.2) \\
& =\left.\partial\right|_{0}\left(q^{\sharp} s\right)\left(\mathrm{Fl}_{-t}^{\zeta_{\Omega(X, Y)}}(u)\right) \\
& =\left.\partial\right|_{0} \tau^{W}(u \cdot \exp (-t \Omega(X, Y)), s(p(u \cdot \exp (-t \Omega(X, Y))))) \\
& =\left.\partial\right|_{0} \tau^{W}(u \cdot \exp (-t \Omega(X, Y)), s(p(u))) \\
& =\left.\partial\right|_{0} \rho(\exp t \Omega(X, Y)) \tau^{W}(u, s(p(u))) \quad \text { by }(18.7) \\
& =\rho^{\prime}(\Omega(X, Y))\left(q^{\sharp} s\right)(u) . \quad \square
\end{aligned}
$$




\section{Characteristic Classes}

20.1. Invariants of Lie algebras. Let $G$ be a Lie group with Lie algebra $\mathfrak{g}$; let $\otimes \mathfrak{g}^{*}$ be the tensor algebra over the dual space $\mathfrak{g}^{*}$, the graded space of all multilinear real (or complex) functionals on $\mathfrak{g}$. Let $S\left(\mathfrak{g}^{*}\right)$ be the symmetric algebra over $\mathfrak{g}^{*}$ which corresponds to the algebra of polynomial functions on $\mathfrak{g}$. The adjoint representation Ad $: G \rightarrow L(\mathfrak{g}, \mathfrak{g})$ induces representations $\mathrm{Ad}^{*}: G \rightarrow L\left(\otimes \mathfrak{g}^{*}, \bigotimes \mathfrak{g}^{*}\right)$ and also $\mathrm{Ad}^{*}: G \rightarrow L\left(S\left(\mathfrak{g}^{*}\right), S\left(\mathfrak{g}^{*}\right)\right)$, which are both given by $\operatorname{Ad}^{*}(g) f=f \circ\left(\operatorname{Ad}\left(g^{-1}\right) \otimes \cdots \otimes \operatorname{Ad}\left(g^{-1}\right)\right)$. A tensor $f \in \otimes \mathfrak{g}^{*}$ (or a polynomial $f \in S\left(\mathfrak{g}^{*}\right)$ ) is called an invariant of the Lie algebra if $\operatorname{Ad}^{*}(g) f=f$ for all $g \in G$. If the Lie group $G$ is connected, $f$ is an invariant if and only if $\mathcal{L}_{X} f=0$ for all $X \in \mathfrak{g}$, where $\mathcal{L}_{X}$ is the restriction of the Lie derivative to left invariant tensor fields on $G$, which coincides with the unique extension of $\operatorname{ad}(X)^{*}: \mathfrak{g}^{*} \rightarrow \mathfrak{g}^{*}$ to a derivation on $\otimes \mathfrak{g}^{*}$ or $S\left(\mathfrak{g}^{*}\right)$, respectively. Compare this with the proof of (14.16.2). Obviously the space of all invariants is a graded subalgebra of $\otimes \mathfrak{g}^{*}$ or $S\left(\mathfrak{g}^{*}\right)$, respectively. The usual notation for the algebra of invariant polynomials is

$$
I(G):=\bigoplus_{k \geq 0} I^{k}(G)=S\left(\mathfrak{g}^{*}\right)^{G}=\bigoplus_{k \geq 0} S^{k}\left(\mathfrak{g}^{*}\right)^{G} .
$$

20.2. The Chern-Weil forms. Let $(P, p, M, G)$ be a principal fiber bundle with principal connection $\Phi=\zeta \circ \omega$ and curvature $R=\zeta \circ \Omega$. For $\psi_{i} \in$ $\Omega^{p_{i}}(P, \mathfrak{g})$ and $f \in S^{k}\left(\mathfrak{g}^{*}\right) \subset \bigotimes^{k} \mathfrak{g}^{*}$ we have the differential forms

$$
\begin{gathered}
\psi_{1} \otimes_{\wedge} \cdots \otimes_{\wedge} \psi_{k} \in \Omega^{p_{1}+\cdots+p_{k}}(P, \mathfrak{g} \otimes \cdots \otimes \mathfrak{g}), \\
f \circ\left(\psi_{1} \otimes_{\wedge} \cdots \otimes_{\wedge} \psi_{k}\right) \in \Omega^{p_{1}+\cdots+p_{k}}(P) .
\end{gathered}
$$

The exterior derivative of the latter one is clearly given by

$$
\begin{aligned}
& d\left(f \circ\left(\psi_{1} \otimes_{\wedge} \cdots \otimes_{\wedge} \psi_{k}\right)\right)=f \circ d\left(\psi_{1} \otimes_{\wedge} \cdots \otimes_{\wedge} \psi_{k}\right) \\
& \quad=f \circ\left(\sum_{i=1}^{k}(-1)^{p_{1}+\cdots+p_{i-1}} \psi_{1} \otimes_{\wedge} \cdots \otimes_{\wedge} d \psi_{i} \otimes_{\wedge} \cdots \otimes_{\wedge} \psi_{k}\right) .
\end{aligned}
$$

Let us now consider an invariant polynomial $f \in I^{k}(G)$ and the curvature form $\Omega \in \Omega_{\text {hor }}^{2}(P, \mathfrak{g})^{G}$. Then the $2 k$-form $f \circ\left(\Omega \otimes_{\wedge} \cdots \otimes_{\wedge} \Omega\right)$ is horizontal since $\Omega$ is horizontal by (19.2.2), It is also $G$-invariant since by (19.2.2) we have

$$
\begin{aligned}
\left(r^{g}\right)^{*}\left(f \circ\left(\Omega \otimes_{\wedge} \cdots \otimes_{\wedge} \Omega\right)\right) & =f \circ\left(\left(r^{g}\right)^{*} \Omega \otimes_{\wedge} \cdots \otimes_{\wedge}\left(r^{g}\right)^{*} \Omega\right) \\
& =f \circ\left(\operatorname{Ad}\left(g^{-1}\right) \Omega \otimes_{\wedge} \cdots \otimes_{\wedge} \operatorname{Ad}\left(g^{-1}\right) \Omega\right) \\
& =f \circ\left(\Omega \otimes_{\wedge} \cdots \otimes_{\wedge} \Omega\right) .
\end{aligned}
$$

So by theorem (19.14) there is a uniquely defined $2 k$-form $\mathrm{cw}(f, P, \omega) \in$ $\Omega^{2 k}(M)$ with $p^{*} \mathrm{cw}(f, P, \omega)=f \circ\left(\Omega \otimes_{\wedge} \cdots \otimes_{\wedge} \Omega\right)$, which we will call the Chern-Weil form of $f$. 
If $h: N \rightarrow M$ is a smooth mapping, then for the pullback bundle $h^{*} P$ the Chern-Weil form is given by $\operatorname{cw}\left(f, h^{*} P, h^{*} \omega\right)=h^{*} \mathrm{cw}(f, P, \omega)$, which is easily seen by applying $p^{*}$.

20.3. Theorem (Chern-Weil homomorphism). In the setting of $(20.2)$ we have:

(1) For $f \in I^{k}(G)$ the Chern-Weil form $\mathrm{cw}(f, P, \omega)$ is a closed differential form: $d \mathrm{cw}(f, P, \omega)=0$. So there is a well defined cohomology class $\mathrm{Cw}(f, P)=[\mathrm{cw}(f, P, \omega)] \in H^{2 k}(M)$, called the characteristic class of the invariant polynomial $f$.

(2) The characteristic class $\mathrm{Cw}(f, P)$ does not depend on the choice of the principal connection $\omega$.

(3) The mapping $\mathrm{Cw}_{P}: I^{*}(G) \rightarrow H^{2 *}(M)$ is a homomorphism of commutative algebras, and it is called the Chern-Weil homomorphism.

(4) If $h: N \rightarrow M$ is a smooth mapping, then the Chern-Weil homomorphism for the pullback bundle $h^{*} P$ is given by

$$
\mathrm{Cw}_{h^{*} P}=h^{*} \circ \mathrm{Cw}_{P}: I^{*}(G) \rightarrow H^{2 *}(N) .
$$

Proof. (11) Since $f \in I^{k}(G)$ is invariant, we have for any $X \in \mathfrak{g}$

$$
\begin{aligned}
0 & =\left.\frac{d}{d t}\right|_{0} \operatorname{Ad}\left(\exp \left(t X_{0}\right)\right)^{*} f\left(X_{1}, \ldots, X_{k}\right) \\
& =\left.\frac{d}{d t}\right|_{0} f\left(\operatorname{Ad}\left(\exp \left(t X_{0}\right)\right) X_{1}, \ldots, \operatorname{Ad}\left(\exp \left(t X_{0}\right)\right) X_{k}\right) \\
& =\sum_{i=1}^{k} f\left(X_{1}, \ldots,\left[X_{0}, X_{i}\right], \ldots, X_{k}\right) \\
& =\sum_{i=1}^{k} f\left(\left[X_{0}, X_{i}\right], X_{1}, \ldots, \widehat{X_{i}} \ldots, X_{k}\right) .
\end{aligned}
$$

This implies that

$$
\begin{aligned}
d\left(f \circ \left(\Omega \otimes_{\wedge} \cdots\right.\right. & \left.\left.\otimes_{\wedge} \Omega\right)\right)=f \circ\left(\sum_{i=1}^{k} \Omega \otimes_{\wedge} \cdots \otimes_{\wedge} d \Omega \otimes_{\wedge} \cdots \otimes_{\wedge} \Omega\right) \\
& =k f \circ\left(d \Omega \otimes_{\wedge} \cdots \otimes_{\wedge} \Omega\right)+k f \circ\left([\omega, \Omega]_{\wedge} \otimes_{\wedge} \cdots \otimes_{\wedge} \Omega\right) \\
& =k f \circ\left(d_{\omega} \Omega \otimes_{\wedge} \Omega \otimes_{\wedge} \cdots \otimes_{\wedge} \Omega\right)=0 \quad \text { by }[(19.5 .6), \\
p^{*} d \operatorname{cw}(f, P, \omega) & =d p^{*} \operatorname{cw}(f, P, \omega) \\
& =d\left(f \circ\left(\Omega \otimes_{\wedge} \cdots \otimes_{\wedge} \Omega\right)\right)=0,
\end{aligned}
$$

and thus $d \operatorname{cw}(f, P, \omega)=0$ since $p^{*}$ is injective.

(2) Let $\omega_{0}, \omega_{1} \in \Omega^{1}(P, \mathfrak{g})^{G}$ be two principal connections. Then we consider the principal bundle $(P \times \mathbb{R}, p \times I d, M \times \mathbb{R}, G)$ and the principal connection $\tilde{\omega}=(1-t) \omega_{0}+t \omega_{1}=(1-t)\left(\mathrm{pr}_{1}\right)^{*} \omega_{0}+t\left(\mathrm{pr}_{1}\right)^{*} \omega_{1}$ on it, where $t$ is the coordinate function on $\mathbb{R}$. Let $\tilde{\Omega}$ be the curvature form of $\tilde{\omega}$. Let ins $s_{s}: P \rightarrow$ $P \times \mathbb{R}$ be the embedding at level $s, \operatorname{ins}_{s}(u)=(u, s)$. Then we have in turn 
by $(19.2 .3)$ for $s=0,1$

$$
\begin{aligned}
\omega_{s} & =\left(\operatorname{ins}_{s}\right)^{*} \tilde{\omega} \\
\Omega_{s} & =d \omega_{s}+\frac{1}{2}\left[\omega_{s}, \omega_{s}\right]_{\wedge} \\
& =d\left(\operatorname{ins}_{s}\right)^{*} \tilde{\omega}+\frac{1}{2}\left[\left(\mathrm{ins}_{s}\right)^{*} \tilde{\omega},\left(\mathrm{ins}_{s}\right)^{*} \tilde{\omega}\right]_{\wedge} \\
& =\left(\operatorname{ins}_{s}\right)^{*}\left(d \tilde{\omega}+\frac{1}{2}[\tilde{\omega}, \tilde{\omega}]_{\wedge}\right) \\
& =\left(\operatorname{ins}_{s}\right)^{*} \tilde{\Omega} .
\end{aligned}
$$

So we get for $s=0,1$

$$
\begin{aligned}
p^{*}\left(\mathrm{ins}_{s}\right)^{*} \mathrm{cw}(f, P \times \mathbb{R}, \tilde{\omega}) & =\left(\operatorname{ins}_{s}\right)^{*}\left(p \times I d_{\mathbb{R}}\right)^{*} \operatorname{cw}(f, P \times \mathbb{R}, \tilde{\omega}) \\
& =\left(\operatorname{ins}_{s}\right)^{*}\left(f \circ\left(\tilde{\Omega} \otimes_{\wedge} \cdots \otimes_{\wedge} \tilde{\Omega}\right)\right) \\
& =f \circ\left(\left(\operatorname{ins}_{s}\right)^{*} \tilde{\Omega} \otimes_{\wedge} \cdots \otimes_{\wedge}\left(\mathrm{ins}_{s}\right)^{*} \tilde{\Omega}\right) \\
& =f \circ\left(\Omega_{s} \otimes_{\wedge} \cdots \otimes_{\wedge} \Omega_{s}\right) \\
& =p^{*} \operatorname{cw}\left(f, P, \omega_{s}\right) .
\end{aligned}
$$

Since $p^{*}$ is injective, we get $\left(\mathrm{ins}_{s}\right)^{*} \operatorname{cw}(f, P \times \mathbb{R}, \tilde{\omega})=\operatorname{cw}\left(f, P, \omega_{s}\right)$ for $s=0,1$, and since $i n s_{0}$ and ins $_{1}$ are smoothly homotopic, the cohomology classes coincide.

(31) and (4) are obvious.

20.4. Local description of characteristic classes. Let $(P, p, M, G)$ be a principal fiber bundle with a principal connection $\omega \in \Omega^{1}(P, \mathfrak{g})^{G}$. Let $s_{\alpha} \in \Gamma\left(P \mid U_{\alpha}\right)$ be a collection of local smooth sections of the bundle such that $\left(U_{\alpha}\right)$ is an open cover of $M$. Recall (from the proof of (18.3) for example) that then $\varphi_{\alpha}=\left(p, \tau^{G}\left(s_{\alpha} \circ p, \quad\right)\right): P \mid U_{\alpha} \rightarrow U_{\alpha} \times G$ is a principal fiber bundle atlas with transition functions $\varphi_{\alpha \beta}(x)=\tau^{G}\left(s_{\alpha}(x), s_{\beta}(x)\right)$.

Then we consider the physicists' version from (19.4) of the connection $\omega$ which is described by the forms $\omega_{\alpha}:=s_{\alpha}^{*} \omega \in \Omega^{1}\left(U_{\alpha}, \mathfrak{g}\right)$. They transform according to $\omega_{\alpha}=\operatorname{Ad}\left(\varphi_{\beta \alpha}^{-1}\right) \omega_{\beta}+\Theta_{\beta \alpha}$, where $\Theta_{\beta \alpha}=\varphi_{\beta \alpha}^{-1} d \varphi_{\alpha \beta}$ if $G$ is a matrix group; see lemma (19.4). This affine transformation law is due to the fact that $\omega$ is not horizontal. Let $\Omega=d \omega+\frac{1}{2}[\omega, \omega]_{\wedge} \in \Omega_{\text {hor }}^{2}(P, \mathfrak{g})^{G}$ be the curvature of $\omega$; then we consider again the local forms of the curvature:

$$
\begin{aligned}
\Omega_{\alpha}: & =s_{\alpha}^{*} \Omega=s_{\alpha}^{*}\left(d \omega+\frac{1}{2}[\omega, \omega]_{\wedge}\right) \\
& =d\left(s_{\alpha}^{*} \omega\right)+\frac{1}{2}\left[s_{\alpha}^{*} \omega, s_{\alpha}^{*} \omega\right]_{\wedge} \\
& =d \omega_{\alpha}+\frac{1}{2}\left[\omega_{\alpha}, \omega_{\alpha}\right]_{\wedge} .
\end{aligned}
$$

Recall from theorem (19.14) that we have an isomorphism

$$
q^{\sharp}: \Omega(M, P[\mathfrak{g}, \mathrm{Ad}]) \rightarrow \Omega_{\mathrm{hor}}(P, \mathfrak{g})^{G} .
$$


Then $\Omega_{\alpha}=s_{\alpha}^{*} \Omega$ is the local frame expression of $\left(q^{\sharp}\right)^{-1}(\Omega)$ for the induced chart $P[\mathfrak{g}] \mid U_{\alpha} \rightarrow U_{\alpha} \times \mathfrak{g}$; thus we have the the simple transformation formula $\Omega_{\alpha}=\operatorname{Ad}\left(\varphi_{\alpha \beta}\right) \Omega_{\beta}$.

If now $f \in I^{k}(G)$ is an invariant of $G$, for the Chern-Weil form $\mathrm{cw}(f, P, \omega)$ we have

$$
\begin{aligned}
\operatorname{cw}(f, P, \omega) \mid U_{\alpha}: & =s_{\alpha}^{*}\left(p^{*} \operatorname{cw}(f, P, \omega)\right)=s_{\alpha}^{*}\left(f \circ\left(\Omega \otimes_{\wedge} \cdots \otimes_{\wedge} \Omega\right)\right) \\
& =f \circ\left(s_{\alpha}^{*} \Omega \otimes_{\wedge} \cdots \otimes_{\wedge} s_{\alpha}^{*} \Omega\right) \\
& =f \circ\left(\Omega_{\alpha} \otimes_{\wedge} \cdots \otimes_{\wedge} \Omega_{\alpha}\right),
\end{aligned}
$$

where $\Omega_{\alpha} \otimes_{\wedge} \cdots \otimes_{\wedge} \Omega_{\alpha} \in \Omega^{2 k}\left(U_{\alpha}, \mathfrak{g} \otimes \cdots \otimes \mathfrak{g}\right)$.

20.5. Characteristic classes for vector bundles. For a real vector bundle $\left(E, p, M, \mathbb{R}^{n}\right)$ the characteristic classes are by definition the characteristic classes of the linear frame bundle $\left(G L\left(\mathbb{R}^{n}, E\right), p, M, G L(n, \mathbb{R})\right)$. We write $\mathrm{Cw}(f, E):=\mathrm{Cw}\left(f, G L\left(\mathbb{R}^{n}, E\right)\right)$ for short and likewise for complex vector bundles.

Let $(P, p, M, G)$ be a principal bundle and let $\rho: G \rightarrow G L(V)$ be a representation in a finite-dimensional vector space. If $\omega$ is a principal connection form on $P$ with curvature form $\Omega$, then for the induced covariant derivative $\nabla$ on the associated vector bundle $P[V]$ and its curvature $R^{P[V]}$ we have $q^{\sharp} R^{P[V]}=\rho^{\prime} \circ \Omega$ by corollary (19.16). So if the representation $\rho$ is infinitesimally effective, i.e., if $\rho^{\prime}: \mathfrak{g} \rightarrow L(V, V)$ is injective, then we see that actually $R^{P[V]} \in \Omega^{2}(M, P[\mathfrak{g}])$. If $f \in I^{k}(G)$ is an invariant, then we have the induced mapping

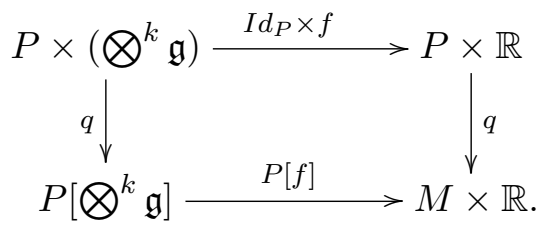

So the Chern-Weil form can also be written as (omitting $P\left[\left(\rho^{\prime}\right)^{-1}\right]$ )

$$
\mathrm{cw}(f, P, \omega)=P[f] \circ\left(R^{P[V]} \otimes_{\wedge} \cdots \otimes_{\wedge} R^{P[V]}\right) .
$$

Sometimes we will make use of this expression.

All characteristic classes for a trivial vector bundle are zero, since the frame bundle is then trivial and admits a principal connection with curvature 0 .

We will determine the classical bases for the algebra of invariants for the matrix groups $G L(n, \mathbb{R}), G L(n, \mathbb{C}), O(n, \mathbb{R}), S O(n, \mathbb{R}), U(n)$, and we will discuss the resulting characteristic classes for vector bundles. 
20.6. The characteristic coefficients. . For a matrix $A \in \mathfrak{g l}(n, \mathbb{R})=$ $L\left(\mathbb{R}^{n}, \mathbb{R}^{n}\right)$ we consider the characteristic coefficients $c_{k}^{n}(A)$ which are given by the implicit equation

$$
\operatorname{det}(A+t \mathbb{I})=\sum_{k=0}^{n} c_{k}^{n}(A) \cdot t^{n-k} .
$$

From lemma (14.19) we have

$$
c_{k}^{n}(A)=\operatorname{Trace}\left(\bigwedge^{k} A: \bigwedge^{k} \mathbb{R}^{n} \rightarrow \bigwedge^{k} \mathbb{R}^{n}\right)
$$

The characteristic coefficient $c_{k}^{n}$ is a homogeneous invariant polynomial of degree $k$, since we have

$$
\operatorname{det}(\operatorname{Ad}(g) A+t \mathbb{I})=\operatorname{det}\left(g A g^{-1}+t \mathbb{I}\right)=\operatorname{det}\left(g(A+t \mathbb{I}) g^{-1}\right)=\operatorname{det}(A+t \mathbb{I}) .
$$

Lemma. We have

$$
c_{k}^{n+m}\left(\left(\begin{array}{cc}
A & 0 \\
0 & B
\end{array}\right)\right)=\sum_{j=0}^{k} c_{j}^{n}(A) c_{k-j}^{m}(B) .
$$

Proof. We have

$$
\begin{aligned}
\operatorname{det}\left(\left(\begin{array}{cc}
A & 0 \\
0 & B
\end{array}\right)+t \mathbb{I}_{n+m}\right) & =\operatorname{det}\left(A+t \mathbb{I}_{n}\right) \operatorname{det}\left(B+t \mathbb{I}_{m}\right) \\
& =\left(\sum_{k=0}^{n} c_{k}^{n}(A) t^{n-k}\right)\left(\sum_{j=0}^{m} c_{j}^{m}(A) t^{m-l}\right) \\
& =\sum_{k=0}^{n+m}\left(\sum_{j=0}^{k} c_{j}^{n}(A) c_{k-j}^{m}(B)\right) t^{n+m-k} .
\end{aligned}
$$

20.7. Pontryagin classes. Let $(E, p, M)$ be a real vector bundle. Then the Pontryagin classes are given by

$$
\begin{aligned}
& p_{k}(E):=\left(\frac{-1}{2 \pi \sqrt{-1}}\right)^{2 k} \mathrm{Cw}\left(c_{2 k}^{\operatorname{dim} E}, E\right) \in H^{4 k}(M ; \mathbb{R}), \\
& p_{0}(E):=1 \in H^{0}(M ; \mathbb{R}) .
\end{aligned}
$$

The factor $\frac{-1}{2 \pi \sqrt{-1}}$ makes this class to be an integer class (in $H^{4 k}(M, \mathbb{Z})$ ) and makes several integral formulas (like the Gauß-Bonnet-Chern formula) more beautiful. In principle one should always replace the curvature $\Omega$ by $\frac{-1}{2 \pi \sqrt{-1}} \Omega$. The inhomogeneous cohomology class

$$
p(E):=\sum_{k \geq 0} p_{k}(E) \in H^{4 *}(M, \mathbb{R})
$$


is called the total Pontryagin class.

Theorem. For the Pontryagin classes we have:

(1) If $E_{1}$ and $E_{2}$ are two real vector bundles over a manifold $M$, then for the fiberwise direct sum we have

$$
p\left(E_{1} \oplus E_{2}\right)=p\left(E_{1}\right) \wedge p\left(E_{2}\right) \in H^{4 *}(M, \mathbb{R}) .
$$

(2) For the pullback of a vector bundle along $f: N \rightarrow M$ we have

$$
p\left(f^{*} E\right)=f^{*} p(E) .
$$

(3) For a real vector bundle and an invariant $f \in I^{k}(G L(n, \mathbb{R}))$ for odd $k$ we have $\mathrm{Cw}(f, E)=0$. Thus the Pontryagin classes exist only in dimension $0,4,8,12, \ldots$.

Proof. (10) If $\omega^{i} \in \Omega^{1}\left(G L\left(\mathbb{R}^{n_{i}}, E_{i}\right), \mathfrak{g l}\left(n_{i}\right)\right)^{G L\left(n_{i}\right)}$ are principal connection forms for the frame bundles of the two vector bundles, then for local frames of the two bundles $s_{\alpha}^{i} \in \Gamma\left(G L\left(\mathbb{R}^{n_{i}}, E_{i} \mid U_{\alpha}\right)\right.$, the forms

$$
\omega_{\alpha}:=\left(\begin{array}{cc}
\omega_{\alpha}^{1} & 0 \\
0 & \omega_{\alpha}^{2}
\end{array}\right) \in \Omega^{1}\left(U_{\alpha}, \mathfrak{g l}\left(n_{1}+n_{2}\right)\right)
$$

are exactly the local expressions of the direct sum connection, and from lemma (20.6) we see that $p_{k}\left(E_{1} \oplus E_{2}\right)=\sum_{j=0}^{k} p_{j}\left(E_{1}\right) p_{k-j}\left(E_{2}\right)$ holds, which implies the desired result.

(2) This follows from (20.3.4)

(3) Choose a fiber Riemann metric $g$ on $E$, consider the corresponding orthonormal frame bundle $\left(O\left(\mathbb{R}^{n}, E\right), p, M, O(n, \mathbb{R})\right)$, and choose a principal connection $\omega$ for it. Then the local expression with respect to local orthonormal frame fields $s_{\alpha}$ are skew-symmetric matrices of 1-forms. So the local curvature forms are also skew-symmetric. As we will show shortly, there exists a matrix $C \in O(n, \mathbb{R})$ such that $C A C^{-1}=A^{\top}=-A$ for any real skew-symmetric matrix; thus $C \Omega_{\alpha} C^{-1}=-\Omega_{\alpha}$. But then

$$
\begin{aligned}
f \circ\left(\Omega_{\alpha} \otimes_{\wedge} \cdots \otimes_{\wedge} \Omega_{\alpha}\right) & =f \circ\left(g_{\alpha} \Omega_{\alpha} g_{\alpha}^{-1} \otimes_{\wedge} \cdots \otimes_{\wedge} g_{\alpha} \Omega_{\alpha} g_{\alpha}^{-1}\right) \\
& =f \circ\left(\left(-\Omega_{\alpha}\right) \otimes_{\wedge} \cdots \otimes_{\wedge}\left(-\Omega_{\alpha}\right)\right) \\
& =(-1)^{k} f \circ\left(\Omega_{\alpha} \otimes_{\wedge} \cdots \otimes_{\wedge} \Omega_{\alpha}\right) .
\end{aligned}
$$

This implies that $\mathrm{Cw}(f, E)=0$ if $k$ is odd.

Claim. There exists a matrix $C \in O(n, \mathbb{R})$ such that $C A C^{-1}=A^{\top}$ for each real matrix with 0 's on the main diagonal.

Note first that

$$
\left(\begin{array}{ll}
0 & 1 \\
1 & 0
\end{array}\right)\left(\begin{array}{ll}
a & b \\
c & d
\end{array}\right)\left(\begin{array}{ll}
0 & 1 \\
1 & 0
\end{array}\right)=\left(\begin{array}{ll}
d & b \\
c & a
\end{array}\right)
$$


Let $E_{i j}$ be the matrix which has 1 in the position $(i, j)$ in the $i$-th row and $j$ th column. Then the $(i j)$-transposition matrix $P_{i j}=\mathbb{I}_{n}-E_{i i}-E_{j j}+E_{i j}+E_{j i}$ acts by conjugation on an arbitrary matrix $A$ by exchanging the pair $A_{i j}$ and $A_{j i}$ and also exchanging the pair $A_{i i}$ and $A_{j j}$ on the main diagonal. So the product $C=\prod_{i<j} P_{i j}$ has the required effect on a matrix with zeros on the main diagonal.

By the way, $\operatorname{Ad}(C)$ acts on the main diagonal via the longest element in the permutation group, with respect to the canonical system of positive roots in $\mathfrak{s l}(n)$ :

$$
\left(\begin{array}{ccccc}
1 & 2 & \ldots & n-1 & n \\
n & n-1 & \ldots & 2 & 1
\end{array}\right)
$$

20.8. Remarks. (1) If two vector bundles $E$ and $F$ are stably equivalent, i.e., $E \oplus\left(M \times \mathbb{R}^{m}\right) \cong F \oplus\left(M \times \mathbb{R}^{n}\right)$ for some $m$ and $n$, then $p(E)=p(F)$. This follows from (20.7.1) and (20.7.2).

(2) If for a vector bundle $E$ for some $k$ the bundle $\overbrace{E \oplus \cdots \oplus E}^{k} \oplus\left(M \times \mathbb{R}^{l}\right)$ is trivial, then $p(E)=1$ since $p(E)^{k}=1$.

(3) Let $(E, p, M)$ be a vector bundle over a compact oriented manifold $M$. For $j_{i} \in \mathbb{N}_{0}$ we put

$$
\lambda_{j_{1}, \ldots, j_{r}}(E):=\int_{M} p_{1}(E)^{j_{1}} \ldots p_{r}(E)^{j_{r}} \in \mathbb{R},
$$

where the integral is set to be 0 on each degree which is not equal to $\operatorname{dim} M$. Then these Pontryagin numbers are indeed integers; see [158]. For example we have

$$
\lambda_{j_{1}, \ldots, j_{r}}\left(T\left(\mathbb{C} P^{n}\right)\right)=\left(\begin{array}{c}
2 n+1 \\
j_{1}
\end{array}\right) \ldots\left(\begin{array}{c}
2 n+1 \\
j_{r}
\end{array}\right) .
$$

20.9. The trace coefficients. For a matrix $A \in \mathfrak{g l}(n, \mathbb{R})=L\left(\mathbb{R}^{n}, \mathbb{R}^{n}\right)$ the trace coefficients are given by

$$
\operatorname{tr}_{k}^{n}(A):=\operatorname{Trace}\left(A^{k}\right)=\operatorname{Trace}(\overbrace{A \circ \ldots \circ A}^{k}) .
$$

Obviously $\operatorname{tr}_{k}^{n}$ is an invariant polynomial, homogeneous of degree $k$. To a direct sum of two matrices $A \in \mathfrak{g l}(n)$ and $B \in \mathfrak{g l}(m)$ it reacts clearly by

$$
\operatorname{tr}_{k}^{n+m}\left(\begin{array}{cc}
A & 0 \\
0 & B
\end{array}\right)=\operatorname{Trace}\left(\begin{array}{cc}
A^{k} & 0 \\
0 & B^{k}
\end{array}\right)=\operatorname{tr}_{k}^{n}(A)+\operatorname{tr}_{k}^{m}(B) .
$$

The tensor product (sometimes also called the Kronecker product) of $A$ and $B$ is given by $A \otimes B=\left(A_{j}^{i} B_{l}^{k}\right)_{(i, k),(j, l) \in n \times m}$ in terms of the canonical bases. 
Since we have $\operatorname{Trace}(A \otimes B)=\sum_{i, k} A_{i}^{i} B_{k}^{k}=\operatorname{Trace}(A) \operatorname{Trace}(B)$, we also get $\operatorname{tr}_{k}^{n m}(A \otimes B)=\operatorname{Trace}\left((A \otimes B)^{k}\right)=\operatorname{Trace}\left(A^{k} \otimes B^{k}\right)=\operatorname{Trace}\left(A^{k}\right) \operatorname{Trace}\left(B^{k}\right)$ $=\operatorname{tr}_{k}^{n}(A) \operatorname{tr}_{k}^{m}(B)$.

Lemma. The trace coefficients and the characteristic coefficients are connected by the following recursive equation:

$$
c_{k}^{n}(A)=\frac{1}{k} \sum_{j=0}^{k-1}(-1)^{k-j-1} c_{j}^{n}(A) \operatorname{tr}_{k-j}^{n}(A) .
$$

Proof. For a matrix $A \in \mathfrak{g l}(n)$ let us denote by $C(A)$ the matrix of the signed algebraic complements of $A$ (also called the classical adjoint) as in (4.33), Then Cramer's rule reads

$$
\text { A.C } C(A)=C(A) \cdot A=\operatorname{det}(A) \cdot \mathbb{I},
$$

and the derivative of the determinant is given by (4.33);

$$
d \operatorname{det}(A) X=\operatorname{Trace}(C(A) X) .
$$

Note that $C(A)$ is a homogeneous matrix valued polynomial of degree $n-1$ in $A$. We define now matrix valued polynomials $a_{k}(A)$ by

$$
C(A+t \mathbb{I})=\sum_{k=0}^{n-1} a_{k}(A) t^{n-k-1} .
$$

We claim that for $A \in \mathfrak{g l}(n)$ and $k=0,1, \ldots, n-1$ we have

$$
a_{k}(A)=\sum_{j=0}^{k}(-1)^{j} c_{k-j}^{n}(A) A^{j} .
$$

We prove this in the following way: From (1) we have

$$
(A+t \mathbb{I}) C(A+t \mathbb{I})=\operatorname{det}(A+t \mathbb{I}) \mathbb{I},
$$

and we insert (3) and (20.6.1) to get in turn

$$
\begin{gathered}
(A+t \mathbb{I}) \sum_{k=0}^{n-1} a_{k}(A) t^{n-k-1}=\sum_{j=0}^{n} c_{j}^{n}(A) t^{n-j} \mathbb{I}, \\
\sum_{k=0}^{n-1} A \cdot a_{k}(A) t^{n-k-1}+\sum_{k=0}^{n-1} a_{k}(A) t^{n-k}=\sum_{j=0}^{n} c_{j}^{n}(A) t^{n-j} \mathbb{I} .
\end{gathered}
$$

We put $a_{-1}(A):=0=: a_{n}(A)$ and compare coefficients of $t^{n-k}$ in the last equation to get the recursion formula

$$
A \cdot a_{k-1}(A)+a_{k}(A)=c_{k}^{n}(A) . \mathbb{I}
$$


which immediately leads to the desired formula (4), even for $k=0,1, \ldots, n$. If we start this computation with the two factors in (11) reversed, we get A. $a_{k}(A)=a_{k}(A)$.A. Note that (44) for $k=n$ is exactly the Caley-Hamilton equation

$$
0=a_{n}(A)=\sum_{j=0}^{n} c_{n-j}^{n}(A) A^{j}
$$

We claim that

$$
\operatorname{Trace}\left(a_{k}(A)\right)=(n-k) c_{k}^{n}(A) .
$$

We use (2) for the proof:

$$
\begin{aligned}
\left.\partial\right|_{0}(\operatorname{det}(A+t \mathbb{I})) & =\left.d \operatorname{det}(A+t \mathbb{I}) \partial\right|_{0}(A+t \mathbb{I})=\operatorname{Trace}(C(A+t \mathbb{I}) \mathbb{I}) \\
& =\operatorname{Trace}\left(\sum_{k=0}^{n-1} a_{k}(A) t^{n-k-1}\right)=\sum_{k=0}^{n-1} \operatorname{Trace}\left(a_{k}(A)\right) t^{n-k-1}, \\
\left.\partial\right|_{0}(\operatorname{det}(A+t \mathbb{I})) & =\left.\partial\right|_{0}\left(\sum_{k=0}^{n} c_{k}^{n}(A) t^{n-k}\right) \\
& =\sum_{k=0}^{n}(n-k) c_{k}^{n}(A) t^{n-k-1} .
\end{aligned}
$$

Comparing coefficients leads to the result (5) .

Now we may prove the lemma itself by the following computation:

$$
\begin{aligned}
(n-k) c_{k}^{n}(A) & =\operatorname{Trace}\left(a_{k}(A)\right) \quad \text { by (5) } \\
& =\operatorname{Trace}\left(\sum_{j=0}^{k}(-1)^{j} c_{k-j}^{n}(A) A^{j}\right) \quad \text { by (4) } \\
& =\sum_{j=0}^{k}(-1)^{j} c_{k-j}^{n}(A) \operatorname{Trace}\left(A^{j}\right) \\
& =n c_{k}^{n}(A)+\sum_{j=1}^{k}(-1)^{j} c_{k-j}^{n}(A) \operatorname{tr}_{j}^{n}(A), \\
c_{k}^{n}(A) & =-\frac{1}{k} \sum_{j=1}^{k}(-1)^{j} c_{k-j}^{n}(A) \operatorname{tr}_{j}^{n}(A) \\
& =\frac{1}{k} \sum_{j=0}^{k-1}(-1)^{k-j-1} c_{j}^{n}(A) \operatorname{tr}_{k-j}^{n}(A) . \quad \square
\end{aligned}
$$


20.10. The trace classes. Let $(E, p, M)$ be a real vector bundle. Then the trace classes are given by

$$
\operatorname{tr}_{k}(E):=\left(\frac{-1}{2 \pi \sqrt{-1}}\right)^{2 k} \mathrm{Cw}\left(\operatorname{tr}_{2 k}^{\operatorname{dim} E}, E\right) \in H^{4 k}(M, \mathbb{R}) .
$$

Between the trace classes and the Pontryagin classes there are the following relations for $k \geq 1$

$$
p_{k}(E)=\frac{-1}{2 k} \sum_{j=0}^{k-1} p_{j}(E) \wedge \operatorname{tr}_{k-j}(E),
$$

which follows directly from lemma (20.9) above.

The inhomogeneous cohomology class

$$
\operatorname{tr}(E)=\sum_{k=0}^{\infty} \frac{1}{(2 k) !} \operatorname{tr}_{k}(E)=\mathrm{Cw}(\text { Trace } \circ \exp , E)
$$

is called the Pontryagin character of $E$. In the second expression we use the smooth invariant function Trace $\circ \exp : \mathfrak{g l}(n) \rightarrow \mathbb{R}$ which is given by

$$
\operatorname{Trace}(\exp (A))=\operatorname{Trace}\left(\sum_{k \geq 0} \frac{A^{k}}{k !}\right)=\sum_{k \geq 0} \frac{1}{k !} \operatorname{Trace}\left(A^{k}\right) \text {. }
$$

Of course one should first take the Taylor series at 0 of Trace $\circ$ exp and then take the Chern-Weil class of each homogeneous part separately.

Theorem. Let $\left(E_{i}, p, M\right)$ be vector bundles over the same base manifold $M$. Then we have:

(4) $\operatorname{tr}\left(E_{1} \oplus E_{2}\right)=\operatorname{tr}\left(E_{1}\right)+\operatorname{tr}\left(E_{2}\right)$.

(5) $\operatorname{tr}\left(E_{1} \otimes E_{2}\right)=\operatorname{tr}\left(E_{1}\right) \wedge \operatorname{tr}\left(E_{2}\right)$.

(6) $\operatorname{tr}\left(g^{*} E\right)=g^{*} \operatorname{tr}(E)$ for any smooth mapping $g: N \rightarrow M$.

Clearly stably equivalent vector bundles have equal Pontryagin characters. Statements (4) and (5) say that one may view the Pontryagin character as a ring homomorphism from the real $K$-theory into cohomology,

$$
\operatorname{tr}: K_{\mathbb{R}}(M) \rightarrow H^{4 *}(M ; \mathbb{R}) .
$$

Statement (6) says that it is even a natural transformation.

Proof. (4) This can be proved in the same way as (20.7.1), but we indicate another method which will be used also in the proof of (5) below. Covariant derivatives for $E_{1}$ and $E_{2}$ induce a covariant derivative on $E_{1} \oplus E_{2}$ by 
$\nabla_{X}^{E_{1} \oplus E_{2}}\left(s_{1}, s_{2}\right)=\left(\nabla_{X}^{E_{1}} s_{1}, \nabla_{X}^{E_{2}}, s_{2}\right)$. For the curvature operators we clearly have

$$
R^{E_{1} \oplus E_{2}}=R^{E_{1}} \oplus R^{E_{2}}=\left(\begin{array}{cc}
R^{E_{1}} & 0 \\
0 & R^{E_{2}}
\end{array}\right) .
$$

So the result follows from (20.9) with the help of (20.5).

(5) We have an induced covariant derivative on $E_{1} \otimes E_{2}$ given by $\nabla_{X}^{E_{1} \otimes E_{2}} s_{1} \otimes$ $s_{2}=\left(\nabla_{X}^{E_{1}} s_{1}\right) \otimes s_{2}+s_{1} \otimes\left(\nabla_{X}^{E_{2}} s_{2}\right)$. Then for the curvatures we get obviously $R^{E_{1} \otimes E_{2}}(X, Y)=R^{E_{1}}(X, Y) \otimes I d_{E_{2}}+I d_{E_{1}} \otimes R^{E_{2}}(X, Y)$. The two summands of the last expression commute, so we get

$$
\left(R^{E_{1}} \otimes I d_{E_{2}}+I d_{E_{1}} \otimes R^{E_{2}}\right)^{{ }_{\wedge}, k}=\sum_{j=0}^{k}\left(\begin{array}{c}
k \\
j
\end{array}\right)\left(R^{E_{1}}\right)^{\diamond_{\wedge}, j} \otimes_{\wedge}\left(R^{E_{2}}\right)^{\diamond_{\wedge}, k-j},
$$

where the product involved is given as in

$$
\left(R^{E} \circ \wedge R^{E}\right)\left(X_{1}, \ldots, X_{4}\right)=\frac{1}{2 ! 2 !} \sum_{\sigma} \operatorname{sign}(\sigma) R^{E}\left(X_{\sigma 1}, X_{\sigma 2}\right) \circ R^{E}\left(X_{\sigma 3}, X_{\sigma 4}\right),
$$

which makes $\left(\Omega(M, L(E, E)), \circ_{\wedge}\right)$ into a graded associative algebra. The next computation takes place in a commutative subalgebra of it:

$$
\begin{aligned}
\operatorname{tr}\left(E_{1} \otimes E_{2}\right) & =\left[\operatorname{Trace} \exp \left(R^{E_{1}} \otimes I d_{E_{2}}+I d_{E_{1}} \otimes R^{E_{2}}\right)\right]_{H(M)} \\
& =\left[\operatorname{Trace}\left(\exp \left(R^{E_{1}}\right) \otimes_{\wedge} \exp \left(R^{E_{2}}\right)\right)\right]_{H(M)} \\
& =\left[\operatorname{Trace}\left(\exp \left(R^{E_{1}}\right)\right) \wedge \operatorname{Trace}\left(\exp \left(R^{E_{2}}\right)\right)\right]_{H(M)} \\
& =\operatorname{tr}\left(E_{1}\right) \wedge \operatorname{tr}\left(E_{2}\right) .
\end{aligned}
$$

(6) This is a general fact.

20.11. The Pfaffian. Let $(V, g)$ be a real Euclidian vector space of dimension $n$, with a positive definite inner product $g$. Then for each $p$ we have an induced inner product on $\bigwedge^{p} V$, see also (25.11), which is given by

$$
\left\langle x_{1} \wedge \cdots \wedge x_{p}, y_{1} \wedge \cdots \wedge y_{p}\right\rangle_{g}=\operatorname{det}\left(g\left(x_{i}, y_{j}\right)_{i, j}\right) .
$$

The inner product $g$, when viewed as a linear isomorphism $g: V \rightarrow V^{*}$, induces an isomorphism $\beta: \bigwedge^{2} V \rightarrow L_{g \text {,skew }}(V, V)$ which is given on decomposable forms by $\beta(x \wedge y)(z)=g(x, z) y-g(y, z) x$. We also have

$$
\begin{gathered}
\beta^{-1}(A)=A \circ g^{-1} \in L_{\text {skew }}\left(V^{*}, V\right)=\left\{B \in L\left(V^{*}, V\right): B^{\top}=-B\right\} \cong \bigwedge^{2} V, \\
\text { where } B^{\top}: V^{*} \stackrel{B^{*}}{\longrightarrow} V^{* *} \stackrel{\cong}{\longrightarrow} .
\end{gathered}
$$

Now we assume that $V$ is of even dimension $n$ and is oriented. Then there is a unique element $e \in \bigwedge^{n} V$ which is positive and normed: $\langle e, e\rangle_{g}=1$. We 
define the Pfaffian of a skew-symmetric matrix $A$ by:

$$
\mathrm{Pf}^{g}(A):=\frac{1}{n !}\langle e, \overbrace{\beta^{-1}(A) \wedge \cdots \wedge \beta^{-1}(A)}^{n / 2}\rangle_{g}, \quad A \in \mathfrak{s o}(n, \mathbb{R}) .
$$

This is a homogeneous polynomial of degree $n / 2$ on $\mathfrak{s o}(n, \mathbb{R})$. Its polarization is the $n / 2$-linear symmetric functional

$$
\mathrm{Pf}^{g}\left(A_{1}, \ldots, A_{n / 2}\right)=\frac{1}{n !}\left\langle e, \beta^{-1}\left(A_{1}\right) \wedge \cdots \wedge \beta^{-1}\left(A_{n / 2}\right)\right\rangle_{g} .
$$

Lemma. For an even-dimensional oriented Euclidean vector space $(V, g)$ and skew-symmetric $A$ we have:

(1) For $B \in L(V, V)$ we have $\operatorname{Pf}^{g}\left(B . A \cdot B^{\top}\right)=\operatorname{det}(B) \operatorname{Pf}(A)$ where $B^{\top}$ is the transpose with respect to $g$.

(2) If $U \in O(V, g)$, then $\mathrm{Pf}^{g}\left(U \cdot A \cdot U^{-1}\right)=\operatorname{det}(U) \mathrm{Pf}^{g}(A)$, so $\mathrm{Pf}^{g}$ is invariant under the adjoint action of $S O(V, g)$.

(3) If $X \in L_{g \text {,skew }}(V, V)=\mathfrak{o}(V, g)$, then we have

$$
\sum_{i=1}^{n / 2} \operatorname{Pf}^{g}\left(A_{1}, \ldots,\left[X, A_{i}\right], \ldots, A_{n / 2}\right)=0 .
$$

(4) $\operatorname{Pf}(r A)=r^{n / 2} \operatorname{Pf}(A)$ for $r \in \mathbb{R}$ and thus also $\operatorname{Pf}\left(A^{\top}\right)=(-1)^{n / 2} \operatorname{Pf}(A)$.

(5) $\operatorname{Pf}(A)^{2}=\operatorname{det}(A)$.

(6) We have

$$
\operatorname{Pf}(A)=\frac{1}{2^{n / 2}(n / 2) !} \sum_{\sigma \in \mathcal{S}_{n}} \operatorname{sign}(\sigma) \prod_{i=1}^{n / 2} A_{\sigma(2 i-1), \sigma(2 i)} .
$$

(7) (191], [57])

$$
\operatorname{Pf}(A)=\sum_{i<j} A_{i, j}(-1)^{i+j} \operatorname{sign}(i-j) \operatorname{Pf}(A(i j, i j))
$$

where $A(i j, i j)$ is the matrix obtained from $A$ by deleting the lines and columns numbered $i$ and $j$.

Proof. (1) The transposed $B^{\top}$ is given by $g(B x, z)=g\left(x, B^{\top} z\right)$. So $\beta(B x \wedge$ $B y)=B \cdot \beta(x \wedge y) \cdot B^{\top}$ and thus $\beta^{-1}\left(B \cdot A \cdot B^{\top}\right)=\bigwedge^{2} B \beta^{-1}(A)$. Then we have:

$$
\begin{aligned}
\operatorname{Pf}^{g}\left(B . A \cdot B^{\top}\right) & =\frac{1}{n !}\left\langle e, \bigwedge^{n}(B)\left(\beta^{-1}(A) \wedge \cdots \wedge \beta^{-1}(A)\right)\right\rangle_{g} \\
& =\frac{1}{n !} \operatorname{det}(B)\left\langle\bigwedge^{n}(U) e, \bigwedge^{n}(U)\left(\beta^{-1}(A) \wedge \cdots \wedge \beta^{-1}(A)\right)\right\rangle_{g} \\
& =\frac{1}{n !} \operatorname{det}(B)\left\langle e, \beta^{-1}(A) \wedge \cdots \wedge \beta^{-1}(A)\right\rangle_{g}
\end{aligned}
$$




$$
=\operatorname{det}(B) \operatorname{Pf}^{g}(A) .
$$

(2) We have $U \in O(V, g)$ if and only if $U^{\top}=U^{-1}$. So this follows from (1).

(3) This follows from (2) by differentiation; see the beginning of the proof of (20.3).

(41) is obvious. The rest is left as an exercise.

20.12. The Pfaffian class. Let $(E, p, M, V)$ be a vector bundle which is fiber oriented and of even fiber dimension. If we choose a fiberwise Riemann metric on $E$, we in fact reduce the linear frame bundle of $E$ to the oriented orthonormal one, $S O\left(\mathbb{R}^{n}, E\right)$. On the Lie algebra $\mathfrak{o}(n, \mathbb{R})$ of the structure group $S O(n, \mathbb{R})$ the Pfaffian form Pf of the standard inner product is an invariant, $\mathrm{Pf} \in I^{n / 2}(S O(n, \mathbb{R}))$. We define the Pfaffian class of the oriented bundle $E$ by

$$
\operatorname{Pf}(E):=\left(\frac{-1}{2 \pi \sqrt{-1}}\right)^{n / 2} \mathrm{Cw}\left(\operatorname{Pf}, S O\left(\mathbb{R}^{n}, E\right)\right) \in H^{n}(M) .
$$

It does not depend on the choice of the Riemann metric on $E$, since for any two fiberwise Riemann metrics $g_{1}$ and $g_{2}$ on $E$ there is an isometric vector bundle isomorphism $f:\left(E, g_{1}\right) \rightarrow\left(E, g_{2}\right)$ covering the identity of $M$, which pulls an $S O(n)$-connection for $\left(E, g_{2}\right)$ to an $S O(n)$-connection for $\left(E, g_{1}\right)$. So the two Pfaffian classes coincide since then $\operatorname{Pf}^{1} \circ\left(f^{*} \Omega_{2} \otimes_{\wedge} \cdots \otimes_{\wedge} f^{*} \Omega_{2}\right)=$ $\operatorname{Pf}^{2} \circ\left(\Omega_{2} \otimes_{\wedge} \cdots \otimes_{\wedge} \Omega_{2}\right)$.

Theorem. The Pfaffian class of oriented even-dimensional vector bundles has the following properties:

(1) $\operatorname{Pf}(E)^{2}=(-1)^{n / 2} p_{n / 2}(E)$ where $n$ is the fiber dimension of $E$.

(2) $\operatorname{Pf}\left(E_{1} \oplus E_{2}\right)=\operatorname{Pf}\left(E_{1}\right) \wedge \operatorname{Pf}\left(E_{2}\right)$.

(3) $\operatorname{Pf}\left(g^{*} E\right)=g^{*} \operatorname{Pf}(E)$ for smooth $g: N \rightarrow M$.

Proof. This is left as an exercise for the reader.

20.13. Chern classes. Let $(E, p, M)$ be a complex vector bundle over the smooth manifold $M$. So the structure group is $G L(n, \mathbb{C})$ where $n$ is the fiber dimension. Recall now the explanation of the characteristic coefficients $c_{k}^{n}$ in (20.6) and insert complex numbers everywhere. Then we get the characteristic coefficients $c_{k}^{n} \in I^{k}(G L(n, \mathbb{C}))$, which are just the extensions of the real ones to the complexification.

We define then the Chern classes by

$$
c_{k}(E):=\left(\frac{-1}{2 \pi \sqrt{-1}}\right)^{k} \mathrm{Cw}\left(c_{k}^{\operatorname{dim} E}, E\right) \in H^{2 k}(M ; \mathbb{R}) .
$$


The total Chern class is again the inhomogeneous cohomology class

$$
c(E):=\sum_{k=0}^{\operatorname{dim}_{\mathbb{C}} E} c_{k}(E) \in H^{2 *}(M ; \mathbb{R}) .
$$

It has the following properties:

$$
\begin{aligned}
c(\bar{E}) & =(-1)^{\operatorname{dim}_{\mathbb{C}} E} c(E), \\
c\left(E_{1} \oplus E_{2}\right) & =c\left(E_{1}\right) \wedge c\left(E_{2}\right), \\
c\left(g^{*} E\right) & =g^{*} c(E) \quad \text { for smooth } g: N \rightarrow M .
\end{aligned}
$$

One can show (see [158]) that (3), (4), (5)), and the following normalization determine the total Chern class already completely: The total Chern class of the canonical complex line bundle over $S^{2}$ (the square root of the tangent bundle with respect to the tensor product) is $1+\omega_{S^{2}}$, where $\omega_{S^{2}}$ is the canonical volume form on $S^{2}$ with total volume 1 .

Lemma. Then Chern classes are real cohomology classes.

Proof. We choose a Hermitian metric on the complex vector bundle $E$, i.e., we reduce the structure group from $G L(n, \mathbb{C})$ to $U(n)$. Then the curvature $\Omega$ of a $U(n)$-principal connection has values in the Lie algebra $\mathfrak{u}(n)$ of skewHermitian matrices $A$ with $A^{*}=-A$. But then we have $c_{k}^{n}(-\sqrt{-1} A) \in \mathbb{R}$ since $\overline{\operatorname{det}_{\mathbb{C}}(-\sqrt{-1} A+t \mathbb{I})}=\operatorname{det}_{\mathbb{C}}(\overline{-\sqrt{-1} A}+t \mathbb{I})=\operatorname{det}_{\mathbb{C}}(-\sqrt{-1} A+t \mathbb{I})$.

20.14. The Chern character. The trace classes of a complex vector bundle are given by

$$
\operatorname{tr}_{k}(E):=\left(\frac{-1}{2 \pi \sqrt{-1}}\right)^{k} \mathrm{Cw}\left(\operatorname{tr}_{k}^{\operatorname{dim} E}, E\right) \in H^{2 k}(M, \mathbb{R}) .
$$

They are also real cohomology classes, and we have $\operatorname{tr}_{0}(E)=\operatorname{dim}_{\mathbb{C}} E$, the fiber dimension of $E$, and $\operatorname{tr}_{1}(E)=c_{1}(E)$. In general we have the following recursive relation between the Chern classes and the trace classes:

$$
c_{k}(E)=\frac{-1}{k} \sum_{j=0}^{k-1} c_{j}(E) \wedge \operatorname{tr}_{k-j}(E),
$$

which follows directly from lemma (20.9). The inhomogeneous cohomology class

$$
\operatorname{ch}(E):=\sum_{k \geq 0} \frac{1}{k !} \operatorname{tr}_{k}(E) \in H^{2 *}(M, \mathbb{R})
$$

is called the Chern character of the complex vector bundle $E$. With the same methods as for the Pontryagin character one can show that the Chern 
character satisfies the following properties:

$$
\begin{aligned}
\operatorname{ch}\left(E_{1} \oplus E_{2}\right) & =\operatorname{ch}\left(E_{1}\right)+\operatorname{ch}\left(E_{2}\right), \\
\operatorname{ch}\left(E_{1} \otimes E_{2}\right) & =\operatorname{ch}\left(E_{1}\right) \wedge \operatorname{ch}\left(E_{2}\right), \\
\operatorname{ch}\left(g^{*} E\right) & =g^{*} \operatorname{ch}(E) .
\end{aligned}
$$

From these it clearly follows that the Chern character can be viewed as a ring homomorphism from complex $K$-theory into even cohomology,

$$
\operatorname{ch}: K_{\mathbb{C}}(M) \rightarrow H^{2 *}(M, \mathbb{R}),
$$

which is natural.

Finally we remark that the Pfaffian class of the underlying real vector bundle of a complex vector bundle $E$ of complex fiber dimension $n$ coincides with the Chern class $c_{n}(E)$. But there is a new class, the Todd class; see below.

20.15. The Todd class. On the vector space $\mathfrak{g l}(n, \mathbb{C})$ of all complex $(n \times$ $n$ )-matrices we consider the smooth function

$$
f(A):=\operatorname{det} \mathbb{C}\left(\sum_{k=0}^{\infty} \frac{(-1)^{k}}{(k+1) !} A^{k}\right) .
$$

It is the unique smooth function which satisfies the functional equation

$$
\operatorname{det}(A) \cdot f(A)=\operatorname{det}(\mathbb{I}-\exp (-A)) .
$$

Clearly $f$ is invariant under $\operatorname{Ad}(G L(n, \mathbb{C}))$ and $f(0)=1$, so we may consider the invariant smooth function, defined near $0, \operatorname{Td}: \mathfrak{g l}(n, \mathbb{C}) \supset U \rightarrow \mathbb{C}$, which is given by $\operatorname{Td}(A)=1 / f(A)$. It is uniquely defined by the functional equations

$$
\begin{gathered}
\operatorname{det}(A)=\operatorname{Td}(A) \operatorname{det}(\mathbb{I}-\exp (-A)), \\
\operatorname{det}\left(\frac{1}{2} A\right) \operatorname{det}\left(\exp \left(\frac{1}{2} A\right)\right)=\operatorname{Td}(A) \operatorname{det}\left(\sinh \left(\frac{1}{2} A\right)\right) .
\end{gathered}
$$

The Todd class of a complex vector bundle is then given by

$$
\begin{aligned}
\operatorname{Td}(E) & =\left[G L\left(\mathbb{C}^{n}, E\right)[\mathrm{Td}]\left(\sum_{k \geq 0}\left(\frac{-1}{2 \pi \sqrt{-1}} R^{E}\right)^{\otimes_{\wedge}, k}\right)\right]_{H^{2 *}(M, \mathbb{R})} \\
& =\mathrm{Cw}(\mathrm{Td}, E) .
\end{aligned}
$$

The Todd class is a real cohomology class since for $A \in \mathfrak{u}(n)$ we have $\operatorname{Td}(-A)=\operatorname{Td}\left(A^{*}\right)=\overline{\operatorname{Td}(A)}$. Since $\operatorname{Td}(0)=1$, the Todd class $\operatorname{Td}(E)$ is an invertible element of $H^{2 *}(M, \mathbb{R})$. 
20.16. The Atiyah-Singer index formula (roughly). Let $E_{i}$ be complex vector bundles over a compact manifold $M$, and let $D: \Gamma\left(E_{1}\right) \rightarrow \Gamma\left(E_{2}\right)$ be an elliptic pseudodifferential operator of order $p$. Then for appropriate Sobolev completions $D$ prolongs to a bounded Fredholm operator between Hilbert spaces $D: \mathcal{H}^{d+p}\left(E_{1}\right) \rightarrow \mathcal{H}^{d}\left(E_{2}\right)$. Its index $\operatorname{index}(D)$ is defined as the dimension of the kernel minus the dimension of the cokernel, which does not depend on $d$ if it is high enough. The Atiyah-Singer index formula says that

$$
\operatorname{index}(D)=(-1)^{\operatorname{dim} M} \int_{T M} \operatorname{ch}(\sigma(D)) \operatorname{Td}(T M \otimes \mathbb{C}),
$$

where $\sigma(D)$ is a virtual vector bundle (with compact support) on $T M \backslash 0$, a formal difference of two vector bundles, the so-called symbol bundle of $D$.

See 21 for a somewhat informal introduction, 208 for a very short introduction, [73 for an analytical treatment using the heat kernel method, [116] for a recent treatment and the papers by Atiyah and Singer for the real thing.

Special cases are the Gauß-Bonnet-Chern formula and the Riemann-RochHirzebruch formula.

\section{Jets}

Jet spaces or jet bundles consist of the invariant expressions of Taylor developments up to a certain order of smooth mappings between manifolds. Their invention goes back to Ehresmann [53. We could have treated them from the beginning and could have mixed them into every chapter, but it is also fine to have all results collected in one place.

21.1. Contact. Recall that smooth functions $f, g: \mathbb{R} \rightarrow \mathbb{R}$ are said to have contact of order $k$ at 0 if all their values and all derivatives up to order $k$ coincide.

Lemma. Let $f, g: M \rightarrow N$ be smooth mappings between smooth manifolds and let $x \in M$. Then the following conditions are equivalent.

(1) For each smooth curve $c: \mathbb{R} \rightarrow M$ with $c(0)=x$ and for each smooth function $h \in C^{\infty}(M)$ the two functions $h \circ f \circ c$ and $h \circ g \circ c$ have contact of order $k$ at 0 .

(2) For each chart $(U, u)$ of $M$ centered at $x$ and each chart $(V, v)$ of $N$ with $f(x) \in V$ the two mappings $v \circ f \circ u^{-1}$ and $v \circ g \circ u^{-1}$, defined near 0 in $\mathbb{R}^{m}$, with values in $\mathbb{R}^{n}$, have the same Taylor development up to order $k$ at 0 . 
(3) For some charts $(U, u)$ of $M$ and $(V, v)$ of $N$ with $x \in U$ and $f(x) \in V$ we have

$$
\left.\frac{\partial^{|\alpha|}}{\partial u^{\alpha}}\right|_{x}(v \circ f)=\left.\frac{\partial^{|\alpha|}}{\partial u^{\alpha}}\right|_{x}(v \circ g)
$$

for all multiindices $\alpha \in \mathbb{N}_{0}^{m}$ with $0 \leq|\alpha| \leq k$.

(4) $T_{x}^{k} f=T_{x}^{k} g$, where $T^{k}$ is the $k$-th iterated tangent bundle functor.

Proof. This is an easy exercise in analysis.

21.2. Definition. If the equivalent conditions of lemma (21.1) are satisfied, we say that $f$ and $g$ have the same $k$-jet at $x$ and we write $j^{k} f(x)$ or $j_{x}^{k} f$ for the resulting equivalence class and call it the $k$-jet at $x$ of $f ; x$ is called the source of the $k$-jet, and $f(x)$ is its target.

The space of all $k$-jets of smooth mappings from $M$ to $N$ is denoted by $J^{k}(M, N)$. We have the source mapping $\alpha: J^{k}(M, N) \rightarrow M$ and the target mapping $\beta: J^{k}(M, N) \rightarrow N$, given by $\alpha\left(j^{k} f(x)\right)=x$ and $\beta\left(j^{k} f(x)\right)=$ $f(x)$. We will also write $J_{x}^{k}(M, N):=\alpha^{-1}(x), J^{k}(M, N)_{y}:=\beta^{-1}(y)$, and $J_{x}^{k}(M, N)_{y}:=J_{x}^{k}(M, N) \cap J^{k}(M, N)_{y}$ for the spaces of jets with source $x$, target $y$, and both, respectively. For $l<k$ we have a canonical surjective mapping $\pi_{l}^{k}: J^{k}(M, N) \rightarrow J^{l}(M, N)$, given by $\pi_{l}^{k}\left(j^{k} f(x)\right):=j^{l} f(x)$. This mapping respects the fibers of $\alpha$ and $\beta$ and $\pi_{0}^{k}=(\alpha, \beta): J^{k}(M, N) \rightarrow$ $M \times N$.

21.3. Jets on vector spaces. Now we look at the case $M=\mathbb{R}^{m}$ and $N=\mathbb{R}^{n}$.

Let $f: \mathbb{R}^{m} \rightarrow \mathbb{R}^{n}$ be a smooth mapping. Then by (21.1.3) the $k$-jet $j^{k} f(x)$ of $f$ at $x$ has a canonical representative, namely the Taylor polynomial of order $k$ of $f$ at $x$ :

$$
\begin{aligned}
f(x+y) & =f(x)+d f(x) \cdot y+\frac{1}{2 !} d^{2} f(x) y^{2}+\cdots+\frac{1}{k !} d^{k} f(x) \cdot y^{k}+o\left(|y|^{k}\right) \\
& =: f(x)+\operatorname{Tay}_{x}^{k} f(y)+o\left(|y|^{k}\right) .
\end{aligned}
$$

Here $y^{k}$ is short for $(y, y, \ldots, y), k$-times. The 'Taylor polynomial without constant'

$$
\operatorname{Tay}_{x}^{k} f: y \mapsto \operatorname{Tay}_{x}^{k}(y):=d f(x) \cdot y+\frac{1}{2 !} d^{2} f(x) \cdot y^{2}+\cdots+\frac{1}{k !} d^{k} f(x) \cdot y^{k}
$$

is an element of the linear space

$$
P^{k}(m, n):=\bigoplus_{j=1}^{k} L_{\text {sym }}^{j}\left(\mathbb{R}^{m}, \mathbb{R}^{n}\right),
$$


where $L_{\text {sym }}^{j}\left(\mathbb{R}^{m}, \mathbb{R}^{n}\right)$ is the vector space of all $j$-linear symmetric mappings $\mathbb{R}^{m} \times \cdots \times \mathbb{R}^{m} \rightarrow \mathbb{R}^{n}$, where we silently use the total polarization of polynomials. Conversely each polynomial $p \in P^{k}(m, n)$ defines a $k$-jet $j_{0}^{k}(y \mapsto z+p(x+y))$ with arbitrary source $x$ and target $z$. So we get canonical identifications $J_{x}^{k}\left(\mathbb{R}^{m}, \mathbb{R}^{n}\right)_{z} \cong P^{k}(m, n)$ and

$$
J^{k}\left(\mathbb{R}^{m}, \mathbb{R}^{n}\right) \cong \mathbb{R}^{m} \times \mathbb{R}^{n} \times P^{k}(m, n) .
$$

If $U \subset \mathbb{R}^{m}$ and $V \subset \mathbb{R}^{n}$ are open subsets, then clearly $J^{k}(U, V) \cong U \times V \times$ $P^{k}(m, n)$ in the same canonical way.

For later uses we consider now the truncated composition

$$
\text { - : } P^{k}(m, n) \times P^{k}(p, m) \rightarrow P^{k}(p, n),
$$

where $p \bullet q$ is just the polynomial $p \circ q$ without all terms of order $>k$. Obviously it is a polynomial, thus a real analytic mapping. Now let $U \subset \mathbb{R}^{m}$, $V \subset \mathbb{R}^{n}$, and $W \subset \mathbb{R}^{p}$ be open subsets and consider the fibered product

$$
\begin{aligned}
J^{k}(U, V) \times{ }_{U} J^{k}(W, U) & =\left\{(\sigma, \tau) \in J^{k}(U, V) \times J^{k}(W, U): \alpha(\sigma)=\beta(\tau)\right\} \\
& =U \times V \times W \times P^{k}(m, n) \times P^{k}(p, m) .
\end{aligned}
$$

Then the mapping

$$
\begin{gathered}
\gamma: J^{k}(U, V) \times_{U} J^{k}(W, U) \rightarrow J^{k}(W, V), \\
\gamma(\sigma, \tau)=\gamma((\alpha(\sigma), \beta(\sigma), \bar{\sigma}),(\alpha(\tau), \beta(\tau), \bar{\tau}))=(\alpha(\tau), \beta(\sigma), \bar{\sigma} \bullet \bar{\tau})
\end{gathered}
$$

is a real analytic mapping, called the fibered composition of jets.

Let $U, U^{\prime} \subset \mathbb{R}^{m}$ and $V \subset \mathbb{R}^{n}$ be open subsets and let $g: U^{\prime} \rightarrow U$ be a smooth diffeomorphism. We define a mapping $J^{k}(g, V): J^{k}(U, V) \rightarrow$ $J^{k}\left(U^{\prime}, V\right)$ by $J^{k}(g, V)\left(j^{k} f(x)\right)=j^{k}(f \circ g)\left(g^{-1}(x)\right)$. Using the canonical representation of jets from above, we get $J^{k}(g, V)(\sigma)=\gamma\left(\sigma, j^{k} g\left(g^{-1}(x)\right)\right)$ or $J^{k}(g, V)(x, y, \bar{\sigma})=\left(g^{-1}(x), y, \bar{\sigma} \bullet \operatorname{Tay}_{g^{-1}(x)}^{k} g\right)$. If $g$ is a $C^{p}$ diffeomorphism, then $J^{k}(g, V)$ is a $C^{p-k}$ diffeomorphism. If $g^{\prime}: U^{\prime \prime} \rightarrow U^{\prime}$ is another diffeomorphism, then clearly $J^{k}\left(g^{\prime}, V\right) \circ J^{k}(g, V)=J^{k}\left(g \circ g^{\prime}, V\right)$ and $J^{k}(, V)$ is a contravariant functor acting on diffeomorphisms between open subsets of $\mathbb{R}^{m}$. Since the truncated composition $\bar{\sigma} \mapsto \bar{\sigma} \bullet \operatorname{Tay}_{g^{-1}(x)}^{k} g$ is linear, the mapping $J_{x}^{k}\left(g, \mathbb{R}^{n}\right):=J^{k}\left(g, \mathbb{R}^{n}\right) \mid J_{x}^{k}\left(U, \mathbb{R}^{n}\right): J_{x}^{k}\left(U, \mathbb{R}^{n}\right) \rightarrow J_{g^{-1}(x)}^{k}\left(U^{\prime}, \mathbb{R}^{n}\right)$ is also linear.

If more generally $g: M^{\prime} \rightarrow M$ is a diffeomorphism between manifolds, the same formula as above defines a bijective mapping $J^{k}(g, N): J^{k}(M, N) \rightarrow$ $J^{k}\left(M^{\prime}, N\right)$ and clearly $J^{k}(, N)$ is a contravariant functor defined on the category of manifolds and diffeomorphisms.

Now let $U \subset \mathbb{R}^{m}, V \subset \mathbb{R}^{n}$, and $W \subset \mathbb{R}^{p}$ be open subsets and let $h: V \rightarrow W$ be a smooth mapping. Then we define $J^{k}(U, h): J^{k}(U, V) \rightarrow J^{k}(U, W)$ by 


$$
\begin{aligned}
J^{k}(U, h)\left(j^{k} f(x)\right)= & j^{k}(h \circ f)(x) \text { or equivalently by } \\
& J^{k}(U, h)(x, y, \bar{\sigma})=\left(x, h(y), \operatorname{Tay}_{y}^{k} h \bullet \bar{\sigma}\right) .
\end{aligned}
$$

If $h$ is $C^{p}$, then $J^{k}(U, h)$ is $C^{p-k}$. Clearly $J^{k}(U, \quad)$ is a covariant functor acting on smooth mappings between open subsets of finite-dimensional vector spaces. The mapping $J_{x}^{k}(U, h)_{y}: J_{x}^{k}(U, V)_{y} \rightarrow J^{k}(U, W)_{h(y)}$ is linear if and only if the mapping $\bar{\sigma} \mapsto \operatorname{Tay}_{y}^{k} h \bullet \bar{\sigma}$ is linear, i.e., if $h$ is affine or if $k=1$. If $h: N \rightarrow N^{\prime}$ is a smooth mapping between manifolds, we have by the same procedure a mapping $J^{k}(M, h): J^{k}(M, N) \rightarrow J^{k}\left(M, N^{\prime}\right)$ and $J^{k}(M, \quad)$ turns out to be a functor on the category of manifolds and smooth mappings.

21.4. The differential group $G_{m}^{k}$. The $k$-jets at 0 of diffeomorphisms of $\mathbb{R}^{m}$ which map 0 to 0 form a group under truncated composition, which will be denoted by $G L^{k}(m, \mathbb{R})$ or $G_{m}^{k}$ for short, and will be called the differential group of order $k$. Clearly an arbitrary 0-respecting $k$-jet $\sigma \in P^{k}(m, m)$ is in $G_{m}^{k}$ if and only if its linear part is invertible; thus

$$
G_{m}^{k}=G L^{k}(m, \mathbb{R})=G L(m) \oplus \bigoplus_{j=2}^{k} L_{\mathrm{sym}}^{j}\left(\mathbb{R}^{m}, \mathbb{R}^{m}\right)=: G L(m) \times P_{2}^{k}(m),
$$

where we put $P_{2}^{k}(m)=\bigoplus_{j=2}^{k} L_{\text {sym }}^{j}\left(\mathbb{R}^{m}, \mathbb{R}^{m}\right)$ for the space of all polynomial mappings without constant and linear term of degree $\leq k$. Since the truncated composition is a polynomial mapping, $G_{m}^{k}$ is a Lie group, and the mapping $\pi_{l}^{k}: G_{m}^{k} \rightarrow G_{m}^{l}$ is a homomorphism of Lie groups, so $\operatorname{ker}\left(\pi_{l}^{k}\right)=\bigoplus_{j=l+1}^{k} L_{\text {sym }}^{j}\left(\mathbb{R}^{m}, \mathbb{R}^{m}\right)=: P_{l+1}^{k}(m)$ is a normal subgroup for all $l$. The exact sequence of groups

$$
\{e\} \rightarrow P_{l+1}^{k}(m) \rightarrow G_{m}^{k} \rightarrow G_{m}^{l} \rightarrow\{e\}
$$

splits if and only if $l=1$; only then do we have a semidirect product.

21.5. Theorem. For smooth manifolds $M$ and $N$ we have:

(1) $J^{k}(M, N)$ is a smooth manifold (it is of class $C^{r-k}$ if $M$ and $N$ are of class $\left.C^{r}\right)$; a canonical atlas is given by all charts $\left(J^{k}(U, V), J^{k}\left(u^{-1}, v\right)\right)$, where $(U, u)$ is a chart on $M$ and $(V, v)$ is a chart on $N$.

(2) $\left(J^{k}(M, N),(\alpha, \beta), M \times N, P^{k}(m, n), G_{m}^{k} \times G_{n}^{k}\right)$ is a fiber bundle with structure group, where $m=\operatorname{dim} M, n=\operatorname{dim} N$, and where $(\gamma, \chi) \in$ $G_{m}^{k} \times G_{n}^{k}$ acts on $\sigma \in P^{k}(m, n)$ by $(\gamma, \chi) . \sigma=\chi \bullet \sigma \bullet \gamma^{-1}$.

(3) If $f: M \rightarrow N$ is a smooth mapping, then $j^{k} f: M \rightarrow J^{k}(M, N)$ is also smooth (it is $C^{r-k}$ if $f$ is $C^{r}$ ), sometimes called the $k$-jet extension of $f$. We have $\alpha \circ j^{k} f=I d_{M}$ and $\beta \circ j^{k} f=f$.

(4) If $g: M^{\prime} \rightarrow M$ is a (C $C^{r}$-)diffeomorphism, then also the induced mapping $J^{k}(g, N): J^{k}(M, N) \rightarrow J^{k}\left(M^{\prime}, N\right)$ is a $\left(C^{r-k}-\right)$ diffeomorphism. 
(5) If $h: N \rightarrow N^{\prime}$ is a (C ${ }^{r}$-) mapping, then

$$
J^{k}(M, h): J^{k}(M, N) \rightarrow J^{k}\left(M, N^{\prime}\right)
$$

is a $\left(C^{r-k}-\right)$ mapping. We get a covariant functor $J^{k}(M, \quad)$ from the category of smooth manifolds and smooth mappings into itself which maps each of the following classes of mappings into itself: immersions, embeddings, closed embeddings, submersions, surjective submersions, fiber bundle projections. Furthermore $J^{k}($, ) is a contra-covariant bifunctor.

(6) The projections $\pi_{l}^{k}: J^{k}(M, N) \rightarrow J^{l}(M, N)$ are smooth and natural, i.e., they commute with the mappings from (4) and (5).

(7) $\left(J^{k}(M, N), \pi_{l}^{k}, J^{l}(M, N), P_{l+1}^{k}(m, n)\right)$ are fiber bundles for all $l$. The bundle $\left(J^{k}(M, N), \pi_{k-1}^{k}, J^{k-1}(M, N), L_{\text {sym }}^{k}\left(\mathbb{R}^{m}, \mathbb{R}^{n}\right)\right)$ is an affine bundle. The first jet space $J^{1}(M, N)$ is a vector bundle, and it is isomorphic to the bundle $\left(L(T M, T N),\left(\pi_{M}, \pi_{N}\right), M \times N\right)$. Moreover we have $J_{0}^{1}(\mathbb{R}, N)=T N$ and $J^{1}(M, \mathbb{R})_{0}=T^{*} M$.

Proof. We use (21.3) heavily. Let $\left(U_{\gamma}, u_{\gamma}\right)$ be an atlas of $M$ and let $\left(V_{\varepsilon}, v_{\varepsilon}\right)$ be an atlas of $N$. Then

$$
J^{k}\left(u_{\gamma}^{-1}, v_{\varepsilon}\right):(\alpha, \beta)^{-1}\left(U_{\gamma} \times V_{\varepsilon}\right) \rightarrow J^{k}\left(u_{\gamma}\left(U_{\gamma}\right), v_{\varepsilon}\left(V_{\varepsilon}\right)\right)
$$

is a bijective mapping and the chart change looks like

$$
J^{k}\left(u_{\gamma}^{-1}, v_{\varepsilon}\right) \circ J^{k}\left(u_{\delta}^{-1}, v_{\nu}\right)^{-1}=J^{k}\left(u_{\delta} \circ u_{\gamma}^{-1}, v_{\varepsilon} \circ v_{\nu}^{-1}\right)
$$

by the functorial properties of $J^{k}\left(\right.$, ). The space $J^{k}(M, N)$ is Hausdorff in the identification topology, since it is a fiber bundle and the usual argument for gluing fiber bundles applies. So (1) follows.

Now we make this manifold atlas into a fiber bundle by using as charts

$$
\begin{gathered}
\left(U_{\gamma} \times V_{\varepsilon}, \psi_{(\gamma, \varepsilon)}: J^{k}(M, N) \mid U_{\gamma} \times V_{\varepsilon} \rightarrow U_{\gamma} \times V_{\varepsilon} \times P^{k}(m, n)\right), \\
\psi_{(\gamma, \varepsilon)}(\sigma)=\left(\alpha(\sigma), \beta(\sigma), J_{\alpha(\sigma)}^{k}\left(u_{\gamma}^{-1}, v_{\varepsilon}\right)_{\beta(\sigma)}\right) .
\end{gathered}
$$

We then get as transition functions

$$
\begin{aligned}
\psi_{(\gamma, \varepsilon)} \psi_{(\delta, \nu)}(x, y, \bar{\sigma}) & =\left(x, y, J_{u_{\delta}(x)}^{k}\left(u_{\delta} \circ u_{\gamma}^{-1}, v_{\varepsilon} \circ v_{\nu}^{-1}\right)(\bar{\sigma})\right) \\
& =\left(x, y, \operatorname{Tay}_{v_{\nu}(y)}^{k}\left(v_{\varepsilon} \circ v_{\nu}^{-1}\right) \bullet \bar{\sigma} \bullet \operatorname{Tay}_{u_{\gamma}(x)}^{k}\left(u_{\delta} \circ u_{\gamma}^{-1}\right)\right),
\end{aligned}
$$

and (2) follows.

(3), (44), and (6) are obvious from (21.3), mainly by the functorial properties of $J^{k}(, \quad)$.

(5) It is clear from (21.3) that $J^{k}(M, h)$ is a smooth mapping. The rest follows by looking at special chart representations of $h$ and the induced chart representations for $J^{k}(M, h)$. 
It remains to show (7) and here we concentrate on the affine bundle. Let $a_{1}+a \in G L(n) \times P_{2}^{k}(n, n), \sigma+\sigma_{k} \in P^{k-1}(m, n) \oplus L_{\mathrm{sym}}^{k}\left(\mathbb{R}^{m}, \mathbb{R}^{n}\right)$, and $b_{1}+b \in G L(m) \times P_{2}^{k}(m, m)$; then the only term of degree $k$ containing $\sigma_{k}$ in $\left(a+a_{k}\right) \bullet\left(\sigma+\sigma_{k}\right) \bullet\left(b+b_{k}\right)$ is $a_{1} \circ \sigma_{k} \circ b_{1}^{k}$, which depends linearly on $\sigma_{k}$. To this the degree $k$ components of compositions of the lower order terms of $\sigma$ with the higher order terms of $a$ and $b$ are added, and these may be quite arbitrary. So an affine bundle results.

We have $J^{1}(M, N)=L(T M, T N)$ since both bundles have the same transition functions. Finally we have $J_{0}^{1}(\mathbb{R}, N)=L\left(T_{0} \mathbb{R}, T N\right)=T N$, and $J^{1}(M, \mathbb{R})_{0}=L\left(T M, T_{0} \mathbb{R}\right)=T^{*} M$.

21.6. Frame bundles and natural bundles. Let $M$ be a manifold of dimension $m$. We consider the jet bundle $J_{0}^{1}\left(\mathbb{R}^{m}, M\right)=L\left(T_{0} \mathbb{R}^{m}, T M\right)$ and the open subset $i n v J_{0}^{1}\left(\mathbb{R}^{m}, M\right)$ of all invertible jets. This is visibly equal to the linear frame bundle of $T M$ as treated in (18.11).

Note that a mapping $f: \mathbb{R}^{m} \rightarrow M$ is locally invertible near 0 if and only if $j^{1} f(0)$ is invertible. A jet $\sigma$ will be called invertible if its order 1 part $\pi_{1}^{k}(\sigma) \in J_{0}^{1}\left(\mathbb{R}^{m}, M\right)$ is invertible. Let us now consider the open subset inv $J_{0}^{k}\left(\mathbb{R}^{m}, M\right) \subset J_{0}^{k}\left(\mathbb{R}^{m}, M\right)$ of all invertible jets and let us denote it by $P^{k} M$. Then by (18.2) we have a principal fiber bundle $\left(P^{k} M, \pi_{M}, M, G_{m}^{k}\right)$ which is called the $k$-th order frame bundle of the manifold $M$. Its principal right action $r$ can be described in several ways: by the fiber composition of jets:

$$
r=\gamma: i n v J_{0}^{k}\left(\mathbb{R}^{m}, \mathbb{R}^{m}\right) \times i n v J_{0}^{k}\left(\mathbb{R}^{m}, M\right)=G_{m}^{k} \times P^{k} M \rightarrow P^{k} M
$$

or by the functorial property of the jet bundle:

$$
r^{j^{k} g(0)}=i n v J_{0}^{k}(g, M)
$$

for a local diffeomorphism $g: \mathbb{R}^{m}, 0 \rightarrow \mathbb{R}^{m}, 0$.

If $h: M \rightarrow M^{\prime}$ is a local diffeomorphism, the induced mapping $J_{0}^{k}\left(\mathbb{R}^{m}, h\right)$ maps the open subset $P^{k} M$ into $P^{k} M^{\prime}$. By the second description of the principal right action this induced mapping is a homomorphism of principal fiber bundles which we will denote by $P^{k}(h): P^{k} M \rightarrow P^{k} M^{\prime}$. Thus $P^{k}$ becomes a covariant functor from the category $\mathcal{M} f_{m}$ of $m$-dimensional manifolds and local diffeomorphisms into the category of all principal fiber bundles with structure group $G_{m}^{k}$ over $m$-dimensional manifolds and homomorphisms of principal fiber bundles covering local diffeomorphisms.

If we are given any smooth left action $\ell: G_{m}^{k} \times S \rightarrow S$ on some manifold $S$, the associated bundle construction from theorem (18.7) gives us a fiber bundle $P^{k} M[S, \ell]=P^{k} M \times_{G_{m}^{k}} S$ over $M$ for each $m$-dimensional manifold $M$; by (18.9.3) this describes a functor $P^{k}(\quad)[S, \ell]$ from the category $\mathcal{M} f_{m}$ into the category of all fiber bundles over $m$-dimensional manifolds 
with standard fiber $S$ and $G_{m}^{k}$-structure, and homomorphisms of fiber bundles covering local diffeomorphisms. These bundles are also called natural bundles or geometric objects.

21.7. Theorem. If $(E, p, M, S)$ is a fiber bundle, let us denote by $J^{k}(E) \rightarrow$ $M$ the space of all $k$-jets of sections of $E$. Then we have:

(1) $J^{k}(E)$ is a closed submanifold of $J^{k}(M, E)$.

(2) The first jet bundle $J^{1}(E) \rightarrow M \times E$ is an affine subbundle of the vector bundle $J^{1}(M, E)=L(T M, T E)$; in fact we have $J^{1}(E)=\{\sigma \in$ $\left.L(T M, T E): T p \circ \sigma=I d_{T M}\right\}$.

(3) $\left(J^{k}(E), \pi_{k-1}^{k}, J^{k-1}(E)\right)$ is an affine bundle.

(4) If $(E, p, M)$ is a vector bundle, then $\left(J^{k}(E), \alpha, M\right)$ is also a vector bundle. If $\phi: E \rightarrow E^{\prime}$ is a homomorphism of vector bundles covering the identity, then $J^{k}(\varphi)$ is of the same kind.

Proof. (1) By (21.5.5) the mapping $J^{k}(M, p)$ is a submersion; thus $J^{k}(E)=$ $J^{k}(M, p)^{-1}\left(j^{k}\left(I d_{M}\right)\right)$ is a submanifold. Part (2) is clear. Parts (3) and (4) are seen by looking at appropriate canonical charts. 


\section{CHAPTER V. Riemann Manifolds}

\section{Pseudo-Riemann Metrics and Covariant Derivatives}

22.1. Riemann metrics. Let $M$ be a smooth manifold of dimension $m$. A Riemann metric $g$ on $M$ is a symmetric $\left(\begin{array}{l}0 \\ 2\end{array}\right)$-tensor field such that $g_{x}$ : $T_{x} M \times T_{x} M \rightarrow \mathbb{R}$ is a positive definite inner product for each $x \in M$. A pseudo-Riemann metric $g$ on $M$ is a symmetric $\left(\begin{array}{l}0 \\ 2\end{array}\right)$-tensor field such that $g_{x}$ is nondegenerate, i.e., $\check{g}_{x}: T x M \rightarrow T_{x}^{*} M$ is bijective for each $x \in M$. If $(U, u)$ is a chart on $M$, then we have

$$
g \mid U=\sum_{i, j=0}^{m} g\left(\frac{\partial}{\partial u^{i}}, \frac{\partial}{\partial u^{j}}\right) d u^{i} \otimes d u^{j}=: \sum_{i, j} g_{i j} d u^{i} \otimes d u^{j} .
$$

Here $\left(g_{i j}(x)\right)$ is a symmetric invertible $(m \times m)$-matrix for each $x \in M$, positive definite in the case of a Riemann metric; thus $\left(g_{i j}\right): U \rightarrow \operatorname{Mat}_{\text {sym }}(m \times$ $m)$. In the case of a pseudo-Riemann metric, the matrix $\left(g_{i j}\right)$ has $p$ positive eigenvalues and $q$ negative ones; $(p, q)$ is called the signature of the metric and $q=m-p$ is called the index of the metric; both are locally constant on $M$ and we shall always assume that it is constant on $M$.

Lemma. One each manifold $M$ there exist many Riemann metrics. But there need not exist a pseudo-Riemann metric of some given signature.

Proof. Let $\left(U_{\alpha}, u_{\alpha}\right)$ be an atlas on $M$ with a subordinated partition of unity $\left(f_{\alpha}\right)$. Choose smooth mappings $\left(g_{i j}^{\alpha}\right)$ from $U_{\alpha}$ to the convex cone of all positive definite symmetric $(m \times m)$-matrices for each $\alpha$ and put $g=$ $\sum_{\alpha} f_{\alpha} \sum_{i j} g_{i j}^{\alpha} d u_{\alpha}^{i} \otimes d_{\alpha}^{j}$. 
For example, on any even-dimensional sphere $S^{2 n}$ there does not exist a pseudo-Riemann metric $g$ of signature $(1,2 n-1)$ : Otherwise there would exist a line subbundle $L \subset T S^{2}$ with $g(v, v)>0$ for $0 \neq v \in L$. But since the Euler characteristic $\chi\left(S^{2 n}\right)=2$, such a line subbundle of the tangent bundle cannot exist; see [80, I, p. 399].

22.2. Length and energy of a curve. Let $c:[a, b] \rightarrow M$ be a smooth curve. In the Riemann case the length of the curve $c$ is then given by

$$
L_{a}^{b}(c):=\int_{a}^{b} g\left(c^{\prime}(t), c^{\prime}(t)\right)^{1 / 2} d t=\int_{a}^{b}\left|c^{\prime}(t)\right|_{g} d t .
$$

In both cases the energy of the curve $c$ is given by

$$
E_{a}^{b}(c):=\frac{1}{2} \int_{a}^{b} g\left(c^{\prime}(t), c^{\prime}(t)\right) d t
$$

In the Riemann case we have by the Cauchy-Schwarz inequality

$$
L_{a}^{b}(c)^{2}=\left(\int_{a}^{b}\left|c^{\prime}\right|_{g} \cdot 1 d t\right)^{2} \leq \int_{a}^{b}\left|c^{\prime}\right|_{g}^{2} d t .(b-a)=2(b-a) E_{a}^{b}(c) .
$$

For piecewise smooth curves the length and the energy are defined by taking it for the smooth pieces and then by summing up over all the pieces. In the pseudo-Riemann case for the length one has to distinguish different classes of curves according to the sign of $g\left(c^{\prime}(t), c^{\prime}(t)\right.$ ) (the sign then should be assumed constant) and by taking an appropriate sign before taking the root. These leads to the concept of 'time-like' curves (with speed less than the speed of light) and 'space-like' curves (travelling faster than light).

The length is invariant under reparameterizations of the curve:

$$
\begin{aligned}
L_{a}^{b}(c \circ f) & =\int_{a}^{b} g\left((c \circ f)^{\prime}(t),(c \circ f)^{\prime}(t)\right)^{1 / 2} d t \\
& =\int_{a}^{b} g\left(f^{\prime}(t) c^{\prime}(f(t)), f^{\prime}(t) c^{\prime}(f(t))\right)^{1 / 2} d t \\
& =\int_{a}^{b} g\left(c^{\prime}(f(t)), c^{\prime}(f(t))\right)^{1 / 2}\left|f^{\prime}(t)\right| d t \\
& =\int_{a}^{b} g\left(c^{\prime}(t), c^{\prime}(t)\right)^{1 / 2} d t=L_{a}^{b}(c) .
\end{aligned}
$$

The energy is not invariant under reparameterizations.

22.3. Theorem (First variational formula). Let $g$ be a pseudo-Riemann metric on an open subset $U \subseteq \mathbb{R}^{m}$. Let $\gamma:[a, b] \times(-\varepsilon, \varepsilon) \rightarrow U$ be a smooth variation of the curve $c=\gamma(, 0):[a, b] \rightarrow U$. Let $r(t)=\left.\frac{\partial}{\partial s}\right|_{0} \gamma(t, s)=$ $T_{(t, 0)} \gamma \cdot(0,1) \in T_{c(t)} U$ be the variational vector field along $c$. 
Then we have:

$$
\begin{aligned}
\left.\frac{\partial}{\partial s}\right|_{0}\left(E_{a}^{b}(\gamma(, s))\right)=\int_{a}^{b}\left(-g(c(t))\left(c^{\prime \prime}(t), r(t)\right)\right. \\
-d g(c(t))\left(c^{\prime}(t)\right)\left(c^{\prime}(t), r(t)\right) \\
\left.\quad+\frac{1}{2} d g(c(t))(r(t))\left(c^{\prime}(t), c^{\prime}(t)\right)\right) d t \\
+g(c(b))\left(c^{\prime}(b), r(b)\right)-g(c(a))\left(c^{\prime}(a), r(a)\right) .
\end{aligned}
$$

Proof. We have the Taylor expansion $\gamma(t, s)=\gamma(t, 0)+s \gamma_{s}(t, 0)+O\left(s^{2}\right)=$ $c(t)+s r(t)+O\left(s^{2}\right)$ where the remainder $O\left(s^{2}\right)=s^{2} R(s, t)$ is smooth and uniformly bounded in $t$. We plug this into the energy and take also the Taylor expansion of $g$ as follows:

$$
\begin{aligned}
& E_{a}^{b}(\gamma(, s))=\frac{1}{2} \int_{a}^{b} g(\gamma(t, s))\left(\gamma_{t}(t, s), \gamma_{t}(t, s)\right) d t \\
& =\frac{1}{2} \int_{a}^{b} g\left(c(t)+s r(t)+O\left(s^{2}\right)\right)\left(c^{\prime}(t)+s r^{\prime}(t)+O\left(s^{2}\right),\right. \\
& \left.c^{\prime}(t)+s r^{\prime}(t)+O\left(s^{2}\right)\right) d t \\
& =\frac{1}{2} \int_{a}^{b}\left(g(c(t))+s g^{\prime}(c(t))(r(t))+O\left(s^{2}\right)\right)(\ldots, \ldots) d t \\
& =\frac{1}{2} \int_{a}^{b}\left(g(c(t))\left(c^{\prime}(t), c^{\prime}(t)\right)+2 s g(c(t))\left(c^{\prime}(t), r^{\prime}(t)\right)\right. \\
& \left.\quad+s g^{\prime}(c(t))(r(t))\left(c^{\prime}(t), c^{\prime}(t)\right)\right) d t+O\left(s^{2}\right) \\
& =E_{a}^{b}(c)+s \int_{a}^{b} g(c(t))\left(c^{\prime}(t), r^{\prime}(t)\right) d t \\
& +\frac{1}{2} s \int_{a}^{b} g^{\prime}(c(t))(r(t))\left(c^{\prime}(t), c^{\prime}(t)\right) d t+O\left(s^{2}\right) .
\end{aligned}
$$

Thus for the derivative we get, using partial integration:

$$
\begin{aligned}
& \left.\frac{\partial}{\partial s}\right|_{0} E_{a}^{b}(\gamma(, s))=\lim _{s \rightarrow 0} \frac{1}{s}\left(E_{a}^{b}(\gamma(, s))-E_{a}^{b}(\gamma(, 0))\right) \\
& =\frac{1}{2} \int_{a}^{b} g^{\prime}(c(t))(r(t))\left(c^{\prime}(t), c^{\prime}(t)\right) d t+\int_{a}^{b} g(c(t))\left(c^{\prime}(t), r^{\prime}(t)\right) d t \\
& =\frac{1}{2} \int_{a}^{b} g^{\prime}(c(t))(r(t))\left(c^{\prime}(t), c^{\prime}(t)\right) d t+\left.g(c(t))\left(c^{\prime}(t), r(t)\right)\right|_{t=a} ^{t=b} \\
& \quad-\int_{a}^{b}\left(g^{\prime}(c(t))\left(c^{\prime}(t)\right)\left(c^{\prime}(t), r(t)\right)+g(c(t))\left(c^{\prime \prime}(t), r(t)\right)\right) d t
\end{aligned}
$$




$$
\begin{aligned}
=\int_{a}^{b}\left(-g(c(t))\left(c^{\prime \prime}(t), r(t)\right)\right. & -g^{\prime}(c(t))\left(c^{\prime}(t)\right)\left(c^{\prime}(t), r(t)\right) \\
& \left.+\frac{1}{2} g^{\prime}(c(t))(r(t))\left(c^{\prime}(t), c^{\prime}(t)\right)\right) d t \\
+ & g(c(b))\left(c^{\prime}(b), r(b)\right)-g(c(a))\left(c^{\prime}(a), r(a)\right) . \quad \square
\end{aligned}
$$

22.4. Christoffel symbols and geodesics. On a pseudo-Riemann manifold $(M, g)$, by theorem $(22.3)$, we have $\left.\frac{\partial}{\partial s}\right|_{0} E_{a}^{b}(\gamma(, s))=0$ for all variations $\gamma$ of the curve $c$ with fixed end points $(r(a)=r(b)=0)$ in a chart $(U, u)$ if and only if the integral in theorem $(22.3)$ vanishes. This is the case if and only if we have in $u(U) \subset \mathbb{R}^{m}$ :

$$
\begin{aligned}
g(c(t))\left(c^{\prime \prime}(t), \quad\right) & =\frac{1}{2} g^{\prime}(c(t))(\quad)\left(c^{\prime}(t), c^{\prime}(t)\right) \\
& -\frac{1}{2} g^{\prime}(c(t))\left(c^{\prime}(t)\right)\left(c^{\prime}(t), \quad\right) \\
& -\frac{1}{2} g^{\prime}(c(t))\left(c^{\prime}(t)\right)\left(\quad, c^{\prime}(t)\right) .
\end{aligned}
$$

For $x \in u(U)$ and $X, Y, Z \in \mathbb{R}^{m}$ we consider the polarized version of the last equation:

$$
\begin{aligned}
g(x)\left(\Gamma_{x}(X, Y), Z\right)= & \frac{1}{2} g^{\prime}(x)(Z)(X, Y) \\
& -\frac{1}{2} g^{\prime}(x)(X)(Y, Z) \\
& -\frac{1}{2} g^{\prime}(x)(Y)(Z, X)
\end{aligned}
$$

which is a well defined smooth mapping

$$
\Gamma: u(U) \rightarrow L_{\mathrm{sym}}^{2}\left(\mathbb{R}^{m} ; \mathbb{R}^{m}\right) .
$$

Back on $U \subset M$ we have in coordinates

$$
\begin{aligned}
\Gamma_{x}(X, Y) & =\Gamma_{x}\left(\sum_{i} X^{i} \frac{\partial}{\partial u^{i}}, \sum_{j} Y^{j} \frac{\partial}{\partial u^{j}}\right)=\sum_{i, j} \Gamma_{x}\left(\frac{\partial}{\partial u^{i}}, \frac{\partial}{\partial u^{j}}\right) X^{i} Y^{j} \\
& =: \sum_{i, j} \Gamma_{i j}(x) X^{i} Y^{j}=: \sum_{i, j, k} \Gamma_{i j}^{k}(x) X^{i} Y^{j} \frac{\partial}{\partial u^{k}},
\end{aligned}
$$

where the $\Gamma_{i j}^{k}: U \rightarrow \mathbb{R}$ are smooth functions, which are called the Christoffel symbols in the chart $(U, u)$. Attention: Most of the literature uses the negative of the Christoffel symbols.

Lemma. If $g \mid U=\sum_{i, j} g_{i j} d u^{i} \otimes d u^{j}$ and if $\left(g_{i j}\right)^{-1}=\left(g^{i j}\right)$ denotes the inverse matrix, then we have

$$
\Gamma_{i j}^{k}=\frac{1}{2} \sum_{l} g^{k l}\left(\frac{\partial g_{i j}}{\partial u^{l}}-\frac{\partial g_{l j}}{\partial u^{i}}-\frac{\partial g_{i l}}{\partial u^{j}}\right) .
$$


Proof. We have

$$
\begin{aligned}
\sum_{k} \Gamma_{i j}^{k} g_{k l} & =\sum_{k} \Gamma_{i j}^{k} g\left(\frac{\partial}{\partial u^{k}}, \frac{\partial}{\partial u^{l}}\right)=g\left(\sum_{k} \Gamma_{i j}^{k} \frac{\partial}{\partial u^{k}}, \frac{\partial}{\partial u^{l}}\right)=g\left(\Gamma\left(\frac{\partial}{\partial u^{i}}, \frac{\partial}{\partial u^{j}}\right), \frac{\partial}{\partial u^{l}}\right) \\
& =\frac{1}{2} g^{\prime}\left(\frac{\partial}{\partial u^{l}}\right)\left(\frac{\partial}{\partial u^{i}}, \frac{\partial}{\partial u^{j}}\right)-\frac{1}{2} g^{\prime}\left(\frac{\partial}{\partial u^{i}}\right)\left(\frac{\partial}{\partial u^{j}}, \frac{\partial}{\partial u^{l}}\right)-\frac{1}{2} g^{\prime}\left(\frac{\partial}{\partial u^{j}}\right)\left(\frac{\partial}{\partial u^{l}}, \frac{\partial}{\partial u^{i}}\right) \\
& =\frac{1}{2} \frac{\partial g_{i j}}{\partial u^{l}}-\frac{1}{2} \frac{\partial g_{l j}}{\partial u^{i}}-\frac{1}{2} \frac{\partial g_{i l}}{\partial u^{j}} . \quad \square
\end{aligned}
$$

Let $c:[a, b] \rightarrow M$ be a smooth curve in the pseudo-Riemann manifold $(M, g)$. The curve $c$ is called a geodesic on $M$ if in each chart $(U, u)$ for the Christoffel symbols of this chart we have

$$
c^{\prime \prime}(t)=\Gamma_{c(t)}\left(c^{\prime}(t), c^{\prime}(t)\right) .
$$

The reason for this name is: If the energy $E_{a}^{b}$ of (each piece of) the curve is minimal under all variations with fixed end points, then by (22.3) the integral

$$
\int_{a}^{b} g_{c(t)}\left(c^{\prime \prime}(t)-\Gamma_{c(t)}\left(c^{\prime}(t), c^{\prime}(t)\right), r(t)\right) d t=0
$$

for each vector field $r$ along $c$ with $r(a)=r(b)=0$. This implies (3). Thus (local) infima of the energy functional $E_{a}^{b}$ are geodesics, and more generally any curve on which the energy functional $E_{a}^{b}$ has vanishing derivative (with respect to local variations with constant ends) is called a geodesic.

Finally we should compute how the Christoffel symbols react to a chart change. Since this is easily done and since we will see soon that the Christoffel symbols indeed are coordinate expressions of an entity which belongs to the second tangent bundle TTM, we leave this exercise to the interested reader.

22.5. Covariant derivatives. Let $(M, g)$ be a pseudo-Riemann manifold. A covariant derivative on $M$ is a mapping $\nabla: \mathfrak{X}(M) \times \mathfrak{X}(M) \rightarrow \mathfrak{X}(M)$, denoted by $(X, Y) \mapsto \nabla_{X} Y$, which satisfies the following conditions:

(1) $\nabla_{X} Y$ is $C^{\infty}(N)$-linear in $X \in \mathfrak{X}(M)$, i.e., $\nabla_{f_{1} X_{1}+f_{2} X_{2}} Y=f_{1} \nabla_{X_{1}} Y+$ $f_{2} \nabla_{X_{2}} Y$. So for a tangent vector $X_{x} \in T_{x} M$ the mapping $\nabla_{X_{x}}$ : $\mathfrak{X}(M) \rightarrow T_{x} M$ makes sense and we have $\left(\nabla_{X} s\right)(x)=\nabla_{X(x)} s$.

(2) $\nabla_{X} Y$ is $\mathbb{R}$-linear in $Y \in \mathfrak{X}(M)$.

(3) $\nabla_{X}(f . Y)=d f(X) . Y+f . \nabla_{X} Y$ for $f \in C^{\infty}(M)$, the derivation property of $\nabla_{X}$.

The covariant derivative $\nabla$ is called symmetric or torsion-free if moreover the following holds:

(4) $\nabla_{X} Y-\nabla_{Y} X=[X, Y]$. 
The covariant derivative $\nabla$ is called compatible with the pseudo-Riemann metric if we have:

(5) $X(g(Y, Z))=g\left(\nabla_{X} Y, Z\right)+g\left(Y, \nabla_{X} Z\right)$ for all $X, Y, Z \in \mathfrak{X}(M)$.

Compare with (19.12) where we treat the covariant derivative on vector bundles.

Theorem. On any pseudo-Riemann manifold $(M, g)$ there exists a unique torsion-free covariant derivative $\nabla=\nabla^{g}$ which is compatible with the metric g. In a chart $(U, u)$ we have

$$
\nabla_{\frac{\partial}{\partial u^{i}}} \frac{\partial}{\partial u^{j}}=-\sum_{k} \Gamma_{i j}^{k} \frac{\partial}{\partial u^{k}}
$$

where the $\Gamma_{i j}^{k}$ are the Christoffel symbols from (22.4).

This unique covariant derivative is called the Levi-Civita covariant derivative.

Proof. We write the cyclic permutations of property (5) equipped with the signs,,++- :

$$
\begin{aligned}
X(g(Y, Z)) & =g\left(\nabla_{X} Y, Z\right)+g\left(Y, \nabla_{X} Z\right) \\
Y(g(Z, X)) & =g\left(\nabla_{Y} Z, X\right)+g\left(Z, \nabla_{Y} X\right) \\
-Z(g(X, Y)) & =-g\left(\nabla_{Z} X, Y\right)-g\left(X, \nabla_{Z} Y\right) .
\end{aligned}
$$

We add these three equations and use the torsion-free property (41) to get

$$
\begin{aligned}
& X(g(Y, Z))+Y(g(Z, X))-Z(g(X, Y)) \\
& =g\left(\nabla_{X} Y+\nabla_{Y} X, Z\right)+g\left(\nabla_{X} Z-\nabla_{Z} X, Y\right)+g\left(\nabla_{Y} Z-\nabla_{Z} Y, X\right) \\
& =g\left(2 \nabla_{X} Y-[X, Y], Z\right)-g([Z, X], Y)+g([Y, Z], X),
\end{aligned}
$$

which we rewrite as an implicit defining equation for $\nabla_{X} Y$ :

$$
\begin{aligned}
2 g\left(\nabla_{X} Y, Z\right) & =X(g(Y, Z))+Y(g(Z, X))-Z(g(X, Y)) \\
& -g(X,[Y, Z])+g(Y,[Z, X])+g(Z,[X, Y]) .
\end{aligned}
$$

This by (7) uniquely determined bilinear mapping $(X, Y) \mapsto \nabla_{X} Y$ indeed satisfies (11)-(5), which is tedious but easy to check. The final assertion of the theorem follows by using (7) once more:

$$
\begin{aligned}
2 g\left(\nabla \frac{\partial}{\partial u^{i}} \frac{\partial}{\partial u^{j}}, \frac{\partial}{\partial u^{l}}\right) & =\frac{\partial}{\partial u^{i}}\left(g\left(\frac{\partial}{\partial u^{j}}, \frac{\partial}{\partial u^{l}}\right)\right)+\frac{\partial}{\partial u^{j}}\left(g\left(\frac{\partial}{\partial u^{l}}, \frac{\partial}{\partial u^{i}}\right)\right)-\frac{\partial}{\partial u^{l}}\left(g\left(\frac{\partial}{\partial u^{i}}, \frac{\partial}{\partial u^{j}}\right)\right) \\
& =-2 \sum_{k} \Gamma_{i j}^{k} g_{k l}, \quad \text { by (22.4.2), }
\end{aligned}
$$


22.6. Geodesic structures and sprays. By (22.5.6) and (22.4.3) we see that a smooth curve $c:(a, b) \rightarrow(M, g)$ is a geodesic in a pseudo-Riemann manifold if $\nabla_{\partial_{t}} c^{\prime}=0$, in a sense which we will make precise later in (22.9.6) when we discuss how we can apply $\nabla$ to vector fields which are only defined along curves or mappings. In each chart $(U, u)$ this is an ordinary differential equation

$$
\begin{gathered}
c^{\prime \prime}(t)=\Gamma_{c(t)}\left(c^{\prime}(t), c^{\prime}(t)\right), \\
\frac{d^{2}}{d t^{2}} c^{k}(t)=\sum_{i, j} \Gamma_{i j}^{k}(c(t)) \frac{d}{d t} c^{i}(t) \frac{d}{d t} c^{j}(t), \quad c=\left(c^{1}, \ldots, c^{m}\right),
\end{gathered}
$$

which is of second order, linear in the second derivative, quadratic in the first derivative, and in general completely nonlinear in $c(t)$ itself. By the theorem of Picard-Lindelöf for ordinary differential equations there exists a unique solution for each given initial condition $c\left(t_{0}\right), c^{\prime}\left(t_{0}\right)$, depending smoothly on the initial conditions. Thus we may piece together the local solutions and get a geodesic structure in the following sense: A geodesic structure on a

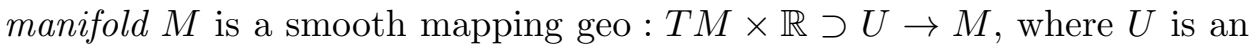
open neighborhood of $T M \times\{0\}$ in $T M \times \mathbb{R}$, which satisfies the following:

(1) $\operatorname{geo}\left(X_{x}\right)(0)=x$ and $\left.\partial\right|_{0} \operatorname{geo}\left(X_{x}\right)(t)=X_{x}$.

(2) $\operatorname{geo}\left(t . X_{x}\right)(s)=\operatorname{geo}\left(X_{x}\right)(t . s)$.

(3) $\operatorname{geo}\left(\operatorname{geo}\left(X_{x}\right)^{\prime}(s)\right)(t)=\operatorname{geo}\left(X_{x}\right)(t+s)$.

(4) $U \cap\left(X_{x} \times \mathbb{R}\right)=\left\{X_{x}\right\} \times$ interval .

One could also require that $U$ be maximal with respect to all these properties. But we shall not elaborate on this since we will reduce everything to the geodesic vector field shortly.

If we are given a geodesic structure geo $: U \rightarrow M$ as above, then the mapping

$$
(X, t) \mapsto \operatorname{geo}(X)^{\prime}(t)=\frac{\partial}{\partial t} \operatorname{geo}(X)(t) \in T M
$$

is the flow for the vector field $S \in \mathfrak{X}(T M)$ which is given by

$$
S(X)=\left.\partial\right|_{0} \frac{\partial}{\partial t} \operatorname{geo}(X)(t) \in T^{2} M,
$$

since we have

$$
\begin{aligned}
\frac{\partial}{\partial t} \frac{\partial}{\partial t} \operatorname{geo}(X)(t) & =\left.\frac{\partial}{\partial s}\right|_{0} \frac{\partial}{\partial s} \operatorname{geo}(X)(t+s) \\
& =\left.\frac{\partial}{\partial s}\right|_{0} \frac{\partial}{\partial s} \operatorname{geo}\left(\frac{\partial}{\partial t} \operatorname{geo}(X)(t)\right)(s) \quad \text { by (주) } \\
& =S\left(\frac{\partial}{\partial t} \operatorname{geo}(X)(t)\right), \\
\operatorname{geo}(X)^{\prime}(0) & =X .
\end{aligned}
$$

The smooth vector field $S \in \mathfrak{X}(T M)$ is called the geodesic spray of the geodesic structure. 
Recall now the chart structure on the second tangent bundle $T^{2} M$ and the canonical flip mapping $\kappa_{M}: T^{2} M \rightarrow T^{2} M$ from (8.12) and (8.13). Let $(U, u)$ be a chart on $M$ and let $c_{(x, y)}(t)=u\left(\operatorname{geo}\left(T u^{-1}(x, y)\right)(t)\right) \in U$. Then we have

$$
\begin{aligned}
T u\left(\operatorname{geo}\left(T u^{-1}(x, y)\right)^{\prime}(t)\right) & =\left(c_{(x, y)}(t), c_{(x, y)}^{\prime}(t)\right), \\
T^{2} u\left(\operatorname{geo}\left(T u^{-1}(x, y)\right)^{\prime \prime}(t)\right) & =\left(c_{(x, y)}(t), c_{(x, y)}^{\prime}(t) ; c_{(x, y)}^{\prime}(t), c_{(x, y)}^{\prime \prime}(t)\right), \\
T^{2} u . S\left(T u^{-1}(x, y)\right) & =T^{2} u\left(\operatorname{geo}\left(T u^{-1}(x, y)\right)^{\prime \prime}(0)\right) \\
& =\left(c_{(x, y)}(0), c_{(x, y)}^{\prime}(0) ; c_{(x, y)}^{\prime}(0), c_{(x, y)}^{\prime \prime}(0)\right) \\
& =(x, y ; y, \bar{S}(x, y)) .
\end{aligned}
$$

Property (2) of the geodesic structure implies in turn

$$
\begin{gathered}
c_{(x, t y)}(s)=c_{(x, y)}(t s), \\
c_{(x, t y)}^{\prime}(s)=t \cdot c_{(x, y)}^{\prime}(t s), \\
c_{(x, t y)}^{\prime \prime}(0)=t^{2} \cdot c_{(x, y)}^{\prime \prime}(0), \\
\bar{S}(x, t y)=t^{2} \bar{S}(x, y),
\end{gathered}
$$

so that $\bar{S}(x, \quad): \mathbb{R}^{m} \rightarrow \mathbb{R}^{m}$ is homogenous of degree 2. By polarizing or taking the second derivative with respect to $y$, we get

$$
\begin{aligned}
\bar{S}(x, y) & =\Gamma_{x}(y, y), \quad \text { for } \quad \Gamma: u(U) \rightarrow L_{\mathrm{sym}}^{2}\left(\mathbb{R}^{m} ; \mathbb{R}^{m}\right), \\
\Gamma_{x}(y, z) & =\frac{1}{2}(\bar{S}(x, y+z)-\bar{S}(x, y)-\bar{S}(x, z)) .
\end{aligned}
$$

If the geodesic structure is induced by a pseudo-Riemann metric on $M$, then we have seen that

$$
c_{(x, y)}^{\prime \prime}(t)=\Gamma_{c_{(x, y)}(t)}\left(c_{(x, y)}^{\prime}(t), c_{(x, y)}^{\prime}(t)\right)
$$

for the Christoffel symbols in the chart $(U, u)$. Thus the geodesic spray is given in terms of the Christoffel symbols by

$$
T^{2} u\left(S\left(T u^{-1}(x, y)\right)\right)=\left(x, y ; y, \Gamma_{x}(y, y)\right) .
$$

22.7. The geodesic exponential mapping. Let $M$ be a smooth manifold and let $S \in \mathfrak{X}(T M)$ be a vector field with the following properties:

(1) $\pi_{T M} \circ S=\operatorname{Id}_{T M} ; S$ is a vector field.

(2) $T\left(\pi_{M}\right) \circ S=\operatorname{Id}_{T M} ; S$ is a 'differential equation of second order'.

(3) Let $m_{t}^{M}: T M \rightarrow T M$ and $m_{t}^{T M}: T^{2} M \rightarrow T^{2} M$ be the scalar multiplications. Then $S \circ m_{t}^{M}=T\left(m_{t}^{M}\right) \circ m_{t}^{T M} \circ S$.

A vector field with these properties is called a spray. 
Theorem. Given a spray $S \in \mathfrak{X}(T M)$ on a manifold $M$, we can write $\operatorname{geo}(X)(t):=\pi_{M}\left(\mathrm{Fl}_{t}^{S}(X)\right)$. Then this is a geodesic structure on $M$ in the sense of $(22.6)$.

If we put $\exp (X):=\pi_{M}\left(\mathrm{Fl}_{1}^{S}(X)\right)=\operatorname{geo}(X)(1)$, then

$$
\exp : T M \supset V \rightarrow M
$$

is a smooth mapping, defined on an open neighborhood $V$ of the zero section in TM, which is called the exponential mapping of the spray $S$ and which has the following properties:

(4) $T_{0_{x}}\left(\exp \mid T_{x} M\right)=\operatorname{Id}_{T_{x} M}$ (via $\left.T_{0_{x}}\left(T_{x} M\right)=T_{x} M\right)$. Thus by the inverse function theorem $\exp _{x}:=\exp \mid T_{x} M: V_{x} \rightarrow W_{x}$ is a diffeomorphism from an open neighborhood $V_{x}$ of $0_{x}$ in TM onto an open neighborhood $W_{x}$ of $x$ in $M$. The chart $\left(W_{x}, \exp _{x}^{-1}\right)$ is called a Riemann normal coordinate system at $x$.

(5) $\operatorname{geo}(X)(t)=\exp (t . X)$.

(6) The mapping

$$
\left(\pi_{M}, \exp \right): T M \supset \tilde{V} \rightarrow M \times M
$$

is a diffeomorphism from an open neighborhood $\tilde{V}$ of the zero section in TM onto an open neighborhood of the diagonal in $M \times M$.

Proof. By properties (11) and (2) the local expression of the spray $S$ is given by $(x, y) \mapsto(x, y ; y, \bar{S}(x, y))$, as in (22.6.5), By (3) we have

$$
(x, t y ; t y, \bar{S}(x, t y))=T\left(m_{t}^{M}\right) \cdot m_{t}^{T M} \cdot(x, y ; y, \bar{S}(x, y))=\left(x, t y ; t y, t^{2} \bar{S}(x, y)\right),
$$

so that $\bar{S}(x, t y)=t^{2} \bar{S}(x, y)$ as in $(22.6)$.

(7) We have $\mathrm{Fl}_{t}^{S}(s . X)=s . \mathrm{Fl}_{\text {s.t }}^{S}(X)$ if one side exists, by uniqueness of solutions of differential equations:

$$
\begin{aligned}
\frac{\partial}{\partial t} s . \mathrm{Fl}_{s . t}^{S}(X) & =\frac{\partial}{\partial t} m_{s}^{M} \mathrm{Fl}_{\text {s.t }}^{S}(X)=T\left(m_{s}^{M}\right) \frac{\partial}{\partial t} \mathrm{Fl}_{\text {s.t }}^{S}(X) \\
& =T\left(m_{s}^{M}\right) \cdot m_{s}^{T M} S\left(\mathrm{Fl}_{\text {s.t }}^{S}(X)\right) \stackrel{(3)}{=} S\left(s . \mathrm{Fl}_{\text {s.t }}^{S}(X)\right), \\
\text { s. } \mathrm{Fl}_{s .0}^{S}(X) & =s . X, \quad \text { thus } \quad \text { s. } \mathrm{Fl}_{\text {s.t }}^{S}(X)=\mathrm{Fl}_{t}^{S}(s . X) .
\end{aligned}
$$

We check that geo $=\pi_{M} \circ \mathrm{Fl}^{S}$ is a geodesic structure, i.e., (22.6.1) (22.6.4) hold:

$$
\begin{aligned}
\operatorname{geo}\left(X_{x}\right)(0) & =\pi_{M}\left(\mathrm{Fl}_{0}^{S}\left(X_{x}\right)\right)=\pi_{M}\left(X_{x}\right)=x \\
\left.\partial\right|_{0} \operatorname{geo}\left(X_{x}\right)(t) & =\left.\partial\right|_{0} \pi_{M}\left(\mathrm{Fl}_{t}^{S}\left(X_{x}\right)\right)=\left.T\left(\pi_{M}\right) \partial\right|_{0} \mathrm{Fl}_{t}^{S}\left(X_{x}\right) \\
& =T\left(\pi_{M}\right) S\left(X_{x}\right) \stackrel{(\underline{\underline{2})}}{=} X_{x}, \\
\operatorname{geo}\left(s . X_{x}\right)(t) & =\pi_{M}\left(\mathrm{Fl}_{t}^{S}\left(s . X_{x}\right)\right)=\pi_{M}\left(s . \mathrm{Fl}_{s . t}^{S}\left(X_{x}\right)\right), \quad \text { see above, } \\
& =\operatorname{geo}\left(X_{x}\right)(s . t),
\end{aligned}
$$




$$
\begin{aligned}
\operatorname{geo}\left(\frac{\partial}{\partial s} \operatorname{geo}\left(X_{x}\right)(s)\right)(t) & =\pi_{M}\left(\mathrm{Fl}_{t}^{S}\left(\frac{\partial}{\partial s} \pi_{M} \mathrm{Fl}_{s}^{S}\left(X_{x}\right)\right)\right) \\
& =\pi_{M}\left(\mathrm{Fl}_{t}^{S}\left(T\left(\pi_{M}\right) S\left(\mathrm{Fl}_{s}^{S}\left(X_{x}\right)\right)\right)\right) \\
& =\pi_{M}\left(\mathrm{Fl}_{t}^{S}\left(\mathrm{Fl}_{s}^{S}\left(X_{x}\right)\right)\right) \quad \text { by (2) } \\
& =\pi_{M}\left(\mathrm{Fl}_{t+s}^{S}\left(X_{x}\right)\right)=\operatorname{geo}\left(X_{x}\right)(t+s) .
\end{aligned}
$$

Let us investigate the exponential mapping. For $\varepsilon>0$ let $X_{x}$ be so small that $\left(\frac{1}{\varepsilon} X_{x}, \varepsilon\right)$ is in the domain of definition of the flow $\mathrm{Fl}^{S}$. Then

$$
\begin{aligned}
\exp _{x}\left(X_{x}\right) & =\pi_{M}\left(\mathrm{Fl}_{1}^{S}\left(X_{x}\right)\right)=\pi_{M}\left(\mathrm{Fl}_{1}^{S}\left(\varepsilon \cdot \frac{1}{\varepsilon} \cdot X_{x}\right)\right) \\
& =\pi_{M}\left(\varepsilon \cdot \mathrm{Fl}_{\varepsilon}^{S}\left(\frac{1}{\varepsilon} \cdot X_{x}\right)\right)=\pi_{M}\left(\mathrm{Fl}_{\varepsilon}^{S}\left(\frac{1}{\varepsilon} \cdot X_{x}\right)\right), \quad \text { by (7) } .
\end{aligned}
$$

We check the properties of the exponential mapping. The tangent mapping satifies:

$$
\begin{aligned}
T_{0_{x}}\left(\exp _{x}\right) . X_{x} & =\left.\partial\right|_{0} \exp _{x}\left(0_{x}+t . X_{x}\right) \\
& =\left.\partial\right|_{0} \pi_{M}\left(\mathrm{Fl}_{1}^{S}\left(t . X_{x}\right)\right) \\
& =\left.\partial\right|_{0} \pi_{M}\left(t . \mathrm{Fl}_{t}^{S}\left(X_{x}\right)\right) \\
& =\left.\partial\right|_{0} \pi_{M}\left(\mathrm{Fl}_{t}^{S}\left(X_{x}\right)\right), \quad \text { by (7) } \\
& =\left.T\left(\pi_{M}\right) \partial\right|_{0}\left(\mathrm{Fl}_{t}^{S}\left(X_{x}\right)\right)=T\left(\pi_{M}\right)\left(S\left(X_{x}\right)\right)=X_{x} .
\end{aligned}
$$

Moreover we have:

(5)

$$
\begin{aligned}
\exp _{x}\left(t . X_{x}\right) & =\pi_{M}\left(\mathrm{Fl}_{1}^{S}\left(t . X_{x}\right)\right) \\
& =\pi_{M}\left(t . \mathrm{Fl}_{t}^{S}\left(X_{x}\right)\right) \\
& =\pi_{M}\left(\mathrm{Fl}_{t}^{S}\left(X_{x}\right)\right)=\operatorname{geo}\left(X_{x}\right)(t) .
\end{aligned}
$$

(6) By (41) we have $T_{0_{x}}\left(\pi_{M}, \exp \right)=\left(\begin{array}{l}\mathbb{I} \\ * \mathbb{I}\end{array}\right)$; thus $\left(\pi_{M}, \exp \right)$ is a local diffeomorphism. Again by (4) the mapping $\left(\pi_{M}, \exp \right)$ is injective on a small neighborhood of the zero section.

22.8. Linear connections and connectors. Let $M$ be a smooth manifold. A smooth mapping $C: T M \times_{M} T M \rightarrow T^{2} M$ is called a linear connection or horizontal lift on $M$ if it has the following properties:

(1) $\left(T\left(\pi_{M}\right), \pi_{T M}\right) \circ C=\operatorname{Id}_{T M \times{ }_{M} T M}$.

(2) $C\left(, X_{x}\right): T_{x} M \rightarrow T_{X_{x}}(T M)$ is linear; this is the first vector bundle structure on $T^{2} M$ treated in (8.13).

(3) $C\left(X_{x}, \quad\right): T_{x} M \rightarrow T\left(\pi_{M}\right)^{-1}\left(X_{x}\right)$ is linear; this is the second vector bundle structure on $T^{2} M$ treated in (8.13)

The connection $C$ is called symmetric or torsion-free if moreover the following property holds: 
(4) $\kappa_{M} \circ C=C \circ$ flip : $T M \times_{M} T M \rightarrow T^{2} M$, where $\kappa_{M}: T^{2} M \rightarrow T^{2} M$ is the canonical flip mapping treated in (8.13).

From the properties (11)-(3) it follows that for a chart $\left(U_{\alpha}, u_{\alpha}\right)$ on $M$ the mapping $C$ is given by

(5) $\left(T^{2}\left(u_{\alpha}\right) \circ C \circ\left(T\left(u_{\alpha}\right)^{-1} \times_{M} T\left(u_{\alpha}\right)^{-1}\right)\right)((x, y),(x, z))=\left(x, z ; y, \Gamma_{x}^{\alpha}(y, z)\right)$,

where the Christoffel symbol $\Gamma_{x}^{\alpha}(y, z) \in \mathbb{R}^{m}(m=\operatorname{dim}(M))$ is smooth in $x \in u_{\alpha}\left(U_{\alpha}\right)$ and is bilinear in $(y, z) \in \mathbb{R}^{m} \times \mathbb{R}^{m}$. For the sake of completeness let us also note the transformation rule of the Christoffel symbols which follows now directly from the chart change of the second tangent bundle in (8.12) and (8.13), The chart change on $M$

$$
u_{\alpha \beta}=u_{\alpha} \circ u_{\beta}^{-1}: u_{\beta}\left(U_{\alpha} \cap U_{\beta}\right) \rightarrow u_{\alpha}\left(U_{\alpha} \cap U_{\beta}\right)
$$

induces the following tranformation of the Christoffel symbols:

$$
\begin{aligned}
& \Gamma_{u_{\alpha \beta}(x)}^{\alpha}\left(d\left(u_{\alpha \beta}\right)(x) y, d\left(u_{\alpha \beta}\right)(x) z\right) \\
& =d\left(u_{\alpha \beta}\right)(x) \Gamma_{x}^{\beta}(y, z)+d^{2}\left(u_{\alpha \beta}\right)(x)(y, z) .
\end{aligned}
$$

We have seen in (22.6.6) that a spray $S$ on a manifold determines symmetric Christoffel symbols and thus a symmetric connection $C$. If the spray $S$ is induced by a pseudo-Riemann metric $g$ on $M$, then the Christoffel symbols are the same as we found by determining the singular curves of the energy in (22.4). The promised geometric description of the Christoffel symbols is (5), which also explains their transformation behavior under chart changes: They belong to the vertical part of the second tangent bundle.

Consider now a linear connection $C: T M \times_{M} T M \rightarrow T^{2} M$. For $\xi \in T^{2} M$ we have

$$
\xi-C\left(T\left(\pi_{M}\right) \cdot \xi, \pi_{T M}(\xi)\right) \in V(T M)=T\left(\pi_{M}\right)^{-1}(0)
$$

which is an element of the vertical bundle, since

$$
T\left(\pi_{M}\right)\left(\xi-C\left(T\left(\pi_{M}\right) \cdot \xi, \pi_{T M}(\xi)\right)\right)=T\left(\pi_{M}\right) \cdot \xi-T\left(\pi_{M}\right) \cdot \xi=0
$$

by (1). Thus we may define the connector $K: T^{2} M \rightarrow T M$ by

$$
K(\xi)=\operatorname{vpr}_{T M}\left(\xi-C\left(T\left(\pi_{M}\right) \cdot \xi, \pi_{T M}(\xi)\right)\right), \quad \text { where } \xi \in T^{2} M,
$$

where the vertical projection $\operatorname{vpr}_{T M}$ was defined in (8.12). In coordinates induced by a chart on $M$ we have

$$
K(x, y ; a, b)=\operatorname{vpr}\left(x, y ; 0, b-\Gamma_{x}(a, y)\right)=\left(x, b-\Gamma_{x}(a, y)\right) .
$$

Obviously the connector $K$ has the following three properties:

(9) We have

$$
K \circ \mathrm{vl}_{T M}=\mathrm{pr}_{2}: T M \times_{M} T M \rightarrow T M
$$

where $\operatorname{vl}_{T M}\left(X_{x}, Y_{x}\right)=\left.\partial\right|_{0}\left(X_{x}+t Y_{x}\right)$ is the vertical lift introduced in $(8.12)$. 
(10) The mapping $K: T T M \rightarrow T M$ is linear for the (first) vector bundle structure on $\pi_{T M}: T T M \rightarrow T M$.

(11) The mapping $K: T T M \rightarrow T M$ is linear for the (second) vector bundle structure on $T\left(\pi_{M}\right): T T M \rightarrow T M$.

A connector, defined as a mapping satisfying (9)-(11), is equivalent to a connection, since one can reconstruct it (which is most easily checked in a chart) by

$$
C\left(\quad, X_{x}\right)=\left(T\left(\pi_{M}\right) \mid \operatorname{ker}\left(K: T_{X_{x}}(T M) \rightarrow T_{x} M\right)\right)^{-1} .
$$

The connecter $K$ is associated to a symmetric connection if and only if $K \circ \kappa_{M}=K$. The connector treated here is a special case of the one in $(19.11)$,

22.9. Covariant derivatives, revisited. We describe here the passage from a linear connection $C: T M \times_{M} T M \rightarrow T^{2} M$ and its associated connector $K: T^{2} M \rightarrow T M$ to the covariant derivative. In the more general setting of vector bundles this is treated in (19.12). Namely, for any manifold $N$, a smooth mapping $s: N \rightarrow T M$ (a vector field along $f:=\pi_{M} \circ s$ ) and a vector field $X \in \mathfrak{X}(N)$ we define

$$
\nabla_{X} s:=K \circ T s \circ X: N \rightarrow T N \rightarrow T^{2} M \rightarrow T M
$$

which is again a vector field along $f$ :

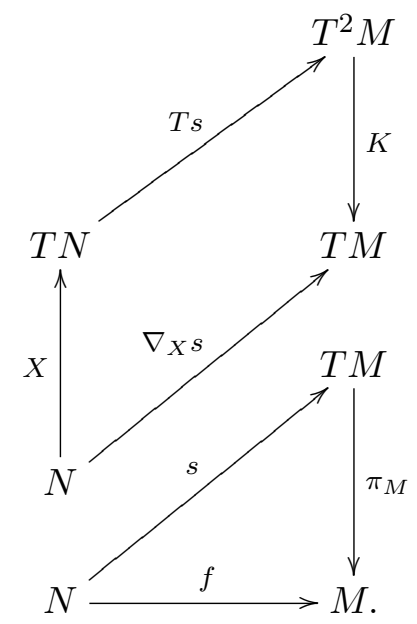

If $f: N \rightarrow M$ is a fixed smooth mapping, let us denote by $C_{f}^{\infty}(N, T M) \cong$ $\Gamma\left(f^{*} T M\right)$ the vector space of all smooth mappings $s: N \rightarrow T M$ with $\pi_{M} \circ s=f$ - vector fields along $f$. Then the covariant derivative may be viewed as a bilinear mapping

$$
\nabla: \mathfrak{X}(N) \times C_{f}^{\infty}(N, T M) \rightarrow C_{f}^{\infty}(N, T M) .
$$

In particular for $f=I d_{M}$ we have $\nabla: \mathfrak{X}(M) \times \mathfrak{X}(M) \rightarrow \mathfrak{X}(M)$ as in (22.5), 
Lemma. This covariant derivative has the following properties:

(3) $\nabla_{X}$ s is $C^{\infty}(N)$-linear in $X \in \mathfrak{X}(N)$. So for a tangent vector $X_{x} \in T_{x} N$ the mapping $\nabla_{X_{x}}: C_{f}^{\infty}(N, T M) \rightarrow T_{f(x)} M$ makes sense and we have $\left(\nabla_{X} s\right)(x)=\nabla_{X(x)} s$.

(4) $\nabla_{X} s$ is $\mathbb{R}$-linear in $s \in C_{f}^{\infty}(N, T M)$.

(5) $\nabla_{X}(h . s)=d h(X) . s+h . \nabla_{X} s$ for $h \in C^{\infty}(N)$; this is the derivation property of $\nabla_{X}$.

(6) For any manifold $Q$ and smooth mapping $g: Q \rightarrow N$ and $Z_{y} \in T_{y} Q$ we have $\nabla_{T g . Z_{y}} s=\nabla_{Z_{y}}(s \circ g)$. If $Z \in \mathfrak{X}(Q)$ and $X \in \mathfrak{X}(N)$ are g-related, then we have $\nabla_{Z}(s \circ g)=\left(\nabla_{X} s\right) \circ g$,

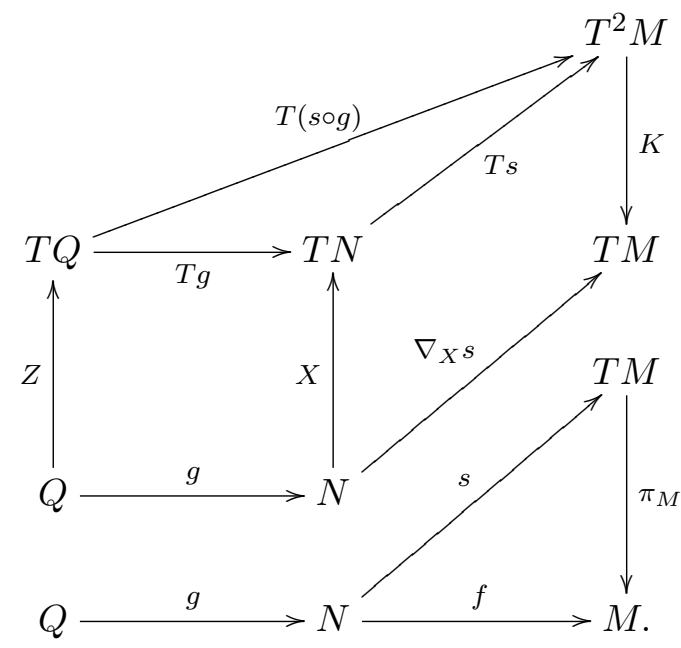

(7) In charts on $N$ and $M$, for $s(x)=(\bar{f}(x), \bar{s}(x))$ and $X(x)=(x, \bar{X}(x))$ we have $\left(\nabla_{X} s\right)(x)=\left(\bar{f}(x), d \bar{s}(x) \cdot \bar{X}(x)-\Gamma_{\bar{f}(x)}(\bar{s}(x), d \bar{f}(x) \bar{X}(x))\right)$.

(8) The connection is symmetric if and only if $\nabla_{X} Y-\nabla_{Y} X=[X, Y]$.

Proof. All these properties follow easily from the definition (1).

Remark. Property (6) is not well understood in some differential geometric literature. It is the reason why in the beginning of (22.6) we wrote $\nabla_{\partial_{t}} c^{\prime}=0$ for the geodesic equation and not $\nabla_{c^{\prime}} c^{\prime}=0$ which one finds in the literature.

22.10. Torsion. Let $\nabla$ be a general covariant derivative on a manifold $M$. Then the torsion is given by

$$
\operatorname{Tor}(X, Y):=\nabla_{X} Y-\nabla_{Y} X-[X, Y], \quad X, Y \in \mathfrak{X}(M) .
$$

It is skew-symmetric and $C^{\infty}(M)$-linear in $X, Y \in \mathfrak{X}(M)$ and is thus a 2form with values in $T M$ : Tor $\in \Omega^{2}(M ; T M)=\Gamma\left(\bigwedge^{2} T^{*} M \otimes T M\right)$, since we 
have

$$
\begin{aligned}
\operatorname{Tor}(f . X, Y) & =\nabla_{f . X} Y-\nabla_{Y}(f . X)-[f . X, Y] \\
& =f . \nabla_{X} Y-Y(f) \cdot X-f \cdot \nabla_{Y}(X)-f \cdot[X, Y]+Y(f) . X \\
& =f . \operatorname{Tor}(X, Y) .
\end{aligned}
$$

Locally on a chart $(U, u)$ we have

$$
\begin{aligned}
\operatorname{Tor} \mid U & =\sum_{i, j} \operatorname{Tor}\left(\frac{\partial}{\partial u^{i}}, \frac{\partial}{\partial u^{j}}\right) \otimes d u^{i} \otimes d u^{j} \\
& =\sum_{i, j}\left(\nabla \frac{\partial}{\partial u^{i}} \frac{\partial}{\partial u^{j}}-\nabla_{\frac{\partial}{\partial u^{j}}} \frac{\partial}{\partial u^{i}}-\left[\frac{\partial}{\partial u^{i}}, \frac{\partial}{\partial u^{j}}\right]\right) \otimes d u^{i} \otimes d u^{j} \\
& =\sum_{i, j}\left(-\Gamma_{i j}^{k}+\Gamma_{j i}^{k}\right) d u^{i} \otimes d u^{j} \otimes \frac{\partial}{\partial u^{k}} \\
& =-\sum_{i, j} \Gamma_{i j}^{k} d u^{i} \wedge d u^{j} \otimes \frac{\partial}{\partial u^{k}}=-2 \sum_{i<j} \Gamma_{i j}^{k} d u^{i} \wedge d u^{j} \otimes \frac{\partial}{\partial u^{k}} .
\end{aligned}
$$

We may add an arbitrary form $T \in \Omega^{2}(M ; T M)$ to a given covariant derivative and we get a new covariant derivative with the same spray and geodesic structure, since the symmetrization of the Christoffel symbols stays the same.

Lemma. Let $K: T T M \rightarrow M$ be the connector of the covariant derivative $\nabla$, and let $X, Y \in \mathfrak{X}(M)$. Then the torsion is given by

$$
\operatorname{Tor}(X, Y)=\left(K \circ \kappa_{M}-K\right) \circ T X \circ Y \text {. }
$$

If moreover $f: N \rightarrow M$ is smooth and $U, V \in \mathfrak{X}(N)$, then we get also

$$
\begin{aligned}
\operatorname{Tor}(T f . U, T f . V) & =\nabla_{U}(T f \circ V)-\nabla_{V}(T f \circ U)-T f \circ[U, V] \\
& =\left(K \circ \kappa_{M}-K\right) \circ T T f \circ T U \circ V .
\end{aligned}
$$

Proof. By (22.9.1), (8.14) (or (8.19), and (22.8.9) we have

$$
\begin{gathered}
\operatorname{Tor}(X, Y)=\nabla_{X} Y-\nabla_{Y} X-[X, Y] \\
=K \circ T Y \circ X-K \circ T X \circ Y-K \circ \operatorname{vl}_{T M} \circ(Y,[X, Y]), \\
K \circ \operatorname{vl}_{T M} \circ(Y,[X, Y])=K \circ\left(T Y \circ X-\kappa_{M} \circ T X \circ Y\right) \\
=K \circ T Y \circ X-K \circ \kappa_{M} \circ T X \circ Y .
\end{gathered}
$$

Similarly we get

$$
\begin{aligned}
K \circ \mathrm{vl}_{T M} \circ & (T f \circ V, T f \circ[U, V])=K \circ T T f \circ \mathrm{vl}_{T N} \circ(V,[U, V]) \\
& =K \circ T T f \circ\left(T V \circ U-\kappa_{N} \circ T U \circ V\right) \\
& =K \circ T T f \circ T V \circ U-K \circ \kappa_{M} \circ T T f \circ T U \circ V,
\end{aligned}
$$


and also

$$
\begin{aligned}
\nabla_{U}(T f \circ V)- & \nabla_{V}(T f \circ U)-T f \circ[X, Y] \\
= & K \circ T T f \circ T V \circ U-K \circ T T f \circ T U \circ V \\
& \quad-K \circ \operatorname{vl}_{T M} \circ(T f \circ V, T f \circ[U, V]) \\
= & \left(K \circ \kappa_{M}-K\right) \circ T T f \circ T U \circ V .
\end{aligned}
$$

The rest will be proved locally, so let us assume now that $M$ is open in $\mathbb{R}^{m}$ and $U(x)=(x, \bar{U}(x))$, etc. Then by (22.8.8) we have

$$
\begin{aligned}
& (T T f \circ T U \circ V)(x) \\
& =T T f(x, \bar{U}(x) ; \bar{V}(x), d \bar{U}(x) \bar{V}(x)) \\
& =\left(f(x), d f(x) \cdot \bar{U}(x) ; d f(x) \cdot \bar{V}(x), d^{2} f(x)(\bar{V}(x), \bar{U}(x))+d f(x) \cdot d \bar{U}(x) \cdot \bar{V}(x)\right)
\end{aligned}
$$

and also

$$
\begin{gathered}
\left(\left(K \circ \kappa_{M}-K\right) \circ T T f \circ T U \circ V\right)(x) \\
=\left(f(x), d^{2} f(x)(\bar{V}(x), \bar{U}(x))+d f(x) \cdot d \bar{U}(x) \cdot \bar{V}(x)\right. \\
\left.\quad-\Gamma_{f(x)}(d f(x) \cdot \bar{U}(x), d f(x) \cdot \bar{V}(x))\right) \\
-\left(f(x), d^{2} f(x)(\bar{V}(x), \bar{U}(x))+d f(x) \cdot d \bar{U}(x) \cdot \bar{V}(x)\right. \\
\left.\quad-\Gamma_{f(x)}(d f(x) \cdot \bar{V}(x), d f(x) \cdot \bar{U}(x))\right) \\
=\left(f(x),-\Gamma_{f(x)}(d f(x) \cdot \bar{U}(x), d f(x) \cdot \bar{V}(x))\right. \\
\left.\quad+\Gamma_{f(x)}(d f(x) \cdot \bar{V}(x), d f(x) \cdot \bar{U}(x))\right) \\
=\operatorname{Tor}(T f \circ U, T f \circ V)(x) . \quad \square
\end{gathered}
$$

22.11. The space of all covariant derivatives. If $\nabla^{0}$ and $\nabla^{1}$ are two covariant derivatives on a manifold $M$, then $\nabla_{X}^{1} Y-\nabla_{X}^{0} Y$ turns out to be $C^{\infty}(M)$-linear in $X, Y \in \mathfrak{X}(M)$ and is thus a $\left(\begin{array}{l}1 \\ 2\end{array}\right)$-tensor field on $M$; see $(22.10)$, Conversely, one may add an arbitrary $\left(\begin{array}{l}1 \\ 2\end{array}\right)$-tensor field $A$ to a given covariant derivative and get a new covariant derivative. Thus the space of all covariant derivatives is an affine space with modeling vector space $\Gamma\left(T^{*} M \otimes T^{*} M \otimes T M\right)$.

22.12. The covariant derivative of tensor fields. Let $\nabla$ be covariant derivative on a manifold $M$, and let $X \in \mathfrak{X}(M)$. Then the $\nabla_{X}$ can be extended uniquely to an operator $\nabla_{X}$ on the space of all tensor fields on $M$ with the following properties:

(1) For $f \in C^{\infty}(M)$ we have $\nabla_{X} f=X(f)=d f(X)$.

(2) $\nabla_{X}$ respects the spaces of $\left(\begin{array}{l}p \\ q\end{array}\right)$-tenor fields. 
(3) $\nabla_{X}(A \otimes B)=\left(\nabla_{X} A\right) \otimes B+A \otimes\left(\nabla_{X} B\right)$, a derivation with respect to the tensor product.

(4) $\nabla_{X}$ commutes with any kind of contraction $C$ (i.e., any trace; see $(8.18)]$ : So for $\omega \in \Omega^{1}(M)$ and $Y \in \mathfrak{X}(M)$ we have

$$
\nabla_{X}(\omega(Y))=\left(\nabla_{X} \omega\right)(Y)+\omega\left(\nabla_{X} Y\right) .
$$

The correct way to understand this is to use the concepts of (19.9) (19.12). Recognize the linear connection as induced from a principal connection on the linear frame bundle $G L\left(\mathbb{R}^{m}, T M\right)$ and induce it then to all vector bundles associated to the representations of the structure group $G L(m, \mathbb{R})$ in all tensor spaces. Contractions are then equivariant mappings and thus intertwine the induced covariant derivatives, which is most clearly seen from $(19.15)$.

Nevertheless, we discuss here the traditional proof, since it helps in actual computations. For $\omega \in \Omega^{1}(M)$ and $Y \in \mathfrak{X}(M)$ and the total contraction $C$ we have

$$
\begin{aligned}
\nabla_{X}(\omega(Y)) & =\nabla_{X}(C(\omega \otimes Y)) \\
& =C\left(\nabla_{X} \omega \otimes Y+\omega \otimes \nabla_{X} Y\right) \\
& =\left(\nabla_{X} \omega\right)(Y)+\omega\left(\nabla_{X} Y\right), \\
\left(\nabla_{X} \omega\right)(Y) & =\nabla_{X}(\omega(Y))-\omega\left(\nabla_{X} Y\right),
\end{aligned}
$$

which is easily seen (as in $(22.10)$ to be $C^{\infty}(M)$-linear in $Y$. Thus $\nabla_{X} \omega$ is again a 1-form.

For a $\left(\begin{array}{l}p \\ q\end{array}\right)$-tensor field $A$ we choose $X_{i} \in \mathfrak{X}(M)$ and $\omega^{j} \in \Omega^{1}(M)$ and arrive (similarly using again the total contraction) at

$$
\begin{aligned}
& \left(\nabla_{X} A\right)\left(X_{1}, \ldots, X_{q}, \omega^{1}, \ldots, \omega^{p}\right)=X\left(A\left(X_{1}, \ldots, X_{q}, \omega^{1}, \ldots, \omega^{p}\right)\right) \\
& \quad-A\left(\nabla_{X} X_{1}, \ldots, X_{q}, \omega^{1}, \ldots, \omega^{p}\right)-\cdots-A\left(X_{1}, \ldots, \nabla_{X} X_{q}, \omega^{1}, \ldots, \omega^{p}\right) \\
& \quad-A\left(X_{1}, \ldots, X_{q}, \nabla_{X} \omega^{1}, \ldots, \omega^{p}\right)-\cdots-A\left(X_{1}, \ldots, X_{q}, \omega^{1}, \ldots, \nabla_{X} \omega^{p}\right) .
\end{aligned}
$$

This expression is again $C^{\infty}(M)$-linear in each entry $X_{i}$ or $\omega^{j}$ and defines thus the $\left(\begin{array}{l}p \\ q\end{array}\right)$-tensor field $\nabla_{X} A$. Obviously $\nabla_{X}$ is a derivation with respect to the tensor product of fields and commutes with all contractions.

For the sake of completeness we also list the local expression

$$
\begin{aligned}
\nabla_{\frac{\partial}{\partial u^{i}}} d u^{j} & =\sum_{k}\left(\nabla_{\frac{\partial}{\partial u^{i}}} d u^{j}\right)\left(\frac{\partial}{\partial u^{k}}\right) d u^{k} \\
& =\sum_{k}\left(\frac{\partial}{\partial u^{i}} \delta_{j}^{k}-d u^{j}\left(\nabla_{\frac{\partial}{\partial u^{i}}} \frac{\partial}{\partial u^{k}}\right)\right) d u^{k}=\sum_{k} \Gamma_{i k}^{j} d u^{k}
\end{aligned}
$$


from which one can easily derive the expression for an arbitrary tensor field:

$$
\begin{aligned}
& \nabla_{\frac{\partial}{\partial u^{i}}} A=\sum\left(\nabla_{\frac{\partial}{\partial u^{i}}} A\right)\left(\frac{\partial}{\partial u^{i_{1}}}, \ldots, \frac{\partial}{\partial u^{i_{q}}}, d u^{j_{1}}, \ldots, d u^{j_{p}}\right) d u^{i_{1}} \otimes \cdots \otimes \frac{\partial}{\partial u^{j^{p}}} \\
& =\sum\left(\frac{\partial}{\partial u^{i}}\left(A\left(\frac{\partial}{\partial u^{i_{1}}}, \ldots, d u^{j_{p}}\right)\right)-A\left(\nabla \frac{\partial}{\partial u^{i}} \frac{\partial}{\partial u^{i_{1}}}, \ldots, d u^{j_{p}}\right)\right. \\
& \left.-\cdots-A\left(\frac{\partial}{\partial u^{i_{1}}}, \ldots, \nabla_{\frac{\partial}{\partial u^{i}}} d u^{j_{p}}\right)\right) d u^{i_{1}} \otimes \cdots \otimes \frac{\partial}{\partial u^{j^{q}}} \\
& =\sum\left(\frac{\partial}{\partial u^{i}} A_{i_{1}, \ldots, i_{q}}^{j_{1}, \ldots, j_{p}}+A_{k, i_{2}, \ldots, i_{q}}^{j_{1}, \ldots, j_{p}} \Gamma_{i, i_{1}}^{k}+\cdots+A_{i_{1}, \ldots, i_{q-1}, k}^{j_{1}, \ldots, j_{p}} \Gamma_{i, i_{q}}^{k}\right. \\
& \left.-A_{i_{1}, \ldots, i_{q}}^{k, j_{2}, \ldots, j_{p}} \Gamma_{i, k}^{j_{1}}-\cdots-A_{i_{1}, \ldots, i_{q}}^{j_{1}, \ldots, j_{p-1}, k} \Gamma_{i, k}^{j_{p}}\right) d u^{i_{1}} \otimes \cdots \otimes \frac{\partial}{\partial u^{j^{q}}} .
\end{aligned}
$$

\section{Geometry of Geodesics}

23.1. Geodesics. On a pseudo-Riemann manifold $(M, g)$ we have a geodesic structure which is described by the flow of the geodesic spray on $T M$. The geodesic with initial value $X_{x} \in T_{x} M$ is denoted by $t \mapsto \exp \left(t . X_{x}\right)$ in terms of the pseudo-Riemann exponential mapping exp and $\exp _{x}=$ $\exp \mid T_{x} M$. We recall the properties of the geodesics which we will use.

(1) $\exp _{x}: T_{x} M \supset U_{x} \rightarrow M$ is defined on a maximal 'radial' open zero neighborhood $U_{x}$ in $T_{x} M$. Here radial means that for $X_{x} \in V_{x}$ we also have $[0,1] . X_{x} \subset V_{x}$. This follows from the flow properties since $\exp _{x}=\pi_{M}\left(\mathrm{Fl}_{1}^{S} \mid T_{x} M\right)$ by (22.7).

(2) $T_{0_{x}}\left(\exp \mid T_{x} M\right)=\operatorname{Id}_{T_{x} M}$; thus $\left.\partial\right|_{0} \exp _{x}\left(t . X_{x}\right)=X_{x}$. See (22.7.4).

(3) $\exp \left(s \cdot\left(\frac{\partial}{\partial t} \exp (t . X)\right)\right)=\exp ((t+s) X)$. See $(22.6 .3)$.

(4) $t \mapsto g\left(\frac{\partial}{\partial t} \exp (t . X), \frac{\partial}{\partial t} \exp (t . X)\right)$ is constant in $t$ : for $c(t)=\exp (t . X)$ we have $\partial_{t} g\left(c^{\prime}, c^{\prime}\right)=2 g\left(\nabla_{\partial_{t}} c^{\prime}, c^{\prime}\right)=0$. Thus in the Riemann case the length $\left|\frac{\partial}{\partial t} \exp (t . X)\right|_{g}=\sqrt{g\left(\frac{\partial}{\partial t} \exp (t . X), \frac{\partial}{\partial t} \exp (t . X)\right)}$ is also constant.

If for a geodesic $c$ the (by (4)) constant $\left|c^{\prime}(t)\right|_{g}$ is 1 , we say that $c$ is parameterized by arc-length.

23.2. Lemma (Gauß). Let $(M, g)$ be a Riemann manifold. For $x \in M$ let $\varepsilon>0$ be so small that $\exp _{x}: D_{x}(\varepsilon):=\left\{X \in T_{x} M:|X|_{g}<\varepsilon\right\} \rightarrow M$ is a diffeomorphism on its image. Then in $\exp _{x}\left(D_{x}(\varepsilon)\right)$ the geodesic rays starting from $x$ are all orthogonal to the 'geodesic spheres' $\left\{\exp _{x}(X):|X|_{g}=k\right\}=$ $\exp _{x}\left(k . S\left(T_{x} M, g\right)\right)$ for $k<\varepsilon$.

On pseudo-Riemann manifolds this result holds too, with the following adaptation: Since the unit spheres in $\left(T_{x} M, g_{x}\right)$ are hyperboloids, they are not small and may not lie in the domain of definition of the geodesic exponential mapping; the result only holds in this domain. 
Proof. $\exp _{x}\left(k . S\left(T_{x} M, g\right)\right)$ is a submanifold of $M$ since $\exp _{x}$ is a diffeomorphism on $D_{x}(\varepsilon)$. Let $s \mapsto v(s)$ be a smooth curve in $k S\left(T_{x} M, g\right) \subset T_{x} M$, and let $\gamma(t, s):=\exp _{x}(t . v(s))$. Then $\gamma$ is a variation of the geodesic $\gamma(t, 0)=$ $\exp _{x}(t . v(0))=: c(t)$. In the energy of the geodesic $t \mapsto \gamma(t, s)$ the integrand is constant by (23.1.4).

$$
\begin{aligned}
E_{0}^{1}(\gamma(, s)) & =\frac{1}{2} \int_{0}^{1} g\left(\frac{\partial}{\partial t} \gamma(t, s), \frac{\partial}{\partial t} \gamma(t, s)\right) d t \\
& =\frac{1}{2} g\left(\left.\partial\right|_{0} \gamma(t, s),\left.\partial\right|_{0} \gamma(t, s)\right) d t \\
& =\frac{1}{2} k^{2} .
\end{aligned}
$$

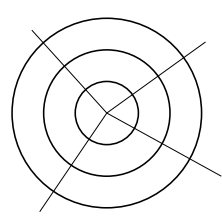

Comparing this with the first variational formula (22.3), i.e.,

$$
\left.\frac{\partial}{\partial s}\right|_{0}\left(E_{0}^{1}(\gamma(\quad, s))\right)=\int_{0}^{1} 0 d t+g(c(1))\left(c^{\prime}(1),\left.\frac{\partial}{\partial s}\right|_{0} \gamma(1, s)\right)-g(c(0))\left(c^{\prime}(0), 0\right),
$$

we get $0=g(c(1))\left(c^{\prime}(1),\left.\frac{\partial}{\partial s}\right|_{0} \gamma(1, s)\right)$, where $\left.\frac{\partial}{\partial s}\right|_{0} \gamma(1, s)$ is an arbitrary tangent vector of $\exp _{x}\left(k S\left(T_{x} M, g\right)\right)$.

23.3. Corollary. Let $(M, g)$ be a Riemann manifold, $x \in M$, and $\varepsilon>0$ be such that $\exp _{x}: D_{x}(\varepsilon):=\left\{X \in T_{x} M:|X|_{g}<\varepsilon\right\} \rightarrow M$ is a diffeomorphism on its image. Let $c:[a, b] \rightarrow \exp _{x}\left(D_{x}(\varepsilon)\right) \backslash\{x\}$ be a piecewise smooth curve, so that $c(t)=\exp _{x}(u(t) . v(t))$ where $0<u(t)<\varepsilon$ and $|v(t)|_{g_{x}}=1$.

Then for the length we have $L_{a}^{b}(c) \geq|u(b)-u(a)|$ with equality if and only if $u$ is monotone and $v$ is constant, so that $c$ is a radial geodesic, reparameterized by $u$.

On pseudo-Riemann manifolds this result holds only for in the domain of definition of the geodesic exponential mapping and only for curves with positive velocity vectors (time-like curves).

Proof. We may assume that $c$ is smooth by treating each smooth piece of $c$ separately. Let $\alpha(u, t):=\exp _{x}(u \cdot v(t))$. Then

$$
\begin{aligned}
c(t) & =\alpha(u(t), t), \\
\frac{\partial}{\partial t} c(t) & =\frac{\partial \alpha}{\partial u}(u(t), t) \cdot u^{\prime}(t)+\frac{\partial \alpha}{\partial t}(u(t), t), \\
\left|\frac{\partial \alpha}{\partial u}\right|_{g_{x}} & =|v(t)|_{g_{x}}=1, \\
0 & =g\left(\frac{\partial \alpha}{\partial u}, \frac{\partial \alpha}{\partial t}\right), \quad \text { by lemma }(23.2),
\end{aligned}
$$

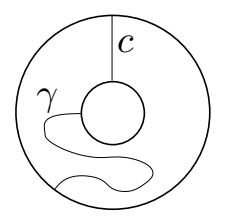

Putting this together, we get

$$
\begin{aligned}
\left|c^{\prime}\right|_{g}^{2} & =g\left(c^{\prime}, c^{\prime}\right)=g\left(\frac{\partial \alpha}{\partial u} \cdot u^{\prime}+\frac{\partial \alpha}{\partial t}, \frac{\partial \alpha}{\partial u} \cdot u^{\prime}+\frac{\partial \alpha}{\partial t}\right) \\
& =\left|u^{\prime}\right|^{2}\left|\frac{\partial \alpha}{\partial u}\right|_{g}^{2}+\left|\frac{\partial \alpha}{\partial t}\right|_{g}^{2}=\left|u^{\prime}\right|^{2}+\left|\frac{\partial \alpha}{\partial t}\right|_{g}^{2} \geq\left|u^{\prime}\right|^{2}
\end{aligned}
$$


with equality if and only if $\left|\frac{\partial \alpha}{\partial t}\right|_{g}=0$; thus $\frac{\partial \alpha}{\partial t}=0$ and $v(t)=$ constant. So finally:

$$
L_{a}^{b}(c)=\int_{a}^{b}\left|c^{\prime}(t)\right|_{g} d t \geq \int_{a}^{b}\left|u^{\prime}(t)\right| d t \geq\left|\int_{a}^{b} u^{\prime}(t) d t\right|=|u(b)-u(a)|
$$

with equality if and only if $u$ is monotone and $v$ is constant.

23.4. Corollary. Let $(M, g)$ be a Riemann manifold. Let $\varepsilon: M \rightarrow \mathbb{R}_{>0}$ be a continuous function such that for $\tilde{V}=\left\{X_{x} \in T_{x} M:\left|X_{x}\right|<\varepsilon(x)\right.$ for all $x \in$ $M\}$ the mapping $\left(\pi_{m}\right.$, exp) :TM $\supseteq \tilde{V} \rightarrow W \subseteq M \times M$ is a diffeomorphism from the open neighborhood $\tilde{V}$ of the zero section in $T M$ onto an open neighborhood $W$ of the diagonal in $M \times M$, as shown in (22.7.6).

Then for each $(x, y) \in W$ there exists a unique geodesic $c$ in $M$ which connects $x$ and $y$ and has minimal length: For each piecewise smooth curve $\gamma$ from $x$ to $y$ we have $L(\gamma) \geq L(c)$ with equality if and only if $\gamma$ is a reparameterization of $c$.

Proof. The set $\tilde{V} \cap T_{x} M=D_{x}(\varepsilon(x))$ satisfies the condition of corollary $(23.3)$. For $X_{x}=\exp _{x}^{-1}(y)=\left(\left(\pi_{M}, \exp \right) \mid \tilde{V}\right)^{-1}(x, y)$ the geodesic $t \mapsto c(t)=\exp _{x}\left(t . X_{x}\right)$ leads from $x$ to $y$. Let $\delta>0$ be small. Then $c$ contains a segment which connects the geodesic spheres $\exp _{x}\left(\delta . S\left(T_{x} M, g\right)\right)$ and $\exp _{x}\left(\left|X_{x}\right|_{g_{x}} \cdot S\left(T_{x} M, g\right)\right)$. By corollary (23.3) the length of this segment is $\geq\left|X_{x}\right|_{g}-\delta$ with equality if and only if this segment is radial, thus a reparameterization of $c$. Since this holds for all $\delta>0$, the result follows.

23.5. The geodesic distance. On a Riemann manifold $(M, g)$ there is a natural topological metric defined by

$$
\begin{aligned}
& \operatorname{dist}^{g}(x, y) \\
& \quad:=\inf \left\{L_{0}^{1}(c): c:[0,1] \rightarrow M \text { piecewise smooth, } c(0)=x, c(1)=y\right\},
\end{aligned}
$$

which we call the geodesic distance (since 'metric' is heavily used). We either assume that $M$ is connected or we take the distance of points in different connected components as $\infty$.

Lemma. On a Riemann manifold $(M, g)$ the geodesic distance is a topological metric which generates the topology of $M$. For $\varepsilon_{x}>0$ small enough the open ball

$$
B_{x}\left(\varepsilon_{x}\right)=\left\{y \in M: \operatorname{dist}^{g}(x, y)<\varepsilon_{x}\right\}
$$

has the property that any two points in it can be connected by a geodesic of minimal length.

Proof. This follows by (23.3) and (23.4), The triangle inequality is easy to check since we admit piecewise smooth curves. 
23.6. Theorem (Hopf-Rinov). For a Riemann manifold $(M, g)$ the following assertions are equivalent:

(1) $\left(M, \operatorname{dist}^{g}\right)$ is a complete metrical space (Cauchy sequences converge).

(2) Each closed subset of $M$ which is bounded for the geodesic distance is compact.

(3) Any geodesic is maximally definable on the whole of $\mathbb{R}$.

(4) $\exp : T M \rightarrow M$ is defined on the whole of $T M$.

(5) There exists a point $x$ such that $\exp _{x}: T_{x} M \rightarrow M$ is defined on the whole of $T_{x} M$, in each connected component of $M$.

If these equivalent conditions are satisfied, then $(M, g)$ is called a complete Riemann manifold. In this case we even have:

(6) On a complete connected Riemann manifold any two points can be connected by a geodesic of minimal length.

Condition (6) does not imply the other conditions: Consider an open convex in $\mathbb{R}^{m}$.

Proof. (2) $\Longrightarrow$ (1) is obvious.

(11) $\Longrightarrow$ (3) Let $c$ be a maximally defined geodesic, parametrized by arclength. If $c$ is defined on the interval $(a, b)$ and if $b<\infty$, say, then by the definition of the distance (23.5) the sequence $c\left(b-\frac{1}{n}\right)$ is a Cauchy sequence; thus by (1), $\lim _{n \rightarrow \infty} c\left(b-\frac{1}{n}\right)=: c(b)$ exists in $M$. For $m, n$ large enough $\left(c\left(b-\frac{1}{n}\right), c\left(b-\frac{1}{m}\right)\right) \in W$ where $W$ is the open neighborhood of the diagonal in $M \times M$ from $(23.4)$ thus the segment of $c$ between $c\left(b-\frac{1}{n}\right)$ and $c\left(b-\frac{1}{m}\right)$ is of minimal length: $\operatorname{dist}^{g}\left(c\left(b-\frac{1}{n}\right), c\left(b-\frac{1}{m}\right)\right)=\left|\frac{1}{n}-\frac{1}{m}\right|$. By continuity $\operatorname{dist}^{g}\left(c\left(b-\frac{1}{n}\right), c(b)\right)=\left|\frac{1}{n}\right|$. Now let us apply corollary [23.3) with center $c(b):$ In $\exp _{c(b)}\left(D_{c(b)}(\varepsilon)\right)$ the curve $t \mapsto c(b+t)$ is a piecewise smooth curve of minimal length; thus by (23.3) a radial geodesic. Thus $\lim _{t \rightarrow b} c^{\prime}(t)=: c^{\prime}(b)$ exists and $t \mapsto \exp _{c(b)}\left((t-b) c^{\prime}(b)\right)$ equals $c(t)$ for $t<b$ and prolongs the geodesic $c$ for $t \geq b$.

(3) $\Longrightarrow$ (4) is obvious.

(41) $\Longrightarrow$ (5) is obvious.

(5) $\Longrightarrow$ (6) for special points, in each connected component separately. In detail: Let $x, y$ be in one connected component of $M$ where $x$ is the special point with $\exp _{x}: T_{x} M \rightarrow M$ defined on the whole of $T_{x} M$. We shall prove that $x$ can be connected to $y$ by a geodesic of minimal length.

Let $\operatorname{dist}^{g}(x, y)=r>0$. Consider the compact set $S:=\exp _{x}\left(\delta . S\left(T_{x} M, g\right)\right) \subset$ $\exp _{x}\left(T_{x} M\right)$ for $0<\delta<r$ so small that $\exp _{x}$ is a diffeomorphism on $\{X \in$ $\left.T_{x} M:|X|_{g}<2 \delta\right\}$. There exists a unit vector $X_{x} \in S\left(T_{x} M, g_{x}\right)$ such that $z=\exp _{x}\left(\delta X_{x}\right)$ has the property that $\operatorname{dist}^{g}(z, y)=\min \left\{\operatorname{dist}^{g}(s, y): s \in S\right\}$. 
(7) Claim. The curve $c(t)=\exp _{x}\left(t . X_{x}\right)$ satisfies the condition

$$
\operatorname{dist}^{g}(c(t), y)=r-t
$$

for all $0 \leq t \leq r$. It will take some effort to prove this claim.

Since any piecewise smooth curve from $x$ to $y$ hits $S$ (its initial segment does so in the diffeomorphic preimage in $\left.T_{x} M\right)$, we have

$$
\begin{aligned}
r=\operatorname{dist}^{g}(x, y) & =\inf _{s \in S}\left(\operatorname{dist}^{g}(x, s)+\operatorname{dist}^{g}(s, y)\right)=\inf _{s \in S}\left(\delta+\operatorname{dist}^{g}(s, y)\right) \\
& =\delta+\min _{s \in S} \operatorname{dist}^{g}(s, y)=\delta+\operatorname{dist}^{g}(z, y), \\
\operatorname{dist}^{g}(z, y) & =r-\delta ; \quad \text { thus }(*) \text { holds for } t=\delta .
\end{aligned}
$$

(8) Claim. If $\left(^{*}\right)$ holds for $t \in[\delta, r]$, then it also holds for all $t^{\prime}$ with $\delta \leq t^{\prime} \leq t$, since we have:

$$
\begin{aligned}
& \operatorname{dist}^{g}\left(c\left(t^{\prime}\right), y\right) \leq \operatorname{dist}^{g}\left(c\left(t^{\prime}\right), c(t)\right)+\operatorname{dist}^{g}(c(t), y) \leq t-t^{\prime}+r-t=r-t^{\prime}, \\
& r=\operatorname{dist}^{g}(x, y) \leq \operatorname{dist}^{g}\left(x, c\left(t^{\prime}\right)\right)+\operatorname{dist}^{g}\left(c\left(t^{\prime}\right), y\right), \\
& \operatorname{dist}^{g}\left(c\left(t^{\prime}\right), y\right) \geq r-\operatorname{dist}^{g}\left(x, c\left(t^{\prime}\right)\right) \geq r-t^{\prime} \quad \Longrightarrow \text { claim (86). }
\end{aligned}
$$

Now let $t_{0}=\sup \left\{t \in[\delta, r]:\left(^{*}\right)\right.$ holds for $\left.t\right\}$. By continuity $(*)$ is then also valid for $t_{0}$. Assume for contradiction that $t_{0}<r$.

Let $S^{\prime}$ be the geodesic sphere with (small) radius $\delta^{\prime}$ centered at $c\left(t_{0}\right)$, and let $z^{\prime} \in S^{\prime}$ be a point with minimal distance to $y$ :

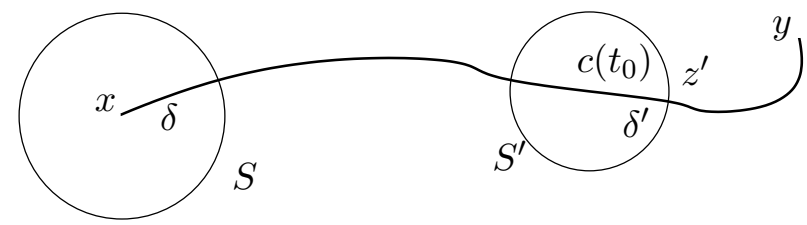

As above we see that

$$
\begin{aligned}
r-t_{0} \stackrel{(*)}{=} \operatorname{dist}^{g}\left(c\left(t_{0}\right), y\right) & =\inf _{s^{\prime} \in S^{\prime}}\left(\operatorname{dist}^{g}\left(c\left(t_{0}\right), s^{\prime}\right)+\operatorname{dist}^{g}\left(s^{\prime}, y\right)\right) \\
& =\delta^{\prime}+\operatorname{dist}^{g}\left(z^{\prime}, y\right), \\
\operatorname{dist}^{g}\left(z^{\prime}, y\right) & =\left(r-t_{0}\right)-\delta^{\prime} \\
\operatorname{dist}^{g}\left(x, z^{\prime}\right) & =\operatorname{dist}^{g}(x, y)-\operatorname{dist}^{g}\left(z^{\prime}, y\right) \\
& =r-\left(r-t_{0}\right)+\delta^{\prime}=t_{0}+\delta^{\prime} .
\end{aligned}
$$

We consider now the piecewise smooth curve $\bar{c}$ which initially follows $c$ from $x$ to $c\left(t_{0}\right)$ and then the minimal geodesic from $c\left(t_{0}\right)$ to $z^{\prime}$, parameterized by arc-length. We just checked that the curve $\bar{c}$ has minimal length $t_{0}+\delta^{\prime}$. Thus each piece of $\bar{c}$ has also minimal length, in particular the piece between $\bar{c}\left(t_{1}\right)$ and $\bar{c}\left(t_{2}\right)$, where $t_{1}<t_{0}<t_{2}$. Since we may choose these two points near to each other, $\bar{c}$ is a minimal geodesic between them by (23.4). Thus $\bar{c}$ 
equals $c, z^{\prime}=c\left(t_{0}+\delta\right)$, $\operatorname{dist}^{g}\left(c\left(t_{0}+\delta^{\prime}\right), y\right)=\operatorname{dist}^{g}\left(z^{\prime}, y\right)=r-\left(\delta^{\prime}+t_{0}\right)$ by $(* *)$, and $\left(^{*}\right)$ holds for $t_{0}+\delta^{\prime}$ also, which contradicts the maximality of $t_{0}$ for the validity of $(*)$. Thus the assumption $t_{0}<r$ is wrong and claim (77) follows.

Finally, by claim (7) we have $\operatorname{dist}^{g}(c(r), y)=r-r=0$; thus $c(t)=$ $\exp _{x}\left(t . X_{x}\right)$ is a geodesic from $x$ to $y$ of length $r=\operatorname{dist}^{g}(x, y)$, thus of minimal length, so (6) for the special points follows.

(44) $\Longrightarrow$ (6), by the foregoing proof, since then any point is special.

(5) $\Longrightarrow$ (2) Let $A \subset M$ be closed and bounded for the geodesic distance. Suppose that $A$ has diameter $r<\infty$. Then $A$ is completely contained in one connected component of $M$, by (23.5), Let $x$ be the special point in this connected component with $\exp _{x}$ defined on the whole of $T_{x} M$. Take $y \in A$.

By (6) for the special point $x$ (which follows from (5)), there exists a geodesic from $x$ to $y$ of minimal length $\operatorname{dist}^{g}(x, y)=: s<\infty$, and each point $z$ of $A$ can be connected to $x$ by a geodesic of minimal length

$$
\operatorname{dist}^{g}(x, z) \leq \operatorname{dist}^{g}(x, y)+\operatorname{dist}^{g}(y, z) \leq r+s .
$$

Thus the compact set (as continuous image of a compact ball) $\exp _{x}\left\{X_{x} \in\right.$ $\left.T_{x} M:\left|X_{x}\right|_{g} \leq r+s\right\}$ contains $A$. Since $A$ is closed, it is compact too.

23.7. Conformal metrics. Two Riemann metrics $g_{1}$ and $g_{2}$ on a manifold $M$ are called conformal if there exists a smooth nowhere vanishing function $f$ with $g_{2}=f^{2} \cdot g_{1}$. Then $g_{1}$ and $g_{2}$ have the same angles, but not the same lengths. A local diffeomorphism $\varphi:\left(M_{1}, g_{1}\right) \rightarrow\left(M_{2}, g_{2}\right)$ is called conformal if $\varphi^{*} g_{2}$ is conformal to $g_{1}$.

As an example, which also explains the name, we mention that any holomorphic mapping with nonvanishing derivative between open domains in $\mathbb{C}$ is conformal for the Euclidean inner product. This is clear from the polar decomposition $\varphi^{\prime}(z)=\left|\varphi^{\prime}(z)\right| e^{i \arg \left(\varphi^{\prime}(z)\right)}$ of the derivative.

As another, not unrelated, example we note that the stereographic projection from (1.2) is a conformal mapping:

$$
u_{+}:\left(S^{n} \backslash\{a\}, g^{S^{n}}\right) \rightarrow\{a\}^{\perp} \rightarrow\left(\mathbb{R}^{n},\langle\quad, \quad\rangle\right), \quad u_{+}(x)=\frac{x-\langle x, a\rangle a}{1-\langle x, a\rangle} .
$$

To see this, take $X \in T_{x} S^{n} \subset T_{x} \mathbb{R}^{n+1}$, so that $\langle X, x\rangle=0$. Then we get:

$$
\begin{aligned}
d u_{+}(x) X & =\frac{(1-\langle x, a\rangle)(X-\langle X, a\rangle a)+\langle X, a\rangle(x-\langle x, a\rangle a)}{(1-\langle x, a\rangle)^{2}} \\
& =\frac{1}{(1-\langle x, a\rangle)^{2}}((1-\langle x, a\rangle) X+\langle X, a\rangle x-\langle x, a\rangle a), \\
\left\langle d u_{+}(x) X, d u_{+}(x) Y\right\rangle & =\frac{1}{(1-\langle x, a\rangle)^{2}}\langle X, Y\rangle=\frac{1}{(1-\langle x, a\rangle)^{2}}\left(g^{S^{n}}\right)_{x}(X, Y) .
\end{aligned}
$$


23.8. Theorem (Nomizu-Ozeki, Morrow). Let $(M, g)$ be a connected Riemann manifold. Then we have:

(1) There exist complete Riemann metrics on $M$ which are conformal to $g$ and are equal to $g$ on any given compact subset of $M$.

(2) There also exist Riemann metrics on $M$ such that $M$ has finite diameter which are conformal to $g$ and are equal to $g$ on any given compact subset of $M$. If $M$ is not compact, then by (23.6.2) a Riemann metric for which $M$ has finite diameter is not complete.

Thus the sets of all complete Riemann metrics and of all Riemann metrics with bounded diameter are both dense in the compact $C^{\infty}$-topology on the space of all Riemann metrics.

Proof of (11). For $x \in M$ let

$$
r(x):=\sup \left\{r: B_{x}(r)=\left\{y \in M: \operatorname{dist}^{g}(x, y) \leq r\right\} \text { is compact in } M\right\} .
$$

If $r(x)=\infty$ for one $x$, then $g$ is a complete metric by (23.6.2). Since $\exp _{x}$ is a diffeomorphism near $0_{x}, r(x)>0$ for all $x$. We assume that $r(x)<\infty$ for all $x$.

Claim. $|r(x)-r(y)| \leq \operatorname{dist}^{g}(x, y)$; thus $r: M \rightarrow \mathbb{R}$ is continuous, since: For small $\varepsilon>0$ the set $B_{x}(r(x)-\varepsilon)$ is compact, $\operatorname{dist}^{g}(z, x) \leq \operatorname{dist}^{g}(z, y)+$ $\operatorname{dist}^{g}(y, x)$ implies that $B_{y}\left(r(x)-\varepsilon-\right.$ dist $\left.^{g}(x, y)\right) \subseteq B_{x}(r(x)-\varepsilon)$ is compact, and thus $r(y) \geq r(x)-\operatorname{dist}^{g}(x, y)-\varepsilon$ and $r(x)-r(y) \leq \operatorname{dist}^{g}(x, y)$. Now interchange $x$ and $y$.

By a partition of unity argument we now construct a smooth function $f \in$ $C^{\infty}\left(M, \mathbb{R}_{>0}\right)$ with $f(x)>\frac{1}{r(x)}$. Consider the Riemann metric $\bar{g}=f^{2} g$.

Claim. $\bar{B}_{x}\left(\frac{1}{4}\right):=\left\{y \in M: \operatorname{dist}^{\bar{g}}(x, y) \leq \frac{1}{4}\right\} \subset B_{x}\left(\frac{1}{2} r(x)\right)$; thus it is compact.

Suppose $y \notin B_{x}\left(\frac{1}{2} r(x)\right)$. For any piecewise smooth curve $c$ from $x$ to $y$ we have

$$
\begin{aligned}
L^{g}(c) & =\int_{0}^{1}\left|c^{\prime}(t)\right|_{g} d t>\frac{r(x)}{2} \\
L^{\bar{g}}(c) & =\int f(c(t)) \cdot\left|c^{\prime}(t)\right|_{g} d t=f\left(c\left(t_{0}\right)\right) \int_{0}^{1}\left|c^{\prime}(t)\right|_{g} d t>\frac{L^{g}(c)}{r\left(c\left(t_{0}\right)\right)},
\end{aligned}
$$

for some $t_{0} \in[0,1]$, by the mean value theorem of integral calculus. Moreover,

$$
\begin{aligned}
\left|r\left(c\left(t_{0}\right)\right)-r(x)\right| & \leq \operatorname{dist}^{g}\left(c\left(t_{0}\right), x\right) \leq L^{g}(c)=: L, \\
r\left(c\left(t_{0}\right)\right) & \leq r(x)+L, \\
L^{\bar{g}}(c) & \geq \frac{L}{r(x)+L} \geq \frac{L}{3 L}=\frac{1}{3},
\end{aligned}
$$


so $y \notin \bar{B}_{x}\left(\frac{1}{4}\right)$ either.

Claim. $(M, \bar{g})$ is a complete Riemann manifold.

Let $X \in T_{x} M$ with $|X|_{\bar{g}}=1$. Then $\exp ^{\bar{g}}(t . X)$ is defined for $|t| \leq \frac{1}{5}<\frac{1}{4}$. But also $\exp ^{\bar{g}}\left(\left.s \cdot \frac{\partial}{\partial t}\right|_{t= \pm 1 / 5} \exp ^{\bar{g}}(t . X)\right)$ is defined for $|s|<\frac{1}{4}$ which equals $\exp ^{\bar{g}}\left(\left( \pm \frac{1}{5}+s\right) X\right)$, and so on. Thus $\exp ^{\bar{g}}(t . X)$ is defined for all $t \in \mathbb{R}$, and by (23.6.4) the metric $\bar{g}$ is complete.

Claim. We may choose $f$ in such a way that $f=1$ on a neighborhood of any given compact set $K \subset M$.

Let $C=\max \left\{\frac{1}{r(x)}: x \in K\right\}+1$. By a partition of unity argument we construct a smooth function $f$ with $f=1$ on a neighborhood of $K$ and $C f(x)>\frac{1}{r(x)}$ for all $x$. By the arguments above, $C^{2} f^{2} g$ is then a complete metric; thus so is $f^{2} g$.

Proof of (2). Let $g$ be a complete Riemann metric on $M$. We choose $x \in M$, a smooth function $h$ with $h(y)>\operatorname{dist}^{g}(x, y)$, and we consider the Riemann metric $\tilde{g}_{y}=e^{-2 h(y)} g_{y}$. By (23.6.6) for any $y \in M$ there exists a minimal $g$-geodesic $c$ from $x$ to $y$, parameterized by arc-length. Then $h(c(s))>\operatorname{dist}^{g}(x, c(s))=s$ for all $s \leq \operatorname{dist}^{g}(x, y)=: L$. But then

$$
L^{\tilde{g}}(c)=\int_{0}^{L} e^{-h(c(s))}\left|c^{\prime}(s)\right|_{g} d s<\int_{0}^{L} e^{-s} 1 d s<\int_{0}^{\infty} e^{-s} d s=1,
$$

so that $M$ has diameter 1 for the Riemann metric $\tilde{g}$. We may also obtain that $\tilde{g}=g$ on a compact set as above.

23.9. Proposition. Let $(M, g)$ be a complete Riemann manifold. Let $X \in \mathfrak{X}(M)$ be a vector field which is bounded with respect to $g,|X|_{g} \leq C$.

Then $X$ is a complete vector field; it admits a global flow.

Proof. The flow of $X$ is given by the differential equation $\frac{\partial}{\partial t} \mathrm{Fl}_{t}^{X}(x)=$ $X\left(\mathrm{Fl}_{t}^{X}(x)\right)$ with initial value $\mathrm{Fl}_{0}^{X}(x)=x$. Suppose that $c(t)=\mathrm{Fl}_{t}^{X}(x)$ is defined on $(a, b)$ and that $b<\infty$, say. Then

$$
\begin{aligned}
\operatorname{dist}^{g}(c(b-1 / n), c(b-1 / m)) \leq L_{b-1 / n}^{b-1 / m}(c)=\int_{b-1 / n}^{b-1 / m}\left|c^{\prime}(t)\right|_{g} d t \\
=\int_{b-1 / n}^{b-1 / m}|X(c(t))|_{g} d t \leq \int_{b-1 / n}^{b-1 / m} C d t=C \cdot\left(\frac{1}{m}-\frac{1}{n}\right) \rightarrow 0,
\end{aligned}
$$

so that $c(b-1 / n)$ is a Cauchy sequence in the complete metrical space $M$ and the limit $c(b)=\lim _{n \rightarrow \infty} c(b-1 / n)$ exists. But then we may continue the flow beyond $b$ by $\mathrm{Fl}_{s}^{X}\left(\mathrm{Fl}_{b}^{X}(x)\right)=\mathrm{Fl}_{b+s}^{X}$. 
23.10. Problem (Unsolved until May 2, 2008, to the author's knowledge). Let $X$ be a complete vector field on a manifold $M$. Does there exist a complete Riemann metric $g$ on $M$ such that the vector field $X$ is bounded with respect to $g$ ?

The only inroad towards this problem is the following:

Proposition ([76]). Let $X$ be a complete vector field on a connected manifold $M$. Then there exists a complete Riemann metric $g$ on the manifold $M \times \mathbb{R}$ such that the vector field $X \times \partial_{t} \in \mathfrak{X}(M \times \mathbb{R})$ is bounded with respect to $g$.

Proof. Since $\mathrm{Fl}_{t}^{X \times \partial_{t}}(x, s)=\left(\mathrm{Fl}_{t}^{X}(x), s+t\right)$, the vector field $X \times \partial_{t}$ is also complete. It is nowhere 0 .

Choose a smooth proper function $f_{1}$ on $M$; for example, if a smooth function $f_{1}$ satisfies $f_{1}(x)>\operatorname{dist}^{\bar{g}}\left(x_{0}, x\right)$ for a complete Riemann metric $\bar{g}$ on $M$, then $f_{1}$ is proper by (23.6.2).

For a Riemann metric $\bar{g}$ on $M$ we consider the Riemann metric $\tilde{g}$ on the product $M \times \mathbb{R}$ which equals $g_{x}$ on $T_{x} M \cong T_{x} M \times 0_{t}=T_{(x, t)}(M \times\{t\})$ and satisfies

$$
\left|X \times \partial_{t}\right|_{\tilde{g}}=1 \quad \text { and } \quad \tilde{g}_{(x, t)}\left(\left(X \times \partial_{t}\right)(x, t), T_{(x, t)}(M \times\{t\})\right)=0 .
$$

We will also use the fiberwise $\tilde{g}$-orthogonal projections

$$
\begin{aligned}
& \operatorname{pr}_{M}: T(M \times \mathbb{R}) \rightarrow T M \times 0 \quad \text { and } \\
& \operatorname{pr}_{X}: T(M \times \mathbb{R}) \rightarrow \mathbb{R} .\left(X \times \partial_{t}\right) \cong \mathbb{R} .
\end{aligned}
$$

The smooth function $f_{2}(x, s)=f_{1}\left(\mathrm{Fl}_{-s}^{X}(x)\right)+s$ satisfies the following and is thus still proper:

$$
\begin{aligned}
\left(\mathcal{L}_{X \times \partial_{t}} f_{2}\right)(x, s) & =\left.\partial\right|_{0} f_{2}\left(\mathrm{Fl}_{t}^{X \times \partial_{t}}(x, s)\right) \\
& =\left.\partial\right|_{0} f_{2}\left(\mathrm{Fl}_{t}^{X}(x), s+t\right) \\
& =\left.\partial\right|_{0}\left(f_{1}\left(\mathrm{Fl}_{-s-t}^{X}\left(\mathrm{Fl}_{t}^{X}(x)\right)\right)+s+t\right) \\
& =\left.\partial\right|_{0} f_{1}\left(\mathrm{Fl}_{-s}^{X}(x)\right)+1=1 .
\end{aligned}
$$

By a partition of unity argument we construct another smooth function $f_{3}: M \times \mathbb{R} \rightarrow \mathbb{R}$ which satisfies

$$
f_{3}(x, s)^{2}>\max \left\{\left|Y\left(f_{2}\right)\right|^{2}: Y \in T_{(x, s)}(M \times\{s\}),|Y|_{\tilde{g}}=1\right\} .
$$

Finally we define a Riemann metric $g$ on $M \times \mathbb{R}$ by

$$
g_{(x, t)}(Y, Z)=f_{3}(x, t)^{2} \tilde{g}_{(x, t)}\left(\operatorname{pr}_{M}(Y), \operatorname{pr}_{M}(Z)\right)+\operatorname{pr}_{X}(Y) \cdot \operatorname{pr}_{X}(Z)
$$

for $Y, Z \in T_{(x, t)}(M \times \mathbb{R})$, which satisfies $\left|X \times \partial_{t}\right|_{g}=1$. 
Claim. $g$ is a complete Riemann metric on $M \times \mathbb{R}$.

Let $c$ be a piecewise smooth curve parameterized by $g$-arc-length. Then

$$
\begin{aligned}
\left|c^{\prime}\right|_{g} & =1, \quad \text { thus also } \quad\left|\operatorname{pr}_{M}\left(c^{\prime}\right)\right|_{g} \leq 1, \quad\left|\operatorname{pr}_{X}\left(c^{\prime}\right)\right| \leq 1, \\
\frac{\partial}{\partial t} f_{2}(c(t)) & =d f_{2}\left(c^{\prime}(t)\right) \\
& =\left(\operatorname{pr}_{M}\left(c^{\prime}(t)\right)\right)\left(f_{2}\right)+\operatorname{pr}_{X}\left(c^{\prime}(t)\right)\left(f_{2}\right), \\
\left|\frac{\partial}{\partial t} f_{2}(c(t))\right| & \leq\left|\frac{\operatorname{pr}_{M}\left(c^{\prime}(t)\right)}{\left|\operatorname{pr}_{M}\left(c^{\prime}(t)\right)\right|_{g}}\left(f_{2}\right)\right|+\left|\frac{\operatorname{pr}_{X}\left(c^{\prime}(t)\right)}{\left|\operatorname{pr}_{X}\left(c^{\prime}(t)\right)\right|_{g}}\left(f_{2}\right)\right| \\
& =\left|\frac{1}{f_{3}(c(t))} \frac{\operatorname{pr}_{M}\left(c^{\prime}(t)\right)}{\left|\operatorname{pr}_{M}\left(c^{\prime}(t)\right)\right|_{\tilde{g}}}\left(f_{2}\right)\right|+\left|\mathcal{L}_{X \times \partial_{t}} f_{2}\right|<2
\end{aligned}
$$

by the definition of $g$ and the properties of $f_{3}$ and $f_{2}$. Thus

$$
\left|f_{2}(c(t))-f_{2}(c(0))\right| \leq \int_{0}^{t}\left|\frac{\partial}{\partial t} f_{2}(c(t))\right| d t \leq 2 t .
$$

Since this holds for every such $c$, we conclude that

$$
\left|f_{2}(x)-f_{2}(y)\right| \leq 2 \operatorname{dist}^{g}(x, y)
$$

and thus each closed and dist ${ }^{g}$-bounded set is contained in some

$$
\left\{y \in M \times \mathbb{R}: \operatorname{dist}^{g}(x, y) \leq R\right\} \subset f_{2}^{-1}\left(\left[f_{2}(x)-\frac{R}{2}, f_{2}(x)+\frac{R}{2}\right]\right)
$$

which is compact since $f_{2}$ is proper. So $(M \times \mathbb{R}, g)$ is a complete Riemann manifold by (23.6.2)

\section{Parallel Transport and Curvature}

24.1. Parallel transport. Let $(M, \nabla)$ be a manifold with a covariant derivative, as treated in (22.7), The pair $(M, \nabla)$ is also sometimes called an affine manifold.

A vector field $Y: N \rightarrow T M$ along a smooth mapping $f=\pi_{M} \circ Y: N \rightarrow M$ is called parallel if $\nabla_{X} Y=0$ for any vector field $X \in \mathfrak{X}(N)$.

If $Y: \mathbb{R} \rightarrow T M$ is a vector field along a given curve $c=\pi_{M} \circ Y: \mathbb{R} \rightarrow M$, then

$$
\nabla_{\partial_{t}} Y=K \circ T Y \circ \partial_{t}=0
$$

takes the following form in a local chart, by (22.7.7).

$$
\begin{aligned}
K \circ T Y \circ \partial_{t} & =K\left(\bar{c}(t), \bar{Y}(t) ; \bar{c}^{\prime}(t), \bar{Y}^{\prime}(t)\right) \\
& =\left(\bar{c}(t), \bar{Y}^{\prime}(t)-\Gamma_{\bar{c}(t)}\left(\bar{Y}(t), \bar{c}^{\prime}(t)\right)\right) .
\end{aligned}
$$

This is a linear ordinary differential equation of first order for $\bar{Y}$ (since $\bar{c}$ is given). Thus for every initial value $Y\left(t_{0}\right)$ for $t_{0} \in \mathbb{R}$ the parallel vector field $Y$ along $c$ is uniquely determined for the whole parameter space $\mathbb{R}$. 
We formalize this by defining the parallel transport along the curve $c: \mathbb{R} \rightarrow$ $M$ as

$$
\operatorname{Pt}(c, t): T_{c(0)} M \rightarrow T_{c(t)} M, \quad \operatorname{Pt}(c, t) . Y(0)=Y(t),
$$

where $Y$ is any parallel vector field along $c$. Note that we treat this notion for principal bundles in (19.6) and for general fiber bundles in (17.8), This is a special case here.

Theorem. On an affine manifold $(M, \nabla)$ the parallel transport has the following properties:

(1) $\operatorname{Pt}(c, t): T_{c(0)} M \rightarrow T_{c(t)} M$ is a linear isomorphism for each $t \in \mathbb{R}$ and each curve $c: \mathbb{R} \rightarrow M$.

(2) For smooth $f: \mathbb{R} \rightarrow \mathbb{R}$ we have $\operatorname{Pt}(c, f(t))=\operatorname{Pt}(c \circ f, t) \operatorname{Pt}(c, f(0))$; the reparameterization invariance.

(3) $\operatorname{Pt}(c, t)^{-1}=\operatorname{Pt}(c(+t),-t)$.

(4) If the covariant derivative is compatible with a pseudo-Riemann metric $g$ on $M$, then $\operatorname{Pt}(c, t)$ is isometric, i.e.,

$$
g_{c(t)}(\operatorname{Pt}(c, t) X, \operatorname{Pt}(c, t) Y)=g_{c(0)}(X, Y) .
$$

Proof. (1) follows from the linearity of the differential equation.

(2) See also (17.8), Let $X$ be parallel along $c, \nabla_{\partial_{t}} X=0$ or $X(t)=$ $\operatorname{Pt}(c, t) X(0)$. Then we have by (22.7.6)

$$
\nabla_{\partial_{t}}(X \circ f)=\nabla_{T_{t} f . \partial_{t}} X=\nabla_{f^{\prime}(t) \partial_{t}} X=f^{\prime}(t) \nabla_{\partial_{t}} X=0
$$

thus $X \circ f$ is also a parallel vector field along $c \circ f$, with initial value $X(f(0))=$ $\operatorname{Pt}(c, f(0)) X(0)$. So

$$
\operatorname{Pt}(c, f(t)) X(0)=X(f(t))=\operatorname{Pt}(c \circ f, t) \operatorname{Pt}(c, f(0)) X(0) .
$$

(3) follows from (2).

(44) Let $X$ and $Y$ be parallel vector fields along $c$, i.e., $\nabla_{\partial_{t}} X=0$, etc. Then

$$
\partial_{t} g(X(t), Y(t))=g\left(\nabla_{\partial_{t}} X(t), Y(t)\right)+g\left(X(t), \nabla_{\partial_{t}} Y(t)\right)=0
$$

thus $g(X(t), Y(t))$ is constant in $t$.

24.2. Flows and parallel transports. Let $X \in \mathfrak{X}(M)$ be a vector field on an affine manifold $(M, \nabla)$. Let $C: T M \times_{M} T M \rightarrow T^{2} M$ be the linear connection for the covariant derivative $\nabla$; see $(22.7)$. The horizontal lift of the vector field $X$ is then given by $C(X, \quad) \in \mathfrak{X}(T M)$ which is $\pi_{M}$-related to $X: T\left(\pi_{M}\right) \circ C(X, \quad)=X \circ \pi_{M}$. A flow line $\mathrm{Fl}_{t}^{C(X,}{ }^{\prime}\left(Y_{x}\right)$ is then a smooth curve in $T M$ whose tangent vector is everywhere horizontal, so the curve is parallel, and $\pi_{M}\left(\mathrm{Fl}_{t}^{C(X,}{ }^{\prime}\left(Y_{x}\right)\right)=\mathrm{Fl}_{t}^{X}(x)$ by (3.14), Thus

$$
\left.\operatorname{Pt}\left(\mathrm{Fl}^{X}, t\right)=\mathrm{Fl}_{t}^{C(X,}\right) \text {. }
$$


Proposition. For vector fields $X, Y \in \mathfrak{X}(M)$ we have:

$$
\begin{aligned}
\nabla_{X} Y & \left.=\left.\partial\right|_{0}\left(\mathrm{Fl}_{-t}^{C(X,}\right) \circ Y \circ \mathrm{Fl}_{t}^{X}\right) \\
& =\left.\partial\right|_{0} \operatorname{Pt}\left(\mathrm{Fl}^{X},-t\right) \circ Y \circ \mathrm{Fl}_{t}^{X} \\
& =:\left.\partial\right|_{0} \operatorname{Pt}\left(\mathrm{Fl}^{X}, t\right)^{*} Y,
\end{aligned}
$$

and more generally,

$$
\begin{aligned}
\frac{\partial}{\partial t} \operatorname{Pt}\left(\mathrm{Fl}^{X},-t\right) & \circ Y \circ \mathrm{Fl}_{t}^{X}=\frac{\partial}{\partial t} \operatorname{Pt}\left(\mathrm{Fl}^{X}, t\right)^{*} Y \\
& =\operatorname{Pt}\left(\mathrm{Fl}^{X}, t\right)^{*} \nabla_{X} Y \\
& =\operatorname{Pt}\left(\mathrm{Fl}^{X},-t\right) \circ \nabla_{X} Y \circ \mathrm{Fl}_{t}^{X} \\
& =\nabla_{X}\left(\operatorname{Pt}\left(\mathrm{Fl}^{X}, t\right)^{*} Y\right) .
\end{aligned}
$$

(4) The local vector bundle isomorphism $\left.\mathrm{Pt}_{(\mathrm{Fl}}^{X}, t\right)$ over $\mathrm{Fl}_{t}^{X}$ induces vector bundle isomorphisms $\mathrm{Pt}^{\otimes}\left(\mathrm{Fl}^{X}, t\right)$ on all tensor bundles $\otimes^{p} T M \otimes \bigotimes^{q} T^{*} M$ over $\mathrm{Fl}_{t}^{X}$. For each tensor field $A$ we have

(2) $\quad \nabla_{X} A=\left.\partial\right|_{0} \mathrm{Pt}^{\otimes}\left(\mathrm{Fl}^{X},-t\right) \circ A \circ \mathrm{Fl}_{t}^{X}=\left.\partial\right|_{0} \mathrm{Pt}^{\otimes}\left(\mathrm{Fl}^{X}, t\right)^{*} A$.

(3) $\quad \frac{\partial}{\partial t} \mathrm{Pt}^{\otimes}\left(\mathrm{Fl}^{X}, t\right)^{*} A=\mathrm{Pt}^{\otimes}\left(\mathrm{Fl}^{X}, t\right)^{*} \nabla_{X} A=\mathrm{Pt}\left(\mathrm{Fl}^{X},-t\right) \circ \nabla_{X} A \circ \mathrm{Fl}_{t}^{X}$

$$
=\nabla_{X}\left(\mathrm{Pt}^{\otimes}\left(\mathrm{Fl}^{X}, t\right)^{*} A\right) \text {. }
$$

Proof. (2) We compute

$$
\begin{aligned}
& \left.\partial\right|_{0} \mathrm{Fl}_{-t}^{C(X,} \quad{ }^{\prime}\left(Y\left(\mathrm{Fl}_{t}^{X}(x)\right)\right) \\
& =-C\left(X, \mathrm{Fl}_{0}^{C(X, \quad)}\left(Y\left(\mathrm{Fl}_{0}^{X}(x)\right)\right)\right)+\left.T\left(\mathrm{Fl}_{0}^{C(X,} \quad\right) \partial\right|_{0}\left(Y\left(\mathrm{Fl}_{t}^{X}(x)\right)\right) \\
& =-C(X(x), Y(x))+T Y . X(x) \\
& =T Y \cdot X(x)-C\left(T\left(\pi_{M}\right) \cdot T Y \cdot X(x), \pi_{T M}(T Y \cdot X(x))\right) \\
& =\left(\operatorname{Id}_{T^{2} M}-(\text { horizontal projection })\right) T Y \cdot X(x) \\
& =\operatorname{vl}(Y(x), K . T Y . X(x))=\operatorname{vl}\left(Y(x),\left(\nabla_{X} Y\right)(x)\right) .
\end{aligned}
$$

The vertical lift disappears if we identify the tangent space to the fiber $T_{x} M$ with the fiber.

(31) We did this several times already; see (3.13), (8.16), and (9.6);

$$
\begin{aligned}
\frac{\partial}{\partial t} \operatorname{Pt}\left(\mathrm{Fl}^{X}, t\right)^{*} Y & =\left.\frac{\partial}{\partial s}\right|_{0}\left(\mathrm{Pt}\left(\mathrm{Fl}^{X},-t\right) \circ \mathrm{Pt}\left(\mathrm{Fl}^{X},-s\right) \circ Y \circ \mathrm{Fl}_{s}^{X} \circ \mathrm{Fl}_{t}^{X}\right) \\
& =\left.\operatorname{Pt}\left(\mathrm{Fl}^{X},-t\right) \circ \frac{\partial}{\partial s}\right|_{0}\left(\mathrm{Pt}^{X}\left(\mathrm{Fl}^{X},-s\right) \circ Y \circ \mathrm{Fl}_{s}^{X}\right) \circ \mathrm{Fl}_{t}^{X} \\
& =\operatorname{Pt}\left(\mathrm{Fl}^{X},-t\right) \circ\left(\nabla_{X} Y\right) \circ \mathrm{Fl}_{t}^{X}=\operatorname{Pt}\left(\mathrm{Fl}^{X}, t\right)^{*} \nabla_{X} Y, \\
\frac{\partial}{\partial t} \operatorname{Pt}\left(\mathrm{Fl}^{X}, t\right)^{*} Y & =\left.\frac{\partial}{\partial s}\right|_{0} \mathrm{Pt}\left(\mathrm{Fl}^{X}, s\right)^{*} \operatorname{Pt}\left(\mathrm{Fl}^{X}, t\right)^{*} Y=\nabla_{X}\left(\mathrm{Pt}\left(\mathrm{Fl}^{X}, t\right)^{*} Y\right) .
\end{aligned}
$$

(41) For a tensor $A$ with foot point $\mathrm{Fl}_{t}^{X}(x)$ let us define $\mathrm{Pt}^{\otimes}\left(\mathrm{Fl}^{X}, t\right)^{*} A$ with foot point $x$ by 


$$
\begin{aligned}
& \left(\mathrm{Pt}^{\otimes}\left(\mathrm{Fl}^{X}, t\right) A\right)\left(X_{1}, \ldots, X_{q}, \omega^{1}, \ldots, \omega^{p}\right) \\
= & A\left(\mathrm{Pt}\left(\mathrm{Fl}^{X}, t\right) X_{1}, \ldots, \operatorname{Pt}\left(\mathrm{Fl}^{X}, t\right) X_{q}, \mathrm{Pt}\left(\mathrm{Fl}^{X},-t\right)^{*} \omega^{1}, \ldots, \mathrm{Pt}\left(\mathrm{Fl}^{X},-t\right)^{*} \omega^{p}\right) .
\end{aligned}
$$

Thus $\mathrm{Pt}^{\otimes}\left(\mathrm{Fl}^{X}, t\right)$ is fiberwise an algebra homomorphism of the tensor algebra which commutes with all contractions. Thus $\left.\partial\right|_{0} \mathrm{Pt}^{\otimes}\left(\mathrm{Fl}^{X}, t\right)^{*}$ becomes a derivation on the algebra of all tensor fields which commutes with contractions and equals $\nabla_{X}$ on vector fields. Thus by (22.12) it coincides with $\nabla_{X}$ on all tensor fields. This implies (2).

(3) can be proved in the same way as (3).

24.3. Curvature. Let $(M, \nabla)$ be an affine manifold. The curvature of the covariant derivative $\nabla$ is given by

$$
\begin{aligned}
R(X, Y) Z & =\nabla_{X} \nabla_{Y} Z-\nabla_{Y} \nabla_{X} Z-\nabla_{[X, Y]} Z \\
& =\left(\left[\nabla_{X}, \nabla_{Y}\right]-\nabla_{[X, Y]}\right) Z,
\end{aligned}
$$

for $X, Y, Z \in \mathfrak{X}(M)$.

A straightforward computation shows that $R(X, Y) Z$ is $C^{\infty}(M)$-linear in each entry; thus $R$ is a $\left(\begin{array}{l}1 \\ 3\end{array}\right)$-tensor field on $M$.

In a local chart $(U, u)$ we have (where $\left.\partial_{i}=\frac{\partial}{\partial u^{i}}\right)$ :

$$
\begin{aligned}
\left.X\right|_{U} & =\sum X^{i} \partial_{i},\left.\quad Y\right|_{U}=\sum Y^{j} \partial_{j},\left.\quad Z\right|_{U}=\sum Z^{k} \partial_{k}, \\
\left.R(X, Y)(Z)\right|_{U}=\sum X^{i} Y^{j} Z^{k} R\left(\partial_{i}, \partial_{j}\right)\left(\partial_{k}\right) & =\left(\sum R_{i, j, k}^{l} d u^{i} \otimes d u^{j} \otimes d u^{k} \otimes \partial_{l}\right)(X, Y, Z), \\
\quad \sum & R_{i, j, k}^{l} \partial_{l}=R\left(\partial_{i}, \partial_{j}\right)\left(\partial_{k}\right) \\
= & \nabla_{\partial_{i}} \nabla_{\partial_{j}} \partial_{k}-\nabla_{\partial_{j}} \nabla_{\partial_{i}} \partial_{k}-0 \\
& =\nabla_{\partial_{i}}\left(-\sum \Gamma_{j, k}^{m} \partial_{m}\right)-\nabla_{\partial_{j}}\left(-\sum \Gamma_{i, k}^{m} \partial_{m}\right) \\
& =-\sum \partial_{i} \Gamma_{j, k}^{m} \partial_{m}-\sum \Gamma_{j, k}^{m} \nabla_{\partial_{i}} \partial_{m}+\sum \partial_{j} \Gamma_{i, k}^{m} \partial_{m}+\sum \Gamma_{i, k}^{m} \nabla_{\partial_{j}} \partial_{m} \\
& =-\sum \partial_{i} \Gamma_{j, k}^{l} \partial_{l}+\sum \Gamma_{j, k}^{m} \Gamma_{i, m}^{l} \partial_{l}+\sum \partial_{j} \Gamma_{i, k}^{l} \partial_{l}-\sum \Gamma_{i, k}^{m} \Gamma_{j, m}^{l} \partial_{l} .
\end{aligned}
$$

We can collect all local formulas here, also from (22.9.7) or (22.5.6) and from (22.4.2) in the case of a Levi-Civita connection (where $X=(x, \bar{X})$, etc.):

$$
\begin{aligned}
\nabla_{\partial_{i}} \partial_{j} & =-\sum \Gamma_{i, j}^{l} \\
\Gamma_{i j}^{k} & =\frac{1}{2} \sum g^{k l}\left(\partial_{l} g_{i j}-\partial_{i} g_{l j}-\partial_{j} g_{i l}\right) \\
R_{i, j, k}^{l} & =-\partial_{i} \Gamma_{j, k}^{l}+\partial_{j} \Gamma_{i, k}^{l}+\sum \Gamma_{j, k}^{m} \Gamma_{i, m}^{l}-\sum \Gamma_{i, k}^{m} \Gamma_{j, m}^{l} \\
\bar{R}(\bar{X}, \bar{Y}) \bar{Z} & =-d \Gamma(x)(\bar{X})(\bar{Y}, \bar{Z})+d \Gamma(x)(\bar{Y})(\bar{X}, \bar{Z}) \\
& +\Gamma_{x}\left(\bar{X}, \Gamma_{x}(\bar{Y}, \bar{Z})\right)-\Gamma_{x}\left(\bar{Y}, \Gamma_{x}(\bar{X}, \bar{Z})\right)
\end{aligned}
$$


24.4. Theorem. Let $\nabla$ be a covariant derivative on a manifold $M$, with torsion Tor; see (22.10). Then the curvature $R$ has the following properties, where $X, Y, Z, U \in \mathfrak{X}(M)$ :

$$
\begin{gathered}
R(X, Y) Z=-R(Y, X) Z . \\
\sum_{\text {cyclic }} R(X, Y) Z=\sum_{\text {cyclic }}\left(\left(\nabla_{X} \operatorname{Tor}\right)(Y, Z)+\operatorname{Tor}(\operatorname{Tor}(X, Y), Z)\right), \\
\text { algebraic Bianchi identity. } \\
\sum_{\text {cyclic }}\left(\left(\nabla_{X} R\right)(Y, Z)+R(\operatorname{Tor}(X, Y), Z)\right)=0, \quad \text { Bianchi identity. }
\end{gathered}
$$

If the connection $\nabla$ is torsion-free, we have:

$$
\sum_{\text {cyclic }} R(X, Y) Z=0, \quad \text { algebraic Bianchi identity. }
$$

(3)

$$
\sum_{\substack{c y c l i c \\ X, Y, Z}}\left(\nabla_{X} R\right)(Y, Z)=0, \quad \text { Bianchi identity. }
$$

If $\nabla$ is the (torsion-free) Levi-Civita connection of a pseudo-Riemann metric $g$, then we have moreover:

$$
\begin{aligned}
& g(R(X, Y) Z, U)=g(R(Z, U) X, Y), \\
& g(R(X, Y) Z, U)=-g(R(X, Y) U, Z) .
\end{aligned}
$$

Proof. (2) The extension of $\nabla_{X}$ to tensor fields was treated in (22.12)

(6) $\left(\nabla_{X} \operatorname{Tor}\right)(Y, Z)=\nabla_{X}(\operatorname{Tor}(Y, Z))-\operatorname{Tor}\left(\nabla_{X} Y, Z\right)-\operatorname{Tor}\left(Y, \nabla_{X} Z\right)$.

From the definition (22.10.1) of the torsion:

$$
\begin{aligned}
\operatorname{Tor}(\operatorname{Tor}(X, Y), Z) & =\operatorname{Tor}\left(\nabla_{X} Y-\nabla_{Y} X-[X, Y], Z\right) \\
& =\operatorname{Tor}\left(\nabla_{X} Y, Z\right)+\operatorname{Tor}\left(Z, \nabla_{Y} X\right)-\operatorname{Tor}([X, Y], Z) .
\end{aligned}
$$

These combine to

$$
\begin{aligned}
\sum_{\text {cyclic }} & \operatorname{Tor}(\operatorname{Tor}(X, Y), Z) \\
& =\sum_{\text {cyclic }}\left(\nabla_{X}(\operatorname{Tor}(Y, Z))-\left(\nabla_{X} \operatorname{Tor}\right)(Y, Z)-\operatorname{Tor}([X, Y], Z)\right)
\end{aligned}
$$

and then

$$
\begin{aligned}
\sum_{\text {cyclic }} & \left(\left(\nabla_{X} \operatorname{Tor}\right)(Y, Z)+\operatorname{Tor}(\operatorname{Tor}(X, Y), Z)\right) \\
& =\sum_{\text {cyclic }}\left(\nabla_{X}(\operatorname{Tor}(Y, Z))-\operatorname{Tor}([X, Y], Z)\right)
\end{aligned}
$$




$$
\begin{aligned}
& =\sum_{\text {cyclic }}\left(\nabla_{X} \nabla_{Y} Z-\nabla_{X} \nabla_{Z} Y-\nabla_{X}[Y, Z]\right. \\
& \left.\quad-\nabla_{[X, Y]} Z+\nabla_{Z}[X, Y]+[[X, Y], Z]\right) \\
& =\sum_{\text {cyclic }}\left(\nabla_{X} \nabla_{Y} Z-\nabla_{X} \nabla_{Z} Y-\nabla_{[X, Y]} Z\right)=\sum_{\text {cyclic }} R(X, Y) Z .
\end{aligned}
$$

(3) We have

$$
\begin{aligned}
\sum_{\text {cyclic }} R(\operatorname{Tor}(X, Y), Z) & =\sum_{\text {cyclic }} R\left(\nabla_{X} Y-\nabla_{Y} X-[X, Y], Z\right) \\
& =\sum_{\text {cyclic }}\left(R\left(\nabla_{X} Y, Z\right)+R\left(Z, \nabla_{Y} X\right)-R([X, Y], Z)\right)
\end{aligned}
$$

and

$$
\begin{aligned}
\sum_{\text {cyclic }} & \left(\nabla_{X} R\right)(Y, Z) \\
& =\sum_{\text {cyclic }}\left(\nabla_{X} R(Y, Z)-R\left(\nabla_{X} Y, Z\right)-R\left(Y, \nabla_{X} Z\right)-R(Y, Z) \nabla_{X}\right)
\end{aligned}
$$

which combines to

$$
\begin{aligned}
\sum_{\text {cyclic }} & \left.\left(\nabla_{X} R\right)(Y, Z)+R(\operatorname{Tor}(X, Y), Z)\right) \\
= & \sum_{\text {cyclic }}\left(\nabla_{X} R(Y, Z)-R(Y, Z) \nabla_{X}-R([X, Y], Z)\right) \\
=\sum_{\text {cyclic }}( & \nabla_{X} \nabla_{Y} \nabla_{Z}-\nabla_{X} \nabla_{Z} \nabla_{Y}-\nabla_{X} \nabla_{[Y, Z]} \\
& -\nabla_{Y} \nabla_{Z} \nabla_{X}+\nabla_{Z} \nabla_{Y} \nabla_{X}+\nabla_{[Y, Z]} \nabla_{X} \\
& \left.-\nabla_{[X, Y]} \nabla_{Z}+\nabla_{Z} \nabla_{[X, Y]}+\nabla_{[[X, Y], Z]}\right)=0 .
\end{aligned}
$$

(51) It suffices to prove $g(R(X, Y) Z, Z)=0$ :

$$
\begin{aligned}
0= & \mathcal{L}_{0}(g(Z, Z)) \\
= & (X Y-Y X-[X, Y]) g(Z, Z) \\
= & 2 X g\left(\nabla_{Y} Z, Z\right)-2 Y g\left(\nabla_{X} Z, Z\right)-2 g\left(\nabla_{[X, Y]} Z, Z\right) \\
= & 2 g\left(\nabla_{X} \nabla_{Y} Z, Z\right)+2 g\left(\nabla_{Y} Z, \nabla_{X} Z\right) \\
& -2 g\left(\nabla_{Y} \nabla_{X} Z, Z\right)-2 g\left(\nabla_{X} Z, \nabla_{Y} Z\right)-2 g\left(\nabla_{[X, Y]} Z, Z\right) \\
= & 2 g\left(\left(\nabla_{X} \nabla_{Y}-\nabla_{Y} \nabla_{X}-\nabla_{[X, Y]}\right) Z, Z\right)=2 g(R(X, Y) Z, Z) .
\end{aligned}
$$


(44) is an algebraic consequence of (11), (2), and (5). Take (2) four times, cyclically permuted, with different signs:

$$
\begin{array}{r}
g(R(X, Y) Z, U)+g(R(Y, Z) X, U)+g(R(Z, X) Y, U)=0, \\
g(R(Y, Z) U, X)+g(R(Z, U) Y, X)+g(R(U, Y) Z, X)=0, \\
-g(R(Z, U) X, Y)-g(R(U, X) Z, Y)-g(R(X, Z) U, Y)=0, \\
-g(R(U, X) Y, Z)-g(R(X, Y) U, Z)-g(R(Y, U) X, Z)=0 .
\end{array}
$$

Add these:

$$
2 g(R(X, Y) Z, U)-2 g(R(Z, U) X, Y)=0
$$

24.5. Theorem. Let $K: T T M \rightarrow T M$ be the connector of the covariant derivative $\nabla$ on $M$. If $s: N \rightarrow T M$ is a vector field along $f:=\pi_{M} \circ s$ : $N \rightarrow M$, then we have for vector fields $X, Y \in \mathfrak{X}(N)$

$$
\begin{aligned}
\nabla_{X} \nabla_{Y} s & -\nabla_{Y} \nabla_{X} s-\nabla_{[X, Y]} s \\
& =\left(K \circ T K \circ \kappa_{T M}-K \circ T K\right) \circ T T s \circ T X \circ Y \\
& =R \circ(T f \circ X, T f \circ Y) s: N \rightarrow T M,
\end{aligned}
$$

where $R \in \Omega^{2}(M ; L(T M, T M))$ is the curvature.

Proof. Recall from (22.9) that $\nabla_{X} s=K \circ T s \circ X$. For $A, B \in T_{Z}(T M)$ we have

$$
\begin{aligned}
\operatorname{vl}_{T M}(K(A), K(B)) & =\left.\partial_{t}\right|_{0}(K(A)+t K(B))=\left.\partial_{t}\right|_{0} K(A+t B) \\
= & \left.T K \circ \partial_{t}\right|_{0}(A+t B)=T K \circ \operatorname{vl}_{\left(T T M, \pi_{T M}, T M\right)}(A, B) .
\end{aligned}
$$

We use then (22.8.9) and some obvious commutation relations:

$$
\begin{aligned}
& \nabla_{X} \nabla_{Y} s-\nabla_{Y} \nabla_{X} s-\nabla_{[X, Y]} s \\
& \quad=K \circ T(K \circ T s \circ Y) \circ X-K \circ T(K \circ T s \circ X) \circ Y-K \circ T s \circ[X, Y], \\
& \left.K \circ T s \circ[X, Y]=K \circ \operatorname{vl}_{T M} \circ(K \circ T s \circ Y, K \circ T s \circ[X, Y]) \quad \text { by }(22.8 .9)\right] \\
& \quad=K \circ T K \circ \operatorname{vl}_{T T M} \circ(T s \circ Y, T s \circ[X, Y]) \\
& \quad=K \circ T K \circ T T s \circ \operatorname{vl}_{T N} \circ(Y,[X, Y]) \\
& \quad=K \circ T K \circ T T s \circ\left(T Y \circ X-\kappa_{N} \circ T X \circ Y\right) \quad \text { by }[(8.14) \\
& \quad=K \circ T K \circ T T s \circ T Y \circ X-K \circ T K \circ T T s \circ \kappa_{N} \circ T X \circ Y .
\end{aligned}
$$

Now we sum up and use TTs $\circ \kappa_{N}=\kappa_{T M} \circ T T s$ to get the first result. If in particular we choose $f=\operatorname{Id}_{M}$ so that $X, Y, s$ are vector fields on $M$, then we get the curvature $R$.

To see that in the general case $\left(K \circ T K \circ \kappa_{E}-K \circ T K\right) \circ T T s \circ T X \circ Y$ coincides with $R(T f \circ X, T f \circ Y) s$, we have to write out the expression $(T T s \circ T X \circ Y)(x) \in T T T M$ in canonical charts induced from charts of 
$N$ and $M$. There we have $X(x)=(x, \bar{X}(x)), Y(x)=(x, \bar{Y}(x))$, and also $s(x)=(f(x), \bar{s}(x))$. So we get:

$(T T s \circ T X \circ Y)(x)=T T s(x, \bar{X}(x) ; \bar{Y}(x), d \bar{X}(x) \bar{Y}(x))$

$$
\begin{gathered}
=(f(x), \bar{s}(x), d f(x) \cdot \bar{X}(x), d \bar{s}(x) \cdot \bar{X}(x) ; d f(x) \cdot \bar{Y}(x), d \bar{s}(x) \cdot \bar{Y}(x), \\
d^{2} f(x)(\bar{Y}(x), \bar{X}(x))+d f(x) \cdot d \bar{X}(x) \cdot \bar{Y}(x), \\
\left.d^{2} \bar{s}(x)(\bar{Y}(x), \bar{X}(x))+d \bar{s}(x) \cdot d \bar{X}(x) \cdot \bar{Y}(x)\right) .
\end{gathered}
$$

Recall (22.8.7) which said $K(x, y ; a, b)=\left(x, b-\Gamma_{x}(a, y)\right)$. Differentiating this, we get

$$
\begin{aligned}
& T K(x, y, a, b ; \xi, \eta, \alpha, \beta) \\
& \quad=\left(x, b-\Gamma_{x}(a, y) ; \xi, \beta-d \Gamma(x)(\xi)(a, y)-\Gamma_{x}(\alpha, y)-\Gamma_{x}(a, \eta)\right) .
\end{aligned}
$$

Thus

$$
\begin{aligned}
&\left(K \circ T K \circ \kappa_{T M}-K \circ T K\right)(x, y, a, b ; \xi, \eta, \alpha, \beta) \\
&=(K \circ T K)(x, y, \xi, \eta ; a, b, \alpha, \beta)-(K \circ T K)(x, y, a, b ; \xi, \eta, \alpha, \beta) \\
&= K\left(x, \eta-\Gamma_{x}(\xi, y) ; a, \beta-d \Gamma(x)(a)(\xi, y)-\Gamma_{x}(\alpha, y)-\Gamma_{x}(\xi, b)\right) \\
& \quad-K\left(x, b-\Gamma_{x}(a, y) ; \xi, \beta-d \Gamma(x)(\xi)(a, y)-\Gamma_{x}(\alpha, y)-\Gamma_{x}(a, \eta)\right) \\
&=(x,-d \Gamma(x)(a)(\xi, y) \\
&\left.\quad+d \Gamma(x)(\xi)(a, y)+\Gamma_{x}\left(a, \Gamma_{x}(\xi, y)\right)-\Gamma_{x}\left(\xi, \Gamma_{x}(a, y)\right)\right) .
\end{aligned}
$$

Now we insert (1) into (2) and get

$$
\left(K \circ T K \circ \kappa_{T M}-K \circ T K\right) \circ T T s \circ T X \circ Y=R \circ(T f \circ X, T f \circ Y) s .
$$

24.6. Curvature and integrability of the horizontal bundle. What is it that the curvature is measuring? We give several answers; one of them is the following, which is intimately related to (16.13), (17.4), (19.2),

Let $C: T M \times_{M} T M \rightarrow T^{2} M$ be the linear connection corresponding to a covariant derivative $\nabla$. For $X \in \mathfrak{X}(M)$ we denoted by $C(X, \quad) \in \mathfrak{X}(T M)$ the horizontal lift of the vector field $X$.

Lemma. In this situation we have for $X, Y \in \mathfrak{X}(M)$ and $Z \in T M$

$$
[C(X, \quad), C(Y, \quad)](Z)-C([X, Y], Z)=-\operatorname{vl}(Z, R(X, Y) Z) .
$$

Proof. We compute locally, in charts induced by a chart $(U, u)$ on $M$. A global proof can be found in (17.4) for general fiber bundles and in (19.2) 
for principal fiber bundles; see also (19.16), Writing $X(x)=(x, \bar{X}(x))$, $Y(x)=(x, \bar{Y}(x))$, and $Z=(x, \bar{Z})$, we have

$$
\begin{aligned}
& C(X, Z)=\left(x, \bar{Z} ; \bar{X}(x), \Gamma_{x}(\bar{X}(x), \bar{Z})\right), \\
& C(Y, Z)=\left(x, \bar{Z} ; \bar{Y}(x), \Gamma_{x}(\bar{Y}(x), \bar{Z})\right), \\
& {[C(X, \quad), C(Y, \quad)](Z)} \\
& =\left(x, \bar{Z} ; d \bar{Y}(x) \cdot \bar{X}(x), d \Gamma(x)(\bar{X}(x))(\bar{Y}(x), \bar{Z})+\Gamma_{x}(d \bar{Y}(x) \cdot \bar{X}(x), \bar{Z})\right. \\
& \left.+\Gamma_{x}\left(\bar{Y}(x), \Gamma_{x}(\bar{X}(x), \bar{Z})\right)\right) \\
& -\left(x, \bar{Z} ; d \bar{X}(x) \cdot \bar{Y}(x), d \Gamma(x)(\bar{Y}(x))(\bar{X}(x), \bar{Z})+\Gamma_{x}(d \bar{X}(x) \cdot \bar{Y}(x), \bar{Z})\right. \\
& \left.+\Gamma_{x}\left(\bar{X}(x), \Gamma_{x}(\bar{Y}(x), \bar{Z})\right)\right) \\
& =(x, \bar{Z} ; d \bar{Y}(x) \cdot \bar{X}(x),-d \bar{X}(x) \cdot \bar{Y}(x), \\
& \Gamma_{x}(d \bar{Y}(x) \cdot \bar{X}(x)-d \bar{X}(x) \cdot \bar{Y}(x), \bar{Z}) \\
& +d \Gamma(x)(\bar{X}(x))(\bar{Y}(x), \bar{Z})-d \Gamma(x)(\bar{Y}(x))(\bar{X}(x), \bar{Z}) \\
& \left.+\Gamma_{x}\left(\bar{Y}(x), \Gamma_{x}(\bar{X}(x), \bar{Z})\right)-\Gamma_{x}\left(\bar{X}(x), \Gamma_{x}(\bar{Y}(x), \bar{Z})\right)\right) \\
& =\left(x, \bar{Z} ; \overline{[X, Y]}(x), \Gamma_{x}(\overline{[X, Y]}(x), \bar{Z})\right) \\
& +(x, \bar{Z} ; 0, d \Gamma(x)(\bar{X}(x))(\bar{Y}(x), \bar{Z})-d \Gamma(x)(\bar{Y}(x))(\bar{X}(x), \bar{Z}) \\
& \left.+\Gamma_{x}\left(\bar{Y}(x), \Gamma_{x}(\bar{X}(x), \bar{Z})\right)-\Gamma_{x}\left(\bar{X}(x), \Gamma_{x}(\bar{Y}(x), \bar{Z})\right)\right) \\
& =C([X, Y], Z)+\mathrm{vl}(Z,-R(X(x), Y(x)) Z), \quad \text { by }(24.3 .2) \text {. }
\end{aligned}
$$

The horizontal lift mapping $C(X, \quad)$ is a section of the horizontal bundle $C(T M, \quad) \subset T(T M)$, and any section is of that form. If the curvature vanishes, then by the theorem of Frobenius (3.20) the horizontal bundle is integrable and we get the leaves of the horizontal foliation.

Lemma. Let $M$ be a manifold and let $\nabla$ be a flat covariant derivative on $M$ (with vanishing curvature). Let $H \subset T M$ be a leaf of the horizontal foliation. Then $\left.\pi_{M}\right|_{H}: H \rightarrow M$ is a covering map.

Proof. Since $T\left(\left.\pi_{M}\right|_{H}\right)=T\left(\pi_{M}\right) \mid C(T M, \quad)$ is fiberwise a linear isomorphism, $\pi_{M}: H \rightarrow M$ is a local diffeomorphism. For $x \in M$ we use a chart $\left(U, u: U \rightarrow u(U)=\mathbb{R}^{m}\right)$ of $M$ centered at $x$ and let $X \in\left(\left.\pi_{M}\right|_{H}\right)^{-1}(x)$. Consider

$$
s: U \rightarrow H, \quad s\left(u^{-1}(z)\right)=\operatorname{Pt}\left(u^{-1}(t \mapsto t . z), 1\right) . X .
$$

Then $\pi_{M} \circ s=\operatorname{Id}_{U}$ and $s(U) \subset H$ is diffeomorphic to $U$, the branch of $H$ through $X$ over $U$. Since $X \in\left(\left.\pi_{M}\right|_{H}\right)^{-1}(x)$ was arbitrary, the set $\left(\left.\pi_{M}\right|_{H}\right)^{-1}(U)$ is the disjoint union of open subsets which are all diffeomorphic via $\pi_{M}$ to $U$. Thus $\pi_{M}: H \rightarrow M$ is a covering map. 
24.7. Theorem. Let $(M, g)$ be a pseudo-Riemann manifold with vanishing curvature. Then $M$ is locally isometric to $\mathbb{R}^{m}$ with the standard inner product of the same signature: For each $x \in M$ there exists a chart $(U, u)$ centered at $x$ such that $g \mid U=u^{*}\langle\quad, \quad\rangle$.

Proof. Choose an orthonormal basis $X_{1}(x), \ldots, X_{m}(x)$ of $\left(T_{x} M, g_{x}\right)$; this means $g_{x}\left(X_{i}(x), X_{j}(x)\right)=\eta_{i i} \delta_{i j}$, where $\eta=\operatorname{diag}(1, \ldots, 1,-1, \ldots,-1)$ is the standard inner product of signature $(p, q)$. Since the curvature $R$ vanishes, we may consider the horizontal foliation of (24.6), Let $H_{i}$ denote the horizontal leaf through $X_{i}(x)$ and define $X_{i}: U \rightarrow T M$ by $X_{i}=$ $\left(\left.\pi_{M}\right|_{H_{i}}\right)^{-1}: U \rightarrow H_{i} \subset T M$, where $U$ is a suitable (simply connected) neighborhood of $x$ in $M$. Since $X_{i} \circ c$ is horizontal in $T M$ for any curve $c$ in $U$, we have $\nabla_{X} X_{i}=0$ for any $X \in \mathfrak{X}(M)$ for the Levi-Civita covariant derivative of $g$. Vector fields $X_{i}$ with this property are called Killing fields. Moreover $X\left(g\left(X_{i}, X_{j}\right)\right)=g\left(\nabla_{X} X_{i}, X_{j}\right)+g\left(X_{i}, \nabla_{X} X_{j}\right)=0$; thus $g\left(X_{i}, X_{j}\right)=$ constant $=g\left(X_{i}(x), X_{j}(x)\right)=\eta_{i i} \delta_{i j}$ and $X_{i}, \ldots, X_{j}$ is an orthonormal frame on $U$. Since $\nabla$ has no torsion, we have

$$
0=\operatorname{Tor}\left(X_{i}, X_{j}\right)=\nabla_{X_{i}} X_{j}-\nabla_{X_{j}} X_{i}-\left[X_{i}, X_{j}\right]=\left[X_{i}, X_{j}\right] .
$$

By theorem (3.17) there exists a chart $(U, u)$ on $M$ centered at $x$ such that $X_{i}=\frac{\partial}{\partial u^{i}}$, i.e., Tu. $X_{i}(x)=\left(u(x), e_{i}\right)$ for the standard basis $e_{i}$ of $\mathbb{R}^{m}$. Thus $T u$ maps an orthonormal frame on $U$ to an orthonormal frame on $u(U) \in \mathbb{R}^{m}$, and $u$ is an isometry.

24.8. Sectional curvature. Let $(M, g)$ be a Riemann manifold, let $P_{x} \subset$ $T_{x} M$ be a 2-dimensional linear subspace of $T_{x} M$, and let $X_{x}, Y_{x}$ be an orthonormal basis of $P_{x}$. Then the number

$$
k\left(P_{x}\right):=-g\left(R\left(X_{x}, Y_{x}\right) X_{x}, Y_{x}\right)
$$

is called the sectional curvature of this subspace. That $k\left(P_{x}\right)$ does not depend on the choice of the orthonormal basis is shown by the following lemma.

For pseudo-Riemann manifolds one can define the sectional curvature only for those subspaces $P_{x}$ on which $g_{x}$ is nondegenerate. This notion is rarely used in general relativity.

\section{Lemma.}

(2) Let $A=\left(A_{j}^{i}\right)$ be a real $(2 \times 2)$-matrix and let $X_{1}, X_{2} \in T_{x} M$. Then for $X_{i}^{\prime}=A_{i}^{1} X_{1}+A_{i}^{2} X_{2}$ we have

$$
g\left(R\left(X_{1}^{\prime}, X_{2}^{\prime}\right) X_{1}^{\prime}, X_{2}^{\prime}\right)=\operatorname{det}(A)^{2} g\left(R\left(X_{1}, X_{2}\right) X_{1}, X_{2}\right) .
$$


(3) Let $X^{\prime}, Y^{\prime}$ be linearly independent in $P_{x} \subset T_{x} M$; then

$$
k\left(P_{x}\right)=-\frac{g\left(R\left(X^{\prime}, Y^{\prime}\right) X^{\prime}, Y^{\prime}\right)}{\left|X^{\prime}\right|^{2}\left|Y^{\prime}\right|^{2}-g\left(X^{\prime}, Y^{\prime}\right)^{2}} .
$$

Proof. (2) Since $g\left(R\left(X_{i}, X_{j}\right) X_{k}, X_{l}\right)=0$ for $i=j$ or $k=l$, we have

$$
\begin{aligned}
& g\left(R\left(X_{1}^{\prime}, X_{2}^{\prime}\right) X_{1}^{\prime}, X_{2}^{\prime}\right)=\sum A_{1}^{i} A_{2}^{j} A_{1}^{k} A_{2}^{l} g\left(R\left(X_{i}, X_{j}\right) X_{k}, X_{l}\right) \\
& =g\left(R\left(X_{1}, X_{2}\right) X_{1}, X_{2}\right) \\
& \quad \cdot\left(A_{1}^{1} A_{2}^{2} A_{1}^{1} A_{2}^{2}-A_{1}^{1} A_{2}^{2} A_{1}^{2} A_{2}^{1}-A_{1}^{2} A_{2}^{1} A_{1}^{1} A_{2}^{2}+A_{1}^{2} A_{2}^{1} A_{1}^{2} A_{2}^{1}\right) \\
& =g\left(R\left(X_{1}, X_{2}\right) X_{1}, X_{2}\right)\left(A_{1}^{1} A_{2}^{2}-A_{2}^{1} A_{1}^{2}\right)^{2} . \quad \square
\end{aligned}
$$

(3) Let $X, Y$ be an orthonormal basis of $P_{x}$, let $X^{\prime}=A_{1}^{1} X+A_{1}^{2} Y$ and let $Y^{\prime}=A_{2}^{1} X+A_{2}^{2} Y$. Then $\operatorname{det}(A)^{2}$ equals the area ${ }^{2}$ of the parallelogram spanned by $X^{\prime}$ and $Y^{\prime}$ which is $\left|X^{\prime}\right|^{2}\left|Y^{\prime}\right|^{2}-g\left(X^{\prime}, Y^{\prime}\right)^{2}$. Now use (2).

24.9. Computing the sectional curvature. Let $g: U \rightarrow S^{2}\left(\mathbb{R}^{m}\right)$ be a pseudo-Riemann metric in an open subset of $\mathbb{R}^{m}$. Then for $X, Y \in T_{x} \mathbb{R}^{m}$ we have:

$$
\begin{aligned}
2 R_{x} & (X, Y, X, Y)=2 g_{x}\left(R_{x}(X, Y) X, Y\right) \\
= & -2 d^{2} g(x)(X, Y)(Y, X)+d^{2} g(x)(X, X)(Y, Y)+d^{2} g(x)(Y, Y)(X, X) \\
& -2 g(\Gamma(Y, X), \Gamma(X, Y))+2 g(\Gamma(X, X), \Gamma(Y, Y)) .
\end{aligned}
$$

Proof. The Christoffels $\Gamma: U \times \mathbb{R}^{m} \times \mathbb{R}^{m} \rightarrow \mathbb{R}^{m}$ are given by (22.4.1)

(1) $2 g_{x}\left(\Gamma_{x}(Y, Z), U\right)=d g(x)(U)(Y, Z)-d g(x)(Y)(Z, U)-d g(x)(Z)(U, Y)$, and the curvature is given in terms of the Christoffels is (24.3.2),

$$
\begin{aligned}
& R(X, Y) Z=\left(\nabla_{X} \nabla_{Y}-\nabla_{Y} \nabla_{X}-\nabla_{[X, Y]}\right) Z \\
& (2) \quad=-d \Gamma(X)(Y, Z)+d \Gamma(Y)(X, Z)+\Gamma(X, \Gamma(Y, Z))-\Gamma(Y, \Gamma(X, Z)) .
\end{aligned}
$$

We differentiate (1) once more:

$$
\begin{aligned}
& 2 d g(x)(X)\left(\Gamma_{x}(Y, Z), U\right)+2 g_{x}(d \Gamma(x)(X)(Y, Z), U) \\
& (3) \quad=+d^{2} g(x)(X, U)(Y, Z)-d^{2} g(x)(X, Y)(Z, U)-d^{2} g(x)(X, Z)(U, Y) .
\end{aligned}
$$

Let us compute the combination from (2), using (3):

$$
\begin{aligned}
- & 2 g_{x}(d \Gamma(x)(X)(Y, Z), U)+2 g_{x}(d \Gamma(x)(Y)(X, Z), U) \\
= & 2 d g(x)(X)\left(\Gamma_{x}(Y, Z), U\right)-2 d g(x)(Y)\left(\Gamma_{x}(X, Z), U\right) \\
& -d^{2} g(x)(X, U)(Y, Z)+d^{2} g(x)(X, Y)(Z, U)+d^{2} g(x)(X, Z)(U, Y) \\
& +d^{2} g(x)(Y, U)(X, Z)-d^{2} g(x)(Y, X)(Z, U)-d^{2} g(x)(Y, Z)(U, X) \\
= & 2 d g(x)(X)\left(\Gamma_{x}(Y, Z), U\right)-2 d g(x)(Y)\left(\Gamma_{x}(X, Z), U\right)
\end{aligned}
$$




$$
\begin{aligned}
& -d^{2} g(x)(X, U)(Y, Z)+d^{2} g(x)(X, Z)(U, Y) \\
& +d^{2} g(x)(Y, U)(X, Z)-d^{2} g(x)(Y, Z)(U, X) .
\end{aligned}
$$

Thus we have

$$
\begin{aligned}
2 R_{x}(X, Y, Z, U):=2 g_{x}\left(R_{x}(X, Y) Z, U\right) \\
=2 g(-d \Gamma(X)(Y, Z)+d \Gamma(Y)(X, Z)+\Gamma(X, \Gamma(Y, Z))-\Gamma(Y, \Gamma(X, Z)), U) \\
=2 d g(x)(X)\left(\Gamma_{x}(Y, Z), U\right)-2 d g(x)(Y)\left(\Gamma_{x}(X, Z), U\right) \\
\quad-d^{2} g(x)(X, U)(Y, Z)+d^{2} g(x)(X, Z)(U, Y) \\
\quad+d^{2} g(x)(Y, U)(X, Z)-d^{2} g(x)(Y, Z)(U, X) \\
\quad+2 g(\Gamma(X, \Gamma(Y, Z)), U)-2 g(\Gamma(Y, \Gamma(X, Z)), U)
\end{aligned}
$$

and for the sectional curvature we get

(4) $2 R_{x}(X, Y, X, Y)=2 g_{x}\left(R_{x}(X, Y) X, Y\right)$

$$
\begin{aligned}
= & 2 d g(x)(X)\left(\Gamma_{x}(Y, X), Y\right)-2 d g(x)(Y)\left(\Gamma_{x}(X, X), Y\right) \\
& -2 d^{2} g(x)(X, Y)(Y, X)+d^{2} g(x)(X, X)(Y, Y)+d^{2} g(x)(Y, Y)(X, X) \\
& +2 g(\Gamma(X, \Gamma(Y, X)), Y)-2 g(\Gamma(Y, \Gamma(X, X)), Y) .
\end{aligned}
$$

Let us check how skew-symmetric the Christoffels are. From (11) we get

$$
\begin{aligned}
& 2 g_{x}\left(\Gamma_{x}(Y, Z), U\right)+2 g_{x}\left(Z, \Gamma_{x}(Y, U)\right)=2 g_{x}\left(\Gamma_{x}(Y, Z), U\right)+2 g_{x}\left(\Gamma_{x}(Y, U), Z\right) \\
& =+d g(x)(U)(Y, Z)-d g(x)(Y)(Z, U)-d g(x)(Z)(U, Y) \\
& \quad+d g(x)(Z)(Y, U)-d g(x)(Y)(U, Z)-d g(x)(U)(Z, Y) \\
& =-2 d g(x)(Y)(Z, U) .
\end{aligned}
$$

Thus

$$
2 d g(x)(Y)(\Gamma(X, V), U)=-2 g(\Gamma(Y, \Gamma(X, V)), U)-2 g(\Gamma(X, V), \Gamma(Y, U)) .
$$

Using this in (4), we get finally

(5) $2 R_{x}(X, Y, X, Y)=2 g_{x}\left(R_{x}(X, Y) X, Y\right)$

$$
\begin{aligned}
= & -2 g(\Gamma(X, \Gamma(Y, X)), Y)-2 g(\Gamma(Y, X), \Gamma(X, Y)) \\
& +2 g(\Gamma(Y, \Gamma(X, X)), Y)+2 g(\Gamma(X, X), \Gamma(Y, Y)) \\
& -2 d^{2} g(x)(X, Y)(Y, X)+d^{2} g(x)(X, X)(Y, Y)+d^{2} g(x)(Y, Y)(X, X) \\
& +2 g(\Gamma(X, \Gamma(Y, X)), Y)-2 g(\Gamma(Y, \Gamma(X, X)), Y) \\
= & -2 d^{2} g(x)(X, Y)(Y, X)+d^{2} g(x)(X, X)(Y, Y)+d^{2} g(x)(Y, Y)(X, X) \\
& -2 g(\Gamma(Y, X), \Gamma(X, Y))+2 g(\Gamma(X, X), \Gamma(Y, Y)) .
\end{aligned}
$$




\section{Computing with Adapted Frames and Examples}

25.1. Frames. We recall that a local frame or frame field $s$ on an open subset $U$ of a pseudo-Riemann manifold $(M, g)$ of dimension $m$ is an $m$ tuple $s_{1}, \ldots, s_{m}$ of vector fields on $U$ such that $s_{1}(x), \ldots, s_{m}(x)$ is a basis of the tangent space $T_{x} M$ for each $x \in U$. Note that then $s$ is a local section of the linear frame bundle $G L\left(\mathbb{R}^{m}, T M\right) \rightarrow M$, a principal fiber bundle, as we treat it in (18.11). We view $s(x)=\left(s_{1}(x), \ldots, s_{m}(x)\right)$ as a linear isomorphism $s(x): \mathbb{R}^{m} \rightarrow T_{x} M$. The frame field $s$ is called an orthonormal frame if $s_{1}(x), \ldots, s_{m}(x)$ is an orthonormal basis of $\left(T_{x} M, g_{x}\right)$ for each $x \in U$. By this we mean that $g_{x}\left(X_{i}(x), X_{j}(x)\right)=\eta_{i i} \delta_{i j}$, where $\eta=\operatorname{diag}(1, \ldots, 1,-1, \ldots,-1)$ is the standard inner product of signature $(p, q=m-p)$.

If $(U, u)$ is a chart on $M$, then $\frac{\partial}{\partial u^{1}}, \ldots, \frac{\partial}{\partial u^{m}}$ is a frame field on $U$. Out of this we can easily build one which contains no isotropic vectors (i.e., ones with $g(X, X)=0)$ and order them in such a way that the fields with $g(X, X)>0$ are at the beginning. Using the Gram-Schmidt orthonormalization procedure, we can change this frame field then into an orthonormal one on a possibly smaller open set $U$. Thus there always exist orthonormal frame fields.

If $s=\left(s_{1}, \ldots, s_{m}\right)$ and $s^{\prime}=\left(s_{1}^{\prime}, \ldots, s_{m}^{\prime}\right)$ are two frame fields on $U, V \subset M$, respectively, then on $U \cap V$ we have

$$
\begin{gathered}
s^{\prime}=s . h, \quad s_{i}^{\prime}=\sum_{j} s_{j} h_{i}^{j}, \quad s_{i}^{\prime}(x)=\sum_{j} s_{j}(x) h_{i}^{j}(x), \\
h=\left(h_{j}^{i}\right): U \cap V \rightarrow G L(m, \mathbb{R}) .
\end{gathered}
$$

25.2. Connection forms. If $s$ is a local frame on an open subset $U$ in a manifold $M$ and if $\nabla$ is a covariant derivative on $M$, we put

$$
\begin{gathered}
\nabla_{X} s_{i}=\sum_{j} s_{j} \cdot \omega_{i}^{j}(X), \quad \nabla_{X} s=s \cdot \omega(X), \quad \nabla s=s . \omega, \\
\omega=\left(\omega_{i}^{j}\right) \in \Omega^{1}(U, \mathfrak{g l}(m)), \quad \text { the connection form of } \nabla .
\end{gathered}
$$

We saw this construction in (19.4) already.

Proposition. We have:

(2) If $Y=\sum s_{j} u^{j} \in \mathfrak{X}(U)$, then

$$
\nabla Y=\sum_{k} s_{k}\left(\sum_{j} \omega_{j}^{k} u^{j}+d u^{k}\right)=s . \omega . u+s . d u .
$$

(3) Let $s$ and $s^{\prime}=s . h$ be two local frames on $U$; then the connection forms $\omega, \omega^{\prime} \in \Omega^{1}(U, \mathfrak{g l}(m))$ are related by

$$
h . \omega^{\prime}=d h+\omega . h .
$$


(4) If $s$ is a local orthonormal frame for a Riemann metric $g$ which is respected by $\nabla$, then

$$
\omega_{i}^{j}=-\omega_{j}^{i}, \quad \omega=\left(\omega_{i}^{j}\right) \in \Omega^{1}(U, \mathfrak{s o}(m)) .
$$

If $s$ is a local orthonormal frame for a pseudo-Riemann metric $g$ which is respected by $\nabla$ and if $\eta_{i j}=g\left(s_{i}, s_{j}\right)=\operatorname{diag}(1, \ldots, 1,-1, \ldots,-1)$ is the standard inner product matrix of the same signature $(p, q)$, then

$$
\eta_{j j} \omega_{i}^{j}=-\eta_{i i} \omega_{j}^{i}, \quad \omega=\left(\omega_{i}^{j}\right) \in \Omega^{1}(U, \mathfrak{s o}(p, q)) .
$$

Proof. We use direct computations.

(21)

(3)

$$
\begin{aligned}
\nabla_{X} Y & =\nabla_{X}\left(\sum_{j} s_{j} u^{j}\right)=\sum_{j}\left(\nabla_{X} s_{j}\right) u^{j}+\sum_{j} s_{j} X\left(u^{j}\right) \\
& =\sum_{k} s_{k} \sum_{j} \omega_{j}^{k}(X) u^{j}+\sum_{k} s_{k} d u^{k}(X) . \\
\nabla s^{\prime} & =s^{\prime} \cdot \omega^{\prime}=s . h \cdot \omega^{\prime}, \\
\nabla s^{\prime} & =\nabla(s . h)=(\nabla s) . h+s . d h=s . \omega . h+s . d h .
\end{aligned}
$$

(41) It suffices to prove the second assertion. We differentiate the constant $\eta_{i j}=g\left(s_{i}, s_{j}\right)$ :

$$
\begin{aligned}
0 & =X\left(g\left(s_{i}, s_{j}\right)\right)=g\left(\nabla_{X} s_{i}, s_{j}\right)+g\left(s_{i}, \nabla_{X} s_{j}\right) \\
& =g\left(\sum s_{k} \omega_{i}^{k}(X), s_{j}\right)+g\left(s_{i}, \sum s_{k} \omega_{j}^{k}(X)\right) \\
& =\sum g\left(s_{k}, s_{j}\right) \omega_{i}^{k}(X)+\sum g\left(s_{i}, s_{k}\right) \omega_{j}^{k}(X)=\eta_{j j} \omega_{i}^{j}(X)+\eta_{i i} \omega_{j}^{i}(X) .
\end{aligned}
$$

25.3. Curvature forms. Let $s$ be a local frame on $U$, and let $\nabla$ be a covariant derivative with curvature $R$. We write

$$
R(X, Y) s=\left(R(X, Y) s_{1}, \ldots, R(X, Y) s_{m}\right) .
$$

Then we have

$$
R s_{j}=\sum s_{k} \cdot\left(d \omega_{j}^{k}+\sum \omega_{l}^{k} \wedge \omega_{j}^{l}\right), \quad R s=s .(d \omega+\omega \wedge \omega),
$$

where $\omega \wedge \omega=\left(\sum \omega_{k}^{i} \wedge \omega_{j}^{k}\right)_{j}^{i} \in \Omega^{2}(U, \mathfrak{g l}(m))$, since

$$
\begin{aligned}
R & X, Y) s=\nabla_{X} \nabla_{Y} s-\nabla_{Y} \nabla_{X} s-\nabla_{[X, Y]} s \\
& =\nabla_{X}(s \cdot \omega(Y))-\nabla_{Y}(s \cdot \omega(X))-s \cdot \omega([X, Y]) \\
& =s \cdot X(\omega(Y))+s \cdot \omega(X) \cdot \omega(Y)-s \cdot Y(\omega(X))-s \cdot \omega(Y) \cdot \omega(X)-s \cdot \omega([X, Y]) \\
& =s \cdot(X(\omega(Y))-Y(\omega(X))-\omega([X, Y])+\omega(X) \cdot \omega(Y)-\omega(Y) \cdot \omega(X)) \\
& =s \cdot(d \omega+\omega \wedge \omega)(X, Y) .
\end{aligned}
$$

We thus get the curvature matrix

$$
\Omega=d \omega+\omega \wedge \omega \in \Omega^{2}(U, \mathfrak{g l}(m)),
$$

and we note its defining equation R.s $=s . \Omega$. 


\section{Proposition.}

(3) If $s$ and $s^{\prime}=s . h$ are two local frames, then the curvature matrices are related by

$$
h . \Omega^{\prime}=\Omega . h .
$$

(4) The second Bianchi identity becomes

$$
d \Omega+\omega \wedge \Omega-\Omega \wedge \omega=0 .
$$

(5) If $s$ is a local orthonormal frame for a Riemann metric $g$ which is respected by $\nabla$, then

$$
\Omega_{i}^{j}=-\Omega_{j}^{i}, \quad \Omega=\left(\Omega_{i}^{j}\right) \in \Omega^{2}(U, \mathfrak{s o}(m)) .
$$

If $s$ is a local orthonormal frame for a pseudo-Riemann metric $g$ which is respected by $\nabla$ and if $\eta_{i j}=g\left(s_{i}, s_{j}\right)=\operatorname{diag}(1, \ldots, 1,-1, \ldots,-1)$ is the standard inner product matrix of the same signature $(p, q)$, then

$$
\eta_{j j} \Omega_{i}^{j}=-\eta_{i i} \Omega_{j}^{i}, \quad \Omega=\left(\Omega_{i}^{j}\right) \in \Omega^{2}(U, \mathfrak{s o}(p, q)) .
$$

Proof. (3) Since $R$ is a tensor field, we have $s \cdot h \cdot \Omega^{\prime}=s^{\prime} \cdot \Omega^{\prime}=R s^{\prime}=R s . h=$ s. S.h.

A second, direct proof goes as follows. By (25.2.3) we have $h \cdot \omega^{\prime}=\omega \cdot h+d h$; thus

$$
\begin{aligned}
h . \Omega^{\prime}= & h \cdot\left(d \omega^{\prime}+\omega^{\prime} \wedge \omega^{\prime}\right) \\
= & h \cdot d\left(h^{-1} \cdot \omega \cdot h+h^{-1} \cdot d h\right)+(\omega \cdot h+d h) \wedge\left(h^{-1} \cdot \omega \cdot h+h^{-1} \cdot d h\right) \\
= & h \cdot\left(-h^{-1} \cdot d h \cdot h\right) \wedge \omega \cdot h+h \cdot h^{-1} \cdot d \omega \cdot h-h \cdot h^{-1} \cdot \omega \wedge d h \\
& +h \cdot\left(-h^{-1} \cdot d h \cdot h^{-1}\right) \wedge d h+h \cdot h^{-1} \cdot d d h \\
& +\omega \wedge h \cdot h^{-1} \cdot \omega+\omega \wedge h \cdot h^{-1} \cdot d h+d h \cdot h^{-1} \wedge \omega \cdot h+d h \cdot h^{-1} \wedge d h \\
= & d \omega \cdot h+\omega \wedge \omega \cdot h=\Omega \cdot h .
\end{aligned}
$$

(44) $d \Omega=d(d \omega+\omega \wedge \omega)=0+d \omega \wedge \omega-\omega \wedge d \omega=(d \omega+\omega \wedge \omega) \wedge \omega-\omega \wedge(d \omega+\omega \wedge \omega)$.

(51) We prove only the second case.

$$
\begin{aligned}
\eta_{j j} \Omega_{i}^{j} & =\eta_{j j} d \omega_{i}^{j}+\sum_{k} \eta_{j j} \omega_{k}^{j} \wedge \omega_{i}^{k}=-\eta_{i i} d \omega_{j}^{i}-\sum_{k} \eta_{k k} \omega_{j}^{k} \wedge \omega_{i}^{k} \\
& =-\eta_{i i} d \omega_{j}^{i}+\sum_{k} \eta_{i i} \omega_{j}^{k} \wedge \omega_{k}^{i}=-\eta_{i i}\left(d \omega_{j}^{i}+\sum_{k} \omega_{k}^{i} \wedge \omega_{j}^{k}\right)=-\eta_{i i} \Omega_{j}^{i} .
\end{aligned}
$$

25.4. Coframes. For a local frame $s=\left(s_{1}, \ldots, s_{m}\right)$ on $U \subset M$ we consider the dual coframe

$$
\sigma=\left(\begin{array}{c}
\sigma^{1} \\
\vdots \\
\sigma^{m}
\end{array}\right), \quad \sigma^{i} \in \Omega^{1}(U)
$$


which forms the dual basis of $T_{x}^{*} M$ for each $x \in U$; it satisfies $\left\langle\sigma^{i}, s_{j}\right\rangle=$ $\sigma^{i}\left(s_{j}\right)=\delta_{j}^{i}$. If $s^{\prime}=s . h$ is another local frame, then its dual coframe is given by

$$
\sigma^{\prime}=h^{-1} . \sigma, \quad \sigma^{i}=\sum_{k}\left(h^{-1}\right)_{k}^{i} \sigma^{k}
$$

since

$$
\left\langle\sum_{k}\left(h^{-1}\right)_{k}^{i} \sigma^{k}, s_{j}^{\prime}\right\rangle=\sum_{k, l}\left(h^{-1}\right)_{k}^{i}\left\langle\sigma^{k}, s_{l}\right\rangle h_{j}^{l}=\delta_{j}^{i} .
$$

Let $s$ be a local frame on $U$, and let $\nabla$ be a covariant derivative. We define the torsion form $\Theta$ by

$$
\text { Tor }=s . \Theta, \quad \operatorname{Tor}(X, Y)=: \sum_{j} s_{j} \Theta^{j}(X, Y), \quad \Theta \in \Omega^{2}\left(U, \mathbb{R}^{m}\right) .
$$

\section{Proposition.}

(3) If $s$ and $s^{\prime}=s . h$ are two local frames, then the torsion forms of a covariant derivative are related by

$$
\Theta^{\prime}=h^{-1} . \Theta .
$$

(4) If $s$ is a local frame with dual coframe $\sigma$, then for a covariant derivative with connection form $\omega \in \Omega^{1}(U, \mathfrak{g l}(m))$ and torsion form $\Theta \in$ $\Omega^{2}\left(U, \mathbb{R}^{m}\right)$ we have

$$
d \sigma=-\omega \wedge \sigma+\Theta, \quad d \sigma^{i}=-\sum_{k} \omega_{k}^{i} \wedge \sigma^{k}+\Theta^{i} .
$$

(5) The algebraic Bianchi identity for a covariant derivative takes the following form:

$$
d \Theta+\omega \wedge \Theta=\Omega \wedge \sigma, \quad d \Theta^{k}+\sum_{l} \omega_{l}^{k} \wedge \Theta^{l}=\sum_{l} \Omega_{l}^{k} \wedge \sigma^{l} .
$$

Proof. (31) Since Tor is a tensor field, we have $s . \Theta=$ Tor $=s^{\prime} \Theta^{\prime}=s . h . \Theta^{\prime}$; thus $h . \Theta^{\prime}=\Theta$ and $\Theta^{\prime}=h^{-1} . \Theta$.

(44) For $X \in \mathfrak{X}(U)$ we have $X=\sum_{i} s_{i} \cdot \sigma^{i}(X)$, for short $X=s . \sigma(X)$. Then

$$
\begin{aligned}
& \nabla_{X} Y=\nabla_{X}(s \cdot \sigma(Y))=\left(\nabla_{X} s\right) \cdot \sigma(Y)+s \cdot X(\sigma(Y)) \\
& \quad=s \cdot \omega(X) \cdot \sigma(Y)+s \cdot X(\sigma(Y)), \\
& \begin{aligned}
s . \Theta & (X, Y)=\operatorname{Tor}(X, Y)=\nabla_{X} Y-\nabla_{Y} X-[X, Y] \\
\quad= & s \cdot \omega(X) \cdot \sigma(Y)+s \cdot X(\sigma(Y))-s \cdot \omega(Y) \cdot \sigma(X)-s \cdot Y(\sigma(X))-s \cdot \sigma([X, Y]) \\
\quad & s \cdot(\omega(X) \cdot \sigma(Y)-\omega(Y) \cdot \sigma(X)+X(\sigma(Y))-Y(\sigma(X))-\sigma([X, Y])) \\
\quad & =s \cdot(\omega \wedge \sigma(X)+d \sigma)(X, Y) .
\end{aligned}
\end{aligned}
$$

Direct proof of (3):

$$
\begin{aligned}
\Theta^{\prime} & =\omega^{\prime} \wedge \sigma^{\prime}+d \sigma^{\prime}=\left(h^{-1} \cdot \omega \cdot h+h^{-1} . d h\right) \wedge h^{-1} \cdot \sigma+d\left(h^{-1} \cdot \sigma\right) \\
& =h^{-1} \cdot \omega \wedge \sigma+h^{-1} . d h \wedge h^{-1} \cdot \sigma-h^{-1} \cdot d h \cdot h^{-1} \cdot \sigma+h^{-1} \cdot d \sigma \\
& =h^{-1}(\omega \wedge \sigma+d \sigma)=h^{-1} \cdot \Theta .
\end{aligned}
$$


(51)

$$
\begin{aligned}
d \Theta & =d(\omega \wedge \sigma+d \sigma)=d \omega \wedge \sigma-\omega \wedge d \sigma+0 \\
& =(d \omega+\omega \wedge \omega) \wedge \sigma-\omega \wedge(\omega \wedge \sigma+d \sigma)=\Omega \wedge \sigma-\omega \wedge \Theta .
\end{aligned}
$$

25.5. Resumé of computing with adapted frames. Let $(M, g)$ be a Riemann manifold, let $s$ be an orthonormal local frame on $U$ with dual coframe $\sigma$, and let $\nabla$ be the Levi-Civita covariant derivative. Then we have:

(1) $\left.g\right|_{U}=\sum_{i} \sigma^{i} \otimes \sigma^{i}$.

(2) $\nabla s=s . \omega, \omega_{j}^{i}=-\omega_{i}^{j}$, so $\omega \in \Omega^{1}(U, \mathfrak{s o}(m))$.

(3) $d \sigma+\omega \wedge \sigma=0, d \sigma^{i}+\sum_{k} \omega_{k}^{i} \wedge \sigma^{k}=0$.

(4) $R s=s . \Omega, \Omega=d \omega+\omega \wedge \omega \in \Omega^{2}(U, \mathfrak{s o}(m)), \Omega_{j}^{i}=d \omega_{j}^{i}+\sum_{k} \omega_{k}^{i} \wedge \omega_{j}^{k}$,

(5) $\Omega \wedge \sigma=0, \sum_{k} \Omega_{k}^{i} \wedge \sigma^{k}=0$, the first Bianchi identity.

(6) $d \Omega+\omega \wedge \Omega-\Omega \wedge \omega=d \Omega+[\omega, \Omega]_{\wedge}=0$, the second Bianchi identity.

For a pseudo-Riemann manifold $(M, g)$ we consider standard inner product matrix $\eta_{i j}=g\left(s_{i}, s_{j}\right)=\operatorname{diag}(1, \ldots, 1,-1, \ldots,-1)$ of the same signature $(p, q)$. Then we have instead:

(11) $g=\sum_{i} \eta_{i i} \sigma^{i} \otimes \sigma^{i}$.

(2) $\eta_{j j} \omega_{i}^{j}=-\eta_{i i} \omega_{j}^{i}$; thus $\omega=\left(\omega_{i}^{j}\right) \in \Omega^{1}(U, \mathfrak{s o}(p, q))$.

(4) $\eta_{j j} \Omega_{i}^{j}=-\eta_{i i} \Omega_{j}^{i}$; thus $\Omega=\left(\Omega_{i}^{j}\right) \in \Omega^{2}(U, \mathfrak{s o}(p, q))$.

25.6. Interpretation in terms of the orthonormal frame bundle. For a pseudo-Riemann manifold $(M, g)$ of dimension $m$ we consider the orthonormal frame bundle

$$
\mathcal{O}(M)=O\left(\mathbb{R}^{m}, T M\right) \stackrel{\pi_{M}}{\longrightarrow} M .
$$

Its fiber $\mathcal{O}(M)_{x}$ consists of all linear isometries $\left(\mathbb{R}^{m}, \eta\right) \rightarrow\left(T_{x} M, g\right)$ where $\eta$ is the standard inner product with the same signature as $g$. It is a principal bundle with structure group $O(p, q)$ (acting by composition from the right), and it has one further structure, the soldering form which encodes the fact that the associated bundle $\mathcal{O}(M) \times_{O(p, q)} \mathbb{R}^{m}$ is the tangent bundle. The soldering form is described as follows: Let $s=\left(s_{1}, \ldots, s_{m}\right) \in \mathcal{O}(M)_{x}$ be an orthonormal frame of $T_{x} M$ with orthonormal coframe (dual basis)

$$
\sigma_{s}=\left(\begin{array}{c}
\sigma_{s}^{1} \\
\vdots \\
\sigma_{s}^{m}
\end{array}\right), \quad \sigma_{s}^{i} \in T_{x}^{*} M .
$$

The soldering form is then given as (with a slight abuse of notation):

$$
\sigma \in \Omega^{1}\left(\mathcal{O}(M), \mathbb{R}^{m}\right),
$$




$$
\sigma_{s}\left(\Xi_{s}\right)=\sigma_{s}\left(T\left(\pi_{m}\right) \cdot \Xi_{s}\right)=\left(\begin{array}{c}
\sigma_{s}^{1}\left(T_{s}\left(\pi_{M}\right) \cdot \Xi_{s}\right) \\
\vdots \\
\sigma_{s}^{m}\left(T_{s}\left(\pi_{M}\right) \cdot \Xi_{s}\right)
\end{array}\right) \in \mathbb{R}^{m} .
$$

For $h \in O(p, q)$ we have

$$
\left(\left(r^{h}\right)^{*} \sigma\right)_{s}\left(\Xi_{s}\right)=\sigma_{s . h}\left(T\left(r^{h}\right) . \Xi_{s}\right)=h^{-1} \cdot \sigma_{s}\left(T\left(\pi_{M}\right) \cdot T\left(r^{h}\right) \Xi\right)=h^{-1} \cdot \sigma_{s}(\Xi) .
$$

So $\sigma$ is $O(p, q)$-equivariant and horizontal: It kills vertical tangent vectors. By (19.14), $\sigma$ induces a differential form on $M$ with values in the associated bundle $O(M) \times_{O(p, q)} \mathbb{R}^{m}$; it is a vector bundle isomorphism $T M \rightarrow O(M) \times_{O(p, q)} \mathbb{R}^{m}$. If $s$ is a local orthonormal frame, i.e., a local section of $\mathcal{O}(M)$, then $s^{*} \sigma=\sigma_{s}$, the dual coframe.

For the description of the principal connection form $\omega$ on $\mathcal{O}(M)$ inducing the Levi-Civita connection $\nabla$ on $M$ we fix an open cover $\left(U_{\alpha}\right)_{\alpha}$ of $M$ and local orthonormal frames $s_{\alpha}: U_{\alpha} \rightarrow \mathcal{O}(M)$. Then $\sigma_{\alpha}=s_{\alpha}{ }^{*} \sigma$ are the dual coframes, and by (25.5) the connection form $\omega_{\alpha} \in \Omega^{1}\left(U_{\alpha}, \mathfrak{o}(p, q)\right)$ is determined by $d \sigma_{\alpha}+\omega_{\alpha} \wedge \sigma_{\alpha}=0$. The local frames $s_{\alpha}$ induce a principal fiber bundle atlas

$$
\left(U_{\alpha}, \varphi_{\alpha}:\left.\mathcal{O}(M)\right|_{U_{\alpha}} \rightarrow U_{\alpha} \times O(p, q)\right), \quad \varphi_{\alpha}\left(u_{x}\right)=\left(x,\left(\left.s_{\alpha}\right|_{x}\right)^{-1} \cdot u_{x}\right) .
$$

For $X \in \mathfrak{o}(p, q)$ the fundamental vector field for the principal right action is given by $\zeta_{X}\left(u_{x}\right)=\left.\partial_{t}\right|_{0} u_{x} \circ \exp (t X)=\operatorname{vl}\left(u_{x}, u_{x} . X\right)$ in terms of the vertical lift vl from (8.12).

The local expression $\gamma_{\alpha}=\left(\varphi_{\alpha}^{-1}\right)^{*} \omega \in \Omega^{1}\left(U_{\alpha} \times O(p, q), \mathfrak{o}(p, q)\right)$ of the principal connection $\omega$ is given by (19.4.6), where $\xi_{x} \in T_{x} M, h \in O(p, q)$, and $X \in$ $\mathfrak{o}(p, q)$. Thus we have:

$$
\begin{aligned}
\omega\left(T\left(\varphi_{\alpha}^{-1}\right)\left(\xi_{x}, T\left(\mu_{h}\right) \cdot X\right)\right) & =\gamma_{\alpha}\left(\xi_{x}, T\left(\mu_{h}\right) \cdot X\right) \\
& =\gamma_{\alpha}\left(\xi_{x}, 0_{h}\right)+X=\operatorname{Ad}\left(h^{-1}\right) \omega_{\alpha}\left(\xi_{x}\right)+X \\
& =h^{-1} \cdot \omega_{\alpha}\left(\xi_{x}\right) \cdot h+X .
\end{aligned}
$$

25.7. Example: The sphere $S^{2} \subset \mathbb{R}^{3}$. We consider the parameterization (leaving out one longitude):

$$
\begin{aligned}
& f:(0,2 \pi) \times(-\pi, \pi) \rightarrow \mathbb{R}^{3}, \\
& f(\varphi, \vartheta)=\left(\begin{array}{cc}
\cos \varphi & \cos \vartheta \\
\sin \varphi & \cos \vartheta \\
\sin \vartheta
\end{array}\right), \\
& g=f^{*}(\text { metric })=f^{*}\left(\sum_{i} d x^{i} \otimes d x^{i}\right) \\
& \quad=\sum_{i=1}^{3} d f^{i} \otimes d f^{i}=\cos ^{2} \vartheta d \varphi \otimes d \varphi+d \vartheta \otimes d \vartheta
\end{aligned}
$$

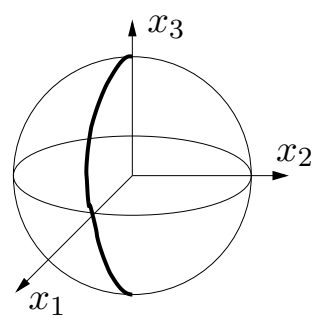


From this we can read off the orthonormal coframe and then the orthonormal frame:

$$
\sigma^{1}=d \vartheta, \quad \sigma^{2}=\cos \vartheta d \varphi, \quad s_{1}=\frac{\partial}{\partial \vartheta}, \quad s_{2}=\frac{1}{\cos \vartheta} \frac{\partial}{\partial \varphi} .
$$

We compute $d \sigma^{1}=0$ and $d \sigma^{2}=-\sin \vartheta d \vartheta \wedge d \varphi=-\tan \vartheta \sigma^{1} \wedge \sigma^{2}$. For the connection forms we have $\omega_{1}^{1}=\omega_{2}^{2}=0$ by skew-symmetry. The off-diagonal terms we compute from (25.5.3), i.e., $d \sigma+\omega \wedge \sigma=0$ :

$$
\begin{aligned}
-d \sigma^{1} & =0+\omega_{2}^{1} \wedge \sigma^{2}=0 & \Rightarrow \omega_{2}^{1}=c(\varphi, \vartheta) \sigma^{2}, \\
-d \sigma^{2} & =\omega_{1}^{2} \wedge \sigma^{1}+0=\tan \vartheta \sigma^{1} \wedge \sigma^{2} & \Rightarrow \omega_{2}^{1}=\tan \vartheta \sigma^{2}=\sin \vartheta d \varphi \\
\omega & =\left(\begin{array}{cc}
0 & \sin \vartheta d \varphi \\
-\sin \vartheta d \varphi & 0
\end{array}\right) . &
\end{aligned}
$$

For the curvature forms we have again $\Omega_{1}^{1}=\Omega_{2}^{2}=0$ by skew-symmetry, and then we may compute the curvature:

$$
\begin{aligned}
& \Omega_{2}^{1}=d \omega_{2}^{1}+\omega_{1}^{1} \wedge \omega_{2}^{1}+\omega_{2}^{1} \wedge \omega_{2}^{2}=d(\sin \vartheta d \varphi)=\cos \vartheta d \vartheta \wedge d \varphi=\sigma^{1} \wedge \sigma^{2}, \\
& \Omega=\left(\begin{array}{cc}
0 & \sigma^{1} \wedge \sigma^{2} \\
-\sigma^{1} \wedge \sigma^{2} & 0
\end{array}\right) .
\end{aligned}
$$

For the sectional curvature we get

$$
\begin{aligned}
k\left(S^{2}\right) & =-g\left(R\left(s_{1}, s_{2}\right) s_{1}, s_{2}\right)=-g\left(\sum_{k} s_{k} \Omega_{1}^{k}\left(s_{1}, s_{2}\right), s_{2}\right) \\
& =-g\left(s_{2}\left(-\sigma^{1} \wedge \sigma^{2}\right)\left(s_{1}, s_{2}\right), s_{2}\right)=1 .
\end{aligned}
$$

\subsection{Example: The Poincaré upper half-plane.}

This is the set $H_{+}^{2}=\left\{(x, y) \in \mathbb{R}^{2}: y>0\right\}$ with metric $d s^{2}=\frac{1}{y^{2}}\left(d x^{2}+d y^{2}\right)$ or

$$
g=\frac{1}{y} d x \otimes \frac{1}{y} d x+\frac{1}{y} d y \otimes \frac{1}{y} d y,
$$

which is conformal with the standard inner product.

The curvature. The orthonormal coframe and frame are then, by (25.5.1)

$$
\sigma^{1}=\frac{1}{y} d x, \quad \sigma^{2}=\frac{1}{y} d y, \quad s_{1}=y \frac{\partial}{\partial x}, \quad s_{2}=y \frac{\partial}{\partial y} .
$$

We have $d \sigma^{1}=d\left(\frac{1}{y} d x\right)=\frac{1}{y^{2}} d x \wedge d y=\sigma^{1} \wedge \sigma^{2}$ and $d \sigma^{2}=0$. The connection forms we compute from (25.5.3), i.e, $d \sigma+\omega \wedge \sigma=0$ :

$$
\begin{aligned}
-d \sigma^{1} & =0+\omega_{2}^{1} \wedge \sigma^{2}=-\sigma^{1} \wedge \sigma^{2} \\
-d \sigma^{2} & =\omega_{1}^{2} \wedge \sigma^{1}+0=0 \quad \Rightarrow \omega_{2}^{1}=-\sigma^{1}=-y^{-1} d x \\
\omega & =\left(\begin{array}{cc}
0 & -\sigma^{1} \\
\sigma^{1} & 0
\end{array}\right) .
\end{aligned}
$$


For the curvature forms we get

$$
\begin{aligned}
& \Omega_{2}^{1}=d \omega_{2}^{1}+\omega_{1}^{1} \wedge \omega_{2}^{1}+\omega_{2}^{1} \wedge \omega_{2}^{2}=d\left(-y^{-1} d x\right)=-\sigma^{1} \wedge \sigma^{2} \\
& \Omega=\left(\begin{array}{cc}
0 & -\sigma^{1} \wedge \sigma^{2} \\
+\sigma^{1} \wedge \sigma^{2} & 0
\end{array}\right) .
\end{aligned}
$$

For the sectional curvature we get

$$
\begin{aligned}
k\left(H_{+}^{2}\right) & =-g\left(R\left(s_{1}, s_{2}\right) s_{1}, s_{2}\right)=-g\left(\sum_{k} s_{k} \Omega_{1}^{k}\left(s_{1}, s_{2}\right), s_{2}\right) \\
& =-g\left(s_{2}\left(\sigma^{1} \wedge \sigma^{2}\right)\left(s_{1}, s_{2}\right), s_{2}\right)=-1 .
\end{aligned}
$$

The geodesics. For deriving the geodesic equation let:

$c(t)=\left(\begin{array}{l}x(t) \\ y(t)\end{array}\right), \quad c^{\prime}(t)=\left(\begin{array}{l}x^{\prime}(t) \\ y^{\prime}(t)\end{array}\right)=\frac{x^{\prime}}{y} y \frac{\partial}{\partial x}+\frac{y^{\prime}}{y} y \frac{\partial}{\partial y}=\frac{x^{\prime}}{y} s_{1}+\frac{y^{\prime}}{y} s_{2}=:(s \circ c) . u$.

The geodesic equation is then

$$
\begin{aligned}
\nabla_{\partial_{t}} c^{\prime} & =\nabla_{\partial_{t}}((s \circ c) \cdot u)=s \cdot \omega\left(c^{\prime}\right) \cdot u+s \cdot d u\left(\partial_{t}\right) \\
& =\left(s_{1}, s_{2}\right)\left(\begin{array}{cc}
0 & \omega_{2}^{1}\left(c^{\prime}\right) \\
-\omega_{2}^{1}\left(c^{\prime}\right) & 0
\end{array}\right)\left(\begin{array}{c}
\frac{x^{\prime}}{y} \\
\frac{y^{\prime}}{y}
\end{array}\right)+\left(s_{1}, s_{2}\right)\left(\begin{array}{l}
\left(\frac{x^{\prime}}{y}\right)^{\prime} \\
\left(\frac{y^{\prime}}{y}\right)^{\prime}
\end{array}\right) \\
& =\frac{x^{\prime 2}}{y} \frac{\partial}{\partial y}-\frac{x^{\prime} y^{\prime}}{y} \frac{\partial}{\partial x}+\frac{x^{\prime \prime} y-x^{\prime} y^{\prime}}{y} \frac{\partial}{\partial x}+\frac{y^{\prime \prime} y-y^{\prime 2}}{y} \frac{\partial}{\partial y}=0, \\
& \left\{\begin{array}{l}
x^{\prime \prime} y-2 x^{\prime} y^{\prime}=0 \\
x^{\prime 2}+y^{\prime \prime} y-y^{\prime 2}=0 .
\end{array}\right.
\end{aligned}
$$

To see the shape of the geodesics, we first investigate $x(t)=$ constant. Then $y^{\prime \prime} y-y^{\prime 2}=0$ has a unique solution for each initial value $y(0), y^{\prime}(0)$; thus the verticals $t \mapsto\left(\begin{array}{c}\text { constant } \\ y(t)\end{array}\right)$ are geodesics. If $x^{\prime}(t)=0$ for a single $t$, then it is for all $t$ since then the geodesic is already vertical. If $x^{\prime}(t) \neq 0$, we claim that the geodesics are upper half-circles with center $M(t)$ on the $x$-axis:

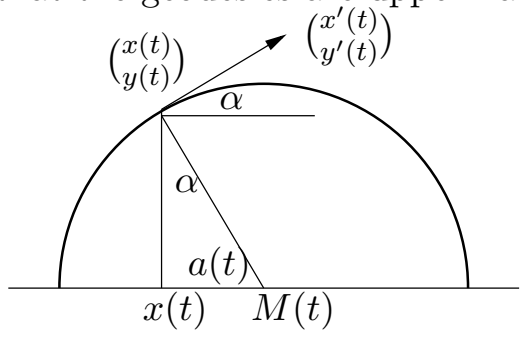

$$
\begin{aligned}
\frac{y^{\prime}(t)}{x^{\prime}(t)} & =\tan \alpha(t)=\frac{a(t)}{y(t)} \Rightarrow a=\frac{y^{\prime} y}{x^{\prime}}, \\
M(t) & =x+\frac{y^{\prime} y}{x^{\prime}}=\frac{x^{\prime} x+y^{\prime} y}{x^{\prime}}, \\
M^{\prime}(t) & =\left(\frac{x^{\prime} x+y^{\prime} y}{x^{\prime}}\right)^{\prime}=\cdots=0 .
\end{aligned}
$$

Thus $M(t)=M$, a constant. Moreover,

$$
\begin{gathered}
\left|\left(\begin{array}{c}
x(t) \\
y(t)
\end{array}\right)-\left(\begin{array}{c}
M \\
0
\end{array}\right)\right|^{2}=(x-M)^{2}+y^{2}=\left(\frac{y^{\prime} y}{x^{\prime}}\right)^{2}+y^{2}, \\
\frac{d}{d t}\left|\left(\begin{array}{c}
x(t) \\
y(t)
\end{array}\right)-\left(\begin{array}{c}
M \\
0
\end{array}\right)\right|^{2}=\left(\left(\frac{y^{\prime} y}{x^{\prime}}\right)^{2}+y^{2}\right)^{\prime}=\cdots=0 .
\end{gathered}
$$


Thus the geodesics are half-circles as asserted. Note that this violates Euclid's parallel axiom: We have a non-Euclidean geometry.

Isometries and the Poincaré upper half-plane as symmetric space. The projective action of the Lie group $S L(2, \mathbb{R})$ on $\mathbb{C} P^{1}$, viewed in the projective chart $\mathbb{C} \ni z \mapsto[z: 1]$, preserves the upper half-plane: A matrix $\left(\begin{array}{ll}a & b \\ c & d\end{array}\right)$ acts by $[z: 1] \mapsto[a z+b: c z+d]=\left[\frac{a z+b}{c z+d}: 1\right]$. Moreover for $z=x+i y$ the expression

$$
\begin{aligned}
\frac{a z+b}{c z+d} & =\frac{(a z+b)(c \bar{z}+d)}{|c z+d|^{2}} \\
& =\frac{a c\left(x^{2}+y^{2}\right)+(a d+b c) x+d b}{(c x+d)^{2}+(c y)^{2}}+i \frac{(a d-b c) y}{(c x+d)^{2}+(c y)^{2}}
\end{aligned}
$$

has imaginary part $>0$ if and only if $y>0$. We denote the action by $m: S L(2, \mathbb{R}) \times H_{+}^{2} \rightarrow H_{+}^{2}$, so that $m\left(\begin{array}{ll}a & b \\ c & d\end{array}\right)(z)=\frac{a z+b}{c z+d}$. Transformations of this form are called a fractional linear transformations or Möbius transformations.

(1) $S L(2, \mathbb{R})$ acts transitively on $H_{+}^{2}$, since $m\left(\begin{array}{cc}\sqrt{y} & x / \sqrt{y} \\ 0 & 1 / \sqrt{y}\end{array}\right)(i)=x+i y$. The isotropy group fixing $i$ is $S O(2) \subset S L(2)$, since $i=\frac{a i+b}{c i+d}=\frac{b d+a c+i}{c^{2}+d^{2}}$ if and only if $c d+a c=0$ and $c^{2}+d^{2}=1$. Thus

$$
H_{+}^{2}=S L(2, \mathbb{R}) / S O(2, \mathbb{R}) .
$$

Any Möbius transformation by an element of $S L(2)$ is an isometry:

$$
\begin{gathered}
A:=\left(\begin{array}{ll}
a & b \\
c & d
\end{array}\right) \in S L(2, \mathbb{R}), \\
m_{A}(z)-m_{A}\left(z^{\prime}\right)=\frac{a z+b}{c z+d}-\frac{a z^{\prime}+b}{c z^{\prime}+d}=\cdots=\frac{z-z^{\prime}}{(c z+d)\left(c z^{\prime}+d\right)}, \\
\left(m_{A}\right)^{\prime}(z)=\lim _{z^{\prime} \rightarrow z} \frac{1}{z-z^{\prime}} \frac{z-z^{\prime}}{(c z+d)\left(c z^{\prime}+d\right)}=\frac{1}{(c z+d)^{2}}, \\
m_{A}(z)-m_{A}\left(z^{\prime}\right)=\sqrt{\left(m_{A}\right)^{\prime}(z)} \sqrt{\left(m_{A}\right)^{\prime}\left(z^{\prime}\right)}\left(z-z^{\prime}\right),
\end{gathered}
$$

always for the same branch of $\sqrt{\left(m_{A}\right)^{\prime}(z)}$. Expressing the metric in the complex variable, we then have

$$
\begin{aligned}
g & =\frac{1}{y^{2}}\left(d x^{2}+d y^{2}\right)=\frac{1}{\operatorname{Im}(z)^{2}} \operatorname{Re}(d z \cdot d \bar{z}) \\
\left(m_{A}\right)^{*} g & =\left(m_{A}\right)^{*}\left(\frac{1}{\operatorname{Im}(z)^{2}} \operatorname{Re}(d z \cdot d \bar{z})\right) \\
& =\frac{1}{\operatorname{Im}\left(\left(m_{A}\right)(z)\right)^{2}} \operatorname{Re}\left(\left(m_{A}\right)^{\prime}(z) d z \cdot\left(m_{A}\right)^{\prime}(\bar{z}) d \bar{z}\right) \\
& =\operatorname{Im}\left(\left(m_{A}\right)(z)\right)^{-2}|c z+d|^{-4} \operatorname{Re}(d z \cdot d \bar{z})=\frac{1}{\operatorname{Im}(z)^{2}} \operatorname{Re}(d z \cdot d \bar{z})
\end{aligned}
$$


since

$$
\begin{aligned}
\operatorname{Im}\left(\left(m_{A}\right)(z)\right)|c z+d|^{2} & =\frac{1}{2 i}\left(m_{A}(z)-m_{A}(\bar{z})\right)|c z+d|^{2} \\
& =\frac{1}{2 i} \frac{z-\bar{z}}{(c z+d)(c \bar{z}+d)}|c z+d|^{2}=\operatorname{Im}(z) .
\end{aligned}
$$

(2) For further use we note the Möbius transformations

$$
\begin{aligned}
& m_{1}=m\left(\begin{array}{cc}
1 & r \\
0 & 1
\end{array}\right): z \mapsto z+r, \quad r \in \mathbb{R}, \\
& m_{2}=m\left(\begin{array}{cc}
\sqrt{r} & 0 \\
0 & 1 / \sqrt{r}
\end{array}\right): z \mapsto r . z, \quad r \in \mathbb{R}_{>0}, \\
& m_{3}=m\left(\begin{array}{cc}
0 & -1 \\
1 & 0
\end{array}\right): z \mapsto \frac{-1}{z}=\frac{-\bar{z}}{|z|^{2}}=\frac{-x+i y}{x^{2}+y^{2}} .
\end{aligned}
$$

We can now use these three isometries to determine again the form of all geodesics in $H_{+}^{2}$. For this note that: If the fixed point set $\left(H_{x}^{2}\right)^{m}=\{z \in$ $\left.H_{+}^{2}: m(z)=z\right\}$ of an isometry $m$ is a connected 1-dimensional submanifold, then this is the image of a geodesic, since for any vector $X_{z} \in T_{z} H_{+}^{2}$ tangent to the fixed point set we have $m(\exp (t X))=\exp \left(t T_{z} m . X\right)=\exp (t X)$. We first use the isometry $\psi(x, y)=(-x, y)$ which is not a Möbius transformation since it reverses the orientation. Its fixed point set is the vertical line $\{(0, y)$ : $y>0\}$ which thus is a geodesic. The image under $m_{1}$ is then the geodesic $\{(r, y): y>0\}$. The fixed point set of the isometry $\psi \circ m_{3}$ is the upper half of the unit circle, which thus is a geodesic. By applying $m_{1}$ and $m_{2}$, we may map it to any upper half-circle with center in the real axis.

(3) The group $S L(2, \mathbb{R})$ acts isometrically doubly transitively on $H_{+}^{2}$ : Any two pairs of points with the same geodesic distance can be mapped to each other by a Möbius transformation. For $A=\left(\begin{array}{ll}a & b \\ c & d\end{array}\right)$ in the isotropy group $S O(2)$ of $i$ we have $m_{A}^{\prime}(i)=\frac{1}{(c i+d)^{2}}$; it double covers the unit circle in $T_{i}\left(H_{+}^{2}\right)$. Thus $S L(2, \mathbb{R})$ acts transitively on the set of all unit tangent vectors in $H_{+}^{2}$, and a shortest geodesic from $z_{1}$ to $z_{2}$ can thus be mapped by a Möbius transformation to a shortest geodesic of the same length from $z_{1}^{\prime}$ to $z_{2}^{\prime}$.

(4) $H_{+}^{2}$ is a complete Riemann manifold, and the geodesic distance is given by

$$
\operatorname{dist}\left(z_{1}, z_{2}\right)=2 \operatorname{artanh}\left|\frac{z_{1}-z_{2}}{z_{1}-\bar{z}_{2}}\right|
$$

The shortest curve from $i y_{1}$ to $i y_{2}$ is obviously on the vertical line since for $z(t)=x(t)+i y(t)$ the length

$$
L(c)=\int_{0}^{1} \frac{1}{y(t)} \sqrt{x^{\prime}(t)^{2}+y^{\prime}(t)^{2}} d t
$$


is minimal for $x^{\prime}(t)=0$; thus $x(t)=$ constant. By the invariance under reparameterizations of the length we have

$$
\operatorname{dist}\left(i y_{1}, i y_{2}\right)=\left|\int_{y_{1}}^{y_{2}} \frac{1}{t} d t\right|=\left|\log y_{2}-\log y_{1}\right|=\left|\log \left(\frac{y_{2}}{y_{1}}\right)\right| .
$$

From the formulas in (11) we see that the double ratio $\left|\frac{z_{1}-z_{2}}{z_{1}-\bar{z}_{2}}\right|$ is invariant under $S L(2, \mathbb{R})$ since:

$$
\left|\frac{m_{A}\left(z_{1}\right)-m_{A}\left(z_{2}\right)}{m_{A}\left(z_{1}\right)-\overline{m_{A}\left(z_{2}\right)}}\right|=\left|\frac{\frac{z_{1}-z_{2}}{\left(c z_{1}+d\right)\left(c z_{2}+d\right)}}{\frac{z_{1}-\bar{z}_{2}}{\left(c z_{1}+d\right)\left(c \bar{z}_{2}+d\right)}}\right|=\left|\frac{z_{1}-z_{2}}{z_{1}-\bar{z}_{2}}\right| .
$$

On the vertical geodesic we have

$$
\begin{aligned}
\left|\frac{i y_{1}-i y_{2}}{i y_{1}+i y_{2}}\right| & =\left|\frac{\frac{y_{1}}{y_{2}}-1}{\frac{y_{1}}{y_{2}}+1}\right|=\left|\frac{e^{\log \left(\frac{y_{1}}{y_{2}}\right)}-1}{e^{\log \left(\frac{y_{1}}{y_{2}}\right)}+1}\right|=\left|\frac{e^{\frac{1}{2}\left|\log \left(\frac{y_{1}}{y_{2}}\right)\right|}-e^{-\frac{1}{2}\left|\log \left(\frac{y_{1}}{y_{2}}\right)\right|}}{e^{\frac{1}{2}\left|\log \left(\frac{y_{1}}{y_{2}}\right)\right|}+e^{-\frac{1}{2}\left|\log \left(\frac{y_{1}}{y_{2}}\right)\right|}}\right| \\
& =\tanh \left(\frac{1}{2} \operatorname{dist}\left(i y_{1}, i y_{2}\right)\right) .
\end{aligned}
$$

Since $S L(2, \mathbb{R})$ acts isometrically doubly transitively by (3) and since both sides are invariant, the result follows.

(5) The geodesic exponential mapping. We have $\exp _{i}(t i)=e^{t} \cdot i$ since by (4) we have $\operatorname{dist}\left(i, e^{t} i\right)=\log \frac{e^{t} i}{i}=t$. Now let $X \in T_{i}\left(H_{+}^{2}\right)$ with $|X|=1$. In (3) we saw that there exists $\varphi$ with

$$
\begin{aligned}
m\left(\begin{array}{cc}
\cos \varphi-\sin \varphi \\
\sin \varphi & \cos \varphi
\end{array}\right)^{\prime}(i) i & =\frac{i}{(i \sin \varphi+\cos \varphi)^{2}}=e^{-2 i \varphi} \cdot i=X, \\
\varphi & =\frac{\pi}{4}-\frac{\arg (X)}{2}+\pi \mathbb{Z}, \\
\exp _{i}(t X) & =m\left(\begin{array}{cc}
\begin{array}{c}
\cos \varphi-\sin \varphi \\
\sin \varphi
\end{array} & \cos \varphi
\end{array}\right)\left(e^{t} i\right)=\frac{\cos \varphi \cdot e^{t} \cdot i-\sin \varphi}{\sin \varphi \cdot e^{t} i+\cos \varphi}
\end{aligned}
$$

(6) Hyperbolic area of a geodesic polygon. By (10.5) the Riemann metric $g=\frac{1}{y^{2}}\left(d x^{2}+d y^{2}\right)$ has density $\operatorname{vol}(g)=\sqrt{\operatorname{det} g_{i j} d x d y}=\frac{1}{y^{2}} d x d y$; thus:

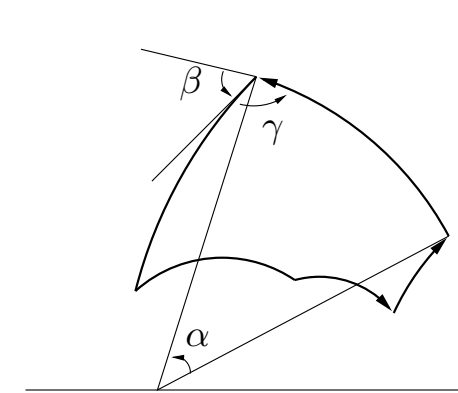

$$
\begin{gathered}
\mathrm{Vol}^{H_{+}^{2}}(P)=\int_{P} \frac{d x \wedge d y}{y^{2}}=\int_{P} d\left(\frac{d x}{y}\right) \\
=\int_{\partial P} \frac{d x}{y}=-\int_{\partial P} d \theta
\end{gathered}
$$

since each geodesic is part of a circle $z-a=r e^{i \theta}, \quad a \in \mathbb{R} . \quad$ On it we have $\frac{d x}{y}=\frac{d(r \cos \theta+a)}{r \sin \theta}=\frac{-r \sin \theta d \theta}{r \sin \theta}=-d \theta$.

The integral is thus the total increase of the tangent angle. For a simply connected polygon the total increase of the tangent angle is $2 \pi$ if we also 
add the exterior angles at the corners: $\int_{\partial P} d \vartheta+\sum_{i} \beta_{i}=\sum_{i} \alpha_{i}+\sum_{i} \beta_{i}=2 \pi$. We change to the inner angles $\gamma_{i}=\pi-\beta_{i}$ and get:

$$
\mathrm{Vol}_{+}^{H_{+}^{2}}(P)=-\int_{\partial P} d \vartheta=-2 \pi+\sum_{i} \beta_{i}=(n-2) \pi-\sum_{i} \gamma_{i} .
$$

This is a particular instance of the theorem of Gauß-Bonnet.

25.9. The 3-sphere $S^{3}$. We use the parameterization of $S^{3} \subset \mathbb{R}^{4}$ given by

$$
f(\varphi, \vartheta, \tau)=\left(\begin{array}{c}
\cos \varphi \cos \vartheta \cos \tau \\
\sin \varphi \cos \vartheta \cos \tau \\
\sin \vartheta \cos \tau \\
\sin \tau
\end{array}\right), \quad \begin{gathered}
0<\varphi<2 \pi \\
-\frac{\pi}{2}<\vartheta<\frac{\pi}{2} \\
-\frac{\pi}{2}<\tau<\frac{\pi}{2}
\end{gathered}
$$

We write $f_{1}^{1}=\partial_{\varphi} f^{1}$, etc. Then the induced metric is given by:

$$
\begin{aligned}
g_{11} & =\left\langle f_{1}, f_{1}\right\rangle=f_{1}^{1} f_{1}^{1}+f_{1}^{2} f_{1}^{2}+f_{1}^{3} f_{1}^{3}+f_{1}^{4} f_{1}^{4}=\cos ^{2} \vartheta \cos ^{2} \tau, \\
g_{12} & =\left\langle f_{1}, f_{2}\right\rangle=0, \quad g_{13}=0, \quad g_{22}=\cos ^{2} \tau, \quad g_{23}=0, \quad g_{33}=1 . \\
g & =\cos ^{2} \vartheta \cos ^{2} \tau d \varphi \otimes d \varphi+\cos ^{2} \tau d \vartheta \otimes d \vartheta+d \tau \otimes d \tau . \\
\sigma^{1} & =\cos \vartheta \cos \tau d \varphi, \quad \sigma^{2}=\cos \tau d \vartheta, \quad \sigma^{3}=d \tau . \\
d \sigma^{1} & =-\sin \vartheta \cos \tau d \vartheta \wedge d \varphi-\cos \vartheta \sin \tau d \tau \wedge d \varphi, \\
d \sigma^{2} & =-\sin \tau d \tau \wedge d \vartheta, \quad d \sigma^{3}=0 .
\end{aligned}
$$

Now we use the first structure equation (25.5.3), i.e., $d \sigma+\omega \wedge \sigma=0$ :

$$
\begin{aligned}
& d \sigma^{1}=-0-\omega_{2}^{1} \wedge \sigma^{2}-\omega_{3}^{1} \wedge \sigma^{3}=\sin \vartheta \cos \tau d \varphi \wedge d \vartheta+\cos \vartheta \sin \tau d \varphi \wedge d \tau, \\
& d \sigma^{2}=-\omega_{1}^{2} \wedge \sigma^{1}-0-\omega_{3}^{2} \wedge \sigma^{3}=\sin \tau d \vartheta \wedge d \tau, \\
& d \sigma^{3}=-\omega_{1}^{3} \wedge \sigma^{1}-\omega_{2}^{3} \wedge \sigma^{2}-0=0 . \\
& -\omega_{2}^{1} \wedge \cos \tau d \vartheta-\omega_{3}^{1} \wedge d \tau=\sin \vartheta \cos \tau d \varphi \wedge d \vartheta+\cos \vartheta \sin \tau d \varphi \wedge d \tau, \\
& -\omega_{1}^{2} \wedge \cos \vartheta \cos \tau d \varphi-\omega_{3}^{2} \wedge d \tau=\sin \tau d \vartheta \wedge d \tau, \\
& -\omega_{1}^{3} \wedge \cos \vartheta \cos \tau d \varphi-\omega_{2}^{3} \wedge \cos \tau d \vartheta=0 . \\
& \left\{\begin{array}{l}
\omega_{3}^{1}=-\cos \vartheta \sin \tau d \varphi \\
\omega_{3}^{2}=-\sin \tau d \vartheta \\
\omega_{2}^{1}=-\sin \vartheta d \varphi
\end{array}\right. \\
& \omega=\left(\begin{array}{ccc}
0 & -\sin \vartheta d \varphi & -\cos \vartheta \sin \tau d \varphi \\
\sin \vartheta d \varphi & 0 & -\sin \tau d \vartheta \\
\cos \vartheta \sin \tau d \varphi & \sin \tau d \vartheta & 0
\end{array}\right) .
\end{aligned}
$$


From this we can compute the curvature:

$$
\begin{aligned}
& \Omega_{2}^{1}=d \omega_{2}^{1}+0+0+\omega_{3}^{1} \wedge \omega_{2}^{3} \\
& =-\cos \vartheta d \vartheta \wedge d \varphi-\cos \vartheta \sin \tau d \varphi \wedge \sin \tau d \vartheta \\
& =\cos \vartheta \cos ^{2} \tau d \varphi \wedge d \vartheta=\sigma^{1} \wedge \sigma^{2}, \\
& \Omega_{3}^{1}=d \omega_{3}^{1}+0+\omega_{2}^{1} \wedge \omega_{3}^{2}+0 \\
& =\sin \vartheta \sin \tau d \vartheta \wedge d \varphi-\cos \vartheta \cos \tau d \tau \wedge d \varphi+\sin \vartheta d \varphi \wedge \sin \tau d \vartheta \\
& =\cos \vartheta \cos \tau d \varphi \wedge d \tau=\sigma^{1} \wedge \sigma^{3}, \\
& \Omega_{3}^{2}=d \omega_{3}^{2}+\omega_{1}^{2} \wedge \omega_{3}^{1}+0+0 \\
& =-\cos \tau d \tau \wedge d \vartheta+0 \\
& =\cos \tau d \vartheta \wedge d \tau=\sigma^{2} \wedge \sigma^{3} \text {, } \\
& \Omega=\left(\begin{array}{ccc}
0 & \sigma^{1} \wedge \sigma^{2} & \sigma^{1} \wedge \sigma^{3} \\
-\sigma^{1} \wedge \sigma^{2} & 0 & \sigma^{2} \wedge \sigma^{3} \\
-\sigma^{1} \wedge \sigma^{3} & -\sigma^{2} \wedge \sigma^{3} & 0
\end{array}\right)=\left(\begin{array}{l}
\sigma^{1} \\
\sigma^{2} \\
\sigma^{3}
\end{array}\right) \wedge\left(\sigma^{1}, \sigma^{2}, \sigma^{3}\right) .
\end{aligned}
$$

Another representation of the 3 -sphere with radius $1 / \sqrt{k}$. The induced metric is given by

$$
g=\frac{1}{k}\left(\cos ^{2} \vartheta \cos ^{2} \tau d \varphi \otimes d \varphi+\cos ^{2} \tau d \vartheta \otimes d \vartheta+d \tau \otimes d \tau\right),
$$

where $0<\varphi<2 \pi,-\frac{\pi}{2}<\vartheta<\frac{\pi}{2}$, and $-\frac{\pi}{2}<\tau<\frac{\pi}{2}$. Now we introduce the coordinate function $r$ by $\cos ^{2} \tau=k r^{2}$, more precisely by

$$
r=\left\{\begin{array}{lll}
-\frac{1}{\sqrt{k}} \cos \tau & \text { for } \quad-\frac{\pi}{2}<\tau<0, & 0<|r|<\frac{1}{\sqrt{k}} . \\
\frac{1}{\sqrt{k}} \cos \tau & \text { for } \quad 0<\tau<\frac{\pi}{2},
\end{array}\right.
$$

Then $\operatorname{sign} \tau \cos \tau=\sqrt{k} r$; thus $-\operatorname{sign} \tau \sin \tau d \tau=\sqrt{k} d r$, and $\operatorname{since~}^{2} \sin ^{2} \tau=$ $1-\cos ^{2} \tau=1-k r^{2}$, we finally get

$$
\left(1-k r^{2}\right) d \tau \otimes d \tau=\sin ^{2} \tau d \tau \otimes d \tau=k d r \otimes d r .
$$

Furthermore we replace $\vartheta$ by $\vartheta+\frac{\pi}{2}$. Then the metric becomes:

$$
\begin{aligned}
g= & \frac{1}{k}\left(\sin ^{2} \vartheta k r^{2} d \varphi \otimes d \varphi+k r^{2} d \vartheta \otimes d \vartheta+\frac{k}{1-k r^{2}} d r \otimes d r\right) \\
= & \frac{1}{1-k r^{2}} d r \otimes d r+r^{2} d \vartheta \otimes d \vartheta+r^{2} \sin ^{2} \vartheta d \varphi \otimes d \varphi, \quad \text { where } \\
& 0<\varphi<2 \pi, \quad 0<\vartheta<\pi, \quad 0<|r|<\frac{1}{\sqrt{k}} .
\end{aligned}
$$


25.10. The Robertson-Walker metric in general relativity. This is the metric of signature $(+---)$ of the form

$$
\begin{aligned}
g & =d t \otimes d t-R(t)^{2}\left(\frac{1}{1-k r^{2}} d r \otimes d r+r^{2} d \vartheta \otimes d \vartheta+r^{2} \sin ^{2} \vartheta d \varphi \otimes d \varphi\right) \\
& \text { for } 0<\varphi<2 \pi, \quad 0<\vartheta<\pi, \quad 0<|r|<\frac{1}{\sqrt{k}}, \\
& =\rho^{0} \otimes \rho^{0}-\rho^{1} \otimes \rho^{1}-\rho^{2} \otimes \rho^{2}-\rho^{3} \otimes \rho^{3}, \\
\rho^{0} & =d t, \quad \rho^{1}=\frac{R}{w} d r, \quad \text { where } w:=\sqrt{1-k r^{2}}, \\
\rho^{2} & =R r d \vartheta, \quad \rho^{3}=R r \sin \vartheta d \varphi .
\end{aligned}
$$

The differential of the coframe is:

$$
\begin{aligned}
d \rho^{0} & =0, \\
d \rho^{1} & =\frac{\dot{R}}{w} d t \wedge d r=\frac{\dot{R}}{R} \rho^{0} \wedge \rho^{1}, \\
d \rho^{2} & =\dot{R} r d t \wedge d \vartheta+R d r \wedge d \vartheta=\frac{\dot{R}}{R} \rho^{0} \wedge \rho^{2}+\frac{w}{R r} \rho^{1} \wedge \rho^{2}, \\
d \rho^{3} & =\dot{R} r \sin \vartheta d \vartheta \wedge d \varphi+R \sin \vartheta d r \wedge d \varphi+R r \cos \vartheta d \vartheta \wedge d \varphi \\
& =\frac{\dot{R}}{R} \rho^{0} \wedge \rho^{3}+\frac{w}{R r} \rho^{1} \wedge \rho^{3}+\frac{\operatorname{cotan} \vartheta}{R r} \rho^{2} \wedge \rho^{3} .
\end{aligned}
$$

Now we use $d \rho+\omega \wedge \rho=0, \omega_{j}^{i}=-\omega_{i}^{j}$ for $1 \leq i, j \leq 3, \omega_{i}^{i}=0$, and $\omega_{i}^{0}=\omega_{0}^{i}$ :

$$
\begin{aligned}
d \rho^{0} & =-\omega_{1}^{0} \wedge \rho^{1}-\omega_{2}^{0} \wedge \rho^{2}-\omega_{3}^{0} \wedge \rho^{3}=0, \\
d \rho^{1} & =-\omega_{0}^{1} \wedge \rho^{0}-\omega_{2}^{1} \wedge \rho^{2}-\omega_{3}^{1} \wedge \rho^{3}=\frac{\dot{R}}{R} \rho^{0} \wedge \rho^{1}, \\
d \rho^{2} & =-\omega_{0}^{2} \wedge \rho^{0}-\omega_{1}^{2} \wedge \rho^{1}-\omega_{3}^{2} \wedge \rho^{3}=\frac{\dot{R}}{R} \rho^{0} \wedge \rho^{2}+\frac{w}{R r} \rho^{1} \wedge \rho^{2}, \\
d \rho^{3} & =-\omega_{0}^{3} \wedge \rho^{0}-\omega_{1}^{3} \wedge \rho^{1}-\omega_{2}^{3} \wedge \rho^{2} \\
& =\frac{\dot{R}}{R} \rho^{0} \wedge \rho^{3}+\frac{w}{R r} \rho^{1} \wedge \rho^{3}+\frac{\operatorname{cotan} \vartheta}{R r} \rho^{2} \wedge \rho^{3} .
\end{aligned}
$$

This is a linear system of equations with a unique solution for the $\omega_{j}^{i}$. Guided by (25.9) we assume that $\omega_{1}^{0}$ is a multiple of $\rho^{1}$, etc., and we get the solutions

$$
\begin{array}{rlrl}
\omega_{0}^{1} & =\frac{\dot{R}}{R} \rho^{1}=\frac{\dot{R}}{w} d r, & \omega_{0}^{2}=\frac{\dot{R}}{R} \rho^{2}=\dot{R} r d \vartheta, \\
\omega_{0}^{3}=\frac{\dot{R}}{R} \rho^{3}=\dot{R} r \sin \vartheta d \varphi, & \omega_{1}^{2}=\frac{w}{R r} \rho^{2}=w d \vartheta, \\
\omega_{1}^{3}=\frac{w}{R r} \rho^{3}=w \sin \vartheta d \varphi, & \omega_{2}^{3}=\frac{\operatorname{cotan} \vartheta}{R r} \rho^{3}=\cos \vartheta d \varphi .
\end{array}
$$


From these we can compute the curvature 2 -forms, using (25.5.5), that is, $\Omega=d \omega+\omega \wedge \omega:$

$$
\begin{array}{ll}
\Omega_{0}^{1}=-\frac{\ddot{R}}{R} \rho^{1} \wedge \rho^{0}, & \Omega_{0}^{2}=-\frac{\ddot{R}}{R} \rho^{2} \wedge \rho^{0}, \\
\Omega_{0}^{3}=-\frac{\ddot{R}}{R} \rho^{3} \wedge \rho^{0}, & \Omega_{1}^{2}=\frac{k+\dot{R}^{2}}{R^{2}} \rho^{2} \wedge \rho^{1}, \\
\Omega_{1}^{3}=-\frac{-k+\dot{R}^{2}}{R^{2}} \rho^{3} \wedge \rho^{1}, & \Omega_{2}^{3}=\frac{k+\dot{R}^{2}}{R^{2}} \rho^{3} \wedge \rho^{2} .
\end{array}
$$

25.11. The Hodge $*$-operator. Let $(M, g)$ be an oriented pseudo-Riemann manifold of signature $(p, q)$. Viewing $g: T M \rightarrow T^{*} M$, we let $g^{-1}$ : $T^{*} M \rightarrow T M$ denote the dual bundle metric on $T^{*} M$. Then $g^{-1}$ induces a symmetric nondegenerate bundle metric

$$
\bigwedge^{k} g^{-1}: \bigwedge^{k} T^{*} M \rightarrow \bigwedge^{k} T M
$$

on the bundle $\bigwedge^{k} T^{*} M$ of $k$-forms which is given by

$$
g^{-1}\left(\varphi_{1} \wedge \cdots \wedge \varphi_{k}, \psi_{1} \wedge \cdots \wedge \psi_{k}\right)=\operatorname{det}\left(g^{-1}\left(\varphi_{i}, \psi_{j}\right)_{i, j=1}^{k}\right), \quad \varphi_{i}, \psi_{j} \in \Omega^{1}(M) .
$$

Let $\eta_{i j}=g\left(s_{i}, s_{j}\right)=\operatorname{diag}(1, \ldots, 1,-1, \ldots,-1)$ be the standard inner product matrix of the same signature $(p, q)$, and let $s=\left(s_{1}, \ldots, s_{m}\right)$ be an orthonormal frame on $U \subseteq M$ with orthonormal coframe $\sigma=\left(\sigma_{1}, \ldots, \sigma_{m}\right)$ as in (25.5) so that $g=\sum_{i} \eta_{i i} \sigma^{i} \otimes \sigma^{i}$; then for $\varphi^{k}, \psi^{k} \in \Omega^{k}(M)$ we have

$$
g^{-1}\left(\varphi^{k}, \psi^{k}\right)=\sum_{\substack{i_{1}<\cdots<i_{k} \\ j_{1}<\cdots<j_{k}}} \varphi^{k}\left(s_{i_{1}}, \ldots, s_{i_{k}}\right) \psi^{k}\left(s_{j_{1}}, \ldots, s_{j_{k}}\right) \eta^{i_{1} j_{1}} \ldots \eta^{i_{k} j_{k}} .
$$

Note that $g^{-1}\left(\sigma^{1} \wedge \cdots \wedge \sigma^{m}, \sigma^{1} \wedge \cdots \wedge \sigma^{m}\right)=(-1)^{q}$.

If $M$ is also oriented, then the volume form $\operatorname{vol}(g)$ from (10.5) agrees with the positively oriented $m$-form of length \pm 1 . We have $\operatorname{vol}(g)=\sigma^{1} \wedge \cdots \wedge \sigma^{m}$ if the frame $s=\left(s_{1}, \ldots, s_{m}\right)$ is positively oriented.

We shall use the following notation:

If $I=\left(i_{1}<\cdots<i_{k}\right)$ and $I^{\prime}=\left(j_{1}<\cdots<j_{m-k}\right)$ are the ordered tuples with $I \cap I^{\prime}=\emptyset$ and $I \sqcup I^{\prime}=\{1, \ldots, m\}$, then we put $\sigma^{I}:=\sigma^{i_{1}} \wedge \cdots \wedge \sigma^{i_{k}}$.

Exercise. The $k$-forms $\sigma^{I}$ for all $I$ as above of length $k$ give an orthonormal basis of $g^{-1}$ on $\Omega^{k}(U)$. The signature of $g^{-1}$ on $\bigwedge^{k} T_{x}^{*} M$ is

$$
\left(P_{+}(p, q, k), P_{-}(p, q, k)\right)=\left(\sum_{j=0, j \text { even }}^{k}\left(\begin{array}{c}
p \\
k-j
\end{array}\right)\left(\begin{array}{l}
q \\
j
\end{array}\right), \sum_{j=0, j \text { odd }}^{k}\left(\begin{array}{c}
p \\
k-j
\end{array}\right)\left(\begin{array}{l}
q \\
j
\end{array}\right)\right) .
$$


On an oriented pseudo-Riemann manifold $(M, g)$ of dimension $m$ and signature $(p, q)$ we have the Hodge isomorphism * with its elementary properties:

$$
\begin{aligned}
& *: \bigwedge^{k} T^{*} M \rightarrow \bigwedge^{m-k} T^{*} M, \\
& \left(* \varphi^{k}\right)\left(X_{k+1}, \ldots, X_{m}\right) \operatorname{vol}(g)=\varphi \wedge g\left(X_{k+1}\right) \wedge \cdots \wedge g\left(X_{m}\right), \\
& \varphi^{k} \wedge \psi^{m-k}=g^{-1}\left(* \varphi^{k}, \psi^{m-k}\right) \operatorname{vol}(g), \\
& g^{-1}\left(* \varphi^{k}, * \psi^{k}\right)=(-1)^{q} g^{-1}\left(\varphi^{k}, \psi^{k}\right), \\
& * * \varphi^{k}=(-1)^{k(m-k)+q} \varphi^{k} \\
& \left(* \varphi^{k}\right) \wedge \psi^{k}=\left(* \psi^{k}\right) \wedge \varphi^{k} .
\end{aligned}
$$

In the local orthonormal frame we get

$$
\begin{aligned}
& \left(* \sigma^{I}\right)\left(s_{j_{1}}, \ldots, s_{j_{m-k}}\right) \operatorname{vol}(g)=\sigma^{I} \wedge g\left(s_{j_{1}}\right) \wedge \cdots \wedge g\left(s_{j_{m-k}}\right) \\
& \quad=\sigma^{I} \wedge g\left(s_{j_{1}}\right) \wedge \cdots \wedge g\left(s_{j_{m-k}}\right)=\sigma^{I} \wedge \eta_{j_{1} j_{1}} \sigma^{j_{1}} \wedge \cdots \wedge \eta_{j_{m-k} j_{m-k}} \sigma^{j_{m-k}} \\
& * \sigma^{I}=\operatorname{sign}\left(\begin{array}{cc}
1 \ldots m \\
I & I^{\prime}
\end{array}\right) \eta_{j_{1} j_{1}} \ldots \eta_{j_{m-k} j_{m-k}} \sigma^{I^{\prime}} .
\end{aligned}
$$

To get a geometric interpretation of $* \varphi^{k}$, we consider

$$
\begin{aligned}
i(X)\left(* \varphi^{k}\right)\left(X_{k+2}, \ldots\right. & \left., X_{m}\right) \operatorname{vol}(g)=\left(* \varphi^{k}\right)\left(X, X_{k+2}, \ldots, X_{m}\right) \operatorname{vol}(g) \\
& =\varphi^{k} \wedge g(X) \wedge g\left(X_{k+2}\right) \wedge \cdots \wedge g\left(X_{m}\right) \\
& =*\left(\varphi^{k} \wedge g(X)\right)\left(X_{k+2}, \ldots, X_{m}\right) \operatorname{vol}(g)
\end{aligned}
$$

so that

$$
\begin{aligned}
i(X)\left(* \varphi^{k}\right) & =*\left(\varphi^{k} \wedge g(X)\right), \\
\left\{X: i_{X} \varphi^{k}=0\right\}^{\perp, g} & =\left\{Y: i_{Y}\left(* \varphi^{k}\right)=0\right\} .
\end{aligned}
$$

25.12. Relations to vector analysis. We consider an oriented pseudoRiemann manifold $(M, g)$ of signature $(p, q)$. For functions $f \in C^{\infty}(M, \mathbb{R})$ and vector fields $X \in \mathfrak{X}(M)$ we have the following operations, gradient and divergence, and their elementary properties:

$$
\begin{aligned}
& \operatorname{grad}^{g}(f)=g^{-1} \circ d f \in \mathfrak{X}(M), \\
& g(X) \in \Omega^{1}(M), \quad * g(X)=(-1)^{q} i_{X} \operatorname{vol}(g), \\
& * d f=* g\left(\operatorname{grad}^{g}(f)\right)=(-1)^{q} i_{\operatorname{grad}^{g}(f)} \operatorname{vol}(g), \\
& \operatorname{div}^{g}(X) \cdot \operatorname{vol}(g)=(-1)^{q} d i_{X} \operatorname{vol}(g)=d * g(X), \\
& \operatorname{grad}^{g}(f \cdot h)=f \cdot \operatorname{grad}^{g}(h)+h \cdot \operatorname{grad}^{g}(f), \\
& \operatorname{div}^{g}(f \cdot X)=f \operatorname{div}^{g}(X)+(-1)^{q} d f(X), \\
& \left.\operatorname{grad}^{g}(f)\right|_{U}=\sum_{i} \eta_{i i} s_{i}(f) \cdot s_{i}, \\
& \operatorname{div}^{g}(X)=\operatorname{Trace}(\nabla X) .
\end{aligned}
$$


Some authors take the negative of our definition of the divergence, so that later the Laplace-Beltrami operator $\Delta f=\left(-\operatorname{div}^{g}\right) \operatorname{grad}^{g}(f)$ is positive definite on any oriented Riemann manifold.

25.13. In dimension three. On an oriented 3-dimensional pseudo-Riemann manifold we have another operator on vector fields, curl, given by

$$
\begin{aligned}
& * g\left(\operatorname{curl}^{g}(X)\right)=(-1)^{q} i_{\operatorname{curl}^{g}(X)} \operatorname{vol}(g)=d g(X), \\
& \operatorname{curl}^{g}(X)=(-1)^{q} g^{-1} * d g(X),
\end{aligned}
$$

and from $d^{2}=0$ we have $\operatorname{curl}^{g} \operatorname{grad}^{g}=0$ and $\operatorname{div}^{g} \operatorname{curl}^{g}=0$.

On the oriented Euclidean space $\mathbb{R}^{3}$ we have

$$
\begin{aligned}
\operatorname{grad}(f) & =\frac{\partial f}{\partial x^{1}} \frac{\partial}{\partial x^{1}}+\frac{\partial f}{\partial x^{2}} \frac{\partial}{\partial x^{2}}+\frac{\partial f}{\partial x^{3}} \frac{\partial}{\partial x^{3}}, \\
\operatorname{curl}(X) & =\left(\frac{\partial X^{3}}{\partial x^{2}}-\frac{\partial X^{2}}{\partial x^{3}}\right) \frac{\partial}{\partial x^{1}}+\left(\frac{\partial X^{1}}{\partial x^{3}}-\frac{\partial X^{3}}{\partial x^{1}}\right) \frac{\partial}{\partial x^{2}}+\left(\frac{\partial X^{2}}{\partial x^{1}}-\frac{\partial X^{1}}{\partial x^{2}}\right) \frac{\partial}{\partial x^{3}}, \\
\operatorname{div}(X) & =\frac{\partial X^{1}}{\partial x^{1}}+\frac{\partial X^{2}}{\partial x^{2}}+\frac{\partial X^{3}}{\partial x^{3}} .
\end{aligned}
$$

Note also that $\operatorname{curl}(f \cdot X)=f \cdot \operatorname{rot}(X)+\operatorname{grad}(f) \times X$ where $\times$ denotes the vector product in $\mathbb{R}^{3}$.

25.14. The Maxwell equations. Let $U \subset \mathbb{R}^{3}$ be an open set in the oriented Euclidean 3-space. We will later assume that the first cohomology vanishes: $H^{1}(U)=0$. We consider three time dependent vector fields and a function,

$$
\begin{aligned}
& E: U \times \mathbb{R} \rightarrow \mathbb{R}^{3}, \quad \text { the electric field, } \\
& B: U \times \mathbb{R} \rightarrow \mathbb{R}^{3}, \quad \text { the magnetic field, } \\
& J: U \times \mathbb{R} \rightarrow \mathbb{R}^{3}, \quad \text { the current field, } \\
& \rho: U \times \mathbb{R} \rightarrow \mathbb{R}, \quad \text { the density function of the electric charge. }
\end{aligned}
$$

Then the Maxwell equations are (where $c$ is the speed of light)

$$
\begin{aligned}
\operatorname{curl}(E) & =-\frac{1}{c} \frac{d}{d t} B, & \operatorname{div}(B) & =0, \\
\operatorname{curl}(B) & =\frac{1}{c} \frac{d}{d t} E+\frac{4 \pi}{c} J, & \operatorname{div}(E) & =4 \pi \rho .
\end{aligned}
$$

Now let $\eta$ be the standard positive definite inner product on $\mathbb{R}^{3}$. From (25.13) we see that the Maxwell equations can be written as

$$
\begin{aligned}
* d \eta(E) & =-\frac{1}{c} \frac{d}{d t} \eta(B), & d * \eta(B) & =0, \\
* d \eta(B) & =\frac{1}{c} \frac{d}{d t} \eta(E)+\frac{4 \pi}{c} \eta(J), & d * \eta(E) & =4 \pi \rho \cdot \operatorname{vol}(\eta) .
\end{aligned}
$$


Now we assume that $H^{1}(U)=0$. Since $d * \eta(B)=0$, we have

$$
* \eta(B)=d A \text { for a function } A, \text { the magnetic potential. }
$$

Then the first Maxwell equation can be written as

$$
d\left(\eta(E)+\frac{1}{c} \frac{d}{d t} A\right)=0 .
$$

Using again $H^{1}(U)=0$, there exists a function $\Phi: U \times \mathbb{R} \rightarrow \mathbb{R}$, called the electric potential, such that

$$
\eta(E)=-\frac{1}{c} \frac{d}{d t} A-d \Phi .
$$

Starting from the magnetic and electric potentials $A, \Phi: U \times \mathbb{R} \rightarrow \mathbb{R}$, the electric and magnetic fields are given by

$$
\eta(E)=-\frac{1}{c} \frac{d}{d t} A-d \Phi, \quad \eta(B)=* d A,
$$

where all terms are viewed as time dependent functions of forms on $\mathbb{R}^{3}$. Then the first row of the Maxwell equations is automatically satisfied. The second row then looks like

$$
-* d * d A=-\frac{1}{c^{2}} \frac{d^{2}}{d t^{2}} A-\frac{1}{c} \frac{d}{d t} d \Phi+\frac{4 \pi}{c} \eta(J), \quad \frac{1}{c} \frac{d}{d t}(* d * A)-\Delta \Phi=4 \pi \rho .
$$

\section{Riemann Immersions and Submersions}

26.1. Riemann submanifolds and isometric immersions. Let $(\bar{M}, \bar{g})$ be a Riemann manifold of dimension $m+p$, and let $M \stackrel{i}{\longrightarrow} \bar{M}$ be a manifold of dimension $m$ with an immersion $i$. Let $g:=i^{*} \bar{g}$ be the induced Riemann metric on $M$. Let $\bar{\nabla}$ be the Levi-Civita covariant derivative on $\bar{M}$, and let $\nabla$ be the Levi-Civita covariant derivative on $M$. We denote by $T i^{\perp}=T M^{\perp}:=$ $\left\{X \in T_{i(x)} \bar{M}, x \in M, \bar{g}\left(X, \operatorname{Ti}\left(T_{x} M\right)\right)=0\right\}$ the normal bundle (over $M$ ) of the immersion $i$ or the immersed submanifold $M$.

Let $X, Y \in \mathfrak{X}(M)$. We may regard $T i . Y$ as vector field with values in $T \bar{M}$ defined along $i$ and thus consider $\bar{\nabla}_{X}(T i . Y): M \rightarrow i^{*} T \bar{M}$.

Lemma. Gauß's formula. If $X, Y \in \mathfrak{X}(M)$, then

$$
\bar{\nabla}_{X}(T i . Y)-T i \circ \nabla_{X} Y=: S(X, Y)
$$

is normal to $M$, and $S: T M \times_{M} T M \rightarrow T i^{\perp}$ is a symmetric tensor field, which is called the second fundamental form or the shape form of $M$.

Proof. For $X, Y, Z \in \mathfrak{X}(M)$ and a suitable open set $U \subset M$ we may choose an open subset $\bar{U} \subset \bar{M}$ with $i(U)$ closed in $\bar{U}$ such that $i: U \rightarrow \bar{U}$ is an 
embedding, and then extensions $\bar{X}, \bar{Y}, \bar{Z} \in \mathfrak{X}(\bar{U})$ with $\left.\bar{X} \circ i\right|_{U}=$ Ti.X $\left.\right|_{U}$, etc. By (22.5.7) we have

$$
\begin{aligned}
& 2 \bar{g}(\bar{\nabla}\bar{X} \bar{Y}, \bar{Z}) \\
&=\bar{X}(\bar{g}(\bar{Y}, \bar{Z}))+\bar{Y}(\bar{g}(\bar{Z}, \bar{X}))-\bar{Z}(\bar{g}(\bar{X}, \bar{Y})) \\
&+\bar{g}([\bar{X}, \bar{Y}], \bar{Z})+\bar{g}([\bar{Z}, \bar{X}], \bar{Y})-\bar{g}([\bar{Y}, \bar{Z}], \bar{X}) .
\end{aligned}
$$

Composing this formula with $\left.i\right|_{U}$, we get on $U$

$$
\begin{aligned}
2 \bar{g}\left(\bar{\nabla}_{X}(T i . Y), T i . Z\right)= & X(g(Y, Z))+Y(g(Z, X))-Z(g(X, Y)) \\
& +g([X, Y], Z)+g([Z, X], Y)-g([Y, Z], X) \\
= & 2 g\left(\nabla_{X} Y, Z\right),
\end{aligned}
$$

again by (22.5.7). Since this holds for all $Z \in \mathfrak{X}(U)$, the orthonormal projection of $\bar{\nabla}_{X} Y$ to $T M$ is just $\nabla_{X} Y$. Thus $S(X, Y):=\bar{\nabla}_{X}(T i . Y)-T i . \nabla_{X} Y$ is a section of $T i^{\perp}$, and it is symmetric in $X, Y$ since

$$
\begin{aligned}
S(X, Y) & =\bar{\nabla}_{X}(T i . Y)-T i \circ \nabla_{X} Y=\left(\bar{\nabla}_{\bar{X}} \bar{Y}\right) \circ i-T i \circ \nabla_{X} Y \\
& =\left(\bar{\nabla}_{\bar{Y}} \bar{X}+[\bar{X}, \bar{Y}]\right) \circ i-T i \cdot\left(\nabla_{Y} X+[X, Y]\right)=S(Y, X) .
\end{aligned}
$$

For $f \in C^{\infty}(M)$ we have

$$
\begin{aligned}
S(f X, Y) & =\bar{\nabla}_{f X}(T i . Y)-T i \circ \nabla_{f X Y} \\
& =f \bar{\nabla}_{X}(T i . Y)-f T i \circ \nabla_{X} Y=f S(X, Y),
\end{aligned}
$$

and $S(X, f Y)=f S(X, Y)$ follows by symmetry.

26.2. Corollary. Let $c:[a, b] \rightarrow M$ be a smooth curve. Then we have

$$
\bar{\nabla}_{\partial_{t}}\left(T i . c^{\prime}\right)=\bar{\nabla}_{\partial_{t}}(i \circ c)^{\prime}=T i \circ \nabla_{\partial_{t}} c^{\prime}+S\left(c^{\prime}, c^{\prime}\right) .
$$

Consequently $c$ is a geodesic in $M$ if and only if

$$
\bar{\nabla}_{\partial t}(i \circ c)^{\prime}=S\left(c^{\prime}, c^{\prime}\right) \in T i^{\perp}
$$

i.e., the acceleration of $i \circ c$ in $\bar{M}$ is orthogonal to $M$.

Let $i: M \rightarrow \bar{M}$ be an isometric immersion. Then the following conditions are equivalent:

(1) Any geodesic in $\bar{M}$ which starts in $i(M)$ in a direction tangent to $i(M)$ stays in $i(M)$; it is then a geodesic in $i(M)$. We call $i: M \rightarrow \bar{M} a$ totally geodesic immersion.

(2) The second fundamental form $S$ of $i: M \rightarrow \bar{M}$ vanishes. 
26.3. In the setting of (26.1) we now investigate $\bar{\nabla}_{X} \xi$ where $X \in \mathfrak{X}(M)$ and where $\xi \in \Gamma\left(T i^{\perp}\right)$ is a normal field. We split it into tangential and normal components:

(1) $\bar{\nabla}_{X} \xi=-T i . L_{\xi}(X)+\nabla_{X}^{\perp} \xi \in \mathfrak{X}(M) \oplus \Gamma\left(T i^{\perp}\right) \quad$ (Weingarten formula).

\section{Proposition.}

(2) The mapping $(\xi, X) \mapsto L_{\xi}(X)$ is $C^{\infty}(M)$-bilinear; thus $L: T i^{\perp} \times_{M}$ $T M \rightarrow T M$ is a tensor field, called the Weingarten mapping or shape operator and we have:

$$
g\left(L_{\xi}(X), Y\right)=\bar{g}(S(X, Y), \xi), \quad \xi \in \Gamma\left(T i^{\perp}\right), X, Y \in \mathfrak{X}(M) .
$$

By the symmetry of $S, L_{\xi}: T M \rightarrow T M$ is a symmetric endomorphism with respect to $g$, i.e., $g\left(L_{\xi}(X), Y\right)=g\left(X, L_{\xi}(Y)\right)$.

(3) The mapping $(X, \xi) \mapsto \nabla_{X}^{\perp} \xi$ is a covariant derivative in the normal bundle $T i^{\perp} \rightarrow M$ which respects the metric $g^{\perp}:=\bar{g} \mid T i^{\perp} \times_{M} T i^{\perp}$; i.e.,

$$
\begin{aligned}
& \nabla^{\perp}: \mathfrak{X}(M) \times \Gamma\left(T i^{\perp}\right) \rightarrow \Gamma\left(T i^{\perp}\right) \quad \text { is } \mathbb{R} \text {-bilinear, } \\
& \nabla_{f \cdot X}^{\perp} \xi=f . \nabla_{X}^{\perp} \xi, \quad \nabla_{X}^{\perp}(f . \xi)=d f(X) . \xi+\nabla_{X}^{\perp} \xi, \\
& X\left(g^{\perp}(\xi, \eta)\right)=g^{\perp}\left(\nabla_{X}^{\perp} \xi, \eta\right)+g^{\perp}\left(\xi, \nabla_{X}^{\perp} \eta\right) .
\end{aligned}
$$

Note that there does not exist torsion for $\nabla^{\perp}$.

Proof. The mapping $(\xi, X) \mapsto L_{\xi}(X)$ is obviously $\mathbb{R}$-bilinear. Moreover,

$$
-T i . L_{\xi}(f . X)+\nabla_{f . X}^{\perp} \xi=\bar{\nabla}_{f . X} \xi=f . \bar{\nabla}_{X} \xi=-f .\left(T i . L_{\xi}(X)\right)+f . \nabla_{X}^{\perp} \xi
$$

which implies

$$
L_{\xi}(f . X)=f . L_{\xi}(X), \quad \nabla_{f . X}^{\perp} \xi=f . \nabla_{X}^{\perp} \xi .
$$

Furthermore,

$$
\begin{aligned}
-T i \cdot L_{f . \xi}(X)+\nabla_{X}^{\perp}(f \cdot \xi) & =\bar{\nabla}_{X}(f \cdot \xi)=d f(X) \cdot \xi+f \cdot \bar{\nabla}_{X} \xi \\
& =-f \cdot\left(T i \cdot L_{\xi}(X)\right)+\left(d f(X) \cdot \xi+f \cdot \nabla_{X}^{\perp} \xi\right)
\end{aligned}
$$

implies

$$
L_{f . \xi}(X)=f . L_{\xi}(X), \quad \nabla_{X}^{\perp}(f . \xi)=d f(X) \cdot \xi+f . \nabla_{X}^{\perp} \xi .
$$

For the rest we enlarge $X, Y \in \mathfrak{X}(M)$ and $\xi, \eta \in \Gamma\left(T i^{\perp}\right)$ locally to vector fields $\bar{X}, \bar{Y}, \bar{\xi}, \bar{\eta}$ on $\bar{M}$. Then we have:

$$
\begin{aligned}
X\left(g^{\perp}(\xi, \eta)\right) & =\bar{X}(\bar{g}(\bar{\xi}, \bar{\eta})) \circ i=\left(\bar{g}\left(\bar{\nabla}_{\bar{X}} \bar{\xi}, \bar{\eta}\right)+\bar{g}\left(\bar{\xi}, \bar{\nabla}_{\bar{X}} \bar{\eta}\right)\right) \circ i \\
& =\bar{g}\left(\bar{\nabla}_{X} \xi, \eta\right)+\bar{g}\left(\xi, \bar{\nabla}_{X} \eta\right) \\
& =\bar{g}\left(-T i . L_{\xi}(X)+\nabla_{X}^{\perp} \xi, \eta\right)+\bar{g}\left(\xi,-T i . L_{\eta}(X)+\nabla_{\bar{X}}^{\perp} \eta\right) \\
& =g^{\perp}\left(\nabla_{X}^{\perp} \xi, \eta\right)+g^{\perp}\left(\xi, \nabla_{X}^{\perp} \eta\right), \\
\bar{X}(\bar{g}(\bar{Y}, \bar{\xi})) & =\bar{g}\left(\bar{\nabla}_{\bar{X}} \bar{Y}, \bar{\xi}\right)+\bar{g}\left(\bar{Y}, \bar{\nabla}_{\bar{X}} \bar{\xi}\right) .
\end{aligned}
$$


Pull this back to $M$ :

$$
\begin{aligned}
0 & =X(\bar{g}(Y, \xi))=\bar{g}\left(\bar{\nabla}_{X}(T i . Y), \xi\right)+\bar{g}\left(T i . Y, \bar{\nabla}_{X} \xi\right) \\
& =\bar{g}\left(T i . \nabla_{X} Y+S(X, Y), \xi\right)+\bar{g}\left(T i . Y,-T i . L_{\xi}(X)+\nabla_{X}^{\perp} \xi\right) \\
& =g^{\perp}(S(X, Y), \xi)+g\left(Y,-L_{\xi}(X)\right) .
\end{aligned}
$$

26.4. Theorem. Let $(M, g) \stackrel{i}{\longrightarrow}(\bar{M}, \bar{g})$ be an isometric immersion of Riemann manifolds with Riemann curvatures $R$ and $\bar{R}$, respectively. Then we have:

(1) For $X_{i} \in \mathfrak{X}(M)$ or $T_{x} M$ we have (Gauß's equation, theorema egregium)

$$
\begin{aligned}
\bar{g}\left(\bar{R}\left(T i . X_{1}, T i . X_{2}\right)\left(T i . X_{3}\right), T i . X_{4}\right)=g\left(R\left(X_{1}, X_{2}\right) X_{3}, X_{4}\right) \\
+g^{\perp}\left(S\left(X_{1}, X_{3}\right), S\left(X_{2}, X_{4}\right)\right)-g^{\perp}\left(S\left(X_{2}, X_{3}\right), S\left(X_{1}, X_{4}\right)\right) .
\end{aligned}
$$

(2) The tangential part of $\bar{R}\left(X_{1}, X_{2}\right) X_{3}$ is given by:

$$
\begin{aligned}
\left(\bar{R}\left(T i . X_{1}, T i . X_{2}\right)\right. & \left.\left(T i . X_{3}\right)\right)^{\top} \\
& =R\left(X_{1}, X_{2}\right) X_{3}+L_{S\left(X_{1}, X_{3}\right)}\left(X_{2}\right)-L_{S\left(X_{2}, X_{3}\right)}\left(X_{1}\right) .
\end{aligned}
$$

(3) The normal part of $\bar{R}\left(X_{1}, X_{2}\right) X_{3}$ is (Codazzi-Mainardi equation):

$$
\begin{aligned}
& \left(\bar{R}\left(T i . X_{1}, T i . X_{2}\right)\left(T i . X_{3}\right)\right)^{\perp} \\
& \quad=\left(\nabla_{X_{1}}^{T i^{\perp} \otimes T^{*} M \otimes T^{*} M} S\right)\left(X_{2}, X_{3}\right)-\left(\nabla_{X_{2}}^{T i^{\perp} \otimes T^{*} M \otimes T^{*} M} S\right)\left(X_{1}, X_{3}\right) .
\end{aligned}
$$

(4) The tangential and the normal parts of $\bar{R}\left(T i . X_{1}, T i . X_{2}\right) \xi$ (where $\xi$ is a normal field along $i)$ are given by:

$$
\begin{aligned}
& \left(\bar{R}\left(T i . X_{1}, T i . X_{2}\right) \xi\right)^{\top} \\
& \quad=T i .\left(\left(\nabla_{X_{2}}^{T M \otimes\left(T i^{\perp}\right)^{*} \otimes T^{*} M} L\right)_{\xi}\left(X_{1}\right)-\left(\nabla_{X_{1}}^{T M \otimes\left(T i^{\perp}\right)^{*} \otimes T^{*} M} L\right)_{\xi}\left(X_{2}\right)\right), \\
& \quad\left(\bar{R}\left(T i . X_{1}, T i . X_{2}\right) \xi\right)^{\perp} \\
& \quad=R^{\nabla^{\perp}}\left(X_{1}, X_{2}\right) \xi+S\left(L_{\xi}\left(X_{1}\right), X_{2}\right)-S\left(L_{\xi}\left(X_{2}\right), X_{1}\right) .
\end{aligned}
$$

Proof. Every $x \in M$ has an open neighborhood $U$ such that $i: U \rightarrow \bar{M}$ is an embedding. Since the assertions are local, we may thus assume that $i$ is an embedding, and we may suppress $i$ in the following proof. For the proof we need vector fields $X_{i} \in \mathfrak{X}(M)$. We start from the Gauß formula (26.1).

$$
\begin{aligned}
\bar{\nabla}_{X_{1}}\left(\bar{\nabla}_{X_{2}} X_{3}\right) & =\bar{\nabla}_{X_{1}}\left(\nabla_{X_{2}} X_{3}+S\left(X_{2}, X_{3}\right)\right) \\
& =\nabla_{X_{1}} \nabla_{X_{2}} X_{3}+S\left(X_{1}, \nabla_{X_{2}} X_{3}\right)+\bar{\nabla}_{X_{1}} S\left(X_{2}, X_{3}\right), \\
\bar{\nabla}_{X_{2}}\left(\bar{\nabla}_{X_{1}} X_{3}\right) & =\nabla_{X_{2}} \nabla_{X_{1}} X_{3}+S\left(X_{2}, \nabla_{X_{1}} X_{3}\right)+\bar{\nabla}_{X_{2}} S\left(X_{1}, X_{3}\right), \\
\bar{\nabla}_{\left[X_{1}, X_{2}\right]} X_{3} & =\nabla_{\left[X_{1}, X_{2}\right]} X_{3}+S\left(\left[X_{1}, X_{2}\right], X_{3}\right) \\
& =\nabla_{\left[X_{1}, X_{2}\right]} X_{3}+S\left(\nabla_{X_{1}} X_{2}, X_{3}\right)-S\left(\nabla_{X_{2}} X_{1}, X_{3}\right) .
\end{aligned}
$$


Inserting this, we get for the part which is tangent to $M$ :

$$
\begin{aligned}
\bar{g}( & \bar{R} \\
= & \left.\left.X_{1}, X_{2}\right) X_{3}, X_{4}\right)=\bar{g}\left(\bar{\nabla}_{X_{1}} \bar{\nabla}_{X_{2}} X_{3}-\bar{\nabla}_{X_{2}} \bar{\nabla}_{X_{1}} X_{3}-\bar{\nabla}_{\left[X_{1}, X_{2}\right]} X_{3}, X_{4}\right) \\
& \quad+\bar{g}\left(S\left(X_{X_{2}}, X_{3}-\nabla_{X_{2}} \nabla_{X_{1}} X_{3}-\nabla_{\left[X_{1}, X_{2}\right]} X_{3}, X_{4}\right)\right. \\
& \quad+\bar{g}\left(\bar{\nabla}_{X_{1}} S\left({X_{2}}_{X_{1}} X_{3}\right)-S\left(\left[X_{1}, X_{2}\right], X_{3}\right), X_{4}\right) \quad(=0) \\
= & g\left(R\left(X_{1}, X_{2}\right) X_{3}, X_{4}\right) \\
& \left.+g^{\perp}\left(S\left(X_{1}, X_{3}\right), X_{4}\right), S\left(X_{2}, X_{4}\right)\right)-g^{\perp}\left(S\left(X_{2}, X_{3}\right), S\left(X_{1}, X_{4}\right)\right),
\end{aligned}
$$

where we also used (26.3.1) and (26.3.2) in:

$$
\begin{aligned}
\bar{g}\left(\bar{\nabla}_{X_{1}} S\left(X_{2}, X_{3}\right), X_{4}\right) & =\bar{g}\left(\nabla_{X_{1}}^{\perp} S\left(X_{2}, X_{3}\right)-L_{S\left(X_{2}, X_{3}\right)}\left(X_{1}\right), X_{4}\right) \\
& =0-g^{\perp}\left(S\left(X_{1}, X_{4}\right), S\left(X_{2}, X_{3}\right)\right) .
\end{aligned}
$$

So (11) and (2) follow. For equation (3) we have to compute the normal components of the +-- sum of the first three equations in this proof:

$$
\begin{aligned}
&\left(\bar{R}\left(X_{1}, X_{2}\right) X_{3}\right)^{\perp} \\
&=0+S\left(X_{1}, \nabla_{X_{2}} X_{3}\right)+\left(\bar{\nabla}_{X_{1}} S\left(X_{2}, X_{3}\right)\right)^{\perp}-0-S\left(X_{2}, \nabla_{X_{1}} X_{3}\right) \\
&-\left(\bar{\nabla}_{X_{2}} S\left(X_{1}, X_{3}\right)\right)^{\perp}-0-S\left(\nabla_{X_{1}} X_{2}, X_{3}\right)+S\left(\nabla_{X_{2}} X_{1}, X_{3}\right) \\
&=\left(\nabla_{X_{1}}^{\perp} S\left(X_{2}, X_{3}\right)-S\left(\nabla_{X_{1}} X_{2}, X_{3}\right)-S\left(X_{2}, \nabla_{X_{1}} X_{3}\right)\right) \\
&-\left(\nabla_{X_{2}}^{\perp} S\left(X_{1}, X_{3}\right)-S\left(\nabla_{X_{2}} X_{1}, X_{3}\right)-S\left(X_{1}, \nabla_{X_{2}} X_{3}\right)\right) \\
&=\left(\nabla_{X_{1}}^{T i \perp \otimes T^{*} M \otimes T^{*} M} S\right)\left(X_{2}, X_{3}\right)-\left(\nabla_{X_{2}}^{T i^{\perp} \otimes T^{*} M \otimes T^{*} M} S\right)\left(X_{1}, X_{3}\right) .
\end{aligned}
$$

For the proof of (4) we start from the Weingarten formula (26.3.1) and use $(26.1)$

$$
\begin{aligned}
\bar{\nabla}_{X_{1}}\left(\bar{\nabla}_{X_{2}} \xi\right) & =\bar{\nabla}_{X_{1}}\left(\nabla_{X_{2}}^{\perp} \xi-L_{\xi}\left(X_{2}\right)\right) \\
& =\nabla_{X_{1}}^{\perp} \nabla_{X_{2}}^{\perp} \xi-L_{\nabla_{X_{2}}} \xi \\
\bar{\nabla}_{X_{2}}\left(\bar{\nabla}_{X_{1}} \xi\right) & =\nabla_{X_{2}}^{\perp} \nabla_{X_{1}}^{\perp} \xi-L_{\nabla_{X_{1}}} \xi \\
\bar{\nabla}_{\left[X_{1}, X_{2}\right]} \xi & \left.=\nabla_{\left[X_{1}, X_{2}\right]}^{\perp} \xi-L_{\xi}\left(X_{2}\right)\right)-S\left(X_{X_{2}}\left(L_{\xi}, L_{\xi}\left(X_{1}\right)\right)-S\left(X_{2}\right)\right) \\
& \left.=\nabla_{\left[X_{1}, X_{2}\right]}^{\perp} \xi-L_{\xi}\left(X_{1}\right)\right),
\end{aligned}
$$

Inserting this, we get for the tangential part:

$$
\begin{aligned}
\left(\bar{R}\left(X_{1}, X_{2}\right) \xi\right)^{\top}=L_{\nabla_{X_{1}} \xi}\left(X_{2}\right)-L_{\nabla_{X_{2}} \xi}\left(X_{1}\right) & \\
& +\nabla_{X_{2}}\left(L_{\xi}\left(X_{1}\right)\right)-L_{\xi}\left(\nabla_{X_{2}} X_{1}\right)-\nabla_{X_{1}}\left(L_{\xi}\left(X_{2}\right)\right)+L_{\xi}\left(\nabla_{X_{1}} X_{2}\right) \\
= & -\left(\nabla_{X_{1}}^{T M \otimes\left(T i^{\perp}\right)^{*} \otimes T^{*} M} L\right)_{\xi}\left(X_{2}\right)+\left(\nabla_{X_{2}}^{T M \otimes\left(T i^{\perp}\right)^{*} \otimes T^{*} M} L\right)_{\xi}\left(X_{1}\right) .
\end{aligned}
$$


For the normal part we get:

$$
\begin{gathered}
\left(\bar{R}\left(X_{1}, X_{2}\right) \xi\right)^{\perp}=\nabla_{X_{1}}^{\perp} \nabla_{X_{2}}^{\perp} \xi-\nabla_{X_{2}}^{\perp} \nabla_{X_{1}}^{\perp} \xi-\nabla_{\left[X_{1}, X_{2}\right]}^{\perp} \xi \\
-S\left(X_{1}, L_{\xi}\left(X_{2}\right)\right)+S\left(X_{2}, L_{\xi}\left(X_{1}\right)\right) . \quad \square
\end{gathered}
$$

26.5. Hypersurfaces. Let $i:(M, g) \rightarrow(\bar{M}, \bar{g})$ be an isometrically embedded hypersurface, so that $\operatorname{dim}(\bar{M})=\operatorname{dim}(M)+1$. Let $\nu$ be a local unit normal field along $M$, i.e., $\nu \in \Gamma\left(T i^{\perp} \mid U\right)$ with $|\nu|_{\bar{g}}=1$. There are two choices for $\nu$.

Theorem. In this situation we have:

(1) $\bar{\nabla}_{X} \nu \in T M$ for all $X \in T M$.

(2) For $X, Y \in \mathfrak{X}(M)$ we have (Weingarten equation):

$$
\bar{g}\left(\bar{\nabla}_{X} \nu, Y\right)=-\bar{g}\left(\nu, \bar{\nabla}_{X} Y\right)=-g^{\perp}(\nu, S(X, Y)) .
$$

(3) $\bar{g}\left(\bar{\nabla}_{X} \nu, Y\right)=\bar{g}\left(\bar{\nabla}_{Y} \nu, X\right)$.

(4) If we put $s(X, Y):=g^{\perp}(\nu, S(X, Y))$, then $s$ is called the classical second fundamental form and the Weingarten equation (2) takes the following form:

$$
\bar{g}\left(\bar{\nabla}_{X} \nu, Y\right)=-s(X, Y) .
$$

(5) For hypersurfaces the Codazzi-Mainardi equation takes the following form:

$$
\bar{g}\left(\bar{R}\left(X_{1}, X_{2}\right) X_{3}, \nu\right)=\left(\nabla_{X_{1}} s\right)\left(X_{2}, X_{3}\right)-\left(\nabla_{X_{2}} s\right)\left(X_{1}, X_{3}\right) .
$$

Proof. (1) Since $1=\bar{g}(\nu, \nu)$, we get $0=X(\bar{g}(\nu, \nu))=2 \bar{g}\left(\bar{\nabla}_{X} \nu, \nu\right)$; thus $\bar{\nabla}_{X} \nu$ is tangent to $M$.

(2) Since $0=\bar{g}(\nu, Y)$, we get $0=X(\bar{g}(\nu, Y))=\bar{g}\left(\bar{\nabla}_{X} \nu, Y\right)+\bar{g}\left(\nu, \bar{\nabla}_{X} Y\right)$ and thus $\bar{g}\left(\bar{\nabla}_{X} \nu, Y\right)=-\bar{g}\left(\nu, \bar{\nabla}_{X} Y\right)=-\bar{g}\left(\nu, \nabla_{X} Y+S(X, Y)\right)=-\bar{g}(\nu, S(X, Y))$. (3) follows from (2) and symmetry of $S(X, Y)$. (4) is a reformulation.

(5) We put ourselves back into the proof of (26.4.3) and use $S(X, Y)=$ $s(X, Y) . \nu$ and the fact that $s \in \Gamma\left(S^{2} T^{*} M \mid U\right)$ is a $\left(\begin{array}{l}0 \\ 2\end{array}\right)$-tensor field so that $\nabla_{X} s$ makes sense. We have

$$
\bar{\nabla}_{X_{1}}\left(S\left(X_{2}, X_{3}\right)\right)=\bar{\nabla}_{X_{1}}\left(s\left(X_{2}, X_{3}\right) \cdot \nu\right)=X_{1}\left(s\left(X_{2}, X_{3}\right) \cdot \nu+s\left(X_{2}, X_{3}\right) \cdot \bar{\nabla}_{X_{1}} \nu\right.
$$

and $\bar{\nabla}_{X_{1}} \nu$ is tangential to $M$ by (1). Thus the normal part is:

$$
\begin{aligned}
& \left(\bar{\nabla}_{X_{1}}\left(S\left(X_{2}, X_{3}\right)\right)\right)^{\perp}=X_{1}\left(s\left(X_{2}, X_{3}\right)\right) . \nu \\
& \quad=\left(\nabla_{X_{1}} s\right)\left(X_{2}, X_{3}\right) . \nu+s\left(\nabla_{X_{1}} X_{2}, X_{3}\right) . \nu+s\left(X_{2}, \nabla_{X_{1}} X_{3}\right) . \nu
\end{aligned}
$$

Now we put this into the formula of the proof of (26.4.3):

$$
\left(\bar{R}\left(X_{1}, X_{2}\right) X_{3}\right)^{\perp}=S\left(X_{1}, \nabla_{X_{2}} X_{3}\right)+\left(\bar{\nabla}_{X_{1}}\left(S\left(X_{2}, X_{3}\right)\right)\right)^{\perp}-S\left(X_{2}, \nabla_{X_{1}} X_{3}\right)
$$




$$
\begin{aligned}
& -\left(\bar{\nabla}_{X_{2}}\left(S\left(X_{1}, X_{3}\right)\right)\right)^{\perp}-S\left(\nabla_{X_{1}} X_{2}, X_{3}\right)+S\left(\nabla_{X_{2}} X_{1}, X_{3}\right) \\
= & \left(\left(\nabla_{X_{1}} s\right)\left(X_{2}, X_{3}\right)-\left(\nabla_{X_{2}} s\right)\left(X_{1}, X_{3}\right)\right) \nu . \quad \square
\end{aligned}
$$

26.6. Remark (Theorema egregium proper). Let $M$ be a surface in $\mathbb{R}^{3}$; then $\bar{R}=0$ and by (26.4.1) we have for $X, Y \in T_{x} M$ :

$0=\langle\bar{R}(X, Y) X, Y\rangle=\langle R(X, Y) X, Y\rangle+s(X, X) . s(Y, Y)-s(Y, X) . s(X, Y)$.

Let us now choose a local coordinate system $(U,(x, y))$ on $M$ and put

$$
\begin{aligned}
& g=i^{*}\langle\quad\rangle=: E d x \otimes d x+F d x \otimes d y+F d y \otimes d x+G d y \otimes d y, \\
& s=: l d x \otimes d x+m d x \otimes d y+m d y \otimes d x+n d y \otimes d y,
\end{aligned}
$$

then $K=$ Gauß's curvature $=$ sectional curvature

$$
\begin{aligned}
& =-\frac{\left\langle R\left(\partial_{x}, \partial_{y}\right) \partial_{x}, \partial_{y}\right\rangle}{\left|\partial_{x}\right|^{2}\left|\partial_{y}\right|^{2}-\left\langle\partial_{x}, \partial_{y}\right\rangle^{2}}=\frac{s\left(\partial_{x}, \partial_{x}\right) \cdot s\left(\partial_{y}, \partial_{y}\right)-s\left(\partial_{x}, \partial_{y}\right)^{2}}{E G-F^{2}} \\
& =\frac{\ln -m^{2}}{E G-F^{2}},
\end{aligned}
$$

which is Gauß's formula for his curvature in his notation.

26.7. Adapted frames for isometric embeddings. All the following also hold for immersions. For notational simplicity we stick with embeddings. Let $e:(M, g) \rightarrow(\bar{M}, \bar{g})$ be an isometric embedding of Riemann manifolds, and let $\operatorname{dim}(\bar{M})=m+p$ and $\operatorname{dim}(M)=m$. An adapted orthonormal frame $\bar{s}=\left(\bar{s}_{1}, \ldots, \bar{s}_{m+p}\right)$ is an orthonormal frame for $\bar{M}$ over $\bar{U} \subset \bar{M}$ such that for $U=\bar{U} \cap M \subset M$ the fields $s_{1}=\left.\bar{s}_{1}\right|_{U}, \ldots, s_{m}=\left.\bar{s}_{m}\right|_{U}$ are tangent to $M$. Thus $s=\left(s_{1}, \ldots, s_{m}\right)$ is an orthonormal frame for $M$ over $U$. The orthonormal coframe

$$
\bar{\sigma}=\left(\begin{array}{c}
\bar{\sigma}^{1} \\
\vdots \\
\bar{\sigma}^{m+p}
\end{array}\right)=\left(\bar{\sigma}^{1}, \ldots, \bar{\sigma}^{m+p}\right)^{\top}
$$

for $\bar{M}$ over $\bar{U}$ dual to $\bar{s}$ is then given by $\bar{\sigma}^{\bar{\imath}}\left(\bar{s}_{\bar{\jmath}}\right)=\delta_{\bar{\jmath}}$. We recall from (25.5)

(1) $\bar{g}=\sum_{\bar{\imath}=1}^{m+p} \bar{\sigma}^{\bar{\imath}} \otimes \bar{\sigma}^{\bar{\imath}}$,

$$
\begin{aligned}
& \bar{\nabla} \bar{s}=\bar{s} \cdot \bar{\omega}, \quad \bar{\omega}_{\bar{\jmath}}^{\bar{c}}=-\bar{\omega}_{\bar{\imath}}^{\bar{\jmath}}, \quad \text { so } \bar{\omega} \in \Omega^{1}(\bar{U}, \mathfrak{s o}(m+p)) \\
& d \bar{\sigma}+\bar{\omega} \wedge \bar{\sigma}=0, \quad d \bar{\sigma}^{\bar{\imath}}+\sum_{\bar{k}=1}^{m+p} \bar{\omega}_{\bar{k}}^{\bar{c}} \wedge \bar{\sigma}^{\bar{k}}=0, \\
& \bar{R} \bar{s}=\bar{s} . \bar{\Omega}, \quad \bar{\Omega}=d \bar{\omega}+\bar{\omega} \wedge \bar{\omega} \in \Omega^{2}(\bar{U}, \mathfrak{s o}(m+p)) \\
& \quad \bar{\Omega}_{\bar{\jmath}}^{\bar{\imath}}=d \bar{\omega}_{\bar{\jmath}}^{\bar{\imath}}+\sum_{\bar{k}=1}^{m+p} \bar{\omega}_{\bar{k}}^{\bar{\imath}} \wedge \bar{\omega}_{\bar{\jmath}}^{\bar{k}}, \\
& \bar{\Omega} \wedge \bar{\sigma}=0, \quad \sum_{\bar{k}=1}^{m+p} \bar{\Omega}_{\bar{k}}^{\bar{l}} \wedge \bar{\sigma}^{\bar{k}}=0, \quad \text { first Bianchi identity, } \\
& d \bar{\Omega}+\bar{\omega} \wedge \bar{\Omega}-\bar{\Omega} \wedge \bar{\omega}=d \bar{\Omega}+[\bar{\omega}, \bar{\Omega}]_{\wedge}=0, \quad \text { second Bianchi identity. }
\end{aligned}
$$


Likewise, the orthonormal coframe $\sigma=\left(\sigma^{1}, \ldots, \sigma^{m}\right)^{\top}$ for $M$ over $U$ dual to $s$ is then given by $\sigma^{i}\left(s_{j}\right)=\delta_{j}^{i}$. Recall again from (25.5),

$$
\begin{aligned}
& g=\sum_{i=1}^{m} \sigma^{i} \otimes \sigma^{i}, \\
& \nabla s=s . \omega, \quad \omega_{j}^{i}=-\omega_{i}^{j}, \quad \text { so } \omega \in \Omega^{1}(U, \mathfrak{s o}(m)), \\
& d \sigma+\omega \wedge \sigma=0, \quad d \sigma^{i}+\sum_{k=1}^{m} \omega_{k}^{i} \wedge \sigma^{k}=0, \\
& R s=s . \Omega, \quad \Omega=d \omega+\omega \wedge \omega \in \Omega^{2}(U, \mathfrak{s o}(m)), \\
& \quad \Omega_{j}^{i}=d \omega_{j}^{i}+\sum_{k=1}^{m} \omega_{k}^{i} \wedge \omega_{j}^{k}, \\
& \Omega \wedge \sigma=0, \quad \sum_{k=1}^{m} \Omega_{k}^{i} \wedge \sigma^{k}=0, \quad \text { first Bianchi identity, } \\
& d \Omega+\omega \wedge \Omega-\Omega \wedge \omega=d \Omega+[\omega, \Omega]_{\wedge}=0, \quad \text { second Bianchi identity. }
\end{aligned}
$$

Obviously we have $\left.\bar{\sigma}^{i}\right|_{U}=\sigma^{i}$, more precisely $e^{*} \bar{\sigma}^{i}=\sigma^{i}$, for $i=1, \ldots, m$, and $e^{*} \bar{\sigma}^{\bar{\imath}}=0$ for $\bar{\imath}=m+1, \ldots, m+p$. We want to compute $e^{*} \overline{\boldsymbol{\omega}}$. From $d \bar{\sigma}^{\bar{\imath}}+\sum_{\bar{k}=1}^{m+p} \bar{\omega}_{\bar{k}}^{\bar{\imath}} \wedge \bar{\sigma}^{\bar{k}}=0$ we get

$$
\begin{aligned}
& d \sigma^{i}=-\sum_{\bar{k}=1}^{m+p} e^{*} \bar{\omega}_{\bar{k}}^{i} \wedge e^{*} \bar{\sigma}^{\bar{k}}=-\sum_{k=1}^{m} e^{*} \bar{\omega}_{k}^{i} \wedge \sigma^{k} \quad \text { for } i=1, \ldots, m, \\
& 0=-\sum_{\bar{k}=1}^{m+p} e^{*} \bar{\omega}_{\bar{k}}^{\bar{\imath}} \wedge e^{*} \bar{\sigma}^{\bar{k}}=-\sum_{k=1}^{m} e^{*} \bar{\omega}_{k}^{\bar{l}} \wedge \sigma^{k} \quad \text { for } m+1 \leq \bar{\imath} .
\end{aligned}
$$

Since also $e^{*} \bar{\omega}_{j}^{i}=-e^{*} \bar{\omega}_{i}^{j}$, the forms $e^{*} \bar{\omega}_{j}^{i}$ for $1 \leq i, j \leq m$ satisfy the defining equations for $\omega_{j}^{i}$; thus we have:

$$
\omega_{j}^{i}=e^{*} \bar{\omega}_{j}^{i}, \quad \text { for } 1 \leq i, j \leq m .
$$

Since $\bar{g}\left(\bar{\nabla}_{X} s_{i}, s_{j}\right)=\bar{\omega}_{i}^{j}(X)=\omega_{i}^{j}(X)=g\left(\nabla_{X} s_{i}, s_{j}\right)$ for $X \in \mathfrak{X}(M)$, equation (41) also expresses the fact that the tangential part $\left(\bar{\nabla}_{X} s_{i}\right)^{\top}=\nabla_{X} s_{i}$.

Next we want to investigate the forms $e^{*} \bar{\omega}_{\bar{\jmath}}^{i}=-e^{*} \bar{\omega}_{i}^{\bar{\jmath}}$ for $1 \leq i \leq m$ and $m+1 \leq \bar{\jmath} \leq m+p$. We shall need the following result.

(5) Lemma (E. Cartan). For $\bar{U}$ open in $\bar{M}^{m+p}$, let $\lambda^{1}, \ldots, \lambda^{m} \in \Omega^{1}(\bar{U})$ be everywhere linearly independent, and consider 1 -forms $\mu_{1}, \ldots, \mu_{m} \in \Omega^{1}(\bar{U})$ such that $\sum_{i=1}^{m} \mu_{i} \wedge \lambda^{i}=0$. Then there exist unique smooth functions $f_{i j} \in$ $C^{\infty}(\bar{U})$ satisfying $\mu_{i}=\sum_{j=1}^{m} f_{i j} \lambda^{j}$ and $f_{i j}=f_{j i}$.

Proof. Near each point we may find $\lambda^{m+1}, \ldots, \lambda^{m+p}$ such that $\lambda^{1}, \ldots, \lambda^{m+p}$ are everywhere linearly independent; thus they form a coframe. Then there exist unique $f_{i j}$ such that $\mu_{i}=\sum_{\bar{k}=1}^{m+p} f_{i \bar{\jmath}} \lambda^{\bar{\jmath}}$. But we have

$$
\begin{aligned}
0 & =\sum_{i=1}^{m} \mu_{i} \wedge \lambda^{i}=\sum_{i=1}^{m} \sum_{\bar{k}=1}^{m+p} f_{i \bar{k}} \lambda^{\bar{k}} \wedge \lambda^{i} \\
& =\sum_{1 \leq k<i \leq m}\left(f_{i k}-f_{k i}\right) \lambda^{k} \wedge \lambda^{i}+\sum_{i=1}^{m} \sum_{\bar{k}=m+1}^{m+p} f_{i \bar{k}} \lambda^{\bar{k}} \wedge \lambda^{i}
\end{aligned}
$$


Since the $\lambda^{\bar{k}} \wedge \lambda^{\bar{\imath}}$ for $\bar{k}<\bar{\imath}$ are linearly independent, we conclude that $f_{i k}=f_{k i}$ for $1 \leq i, k \leq m$ and $f_{i \bar{k}}=0$ for $1 \leq i \leq m<\bar{k} \leq m+p$.

By (3) we have $0=\sum_{k=1}^{m} e^{*} \bar{\omega}_{k}^{\bar{\imath}} \wedge \sigma^{k}$ for $\bar{\imath}=m+1 \ldots m+p$. We use now lemma (5) to see that there exist unique functions $s_{k j}^{\bar{i}} \in C^{\infty}(U)$ for $1 \leq j, k \leq m$ and $\bar{\imath}=m+1, \ldots, m+p$ with:

$$
e^{*} \bar{\omega}_{k}^{\bar{\imath}}=\sum_{j=1}^{m} s_{k j}^{\bar{\imath}} \sigma^{j}, \quad s_{k j}^{\bar{i}}=s_{j k}^{\bar{\imath}} .
$$

This is equivalent to the Weingarten formula (26.3.1).

Since $\bar{g}\left(\bar{\nabla}_{s_{k}} s_{j}, \bar{s}_{\bar{\imath}}\right)=\bar{\omega}_{j}^{\bar{\imath}}\left(s_{k}\right)=\left(e^{*} \bar{\omega}_{j}^{\bar{\imath}}\right)\left(s_{k}\right)=s_{j k}^{\bar{\imath}}$, we have by $(26.1)$

$$
S\left(s_{i}, s_{j}\right)=\sum_{\bar{k}=m+1}^{m+p}\left(\bar{s}_{\bar{k}} \mid U\right)\left(e^{*} \omega_{j}^{\bar{k}}\right)\left(s_{i}\right)=\sum_{\bar{k}=m+1}^{m+p}\left(\bar{s}_{\bar{k}} \mid U\right) s_{i j}^{\bar{k}} .
$$

Let us now investigate the second structure equation $\bar{\Omega}_{\bar{\jmath}}^{\bar{\imath}}=d \bar{\omega}_{\bar{\jmath}}^{\bar{\imath}}+\sum_{\bar{k}=1}^{m+p} \bar{\omega}_{\bar{k}}^{\bar{\imath}} \wedge$ $\bar{\omega} \overline{\bar{j}}$. We look first at indices $1 \leq i, j \leq m$ and restrict it to $M$ :

$$
\begin{aligned}
e^{*} \bar{\Omega}_{j}^{i} & =d e^{*} \bar{\omega}_{j}^{i}+\sum_{k=1}^{m} e^{*} \bar{\omega}_{k}^{i} \wedge e^{*} \bar{\omega}_{j}^{k}+\sum_{\bar{k}=m+1}^{m+p} e^{*} \bar{\omega}_{\bar{k}}^{i} \wedge e^{*} \bar{\omega}_{j}^{\bar{k}} \\
& =d \omega_{j}^{i}+\sum_{k=1}^{m} \omega_{k}^{i} \wedge \omega_{j}^{k}+\sum_{\bar{k}=m+1}^{m+p} e^{*} \bar{\omega}_{\bar{k}}^{i} \wedge e^{*} \bar{\omega}_{j}^{\bar{k}}, \\
e^{*} \bar{\Omega}_{j}^{i} & =\Omega_{j}^{i}+\sum_{\bar{k}=m+1}^{m+p} e^{*} \bar{\omega}_{\bar{k}}^{i} \wedge e^{*} \omega_{j}^{\bar{k}}=\Omega_{j}^{i}-\sum_{\bar{k}=m+1}^{m+p} \sum_{l, n=1}^{m} s_{i l}^{\bar{k}} s_{j n}^{\bar{k}} \sigma^{l} \wedge \sigma^{n} .
\end{aligned}
$$

This is equivalent to the Gauß equation (26.4.1).

Then we look at the indices $1 \leq j \leq m<\bar{\imath} \leq m+p$ and restrict the second structure equation to $M$ :

$$
\begin{aligned}
e^{*} \bar{\Omega}_{j}^{\bar{\imath}} & =d e^{*} \bar{\omega}_{j}^{\bar{\imath}}+\sum_{k=1}^{m} e^{*} \bar{\omega}_{k}^{\bar{\imath}} \wedge e^{*} \bar{\omega}_{j}^{k}+\sum_{\bar{k}=m+1}^{m+p} e^{*} \bar{\omega}_{\bar{k}}^{\bar{\tau}} \wedge e^{*} \bar{\omega}_{j}^{\bar{k}} \\
& =d e^{*} \bar{\omega}_{j}^{\bar{\imath}}+\sum_{k=1}^{m} e^{*} \bar{\omega}_{k}^{\bar{\imath}} \wedge \omega_{j}^{k}+\sum_{\bar{k}=m+1}^{m+p} e^{*} \bar{\omega}_{\bar{k}}^{\bar{\imath}} \wedge e^{*} \bar{\omega}_{j}^{\bar{k}}
\end{aligned}
$$

which is equivalent to the Codazzi-Mainardi equation. In the case of a hypersurface this takes the simpler form:

$$
e^{*} \bar{\Omega}_{j}^{m+1}=d e^{*} \bar{\omega}_{j}^{m+1}+\sum_{k=1}^{m} e^{*} \bar{\omega}_{k}^{m+1} \wedge \omega_{j}^{k} .
$$


26.8. Resumé of computing with adapted frames for submanifolds. Let $e:(M, g) \rightarrow(\bar{M}, \bar{g})$ be an isometric embedding between Riemann manifolds. Let $\bar{s}=\left(\bar{s}_{1}, \ldots, \bar{s}_{m+p}\right)$ be an orthonormal local frame on $\bar{M}$ over $\bar{U} \subset \bar{M}$ with connection 1-form $\bar{\omega}=\left(\bar{\omega}_{\bar{\jmath}}^{\bar{\imath}}\right) \in \Omega^{1}(U, \mathfrak{s o}(m+p))$ and curvature 2 -form $\bar{\Omega}=\left(\bar{\Omega}_{\bar{\jmath}}^{\bar{\imath}}\right) \in \Omega^{2}(U, \mathfrak{s o}(m+p))$, such that the $s_{i}:=\bar{s}_{i} \mid U$ form a local orthonormal frame $s=\left(s_{1}, \ldots, s_{m}\right)$ of $T M$ over $U=\bar{U} \cap M$, with connection 1-form $\omega=\left(\omega_{j}^{i}\right) \in \Omega^{1}(U, \mathfrak{s o}(m))$ and curvature 2-form $\Omega=\left(\Omega_{j}^{i}\right) \in \Omega^{2}(U, \mathfrak{s o}(m))$. Let

$$
\bar{\sigma}=\left(\begin{array}{c}
\bar{\sigma}^{1} \\
\vdots \\
\bar{\sigma}^{m+p}
\end{array}\right), \quad \sigma=\left(\begin{array}{c}
\sigma^{1} \\
\vdots \\
\sigma^{m}
\end{array}\right)
$$

be the dual coframes. Using the ranges of indices $1 \leq i, j, k, l \leq m$ and $m+1 \leq \bar{\imath}, \bar{\jmath}, \bar{k} \leq m+p$, we then have:

$$
\begin{aligned}
& e^{*} \bar{\sigma}^{i}=\sigma^{i}, \quad e^{*} \bar{\sigma}^{\bar{\imath}}=0, \\
& e^{*} \bar{\omega}_{j}^{i}=\omega_{j}^{i}, \quad e^{*} \bar{\omega}_{j}^{\bar{\imath}}=\sum_{k \leq m} s_{j k}^{\bar{\imath}} \sigma^{k}, \quad s_{j k}^{\bar{\imath}}=s_{k j}^{\bar{\imath}}, \\
& e^{*} \bar{\Omega}_{j}^{i}=\Omega_{j}^{i}+\sum_{m<\bar{k}} e^{*} \bar{\omega}_{\bar{k}}^{i} \wedge e^{*} \bar{\omega}_{j}^{\bar{k}}=\Omega_{j}^{i}-\sum_{\bar{k}=m+1}^{m+p} \sum_{l, n=1}^{m} s_{i l}^{\bar{k}} s_{j n}^{\bar{k}} \sigma^{l} \wedge \sigma^{n}, \\
& e^{*} \bar{\Omega}_{j}^{\bar{\imath}}=d e^{*} \bar{\omega}_{j}^{\bar{\imath}}+\sum_{k=1}^{m} e^{*} \bar{\omega}_{k}^{\bar{c}} \wedge \omega_{j}^{k}+\sum_{\bar{k}=m+1}^{m+p} e^{*} \bar{\omega}_{\bar{k}}^{\bar{c}} \wedge e^{*} \bar{\omega}_{j}^{\bar{k}} .
\end{aligned}
$$

26.9. Definitions. Let $p: E \rightarrow B$ be a submersion of smooth manifolds, that is, $T p: T E \rightarrow T B$ is surjective. Then

$$
V=V(p)=V(E):=\operatorname{ker}(T p)
$$

is called the vertical subbundle of $E$. If $E$ is a Riemann manifold with metric $g$, then we can go on to define the horizontal subbundle of $E$ :

$$
\operatorname{Hor}=\operatorname{Hor}(p)=\operatorname{Hor}(E)=\operatorname{Hor}(E, g):=V(p)^{\perp} .
$$

If both $\left(E, g_{E}\right)$ and $\left(B, g_{B}\right)$ are Riemann manifolds, then we will call $p$ a Riemann submersion if

$$
T_{x} p: \operatorname{Hor}(p)_{x} \rightarrow T_{p(x)} B
$$

is an isometric isomorphism for all $x \in E$.

Examples. For any two Riemann manifolds $M, N$, the projection $p r_{1}$ : $M \times N \rightarrow M$ is a Riemann submersion. Here the Riemann metric on the product $M \times N$ is given by

$$
g_{M \times N}\left(X_{M}+X_{N}, Y_{M}+Y_{N}\right):=g_{M}\left(X_{M}, Y_{M}\right)+g_{N}\left(X_{N}, Y_{N}\right)
$$

using $T(M \times N) \cong T M \oplus T N$. In particular, $\mathbb{R}^{m+n} \rightarrow R^{m}$ with the usual metric, or $p r_{2}: S^{n} \times \mathbb{R}^{+} \rightarrow R^{+}$are Riemann submersions. 
26.10. Definition. Let $p: E \rightarrow B$ be a Riemann submersion. A vector field $X \in \mathfrak{X}(E)$ is called:

- vertical if $X(x) \in V_{x}(p)$ for all $x$ (i.e., if $T p . X(x)=0$ ),

- horizontal if $X(x) \in \operatorname{Hor}_{x}(p)$ for all $x$ (i.e., if $X(x) \perp V_{x}(p)$ ),

- projectable if there is an $\eta \in \mathfrak{X}(B)$, such that Tp.X $=\eta \circ p$,

- basic if it is horizontal and projectable.

Any vector field $Y \in \mathfrak{X}(E)$ can be uniquely decomposed as

$$
Y=Y^{\mathrm{ver}}+Y^{\text {hor }}
$$

into its vertical and horizontal components. The orthogonal projection $\Phi: T E \rightarrow V(E)$ with respect to the Riemann metric is a (generalized) connection on the bundle $(E, p, B)$ in the sense of (17.3) and defines a local parallel transport over each curve in $B$ (denoted by $\mathrm{Pt}^{\Phi}(c,$.$) ) as well as the$ horizontal lift of tangent vectors:

$$
C: T B \times{ }_{B} E \longrightarrow E, \quad\left(X_{b}, e\right) \mapsto Y_{e},
$$

where $Y_{e} \in \operatorname{Hor}_{e}(p)$ with $T_{e} p . Y_{e}=X_{b}$. This map also gives us an isomorphism $C_{*}: \mathfrak{X}(B) \rightarrow \mathfrak{X}_{\text {basic }}(E)$ between the vector fields on $B$ and the basic vector fields.

26.11. Lemma. Consider a Riemann submersion $p:\left(E, g_{E}\right) \rightarrow\left(B, g_{B}\right)$ with connection $\Phi: T E \rightarrow V(p)$ and $c:[0,1] \rightarrow B$, a geodesic. Then we have:

(1) The length is preserved by lifting curves horizontally:

$$
L_{0}^{t}(c)=L_{0}^{t}\left(\mathrm{Pt}^{\Phi}(c, ., u)\right),
$$

where $u \in E_{c(0)}$ is the starting point of the parallel transport. Also the energy is preserved, $E_{0}^{t}(c)=E_{0}^{t}\left(\mathrm{Pt}^{\Phi}(c, ., u)\right)$.

(2) $\operatorname{Pt}^{\Phi}(c, ., u) \perp E_{c(t)}$ for all $t$.

(3) If $c$ is a geodesic of minimal length in $B$, then we have

$$
L_{0}^{1}\left(\mathrm{Pt}^{\Phi}(c, ., u)\right)=\operatorname{dist}\left(E_{c(0)}, E_{c(1)}\right) .
$$

(4) If $c$ is a geodesic in $B$, then $t \mapsto \mathrm{Pt}^{\Phi}(c, t, u)$ is a geodesic in $E$.

(5) For vector fields $\xi, \eta \in \mathfrak{X}(B)$ and the corresponding horizontal lifts $C(\xi), C(\eta) \in \mathfrak{X}(E)$, we have

$$
\left(\nabla_{C(\xi)}^{E} C(\eta)\right)^{h o r}=C\left(\nabla_{\xi}^{B} \eta\right) .
$$


Proof. (11) Since $\partial_{s} \operatorname{Pt}^{\Phi}(c, s, u)$ is a horizontal vector and by the property of $p$ as Riemann submersion, we have

$$
\begin{aligned}
L_{0}^{t}\left(\mathrm{Pt}^{\Phi}(c, ., u)\right) & =\int_{0}^{t} g_{E}\left(\partial_{s} \mathrm{Pt}^{\Phi}(c, s, u), \partial_{s} \mathrm{Pt}^{\Phi}(c, s . u)\right)^{\frac{1}{2}} d s \\
& =\int_{0}^{t} g_{B}\left(c^{\prime}(s), c^{\prime}(s)\right)^{\frac{1}{2}} d s=L_{0}^{t}(c), \\
E_{0}^{t}\left(\mathrm{Pt}^{\Phi}(c, ., u)\right) & =\frac{1}{2} \int_{0}^{t} g_{E}\left(\partial_{s} \mathrm{Pt}^{\Phi}(c, s, u), \partial_{s} \mathrm{Pt}^{\Phi}(c, s . u)\right) d s=E_{0}^{t}(c) .
\end{aligned}
$$

(2) This is due to our choice of $\Phi$ as orthogonal projection onto the vertical bundle in terms of the given metric on $E$. By this choice, the parallel transport is the unique horizontal curve covering $c$, so it is orthogonal to each fiber $E_{c(t)}$ it meets.

(3) Consider a (piecewise) smooth curve $e:[0,1] \rightarrow E$ from $E_{c(0)}$ to $E_{c(1)}$; then $p \circ e$ is a (piecewise) smooth curve from $c(0)$ to $c(1)$. Since $c$ is a minimal geodesic, we have $L_{0}^{1}(c) \leq L_{0}^{1}(p \circ e)$. Furthermore, we can decompose the vectors tangent to $e$ into horizontal and vertical components and use the fact that $T p$ is an isometry on horizontal vectors to show that $L_{0}^{1}(e) \geq L_{0}^{1}(p \circ e)$ :

$$
\begin{aligned}
L_{0}^{1}(e)=\int_{0}^{1} \mid e^{\prime}(t)^{\mathrm{ver}} & +\left.e^{\prime}(t)^{\mathrm{hor}}\right|_{g_{E}} d t \\
& \geq \int_{0}^{1}\left|e^{\prime}(t)^{\mathrm{hor}}\right|_{g_{E}} d t=\int_{0}^{1}\left|(p \circ e)^{\prime}(t)\right|_{g_{M}} d t=L_{0}^{1}(p \circ e) .
\end{aligned}
$$

Now with (1) we can conclude that for all (piecewise) smooth curves $e$ from $E_{c(0)}$ to $E_{c(1)}$ we have:

$$
\begin{aligned}
& L_{0}^{1}(e) \geq L_{0}^{1}(p \circ e) \geq L_{0}^{1}(c)=L_{0}^{1}\left(\mathrm{Pt}^{\Phi}(c, ., u)\right) ; \\
& \text { thus } \quad L_{0}^{1}\left(\mathrm{Pt}^{\Phi}(c, ., u)\right)=\operatorname{dist}\left(E_{c(0)}, E_{c(1)}\right) .
\end{aligned}
$$

(41) This is a consequence of (3) and the observation from (22.4) that every curve which minimizes length or energy locally is a geodesic.

(5) Since $g_{E}(C(\xi), C(\eta))=g_{B}(\xi, \eta) \circ p$ and since $C(\xi)$ is $p$-related to $\xi$ and $C(\eta)$ is $p$-related to $\eta$, we get that $[C(\xi), C(\eta)]$ is $p$-related to $[\xi, \eta]$. We can then apply the implicit equation (22.5.7) for the covariant derivative twice:

$$
\begin{aligned}
& 2 g_{E}\left(\left(\nabla_{C(\xi)}^{E} C(\eta)\right)^{\mathrm{hor}}, C(\zeta)\right)=2 g_{E}\left(\nabla_{C(\xi)}^{E} C(\eta), C(\zeta)\right) \\
& =C(\xi)\left(g_{E}(C(\eta), C(\zeta))\right)+C(\eta)\left(g_{E}(C(\zeta), C(\xi))\right)-C(\zeta)\left(g_{E}(C(\xi), C(\eta))\right) \\
& -g_{E}(C(\xi),[C(\eta), C(\zeta)])+g_{E}(C(\eta),[C(\zeta), C(\xi)])+g_{E}(C(\zeta),[C(\xi), C(\eta)]) \\
& =\left(\xi\left(g_{B}(\eta, \zeta)\right)+\eta\left(g_{B}(\zeta, \xi)\right)-\zeta\left(g_{B}(\xi, \eta)\right)\right. \\
& \left.\quad-g_{B}(\xi,[\eta, \zeta])+g_{B}(\eta,[\zeta, \xi])+g_{B}(\zeta,[\xi, \eta])\right) \circ p=2 g_{B}\left(\nabla_{\xi}^{B} \eta, \zeta\right) \circ p .
\end{aligned}
$$


Since this holds for any $\zeta \in \mathfrak{X}(B)$, we conclude

$$
\left(\nabla_{C(\xi)}^{E} C(\eta)\right)^{\text {hor }}=C\left(\nabla_{\xi}^{B} \eta\right) \text {. }
$$

26.12. Corollary. Consider a Riemann submersion $p: E \rightarrow B$, and let $c:[0,1] \rightarrow E$ be a geodesic in $E$ with the property $c^{\prime}\left(t_{0}\right) \perp E_{p\left(c\left(t_{0}\right)\right)}$ for some $t_{0}$. Then $c^{\prime}(t) \perp E_{p(c(t))}$ for all $t \in[0,1]$ and $p \circ c$ is a geodesic in $B$.

Proof. Consider the curve $f: t \mapsto \exp _{p\left(c\left(t_{0}\right)\right)}^{B}\left(t T_{c\left(t_{0}\right)} p \cdot c^{\prime}\left(t_{0}\right)\right)$. It is a geodesic in $B$ and therefore lifts to a geodesic $e(t)=\mathrm{Pt}^{\Phi}\left(f, t-t_{0}, c\left(t_{0}\right)\right)$ in $E$ by (26.11.4). Also $e\left(t_{0}\right)=c\left(t_{0}\right)$ and $e^{\prime}\left(t_{0}\right)=C\left(T_{c\left(t_{0}\right)} p \cdot c^{\prime}\left(t_{0}\right), c\left(t_{0}\right)\right)=c^{\prime}\left(t_{0}\right)$ since $c^{\prime}\left(t_{0}\right) \perp E_{p\left(c\left(t_{0}\right)\right)}$ is horizontal. But geodesics are uniquely determined by their starting point and starting vector. Therefore $e=c$; thus $e$ is orthogonal to each fiber it meets by (26.11.2) and it projects onto the geodesic $f$ in $B$.

26.13. Corollary. Let $p: E \rightarrow B$ be a Riemann submersion. If $\operatorname{Hor}(E)$ is integrable, then:

(1) Every leaf is totally geodesic in the sense of (26.2).

(2) For each leaf $L$ the restriction $p: L \rightarrow B$ is a local isometry.

Proof. (11) follows from corollary (26.12), while (2) is just a direct consequence of the definitions.

26.14. Remark. If $p: E \rightarrow B$ is a Riemann submersion, then $\left.\operatorname{Hor}(E)\right|_{E_{b}}=$ $\operatorname{Nor}\left(E_{b}\right)$ for all $b \in B$ and $p$ defines a global parallelism as follows. A section $\tilde{v} \in C^{\infty}\left(\operatorname{Nor}\left(E_{b}\right)\right)$ is called $p$-parallel if $T_{e} p . \tilde{v}(e)=v \in T_{b} B$ is the same point for all $e \in E_{b}$. There is also a second parallelism. It is given by the induced covariant derivative: A section $\tilde{v} \in C^{\infty}\left(\operatorname{Nor}\left(E_{b}\right)\right)$ is called parallel if $\nabla^{\text {Nor }} \tilde{v}=0$. The $p$-parallelism is always flat and with trivial holonomy which is not generally true for $\nabla^{\text {Nor }}$. Yet we will see later on that if $\operatorname{Hor}(E)$ is integrable, then the two parallelisms coincide.

26.15. Definition. A Riemann submersion $p: E \rightarrow B$ is called integrable if $\operatorname{Hor}(E)=(\operatorname{ker} T p)^{\perp}$ is an integrable distribution.

26.16. Structure theory of Riemann submersions. Let $p:\left(E, g^{E}\right) \rightarrow$ $\left(B, g^{B}\right)$ be a Riemann submersion. We consider first the second fundamental form $S^{E_{b}}: T E_{b} \times_{E_{b}} T E_{b} \rightarrow \operatorname{Hor}(E)$ of the submanifold $E_{b}:=p^{-1}(b)$ in $E$. By (26.1), $S^{E_{b}}$ is given as:

$$
\begin{gathered}
S^{E_{b}}\left(X^{\mathrm{ver}}, Y^{\mathrm{ver}}\right)=\nabla_{X}^{E \text { ver }} Y^{\mathrm{ver}}-\nabla_{X^{\mathrm{ver}}}^{E_{b}} Y^{\mathrm{ver}}=\nabla_{X}^{E \text { ver }} Y^{\mathrm{ver}}-\left(\nabla_{X^{\mathrm{ver}}}^{E} Y^{\mathrm{ver}}\right)^{\mathrm{ver}} \\
=\left(\nabla_{X^{\mathrm{ver}}}^{E} Y^{\mathrm{ver}}\right)^{\mathrm{hor}}=\left(\nabla_{X^{\mathrm{ver}}}^{E} Y^{\mathrm{ver}}\right)^{\text {hor }} .
\end{gathered}
$$


The covariant derivative on the normal bundle $\operatorname{Nor}\left(E_{b}\right)=\left.\operatorname{Hor}(E)\right|_{E_{b}} \rightarrow E_{b}$ is given by the Weingarten formula (26.3) as the corresponding projection:

$$
\begin{aligned}
\nabla^{\text {Nor }}: \mathfrak{X}\left(E_{b}\right) \times \Gamma\left(\operatorname{Nor}\left(E_{b}\right)\right) \rightarrow \Gamma\left(\operatorname{Nor}\left(E_{b}\right)\right), \\
\nabla_{X^{\text {ver }}}^{\text {Nor }} Y^{\text {hor }}=\left(\nabla_{X^{\text {ver }}}^{E} Y^{\text {hor }}\right)^{\text {hor }} .
\end{aligned}
$$

Yet in the decomposition

$$
\nabla_{X}^{E} Y=\left(\nabla_{X^{\text {ver }}+X^{\text {hor }}}^{E}\left(Y^{\text {ver }}+Y^{\text {hor }}\right)\right)^{\text {ver }+ \text { hor }}
$$

we can find two more tensor fields (besides $S$ ), the so-called O'Neill tensor fields (see [181]):

$$
\begin{aligned}
& X, Y \in \mathfrak{X}(E), \\
& T(X, Y):=\left(\nabla_{X}^{E \text { ver }} Y^{\text {ver }}\right)^{\text {hor }}+\left(\nabla_{X}^{E}{ }^{\text {ver }} Y^{\text {hor }}\right)^{\text {ver }}, \\
& A(X, Y):=\left(\nabla_{X^{\text {hor }}}^{E} Y^{\text {hor }}\right)^{\text {ver }}+\left(\nabla_{X^{\text {hor }}}^{E} Y^{\text {ver }}\right)^{\text {hor }} .
\end{aligned}
$$

Each of these four terms making up $A$ and $T$ is a tensor field by itself the first one restricting to $S$ on $E_{b}$. They are combined as two tensors in just this way because of the results below.

Theorem ([181]). For horizontal vectors $X, Y, Z, H \in \operatorname{Hor}(p)_{x}$ we have

$$
\begin{gathered}
g_{x}^{E}\left(R_{x}(X, Y) Z, H\right)=g_{p(x)}^{B}\left(R_{p(x)}^{B}\left(T_{x} p \cdot X, T_{x} p . Y\right) T_{x} p . Z, T_{p} H\right) \\
+2 g_{x}^{E}(A(X, Y), A(Z, H))-g_{x}^{E}(A(Y, Z), A(X, H)) \\
-g_{x}^{E}(A(Z, X), A(Y, H)) .
\end{gathered}
$$

Proof. Since this is of tensorial character, we can assume that $X, Y, Z, U$ are basic local vector fields which are horizontal lifts of commuting vector fields $\xi, \eta, \zeta, \chi \in \mathfrak{X}(B)$; so $X=C(\xi), Y=C(\eta), Z=C(\zeta), H=C(\chi)$ (see $(26.10)$ and all Lie brackets $[\xi, \eta]$, etc., on $B$ vanish. Note first that for a vertical field $V=V^{\text {ver }}$ we have

$$
\nabla_{V}^{E} C(\xi)-\nabla_{C(\xi)}^{E} V=[V, C(\xi)]=0
$$

since $V$ is projectable to 0 . But then

$$
\begin{aligned}
0 & =\frac{1}{2} V g^{E}(X, X)=g^{E}\left(\nabla_{V}^{E} X, X\right) \\
& =g^{E}\left(\nabla_{X}^{E} V, X\right)=0-g^{E}\left(V, \nabla_{X}^{E} X\right) \\
& =g^{E}(V, A(X, X))
\end{aligned}
$$

and since $A(X, X)$ is vertical for horizontal $X$, this implies $A(X, X)=0$. Thus $A(X, Y)=-A(Y, X)$ for basic fields $X, Y$. 
Then $[X, Y]=[C(\xi), C(\eta)]$ is vertical since it projects to $[\xi, \eta]=0$, and moreover

$$
\begin{aligned}
{[X, Y] } & =\nabla_{X} Y-\nabla_{Y} X=\left(\nabla_{X} Y\right)^{\text {ver }}-\left(\nabla_{Y} X\right)^{\text {ver }} \\
& =A(X, Y)-A(Y, X)=2 A(X, Y), \\
\nabla_{[X, Y]}^{E} Z & =\nabla_{[X, Y]^{\text {ver }}}\left(Z^{\text {hor }}\right)=T\left([X, Y]^{\text {ver }}, Z^{\text {hor }}\right)+A\left([X, Y]^{\text {ver }}, Z^{\text {hor }}\right) \\
& =2 T(A(X, Y), Z)+2 A(A(X, Y), Z) .
\end{aligned}
$$

By (26.11.5) we have

$$
\begin{aligned}
& \nabla_{Y}^{E} Z=A(Y, Z)+\left(\nabla_{Y}^{E} Z\right)^{\text {hor }}=A(Y, Z)+C\left(\nabla_{\eta}^{B} \zeta\right) \\
& \nabla_{X}^{E} \nabla_{Y}^{E} Z=\nabla_{X}^{E}(A(Y, Z))+\nabla_{C(\xi)}^{E}\left(C\left(\nabla_{\eta}^{B} \zeta\right)\right) \\
& \quad=A(X, A(Y, Z))+\left(\nabla_{X}^{E}(A(Y, Z))\right)^{\mathrm{ver}}+C\left(\nabla_{\xi}^{B} \nabla_{\eta}^{B} \zeta\right)+A\left(X, C\left(\nabla_{\eta}^{B} \zeta\right)\right) .
\end{aligned}
$$

Combining, we get

$$
\begin{aligned}
R^{E} & (X, Y) Z=\nabla_{X}^{E} \nabla_{Y}^{E} Z-\nabla_{X}^{E} \nabla_{Y}^{E} Z-\nabla_{[X, Y]}^{E} Z \\
= & A(X, A(Y, Z))+\left(\nabla_{X}^{E}(A(Y, Z))\right)^{\mathrm{ver}}+C\left(\nabla_{\xi}^{B} \nabla_{\eta}^{B} \zeta\right)+A\left(X, C\left(\nabla_{\eta}^{B} \zeta\right)\right) \\
& -A(Y, A(X, Z))-\left(\nabla_{Y}^{E}(A(X, Z))\right)^{\mathrm{ver}}-C\left(\nabla_{\eta}^{B} \nabla_{\xi}^{B} \zeta\right)-A\left(Y, C\left(\nabla_{\xi}^{B} \zeta\right)\right) \\
& -2 T(A(X, Y), Z)-2 A(A(X, Y), Z) \\
= & C\left(\nabla_{\xi}^{B} \nabla_{\eta}^{B} \zeta-\nabla_{\eta}^{B} \nabla_{\xi}^{B} \zeta-\nabla_{[\xi, \eta]} \zeta\right) \\
& +A(X, A(Y, Z))-A(Y, A(X, Z))-2 A(A(X, Y), Z) \\
& +A\left(X, C\left(\nabla_{\eta}^{B} \zeta\right)\right)-A\left(Y, C\left(\nabla_{\xi}^{B} \zeta\right)\right)-2 T(A(X, Y), Z) \\
& +\left(\nabla_{X}^{E}(A(Y, Z))\right)^{\mathrm{ver}}-\left(\nabla_{Y}^{E}(A(X, Z))\right)^{\mathrm{ver}}
\end{aligned}
$$

using $[\xi, \eta]=0$, where the first two lines are horizontal and the last two lines are vertical. Take the inner product in $E$ with the horizontal $H$ and use

$$
\begin{aligned}
g^{E}(A(X, A(Y, Z)), H) & =g^{E}\left(\nabla_{X}\left(A(Y, Z)^{\mathrm{ver}}\right), H\right)=g^{E}\left(A(Y, Z)^{\mathrm{ver}}, \nabla_{X} H\right) \\
& =g^{E}\left(A(Y, Z)^{\mathrm{ver}},\left(\nabla_{X} H\right)^{\mathrm{ver}}\right)=g^{E}(A(Y, Z), A(X, H))
\end{aligned}
$$

to get the desired formula.

26.17. Corollary. Let $p: E \rightarrow B$ be a Riemann submersion between manifolds with connections, and consider horizontal vectors $X, Y, Z, H \in$ $\operatorname{Hor}(p)_{x}$. Then the sectional curvature expression becomes

$$
\begin{aligned}
g_{x}^{E} & \left.R^{E}(X, Y) X, Y\right) \\
& =g_{p(x)}^{B}\left(R^{B}\left(T_{x} p \cdot X, T_{x} p \cdot Y\right) T_{x} p \cdot X, T_{p} Y\right)+3\left\|A_{x}(X, Y)\right\|_{g^{E}}^{2} \\
& =g_{p(x)}^{B}\left(R^{B}\left(T_{x} p \cdot X, T_{x} p \cdot Y\right) T_{x} p \cdot X, T_{p} Y\right)+\frac{3}{4}\left\|[\bar{X}, \bar{Y}]^{v e r}\right\|_{g^{E}}^{2}
\end{aligned}
$$

for basic horizontal extensions $\bar{X}, \bar{Y}$ of $X, Y$. 
Proof. Again we extend everything to basic horizontal vector fields projecting to fields on $B$. From a slight generalization of (26.16.6) we have $[X, Y]^{\text {ver }}=2 A(X, Y)$ in this case. By theorem $(26.16)$ we have

$$
\begin{aligned}
g^{E}(R(X, Y) X, Y) & =g^{B}\left(R^{B}(\xi, \eta) \xi, \eta\right)+3 g^{E}(A(X, Y), A(X, Y)) \\
& =g^{B}\left(R^{B}(\xi, \eta) \xi, \eta\right)+\frac{3}{4}\left\|[X, Y]^{\text {ver }}\right\|^{2} .
\end{aligned}
$$

Note that the last expression gives another formula in the case where $X, Y$ are horizontal and project to commuting fields.

26.18. Riemann submersions via local frames. Let $p:\left(E, g_{E}\right) \rightarrow$ $\left(B, g_{B}\right)$ be a Riemann submersion. Choose for an open neighborhood $U$ in $E$ an orthonormal frame field $s=\left(s_{1}, \ldots, s_{m+k}\right) \in \Gamma(T E \mid U)^{m+k}$ in such a way that $s_{1}, \ldots, s_{m}$ are vertical and $s_{m+1}, \ldots, s_{m+k}$ are basic (horizontal and projectable). That way, if we project $s_{m+1}, \ldots, s_{m+k}$ onto $T B \mid p(U)$, we get another orthonormal frame field, $\bar{s}=\left(\bar{s}_{m+1}, \ldots, \bar{s}_{m+k}\right) \in C^{\infty}(T B \mid p(U))^{k}$, since $p$, as Riemann submersion, is isometric on horizontal vectors. The indices will always run in the domain indicated:

$$
1 \leq i, j, k \leq m, \quad m+1 \leq \bar{a}, \bar{b}, \bar{c} \leq m+k, \quad 1 \leq A, B, C \leq m+k .
$$

The orthonormal coframe dual to $s$ is given by

$$
\sigma^{A}\left(s_{B}\right)=\delta_{B}^{A}, \quad \sigma=\left(\begin{array}{c}
\sigma^{1} \\
\vdots \\
\sigma^{m+k}
\end{array}\right) \in \Omega^{1}(U)^{m+k} .
$$

Analogously, we have the orthonormal coframe $\bar{\sigma}^{\bar{a}} \in \Omega^{1}(p(U))$ on $p(U) \subseteq B$, with $\bar{\sigma}^{\bar{a}}\left(\bar{s}_{\bar{b}}\right)=\delta_{\bar{b}}^{\bar{a}}$. It is related to $\sigma^{\bar{a}}$ by $p^{*} \bar{\sigma}^{\bar{a}}=\sigma^{\bar{a}}$. By $(25.5)$ we have on $\left(U \subset E, g_{E}\right)$

$$
\begin{aligned}
& \left.g_{E}\right|_{U}=\sum_{A} \sigma^{A} \otimes \sigma^{A}, \\
& \nabla^{E} s=s . \omega \quad \text { where } \quad \omega_{B}^{A}=-\omega_{A}^{B}, \quad \text { so } \quad \omega \in \Omega^{1}(U, \mathfrak{s o}(n+k)), \\
& d \sigma+\omega \wedge \sigma=0, \quad \text { i.e., } \quad d \sigma^{A}+\sum_{C} \omega_{C}^{A} \wedge \sigma^{C}=0, \\
& R s=s . \Omega \quad \text { where } \quad \Omega=d \omega+\omega \wedge \omega \in \Omega^{2}(U, \mathfrak{s o}(n+k)), \\
& \quad \text { or } \quad \Omega_{B}^{A}=d \omega_{B}^{A}+\sum_{C} \omega_{C}^{A} \wedge \omega_{B}^{C}, \\
& \Omega \wedge \sigma=0 \quad \text { or } \quad \sum_{C} \Omega_{C}^{A} \wedge \sigma^{C}=0, \quad \text { the first Bianchi identity, } \\
& d \Omega+\omega \wedge \Omega-\Omega \wedge \omega=d \Omega+[\omega, \Omega]_{\wedge}=0, \quad \text { the second Bianchi identity, }
\end{aligned}
$$

and similarly on $\left(p(U) \subset B, g^{B}\right)$ with bars on all forms.

For the following it will be faster to rederive some results than compiling them from (26.7) and (26.8). We start by pulling back the structure equation 


$$
\begin{aligned}
d \bar{\sigma}+\bar{\omega} \wedge \bar{\sigma} & =0 \text { from } B \text { to } E \text { via } p^{*}: \\
0 & =p^{*}\left(d \bar{\sigma}^{\bar{a}}+\sum \bar{\omega}_{\bar{b}}^{\bar{a}} \wedge \bar{\sigma}^{\bar{b}}\right) \\
& =d p^{*} \bar{\sigma}^{\bar{a}}+\sum\left(p^{*} \bar{\omega}_{\bar{b}}^{\bar{a}}\right) \wedge\left(p^{*} \bar{\sigma}^{\bar{b}}\right)=d \sigma^{\bar{a}}+\sum\left(p^{*} \bar{\omega}_{\bar{b}}^{\bar{a}}\right) \wedge \sigma^{\bar{b}} .
\end{aligned}
$$

The $\bar{a}$-part of the structure equation on $E, d \sigma^{\bar{a}}+\sum \omega_{\bar{b}}^{\bar{a}} \wedge \sigma^{\bar{b}}+\sum \omega_{i}^{\bar{a}} \wedge \sigma^{i}=0$, combines with this to give

$$
\sum\left(p^{*} \bar{\omega}_{\bar{b}}^{\bar{a}}\right) \wedge \sigma^{\bar{b}}=\sum \omega_{\bar{b}}^{\bar{a}} \wedge \sigma^{\bar{b}}+\sum \omega_{i}^{\bar{a}} \wedge \sigma^{i}
$$

The left hand side of this equation contains no $\sigma^{i} \wedge \sigma^{\bar{a}}$ - or $\sigma^{i} \wedge \sigma^{j}$-terms. Let us write out $\omega_{\bar{b}}^{\bar{a}}$ and $\omega_{i}^{\bar{a}}$ in this basis:

$$
\omega_{\bar{b}}^{\bar{a}}=-\omega_{\bar{a}}^{\bar{b}}=: \sum q_{\bar{b} \bar{c}}^{\bar{a}} \sigma^{\bar{c}}+\sum b_{\bar{b} i}^{\bar{a}} \sigma^{i}, \quad \omega_{i}^{\bar{a}}=-\omega_{\bar{a}}^{i}=: \sum a_{i \bar{b}}^{\bar{a}} \sigma^{\bar{b}}+\sum r_{i j}^{\bar{a}} \sigma^{j}
$$

This gives us for the right hand side of (11):

$$
\begin{aligned}
\sum q_{\bar{b} \bar{c}}^{\bar{a}} \sigma^{\bar{c}} \wedge \sigma^{\bar{b}}+\sum b_{\bar{b} i}^{\bar{a}} \sigma^{i} \wedge \sigma^{\bar{b}}+\sum a_{i \bar{b}}^{\bar{a}} \sigma^{\bar{b}} \wedge \sigma^{i}+\sum r_{i j}^{\bar{a}} \sigma^{j} \wedge \sigma^{i} \\
\quad=\sum q_{\bar{b} \bar{c}}^{\bar{a}} \sigma^{\bar{c}} \wedge \sigma^{\bar{b}}+\sum\left(b_{\bar{b} i}^{\bar{a}}-a_{i \bar{b}}^{\bar{a}}\right) \sigma^{i} \wedge \sigma^{\bar{b}}+\frac{1}{2} \sum\left(r_{i j}^{\bar{a}}-r_{j i}^{\bar{a}}\right) \sigma^{j} \wedge \sigma^{i} .
\end{aligned}
$$

So we have found $a_{i \bar{b}}^{\bar{a}}=b_{\bar{b} i}^{\bar{a}}$ and $r_{i j}^{\bar{a}}=r_{j i}^{\bar{a}}$ or, in other words, $\omega_{i}^{\bar{a}}\left(s_{\bar{b}}\right)=\omega_{\bar{b}}^{\bar{a}}\left(s_{i}\right)$ and $\omega_{i}^{\bar{a}}\left(s_{j}\right)=\omega_{j}^{\bar{a}}\left(s_{i}\right)$. That is, $\omega_{i}^{\bar{a}}\left(s_{A}\right)=\omega_{A}^{\bar{a}}\left(s_{i}\right)$, and this just means that the horizontal part of $\left[s_{A}, s_{i}\right]$ is 0 , or $\left[s_{A}, s_{i}\right]$ is always vertical:

$$
0=\sum s_{\bar{a}} \omega_{i}^{\bar{a}}\left(s_{A}\right)-\sum s_{\bar{a}} \omega_{A}^{\bar{a}}\left(s_{i}\right)=\left(\nabla_{s_{A}} s_{i}-\nabla_{s_{i}} s_{A}\right)^{\text {hor }}=\left[s_{A}, s_{i}\right]^{\text {hor }} .
$$

Now we consider again the second fundamental form $S^{E_{b}}: T E_{b} \times_{E_{b}} T E_{b} \rightarrow$ $\operatorname{Hor}(E)$ of the submanifold $E_{b}:=p^{-1}(b)$ in $E$. By (26.1), $S^{E_{b}}$ is given as:

$$
\begin{aligned}
S^{E_{b}}\left(X^{\mathrm{ver}}, Y^{\mathrm{ver}}\right)=\nabla_{X}^{E}{ }^{\mathrm{ver}} Y^{\mathrm{ver}}-\nabla_{X^{\mathrm{ver}}}^{E_{b}} Y^{\mathrm{ver}}=\nabla_{X^{\mathrm{ver}}}^{E} Y^{\mathrm{ver}}-\left(\nabla_{X^{\mathrm{ver}}}^{E} Y^{\mathrm{ver}}\right)^{\mathrm{ver}} \\
=\left(\nabla_{X^{\mathrm{ver}}}^{E} Y^{\mathrm{ver}}\right)^{\text {hor }} \\
=\left(\nabla_{X^{\mathrm{ver}}}^{E}\left(\sum s_{i} \sigma^{i}\left(Y^{\mathrm{ver}}\right)\right)\right)^{\text {hor }} \\
=\left(\sum\left(\nabla_{X^{\mathrm{ver}}}^{E} s_{i}\right) \sigma^{i}\left(Y^{\mathrm{ver}}\right)+\sum s_{i} d\left(\sigma^{i}\left(Y^{\mathrm{ver}}\right)\right) \cdot X^{\mathrm{ver}}\right) \\
=\left(\sum s_{A} \omega_{i}^{A}\left(X^{\mathrm{ver}}\right) \sigma^{i}\left(Y^{\mathrm{ver}}\right)\right)^{\mathrm{hor}}+0=\sum s_{\bar{a}} \omega_{i}^{\bar{a}}\left(X^{\mathrm{ver}}\right) \sigma^{i}\left(Y^{\mathrm{ver}}\right) \\
=\sum r_{i j}^{\bar{a}}\left(s_{\bar{a}} \otimes \sigma^{j} \otimes \sigma^{i}\right)\left(X^{\mathrm{ver}}, Y^{\mathrm{ver}}\right) .
\end{aligned}
$$

So

$$
\sum s_{\bar{a}} \sigma^{\bar{a}}\left(S^{E_{b}}\right)=\sum r_{i j}^{\bar{a}} s_{\bar{a}} \otimes \sigma^{j} \otimes \sigma^{i} .
$$

Note that $r_{i j}^{\bar{a}}=r_{j i}^{\bar{a}}$ from above corresponds to symmetry of $S$. The covariant derivative on the normal bundle $\operatorname{Nor}\left(E_{b}\right)=\left.\operatorname{Hor}(E)\right|_{E_{b}} \rightarrow E_{b}$ is given by the Weingarten formula (26.3) as the corresponding projection:

$$
\begin{gathered}
\nabla^{\text {Nor }}: \mathfrak{X}\left(E_{b}\right) \times \Gamma\left(\operatorname{Nor}\left(E_{b}\right)\right) \rightarrow \Gamma\left(\operatorname{Nor}\left(E_{b}\right)\right), \\
\nabla_{X^{\text {ver }}}^{\text {Nor }} Y^{\text {hor }}=\left(\nabla_{X^{\text {ver }}}^{E} Y^{\text {hor }}\right)^{\text {hor }}=\left(\nabla_{X}^{E} E^{\text {ver }}\left(\sum s_{\bar{b}} \sigma^{\bar{b}}\left(Y^{\text {hor }}\right)\right)\right)^{\text {hor }}
\end{gathered}
$$




$$
\begin{aligned}
& =\left(\sum\left(\nabla_{X^{\mathrm{ver}}}^{E} s_{\bar{b}}\right) \sigma^{\bar{b}}\left(Y^{\mathrm{hor}}\right)\right)^{\text {hor }}+\sum s_{\bar{b}} d \sigma^{\bar{b}}\left(Y^{\mathrm{hor}}\right) \cdot X^{\mathrm{ver}} \\
& =\sum s_{\bar{a}} \omega_{\bar{b}}^{\bar{a}}\left(X^{\mathrm{ver}}\right) \sigma^{\bar{b}}\left(Y^{\mathrm{hor}}\right)+\sum s_{\bar{b}} d \sigma^{\bar{b}}\left(Y^{\mathrm{hor}}\right) \cdot X^{\mathrm{ver}} \\
& =\sum b_{\bar{b}}^{\bar{a}} s_{\bar{a}} \otimes \sigma^{i} \otimes \sigma^{\bar{b}}\left(X^{\mathrm{ver}}, Y^{\mathrm{hor}}\right)+\sum s_{\bar{a}} \otimes d \sigma^{\bar{a}}\left(Y^{\mathrm{hor}}\right)\left(X^{\mathrm{ver}}\right), \\
\nabla^{\mathrm{Nor}} Y^{\mathrm{hor}} & =\sum\left(b_{\bar{b} i}^{\bar{a}} \sigma^{\bar{b}}\left(Y^{\mathrm{hor}}\right) \sigma^{i}+d \sigma^{\bar{a}}\left(Y^{\mathrm{hor}}\right)\right) \otimes s_{\bar{a}} .
\end{aligned}
$$

We consider now the O'Neill tensor fields from (26.16)

$$
\begin{aligned}
X & , Y \in \mathfrak{X}(E), \\
A(X, Y) & =\left(\nabla_{X^{\mathrm{hor}}}^{E} Y^{\mathrm{hor}}\right)^{\mathrm{ver}}+\left(\nabla_{X^{\mathrm{hor}}}^{E} Y^{\mathrm{ver}}\right)^{\text {hor }} \\
& =\left(\nabla_{X^{\mathrm{hor}}}^{E}\left(\sum s_{\bar{a}} \sigma^{\bar{a}}(Y)\right)\right)^{\mathrm{ver}}+\left(\nabla_{X^{\mathrm{hor}}}^{E}\left(\sum s_{i} \sigma^{i}(Y)\right)\right)^{\mathrm{hor}} \\
& =\sum s_{i} \omega_{\bar{a}}^{i}\left(X^{\mathrm{hor}}\right) \sigma^{\bar{a}}(Y)+0+\sum s_{\bar{a}} \omega_{i}^{\bar{a}}\left(X^{\mathrm{hor}}\right) \sigma^{i}(Y)+0 \\
& =\sum s_{i}\left(-a_{i \bar{b}}^{\bar{a}}\right) \sigma^{\bar{b}}(X) \sigma^{\bar{a}}(Y)+\sum s_{\bar{a}} a_{i \overline{\bar{a}}}^{\bar{a}} \sigma^{\bar{b}}(X) \sigma^{i}(Y) \\
& =\sum a_{i \bar{b}}^{\bar{a}}\left(\sigma^{\bar{b}} \otimes \sigma^{i} \otimes s_{\bar{a}}-\sigma^{\bar{b}} \otimes \sigma^{\bar{a}} \otimes s_{i}\right)(X, Y) .
\end{aligned}
$$

Analogously:

$$
T=\sum r_{i j}^{\bar{a}}\left(\sigma^{j} \otimes \sigma^{i} \otimes s_{\bar{a}}-\sigma^{j} \otimes \sigma^{\bar{a}} \otimes s_{i}\right) .
$$

If $\operatorname{Hor}(E)$ is integrable, then every leaf $L$ is totally geodesic by (26.13.1), and the $\left.s_{\bar{a}}\right|_{L}$ are a local orthonormal frame field on $L$. The leaf $L$ is totally geodesic if and only if its second fundamental form which is given by

$$
S^{L}\left(X^{\text {hor }}, Y^{\text {hor }}\right):=\left(\nabla_{X}^{E \text { hor }} Y^{\text {hor }}\right)^{\text {ver }}
$$

vanishes. So it is a necessary condition for the integrability of $\operatorname{Hor}(E)$ that $S^{L}=0$, that is,

$0=S^{L}\left(s_{\bar{a}}, s_{\bar{b}}\right)=\left(\nabla_{s_{\bar{a}}} s_{\bar{b}}\right)^{\mathrm{ver}}=\sum s_{i} \omega_{\bar{b}}^{i}\left(s_{\bar{a}}\right)=\sum s_{i}\left(-a_{i \bar{c}}^{\bar{b}}\right) \sigma^{\bar{c}}\left(s_{\bar{a}}\right)=-\sum_{i} s_{i} a_{i \bar{a}}^{\bar{b}}$.

This is equivalent to the condition $a_{i \bar{b}}^{\bar{a}}=0$ for all ${ }_{i \bar{b}}{ }_{i \bar{b}}$ or to $A=0$.

Let us now prove the converse: If $A$ vanishes, then the horizontal distribution on $E$ is integrable. In this case, we have $0=A\left(s_{\bar{a}}, s_{\bar{b}}\right)=\left(\nabla_{s_{\bar{E}}}^{E} s_{\bar{b}}\right)^{\text {ver }}+0$, as well as $0=A\left(s_{\bar{b}}, s_{\bar{a}}\right)=\left(\nabla_{s_{\bar{b}}}^{E} s_{\bar{a}}\right)^{\text {ver }}+0$. Therefore, $\left[s_{\bar{a}}, s_{\bar{b}}\right]=\nabla_{s_{\bar{a}}}^{E} s_{\bar{b}}-\nabla_{s_{\bar{b}}}^{E} s_{\bar{a}}$ is horizontal, and the horizontal distribution is integrable.

26.19. Theorem. Let $p: E \rightarrow B$ be a Riemann submersion; then the following conditions are equivalent:

(1) $p$ is integrable (that is, $\operatorname{Hor}(p)$ is integrable).

(2) Every p-parallel normal field along $E_{b}$ is $\nabla^{\text {Nor }}$-parallel.

(3) The O'Neill tensor $A$ is zero.

Proof. We already saw (11) $\Longleftrightarrow(3)$ above. 
(3) $\Longrightarrow$ (2) Take $s_{\bar{a}}$ for a $p$-parallel normal field $X$ along $E_{b}$. The condition $A=0$ implies $A\left(s_{\bar{a}}, s_{i}\right)=0+\left(\nabla_{s_{\bar{a}}} s_{i}\right)^{\text {hor }}=0$. Recall that, as we showed in $(26.18 .2)$ above, $\left[s_{i}, s_{\bar{a}}\right]$ is vertical. Therefore,

$$
\nabla_{s_{i}}^{\mathrm{Nor}} s_{\bar{a}}=\left(\nabla_{s_{i}}^{E} s_{\bar{a}}\right)^{\mathrm{hor}}=\left(\left[s_{i}, s_{\bar{a}}\right]+\nabla_{s_{\bar{a}}}^{E} s_{i}\right)^{\mathrm{hor}}=0 .
$$

Since for any $e \in E_{b},\left.T_{e} p\right|_{\text {Nor }_{b}\left(E_{b}\right)}$ is an isometric isomorphism, a $p$-parallel normal field $X$ along $E_{b}$ is determined completely by the equation $X(e)=$ $\sum X^{\bar{a}}(e) s_{\bar{a}}(e)$. Therefore it is always a linear combination of the $s_{\bar{a}}$ with constant coefficients and we are done.

(2) $\Longrightarrow$ (3) By (2),$\nabla_{s_{i}}^{\text {Nor }} s_{\bar{a}}=\left(\nabla_{s_{i}}^{E} s_{\bar{a}}\right)^{\text {hor }}=0$. Therefore, as above, we have that $\left(\left[s_{i}, s_{\bar{a}}\right]+\nabla_{s_{\bar{a}}}^{E} s_{i}\right)^{\text {hor }}=0+\left(\nabla_{s_{\bar{a}}}^{E} s_{i}\right)^{\text {hor }}=A\left(s_{\bar{a}}, s_{i}\right)=0$. Thus $\sigma^{\bar{b}} A\left(s_{\bar{a}}, s_{i}\right)=a_{\bar{a} i}^{\bar{b}}=0$, so $A$ vanishes completely.

\section{Jacobi Fields}

27.1. Jacobi fields. Let $(M, \nabla)$ be a manifold with covariant derivative $\nabla$, with curvature $R$ and torsion Tor. Let us consider a smooth mapping $\gamma:(-\varepsilon, \varepsilon) \times[0,1] \rightarrow M$ such that $t \mapsto \gamma(s, t)$ is a geodesic for each $s \in(-\varepsilon, \varepsilon)$; we call this a 1-parameter variation through geodesics. Let us write $\partial_{s} \gamma=: \gamma^{\prime}$ and $\partial_{t} \gamma=: \dot{\gamma}$ in the following. Our aim is to investigate the variation vector field $\left.\partial_{s}\right|_{0} \gamma(s, \quad)=\gamma^{\prime}(0, \quad)$.

We first note that by $(22.10 .4)$ we have

$$
\begin{aligned}
\nabla_{\partial_{s}} \dot{\gamma} & =\nabla_{\partial_{s}}\left(T \gamma \cdot \partial_{t}\right)=\nabla_{\partial_{t}}\left(T \gamma \cdot \partial_{s}\right)+T \gamma \cdot\left[\partial_{s}, \partial_{t}\right]+\operatorname{Tor}\left(T \gamma \cdot \partial_{s}, T \gamma \cdot \partial_{t}\right) \\
& =\nabla_{\partial_{t}} \gamma^{\prime}+\operatorname{Tor}\left(\gamma^{\prime}, \dot{\gamma}\right)
\end{aligned}
$$

We have $\nabla_{\partial_{t}} \dot{\gamma}=\nabla_{\partial_{t}}\left(\partial_{t} \gamma\right)=0$ since $\gamma(s, \quad)$ is a geodesic for each $s$. Thus by using (24.5), we get

$$
\begin{aligned}
0 & =\nabla_{\partial_{s}} \nabla_{\partial_{t}} \dot{\gamma}=R\left(T \gamma \cdot \partial_{s}, T \gamma \cdot \partial_{t}\right) \dot{\gamma}+\nabla_{\partial_{t}} \nabla_{\partial_{s}} \dot{\gamma}+\nabla_{\left[\partial_{s}, \partial_{t}\right]} \dot{\gamma} \\
& =R\left(\gamma^{\prime}, \dot{\gamma}\right) \dot{\gamma}+\nabla_{\partial_{t}} \nabla_{\partial_{t}} \gamma^{\prime}+\nabla_{\partial_{t}} \operatorname{Tor}\left(\gamma^{\prime}, \dot{\gamma}\right) .
\end{aligned}
$$

Inserting $s=0$, along the geodesic $c=\gamma(0, \quad)$ we get the Jacobi differential equation for the variation vector field $Y=\left.\partial_{s}\right|_{0} \gamma=\gamma^{\prime}(0, \quad)$ :

$$
0=R(Y, \dot{c}) \dot{c}+\nabla_{\partial_{t}} \nabla_{\partial_{t}} Y+\nabla_{\partial_{t}} \operatorname{Tor}(Y, \dot{c})
$$

This is a linear differential equation of second order for vector fields $Y$ along the fixed geodesic $c:[0,1] \rightarrow M$. Thus for any $t_{0} \in[0,1]$ and any initial values $\left(Y\left(t_{0}\right),\left(\nabla_{\partial_{t}}\right)\left(t_{0}\right)\right) \in T_{c\left(t_{0}\right)} M \times T_{c\left(t_{0}\right)} M$ there exists a unique global solution $Y$ of (3) along $c$. These solutions are called Jacobi fields along c; they form a $2 m$-dimensional vector space. 
27.2. The Jacobi flow. Consider a linear connector $K: T T M \rightarrow M$ on the tangent bundle with its corresponding horizontal lift mapping $C$ : $T M \times M T M \rightarrow T T M$ (see (22.8)], its spray $S: T M \rightarrow T T M$ given by $S(X):=C(X, X)\left(\right.$ see $(22.7)$ and its covariant derivative $\nabla_{X} Y=K \circ T Y \circ X$ $(\operatorname{see}(22.9)$.

Theorem (155]). Let $S: T M \rightarrow T T M$ be a spray on a manifold $M$. Then $\kappa_{T M} \circ T S: T T M \rightarrow T T T M$ is a vector field. Consider a flow line

$$
J(t)=\mathrm{Fl}_{t}^{\kappa_{T M} \circ T S}(J(0))
$$

of this field. Then we have:

- $c:=\pi_{M} \circ \pi_{T M} \circ J$ is a geodesic on $M$,

- $\dot{c}=\pi_{T M} \circ J$ is the velocity field of $c$,

- $Y:=T\left(\pi_{M}\right) \circ J$ is a Jacobi field along $c$,

- $\dot{Y}=\kappa_{M} \circ J$ is the velocity field of $Y$,

- $\nabla_{\partial_{t}} Y=K \circ \kappa_{M} \circ J$ is the covariant derivative of $Y$.

The Jacobi equation is given by:

$$
\begin{aligned}
0 & =\nabla_{\partial_{t}} \nabla_{\partial_{t}} Y+R(Y, \dot{c}) \dot{c}+\nabla_{\partial_{t}} \operatorname{Tor}(Y, \dot{c}) \\
& =K \circ T K \circ T S \circ J .
\end{aligned}
$$

This implies that in a canonical chart induced from a chart on $M$ the curve $J(t)$ is given by

$$
(c(t), \dot{c}(t) ; Y(t), \dot{Y}(t)) \text {. }
$$

Proof. Consider a curve $s \mapsto X(s)$ in $T M$. Then each $t \mapsto \pi_{M}\left(\mathrm{Fl}_{t}^{S}(X(s))\right)$ is a geodesic in $M$, and in the variable $s$ it is a variation through geodesics. Thus $Y(t):=\left.\partial_{s}\right|_{0} \pi_{M}\left(\mathrm{Fl}_{t}^{S}(X(s))\right)$ is a Jacobi field along the geodesic $c(t):=$ $\pi_{M}\left(\mathrm{Fl}_{t}^{S}(X(0))\right)$ by $(27.1)$, and each Jacobi field is of this form, for a suitable curve $X(s)$; see $(27.5 .4)$ below. We consider now the curve $J(t):=$ $\left.\partial_{s}\right|_{0} \mathrm{Fl}_{t}^{S}(X(s))$ in $T T M$. Then by (8.13.6) we have

$$
\begin{aligned}
\partial_{t} J(t) & =\left.\partial_{t} \partial_{s}\right|_{0} \mathrm{Fl}_{t}^{S}(X(s))=\left.\kappa_{T M} \partial_{s}\right|_{0} \partial_{t} \mathrm{Fl}_{t}^{S}(X(s))=\left.\kappa_{T M} \partial_{s}\right|_{0} S\left(\mathrm{Fl}_{t}^{S}(X(s))\right) \\
& =\left(\kappa_{T M} \circ T S\right)\left(\left.\partial_{s}\right|_{0} \mathrm{Fl}_{t}^{S}(X(s))\right)=\left(\kappa_{T M} \circ T S\right)(J(t)),
\end{aligned}
$$

so that $J(t)$ is a flow line of the vector field $\kappa_{T M} \circ T S: T T M \rightarrow T T T M$. Moreover using the properties of $\kappa$ from (8.13) and of $S$ from (22.7), we get

$$
\begin{aligned}
T \pi_{M} \cdot J(t) & =\left.T \pi_{M} \cdot \partial_{s}\right|_{0} \mathrm{Fl}_{t}^{S}(X(s))=\left.\partial_{s}\right|_{0} \pi_{M}\left(\mathrm{Fl}_{t}^{S}(X(s))\right)=Y(t), \\
\pi_{M} T \pi_{M} J(t) & =c(t), \text { the geodesic, } \\
\partial_{t} Y(t) & =\left.\partial_{t} T \pi_{M} \cdot \partial_{s}\right|_{0} \mathrm{Fl}_{t}^{S}(X(s))=\left.\partial_{t} \partial_{s}\right|_{0} \pi_{M}\left(\mathrm{Fl}_{t}^{S}(X(s))\right) \\
& =\left.\kappa_{M} \partial_{s}\right|_{0} \partial_{t} \pi_{M}\left(\mathrm{Fl}_{t}^{S}(X(s))\right)=\left.\kappa_{M} \partial_{s}\right|_{0} \partial_{t} \pi_{M}\left(\mathrm{Fl}_{t}^{S}(X(s))\right)
\end{aligned}
$$




$$
\begin{aligned}
& =\left.\kappa_{M} \partial_{s}\right|_{0} T \pi_{M} \cdot \partial_{t} \mathrm{Fl}_{t}^{S}(X(s))=\left.\kappa_{M} \partial_{s}\right|_{0}\left(T \pi_{M} \circ S\right) \mathrm{Fl}_{t}^{S}(X(s)) \\
& =\left.\kappa_{M} \partial_{s}\right|_{0} \mathrm{Fl}_{t}^{S}(X(s))=\kappa_{M} J(t), \\
\nabla_{\partial_{t}} Y & =K \circ \partial_{t} Y=K \circ \kappa_{M} \circ J .
\end{aligned}
$$

Finally let us express the Jacobi equation (27.1.3), For the sake of shortness we write $\gamma(s, t):=\pi_{M}\left(\mathrm{Fl}_{t}^{S}(X(s))\right)$ :

$$
\begin{aligned}
& \nabla_{\partial_{t}} \nabla_{\partial_{t}} Y+R(Y, \dot{c}) \dot{c}+\nabla_{\partial_{t}} \operatorname{Tor}(Y, \dot{c}) \\
&=\nabla_{\partial_{t}} \nabla_{\partial_{t}} \cdot T \gamma \cdot \partial_{s}+R\left(T \gamma \cdot \partial_{s}, T \gamma \cdot \partial_{t}\right) T \gamma \cdot \partial_{t}+\nabla_{\partial_{t}} \operatorname{Tor}\left(T \gamma \cdot \partial_{s}, T \gamma \cdot \partial_{t}\right) \\
&= K \cdot T\left(K \cdot T\left(T \gamma \cdot \partial_{s}\right) \cdot \partial_{t}\right) \cdot \partial_{t} \\
&+\left(K . T K \cdot \kappa_{T M}-K \cdot T K\right) \cdot T T\left(T \gamma \cdot \partial_{t}\right) \cdot T \partial_{s} \cdot \partial_{t} \\
&+K \cdot T\left(\left(K \cdot \kappa_{M}-K\right) \cdot T T \gamma \cdot T \partial_{s} \cdot \partial_{t}\right) \cdot \partial_{t} .
\end{aligned}
$$

Note that for example for the term in the second summand we have

$$
\begin{aligned}
\operatorname{TTT} \gamma \cdot T T \partial_{t} \cdot T \partial_{s} . \partial_{t} & =T\left(T\left(\partial_{t} \gamma\right) \cdot \partial_{s}\right) \cdot \partial_{t}=\partial_{t} \partial_{s} \partial_{t} \gamma \\
& =\partial_{t} \cdot \kappa_{M} \cdot \partial_{t} \cdot \partial_{s} \gamma=T \kappa_{M} \cdot \partial_{t} \cdot \partial_{t} \cdot \partial_{s} \gamma
\end{aligned}
$$

which at $s=0$ equals $T \kappa_{M} \ddot{Y}$. Using this, we get for the Jacobi equation at $s=0$ :

$$
\begin{aligned}
& \nabla_{\partial_{t}} \nabla_{\partial_{t}} Y+R(Y, \dot{c}) \dot{c}+\nabla_{\partial_{t}} \operatorname{Tor}(Y, \dot{c}) \\
& =\left(K . T K+K . T K . \kappa_{T M} \cdot T \kappa_{M}-K . T K . T \kappa_{M}+K . T K . T \kappa_{M}-K . T K\right) . \partial_{t} \partial_{t} Y \\
& =K . T K . \kappa_{T M} . T \kappa_{M} . \partial_{t} \partial_{t} Y=K . T K . \kappa_{T M} . \partial_{t} J=K . T K . T S . J
\end{aligned}
$$

where we used $\partial_{t} \partial_{t} Y=\partial_{t}\left(\kappa_{M} . J\right)=T \kappa_{M} \partial_{t} J=T \kappa_{M} \cdot \kappa_{T M} . T S . J$. Finally the validity of the Jacobi equation $0=K . T K . T S . J$ follows trivially from $K \circ S=0_{T M}$.

Note that the system of Jacobi fields depends only on the geodesic structure, thus on the spray induced by the given covariant derivative. So we may assume that the covariant derivative is torsion-free without loss; we do this from now on.

27.3. Fermi charts. Let $(M, g)$ be a Riemann manifold. Let $c:(-2 \varepsilon, 1+$ $2 \varepsilon) \rightarrow M$ be a geodesic (for $\varepsilon>0$ ). We define the Fermi chart along $c$ as follows.

Since $c([-\varepsilon, 1+\varepsilon])$ is compact in $M$, there exists $\rho>0$ such that

$$
\begin{aligned}
& B_{c(0)}^{\perp}(\rho):=\left\{X \in T_{c(0)}^{\perp} c:=\left\{Y \in T_{c(0)} M: g\left(Y, c^{\prime}(0)\right)=0\right\},|X|_{g}<\rho\right\}, \\
& \exp \circ \operatorname{Pt}(c, \quad):(-\varepsilon, 1+\varepsilon) \times B_{c}^{\perp}(0)(\rho) \rightarrow M, \\
& (t, X) \mapsto \exp _{c(t)}(\operatorname{Pt}(c, t) X)
\end{aligned}
$$

is everywhere defined. 
Since its tangent mapping along $(-\varepsilon, 1+\varepsilon) \times\{0\}$,

$$
\begin{gathered}
T_{t, 0}(\exp \circ \operatorname{Pt}(c, \quad)): \mathbb{R} \times T_{c(0)}^{\perp} c \rightarrow T_{c}(t) M=T_{c(t)}(c([0,1])) \times T_{c(t)}^{\perp} c, \\
(s, Y) \mapsto s . c^{\prime}(t)+\operatorname{Pt}(c, t) Y,
\end{gathered}
$$

is a linear isomorphism, we may assume (by choosing $\rho$ smaller if necessary using (22.7.6) that the mapping $\exp \circ \operatorname{Pt}(c, \quad)$ in (1) is a diffeomorphism onto its image. Its inverse,

$$
\begin{gathered}
u_{c, \rho}:=(\exp \circ \operatorname{Pt}(c, \quad))^{-1}: U_{c . \rho} \rightarrow(-\varepsilon, 1+\varepsilon) \times B_{c(0)}^{\perp}(\rho), \\
U_{c . \rho}:=(\exp \circ \operatorname{Pt}(c, \quad))\left((-\varepsilon, 1+\varepsilon) \times B_{c(0)}^{\perp}(\rho)\right),
\end{gathered}
$$

is called the Fermi chart along $c$. Its importance is due to the following result.

27.4. Lemma. Let $X$ be a vector field along the geodesic $c$. For the Fermi chart along c put $T_{c(t)}\left(u_{c, \rho}\right)^{-1} \cdot X(t)=:(t, \bar{X}(t))$. Then we have

$$
T_{c(t)} u_{c, \rho} \cdot\left(\nabla_{\partial t} X\right)(t)=\left(t, \bar{X}^{\prime}(t)\right) .
$$

So in the Fermi chart the covariant derivative $\nabla_{\partial_{t}}$ along $c$ is just the ordinary derivative. More is true: The Christoffel symbol in the Fermi chart vanishes along $(-\varepsilon, 1+\varepsilon) \times\{0\}$.

The last statement is a generalization of the property of Riemann normal coordinates $\exp _{x}^{-1}$ that the Christoffel symbol vanishes at 0 ; see (22.7).

Proof. In terms of the Christoffel symbol of the Fermi chart the geodesic equation is given by $\bar{c}^{\prime \prime}(t)=\Gamma_{\bar{c}(t)}\left(\bar{c}^{\prime}(t), \bar{c}^{\prime}(t)\right)$; see (22.4), But in the Fermi chart the geodesic $c$ is given by $u_{c, \rho}(c(t))=(t, 0)$, so the geodesic equation becomes $0=\Gamma_{\bar{c}(t)}((1,0),(1,0))=\Gamma_{\bar{c}(t)}\left(\bar{c}^{\prime}(t), \bar{c}^{\prime}(t)\right)$. For $Y_{0} \in T_{c(0)}^{\perp} c$ the parallel vector field $Y(t)=\operatorname{Pt}(c, t) Y_{0}$ is represented by $\left(t, 0 ; 0, Y_{0}\right)$ in the Fermi chart; thus we get $0=\Gamma_{\bar{c}(t)}\left(\bar{c}^{\prime}(t), Y_{0}\right)$. The geodesic $s \mapsto \exp _{c(t)}(s \cdot \operatorname{Pt}(c, t) . Y)$ for $Y \in T_{c(0)}^{\perp} c$ is represented by $s \mapsto(t, s . Y)$ in the Fermi chart. The corresponding geodesic equation is $0=\frac{\partial^{2}}{\partial s^{2}}(t, s . Y)=\Gamma_{(t, s . Y)}(Y, Y)$. By symmetry of $\Gamma_{(t, 0)}$ these facts imply that $\Gamma_{(t, 0)}=0$. Finally,

$$
T u_{c, \rho} \cdot\left(\nabla_{\partial_{t}} X\right)(t)=\bar{X}^{\prime}(t)-\Gamma_{(t, 0)}\left(\bar{c}^{\prime}(t), \bar{X}(t)\right)=\bar{X}^{\prime}(t) .
$$

27.5. Let $\left(M^{m}, g\right)$ be a Riemann manifold, and let $c:[0,1] \rightarrow M$ be a geodesic which might be constant. Let us denote by $\mathcal{J}_{c}$ the $2 m$-dimensional real vector space of all Jacobi fields along $c$, i.e., all vector fields $Y$ along $c$ satisfying

$$
\nabla_{\partial_{t}} \nabla_{\partial_{t}} Y+R(Y, \dot{c}) \dot{c}=0
$$




\section{Theorem.}

(1) The vector space $\mathcal{J}_{c}$ is canonically isomorphic to the vector space $T_{c(t)} M \times$ $T_{c(t)} M$ via $\mathcal{J}_{c} \ni Y \mapsto\left(Y(t),\left(\nabla_{\partial_{t}} Y\right)(t)\right)$, for each $t \in[0,1]$.

(2) The vector space $\mathcal{J}_{c}$ carries a canonical symplectic structure (see (20.4)):

$$
\omega_{c}(Y, Z)=g\left(Y(t),\left(\nabla_{\partial_{t}} Z\right)(t)\right)-g\left(Z(t),\left(\nabla_{\partial_{t}} Y\right)(t)\right)=\text { constant in } t .
$$

(3) Now let $c^{\prime} \neq 0$. Then $\mathcal{J}_{c}$ splits naturally into the direct sum $\mathcal{J}_{c}=$ $\mathcal{J}_{c}^{\top} \oplus \mathcal{J}_{c}^{\perp}$. Here $\mathcal{J}_{c}^{\top}$ is the 2-dimensional $\omega_{c}$-nondegenerate subspace of all Jacobi fields which are tangent to $c$. All these are of the form $t \mapsto(a+t b) c^{\prime}(t)$ for $(a, b) \in \mathbb{R}^{2}$. Also, $\mathcal{J}_{c}^{\perp}$ is the $(2 m-2)$-dimensional $\omega_{c}$-nondegenerate subspace consisting of all Jacobi fields $Y$ satisfying $g\left(Y(t), c^{\prime}(t)\right)=0$ for all t. Moreover, $\omega_{c}\left(\mathcal{J}_{c}^{\top}, \mathcal{J}_{c}^{\perp}\right)=0$.

(4) Each Jacobi field $Y \in \mathcal{J}_{c}$ is the variation vector field of a 1-parameter variation of $c$ through geodesics, and conversely.

(5) Let $\mathcal{J}_{c}^{0}$ be the $m$-dimensional vector space consisting of all Jacobi fields $Y$ with $Y(0)=0$. Then $\omega_{c}\left(\mathcal{J}_{c}^{0}, \mathcal{J}_{c}^{0}\right)=0$, so $\mathcal{J}_{c}^{0}$ is a Lagrangian subspace (see (20.4)).

Proof. First let $c^{\prime}(t)=0$ so $c(t)=c(0)$. Then $Y(t) \in T_{c(0)} M$ for all $t$. The Jacobi equation becomes $\nabla_{t} \nabla_{t} Y=Y^{\prime \prime}$ so $Y(t)=A+t B$ for $A, B \in T_{c(0)} M$. Then (11), (2), and (5) hold.

Let us now assume that $c^{\prime} \neq 0$. Part (1) follows from (27.1)

(2) For $Y, Z \in \mathcal{J}_{c}$ consider:

$$
\begin{aligned}
\omega_{c}(Y, Z)(t)= & g\left(Y(t),\left(\nabla_{\partial_{t}} Z\right)(t)\right)-g\left(Z(t),\left(\nabla_{\partial_{t}} Y\right)(t)\right), \\
\partial_{t} \omega_{c}(Y, Z)= & g\left(\nabla_{\partial_{t}} Y, \nabla_{\partial_{t}} Z\right)+g\left(Y, \nabla_{\partial_{t}} \nabla_{\partial_{t}} Z\right) \\
& \quad-g\left(\nabla_{\partial_{t}} Z, \nabla_{\partial_{t}} Y\right)-g\left(Z, \nabla_{\partial_{t}} \nabla_{\partial_{t}} Y\right) \\
= & -g\left(Y, R\left(Z, c^{\prime}\right) c^{\prime}\right)+g\left(Z, R\left(Y, c^{\prime}\right) c^{\prime}\right) \\
= & -g\left(R\left(Z, c^{\prime}\right) c^{\prime}, Y\right)+g\left(R\left(Y, c^{\prime}\right) c^{\prime}, Z\right) \\
= & g\left(R\left(Z, c^{\prime}\right) Y, c^{\prime}\right)-g\left(R\left(Y, c^{\prime}\right) Z, c^{\prime}\right)=0,
\end{aligned}
$$

where we used (24.4.4) and (24.4.5), Thus $\omega_{c}(Y, Z)(t)$ is constant in $t$. Also it is the standard symplectic structure (see (20.5) on $T_{c(t)} M \times T_{c(t)} M$ induced by $g_{c(t)}$ via (1).

(33) We have $c^{\prime} \neq 0$. In the Fermi chart $\left(U_{c, \rho}, u_{c, \rho}\right)$ along $c$ we have $c^{\prime}=e_{1}$, the first unit vector, and the Jacobi equation becomes

$$
Y \in \mathcal{J}_{c} \Longleftrightarrow Y^{\prime \prime}(t)+R\left(Y, e_{1}\right) e_{1}=0 .
$$

Consider first a Jacobi field $Y(t)=f(t) \cdot c^{\prime}(t)$ which is tangential to $c^{\prime}$. From (6) we get

$$
0=Y^{\prime \prime}(t)+R\left(Y(t), e_{1}\right) e_{1}=f^{\prime \prime}(t) \cdot e_{1}+f(t) \cdot R\left(e_{1}, e_{1}\right) e_{1}=f^{\prime \prime}(t) \cdot e_{1}
$$


so that $f(t)=a+t b$ for $a, b \in \mathbb{R}$. Let $g(t)=a^{\prime}+t b^{\prime}$. We use the symplectic structure at $t=0$ to get $\omega_{c}\left(f . c^{\prime}, g . c^{\prime}\right)=g\left(a . c^{\prime}, b \cdot c^{\prime}\right)-g\left(a^{\prime} \cdot c^{\prime}, b . c^{\prime}\right)=\left(a b^{\prime}-\right.$ $\left.a^{\prime} b\right)\left|c^{\prime}\right|^{2}$, a multiple of the canonical symplectic structure on $\mathbb{R}^{2}$.

For an arbitrary $Y \in \mathcal{J}_{c}$ we can then write $Y=Y_{1}+Y_{2}$ uniquely where $Y_{1} \in \mathcal{J}_{c}^{\top}$ is tangent to $c^{\prime}$ and where $Y_{2}$ is in the $\omega_{c}$-orthogonal complement to $\mathcal{J}_{c}^{\top}$ in $\mathcal{J}_{c}$ :

$0=\omega_{c}\left(c^{\prime}, Y_{2}\right)=g\left(c^{\prime}, \nabla_{\partial_{t}} Y_{2}\right)-g\left(\nabla_{\partial_{t}} c^{\prime}, Y_{2}\right)=g\left(c^{\prime}, \nabla_{\partial_{t}} Y_{2}\right) \quad \Longrightarrow \nabla_{\partial_{t}} Y_{2} \perp c^{\prime}$,

$0=\omega_{c}\left(t . c^{\prime}, Y_{2}\right)=g\left(t . c^{\prime}, \nabla_{\partial_{t}} Y_{2}\right)-g\left(c^{\prime}, Y_{2}\right)=-g\left(c^{\prime}, Y_{2}\right) \quad \Longrightarrow Y_{2} \perp c^{\prime}$.

Conversely, $Y_{2} \perp{ }^{g} c^{\prime}$ implies $0=\partial_{t} g\left(c^{\prime}, Y_{2}\right)=g\left(c^{\prime}, \nabla_{\partial_{t}} Y_{2}\right)$ so that $Y_{2} \in \mathcal{J}_{c}^{\perp}$ and $\mathcal{J}_{c}^{\perp}$ equals the $\omega_{c}$-orthogonal complement of $\mathcal{J}_{c}^{\top}$. By symplectic linear algebra the latter space is $\omega_{c}$-nondegenerate.

(44) for $\dot{c} \neq 0$ and $\dot{c}=0$. Let $Y \in \mathcal{J}_{c}$ be a Jacobi field. Consider $b(s):=$ $\exp _{c(0)}(s . Y(0))$. We look for a vector field $X$ along $b$ such that $\left(\nabla_{\partial_{s}} X\right)(0)=$ $\nabla_{\partial_{t}} Y(0)$. We try $X(s):=\operatorname{Pt}(b, s)\left(\dot{c}(0)+s \cdot\left(\nabla_{\partial_{t}} Y\right)(0)\right)$, then

$$
\begin{aligned}
X^{\prime}(0) & =\left.\partial_{s}\right|_{0}\left(\operatorname{Pt}(b, s)\left(\dot{c}(0)+s \cdot\left(\nabla_{\partial_{t}} Y\right)(0)\right)\right) \\
& =\left.\partial_{s}\right|_{0}\left(\operatorname{Pt}(b, s)(\dot{c}(0))+\left.T(\operatorname{Pt}(b, 0)) \partial_{s}\right|_{0}\left(\dot{c}(0)+s \cdot\left(\nabla_{\partial_{t}} Y\right)(0)\right)\right. \\
& =C\left(b^{\prime}(0), \dot{c}(0)\right)+\operatorname{vl}_{T M}\left(\dot{c}(0),\left(\nabla_{\partial_{t}} Y\right)(0)\right) \quad u \operatorname{using}(24.2)
\end{aligned}
$$

Now we put $\gamma(s, t):=\exp _{b(s)}(t . X(s))$; then $\gamma(0, t)=\exp _{c(0)}(t . X(0))=$ $\exp _{c(0)}(t . \dot{c}(0))=c(t)$. Obviously, $\gamma$ is a 1-parameter variation of $c$ through geodesics; thus the variation vector field $Z(t)=\left.\partial_{s}\right|_{0} \gamma(s, t)$ is a Jacobi vector field. We have

$$
\begin{aligned}
Z(0) & =\left.\partial_{s}\right|_{0} \gamma(s, 0)=\left.\partial_{s}\right|_{0} \exp _{b(s)}\left(0_{b(s)}\right)=\left.\partial_{s}\right|_{0} b(s)=Y(0), \\
\left(\nabla_{\partial_{t}} Z\right)(0) & =\left.\nabla_{\partial_{t}}\left(T \gamma \cdot \partial_{s}\right)\right|_{s=0, t=0} \\
& =\left.\nabla_{\partial_{s}}\left(T \gamma \cdot \partial_{t}\right)\right|_{s=0, t=0} \quad \text { by }(22.10 .4) \text { or }(27.1 .1) \\
& =\left.\nabla_{\partial_{s}}\left(\left.\partial_{t}\right|_{0} \exp _{b(s)}(t . X(s))\right)\right|_{s=0}=\left.\nabla_{\partial_{s}} X\right|_{s=0} \\
& =K\left(\left.\partial_{s}\right|_{0} X(s)\right)=K\left(C\left(b^{\prime}(0), \dot{c}(0)\right)+\operatorname{vl}\left(\dot{c}(0),\left(\nabla_{\partial_{t}} Y\right)(0)\right)\right) \\
& =0+\left(\nabla_{\partial_{t}} Y\right)(0) .
\end{aligned}
$$

Thus $Z=Y$ by (1).

(15) follows from (11) and symplectic linear algebra; see (20.5).

27.6. Lemma. Let $c$ be a geodesic with $c^{\prime} \neq 0$ in a Riemann manifold $(M, g)$ and let $Y \in \mathcal{J}_{c}^{0}$ be a Jacobi field along $c$ with $Y(0)=0$. Then we have

$$
Y(t)=T_{t . \dot{c}(0)}\left(\exp _{c(0)}\right) \operatorname{vl}\left(t . \dot{c}(0), t .\left(\nabla_{\partial_{t}} Y\right)(0)\right) .
$$

Proof. Let us step back into the proof of (27.5.4), There we had

$$
b(s)=\exp _{c(0)}(s . Y(0))=c(0),
$$




$$
\begin{aligned}
X(s) & =\operatorname{Pt}(c, s)\left(\dot{c}(0)+s \cdot\left(\nabla_{\partial_{t}} Y\right)(0)\right)=\dot{c}(0)+s \cdot\left(\nabla_{\partial_{t}} Y\right)(0) \\
Y(t) & =\left.\partial_{s}\right|_{0} \gamma(s, t)=\left.\partial_{s}\right|_{0} \exp _{b(s)}(t \cdot X(s))=\left.T_{t \cdot \dot{c}(0)}\left(\exp _{c(0)}\right) \partial_{s}\right|_{0} m_{t} X(s) \\
& =\left.T_{t . \dot{c}(0)}\left(\exp _{c(0)}\right) \cdot T\left(m_{t}\right) \partial_{s}\right|_{0}\left(\dot{c}(0)+s \cdot\left(\nabla_{\partial_{t}} Y\right)(0)\right) \\
& =T_{t . \dot{c}(0)}\left(\exp _{c(0)}\right) \cdot T\left(m_{t}\right) \cdot \operatorname{vl}\left(\dot{c}(0),\left(\nabla_{\partial_{t}} Y\right)(0)\right) \\
& =T_{t . \dot{c}(0)}\left(\exp _{c(0)}\right) \cdot \operatorname{vl}\left(t \cdot \dot{c}(0), t \cdot\left(\nabla_{\partial_{t}} Y\right)(0)\right) \cdot
\end{aligned}
$$

27.7. Corollary. On a Riemann manifold $(M, g)$ consider $\exp _{x}: T_{x} M \rightarrow$ $M$. Then for $X \in T_{x} M$ the kernel of $T_{X}\left(\exp _{x}\right): T_{X}\left(T_{x} M\right) \rightarrow T_{\exp _{x}(X)} M$ is isomorphic to the linear space consisting of all Jacobi fields $Y \in \mathcal{J}_{c}^{0}$ for $c(t)=\exp \mid{ }_{x}(t X)$ which satisfy $Y(0)=0$ and $Y(1)=0$.

Proof. By (27.6), $Y(t)=T_{t X}\left(\exp _{x}\right) \cdot \operatorname{vl}\left(t X, t\left(\nabla_{\partial_{t}} Y\right)(0)\right)$ is a Jacobi field with $Y(0)=0$. But then $0=Y(1)=T_{X}\left(\exp _{x}\right) \operatorname{vl}\left(X,\left(\nabla_{\partial_{t}} Y\right)(0)\right)$ holds if and only if $\left(\nabla_{\partial_{t}} Y\right)(0) \in \operatorname{ker}\left(T_{X}\left(\exp _{x}\right)\right)$.

27.8. Let $(M, g)$ and $(\tilde{M}, \tilde{g})$ be two Riemann manifolds of the same dimension. Let $c:[0,1] \rightarrow M$ and $\tilde{c}:[0,1] \rightarrow \tilde{M}$ be two geodesics of the same length. We choose a linear isometry $I_{0}:\left(T_{c(0)} M, g_{c(0)}\right) \rightarrow\left(T_{\tilde{c}(0)} \tilde{M}, \tilde{g}_{\tilde{c}(0)}\right)$ and define the linear isometries:

$$
I_{t}:=\tilde{\operatorname{Pt}}(\tilde{c}, t) \circ I_{0} \circ \operatorname{Pt}(c, t)^{-1}: T_{c(t)} M \rightarrow T_{\tilde{c}(t)} \tilde{M} .
$$

Lemma. If $Y$ is a vector field along $c$, then $t \mapsto\left(I_{*} Y\right)(t)=I_{t} Y(t)$ is a vector field along $\tilde{c}$ and we have $\tilde{\nabla}_{\partial_{t}}\left(I_{*} Y\right)=I_{*}\left(\nabla_{\partial_{t}} Y\right)$ so that $\tilde{\nabla}_{\partial_{t}} \circ I_{*}=$ $I_{*} \circ \nabla_{\partial_{t}}$.

Proof. We use Fermi charts (with the minimum of the two $\rho$ 's)

$$
\begin{gathered}
M \supset U_{c, \rho} \stackrel{u_{c, \rho} \longrightarrow}{\longrightarrow}(-\varepsilon, 1+\varepsilon) \times B_{c(0)}^{\perp}(\rho) \\
\operatorname{Id} \times I_{0} \downarrow \text { linear } \\
\tilde{M} \supset U_{\tilde{c}, \rho} \stackrel{u_{\tilde{c}, \rho}}{\longrightarrow}(-\varepsilon, 1+\varepsilon) \times B_{\tilde{c}(0)}^{\perp}(\rho) .
\end{gathered}
$$

By construction of the Fermi charts we have $\left(I_{*} Y\right)(t)=T\left(u_{\tilde{c}, \rho}^{-1} \circ\left(\operatorname{Id} \times I_{0}\right) \circ\right.$ $\left.u_{c, \rho}\right) . Y(t)$. Thus

$$
\begin{array}{rlr}
\tilde{\nabla}_{\partial_{t}}\left(I_{*} Y\right)(t) & =\tilde{\nabla}_{\partial_{t}}\left(T\left(u_{\tilde{c}, \rho}^{-1} \circ\left(\operatorname{Id} \times I_{0}\right) \circ u_{c, \rho}\right) \cdot Y\right)(t) & \\
& =T\left(u_{\tilde{c}, \rho}\right)^{-1} \partial_{t}\left(\left(\operatorname{Id} \times I_{0}\right) \circ T\left(u_{c, \rho}\right) \cdot Y(t)\right) & \text { by }(27.4) \\
& =T\left(u_{\tilde{c}, \rho}\right)^{-1} \cdot\left(\operatorname{Id} \times I_{0}\right) \cdot \partial_{t} T\left(u_{c, \rho}\right) \cdot Y(t) & \\
& =T\left(u_{\tilde{c}, \rho}\right)^{-1} \cdot\left(\operatorname{Id} \times I_{0}\right) \cdot T\left(u_{c, \rho}\right) \cdot\left(\nabla_{\partial_{t}} Y\right)(t) & \text { by }(27.4) \\
& =I_{*}\left(\nabla_{\partial_{t}} Y\right)(t) .
\end{array}
$$


27.9. Jacobi operators. On a Riemann manifold $(M, g)$ with curvature $R$ we consider for each vector field $X \in \mathfrak{X}(M)$ the corresponding Jacobi operator $R_{X}: T M \rightarrow T M$ which is given by $R_{X}(Y)=R(Y, X) X$. It turns out that each $R_{X}$ is a self-adjoint endomorphism, $g\left(R_{X}(Y, Z)\right)=g\left(Y, R_{X}(Z)\right)$, since we have $g(R(Y, X) X, Z)=g(R(X, Z) Y, X)=g(R(Z, X) X, Y)$ by $(24.4 .4)$ and (24.4.5), One can reconstruct the curvature $R$ from the family of Jacobi operators $R_{X}$ by polarization and the properties from (24.4).

27.10 Theorem (E. Cartan). Let $(M, g)$ and $(\tilde{M}, \tilde{g})$ be Riemann manifolds of the same dimension. Let $x \in M, \tilde{x} \in \tilde{M}$, and $\varepsilon>0$ be such that $\exp _{x}: B_{0_{x}}(\varepsilon) \rightarrow M$ and $\exp _{\tilde{x}}: B_{0_{\tilde{x}}}(\varepsilon) \rightarrow \tilde{M}$ are both diffeomorphisms onto their images. Let $I_{x}:\left(T_{x} M, g_{x}\right) \rightarrow\left(T_{\tilde{x}} \tilde{M}, \tilde{g}_{\tilde{x}}\right)$ be a linear isometry. Then the following hold:

The mapping $\Phi:=\exp _{\tilde{x}} \circ I_{x} \circ\left(\exp _{x} \mid B_{0_{x}}(\varepsilon)\right)^{-1}: B_{x}(\varepsilon) \rightarrow B_{0_{x}}(\varepsilon) \rightarrow B_{0_{\tilde{x}}}(\varepsilon) \rightarrow$ $B_{\tilde{x}}(\varepsilon)$ is a diffeomorphism which maps radial geodesics to radial geodesics. The tangent mapping $T \Phi$ maps Jacobi fields $Y$ along radial geodesics with $Y(0)=0$ to Jacobi fields $\tilde{Y}$ along radial geodesics with $\tilde{Y}(0)=0$.

Suppose that moreover for all radial geodesics $c$ in $B_{x}(\varepsilon)$ and their images $\tilde{c}=\Phi \circ c$ the property

$$
I_{t} \circ R_{\dot{c}(t)}=\tilde{R}_{\dot{\tilde{c}}(t)} \circ I_{t}
$$

holds where $I_{t}: T_{c(t)} M \rightarrow T_{\tilde{c}(t)} \tilde{M}$ is defined in (27.8). Then $\Phi$ is an isometry. Conversely, if $\Phi$ is an isometry, then (1) holds.

Proof. It is clear that $\Phi$ maps radial geodesics in $B_{x}(\varepsilon) \subset M$ to radial geodesics in $B_{\tilde{x}}(\varepsilon) \subset \tilde{M}$. Any Jacobi field $Y$ along a radial geodesic $c$ can be written as variation vector field $Y(t)=\left.\partial_{s}\right|_{0} \gamma(s, t)$ where $\gamma(s, \quad)$ is a radial geodesic for all $s$ and $\gamma(0, t)=c(t)$. Then $T \Phi . Y(t)=T \Phi .\left.\partial_{s}\right|_{0} \gamma(s, t)=$ $\left.\partial_{s}\right|_{0}(\Phi \gamma(s, t))$, and any $\Phi \gamma(s, \quad)$ is a radial geodesic in $B_{\tilde{x}}(\varepsilon)$. Thus $T \Phi . Y$ is a Jacobi field along the radial geodesic $\Phi \circ c$ with $T \Phi . Y(0)=0$. This proves the first assertion.

Now let $Y$ be a Jacobi field along the radial geodesic $c$ with $Y(0)=0$. Then the Jacobi equation $0=\nabla_{\partial_{t}} \nabla_{\partial_{t}} Y+R_{\dot{c}}(Y)$ holds. Consider $\left(I_{*} Y\right)(t)=$ $I_{t} Y(t)$. By (27.8) and (1) we then have

$$
\tilde{\nabla}_{\partial_{t}} \tilde{\nabla}_{\partial_{t}}\left(I_{*} Y\right)+\tilde{R}_{\dot{\tilde{c}}}\left(I_{*} Y\right)=I_{*}\left(\nabla_{\partial_{t}} \nabla_{\partial_{t}} Y+R_{\dot{c}} Y\right)=0 .
$$

Thus $I_{*} Y$ is again a Jacobi field along the radial geodesic $\tilde{c}$ with $\left(I_{*} Y\right)(0)=$ 0 . Since also $\tilde{\nabla}_{\partial_{t}}\left(I_{*} Y\right)(0)=I_{*}\left(\nabla_{\partial_{t}} Y\right)(0)=I_{0}\left(\nabla_{\partial_{t}} Y\right)(0)=T \Phi .\left(\nabla_{\partial_{t}} Y\right)(0)$, we get $I_{*} Y=T \Phi . Y$. Since the vectors $Y(t)$ for Jacobi fields $Y$ along $c$ with $Y(0)=0$ span $T_{c(t)} M$ by (27.6), we may conclude that $T_{c(t)} \Phi=I_{t}$ : $T_{c(t)} M \rightarrow T_{\tilde{c}(t)} \tilde{M}$ is an isometry. The converse statement is obvious since an isometry intertwines the curvatures. 
27.11. Conjugate points. Let $c:[0, a] \rightarrow M$ be a geodesic on a Riemann manifold $(M, g)$ with $c(0)=x$. A parameter $t_{0} \in[0, a]$ or its image $c\left(t_{0}\right) \in$ $c([0, a])$ is called a conjugate point for $x=c(0)$ on $c([0, a])$ if the tangent mapping

$$
T_{t_{0} \dot{c}(0)}\left(\exp _{x}\right): T_{t_{0} \dot{c}(0)}\left(T_{x} M\right) \rightarrow T_{c\left(t_{0}\right)} M
$$

is not an isomorphism. Then $t_{0}>0$. The multiplicity of the conjugate point is the dimension of the kernel of $T_{t_{0} \dot{c}(0)}\left(\exp _{x}\right)$ which equals the dimension of the subspace of all Jacobi fields $Y$ along $c$ with $Y(0)=0$ and $Y\left(t_{0}\right)=0$, by $(27.7)$

27.12. Example. Let $M=\rho \cdot S^{m} \subset \mathbb{R}^{M+1}$, the sphere of radius $\rho>$ 0 . Then any geodesic $c$ with $|\dot{c}|=1$ satisfies $c(\rho \pi)=-c(0)$, so $-c(0)$ is conjugate to $c(0)$ along $c$ with multiplicity $m-1$.

27.13. Lemma. Let $c:[0, a] \rightarrow M$ be a geodesic in a Riemann manifold $(M, g)$. Then the vector $\left.\partial_{t}(t . \dot{c}(0))\right|_{t=t_{0}}=\operatorname{vl}\left(t_{0} \cdot \dot{c}(0), \dot{c}(0)\right) \in T_{t_{0} \cdot \dot{c}(0)}\left(T_{c(0)} M\right)$ is orthogonal to the kernel $\operatorname{ker}\left(T_{t_{0} \dot{c}(0)}\left(\exp _{c(0)}\right)\right)$, for any $t_{0} \in[0, a]$.

Proof. If $c\left(t_{0}\right)$ is not a conjugate point to $x=c(0)$ of $c$, this is clearly true. If it is, let $Y$ be the Jacobi field along $c$ with $Y(0)=0$ and $\left(\nabla_{\partial_{t}} Y\right)(0)=X \neq 0$ where $\operatorname{vl}\left(t_{0} \dot{c}(0), X\right) \in \operatorname{ker}\left(T_{t_{0} \dot{c}(0)}\left(\exp _{x}\right)\right)$. Then we have

$$
T_{t_{0} \dot{c}(0)}\left(\exp _{x}\right) \operatorname{vl}\left(t_{0} \dot{c}(0), X\right)=Y\left(t_{0}\right)=0 .
$$

Let $\hat{c}(t)=\left(t-t_{0}\right) \dot{c}(0) \in \mathcal{J}_{c}^{\top}$, a tangential Jacobi field along $c$. By (27.5.2) applied for $t=0$ and for $t-t_{0}$ we get

$$
\begin{aligned}
\omega_{c}(\hat{c}, Y) & =g\left(\hat{c}(0),\left(\nabla_{\partial_{t}}\right) Y(0)\right)-g\left(Y(0),\left(\nabla_{\partial_{t}} Y\right)(0)\right)=g\left(t_{0} \cdot \dot{c}(0), X\right)-0 \\
& =g\left(\hat{c}\left(t_{0}\right),\left(\nabla_{\partial_{t}}\right) Y\left(t_{0}\right)\right)-g\left(Y\left(t_{0}\right),\left(\nabla_{\partial_{t}} Y\right)\left(t_{0}\right)\right)=0 .
\end{aligned}
$$

Thus $t_{0} . g(\dot{c}(0), X)=0$ and since $t_{0}>0$, we get $X \perp \dot{c}(0)$.

We can extract more information about the Jacobi field $Y$ from this proof. We showed that then $\left(\nabla_{\partial_{t}} Y\right)(0) \perp{ }^{g} \dot{c}(0)$. We use this in the following application of (27.5.2) for $t=0$ : Now

$$
\omega_{c}(\dot{c}, Y)=g\left(\dot{c}(0),\left(\nabla_{\partial_{t}} Y\right)(0)\right)-g\left(Y\left(0,\left(\nabla_{\partial_{t}} \dot{c}\right)(0)\right)\right)=0 .
$$

Together with $\omega_{c}(\hat{c}, Y)=0$ from the proof this says that $Y \in \mathcal{J}_{c}^{\perp}$, so by $(27.5 .3), Y(t) \perp{ }^{g} \dot{c}(t)$ for all $t$.

Let us denote by $\mathcal{J}_{c}^{\perp, 0}=\mathcal{J}_{c}^{\perp} \cap \mathcal{J}_{c}^{0}$ the space of all Jacobi fields $Y$ with $Y(0)=0$ and $Y(t) \perp{ }^{g} \dot{c}(t)$ for all $t$. Then the dimension of the kernel of $T_{t_{0} \dot{c}(0)}\left(\exp _{x}\right)$ equals the dimension of the space of all $Y \in \mathcal{J}_{c}^{\perp, 0}$ which satisfy $Y\left(t_{0}\right)=0$.

Thus, if $c(0)$ and $c\left(t_{0}\right)$ are conjugate, then there are 1-parameter variations of $c$ through geodesics which all start at $c(0)$ and end at $c\left(t_{0}\right)$, at least 
infinitesimally in the variation parameter. For this reason conjugate points are also called focal points. We will strengthen this later on.

27.14. The Hessian of the energy alias second variation formulas. Let $(M, g)$ be a Riemann manifold. Let $c:[0, a] \rightarrow M$ be a geodesic with $c(0)=x$ and $c(a)=y$. A smooth variation of $c$ with fixed ends is a smooth mapping $F:(-\varepsilon, \varepsilon) \times[0, a] \rightarrow M$ with $F(0, t)=c(t), F(s, 0)=x$, and $F(s, a)=y$. The variation vector field for $F$ is the vector field $X=$ $\left.\partial_{s}\right|_{0} F(s, \quad)$ along $c$, with $X(0)=0$ and $X(a)=0$.

The space $C^{\infty}(([0, a], 0, a),(M, x, y))$ of all smooth curves $\gamma:[0, a] \rightarrow M$ with $c(0)=x$ and $c(a)=y$ is an infinite-dimensional smooth manifold modeled on Fréchet spaces. See 113 for a thorough account of this. The tangent space $T_{c}\left(C^{\infty}(([0, a], 0, a),(M, x, y))\right)$ at the geodesic $c$ of this infinitedimensional manifold consists of all variation vector fields along $c$ as above. We consider again the energy as a smooth function

$$
E: C^{\infty}(([0, a], 0, a),(M, x, y)) \rightarrow \mathbb{R}, \quad E(\gamma)=\frac{1}{2} \int_{0}^{a}|\dot{\gamma}(t)|_{g}^{2} d t
$$

Let now $F$ be a variation with fixed ends of the geodesic $c$. Then we have:

$$
\begin{aligned}
\partial_{s} E(F(s, \quad)) & =\frac{1}{2} \int_{0}^{a} \partial_{s} g\left(\partial_{t} F, \partial_{t} F\right) d t=\int_{0}^{a} g\left(\nabla_{\partial_{s}} \partial_{t} F, \partial_{t} F\right) d t \\
& =\int_{0}^{a} g\left(\nabla_{\partial_{t}} \partial_{s} F, \partial_{t} F\right) d t, \quad \text { by (22.10.4) or (27.1.1). }
\end{aligned}
$$

Therefore,

$$
\begin{aligned}
&\left.\partial_{s}^{2}\right|_{0} E(F(s, \quad))=\left.\int_{0}^{a}\left(g\left(\nabla_{\partial_{s}} \nabla_{\partial_{t}} \partial_{s} F, \partial_{t} F\right)+g\left(\nabla_{\partial_{t}} \partial_{s} F, \nabla_{\partial_{s}} \partial_{t} F\right)\right)\right|_{s=0} d t \\
&= \int_{0}^{a}\left(g\left(\nabla_{\partial_{t}} \nabla_{\partial_{s}} \partial_{s} F, \partial_{t} F\right)+g\left(R\left(\partial_{s} F, \partial_{t} F\right) \partial_{s} F, \partial_{t} F\right)\right. \\
&\left.\quad+g\left(\nabla_{\partial_{t}} \partial_{s} F, \nabla_{\partial_{t}} \partial_{s} F\right)\right)\left.\right|_{s=0} d t \quad \text { by } \underbrace{(24.5)} \text { and }(22.10 .4) \\
&=\left.\int_{0}^{a}\left(g\left(\nabla_{\partial_{t}} \partial_{s} F, \nabla_{\partial_{t}} \partial_{s} F\right)+g\left(R\left(\partial_{s} F, \partial_{t} F\right) \partial_{s} F, \partial_{t} F\right)\right)\right|_{s=0} d t \\
& \quad+\int_{0}^{a}\left(\left.g\left(\nabla_{\partial_{t}} \nabla_{\partial_{s}} \partial_{s} F, \partial_{t} F\right)\right|_{s=0}+g(\left.\nabla_{\partial_{s}} \partial_{s} F\right|_{s=0}, \underbrace{\left.\nabla_{\partial_{t}} \partial_{t} F\right|_{s=0}}_{\nabla_{\partial_{t}} \dot{c}=0})\right) d t
\end{aligned}
$$

The last summand equals $\left.\int_{0}^{a} \partial_{t} g\left(\nabla_{\partial_{s}} \partial_{s} F, \partial_{t} F\right)\right|_{s=0} d t$ which vanishes since we have a variation with fixed ends and thus $\left(\nabla_{\partial_{s}} \partial_{s} F\right)(s, 0)=0$ and also $\left(\nabla_{\partial_{s}} \partial_{s} F\right)(s, a)=0$. Recall $X=\left.\partial_{s}\right|_{0} F$, a vector field along $c$ with $X(0)=0$ and $X(a)=0$. Thus

$d^{2} E(c)(X, X)=\left.\partial_{s}^{2}\right|_{0} E(F(s, \quad))=\int_{0}^{a}\left(g\left(\nabla_{\partial_{t}} X, \nabla_{\partial_{t}} X\right)+g(R(X, \dot{c}) X, \dot{c})\right) d t$. 
If we polarize this, we get the Hessian of the energy at a geodesic $c$ as follows (the boundary terms vanish since $X, Y$ vanish at the ends 0 and $a$ ):

$$
\begin{aligned}
d E(c)(X) & =\int_{0}^{a} g\left(\nabla_{\partial_{t}} X, \dot{c}\right) d t=-\int_{0}^{a} g\left(X, \nabla_{\partial_{t}} \dot{c}\right) d t=0, \\
d^{2} E(c)(X, Y) & =\int_{0}^{a}\left(g\left(\nabla_{\partial_{t}} X, \nabla_{\partial_{t}} Y\right)-g\left(R_{\dot{c}}(X), Y\right)\right) d t \\
d^{2} E(c)(X, Y) & =-\int_{0}^{a} g\left(\nabla_{\partial_{t}} \nabla_{\partial_{t}} X+R_{\dot{c}}(X), Y\right) d t .
\end{aligned}
$$

We see that among all vector fields $X$ along $c$ with $X(0)=0$ and $X(a)=0$ those which satisfy $d^{2} E(c)(X, Y)=0$ for all $Y$ are exactly the Jacobi fields. We shall need a slight generalization. Let $X, Y$ be continuous vector fields along $c$ which are smooth on $\left[t_{i}, t_{i+1}\right]$ for $0=t_{0}<t_{1}<\cdots<t_{k}=a$, and which vanish at 0 and $a$. These are tangent vectors at $c$ to the smooth manifold of all curves from $x$ to $y$ which are piecewise smooth in the same manner. Then we take the following formula as a definition, which can be motivated by the computations above (with considerable care). We will just need that $d^{2} E(c)$, to be defined below, is continuous in the natural uniform $C^{2}$-topology on the space of piecewise smooth vector fields.

$$
\begin{aligned}
d^{2} E(c)(X, Y)=\int_{0}^{a}\left(g\left(\nabla_{\partial_{t}} X, \nabla_{\partial_{t}} Y\right)+g(R(X, \dot{c}) Y, \dot{c})\right) d t \\
=\sum_{i=0}^{k-1} \int_{t_{i}}^{t_{i+1}}\left(g\left(\nabla_{\partial_{t}} X, \nabla_{\partial_{t}} Y\right)+g(R(X, \dot{c}) Y, \dot{c})\right) d t \\
=\sum_{i=0}^{k-1} \int_{t_{i}}^{t_{i+1}}\left(\partial_{t} g\left(\nabla_{\partial_{t}} X, Y\right)-g\left(\nabla_{\partial_{t}} \nabla_{\partial_{t}} X, Y\right)-g(R(X, \dot{c}) \dot{c}, Y)\right) d t \\
=-\int_{0}^{a} g\left(\nabla_{\partial_{t}} \nabla_{\partial_{t}} X+R_{\dot{c}}(X), Y\right) d t \\
\quad+\sum_{i=0}^{k-1}\left(g\left(\left(\nabla_{\partial_{t}} X\right)\left(t_{i+1}-\right), Y\left(t_{i+1}\right)\right)-g\left(\left(\nabla_{\partial_{t}} X\right)\left(t_{i}+\right), Y\left(t_{i+1}\right)\right)\right) .
\end{aligned}
$$

27.15. Theorem. Let $(M, g)$ be a Riemann manifold and let $c:[0, a] \rightarrow M$ be a geodesic with $c(0)=x$ and $c(a)=y$.

(1) If $T_{t \dot{c}(0)}\left(\exp _{x}\right): T_{t \dot{c}(0)}\left(T_{x} M\right) \rightarrow T_{c(t)} M$ is an isomorphism for all $t \in$ $[0, a]$, then for any smooth curve e from $x$ to $y$ which is near enough to $c$ the length $L(e) \geq L(c)$ with equality if and only if $e$ is a reparameterization of c. Moreover, $d^{2} E(c)(X, X) \geq 0$ for each smooth vector field $X$ along $c$ which vanishes at the ends.

(2) If there are conjugate points $c(0), c\left(t_{1}\right)$ along $c$ with $0<t_{1}<a$, then there exists a smooth vector field $X$ along $c$ with $X(0)=0$ and $X(a)=0$ 
such that $d^{2} E(c)(X, X)<0$. Thus for any smooth variation $F$ of $c$ with $\left.\partial_{s}\right|_{0} F(s, \quad)=X$ the curve $F(s, \quad)$ from $x$ to $y$ is shorter than $c$ for all $0<|s|<\varepsilon$.

Proof. (11) Since $T_{t \dot{c}(0)}\left(\exp _{x}\right): T_{t \dot{c}(0)}\left(T_{x} M\right) \rightarrow T_{c(t)} M$ is an isomorphism, for each $t \in[0, a]$ there exist an open neighborhood $U(t . \dot{c}(0)) \subset T_{x} M$ of $t \dot{c}(0)$ such that $\exp _{x} \mid U(t . \dot{c}(0))$ is a diffeomorphism onto its image. Since $[0, a] . \dot{c}(0)$ is compact in $T_{x} M$, there exists an $\varepsilon>0$ such that $U(t . \dot{c}(0)) \supset B_{t \dot{c}(0)}(\varepsilon)$ for all $t$.

Now let $e:[0, a] \rightarrow M$ be a smooth curve with $e(0)=x$ and $e(a)=y$ which is near $c$ in the sense that there exists a subdivision $0=t_{0}<t_{1}<\cdots<t_{k}=a$ with $e\left(\left[t_{i}, t_{i+1}\right]\right) \subset \exp _{x}\left(B_{t_{i} \dot{c}(0)}(\varepsilon)\right)$. We put:

$$
\begin{aligned}
& \tilde{e}:[0, a] \rightarrow T_{x} M, \\
& \tilde{e}(t):=\left(\exp _{x} \mid B_{t_{i} \dot{c}(0)}(\varepsilon)\right)^{-1}(e(t)), \quad t \in\left[t_{i}, t_{i+1}\right] .
\end{aligned}
$$

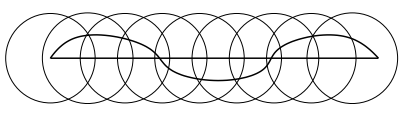

Then $\tilde{e}$ is smooth, $\tilde{e}(0)=0_{x}, \tilde{e}(a)=a . \dot{c}(0)$, and $\exp _{x}(\tilde{e}(t))=e(t)$. We consider the polar representation $\tilde{e}(t)=r(t) \cdot \varphi(t)$ in $T_{x} M$ where $\varphi(t)=\frac{\tilde{e}(t)}{|\tilde{e}(t)|}$ and $r(t)=|\tilde{e}(t)|$. Let $r=|\tilde{e}(a)|=a|\dot{c}(0)|$. Then we put:

$$
\gamma(s, t)=\exp _{x}(\text { r.t. } \varphi(s))
$$

which implies

$$
e(t)=\gamma\left(t, \frac{r(t)}{r}\right)=\exp _{x}(r(t) \cdot \varphi(t)), \quad \dot{e}(t)=\partial_{s} \gamma\left(t, \frac{r(t)}{r}\right)+\partial_{t} \gamma\left(t, \frac{r(t)}{r}\right) \frac{\dot{r}(t)}{r} .
$$

Note that $\nabla_{\partial_{t}} \partial_{t} \gamma=0$ since $\gamma(s, \quad)$ is a geodesic. From

$$
\begin{aligned}
& \partial_{t} g\left(\partial_{s} \gamma, \partial_{t} \gamma\right)=g\left(\nabla_{\partial_{t}} \partial_{s} \gamma, \partial_{t} \gamma\right)+g\left(\partial_{s} \gamma, \nabla_{\partial_{t}} \partial_{t} \gamma\right) \\
& =g\left(\nabla_{\partial_{s}} \partial_{t} \gamma, \partial_{t} \gamma\right)+0 \quad \text { by }(22.10 .1) \\
& =\frac{1}{2} \partial_{s} g\left(\partial_{t} \gamma, \partial_{t} \gamma\right)=\frac{1}{2} \partial_{s}\left|\partial_{t} \gamma(s, \quad)\right|^{2}=\frac{1}{2} \partial_{s} r^{2}|\varphi(s)|^{2}=\frac{1}{2} \partial_{s} r^{2}=0
\end{aligned}
$$

we get that $g\left(\partial_{s} \gamma(s, t), \partial_{t} \gamma(s, t)\right)=g\left(\partial_{s} \gamma(s, 0), \partial_{t} \gamma(s, 0)\right)=g(0, r . \varphi(s))=0$. Thus

$$
g_{\gamma(s, t)}\left(\partial_{s} \gamma(s, t), \partial_{t} \gamma(s, t)\right)=0 \quad \text { for all } s, t .
$$

By Pythagoras

$$
\begin{aligned}
|\dot{e}(t)|_{g}^{2} & =\left|\partial_{s} \gamma\left(t, \frac{r(t)}{r}\right)\right|_{g}^{2}+\left|\partial_{t} \gamma\left(t, \frac{r(t)}{r}\right)\right|_{g}^{2} \frac{|\dot{r}(t)|^{2}}{r^{2}} \\
& =\left|\partial_{s} \gamma\left(t, \frac{r(t)}{r}\right)\right|_{g}^{2}+r^{2}|\varphi(t)|_{g}^{2} \frac{|\dot{r}(t)|^{2}}{r^{2}} \geq|\dot{r}(t)|^{2}
\end{aligned}
$$

with equality if and only if $\partial_{s} \gamma\left(t, \frac{r(t)}{r}\right)=0$, i.e., $\varphi(t)$ is constant in $t$. So (4)

$$
L(e)=\int_{0}^{a}|\dot{e}(t)|_{g} d t \geq \int_{0}^{a}|\dot{r}(t)| d t \geq \int_{0}^{a} \dot{r}(t) d t=r(a)-r(0)=r=L(c)
$$


with equality if and only if $\dot{r}(t) \geq 0$ and $\varphi(t)$ is constant, i.e., $e$ is a reparameterization of $c$.

Note that (3) and (4) generalize Gauß's lemma (23.2) and its corollary (23.3) to more general assumptions.

Now consider a vector field $X$ along $c$ with $X(0)=0$ and $X(a)=0$ and let $F:(-\varepsilon, \varepsilon) \times[0, a] \rightarrow M$ be a smooth variation of $c$ with $F(s, 0)=x$, $F(s, a)=y$, and $\left.\partial_{s}\right|_{0} F=X$. We have

$$
\begin{aligned}
2 E(F(s, \quad)) . a & =\int_{0}^{a}\left|\partial_{t} F\right|_{g}^{2} d t \cdot \int_{0}^{a} 1^{2} d t \geq\left(\int_{0}^{a}\left|\partial_{t} F\right|_{g} \cdot 1 d t\right)^{2} \\
& =L(F(s, \quad))^{2} \geq L(c)^{2} \quad \text { by (4) } \\
& =\left(\int_{0}^{a}|\dot{c}(0)|_{g} d t\right)^{2}=|\dot{c}(0)|^{2} \cdot a^{2}=\int_{0}^{a}|\dot{c}(0)|^{2} d t \cdot a=2 E(c) . a .
\end{aligned}
$$

Since $c$ is a geodesic, we have $\left.\partial_{s}\right|_{0} E(F(s, \quad))=0$ and therefore we also get $d^{2} E(c)(X, X)=\left.\partial_{s}^{2}\right|_{0} E(F(s, \quad)) \geq 0$.

(2) Let $c(0), c\left(t_{1}\right)$ be conjugate points along $c$ with $0<t_{1}<a$. By $(27.11)$ there exists a Jacobi field $Y \neq 0$ along $c$ with $Y(0)=0$ and $Y\left(t_{1}\right)=0$. Choose $0<t_{0}<t_{1}<t_{2}<a$ and a vector field $Z$ along $c$ with $Z \mid\left[0, t_{0}\right]=0$, $Z \mid\left[t_{2}, a\right]=0$, and $Z\left(t_{1}\right)=-\left(\nabla_{\partial_{t}} Y\right)\left(t_{1}\right) \neq 0($ since $Y \neq 0)$. Let $\tilde{Y}$ be the continuous piecewise smooth vector field along $c$ which is given by $\tilde{Y} \mid\left[0, t_{1}\right]=$ $Y \mid\left[0, t_{1}\right]$ and $\tilde{Y} \mid\left[t_{1}, a\right]=0$. Then $\tilde{Y}+\eta Z$ is a continuous piecewise smooth vector field along $c$ which is broken at $t_{1}$ and vanishes at 0 and at $a$. Then we have

$d^{2} E(c)(\tilde{Y}+\eta Z, \tilde{Y}+\eta Z)=d^{2} E(c)(\tilde{Y}, \tilde{Y})+\eta^{2} d^{2} E(c)(Z, Z)+2 \eta d^{2} E(c)(\tilde{Y}, Z)$

and by (22.12.3)

$$
\begin{aligned}
& d^{2} E(c)(\tilde{Y}, \tilde{Y})=-\int_{0}^{t_{1}} g\left(\nabla_{\partial_{t}} \nabla_{\partial_{t}} Y+R_{\dot{c}}(Y), Y\right) \\
&-\int_{t_{1}}^{a} g\left(\nabla_{\partial_{t}} \nabla_{\partial_{t}} 0+R_{\dot{c}}(0), 0\right) \\
&+g\left(\left(\nabla_{\partial_{t}} Y\right)\left(t_{1}-\right), 0\right)-g\left(\left(\nabla_{\partial_{t}} Y\right)(0+), 0\right) \\
&+g\left(\left(\nabla_{\partial_{t}} \tilde{Y}\right)(a-), 0\right)-g\left(\left(\nabla_{\partial_{t}} \tilde{Y}\right)\left(t_{1}+\right), 0\right)=0, \\
& d^{2} E(c)(\tilde{Y}, \tilde{Z})=-\int_{0}^{t_{1}} g\left(\nabla_{\partial_{t}} \nabla_{\partial_{t}} Y+R_{\dot{c}}(Y), Z\right) \\
& \quad-\int_{t_{1}}^{a} g\left(\nabla_{\partial_{t}} \nabla_{\partial_{t}} 0+R_{\dot{c}}(0), Z\right) \\
& \quad+g\left(\left(\nabla_{\partial_{t}} Y\right)\left(t_{1}-\right), Z\left(t_{1}\right)\right)-g\left(\left(\nabla_{\partial_{t}} Y\right)(0+), 0\right) \\
& \quad+g\left(\left(\nabla_{\partial_{t}} \tilde{Y}\right)(a-), 0\right)-g\left(\left(\nabla_{\partial_{t}} 0\right)\left(t_{1}+\right), Z\left(t_{1}\right)\right) \\
&= g\left(\left(\nabla_{\partial_{t}} Y\right)\left(t_{1}\right), Z\left(t_{1}\right)\right)=-g\left(\left(\nabla_{\partial_{t}} Y\right)\left(t_{1}\right),\left(\nabla_{\partial_{t}} Y\right)\left(t_{1}\right)\right)
\end{aligned}
$$




$$
\begin{gathered}
=-\left|\left(\nabla_{\partial_{t}} Y\right)\left(t_{1}\right)\right|_{g}^{2}<0, \\
d^{2} E(c)(\tilde{Y}+\eta Z, \tilde{Y}+\eta Z)=\eta^{2} d^{2} E(c)(Z, Z)-2 \eta\left|\left(\nabla_{\partial_{t}} Y\right)\left(t_{1}\right)\right|_{g}^{2} .
\end{gathered}
$$

The last expression will be negative for $\eta$ small enough. Since $d^{2} E(c)$ is continuous in the $C^{2}$-topology for continuous piecewise smooth vector fields along $c$, we can approximate $\tilde{Y}+\eta Z$ by a smooth vector field $X$ vanishing at the ends such that we still have $d^{2} E(c)(X, X)<0$.

Finally, let $F:(-\varepsilon, \varepsilon) \times[0, a] \rightarrow M$ be any smooth variation of $c$ with fixed ends and $\left.\partial_{s}\right|_{0} F=X$. Consider the Taylor expansion

$$
E(F(s, \quad))=E(c)+s d E(c)(X)+\frac{s^{2}}{2} d^{2} E(c)(X, X)+s^{3} h(s)
$$

where $h(s)=\left.\int_{0}^{1} \frac{(1-u)^{2}}{2} \partial_{v}^{3} E(F(v, \quad))\right|_{v=u s} d u$. Since $d E(c)(X)=0$, this implies $E(F(s))<,E(c)$ for $s \neq 0$ small enough. Using both halves of (5), this implies $L(F(s, \quad))^{2} \leq 2 E(F(s, \quad)) a<2 E(c) a=L(c)^{2}$.

27.16. Theorem. Let $(M, g)$ be a Riemann manifold with sectional curvature $k \geq k_{0}>0$. Then for any geodesic $c$ in $M$ the distance between two conjugate points along $c$ is $\leq \frac{\pi}{\sqrt{k_{0}}}$.

Proof. Let $c:[0, a] \rightarrow M$ be a geodesic with $|\dot{c}|=1$ such that $c(a)$ is the first point which is conjugate to $c(0)$ along $c$. We choose a parallel unit vector field $Z$ along $c, Z(t)=\operatorname{Pt}(c, t) . Z(0),|Z(0)|_{g}=1, Z(t) \perp{ }^{g} \dot{c}(t)$, so that $\nabla_{\partial_{t}} Z=0$. Consider $f \in C^{\infty}([0, a], \mathbb{R})$ with $f(0)=0$ and $f(a)=0$, and let $0<b<a$. By (27.15.1) we have $d^{2} E_{0}^{b}(c)(f Z, f Z) \geq 0$. By (27.14.1) we have

$$
\begin{aligned}
d^{2} E_{0}^{b}(c)(f Z, f Z) & =\int_{0}^{b}\left(g\left(\nabla_{\partial_{t}}(f Z), \nabla_{\partial_{t}}(f Z)\right)-g(R(f Z, \dot{c}) \dot{c}, f Z)\right) d t \\
& =\int_{0}^{b}\left(f^{\prime 2}-f^{2} k(Y \wedge \dot{c})\right) d t \leq \int_{0}^{b}\left(f^{\prime 2}-f^{2} k_{0}\right) d t
\end{aligned}
$$

since $Y, \dot{c}$ form an orthonormal basis. Now we choose $f(t)=\sin (\pi \mathfrak{t} b)$ so that $\int_{0}^{b} f^{2} d t=\frac{b}{2}$ and $\int_{0}^{b} f^{\prime 2} d t=\frac{\pi^{2}}{2 b}$. Thus

$$
0 \leq \int_{0}^{b}\left(f^{\prime 2}-f^{2} k_{0}\right) d t=\frac{\pi^{2}}{2 b}-\frac{b}{2} k_{0}
$$

which implies $b \leq \frac{\pi}{\sqrt{k_{0}}}$. Since this holds for all $b<a$, we get $a \leq \frac{\pi}{\sqrt{k_{0}}}$.

27.17. Corollary (Myers, 1935). Let $M$ be a complete connected Riemann manifold with sectional curvature $k \geq k_{0}>0$. Then the diameter of $M$ is bounded:

$$
\operatorname{diam}(M):=\sup \{\operatorname{dist}(x, y): x, y \in M\} \leq \frac{\pi}{\sqrt{k_{0}}} .
$$

Thus $M$ is compact and each covering space of $M$ is also compact, so the the fundamental group $\pi_{1}(M)$ is finite. 
Proof. By (23.6.6) any two points $x, y \in M$ can be connected by a geodesic $c$ of minimal length. Assume for contradiction that $\operatorname{dist}(x, y)>\frac{\pi}{\sqrt{k_{0}}}$; then by (27.16) there exists an interior point $z$ on the geodesic $c$ which is conjugate to $x$. By (27.15.2) there exist smooth curves in $M$ from $x$ to $y$ which are shorter than $c$, contrary to the minimality of $c$.

27.18. Theorem. Let $M$ be a connected complete Riemann manifold with sectional curvature $k \leq 0$. Then $\exp _{x}: T_{x} M \rightarrow M$ is a covering mapping for each $x \in M$. If $M$ is also simply connected, then $\exp _{x}: T_{x} M \rightarrow M$ is a diffeomorphism.

This result is due to $8 \mathbf{8 1}$ for surfaces and to 33 in the general case.

Proof. Let $c:[0, \infty) \rightarrow M$ be a geodesic with $c(0)=x$. If $c(a)$ is a point conjugate to $c(0)$ along $c$, then by (27.11) and (27.7) there exists a Jacobi field $Y \neq 0$ along $c$ with $Y(0)=0$ and $Y(a)=0$. By (27.13) we have $Y(t) \perp{ }^{g} \dot{c}(t)$ for all $t$. Now use (27.14.2) and (27.14.1) to get

$$
\begin{aligned}
d^{2} E(c)(Y, Y) & =-\int_{0}^{a} g\left(\nabla_{\partial_{t}} \nabla_{\partial_{t}} Y+R_{\dot{c}}(Y), Y\right) d t=0 \\
d^{2} E_{0}^{a}(c)(Y, Y) & =\int_{0}^{a}\left(g\left(\nabla_{\partial_{t}} Y, \nabla_{\partial_{t}} Y\right)-g(R(Y, \dot{c}) \dot{c}, Y)\right) d t \\
& =\int_{0}^{a}\left(\left|\nabla_{\partial_{t}} Y\right|_{g}^{2}-k(Y \wedge \dot{c})\left(|Y|^{2}|\dot{c}|^{2}-g(Y, \dot{c})\right)\right) d t>0,
\end{aligned}
$$

a contradiction. Thus there are no conjugate points. Thus the surjective (by (23.6) mapping $\exp _{x}: T_{x} M \rightarrow M$ is a local diffeomorphism by (27.11), Lemma (27.20) below then finishes the proof.

27.19. A smooth mapping $f:(M, g) \rightarrow(\bar{M}, \bar{g})$ between Riemann manifolds is called distance increasing if $f^{*} \bar{g} \geq g$; in detail, $\bar{g}_{f(x)}\left(T_{x} f . X, T_{x} f . X\right) \geq$ $g_{x}(X, X)$ for all $X \in T_{x} M$, all $x \in M$.

Lemma. Let $(M, g)$ be a connected complete Riemann manifold. If a smooth mapping $f:(M, g) \rightarrow(\bar{M}, \bar{g})$ is surjective and distance increasing, then $f$ is a covering mapping.

Proof. Obviously, $f$ is locally injective; thus $T_{x} f$ is injective for all $x$ and $\operatorname{dim}(M) \leq \operatorname{dim}(\bar{M})$. Since $f$ is surjective, $\operatorname{dim}(M) \geq \operatorname{dim}(\bar{M})$ by the theorem of Sard (1.18).

For each curve $c:[0,1] \rightarrow M$ we have

$$
L_{g}(c)=\int_{0}^{1}\left|c^{\prime}\right|_{g} d t \leq \int_{0}^{1}\left|c^{\prime}\right|_{f^{*} \bar{g}} d t=L_{f^{*} \bar{g}}(c) ;
$$

thus $\operatorname{dist}_{g}(x, y) \leq \operatorname{dist}_{f^{*} \bar{g}}(x, y)$ for $x, y \in M$. So $\left(M, \operatorname{dist}_{f^{*} \bar{g}}\right)$ is a complete metric space and $\left(M, f^{*} \bar{g}\right)$ is a complete Riemann manifold also. Without 
loss we may thus assume that $g=f^{*} \bar{g}$, so that $f$ is a local isometry. Then $(\bar{M}=f(M), \bar{g})$ is also complete.

For fixed $\bar{x} \in \bar{M}$ let $r>0$ such that $\exp _{\bar{x}}: B_{0_{\bar{x}}}(2 r) \rightarrow B_{\bar{x}}(2 r) \subset \bar{M}$ is a diffeomorphism. Let $f^{-1}(\bar{x})=\left\{x_{1}, x_{2}, \ldots\right\}$. For each $i$ the following diagram commutes:

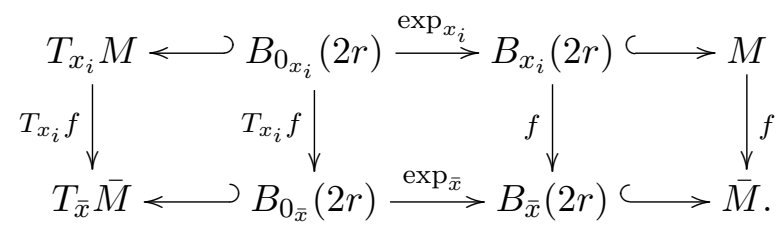

We claim (which finishes the proof):

(1) $f: B_{x_{i}}(2 r) \rightarrow B_{\bar{x}}(2 r)$ is a diffeomorphism for each $i$,

(2) $f^{-1}\left(B_{\bar{x}}(r)\right)=\bigcup_{i} B_{x_{i}}(r)$,

(3) $B_{x_{i}}(r) \cup B_{x_{j}}(r)=\emptyset$ for $i \neq j$.

(11) From the diagram we conclude that $\exp _{x_{i}}$ is injective and $f$ is surjective. Since $\exp _{x_{i}}: B_{0_{x_{i}}}(r) \rightarrow B_{x_{i}}(r)$ is also surjective (by completeness), $f:$ $B_{x_{i}}(r) \rightarrow B_{\bar{x}}(r)$ is injective too and thus a diffeomorphism.

(2) From the diagram (with $2 r$ replaced by $r$ ) we see that $f^{-1}\left(B_{\bar{x}}(r)\right) \supseteq$ $B_{x_{i}}(r)$ for all $i$. If conversely $y \in f^{-1}\left(B_{\bar{x}}(r)\right)$, let $\bar{c}:[0, s] \rightarrow B_{\bar{x}}(r)$ be the minimal geodesic from $f(y)$ to $\bar{x}$ in $\bar{M}$ where $s=\operatorname{dist}_{\bar{g}}(f(y), \bar{x})$. Let $c$ be the geodesic in $M$ which starts at $y$ and satisfies $T_{y} f \cdot c^{\prime}(0)=\bar{c}^{\prime}(0)$. Since $f$ is an infinitesimal isometry, $f \circ c=\bar{c}$ and thus $f(c(s))=\bar{x}$. So $c(s)=x_{i}$ for some $i$. Since $\operatorname{dist}_{g}\left(y, x_{i}\right) \leq s<r$, we have $y \in B_{0_{x_{i}}}(r)$. Thus $f^{-1}\left(B_{\bar{x}}(r)\right) \subseteq \bigcup_{i} B_{x_{i}}(r)$.

(3) If $y \in B_{x_{i}}(r) \cup B_{x_{j}}(r)$, then $x_{j} \in B_{x_{i}}(2 r)$ and by (11) we get $x_{j}=x_{i}$.

27.20. Lemma ([106]). If $M$ is a connected complete Riemann manifold without conjugate points, then $\exp _{x}: T_{x} M \rightarrow M$ is a covering mapping.

Proof. Since $(M, g)$ is complete and connected, $\exp _{x}: T_{x} M \rightarrow M$ is surjective; and it is also a local diffeomorphism by (27.11) since $M$ has no conjugate points. We will construct a complete Riemann metric $\tilde{g}$ on $T_{x} M$ such that $\exp _{x}:\left(T_{x} M, \tilde{g}\right) \rightarrow(M, g)$ is distance increasing. By (27.19) this finishes the proof.

Define the continuous function $h: T_{x} M \rightarrow \mathbb{R}_{>0}$ by

$$
\begin{aligned}
h(X) & =\sup \left\{r:\left|T_{X}\left(\exp _{x}\right) \cdot \xi\right|_{\exp _{x}(X)}^{2} \geq r|\xi|_{g_{x}}^{2} \text { for all } \xi \in T_{x} M\right\} \\
& =\min \left\{\left|T_{X}\left(\exp _{x}\right) \cdot \xi\right|_{\exp _{x}(X)}^{2}:|\xi|_{g_{x}}=1\right\} \\
& =1 / \sqrt{\text { operator norm }\left(T_{X}\left(\exp _{x}\right)^{-1}: T_{\exp _{x}(X)} M \rightarrow T_{x} M\right)} .
\end{aligned}
$$


We use polar coordinates $\varphi: \mathbb{R}_{>0} \times S^{m-1} \rightarrow T_{x} M \backslash\left\{0_{x}\right\}$ given by $\varphi(r, \vartheta)=$ $r . \vartheta$ and express the metric by $\varphi^{*}\left(g_{x}\right)=d r^{2}+r^{2} g^{S}$ where $g^{S}$ is the metric on the sphere. Now we choose an even smooth function $f: \mathbb{R} \rightarrow \mathbb{R}$ which satisfies $0<f(r(X)) \leq h(X)$. Consider the Riemann metric $\tilde{g}=d r^{2}+$ $r^{2} f(r)$ on $T_{x} M$.

For every $R>0$ we have

$$
\bar{B}_{0_{x}}^{\tilde{g}}(R)=\left\{X \in T_{x} M: \operatorname{dist}_{\tilde{g}}\left(X, 0_{x}\right) \leq R\right\} \subseteq\left\{X \in T_{x} M: r(X) \leq R\right\}
$$

which is compact; thus $\left(T_{x} M, \tilde{g}\right)$ is complete.

It remains to check that $\exp _{x}:\left(T_{x} M, \tilde{g}\right) \rightarrow(M, g)$ is distance increasing. Let $\xi \in T_{X}\left(T_{x} M\right)$. If $X=0_{x}$, then $T_{0_{x}}\left(\exp _{x}\right) \cdot \xi=\xi$, so $\exp _{x}$ is distance increasing at $0_{x}$ since $f(0) \leq 1$.

So let $X \neq 0_{x}$. Then $\xi=\xi_{1}+\xi_{2}$ where $d r\left(\xi_{2}\right)=0$; thus $\xi_{2}$ tangent to the sphere through $X$, and $\xi_{1} \perp \xi_{2}$ (with respect to both $g_{x}$ and $\tilde{g}_{X}$ ). Then

$|\xi|_{g_{x}}^{2}=\left|\xi_{1}\right|_{g_{x}}^{2}+\left|\xi_{2}\right|_{g_{x}}^{2}, \quad|\xi|_{\tilde{g}}^{2}=\left|\xi_{1}\right|_{\tilde{g}}^{2}+\left|\xi_{2}\right|_{\tilde{g}}^{2}, \quad|\xi|_{g_{x}}=|\xi|_{\tilde{g}}=\left|\operatorname{dr}\left(\xi_{1}\right)\right|=|d r(\xi)|$.

By variant (27.15.3) of Gauß's lemma the vector $T_{X}\left(\exp _{x}\right) \cdot \xi_{1} \in T_{\exp _{x}(X)} M$ is tangent to the geodesic $t \mapsto \exp _{x}(t . X)$ in $(M, g)$ and $T_{X}\left(\exp _{x}\right) \cdot \xi_{2}$ is normal to it. Thus $\left|T_{X}\left(\exp _{x}\right) \cdot \xi_{1}\right|_{g}=\left|\xi_{1}\right|_{g}=\left|\xi_{1}\right|_{\tilde{g}}$ and

$$
\begin{gathered}
\left|T_{X}\left(\exp _{x}\right) \cdot \xi\right|_{g}^{2}=\left|T_{X}\left(\exp _{x}\right) \cdot \xi_{1}\right|_{g}^{2}+\left|T_{X}\left(\exp _{x}\right) \cdot \xi_{2}\right|_{g}^{2}=\left|\xi_{1}\right|_{\tilde{g}}+\left|T_{X}\left(\exp _{x}\right) \cdot \xi_{2}\right|_{g}^{2}, \\
\left|T_{X}\left(\exp _{x}\right) \cdot \xi\right|_{g}^{2}-|\xi|_{\tilde{g}}^{2}=\left|T_{X}\left(\exp _{x}\right) \cdot \xi_{2}\right|_{g}^{2}-\left|\xi_{2}\right|_{\tilde{g}}^{2} .
\end{gathered}
$$

In order to show that $\left|T_{X}\left(\exp _{x}\right) \cdot \xi\right|_{g} \geq|\xi|_{\tilde{g}}$, we can thus assume that $\xi=\xi_{2}$ is normal to the ray $t \mapsto t$.X. But for these $\xi$ we have $|\xi|_{\tilde{g}}^{2}=f(r(X))|\xi|_{g_{x}}^{2}$ by construction of $\tilde{g}$ and

$$
\left|T_{X}\left(\exp _{x}\right) \cdot \xi\right|_{g}^{2} \geq h(X)|\xi|_{g_{x}}^{2} \geq f(r(X))|\xi|_{g_{x}}^{2}=|\xi|_{\tilde{g}}^{2}
$$

So $\exp _{x}:\left(T_{x} M, \tilde{g}\right) \rightarrow(M, g)$ is distance increasing. 



\section{CHAPTER VI. Isometric Group Actions or Riemann $G$-Manifolds}

In this chapter, a Riemann or pseudo-Riemann metric will usually be called $\gamma$ since $g$ is usually a group element.

\section{Isometries, Homogeneous Manifolds, and Symmetric Spaces}

28.1. The group of isometries. Let $(M, \gamma)$ be a connected pseudo-Riemann manifold. Recall that a diffeomorphism $\varphi: M \rightarrow M$ is an isometry if

$$
\varphi^{*} \gamma=\gamma
$$

A vector field $\xi \in \mathfrak{X}(M)$ is called a Killing vector field if its flow $\mathrm{Fl}_{t}^{\xi}$ consists of local isometries. By (8.16.3) this is the case if and only if the Lie derivative satisfies $\mathcal{L}_{\xi} \gamma=0$. By (8.20) the space of all Killing vector fields is a Lie algebra.

Theorem. The space $\mathfrak{X}(M, \gamma)=\left\{\xi \in \mathfrak{X}(M): \mathcal{L}_{\xi} \gamma=0\right\}$ of all Killing vector fields on a pseudo-Riemann manifold $(M, \gamma)$ is a finite-dimensional Lie algebra of dimension at most $m^{2}+m$ where $m=\operatorname{dim}(M)$.

The subspace of all complete Killing fields $\xi$ (see (3.8)) is also a finitedimensional Lie algebra. 
The group $\operatorname{Isom}(M, \gamma)$ of all isometries of $(M, \gamma)$ is a Lie group with Lie algebra the algebra of all complete Killing fields. It acts smoothly on $M$. The Lie group topology equals the pointwise open topology and also the compact open toplogy in the Riemann case.

Proof. Any Killing vector field $\xi$ is a Jacobi field along each geodesic since its flow $\mathrm{Fl}_{t}^{\xi}$ deforms geodesics through geodesics; see (27.1), Let us fix a point $x_{0} \in M$. By (27.5.1) the field $\xi$ is then uniquely determined by its value $\xi\left(x_{0}\right)$ and by its covariant derivative $T_{x_{0}} M \ni X \mapsto \nabla_{X} \xi \in T_{x_{0}} M$ : First we know $\xi$ on $\exp _{x_{0}}^{\gamma}\left(U_{x_{0}}\right)$ for a 0-neighborhood $U_{x_{0}}$, and each point $x \in M$ can be connected to $x_{0}$ by a curve consisting of geodesic arcs. Thus the dimension of the Lie algebra $\mathfrak{X}(M, \gamma)=\left\{\xi \in \mathfrak{X}(M): \mathcal{L}_{\xi} \gamma=0\right\}$ of all Killing vector fields is at most $m^{2}+m$ if $\operatorname{dim}(M)=m$.

We now use theorem (6.5) Let $G$ be a simply connected Lie group with Lie algebra $\mathfrak{X}(M, \gamma)$. Then there is a smooth local action $G \times M \supseteq U \stackrel{\ell}{\longrightarrow} M$.

Let $H$ be the subgroup of $G$ consisting of all $g \in G$ with $\ell_{g}$ defined on the whole of $M$. Let $\mathfrak{g} \subset \mathfrak{X}(M, \gamma)$ be the vector space of all $\xi$ such that $\exp (t \xi) \in H$ for all $t$. By (4.30.3) the space $\mathfrak{g}$ is a Lie subalgebra of $\mathfrak{X}(M, \gamma)$, and $\mathfrak{g}$ consists of complete vector fields. By theorem (6.5) again, there is a Lie group $\operatorname{Isom}_{0}(M, \gamma)$ consisting of diffeomorphisms of $M$ which implements the infinitesimal action of the Lie algebra $\mathfrak{g}$ on $M$. This is the connected component of the isometry group $\operatorname{Isom}(M, \gamma)$. Therefore the isometry group is a Lie group.

Obviously, the pointwise open topology on $\operatorname{Isom}(M, \gamma)$ equals the topology as a Lie group. In the Riemann case, $\operatorname{Isom}(M, \gamma)$ consists of equicontinuous mappings (namely isometries) for the Riemann distance function on $M$; thus the pointwise open topology equals the compact open topology.

28.2. Invariant covariant derivatives on homogeneous spaces. Let $G$ be a Lie group and $H$ a closed subgroup with Lie algebras $\mathfrak{g}$ and $\mathfrak{h}$, respectively. Let $\ell: G \times G / H$ be the left action of $G$ on the homogeneous space $G / H$ with notation $\ell_{g}(x)=\ell^{x}(g)=\ell(g, x)=g . x$ for $g \in G, x \in G / H$. Let $\zeta: \mathfrak{g} \rightarrow \mathfrak{X}(G / H)$ be the corresponding infinitesimal action, called the fundamental vector field mapping. It is a Lie algebra antihomomorphism. Let $p: G \rightarrow G / H$ be the projection, $p(e)=o \in G / H$. Then $T_{e} p: \mathfrak{g} \rightarrow$ $T_{o}(G / H)$ factors to a linear isomorphism $\bar{p}: \mathfrak{g} / \mathfrak{h} \rightarrow T_{o}(G / H)$ which is equivariant under $\underline{\operatorname{Ad}}: H \rightarrow \operatorname{Aut}(\mathfrak{g} / \mathfrak{h})$ (induced from the adjoint action) and $h \mapsto T_{o} \ell_{h} \in G L\left(T_{o}(G / H)\right)$. We shall also use $T_{e}(\underline{\mathrm{Ad}})=:$ ad $: \mathfrak{h} \rightarrow$ $L(\mathfrak{g} / \mathfrak{h}, \mathfrak{g} / \mathfrak{h})$.

Let $\nabla$ be a $G$-invariant linear connection for $T(G / H)$, and let $\xi, \eta \in \mathfrak{X}(G / H)$. Recall its properties: $\nabla: \mathfrak{X}(G / H) \times \mathfrak{X}(G / H) \rightarrow \mathfrak{X}(G / H)$ is $\mathbb{R}$-bilinear, 
$\nabla_{f \xi} \eta=f . \nabla_{\xi} \eta$, and $\nabla_{\xi}(f . \eta)=d f(\xi) \cdot \eta+f . \nabla_{\xi} \eta$. The Nomizu operator $N_{\xi}: \mathfrak{X}(G / H) \rightarrow \mathfrak{X}(G / H)$ is defined by

$$
N_{\xi} \eta:=\nabla_{\xi} \eta-[\xi, \eta]
$$

Then $N_{\xi}(f . \eta)=f . N_{\xi} \eta$, so $N_{\xi}$ is tensorial, $N_{\xi}: T G / H \rightarrow T G / H$. Moreover, $N_{f . \xi} \eta=f \cdot N_{\xi} \eta+d f(\eta) . \xi$. Now $G$-invariance for $\nabla$ means $\ell_{g^{-1}}^{*}\left(\nabla_{\ell_{g}^{*} \xi} \ell_{g}^{*} \eta\right)=$ $\nabla_{\xi} \eta$ so that $\nabla_{\ell_{g}^{*} \xi} \ell_{g}^{*} \eta=\ell_{g}^{*} \nabla_{\xi} \eta$ implies

$$
N_{\ell_{g}^{*} \xi} \ell_{g}^{*} \eta=\nabla_{\ell_{g}^{*} \xi} \ell_{g}^{*} \eta-\left[\ell_{g}^{*} \xi, \ell_{g}^{*} \eta\right]=\ell_{g}^{*} \nabla_{\xi} \eta-\ell_{g}^{*}[\xi, \eta]=\ell_{g}^{*} N_{\xi} \eta .
$$

Let us apply this for a fundamental vector field $\xi=\zeta_{X}$ :

$T\left(\ell_{g^{-1}}\right) \circ\left(N_{\zeta_{X}} \eta\right) \circ \ell_{g}=\ell_{g}^{*}\left(N_{\zeta_{X}} \eta\right)=N_{\ell_{g}^{*} \zeta_{X}}\left(\ell_{g}^{*} \eta\right)=N_{\zeta_{\mathrm{Ad}\left(g^{-1}\right) X}}\left(T\left(\ell_{g^{-1}}\right) \circ \eta \circ \ell_{g}\right)$.

We evaluate this at the origin $o \in G / H$ :

$$
\begin{gathered}
\left.T\left(\ell_{g^{-1}}\right) \cdot\left(N_{\zeta_{X}} \eta\right)\right|_{g H}=N_{\zeta_{\mathrm{Ad}\left(g^{-1}\right) X}} \cdot\left(\left.T_{g H}\left(\ell_{g^{-1}}\right) \cdot \eta\right|_{g H}\right), \\
\left.N_{\zeta_{X}}\right|_{g H}=\left.T_{o}\left(\ell_{g}\right) \cdot N_{\zeta_{\mathrm{Ad}\left(g^{-1}\right) X}}\right|_{o} \cdot T_{g H}\left(\ell_{g^{-1}}\right): T_{g H} G / H \rightarrow T_{g H} G / H .
\end{gathered}
$$

Let us now define

$$
\Phi: \mathfrak{g} \rightarrow L(\mathfrak{g} / \mathfrak{h}, \mathfrak{g} / \mathfrak{h}) \quad \text { by } \quad \Phi_{X}(Y+\mathfrak{h})=\left.\bar{p}^{-1} \cdot N_{\zeta_{X}}\right|_{o} \cdot \bar{p} \cdot(Y+\mathfrak{h}) .
$$

We have $\zeta_{Z}(g H)=\left.T_{o}\left(\ell_{g}\right) \cdot \zeta_{\operatorname{Ad}\left(g^{-1}\right) Z}\right|_{o}$, and for $h \in H$ we have $T_{o}\left(\ell_{h}\right) \cdot \bar{p}=$ $\underline{\operatorname{Ad}}(h) \cdot \bar{p}$. Using (2) we obtain:

$$
\begin{aligned}
\Phi_{X} & =\left.\bar{p}^{-1} \cdot N_{\zeta_{X}}\right|_{o} \cdot \bar{p}=\left.\bar{p}^{-1} \cdot T_{o}\left(\ell_{h}\right) \cdot N_{\zeta_{\operatorname{Ad}\left(h^{-1}\right) X}}\right|_{o} \cdot T_{o}\left(\ell_{h^{-1}}\right) \cdot \bar{p} \\
& =\left.\underline{\operatorname{Ad}}(h) \cdot \bar{p}^{-1} \cdot N_{\zeta_{\operatorname{Ad}\left(h^{-1}\right) X}}\right|_{o} \cdot \bar{p} \cdot \underline{\operatorname{Ad}}\left(h^{-1}\right)=\underline{\operatorname{Ad}}(h) \cdot \Phi_{\operatorname{Ad}\left(h^{-1}\right) X} \cdot \underline{\operatorname{Ad}}\left(h^{-1}\right) .
\end{aligned}
$$

$$
\Phi_{\operatorname{Ad}(h) X}=\underline{\operatorname{Ad}}(h) \cdot \Phi_{X} \cdot \underline{\operatorname{Ad}}\left(h^{-1}\right), \quad h \in H, X \in \mathfrak{g} .
$$

If $X \in \mathfrak{h}$, then $\zeta_{X}(o)=0$ so $\left.\left(N_{\zeta_{X}} \eta\right)\right|_{o}=\nabla_{\left.\zeta_{X}\right|_{o}} \eta-\left.\left[\zeta_{X}, \eta\right]\right|_{o}=0-\left.\left[\zeta_{X}, \eta\right]\right|_{o}$ which depends only on $\eta(o)$ since $\zeta_{X}(o)=0$ so $\left.\left[\zeta_{X}, f . \eta\right]\right|_{o}=f(o) .\left.\left[\zeta_{X}, \eta\right]\right|_{o}+$ $d f\left(\left.\zeta_{X}\right|_{o}\right) \cdot \eta(o)$. Thus for $X \in \mathfrak{h}$ :

$$
\begin{aligned}
\Phi_{X}(Y+\mathfrak{h}) & =\left.\bar{p}^{-1} \cdot N_{\zeta_{X}}\right|_{o} \cdot \bar{p} \cdot(Y+\mathfrak{h})=\left.\bar{p}^{-1} \cdot N_{\zeta_{X}}\right|_{o} \cdot T_{e} p \cdot Y=\left.\bar{p}^{-1} \cdot N_{\zeta_{X}}\right|_{o}\left(\left.\zeta_{Y}\right|_{o}\right) \\
& =-\left.\bar{p}^{-1} \cdot\left[\zeta_{X}, \zeta_{Y}\right]\right|_{o}=\left.\bar{p}^{-1} \cdot \zeta_{[X, Y]}\right|_{o}=\underline{\operatorname{ad}}(X)(Y+\mathfrak{h}) .
\end{aligned}
$$

Theorem. G-invariant linear connections $\nabla$ on a homogeneous space $G / H$ correspond bijectively to $H$-homomorphisms $\Phi: \mathfrak{g} \rightarrow L(\mathfrak{g} / \mathfrak{h}, \mathfrak{g} / \mathfrak{h})$ such that $\Phi_{X}=\underline{\operatorname{ad}}(X)$ for $X \in \mathfrak{h}$. If one such homomorphism exists, then the space of all $G$-invariant linear connections on $G / H$ is an affine space modeled on $\operatorname{Hom}_{H}\left(\otimes^{2}(\mathfrak{g} / \mathfrak{h}), \mathfrak{g} / \mathfrak{h}\right)$.

The torsion of $\nabla$ corresponds to the linear mapping $\bigwedge^{2} \mathfrak{g} / \mathfrak{h} \rightarrow \mathfrak{g} / \mathfrak{h}$ which is induced by

$$
(X, Y) \mapsto \Phi_{X}(Y+\mathfrak{h})-\Phi_{Y}(X+\mathfrak{h})+([X, Y]+\mathfrak{h}) .
$$


The curvature of $\nabla$ corresponds to the mapping $\bigwedge^{2} \mathfrak{g} / \mathfrak{h} \rightarrow L(\mathfrak{g} / \mathfrak{h}, \mathfrak{g} / \mathfrak{h})$ which is induced by

$$
(X, Y) \mapsto \Phi_{X} \circ \Phi_{Y}-\Phi_{Y} \circ \Phi_{X}+\Phi_{[X, Y]}
$$

Proof. Just unravel all computations from before backwards. Note that

$$
\begin{aligned}
\left.\left(\nabla_{\zeta_{X}} \zeta_{Y}\right)\right|_{g H}=\left.\left(N_{\zeta_{X}} \zeta_{Y}\right)\right|_{g H}+\left.\left[\zeta_{X}, \zeta_{Y}\right]\right|_{g H} & \\
= & \left.\left.T_{o}\left(\ell_{g}\right) \cdot N_{\zeta_{\operatorname{Ad}\left(g^{-1}\right) X}}\right|_{o} \cdot T_{g H}\left(\ell_{g^{-1}}\right) \cdot \zeta_{Y}\right|_{g H}-\left.\zeta_{[X, Y]}\right|_{g H} \\
= & \left.\left.T_{o}\left(\ell_{g}\right) \cdot N_{\zeta_{\operatorname{Ad}\left(g^{-1}\right) X}}\right|_{o} \cdot \zeta_{\operatorname{Ad} g^{-1} Y}\right|_{o}-\left.T_{o}\left(\ell_{g}\right) \cdot T_{g H}\left(\ell_{g^{-1}}\right) \cdot \zeta_{[X, Y]}\right|_{g H} \\
= & T_{o}\left(\ell_{g}\right) \cdot \bar{p} \cdot \Phi_{\operatorname{Ad}\left(g^{-1}\right) X}\left(\operatorname{Ad} g^{-1} Y+\mathfrak{h}\right)-\left.T_{o}\left(\ell_{g}\right) \cdot \zeta_{\operatorname{Ad}\left(g^{-1}\right)[X, Y]}\right|_{o} \\
= & T_{o}\left(\ell_{g}\right) \cdot \bar{p} \cdot\left(\Phi_{\operatorname{Ad}\left(g^{-1}\right) X}\left(\operatorname{Ad}\left(g^{-1}\right) Y+\mathfrak{h}\right)\right. \\
& \left.\quad-\left(\left[\operatorname{Ad}\left(g^{-1}\right) X, \operatorname{Ad}\left(g^{-1}\right) Y\right]+\mathfrak{h}\right)\right) \cdot
\end{aligned}
$$

28.3. Invariant pseudo-Riemann metrics on homogenous spaces. Let $G$ be a Lie group and let $H$ be a closed subgroup. A $G$-invariant pseudo-Riemann metric $\gamma$ on $G / H$ (if it exists) is uniquely determined by the $H$-invariant nondegenerate bilinear form $\gamma_{o}$ on $T_{o} G / H$, and this in turn is determined by the $\underline{\operatorname{Ad}}(H)$-invariant bilinear form $B=\bar{p}^{*} \gamma_{o}$ on $\mathfrak{g} / \mathfrak{h}$, if it exists. Suppose that such a nondegenerate bilinear form exists. Then

$$
\gamma_{g H}(\xi, \eta)=\left(\ell_{g^{-1}}^{*} \gamma\right)_{g H}(\xi, \eta)=\gamma_{o}\left(T\left(\ell_{g^{-1}}\right) \cdot \xi, T\left(\ell_{g^{-1}}\right) . \eta\right) .
$$

For fundamental vector fields we get:

$$
\begin{aligned}
& \gamma_{g H}\left(\zeta_{X}(g H), \zeta_{Y}(g H)\right)=\gamma_{o}\left(T\left(\ell_{g^{-1}}\right) \cdot \zeta_{X}(g H), T\left(\ell_{g^{-1}}\right) \cdot \zeta_{Y}(g H)\right) \\
& =B\left(\operatorname{Ad}\left(g^{-1}\right) X+\mathfrak{h}, \operatorname{Ad}\left(g^{-1}\right) Y+\mathfrak{h}\right), \\
& \left.d(\operatorname{Ad} \circ \nu)\right|_{g}\left(T_{e}\left(\mu_{g}\right) X\right)=\operatorname{Ad}(\nu(g)) \cdot\left(\operatorname{ad} \circ \kappa^{l}\right)\left(T_{g} \nu \cdot T_{e}\left(\mu_{g}\right) \cdot X\right) \\
& =\operatorname{Ad}\left(g^{-1}\right) \cdot \operatorname{ad}(\operatorname{Ad}(g) \cdot X), \\
& \left.d B((\operatorname{Ad} \circ \nu) \cdot X+\mathfrak{h},(\operatorname{Ad} \circ \nu) \cdot X+\mathfrak{h})\right|_{g}\left(T_{e}\left(\mu_{g}\right) \cdot Z\right) \\
& =-2 B\left(\operatorname{Ad}\left(g^{-1}\right) \cdot \operatorname{ad}(\operatorname{Ad}(g) \cdot Z) \cdot X+\mathfrak{h}, \operatorname{Ad}\left(g^{-1}\right) X+\mathfrak{h}\right) \\
& =-2 B\left(\operatorname{Ad}\left(g^{-1}\right) \cdot[\operatorname{Ad}(g) \cdot Z, X]+\mathfrak{h}, \operatorname{Ad}\left(g^{-1}\right) X+\mathfrak{h}\right) \\
& =-2 B\left(\left[Z, \operatorname{Ad}\left(g^{-1}\right) \cdot X\right]+\mathfrak{h}, \operatorname{Ad}\left(g^{-1}\right) X+\mathfrak{h}\right), \\
& d \gamma\left(\zeta_{X}, \zeta_{X}\right)\left(\zeta_{Z}(g H)\right)=\left.d \gamma\left(\zeta_{X}, \zeta_{X}\right)\right|_{g H}\left(T_{o}\left(\ell_{g}\right) \cdot \zeta_{\mathrm{Ad}\left(g^{-1}\right) Z}(o)\right) \\
& =\left.d \gamma\left(\zeta_{X}, \zeta_{X}\right)\right|_{g H}\left(T p \cdot T_{e}\left(\mu_{g}\right) \cdot \operatorname{Ad}\left(g^{-1}\right) \cdot Z\right) \\
& =\left.d B((\operatorname{Ad} \circ \nu) \cdot X+\mathfrak{h},(\operatorname{Ad} \circ \nu) \cdot X+\mathfrak{h})\right|_{g}\left(T_{e}\left(\mu_{g}\right) \cdot \operatorname{Ad}\left(g^{-1}\right) Z\right) \\
& =-2 B\left(\left[\operatorname{Ad}\left(g^{-1}\right) Z, \operatorname{Ad}\left(g^{-1}\right) \cdot X\right]+\mathfrak{h}, \operatorname{Ad}\left(g^{-1}\right) X+\mathfrak{h}\right) \\
& =-B\left(\operatorname{Ad}\left(g^{-1}\right)[Z, X]+\mathfrak{h}, \operatorname{Ad}\left(g^{-1}\right) X+\mathfrak{h}\right) \text {. }
\end{aligned}
$$


On the other hand we have for a $G$-invariant linear connection on $G / H$ corresponding to $\Phi: \mathfrak{g} \rightarrow L(\mathfrak{g} / \mathfrak{h}, \mathfrak{g} / \mathfrak{h})$ as in (28.2.3).

$$
\begin{array}{rl}
\left.2 \gamma\left(\nabla_{\zeta_{Z}} \zeta_{X}, \zeta_{X}\right)\right|_{g H}=2 & B\left(\Phi_{\operatorname{Ad}\left(g^{-1}\right) Z}\left(\operatorname{Ad} g^{-1} X+\mathfrak{h}\right)\right. \\
& \left.-\left(\left[\operatorname{Ad}\left(g^{-1}\right) Z, \operatorname{Ad}\left(g^{-1}\right) X\right]+\mathfrak{h}\right), \operatorname{Ad}\left(g^{-1}\right) X+\mathfrak{h}\right) .
\end{array}
$$

Collecting, we get

$$
\begin{aligned}
d \gamma\left(\zeta_{X}, \zeta_{X}\right)\left(\zeta_{Z}(g H)\right)- & \left.2 \gamma\left(\nabla_{\zeta_{Z}} \zeta_{X}, \zeta_{X}\right)\right|_{g H} \\
= & 2 B\left(\Phi_{\operatorname{Ad}\left(g^{-1}\right) Z}\left(\operatorname{Ad} g^{-1} X+\mathfrak{h}\right), \operatorname{Ad}\left(g^{-1}\right) X+\mathfrak{h}\right)
\end{aligned}
$$

so that the connection

(1) $\nabla$ respects $\gamma \Longleftrightarrow B\left(\Phi_{Z}(X+\mathfrak{h}), Y+\mathfrak{h}\right)+B\left(X+\mathfrak{h}, \Phi_{Z}(Y+\mathfrak{h})\right)=0$.

The Levi-Civita connection $\nabla$ is uniquely determined by the pseudo-metric $\gamma$. We now derive a formula for $\Phi$ corresponding to the connection $\nabla$ directly from $B . \nabla$ is torsion-free iff

$$
0=\Phi_{X}(Y+\mathfrak{h})-\Phi_{Y}(X+\mathfrak{h})+([X, Y]+\mathfrak{h}) \text { for all } X, Y \in \mathfrak{g}
$$

Consider the symmetric bilinear form $\tilde{B}: \mathfrak{g} \times \mathfrak{g} \rightarrow \mathfrak{g} / \mathfrak{h} \times \mathfrak{g} / \mathfrak{h} \stackrel{B}{\longrightarrow} \mathbb{R}$ on $\mathfrak{g}$. Then torsion-freeness corresponds to

$$
\tilde{B}([X, Y], Z)=-B\left(\Phi_{X}(Y+\mathfrak{h}), Z+\mathfrak{h}\right)+B\left(\Phi_{Y}(X+\mathfrak{h}), Z+\mathfrak{h}\right) .
$$

We permute this cyclically:

$$
\begin{aligned}
& +\tilde{B}([X, Y], Z)=-B\left(\Phi_{X}(Y+\mathfrak{h}), Z+\mathfrak{h}\right)+B\left(\Phi_{Y}(X+\mathfrak{h}), Z+\mathfrak{h}\right), \\
& +\tilde{B}([Y, Z], X)=-B\left(\Phi_{Y}(Z+\mathfrak{h}), X+\mathfrak{h}\right)+B\left(\Phi_{Z}(Y+\mathfrak{h}), X+\mathfrak{h}\right), \\
& -\tilde{B}([Z, X], Y)=+B\left(\Phi_{Z}(X+\mathfrak{h}), Y+\mathfrak{h}\right)-B\left(\Phi_{X}(Z+\mathfrak{h}), Y+\mathfrak{h}\right) .
\end{aligned}
$$

We add, using (1):

(2) $-\tilde{B}([X, Y], Z)-\tilde{B}([Y, Z], X)+\tilde{B}([Z, X], Y)=2 B\left(\Phi_{Y}(Z+\mathfrak{h}), X+\mathfrak{h}\right)$.

It remains to check that the trilinear expression

$$
(X, Y, Z) \mapsto-\tilde{B}([X, Y], Z)-\tilde{B}([Y, Z], X)+\tilde{B}([Z, X], Y)
$$

factors to $\mathfrak{g} / \mathfrak{h} \times \mathfrak{g} \times \mathfrak{g} / \mathfrak{h} \rightarrow \mathbb{R}$. If $X$ is in $\mathfrak{h}$, then the second term vanishes and the first term cancels with the third one since $B$ is $\underline{\operatorname{Ad}}(\mathfrak{h})$-invariant. Similarly for $Z \in \mathfrak{h}$. So (2) defines a mapping $\Phi$ which in turn gives rise to the Levi-Civita connection $\nabla$. 
28.4. Pseudo-Riemann locally symmetric spaces. Let $(M, \gamma)$ be a connected pseudo-Riemann manifold. For $x \in M$ let $U_{x}$ be an open neighborhood of $x$ in $M$ and let $B_{0_{x}}$ be an open absolutely convex 0-neighborhood in $T_{x} M$ such that $\exp _{x}^{\gamma}: B_{0_{x}} \rightarrow U_{x}$ is a diffeomorphism. We consider the exponential mapping

$$
T_{x} M \supset B_{0_{x}} \stackrel{\exp _{x}^{\gamma}}{\longrightarrow} U_{x} \subset M
$$

and the local geodesic symmetry

$$
s_{x}: U_{x} \rightarrow U_{x}, \quad s_{x}\left(\exp _{x}^{\gamma}(X)\right)=\exp _{x}^{\gamma}(-X) .
$$

Note that $T_{x}\left(s_{x}\right)=-\operatorname{Id}$ on $T_{x} M$. The pseudo-Riemann manifold $(M, \gamma)$ is called a pseudo-Riemann locally symmetric space if each local symmetry $s_{x}$ is a local isometry, i.e., $s_{x}^{*} \gamma=\gamma$.

Proposition. A pseudo-Riemann manifold $(M, \gamma)$ is locally symmetric if and only if its curvature is parallel: $\nabla R^{\nabla}=0$.

Proof. If $(M, \gamma)$ is locally symmetric, we have $s_{x}^{*}(\nabla R)=\left(s^{*} \nabla\right)\left(s^{*} R\right)=$ $\nabla R$, but $\left(s_{x}^{*}(\nabla R)\right)_{x}=(-1)^{5}(\nabla R)_{x}$ so that $(\nabla R)_{x}=0$ for all $x \in M$. If conversely $\nabla R=0$, then $R$ is constant under parallel transport. Thus by theorem (27.10) each local symmetry is an isometry.

28.5. Symmetric spaces. A connected (pseudo-)Riemann manifold $(M, \gamma)$ is called a (pseudo-)Riemann symmetric space if for each $x \in M$ the local symmetry extends to a globally defined isometry $s_{x}: M \rightarrow M$. Let us choose a point $o \in M$ which we call the origin.

(1) An isometry $f$ on $(M, \gamma)$ which is involutive $\left(f^{2}=\mathrm{Id}\right)$ and has $x$ as isolated fixed point equals $s_{x}$, by considering the linear involution $T_{x} f$ : Among the possible eigenvalues \pm 1 only -1 is admissible since $x$ is a locally isolated fixed point.

(2) $(M, \gamma)$ is a geodesically complete Riemann manifold.

Namely, let $c:(a, b) \rightarrow M$ be a geodesic. Then $s_{c(t)}$ maps the geodesic to itself (suitably reparameterized) and thus prolongs $c$ (if $t$ is not the midpoint). So any geodesic is extendable to $\mathbb{R}$ and by $(23.6)$, $(M, \gamma)$ is a complete Riemann manifold.

(3) The group $\operatorname{Isom}(M, \gamma)$ of all isometries of $M$ acts transitively on $M$. In the Riemann case, by (23.6.6) for any point $x \in M$ there exists a geodesic $c:[0,1] \rightarrow M$ with $c(0)=o$ and $c(1)=x$. But then $s_{c(1 / 2)}(o)=x$. So every point of $M$ lies in the orbit through $o$. In the pseudo-Riemann case where the Hopf-Rinov theorem (23.6) does not hold, we can choose a piecewise smooth curve $c$ from $o$ to $x$ which consists of geodesic segments. Then we 
can apply the reflections $s_{y}$ with respect to the midpoint of each geodesic segment, iteratively, to map $o$ to $x$.

(4) The group $G:=\operatorname{Isom}(M, \gamma)$ of isometries of $M$ is a Lie group and the action $\ell: G \times M \rightarrow M$ is smooth. This is an immediate consequence of theorem (28.1),

(5) The mapping $s: M \times M \rightarrow M$ given by $(x, y) \mapsto s_{x}(y)$ is smooth. This is obvious.

(6) Let $\sigma: G \rightarrow G$ be given by $\sigma(\varphi)=s_{o} \circ \varphi \circ s_{o}$. Let $H=G_{o}$ be the isotropy group of the origin $o$. Then $\sigma$ is an involutive automorphism of $G$ and we have $G^{\sigma}=\{g \in G: \sigma(g)=g\} \supseteq H \supseteq G_{0}^{\sigma}$. Namely, for $g \in H$ we have

$$
\begin{aligned}
\left(s_{o} \circ g \circ s_{o}\right)\left(\exp _{o}^{\gamma}(t \cdot X)\right) & =\left(s_{o} \circ g\right)\left(\exp _{o}^{\gamma}(-t \cdot X)\right)=s_{o}\left(\exp _{o}^{\gamma}\left(-t \cdot T_{o} g \cdot X\right)\right) \\
& =\exp _{o}^{\gamma}\left(t \cdot T_{o} g \cdot X\right)=g\left(\exp _{o}^{\gamma}(t \cdot X)\right)
\end{aligned}
$$

so that $\sigma(g)=g$ near $o$ and thus everywhere, since $g$ is an isometry. The Lie algebra of $G^{\sigma}$ is $\mathfrak{g}^{\sigma^{\prime}}$, the space of all $\xi \in \mathfrak{g}=\mathfrak{X}(M, \gamma)$ such that $\sigma^{\prime}(\xi)=\xi$. But if $\xi=\sigma^{\prime}(\xi)=T s_{o} \circ \xi \circ s_{o}$, then at $o$ we have $\xi(o)=T_{o}\left(s_{o}\right) \cdot \xi(o)=-\xi(o)$ so that $\xi$ vanishes at $o$. But then $\exp ^{G}(t \xi)=\mathrm{Fl}_{t}^{\xi}$ has $o$ as fixed point.

(7) For $x, y \in M$ we have $s_{x} \circ s_{y}=\ell_{g}$ for some $g \in G$. Choose $g_{x}, g_{y} \in G$ with $g_{x} . o=x$ and $g_{y} \cdot o=y$. Then

$$
\begin{aligned}
s_{x} & =\ell_{g_{x}} \circ s_{o} \circ \ell_{g_{x}^{-1}}, \quad s_{y}=\ell_{g_{y}} \circ s_{o} \circ \ell_{g_{y}^{-1}}, \\
s_{x} \circ s_{y} & =\ell_{g_{x}} \circ s_{o} \circ \ell_{g_{x}^{-1}} \circ \ell_{g_{y}} \circ s_{o} \circ \ell_{g_{y}^{-1}} \\
& =\ell_{g_{x}} \circ\left(s_{o} \circ \ell_{g_{x}^{-1} \cdot g_{y}} \circ s_{o}\right) \circ \ell_{g_{y}^{-1}} \\
& =\ell_{g_{x}} \circ \ell_{\sigma\left(g_{x}^{-1} \cdot g_{y}\right)} \circ \ell_{g_{y}^{-1}}=\ell_{g_{x} \cdot \sigma\left(g_{x}^{-1} \cdot g_{y}\right) \cdot g_{y}^{-1}}
\end{aligned}
$$

(8) Since $\sigma^{\prime}: \mathfrak{g} \rightarrow \mathfrak{g}$ is an involutive automorphism of $\mathfrak{g}=\mathfrak{X}(M, \gamma)$, we can decompose $\mathfrak{g}$ into the \pm 1 eigenspaces of $\sigma^{\prime}$ and obtain

$$
\mathfrak{g}=\mathfrak{X}(M, \gamma)=\mathfrak{h} \oplus \mathfrak{m}, \quad[\mathfrak{h}, \mathfrak{h}] \subset \mathfrak{h}, \quad[\mathfrak{h}, \mathfrak{m}] \subset \mathfrak{m}, \quad[\mathfrak{m}, \mathfrak{m}] \subset \mathfrak{h},
$$

which is called a reductive decomposition of $\mathfrak{g}$. Note that this decomposition is invariant under $\operatorname{ad}(H)$ acting on $\mathfrak{g}$.

(9) Let $p: G \rightarrow M \cong G / H$ be the submersion $p(g)=g . o=\ell(g, o)=\ell^{o}(g)$. Then $T_{e} p: \mathfrak{g} \rightarrow T_{o} M$ induces a linear isomorphism $p^{\prime}:=\left.T_{e} p\right|_{\mathfrak{m}}: \mathfrak{m} \rightarrow T_{o} M$ which is equivariant for the action of $H=G_{o}$ on $\mathfrak{m}$ via ad and on $T_{o} M$ via $h \mapsto T_{o} \ell_{h}$. The bilinear form $B:=\left(p^{\prime}\right)^{*} \gamma_{o}$ on $\mathfrak{m}$ is nondegenerate and $\operatorname{ad}(H)$-invariant. We identify $\mathfrak{m} \cong \mathfrak{g} / \mathfrak{h} \cong T_{o} M$ and make use of (28.3). From now on let $X, X_{i} \in \mathfrak{h}, Y, Y_{i} \in \mathfrak{m}$. If $B$ is an $H$-invariant inner product on $\mathfrak{m}$, we have $B\left(\operatorname{ad}_{X} Y_{1}, Y_{2}\right)+B\left(Y_{1}, \operatorname{ad}_{X} Y_{2}\right)=0$. We extend $B$ to a bilinear 
form $\tilde{B}$ on $\mathfrak{g}$ which has $\mathfrak{h}$ as its kernel. Equation (28.3.1) then becomes

$$
\begin{aligned}
B\left(\Phi_{X} Y_{1}, Y_{2}\right) & =-\frac{1}{2} \tilde{B}\left(\left[Y_{2}, X\right], Y_{1}\right)-\frac{1}{2} \tilde{B}\left(\left[X, Y_{1}\right], Y_{2}\right)+\frac{1}{2} \tilde{B}\left(\left[Y_{1}, Y_{2}\right], X\right) \\
& =-B\left(\operatorname{ad}_{X} Y_{1}, Y_{2}\right) \\
B\left(\Phi_{Y} Y_{1}, Y_{2}\right) & =-\frac{1}{2} \tilde{B}\left(\left[Y_{2}, Y\right], Y_{1}\right)-\frac{1}{2} \tilde{B}\left(\left[Y, Y_{1}\right], Y_{2}\right)+\frac{1}{2} \tilde{B}\left(\left[Y_{1}, Y_{2}\right], Y\right)=0 \\
\Phi_{X+Y} Y_{1} & =-\operatorname{ad}_{X} Y_{1}
\end{aligned}
$$

Note that the $G$-invariant connection on the symmetric space $M=G / H$ is prescribed by $\Phi$ uniquely and is independent of the choice of the metric $\gamma$. From theorem (28.2) we conclude that the curvature operator is given by

$$
R\left(Y_{1}, Y_{2}\right) Y_{3}=\Phi_{Y_{1}} \cdot \Phi_{Y_{2}} \cdot Y_{3}-\Phi_{Y_{2}} \cdot \Phi_{Y_{1}} \cdot Y_{3}+\Phi_{\left[Y_{1}, Y_{2}\right]} Y_{3}=-\left[\left[Y_{1}, Y_{2}\right], Y_{3}\right] .
$$

(10) Geodesics emanating from o are given by

$$
\exp _{o}^{M}\left(t \cdot p^{\prime} . Y\right)=p\left(\exp ^{G}(t Y)\right), \quad Y \in \mathfrak{m} .
$$

The fundamental vector field $\zeta_{Y} \in \mathfrak{X}(M)$ is

$$
\begin{aligned}
\zeta_{Y}\left(\ell_{g}(o)\right) & =T_{o}\left(\ell_{g}\right) \cdot \zeta_{\mathrm{ad}\left(g^{-1}\right) Y}(o) \text { by }(6.2 .2) \\
& =T_{o}\left(\ell_{g}\right) \cdot T_{e}\left(\ell^{o}\right) \operatorname{ad}\left(g^{-1}\right) Y=T_{o}\left(\ell_{g}\right) \cdot p^{\prime} \cdot \operatorname{ad}\left(g^{-1}\right) Y .
\end{aligned}
$$

By (28.2.6) for $g=\exp (t Y)$ we have

$$
\begin{aligned}
\left.\nabla_{\zeta_{Y}} \zeta_{Y}\right|_{\exp (t Y) . o}= & T_{o}\left(\ell_{\exp (t Y)}\right) \cdot \bar{p} \cdot\left(\Phi_{\operatorname{Ad}(\exp (-t Y)) Y}(\operatorname{Ad}(\exp (-t Y)) Y+\mathfrak{h})\right. \\
& -([\operatorname{Ad}(\exp (-t Y)) Y, \operatorname{Ad}(\exp (-t Y)) Y]+\mathfrak{h})) \\
= & T_{o}\left(\ell_{\exp (t Y)}\right) \cdot \bar{p} \cdot\left(\Phi_{Y}(Y)-([Y, Y]+\mathfrak{h})\right)=0 .
\end{aligned}
$$

So $\zeta_{Y}$ is parallel along the flowline $\mathrm{Fl}_{t}^{\zeta_{Y}}(o)=\exp (t Y) . o$ and thus $\exp (t Y) . o$ is a geodesic in $M$.

(11) We consider now a geodesically complete connected submanifold $N$ of $M$. Without loss we assume that $o \in N$. If $N$ is totally geodesic, then $N$ is itself a symmetric space with group of isometries $G^{\prime}=N_{G}(N)=\{g \in G$ : $\left.\ell_{g}(N) \subset N\right\}$ and isotropy group $G_{o}^{\prime}=H \cap G^{\prime}$. For $x \in N$ the submanifolds $N$ and $s_{x}(N)$ are both totally geodesic with the same tangent space at $x$; thus $s_{x}(N)=N$. For $g \in G^{\prime}$ we have $\ell_{\sigma(g)}=s_{o} \circ \ell_{g} \circ s_{o}: N \rightarrow N$, so $\sigma(g) \in G^{\prime}$. Finally, $G^{\prime}$ acts transitively on $N$ : For $x \in N$ choose a piecewise geodesic in $N$ from $o$ to $x$. For each geodesic piece $c:[0,1] \rightarrow N$ the mapping $s_{c(3 / 4)} \circ s_{c(1 / 4)}$ is in $G^{\prime}$ by (7) and maps $c(0)$ to $c(1)$.

(12) There is a bijective correspondence between

- totally geodesic connected geodesically complete submanfolds $N$ of $M$ containing o and

- linear subspaces $\mathfrak{n} \subseteq \mathfrak{m}$ with $[[\mathfrak{n}, \mathfrak{n}], \mathfrak{n}] \subseteq \mathfrak{n}$. 
The correspondence is given by $\mathfrak{n}=\left(\left.T_{e} p\right|_{\mathfrak{m}}\right)^{-1}\left(T_{o} N\right)$. The submanifold $N$ is flat if and only if $[[\mathfrak{n}, \mathfrak{n}], \mathfrak{n}]=0$. Given $N$, then by (11) we have $N \cong G^{\prime} / H^{\prime}$ where $H^{\prime}=G \cap H$, and $\sigma \in \operatorname{Aut}(\mathfrak{g})$ respects the Lie subalgebra $\mathfrak{g}^{\prime}=\operatorname{Lie}\left(G^{\prime}\right)$. Thus $\mathfrak{g}^{\prime}=\mathfrak{h}^{\prime} \oplus \mathfrak{n} \subseteq \mathfrak{h} \oplus \mathfrak{m}$ are compatible reductive decompositions and thus $[\mathfrak{n}, \mathfrak{n}] \subset \mathfrak{h}^{\prime}$ and $\left[\mathfrak{h}^{\prime}, \mathfrak{n}\right] \subset \mathfrak{n}$.

Conversely, given $\mathfrak{n} \subseteq \mathfrak{m}$ with $[[\mathfrak{n}, \mathfrak{n}], \mathfrak{n}] \subseteq \mathfrak{n}$, we put $\mathfrak{h}^{\prime}:=[\mathfrak{n}, \mathfrak{n}] \subset \mathfrak{h}^{\prime}$ and $\mathfrak{g}^{\prime}=\mathfrak{h}^{\prime} \oplus \mathfrak{n}$. This is a Lie subalgebra of $\mathfrak{g}$. Let $G^{\prime}$ be the connected Lie subgroup of $G$ with Lie algebra $\mathfrak{h}^{\prime}$. Then $G^{\prime} . o=\ell\left(G^{\prime}, o\right)$ is a connected geodesically complete submanifold of $M$ which is a symmetric space and thus totally geodesic in $M$ by (10).

This ends our very short treatment of symmetric spaces. From here on it becomes quite algebraic, and there are many good books on this subject; [82] is the standard reference. The theory of homogeneous manifolds however is best understood by using Cartan connections; for this see the book [32].

\section{Riemann $G$-Manifolds}

29.1. Preliminaries. Let $(M, \gamma)$ be a Riemann $G$-manifold. If $\varphi: M \rightarrow M$ is an isometric diffeomorphism, then

(1) $\varphi\left(\exp _{x}^{M}(t X)\right)=\exp _{\varphi(x)}^{M}\left(t T_{x} \varphi \cdot X\right)$. This is due to the fact that isometries map geodesics to geodesics, and the starting vector of the geodesic $t \mapsto \varphi\left(\exp _{x}^{M}(t . X)\right)$ is $T_{x} \varphi \cdot X$.

(2) If $\varphi(x)=x$, then, in the chart $\left(U_{x},\left(\exp _{x}^{M}\right)^{-1}\right), \varphi$ is a linear isometry (where $U_{x}$ is a neighborhood of $x$ so small that $\left(\exp _{x}^{M}\right)^{-1}: U_{x} \rightarrow T_{x} M$ is a diffeomorphism onto a neighborhood of 0 in $\left.T_{x} M\right)$ :

$$
\begin{aligned}
\bar{\varphi}(X): & =\left(\exp _{x}^{M}\right)^{-1} \circ \varphi \circ \exp _{x}^{M}(X) \\
& =\left(\exp _{x}^{M}\right)^{-1} \exp _{x}^{M}\left(T_{x} \varphi \cdot X\right)=T_{x} \varphi \cdot X .
\end{aligned}
$$

(3) $M^{\varphi}:=\{x \in M: \varphi(x)=x\}$ is a totally geodesic submanifold of $M$ :

If we choose $X \in T_{x}\left(M^{\varphi}\right)$, then, since $T_{x} \varphi \cdot X=X$ and by (1), we have

$$
\varphi\left(\exp _{x}^{M}(t X)\right)=\exp _{x}^{M}\left(T_{x} \varphi \cdot t X\right)=\exp _{x}^{M}(t X) .
$$

So the geodesic through $x$ with starting vector $X$ stays in $M^{\varphi}$.

(4) If $H$ is a set of isometries, then $M^{H}=\{x \in M: \varphi(x)=x$ for all $\varphi \in$ $H\}$ is also a totally geodesic submanifold in $M$.

29.2. Definition. Let $M$ be a proper Riemann $G$-manifold, $x \in M$. The normal bundle to the orbit $G . x$ is defined as

$$
\operatorname{Nor}(G . x):=T(G \cdot x)^{\perp} .
$$

Let $\operatorname{Nor}_{\varepsilon}(G . x)=\left\{X \in \operatorname{Nor}(G . x):\|X\|_{\gamma}<\varepsilon\right\}$, and choose $r>0$ small enough for $\exp _{x}: T_{x} M \supseteq B_{r}\left(0_{x}\right) \rightarrow M$ to be a diffeomorphism onto its 
image and for $\exp _{x}\left(B_{r}\left(0_{x}\right)\right) \cap G . x$ to have only one component. Then, since the action of $G$ is isometric, exp defines a diffeomorphism from $\operatorname{Nor}_{r / 2}(G . x)$ onto an open neighborhood of $G . x$, so $\exp \left(\operatorname{Nor}_{r / 2}(G . x)\right)=: U_{r / 2}(G . x)$ is a tubular neighborhood of G.x. We define the normal slice at $x$ by

$$
S_{x}:=\exp _{x}\left(\operatorname{Nor}_{r / 2}(G \cdot x)_{x}\right) \text {. }
$$

29.3. Lemma. Under these conditions we have that

(1) $S_{g . x}=g \cdot S_{x}$,

(2) $S_{x}$ is a slice at $x$.

Proof. (1) Since $G$ acts isometrically and by (29.1.1),

$$
S_{g \cdot x}=\exp _{g \cdot x}\left(T_{x} \ell_{g}\left(\operatorname{Nor}_{r / 2}(G . x)\right)_{x}\right)=\ell_{g} \exp _{x}\left(\operatorname{Nor}_{r / 2}(G \cdot x)_{x}\right)=g \cdot S_{x} .
$$

(2) The mapping $r: G . S_{x} \rightarrow G . x$ given by $\exp _{g . x} X \mapsto g . x$ defines a smooth equivariant retraction (note that $S_{x}$ and $S_{y}$ are disjoint if $x \neq y$ ).

29.4. Isotropy representation. Let $M$ be a $G$-manifold and $x \in M$; then the representation of the isotropy group

$$
G_{x} \longrightarrow G L\left(T_{x} M\right), \quad g \mapsto T_{x} \ell_{g},
$$

is called the isotropy representation. If $M$ is a Riemann $G$-manifold, then the isotropy representation is orthogonal and $T_{x}(G . x)$ is an invariant subspace under $G_{x}$. So $T_{x}(G . x)^{\perp}$ is also invariant, and

$$
G_{x} \longrightarrow\left(\operatorname{Nor}_{x}(G . x)\right), \quad g \mapsto T_{x} \ell_{g},
$$

is called the slice representation.

29.5. Example. Let $M=G$ be a compact Lie group with a bi-invariant metric. Then $G \times G$ acts on $G$ by $\left(g_{1}, g_{2}\right) . g:=g_{1} g g_{2}^{-1}$, making $G$ a Riemann $(G \times G)$-space. The isotropy group of $e$ is $(G \times G)_{e}=\{(g, g): g \in G\}$, and the isotropy representation coincides with the adjoint representation of $G \cong(G \times G)_{e}$ on $\mathfrak{g}=T_{e}(G)$.

29.6. Example. Let $G / K$ be a semisimple symmetric space ( $K$ compact) and let $\mathfrak{g}=\mathfrak{k}+\mathfrak{m}$ be the corresponding reductive decomposition of the Lie algebra $\mathfrak{g} ;$ see $(28.5)$. Then $T_{e}(G / K) \cong \mathfrak{g} / \mathfrak{k} \cong \mathfrak{m}$, and the isotropy subgroup of $G$ at $e$ is $K$. The isotropy representation is $\operatorname{Ad}_{K, G}^{\perp}: K \rightarrow(\mathfrak{m})$. The slices are points since the action is transitive.

29.7. Lemma. Let $M$ be a proper Riemann $G$-manifold, $x \in M$. Then the following three statements are equivalent:

(1) $x$ is a regular point.

(2) The slice representation at $x$ is trivial.

(3) $G_{y}=G_{x}$ for all $y \in S_{x}$ for a sufficiently small slice $S_{x}$. 
Proof. Clearly, (2) $\Longleftrightarrow$ (3). To see (3) $\Longrightarrow$ (11), let $S_{x}$ be a small slice at $x$. Then $U:=G . S$ is an open neighborhood of $G . x$ in $M$, and for all g.s $\in U$ we have $G_{g . s}=g G_{s} g^{-1}=g G_{x} g^{-1}$. Therefore $G . x$ is a principal orbit. The converse is true by (6.16.3), since $G_{x}$ is compact.

29.8. Definition. Let $M$ be a Riemann $G$-manifold and G.x some orbit; then a smooth section $u$ of the normal bundle $\operatorname{Nor}(G . x)$ is called an equivariant normal field if

$$
T_{y}\left(\ell_{g}\right) \cdot u(y)=u(g . y) \quad \text { for all } y \in G . x, g \in G .
$$

29.9. Corollary. Let $M$ be a proper Riemann $G$-manifold and $x$ a regular point. If $X \in \operatorname{Nor}_{x}(G . x)$, then $\hat{X}(g \cdot x):=T_{x}\left(\ell_{g}\right) . X$ is a well defined equivariant normal field along G.x in $M$.

Proof. If $g . x=h . x$, then $h^{-1} g \in G_{x} \Rightarrow T_{x}\left(\ell_{h^{-1} g}\right) \cdot X=X$, since the slice representation is trivial by (29.7.2). Now by the chain rule, $T_{x}\left(\ell_{g}\right) \cdot X=$ $T_{x}\left(\ell_{h}\right) . X$. Therefore $\hat{X}$ is a well defined, smooth section of $\operatorname{Nor}(G . x)$. It is equivariant by definition.

29.10. Corollary. Let $M$ be a Riemann $G$-manifold, G.x a principal orbit, and $\left(u_{1}, \ldots, u_{n}\right)$ an orthonormal basis of $\operatorname{Nor}_{x}(G . x)$. By corollary (29.9), each $u_{i}$ defines an equivariant normal field $\hat{u}_{i}$. So $\left(\hat{u}_{1}, \ldots, \hat{u}_{n}\right)$ is a global equivariant orthonormal frame field for $\operatorname{Nor}(G . x)$, and $\operatorname{Nor}(G . x)$ is a trivial bundle.

This follows also from the tubular neighborhood description $G . S_{x} \cong G \times{ }_{G_{x}}$ $S_{x}$, where $S_{x}$ is a normal slice at $x$ with trivial $G_{x}$-action; see $(29.7)$.

29.11. Orbits as Riemann submanifolds. Let $(M, \gamma)$ be a Riemann $G$-manifold and $u$ an equivariant normal field along an orbit G.x. $x_{0}$ Considering this orbit as a Riemann submanifold, we recall from (26.1) the second fundamental form $S \in \Gamma\left(S^{2} T^{*}\left(G . x_{0}\right) \otimes \operatorname{Nor}\left(G . x_{0}\right)\right)$ and from (26.3.2) the Weingarten mapping or shape operator $L_{u}: T\left(G . x_{0}\right) \rightarrow T\left(G . x_{0}\right)$ along the normal field, which are related by

$$
\left.\gamma\right|_{T P}\left(L_{u(x)}\left(X_{x}\right), Y_{x}\right)=\gamma\left(S\left(X_{x}, Y_{x}\right), u(x)\right), \quad X_{x}, Y_{x} \in T_{x}\left(G . x_{0}\right), x \in G . x_{0} .
$$

Its eigenvalues are called the main curvatures of $G . x_{0}$ along $u$. Since $\gamma$ and the submanifold $G>x_{0}$ are $G$-invariant, the second fundamental form $S$ is $G$-equivariant. Since $u$ is an equivariant normal form, the shape operator $L_{u}$ along $u$ is also $G$-equivariant. 
29.12. Lemma. Let $u$ be an equivariant normal field along an orbit G.x; then

(1) $L_{u(g \cdot x)}=T_{x}\left(\ell_{g}\right) \cdot L_{u(x)} \cdot T_{g \cdot x}\left(\ell_{g^{-1}}\right)$,

(2) the main curvatures of $G . x$ along $u$ are all constant,

(3) $\left\{\exp ^{M}(u(y)): y \in G . x\right\}$ is another G-orbit.

Proof. (1) was already proved in (29.11) above. This implies (2) since the eigenvalues are invariant under conjugation.

(3) $\left\{\exp ^{M}(u(y)): y \in G \cdot x\right\}=G \cdot \exp ^{M}(u(x))$, since

$$
g \cdot \exp ^{M}(u(x))=\exp ^{M}\left(T \ell_{g} \cdot u(x)\right)=\exp ^{M}(u(g \cdot x))
$$

29.13. Example. Let $N^{n}(c)$ be the simply connected space form with constant sectional curvature $c$, that is,

$$
N^{n}(c)= \begin{cases}S^{n}, \text { sphere with radius } \frac{1}{c}, & \text { if } c>0, \\ \mathbb{R}^{n}, & \text { if } c=0, \\ H^{n}, \text { hyperbolic sphere with radius } \frac{1}{|c|}, & \text { if } c<0 .\end{cases}
$$

Let $G$ be a closed subgroup of $\operatorname{Isom}\left(N^{n}(c)\right)$. If $P$ is a $G$-orbit, then so is the subset $\{\exp (u(x)): x \in P\}$ for any equivariant normal field $u$ along $P$. For instance:

(1) If $G=S O(n) \subset \operatorname{Isom}\left(\mathbb{R}^{n}\right)$, then the $G$-orbits are the spheres with center 0 . A radial vector field with constant length on each sphere, $u(x):=f(|x|) . x$, defines an equivariant normal field on each orbit. Clearly its flow carries orbits to orbits.

(2) Another example is the subgroup of $\operatorname{Isom}\left(\mathbb{R}^{n}\right)$ consisting only of affine translations in directions corresponding to a linear subspace $V \subset \mathbb{R}^{n}$. Here the orbits of $G$ are then affine planes parallel to $V$. An equivariant normal field on an orbit is a constant vector field orthogonal to $V$.

29.14. Theorem. Let $M$ be a proper $G$-manifold; then the set of all regular points $M_{\text {reg }}$ is open and dense in $M$. In particular, there is always a principal orbit type.

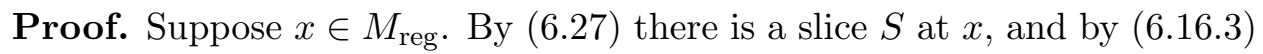
$S$ can be chosen small enough for all orbits through $S$ to be principal as well. Therefore G.S is an open neighborhood of $x$ in $M_{\text {reg }}$ which itself is open by (6.15.3).

To see that $M_{\text {reg }}$ is dense, let $U \subseteq M$ be open, $x \in U$, and $S$ be a slice at $x$. Now choose a $y \in G . S \cap U$ for which the isotropy group $G_{y}$ has minimal dimension and the smallest number of connected components for 
this dimension in all of $G . S \cap U$. Let $S_{y}$ be a slice at $y$; then G. $S_{y} \cap G . S \cap U$ is open, and for any $z \in G . S_{y} \cap G . S \cap U$ we have $z \in g . S_{y}=S_{g . y}$, so $G_{z} \subseteq G_{g . y}=g G_{y} g^{-1}$. By choice of $y$, this implies $G_{z}=g G_{y} g^{-1}$ for all $z \in G . S_{y} \cap G . S \cap U$, and $G . y$ is a principal orbit.

29.15. Theorem. Let $M$ be a proper $G$-manifold and $x \in M$. Then there is a $G$-invariant neighborhood $U$ of $x$ in which only finitely many orbit types occur.

Proof. By theorem (6.30) there is a $G$-invariant Riemann metric on $M$. Let $S$ be the normal slice at $x$. Then $S$ is again a Riemann manifold, and the compact group $G_{x}$ acts isometrically on $S$. In (6.16.4) we saw that if $G_{x} . s_{1}$ and $G_{x} . s_{2}$ have the same orbit type in $S$, then $G . s_{1}$ and $G . s_{2}$ have the same orbit type in $M$. So the number of $G$-orbit types in $G$. $S$ can be no more than the number of $G_{x}$-orbit types in $S$. Therefore it is sufficient to consider the case where $G$ is a compact Lie group. Let us now prove the assertion under this added assumption. We carry out induction on the dimension of $M$.

For $n=0$ there is nothing to prove. Suppose the assertion is proved for $\operatorname{dim} M<n$. Again, it will do to find a slice $S$ at $x$ with only a finite number of $G_{x}$-orbit types. If $\operatorname{dim} S<\operatorname{dim} M$, this follows from the induction hypothesis. Now suppose $\operatorname{dim} S=n$. The slice $S$ is equivariantly diffeomorphic (by $\exp ^{\gamma}$ ) to an open ball in $T_{x} M$ under the slice representation. Since the slice representation is orthogonal, it restricts to a $G_{x^{-}}$action on each sphere r. $S^{n-1}$ in this ball. By the induction hypothesis, locally, $S^{n-1}$ has only finitely many $G_{x}$-orbit types. Since $S^{n-1}$ is compact, it has only finitely many orbit types globally. The orbit types are the same on all spheres $r . S^{n-1}(r>0)$, since $x \mapsto \frac{1}{r} x$ is $G$-equivariant. Therefore, $S$ has only finitely many orbit types: those of $S^{n-1}$ and the 0-orbit.

29.16. Theorem. If $M$ is a proper $G$-manifold, then the set $M_{\text {sing }} / G$ of all singular $G$-orbits does not locally disconnect the orbit space $M / G$ (that is, to every point in $M / G$ the connected neighborhoods remain connected even after removal of all singular orbits).

Proof. As in the previous theorem, we will reduce the statement to an assertion about the slice representation. By theorem (6.30), there is a $G$ invariant Riemann metric on $M$. Let $S$ be the normal slice at $x$. Then $S$ is again a Riemann manifold, and the compact group $G_{x}$ acts isometrically on $S$. A principal $G_{x}$-orbit is the restriction of a principal $G$-orbit, since $G_{x} . s$ is principal means that all orbits in a sufficiently small neighborhood of $G_{x} . s$ have the same orbit type as the orbit $G_{x} . s$; see (29.7). Therefore, by (6.16.4), the corresponding orbits in $G . U$ are also of the same type, and 
$G . s$ is principal as well. So there are 'fewer' singular $G$-orbits in $G$.S than there are singular $G_{x}$-orbits in $S$. Now cover $M$ with tubular neighborhoods like $G . S_{x}$, and recall that $G . S_{x} / G \cong S_{x} / G_{x}$ by (6.16.5). This together with the above argument shows us that it will suffice to prove the statement for the slice action. Furthermore, as in the proof of theorem (29.15), we can restrict our considerations to the slice representation. So we have reduced the statement to the following:

If $V$ is a real, $n$-dimensional vector space and $G$ a compact Lie group acting on $V$, then the set $V_{\text {sing }} / G$ of all singular $G$-orbits does not locally disconnect the orbit space $V / G$ (that is, to every point in $V / G$ the connected neighborhoods remain connected even after removal of all singular orbits).

We will prove this by induction on the dimension $n$ of $V$. For $n=1$, i.e., $V=\mathbb{R}$, the only nontrivial choice for $G$ is $O(1) \cong \mathbb{Z}_{2}$. In this case, $\mathbb{R} / G=[0, \infty)$ and $\mathbb{R}_{\text {sing }} / G=\{0\}$. Clearly, $\{0\}$ does not locally disconnect $[0, \infty)$, and we can proceed to the general case.

Suppose the assertion is proved for all dimensions smaller than $n$. Now for $G \subseteq O(n)$ we consider the induced action on the invariant submanifold $S^{n-1}$. For any $x \in S^{n-1}$ we can apply the induction hypothesis to the slice representation $G_{x} \rightarrow\left(\operatorname{Nor}_{x}(G . x)\right)$. This implies for the $G_{x}$-action on $S_{x}$ that $S_{x} / G_{x} \cong G . S_{x} / G$ is not locally disconnected after removing all its singular points. As above, we can again cover $S^{n-1}$ with tubular neighborhoods like $G . S_{x}$, and we see that all of $S^{n-1} / G$ is not locally disconnected by its singular orbits. Now we need to verify that the orbit space of the unit ball $D^{n}$ is not locally disconnected by its singular orbits. Since scalar multiplication is a $G$-equivariant diffeomorphism, the singular orbits in $V$ (not including $\{0\})$ project radially onto singular orbits in $S^{n-1}$. So if we view the ball $D^{n}$ as cone over $S^{n-1}$ and denote the cone construction by cone $\left(S^{n-1}\right)$, then $D_{\text {sing }}^{n}=\operatorname{cone}\left(S_{\text {sing }}^{n-1}\right)$. Furthermore, we have a homeomorphism

$$
\operatorname{cone}\left(S^{n-1}\right) / G \longrightarrow \operatorname{cone}\left(S^{n-1} / G\right), \quad G .[x, t] \mapsto[G . x, t]
$$

since $G$ preserves the 'radius' $t$. Therefore

$$
\begin{aligned}
D^{n} / G & =\left(\operatorname{cone}\left(S^{n-1}\right)\right) / G \cong \operatorname{cone}\left(S^{n-1} / G\right), \\
D_{\text {sing }}^{n} / G & =\operatorname{cone}\left(S_{\text {sing }}^{n-1}\right) / G \cong \operatorname{cone}\left(S_{\text {sing }}^{n-1} / G\right) .
\end{aligned}
$$

Since $S_{\text {sing }}^{n-1} / G$ does not locally disconnect $S^{n-1} / G$, we also see that

$$
\operatorname{cone}\left(S_{\text {sing }}^{n-1} / G\right) \cong D_{\text {sing }}^{n} / G
$$

does not locally disconnect $\operatorname{cone}\left(S^{n-1} / G\right) \cong D^{n} / G$. 
29.17. Corollary. Let $M$ be a connected proper $G$-manifold; then:

(1) $M / G$ is connected.

(2) $M$ has precisely one principal orbit type.

Proof. (1) Since $M$ is connected and the quotient map $\pi: M \rightarrow M / G$ is continuous, its image $M / G$ is connected as well.

(2) By the theorem (29.16) we have that $M / G \backslash M_{\text {sing }} / G=M_{\mathrm{reg}} / G$ is connected. On the other hand by (29.7), the orbits of a certain principal orbit type form an open subset of $M / G$, in particular of $M_{\mathrm{reg}} / G$. Therefore if there were more than one principal orbit type, these orbit types would partition $M_{\mathrm{reg}} / G$ into disjoint nonempty open subsets contradicting the fact that $M_{\mathrm{reg}} / G$ is connected.

29.18. Corollary. Let $M$ be a connected, proper $G$-manifold of dimension $n$ and let $k$ be the least number of connected components of all isotropy groups of dimension $m:=\inf \left\{\operatorname{dim} G_{x} \mid x \in M\right\}$. Then the following two assertions are equivalent:

(1) G. $x_{0}$ is a principal orbit.

(2) The isotropy group $G_{x_{0}}$ has dimension $m$ and $k$ connected components.

If furthermore $G$ is connected and simply connected, these conditions are again equivalent to:

(3) The orbit $G . x_{0}$ has dimension $n-m$ and for the order of the fundamental group we have $\left|\pi_{1}\left(G \cdot x_{0}\right)\right|=k$.

Proof. Recall that we proved the existence of a principal orbit in (29.14) just by taking a $G_{x_{0}}$ as described above. The other direction of the proof follows from corollary (29.17). Since there is only one principal orbit type, this must be it.

If moreover $G$ is connected and simply connected, we look at the fibration $G_{x_{0}} \rightarrow G \rightarrow G / G_{x_{0}}=G \cdot x_{0}$ and at the following portion of its long exact homotopy sequence:

$$
0=\pi_{1}(G) \rightarrow \pi_{1}\left(G \cdot x_{0}\right) \rightarrow \pi_{0}\left(G_{x_{0}}\right) \rightarrow \pi_{0}(G)=0
$$

from which we see that $\left|\pi_{1}\left(G . x_{0}\right)\right|=k$ if and only if the isotropy group $G_{x_{0}}$ has $k$ connected components.

29.19. Theorem ([198). Let $\pi: G \rightarrow O(V)$ be an orthogonal, real, finite-dimensional representation of a compact Lie group $G$. Let $\rho_{1}, \ldots, \rho_{k} \in$ $\mathbb{R}[V]^{G}$ be homogeneous generators for the algebra $\mathbb{R}[V]^{G}$ of invariant polynomials on $V$. For $v \in V$, let $\operatorname{Nor}_{v}(G . v):=T_{v}(G . v)^{\perp}$ be the normal space 
to the orbit at $v$, and let $\operatorname{Nor}_{v}(G \cdot v)^{G_{v}}$ be the subspace of those vectors which are invariant under the isotropy group $G_{v}$.

Then $\operatorname{grad} \rho_{1}(v), \ldots, \operatorname{grad} \rho_{k}(v)$ span $\operatorname{Nor}_{v}(G . v)^{G_{v}}$ as a real vector space.

Proof. Clearly each $\operatorname{grad} \rho_{i}(v) \in \operatorname{Nor}_{v}(G . v)^{G_{v}}$. In the following we will identify $G$ with its image $\pi(G) \subseteq O(V)$. Its Lie algebra is then a subalgebra of $\mathfrak{o}(V)$ and can be realized as a Lie algebra consisting of skew-symmetric matrices. Let $v \in V$, and let $S_{v}$ be the normal slice at $v$ which is chosen so small that the projection of the tubular neighborhood (see (6.18) $p_{G . v}$ : $G . S_{v} \rightarrow G . v$ from the diagram

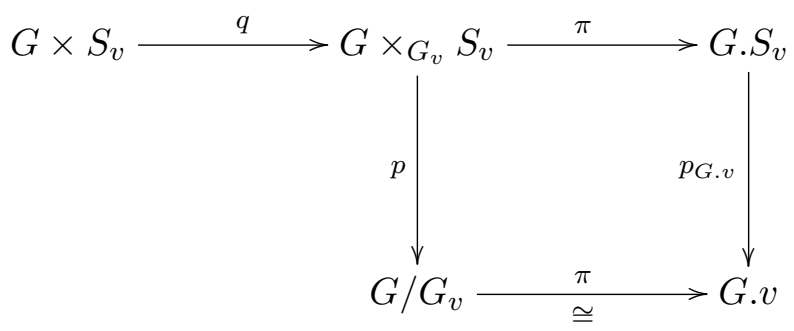

has the property that for any $w \in G . S_{v}$ the point $p_{G . v}(w) \in G . v$ is the unique point in the orbit $G . v$ which minimizes the distance between $w$ and the orbit G.v.

Choose $n \in \operatorname{Nor}_{v}(G . v)^{G_{v}}$ so small that $x:=v+n \in S_{v}$. So $p_{G . v}(x)=v$. For the isotropy groups we have $G_{x} \subseteq G_{v}$ by (6.16.2). But we have also $G_{v} \subseteq G_{v} \cap G_{n} \subseteq G_{x}$, so that $G_{v}=G_{x}$. Let $S_{x}$ be the normal slice at $x$ which we also choose so small that $p_{G . x}: G . S_{x} \rightarrow G . x$ has the same minimizing property as $p_{G . v}$ above, but so large that $v \in G . S_{x}$ (choose $n$ smaller if necessary). We also have $p_{G . x}(v)=x$ since for the Euclidean distance in $V$ we have

$$
\begin{aligned}
|v-x| & =\min _{g \in G}|g \cdot v-x| & & \text { since } v=p_{G \cdot v}(x) \\
& =\min _{g \in G}|h \cdot g \cdot v-h \cdot x| & & \text { for all } h \in G \\
& =\min _{g \in G}\left|v-g^{-1} \cdot x\right| & & \text { by choosing } h=g^{-1} .
\end{aligned}
$$

For $w \in G$. $S_{x}$ we consider the local, smooth, $G$-invariant function

$$
\begin{aligned}
\operatorname{dist}(w, G . x)^{2} & =\operatorname{dist}\left(w, p_{G . x}(w)\right)^{2}=\left\langle w-p_{G . x}(w), w-p_{G . x}(w)\right\rangle \\
& =\langle w, w\rangle+\left\langle p_{G . x}(w), p_{G . x}(w)\right\rangle-2\left\langle w, p_{G . x}(w)\right\rangle \\
& =\langle w, w\rangle+\langle x, x\rangle-2\left\langle w, p_{G . x}(w)\right\rangle .
\end{aligned}
$$

Its derivative with respect to $w$ is

$$
d\left(\operatorname{dist}(\quad, G . x)^{2}\right)(w) y=2\langle w, y\rangle-2\left\langle y, p_{G . x}(w)\right\rangle-2\left\langle w, d p_{G . x}(w) y\right\rangle .
$$


We shall show below that

$$
\left\langle v, d p_{G . x}(v) y\right\rangle=0 \quad \text { for all } y \in V,
$$

so that the derivative at $v$ is given by

(3) $d\left(\operatorname{dist}(\quad, G . x)^{2}\right)(v) y=2\langle v, y\rangle-2\left\langle y, p_{G . x}(v)\right\rangle=2\langle v-x, y\rangle=-2\langle n, y\rangle$.

Now choose a smooth $G_{x}$-invariant function $f: S_{x} \rightarrow \mathbb{R}$ with compact support which equals 1 in an open ball around $x$ and extend it smoothly (see the diagram above, but for $S_{x}$ ) to $G . S_{x}$ and then to the whole of $V$. We assume that $f$ is still equal to 1 in a neighborhood of $v$. Then $g=$ $f$. dist $(, G . x)^{2}$ is a smooth $G$-invariant function on $V$ which coincides with $\operatorname{dist}(, G \cdot x)^{2}$ near $v$. By the theorem of Schwarz (7.13) there is a smooth function $h \in C^{\infty}\left(\mathbb{R}^{k}, \mathbb{R}\right)$ such that $g=h \circ \rho$, where $\rho=\left(\rho_{1}, \ldots, \rho_{k}\right): V \rightarrow$ $\mathbb{R}^{k}$. Then we have finally by (3)

$$
\begin{aligned}
-2 n & =\operatorname{grad}\left(\operatorname{dist}(, G \cdot x)^{2}\right)(v)=\operatorname{grad} g(v) \\
& =\operatorname{grad}(h \circ \rho)(v)=\sum_{i=1}^{k} \frac{\partial h}{\partial y_{i}}(\rho(v)) \operatorname{grad} \rho_{i}(v),
\end{aligned}
$$

which proves the result.

It remains to check equation (2). Since $T_{v} V=T_{v}(G \cdot v) \oplus \operatorname{Nor}_{v}(G \cdot v)$, the normal space $\operatorname{Nor}_{x}(G . x)=\operatorname{ker} d p_{G . x}(v)$ is still transversal to $T_{v}(G . v)$ if $n$ is small enough; so it remains to show that $\left\langle v, d p_{G . x}(v) . X . v\right\rangle=0$ for each $X \in \mathfrak{g}$. Since $x=p_{G . x}(v)$, we have $|v-x|^{2}=\min _{g \in G}|v-g . x|^{2}$, and thus the derivative of $g \mapsto\langle v-g \cdot x, v-g \cdot x\rangle$ at $e$ vanishes: For all $X \in \mathfrak{g}$ we have

$$
0=2\langle-X . x, v-x\rangle=2\langle X . x, x\rangle-2\langle X . x, v\rangle=0-2\langle X . x, v\rangle,
$$

since the action of $X$ on $V$ is skew-symmetric. Now we consider the equation $p_{G . x}(g \cdot v)=g \cdot p_{G . x}(v)$ and differentiate it with respect to $g$ at $e \in G$ in the direction $X \in \mathfrak{g}$ to obtain in turn

$$
\begin{aligned}
d p_{G . x}(v) . X . v & =X \cdot p_{G . x}(v)=X . x, \\
\left\langle v, d p_{G . x}(v) . X . v\right\rangle & =\langle v, X . x\rangle=0, \quad \text { by }(4) .
\end{aligned}
$$

29.20. Lemma. Let $\pi: G \rightarrow O(V)$ be an orthogonal representation. Let $\omega \in \Omega_{\text {hor }}^{p}(V)^{G}$ be an invariant differential form on $V$ which is horizontal in the sense that $i_{w} \omega_{x}=0$ if $w$ is tangent to the orbit $G . x$. Let $v \in V$ and let $w \in T_{v} V$ be orthogonal to the space $\operatorname{Nor}_{v}(G . v)^{G_{v}^{0}}$ of those orthogonal vectors which are invariant under the connected component $G_{v}^{0}$ of the isotropy group $G_{v}$. Then $i_{w} \omega_{v}=0$.

Proof. We consider the orthogonal decomposition

$$
T_{v} V=T_{v}(G . v) \oplus W \oplus \operatorname{Nor}_{v}(G . v)^{G_{v}^{0}} .
$$


We may assume that $w \in W$ since $i_{u} \omega_{v}=0$ for $u \in T_{v}(G . v)$.

We claim that each $w \in W$ is a linear combination of elements of the form $X . u$ for $u \in W$ and $X \in \mathfrak{g}_{v}:=\operatorname{ker}(d \pi(\quad) v)$. Since $G_{v}^{0}$ is compact, the representation space $W$ has no fixed point other than zero and is completely reducible under $G_{v}^{0}$ and thus also under its Lie algebra $\mathfrak{g}_{v}$, and we may treat each irreducible component separately or assume that $W$ is irreducible. Then $\mathfrak{g}_{v}(W)$ is an invariant subspace which is not 0 . So it agrees with $W$, and the claim follows.

So we may assume that $w=X . u$ for $u \in W$. But then

$$
\left(v+\frac{1}{n} u, X . u=n X .\left(v+\frac{1}{n} u\right)\right) \in T_{v+\frac{1}{n} u}\left(G \cdot\left(v+\frac{1}{n} u\right)\right)
$$

satisfies $i_{X . u} \omega_{v+u / n}=0$ by horizontality and thus we have

$$
i_{w} \omega_{v}=i_{X . u} \omega_{v}=\lim _{n} i_{X . u} \omega_{v+u / n}=0 .
$$

29.21. $G$-manifold with a single orbit type as fiber bundle. Let $(M, \gamma)$ be a proper Riemann $G$-manifold and suppose that $M$ has only one orbit type (see 6.11), $(H)$. We then want to study the quotient map $\pi$ : $M \rightarrow M / G$. Let us first consider the orbit space $M / G$. Choose $x \in M$ and let $S_{x}$ denote the normal slice at $x$. Then by (6.16.2) we have $G_{y} \subseteq$ $G_{x}$ for all $y \in S_{x}$. Since $G_{y}$ must additionally be conjugate to $G_{x}$ and both are compact, they must be the same by (6.12). So $G_{x}=G_{y}$ and therefore $G_{x}$ acts trivially on $S_{x}$ (this can also be seen as a special case of (29.7)). From (6.16.5) it follows that $\pi\left(S_{x}\right) \cong S_{x} / G_{x}=S_{x}$, and with (6.18) we have that $G . S_{x}$ is isomorphic to $G / G_{x} \times S_{x}$. Therefore, for any $x \in M$, $\left(\pi\left(S_{x}\right),\left.\exp _{x}^{-1}\right|_{S_{x}}\right)$ can serve as a chart for $M / G$ :

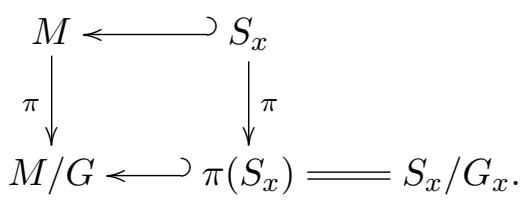

To make an atlas out of these charts, we have to check whether they are compatible - which is obvious. By (6.29), $M / G$ is Hausdorff, and therefore it is a smooth manifold.

Now let us study the smooth submersion $\pi: M \rightarrow M / G$. We want to find a Riemann metric on $M / G$ which will make $\pi$ a Riemann submersion.

Claim. For $X_{x}, Y_{x} \in \operatorname{Hor}_{x}(\pi)=\operatorname{Nor}_{x}(G . x)$, the following inner product is well defined:

$$
\bar{\gamma}_{\pi(x)}\left(T \pi X_{x}, T \pi Y_{x}\right):=\gamma_{x}\left(X_{x}, Y_{x}\right)
$$


Proof. Choose $X_{g x}^{\prime}, Y_{g x}^{\prime} \in \operatorname{Hor}_{g x}(\pi)$ such that $T \pi \cdot X_{g x}^{\prime}=T \pi \cdot X_{x}$ and $T \pi . Y_{g x}^{\prime}=T \pi . Y_{x}$. Then we see that $X_{g x}^{\prime}=T\left(\ell_{g}\right) X_{x}$ by the following argumentation: Clearly $T \pi\left(X_{g x}^{\prime}-T\left(\ell_{g}\right) \cdot X_{x}\right)=0$, so the difference $X_{g x}^{\prime}-T\left(\ell_{g}\right) \cdot X_{x}$ is vertical. The map $\ell_{g}$ leaves $G . x$ invariant; consequently, $T \ell_{g}$ maps vertical vectors to vertical vectors and since it is an isometry, it also maps horizontal vectors to horizontal vectors. Therefore $X_{g x}^{\prime}-T\left(\ell_{g}\right) \cdot X_{x}$ is horizontal as well as vertical and must be zero.

Now we can conclude, that

$$
\gamma_{g x}\left(X_{g x}^{\prime}, Y_{g x}^{\prime}\right)=\gamma_{g x}\left(T\left(\ell_{g}\right) X_{x}, T\left(\ell_{g}\right) Y_{x}\right)=\gamma_{x}\left(X_{x}, Y_{x}\right) .
$$

So we have found a Riemann metric $\bar{\gamma}$ on $M / G$ which makes $\pi$ a Riemann submersion.

Let us finally try to understand in which sense $\pi: M \rightarrow M / G$ is an associated bundle. Let $x \in M$ be such that $G_{x}=H$. By (29.1.4) the set $M^{H}=\{x \in M: g \cdot x=x$ for all $g \in H\}$ is a geodesically complete submanifold of $M$. It is $N_{G}(H)$-invariant, and the restriction $\pi: M^{H} \rightarrow M / G$ is a smooth submersion since for each $y \in M^{H}$ the slice $S_{y}$ is also contained in $M^{H}$. The fiber of $\pi: M^{H} \rightarrow M / G$ is a free $N_{G}(H) / H$-orbit: If $\pi(x)=\pi(y)$ and $G_{x}=H=G_{y}$, then $g \in N_{G}(H)$. So $\pi: M^{H} \rightarrow M / G$ is a principal $N_{G}(H) / H$-bundle, and $M$ is the associated bundle with fiber $G / H$ as follows:

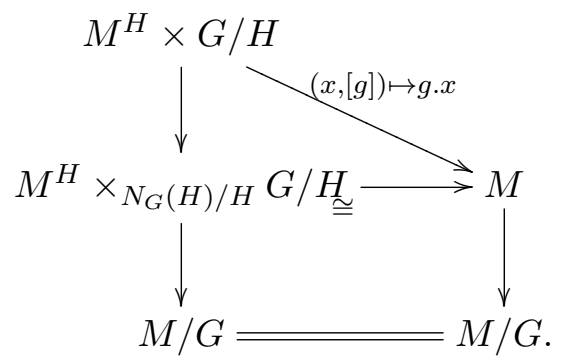

29.22. Another fiber bundle construction. Let $M$ again be a proper Riemann $G$-manifold with only one orbit type. Then we can 'partition' $M$ into the totally geodesic submanifolds

$$
M^{g H g^{-1}}:=\left\{x \in M: g h g^{-1} \cdot x=x \text { for all } h \in H\right\}
$$

where $H=G_{x_{0}}$ ( $x_{0} \in M$ arbitrary) is fixed and $g$ varies. This is not a proper partitioning in the sense that if $g \neq e$ commutes with $H$, for instance, then $M^{g H g^{-1}}=M^{e H e^{-1}}$. We want to find out just which $g$ give the same sets $M^{g H g^{-1}}$.

Claim.

$$
M^{g H g^{-1}}=M^{g^{\prime} H g^{\prime-1}} \quad \Longleftrightarrow \quad g N(H)=g^{\prime} N(H)
$$

where $N(H)$ denotes the normalizer of $H$ in $G$. 
Proof. First let us show the following identity:

$$
N(H)=\left\{g \in G: g\left(M^{H}\right) \subseteq M^{H}\right\} .
$$

$(\subseteq)$ Let $n \in N(H)$ and $y \in M^{H}$. Then $n . y$ is $H$-invariant:

$$
h n . y=n n^{-1} h n . y=n\left(n^{-1} h n\right) . y=n . y .
$$

() $g M^{H} \subseteq M^{H}$ implies that $h g . y=g . y$, or equivalently $g^{-1} h g . y=y$, for any $y \in M^{H}$ and $h \in H$. Recall at this point that $H=G_{x_{0}}$ for some $x_{0} \in M$. Therefore, we have $g^{-1} h g . x_{0}=x_{0}$ and consequently $g^{-1} h g \in G_{x_{0}}=H$.

Using this characterization for $N(H)$ and the identity

$$
g^{\prime}\left\{g \in G: g M^{H} \subseteq M^{H}\right\}=\left\{g \in G: g M^{H} \subseteq g^{\prime} M^{H}\right\},
$$

we can convert the right hand side of our equality, $g N(H)=g^{\prime} N(H)$, to the following:

$$
\left\{a \in G: a M^{H} \subseteq g \cdot M^{H}\right\}=\left\{a \in G: a M^{H} \subseteq g^{\prime} \cdot M^{H}\right\} .
$$

In particular, this is the case if

$$
g \cdot M^{H}=g^{\prime} \cdot M^{H} .
$$

In fact, let us show that the two equations are equivalent. Suppose indirectly that $g . y \notin g^{\prime} \cdot M^{H}$ for some $y \in M^{H}$. Then $a=g$ has the property $a . M^{H} \nsubseteq$ $g^{\prime} \cdot M^{H}$, so $\left\{a \in G: a M^{H} \subseteq g \cdot M^{H}\right\} \neq\left\{a \in G: a M^{H} \subseteq g^{\prime} \cdot M^{H}\right\}$.

So far we have shown that $g N(H)=g^{\prime} N(H) \Leftrightarrow g \cdot M^{H}=g^{\prime} \cdot M^{H}$. To complete the proof, it only remains to check whether

$$
M^{g H g^{-1}}=g \cdot M^{H} .
$$

This is easily done (as well as plausible, since it strongly resembles the 'dual' notion $\left.G_{g x}=g G_{x} g^{-1}\right)$ :

$$
\begin{aligned}
y \in M^{g H g^{-1}} & \Longleftrightarrow g h g^{-1} \cdot y=y \text { for all } h \in H \\
& \Longleftrightarrow h g^{-1} \cdot y=g^{-1} y \text { for all } h \in H \\
& \Longleftrightarrow g^{-1} \cdot y \in M^{H} \\
& \Longleftrightarrow y \in g M^{H} .
\end{aligned}
$$

Claim. The map $\bar{\pi}: M \rightarrow G / N(H)$ defined by $M^{g H g^{-1}} \ni x \mapsto g \cdot N(H)$ is a fiber bundle with typical fiber $M^{H}$. 
Proof. To prove this, let us consider the following diagram:

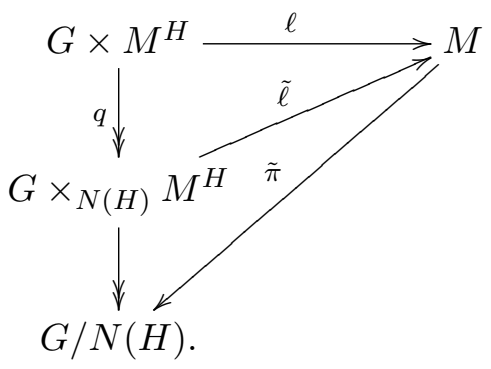

Here we use the restricted action $\ell: N(H) \times M^{H} \rightarrow M^{H}$ to associate to the principal bundle $G \rightarrow G / N(H)$ the bundle $G\left[M^{H}, \ell\right]=G \times_{N(H)} M^{H}$. It remains to show that $\tilde{\ell}$ is a diffeomorphism, since then $\tilde{\pi}$ has the desired fiber bundle structure. The map $\tilde{\ell}$ is smooth, since $\tilde{\ell} \circ q=\ell$ is smooth and $q$ is a submersion. Now let us show that $\tilde{\ell}$ is bijective.

(1) $\tilde{\ell}$ is surjective: Since $H$ is the only orbit type, for every $x \in M$ there is a $g \in G$, such that $G_{x}=g H g^{-1}$, which implies $x \in M^{g H g^{-1}}=g M^{H} \subseteq$ $\ell\left(G \times M^{H}\right)$. So $\ell$ is surjective and, by the commutativity of the diagram, so is $\tilde{\ell}$.

(2) $\tilde{\ell}$ is injective: Suppose $\ell(a, x)=a . x=b . y=\ell(b, y)$, for some $a, b \in$ $G, x, y \in M^{H}$. Then $b^{-1} a . x=y \in M^{H}$ implies $h b^{-1} a . x=y=b^{-1} a . x$ which implies again $\left(b^{-1} a\right)^{-1} h b^{-1} a . x=x$. Since there is only one orbit type and all isotropy groups are compact, we know that $x \in M^{H} \Rightarrow H=G_{x}$ (by $(6.12)$. So $\left(b^{-1} a\right)^{-1} h b^{-1} a$ is again in $H$, and $b^{-1} a \in N(H)$. In this case, $q(a, x)=[a, x]=\left[b b^{-1} a, x\right]=\left[b, b^{-1} a . x\right]=[b, y]=q(b, y)$.

The inverse map $\tilde{\ell}^{-1}$ is smooth, since $\ell$ is a submersion. So $\tilde{\ell}$ is a diffeomorphism and $\bar{\pi}$ a fiber bundle with typical fiber $M^{H}$.

29.23. Construction for more than one orbit type. Let $(H)$ be one particular orbit type $\left(H=G_{x}\right)$. To reduce the case at hand to the previous one, we must partition the points in $M$ into sets with common orbit type:

$$
M_{(H)}:=\left\{x \in M:\left(G_{x}\right)=(H)\right\} .
$$

Claim. For a proper Riemann $G$-manifold, the space $M_{(H)}$ as defined above is a smooth $G$-invariant submanifold.

Proof. The set $M_{(H)}$ is of course $G$-invariant as a collection of orbits of a certain type. We only have to prove that it is a smooth submanifold. Take any $x$ in $M_{(H)}$ (then, without loss, $H=G_{x}$ ), and let $S_{x}$ be a slice at $x$. Consider the tubular neighborhood $G . S \cong G \times_{H} S_{x}$ (see (6.18). Then the orbits of type $(H)$ in $G . S$ are just those orbits that meet $S_{x}$ in $S_{x}^{H}$ 
(where $S_{x}^{H}$ shall denote the fixed point set of $H$ in $S_{x}$ ). Or, equivalently, $\left(G \times_{H} S_{x}\right)_{(H)}=G \times_{H} S_{x}^{H}$ :

$(\subseteq)[g, s] \in\left(G \times_{H} S_{x}\right)_{(H)} \Rightarrow g . s \in G . S_{(H)} \Rightarrow g H g^{-1}=G_{s} \subseteq H \Rightarrow G_{s}=$ $H \Rightarrow s \in S_{x}^{H} \Rightarrow[g, s] \in G \times{ }_{H} S_{x}^{H}$.

() $[g, s] \in G \times_{H} S_{x}^{H} \Rightarrow s \in S_{x}^{H} \Rightarrow H \subseteq G_{s}$, but since $s \in S_{x}$, we have $G_{s} \subseteq G_{x}=H$ by (6.16.2), therefore $G_{s}=H$ and $[g, s] \in\left(G \times_{H} S_{x}\right)_{(H)}$.

Now, let $S_{x}=\exp _{x}\left(\operatorname{Nor}_{r}(G . x)\right)$ be the normal slice at $x$. That is, $r$ is chosen so small that $\exp _{x}$ is a diffeomorphism on $\operatorname{Nor}_{r}(G \cdot x)=: V$. Notice that $V$ is not only diffeomorphic to $S_{x}$, but $G$-equivariantly so, if we let $G$ act on $\operatorname{Nor}_{x}(G . x)$ via the slice representation. Since the slice action is orthogonal, in particular linear, the set of points fixed by the action of $H$ is a linear subspace of $\operatorname{Nor}_{x}(G . x)$ and its intersection with $V$, a "linear" submanifold. Therefore $S_{x}^{H}$ is also a submanifold of $S_{x}$. Now consider the diagram

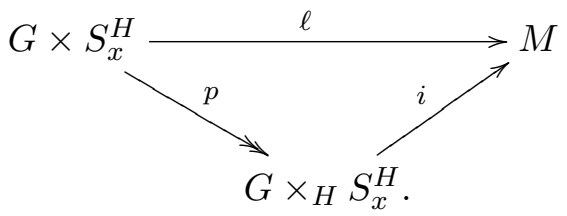

The map $i$ is well defined, injective and smooth, since $p$ is a submersion and $\ell$ is smooth. Furthermore, $p$ is open, and so is $\ell$. Just consider any open set of the form $U \times W$ in $G \times S_{x}^{H}$. Then $\ell(U \times W)$ is the union of all sets $\ell_{u}(W)$ for $u \in U$. Since $\ell_{u}$ is a diffeomorphism, each one of these is open, so $\ell(U \times W)$ is open as well. Therefore, $i$ must be open, and so $i$ is an embedding and $G . S^{H} \cong G \times_{H} S_{x}^{H}$ is an embedded submanifold of $M$.

Let $(H)$ be one particular orbit type $\left(H=G_{x}\right)$; then $M^{H}$ is again a closed, totally geodesic submanifold of $M$; see (29.1.3).

Claim. $\left\{x \in M: G_{x}=H\right\}$ is an open submanifold of $M^{H}$.

For one orbit type, $x \in M^{H}$ implied $H=G_{x}$, and thus $\left\{x \in M: G_{x}=\right.$ $H\}=M^{H}$. For more than one orbit type, $M^{H}$ is not necessarily contained in $M_{(H)}$. Therefore, it is necessary to study $\left\{x \in M: G_{x}=H\right\}=M^{H} \cap M_{(H)}$.

Proof. In (29.22) we saw that $N(H)$ is the largest subgroup of $G$ acting on $M^{H}$. It induces a proper $N(H) / H$-action on $M^{H}$. Now, $\left\{x \in M: G_{x}=H\right\}$ is the set of all points in $M^{H}$ with trivial isotropy group with respect to this action. So by (29.18) it is simply the set of all regular points. Therefore, by (29.14), $\left\{x \in M: G_{x}=H\right\}$ is an open, dense submanifold of $M^{H}$.

Now, $M_{(H)}$ can be turned into a fiber bundle over $G / N(H)$ with typical fiber $\left\{x \in M: G_{x}=H\right\}$ just as before. On the other hand, $M_{(H)}$ is a fiber bundle over $M_{(H)} / G$ with typical fiber $G / H$. The partition of $M$ into submanifolds 
$M_{(H)}$ and that of $M / G$ into the different orbit types is locally finite by $(29.15)$. So $M$ and $M / G$ are in a sense stratified, and $\pi: M \rightarrow M / G$ is a stratified Riemann submersion (see also [40]).

29.24. Remark. Let $M$ be a connected Riemann $G$-manifold and $(H)$ the principal orbit type, then we saw in (29.23) that $\pi: M_{(H)} \rightarrow M_{(H)} / G$ is a Riemann submersion. Now we can prove:

Claim. For $x \in M_{\text {reg }}=M_{(H)}$ a vector field $\xi \in \Gamma(\operatorname{Nor}(G . x))$ is $\pi$-parallel if and only if $\xi$ is $G$-equivariant.

Proof. $(\Longleftarrow)$ If $\xi(g \cdot x)=T_{x} \ell_{g} \cdot \xi(x)$, then $T_{g \cdot x} \pi \cdot \xi(g \cdot x)=T_{g \cdot x} \pi \circ T_{x} \ell_{g} \cdot \xi(x)=$ $T_{x} \pi . \xi(x)$ for all $g \in G$. Therefore $\xi$ is $\pi$-parallel.

( $\Longrightarrow$ ) The tangent vectors $\xi(g . x)$ and $T_{x} \ell_{g} \xi(x)$ are both in $\operatorname{Nor}_{g . x}(G . x)$, and since $\xi$ is $\pi$-parallel, we have $T_{g . x} \pi \cdot \xi(g . x)=T_{x} \pi \cdot \xi(x)=T_{g \cdot x} \pi \circ T_{x} \ell_{g} \cdot \xi(x)$. So $\xi(g . x)$ and $T_{x} \ell_{g} \cdot \xi(x)$ both have the same image under $T_{g . x} \pi$. Because $T_{g . x} \pi$ restricted to $\operatorname{Nor}_{g . x}(G . x)$ is an isomorphism, $\xi(g . x)=T_{x} \ell_{g} \cdot \xi(x)$.

\section{Polar Actions}

In this chapter, let $(M, \gamma)$ always denote a connected, complete Riemann $G$ manifold, and assume that the action of $G$ on $M$ is effective and isometric.

30.1. Lemma. Consider $X \in \mathfrak{g}$, the Lie algebra of $G, \zeta_{X}$, the associated fundamental vector field to $X$, and $c$, a geodesic in $M$. Then $\gamma\left(c^{\prime}(t), \zeta_{X}(c(t))\right)$ is constant in $t$.

This is an example of a momentum mapping if we lift the whole situation to the symplectic manifold $T^{*} M$ and identify this with $T M$ via $\gamma$. See section $(34)$.

Proof. Let $\nabla$ be the Levi-Civita covariant derivative on $M$. Then

$$
\partial_{t} \cdot \gamma\left(c^{\prime}(t), \zeta_{X}(c(t))\right)=\gamma\left(\nabla_{\partial_{t}} c^{\prime}(t), \zeta_{X}(c(t))\right)+\gamma\left(c^{\prime}(t), \nabla_{\partial_{t}}\left(\zeta_{X} \circ c\right)\right) .
$$

Since $c$ is a geodesic, $\nabla_{\partial_{t}} c^{\prime}(t)=0$, and so is the entire first summand. So it remains to show that $\gamma\left(c^{\prime}(t), \nabla_{\partial_{t}}\left(\zeta_{X} \circ c\right)\right)$ vanishes as well.

Let $s_{1}, \ldots, s_{n}$ be a local orthonormal frame field on an open neighborhood $U$ of $c(t)$, and let $\sigma^{1}, \ldots, \sigma^{n}$ be the orthonormal coframe. Then $\gamma=\sum \sigma^{i} \otimes \sigma^{i}$. Let us use the notation

$$
\begin{gathered}
\left.\zeta_{X}\right|_{U}=: \sum s_{i} X^{i} \\
\left.\nabla \zeta_{X}\right|_{U}=: \sum X_{i}^{j} s_{j} \otimes \sigma^{i} .
\end{gathered}
$$

Then we have

$$
\nabla_{\partial_{t}}\left(\zeta_{X} \circ c\right)=\sum X_{i}^{j}(c(t)) s_{j}(c(t)) \sigma^{i}\left(c^{\prime}(t)\right)
$$


So

$$
\begin{aligned}
\gamma\left(c^{\prime}(t), \nabla_{\partial_{t}}\left(\zeta_{X} \circ c\right)\right) & =\sum \sigma^{j}\left(c^{\prime}(t)\right) \sigma^{j}\left(\nabla_{\partial_{t}}\left(\zeta_{X} \circ c\right)\right) \\
& =\sum X_{i}^{j}(c(t)) \sigma^{j}\left(c^{\prime}(t)\right) \sigma^{i}\left(c^{\prime}(t)\right) .
\end{aligned}
$$

If we now show that $X_{i}^{j}+X_{j}^{i}=0$, then $\gamma\left(c^{\prime}(t), \nabla_{\partial_{t}}\left(\zeta_{X} \circ c\right)\right)$ will be zero, and the proof will be complete. Since the action of $G$ is isometric, $\zeta_{X}$ is a Killing vector field; that is, $\mathcal{L}_{\zeta_{X}} \gamma=0$. So we have

$$
\sum \mathcal{L}_{\zeta_{X}} \sigma^{i} \otimes \sigma^{i}+\sum \sigma^{i} \otimes \mathcal{L}_{\zeta_{X}} \sigma^{i}=0
$$

Now we must try to express $\mathcal{L}_{\zeta_{X}} \sigma^{i}$ in terms of $X_{i}^{j}$. For this, recall the structure equation: $d \sigma^{k}+\sum \omega_{j}^{k} \wedge \sigma^{j}=0$. We have

$$
\begin{aligned}
\mathcal{L}_{\zeta_{X}} \sigma^{i} & =i_{\zeta_{X}} d \sigma^{i}+d\left(i_{\zeta_{X}} \sigma^{i}\right)=-i_{\zeta_{X}}\left(\sum \omega_{j}^{i} \wedge \sigma^{j}\right)+d\left(\sigma^{i}\left(\zeta_{X}\right)\right) \\
& =-i_{\zeta_{X}} \sum \omega_{j}^{i} \wedge \sigma^{j}+d X^{i}=\sum \omega_{j}^{i} \cdot X^{j}-\sum \omega_{j}^{i}\left(\zeta_{X}\right) \sigma^{j}+d X^{i} .
\end{aligned}
$$

Since

$$
\left.\nabla \zeta_{X}\right|_{U}=\nabla\left(\sum s_{j} X^{j}\right)=\sum s_{i} \cdot \omega_{j}^{i} \cdot X^{j}+\sum s_{i} \otimes d X^{i}=\sum X_{j}^{i} s_{i} \otimes \sigma^{j},
$$

we can replace $\sum \omega_{j}^{i} \cdot X^{j}$ by $\sum X_{j}^{i} \sigma^{j}-d X^{i}$. Therefore,

$$
\mathcal{L}_{\zeta_{X}} \sigma^{i}=\sum\left(X_{j}^{i} \sigma^{j}-\omega_{j}^{i}\left(\zeta_{X}\right) \sigma^{j}\right)=\sum\left(X_{j}^{i}-\omega_{j}^{i}\left(\zeta_{X}\right)\right) \sigma^{j} .
$$

Now, let us insert this into $0=\mathcal{L}_{\zeta_{X}} \gamma$ :

$$
\begin{aligned}
0 & =\sum \mathcal{L}_{\zeta_{X}} \sigma^{i} \otimes \sigma^{i}+\sum \sigma^{i} \otimes \mathcal{L}_{\zeta_{X}} \sigma^{i} \\
& =\sum\left(X_{j}^{i}-\omega_{j}^{i}\left(\zeta_{X}\right)\right) \sigma^{j} \otimes \sigma^{i}+\sum\left(X_{j}^{i}-\omega_{j}^{i}\left(\zeta_{X}\right)\right) \sigma^{i} \otimes \sigma^{j} \\
& =\sum\left(X_{j}^{i}+X_{i}^{j}\right) \sigma^{j} \otimes \sigma^{i}-\sum\left(\omega_{j}^{i}\left(\zeta_{X}\right)+\omega_{i}^{j}\left(\zeta_{X}\right)\right) \sigma^{j} \otimes \sigma^{i} \\
& =\sum\left(X_{j}^{i}+X_{i}^{j}\right) \sigma^{j} \otimes \sigma^{i}-0
\end{aligned}
$$

since $\omega(Y)$ is skew-symmetric. This implies $X_{j}^{i}+X_{i}^{j}=0$, and we are done.

30.2. Definition. For any $x$ in $M_{\text {reg }}$ we define:

$$
\begin{aligned}
E(x) & :=\exp _{x}^{\gamma}\left(\operatorname{Nor}_{x}(G . x)\right) \subseteq M, \\
E_{r e g}(x) & :=E(x) \cap M_{\text {reg }} .
\end{aligned}
$$

In a neighborhood of $x, E(x)$ is a manifold; globally, it can intersect itself.

30.3. Lemma. Let $x \in M_{\text {reg }}$, then:

(1) $g \cdot E(x)=E(g . x), g \cdot E_{r e g}(x)=E_{\text {reg }}(g \cdot x)$. 
(2) For $X_{x} \in \operatorname{Nor}(G . x)$ the geodesic $c: t \mapsto \exp \left(t . X_{x}\right)$ is orthogonal to every orbit it meets.

(3) If $G$ is compact, then $E(x)$ meets every orbit in $M$.

Proof. (1) This is a direct consequence of (29.1.1).

$$
g . \exp _{x}(t . X)=\exp _{g \cdot x}\left(t \cdot T_{x} \ell_{g} \cdot X\right) .
$$

(2) By choice of starting vector $X_{x}$, the geodesic $c$ is orthogonal to the orbit $G . x$, which it meets at $t=0$. Therefore it intersects every orbit it meets orthogonally, by lemma (30.1),

(3) For arbitrary $x, y \in M$, we will prove that $E(x)$ intersects $G$. $y$. Since $G$ is compact, by continuity of $\ell^{y}: G \rightarrow M$, the orbit $G . y$ is compact as well. Therefore we can choose $g \in G$ in such a way that $\operatorname{dist}(x, G . y)=\operatorname{dist}(x, g . y)$. Let $c(t):=\exp _{x}\left(t . X_{x}\right)$ be a minimal geodesic connecting $x=c(0)$ with $g . y=c(1)$. We now have to show that $X_{x} \in \operatorname{Nor}_{x}(G . x)$ : Take a point $p=c(t)$ on the geodesic very close to $g . y$ - close enough so that $\exp _{p}$ is a diffeomorphism into a neighborhood $U_{p}$ of $p$ containing $g . y$ (it shall have domain $\left.V \subseteq T_{p} M\right)$. In this situation Gauß's lemma (23.2) states that all geodesics through $p$ are orthogonal to the geodesic $\operatorname{spheres:~} \exp _{p}\left(k . S^{m-1}\right)$ (where $S^{m-1}:=\left\{X_{p} \in T_{p} M: \gamma\left(X_{p}, X_{p}\right)=1\right\}$, and $k>0$ is small enough for $k . S^{m-1} \subseteq V$ to hold). From this it can be concluded that $c$ is orthogonal to G.y: Take the smallest geodesic sphere around $p$ touching G.y. By the minimality of $c, c$ must leave the geodesic sphere at a touching point, and by Gauß's lemma, it must leave at a right angle to the geodesic sphere. Clearly, the touching point is just $g . y=c(1)$, and there $c$ also meets $G . y$ at a right angle. By (2), $c$ encloses a right angle with every other orbit it meets as well. In particular, $c$ starts orthogonally to G.x. Therefore, $X_{x}$ is in $\operatorname{Nor}_{x}(G . x)$, and $g . y=c(1) \in E(x)$.

30.4. Remark. Let $x \in M$ be a regular point and $S_{x}$ the normal slice at $x$. If $S_{x}$ is orthogonal to every orbit it meets, then so are all $g . S_{x}(g \in G$ arbitrary). So the submanifolds $g . S_{x}$ can be considered as leaves of the horizontal foliation (local solutions of the horizontal distribution - which has constant rank in a neighborhood of a regular point), and the Riemann submersion $\pi: M_{\text {reg }} \rightarrow M_{\mathrm{reg}} / G$ is integrable. Since this is not always the case (the horizontal distribution is not generally integrable), it must also be false, in general, that the normal slice is orthogonal to every orbit it meets. But it does always meet orbits transversally.

Example. Consider the isometric action of the circle group $S^{1}$ on $\mathbb{C} \times \mathbb{C}$ (as real vector spaces) defined by $e^{i t} \cdot\left(z_{1}, z_{2}\right):=\left(e^{i t} \cdot z_{1}, e^{i t} \cdot z_{2}\right)$. Then $p=(0,1)$ is a regular point: $G_{p}=\{1\}$. The subspace $\operatorname{Nor}_{p}\left(S^{1} . p\right)$ of $T_{p} \mathbb{C} \times \mathbb{C}$ takes on the following form: $\operatorname{Nor}_{p}\left(S^{1} . p\right)=\langle(1,0),(i, 0),(0,1)\rangle_{\mathbb{R}}=\mathbb{C} \times \mathbb{R}$. Therefore, we 
get $E(0,1)=\{(u, 1+r): u \in \mathbb{C}, r \in \mathbb{R}\}$. In particular, $y=(1,1) \in E(0,1)$, but $S^{1} . y=\left\{\left(e^{i t}, e^{i t}\right): t \in \mathbb{R}\right\}$ is not orthogonal to $E(0,1)$. Its tangent space, $T_{y}\left(S^{1} \cdot y\right)=\mathbb{R} .(i, i)$, is not orthogonal to $\mathbb{C} \times \mathbb{R}$.

30.5. Definition. A connected closed complete submanifold $\Sigma \subset M$ is called a section for the $G$-action, and the action is called a polar action if:

(1) $\Sigma$ meets every orbit, or equivalently, $G \cdot \Sigma=M$.

(2) Where $\Sigma$ meets an orbit, it meets it orthogonally: For $x \in \Sigma$ we have $T_{x} \Sigma \subseteq \operatorname{Nor}_{x}(G . x)$; equivalently, for $x \in \Sigma, X \in \mathfrak{g}$ we have $\zeta_{X}(x) \perp T_{x} \Sigma$.

If $\Sigma$ is a section, then so is $g . \Sigma$ for all $g$ in $G$. Since $G . \Sigma=M$, there is a section through every point in $M$. We say that $M$ admits sections.

The notion of a section was introduced in [216, 217] and in slightly different form in 189, 190. The case of linear representations was considered in 23, 36, and then in 38 where representations admitting sections were called polar representations (see (30.16) and where all polar representations of connected Lie groups were completely classified. Also, [35. considered Riemann manifolds admitting flat sections. We follow here the notion of 189.

30.6. Examples. For the standard action of $O(n)$ on $\mathbb{R}^{n}$ the orbits are spheres, and every line through 0 is a section.

If $G$ is a compact, connected Lie group with bi-invariant metric, then conj : $G \times G \rightarrow G, \operatorname{conj}_{g}(h)=g h g^{-1}$ is an isometric action on $G$. The orbits are just the conjugacy classes of elements.

Proposition. Every maximal torus $H$ of a compact connected Lie group $G$ is a section.

A torus is a product of circle groups, or equivalently, a compact connected abelian Lie group; a maximal torus of a compact Lie group is a toral subgroup which is not properly contained in any larger toral subgroup.

Proof. We check (30.5.1) $\operatorname{conj}(G) \cdot H=G$ : This states that any $g \in G$ can be found in some subgroup which is conjugate to $H, g \in a H a^{-1}$. This is equivalent to $g a \in a H$ or $g a H=a H$. So the claim now presents itself as a fixed point problem: Does the map $\ell_{g}: G / H \rightarrow G / H: a H \mapsto g a H$ have a fixed point? It is solved in the following way:

The fixed point theorem of Lefschetz [215, 11.6.2, p. 297] says that $a$ smooth mapping $f: M \rightarrow M$ from a connected compact manifold to itself has no fixed point if and only if

$$
\sum_{i=0}^{\operatorname{dim} M}(-1)^{i} \operatorname{Trace}\left(H^{i}(f): H^{i}(M) \rightarrow H^{i}(M)\right)=0 .
$$


Since $G$ is connected, $\ell_{g}$ is homotopic to the identity, so

$$
\begin{aligned}
& \sum_{i=0}^{\operatorname{dim} G / H}(-1)^{i} \operatorname{Trace}\left(H^{i}\left(\ell_{g}\right): H^{i}(G / H) \rightarrow H^{i}(G / H)\right) \\
= & \sum_{i=0}^{\operatorname{dim} G / H}(-1)^{i} \operatorname{Trace}\left(H^{i}(\mathrm{Id})\right)=\sum_{i=0}^{\operatorname{dim} G / H}(-1)^{i} \operatorname{dim} H^{i}(G / H)=\chi(G / H),
\end{aligned}
$$

the Euler characteristic of $G / H$. This is given by the following theorem $\mathbf{1 8 2}$, Sec. 13, Theorem 2, p. 217]: If $G$ is a connected compact Lie group and $H$ is a connected compact subgroup, then the Euler characteristic $\chi(G / H) \geq 0$. Moreover $\chi(G / H)>0$ if and only if the rank of $G$ equals the rank of $H$. In the case when $\chi(G / H)>0$, then $\chi(G / H)=\left|W_{G}\right| /\left|W_{H}\right|$, the quotient of the respective Weyl groups.

Since the Weyl group of a torus is trivial, in our case we have $\chi(G / H)=$ $\left|W_{G}\right|>0$, and thus there exists a fixed point.

Now we show that (30.5.2) holds, $h \in H, X \in \mathfrak{g} \Rightarrow \zeta_{X}(h) \perp T_{h} H$ : We have

$$
\zeta_{X}(h)=\left.\partial_{t}\right|_{0} \exp (t X) h \exp (-t X)=T_{e} \mu^{h} \cdot X-T_{e} \mu_{h} . X .
$$

Now choose $Y \in \mathfrak{h}$. Then we have $T_{e} \mu_{h} . Y \in T_{h} H$, and

$$
\begin{aligned}
\gamma_{h}\left(T_{e} \mu_{h} \cdot Y, T_{e} \mu^{h} \cdot X-T_{e} \mu_{h} \cdot X\right) & =\gamma_{e}(Y, \operatorname{Ad}(h) \cdot X-X) \\
& =\gamma_{e}(Y, \operatorname{Ad}(h) \cdot X)-\gamma_{e}(Y, X) \\
& =\gamma_{e}(\operatorname{Ad}(h) \cdot Y, \operatorname{Ad}(h) \cdot X)-\gamma_{e}(Y, X)=0
\end{aligned}
$$

by the right, left, and therefore Ad-invariance of $\gamma$ and by the commutativity of $H$.

30.7. Example. Let $G$ be a compact semisimple Lie group acting on its Lie algebra by the adjoint action Ad : $G \times \mathfrak{g} \rightarrow \mathfrak{g}$. Then every Cartan subalgebra $\mathfrak{h}$ of $\mathfrak{g}$ is a section.

Proof. Every element of a semisimple Lie algebra $\mathfrak{g}$ is contained in a Cartan subalgebra, and any two Cartan subalgebras are conjugated by an element $g \in G$, since $G$ is compact. This is a consequence of (30.6) above, since the subgroup in $G$ corresponding to a Cartan subalgebra is a maximal torus. Thus every $\operatorname{Ad}_{G}$-orbit meets the Cartan subalgebra $\mathfrak{h}$. It meets orthogonally with respect to the Cartan-Killing form $B$ : Let $H_{1}, H_{2} \in \mathfrak{h}$ and $X \in \mathfrak{g}$. Then $\left.\partial_{t}\right|_{0} \operatorname{Ad}(\exp (t X)) \cdot H_{1}=\operatorname{ad}(X) H_{1}$ is a typical vector tangent to the orbit through $H_{1} \in \mathfrak{h}$, and $H_{2}$ is tangent to $\mathfrak{h}$. Then

$$
B\left(\operatorname{ad}(X) H_{1}, H_{2}\right)=B\left(\left[X, H_{1}\right], H_{2}\right)=B\left(X,\left[H_{1}, H_{2}\right]\right)=0
$$

since $\mathfrak{h}$ is commutative. 
30.8. Example. In (7.1) we showed that for the $O(n)$-action on $S(n)$ by conjugation the space $\Sigma$ of all diagonal matrices is a section.

Similarly, when the $S U(n)$ act on the Hermitian matrices by conjugation, the (real) diagonal matrices turn out to be a section.

30.9. Definition. The principal horizontal distribution on a Riemann $G$-manifold $M$ is the horizontal distribution on $\pi: M_{\text {reg }} \rightarrow M_{\mathrm{reg}} / G$.

Theorem. If a connected, complete Riemann $G$-manifold $M$ has a section $\Sigma$, then:

(1) The principal horizontal distribution is integrable.

(2) Every connected component of $\Sigma_{\text {reg }}$ is a leaf for the principal horizontal distribution.

(3) If $L$ is the leaf of $\operatorname{Hor}\left(M_{\text {reg }}\right)$ through $x \in M_{\text {reg }}$, then $\left.\pi\right|_{L}: L \rightarrow M_{\text {reg }} / G$ is an isometric covering map.

(4) $\Sigma$ is totally geodesic.

(5) Every regular point $x \in M$ is contained in a unique section $E(x)=$ $\exp _{x}^{\gamma}\left(\operatorname{Nor}_{x}(G \cdot x)\right)$.

(6) A G-equivariant normal field along a principal orbit is parallel in terms of the induced covariant derivative $\nabla^{\text {Nor }}$.

Proof. (11) The submanifolds $g \cdot \Sigma_{\text {reg }}$ of $M_{\text {reg }}$ are integral manifolds to the horizontal distribution, since they are orthogonal to each orbit and by an argument of dimension.

(2) is clear. (3) is (26.13.2), (41) follows from (26.13.1),

(5) For $x \in M$ choose $g \in G$ such that $g . x \in \Sigma \cap G$. $x$; then $g^{-1} . \Sigma$ is a section through $x$. By (2) and (4) we have $E(x) \subseteq g^{-1} \cdot \Sigma$. The converse can be seen as follows: Let $y \in g^{-1} . \Sigma$ and choose a minimal geodesic from $x$ to $y$. By the argument given in the proof of (30.3.2) this geodesic is orthogonal to the orbit through $x$ and thus lies in $E(x)$. So $y \in E(x)$.

(6) See (26.19) and recall that by (29.24) a normal field is $G$-equivariant if and only if it is $\pi$-parallel, where $\pi: M \rightarrow M / G$ is the orbit map.

30.10. Remark. The converse of (30.9.1) is not true. Namely, an integral manifold of $\operatorname{Hor}\left(M_{\mathrm{reg}}\right)$ is not, in general, a section.

Example. Consider the Lie group $G=S^{1} \times\{1\}$, and let it act on $M:=$ $S^{1} \times S^{1}$ by translation. Let $\xi=(1,0)$ denote the fundamental vector field of the action, and choose any $\eta \in \operatorname{Lie}\left(S^{1} \times S^{1}\right)=\mathbb{R} \times \mathbb{R}$ which generates a 1-parameter subgroup $c$ which is dense in $S^{1} \times S^{1}$ (irrational ascent). Now, endow $S^{1} \times S^{1}$ with a Riemann metric making $\xi$ and $\eta$ an orthonormal frame 
field. Any section of $M$ would then have to be a coset of $c$, and therefore dense. This contradicts the assumption that a section is a closed embedded submanifold.

30.11. Example. If $(G / H, \sigma)$ is a symmetric space, then the totally geodesic connected submanifolds $N$ of $G / H$ through $e \in G / H$ correspond exactly to the linear subspaces

$$
T_{e} N=\mathfrak{n} \subseteq \mathfrak{m}:=T_{e} G / H \cong\left\{X \in \mathfrak{g}: \sigma^{\prime}(X)=-X\right\}
$$

which fulfill $[[\mathfrak{n}, \mathfrak{n}], \mathfrak{n}] \subseteq \mathfrak{n} ;$ see $(28.5 .12)$.

This implies that a locally totally geodesic submanifold of a simply connected symmetric space can be extended uniquely to a complete, totally geodesic submanifold. Here we mean by locally geodesic submanifold that a geodesic can leave the submanifold only at its "boundary". In other words, the second fundamental form must be zero.

30.12. Corollary. Let $M=G / H$ be a simply connected, complete symmetric space, and let $K \subseteq G$ be a Lie subgroup. Then the action of $K$ on $G / H$ admits sections if and only if $\operatorname{Hor}\left(M_{\text {reg }}\right)$ is integrable. In particular, if the principal $K$-orbits have codimension 1 , there exist sections.

30.13. Theorem. Consider any Riemann $G$-manifold $M$. Then the following statements are equivalent.

(1) $\operatorname{Hor}\left(M_{\text {reg }}\right)$ is integrable.

(2) Each $G$-equivariant normal field along a principal orbit is $\nabla^{\mathrm{Nor}}$-parallel.

(3) For $x \in M_{\text {reg }}$, $S$ the normal slice at $x$ and $X \in \mathfrak{g}$ and $s \in S$ arbitrary, $\zeta_{X}(s) \perp T_{s}(S)$.

Proof. The equivalence of (1) and (2) is a direct consequence of (26.19) and remark (29.24). Furthermore, suppose (1); then there is an integral submanifold $H$ of the horizontal distribution going through $x$. The submanifold $H$ is totally geodesic by (26.13.1), and so $S=\exp _{x}\left(\operatorname{Nor}_{r}(G . x)\right)$ is contained in $H$. Therefore, (3) holds: The fundamental vector field $\zeta_{X}$ is tangent to the orbit G.s and so it is perpendicular to the horizontal distribution and to $T_{s}(S)$. Now if we suppose (3) , then $S$ is an integral submanifold of $\operatorname{Hor}\left(M_{\mathrm{reg}}\right)$, and (1) holds.

30.14. Remark. We already saw in (29.10) that Nor G.x is a trivial bundle. Now we even have a parallel global frame field. So the normal bundle to a regular orbit is flat. 
30.15. Corollary. Consider an orthogonal representation $\rho: G \rightarrow O(V)$ Let $x \in V$ be any regular point and $\Sigma$ the linear subspace of $V$ that is orthogonal to the orbit through $x$. Then the following statements are equivalent:

(1) $V$ admits sections.

(2) $\Sigma$ is a section.

(3) For all $y \in \Sigma$ and $X \in \mathfrak{g}$ we have $\zeta_{X}(y) \perp \Sigma$.

Proof. (3) implies that the horizontal bundle is integrable (see (30.13)). In this case (30.11) implies (11). Also, (1) implies (2) by (30.9.5), and (2) obviously implies (3).

30.16. Definition. An orthogonal representation of $G$ is called a polar representation if it admits sections.

Corollary. Let $G \subset O(V)$ be a polar representation of a compact Lie group, and let $v \in V$ be a regular point. Then

$$
\Sigma:=\left\{w \in V: \zeta_{\mathfrak{g}}(w) \subseteq \zeta_{\mathfrak{g}}(v)\right\}
$$

is the section through $v$, where $\zeta_{\mathfrak{g}}(w):=\left\{\zeta_{X}(w): X \in \mathfrak{g}\right\} \subseteq V$.

Proof. Since $\zeta_{\mathfrak{g}}(v)=T_{v}(G . v)$ and by (30.15), a section through $v$ is given by $\Sigma^{\prime}:=\zeta_{\mathfrak{g}}(v)^{\perp}$. If $z \in \Sigma^{\prime}$, then $\zeta_{\mathfrak{g}}(z) \subseteq\left(\Sigma^{\prime}\right)^{\perp}$, which in our case implies that $\zeta_{\mathfrak{g}}(z) \subseteq \zeta_{\mathfrak{g}}(v)$. So $z \in \Sigma$.

Conversely, suppose $z$ is a regular point in $\Sigma$. Consider the section $\Sigma^{\prime \prime}=$ $\zeta_{\mathfrak{g}}(z)^{\perp}$ through $z$. Then, since $\zeta_{\mathfrak{g}}(z) \subseteq \zeta_{\mathfrak{g}}(v)$, we also have that $\Sigma^{\prime}=\zeta_{\mathfrak{g}}(v)^{\perp} \subseteq$ $\zeta_{\mathfrak{g}}(z)^{\perp}=\Sigma^{\prime \prime}$. Therefore $\Sigma^{\prime}=\Sigma^{\prime \prime}$ and, in particular, $z \in \Sigma^{\prime}$.

30.17. Lemma. Let $G \subset O(V)$ be an orthogonal representation of a compact Lie group. Then for every $v \in V$ the normal space to the orbit

$$
\operatorname{Nor}_{v}:=\operatorname{Nor}_{v}(G \cdot v)=T_{v}(G \cdot v)^{\perp}
$$

meets every orbit.

Proof. Let $w \in V$ and consider $f: G \rightarrow \mathbb{R}, f(g)=\langle g . w, v\rangle$. Let $g_{0}$ be a critical point, e.g., a minimum on the compact group $G$; then $0=$ $d f\left(g_{0}\right) \cdot\left(X . g_{0}\right)=\left\langle X . g_{0} \cdot w, v\right\rangle=-\left\langle g_{0} \cdot w, X . v\right\rangle$ for all $X \in \mathfrak{g}$. Thus $g_{0} \cdot w \in$ $\operatorname{Nor}_{v}(G \cdot v)$.

30.18. Lemma. Let $G \subset O(V)$ be an orthogonal representation of a compact Lie group. For any regular $v_{0} \in V$ the following assertions are equivalent:

(1) For any $v \in V_{\text {reg }}$ there exists $g \in G$ with $g \cdot T_{v}(G \cdot v)=T_{v_{0}}\left(G \cdot v_{0}\right)$.

(2) $\operatorname{Nor}_{v_{0}}\left(G . v_{0}\right)=T_{v_{0}}\left(G . v_{0}\right)^{\perp}$ is a section. 
Proof. (11) $\Rightarrow$ (2) Let $\mathfrak{g} \subset \mathfrak{o}(V)$ be the Lie algebra of $G$. Consider the linear subspace

$$
A:=\left\{v \in \operatorname{Nor}_{v_{0}}\left(G \cdot v_{0}\right):\left\langle\mathfrak{g} \cdot v, \operatorname{Nor}_{v_{0}}\left(G \cdot v_{0}\right)\right\rangle=0\right\}
$$

of $\operatorname{Nor}_{v_{0}}\left(G . v_{0}\right) \subset V$. If (2) does not hold, then $A \subsetneq \operatorname{Nor}_{v_{0}}\left(G . v_{0}\right)$, and then $\operatorname{dim}(G . A)<\operatorname{dim}(V)$. So there exists $w \in V_{\text {reg }} \backslash G$.A, and by lemma (30.17) we may assume that $w \in \operatorname{Nor}_{v_{0}}\left(G \cdot v_{0}\right)$. By (11) there exists $g \in G$ with $g . \operatorname{Nor}_{w}(G . w)=\operatorname{Nor}_{v_{0}}\left(G . v_{0}\right)$. This means $\operatorname{Nor}_{g \cdot w}(G . w)=\operatorname{Nor}_{v_{0}}\left(G . v_{0}\right)$, a contradiction to $g . w \notin A$.

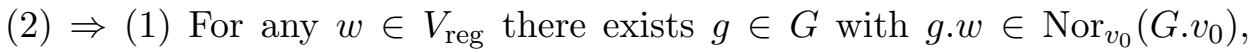
by (3.18), But then $g . \operatorname{Nor}_{w}(G . w)=\operatorname{Nor}_{g . w}(G . w)=\operatorname{Nor}_{v_{0}}\left(G . v_{0}\right)$, so (1) holds.

30.19. Theorem. If $G \subset O(V)$ is a polar representation, then for any $v \in V$ with a section $\Sigma \subset \operatorname{Nor}_{v}(G . v)$, the isotropy representation $G_{v} \subset$ $O\left(\operatorname{Nor}_{v}(G . v)\right)$ is also polar with the same section $\Sigma \subset \operatorname{Nor}_{v}(G . v)$.

Conversely, if there exists some $v \in V$ such that the isotropy representation $G_{v} \subset O\left(\operatorname{Nor}_{v}(G . v)\right)$ is polar with section $\Sigma \subset \operatorname{Nor}_{v}(G . v)$, then also $G \subset$ $O(V)$ is polar with the same section $\Sigma \subset V$.

Proof. Let $G \subset O(V)$ be polar with section $\Sigma$, and let $v \in \Sigma$ and $w \in$ $\Sigma_{\text {reg }}=\Sigma \cap V_{\text {reg. }}$.

Claim. Then $V=\Sigma \oplus \mathfrak{g}_{v} \cdot w \oplus \mathfrak{g} \cdot v$ is an orthogonal direct sum decomposition. Namely, we have $\langle\mathfrak{g} . \Sigma, \Sigma\rangle=0$ so that

$$
\left\langle\mathfrak{g}_{v} \cdot w, \mathfrak{g} \cdot v\right\rangle=\langle w, \mathfrak{g} \cdot \underbrace{\mathfrak{g}_{v} \cdot v}_{0}\rangle-\langle w, \underbrace{\left[\mathfrak{g}_{v}, \mathfrak{g}\right]}_{\subset \mathfrak{g}} \cdot v\rangle=0 .
$$

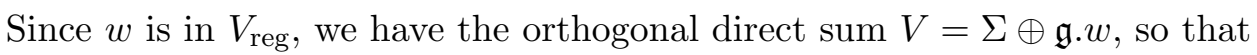
$\operatorname{dim}(V)=\operatorname{dim}(\Sigma)+\operatorname{dim}(\mathfrak{g})-\operatorname{dim}\left(\mathfrak{g}_{w}\right) ;$ and also we have $\left(\mathfrak{g}_{v}\right)_{w}=\mathfrak{g}_{w}$. Thus we get

$$
\begin{aligned}
\operatorname{dim}\left(\Sigma \oplus \mathfrak{g}_{v} . w \oplus \mathfrak{g} \cdot v\right) & =\operatorname{dim}(\Sigma)+\operatorname{dim}\left(\mathfrak{g}_{v}\right)-\operatorname{dim}\left(\left(\mathfrak{g}_{v}\right)_{w}\right)+\operatorname{dim}(\mathfrak{g})-\operatorname{dim}\left(\mathfrak{g}_{v}\right) \\
& =\operatorname{dim}(\Sigma)+\operatorname{dim}\left(\mathfrak{g}_{v}\right)-\operatorname{dim}\left(\mathfrak{g}_{w}\right)+\operatorname{dim}(\mathfrak{g})-\operatorname{dim}\left(\mathfrak{g}_{v}\right) \\
& =\operatorname{dim}(V)
\end{aligned}
$$

and the claim follows.

But then we see from the claim that $\operatorname{Nor}_{v}=\Sigma \oplus \mathfrak{g}_{v} \cdot w$ is an orthogonal decomposition and that (30.18.1) holds, so that $G_{v} \subset$ Nor $_{v}$ is polar with section $\Sigma$.

Conversely, if $G_{v} \subset \operatorname{Nor}_{v}$ is polar with section $\Sigma$, we get the orthogonal decomposition $\operatorname{Nor}_{v}(G . v)=\Sigma \oplus \mathfrak{g}_{v} . \Sigma$ of $\operatorname{Nor}_{v}(G . v)$. This implies $\langle\Sigma, \mathfrak{g} . \Sigma\rangle=$ 0 . By lemma (30.17) we have $G$. Nor $_{v}=V$. By polarity we have $G_{v} \cdot \Sigma=$ Nor $_{v}$; thus finally $G . \Sigma=V$. So $G \subset O(V)$ is polar. 
30.20. Theorem. Let $G$ be connected and $G \subset O\left(V=V_{1} \oplus V_{2}\right)$ be a polar reducible representation, which is decomposed as $V=V_{1} \oplus V_{2}$ as $G$-module. Then we have:

(1) Both $G$-modules $V_{1}$ and $V_{2}$ are polar, and any section $\Sigma$ of $V$ is of the form $\Sigma=\Sigma_{1} \oplus \Sigma_{2}$ for sections $\Sigma_{i}$ in $V_{i}$.

(2) Consider the connected subgroups

$$
G_{1}:=\left\{g \in G: g \mid \Sigma_{2}=0\right\}^{o}, \quad G_{2}:=\left\{g \in G: g \mid \Sigma_{1}=0\right\}^{o} .
$$

Then $G=G_{1} \cdot G_{2}$, and $G_{1} \times G_{2}$ acts on $V=V_{1} \oplus V_{2}$ componentwise by $\left(g_{1}, g_{2}\right)\left(v_{1}+v_{2}\right)=g_{1} \cdot v_{1}+g_{2} \cdot v_{2}$, with the same orbits as $G: G . v=$ $\left(G_{1} \times G_{2}\right)$.v for any $v$.

Proof. Let $v=v_{1}+v_{2} \in \Sigma \cap V_{\text {reg }} \subset V=V_{1} \oplus V_{2}$. Then $V=\Sigma \oplus \mathfrak{g} \cdot v$; thus $v_{i}=s_{i}+X_{i} . v$ for $s_{i} \in \Sigma_{i}$ and $X_{i} \in \mathfrak{g}$. But then $s_{i} \in \Sigma_{i} \cap V_{i}=: \Sigma_{i}$ and $V_{i}=\left(\Sigma \cap V_{i}\right) \oplus \mathfrak{g} \cdot v_{i}$ and the assertion (1) follows.

Moreover $\operatorname{Nor}_{v_{1}}=\left(\mathfrak{g} \cdot v_{1}\right)^{\perp}=\Sigma_{1} \oplus V_{2}$, and by theorem (30.19) the action of $G_{v_{1}}$ on this space is polar with section $\Sigma_{1} \oplus \Sigma_{2}$. Thus we have $\mathfrak{g}_{v_{1}}=\mathfrak{g}_{2}:=\mathfrak{g}_{\Sigma_{1}}$ and $\mathfrak{g}_{v_{1}}$ acts only on $V_{2}$ and vanishes on $V_{1}$ and we get $V_{2}=\Sigma_{2} \oplus \mathfrak{g}_{v_{1}} v_{2}=$ $\Sigma_{2} \oplus \mathfrak{g} \cdot v_{2}$. Similarly $\mathfrak{g}_{v_{2}}=\mathfrak{g}_{1}:=\mathfrak{g}_{\Sigma_{2}}$ and $\mathfrak{g}_{v_{2}}$ acts only on $V_{1}$ and vanishes on $V_{2}$, and $V_{1}=\Sigma_{1} \oplus \mathfrak{g}_{v_{2}} v_{1}=\Sigma_{1} \oplus \mathfrak{g} \cdot v_{1}$. Thus $\mathfrak{g}=\mathfrak{g}_{1}+\mathfrak{g}_{2}$ and consequently $G=G_{1} \cdot G_{2}=G_{2} \cdot G_{1}$ by compactness of $G_{i}$. For any $g \in G$ we have $g=$ $g_{1} . g_{2}=g_{2}^{\prime} . g_{1}^{\prime}$ for $g_{i}, g_{i}^{\prime} \in G_{i}$. For $u=u_{1}+u_{2} \in V_{1} \oplus V_{2}=V$ we then have $g \cdot\left(u_{1}+u_{2}\right)=g_{1} \cdot g_{2} \cdot u_{1}+g_{2}^{\prime} \cdot g_{1}^{\prime} \cdot u_{2}=g_{1} \cdot u_{1}+g_{2}^{\prime} \cdot u_{2}$; thus $G \cdot u \subseteq\left(G_{1} \times G_{2}\right) \cdot u$. Since both orbits have the same dimension, $G$. $u$ is open in $\left(G_{1} \times G_{2}\right) . u$; since all groups are compact and connected, the orbits coincide.

30.21. The generalized Weyl group of a section. Consider a complete Riemann $G$-manifold $M$ which admits sections. For any closed subset $S$ of $M$ we define the largest subgroup of $G$ which induces an action on $S$ :

$$
N(S):=\left\{g \in G: \ell_{g}(S)=S\right\}
$$

and the subgroup consisting of all $g \in G$ which act trivially on $S$ :

$$
Z(S):=\left\{g \in G: \ell_{g}(s)=s, \text { for all } s \in S\right\} .
$$

Then, since $S$ is closed, $N(S)$ is closed, hence a Lie subgroup of $G$. The centralizer $Z(S)=\bigcap_{s \in S} G_{s}$ is closed as well and is a normal subgroup of $N(S)$. Therefore, $N(S) / Z(S)$ is a Lie group, and it acts on $S$ effectively.

If we take for $S$ a section $\Sigma$, then the above constructed group is called the generalized Weyl group of $\Sigma$ and is denoted by

$$
W(\Sigma)=N(\Sigma) / Z(\Sigma) .
$$


For any regular point $x \in \Sigma, G_{x}$ acts trivially on the normal slice $S_{x}$ at $x$ (by (29.7)]. Since $\Sigma=\exp _{x} \operatorname{Nor}_{x}(G . x)$ by (30.9.5), $S_{x}$ is an open subset of $\Sigma$, and we see that $G_{x}$ acts trivially on all of $\Sigma$. So we have $G_{x} \subseteq Z(\Sigma)$. On the other hand, $Z(\Sigma) \subseteq G_{x}$ is obvious; therefore

$$
Z(\Sigma)=G_{x} \quad \text { for } x \in \Sigma \cap M_{\text {reg }} .
$$

Now, since $Z(\Sigma)$ is a normal subgroup of $N(\Sigma)$, we have $N(\Sigma) \subseteq N\left(G_{x}\right)$ where the second $N$ stands for the normalizer in $G$. So we have

$$
W(\Sigma) \subseteq N\left(G_{x}\right) / G_{x} \quad \text { for } x \in \Sigma \cap M_{\text {reg. }} .
$$

30.22. Proposition. Let $M$ be a proper Riemann $G$-manifold and let $\Sigma$ be a section; then the associated Weyl group $W(\Sigma)$ is discrete. If $\Sigma^{\prime}$ is a different section, then there is an isomorphism $W(\Sigma) \rightarrow W\left(\Sigma^{\prime}\right)$ induced by an inner automorphism of $G$. It is uniquely determined up to an inner automorphism of $W(\Sigma)$.

Proof. Take a regular point $x \in \Sigma$ and consider the normal slice $S_{x}$. Then $S_{x} \subseteq \Sigma$ is open. Therefore, any $g$ in $N(\Sigma)$ close to the identity element maps $x$ back into $S_{x}$. By (6.15.2), the element $g$ then lies in $G_{x}=Z(\Sigma)$. So $Z(\Sigma)$ is an open subset of $N(\Sigma)$, and the quotient $W(\Sigma)$ is discrete.

If $\Sigma^{\prime}$ is another section, then $\Sigma^{\prime}=g . \Sigma$ where $g \in G$ is uniquely determined up to $N(\Sigma)$. Clearly, $\operatorname{conj}_{g}: G \rightarrow G$ induces isomorphisms

$$
\begin{aligned}
& \operatorname{conj}_{g}: N(\Sigma) \cong N\left(\Sigma^{\prime}\right), \\
& Z(\Sigma) \stackrel{\cong}{\longrightarrow} Z\left(\Sigma^{\prime}\right),
\end{aligned}
$$

and therefore it factors to an isomorphism $W(\Sigma) \stackrel{\cong}{\longrightarrow} W\left(\Sigma^{\prime}\right)$.

30.23. Example. Any finite group is a generalized Weyl group in the appropriate setting. That is, to an arbitrary finite group $W$ we will now construct a setting in which it occurs as a Weyl group. Let $G$ be a compact Lie group and $H$ a closed subgroup such that $W \subseteq N(H) / H$ (this is always possible since any finite group can be regarded as a subgroup of $O(V)=$ : $G$ so we need only choose $H=\{e\})$. Next, take a smooth manifold $\Sigma$ on which $W$ acts effectively. Consider the inverse image of $W$ under the quotient map $\pi: N(H) \rightarrow N(H) / H, K:=\pi^{-1}(W)$. Then the action of $W$ induces a $K$-action on $\Sigma$ as well. The smooth manifold $M:=G \times_{K} \Sigma$ has a left $G$ action. Let $-B$ denote the $G$-invariant Riemann metric on $G$ induced by the Cartan-Killing form on the semisimple part and any inner product on the center, and let $\gamma_{\Sigma}$ be a $W$-invariant Riemann metric on $\Sigma$. Then the Riemann metric $-B \times \gamma_{\Sigma}$ on $G \times \Sigma$ induces a $G$-invariant Riemann metric on the quotient space $G \times_{K} \Sigma$. With this, $G \times_{K} \Sigma$ is a Riemann $G$-manifold, 
and if $q: G \times \Sigma \rightarrow G \times \times_{K} \Sigma$ is the quotient map, then $q(\{e\} \times \Sigma) \cong \Sigma$ meets every $G$-orbit orthogonally. So it is a section. The largest subgroup of $G$ acting on $\Sigma$ is $K$ and the largest one acting trivially on $\Sigma$ is $H$. Therefore, $W(\Sigma)=K / H=W$ is the Weyl group associated to the section $\Sigma$.

30.24. Theorem. Let $M$ be a proper Riemann $G$-manifold with sections. Then, for any $x \in M$, the slice representation $G_{x} \rightarrow O\left(\operatorname{Nor}_{x}(G . x)\right)$ is a polar representation. If $\Sigma$ is a section through $x$ in $M$, then $T_{x} \Sigma$ is a section in $\operatorname{Nor}_{x}(G . x)$ for the slice representation. Furthermore,

$$
W\left(T_{x} \Sigma\right)=W(\Sigma)_{x}
$$

Proof. Clearly $T_{x} \Sigma \subseteq \operatorname{Nor}_{x}(G . x)$. We begin by showing that it has the right codimension. Take a $\xi \in \operatorname{Nor}_{x}(G . x)$ close to $0_{x}$; then $\left(G_{x}\right)_{\xi}=G_{y}$ for $y=\exp _{x}^{\gamma} \xi$, since $\exp _{x}$ is a $G_{x}$-equivariant diffeomorphism in a neighborhood of $0_{x}$. So $G_{x} \cdot \xi \cong G_{x} /\left(G_{x}\right)_{\xi}=G_{x} / G_{y}$. Let us now calculate the codimension of $G_{x} \cdot \xi$ in $\operatorname{Nor}_{x}(G \cdot x)$ :

$$
\begin{aligned}
& \operatorname{dim}\left(\operatorname{Nor}_{x}(G . x)\right)-\operatorname{dim}\left(G_{x} \cdot \xi\right)=\operatorname{dim}\left(\operatorname{Nor}_{x}(G . x)\right)-\operatorname{dim}\left(G_{x}\right)+\operatorname{dim}\left(G_{y}\right) \\
& =\underbrace{\operatorname{dim}\left(\operatorname{Nor}_{x}(G . x)\right)+\operatorname{dim}\left(G / G_{x}\right)}_{=\operatorname{dim} M}-\underbrace{\left(\operatorname{dim} G-\operatorname{dim}\left(G_{y}\right)\right)}_{=\operatorname{dim} G / G_{y}}=\operatorname{codim}_{M}(G . y) .
\end{aligned}
$$

Since regular points form a dense subset, we can choose $\xi \in T_{x} \Sigma$ regular by assuming that $y=\exp _{x}^{\gamma}(X)$ is regular in $\Sigma$. Then $y$ is regular as well and we get

$$
\operatorname{codim}_{\operatorname{Nor}_{x}(G . x)}\left(G_{x} \cdot \xi\right)=\operatorname{codim}_{M}(G \cdot y)=\operatorname{dim} \Sigma=\operatorname{dim}\left(T_{x} \Sigma\right) .
$$

So $T_{x} \Sigma$ is a linear subspace of $\operatorname{Nor}_{x} G . x$ with the right codimension for a section. Therefore, if we show that $T_{x} \Sigma$ is orthogonal to each orbit it meets, then it is already the entire orthogonal complement of a regular orbit, and by corollary (30.15), we know that it meets every orbit.

Denote the $G$-action on $M$ by $\ell: G \rightarrow \operatorname{Isom}(M)$. If $\xi \in T_{x} \Sigma$ is arbitrary, then it remains to prove that for all $\eta \in T_{x} \Sigma$ and $X \in \mathfrak{g}_{x}$ :

$$
\gamma_{x}\left(\eta, \zeta_{X}^{\left.T \ell\right|_{G_{x}}}(\xi)\right)=0
$$

To do this, choose a smooth 1-parameter family $\eta(t) \in T_{\exp (t \xi)} \Sigma$ such that $\eta(0)=\eta$ and $\nabla_{\partial_{t}} \eta=0$. Since $\Sigma$ is a section in $M$, we know for each single $t$ that

$$
\gamma_{\exp (t \xi)}\left(\zeta_{X}^{\ell}\left(\exp ^{\gamma}(t \xi)\right), \eta(t)\right)=0
$$

If we differentiate this equation, we get

$$
0=\left.\partial_{s}\right|_{0} \gamma\left(\zeta_{X}^{\ell}\left(\exp ^{\gamma}(s \xi)\right), \eta(s)\right)=\gamma\left(\nabla_{\partial_{s}} \zeta_{X}^{\ell}\left(\exp ^{\gamma}(s \xi)\right), \eta(0)\right)
$$


So it remains to show that $\nabla_{\partial_{s}} \zeta_{X}^{\ell}\left(\exp ^{\gamma}(s \xi)\right)$ is the fundamental vector field of $X$ at $\xi$ for the slice representation:

$$
\begin{aligned}
\nabla_{\partial_{s}} \zeta_{X}^{\ell}\left(\exp ^{\gamma}(s \xi)\right) & =\nabla_{\xi} \zeta_{X}^{\ell}=K \circ T \zeta_{X}^{\ell} \cdot \xi \\
& =\left.K \circ T\left(\left.\partial_{t}\right|_{0} \ell_{\exp ^{G}(t X)}\right) \cdot \partial_{s}\right|_{0} \exp _{x}^{\gamma}(s \xi) \\
& =\left.\left.K \cdot \partial_{s}\right|_{0} \cdot \partial_{t}\right|_{0} \ell_{\exp ^{G}(t X)}\left(\exp _{x}^{\gamma}(s \xi)\right) \\
& =\left.\left.K \cdot \kappa_{M} \cdot \partial_{t}\right|_{0} \cdot \partial_{s}\right|_{0} \ell_{\exp ^{G}(t X)}\left(\exp _{x}^{\gamma}(s \xi)\right) \\
& =\left.K \cdot \kappa_{M} \cdot \partial_{t}\right|_{0} \cdot T\left(\ell_{\exp ^{G}(t X)}\right)(\xi) .
\end{aligned}
$$

Here, $K$ denotes the connector and $\kappa_{M}$ the canonical flip between the two structures of $T T M$, and we use the identity $K \circ \kappa=K$, which is a consequence of the symmetry of the Levi-Civita connection. The argument of $K$ in the last expression is vertical already since $X \in \mathfrak{g}_{x}$. Therefore we can replace $K$ by the vertical projection and get

$$
\nabla_{\partial_{s}} \zeta_{X}^{\ell}\left(\exp ^{\gamma}(s \xi)\right)=\left.\operatorname{vpr} \partial_{t}\right|_{0} T_{x}\left(\ell_{\exp ^{G}(t X)}\right) \cdot \xi=\zeta_{X}^{T_{2} \ell_{G_{x}}}(\xi)
$$

So $\zeta_{X}^{T_{2} \ell_{G_{x}}}(\xi)$ intersects $T_{x} \Sigma$ orthogonally, and therefore $T_{x} \Sigma$ is a section.

Now consider $N_{G_{x}}\left(T_{x}(\Sigma)\right)=\left\{g \in G_{x}: T_{x}\left(\ell_{g}\right) \cdot T_{x} \Sigma=T_{x} \Sigma\right\}$. Clearly, $N_{G}(\Sigma) \cap G_{x} \subseteq N_{G_{x}}\left(T_{x}(\Sigma)\right)$. On the other hand, any $g \in N_{G_{x}}\left(T_{x}(\Sigma)\right)$ leaves $\Sigma$ invariant as the following argument shows.

For any regular $y \in \Sigma$ we have $\Sigma=\exp _{y} \operatorname{Nor}(G . y)$. Therefore $x=\exp _{y} \eta$ for a suitable $\eta \in T_{y} \Sigma$, and conversely, $y$ can be written as $y=\exp _{x} \xi$ for $\xi=-\left.\partial_{t}\right|_{1} \exp _{y}(t \eta) \in T_{x} \Sigma$. Now $g . y=g$. $\exp _{x} \xi=\exp _{x} T_{x} \ell_{g} \cdot \xi$ lies in $\Sigma$, since $T_{x} \ell_{g} . \xi$ lies in $T_{x} \Sigma$. So $g$ maps all regular points in $\Sigma$ back into $\Sigma$. Since these form a dense subset and since $\ell_{g}$ is continuous, we get $g \in N_{G}(\Sigma)$.

We have now shown that

$$
N_{G_{x}}\left(T_{x} \Sigma\right)=N_{G}(\Sigma) \cap G_{x} .
$$

Analogous arguments used on $Z_{G_{x}}\left(T_{x} \Sigma\right)$ give

$$
Z_{G_{x}}\left(T_{x} \Sigma\right)=Z_{G}(\Sigma)
$$

and we see that

$$
W_{G_{x}}\left(T_{x} \Sigma\right)=\left(N(\Sigma) \cap G_{x}\right) / Z(\Sigma)=W(\Sigma)_{x} .
$$

30.25. Corollary. Let $M$ be a Riemann $G$-manifold admitting sections and let $x \in M$. Then for any section $\Sigma$ through $x$ we have

$$
\operatorname{Nor}_{x}(G \cdot x)^{G_{x}^{0}} \subseteq T_{x} \Sigma,
$$

where $G_{x}^{0}$ is the connected component of the isotropy group $G_{x}$ at $x$.

Proof. By theorem (30.24) the tangent space $T_{x} \Sigma$ is a section for the slice representation $G_{x} \rightarrow O\left(\operatorname{Nor}_{x}(G . x)\right)$. Let $\xi \in T_{x} \Sigma$ be a regular vector for the 
slice representation. By corollary (30.16) we have $T_{x} \Sigma=\left\{\eta \in \operatorname{Nor}_{x}(G . x)\right.$ : $\left.\zeta_{\mathfrak{g}_{x}}(\eta) \subset \zeta_{\mathfrak{g}_{x}}(\xi)\right\}$. Since $\operatorname{Nor}_{x}(G \cdot x)^{G_{x}^{0}}$ consists of all $\eta$ in $\operatorname{Nor}_{x}(G . x)$ with $\zeta_{\mathfrak{g}_{x}}(\eta)=0$, the result follows.

30.26. Corollary. Let $M$ be a proper Riemann G-manifold admitting sections and let $x \in M$. Then $G_{x}$ acts transitively on the set of all sections through $x$.

Proof. Consider two arbitrary sections $\Sigma_{1}$ and $\Sigma_{2}$ through $x$ and a normal slice $S_{x}$ at $x$. By theorem (30.24), $T_{x} \Sigma_{2}$ is a section for the slice representation. Since $\exp _{x}$ can be restricted to a $G_{x}$-equivariant diffeomorphism onto $S_{x}, \Sigma_{2} \cap S_{x}$ is a section for the $G_{x}$-action on $S_{x}$. Next, choose a regular point $y \in \Sigma_{1} \cap S_{x}$. Its $G_{x}$-orbit meets the section $\Sigma_{2} \cap S_{x}$, that is, we can find a $g \in G_{x}$ such that $g . y \in \Sigma_{2}$. Now $\Sigma_{2}$ and $g . \Sigma_{1}$ are both sections containing the regular point $g . y$. Therefore they are equal.

30.27. Corollary. Let $M$ be a proper $G$-manifold with sections, let $\Sigma$ be a section of $M$ and let $x \in \Sigma$. Then

$$
G . x \cap \Sigma=W(\Sigma) \cdot x .
$$

Proof. The inclusion $(\supseteq)$ is clear. Now we have

$$
y \in G . x \cap \Sigma \quad \Longleftrightarrow \quad y=g . x \in \Sigma \text { for some } g \in G .
$$

Take this $g$ and consider the section $\Sigma^{\prime}:=g . \Sigma$. Then $\Sigma$ and $\Sigma^{\prime}$ are both sections through $y$, and by (30.26) there is a $g^{\prime} \in G_{y}$ which carries $\Sigma^{\prime}$ back into $\Sigma$. Now $g^{\prime} g \cdot \Sigma=\Sigma$, that is, $g^{\prime} g \in N(\Sigma)$, and $g^{\prime} g \cdot x=g^{\prime} \cdot y=y$. So $y \in N(\Sigma) \cdot x=W(\Sigma) \cdot x$.

30.28. Corollary. If $M$ is a proper $G$-manifold with section $\Sigma$, then the inclusion of $\Sigma$ into $M$ induces a homeomorphism $j$ between the orbit spaces:

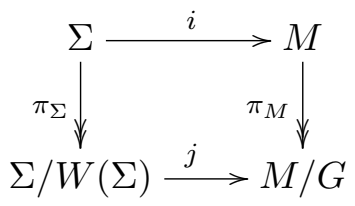

(but it does not necessarily preserve orbit types; see remark (6.17).

Proof. By the preceding corollary there is a one to one correspondence between the $G$-orbits in $M$ and the $W(G)$-orbits in $\Sigma$, so $j$ is well defined and bijective. Since $j \circ \pi_{\Sigma}=\pi_{M} \circ i$ and $\pi_{\Sigma}$ is open, $j$ is continuous.

Consider any open set $U \subseteq \Sigma / W(\Sigma)$. We now have to show that

$$
\pi_{M}^{-1} j(U)=G \cdot \pi_{\Sigma}^{-1}(U)
$$


is an open subset of $M$ (since then $j(U)$ is open and $j^{-1}$ is continuous). Take any $x \in \pi_{M}^{-1} j(U)$. We assume $x \in \Sigma$ (otherwise it can be replaced by a suitable $g . x \in \Sigma)$. So $x \in \pi_{\Sigma}^{-1}(U)$. Let $S_{x}$ be a normal slice at $x$; then $\Sigma \cap S_{x}$ is a submanifold of $S_{x}$ of dimension $\operatorname{dim} \Sigma$. In $S_{x}, x$ has arbitrarily small $G_{x}$-invariant neighborhoods, since the slice action is orthogonal and $S_{x}$ is $G$-equivariantly diffeomorphic to an open ball in $\operatorname{Nor}_{x}(G . x)$. Let $V_{x}$ be such an open neighborhood of $x$, small enough for $V_{x} \cap \Sigma$ to be contained in $\pi_{\Sigma}^{-1}(U)$. Then $V_{x}$ is again a slice; therefore $G \cdot V_{x}$ is open in $M$ by (6.15.3). Now we have to check whether $G . V_{x}$ is really a subset of $\pi_{M}^{-1} j(U)$. Using corollary (30.26), we get

$$
G .\left(V_{x} \cap \Sigma\right)=G \cdot G_{x}\left(V_{x} \cap \Sigma\right)=G \cdot\left(V_{x} \cap G_{x} \cdot \Sigma\right)=G \cdot V_{x} .
$$

Therefore, $G . V_{x} \subseteq G \cdot \pi_{\Sigma}^{-1}(U)=\pi_{M}^{-1} j(U)$ where it is an open neighborhood of $x$. So $\pi_{M}^{-1} j(U)$ is an open subset of $M, j(U)$ is open in $M / G$, and $j^{-1}$ is continuous.

30.29. Corollary. Let $M$ be a proper Riemann $G$-manifold and $\Sigma \subseteq M$ a section with Weyl group $W$. Then the inclusion $i: \Sigma \hookrightarrow M$ induces an isomorphism

$$
C^{0}(M)^{G} \stackrel{i^{*}}{\longrightarrow} C^{0}(\Sigma)^{W}
$$

Proof. By corollary (30.27) we see that every $f \in C^{0}(\Sigma)^{W}$ has a unique $G$-equivariant extension $f$ onto $V$. If we consider once more the diagram

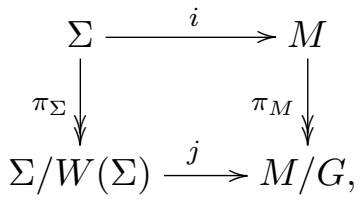

we see that $f$ factors over $\pi_{\Sigma}$ to a map $f^{\prime} \in C^{0}(\Sigma / W(\Sigma))$, and since $j$ is a homeomorphism by (30.28), we get for the $G$-invariant extension $\tilde{f}$ of $f$ :

$$
\tilde{f}=f^{\prime} \circ j^{-1} \circ \pi_{M} \in C^{0}(M)^{G} .
$$

30.30. Theorem ([189, 4.12] or [220, theorem D]). Let $G \rightarrow G L(V)$ be a polar representation of a compact Lie group $G$, with section $\Sigma$ and generalized Weyl group $W=W(\Sigma)$.

Then the algebra $\mathbb{R}[V]^{G}$ of $G$-invariant polynomials on $V$ is isomorphic to the algebra $\mathbb{R}[\Sigma]^{W}$ of $W$-invariant polynomials on the section $\Sigma$, via the restriction mapping $\left.f \mapsto f\right|_{\Sigma}$. 
Remark. This seemingly very algebraic theorem is actually a consequence of the geometry of the orbits. This already becomes evident in the case of a degree 1 homogeneous polynomial. To see that the $G$-invariant extension of $p \in \mathbb{B}[\Sigma]_{1}^{W}$ to $V$ is again a polynomial (and again of degree 1 ), we we must assume the following convexity result of Terng.

Under the conditions of the theorem, for every regular orbit $G . x$ the orthogonal projection onto $\Sigma, \operatorname{pr}(G . x)$, is contained in the convex hull of $G . x \cap \Sigma$ (this is a finite subset of $\Sigma$ by (30.27) since $G$ is compact and $W(\Sigma)$ is discrete).

Let us make this assumption. Denote by $\tilde{p}$ the unique $G$-invariant extension of $p$; then clearly $\tilde{p}$ is homogeneous. Now, notice that for any orbit G.x, $p$ is constant on the convex hull of $G . x \cap \Sigma=:\left\{g_{1} . x, g_{2} . x, \ldots, g_{k} \cdot x\right\}$. Just take any $s=\sum \lambda_{i} g_{i} . x$ with $\sum \lambda_{i}=1$; then

$$
p(s)=\sum \lambda_{i} p\left(g_{i} \cdot x\right)=p\left(g_{1} \cdot x\right) \sum \lambda_{i}=p\left(g_{1} \cdot x\right) .
$$

With this and with our assumption we can show that for regular points $u, v \in M, \tilde{p}(u+v)=\tilde{p}(u)+\tilde{p}(v)$. Suppose without loss of generality that $u+v \in \Sigma ;$ then

$$
p(u+v)=p(\operatorname{pr}(u)+\operatorname{pr}(v))=p(\operatorname{pr}(u))+p(\operatorname{pr}(v)) .
$$

At this point, the convexity theorem asserts that $\operatorname{pr}(u)$ and $\operatorname{pr}(v)$ can be written as convex combinations of elements of $G . u \cap \Sigma$ and $G . v \cap \Sigma$, respectively. If we fix an arbitrary $g_{u}$ (resp. $g_{v}$ ) in $G$ such that $g_{u} \cdot u$ (resp. $g_{v} \cdot v$ ) lie in $\Sigma$, then by the above argument we get

$$
p(\operatorname{pr}(u))=p\left(g_{u} \cdot u\right) \quad \text { and } \quad p(\operatorname{pr}(v))=p\left(g_{v} \cdot v\right) .
$$

So we have

$$
p(u+v)=p\left(g_{u} \cdot u\right)+p\left(g_{v} \cdot v\right)=\tilde{p}(u)+\tilde{p}(v),
$$

and $\tilde{p}$ is linear on $V_{\text {reg. }}$. Since the regular points are a dense subset of $V$ and since $\tilde{p}$ is continuous by (30.29) $\tilde{p}$ is linear altogether.

A proof of the convexity theorem can be found in [219] or again in [190, pp. 168-170]. For a proof of theorem (30.30) we refer to [220. In both sources the assertions are shown for the more general case where the principal orbits are replaced by isoparametric submanifolds (i.e., submanifolds of a space form with flat normal bundle and whose principal curvatures along any parallel normal field are constant; compare (29.12) and (30.14). To any isoparametric submanifold there is a singular foliation which generalizes the orbit foliation of a polar action but retains many of its fascinating properties. In connection with the example we studied in (7.1), the convexity theorem from above yields the following classical result of [203]: 
Let $M \subseteq S(n)$ be the subset of all symmetric matrices with fixed distinct eigenvalues $a_{1}, \ldots, a_{n}$ and $\operatorname{pr}: S(n) \rightarrow R^{n}$ defined by

$$
\operatorname{pr}\left(x_{i j}\right):=\left(x_{11}, x_{22}, \ldots, x_{n n}\right) ;
$$

then $\operatorname{pr}(M)$ is contained in the convex hull of the permutation group orbit $\mathcal{S}_{n} . a$ through $a=\left(a_{1}, \ldots, a_{n}\right)$.

30.31. Theorem. Let $M$ be a proper Riemann $G$-manifold with section $\Sigma$ and Weyl group $W$. Then the inclusion $i: \Sigma \hookrightarrow M$ induces an isomorphism

$$
C^{\infty}(M)^{G} \stackrel{i^{*}}{\longrightarrow} C^{\infty}(\Sigma)^{W(\Sigma)}
$$

Proof. Clearly $f \in C^{\infty}(M)^{G}$ implies $i^{*} f \in C^{\infty}(\Sigma)^{W}$. By (30.29) we know that every $f \in C^{\infty}(\Sigma)^{W}$ has a unique continuous $G$-invariant extension $\tilde{f}$. We now have to show that $\tilde{f} \in C^{\infty}(M)^{G}$.

Let us take an $x \in M$ and show that $\tilde{f}$ is smooth at $x$. Actually, we can assume $x \in \Sigma$, because if $\tilde{f}$ is smooth at $x$, then $\tilde{f} \circ \ell_{g^{-1}}$ is smooth at $g . x$, so $\tilde{f}$ is smooth at $g . x$ as well. Now let $S_{x}$ denote a normal slice at $x$. Then we have

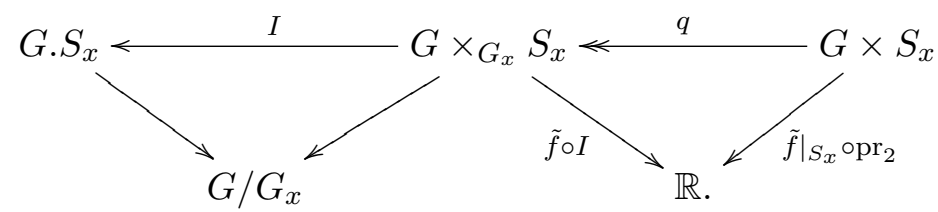

Since in the above diagram $I$ is an isomorphism and $q$ a submersion, it is sufficient to show that $\left.\tilde{f}\right|_{S_{x}} \circ \mathrm{pr}_{2}$, or equivalently, that $\left.\tilde{f}\right|_{S_{x}}$ is smooth at $x$. Let $B \subseteq T_{x} S_{x}$ be a ball around $0_{x}$ such that $B \cong S_{x}$ and $T_{x} \Sigma \cap B \cong \Sigma \cap S_{x}$. Then, by theorem (30.24), the $G_{x}$-action on $S_{x}$ is basically a polar representation (up to diffeomorphism). So it remains to show the following:

Claim. If $\Sigma$ is a section of a polar representation $G_{x} \rightarrow O(V)$ with Weyl group $W_{x}$ and $f$ is a smooth $W_{x}$-invariant function on $\Sigma$, then $f$ extends to a smooth $G_{x}$-invariant function $\tilde{f}$ on $V$.

In order to show this, let $\rho_{1}, \ldots, \rho_{k}$ be a system of homogeneous Hilbert generators for $\mathbb{R}[\Sigma]^{W_{x}}$. Then, by Schwarz's theorem (7.13), there is an $f^{\prime} \in C^{\infty}\left(\mathbb{R}^{k}\right)$ such that $f=f^{\prime} \circ\left(\rho_{1}, \ldots, \rho_{k}\right)$. By theorem (30.30), each $\rho_{i}$ extends to a polynomial $\tilde{\rho}_{i} \in \mathbb{R}[V]^{G_{x}}$. Therefore we get that

$$
\tilde{f}:=f^{\prime} \circ\left(\tilde{\rho}_{1}, \ldots, \tilde{\rho}_{k}\right): V \rightarrow \mathbb{R}
$$

is a smooth $G_{x}$-invariant extension of $f$. 
30.32. Basic differential forms. Our next aim is to show that pullback along the embedding $\Sigma \rightarrow M$ induces an isomorphism $\Omega_{\text {hor }}^{p}(M)^{G} \cong$ $\Omega^{p}(\Sigma)^{W(\Sigma)}$ for each $p$, where a differential form $\omega$ on $M$ is called horizontal if it kills each vector tangent to some orbit. For each point $x$ in $M$, the slice representation of the isotropy group $G_{x}$ on the normal space $T_{x}(G \cdot x)^{\perp}$ to the tangent space to the orbit through $x$ is a polar representation. The first step is to show that the result holds for polar representations. This is done in theorem (30.40), The method used there is inspired by [212]. Then the general result is proven, following [154, 156].

As usual, for a Lie group $G$ we denote by $\mathfrak{g}$ its Lie algebra, the multiplication by $\mu: G \times G \rightarrow G$; for $g \in G$ let $\mu_{g}, \mu^{g}: G \rightarrow G$ denote the left and right translation. Let $\ell: G \times M \rightarrow M$ be a left action of the Lie group $G$ on a smooth manifold $M$. We consider the partial mappings $\ell_{g}: M \rightarrow M$ for $g \in G$ and $\ell^{x}: G \rightarrow M$ for $x \in M$ and the fundamental vector field mapping $\zeta: \mathfrak{g} \rightarrow \mathfrak{X}(M)$ given by $\zeta_{X}(x)=T_{e}\left(\ell^{x}\right) X$. Since $\ell$ is a left action, the negative $-\zeta$ is a Lie algebra homomorphism.

A differential form $\varphi \in \Omega^{p}(M)$ is called $G$-invariant if $\left(\ell_{g}\right)^{*} \varphi=\varphi$ for all $g \in G$ and horizontal if $\varphi$ kills each vector tangent to a $G$-orbit: $i_{\zeta_{X}} \varphi=0$ for all $X \in \mathfrak{g}$. We denote by $\Omega_{\mathrm{hor}}^{p}(M)^{G}$ the space of all horizontal $G$-invariant $p$-forms on $M$. They are also called basic forms.

30.33. Lemma. Under the exterior differential, the space $\Omega_{h o r}(M)^{G}$ of basic forms is a subcomplex of $\Omega(M)$.

The cohomology of the complex $\left(\Omega_{\mathrm{hor}}(M)^{G}, d\right)$ is called the basic cohomology of the $G$-manifold $M$.

Proof. If $\varphi \in \Omega_{\mathrm{hor}}(M)^{G}$, then the exterior derivative $d \varphi$ is clearly $G$ invariant. For $X \in \mathfrak{g}$ we have

$$
i_{\zeta_{X}} d \varphi=i_{\zeta_{X}} d \varphi+d i_{\zeta_{X}} \varphi=\mathcal{L}_{\zeta_{X}} \varphi=0
$$

so $d \varphi$ is also horizontal.

30.34. Lemma. Let $f, g: M \rightarrow N$ be smooth $G$-equivariant mappings between $G$-manifolds which are $G$-equivariantly $C^{\infty}$-homotopic: $\mathbb{R} \times M$ is again a $G$-manifold (with the action on $M$ only), and there exists a $G$-equivariant $h \in C^{\infty}(\mathbb{R} \times M, N)$ with $h(0, x)=f(x)$ and $h(1, x)=g(x)$.

Then $f$ and $g$ induce the same mapping in basic cohomology:

$$
f^{*}=g^{*}: H_{\text {basic }}(N) \rightarrow H_{\text {basic }}(M) \text {. }
$$

Proof. We recall the proof of (11.4) where we showed this without $G$. For $\omega \in \Omega_{\mathrm{hor}}^{k}(N)^{G}$ we have $h^{*} \omega \in \Omega_{\mathrm{hor}}^{k}(\mathbb{R} \times M)^{G}$ since $h$ is equivariant. The insertion operator inst $: M \rightarrow \mathbb{R} \times M$, given by $\operatorname{ins}_{t}(x)=(t, x)$, is also 
equivariant. The integral operator $I_{0}^{1}(\varphi):=\int_{0}^{1} \operatorname{ins}_{t}^{*} \varphi d t$ commutes with the insertion of fundamental vector fields and with the $G$-action, so it induces an operator $I_{0}^{1}: \Omega_{\text {hor }}^{k}(\mathbb{R} \times M)^{G} \rightarrow \Omega_{\text {hor }}^{k}(M)^{G}$. Let $T:=\frac{\partial}{\partial t} \in \mathfrak{X}(\mathbb{R} \times M)$ be the unit vector field in direction $\mathbb{R}$. Thus the homotopy operator $\bar{h}:=$ $I_{0}^{1} \circ i_{T} \circ h^{*}: \Omega_{\mathrm{hor}}^{k}(N)^{G} \rightarrow \Omega_{\mathrm{hor}}^{k-1}(M)^{G}$ is well defined, and from the proof of (11.4) we conclude that it still satisfies

$$
\begin{aligned}
g^{*}-f^{*} & =\left(h \circ \operatorname{ins}_{1}\right)^{*}-\left(h \circ \operatorname{ins}_{0}\right)^{*} \\
& =\left(\text { ins }_{1}^{*}-\text { ins }_{0}^{*}\right) \circ h^{*} \\
& =\left(d \circ I_{0}^{1} \circ i_{T}+I_{0}^{1} \circ i_{T} \circ d\right) \circ h^{*} \\
& =d \circ \bar{h}-\bar{h} \circ d,
\end{aligned}
$$

which implies the desired result since for $\omega \in \Omega_{\mathrm{hor}}^{k}(M)^{G}$ with $d \omega=0$ we have $g^{*} \omega-f^{*} \omega=\bar{h} d \omega+d \bar{h} \omega=d \bar{h} \omega$.

30.35. Basic lemma of Poincaré (111]). Let $\ell: G \times M \rightarrow M$ be a proper $G$-manifold. For $k>0$ let $\omega \in \Omega_{h o r}^{k}(M)^{G}$ be a basic $k$-form on $M$ with $d \omega=0$ in a G-invariant neighborhood of an orbit $G . x$. Then there exists a basic form $\varphi \in \Omega_{\text {hor }}^{k-1}(M)^{G}$ such that $d \omega=\varphi$ in a G-invariant neighborhood of G.x.

Proof. By (6.30) we may assume that $M$ is a Riemann $G$-manifold. Let $S_{x}$ be a slice with center $x$ which is diffeomorphic to a small ball in $T_{x}(G . x)^{\perp} \subset$ $T_{x} M$ and thus contractible.

We denote again by $\ell: G_{x} \times S_{x} \rightarrow S_{x}$ the induced action of the isotropy group $G_{x}$ on the slice. Then $d \omega=0$ on the $G$-invariant neighborhood G.S $S_{x}$ which is $G$-equivariantly diffeomorphic to the associated bundle $G \times{ }_{G_{x}} S_{x}$ by (6.18), The quotient mapping

$$
q: G \times S_{x} \rightarrow G \times{ }_{G_{x}} S_{x}
$$

is the projection of a principal $G_{x}$ bundle by (18.7.3) for the right action $R^{h}(g, y)=\left(g h, h^{-1} . y\right)$, and it is equivariant for the left $G$-action (acting on $G$ alone). Thus $q^{*} \omega$ is still $G$-horizontal and $G$-invariant on $G \times S_{x}$ and thus $q^{*} \omega$ is of the form $\operatorname{pr}_{S_{x}} \alpha$ for a unique form $\alpha$ on $S_{x}$. Moreover $q^{*} \omega$ is also horizontal and invariant for the right $G_{x}$-action by (19.14). So $\alpha$ is a $G_{x}$-basic form on $S_{x}$.

Now $S_{x}$ is $G_{x}$-equivariantly diffeomorphic to a ball in a vector space where $G_{x}$ acts linearly and isometrically. This ball is $G_{x}$-equivariantly contractible to 0 via $v \rightarrow r . v, r \in[0,1]$. Thus the basic cohomology of $H_{\text {basic }}^{k}\left(S_{x}\right)$ vanishes for $k>0$, and there exists a $G_{x}$-basic form $\beta \in \Omega_{\text {hor }}^{k-1}\left(S_{x}\right)^{G_{x}}$ with $d \beta=\alpha$. Then $\operatorname{pr}_{S_{x}}^{*} \beta$ is $G_{x}$-basic and $G$-basic on $G \times S_{x}$, so it induces a form $\varphi \in \Omega_{\mathrm{hor}}^{k-1}\left(G \times_{G_{x}} S_{x}\right)^{G}$ which satisfies $d \psi=\omega$. 
30.36. Theorem. For a proper $G$-manifold $M$ the basic cohomology $H_{\text {basic }}^{*}(M)$ coincides with the real cohomology of the Hausdorff orbit space $M / G$ in the sense of Čech or in the sense of singular cohomology.

Sketch of proof. On the category of proper $G$-manifolds and smooth $G$-equivariant mappings the basic cohomology satisfies the axioms for cohomology listed in (11.11). We proved all but the Mayer-Vietoris property, for which the proof (11.10) applies without any change. Pushing these properties down to the orbit spaces, they suffice to prove that basic cohomology equals singular or Čech cohomology of the orbit space with real coefficients, via the abstract theorem of de Rham in sheaf theory.

30.37. Theorem ([154, 156]). Let $M \times G \rightarrow M$ be a proper isometric right action of a Lie group $G$ on a smooth Riemann manifold $M$, which admits a section $\Sigma$.

Then the restriction of differential forms induces an isomorphism

$$
\Omega_{h o r}^{p}(M)^{G} \stackrel{\cong}{\longrightarrow} \Omega^{p}(\Sigma)^{W(\Sigma)}
$$

between the space of horizontal $G$-invariant differential forms on $M$ and the space of all differential forms on $\Sigma$ which are invariant under the action of the generalized Weyl group $W(\Sigma)$ of the section $\Sigma$.

Proof of injectivity in (30.37), Let $i: \Sigma \rightarrow M$ be the embedding of the section. It clearly induces a linear mapping $i^{*}: \Omega_{\mathrm{hor}}^{p}(M)^{G} \rightarrow \Omega^{p}(\Sigma)^{W(\Sigma)}$ which is injective by the following argument: Let $\omega \in \Omega_{\mathrm{hor}}^{p}(M)^{G}$ with $i^{*} \omega=$ 0 . For $x \in \Sigma$ we have $i_{X} \omega_{x}=0$ for $X \in T_{x} \Sigma$ since $i^{*} \omega=0$ and also for $X \in T_{x}(G . x)$ since $\omega$ is horizontal. Let $x \in \Sigma \cap M_{\text {reg }}$ be a regular point; then $T_{x} \Sigma=\left(T_{x}(G . x)\right)^{\perp}$ and so $\omega_{x}=0$. This holds along the whole orbit through $x$ since $\omega$ is $G$-invariant. Thus $\omega \mid M_{\text {reg }}=0$, and since $M_{\text {reg }}$ is dense in $M, \omega=0$.

So it remains to show that $i^{*}$ is surjective. This will be done in (30.44) below.

30.38. Lemma. Let $\ell \in V^{*}$ be a linear functional on a finite-dimensional vector space $V$, and let $f \in C^{\infty}(V, \mathbb{R})$ be a smooth function which vanishes on the kernel of $\ell$, so that $f \mid \ell^{-1}(0)=0$. Then there is a unique smooth function $g$ such that $f=\ell . g$.

Proof. Choose coordinates $x^{1}, \ldots, x^{n}$ on $V$ such that $\ell=x^{1}$. Then we have $f\left(0, x^{2}, \ldots, x^{n}\right)=0$ and therefore

$$
f\left(x^{1}, \ldots, x^{n}\right)=\int_{0}^{1} \partial_{1} f\left(t x^{1}, x^{2}, \ldots, x^{n}\right) d t \cdot x^{1}=g\left(x^{1}, \ldots, x^{n}\right) \cdot x^{1} .
$$


30.39. Question. Let $G \rightarrow G L(V)$ be a representation of a compact Lie group in a finite-dimensional vector space $V$. Let

$$
\rho=\left(\rho_{1}, \ldots, \rho_{m}\right): V \rightarrow \mathbb{R}^{m}
$$

be the polynomial mapping whose components $\rho_{i}$ are a minimal set of homogeneous generators for the algebra $\mathbb{R}[V]^{G}$ of invariant polynomials.

We consider the pullback homomorphism $\rho^{*}: \Omega^{p}\left(\mathbb{R}^{m}\right) \rightarrow \Omega^{p}(V)$. Is it surjective onto the space $\Omega_{h o r}^{p}(V)^{G}$ of $G$-invariant horizontal smooth p-forms on $V$ ?

See remark (30.41) for a class of representations where the answer is yes.

In general the answer is no. A counterexample is the following: Let the cyclic group $\mathbb{Z}_{n}=\mathbb{Z} / n \mathbb{Z}$ of order $n$, viewed as the group of $n$-th roots of unity, act on $\mathbb{C}=\mathbb{R}^{2}$ by complex multiplication. A generating system of polynomials consists of $\rho_{1}=|z|^{2}, \rho_{2}=\operatorname{Re}\left(z^{n}\right), \rho_{3}=\operatorname{Im}\left(z^{n}\right)$. But then each $d \rho_{i}$ vanishes at 0 and there is no chance of having the horizontal invariant volume form $d x \wedge d y$ in $\rho^{*} \Omega\left(\mathbb{R}^{3}\right)$.

30.40. Theorem ([154, 156]). Let $G \rightarrow G L(V)$ be a polar representation of a compact Lie group $G$, with section $\Sigma$ and generalized Weyl group $W=$ $W(\Sigma)$. Then the pullback to $\Sigma$ of differential forms induces an isomorphism

$$
\Omega_{h o r}^{p}(V)^{G} \stackrel{\cong}{\longrightarrow} \Omega^{p}(\Sigma)^{W(\Sigma)} .
$$

According to [38, remark after proposition 6], for any polar representation of a connected Lie group the generalized Weyl group $W(\Sigma)$ is a reflection group. This theorem is true for polynomial differential forms and also for real analytic differential forms, by essentially the same proof.

Proof. Let $i: \Sigma \rightarrow V$ be the embedding. It is proved in (30.37) that the restriction $i^{*}: \Omega_{\mathrm{hor}}^{p}(V)^{G} \rightarrow \Omega^{p}(\Sigma)^{W(G)}$ is injective, so it remains to prove surjectivity.

Let us first suppose that $W=W(\Sigma)$ is generated by reflections (a reflection group or Coxeter group). Let $\rho_{1}, \ldots, \rho_{n}$ be a minimal set of homogeneous generators of the algebra $\mathbb{R}[\Sigma]^{W}$ of $W$-invariant polynomials on $\Sigma$. Then this is a set of algebraically independent polynomials, $n=\operatorname{dim} \Sigma$, and their degrees $d_{1}, \ldots, d_{n}$ are uniquely determined up to order. We even have (see [95])

(1) $d_{1} \ldots d_{n}=|W|$, the order of $W$

(2) $d_{1}+\cdots+d_{n}=n+N$, where $N$ is the number of reflections in $W$,

(3) $\prod_{i=1}^{n}\left(1+\left(d_{i}-1\right) t\right)=a_{0}+a_{1} t+\cdots+a_{n} t^{n}$, where $a_{i}$ is the number of elements in $W$ whose fixed point set has dimension $n-i$. 
Let us consider the mapping $\rho=\left(\rho_{1}, \ldots, \rho_{n}\right): \Sigma \rightarrow \mathbb{R}^{n}$ and its Jacobian $J(x)=\operatorname{det}(d \rho(x))$. Let $x^{1}, \ldots, x^{n}$ be coordinate functions in $\Sigma$. Then for each $\sigma \in W$ we have

$$
\begin{aligned}
J . d x^{1} \wedge \cdots \wedge d x^{n} & =d \rho_{1} \wedge \cdots \wedge d \rho_{n}=\sigma^{*}\left(d \rho_{1} \wedge \cdots \wedge d \rho_{n}\right) \\
& =(J \circ \sigma) \sigma^{*}\left(d x^{1} \wedge \cdots \wedge d x^{n}\right) \\
& =(J \circ \sigma) \operatorname{det}(\sigma)\left(d x^{1} \wedge \cdots \wedge d x^{n}\right) \\
J \circ \sigma & =\operatorname{det}\left(\sigma^{-1}\right) J .
\end{aligned}
$$

If $J(x) \neq 0$, then in a neighborhood of $x$ the mapping $\rho$ is a diffeomorphism by the inverse function theorem, so that the 1 -forms $d \rho_{1}, \ldots, d \rho_{n}$ are a local coframe there. Since the generators $\rho_{1}, \ldots, \rho_{n}$ are algebraically independent over $\mathbb{R}, J \neq 0$. Since $J$ is a polynomial of degree $\left(d_{1}-1\right)+\cdots+\left(d_{n}-1\right)=N$ (see $(2))$, the set $U=\Sigma \backslash J^{-1}(0)$ is open and dense in $\Sigma$, and $d \rho_{1}, \ldots, d \rho_{n}$ form a coframe on $U$.

Now let $\left(\sigma_{\alpha}\right)_{\alpha=1, \ldots, N}$ be the set of reflections in $W$, with reflection hyperplanes $H_{\alpha}$. Let $\ell_{\alpha} \in \Sigma^{*}$ be a linear functional with $H_{\alpha}=\ell^{-1}(0)$. If $x \in H_{\alpha}$, we have $J(x)=\operatorname{det}\left(\sigma_{\alpha}\right) J\left(\sigma_{\alpha} \cdot x\right)=-J(x)$, so that $J \mid H_{\alpha}=0$ for each $\alpha$, and by lemma (30.38) we have

$$
J=c . \ell_{1} \ldots \ell_{N}
$$

Since $J$ is a polynomial of degree $N, c$ must be a constant. Repeating the last argument for an arbitrary function $g$ and using (5), we get:

(6) If $g \in C^{\infty}(\Sigma, \mathbb{R})$ satisfies $g \circ \sigma=\operatorname{det}\left(\sigma^{-1}\right) g$ for each $\sigma \in W$, we have $g=J . h$ for $h \in C^{\infty}(\Sigma, \mathbb{R})^{W}$.

(7) Claim. Let $\omega \in \Omega^{p}(\Sigma)^{W}$. Then we have

$$
\omega=\sum_{j_{1}<\cdots<j_{p}} \omega_{j_{1} \ldots j_{p}} d \rho_{j_{1}} \wedge \cdots \wedge d \rho_{j_{p}}
$$

where $\omega_{j_{1} \ldots j_{p}} \in C^{\infty}(\Sigma, \mathbb{R})^{W}$.

Since $d \rho_{1}, \ldots, d \rho_{n}$ form a coframe on the $W$-invariant dense open set $U=$ $\{x: J(x) \neq 0\}$, we have

$$
\omega\left|U=\sum_{j_{1}<\cdots<j_{p}} g_{j_{1} \ldots j_{p}} d \rho_{j_{1}}\right| U \wedge \cdots \wedge d \rho_{j_{p}} \mid U
$$

for $g_{j_{1} \ldots j_{p}} \in C^{\infty}(U, \mathbb{R})$. Since $\omega$ and all $d \rho_{i}$ are $W$-invariant, we may replace $g_{j_{1} \ldots j_{p}}$ by

$$
\frac{1}{|W|} \sum_{\sigma \in W} g_{j_{1} \ldots j_{p}} \circ \sigma \in C^{\infty}(U, \mathbb{R})^{W},
$$

or assume without loss that $g_{j_{1} \ldots j_{p}} \in C^{\infty}(U, \mathbb{R})^{W}$. 
Let us choose now a form index $i_{1}<\cdots<i_{p}$ with

$$
\left\{i_{p+1}<\cdots<i_{n}\right\}=\{1, \ldots, n\} \backslash\left\{i_{1}<\cdots<i_{p}\right\} .
$$

Then for some $\operatorname{sign} \varepsilon= \pm 1$ we have

$$
\begin{aligned}
\omega \mid U \wedge d \rho_{i_{p+1}} \wedge \cdots \wedge d \rho_{i_{n}} & =\varepsilon \cdot g_{i_{1} \ldots i_{p}} . d \rho_{1} \wedge \cdots \wedge d \rho_{n} \\
& =\varepsilon \cdot g_{i_{1} \ldots i_{p}} . J . d x^{1} \wedge \cdots \wedge d x^{n}, \\
\omega \wedge d \rho_{i_{p+1}} \wedge \cdots \wedge d \rho_{i_{n}} & =\varepsilon \cdot k_{i_{1} \ldots i_{p}} d x^{1} \wedge \cdots \wedge d x^{n}
\end{aligned}
$$

for a function $k_{i_{1} \ldots i_{p}} \in C^{\infty}(\Sigma, \mathbb{R})$. Thus

$$
k_{i_{1} \ldots i_{p}}\left|U=g_{i_{1} \ldots i_{p}} . J\right| U .
$$

Since $\omega$ and all $d \rho_{i}$ are $W$-invariant, by (8) we get $k_{i_{1} \ldots i_{p}} \circ \sigma=\operatorname{det}\left(\sigma^{-1}\right) k_{i_{1} \ldots i_{p}}$ for each $\sigma \in W$. But then by (6) we have $k_{i_{1} \ldots i_{p}}=\omega_{i_{1} \ldots i_{p}} . J$ for unique $\omega_{i_{1} \ldots i_{p}} \in C^{\infty}(\Sigma, \mathbb{R})^{W}$, and (9) then implies $\omega_{i_{1} \ldots i_{p}} \mid U=g_{i_{1} \ldots i_{p}}$, so that the claim (7) follows since $U$ is dense.

Now we may finish the proof of the theorem in the case where $W=W(\Sigma)$ is a reflection group. Let $i: \Sigma \rightarrow V$ be the embedding. By theorem $(30.30)$ the algebra $\mathbb{R}[V]^{G}$ of $G$-invariant polynomials on $V$ is isomorphic to the algebra $\mathbb{R}[\Sigma]^{W}$ of $W$-invariant polynomials on the section $\Sigma$, via the restriction mapping $i^{*}$. Choose polynomials $\tilde{\rho}_{1}, \ldots, \tilde{\rho}_{n} \in \mathbb{R}[V]^{G}$ with $\tilde{\rho}_{i} \circ i=\rho_{i}$ for all $i$. Put $\tilde{\rho}=\left(\tilde{\rho}_{1}, \ldots, \tilde{\rho}_{n}\right): V \rightarrow \mathbb{R}^{n}$. In the setting of claim (7), use Schwarz's theorem (7.13) to find $h_{i_{1}, \ldots, i_{p}} \in C^{\infty}\left(\mathbb{R}^{n}, \mathbb{R}\right)$ with $h_{i_{1}, \ldots, i_{p}} \circ \rho=\omega_{i_{1}, \ldots, i_{p}}$ and consider

$$
\tilde{\omega}=\sum_{j_{1}<\cdots<j_{p}}\left(h_{j_{1} \ldots j_{p}} \circ \tilde{\rho}\right) d \tilde{\rho}_{j_{1}} \wedge \cdots \wedge d \tilde{\rho}_{j_{p}}
$$

which is in $\Omega_{\text {hor }}^{p}(V)^{G}$ and satisfies $i^{*} \tilde{\omega}=\omega$.

Thus the mapping $i^{*}: \Omega_{\text {hor }}^{p}(V)^{G} \rightarrow \Omega_{\text {hor }}^{p}(\Sigma)^{W}$ is surjective in the case where $W=W(\Sigma)$ is a reflection group.

Now we treat the general case. Let $G_{0}$ be the connected component of $G$. From (30.15.3) one concludes: $A$ subspace $\Sigma$ of $V$ is a section for $G$ if and only if it is a section for $G_{0}$. Thus $\rho$ is a polar representation for $G$ if and only if it is a polar representation for $G_{0}$.

The generalized Weyl groups of $\Sigma$ with respect to $G$ and to $G_{0}$ are related by

$$
W\left(G_{0}\right)=N_{G_{0}}(\Sigma) / Z_{G_{0}}(\Sigma) \subset W(G)=N_{G}(\Sigma) / Z_{G}(\Sigma),
$$

since $Z_{G}(\Sigma) \cap N_{G_{0}}(\Sigma)=Z_{G_{0}}(\Sigma)$.

Let $\omega \in \Omega^{p}(\Sigma)^{W(G)} \subset \Omega^{p}(\Sigma)^{W\left(G_{0}\right)}$. Since $G_{0}$ is connected, the generalized Weyl group $W\left(G_{0}\right)$ is generated by reflections (a Coxeter group) by $\mathbf{3 8}$, 
remark after proposition 6]. Thus by the first part of the proof

$$
i^{*}: \Omega_{\mathrm{hor}}^{p}(V)^{G_{0}} \stackrel{\cong}{\longrightarrow} \Omega^{p}(\Sigma)^{W\left(G_{0}\right)}
$$

is an isomorphism, and we get $\varphi \in \Omega_{\text {hor }}^{p}(M)^{G_{0}}$ with $i^{*} \varphi=\omega$. Let us consider

$$
\psi:=\int_{G} g^{*} \varphi d g \in \Omega_{\mathrm{hor}}^{p}(V)^{G},
$$

where $d g$ denotes Haar measure on $G$. In order to show that $i^{*} \psi=\omega$, it suffices to check that $i^{*} g^{*} \varphi=\omega$ for each $g \in G$. Now $g(\Sigma)$ is again a section of $G$, thus also of $G_{0}$. Since any two sections are related by an element of the group, there exists $h \in G_{0}$ such that $h g(\Sigma)=\Sigma$. Then $h g \in N_{G}(\Sigma)$ and we denote by $[h g]$ the coset in $W(G)$, and we may compute as follows:

$$
\begin{aligned}
\left(i^{*} g^{*} \varphi\right)_{x} & =\left(g^{*} \varphi\right)_{x} \cdot \bigwedge^{p} T i=\varphi_{g(x)} \cdot \bigwedge^{p} T g \cdot \bigwedge^{p} T i \\
& =\left(h^{*} \varphi\right)_{g(x)} \cdot \bigwedge_{p}^{p} T g \cdot \bigwedge^{p} T i, \quad \text { since } \varphi \in \Omega_{\mathrm{hor}}^{p}(M)^{G_{0}} \\
& =\varphi_{h g(x)} \cdot \bigwedge_{p}^{p} T(h g) \cdot \bigwedge_{p}^{p} T i=\varphi_{i[h g](x)} \cdot \bigwedge^{p} T i \cdot \bigwedge_{p}^{p} T([h g]) \\
& =\varphi_{i[h g](x)} \cdot \bigwedge_{p}^{p} T i \cdot \bigwedge_{p}^{p} T([h g])=\left(i^{*} \varphi\right)_{[h g]}(x) \cdot \bigwedge^{p} T([h g]) \\
& =\omega_{[h g](x)} \cdot \bigwedge^{p} T([h g])=[h g]^{*} \omega=\omega .
\end{aligned}
$$

30.41. Remark. The proof of theorem (30.40) shows that the answer to question (30.39) is yes for the representations treated in (30.40).

30.42. Corollary. Let $\rho: G \rightarrow O(V,\langle\rangle$,$) be an orthogonal polar$ representation of a compact Lie group $G$, with section $\Sigma$ and generalized Weyl group $W=W(\Sigma)$. Let $B \subset V$ be an open ball centered at 0 .

Then the restriction of differential forms induces an isomorphism

$$
\Omega_{h o r}^{p}(B)^{G} \stackrel{\cong}{\longrightarrow} \Omega^{p}(\Sigma \cap B)^{W(\Sigma)} .
$$

Proof. Check the proof of (30.40) or use the following argument. Suppose that $B=\{v \in V:|v|<1\}$ and consider a smooth diffeomorphism $f$ : $[0,1) \rightarrow[0, \infty)$ with $f(t)=t$ near 0 . Then $g(v):=\frac{f(|v|)}{|v|} v$ is a $G$-equivariant diffeomorphism $B \rightarrow V$ and by $(30.40)$ we get

$$
\Omega_{\mathrm{hor}}^{p}(B)^{G} \stackrel{\left(g^{-1}\right)^{*}}{\longrightarrow} \Omega_{\mathrm{hor}}^{p}(V)^{G} \stackrel{\cong}{\longrightarrow} \Omega^{p}(\Sigma)^{W(\Sigma)} \stackrel{g^{*}}{\longrightarrow} \Omega^{p}(\Sigma \cap B)^{W(\Sigma)} .
$$


30.43. Let us assume that we are in the situation of the main theorem (30.37) for the rest of this section. For $x \in M$ let $S_{x}$ be a (normal) slice and $G_{x}$ the isotropy group, which acts on the slice. Then $G . S_{x}$ is open in $M$ and $G$-equivariantly diffeomorphic to the associated bundle $G \rightarrow G / G_{x}$ via

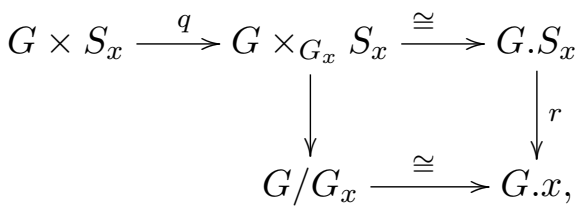

where $r$ is the projection of a tubular neighborhood. Since $q: G \times S_{x} \rightarrow$ $G \times{ }_{G_{x}} S_{x}$ is a principal $G_{x}$-bundle with principal right action $(g, s) \cdot h=$ $\left(g h, h^{-1} . s\right)$, we have an isomorphism $q^{*}: \Omega\left(G \times{ }_{G_{x}} S_{x}\right) \rightarrow \Omega_{G_{x}-\mathrm{hor}}\left(G \times S_{x}\right)^{G_{x}}$. Since $q$ is also $G$-equivariant for the left $G$-actions, the isomorphism $q^{*}$ maps the subalgebra $\Omega_{\text {hor }}^{p}\left(G . S_{x}\right)^{G} \cong \Omega_{\text {hor }}^{p}\left(G \times_{G_{x}} S_{x}\right)^{G}$ of $\Omega\left(G \times_{G_{x}} S_{x}\right)$ to the subalgebra $\Omega_{G_{x}-\text { hor }}^{p}\left(S_{x}\right)^{G_{x}}$ of $\Omega_{G_{x}-\text { hor }}\left(G \times S_{x}\right)^{G_{x}}$. So we have proved:

Lemma. In this situation there is a canonical isomorphism

$$
\Omega_{h o r}^{p}\left(G . S_{x}\right)^{G} \stackrel{\cong}{\longrightarrow} \Omega_{G_{x}-h o r}^{p}\left(S_{x}\right)^{G_{x}}
$$

which is given by pullback along the embedding $S_{x} \rightarrow G . S_{x}$.

30.44. Rest of the proof of theorem (30.37). Let us consider $\omega \in$ $\Omega^{p}(\Sigma)^{W(\Sigma)}$. We want to construct a form $\tilde{\omega} \in \Omega_{\mathrm{hor}}^{p}(M)^{G}$ with $i^{*} \tilde{\omega}=\omega$. This will finish the proof of theorem (30.40).

Choose $x \in \Sigma$ and an open ball $B_{x}$ with center 0 in $T_{x} M$ such that the Riemann exponential mapping $\exp _{x}: T_{x} M \rightarrow M$ is a diffeomorphism on $B_{x}$. We consider now the compact isotropy group $G_{x}$ and the slice representation $\rho_{x}: G_{x} \rightarrow O\left(V_{x}\right)$, where $V_{x}=\operatorname{Nor}_{x}(G \cdot x)=\left(T_{x}(G \cdot x)\right)^{\perp} \subset T_{x} M$ is the normal space to the orbit. This is a polar representation with section $T_{x} \Sigma$, and its generalized Weyl group is given by $W\left(T_{x} \Sigma\right) \cong N_{G}(\Sigma) \cap G_{x} / Z_{G}(\Sigma)=$ $W(\Sigma)_{x}$ (see (30.24)). Then $\exp _{x}: B_{x} \cap V_{x} \rightarrow S_{x}$ is a diffeomorphism onto a slice and $\exp _{x}: B_{x} \cap T_{x} \Sigma \rightarrow \Sigma_{x} \subset \Sigma$ is a diffeomorphism onto an open neighborhood $\Sigma_{x}$ of $x$ in the section $\Sigma$.

Let us now consider the pullback $\left(\exp \mid B_{x} \cap T_{x} \Sigma\right)^{*} \omega \in \Omega^{p}\left(B_{x} \cap T_{x} \Sigma\right)^{W\left(T_{x} \Sigma\right)}$. By corollary (30.42) there exists a unique form $\varphi^{x} \in \Omega_{G_{x}-\text { hor }}^{p}\left(B_{x} \cap V_{x}\right)^{G_{x}}$ such that $i^{*} \varphi^{x}=\left(\exp \mid B_{x} \cap T_{x} \Sigma\right)^{*} \omega$, where $i_{x}$ is the embedding. Then we have

$$
\left(\left(\exp \mid B_{x} \cap V_{x}\right)^{-1}\right) * \varphi^{x} \in \Omega_{G_{x}-\text { hor }}^{p}\left(S_{x}\right)^{G_{x}}
$$

and by lemma (30.43) this form corresponds uniquely to a differential form $\omega^{x} \in \Omega_{\text {hor }}^{p}\left(G . S_{x}\right)^{G}$ which satisfies $\left(i \mid \Sigma_{x}\right)^{*} \omega^{x}=\omega \mid \Sigma_{x}$, since the exponential mapping commutes with the respective restriction mappings. Now the intersection $G . S_{x} \cap \Sigma$ is the disjoint union of all the open sets $w_{j}\left(\Sigma_{x}\right)$ where 
we pick one $w_{j}$ in each left coset of the subgroup $W(\Sigma)_{x}$ in $W(\Sigma)$. If we choose $g_{j} \in N_{G}(\Sigma)$ projecting on $w_{j}$ for all $j$, then

$$
\begin{aligned}
\left(i \mid w_{j}\left(\Sigma_{x}\right)\right)^{*} \omega^{x} & =\left(\ell_{g_{j}} \circ i \mid \Sigma_{x} \circ w_{j}^{-1}\right)^{*} \omega^{x} \\
& =\left(w_{j}^{-1}\right)^{*}\left(i \mid \Sigma_{x}\right)^{*} \ell_{g_{j}}^{*} \omega^{x} \\
& =\left(w_{j}^{-1}\right)^{*}\left(i \mid \Sigma_{x}\right)^{*} \omega^{x}=\left(w_{j}^{-1}\right)^{*}\left(\omega \mid \Sigma_{x}\right)=\omega \mid w_{j}\left(\Sigma_{x}\right),
\end{aligned}
$$

so that $\left(i \mid G . S_{x} \cap \Sigma\right)^{*} \omega^{x}=\omega \mid G . S_{x} \cap \Sigma$. We can do this for each point $x \in \Sigma$. Using the method of (6.28) and (6.30), we may find a sequence of points $\left(x_{n}\right)_{n \in \mathbb{N}}$ in $\Sigma$ such that the $\pi\left(\Sigma_{x_{n}}\right)$ form a locally finite open cover of the orbit space $M / G \cong \Sigma / W(\Sigma)$ and a smooth partition of unity $f_{n}$ consisting of $G$-invariant functions with $\operatorname{supp}\left(f_{n}\right) \subset G$. $S_{x_{n}}$. Then $\tilde{\omega}:=\sum_{n} f_{n} \omega^{x_{n}} \in$ $\Omega_{\text {hor }}^{p}(M)^{G}$ has the required property $i^{*} \tilde{\omega}=\omega$. 


\section{CHAPTER VII. Symplectic and Poisson Geometry}

\section{Symplectic Geometry and Classical Mechanics}

31.1. Motivation. A particle with mass $m>0$ moves in a potential $V(q)$ along a curve $q(t)$ in $\mathbb{R}^{3}$ in such a way that Newton's second law is satisfied: $m \ddot{q}(t)=-\operatorname{grad} V(q(t))$. Let us consider the the quantity $p_{i}:=m \cdot \dot{q}^{i}$ as an independent variable. It is called the $i$-th momentum. Let us define the energy function (as the sum of the kinetic and potential energy) by

$$
E(q, p):=\frac{1}{2 m}|p|^{2}+V(q)=\frac{m|\dot{q}|^{2}}{2}+V(q) .
$$

Then $m \ddot{q}(t)=-\operatorname{grad} V(q(t))$ is equivalent to

$$
\left\{\begin{array}{l}
\dot{q}^{i}=\frac{\partial E}{\partial p_{i}}, \\
\dot{p}_{i}=-\frac{\partial E}{\partial q^{i}}, \quad i=1,2,3,
\end{array}\right.
$$

which are Hamilton's equations of motion. In order to study this equation for a general energy function $E(q, p)$, we consider the matrix

$$
J=\left(\begin{array}{cc}
0 & \mathbb{I}_{\mathbb{R}^{3}} \\
-\mathbb{I}_{\mathbb{R}^{3}} & 0
\end{array}\right) .
$$

Then the equation is equivalent to $\dot{u}(t)=J \cdot \operatorname{grad} E(u(t))$, where $u=(q, p) \in$ $\mathbb{R}^{6}$. In complex notation, where $z^{i}=q^{i}+\sqrt{-1} p_{i}$, this is equivalent to $\dot{z}^{i}=-2 \sqrt{-1} \frac{\partial E}{\partial \bar{z}^{i}}$. 
Consider the Hamiltonian vector field $H_{E}:=J \cdot \operatorname{grad} E$ associated to the energy function $E$; then we have $\dot{u}(t)=H_{E}(u(t))$, so the orbit of the particle with initial position and momentum $\left(q_{0}, p_{0}\right)=u_{0}$ is given by $u(t)=$ $\mathrm{Fl}_{t}^{H_{E}}\left(u_{0}\right)$.

Let us now consider the symplectic structure

$$
\omega(x, y)=\sum_{i=1}^{3}\left(x^{i} y^{3+i}-x^{3+i} y^{i}\right)=(x \mid J y) \quad \text { for } x, y \in \mathbb{R}^{6} .
$$

Then the Hamiltonian vector field $H_{E}$ is given by

$$
\begin{aligned}
\omega\left(H_{E}(u), v\right) & =\left(H_{E} \mid J v\right)=(J \operatorname{grad} E(u) \mid J v) \\
& =\left(J^{\top} J \operatorname{grad} E(u) \mid v\right)=(\operatorname{grad} E(u) \mid v)=d E(u) v .
\end{aligned}
$$

The Hamiltonian vector field is therefore the 'gradient' of $E$ with respect to the symplectic structure $\omega$; we write $H_{E}=\operatorname{grad}^{\omega} E$.

How does this equation react to coordinate transformations? So let $f$ : $\mathbb{R}^{3} \times \mathbb{R}^{3} \rightarrow \mathbb{R}^{3} \times \mathbb{R}^{3}$ be a (local) diffeomorphism. We consider the energy $E \circ f$ and put $u=f(w)$. Then

$$
\begin{aligned}
\omega\left(\operatorname{grad}^{\omega}\right. & (E \circ f)(w), v)=d(E \circ f)(w) v=d E(f(w)) \cdot d f(w) v \\
& =\omega\left(\operatorname{grad}^{\omega} E(f(w)), d f(w) v\right) \\
& =\omega\left(d f(w) d f(w)^{-1} \operatorname{grad}^{\omega} E(f(w)), d f(w) v\right) \\
& =\omega\left(d f(w)\left(f^{*} \operatorname{grad}^{\omega} E\right)(w), d f(w) v\right) \\
& =\left(f^{*} \omega\right)\left(\left(f^{*} \operatorname{grad}^{\omega} E\right)(w), v\right) .
\end{aligned}
$$

So we see that $f^{*} \operatorname{grad}^{\omega} E=\operatorname{grad}^{\omega}(E \circ f)$ if and only if $f^{*} \omega=\omega$, i.e., $d f(w) \in$ $S p(3, \mathbb{R})$ for all $w$. Such diffeomorphisms are called symplectomorphisms. By (3.14) we have

$$
\mathrm{Fl}_{t}^{f^{*} \operatorname{grad}^{\omega} E}=f^{-1} \circ \mathrm{Fl}_{t}^{\operatorname{grad}^{\omega} E} \circ f
$$

in general.

31.2. Lemma (E. Cartan). Let $V$ be a real finite-dimensional vector space, and let $\omega \in \bigwedge^{2} V^{*}$ be a 2-form on $V$. Consider the linear mapping $\check{\omega}: V \rightarrow V^{*}$ given by $\langle\breve{\omega}(v), w\rangle=\omega(v, w)$.

If $\omega \neq 0$, then the rank of the linear mapping $\check{\omega}: V \rightarrow V^{*}$ is $2 p$, and there exist linearly independent $l^{1}, \ldots, l^{2 p} \in V^{*}$ which form a basis of $\check{\omega}(V) \subset V^{*}$ such that $\omega=\sum_{k=1}^{p} l^{2 k-1} \wedge l^{2 k}$. Furthermore, $l^{2}$ can be chosen arbitrarily in $\check{\omega}(V) \backslash 0$.

Proof. Let $v_{1}, \ldots, v_{n}$ be a basis of $V$ and let $v^{1}, \ldots, v^{n}$ be the dual basis of $V^{*}$. Then $\omega=\sum_{i<j} \omega\left(v_{i}, v_{j}\right) v^{i} \wedge v^{j}=: \sum_{i<j} a_{i j} v^{i} \wedge v^{j}$. Since $\omega \neq 0$, not all 
$a_{i j}=0$. Suppose that $a_{12} \neq 0$. Put

$$
\begin{aligned}
l^{1} & =\frac{1}{a_{12}} \check{\omega}\left(v_{1}\right)=\frac{1}{a_{12}} i\left(v_{1}\right) \omega=\frac{1}{a_{12}} i\left(v_{1}\right)\left(\sum_{i<j} a_{i j} v^{i} \wedge v^{j}\right) \\
& =v^{2}+\frac{1}{a_{12}} \sum_{j=3}^{n} a_{1 j} v^{j}, \\
l^{2} & =\check{\omega}\left(v_{2}\right)=i\left(v_{2}\right) \omega=i\left(v_{2}\right)\left(\sum_{i<j} a_{i j} v^{i} \wedge v^{j}\right)=-a_{12} v^{1}+\sum_{j=3}^{n} a_{2 j} v^{j} .
\end{aligned}
$$

So, $l^{1}, l^{2}, v^{3}, \ldots, v^{n}$ is still a basis of $V^{*}$. Put $\omega_{1}:=\omega-l^{1} \wedge l^{2}$. Then

$$
\begin{aligned}
& i_{v_{1}} \omega_{1}=i_{v_{1}} \omega-i_{v_{1}} l^{1} \wedge l^{2}+l^{1} \wedge i_{v_{1}} l^{2}=a_{12} l^{1}-0-a_{12} l^{1}=0, \\
& i_{v_{2}} \omega_{1}=i_{v_{2}} \omega-i_{v_{2}} l^{1} \wedge l^{2}+l^{1} \wedge i_{v_{2}} l^{2}=l^{2}-l^{2}+0=0 .
\end{aligned}
$$

So the 2 -form $\omega_{1}$ is in the subalgebra of $\bigwedge V^{*}$ generated by $v^{3}, v^{4}, \ldots, v^{n}$. If $\omega_{1}=0$, then $\omega=l^{1} \wedge l^{2}$. If $\omega_{1} \neq 0$, we can repeat the procedure and get the form of $\omega$.

If $l=\check{\omega}(v) \in \check{\omega}(V) \subset V^{*}$ is arbitrary but $\neq 0$, there is some $w \in V$ with $\langle l, w\rangle=\omega(v, w) \neq 0$. Choose a basis $v_{1}, \ldots, v_{n}$ of $V$ with $v_{1}=w$ and $v_{2}=v$. Then $l^{2}=i\left(v_{2}\right) \omega=i(v) \omega=l$.

31.3. Corollary. Let $\omega \in \bigwedge^{2} V^{*}$ and let $2 p=\operatorname{rank}\left(\check{\omega}: V \rightarrow V^{*}\right)$.

Then $p$ is the maximal number $k$ such that $\omega^{\wedge k}=\omega \wedge \cdots \wedge \omega \neq 0$.

Proof. By $(31.2)$ we have $\omega^{\wedge p}=p ! l^{1} \wedge l^{2} \wedge \cdots \wedge l^{2 p}$ and $\omega^{\wedge(2 p+1)}=0$.

31.4. Symplectic vector spaces. A symplectic form on a vector space $V$ is a 2-form $\omega \in \bigwedge^{2} V^{*}$ such that $\check{\omega}: V \rightarrow V^{*}$ is an isomorphism. Then $\operatorname{dim}(V)=2 n$ and there is a basis $l^{1}, \ldots, l^{2 n}$ of $V^{*}$ such that $\omega=\sum_{i=1}^{n} l^{i} \wedge$ $l^{n+i}$, by $(31.2)$.

For a linear subspace $W \subset V$ we define the symplectic orthogonal by $W^{\omega \perp}=$ $W^{\perp}:=\{v \in V: \omega(w, v)=0$ for all $w \in W\}$; which coincides with the annihilator (or polar) $\check{\omega}(W)^{\circ}=\{v \in V:\langle\check{\omega}(w), v\rangle=0$ for all $w \in W\}$ in $V$.

Lemma. For linear subspaces $W, W_{1}, W_{2} \subset V$ we have:

(1) $W^{\perp \perp}=W$.

(2) $\operatorname{dim}(W)+\operatorname{dim}\left(W^{\perp}\right)=\operatorname{dim}(V)=2 n$.

(3) $\check{\omega}\left(W^{\perp}\right)=W^{\circ}$ and $\check{\omega}(W)=\left(W^{\perp}\right)^{\circ}$ in $V^{*}$.

(4) For two linear subspace $W_{1}, W_{2} \subset V$ we have $W_{1} \subseteq W_{2} \Leftrightarrow W_{1}^{\perp} \supseteq W_{2}^{\perp}$, $\left(W_{1} \cap W_{2}\right)^{\perp}=W_{1}^{\perp}+W_{2}^{\perp}$, and $\left(W_{1}+W_{2}\right)^{\perp}=W_{1}^{\perp} \cap W_{2}^{\perp}$.

(5) $\operatorname{dim}\left(W_{1} \cap W_{2}\right)-\operatorname{dim}\left(W_{1}^{\perp} \cap W_{2}^{\perp}\right)=\operatorname{dim} W_{1}+\operatorname{dim} W_{2}-2 n$. 
Proof. (11) - (4) are obvious, using duality and the annihilator. (15) can be seen as follows. By (4) we have

$$
\begin{aligned}
\operatorname{dim}\left(W_{1} \cap W_{2}\right)^{\perp} & =\operatorname{dim}\left(W_{1}^{\perp}+W_{2}^{\perp}\right) \\
& =\operatorname{dim}\left(W_{1}^{\perp}\right)+\operatorname{dim}\left(W_{2}^{\perp}\right)-\operatorname{dim}\left(W_{1}^{\perp} \cap W_{2}^{\perp}\right), \\
\operatorname{dim}\left(W_{1} \cap W_{2}\right) & =2 n-\operatorname{dim}\left(W_{1} \cap W_{2}\right)^{\perp} \quad \text { by (2) } \\
& =2 n-\operatorname{dim}\left(W_{1}^{\perp}\right)-\operatorname{dim}\left(W_{2}^{\perp}\right)+\operatorname{dim}\left(W_{1}^{\perp} \cap W_{2}^{\perp}\right) \\
& =\operatorname{dim}\left(W_{1}\right)+\operatorname{dim}\left(W_{2}\right)-2 n+\operatorname{dim}\left(W_{1}^{\perp} \cap W_{2}^{\perp}\right) .
\end{aligned}
$$

A linear subspace $W \subseteq V$ is called:

$\begin{array}{llll}\text { isotropic } & \text { if } & W \subseteq W^{\perp} & \Rightarrow \operatorname{dim}(W) \leq n, \\ \text { coisotropic } & \text { if } & W \supseteq W^{\perp} & \Rightarrow \operatorname{dim}(W) \geq n, \\ \text { Lagrangian } & \text { if } & W=W^{\perp} & \Rightarrow \operatorname{dim}(W)=n, \\ \text { symplectic } & \text { if } & W \cap W^{\perp}=0 & \Rightarrow \operatorname{dim}(W)=\text { even. }\end{array}$

31.5. Example. Let $W$ be a vector space with dual $W^{*}$. Then $\left(W \times W^{*}, \omega\right)$ is a symplectic vector space where $\omega\left(\left(v, v^{*}\right),\left(w, w^{*}\right)\right)=\left\langle w^{*}, v\right\rangle-\left\langle v^{*}, w\right\rangle$. Choose a basis $w_{1}, \ldots, w_{n}$ of $W=W^{* *}$ and let $w^{1}, \ldots, w^{n}$ be the dual basis. Then $\omega=\sum_{i} w^{i} \wedge w_{i}$. The two subspaces $W \times 0$ and $0 \times W^{*}$ are Lagrangian.

Consider now a symplectic vector space $(V, \omega)$ and suppose that $W_{1}, W_{2} \subseteq V$ are two Lagrangian subspaces such that $W_{1} \cap W_{2}=0$. Then $\omega: W_{1} \times W_{2} \rightarrow \mathbb{R}$ is a duality pairing, so we may identify $W_{2}$ with $W_{1}^{*}$ via $\omega$. Then $(V, \omega)$ is isomorphic to $W_{1} \times W_{1}^{*}$ with the symplectic structure described above.

31.6. Let $\mathbb{R}^{2 n}=\mathbb{R}^{n} \times\left(\mathbb{R}^{n}\right)^{*}$ with the standard symplectic structure $\omega$ from $(31.5)$. Recall from $(4.7)$ the Lie group $S p(n, \mathbb{R})$ of symplectic automorphisms of $\left(\mathbb{R}^{2 n}, \omega\right)$,

$$
S p(n, \mathbb{R})=\left\{A \in L\left(\mathbb{R}^{2 n}, \mathbb{R}^{2 n}\right): A^{\top} J A=J\right\}, \quad \text { where } J=\left(\begin{array}{cc}
0 & \mathbb{I}_{\mathbb{R}^{n}} \\
-\mathbb{I}_{\mathbb{R}^{n}} & 0
\end{array}\right) .
$$

Let $(\mid)$ be the standard inner product on $\mathbb{R}^{2 n}$ and let $\mathbb{R}^{2 n} \cong \sqrt{-1} \mathbb{R}^{n} \oplus$ $\mathbb{R}^{n}=\mathbb{C}^{n}$, where the scalar multiplication by $\sqrt{-1}$ is given by $J\left(\begin{array}{l}x \\ y\end{array}\right)=\left(\begin{array}{c}y \\ -x\end{array}\right)$. Then we have:

$$
\begin{aligned}
\omega\left(\left(\begin{array}{l}
x \\
y
\end{array}\right),\left(\begin{array}{l}
x^{\prime} \\
y^{\prime}
\end{array}\right)\right) & =\left\langle y^{\prime}, x\right\rangle-\left\langle y, x^{\prime}\right\rangle=\left(\left(\begin{array}{l}
x \\
y
\end{array}\right) \mid\left(\begin{array}{c}
y^{\prime} \\
-x^{\prime}
\end{array}\right)\right)=\left(\left(\begin{array}{l}
x \\
y
\end{array}\right) \mid J\left(\begin{array}{l}
x^{\prime} \\
y^{\prime}
\end{array}\right)\right) \\
& =\left(x^{T}, y^{T}\right) J\left(\begin{array}{l}
x^{\prime} \\
y^{\prime}
\end{array}\right) .
\end{aligned}
$$


Since $J^{2}=-\mathbb{I}_{R^{2 n}}$ we have $J \in S p(n, \mathbb{R})$, and since $J^{\top}=-J=J^{-1}$ we also have $J \in O(2 n, \mathbb{R})$. We consider now the Hermitian inner product $h: \mathbb{C}^{n} \times \mathbb{C}^{n} \rightarrow \mathbb{C}$ given by

$$
\begin{aligned}
h(u, v): & =(u \mid v)+\sqrt{-1} \omega(u, v)=(u \mid v)+\sqrt{-1}(u \mid J v), \\
h(v, u) & =(v \mid u)+\sqrt{-1}(v \mid J u)=(u \mid v)+\sqrt{-1}\left(J^{\top} v \mid u\right) \\
& =(u \mid v)-\sqrt{-1}(u \mid J v)=\overline{h(u, v)}, \\
h(J u, v) & =(J u \mid v)+\sqrt{-1}(J u \mid J v)=\sqrt{-1}\left(\left(u \mid J^{\top} J v\right)-\sqrt{-1}\left(u \mid J^{\top} v\right)\right) \\
& =\sqrt{-1}((u \mid v)+\sqrt{-1} \omega(u, v))=\sqrt{-1} h(u, v) .
\end{aligned}
$$

Lemma. The subgroups $S p(n, \mathbb{R}), O(2 n, \mathbb{R})$, and $U(n)$ of $G L(2 n, \mathbb{R})$ acting on $\mathbb{R}^{2 n} \cong \mathbb{C}^{n}$ are related by

$O(2 n, \mathbb{R}) \cap G L(n, \mathbb{C})=S p(n, \mathbb{R}) \cap G L(n, \mathbb{C})=S p(n, \mathbb{R}) \cap O(2 n, \mathbb{R})=U(n)$.

Proof. For $A \in G L\left(2 n, \mathbb{R}\right.$ ) (and all $u, v \in \mathbb{R}^{2 n}$ ) we have in turn

$$
\begin{aligned}
& h(A u, A v)=h(u, v) \quad \Leftrightarrow A \in U(n), \\
& \left\{\begin{array}{cc}
(A u \mid A v)=(u \mid v) & \text { (real part) } \\
\omega(A u, A v) & =\omega(u, v) \quad \text { (imagin. part) }
\end{array}\right\} \Leftrightarrow A \in O(2 n, \mathbb{R}) \cap S p(n, \mathbb{R}), \\
& \left\{\begin{array}{c}
(A u \mid A v)=(u \mid v) \\
J A=A J
\end{array}\right\} \quad \Leftrightarrow A \in O(2 n, \mathbb{R}) \cap G L(n, \mathbb{C}), \\
& \left\{\begin{array}{c}
J A=A J \\
(A u \mid J A v)=(A u \mid A J v)=(u \mid J v)
\end{array}\right\} \Leftrightarrow A \in S p(n, \mathbb{R}) \cap G L(n, \mathbb{C}) .
\end{aligned}
$$

31.7. The Lagrange-Grassmann manifold. Let $L\left(\mathbb{R}^{2 n}, \omega\right)=L(2 n)$ denote the space of all Lagrangian linear subspaces of $\mathbb{R}^{2 n}$; we call it the Lagrange-Grassmann manifold. It is a subset of the Grassmann manifold $G(n, 2 n ; \mathbb{R}) ;$ see $(18.5)$.

In the situation of (31.6) we consider a linear subspace $W \subset\left(\mathbb{R}^{2 n}, \omega\right)$ of dimension $n$. Then we have:

$W$ is a Lagrangian subspace

$$
\begin{aligned}
& \Leftrightarrow \omega|W=0 \Leftrightarrow(\mid J(\quad))| W=0 \\
& \Leftrightarrow J(W) \text { is orthogonal to } W \text { with respect to }(\quad \mid \quad)=\operatorname{Re}(h) .
\end{aligned}
$$

Thus the group $O(2 n, \mathbb{R}) \cap G L(n, \mathbb{C})=U(n)$ acts transitively on the Lagrange-Grassmann manifold $L(2 n)$. The isotropy group of the Lagrangian subspace $\mathbb{R}^{n} \times 0$ is the subgroup $O(n, \mathbb{R}) \subset U(n)$ consisting of all unitary matrices with all entries real. So $L(2 n)=U(n) / O(n, \mathbb{R})$ by $(5.11)$, which 
is a compact homogenous space and a smooth manifold. The dimension of $L(2 n)$ is given by

$$
\operatorname{dim} L(2 n)=\operatorname{dim} U(n)-\operatorname{dim} O(n, \mathbb{R})=\left(n+2 \frac{n(n-1)}{2}\right)-\frac{n(n-1)}{2}=\frac{n(n+1)}{2} .
$$

Which choices did we make in this construction? Starting with a general symplectic vector space $(V, \omega)$, we first choose a basic Lagrangian subspace $L\left(=\mathbb{R}^{n} \times 0\right)$, and then we identify $V / L$ with $L^{*}$ via $\omega$. Next we chose a positive inner product on $L$, transport it to $L^{*}$ via $\omega$ and extend it to $L \times L^{*}$ by putting $L$ and $L^{*}$ orthogonal to each other. All these possible choices are homotopic to each other.

Finally we consider $\operatorname{det}_{\mathbb{C}}=\operatorname{det}: U(n) \rightarrow S^{1} \subset \mathbb{C}$. Then $\operatorname{det}(O(n))=\{ \pm 1\}$. So $\operatorname{det}^{2}: U(n) \rightarrow S^{1}$ and $\operatorname{det}^{2}(O(n))=\{1\}$. For $U \in U(n)$ and $A \in O(n, \mathbb{R})$ we have $\operatorname{det}^{2}(U A)=\operatorname{det}^{2}(U) \operatorname{det}^{2}(A)=\operatorname{det}^{2}(U)$, so this factors to a well defined smooth mapping $\operatorname{det}^{2}: U(n) / O(n)=L(2 n) \rightarrow S^{1}$.

Claim. The group $S U(n)$ acts (from the left) transitively on each fiber of $\operatorname{det}^{2}: L(2 n)=U(n) / O(n) \rightarrow S^{1}$.

Namely, for $U_{1}, U_{2} \in U(n)$ with $\operatorname{det}^{2}\left(U_{1}\right)=\operatorname{det}^{2}\left(U_{2}\right)$ we get $\operatorname{det}\left(U_{1}\right)=$ $\pm \operatorname{det}\left(U_{2}\right)$. There exists $A \in O(n)$ such that $\operatorname{det}\left(U_{1}\right)=\operatorname{det}\left(U_{2} \cdot A\right)$; thus $U_{1}\left(U_{2} A\right)^{-1} \in S U(n)$ and $U_{1}\left(U_{2} A\right)^{-1} U_{2} A O(n)=U_{1} O(n)$. The claim is proved.

Now $S U(n)$ is simply connected and each fiber of $\operatorname{det}^{2}: U(n) / O(n) \rightarrow S^{1}$ is diffeomorphic to $S U(n) / S O(n)$ which again is simply connected by the exact homotopy sequence of the fibration $S O(n) \rightarrow S U(n) \rightarrow S U(n) / S O(N)$,

$$
\cdots \rightarrow\left(0=\pi_{1}(S U(n))\right) \rightarrow \pi_{1}(S U(n) / S O(n)) \rightarrow\left(\pi_{0}(S O(n))=0\right) \rightarrow \cdots .
$$

Now we consider the fibration $S U(n) / S O(n) \rightarrow L(2 n) \rightarrow S^{1}$; from its exact homotopy sequence

$$
\rightarrow 0=\pi_{1}(S U(n) / S O(n)) \rightarrow \pi_{1}(L(2 n)) \rightarrow \pi_{1}\left(S^{1}\right) \rightarrow \pi_{0}(S U(n) / S O(n))=0
$$

we conclude that $\pi_{1}(L(2 n))=\pi_{1}\left(S^{1}\right)=\mathbb{Z}$. Also (by the Hurewicz homomorphism) we have $H^{1}(L(2 n), \mathbb{Z})=\mathbb{Z}$ and thus $H^{1}(L(2 n), \mathbb{R})=\mathbb{R}$.

Let $\left.\frac{d z}{2 \pi \sqrt{-1} z}\right|_{S^{1}}=\left.\frac{x d y-y d x}{2 \pi \sqrt{-1}}\right|_{S^{1}} \in \Omega^{1}\left(S^{1}\right)$ be a generator of $H^{1}\left(S^{1}, \mathbb{Z}\right)$. Then the pullback $\left(\operatorname{det}^{2}\right)^{*} \frac{d z}{2 \pi \sqrt{-1} z}=\left(\operatorname{det}^{2}\right)^{*} \frac{x d y-y d x}{2 \pi \sqrt{-1}} \in \Omega^{1}(L(2 n))$ is a generator of $H^{1}(L(2 n))$. Its cohomology class is called the Maslov class.

31.8. Symplectic manifolds and their submanifolds. A symplectic manifold $(M, \omega)$ is a manifold $M$ together with a 2 -form $\omega \in \Omega^{2}(M)$ such that $d \omega=0$ and $\omega_{x} \in \bigwedge^{2} T_{x}^{*} M$ is a symplectic structure on $T_{x} M$ for each $x \in M$. So $\operatorname{dim}(M)$ is even; $\operatorname{dim}(M)=2 n$, say. Moreover, $\omega^{\wedge n}=\omega \wedge \cdots \wedge \omega$ is a volume form on $M$ (nowhere zero) called the Liouville volume, which fixes also an orientation of $M$. 
Among the submanifolds $N$ of $M$ we can single out those whose tangent spaces $T_{x} N$ have special relations to the symplectic structure $\omega_{x}$ on $T_{x} M$ as listed in (31.4), Thus a submanifold $N$ of $M$ is called:

$$
\begin{array}{llll}
\text { isotropic } & \text { if } \quad T_{x} N \subseteq T_{x} N^{\omega \perp} \text { for each } x \in N & \Rightarrow \operatorname{dim}(N) \leq n, \\
\text { coisotropic } & \text { if } \quad T_{x} N \supseteq T_{x} N^{\omega \perp} \text { for each } x \in N & \Rightarrow \operatorname{dim}(N) \geq n, \\
\text { Lagrangian } & \text { if } \quad T_{x} N=T_{x} N^{\omega \perp} \text { for each } x \in N & \Rightarrow \operatorname{dim}(N)=n, \\
\text { symplectic } & \text { if } \quad T_{x} N \cap T_{x} N^{\omega \perp}=0 \text { for each } x \in N & \Rightarrow \operatorname{dim}(N)=\text { even, }
\end{array}
$$

where for a linear subspace $W \subset T_{x} N$ its symplectic orthogonal is given by $W^{\omega \perp}=\left\{X \in T_{x} M: \omega_{x}(X, Y)=0\right.$ for all $\left.Y \in W\right\}$, as in (31.4).

31.9. The cotangent bundle. Consider the manifold $M=T^{*} Q$, where $Q$ is a manifold. Recall that for any smooth $f: Q \rightarrow P$ which is locally a diffeomorphism we get a homomorphism of vector bundles $T^{*} f: T^{*} Q \rightarrow$ $T^{*} P$ covering $f$ by $T_{x}^{*} f=\left(\left(T_{x} f\right)^{-1}\right)^{*}: T_{x}^{*} Q \rightarrow T_{f(x)}^{*} P$.

There is a canonical 1-form $\vartheta=\vartheta_{Q} \in \Omega^{1}\left(T^{*} Q\right)$, called the Liouville form, which is given by

$$
\vartheta(X)=\left\langle\pi_{T^{*} Q}(X), T\left(\pi_{Q}\right)(X)\right\rangle, \quad X \in T\left(T^{*} Q\right),
$$

where we used the projections (and their local forms):
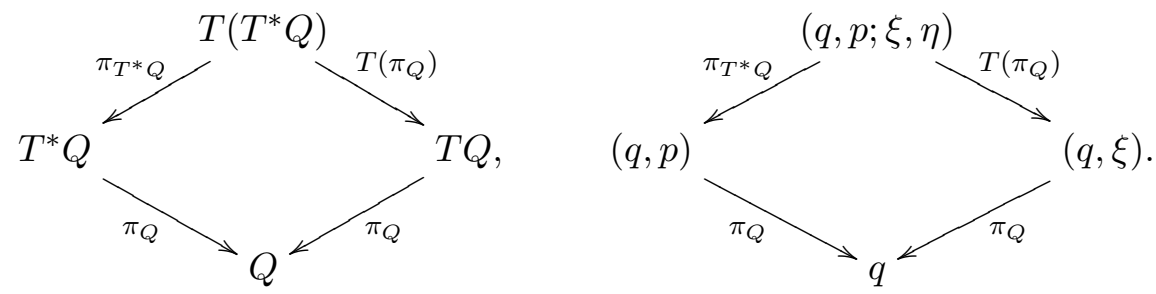

For a chart $q=\left(q^{1}, \ldots, q^{n}\right): U \rightarrow \mathbb{R}^{n}$ on $Q$ and its induced (cotangent) chart $T^{*} q: T^{*} U \rightarrow \mathbb{R}^{n} \times\left(\mathbb{R}^{n}\right)^{*}$, where $T_{x}^{*} q=\left(T_{x} q^{-1}\right)^{*}$, we consider the 'momentum coordinates' $p_{i}:=\left\langle T^{*} q(\quad), e_{i}\right\rangle: T^{*} U \rightarrow \mathbb{R}$. Then $\left(q^{1}, \ldots, q^{n}, p_{1}, \ldots, p_{n}\right): T^{*} U \rightarrow \mathbb{R}^{n} \times\left(\mathbb{R}^{n}\right)^{*}$ are the canonically induced coordinates on the cotangent bundle. In these coordinates we have

$$
\vartheta_{Q}=\sum_{i=1}^{n}\left(\vartheta_{Q}\left(\frac{\partial}{\partial q^{i}}\right) d q^{i}+\vartheta_{Q}\left(\frac{\partial}{\partial p_{i}}\right) d p_{i}\right)=\sum_{i=1}^{n} p_{i} d q^{i}+0,
$$

since $\vartheta_{Q}\left(\frac{\partial}{\partial q^{i}}\right)=\vartheta_{\mathbb{R}^{n}}\left(q, p ; e_{i}, 0\right)=\left\langle p, e_{i}\right\rangle=p_{i}$.

Now we define the canonical symplectic structure $\omega_{Q}=\omega \in \Omega^{2}\left(T^{*} Q\right)$ by

$$
\omega_{Q}:=-d \vartheta_{Q} \stackrel{\text { locally }}{=} \sum_{i=1}^{n} d q^{i} \wedge d p_{i}
$$


Note that $\check{\omega}\left(\frac{\partial}{\partial q^{i}}\right)=d p_{i}$ and $\check{\omega}\left(\frac{\partial}{\partial p_{i}}\right)=-d q^{i}$.

Lemma. The 1-form $\vartheta_{Q} \in \Omega^{1}\left(T^{*} Q\right)$ has the following universal property and is uniquely determined by it:

Any 1-form $\varphi \in \Omega^{1}(Q)$ is a smooth section $\varphi: Q \rightarrow T^{*} Q$ and for the pullback we have $\varphi^{*} \vartheta_{Q}=\varphi \in \Omega^{1}(Q)$. Moreover, $\varphi^{*} \omega_{Q}=-d \varphi \in \Omega^{2}(Q)$.

The 1-form $\vartheta_{Q}$ is natural in $Q \in \mathcal{M} f_{n}$ : For every local diffeomorphism $f: Q \rightarrow P$ the local diffeomorphism $T^{*} f: T^{*} Q \rightarrow T^{*} P$ satisfies $\left(T^{*} f\right)^{*} \vartheta_{P}=$ $\vartheta_{Q}$, and a fortiori $\left(T^{*} f\right)^{*} \omega_{P}=\omega_{Q}$.

In this sense $\vartheta_{Q}$ is a universal 1-form, or a universal connection, and $\omega_{Q}$ is the universal curvature, for $\mathbb{R}^{1}$-principal bundles over $Q$. Compare with section (19).

Proof. For a 1 -form $\varphi \in \Omega^{1}(Q)$ we have

$$
\begin{aligned}
\left(\varphi^{*} \vartheta_{Q}\right)\left(X_{x}\right) & =\left(\vartheta_{Q}\right)_{\varphi_{x}}\left(T_{x} \varphi \cdot X_{x}\right)=\varphi_{x}\left(T_{\varphi_{x}} \pi_{Q} \cdot T_{x} \varphi \cdot X_{x}\right) \\
& =\varphi_{x}\left(T_{x}\left(\pi_{Q} \circ \varphi\right) \cdot X_{x}\right)=\varphi_{x}\left(X_{x}\right) .
\end{aligned}
$$

Thus $\varphi^{*} \vartheta_{Q}=\varphi$. Clearly this equation describes $\vartheta_{Q}$ uniquely. For $\omega$ we have $\varphi^{*} \omega_{Q}=-\varphi^{*} d \vartheta_{Q}=-d \varphi^{*} \vartheta_{Q}=-d \varphi$.

For a local diffeomorphism $f: Q \rightarrow P$, for $\alpha \in T_{x}^{*} Q$, and for $X_{\alpha} \in T_{\alpha}\left(T^{*} Q\right)$ we compute as follows:

$$
\begin{gathered}
\left(\left(T^{*} f\right)^{*} \vartheta_{P}\right)_{\alpha}\left(X_{\alpha}\right)=\left(\vartheta_{P}\right)_{T^{*} f \cdot \alpha}\left(T_{\alpha}\left(T^{*} f\right) \cdot X_{\alpha}\right)=\left(T^{*} f \cdot \alpha\right)\left(T\left(\pi_{P}\right) \cdot T\left(T^{*} f\right) \cdot X_{\alpha}\right) \\
=\left(\alpha \circ T_{x} f^{-1}\right)\left(T\left(\pi_{P} \circ T^{*} f\right) \cdot X_{\alpha}\right)=\alpha \cdot T_{x} f^{-1} \cdot T\left(f \circ \pi_{Q}\right) \cdot X_{\alpha} \\
=\alpha\left(T\left(\pi_{Q}\right) \cdot X_{\alpha}\right)=\vartheta_{Q}\left(X_{\alpha}\right) \cdot \quad \square
\end{gathered}
$$

31.10. Lemma. Let $\varphi: T^{*} Q \rightarrow T^{*} P$ be a (globally defined) local diffeomorphism such that $\varphi^{*} \vartheta_{P}=\vartheta_{Q}$. Then there exists a local diffeomorphism $f: Q \rightarrow P$ such that $\varphi=T^{*} f$.

Proof. Let $\xi_{Q}=-\check{\omega}^{-1} \circ \vartheta_{Q} \in \mathfrak{X}\left(T^{*} Q\right)$ be the so-called Liouville vector field:

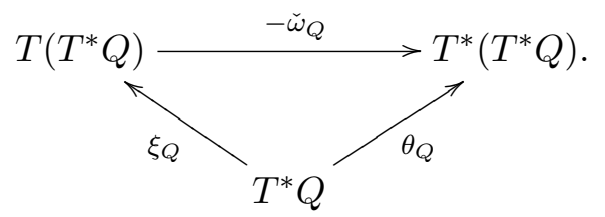

Then locally $\xi_{Q}=\sum_{i=1}^{n} p_{i} \frac{\partial}{\partial p_{i}}$. Its flow is given by $\mathrm{Fl}_{t}^{\xi_{Q}}(\alpha)=e^{t}$. $\alpha$. Since $\varphi^{*} \vartheta_{P}=\vartheta_{Q}$, we also have that the Liouville vector fields $\xi_{Q}$ and $\xi_{P}$ are $\varphi$ dependent. Since $\xi_{Q}=0$ exactly at the zero section, we have $\varphi\left(0_{Q}\right) \subseteq 0_{P}$, so there is a smooth mapping $f: Q \rightarrow P$ with $0_{P} \circ f=\varphi \circ 0_{Q}: Q \rightarrow T^{*} P$. By (3.14) we have $\varphi \circ \mathrm{Fl}_{t}^{\xi_{Q}}=\mathrm{Fl}_{t}^{\xi_{P}} \circ \varphi$, so the image of $\varphi$ of the closure 
of a flow line of $\xi_{Q}$ is contained in the closure of a flow line of $\xi_{P}$. For $\alpha_{x} \in T_{x}^{*} Q$ the closure of the flow line is $[0, \infty) . \alpha_{x}$ and $\varphi\left(0_{x}\right)=0_{f(x)}$; thus $\varphi\left([0, \infty) \cdot \alpha_{x}\right) \subset T_{f(x)}^{*} P$, and $\varphi$ is fiber respecting: $\pi_{P} \circ \varphi=f \circ \pi_{Q}: T^{*} Q \rightarrow P$. Finally, for $X_{\alpha} \in T_{\alpha}\left(T^{*} Q\right)$ we have

$$
\begin{aligned}
\alpha\left(T_{\alpha} \pi_{Q} \cdot X_{\alpha}\right) & =\vartheta_{Q}\left(X_{\alpha}\right)=\left(\varphi^{*} \vartheta_{P}\right)\left(X_{\alpha}\right)=\left(\vartheta_{P}\right)_{\varphi(\alpha)}\left(T_{\alpha} \varphi \cdot X_{\alpha}\right) \\
& =(\varphi(\alpha))\left(T_{\varphi(\alpha)} \pi_{P} \cdot T_{\alpha} \varphi \cdot X_{\alpha}\right)=(\varphi(\alpha))\left(T_{\alpha}\left(\pi_{P} \circ \varphi\right) \cdot X_{\alpha}\right) \\
& =(\varphi(\alpha))\left(T_{\alpha}\left(f \circ \pi_{Q}\right) \cdot X_{\alpha}\right)=(\varphi(\alpha))\left(T f \cdot T_{\alpha} \pi_{Q} \cdot X_{\alpha}\right), \\
\text { thus } \quad \alpha & =\varphi(\alpha) \circ T_{\pi_{Q}(\alpha)} f, \\
\varphi(\alpha) & =\alpha \circ T_{\pi_{Q}(\alpha)} f^{-1}=\left(T_{\pi_{Q}(\alpha)} f^{-1}\right)^{*}(\alpha)=T^{*} f(\alpha) . \quad \square
\end{aligned}
$$

31.11. Time dependent vector fields. Let $f_{t}$ be a smooth curve of diffeomorphisms on a manifold $M$ locally defined for each $t$, with $f_{0}=\operatorname{Id}_{M}$, as in (3.6). We define two time dependent vector fields

$$
\xi_{t}(x):=\left(T_{x} f_{t}\right)^{-1} \frac{\partial}{\partial t} f_{t}(x), \quad \eta_{t}(x):=\left(\frac{\partial}{\partial t} f_{t}\right)\left(f_{t}^{-1}(x)\right) .
$$

Then $T\left(f_{t}\right) \cdot \xi_{t}=\frac{\partial}{\partial t} f_{t}=\eta_{t} \circ f_{t}$, so $\xi_{t}$ and $\eta_{t}$ are $f_{t}$-related.

Lemma. In this situation, for $\omega \in \Omega^{k}(M)$ we have:

(1) $i_{\xi_{t}} f_{t}^{*} \omega=f_{t}^{*} i_{\eta_{t}} \omega$.

(2) $\frac{\partial}{\partial t} f_{t}^{*} \omega=f_{t}^{*} \mathcal{L}_{\eta_{t}} \omega=\mathcal{L}_{\xi_{t}} f_{t}^{*} \omega$

Proof. (11) is by computation:

$$
\begin{aligned}
\left(i_{\xi_{t}} f_{t}^{*} \omega\right)_{x}\left(X_{2}, \ldots, X_{k}\right) & =\left(f_{t}^{*} \omega\right)_{x}\left(\xi_{t}(x), X_{2}, \ldots, X_{k}\right) \\
& =\omega_{f_{t}(x)}\left(T_{x} f_{t} \cdot \xi_{t}(x), T_{x} f_{t} \cdot X_{2}, \ldots, T_{x} f_{t} \cdot X_{k}\right) \\
& =\omega_{f_{t}(x)}\left(\eta_{t}\left(f_{t}(x)\right), T_{x} f_{t} \cdot X_{2}, \ldots, T_{x} f_{t} \cdot X_{k}\right) \\
& =\left(f_{t}^{*} i_{\eta_{t}} \omega\right)_{x}\left(X_{2}, \ldots, X_{k}\right) .
\end{aligned}
$$

(2) We put $\bar{\eta} \in \mathfrak{X}(\mathbb{R} \times M), \bar{\eta}(t, x)=\left(\partial_{t}, \eta_{t}(x)\right)$. We recall from (3.30) the evolution operator for $\eta_{t}$ :

$$
\Phi^{\eta}: \mathbb{R} \times \mathbb{R} \times M \rightarrow M, \quad \frac{\partial}{\partial t} \Phi_{t, s}^{\eta}(x)=\eta_{t}\left(\Phi_{t, s}^{\eta}(x)\right), \quad \Phi_{s, s}^{\eta}(x)=x,
$$

which satisfies

$$
\left(t, \Phi_{t, s}^{\eta}(x)\right)=\mathrm{Fl}_{t-s}^{\bar{\eta}}(s, x), \quad \Phi_{t, s}^{\eta}=\Phi_{t, r}^{\eta} \circ \Phi_{r, s}^{\eta}(x) .
$$

Since $f_{t}$ satisfies $\frac{\partial}{\partial t} f_{t}=\eta_{t} \circ f_{t}$ and $f_{0}=\operatorname{Id}_{M}$, we may conclude that $f_{t}=\Phi_{t, 0}^{\eta}$, or $\left(t, f_{t}(x)\right)=\mathrm{Fl}_{t}^{\bar{\eta}}(0, x)$, so $f_{t}=\mathrm{pr}_{2} \circ \mathrm{Fl}_{t}^{\bar{\eta}} \circ$ ins $_{0}$. Thus

$$
\begin{aligned}
\frac{\partial}{\partial t} f_{t}^{*} \omega & =\frac{\partial}{\partial t}\left(\mathrm{pr}_{2} \circ \mathrm{Fl}_{t}^{\bar{\eta}} \circ \operatorname{ins}_{0}\right)^{*} \omega=\operatorname{ins}_{0}^{*} \frac{\partial}{\partial t}\left(\mathrm{Fl}_{t}^{\bar{\eta}}\right)^{*} \operatorname{pr}_{2}^{*} \omega \\
& =\operatorname{ins}_{0}^{*}\left(\mathrm{Fl}_{t}^{\bar{\eta}}\right)^{*} \mathcal{L}_{\bar{\eta}} \operatorname{pr}_{2}^{*} \omega .
\end{aligned}
$$


For time independent vector fields $X_{i}$ on $M$ we have, using (9.6)

$$
\begin{aligned}
\left.\left(\mathcal{L}_{\bar{\eta}} \operatorname{pr}_{2}^{*} \omega\right)\left(0 \times X_{1}, \ldots, 0 \times X_{k}\right)\right|_{(t, x)}=\left.\bar{\eta}\left(\left(\operatorname{pr}_{2}^{*} \omega\right)\left(0 \times X_{1}, \ldots\right)\right)\right|_{(t, x)} \\
\quad-\left.\sum_{i}\left(\operatorname{pr}_{2}^{*} \omega\right)\left(0 \times X_{1}, \ldots,\left[\bar{\eta}, 0 \times X_{i}\right], \ldots, 0 \times X_{k}\right)\right|_{(t, x)} \\
=\left(\partial_{t}, \eta_{t}(x)\right)\left(\omega\left(X_{1}, \ldots, X_{k}\right)\right)-\left.\sum_{i} \omega\left(X_{1}, \ldots,\left[\eta_{t}, X_{i}\right], \ldots, X_{k}\right)\right|_{x} \\
=\left(\mathcal{L}_{\eta_{t}} \omega\right)_{x}\left(X_{1}, \ldots, X_{k}\right) .
\end{aligned}
$$

This implies for $X_{i} \in T_{x} M$

$$
\begin{aligned}
\left(\frac{\partial}{\partial t} f_{t}^{*} \omega\right)_{x}\left(X_{1}, \ldots, X_{k}\right) & =\left(\operatorname{ins}_{0}^{*}\left(\mathrm{Fl}_{t}^{\bar{\eta}}\right)^{*} \mathcal{L}_{\bar{\eta}} \operatorname{pr}_{2}^{*} \omega\right)_{x}\left(X_{1}, \ldots, X_{k}\right) \\
& =\left(\left(\mathrm{Fl}_{t}^{\bar{\eta}}\right)^{*} \mathcal{L}_{\bar{\eta}} \operatorname{pr}_{2}^{*} \omega\right)_{(0, x)}\left(0 \times X_{1}, \ldots, 0 \times X_{k}\right) \\
& =\left(\mathcal{L}_{\bar{\eta}} \operatorname{pr}_{2}^{*} \omega\right)_{\left(t, f_{t}(x)\right)}\left(0_{t} \times T_{x} f_{t} \cdot X_{1}, \ldots, 0_{t} \times T_{x} t_{x} . X_{k}\right) \\
& =\left(\mathcal{L}_{\eta_{t}} \omega\right)_{f_{t}(x)}\left(T_{x} f_{t} \cdot X_{1}, \ldots, T_{x} t_{x} . X_{k}\right) \\
& =\left(f_{t}^{*} \mathcal{L}_{\eta_{t}} \omega\right)_{x}\left(X_{1}, \ldots, X_{k}\right),
\end{aligned}
$$

which proves the first part of (2). The second part now follows by using (1):

$$
\begin{aligned}
\frac{\partial}{\partial t} f_{t}^{*} \omega & =f_{t}^{*} \mathcal{L}_{\eta_{t}} \omega=f_{t}^{*}\left(d i_{\eta_{t}}+i_{\eta_{t}} d\right) \omega=d f_{t}^{*} i_{\eta_{t}} \omega+f_{t}^{*} i_{\eta_{t}} d \omega \\
& =d i_{\xi_{t}} f_{t}^{*} \omega+i_{\xi_{t}} f_{t}^{*} d \omega=d i_{\xi_{t}} f_{t}^{*} \omega+i_{\xi_{t}} d f_{t}^{*} \omega=\mathcal{L}_{\xi_{t}} f_{t}^{*} \omega
\end{aligned}
$$

31.12. Surfaces. Let $M$ be an orientable 2-dimensional manifold. Let $\omega \in \Omega^{2}(M)$ be a volume form on $M$. Then $d \omega=0$, so $(M, \omega)$ is a symplectic manifold. There are not many different symplectic structures on $M$ if $M$ is compact, since we have:

31.13. Theorem (J. Moser). Let $M$ be a connected compact oriented manifold. Let $\omega_{0}, \omega_{1} \in \Omega^{\operatorname{dim} M}(M)$ be two volume forms (both $>0$ ).

If $\int_{M} \omega_{0}=\int_{M} \omega_{1}$, then there is a diffeomorphism $f: M \rightarrow M$ such that $f^{*} \omega_{1}=\omega_{0}$.

Proof. Put $\omega_{t}:=\omega_{0}+t\left(\omega_{1}-\omega_{0}\right)$ for $t \in[0,1]$; then each $\omega_{t}$ is a volume form on $M$ since these form a convex set.

We look for a curve of diffeomorphisms $t \mapsto f_{t}$ with $f_{t}^{*} \omega_{t}=\omega_{0}$; then $\frac{\partial}{\partial t}\left(f_{t}^{*} \omega_{t}\right)=0$. Since $\int_{M}\left(\omega_{1}-\omega_{0}\right)=0$, we have $\left[\omega_{1}-\omega_{0}\right]=0 \in H^{m}(M)$, so $\omega_{1}-\omega_{0}=d \psi$ for some $\psi \in \Omega^{m-1}(M)$. Put $\eta_{t}:=\left(\frac{\partial}{\partial t} f_{t}\right) \circ f_{t}^{-1}$; then by (31.11) we have:

$$
\begin{aligned}
& 0 \stackrel{\text { wish }}{=} \frac{\partial}{\partial t}\left(f_{t}^{*} \omega_{t}\right)=f_{t}^{*} \mathcal{L}_{\eta_{t}} \omega_{t}+f_{t}^{*} \frac{\partial}{\partial t} \omega_{t}=f_{t}^{*}\left(\mathcal{L}_{\eta_{t}} \omega_{t}+\omega_{1}-\omega_{0}\right), \\
& 0 \stackrel{\text { wish }}{=} \mathcal{L}_{\eta_{t}} \omega_{t}+\omega_{1}-\omega_{0}=d i_{\eta_{t}} \omega_{t}+i_{\eta_{t}} d \omega_{t}+d \psi=d i_{\eta_{t}} \omega_{t}+d \psi .
\end{aligned}
$$

We can choose $\eta_{t}$ uniquely by $i_{\eta_{t}} \omega_{t}=-\psi$, since $\omega_{t}$ is nondegenerate for all $t$. Then the evolution operator $f_{t}=\Phi_{t, 0}^{\eta}$ exists for $t \in[0,1]$ since $M$ is 
compact, by (3.30). We have, using (31.11.2).

$$
\frac{\partial}{\partial t}\left(f_{t}^{*} \omega_{t}\right)=f_{t}^{*}\left(\mathcal{L}_{\eta_{t}} \omega_{t}+d \psi\right)=f_{t}^{*}\left(d i_{\eta_{t}} \omega_{t}+d \psi\right)=0,
$$

so $f_{t}^{*} \omega_{t}=$ constant $=\omega_{0}$.

31.14. Coadjoint orbits of a Lie group. Let $G$ be a Lie group with Lie algebra $\mathfrak{g}$ and dual space $\mathfrak{g}^{*}$, and consider the adjoint representation Ad $: G \rightarrow G L(\mathfrak{g})$. The coadjoint representation $\mathrm{Ad}^{*}: G \rightarrow G L\left(\mathfrak{g}^{*}\right)$ is then given by $\operatorname{Ad}^{*}(g) \alpha:=\alpha \circ \operatorname{Ad}\left(g^{-1}\right)=\operatorname{Ad}\left(g^{-1}\right)^{*}(\alpha)$. For $\alpha \in \mathfrak{g}^{*}$ we consider the coadjoint orbit G. $\alpha \subset \mathfrak{g}^{*}$ which is diffeomorphic to the homogenous space $G / G_{\alpha}$, where $G_{\alpha}$ is the isotropy group $\left\{g \in G: \operatorname{Ad}^{*}(g) \alpha=\alpha\right\}$ at $\alpha$.

As in (6.2), for $X \in \mathfrak{g}$ we consider the fundamental vector field $\zeta_{X}=$ $-\operatorname{ad}(X)^{*} \in \mathfrak{X}\left(\mathfrak{g}^{*}\right)$ of the coadjoint action. For any $Y \in \mathfrak{g}$ we consider the linear function $\operatorname{ev}_{Y}: \mathfrak{g}^{*} \rightarrow \mathbb{R}$. The Lie derivative of the fundamental vector field $\zeta_{X}$ on the function $\mathrm{ev}_{Y}$ is then given by

(1) $\mathcal{L}_{\zeta_{X}} \operatorname{ev}_{Y}=-d \operatorname{ev}_{Y} \circ \operatorname{ad}(X)^{*}=-\operatorname{ev}_{Y} \circ \operatorname{ad}(X)^{*}=\operatorname{ev}_{[Y, X]}, \quad X, Y \in \mathfrak{g}$.

Note that the tangent space to the orbit is $T_{\beta}(G . \alpha)=\left\{\zeta_{X}(\beta): X \in \mathfrak{g}\right\}$. Now we define the symplectic structure on the orbit $O=G . \alpha$ by

$$
\begin{aligned}
\left(\omega_{O}\right)_{\alpha}\left(\zeta_{X}, \zeta_{Y}\right) & =\alpha([X, Y])=\langle\alpha,[X, Y]\rangle, \quad \alpha \in \mathfrak{g}^{*}, \quad X, Y \in \mathfrak{g}, \\
\omega_{O}\left(\zeta_{X}, \zeta_{Y}\right) & =\operatorname{ev}_{[X, Y]} .
\end{aligned}
$$

Theorem (Kirillov, Kostant, Souriau). If $G$ is a Lie group, then any coadjoint orbit $O \subset \mathfrak{g}^{*}$ carries a canonical symplectic structure $\omega_{O}$ which is invariant under the coadjoint action of $G$.

Proof. First we claim that for $X \in \mathfrak{g}$ we have $X \in \mathfrak{g}_{\alpha}=\left\{Z \in \mathfrak{g}: \zeta_{Z}(\alpha)=0\right\}$ if and only if $\alpha([X, \quad])=\left(\omega_{O}\right)_{\alpha}\left(\zeta_{X}, \quad\right)=0$. Indeed, for $Y \in \mathfrak{g}$ we have

$$
\begin{aligned}
\langle\alpha,[X, Y]\rangle & =\left\langle\alpha,\left.\partial\right|_{0} \operatorname{Ad}(\exp (t X)) Y\right\rangle=\left.\partial\right|_{0}\langle\alpha, \operatorname{Ad}(\exp (t X)) Y\rangle \\
& =\left.\partial\right|_{0}\left\langle\operatorname{Ad}^{*}(\exp (-t X)) \alpha, Y\right\rangle=-\left\langle\zeta_{X}(\alpha), Y\right\rangle=0 .
\end{aligned}
$$

This shows that $\omega_{O}$ as defined in (2) is well defined and also nondegenerate along each orbit.

Now we show that $d \omega_{O}=0$, using (2):

$$
\begin{aligned}
\left(d \omega_{O}\right)\left(\zeta_{X}, \zeta_{Y}, \zeta_{Z}\right) & =\sum_{\text {cyclic }} \zeta_{X} \omega_{O}\left(\zeta_{Y}, \zeta_{Z}\right)-\sum_{\text {cyclic }} \omega_{O}\left(\left[\zeta_{X}, \zeta_{Y}\right], \zeta_{Z}\right) \\
& \left.=\sum_{\text {cyclic }} \zeta_{X} \operatorname{ev}_{[Y, Z]}-\sum_{\text {cyclic }} \omega_{O}\left(\zeta_{-[X, Y]}, \zeta_{Z}\right) \quad \text { (now use (10) }\right) \\
& =\sum_{\text {cyclic }} \operatorname{ev}_{[[Y, Z], X]}+\sum_{\text {cyclic }} \operatorname{ev}_{[[X, Y], Z]}=0 \quad \text { by Jacobi. }
\end{aligned}
$$


Finally we show that $\omega_{O}$ is $G$-invariant: For $g \in G$ we have

$$
\begin{aligned}
& \left(\left(\operatorname{Ad}^{*}(g)\right)^{*} \omega_{O}\right)_{\alpha}\left(\zeta_{X}(\alpha), \zeta_{Y}(\alpha)\right) \\
& \quad=\left(\omega_{O}\right)_{\operatorname{Ad}^{*}(g) \alpha}\left(T\left(\operatorname{Ad}^{*}(g)\right) \cdot \zeta_{X}(\alpha), T\left(\operatorname{Ad}^{*}(g)\right) \cdot \zeta_{Y}(\alpha)\right) \\
& \quad=\left(\omega_{O}\right)_{\operatorname{Ad}^{*}(g) \alpha}\left(\zeta_{\operatorname{Ad}(g) X}\left(\operatorname{Ad}^{*}(g) \alpha\right), \zeta_{\operatorname{Ad}(g) Y}\left(\operatorname{Ad}^{*}(g) \alpha\right)\right), \quad \text { by }(6.2 .2), \\
& \quad=\left(\operatorname{Ad}^{*}(g) \alpha\right)([\operatorname{Ad}(g) X, \operatorname{Ad}(g) Y]) \\
& \quad=\left(\alpha \circ \operatorname{Ad}\left(g^{-1}\right)\right)(\operatorname{Ad}(g)[X, Y])=\alpha([X, Y])=\left(\omega_{O}\right)_{\alpha}\left(\zeta_{X}, \zeta_{Y}\right) . \quad \square
\end{aligned}
$$

31.15. Theorem (Darboux). Let $(M, \omega)$ be a symplectic manifold of dimension $2 n$. Then for each $x \in M$ there exists a chart $(U, u)$ of $M$ centered at $x$ such that $\omega \mid U=\sum_{i=1}^{n} d u^{i} \wedge d u^{n+i}$. So each symplectic manifold is locally symplectomorphic to a cotangent bundle.

Proof. Take any chart $\left(U, u: U \rightarrow u(U) \subset \mathbb{R}^{2 n}\right)$ centered at $x$. Choose linear coordinates on $\mathbb{R}^{2 n}$ in such a way that $\omega_{x}=\left.\sum_{i=1}^{n} d u^{i} \wedge d u^{n+i}\right|_{x}$ at $x$ only. Then $\omega_{0}=\omega \mid U$ and $\omega_{1}=\sum_{i=1}^{n} d u^{i} \wedge d u^{n+i}$ are two symplectic structures on the open set $U \subset M$ which agree at $x$. Put $\omega_{t}:=\omega_{0}+t\left(\omega_{1}-\omega_{0}\right)$. By making $U$ smaller if necessary, we may assume that $\omega_{t}$ is a symplectic structure for all $t \in[0,1]$.

We want to find a curve of diffeomorphisms $f_{t}$ near $x$ with $f_{0}=$ Id such that $f_{t}(x)=x$ and $f_{t}^{*} \omega_{t}=\omega_{0}$. Then $\frac{\partial}{\partial t} f_{t}^{*} \omega_{t}=\frac{\partial}{\partial t} \omega_{0}=0$. We may assume that $U$ is contractible; thus $H^{2}(U)=0$, so $d\left(\omega_{1}-\omega_{0}\right)=0$ implies that $\omega_{1}-\omega_{0}=d \psi$ for some $\psi \in \Omega^{1}(U)$. By adding a constant form (in the chart on $U$ ), we may assume that $\psi_{x}=0$. So we get for the time dependent vector field $\eta_{t}=\frac{\partial}{\partial t} f_{t} \circ f_{t}^{-1}$, using (31.11.2),

$$
\begin{aligned}
0 & =\frac{\partial}{\partial t} f_{t}^{*} \omega_{t}=f_{t}^{*}\left(\mathcal{L}_{\eta_{t}} \omega_{t}+\frac{\partial}{\partial t} \omega_{t}\right)=f_{t}^{*}\left(d i_{\eta_{t}} \omega_{t}+i_{\eta_{t}} d \omega_{t}+\omega_{1}-\omega_{0}\right) \\
& =f_{t}^{*} d\left(i_{\eta_{t}} \omega_{t}+\psi\right) .
\end{aligned}
$$

We can now prescribe $\eta_{t}$ uniquely by $i_{\eta_{t}} \omega_{t}=-\psi$, since $\omega_{t}$ is nondegenerate on $x$. Moreover $\eta_{t}(x)=0$ since $\psi_{x}=0$. On a small neighborhood of $x$ the left evolution operator $f_{t}$ of $\eta_{t}$ exists for all $t \in[0,1]$, and then clearly $\frac{\partial}{\partial t}\left(f_{t}^{*} \omega_{t}\right)=0$, so $f_{t}^{*} \omega_{t}=\omega_{0}$ for all $t \in[0,1]$.

31.16. Relative Poincaré lemma. Let $M$ be a smooth manifold, let $N \subset M$ be a submanifold, and let $k \geq 0$. Let $\omega$ be a closed $(k+1)$-form on $M$ which vanishes when pulled back to $N$. Then there exists a $k$-form $\varphi$ on an open neighborhood $U$ of $N$ in $M$ such that $d \varphi=\omega \mid U$ and $\varphi=0$ along $N$. If moreover $\omega=0$ along $N$ (on $\left.\bigwedge^{k} T M \mid N\right)$, then we may choose $\varphi$ such that the first derivatives of $\varphi$ vanish on $N$.

Proof. By restricting to a tubular neighborhood of $N$ in $M$, we may assume that $p: M=: E \rightarrow N$ is a smooth vector bundle and that $i: N \rightarrow E$ is 
the zero section of the bundle. We consider $\mu: \mathbb{R} \times E \rightarrow E$, given by $\mu(t, x)=\mu_{t}(x)=t x$; then $\mu_{1}=\operatorname{Id}_{E}$ and $\mu_{0}=i \circ p: E \rightarrow N \rightarrow E$. Let $\xi \in \mathfrak{X}(E)$ be the vertical vector field $\xi(x)=\operatorname{vl}(x, x)=\left.\partial\right|_{0}(x+t x)$; then $\mathrm{Fl}_{t}^{\xi}=\mu_{e^{t}}$. So locally for $t$ near $(0,1]$ we have

$$
\begin{aligned}
\frac{d}{d t} \mu_{t}^{*} \omega & =\frac{d}{d t}\left(\mathrm{Fl}_{\log t}^{\xi}\right)^{*} \omega=\frac{1}{t}\left(\mathrm{Fl}_{\log t}^{\xi}\right)^{*} \mathcal{L}_{\xi} \omega \text { by }(31.11) \text { or }(8.16) \\
& =\frac{1}{t} \mu_{t}^{*}\left(i_{\xi} d \omega+d i_{\xi} \omega\right)=\frac{1}{t} d \mu_{t}^{*} i_{\xi} \omega .
\end{aligned}
$$

For $x \in E$ and $X_{1}, \ldots, X_{k} \in T_{x} E$ we may compute

$$
\begin{aligned}
\left(\frac{1}{t} \mu_{t}^{*} i_{\xi} \omega\right)_{x}\left(X_{1}, \ldots, X_{k}\right) & =\frac{1}{t}\left(i_{\xi} \omega\right)_{t x}\left(T_{x} \mu_{t} \cdot X_{1}, \ldots, T_{x} \mu_{t} \cdot X_{k}\right) \\
& =\frac{1}{t} \omega_{t x}\left(\xi(t x), T_{x} \mu_{t} \cdot X_{1}, \ldots, T_{x} \mu_{t} \cdot X_{k}\right) \\
& =\omega_{t x}\left(\operatorname{vl}(t x, x), T_{x} \mu_{t} \cdot X_{1}, \ldots, T_{x} \mu_{t} \cdot X_{k}\right) .
\end{aligned}
$$

So if $k \geq 0$, the $k$-form $\frac{1}{t} \mu_{t}^{*} i_{\xi} \omega$ is defined and smooth in $(t, x)$ for all $t$ near $[0,1]$ and describes a smooth curve in $\Omega^{k}(E)$. Note that for $x \in N=0_{E}$ we have $\left(\frac{1}{t} \mu_{t}^{*} i_{\xi} \omega\right)_{x}=0$, and if $\omega=0$ along $N$, then $\frac{1}{t} \mu_{t}^{*} i_{\xi} \omega$ vanishes of second order along $N$. Since $\mu_{0}^{*} \omega=p^{*} i^{*} \omega=0$ and $\mu_{1}^{*} \omega=\omega$, we have

$$
\begin{aligned}
\omega & =\mu_{1}^{*} \omega-\mu_{0}^{*} \omega=\int_{0}^{1} \frac{d}{d t} \mu_{t}^{*} \omega d t \\
& =\int_{0}^{1} d\left(\frac{1}{t} \mu_{t}^{*} i_{\xi} \omega\right) d t=d\left(\int_{0}^{1} \frac{1}{t} \mu_{t}^{*} i_{\xi} \omega d t\right)=: d \varphi .
\end{aligned}
$$

If $x \in N$, we have $\varphi_{x}=0$, and also the last claim is obvious from the explicit form of $\varphi$.

31.17. Lemma. Let $M$ be a smooth finite-dimensional manifold, let $N \subset$ $M$ be a submanifold, and let $\omega_{0}$ and $\omega_{1}$ be symplectic forms on $M$ which are equal along $N$ (on $\left.\bigwedge^{2} T M \mid N\right)$.

Then there exists a diffeomorphism $f: U \rightarrow V$ between two open neighborhoods $U$ and $V$ of $N$ in $M$ which satisfies $f\left|N=\operatorname{Id}_{N}, T f\right|(T M \mid N)=$ $\operatorname{Id}_{T M \mid N}$, and $f^{*} \omega_{1}=\omega_{0}$.

Proof. Let $\omega_{t}=\omega_{0}+t\left(\omega_{1}-\omega_{0}\right)$ for $t \in[0,1]$. Since the restrictions of $\omega_{0}$ and $\omega_{1}$ to $\bigwedge^{2} T M \mid N$ are equal, there is an open neighborhood $U_{1}$ of $N$ in $M$ such that $\omega_{t}$ is a symplectic form on $U_{1}$, for all $t \in[0,1]$. If $i: N \rightarrow M$ is the inclusion, we also have $i^{*}\left(\omega_{1}-\omega_{0}\right)=0$, and by assumption $d\left(\omega_{1}-\omega_{0}\right)=0$. Thus by lemma (31.16) there is a smaller open neighborhood $U_{2}$ of $N$ such that $\omega_{1}\left|U_{2}-\omega_{0}\right| U_{2}=d \varphi$ for some $\varphi \in \Omega^{1}\left(U_{2}\right)$ with $\varphi_{x}=0$ for $x \in N$, such that also all first derivatives of $\varphi$ vanish along $N$.

Let us now consider the time dependent vector field $X_{t}:=-\left(\omega_{t}\right)^{-1} \circ \varphi$ given by $i_{X_{t}} \omega_{t}=\varphi$, which vanishes together with all first derivatives along $N$. Let $f_{t}$ be the curve of local diffeomorphisms with $\frac{\partial}{\partial t} f_{t}=X_{t} \circ f_{t}$; then $f_{t} \mid N=\operatorname{Id}_{N}$ 
and $T f_{t} \mid(T M \mid N)=\mathrm{Id}$. There is a smaller open neighborhood $U$ of $N$ such that $f_{t}$ is defined on $U$ for all $t \in[0,1]$. Then by (31.11) we have

$$
\begin{aligned}
\frac{\partial}{\partial t}\left(f_{t}^{*} \omega_{t}\right) & =f_{t}^{*} \mathcal{L}_{X_{t}} \omega_{t}+f_{t}^{*} \frac{\partial}{\partial t} \omega_{t}=f_{t}^{*}\left(d i_{X_{t}} \omega_{t}+\omega_{1}-\omega_{0}\right) \\
& =f_{t}^{*}\left(-d \varphi+\omega_{1}-\omega_{0}\right)=0,
\end{aligned}
$$

so $f_{t}^{*} \omega_{t}$ is constant in $t$, equals $f_{0}^{*} \omega_{0}=\omega_{0}$, and finally $f_{1}^{*} \omega_{1}=\omega_{0}$ as required.

31.18. Lemma (Ehresmann). Let $(V, \omega)$ be a symplectic vector space of real dimension $2 n$, and let $g$ be a nondegenerate symmetric bilinear form on $V$. Let $K:=\check{g}^{-1} \circ \check{\omega}: V \rightarrow V^{*} \rightarrow V$ so that $g(K v, w)=\omega(v, w)$.

Then $K \in G L(V)$ and the following properties are equivalent:

(1) $K^{2}=-\operatorname{Id}_{V}$, so $K$ is a complex structure.

(2) $\omega(K v, K w)=\omega(v, w)$, so $K \in S p(V, \omega)$.

(3) $g(K v, K w)=g(v, w)$, so $K \in O(V, g)$.

If these conditions are satisfied, any subpair of the triple $\omega, g, J$ is said to be compatible.

Proof. Starting from the definition, we have in turn:

$$
\begin{aligned}
g(K v, w) & =\langle\check{g} K(v), w\rangle=\left\langle\check{g} \check{g}^{-1} \check{\omega}(v), w\right\rangle=\langle\check{\omega}(v), w\rangle=\omega(v, w), \\
\omega(K v, K w) & =g\left(K^{2} v, K w\right)=g\left(K w, K^{2} v\right)=\omega\left(w, K^{2} v\right)=-\omega\left(K^{2} v, w\right), \\
g\left(K^{2} v, w\right) & =\omega(K v, w)=-\omega(w, K v)=-g(K w, K v)=-g(K v, K w) .
\end{aligned}
$$

The second line shows that (11) $\Leftrightarrow(2)$, and the third line shows that (11) $\Leftrightarrow$ (3).

31.19. Lemma (Polar decomposition of $\omega)$. Let $(V, g)$ be a Euclidean real vector space (positive definite). Let $\omega$ be a symplectic structure on $V$, let $A=\check{g}^{-1} \circ \check{\omega} \in G L(V)$, and let $A=B J$ be the polar decomposition from (4.38), Then $A$ is $g$-skew-symmetric, $J$ is a complex structure, and the nondegenerate symmetric inner product $g_{1}(v, w)=\omega(v, J w)$ is positive definite.

Proof. We have $g(A v, w)=\omega(v, w)=-\omega(w, v)=-g(A w, v)=-g(v, A w)$; thus $A^{\top}=-A$. This has the consequence (see the proof of (4.38) that $B=$ $\exp \left(\frac{1}{2} \log \left(A A^{\top}\right)\right)=\exp \left(\frac{1}{2} \log \left(-A^{2}\right)\right)$ commutes with $A$; thus also $J=B^{-1} A$ commutes with $A$ and thus with $B$. Since $B^{\top}=B$, we get $J^{-1}=J^{\top}=$ $\left(B^{-1} A\right)^{\top}=A^{\top}\left(B^{-1}\right)^{\top}=-A B^{-1}=-B^{-1} A=-J$; thus $J$ is a complex structure. Moreover, we have

$$
\omega(J v, J w)=g(A J v, J w)=g(J A v, J w)=g(A v, w)=\omega(v, w) ;
$$


thus by (31.18) the symplectic form $\omega$ and the complex structure $J$ are compatible, and the symmetric (by (31.18) bilinear form $g_{1}$ defined by $g_{1}(v, w)=\omega(v, J w)$ is positive definite: $g_{1}(v, v)=\omega(v, J v)=g(A v, J v)=$ $g(B J v, J v)>0$ since $B$ is positive definite.

31.20. Relative Darboux theorem (Weinstein). Let $(M, \omega)$ be a symplectic manifold, and let $L \subset M$ be a Lagrangian submanifold.

Then there exist a tubular neighborhood $U$ of $L$ in $M$, an open neigborhood $V$ of the zero section $0_{L}$ in $T^{*} L$ and a symplectomorphism

$$
\left(T^{*} L, \omega_{L}\right) \supset\left(V, \omega_{L}\right) \stackrel{\varphi}{\longrightarrow}(U, \omega \mid U) \subset(M, \omega)
$$

such that $\varphi \circ 0_{L}: L \rightarrow V \rightarrow M$ is the embedding $L \subset M$.

Moreover, suppose that for the Lagrangian subbundle $T L$ in the symplectic vector bundle $T M \mid L \rightarrow L$ we are given a complementary Lagrangian subbundle $E \rightarrow L$; then the symplectomorphism $\varphi$ may be chosen in such a way that $T_{0_{x}} \varphi \cdot V_{0_{x}}\left(T^{*} L\right)=E_{\varphi\left(0_{x}\right)}$ for $x \in L$.

Here $V\left(T^{*} L\right)$ denotes the vertical bundle in the tangent bundle of $T^{*} L$.

Proof. The tangent bundle $T L \rightarrow L$ is a Lagrangian subbundle of the symplectic vector bundle $T M \mid L \rightarrow L$.

Claim. There exists a Lagrangian complementary vector bundle $E \rightarrow L$ in the symplectic vector bundle $T M \mid L$. Namely, we choose a fiberwise Riemann metric $g$ in the vector bundle $T M \mid L \rightarrow L$ and consider the vector bundle homomorphism $A=\check{g}^{-1} \check{\omega}: T M\left|L \rightarrow T^{*} M\right| L \rightarrow T M \mid L$ and its polar decomposition $A=B J$ with respect to $g$ as explained in (31.19), Then $J$ is a fiberwise complex structure, and $g_{1}(u, v):=\omega(u, J v)$ defines again a positive definite fiberwise Riemann metric. Since $g_{1}(J, \quad)=\omega(, \quad)$ vanishes on $T L$, the Lagrangian subbundle $E=J T L \subset T M \mid L$ is $g_{1}$-orthogonal to $T L$, thus a complement.

We may use either the constructed or the given Lagrangian complement to $T L$ in what follows.

The symplectic structure $\omega$ induces a duality pairing between the vector bundles $E$ and $T L$; thus we may identify $(T M \mid L) / T L \cong E \rightarrow L$ with the cotangent bundle $T^{*} L$ by $\left\langle X_{x}, \check{\omega}\left(Y_{x}\right)\right\rangle=\omega\left(X_{x}, Y_{x}\right)$ for $x \in L, X_{x} \in T_{x} L$ and $Y_{x} \in E_{x}$.

Let $\psi:=\exp ^{g} \circ \check{\omega}^{-1}: T^{*} L \rightarrow M$ where $\exp ^{g}$ is any geodesic exponential mapping on $T M$ restricted to $E$. Then $\psi$ is a diffeomorphism from a neighborhood $V$ of the zero section in $T^{*} L$ to a tubular neighborhood $U$ of $L$ in $M$, which equals the embedding of $L$ along the zero section.

Let us consider the pullback $\psi^{*} \omega$ and compare it with $\omega_{L}$ on $V$. For $0_{x} \in 0_{L}$ we have $T_{0_{x}} V=T_{x} L \oplus T_{x}^{*} L \cong T_{x} L \oplus E_{x}$. The linear subspace $T_{x} L$ is 
Lagrangian for both $\omega_{L}$ and $\psi^{*} \omega$ since $L$ is a Lagrange submanifold. The linear subspace $T_{x}^{*} L$ is Lagrangian for $\omega_{L}$, and it is also Lagrangian for $\psi^{*} \omega$ since $E$ was a Lagrangian bundle. Both $\left(\omega_{L}\right)_{0_{x}}$ and $\left(\psi^{*} \omega\right)_{0_{x}}$ induce the same duality between $T_{x} L$ and $T_{x}^{*} L$ since the identification $E_{x} \cong T_{x}^{*} L$ was via $\omega_{x}$. Thus $\omega_{L}$ equals $\psi^{*} \omega$ along the zero section.

Finally, by lemma (31.17) the identity of the zero section extends to a diffeomorphism $\rho$ on a neighborhood with $\rho^{*} \psi^{*} \omega=\omega_{L}$. The diffeomorphism $\varphi=\psi \circ \rho$ then satisfies the theorem.

31.21. The Poisson bracket. Let $(M, \omega)$ be a symplectic manifold. For $f \in C^{\infty}(M)$ the Hamiltonian vector field or symplectic gradient $H_{f}=$ $\operatorname{grad}^{\omega}(f) \in \mathfrak{X}(M)$ is defined by any of the following equivalent prescriptions:

$$
i\left(H_{f}\right) \omega=d f, \quad H_{f}=\check{\omega}^{-1} d f, \quad \omega\left(H_{f}, X\right)=X(f) \text { for } X \in T M .
$$

For two functions $f, g \in C^{\infty}(M)$ we define their Poisson bracket $\{f, g\}$ by

$$
\begin{aligned}
\{f, g\}: & =i\left(H_{f}\right) i\left(H_{g}\right) \omega=\omega\left(H_{g}, H_{f}\right) \\
& =H_{f}(g)=\mathcal{L}_{H_{f}} g=d g\left(H_{f}\right) \in C^{\infty}(M) .
\end{aligned}
$$

Let us furthermore put

$$
\mathfrak{X}(M, \omega):=\left\{X \in \mathfrak{X}(M): \mathcal{L}_{X} \omega=0\right\}
$$

and call this the space of locally Hamiltonian vector fields or $\omega$-respecting vector fields.

Theorem. Let $(M, \omega)$ be a symplectic manifold.

Then $\left(C^{\infty}(M),\{, \quad\}\right)$ is a Lie algebra which also satisfies $\{f, g h\}=$ $\{f, g\} h+g\{f, h\}$, i.e., $\operatorname{ad}_{f}=\{f, \quad\}$ is a derivation of $\left(C^{\infty}(M), \cdot\right)$.

Moreover, there is an exact sequence of Lie algebras and Lie algebra homomorphisms

$$
\begin{array}{cccc}
0 \longrightarrow H^{0}(M) \stackrel{\alpha}{\longrightarrow} & C^{\infty}(M) \stackrel{H=\operatorname{grad}^{\omega}}{\longrightarrow} \mathfrak{X}(M, \omega) \stackrel{\gamma}{\longrightarrow} H^{1}(M) \longrightarrow 0 \\
0 & \left\{\begin{array}{c}
4 \\
0
\end{array} \quad[, \quad]\right. & 0
\end{array}
$$

where the brackets are written under the spaces, where $\alpha$ is the embedding of the space of all locally constant functions, and where $\gamma(X):=\left[i_{X} \omega\right] \in$ $H^{1}(M)$.

The whole situation behaves invariantly (resp. equivariantly) under pullback by symplectomorphisms $\varphi: M \rightarrow M:$ For example $\varphi^{*}\{f, g\}=\left\{\varphi^{*} f, \varphi^{*} g\right\}$, $\varphi^{*}\left(H_{f}\right)=H_{\varphi^{*} f}$, and $\varphi^{*} \gamma(X)=\gamma\left(\varphi^{*} X\right)$. Consequently for $X \in \mathfrak{X}(M, \omega)$ we have $\mathcal{L}_{X}\{f, g\}=\left\{\mathcal{L}_{X} f, g\right\}+\left\{f, \mathcal{L}_{X} g\right\}$ and $\gamma\left(\mathcal{L}_{X} Y\right)=0$. 
Proof. The operator $H$ takes values in $\mathfrak{X}(M, \omega)$ since

$$
\mathcal{L}_{H_{f}} \omega=i_{H_{f}} d \omega+d i_{H_{f}} \omega=0+d d f=0 .
$$

The mapping $H$ is a Lie algebra homomorphism, i.e., $H(\{f, g\})=\left[H_{f}, H_{g}\right]$, since by (9.9) and (9.7) we have

$$
\begin{aligned}
i_{H(\{f, g\})} \omega & =d\{f, g\}=d \mathcal{L}_{H_{f}} g=\mathcal{L}_{H_{f}} d g-0=\mathcal{L}_{H_{f}} i_{H_{g}} \omega-i_{H_{g}} \mathcal{L}_{H_{f}} \omega \\
& =\left[\mathcal{L}_{H_{f}}, i_{H_{g}}\right] \omega=i_{\left[H_{f}, H_{g}\right]} \omega .
\end{aligned}
$$

The sequence is exact at $H^{0}(M)$ since the embedding $\alpha$ of the locally constant functions is injective.

The sequence is exact at $C^{\infty}(M)$ : For a locally constant function $c$ we have $H_{c}=\check{\omega}^{-1} d c=\check{\omega}^{-1} 0=0$. If $H_{f}=\check{\omega}^{-1} d f=0$ for $f \in C^{\infty}(M)$, then $d f=0$, so $f$ is locally constant.

The sequence is exact at $\mathfrak{X}(M, \omega)$ : For $X \in \mathfrak{X}(M, \omega)$ we have $d i_{X} \omega=$ $d i_{X} \omega+i_{X} d \omega=\mathcal{L}_{X} \omega=0$; thus $\gamma(X)=\left[i_{X} \omega\right] \in H^{1}(M)$ is well defined. For $f \in C^{\infty}(M)$ we have $\gamma\left(H_{f}\right)=\left[i_{H_{f}} \omega\right]=[d f]=0 \in H^{1}(M)$. If $X \in \mathfrak{X}(M, \omega)$ with $\gamma(X)=\left[i_{X} \omega\right]=0 \in H^{1}(M)$, then $i_{X} \omega=d f$ for some $f \in \Omega^{0}(M)=$ $C^{\infty}(M)$, but then $X=H_{f}$.

The sequence is exact at $H^{1}(M)$ : The mapping $\gamma$ is surjective since for $\varphi \in \Omega^{1}(M)$ with $d \varphi=0$ we may consider $X:=\check{\omega}^{-1} \varphi \in \mathfrak{X}(M)$ which satisfies $\mathcal{L}_{X} \omega=i_{X} d \omega+d i_{X} \omega=0+d \varphi=0$ and $\gamma(X)=\left[i_{X} \omega\right]=[\varphi] \in H^{1}(M)$.

The Poisson bracket $\{\quad, \quad\}$ is a Lie bracket and $\{f, g h\}=\{f, g\} h+g\{f, h\}$ :

$$
\begin{aligned}
\{f, g\} & =\omega\left(H_{g}, H_{f}\right)=-\omega\left(H_{f}, H_{g}\right) \\
& =\{g, f\}, \\
\{f,\{g, h\}\} & =\mathcal{L}_{H_{f}} \mathcal{L}_{H_{g}} h=\left[\mathcal{L}_{H_{f}}, \mathcal{L}_{H_{g}}\right] h+\mathcal{L}_{H_{g}} \mathcal{L}_{H_{f}} h \\
& =\mathcal{L}_{\left[H_{f}, H_{g}\right]} h+\{g,\{f, h\}\} \\
& =\mathcal{L}_{H_{\{f, g\}}} h+\{g,\{f, h\}\} \\
& =\{\{f, g\}, h\}+\{g,\{f, h\}\}, \\
\{f, g h\} & =\mathcal{L}_{H_{f}}(g h)=\mathcal{L}_{H_{f}}(g) h+g \mathcal{L}_{H_{f}}(h) \\
& =\{f, g\} h+g\{f, h\} .
\end{aligned}
$$

All mappings in the sequence are Lie algebra homomorphisms: For local constants $\left\{c_{1}, c_{2}\right\}=H_{c_{1}} c_{2}=0$. We already checked for $H$. For $X, Y \in$ $\mathfrak{X}(M, \omega)$ we have

$i_{[X, Y]} \omega=\left[\mathcal{L}_{X}, i_{Y}\right] \omega=\mathcal{L}_{X} i_{Y} \omega-i_{Y} \mathcal{L}_{X} \omega=d i_{X} i_{Y} \omega+i_{X} d i_{Y} \omega-0=d i_{X} i_{Y} \omega ;$

thus $\gamma([X, Y])=\left[i_{[X, Y]} \omega\right]=0 \in H^{1}(M)$. 
Let us now transform the situation by a symplectomorphism $\varphi: M \rightarrow M$ via pullback. Then

$$
\begin{aligned}
\varphi^{*} \omega & =\omega \Leftrightarrow(T \varphi)^{*} \circ \check{\omega} \circ T \varphi=\check{\omega} \\
\Rightarrow H_{\varphi^{*} f} & =\check{\omega}^{-1} d \varphi^{*} f=\check{\omega}^{-1}\left(\varphi^{*} d f\right) \\
& =\left(T \varphi^{-1} \circ \check{\omega}^{-1} \circ\left(T \varphi^{-1}\right)^{*}\right) \circ\left((T \varphi)^{*} \circ d f \circ \varphi\right) \\
& =\left(T \varphi^{-1} \circ \check{\omega}^{-1} \circ d f \circ \varphi\right)=\varphi^{*}\left(H_{f}\right), \\
\varphi^{*}\{f, g\} & =\varphi^{*}\left(d g\left(H_{f}\right)\right)=\left(\varphi^{*} d g\right)\left(\varphi^{*} H_{f}\right)=d\left(\varphi^{*} g\right)\left(H_{\varphi^{*} f}\right)=\left\{\varphi^{*} f, \varphi^{*} g\right\} .
\end{aligned}
$$

For the assertions about the Lie derivative apply $\mathcal{L}_{X}=\left.\partial\right|_{0}\left(\mathrm{Fl}_{t}^{X}\right)^{*}$.

31.22. Basic example. In the situation of (31.1), where $M=T^{*} \mathbb{R}^{n}$ with $\omega=\omega_{\mathbb{R}^{n}}=-d \vartheta_{\mathbb{R}^{n}}=\sum_{i=1}^{n} d q^{i} \wedge d p_{i}$, we have

$$
\begin{gathered}
\check{\omega}: T\left(T^{*} \mathbb{R}^{n}\right) \rightarrow T^{*}\left(T^{*} \mathbb{R}^{n}\right), \quad \check{\omega}\left(\partial_{q^{i}}\right)=d p_{i}, \quad \check{\omega}\left(\partial_{p^{i}}\right)=-d q_{i}, \\
H_{f}=\check{\omega}^{-1} . d f=\check{\omega}^{-1}\left(\sum_{i}\left(\frac{\partial f}{\partial q^{i}} d q^{i}+\frac{\partial f}{\partial p_{i}} d p_{i}\right)\right)=\sum_{i}\left(\frac{\partial f}{\partial p_{i}} \frac{\partial}{\partial q^{i}}-\frac{\partial f}{\partial q^{i}} \frac{\partial}{\partial p_{i}}\right), \\
\{f, g\}=H_{f} g=\sum_{i}\left(\frac{\partial f}{\partial p_{i}} \frac{\partial g}{\partial q^{i}}-\frac{\partial f}{\partial q^{i}} \frac{\partial g}{\partial p_{i}}\right), \\
\left\{p_{i}, p_{j}\right\}=0, \quad\left\{q^{i}, q^{j}\right\}=0, \quad\left\{q^{i}, p_{j}\right\}=-\delta_{j}^{i} .
\end{gathered}
$$

31.23. Kepler's laws: Elementary approach. Here we give an elementary approach to the derivation of Kepler's laws.

Let us choose the orthonormal coordinate system in the oriented Euclidean space $\mathbb{R}^{3}$ with standard inner product ( $\mid \quad$ ) and vector product $q \times q^{\prime}$ in such a way that the sun with mass $M$ is at $0 \in \mathbb{R}^{3}$. A planet now moves in a force field $F$ on an orbit $q(t)$ according to Newton's law:

$$
F(q(t))=m \ddot{q}(t) .
$$

(2) If the force field is centripetal, $F(q)=-f(q) q$ for $f \geq 0$, then the angular momentum $q(t) \times \dot{q}(t)=J$ is a constant vector, since

$$
\partial_{t}(q \times \dot{q})=\dot{q} \times \dot{q}+q \times \ddot{q}=0+\frac{1}{m} f(q) q \times q=0 .
$$

Thus the planet moves in the plane orthogonal to the angular momentum vector $J$ and we may choose coordinates such that this is the plane $q^{3}=0$. Let $z=q^{1}+i q^{2}=r e^{i \varphi}$; then

$$
\begin{aligned}
J & =\left(\begin{array}{l}
0 \\
0 \\
j
\end{array}\right)=z \times \dot{z}=\left(\begin{array}{c}
q^{1} \\
q^{2} \\
0
\end{array}\right) \times\left(\begin{array}{c}
\dot{q}^{1} \\
\dot{q}^{2} \\
0
\end{array}\right)=\left(\begin{array}{c}
0 \\
0 \\
q^{1} \dot{q}^{2}-q^{2} \dot{q}^{1}
\end{array}\right), \\
j & =q^{1} \dot{q}^{2}-q^{2} \dot{q}^{1}=\operatorname{Im}(\bar{z} \cdot \dot{z})=\operatorname{Im}\left(r e^{-i \varphi}\left(\dot{r} e^{i \varphi}+i r \dot{\varphi} e^{i \varphi}\right)\right) \\
& =\operatorname{Im}\left(r \dot{r}+i r^{2} \dot{\varphi}\right)=r^{2} \dot{\varphi}
\end{aligned}
$$


(3) Thus in a centripetal force field area is swept out at a constant rate $j=r^{2} \dot{\varphi}$ (2nd law of Kepler, 1602, published 1606), since

$$
\begin{aligned}
\operatorname{Area}\left(t_{1}, t_{2}\right) & =\int_{\varphi\left(t_{1}\right)}^{\varphi\left(t_{2}\right)} \int_{0}^{r(\varphi)} r d r d \varphi=\int_{\varphi\left(t_{1}\right)}^{\varphi\left(t_{2}\right)} \frac{1}{2} r(\varphi)^{2} d \varphi \\
& =\int_{t_{1}}^{t_{2}} \frac{1}{2} r(\varphi(t))^{2} \dot{\varphi}(t) d t=\frac{j}{2}\left(t_{2}-t_{1}\right) .
\end{aligned}
$$

Now we specify the force field. According to Newton's law of gravity the sun acts on a planet of mass $m$ at the point $0 \neq q \in \mathbb{R}^{3}$ by the force

$$
\begin{aligned}
& F(q)=-G \frac{M m}{|q|^{3}} q=-\operatorname{grad} U(q), \\
& U(q)=-G \frac{M m}{|q|},
\end{aligned}
$$

where $G=(6.67428 \pm 0.00067) \cdot 10^{-11} \mathrm{~m}^{3} \mathrm{~kg}^{-1} \mathrm{~s}^{-2}$ is the gravitational constant and $U$ is the gravitational potential. We consider now the energy function (compare with $(31.1)$ along the orbit as the sum of the kinetic and the potential energies

$$
E(t):=\frac{m}{2}|\dot{q}(t)|^{2}+U(q(t))=\frac{m}{2}|\dot{q}(t)|^{2}-G \frac{M m}{|q(t)|}
$$

which is constant along the orbit, since

$$
\partial_{t} E(t)=m(\ddot{q}(t) \mid \dot{q}(t))+(\operatorname{grad} U(q(t)) \mid \dot{q}(t))=0 .
$$

We have in the coordinates specified above for the velocity $v=|\dot{q}|$

$$
v^{2}=|\dot{q}|^{2}=\operatorname{Re}(\overline{\dot{z}} \dot{z})=\operatorname{Re}\left(\left(\dot{r} e^{-i \varphi}-i r \dot{\varphi} e^{-i \varphi}\right)\left(\dot{r} e^{i \varphi}+i r \dot{\varphi} e^{i \varphi}\right)\right)=\dot{r}^{2}+r^{2} \dot{\varphi}^{2} .
$$

We look now for a solution in the form $r=r(\varphi)$. From (3) we have $\dot{\varphi}=j / r^{2}$ so that

$$
v^{2}=\dot{r}^{2}+r^{2} \dot{\varphi}^{2}=\left(\frac{d r}{d \varphi}\right)^{2} \dot{\varphi}^{2}+r^{2} \dot{\varphi}^{2}=\left(\frac{d r}{d \varphi}\right)^{2} \frac{j^{2}}{r^{4}}+\frac{j^{2}}{r^{2}}
$$

Plugging into the conservation of energy (5), we get

$$
\begin{gathered}
\left(\frac{d r}{d \varphi}\right)^{2} \frac{j^{2}}{r^{4}}+\frac{j^{2}}{r^{2}}-2 G M \frac{1}{r(t)}=\gamma=\text { constant } \\
\frac{1}{r^{4}}\left(\frac{d r}{d \varphi}\right)^{2}=\frac{\gamma}{j^{2}}+\frac{2 G M}{j^{2}} \frac{1}{r(t)}-\frac{1}{r^{2}}
\end{gathered}
$$

Excluding the catastrophe of the planet falling into the sun, we may assume that $r$ is never 0 and substitute

$$
u(\varphi)=\frac{1}{r(\varphi)}, \quad \frac{d u}{d \varphi}=-\frac{1}{r^{2}} \frac{d r}{d \varphi}
$$


into (6) to obtain

$$
\begin{aligned}
\left(\frac{d u}{d \varphi}\right)^{2} & =\frac{\gamma}{j^{2}}+\frac{2 G M}{j^{2}} u-u^{2}=\frac{G^{2} M^{2}}{j^{4}}\left(1+\frac{\gamma j^{2}}{G^{2} M^{2}}\right)-\left(u-\frac{G M}{j^{2}}\right)^{2}, \\
\text { (7) }\left(\frac{d u}{d \varphi}\right)^{2} & =\frac{\varepsilon^{2}}{p^{2}}-\left(u-\frac{1}{p}\right)^{2}, \quad \text { where } p:=\frac{j^{2}}{G M}, \quad \varepsilon:=\sqrt{1+\frac{\gamma j^{2}}{G^{2} M^{2}}}
\end{aligned}
$$

are parameters suitable to describe conic sections.

If $\varepsilon=0$, then $\left(\frac{d u}{d \varphi}\right)^{2}=-\left(u-\frac{1}{p}\right)^{2}$ so that both sides have to be zero: $u=1 / p$ or $r=p=$ constant and the planet moves on a circle.

If $\varepsilon>0$, then (7) becomes

$$
\begin{aligned}
\frac{d u}{d \varphi} & =\sqrt{\frac{\varepsilon^{2}}{p^{2}}-\left(u-\frac{1}{p}\right)^{2}} \quad \text { or } \quad d \varphi=\frac{d u}{\sqrt{\frac{\varepsilon^{2}}{p^{2}}-\left(u-\frac{1}{p}\right)^{2}}}, \\
\varphi+C & =\int d \varphi=\int \frac{d u}{\sqrt{\frac{\varepsilon^{2}}{p^{2}}-\left(u-\frac{1}{p}\right)^{2}}} \quad \text { now use } w=u-\frac{1}{p} \\
& =\int \frac{d w}{\sqrt{\frac{\varepsilon^{2}}{p^{2}}-w^{2}}}=\frac{p}{\varepsilon} \int \frac{d w}{\sqrt{1-\left(\frac{p w}{\varepsilon}\right)^{2}}} \quad \text { now use } z=\frac{p w}{\varepsilon} \\
& =\int \frac{d z}{\sqrt{1-z^{2}}}=\arcsin (z)=\arcsin \left(\frac{p w}{\varepsilon}\right)=\arcsin \left(\frac{p u-1}{\varepsilon}\right) .
\end{aligned}
$$

This implies

$$
\begin{aligned}
\sin (\varphi+C) & =\frac{p u-1}{\varepsilon}, \quad u=\frac{1+\varepsilon \sin (\varphi+C)}{p}, \\
r & =\frac{1}{u}=\frac{p}{1+\varepsilon \sin (\varphi+C)} .
\end{aligned}
$$

We choose the parameter $C$ such that the minimal distance $\frac{p}{1+\varepsilon}$ of the planet from the sun (its perihel) is attained at $\varphi=0$ so that $\sin (C)=1$ or $C=\pi / 2$; then $\sin (\varphi+\pi / 2)=\cos (\varphi)$ and the planetary orbit is described by the equation

$$
r=\frac{p}{1+\varepsilon \cos \varphi}, \quad p>0, \quad \varepsilon \geq 0 .
$$

Equation (8) describes a conic section in polar coordinates with one focal point at 0 . We have:

- a circle for $\varepsilon=0$,

- an ellipse for $0 \leq \varepsilon<1$,

- a parabola for $\varepsilon=1$,

- the left branch of a hyperbola for $\varepsilon>1$. 
For the ellipse with the right hand focal point at 0 :

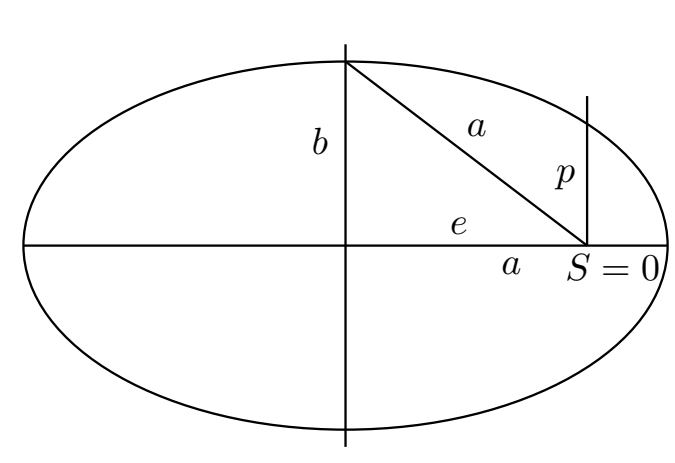

$$
\begin{aligned}
& \frac{\left(q_{1}+e\right)^{2}}{a^{2}}+\frac{q_{2}^{2}}{b^{2}}=1, \\
& e=\sqrt{a^{2}-b^{2}}, \\
& \frac{(r \cos \varphi+e)^{2}}{a^{2}}+\frac{r^{2} \sin ^{2} \varphi}{b^{2}}=1, \\
& \left(b^{2}-a^{2}\right) r^{2} \cos ^{2} \varphi \\
& \quad+2 b^{2} r \sqrt{a^{2}-b^{2}} \cos \varphi \\
& +a^{2} r^{2}-b^{4}=0 .
\end{aligned}
$$

Solving for $\cos \varphi$, we get

$$
\begin{aligned}
\cos \varphi & =\frac{-2 b^{2} r \sqrt{a^{2}-b^{2}} \pm \sqrt{4 b^{4} r^{2}\left(a^{2}-b^{2}\right)+4\left(a^{2}-b^{2}\right) r^{2}\left(a^{2} r^{2}-b^{4}\right)}}{-2\left(a^{2}-b^{2}\right) r^{2}} \\
& =\frac{-2 b^{2} r e \pm 2 r^{2} e a}{-2 r^{2} e^{2}}=\frac{b^{2}}{r e} \pm \frac{a}{e} \\
\frac{b^{2}}{r e} & =\cos \varphi \pm \frac{a}{e}, \\
r & =\frac{b^{2}}{e\left(\cos \varphi \pm \frac{a}{e}\right)}=\frac{b^{2}}{e \frac{a}{e}\left( \pm 1+\frac{e}{a} \cos \varphi\right)}=\frac{\frac{b^{2}}{a}}{ \pm 1+\frac{e}{a} \cos \varphi} .
\end{aligned}
$$

Put $p=b^{2} / a$ and $0 \leq \varepsilon=\sqrt{1-b^{2} / a^{2}}=e / a \leq 1$ and note that $r>0$ to obtain the desired equation (8), i.e.,

$$
r=\frac{p}{1+\varepsilon \cos \varphi} .
$$

For the parabola with focal point at 0 :

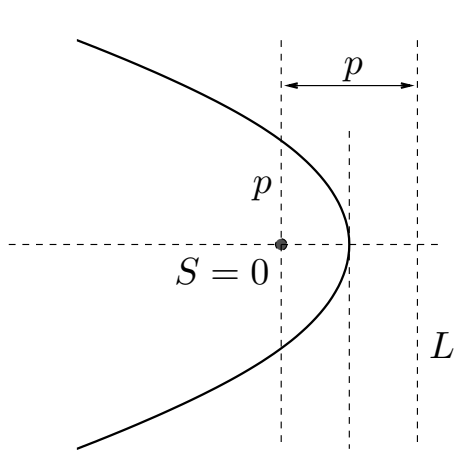

$$
\begin{gathered}
q_{2}{ }^{2}=-2 p\left(q_{1}-\frac{p}{2}\right)=-2 p q_{1}+p^{2}, \\
r^{2}\left(1-\cos ^{2} \varphi\right)=-2 p r \cos \varphi+p^{2}, \\
r^{2} \cos ^{2} \varphi-2 p r \cos \varphi+p^{2}-r^{2}=0, \\
\cos \varphi=\frac{2 p r \pm \sqrt{4 p^{2} r^{2}-4 r^{2}\left(p^{2}-r^{2}\right)}}{2 r^{2}} \\
=\frac{p \pm r}{r}=\frac{p}{r} \pm 1, \\
r=\frac{p}{1+\cos \varphi} \quad>0 .
\end{gathered}
$$


For the hyperbola with left hand focal point at 0 :

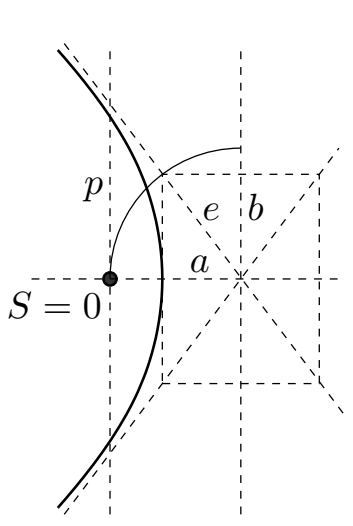

$$
\begin{aligned}
& \frac{\left(q_{1}-e\right)^{2}}{a^{2}}-\frac{q_{2}^{2}}{b^{2}}=1, \\
& e=\sqrt{a^{2}+b^{2}}, \\
& \frac{(r \cos \varphi-e)^{2}}{a^{2}}-\frac{r^{2} \sin ^{2} \varphi}{b^{2}}=1, \\
& b^{2} r^{2} \cos ^{2} \varphi-2 b^{2} r \sqrt{a^{2}+b^{2}} \cos \varphi \\
& \quad+a^{2} b^{2}+b^{4}-a^{2} r^{2}\left(1-\cos ^{2} \varphi\right)=a^{2} b^{2}, \\
& \left(b^{2}+a^{2}\right) r^{2} \cos ^{2} \varphi \\
& \quad-2 b^{2} r \sqrt{a^{2}+b^{2}} \cos \varphi+b^{4}-a^{2} r^{2}=0 .
\end{aligned}
$$

Solving again for $\cos \varphi$, we get

$$
\begin{aligned}
\cos \varphi & =\frac{2 b^{2} r \sqrt{a^{2}+b^{2}} \pm \sqrt{4 b^{4} r^{2}\left(a^{2}+b^{2}\right)-4\left(a^{2}+b^{2}\right) r^{2}\left(b^{4}-a^{2} r^{2}\right)}}{2\left(a^{2}+b^{2}\right) r^{2}} \\
& =\frac{2 b^{2} r e \pm 2 r^{2} e a}{2 r^{2} e^{2}} .
\end{aligned}
$$

Put $p=b^{2} / a$ and $\varepsilon=\sqrt{1+b^{2} / a^{2}}=e / a>1$ and note that $r>0$ to obtain the desired equation (8), i.e., $r=\frac{p}{1+\varepsilon \cos \varphi}$.

(Kepler's 3rd law) If $T$ is the orbital period of a planet on an elliptic orbit with major half-axis a, then:

$$
\frac{T^{2}}{a^{3}}=\frac{(2 \pi)^{2}}{G M}
$$

is a constant depending only on the mass of the sun and not on the planet. Let $a$ and $b$ be the major and minor half-axes of an elliptic planetary orbit with period $T$. The area of this ellipse is $a b \pi$. But by (3) this area equals $a b \pi=j T / 2$. In (7) we had $p=j^{2} /(G M)$, and for an ellipse we have $p=b^{2} / a$; thus we get

$$
\frac{j}{2} T=a b \pi=a^{3 / 2} p^{1 / 2} \pi=a^{3 / 2} \frac{j}{\sqrt{G M}} \pi, \quad T=\frac{2 \pi a^{3 / 2}}{\sqrt{G M}}, \quad \frac{T^{2}}{a^{3}}=\frac{(2 \pi)^{2}}{G M} .
$$

31.24. Kepler's laws II: The 2-body system. Here we start to treat the 2-body system with methods like Poisson brackets, etc., as explained in $(31.22)$. So the symplectic manifold (the phase space) is $T^{*}\left(\mathbb{R}^{3} \backslash\{0\}\right)$ with symplectic form $\omega=\omega_{\mathbb{R}^{3}}=-d \vartheta_{\mathbb{R}^{3}}=\sum_{i=1}^{3} d q^{i} \wedge d p_{i}$. As in (31.1) we use the canonical coordinates $q^{i}$ on $\mathbb{R}^{3}$ and $p_{i}:=m \cdot \dot{q}^{i}$ on the cotangent fiber. The Hamiltonian function of the system is the energy from (31.23.5) written in 
these coordinates:

(1) $E(q, p):=\frac{1}{2 m}|p|^{2}+U(q)=\frac{1}{2 m}|p|^{2}-G \frac{M m}{|q|}=\frac{1}{2 m} \sum p_{i}^{2}-G \frac{M m}{\sqrt{\sum\left(q^{i}\right)^{2}}}$.

The Hamiltonian vector field is then given by

$$
H_{E}=\sum_{i=1}^{3}\left(\frac{\partial E}{\partial p_{i}} \frac{\partial}{\partial q^{i}}-\frac{\partial E}{\partial q^{i}} \frac{\partial}{\partial p_{i}}\right)=\sum_{i=1}^{3}\left(\frac{1}{m} p_{i} \frac{\partial}{\partial q^{i}}-\frac{G M m}{|q|^{3}} q^{i} \frac{\partial}{\partial p_{i}}\right) .
$$

The flow lines of this vector field can be expressed in terms of elliptic functions. Briefed by (31.23.2), we consider the 3 components of the vector product $J(q, p)=q \times p$ and we may compute that

$$
\begin{gathered}
J^{1}=q^{2} p_{3}-q^{3} p_{2}, \quad J^{2}=-q^{1} p_{3}+q^{3} p_{1}, \quad J^{3}=q^{1} p_{2}-q^{2} p_{1}, \\
\left\{E, J^{i}\right\}=0, \quad\left\{J^{1}, J^{2}\right\}=-J^{3}
\end{gathered}
$$

We shall later interpret $\left(J^{1}, J^{2}, J^{3}\right)$ as a momentum mapping.

\section{Completely Integrable Hamiltonian Systems}

32.1. Introduction. The pioneers of analytical mechanics, Euler, Lagrange, Jacobi, Kowalewska, ..., were deeply interested in completely integrable systems, of which they discovered many examples: the motion of a rigid body with a fixed point in the three classical cases (the Euler-Lagrange, Euler-Poisot, and Kowalewska cases), Kepler's system, the motion of a massive point in the gravitational field created by fixed attracting points, geodesics on an ellipsoid, etc. To analyze such systems, Jacobi developed a method which now bears his name, based on a search for a complete integral of the first order partial differential equation associated with the Hamitonian system under consideration, called the Hamilton-Jacobi equation. Later it turned out, with many contributions by Poincaré, that complete integrability is very exceptional: A small perturbation of the Hamiltonian function can destroy it. Thus this topic fell into disrespect.

Later Kolmogorov, Arnold, and Moser showed that certain qualitative properties of completely integrable systems persist after perturbation: certain invariant tori on which the quasi-periodic motion of the nonperturbed, completely integrable system takes place survive the perturbation.

More recently it has been shown that certain nonlinear partial differential equations such as the Korteweg-de Vries equation $u_{t}+3 u_{x} u+a u_{x x x}=0$ or the Camassa-Holm equation $u_{t}-u_{t x x}=u_{x x x} . u+2 u_{x x} . u_{x}-3 u_{x} . u$ may be regarded as infinite-dimensional ordinary differential equations which have many properties of completely integrable Hamiltonian systems. This started new, very active research in completely integrable systems. See [68, 69] for an overview. 
32.2. Completely integrable systems. Let $(M, \omega)$ be a symplectic manifold with $\operatorname{dim}(M)=2 n$ with a Hamiltonian function $h \in C^{\infty}(M)$.

(1) The Hamiltonian system $(M, \omega, h)$ is called completely integrable if there are $n$ functions $f_{1}, \ldots, f_{n} \in C^{\infty}(M)$ which

- are pairwise in involution: $\left\{f_{i}, f_{j}\right\}=0$ for all $i, j$,

- are first integrals of the system: $\left\{h, f_{i}\right\}=0$ for all $i$,

- are nondegenerate: their differentials are linearly independent on a dense open subset of $M$.

We shall keep this notation throughout this section.

(2) The $n+1$ functions $h, f_{1}, \ldots, f_{n} \in C^{\infty}(M)$ are pairwise in involution. At each point $x \in M$ the Hamiltonian fields $H_{h}(x), H_{f_{1}}(x), \ldots, H_{f_{n}}(x)$ span an isotropic subset of $T_{x} M$ which has dimension $\leq n$; thus they are linearly dependent. On the dense open subset $U \subseteq M$ where the differentials $d f_{i}$ are linearly independent, $d h(x)$ is a linear combination of $d f_{1}(x), \ldots, d f_{n}(x)$. Thus each $x \in U$ has an open neighborhood $V \subset U$ such that $h \mid V=\tilde{h} \circ$ $\left(f_{1}, \ldots, f_{n}\right) \mid V$ for a smooth local function on $\mathbb{R}^{n}$. To see this, note that the $H_{f_{i}}$ span an integrable distribution of constant rank in $U$ whose leaves are given by the connected components of the sets described by the equations $f_{i}=c_{i}, c_{i}$ constant, for $i=1, \ldots, n$ of maximal rank. Since $\left\{h, f_{i}\right\}=0$, the function $h$ is constant along each leaf and thus factors locally over the mapping $f:=\left(f_{1}, \ldots, f_{n}\right): U \rightarrow f(U) \subset \mathbb{R}^{n}$. The Hamiltonian vector field $H_{h}$ is then a linear combination of the Hamiltonian fields $H_{f_{i}}$,

$$
H_{h}=\check{\omega}^{-1}(d h)=\check{\omega}^{-1}\left(\sum_{i=1}^{n} \frac{\partial \tilde{h}}{\partial f_{i}}\left(f_{1}, \ldots, f_{n}\right) d f_{i}\right)=\sum_{i=1}^{n} \frac{\partial \tilde{h}}{\partial f_{i}}\left(f_{1}, \ldots, f_{n}\right) H_{f_{i}} .
$$

whose coefficients $\frac{\partial \tilde{h}}{\partial f_{i}}\left(f_{1}, \ldots, f_{n}\right)$ depend only on the first integrals $f_{1}, \ldots, f_{n}$. The $f_{i}$ are constant along the flow lines of $H_{h}$ since $\left\{h, f_{i}\right\}=0$ implies $\left(\mathrm{Fl}_{t}^{H_{h}}\right)^{*} f_{i}=f_{i}$ and $\left(\mathrm{Fl}_{t}^{H_{h}}\right)^{*} H_{f_{i}}=H_{f_{i}}$. This last argument also shows that a trajectory of $H_{h}$ intersecting $U$ is completely contained in $U$. Therefore these coefficients $\frac{\partial \tilde{h}_{i}}{\partial f_{i}}\left(f_{1}, \ldots, f_{n}\right)$ are constant along each trajectory of $H_{h}$ which is contained in $U$.

(3) The Hamiltonian vector fields $H_{f_{1}}, \ldots, H_{f_{n}}$ span a smooth integrable distribution of nonconstant rank on $M$ according to (3.28), since $\left[H_{f_{i}}, H_{f_{j}}\right]=$ $H_{\left\{f_{i}, f_{j}\right\}}=0$ and $\left(\mathrm{Fl}_{t}^{H_{f_{i}}}\right)^{*} H_{f_{j}}=H_{f_{j}}$, so the dimension of the span is constant along each flow. Thus we have a foliation of jumping dimension on $M$ : Each point of $M$ lies in an initial submanifold which is an integral manifold for the distribution spanned by the $H_{f_{i}}$. Each trajectory of $H_{h}$ or of any $H_{f_{i}}$ is completely contained in one of these leaves. The restriction of this foliation to the open set $U$ is a foliation of $U$ by Lagrangian submanifolds, whose 
leaves are defined by the equations $f_{i}=c_{i}, i=1, \ldots, n$, where the $c_{i}$ are constants.

32.3. Lemma ([10]). Let $\mathbb{R}^{2 n}=\mathbb{R}^{n} \times \mathbb{R}^{n}$ be the standard symplectic vector space with standard basis $e_{i}$ such that $\omega=\sum_{i=1}^{n} e^{i} \wedge e^{n+i}$. Let $W \subset \mathbb{R}^{2 n}$ be a Lagrangian subspace.

Then there is a partition $\{1, \ldots, n\}=I \sqcup J$ such that the Lagrangian subspace $U$ of $\mathbb{R}^{2 n}$ spanned by the $e_{i}$ for $i \in I$ and the $e_{n+j}$ for $j \in J$ is a complement to $W$ in $\mathbb{R}^{2 n}$.

Proof. Let $k=\operatorname{dim}\left(W \cap\left(\mathbb{R}^{n} \times 0\right)\right)$. If $k=n$, we may take $I=\emptyset$. If $k<n$, there exist $n-k$ elements $e_{i_{1}}, \ldots, e_{i_{n-k}}$ of the basis $e_{1}, \ldots, e_{n}$ of $\mathbb{R}^{n} \times 0$ which span a complement $U^{\prime}$ of $W \cap\left(\mathbb{R}^{n} \times 0\right)$ in $\mathbb{R}^{n} \times 0$. Put $I=\left\{i_{1}, \ldots, i_{n-k}\right\}$ and let $J$ be the complement. Let $U^{\prime \prime}$ be the span of the $e_{n+j}$ for $j \in J$, and let $U=U^{\prime} \oplus U^{\prime \prime}$. Then $U$ is a Lagrangian subspace. We have

$\mathbb{R}^{n} \times 0=\left(W \cap\left(\mathbb{R}^{n} \times 0\right)\right) \oplus U^{\prime}, \quad W \cap\left(\mathbb{R}^{n} \times 0\right) \subset W, \quad U^{\prime}=U \cap\left(\mathbb{R}^{n} \times 0\right) \subset U$.

Thus $\mathbb{R}^{n} \times 0 \subset W+U$. Since $\mathbb{R}^{n} \times 0, W, U$ are Lagrangian, by (31.4.4) we have $W \cap U=W^{\perp} \cap U^{\perp}=(W+U)^{\perp} \subset\left(\mathbb{R}^{n} \times 0\right)^{\perp}=\mathbb{R}^{n} \times 0$; thus $W \cap U=\left(W \cap\left(\mathbb{R}^{n} \times 0\right)\right) \cap\left(U \cap\left(\mathbb{R}^{n} \times 0\right)\right)=W \cap\left(\mathbb{R}^{n} \times 0\right) \cap U^{\prime}=0$, and $U$ is a complement of $W$.

32.4. Lemma. Let $(M, \omega)$ be a symplectic manifold of dimension $2 n$, and let $x \in M$. Suppose that $2 n$ smooth functions $u^{1}, \ldots, u^{n}, f_{1}, \ldots, f_{n}$ are given near $x$, that their differentials are linearly independent, and that they satisfy the following properties:

- The submanifold defined by the equations $u^{i}=u^{i}(x)$ for $i=1, \ldots, n$ is Lagrangian.

- The functions $f_{1}, \ldots, f_{n}$ are pairwise in involution: $\left\{f_{i}, f_{j}\right\}=0$ for all $i, j$.

Then on an open neighborhood $U$ of $x$ in $M$ we may determine $n$ other smooth functions $g_{1}, \ldots, g_{n}$ such that

$$
\omega \mid U=\sum_{i=1}^{n} d f_{i} \wedge d g_{i} .
$$

The determination of $g_{i}$ uses exclusively the operations of integration, elimination (solving linear equations), and partial differentiation.

Proof. Without loss we may assume that $u^{i}(x)=0$ for all $i$. There exists a contractible open neighborhood $U$ of $x$ in $M$ such that $(u, f):=$ $\left(u^{1}, \ldots, u^{n}, f_{1}, \ldots, f_{n}\right)$ is a chart defined on $U$ and such that each diffeomorphism $\psi_{t}(u, f):=(t u, f)$ is defined on the whole of $U$ for $t$ near $[0,1]$ and maps $U$ into itself. Since $\psi_{0}$ maps $U$ onto the Lagrange submanifold 
$N:=\left\{y \in U: u^{i}(y)=0\right.$ for $\left.i=1, \ldots, n\right\}$, we have $\psi_{0}^{*} \omega=0$. Using the homotopy invariance (11.4), we have

$$
\omega \mid U=\psi_{1}^{*} \omega=\psi_{0}^{*} \omega+d \bar{h}(\omega)-\bar{h}(d \omega)=0+d \bar{h}(\omega)+0,
$$

where $\bar{h}(\omega)=\int_{0}^{1}$ ins $_{t}^{*} i_{\partial_{t}} \psi^{*} \omega d t$ is from the proof of (11.4).

Since $f_{1}, \ldots, f_{n}$ are pairwise in involution and have linearly independent differentials, $\omega \mid U$ belongs to the ideal in $\Omega^{*}(U)$ generated by $d f_{1}, \ldots, d f_{n}$. This is a pointwise property. At $y \in U$ the tangent vectors $H_{f_{1}}(y), \ldots, H_{f_{n}}(y)$ span a Lagrangian vector subspace $L$ of $T_{y} M$ with annihilator $L^{o} \subset T_{y}^{*} M$ spanned by $d f_{1}(y), \ldots, d f_{n}(y)$. Choose a complementary Lagrangian subspace $W \subset T_{y} M$; see the proof of (31.20), Let $\alpha_{1}, \ldots, \alpha_{n} \in T_{y}^{*} M$ be a basis of the annihilator $W^{o}$. Then $\omega_{y}=\sum_{i, j=1}^{n} \omega_{i j} \alpha_{i} \wedge d f_{j}(y)$ since $\omega$ vanishes on $L$, on $W$, and induces a duality between $L$ and $W$.

From the form of $\bar{h}(\omega)$ above we then see that $\bar{h}(\omega)$ also belongs to this ideal, since $\psi_{t}^{*} f_{i}=f_{i}$ for all $i$. Namely,

$$
\bar{h}(\omega)=\sum_{i, j=1}^{n} \int_{0}^{1}\left(\operatorname{ins}_{t}^{*} i_{\partial_{t}} \psi^{*}\left(\omega_{i j} \cdot \alpha_{i}\right)\right) d f_{j} d t=:-\sum_{j=1}^{n} g_{j} d f_{j}
$$

for smooth functions $g_{i}$. Finally we remark that the determination of the components of $\omega$ in the chart $(u, f)$ uses partial differentiations and eliminations, whereas the calculation of the components of $\bar{h}(\omega)$ uses integration.

32.5. Lemma. Let $(M, \omega)$ be a symplectic manifold of dimension $2 n$. We assume that the following data are known on an open subset $U$ of $M$ :

- a canonical system of local coordinates $\left(q^{1}, \ldots, q^{n}, p_{1}, \ldots, p_{n}\right)$ on $U$ such that the symplectic form is given by $\omega \mid U=\sum_{i=1}^{n} d q^{i} \wedge d p_{i}$,

- smooth functions $f_{1}, \ldots, f_{n}$ which are pairwise in involution, $\left\{f_{i}, f_{j}\right\}=$ 0 for all $i, j$, and whose differentials are linearly independent.

Then each $x \in U$ admits an open neighborhood $V \subseteq U$ on which we can determine other smooth functions $g_{1}, \ldots, g_{n}$ such that

$$
\left.\omega\right|_{V}=\sum_{i=1}^{n} d f_{i} \wedge d g_{i}
$$

The determination of $g_{i}$ uses exclusively the operations of integration, elimination (use of the implicit function theorem), and partial differentiation.

Proof. If the functions $q^{1}, \ldots, q^{n}, f_{1}, \ldots, f_{n}$ have linearly independent differentials at a point $x \in U$, the result follows from (32.4). In the general case consider the Lagrangian subspace $L \subset T_{x} M$ spanned by $H_{f_{1}}(x), \ldots, H_{f_{n}}(x)$. By lemma (32.3) there exists a partition $\{1, \ldots, n\}=I \sqcup J$ such that the 
Langrangian subspace $W \subset T_{x} M$ spanned by $H_{q^{i}}(x)$ for $i \in I$ and $H_{p_{j}}(x)$ for $j \in J$ is complementary to $L$. Now the result follows from lemma (32.4) by calling $u^{k}, k=1, \ldots, n$, the functions $q^{i}$ for $i \in I$ and $p_{j}$ for $j \in J$.

32.6. Proposition. Let $(M, \omega, h)$ be a Hamiltonian system on a symplectic manifold of dimension $2 n$. We assume that the following data are known on an open subset $U$ of $M$ :

- a canonical system of local coordinates $\left(q^{1}, \ldots, q^{n}, p_{1}, \ldots, p_{n}\right)$ on $U$ such that the symplectic form is given by $\omega \mid U=\sum_{i=1}^{n} d q^{i} \wedge d p_{i}$,

- a family $f=\left(f_{1}, \ldots, f_{n}\right)$ of smooth first integrals for the Hamiltonian function $h$ which are pairwise in involution, i.e., $\left\{h, f_{i}\right\}=0$ and $\left\{f_{i}, f_{j}\right\}=0$ for all $i, j$, and whose differentials are linearly independent.

Then for each $x \in U$ the integral curve of $H_{h}$ passing through $x$ can be determined locally by using exclusively the operations of integration, elimination, and partial differentiation.

Proof. By lemma (32.5) there exists an open neighborhood $V$ of $x$ in $U$ and functions $g_{1}, \ldots, g_{n} \in C^{\infty}(V)$ such that $\omega \mid V=\sum_{i=1}^{n} d f_{i} \wedge d g_{i}$. The determination uses only integration, partial differentiation, and elimination. We may choose $V$ so small that $(f, g):=\left(f_{1}, \ldots, f_{n}, g_{1}, \ldots, g_{n}\right)$ is a chart on $V$ with values in a cube in $\mathbb{R}^{2 n}$.

We have already seen in (32.2.2) that $h \mid V=\tilde{h} \circ(f, g)$ where $\tilde{h}=h \circ(f, g)^{-1}$ is a smooth function on the cube which does not depend on the $g_{i}$. In fact $\tilde{h}$ may be determined by elimination since $h$ is constant on the leaves of the foliation given by $f_{i}=c_{i}, c_{i}$ constant.

The differential equation for the trajectories of $H_{h}$ in $V$ is given by

$$
\dot{f}_{k}=\frac{\partial \tilde{h}}{\partial g_{k}}=0, \quad \dot{g}_{k}=-\frac{\partial \tilde{h}}{\partial f_{k}}, \quad k=1, \ldots, n
$$

thus the integral curve $\mathrm{Fl}_{t}^{H_{h}}(x)$ is given by

$$
\begin{aligned}
& f_{k}\left(\mathrm{Fl}_{t}^{H_{h}}(x)\right)=f_{k}(x), \\
& g_{k}\left(\mathrm{Fl}_{t}^{H_{h}}(x)\right)=g_{k}(x)-t \frac{\partial \tilde{h}}{\partial f_{k}}(f(x)), \quad k=1, \ldots, n .
\end{aligned}
$$

32.7. Proposition. Let $(M, \omega, h)$ be a Hamiltonian system with $\operatorname{dim}(M)=$ $2 n$ and let $f=\left(f_{1}, \ldots, f_{n}\right)$ be a family first integrals of $h$ which are pairwise in involution, $\left\{h, f_{i}\right\}=0$ and $\left\{f_{i}, f_{j}\right\}=0$ for all $i, j$. Suppose that all Hamiltonian vector fields $H_{f_{i}}$ are complete. Then we have:

(1) The vector fields $H_{f_{i}}$ are the infinitesimal generators of a smooth action $\ell: \mathbb{R}^{n} \times M \rightarrow M$ whose orbits are the isotropic leaves of the 
foliation with jumping dimension described in (32.2.3) and which can be described by

$$
\ell_{\left(t_{1}, \ldots, t_{n}\right)}(x)=\left(\mathrm{Fl}_{t_{1}}^{H_{f_{1}}} \circ \ldots \circ \mathrm{Fl}_{t_{n}}^{H_{f_{n}}}\right)(x) .
$$

Each orbit is invariant under the flow of $H_{h}$.

(2) (Liouville's theorem) If $a \in f(M) \subset \mathbb{R}^{n}$ is a regular value of $f$ and if $N \subseteq f^{-1}(a)$ is a connected component, then $N$ is a Lagrangian submanifold and is an orbit of the action of $\mathbb{R}^{n}$ which acts transitively and locally freely on $N$ : For any point $x \in N$ the isotopy subgroup $\left(\mathbb{R}^{n}\right)_{x}:=\left\{t \in \mathbb{R}^{n}: \ell_{t}(x)=x\right\}$ is a discrete subgroup of $\mathbb{R}^{n}$. Thus it is a lattice $\sum_{i=1}^{k} 2 \pi \mathbb{Z} v_{i}$ generated by $k=\operatorname{rank}\left(\mathbb{R}^{n}\right)_{x}$ linearly independent vectors $2 \pi v_{i} \in \mathbb{R}^{n}$. The orbit $N$ is diffeomorphic to the quotient group $\mathbb{R}^{n} /\left(\mathbb{R}^{n}\right)_{x} \cong \mathbb{T}^{k} \times \mathbb{R}^{n-k}$, a product of the $k$-dimensional torus by an $(n-k)$-dimensional vector space.

Moreover, there exist constants $\left(w_{1}, \ldots, w_{n}\right) \in \mathbb{R}^{n}$ such that the flow of the Hamiltonian $h$ on $N$ is given by $\mathrm{Fl}_{t}^{H_{h}}=\ell_{\left(t w_{1}, \ldots, t w_{n}\right)}$. If we use coordinates $\left(b_{1} \bmod 2 \pi, \ldots, b_{k} \bmod 2 \pi, b_{k+1}, \ldots, b_{n}\right)$ corresponding to the diffeomorphic description $N \cong \mathbb{T}^{k} \times \mathbb{R}^{n-k}$, the flow of $h$ is given by

$\mathrm{Fl}_{t}^{H_{h}}\left(b_{1} \bmod 2 \pi, \ldots, b_{k} \bmod 2 \pi, b_{k+1}, \ldots, b_{n}\right)$

$=\left(b_{1}+t c_{1} \bmod 2 \pi, \ldots, b_{k}+t c_{k} \bmod 2 \pi, b_{k+1}+t c_{k+1}, \ldots, b_{n}+t c_{n}\right)$

for constant $c_{i}=w_{i} /\left|v_{i}\right|$ for $i \leq k$ and $c_{j}=w_{j}$ for $j>k$. If $N$ is compact so that $k=n$, this is called a quasi-periodic flow.

Proof. The action $\ell$ is well defined since the complete vector fields $H_{f_{i}}$ commute; see the proof of (3.17). Or we conclude the action directly from theorem (6.5), The rest of this theorem follows already from (32.2), or it is obvious. The form of discrete subgroups of $\mathbb{R}^{n}$ is proved in the next lemma.

32.8. Lemma. Let $G$ be a discrete subgroup of $\mathbb{R}^{n}$. Then $G$ is the lattice $\sum_{i=1}^{k} \mathbb{Z} v_{i}$ generated by $0 \leq k=\operatorname{rank}(G) \leq n$ linearly independent vectors $v_{i} \in \mathbb{R}^{n}$.

Proof. We use the standard Euclidean structure of $\mathbb{R}^{n}$. If $G \neq 0$, there is $0 \neq v \in G$. Let $v_{1}$ be the point in $\mathbb{R} v \cap G$ which is nearest to 0 but nonzero. Then $G \cap \mathbb{R} v=\mathbb{Z} v_{1}$ : If there were $w \in G$ in one of the intervals $(m, m+1) v_{1}$, then $w-m v_{1} \in \mathbb{R} v_{1}$ would be nonzero and closer to 0 than $v_{1}$.

If $G \neq \mathbb{Z} v_{1}$, there exists $v \in G \backslash \mathbb{R} v_{1}$. We will show that there exists a point $v_{2}$ in $G$ with minimal distance to the line $\mathbb{R} v_{1}$ but not in the line. Suppose that the orthogonal projection $\operatorname{pr}_{\mathbb{R}_{1}}(v)$ of $v$ onto $\mathbb{R} v_{1}$ lies in the interval 
$P=[m, m+1] v_{1}$ for $m \in \mathbb{Z}$, consider the cylinder

$$
C=\left\{z \in \operatorname{pr}_{\mathbb{R} v_{1}}^{-1}(P): \operatorname{dist}(z, P) \leq \operatorname{dist}(v, P)\right\}
$$

and choose a point $v_{2} \in G \backslash \mathbb{R} v_{1}$ in this cylinder nearest to $P$. Then $v_{2}$ has minimal distance to $\mathbb{R} v_{1}$ in $G \backslash\left(\mathbb{R} v_{1}\right)$ since any other point in $G$ with smaller distance can be shifted into the cylinder $C$ by adding some suitable $m v_{1}$.

Then $\mathbb{Z} v_{1}+\mathbb{Z} v_{2}$ forms a lattice in the plane $\mathbb{R} v_{1}+\mathbb{R} v_{2}$ which is partitioned into parallelograms $Q=\left\{a_{1} v_{1}+a_{2} v_{2}: m_{i} \leq a_{i}<m_{i}+1\right\}$ for $m_{i} \in \mathbb{Z}$. If there is a point $w \in G$ in one of these parallelograms $Q$, then a suitable translate $w-n_{1} v_{1}-n_{2} v_{2}$ would be nearer to $\mathbb{R} v_{1}$ than $v_{2}$. Thus $G \cap\left(\mathbb{R} v_{1}+\mathbb{R} v_{2}\right)=$ $\mathbb{Z} v_{1}+\mathbb{Z} v_{2}$

If there is a point of $G$ outside this plane, we may find as above a point $v_{3}$ of $G$ with minimal distance to the plane, and by covering the 3 -space $\mathbb{R} v_{1}+\mathbb{R} v_{2}+\mathbb{R} v_{3}$ with parallelepipeds, we may show as above that $G \cap$ $\left(\mathbb{R} v_{1}+\mathbb{R} v_{2}+\mathbb{R} v_{3}\right)=\mathbb{Z} v_{1}+\mathbb{Z} v_{2}+\mathbb{Z} v_{3}$, and so on.

\section{Poisson Manifolds}

33.1. Poisson manifolds. A Poisson structure on a smooth manifold $M$ is a Lie bracket $\{, \quad\}$ on the vector space of smooth functions $C^{\infty}(M)$ also satisfying

$$
\{f, g h\}=\{f, g\} h+g\{f, h\} .
$$

This means that for each $f \in C^{\infty}(M)$ the mapping $\operatorname{ad}_{f}=\{f, \quad\}$ is a derivation of $\left(C^{\infty}(M), \cdot\right)$, so by $(3.3)$ there exists a unique vector field $H(f)=H_{f} \in \mathfrak{X}(M)$ such that $\{f, h\}=H_{f}(h)=d h\left(H_{f}\right)$ holds for each $h \in C^{\infty}(M)$. We also have $H(f g)=f H_{g}+g H_{f}$ since

$$
H_{f g}(h)=\{f g, h\}=f\{g, h\}+g\{f, h\}=\left(f H_{g}+g H_{f}\right)(h) .
$$

Thus there exists a unique tensor field $P \in \Gamma\left(\bigwedge^{2} T M\right)$ such that

$$
\{f, g\}=H_{f}(g)=P(d f, d g)=\langle d f \wedge d g, P\rangle .
$$

The choice of sign is motivated by the following. If $\omega$ is a symplectic form on $M$, we consider, using (31.21).

$$
\begin{aligned}
\check{\omega} & : T M \rightarrow T^{*} M, \quad\langle\check{\omega}(X), Y\rangle=\omega(X, Y), \\
\check{P} & =\check{\omega}^{-1}: T^{*} M \rightarrow T M, \quad\langle\psi, \check{P}(\varphi)\rangle=P(\varphi, \psi), \\
H_{f} & =\check{\omega}^{-1}(d f)=\check{P}(d f), \quad i_{H_{f}} \omega=d f, \\
\{f, g\} & =H_{f}(g)=i_{H_{f}} d g=i_{H_{f}} i_{H_{g}} \omega=\omega\left(H_{g}, H_{f}\right) \\
& =\left\langle d g, H_{f}\right\rangle=\langle d g, \check{P}(d f)\rangle=P(d f, d g)=\frac{1}{2}\langle d f \wedge d g, P\rangle .
\end{aligned}
$$


33.2. Proposition. Schouten-Nijenhuis bracket. Let $M$ be a smooth manifold. We consider the space $\Gamma(\bigwedge T M)$ of multivector fields on $M$. This space carries a graded Lie bracket for the grading $\Gamma\left(\bigwedge^{*+1} T M\right)$,* = $-1,0,1,2, \ldots$, called the Schouten-Nijenhuis bracket, which is given by

(1) $\left[X_{1} \wedge \cdots \wedge X_{p}, Y_{1} \wedge \cdots \wedge Y_{q}\right]$

$$
=\sum_{i, j}(-1)^{i+j}\left[X_{i}, Y_{j}\right] \wedge X_{1} \wedge \cdots \widehat{X}_{i} \cdots \wedge X_{p} \wedge Y_{1} \wedge \cdots \widehat{Y}_{j} \cdots \wedge Y_{q}
$$

(2) $[f, U]=-\bar{\imath}(d f) U$,

where $\bar{\imath}(d f)$ is the insertion operator $\bigwedge^{k} T M \rightarrow \bigwedge^{k-1} T M$, the adjoint of df $\wedge(\quad): \bigwedge^{l} T^{*} M \rightarrow \bigwedge^{l+1} T^{*} M$.

Let $U \in \Gamma\left(\bigwedge^{u} T M\right), V \in \Gamma\left(\bigwedge^{v} T M\right), W \in \Gamma\left(\bigwedge^{w} T M\right)$, and $f \in C^{\infty}(M, \mathbb{R})$. Then we have:

$$
\begin{aligned}
{[U, V] } & =-(-1)^{(u-1)(v-1)}[V, U] . \\
{[U,[V, W]] } & =[[U, V], W]+(-1)^{(u-1)(v-1)}[V,[U, W]] . \\
{[U, V \wedge W] } & =[U, V] \wedge W+(-1)^{(u-1) v} V \wedge[U, W] . \\
{[X, U] } & =\mathcal{L}_{X} U .
\end{aligned}
$$

(7) Let $P \in \Gamma\left(\bigwedge^{2} T M\right)$. Then the product $\{f, g\}:=\frac{1}{2}\langle d f \wedge d g, P\rangle$ on $C^{\infty}(M)$ satisfies the Jacobi identity if and only if $[P, P]=0$.

Proof. The bilinear mapping $\bigwedge^{k} \Gamma(T M) \times \bigwedge^{l} \Gamma(T M) \rightarrow \bigwedge^{k+l-1} \Gamma(T M)$ given by (11) factors over $\bigwedge^{k} \Gamma(T M) \rightarrow \bigwedge_{C^{\infty}(M)}^{k} \Gamma(T M)=\Gamma\left(\bigwedge^{k} T M\right)$ since we may easily compute that

$$
\begin{aligned}
& {\left[X_{1} \wedge \cdots \wedge X_{p}, Y_{1} \wedge \cdots \wedge f Y_{j}\right.}\left.\wedge \cdots \wedge Y_{q}\right]=f\left[X_{1} \wedge \cdots \wedge X_{p}, Y_{1} \wedge \cdots \wedge Y_{q}\right] \\
&+(-1)^{p} \bar{\imath}(d f)\left(X_{1} \wedge \cdots \wedge X_{p}\right) \wedge Y_{1} \wedge \cdots \wedge Y_{q}
\end{aligned}
$$

So the bracket [ , ] : $\Gamma\left(\bigwedge^{k-1} T M\right) \times \Gamma\left(\bigwedge^{l-1} T M\right) \rightarrow \Gamma\left(\bigwedge^{k+l-1} T M\right)$ is a well defined operation. Properties (3) $-(\underline{6})$ have to be checked by direct computations.

Property (7) can be seen as follows: We have

$$
2\{f, g\}=\langle d f \wedge d g, P\rangle=\langle d g, \bar{\imath}(d f) P\rangle=-\langle d g,[f, P]\rangle=[g,[f, P]] .
$$

Now a straightforward computation involving the graded Jacobi identity and the graded skew-symmetry of the Schouten-Nijenhuis bracket gives

$$
[h,[g,[f,[P, P]]]]=-8(\{f,\{g, h\}\}+\{g,\{h, f\}\}+\{h,\{f, g\}\}) .
$$

Since $[h,[g,[f,[P, P]]]]=\langle d f \wedge d g \wedge d h,[P, P]\rangle$, the result follows.

In $\left[200\right.$ there is an expression for $(-1)^{u-1}[U, V]$ in terms of covariant derivatives which does not depend on the covariant derivative, and in [176] it is 
found that it satisfied the graded Jacobi identity. In [124] the relation of the Schouten-Nijenhuis bracket to Poisson manifolds was spelled out. See also [222], 147] for the version presented here and [223] for more information.

Let us point out here that the skew-symmetric Schouten-Nijenhuis bracket has a symmetric counterpart. It is an ordinary (non-graded) Lie bracket extending the Lie bracket from the space of vector fields to the space $\Gamma(\bigvee T M)$ of symmetric multivector fields. It satisfies $[X, f]=\mathcal{L}_{X} f$ for $X \in \mathfrak{X}(M)$ and

$$
\begin{aligned}
& {\left[X_{1} \vee \cdots \vee X_{p}, Y_{1} \vee \cdots \vee Y_{q}\right]} \\
& \qquad \sum_{i, j}\left[X_{i}, Y_{j}\right] \vee X_{1} \vee \cdots \widehat{X}_{i} \cdots \vee X_{p} \vee Y_{1} \vee \cdots \widehat{Y}_{j} \cdots \vee Y_{q} .
\end{aligned}
$$

A symmetric multivector field on $M$ can be viewed as a smooth function on $T^{*} M$ which is a homogeneous polynomial on each fiber. The symmetric Schouten-Nijenhuis bracket is then just the restriction of the canonical Poisson bracket on $C^{\infty}\left(T^{*} M\right)$ to the subalgebra of these fiberwise polynomial functions.

33.3. Hamiltonian vector fields for Poisson structures. Let $(M, P)$ be a Poisson manifold. As usual we denote by $\check{P}: T^{*} M \rightarrow T M$ the associated skew-symmetric homomorphism of vector bundles. Let $\mathfrak{X}(M, P):=$ $\left\{X \in \mathfrak{X}(M): \mathcal{L}_{X} P=0\right\}$ be the Lie algebra of infinitesimal automorphisms of the Poisson structure. For $f \in C^{\infty}(M)$ we define the Hamiltonian vector field by

$$
\operatorname{grad}^{P}(f)=H_{f}=\check{P}(d f)=-[f, P]=-[P, f] \in \mathfrak{X}(M),
$$

and we recall the relation between Poisson structure and Poisson bracket, $(33.1 .2)$ and (33.2.8)

$$
\{f, g\}=H_{f}(g)=P(d f, d g)=\frac{1}{2}\langle d f \wedge d g, P\rangle=[g,[f, P]] .
$$

Lemma. The Hamiltonian vector field mapping takes values in $\mathfrak{X}(M, P)$ and is a Lie algebra homomorphism

$$
\left(C^{\infty}(M),\{\quad, \quad\}_{P}\right) \stackrel{H=\operatorname{grad}^{P}}{\longrightarrow} \mathfrak{X}(M, P) .
$$

Proof. For $f \in C^{\infty}(M)$ we have:

$$
\begin{gathered}
0=[f,[P, P]]=[[f, P], P]-[P,[f, P]]=2[[f, P], P], \\
\mathcal{L}_{H_{f}} P=\left[H_{f}, P\right]=-[[f, P], P]=0 .
\end{gathered}
$$

For $f, g \in C^{\infty}(M)$ we get

$$
\begin{aligned}
{\left[H_{f}, H_{g}\right] } & =[[f, P],[g, P]]=[g,[[f, P], P]]-[[g,[f, P]], P] \\
& =\left[g,-\mathcal{L}_{H_{f}} P\right]-[\{f, g\}, P]=0+H(\{f, g\}) .
\end{aligned}
$$


33.4. Theorem. Let $(M, P)$ be a Poisson manifold. Then $\check{P}\left(T^{*} M\right) \subseteq T M$ is an integrable smooth distribution (with jumping dimension) in the sense of (3.23). On each leaf $L$ (which is an initial submanifold of $M$ by (3.25). the Poisson structure $P$ induces the inverse of a symplectic structure on $L$.

One says that the Poisson manifold $M$ is stratified into symplectic leaves.

Proof. We use theorem (3.28), Consider the set

$$
\mathcal{V}:=\left\{\check{P}(d f)=H_{f}=-[f, P]: f \in C^{\infty}(M)\right\} \subset \mathfrak{X}\left(\check{P}\left(T^{*} M\right)\right)
$$

of sections of the distribution. The set $\mathcal{V}$ spans the distribution since through each point in $T^{*} M$ we may find a form $d f$. The set $\mathcal{V}$ is involutive since $\left[H_{f}, H_{g}\right]=H_{\{f, g\}}$. Finally we have to check that the dimension of $\check{P}\left(T^{*} M\right)$ is constant along flow lines of vector fields in $\mathcal{V}$, i.e., of vector fields $H_{f}$ :

$$
\begin{aligned}
\check{P} & =\left(\mathrm{Fl}_{t}^{H_{f}}\right)^{*} \check{P}=T\left(\mathrm{Fl}_{-t}^{H_{f}}\right) \circ \check{P} \circ\left(T \mathrm{Fl}_{-t}^{H_{f}}\right)^{*} \quad \text { since } \mathcal{L}_{H_{f}} P=0 \\
& \Longrightarrow \operatorname{dim} \check{P}\left(T_{\mathrm{Fl}_{t}^{*}{ }^{H_{f}}(x)}^{M}\right)=\text { constant in } t .
\end{aligned}
$$

So all assumptions of theorem (3.28) are satisfied and thus the distribution $P\left(T^{*} M\right)$ is integrable.

Now let $L$ be a leaf of the distribution $P\left(T^{*} M\right)$, a maximal integral manifold. The 2 -vector field $P \mid L$ is tangent to $L$, since a local smooth function $f$ on $M$ is constant along each leaf if and only if $\check{P}(d f)=-d f \circ \check{P}: T^{*} M \rightarrow \mathbb{R}$ vanishes. Therefore, $\check{P} \mid L: T^{*} L \rightarrow T L$ is an injective homomorphism of vector bundles of the same fiber dimension and is thus an isomorphism. Then $\check{\omega}_{L}:=(\check{P} \mid L)^{-1}: T L \rightarrow T^{*} L$ defines a 2 -form $\omega_{L} \in \Omega^{2}(L)$ which is nondegenerate. It remains to check that $\omega_{L}$ is closed. For each $x \in L$ there exists an open neighborhood $U \subset M$ and functions $f, g, h \in C^{\infty}(U)$ such that the vector fields $H_{f}=\check{P}(d f) \mid L, H_{g}$, and $H_{h}$ on $L$ take arbitrary prescribed values in $T_{x} L$ at $x \in L$. Thus $d \omega_{L}=0 \in \Omega^{3}(L)$ results from the following computation:

$$
\begin{aligned}
\omega_{L}\left(H_{f}, H_{g}\right)= & \left(i_{H_{f}} \omega_{L}\right)\left(H_{g}\right)=\check{\omega}_{L}\left(H_{f}\right)\left(H_{g}\right) \\
= & d f\left(H_{g}\right)=\{g, f\} \\
d \omega_{L}\left(H_{f}, H_{g}, H_{h}\right)= & H_{f}\left(\omega_{L}\left(H_{g}, H_{h}\right)\right)+H_{g}\left(\omega_{L}\left(H_{h}, H_{f}\right)\right) \\
& +H_{h}\left(\omega_{L}\left(H_{f}, H_{g}\right)\right)-\omega_{L}\left(\left[H_{f}, H_{g}\right], H_{h}\right) \\
& -\omega_{L}\left(\left[H_{g}, H_{h}\right], H_{f}\right)-\omega_{L}\left(\left[H_{h}, H_{f}\right], H_{g}\right) \\
= & \{\{h, g\}, f\}+\{\{f, h\}, g\}+\{\{g, f\}, h\} \\
& -\{h,\{f, g\}\}-\{f,\{g, h\}\}-\{g,\{h, f\}\}=0 .
\end{aligned}
$$


33.5. Proposition. Poisson morphisms. Let $\left(M_{1}, P_{1}\right)$ and $\left(M_{2}, P_{2}\right)$ be two Poisson manifolds. A smooth mapping $\varphi: M_{1} \rightarrow M_{2}$ is called a Poisson morphism if any of the following equivalent conditions is satisfied:

(1) For all $f, g \in C^{\infty}\left(M_{2}\right)$ we have $\varphi^{*}\{f, g\}_{2}=\left\{\varphi^{*} f, \varphi^{*} g\right\}_{1}$.

(2) For all $f \in C^{\infty}\left(M_{2}\right)$ the Hamiltonian vector fields $H_{\varphi^{*} f}^{1} \in \mathfrak{X}\left(M_{1}, P_{1}\right)$ and $H_{f}^{2} \in \mathfrak{X}\left(M_{2}, P_{2}\right)$ are $\varphi$-related.

(3) We have $\bigwedge^{2} T \varphi \circ P_{1}=P_{2} \circ \varphi: M_{1} \rightarrow \bigwedge^{2} T M_{2}$.

(4) For each $x \in M_{1}$ we have

$$
T_{x} \varphi \circ\left(\check{P}_{1}\right)_{x} \circ\left(T_{x} \varphi\right)^{*}=\left(\check{P}_{2}\right)_{\varphi(x)}: T_{\varphi(x)}^{*} M_{2} \rightarrow T_{\varphi(x)} M_{2} .
$$

Proof. For $x \in M_{1}$ we have

$$
\begin{aligned}
\left\{\varphi^{*} f, \varphi^{*} g\right\}_{1}(x) & =\left(P_{1}\right)_{x}\left(\left.d(f \circ \varphi)\right|_{x},\left.d(g \circ \varphi)\right|_{x}\right) \\
& =\left(P_{1}\right)_{x}\left(\left.d f\right|_{\varphi(x)} \cdot T_{x} \varphi,\left.d g\right|_{\varphi(x)} \cdot T_{x} \varphi\right) \\
& =\frac{1}{2}\left(P_{1}\right)_{x} \cdot \bigwedge^{2}\left(T_{x} \varphi\right)^{*} \cdot\left(\left.\left.d f\right|_{\varphi(x)} \wedge d g\right|_{\varphi(x)}\right) \\
& =\left(\bigwedge T_{x} \varphi \cdot\left(P_{1}\right)_{x}\right)\left(\left.d f\right|_{\varphi(x)},\left.d g\right|_{\varphi(x)}\right), \\
\varphi^{*}\{f, g\}_{2}(x) & =\{f, g\}_{2}(\varphi(x))=\left(P_{2}\right)_{\varphi(x)}\left(\left.d f\right|_{\varphi(x)},\left.d g\right|_{\varphi(x)}\right) .
\end{aligned}
$$

This shows that (11) and (3) are equivalent since $d f(y)$ meets each point of $T^{*} M_{2}$. (3) and (4) are obviously equivalent.

(21) and (4) are equivalent since we have

$$
\begin{aligned}
T_{x} \varphi \cdot H_{\varphi^{*} f}^{1}(x) & =\left.T_{x} \varphi \cdot\left(\check{P}_{1}\right)_{x} \cdot d(f \circ \varphi)\right|_{x}=\left.T_{x} \varphi \cdot\left(\check{P}_{1}\right)_{x} \cdot\left(T_{x} \varphi\right)^{*} \cdot d f\right|_{\varphi(x)}, \\
H_{f}^{2}(\varphi(x)) & =\left.\left(\check{P}_{2}\right)_{\varphi(x)} \cdot d f\right|_{\varphi(x)} \cdot \quad \square
\end{aligned}
$$

33.6. Proposition. Let $\left(M_{1}, P_{1}\right),\left(M_{2}, P_{2}\right)$, and $\left(M_{3}, P_{3}\right)$ be Poisson manifolds and let $\varphi: M_{1} \rightarrow M_{2}$ and $\psi: M_{2} \rightarrow M_{3}$ be smooth mappings.

(1) If $\varphi$ and $\psi$ are Poisson morphisms, then also $\psi \circ \varphi$ is a Poisson morphism.

(2) If $\varphi$ and $\psi \circ \varphi$ are Poisson morphisms and if $\varphi$ is surjective, then also $\psi$ is a Poisson morphism. In particular, if $\varphi$ is Poisson and a diffeomorphism, then also $\varphi^{-1}$ is Poisson.

Proof. Part (11) follows from (33.5.1), say. For (2) we use (33.5.3) as follows:

$$
\begin{aligned}
\bigwedge^{2} T \varphi \circ P_{1}=P_{2} \circ \varphi \quad \text { and } \quad \bigwedge^{2} T(\psi \circ \varphi) \circ P_{1}=P_{3} \circ \psi \circ \varphi \\
\operatorname{imply} \bigwedge^{2} T \psi \circ P_{2} \circ \varphi=\bigwedge^{2} T \psi \circ \bigwedge^{T} \varphi \circ P_{1}=\bigwedge^{2} T(\psi \circ \varphi) \circ P_{1}=P_{3} \circ \psi \circ \varphi,
\end{aligned}
$$


which implies the result since $\varphi$ is surjective.

33.7. Example and theorem. For a Lie algebra $\mathfrak{g}$ there is a canonical Poisson structure $P$ on the dual $\mathfrak{g}^{*}$, given by the dual of the Lie bracket:

$$
\begin{gathered}
{[, \quad]: \bigwedge^{2} \mathfrak{g} \rightarrow \mathfrak{g}, \quad P=-[\quad, \quad]^{*}: \mathfrak{g}^{*} \rightarrow \bigwedge^{2} \mathfrak{g}^{*},} \\
\{f, g\}(\alpha)=\langle\alpha,[d g(\alpha), d f(\alpha)]\rangle \quad \text { for } f, g \in C^{\infty}\left(\mathfrak{g}^{*}\right), \alpha \in \mathfrak{g}^{*} .
\end{gathered}
$$

The symplectic leaves are exactly the connected components of coadjoint orbits with their symplectic structures from (31.14).

Proof. We check directly the properties (33.1) of a Poisson structure. Skew symmetry is clear. The derivation property (33.1.1) is:

$$
\begin{aligned}
\{f, g h\}(\alpha) & =\langle\alpha,[h(\alpha) d g(\alpha)+g(\alpha) d h(\alpha), d f(\alpha)]\rangle \\
& =\langle\alpha,[d g(\alpha), d f(\alpha)]\rangle h(\alpha)+g(\alpha)\langle\alpha,[d h(\alpha), d f(\alpha)]\rangle \\
& =(\{f, g\} h+g\{f, h\})(\alpha) .
\end{aligned}
$$

For the Jacobi identity (33.1.1) we compute

$$
\begin{aligned}
&\left\langle\beta,\left.d\{g, h\}\right|_{\alpha}\right\rangle=\langle\beta,[d h(\alpha), d g(\alpha)]\rangle+\left\langle\alpha,\left[d^{2} h(\alpha)(\beta, \quad), d g(\alpha)\right]\right\rangle \\
&+\left\langle\alpha,\left[d h(\alpha), d^{2} g(\alpha)(\beta, \quad)\right]\right\rangle \\
&=\langle\beta,[d h(\alpha), d g(\alpha)]\rangle-\left\langle\left(\operatorname{ad}_{d g(\alpha)}\right)^{*} \alpha, d^{2} h(\alpha)(\beta, \quad)\right\rangle \\
& \quad+\left\langle\left(\operatorname{ad}_{d h(\alpha)}\right)^{*} \alpha, d^{2} g(\alpha)(\beta, \quad)\right\rangle \\
&=\langle\beta,[d h(\alpha), d g(\alpha)]\rangle-d^{2} h(\alpha)\left(\beta,\left(\operatorname{ad}_{d g(\alpha)}\right)^{*} \alpha\right) \\
&+d^{2} g(\alpha)\left(\beta,\left(\operatorname{ad}_{d h(\alpha)}\right)^{*} \alpha\right)
\end{aligned}
$$

and we use this to obtain

$$
\begin{aligned}
&\{f,\{g, h\}\}(\alpha)=\langle\alpha,[d\{g, h\}(\alpha), d f(\alpha)]\rangle \\
&=\langle\alpha,[[d h(\alpha), d g(\alpha)], d f(\alpha)]\rangle-\left\langle\alpha,\left[d^{2} h(\alpha)\left(\quad,\left(\operatorname{ad}_{d g(\alpha)}\right)^{*} \alpha\right), d f(\alpha)\right]\right\rangle \\
& \quad+\left\langle\alpha,\left[d^{2} g(\alpha)\left(,,\left(\operatorname{ad}_{d h(\alpha)}\right)^{*} \alpha\right), d f(\alpha)\right]\right\rangle \\
&=\langle\alpha,[[d h(\alpha), d g(\alpha)], d f(\alpha)]\rangle-d^{2} h(\alpha)\left(\left(\operatorname{ad}_{d f(\alpha)}\right)^{*} \alpha,\left(\operatorname{ad}_{d g(\alpha)}\right)^{*} \alpha\right) \\
& \quad+d^{2} g(\alpha)\left(\left(\operatorname{ad}_{d f(\alpha)}\right)^{*} \alpha,\left(\operatorname{ad}_{d h(\alpha)}\right)^{*} \alpha\right) .
\end{aligned}
$$

The cyclic sum over the last expression vanishes. Comparing with (31.14) and $(31.21 .2)$, we see that the symplectic leaves are exactly the coadjoint orbits, since

$$
\begin{aligned}
\left\langle H_{f}(\alpha), d g(\alpha)\right\rangle & =\left.H_{f}(g)\right|_{\alpha}=\{f, g\}(\alpha)=\langle\alpha,[d g(\alpha), d f(\alpha)]\rangle \\
& =-\left\langle\left(\operatorname{ad}_{d f(\alpha)}\right)^{*} \alpha, d g(\alpha)\right\rangle, \\
H_{f}(\alpha) & =-\left(\operatorname{ad}_{d f(\alpha)}\right)^{*} \alpha .
\end{aligned}
$$


The symplectic structure on an orbit $O=\operatorname{Ad}(G)^{*} \alpha$ is the same as in (31.14) which was given by $\omega_{O}\left(\zeta_{X}, \zeta_{Y}\right)=\operatorname{ev}_{[X, Y]}$ where $\zeta_{X}=-\operatorname{ad}(X)^{*}$ is the fundamental vector field of the (left) adjoint action. But then $d \operatorname{ev}_{Y}\left(\zeta_{X}(\alpha)\right)=$ $-\left\langle\operatorname{ad}(X)^{*} \alpha, Y\right\rangle=\langle\alpha,[Y, X]\rangle=\omega_{O}\left(\zeta_{Y}, \zeta_{X}\right)$ so that on the orbit the Hamiltonian vector field is given by $H_{\mathrm{ev}_{Y}}=\zeta_{Y}=-\operatorname{ad}(Y)^{*}=-\operatorname{ad}\left(d \operatorname{ev}_{Y}(\alpha)\right)^{*}$, as for the Poisson structure above.

33.8. Theorem. Poisson reduction. Let $(M, P)$ be a Poisson manifold and let $r: M \times G \rightarrow M$ be the right action of a Lie group on $M$ such that each $r^{g}: M \rightarrow M$ is a Poisson morphism. Let us suppose that the orbit space $M / G$ is a smooth manifold such that the projection $p: M \rightarrow M / G$ is a submersion.

Then there exists a unique Poisson structure $\bar{P}$ on $M / G$ such that $p$ : $(M, P) \rightarrow(M / G, \bar{P})$ is a Poisson morphism.

The quotient $M / G$ is a smooth manifold if the action is proper and all orbits of $G$ are of the same type: All isotropy groups $G_{x}$ are conjugate in $G$. See $(29.21)$

Proof. We work with Poisson brackets. A function $f \in C^{\infty}(M)$ is of the form $f=\bar{f} \circ p$ for $\bar{f} \in C^{\infty}(M / G)$ if and only if $f$ is $G$-invariant. Thus $p^{*}: C^{\infty}(M / G) \rightarrow C^{\infty}(M)$ is an algebra isomorphism onto the subalgebra $C^{\infty}(M)^{G}$ of $G$-invariant functions. If $f, h \in C^{\infty}(M)$ are $G$-invariant, then so is $\{f, h\}$ since $\left(r^{g}\right)^{*}\{f, h\}=\left\{\left(r^{g}\right)^{f},\left(r^{g}\right)^{*} h\right\}=\{f, h\}$ by (33.5), for all $g \in G$. So $C^{\infty}(M)^{G}$ is a subalgebra for the Poisson bracket which we may regard as a Poisson bracket on $C^{\infty}(M / G)$.

33.9. Poisson cohomology. Let $(M, P)$ be a Poisson manifold. We consider the mapping

$$
\delta_{P}:=[P, \quad]: \Gamma\left(\bigwedge^{k-1} T M\right) \rightarrow \Gamma\left(\bigwedge^{k} T M\right)
$$

which satisfies $\delta_{P} \circ \delta_{P}=0$ since $[P,[P, U]]=[[P, P], U]+(-1)^{1.1}[P,[P, U]]$ by the graded Jacobi identity. Thus we define the Poisson cohomology by

$$
H_{\text {Poisson }}^{k}(M):=\frac{\operatorname{ker}\left(\delta_{P}: \Gamma\left(\bigwedge^{k} T M\right) \rightarrow \Gamma\left(\bigwedge^{k+1} T M\right)\right)}{\operatorname{im}\left(\delta_{P}: \Gamma\left(\bigwedge^{k-1} T M\right) \rightarrow \Gamma\left(\bigwedge^{k} T M\right)\right)} .
$$

The direct sum

$$
H_{\text {Poisson }}^{*}(M)=\bigoplus_{k=0}^{\operatorname{dim}(M)} H_{\text {Poisson }}^{k}(M)
$$

is a graded commutative algebra via $U \wedge V$ since $\operatorname{im}\left(\delta_{P}\right)$ is an ideal in $\operatorname{ker}\left(\delta_{P}\right)$ by (33.2.5), The degree 0 part of Poisson cohomology is given by

$$
H_{\text {Poisson }}^{0}(M)=\left\{f \in C^{\infty}(M): H_{f}=\{f, \quad\}=0\right\},
$$


i.e., the vector space of all functions which are constant along each symplectic leaf of the Poisson structure, since $[P, f]=[f, P]=-\bar{\imath}(d f) P=-\check{P}(d f)=$ $-H_{f}=-\{f, \quad\}$ by (33.2.2), (33.2.8), and (33.1.2), The degree 1 part of Poisson cohomology is given by

$$
\begin{aligned}
H_{\text {Poisson }}^{1}(M) & =\frac{\left\{X \in \mathfrak{X}(M):[P, X]=-\mathcal{L}_{X} P=0\right\}}{\left\{[P, f]: f \in C^{\infty}(M)\right\}} \\
& =\frac{\mathfrak{X}(M, P)}{\left\{H_{f}: f \in C^{\infty}(M)\right\}} .
\end{aligned}
$$

Thus we get the following refinement of lemma (33.3). There exists an exact sequence of homomorphisms of Lie algebras:

$$
\begin{gathered}
0 \longrightarrow H_{\text {Poisson }}^{0}(M) \stackrel{\alpha}{\longrightarrow} C^{\infty}(M) \underset{\operatorname{grad}^{P}}{H=} \mathfrak{X}(M, P) \stackrel{\gamma}{\longrightarrow} H_{\text {Poisson }}^{1}(M) \longrightarrow 0, \\
0 \\
\{,\} \quad[,] \quad[,]
\end{gathered}
$$

where the brackets are written under the spaces, where $\alpha$ is the embedding of the space of all functions which are constant on all symplectic leaves, and where $\gamma$ is the quotient mapping from (3). The bracket on $H_{\text {Poisson }}^{1}(M)$ is induced by the Lie bracket on $\mathfrak{X}(M, P)$ since $\left\{H_{f}: f \in C^{\infty}(M)\right\}$ is an ideal: $\left[H_{f}, X\right]=[-[f, P], X]=-[f,[P, X]]-[P,[f, X]]=0+[X(f), P]=-H_{X(f)}$.

33.10. Lemma ([67, [130]). Let $(M, P)$ be a Poisson manifold.

Then there exists a Lie bracket $\{\quad, \quad\}^{1}: \Omega^{1}(M) \times \Omega^{1}(M) \rightarrow \Omega^{1}(M)$ which is given by

$$
\begin{aligned}
\{\varphi, \psi\}^{1} & =\mathcal{L}_{\check{P}(\varphi)} \psi-\mathcal{L}_{\check{P}(\psi)} \varphi-d(P(\varphi, \psi)) \\
& =\mathcal{L}_{\check{P}(\varphi)} \psi-\mathcal{L}_{\check{P}(\psi)} \varphi-d i_{\check{P}(\varphi)} \psi .
\end{aligned}
$$

It is the unique $\mathbb{R}$-bilinear skew-symmetric bracket satisfying

$$
\begin{gathered}
\{d f, d g\}^{1}=d\{f, g\} \quad \text { for } f, g \in C^{\infty}(M), \\
\{\varphi, f \psi\}^{1}=f\{\varphi, \psi\}^{1}+\mathcal{L}_{\check{P}(\varphi)}(f) \psi \quad \text { for } \varphi, \psi \in \Omega^{1}(M) .
\end{gathered}
$$

Furthermore $\check{P}_{*}: \Omega^{1}(M) \rightarrow \mathfrak{X}(M)$ is a homomorphism of Lie algebras:

$$
\check{P}\left(\{\varphi, \psi\}^{1}\right)=[\check{P}(\varphi), \check{P}(\psi)] \text { for } \varphi, \psi \in \Omega^{1}(M) .
$$

The coboundary operator of Poisson cohomology has a similar form in terms of the bracket $\{,\}^{1}$ as the exterior derivative has in terms of the usual Lie bracket. Namely, for $U \in \Gamma\left(\bigwedge^{k} T M\right)$ and $\varphi_{0}, \ldots, \varphi_{k} \in \Omega^{1}(M)$ we have

$$
\begin{aligned}
(-1)^{k}\left(\delta_{P} U\right)\left(\varphi_{0}, \ldots, \varphi_{k}\right):=\sum_{i=0}^{k}(-1)^{i} \mathcal{L}_{P\left(\varphi_{i}\right)}\left(U\left(\varphi_{0}, \ldots, \widehat{\varphi}_{i}, \ldots, \varphi_{k}\right)\right) \\
+\sum_{i<j}(-1)^{i+j} U\left(\left\{\varphi_{i}, \varphi_{j}\right\}^{1}, \varphi_{0}, \ldots, \widehat{\varphi}_{i}, \ldots, \widehat{\varphi_{j}}, \ldots, \varphi_{k}\right) .
\end{aligned}
$$


Proof. (11) is skew-symmetric $\mathbb{R}$-bilinear and satisfies (2) and (3) since by (33.3) we have

$$
\begin{aligned}
\{d f, d g\}^{1}= & \mathcal{L}_{\check{P}(d f)} d g-\mathcal{L}_{\check{P}(d g)} d f-d(P(d f, d g))=d \mathcal{L}_{H_{f}} g-d \mathcal{L}_{H_{g}} f-d\{f, g\} \\
= & d\{f, g\} \\
\{\varphi, f \psi\}^{1}= & \mathcal{L}_{\check{P}(\varphi)}(f \psi)-\mathcal{L}_{f \check{P}(\psi)} \varphi-d(f P(\varphi, \psi)) \\
= & \mathcal{L}_{\check{P}(\varphi)}(f) \psi+f \mathcal{L}_{\check{P}(\varphi)}(\psi)-f \mathcal{L}_{\check{P}(\psi)} \varphi-\varphi(\check{P}(\psi)) d f \\
& -P(\varphi, \psi) d f-f d(P(\varphi, \psi)) \\
= & f\{\varphi, \psi\}^{1}+\mathcal{L}_{\check{P}(\varphi)}(f) \psi
\end{aligned}
$$

So an $\mathbb{R}$-bilinear and skew-symmetric operation satisfying (2) and (3) exists. It is uniquely determined since from (3) we see that is local in $\psi$, i.e., if $\psi \mid U=0$ for some open $U$, then also $\{\varphi, \psi\}^{1} \mid U=0$ by using appropriate bump functions. By skew-symmetry it is also local in $\varphi$. But locally each 1form is a linear combination of expressions $f d f^{\prime}$. Thus (2) and (3) determine the bracket $\{,\}^{1}$ uniquely. By locality it suffices to check condition (44) for 1-forms $f d f^{\prime}$ only:

$$
\begin{aligned}
\check{P}\left(\left\{f d f^{\prime}, g d g^{\prime}\right\}^{1}\right) & =\check{P}\left(f g\left\{d f^{\prime}, d g^{\prime}\right\}^{1}+f H_{f^{\prime}}(g) d g^{\prime}-g H_{g^{\prime}}(f) d f^{\prime}\right) \\
& =f g \check{P}\left(d\left\{f^{\prime}, g^{\prime}\right\}\right)+f H_{f^{\prime}}(g) \check{P}\left(d g^{\prime}\right)-g H_{g^{\prime}}(f) \check{P}\left(d f^{\prime}\right) \\
& =f g H_{\left\{f^{\prime}, g^{\prime}\right\}}+f H_{f^{\prime}}(g) \check{P}\left(d g^{\prime}\right)-g H_{g^{\prime}}(f) \check{P}\left(d f^{\prime}\right) \\
& =f g\left[H_{f^{\prime}}, H_{g^{\prime}}\right]+f H_{f^{\prime}}(g) H_{g^{\prime}}-g H_{g^{\prime}}(f) H_{f^{\prime}} \\
& =\left[f H_{f^{\prime}}, g H_{g^{\prime}}\right]=\left[\check{P}\left(f d f^{\prime}\right), \check{P}\left(g d g^{\prime}\right)\right] .
\end{aligned}
$$

Now we can check the Jacobi identity. Again it suffices to do this for 1-forms $f d f^{\prime}$. We shall use:

$$
\begin{aligned}
\left\{f d f^{\prime}, g d g^{\prime}\right\}^{1} & =f g\left\{d f^{\prime}, d g^{\prime}\right\}^{1}+f H_{f^{\prime}}(g) d g^{\prime}-g H_{g^{\prime}}(f) d f^{\prime} \\
& =f g d\left\{f^{\prime}, g^{\prime}\right\}+f\left\{f^{\prime}, g\right\} d g^{\prime}-g\left\{g^{\prime}, f\right\} d f^{\prime}
\end{aligned}
$$

in order to compute

$$
\begin{aligned}
& \left\{\left\{f d f^{\prime}, g d g^{\prime}\right\}^{1}, h d h^{\prime}\right\}^{1}=\left\{f g d\left\{f^{\prime}, g^{\prime}\right\}+f\left\{f^{\prime}, g\right\} d g^{\prime}-g\left\{g^{\prime}, f\right\} d f^{\prime}, h d h^{\prime}\right\}^{1} \\
& =\left\{f g d\left\{f^{\prime}, g^{\prime}\right\}, h d h^{\prime}\right\}^{1}+\left\{f\left\{f^{\prime}, g\right\} d g^{\prime}, h d h^{\prime}\right\}^{1}-\left\{g\left\{g^{\prime}, f\right\} d f^{\prime}, h d h^{\prime}\right\}^{1} \\
& =f g h d\left\{\left\{f^{\prime}, g^{\prime}\right\}, h^{\prime}\right\}+f g\left\{\left\{f^{\prime}, g^{\prime}\right\}, h\right\} d h^{\prime}-h\left\{h^{\prime}, f g\right\} d\left\{f^{\prime}, g^{\prime}\right\} \\
& +f\left\{f^{\prime}, g\right\} h d\left\{g^{\prime}, h^{\prime}\right\}+f\left\{f^{\prime}, g\right\}\left\{g^{\prime}, h\right\} d h^{\prime}-h\left\{h^{\prime}, f\left\{f^{\prime}, g\right\}\right\} d g^{\prime} \\
& -g\left\{g^{\prime}, f\right\} h d\left\{f^{\prime}, h^{\prime}\right\}-g\left\{g^{\prime}, f\right\}\left\{f^{\prime}, h\right\} d h^{\prime}+h\left\{h^{\prime}, g\left\{g^{\prime}, f\right\}\right\} d f^{\prime} \\
& =f g h d\left\{\left\{f^{\prime}, g^{\prime}\right\}, h^{\prime}\right\}+\left(f g\left\{f^{\prime},\left\{g^{\prime}, h\right\}\right\} d h^{\prime}-f g\left\{g^{\prime}\left\{f^{\prime}, h\right\}\right\} d h^{\prime}\right) \\
& +\left(-g h\left\{h^{\prime}, f\right\} d\left\{f^{\prime}, g^{\prime}\right\}-f h\left\{h^{\prime}, g\right\} d\left\{f^{\prime}, g^{\prime}\right\}\right) \\
& +h f\left\{f^{\prime}, g\right\} d\left\{g^{\prime}, h^{\prime}\right\}+f\left\{f^{\prime}, g\right\}\left\{g^{\prime}, h\right\} d h^{\prime}
\end{aligned}
$$




$$
\begin{aligned}
& +\left(-h\left\{h^{\prime}, f\right\}\left\{f^{\prime}, g\right\} d g^{\prime}-h f\left\{h^{\prime},\left\{f^{\prime}, g\right\}\right\} d g^{\prime}\right) \\
- & h g\left\{g^{\prime}, f\right\} d\left\{f^{\prime}, h^{\prime}\right\}-g\left\{g^{\prime}, f\right\}\left\{f^{\prime}, h\right\} d h^{\prime} \\
+ & \left(h\left\{h^{\prime}, g\right\}\left\{g^{\prime}, f\right\} d f^{\prime}+g h\left\{h^{\prime},\left\{g^{\prime}, f\right\}\right\} d f^{\prime}\right) .
\end{aligned}
$$

The cyclic sum over these expressions vanishes by using once the Jacobi identity for the Poisson bracket and many pairwise cancellations.

It remains to check formula (5) for the coboundary operator of Poisson cohomology. We use induction on $k$. For $k=0$ we have

$$
\left(\delta_{P} f\right)(d g)=\mathcal{L}_{H_{g}} f=\{g, f\}=-\mathcal{L}_{H_{f}} g=-H_{f}(d g)=[P, f](d g) .
$$

For $k=1$ we have

$$
\begin{aligned}
\left(\delta_{P} X\right)(d f, d g) & =\mathcal{L}_{H_{f}}(X(d g))-\mathcal{L}_{H_{g}}(X(d f))-X\left(\{d f, d g\}^{1}\right) \\
& =\mathcal{L}_{H_{f}}(X(d g))-\mathcal{L}_{H_{g}}(X(d f))-X(d\{f, g\}), \\
{[P, X](d f, d g) } & =-\left(\mathcal{L}_{X} P\right)(d f, d g) \\
& =-\mathcal{L}_{X}(P(d f, d g))+P\left(\mathcal{L}_{X} d f, d g\right)+P\left(d f, \mathcal{L}_{X} d g\right) \\
& =-X(d\{g, f\})+\{g, X(d f)\}+\{X(d g), f\} \\
& =-\left(X(d\{f, g\})-\mathcal{L}_{H_{g}}(X(d f))-\mathcal{L}_{H_{f}}(X(d g))\right) \\
& =-\left(\delta_{P} X\right)(d f, d g) .
\end{aligned}
$$

Finally we note that the algebraic consequences of the definition of $\delta_{P}$ are the same as for the exterior derivative $d$; in particular, we have $\delta_{P}(U \wedge V)=$ $\left(\delta_{P} U\right) \wedge V+(-1)^{u} U \wedge\left(\delta_{P} V\right)$. So formula (5) now follows since both sides are graded derivations and agree on the generators of $\Gamma\left(\bigwedge^{*} T M\right)$, namely on $C^{\infty}(M)$ and on $\mathfrak{X}(M)$.

33.11. Remark: The Koszul bracket. Lemma (33.10) has the following generalization which we present without proof. For a Poisson field $P \in$ $\Gamma\left(\bigwedge^{2} T M\right)$, the insertion operator $i_{P}: \Omega^{k}(M) \rightarrow \Omega^{k-2}(M)$ is the adjoint of multiplication by $P$ :

$$
\left\langle i_{P} \varphi, U\right\rangle=\langle\varphi \cdot P \wedge U\rangle \quad \text { for } \varphi \in \Omega^{p}(M) \text { and } U \in \Gamma\left(\bigwedge^{p-2} T M\right) .
$$

Then $\partial_{P}:=\left[i_{P}, d\right]=i_{P} \circ d-d \circ i_{P}$ is the Poisson homology operator of Koszul and satisfies $\partial_{P} \circ \partial_{P}=0$.

Result (112]). Let $(M, P)$ be a Poisson manifold. On the exterior algebra $\Omega^{*+1}(M)$ of differential forms,

$$
\{\varphi, \psi\}^{1}:=(-1)^{p}\left(\partial_{P}(\varphi \wedge \psi)-\partial_{P}(\varphi) \wedge \psi-(-1)^{p} \varphi \wedge \partial_{P}(\psi)\right)
$$

defines a graded Lie bracket, called the Koszul bracket. It satisfies the Leibniz rule

$$
\{\varphi, \psi \wedge \tau\}^{1}=\{\varphi, \psi\}^{1} \wedge \tau+(-1)^{(p-1) q} \psi \wedge\{\varphi, \tau\}^{1}
$$


where $\varphi \in \Omega^{p}(M), \psi \in \Omega^{q}(M)$, and $\tau \in \Omega(M)$. The exterior derivative is a derivation of the bracket

$$
d\{\varphi, \psi\}^{1}=\{d \varphi, \psi\}^{1}+(-1)^{p-1}\{\varphi, d \psi\}^{1} .
$$

On the space $\Omega^{1}(M)$ of 1 -forms this bracket coincides with the Lie bracket from lemma (33.10). Moreover, the algebra homomorphism (for the wedge product)

$$
\bigwedge \check{P}: \Omega(M) \rightarrow \Gamma(\bigwedge T M)
$$

is a homomorphism of graded Lie algebras from the Koszul bracket into the Schouten-Nijenhuis bracket.

33.12. The graded Poisson bracket for differential forms. We consider a Poisson manifold $(M, P)$. Recall $\Omega(M ; T M)=\Gamma\left(\Lambda^{*} T^{*} M \otimes T M\right)$, the space of tangent bundle valued differential forms on $M$, equipped with the Frölicher-Nijenhuis bracket [ , ]; see (16.5). Recall for $K \in \Omega^{k}(M ; T M)$ the graded Lie derivative $\mathcal{L}_{K} \in \operatorname{Der}_{k} \Omega(M)$ from (16.3) and the graded algebraic derivative $i_{K} \in \operatorname{Der}_{k-1} \Omega(M)$ from (16.2),

We first extend $\check{P}: T^{*} M \rightarrow T M$ to a linear mapping

$$
\check{P}: \Omega(M) \rightarrow \Omega(M ; T M)
$$

of degree -1 by $\check{P} \mid \Omega^{0}(M)=0$, and for $\varphi_{i} \in \Omega^{1}(M)$ by

$$
\check{P}\left(\varphi_{1} \wedge \cdots \wedge \varphi_{k}\right)=\sum_{i=1}^{k}(-1)^{i-1} \varphi_{1} \wedge \ldots \widehat{\varphi}_{i} \cdots \wedge \varphi_{k} \otimes \check{P}\left(\varphi_{i}\right) .
$$

This extension is an $\Omega(M)$-bimodule valued graded derivation of degree -1 , i.e., for $\varphi \in \Omega^{p}(M)$ and $\psi \in \Omega^{q}(M)$ we have:

$$
\check{P}(\varphi \wedge \psi)=\check{P}(\varphi) \wedge \psi+(-1)^{p} \varphi \wedge \check{P}(\psi) .
$$

Then we have the Hamiltonian mapping:

$$
H=\operatorname{grad}^{\check{P}}: \Omega(M) \rightarrow \Omega(M ; T M), \quad H(\psi):=\check{P}(d \psi) .
$$

Result ([79]). The Poisson bracket on $C^{\infty}(M)=\Omega^{0}(M)$ extends to a graded Lie bracket $\{\quad, \quad\}$ on the space $\Omega(M)$ of all differential forms which is given by

$$
\begin{aligned}
\{\varphi, \psi\}: & =\mathcal{L}_{H_{\varphi}} \psi+d \mathcal{L}_{\check{P}(\varphi)} \psi \\
& =i_{\check{P}(d \varphi)} d \psi+d i_{\check{P}(\varphi)} d \psi-(-1)^{p q} d i_{\check{P}(\psi)} d \varphi
\end{aligned}
$$

such that the Hamiltonian mapping

$$
H:(\Omega(M),\{\quad, \quad\}) \rightarrow(\Omega(M ; T M),[\quad, \quad])
$$


is a Lie algebra homomorphism into the Frölicher-Nijenhuis bracket. Moreover we have

$$
\begin{aligned}
\{f, \psi\} & =\mathcal{L}_{H_{f}} \psi \quad \text { for } f \in C^{\infty}(M), \\
d\{\varphi, \psi\} & =\{d \varphi, \psi\}=(-1)^{p}\{\varphi, d \psi\} .
\end{aligned}
$$

Thus $Z(M)=\operatorname{ker}(d: \Omega(M) \rightarrow \Omega(M))$ is a commutative Lie ideal. Exterior derivative $d:\left(\Omega^{*}(M),\{\},\right) \rightarrow\left(\Omega^{*+1}(M),\{,\}^{1}\right)$ is a Lie algebra homomorphism into the Koszul bracket from (33.11). But this bracket does not act as a derivation for the exterior product; there is no extension of the Poisson bracket doing this and mapping to the Koszul bracket via d.

In 145, for the case of symplectic manifolds, it was shown that the Poisson bracket on $C^{\infty}(M)=\Omega^{0}(M)$ extends to a graded Lie bracket on the space $\Omega(M) / B(M)$ of differential forms modulo exact forms such that the Hamiltonian mapping $H$ is a homomorphism of Lie algebras. This bracket was given by the quotient modulo $B(M)$ of either $i\left(H_{\varphi}\right) d \psi$ or $\mathcal{L}_{H(\varphi)} \psi$. The first bracket is graded anticommutative, the second satisfies one form of the graded Jacobi identity, and the two differ by something exact. See also [161] and [30]. Later Grabowski in [79] found the correct expression for the bracket on $\Omega(M)$. See also 109 for a still more general view on this.

33.13. Dirac structures - a common generalization of symplectic and Poisson structures ([37, [29, [28]). Let $M$ be a smooth manifold of dimension $m$. By a Dirac structure on $M$ we mean a vector subbundle $D \subset T M \times_{M} T^{*} M$ with the following two properties:

(1) Each fiber $D_{x}$ is maximally isotropic with respect to the metric of signature $(m, m)$ on $T M \times_{M} T^{*} M$ given by

$$
\left\langle(X, \alpha),\left(X^{\prime}, \alpha^{\prime}\right)\right\rangle_{+}=\alpha\left(X^{\prime}\right)+\alpha^{\prime}(X) .
$$

So $D$ is of fiber dimension $m$.

(2) The space of sections of $D$ is closed under the non-skew-symmetric version of the Courant bracket

$$
\left[(X, \alpha),\left(X^{\prime}, \alpha^{\prime}\right)\right]=\left(\left[X, X^{\prime}\right], \mathcal{L}_{X} \alpha^{\prime}-i_{X^{\prime}} d \alpha\right) .
$$

If $(X, \alpha)$ and $\left(X^{\prime}, \alpha^{\prime}\right)$ are sections of $D$, then $i_{X} \alpha^{\prime}=-i_{X^{\prime}} \alpha$ by isotropy; thus $\mathcal{L}_{X} \alpha^{\prime}-i_{X^{\prime}} d \alpha=i_{X} d \alpha^{\prime}+\frac{1}{2} d\left(i_{X} \alpha^{\prime}-i_{X^{\prime}} \alpha\right)-i_{X^{\prime}} d \alpha$ so the Courant bracket is skew-symmetric on $\Gamma(D)$.

Natural examples of Dirac structures are the following:

(3) Symplectic structures $\omega$ on $M$, where $D=D^{\omega}=\{(X, \check{\omega}(X)): X \in T M\}$ is just the graph of $\check{\omega}: T M \rightarrow T^{*} M$.

More generally, for a 2 -form $\omega$ on $M$ the graph $D^{\omega}$ of $\check{\omega}: T M \rightarrow T^{*} M$ is a Dirac structure if and only if $d \omega=0$ (a presymplectic structure); these are 
precisely the Dirac structures $D$ with $T M \cap D=\{0\}$. Namely,

$$
\begin{aligned}
\langle(X, \check{\omega}(X)),(Y, \check{\omega}(Y))\rangle_{+} & =\omega(X, Y)+\omega(Y, X)=0, \\
{\left[\left(X, i_{X} \omega\right),\left(Y, i_{Y} \omega\right)\right] } & =\left([X, Y], \mathcal{L}_{X} i_{Y} \omega-i_{Y} d i_{X} \omega\right) \\
& =\left([X, Y], i_{[X, Y]} \omega\right) .
\end{aligned}
$$

(4) Poisson structures $P$ on $M$ where $D=D^{P}=\left\{(\check{P}(\alpha), \alpha): \alpha \in T^{*} M\right\}$ is the graph of $P: T^{*} M \rightarrow T M$; these are precisely the Dirac structures $D$ which are transversal to $T^{*} M$. Namely,

$$
\begin{aligned}
\langle(\check{P}(\alpha), \alpha),(\check{P}(\beta), \beta)\rangle_{+} & =P(\alpha, \beta)+P(\beta, \alpha)=0, \\
{[(\check{P}(\alpha), \alpha),(\check{P}(\beta), \beta)] } & =\left([\check{P}(\alpha), \check{P}(\beta)], \mathcal{L}_{\check{P}(\alpha)} \beta-i_{\check{P}(\beta)} d \alpha\right) \\
& =\left(\check{P}\left(\{\alpha, \beta\}^{1}\right),\{\alpha, \beta\}^{1}\right), \quad \operatorname{using}(33.10),
\end{aligned}
$$

Given a Dirac structure $D$ on $M$, we consider its range $R(D)=\operatorname{pr}_{T M}(D)=$ $\left\{X \in T M:(X, \alpha) \in D\right.$ for some $\left.\alpha \in T^{*} M\right\}$. There is a skew-symmetric 2 -form $\Theta_{D}$ on $R(D)$ which is given by $\Theta_{D}\left(X, X^{\prime}\right)=\alpha\left(X^{\prime}\right)$ where $\alpha \in T^{*} M$ is such that $(X, \alpha) \in D$. The range $R(D)$ is an integrable distribution of nonconstant rank in the sense of (3.28), so $M$ is foliated into maximal integral submanifolds $L$ of $R(D)$ of varying dimensions, which are all initial submanifolds. The form $\Theta_{D}$ induces a closed 2-form on each leaf $L$ and $\left(L, \Theta_{D}\right)$ is thus a presymplectic manifold $\left(\Theta_{D}\right.$ might be degenerate on some $L)$. If the Dirac structure corresponds to a Poisson structure, then the $\left(L, \Theta_{D}\right)$ are exactly the symplectic leaves of the Poisson structure.

The main advantage of Dirac structures is that one can apply arbitrary pushforwards and pullbacks to them. So if $f: N \rightarrow M$ is a smooth mapping and $D_{M}$ is a Dirac structure on $M$, then the pullback is defined by $f^{*} D_{M}=$ $\left\{\left(X, f^{*} \alpha\right) \in T N \times{ }_{N} T^{*} N:(T f . X, \alpha) \in D_{M}\right\}$. Likewise the pushforward of a Dirac structure $D_{N}$ on $N$ is given by $f_{*} D_{N}=\left\{(T f . X, \alpha) \in T M \times{ }_{M} T^{*} M\right.$ : $\left.\left(X, f^{*} \alpha\right) \in D_{N}\right\}$. If $D=D^{\omega}$ for a closed 2-form $\omega$ on $M$, then $f^{*}\left(D^{\omega}\right)=$ $D^{f^{*} \omega}$. If $P_{N}$ and $P_{M}$ are Poisson structures on $N$ and $M$, respectively, which are $f$-related, then $f_{*} D^{P_{N}}=D^{f_{*} P_{N}}=D^{P_{M}}$.

\section{Hamiltonian Group Actions and Momentum Mappings}

34.1. Symplectic and Hamiltonian group actions. Let us suppose that a Lie group $G$ acts from the right on a symplectic manifold $(M, \omega)$ by $r: M \times G \rightarrow M$ in a way which respects $\omega$, so that each transformation $r^{g}$ is a symplectomorphism. This is called a symplectic group action. Let us list some immediate consequences:

(1) The space $C^{\infty}(M)^{G}$ of $G$-invariant smooth functions is a Lie subalgebra for the Poisson bracket, since $\left(r^{g}\right)^{*}\{f, h\}=\left\{\left(r^{g}\right)^{*} f,\left(r^{g}\right)^{*} h\right\}=\{f, h\}$ holds for each $g \in G$ and $f, h \in C^{\infty}(M)^{G}$. 
(2) For $x \in M$ the pullback of $\omega$ to the orbit $x . G$ is a closed 2-form of constant rank and is invariant under the action of $G$ on the orbit. Note first that the orbit is an initial submanifold by (6.4). If $i: x . G \rightarrow M$ is the embedding of the orbit, then $r^{g} \circ i=i \circ r^{g}$, so that $i^{*} \omega=i^{*}\left(r^{g}\right)^{*} \omega=\left(r^{g}\right)^{*} i^{*} \omega$ holds for each $g \in G$ and thus $i^{*} \omega$ is invariant. Since $G$ acts transitively on the orbit, $i^{*} \omega$ has constant rank (as a mapping $T(x . G) \rightarrow T^{*}(x \cdot G)$ ).

(3) By (6.3) the fundamental vector field mapping $\zeta: \mathfrak{g} \rightarrow \mathfrak{X}(M, \omega)$, given by $\zeta_{X}(x)=T_{e}(r(x, \quad)) X$ for $X \in \mathfrak{g}$ and $x \in M$, is a homomorphism of Lie algebras, where $\mathfrak{g}$ is the Lie algebra of $G$. (For a left action we get an antihomomorphism of Lie algebras; see (6.2) . Moreover, $\zeta$ takes values in $\mathfrak{X}(M, \omega)$. Let us consider again the exact sequence of Lie algebra homomorphisms from $(31.21)$

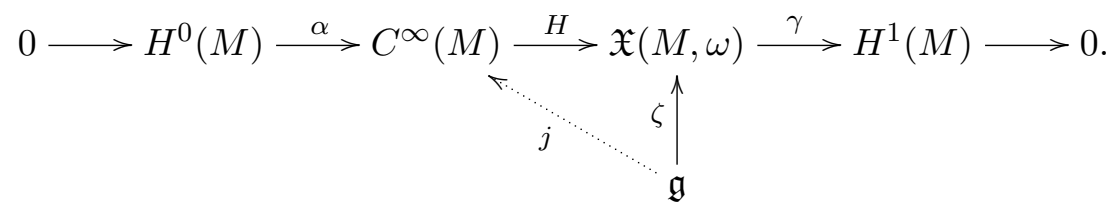

One can lift $\zeta$ to a linear mapping $j: \mathfrak{g} \rightarrow C^{\infty}(M)$ if and only if $\gamma \circ \zeta=0$. In this case the action of $G$ is called a Hamiltonian group action, and the linear mapping $j: \mathfrak{g} \rightarrow C^{\infty}(M)$ is called a generalized Hamiltonian function for the group action. It is unique up to addition of a mapping $\alpha \circ \tau$ for $\tau: \mathfrak{g} \rightarrow H^{0}(M)$.

(4) If $H^{1}(M)=0$, then any symplectic action on $(M, \omega)$ is a Hamiltonian action. If not and $\gamma \circ \zeta \neq 0$, we may either (a) lift $\omega$ to the universal cover of $M$ for which the first cohomology then vanishes, and try to lift the group action also (where we might have to enlarge the group by the discrete group of deck transformations), or (b) replace $\mathfrak{g}$ by its Lie subalgebra $\operatorname{ker}(\gamma \circ \zeta) \subset \mathfrak{g}$ and consider the corresponding Lie subgroup $G$; in both cases we get a Hamiltonian action.

(5) If the Lie algebra $\mathfrak{g}$ is equal to its commutator subalgebra $[\mathfrak{g}, \mathfrak{g}]$ (i.e., to the linear span of all $[X, Y]$ for $X, Y \in \mathfrak{g})$, then any infinitesimal symplectic action $\zeta: \mathfrak{g} \rightarrow \mathfrak{X}(M, \omega)$ is a Hamiltonian action, since then any $Z \in \mathfrak{g}$ can be written as $Z=\sum_{i}\left[X_{i}, Y_{i}\right]$ so that $\zeta_{Z}=\sum\left[\zeta_{X_{i}}, \zeta_{Y_{i}}\right] \in \operatorname{im}\left(\operatorname{grad}^{\omega}\right)$ since $\gamma: \mathfrak{X}(M, \omega) \rightarrow H^{1}(M)$ is a homomorphism into the zero Lie bracket.

34.2. Lemma. Momentum mappings. For an infinitesimal symplectic action, i.e., a homomorphism $\zeta: \mathfrak{g} \rightarrow \mathfrak{X}(M, \omega)$ of Lie algebras, we can find a linear lift $j: \mathfrak{g} \rightarrow C^{\infty}(M)$ if and only if there exists a mapping $J: M \rightarrow \mathfrak{g}^{*}$ such that

$$
H_{\langle J, X\rangle}=\zeta_{X} \quad \text { for all } X \in \mathfrak{g} \text {. }
$$


Proof. Namely, for $y \in M$ we have

$$
J: M \rightarrow \mathfrak{g}^{*}, \quad\langle J(y), X\rangle=j(X)(y) \in \mathbb{R}, \quad j: \mathfrak{g} \rightarrow C^{\infty}(M) .
$$

The mapping $J: M \rightarrow \mathfrak{g}^{*}$ is called the momentum mapping for the infinitesimal action $\zeta: \mathfrak{g} \rightarrow \mathfrak{X}(M, \omega)$. This holds even for a Poisson manifold $(M, P)$ (see section $(33)$ and an infinitesimal action of a Lie algebra $\zeta: \mathfrak{g} \rightarrow \mathfrak{X}(M, P)$ by Poisson morphisms. Let us note again the relations between the generalized Hamiltonian $j$ and the momentum mapping $J$ :

$$
\begin{gathered}
J: M \rightarrow \mathfrak{g}^{*}, \quad j: \mathfrak{g} \rightarrow C^{\infty}(M), \quad \zeta: \mathfrak{g} \rightarrow \mathfrak{X}(M, P), \\
\langle J, X\rangle=j(X) \in C^{\infty}(M), \quad H_{j(X)}=\zeta(X), \quad X \in \mathfrak{g},
\end{gathered}
$$

where $\langle$,$\rangle is the duality pairing.$

34.3. Basic properties of the momentum mapping. Consider a Hamiltonian right action $r: M \times G \rightarrow M$ of a Lie group $G$ on a symplectic manifold $M$, let $j: \mathfrak{g} \rightarrow C^{\infty}(M)$ be a generalized Hamiltonian and let $J: M \rightarrow \mathfrak{g}^{*}$ be the associated momentum mapping.

(1) For $x \in M$, the transposed mapping of $d J(x): T_{x} M \rightarrow \mathfrak{g}^{*}$ is

$$
d J(x)^{\top}: \mathfrak{g} \rightarrow T_{x}^{*} M, \quad d J(x)^{\top}=\check{\omega}_{x} \circ \zeta,
$$

since for $\xi \in T_{x} M$ and $X \in \mathfrak{g}$ we have

$$
\langle d J(\xi), X\rangle=\left\langle i_{\xi} d J, X\right\rangle=i_{\xi} d\langle J, X\rangle=i_{\xi} i_{\zeta_{X}} \omega=\left\langle\check{\omega}_{x}\left(\zeta_{X}(x)\right), \xi\right\rangle .
$$

(2) The image $d J\left(T_{x} M\right)$ of $d J(x): T_{x} M \rightarrow \mathfrak{g}^{*}$ is the annihilator $\mathfrak{g}_{x}^{\circ}$ of the isotropy Lie algeba $\mathfrak{g}_{x}:=\left\{X \in \mathfrak{g}: \zeta_{X}(x)=0\right\}$ in $\mathfrak{g}^{*}$, since the annihilator of the image is the kernel of the transposed mapping,

$$
\operatorname{im}(d J(x))^{\circ}=\operatorname{ker}\left(d J(x)^{\top}\right)=\operatorname{ker}\left(\check{\omega}_{x} \circ \zeta\right)=\operatorname{ker}\left(\operatorname{ev}_{x} \circ \zeta\right)=\mathfrak{g}_{x} .
$$

(3) The kernel of $d J(x)$ is the symplectic orthogonal $\left(T_{x}(x . G)\right)^{\perp} \subseteq T_{x} M$, since for the annihilator of the kernel we have

$$
\begin{aligned}
\operatorname{ker}(d J(x))^{\circ} & =\operatorname{im}\left(d J(x)^{\top}\right)=\operatorname{im}\left(\check{\omega}_{x} \circ \zeta\right)=\left\{\check{\omega}_{x}\left(\zeta_{X}(x)\right): X \in \mathfrak{g}\right\} \\
& =\check{\omega}_{x}\left(T_{x}(x \cdot G)\right) .
\end{aligned}
$$

(4) For each $x \in M$ the rank of $d J(x): T_{x} M \rightarrow \mathfrak{g}^{*}$ equals the dimension of the orbit $x . G$, i.e., the codimension in $\mathfrak{g}$ of the isotropy Lie algebra $\mathfrak{g}_{x}$. This follows from (3) since

$\operatorname{rank}(d J(x))=\operatorname{codim}_{T_{x} M}(\operatorname{ker} d J(x))=\operatorname{dim}\left(\operatorname{ker}(d J(x))^{\circ}\right)=\operatorname{dim}\left(T_{x}(x \cdot G)\right)$.

(5) The momentum mapping $J: M \rightarrow \mathfrak{g}^{*}$ is a submersion at $x \in M$ if and only if the isotropy group $G_{x}$ is discrete.

(6) If $G$ is connected, $x \in M$ is a fixed point for the $G$-action if and only if $x$ is a critical point of $J$, i.e., $d J(x)=0$. 
(7) Suppose that all orbits of the $G$-action on $M$ have the same dimension. Then $J: M \rightarrow \mathfrak{g}^{*}$ is of constant rank. Moreover, the distribution $\mathcal{F}$ of all symplectic orthogonals to the tangent spaces to all orbits is then an integrable distribution of constant rank and its leaves are exactly the connected components of the fibers of $J$. Namely, the rank of $J$ is constant by (3). For each $x \in M$ the subset $J^{-1}(J(x))$ is then a submanifold by (1.13), and by using (3) we see that $J^{-1}(J(x))$ is a maximal integral submanifold of $\mathcal{F}$ through $x$.

A direct proof that the distribution $\mathcal{F}$ is integrable is as follows: It has constant rank and is involutive, since for $\xi \in \mathfrak{X}(M)$ we have $\xi \in \mathfrak{X}(\mathcal{F})$ if and only if $i_{\xi} i_{\zeta_{X}} \omega=-\omega\left(\xi, \zeta_{X}\right)=0$ for all $X \in \mathfrak{g}$. For $\xi, \eta \in \mathfrak{X}(\mathcal{F})$ and $X \in \mathfrak{g}$ we have

$$
\begin{aligned}
i_{[\xi, \eta]} i_{\zeta_{X}} \omega & =\left[\mathcal{L}_{\xi}, i_{\eta}\right] i_{\zeta_{X}} \omega=\mathcal{L}_{\xi} i_{\eta} i_{\zeta_{X}} \omega-i_{\eta} \mathcal{L}_{\xi} i_{\zeta_{X}} \omega \\
& =0-i_{\eta} i_{\xi} d i_{\zeta_{X}} \omega-i_{\eta} d i_{\xi} i_{\zeta_{X}} \omega=0 .
\end{aligned}
$$

(8) (E. Noether's theorem) Let $h \in C^{\infty}(M)$ be a Hamiltonian function which is invariant under the Hamiltonian $G$ action. Then the momentum mapping $J: M \rightarrow \mathfrak{g}^{*}$ is constant on each trajectory of the Hamiltonian vector field $H_{h}$. Namely,

$$
\begin{aligned}
\frac{d}{d t}\left\langle J \circ \mathrm{Fl}_{t}^{H_{h}}, X\right\rangle & =\left\langle d J \circ \frac{d}{d t} \mathrm{Fl}_{t}^{H_{h}}, X\right\rangle=\left\langle d J\left(H_{h}\right) \circ \mathrm{Fl}_{t}^{H_{h}}, X\right\rangle \\
& =\left(i_{H_{h}} d\langle J, X\rangle\right) \circ \mathrm{Fl}_{t}^{H_{h}}=\{h,\langle J, X\rangle\} \circ \mathrm{Fl}_{t}^{H_{h}} \\
& =-\{\langle J, X\rangle, h\} \circ \mathrm{Fl}_{t}^{H_{h}}=-\left(\mathcal{L}_{\zeta_{X}} h\right) \circ \mathrm{Fl}_{t}^{H_{h}}=0 .
\end{aligned}
$$

E. Noether's theorem admits the following generalization.

34.4. Theorem (Marsden and Weinstein). Let $G_{1}$ and $G_{2}$ be two Lie groups which act by Hamiltonian actions $r_{1}$ and $r_{2}$ on the symplectic manifold $(M, \omega)$, with momentum mappings $J_{1}$ and $J_{2}$, respectively. We assume that $J_{2}$ is $G_{1}$-invariant, i.e., $J_{2}$ is constant along all $G_{1}$-orbits and that $G_{2}$ is connected.

Then $J_{1}$ is constant on the $G_{2}$-orbits and the two actions commute.

Proof. Let $\zeta^{i}: \mathfrak{g}_{i} \rightarrow \mathfrak{X}(M, \omega)$ be the two infinitesimal actions. Then for $X_{1} \in \mathfrak{g}_{1}$ and $X_{2} \in \mathfrak{g}_{2}$ we have

$$
\begin{aligned}
\mathcal{L}_{\zeta_{X_{2}}^{2}}\left\langle J_{1}, X_{1}\right\rangle & =i_{\zeta_{X_{2}}^{2}} d\left\langle J_{1}, X_{1}\right\rangle=i_{\zeta_{X_{2}}^{2}} i_{\zeta_{X_{1}}^{1}} \omega \\
& =\left\{\left\langle J_{2}, X_{2}\right\rangle,\left\langle J_{1}, X_{1}\right\rangle\right\}=-\left\{\left\langle J_{1}, X_{1}\right\rangle,\left\langle J_{2}, X_{2}\right\rangle\right\} \\
& =-i_{\zeta_{X_{1}}^{1}} d\left\langle J_{2}, X_{2}\right\rangle=-\mathcal{L}_{\zeta_{X_{1}}^{1}}\left\langle J_{2}, X_{2}\right\rangle=0
\end{aligned}
$$

since $J_{2}$ is constant along each $G_{1}$-orbit. Since $G_{2}$ is assumed to be connected, $J_{1}$ is also constant along each $G_{2}$-orbit. We also saw that each Poisson bracket $\left\{\left\langle J_{2}, X_{2}\right\rangle,\left\langle J_{1}, X_{1}\right\rangle\right\}$ vanishes; by $H_{\left\langle J_{i}, X_{i}\right\rangle}=\zeta_{X_{i}}^{i}$ we conclude 
that $\left[\zeta_{X_{1}}^{1}, \zeta_{X_{2}}^{2}\right]=0$ for all $X_{i} \in \mathfrak{g}_{i}$ which implies the result if also $G_{1}$ is connected. In the general case we can argue as follows:

$$
\begin{aligned}
\left(r_{1}^{g_{1}}\right)^{*} \zeta_{X_{2}}^{2} & =\left(r_{1}^{g_{1}}\right)^{*} H_{\left\langle J_{2}, X_{2}\right\rangle}=\left(r_{1}^{g_{1}}\right)^{*}\left(\check{\omega}^{-1} d\left\langle J_{2}, X_{2}\right\rangle\right) \\
& =\left(\left(\left(r_{1}^{g_{1}}\right)^{*} \omega\right)^{-1}\right)^{-1} d\left\langle\left(r_{1}^{g_{1}}\right)^{*} J_{2}, X_{2}\right\rangle=\check{\omega}^{-1} d\left\langle J_{2}, X_{2}\right\rangle=H_{\left\langle J_{2}, X_{2}\right\rangle}=\zeta_{X_{2}}^{2} .
\end{aligned}
$$

Thus $r_{1}^{g_{1}}$ commutes with each $r_{2}^{\exp \left(t X_{2}\right)}$ and thus with each $r_{2}^{g_{2}}$, since $G_{2}$ is connected.

34.5. Remark. The classical first integrals of mechanical systems can be derived by Noether's theorem, where the group $G$ is the group of isometries of Euclidean 3 -space $\mathbb{R}^{3}$, the semidirect product $\mathbb{R}^{3} \rtimes S O(3)$. Let $(M, \omega, h)$ be a Hamiltonian mechanical system consisting of several rigid bodies moving in physical 3-space. Then the Hamiltonian function is the sum of the kinetic energy and the potential energy. This system is said to be free if the Hamiltonian function $h$ describing the movement of the system is invariant under the group of isometries acting on $\mathbb{R}^{3}$ and its induced action on phase space $M \subseteq T^{*}\left(\mathbb{R}^{3 k}\right)$. This action is Hamiltonian since for the motion group $G$ we have $[\mathfrak{g}, \mathfrak{g}]=\mathfrak{g}$, by (34.1.5). Equivalently, the action is free if there is no potential. Then there exists a momentum mapping $J=\left(J_{l}, J_{a}\right): M \rightarrow\left(\mathbb{R}^{3} \rtimes \mathfrak{s o}(3)\right)^{*}=\left(\mathbb{R}^{3}\right)^{*} \times \mathfrak{s o}(3)^{*}$. Its component $J_{l}$ is the momentum mapping for the action of the translation group and is called the linear momentum; the component $J_{a}$ is the momentum mapping for the action of the rotation group and is called the angular momentum.

The momentum map is essentially due to Lie [125, pp. 300-343]. The modern notion is due to [110, 213, and [105. Also, 133, [123] and [135] are convenient references, and [135] has a large and updated bibliography. The momentum map has a strong tendency to have convex image and is important for representation theory; see [105] and [172. Recently, there has also been a proposal for a group valued momentum mapping; see [3].

34.6. Strongly Hamiltonian group actions. Suppose that we have a Hamiltonian action $M \times G \rightarrow M$ on the symplectic manifold $(M, \omega)$, and consider a generalized Hamiltonian $j: \mathfrak{g} \rightarrow C^{\infty}(M)$, which is unique up to addition of $\alpha \circ \tau$ for some $\tau: \mathfrak{g} \rightarrow H^{0}(M)$ :

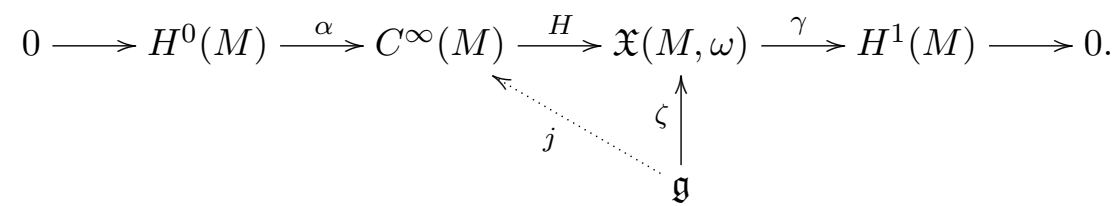

We want to investigate whether we can change $j$ into a homomorphism of Lie algebras. 
(1) The map $\mathfrak{g} \ni X, Y \mapsto\{j X, j Y\}-j([X, Y])=: \bar{\jmath}(X, Y)$ takes values in $\operatorname{ker}(H)=\operatorname{im}(\alpha)$ since

$H(\{j X, j Y\})-H(j([X, Y]))=\left[H_{j X}, H_{j Y}\right]-\zeta_{[X, Y]}=\left[\zeta_{X}, \zeta_{Y}\right]-\zeta_{[X, Y]}=0$.

Moreover, $\bar{\jmath}: \bigwedge^{2} \mathfrak{g} \rightarrow H^{0}(M)$ is a cocycle for the Chevalley cohomology of the Lie algebra $\mathfrak{g}$, as explained in (14.14).

$$
\begin{aligned}
d \bar{\jmath}(X, Y, Z) & =-\sum_{\text {cyclic }} \bar{\jmath}([X, Y], Z)=-\sum_{\text {cyclic }}(\{j([X, Y]), j Z\}-j([[X, Y], Z])) \\
& =-\sum_{\text {cyclic }}\{\{j X, j Y\}-\bar{\jmath}(X, Y), j Z\}-0 \\
& =-\sum_{\text {cyclic }}(\{\{j X, j Y\}, j Z\}-\{\bar{\jmath}(X, Y), j Z\})=0,
\end{aligned}
$$

by the Jacobi identity and since $\bar{\jmath}(X, Y) \in H^{0}(M)$ which equals the center of the Poisson algebra. Recall that the linear mapping $j: \mathfrak{g} \rightarrow C^{\infty}(M)$ was unique up to addition of a mapping $\alpha \circ \tau$ for $\tau: \mathfrak{g} \rightarrow H^{0}(M)$. But

$$
\begin{aligned}
\overline{j+\tau}(X, Y) & =\{(j+\tau) X,(j+\tau) Y\}-(j+\tau)([X, Y]) \\
& =\{j X, j Y\}+0-j([X, Y])-\tau([X, Y])=(\bar{\jmath}+d \tau)(X, Y) .
\end{aligned}
$$

Thus, if $\gamma \circ \zeta=0$, there is a unique Chevalley cohomology class $\tilde{\zeta}:=[\bar{\jmath}] \in$ $H^{2}\left(\mathfrak{g}, H^{0}(M)\right)$.

(2) The cohomology class $\tilde{\zeta}=[\bar{\jmath}]$ is automatically zero if $H^{2}\left(\mathfrak{g}, H^{0}(M)\right)=$ $H^{2}(\mathfrak{g}) \otimes H^{0}(M)=0$. This is the case for semisimple $\mathfrak{g}$, by the Whitehead lemmas; see [83, p. 249].

(3) The cohomology class $\tilde{\zeta}=[\bar{\jmath}]$ is automatically zero if the symplectic structure $\omega$ on $M$ is exact, $\omega=-d \vartheta$ for $\vartheta \in \Omega^{1}(M)$, and $\mathcal{L}_{\zeta_{X}} \vartheta=0$ for each $X \in \mathfrak{g}$ : Then we may use $j(X)=i_{\zeta_{X}} \vartheta=\vartheta\left(\zeta_{X}\right)$, since $i\left(H_{j X}\right) \omega=d(j X)=$ $d i_{\zeta_{X}} \vartheta=\mathcal{L}_{\zeta_{X}} \vartheta-i_{\zeta_{X}} d \vartheta=0+i_{\zeta_{X}} \omega$ implies $H_{j X}=\zeta_{X}$. For this choice of $j$ we have

$$
\begin{aligned}
\bar{\jmath}(X, Y) & =\{j X, j Y\}-j([X, Y])=\mathcal{L}_{H_{j X}}(j Y)-i_{\zeta([X, Y])} \vartheta \\
& =\mathcal{L}_{\zeta_{X}} i_{\zeta_{Y}} \vartheta-i_{\left[\zeta_{X}, \zeta_{Y}\right]} \vartheta=\mathcal{L}_{\zeta_{X}} i_{\zeta_{Y}} \vartheta-\left[\mathcal{L}_{\zeta_{X}}, i_{\zeta_{Y}}\right] \vartheta=-i_{\zeta_{Y}} \mathcal{L}_{\zeta_{X}} \vartheta=0 .
\end{aligned}
$$

(4) The condition of (3) holds if $M=T^{*} Q$ is a cotantent bundle and if $\zeta: \mathfrak{g} \rightarrow \mathfrak{X}\left(T^{*} Q, \omega_{Q}\right)$ is induced by $\sigma: \mathfrak{g} \rightarrow \mathfrak{X}(Q)$ in the sense that its flow is given by $\mathrm{Fl}_{t}^{\zeta_{X}}=T^{*}\left(\mathrm{Fl}_{t}^{\sigma_{X}}\right)=T\left(\mathrm{Fl}_{-t}^{\sigma_{X}}\right)^{*}$. Namely, by (31.9) we have:

$$
\mathcal{L}_{\zeta_{X}} \vartheta_{Q}=\left.\partial\right|_{0}\left(\mathrm{Fl}_{t}^{\zeta_{X}}\right)^{*} \vartheta_{Q}=\left.\partial\right|_{0}\left(T^{*}\left(\mathrm{Fl}_{t}^{\sigma_{X}}\right)\right)^{*} \vartheta_{Q}=0
$$

Let us note here for further use that the $j$ is given by the following formula: For $p_{q} \in T_{q}^{*} Q$ we have:

$$
j(X)\left(p_{q}\right)=\vartheta\left(\zeta_{X}\left(p_{q}\right)\right)=\left\langle\pi_{T^{*} Q}\left(\zeta_{X}\left(p_{q}\right)\right), T\left(\pi_{Q}\right)\left(\zeta_{X}\left(p_{q}\right)\right)\right\rangle=\left\langle p_{q}, \sigma_{X}(q)\right\rangle .
$$


(5) An example where the cohomology class $\tilde{\zeta}=[\bar{\jmath}] \in H^{2}\left(\mathfrak{g}, H^{0}(M)\right)$ does not vanish: Let $\mathfrak{g}=\left(\mathbb{R}^{2},[, \quad]=0\right)$ with coordinates $a, b$. Let $M=T^{*} \mathbb{R}$ with coordinates $q, p$, and let $\omega=d q \wedge d p$. Let $\zeta_{(a, b)}=a \partial_{q}+b \partial_{p}$. A lift is given by $j(a, b)(q, p)=a p-b q$. Then

$$
\begin{aligned}
\bar{\jmath}\left(\left(a_{1}, b_{1}\right),\left(a_{2}, b_{2}\right)\right) & =\left\{j\left(a_{1}, b_{1}\right), j\left(a_{2}, b_{2}\right)\right\}-j(0)=\left\{a_{1} p-b_{1} q, a_{2} p-b_{2} q\right\} \\
& =-a_{1} b_{2}+a_{2} b_{1} .
\end{aligned}
$$

(6) For a symplectic group action $r: M \times G \rightarrow M$ of a Lie group $G$ on a symplectic manifold $M$, let us suppose that the cohomology class $\tilde{\zeta}=[\bar{\jmath}] \in$ $H^{2}\left(\mathfrak{g}, H^{0}(M)\right)$ from $(34.1 .1)$ vanishes. Then there exists $\tau \in L\left(\mathfrak{g}, H^{0}(M)\right)$ with $d \tau=\bar{\jmath}$, i.e.,

$$
\begin{aligned}
d \tau(X, Y) & =-\tau([X, Y])=\bar{\jmath}(X, Y)=\{j X, j Y\}-j([X, Y]), \\
\overline{j-\tau}(X, Y) & =\{(j-\tau) X,(j-\tau) Y\}-(j-\tau)([X, Y]) \\
& =\{j X, j Y\}+0-j([X, Y])+\tau([X, Y])=0,
\end{aligned}
$$

so that $j-\tau: \mathfrak{g} \rightarrow C^{\infty}(M)$ is a homomorphism of Lie algebras. Then the action of $G$ is called a strongly Hamiltonian group action and the homomorphism $j-\tau: \mathfrak{g} \rightarrow C^{\infty}(M)$ is called the associated infinitesimal strongly Hamiltonian action.

34.7. Theorem. The momentum mapping $J: M \rightarrow \mathfrak{g}^{*}$ for an infinitesimal strongly Hamiltonian action $j: \mathfrak{g} \rightarrow C^{\infty}(M)$ on a Poisson manifold $\left(M, P^{M}\right)$ has the following properties:

(1) $J$ is infinitesimally equivariant: For each $X \in \mathfrak{g}$ the Hamiltonian vector fields $H_{j(X)}=\zeta_{X} \in \mathfrak{X}(M, P)$ and $\operatorname{ad}(X)^{*}: \mathfrak{g}^{*} \rightarrow \mathfrak{g}^{*}$ are $J$-related.

(2) $J$ is a Poisson morphism $J:\left(M, P^{M}\right) \rightarrow\left(\mathfrak{g}^{*}, P^{\mathfrak{g}^{*}}\right)$ into the canonical Poisson structure on $\mathfrak{g}^{*}$ from (33.7),

(3) The momentum mapping for a strongly Hamiltonian action of a connected Lie group $G$ on a Poisson manifold is $G$-equivariant: $J(x . g)=$ $\operatorname{Ad}(g)^{*} . J(x)$.

Proof. (1) By definition (34.2.1) we have $\langle J(x), X\rangle=j(X)(x)$; differentiating this, we get $\left\langle d J(x)\left(\xi_{x}\right), X\right\rangle=d(j(X))\left(\xi_{x}\right)$ or $d\langle J, X\rangle=d j(X) \in \Omega^{1}(M)$. Then we have

$$
\begin{aligned}
\left\langle d J\left(\zeta_{X}\right), Y\right\rangle & =d j(Y)\left(\zeta_{X}\right)=H_{j(X)}(j(Y)) \\
& =\{j(X), j(Y)\}=j[X, Y], \\
\left\langle\operatorname{ad}(X)^{*} \circ J, Y\right\rangle & =\langle J, \operatorname{ad}(X) Y\rangle=\langle J,[X, Y]\rangle, \\
d J . \zeta_{X} & =\operatorname{ad}(X)^{*} \circ J .
\end{aligned}
$$


(2) We have to show that $\bigwedge^{2} d J(x) \cdot P^{M}=P^{\mathfrak{g}^{*}}(J(x))$, according to (33.5.3)

$$
\begin{aligned}
\left\langle P^{\mathfrak{g}^{*}} \circ J, X \wedge Y\right\rangle & =2\langle J,[X, Y]\rangle \quad \text { by (33.7) } \\
& =j[X, Y]=\{j(X), j(Y)\}, \\
\left\langle\bigwedge d J(x) . P^{M}, X \wedge Y\right\rangle & =\left\langle\bigwedge d J(x)^{*} \cdot(X \wedge Y), P^{M}\right\rangle \\
& =\left\langle d J(x)^{*} X \wedge d J(x)^{*} Y, P^{M}\right\rangle \\
& =\left\langle P^{M}, d\langle J, X\rangle \wedge d\langle J, Y\rangle\right\rangle(x) \\
& =\left\langle P^{M}, d j(X) \wedge d j(Y)\right\rangle(x)=2\{j(X), j(Y)\}(x) .
\end{aligned}
$$

(3) is an immediate consequence of (1).

34.8. Equivariance of momentum mappings. Let $J: M \rightarrow \mathfrak{g}^{*}$ be a momentum mapping for a Hamiltonian right group action $r: M \times G \rightarrow M$ on a symplectic manifold $(M, \omega)$. We do not assume here that the lift $j: \mathfrak{g} \rightarrow C^{\infty}(M)$ given by $j(X)=\langle J, X\rangle$ is a Lie algebra homomorphism. Recall that for the fundamental vector field mapping $\zeta: \mathfrak{g} \rightarrow \mathfrak{X}(M, \omega)$ we have $\zeta_{X}=H_{j(X)}=H_{\langle J, X\rangle}$. We also assume that $M$ is connected; otherwise one has to treat each connected component separately.

For $X \in \mathfrak{g}$ and $g \in G$ we have (compare with the proof of (34.4)

$$
\begin{aligned}
& \left(r^{g}\right)^{*} \zeta_{X}=\left(r^{g}\right)^{*} H_{\langle J, X\rangle}=\left(r^{g}\right)^{*}\left(\check{\omega}^{-1} d\langle J, X\rangle\right) \\
& =\left(\left(\left(r^{g}\right)^{*} \omega\right)^{-}\right)^{-1} d\left\langle\left(r^{g}\right)^{*} J, X\right\rangle=\check{\omega}^{-1} d\left\langle J \circ r^{g}, X\right\rangle=H_{\left\langle J \circ r^{g}, X\right\rangle}, \\
& \left(r^{g}\right)^{*} \zeta_{X}=T\left(r^{g^{-1}}\right) \circ \zeta_{X} \circ r^{g}=\zeta_{\operatorname{Ad}(g) X} \quad \text { by (6.3.2) } \\
& =H_{\langle J, \operatorname{Ad}(g) X\rangle}=H_{\left\langle\operatorname{Ad}(g)^{*} J, X\right\rangle} \cdot
\end{aligned}
$$

So we conclude that $\left\langle J \circ r^{g}-\operatorname{Ad}(g)^{*} J, X\right\rangle \in H^{0}(M)$ is a constant function on $M$ (which we assumed to be connected) for every $X \in \mathfrak{g}$ and we get a smooth mapping

$$
\bar{J}: G \rightarrow \mathfrak{g}^{*},
$$

$\bar{J}(g):=J \circ r^{g}-\operatorname{Ad}(g)^{*} \circ J=J(x . g)-\operatorname{Ad}(g)^{*} J(x) \in \mathfrak{g}^{*} \quad$ for each $x \in M$,

which satisfies for $g_{1}, g_{2} \in G$ and each $x \in M$

$$
\begin{aligned}
& \bar{J}\left(g_{0} g_{1}\right)=J\left(x . g_{0} g_{1}\right)-\operatorname{Ad}\left(g_{0} g_{1}\right)^{*} J(x) \\
& \quad=J\left(\left(x . g_{0}\right) \cdot g_{1}\right)-\operatorname{Ad}\left(g_{1}\right)^{*} \operatorname{Ad}\left(g_{0}\right)^{*} J(x) \\
& \quad=J\left(\left(x \cdot g_{0}\right) \cdot g_{1}\right)-\operatorname{Ad}\left(g_{1}\right)^{*} J\left(x \cdot g_{0}\right)+\operatorname{Ad}\left(g_{1}\right)^{*}\left(J\left(x \cdot g_{0}\right)-\operatorname{Ad}\left(g_{0}\right)^{*} J(x)\right) \\
& \quad=\bar{J}\left(g_{1}\right)+\operatorname{Ad}\left(g_{1}\right)^{*} \bar{J}\left(g_{0}\right)=\bar{J}\left(g_{1}\right)+\bar{J}\left(g_{0}\right) \cdot \operatorname{Ad}\left(g_{1}\right) .
\end{aligned}
$$

This equation says that $\bar{J}: G \rightarrow \mathfrak{g}^{*}$ is a smooth 1-cocycle with values in the right $G$-module $\mathfrak{g}^{*}$ for the smooth group cohomology which is given by the following coboundary operator, which for completeness sake we write for a 
$G$-bimodule $V$, i.e., a vector space $V$ with a linear left action $\lambda: G \times V \rightarrow V$ and a linear right action $\rho: V \times G \rightarrow V$ which commute:

$$
\begin{aligned}
(3) C^{k}(G, V):= & C^{\infty}\left(G^{k}=G \times \ldots \times G, V\right), \quad C^{0}(G, V)=V, \quad k \geq 0 \\
\delta: C^{k}(G, V) \rightarrow & C^{k+1}(G, V), \\
\delta \Phi\left(g_{0}, \ldots, g_{k}\right)= & g_{0} . \Phi\left(g_{1}, \ldots, g_{k}\right)+\sum_{i=1}^{k}(-1)^{i} \Phi\left(g_{0}, \ldots, g_{i-2}, g_{i-1} g_{i}, \ldots, g_{k}\right) \\
& +(-1)^{k+1} \Phi\left(g_{0}, \ldots, g_{k-1}\right) . g_{k} .
\end{aligned}
$$

It is easy to check that $\delta \circ \delta=0$. As noted in (15.16), the group cohomology is defined by

$$
H^{k}(G ; V):=\frac{\operatorname{ker}\left(\delta: C^{k}(G, V) \rightarrow C^{k+1}(G, V)\right)}{\operatorname{im}\left(\delta: C^{k-1}(G, V) \rightarrow C^{k}(G, V)\right)} .
$$

Since for $v \in V=C^{0}(G, V)$ we have $\delta v\left(g_{0}\right)=g_{0} \cdot v-v \cdot g_{0}$, it follows that $H^{0}(G, V)=\{v \in V: g . v=v \cdot g\}=Z_{V}(G)$. A smooth mapping $\Phi: G \rightarrow V$ is a cocycle $\delta \Phi=0$ if and only if $\Phi\left(g_{0} g_{1}\right)=g_{0} . \Phi\left(g_{1}\right)+\Phi\left(g_{0}\right) \cdot g_{1}$, i.e., $\Phi$ is a 'derivation'.

In our case $V=\mathfrak{g}^{*}$ with trivial left $G$-action (each $g \in G$ acts by the identity) and right action $\operatorname{Ad}(\quad)^{*}$. Any other moment mapping $J^{\prime}: M \rightarrow \mathfrak{g}^{*}$ is of the form $J^{\prime}=J+\alpha$ for constant $\alpha \in \mathfrak{g}^{*}$ since $M$ is connected. The associated group cocycle is then

$$
\begin{aligned}
\overline{J+\alpha}(g) & =J(x . g)+\alpha-\operatorname{Ad}(g)^{*}(J(x)+\alpha)=\bar{J}(g)+\alpha-\alpha \cdot \operatorname{Ad}(g) \\
& =(\bar{J}+\delta \alpha)(g),
\end{aligned}
$$

so that the group cohomology class $\tilde{r}=[\bar{J}] \in H^{1}\left(G, \mathfrak{g}^{*}\right)$ of the Hamiltonian $G$-action does not depend on the choice of the momentum mapping.

(5) The differential $d \bar{J}(e): \mathfrak{g} \rightarrow \mathfrak{g}^{*}$ at $e \in G$ of the group cocycle $\bar{J}: G \rightarrow \mathfrak{g} *$ satisfies

$$
\langle d \bar{J}(e) X, Y\rangle=\bar{j}(X, Y),
$$

where $\bar{j}$, given by $\bar{j}(X, Y)=\{j(X), j(Y)\}-j([X, Y])$, is the Lie algebra cocycle from (34.6.1), since

$$
\begin{aligned}
\{j(X), j(Y)\}(x)=H_{j(X)}(j(Y))(x)=i\left(H_{\langle J, X\rangle}(x)\right) d\langle J, Y\rangle & \\
& =\left\langle d J\left(\zeta_{X}(x)\right), Y\right\rangle=\left.\partial\right|_{0}\langle J(x . \exp (t X)), Y\rangle \\
& =\left.\partial\right|_{0}\left\langle\operatorname{Ad}(\exp (t X))^{*} J(x)+\bar{J}(\exp (t X)), Y\right\rangle \\
& =\left\langle\operatorname{ad}(X)^{*} J(x)+d \bar{J}(e)(X), Y\right\rangle=\langle J(x), \operatorname{ad}(X) Y\rangle+\langle d \bar{J}(e)(X), Y\rangle \\
& =j[X, Y](x)+\langle d \bar{J}(e)(X), Y\rangle .
\end{aligned}
$$


(6) If the group cohomology class $\tilde{r}$ of the Hamiltonian group action vanishes, then there exists a $G$-equivariant momentum mapping $J: M \rightarrow \mathfrak{g}^{*}$, i.e.,

$$
J(x . g)=\operatorname{Ad}(g)^{*} J(x) .
$$

Namely, let the group cohomology class be given by $\tilde{r}=[\bar{J}] \in H^{1}\left(G, \mathfrak{g}^{*}\right)$. Then $\bar{J}=\delta \alpha$ for some constant $\alpha \in \mathfrak{g}^{*}$. Then $J_{1}=J-\alpha$ is a $G$-equivariant momentum mapping since $J_{1}(x . g)=J(x . g)-\alpha=\operatorname{Ad}(g)^{*} J(x)+\bar{J}(g)-\alpha=$ $\operatorname{Ad}(g)^{*} J(x)+\delta \alpha(g)-\alpha=\operatorname{Ad}(g)^{*} J(x)-\operatorname{Ad}(g)^{*} \alpha=\operatorname{Ad}(g)^{*} J_{1}(x)$.

For $X, Y \in \mathfrak{g}$ and $g \in G$ we have

$$
\langle\bar{J}(g),[X, Y]\rangle=-\bar{\jmath}(X, Y)+\bar{\jmath}(\operatorname{Ad}(g) X, \operatorname{Ad}(g) Y) .
$$

To see this, we use the cocycle property $\bar{J}\left(g_{0} g_{1}\right)=\bar{J}\left(g_{1}\right)+\operatorname{Ad}\left(g_{1}\right)^{*} \bar{J}\left(g_{0}\right)$ from part (2) to get

$$
\begin{aligned}
d \bar{J}(g)\left(T\left(\mu^{g}\right) X\right) & =\left.\partial\right|_{0} \bar{J}(\exp (t X) g)=\left.\partial\right|_{0}\left(\bar{J}(g)+\operatorname{Ad}(g)^{*} \bar{J}(\exp (t X))\right) \\
& =\operatorname{Ad}(g)^{*} d \bar{J}(e) X, \\
\langle\bar{J}(g),[X, Y]\rangle & =\left.\partial\right|_{0}\langle\bar{J}(g), \operatorname{Ad}(\exp (t X)) Y\rangle=\left.\partial\right|_{0}\left\langle\operatorname{Ad}(\exp (t X))^{*} \bar{J}(g), Y\right\rangle \\
& =\left.\partial\right|_{0}\langle\bar{J}(g \exp (t X))-\bar{J}(\exp (t X)), Y\rangle \\
& =\left\langle\left.\partial\right|_{0} \bar{J}\left(g \exp (t X) g^{-1} g\right)-\left.\partial\right|_{0} \bar{J}(\exp (t X)), Y\right\rangle \\
& =\left\langle\left.\partial\right|_{0} \bar{J}(\exp (t \operatorname{Ad}(g) X) g)-d \bar{J}(e) X, Y\right\rangle \\
& =\left\langle\operatorname{Ad}(g)^{*} d \bar{J}(e) \operatorname{Ad}(g) X-d \bar{J}(e) X, Y\right\rangle \\
& =\bar{\jmath}(\operatorname{Ad}(g) X, \operatorname{Ad}(g) Y)-\bar{J}(X, Y)
\end{aligned}
$$

34.9. Theorem. Let $J: M \rightarrow \mathfrak{g}^{*}$ be a momentum mapping for a Hamiltonian right group action $r: M \times G \rightarrow M$ on a connected symplectic manifold $(M, \omega)$ with group 1-cocycle $\bar{J}: G \rightarrow \mathfrak{g}^{*}$ and Lie algebra 2-cocycle $\bar{\jmath}: \bigwedge^{2} \mathfrak{g} \rightarrow \mathbb{R}$. Then we have:

(1) There is a unique affine right action of $G$ on $\mathfrak{g}^{*}$ with linear part the coadjoint action,

$$
a^{g}=a_{\bar{J}}^{g}: \alpha \mapsto \operatorname{Ad}(g)^{*} \alpha+\bar{J}(g),
$$

such that $J: M \rightarrow \mathfrak{g}^{*}$ is $G$-equivariant.

(2) There is a Poisson structure on $\mathfrak{g}^{*}$, given by

$$
\{f, h\}_{\bar{\jmath}}(\alpha)=\left\langle\alpha,[d f(\alpha), d h(\alpha)]_{\mathfrak{g}}\right\rangle+\bar{\jmath}(d f(\alpha), d h(\alpha)),
$$

which is invariant under the affine $G$-action $a_{\bar{J}}$ from (11) and has the property that the momentum mapping $J:(M, \omega) \rightarrow\left(\mathfrak{g}^{*},\{, \quad\}_{\bar{\jmath}}\right)$ is a Poisson morphism. The symplectic leaves of this Poisson structure are exactly the orbits under the connected component $G_{0}$ of e for the affine action in (1). 
Proof. (11) By (34.8.1), $J$ is $G$-equivariant. It remains to check that we have a right action:

$$
\begin{aligned}
a^{g_{2}} a^{g_{1}}(\alpha) & =a^{g_{2}}\left(\operatorname{Ad}\left(g_{1}\right)^{*} \alpha+\bar{J}\left(g_{1}\right)\right) \\
& =\operatorname{Ad}\left(g_{2}\right)^{*} \operatorname{Ad}\left(g_{1}\right)^{*} \alpha+\operatorname{Ad}\left(g_{2}\right)^{*} \bar{J}\left(g_{1}\right)+\bar{J}\left(g_{2}\right) \\
& =\operatorname{Ad}\left(g_{1} g_{2}\right)^{*} \alpha+\bar{J}\left(g_{1} g_{2}\right) \\
& =a^{g_{1} g_{2}} \alpha, \quad \text { by }(34.8 .2) .
\end{aligned}
$$

(2) Let $X_{1}, \ldots, X_{n}$ be a basis of $\mathfrak{g}$ with dual basis $\xi^{1}, \ldots, \xi^{n}$ of $\mathfrak{g}^{*}$. Then we have in terms of the structure constants of the Lie algebra $\mathfrak{g}$

$$
\begin{aligned}
{\left[X_{i}, X_{j}\right] } & =\sum_{k} c_{i j}^{k} X_{k}, \\
{[, \quad] } & =\frac{1}{2} \sum_{i j k} c_{i j}^{k} X_{k} \otimes\left(\xi^{i} \wedge \xi^{j}\right), \\
P^{\mathfrak{g}^{*}} & =-[\quad, \quad]^{*}=-\frac{1}{2} \sum_{i j k} c_{i j}^{k}\left(\xi^{i} \otimes X_{k}\right) \wedge \xi^{j}, \\
\bar{\jmath} & =\frac{1}{2} \sum_{i j} \bar{\jmath}_{i j} \xi^{i} \wedge \xi^{j}, \\
P_{\bar{\jmath}}^{\mathfrak{g}^{*}} & =-\frac{1}{2} \sum_{i j k} c_{i j}^{k}\left(\xi^{i} \otimes X_{k}\right) \wedge \xi^{j}+\frac{1}{2} \sum_{i j} \bar{\jmath}_{i j} \xi^{i} \wedge \xi^{j}: \mathfrak{g}^{*} \rightarrow \bigwedge^{2} \mathfrak{g}^{*} .
\end{aligned}
$$

Let us now compute the Schouten bracket. We note that $\left[P^{\mathfrak{g}^{*}}, P^{\mathfrak{g}^{*}}\right]=0$ since this is a Poisson structure, and $[\bar{\jmath}, \bar{\jmath}]=0$ since it is a constant 2 -vector field on the vector space $\mathfrak{g}^{*}$. Then

$$
\begin{aligned}
& {\left[P_{\bar{\jmath}}^{\mathfrak{g}^{*},}, P_{\bar{\jmath}}^{\mathfrak{g}^{*}}\right]=\left[P^{\mathfrak{g}^{*}}+\bar{\jmath}, P^{\mathfrak{g}^{*}}+\bar{\jmath}\right]} \\
& =\left[P^{\mathfrak{g}^{*}}, P^{\mathfrak{g}^{*}}\right]+2\left[P^{\mathfrak{g}^{*}}, \bar{\jmath}\right]+[\bar{\jmath}, \bar{\jmath}] \\
& =0+2\left[P^{\mathfrak{g}^{*}}, \bar{\jmath}\right]+0 \\
& =-\frac{1}{2} \sum_{i j k l m} c_{i j}^{k} \bar{\jmath}_{l m}\left(\left[\xi^{i} \otimes X_{k}, \xi^{l}\right] \wedge \xi^{j} \wedge \xi^{m}-\left[\xi^{i} \otimes X_{k}, \xi^{m}\right] \wedge \xi^{j} \wedge \xi^{l}\right. \\
& \left.\quad-\left[\xi^{j}, \xi^{l}\right] \wedge\left(\xi^{i} \otimes X_{k}\right) \wedge \xi^{m}+\left[\xi^{j}, \xi^{m}\right] \wedge\left(\xi^{i} \otimes X_{k}\right) \wedge \xi^{l}\right) \\
& =-\frac{1}{2} \sum_{i j k l m} c_{i j}^{k} \bar{\jmath}_{l m}\left(-\delta_{k}^{l} \xi^{i} \wedge \xi^{j} \wedge \xi^{m}+\delta_{k}^{m} \xi^{i} \wedge \xi^{j} \wedge \xi^{l}-0+0\right) \\
& =\sum_{i j k m} c_{i j}^{k} \bar{\jmath}_{k m} \xi^{i} \wedge \xi^{j} \wedge \xi^{m}=-2 d \bar{\jmath}=0 .
\end{aligned}
$$

which is zero since $\bar{\jmath}$ is a Lie algebra cocycle. Thus $P_{\bar{\jmath}}^{\mathfrak{g}^{*}}$ is a Poisson structure. 
The Poisson structure $P_{\overline{\mathfrak{g}}}^{\mathfrak{g}^{*}}$ is invariant under the affine action since

$$
\begin{aligned}
& \left\{f \circ a^{g}, h \circ a^{g}\right\}_{\bar{\jmath}}(\alpha)=\left\langle\alpha,\left[d f\left(a^{g}(\alpha)\right) \cdot T\left(a^{g}\right), d h\left(a^{g}(\alpha)\right) \cdot T\left(a^{g}\right)\right]\right\rangle \\
& \quad+\bar{\jmath}\left(d f\left(a^{g}(\alpha)\right) \cdot T\left(a^{g}\right), d h\left(a^{g}(\alpha)\right) \cdot T\left(a^{g}\right)\right) \\
& =\left\langle\alpha,\left[d f\left(a^{g}(\alpha)\right) \cdot \operatorname{Ad}(g)^{*}, d h\left(a^{g}(\alpha)\right) \cdot \operatorname{Ad}(g)^{*}\right]\right\rangle \\
& \quad+\bar{\jmath}\left(d f\left(a^{g}(\alpha)\right) \cdot \operatorname{Ad}(g)^{*}, d h\left(a^{g}(\alpha)\right) \cdot \operatorname{Ad}(g)^{*}\right) \\
& =\left\langle\alpha, \operatorname{Ad}(g)\left[d f\left(a^{g}(\alpha)\right), d h\left(a^{g}(\alpha)\right)\right]\right\rangle+\bar{\jmath}\left(\operatorname{Ad}(g) d f\left(a^{g}(\alpha)\right), \operatorname{Ad}(g) d h\left(a^{g}(\alpha)\right)\right) \\
& =\left\langle\operatorname{Ad}(g)^{*} \alpha,\left[d f\left(a^{g}(\alpha)\right), d h\left(a^{g}(\alpha)\right)\right]\right\rangle+\left\langle\bar{J}(g),\left[d f\left(a^{g}(\alpha)\right), d h\left(a^{g}(\alpha)\right)\right]\right\rangle \\
& \left.\quad+\bar{\jmath}\left(d f\left(a^{g}(\alpha)\right), d h\left(a^{g}(\alpha)\right)\right), \quad \text { by }[34.8 \cdot 7)\right] \\
& =\left\langle a^{g}(\alpha),\left[d f\left(a^{g}(\alpha)\right), d h\left(a^{g}(\alpha)\right)\right]\right\rangle+\bar{\jmath}\left(d f\left(a^{g}(\alpha)\right), d h\left(a^{g}(\alpha)\right)\right) \\
& =\{f, g\}_{\bar{\jmath}}\left(a^{g}(\alpha)\right) .
\end{aligned}
$$

To see that the momentum mapping $J:(M, \omega) \rightarrow\left(\mathfrak{g}^{*}, P_{\bar{j}}^{\mathfrak{g}^{*}}\right)$ is a Poisson morphism, we have to show that $\bigwedge^{2} d J(x) \cdot P^{\omega}(x)=P_{\bar{\jmath}}^{\mathfrak{g}^{*}}(J(x)) \in \bigwedge^{2} \mathfrak{g}^{*}$ for $x \in M$, according to (33.5.3). Recall from the definition (34.2.1) that $\langle J, X\rangle=j(X) ;$ thus also $\langle d J(x), X\rangle=d j(X)(x): T_{x} M \rightarrow \mathbb{R}$.

$$
\begin{aligned}
\left\langle\bigwedge^{2} d J(x)\right. & \left.. P^{\omega}(x), X \wedge Y\right\rangle=\left\langle P^{\omega}(x), \bigwedge^{2} d J(x)^{*}(X \wedge Y)\right\rangle \\
& =\left\langle P^{\omega}(x), d J(x)^{*} X \wedge d J(x)^{*} Y\right\rangle=\left\langle P^{\omega}(x), d\langle J, X\rangle \wedge d\langle J, Y\rangle\right\rangle \\
& =\left\langle P^{\omega}(x), d j(X) \wedge d j(Y)\right\rangle=2\{j(X), j(Y)\}_{\omega} \\
& =2 \bar{\jmath}(X, Y)+2 j([X, Y])(x) \quad \text { by }(34.6 .1) \\
& =2\langle J(x),[X, Y]\rangle+2 \bar{\jmath}(X, Y) \\
& =\left\langle P_{\bar{\jmath}}^{\mathfrak{g}^{*}}(J(x)), X \wedge Y\right\rangle .
\end{aligned}
$$

It remains to investigate the symplectic leaves of the Poisson structure $P_{\bar{\jmath}}^{\mathfrak{g}^{*}}$. The fundamental vector fields for the twisted right action $a_{\bar{J}}$ is given by

$$
\zeta_{X}^{a_{\bar{J}}}(\alpha)=\left.\partial\right|_{0}\left(\operatorname{Ad}(\exp (t X))^{*} \alpha+\bar{J}(\exp (t X))\right)=\operatorname{ad}(X)^{*} \alpha+d \bar{J}(e) X .
$$

This fundamental vector field is also the Hamiltonian vector field for the function $\mathrm{ev}_{X}: \mathfrak{g}^{*} \rightarrow \mathbb{R}$ since

$$
\begin{aligned}
H_{\mathrm{ev}_{X}}^{\bar{\jmath}}(f)(\alpha) & =\left\{\operatorname{ev}_{X}, f\right\}_{\bar{\jmath}}(\alpha)=\langle\alpha,[X, d f(\alpha)]\rangle+\bar{\jmath}(X, d f(\alpha)) \\
& =\left\langle\operatorname{ad}(X)^{*} \alpha, d f(\alpha)\right\rangle+\langle d \bar{J}(e) X, d f(\alpha)\rangle \\
& =\zeta_{X}^{a_{\bar{J}}}(f)(\alpha) .
\end{aligned}
$$

Hamiltonian vector fields of linear functions suffice to span the integrable distribution with jumping dimension which generates the symplectic leaves. Thus the symplectic leaves are exactly the orbits of the $G_{0}$-action $a_{\bar{J}}$. 
34.10. Corollary (Kostant, Souriau). Let $J: M \rightarrow \mathfrak{g}^{*}$ be a momentum mapping for a transitive Hamiltonian right group action $r: M \times G \rightarrow M$ on a connected symplectic manifold $(M, \omega)$ with group 1 -cocycle $\bar{J}: G \rightarrow \mathfrak{g}^{*}$ and Lie algebra 2 -cocycle $\bar{\jmath}: \bigwedge^{2} \mathfrak{g} \rightarrow \mathbb{R}$.

Then the image $J(M)$ of the momentum mapping is an orbit $O$ of the affine action $a_{\bar{J}}$ of $G$ on $\mathfrak{g}^{*}$ for which $J$ is equivariant, and the map $J: M \rightarrow O$ is locally a symplectomorphism and a covering mapping of $O$.

Proof. Since $G$ acts transitively on $M$ and $J$ is $G$-equivariant, $J(M)=O$ is an orbit for the twisted action $a_{J}$ of $G$ on $\mathfrak{g}^{*}$. Since $M$ is connected, $O$ is connected and is thus a symplectic leaf of the twisted Poisson structure $P_{\bar{j}}^{\mathfrak{g}^{*}}$ for which $J: M \rightarrow \mathfrak{g}^{*}$ is a Poisson mapping. Along $O$ the Poisson structure is symplectic and its pullback via $J$ equals $\omega$; thus $T_{x} J: T_{x} M \rightarrow T_{J(x)} O$ is invertible for each $x \in M$ and $J$ is a local diffeomorphism. Since $J$ is equivariant, it is diffeomorphic to a mapping $M \cong G / G_{x} \rightarrow G / G_{J(x)}$ and is thus a covering mapping.

34.11. Let us suppose that for some symplectic infinitesimal action of a Lie algebra $\zeta: \mathfrak{g} \rightarrow \mathfrak{X}(M, \omega)$ the cohomology class $\tilde{\zeta}=[\bar{\jmath}] \in H^{2}\left(\mathfrak{g}, H^{0}(M)\right)$ does not vanish. Then we replace the Lie algebra $\mathfrak{g}$ by the central extension, see section (15),

$$
0 \rightarrow H^{0}(M) \rightarrow \tilde{\mathfrak{g}} \rightarrow \mathfrak{g} \rightarrow 0
$$

which is defined by $\tilde{\zeta}=[\bar{\jmath}]$ in the following way: $\tilde{\mathfrak{g}}=H^{0}(M) \times \mathfrak{g}$ with bracket $[(a, X),(b, Y)]:=(\bar{\jmath}(X, Y),[X, Y])$. This satisfies the Jacobi identity since

$$
\begin{aligned}
{[[(a, X),(b, Y)],(c, Z)] } & =[(\bar{\jmath}(X, Y),[X, Y]),(c, Z)] \\
& =(\bar{\jmath}([X, Y], Z),[[X, Y], Z])
\end{aligned}
$$

and the cyclic sum of this expression vanishes. The map $j_{1}: \tilde{g} \rightarrow C^{\infty}(M)$, given by $j_{1}(a, X)=j(X)+a$, fits into the diagram

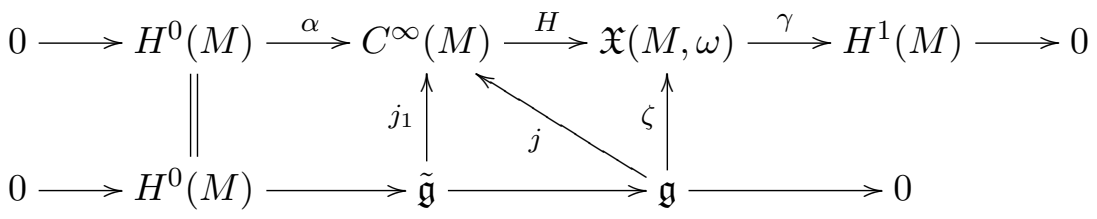

and is a homomorphism of Lie algebras since

$$
\begin{aligned}
j_{1}([(a, X),(b, Y)]) & =j_{1}(\bar{\jmath}(X, Y),[X, Y])=j([X, Y])+\bar{\jmath}(X, Y) \\
& =j([X, Y])+\{j X, j Y\}-j([X, Y]) \\
& =\{j X, j Y\}=\{j X+a, j Y+b\} \\
& =\left\{j_{1}(a, X), j_{1}(b, Y)\right\} .
\end{aligned}
$$


In this case we can consider the momentum mapping

$$
\begin{aligned}
J_{1}: M & \rightarrow \tilde{\mathfrak{g}}^{*}=\left(H^{0}(M) \times \mathfrak{g}\right)^{*}, \\
\left\langle J_{1}(x),(a, X)\right\rangle & =j_{1}(a, X)(x) \\
& =j(X)(x)+a, \\
H_{j_{1}(a, X)} & =\zeta_{X}, \quad x \in M, \quad X \in \mathfrak{g}, \quad a \in H^{0}(M),
\end{aligned}
$$

which has all the properties of theorem (34.7).

Let us describe this in more detail. Property (34.7.1) says that for all $(a, X) \in H^{0}(M) \times \mathfrak{g}$ the vector fields $H_{j(X)+a}=\zeta_{X} \in \mathfrak{X}(M)$ and $\operatorname{ad}(a, X)^{*} \in$ $\mathfrak{X}\left(\tilde{g}^{*}\right)$ are $J_{1}$-related. We have

$$
\begin{aligned}
\left\langle\operatorname{ad}(a, X)^{*}(\alpha, \xi),\right. & (b, Y)\rangle=\langle(\alpha, \xi),[(a, X)(b, Y)]\rangle \\
& =\langle(\alpha, \xi),(\bar{\jmath}(X, Y),[X, Y])\rangle \\
& =\alpha \bar{\jmath}(X, Y)+\langle\xi,[X, Y]\rangle \\
& =\alpha \bar{\jmath}(X, Y)+\left\langle\operatorname{ad}(X)^{*} \xi, Y\right\rangle \\
& =\left\langle\left(0, \alpha \bar{\jmath}(X, \quad)+\operatorname{ad}(X)^{*} \xi\right),(b, Y)\right\rangle, \\
\operatorname{ad}(a, X)^{*}(\alpha, \xi) & =\left(0, \alpha \bar{\jmath}(X, \quad)+\operatorname{ad}(X)^{*} \xi\right) .
\end{aligned}
$$

This is related to formula (34.9.3) which describes the infinitesimal twisted right action corresponding to the twisted group action of (34.9.1).

The Poisson bracket on $\tilde{\mathfrak{g}}^{*}=\left(H^{0}(M) \times \mathfrak{g}\right)^{*}=H^{0}(M)^{*} \times \mathfrak{g}^{*}$ is given by

$$
\begin{aligned}
\{f, h\}^{\tilde{\mathfrak{g}}^{*}}(\alpha, \xi) & =\left\langle(\alpha, \xi),\left[\left(d_{1} f(\alpha, \xi), d_{2} f(\alpha, \xi)\right),\left(d_{1} h(\alpha, \xi), d_{2} h(\alpha, \xi)\right)\right]\right\rangle \\
& =\left\langle(\alpha, \xi),\left(\bar{\jmath}\left(d_{2} f(\alpha, \xi), d_{2} h(\alpha, \xi)\right),\left[d_{2} f(\alpha, \xi), d_{2} h(\alpha, \xi)\right]\right)\right\rangle \\
& =\alpha \bar{\jmath}\left(d_{2} f(\alpha, \xi), d_{2} h(\alpha, \xi)\right)+\left\langle\xi,\left[d_{2} f(\alpha, \xi), d_{2} h(\alpha, \xi)\right]\right\rangle
\end{aligned}
$$

which for $\alpha=1$ and connected $M$ is the twisted Poisson bracket in (34.9.2). We may continue and derive all properties of (34.9) for a connected Lie group from here, with some interpretation.

34.12. Symplectic reduction. Let $J: M \rightarrow \mathfrak{g}^{*}$ be a momentum mapping for a Hamiltonian right group action $r: M \times G \rightarrow M$ on a connected symplectic manifold $(M, \omega)$ with group 1-cocycle $\bar{J}: G \rightarrow \mathfrak{g}^{*}$ and Lie algebra 2-cocycle $\bar{\jmath}: \bigwedge^{2} \mathfrak{g} \rightarrow \mathbb{R}$.

(1) ([22]) A point $\alpha \in J(M) \subset \mathfrak{g}^{*}$ is called a weakly regular value for $J$ if $J^{-1}(\alpha) \subset M$ is a submanifold such that for each $x \in J^{-1}(\alpha)$ we have $T_{x} J^{-1}(\alpha)=\operatorname{ker}\left(T_{x} J\right)$.

This is the case if $\alpha$ is a regular value for $J$, or if $J$ is of constant rank in a neighborhood of $J^{-1}(\alpha)$, by (1.13). Let us fix a weakly regular value $\alpha \in \mathfrak{g}^{*}$ of $J$ for the following. The submanifold $J^{-1}(\alpha) \subset M$ then has the following properties: 
(2) For a weakly regular value $\alpha$ of $J$, the submanifold $J^{-1}(\alpha)$ is invariant under the action of the isotropy group $G_{\alpha}=\left\{g \in G: a_{\bar{J}}^{g}(\alpha)=\alpha\right\}$. The dimension of the the isotropy group $G_{x}$ of $x \in J^{-1}(\alpha)$ does not depend on $x \in J^{-1}(\alpha)$ and is given by

$$
\operatorname{dim}\left(G_{x}\right)=\operatorname{dim}(G)-\operatorname{dim}(M)+\operatorname{dim}\left(J^{-1}(\alpha)\right) .
$$

Namely, $J: M \rightarrow \mathfrak{g}^{*}$ is equivariant for these actions by (34.9.1). Thus $J^{-1}(\alpha)$ is invariant under $G_{\alpha}$ and $G_{x} \subseteq G_{\alpha}$. For each $x \in J^{-1}(\alpha)$, by $(34.3 .4)$ we have $\operatorname{im}(d J(x))=\mathfrak{g}_{x}^{\circ} \subset \mathfrak{g}^{*}$. Since $T_{x}\left(J^{-1}(\alpha)\right)=\operatorname{ker}(d J(x))$, we get

$$
\begin{aligned}
\operatorname{dim}\left(T_{x} M\right) & =\operatorname{dim}\left(T_{x} J^{-1}(\alpha)\right)+\operatorname{rank}(d J(x)), \\
\operatorname{dim}\left(G_{x}\right) & =\operatorname{dim}(G)-\operatorname{dim}(x \cdot G) \\
& =\operatorname{dim}(G)-\operatorname{dim}\left(\mathfrak{g}_{x}^{\circ}\right) \\
& =\operatorname{dim}(G)-\operatorname{rank}(d J(x)) \\
& =\operatorname{dim}(G)-\operatorname{dim}(M)+\operatorname{dim}\left(J^{-1}(\alpha)\right) .
\end{aligned}
$$

(3) At any $x \in J^{-1}(\alpha)$ the kernel of the pullback $\omega^{J^{-1}(\alpha)}$ of the symplectic form $\omega$ equals $T_{x}\left(x . G_{\alpha}\right)$ and its rank is constant and is given by

$$
\operatorname{rank}\left(\omega^{J^{-1}(\alpha)}\right)=2 \operatorname{dim}\left(J^{-1}(\alpha)\right)+\operatorname{dim}\left(a_{\bar{J}}^{G}(\alpha)\right)-\operatorname{dim}(M) .
$$

Namely, $T_{x} J^{-1}(\alpha)=\operatorname{ker}(d J(x))$ implies

$$
\begin{array}{rlr}
\operatorname{ker}\left(\omega^{J^{-1}(\alpha)}\right) & =T_{x}\left(J^{-1}(\alpha)\right) \cap T_{x}\left(J^{-1}(\alpha)\right)^{\perp} \\
& =T_{x}\left(J^{-1}(\alpha)\right) \cap \operatorname{ker}(d J(x))^{\perp} \\
& =T_{x}\left(J^{-1}(\alpha)\right) \cap T_{x}(x . G), \quad \text { by }(34.3 .3) \\
& =T_{x}\left(x \cdot G_{\alpha}\right),
\end{array}
$$

$$
\begin{aligned}
\operatorname{rank} & \left(\omega_{x}^{J^{-1}(\alpha)}\right)=\operatorname{dim}\left(J^{-1}(\alpha)\right)-\operatorname{dim}\left(x \cdot G_{\alpha}\right) \\
& =\operatorname{dim}\left(J^{-1}(\alpha)\right)-\operatorname{dim}\left(G_{\alpha}\right)+\operatorname{dim}\left(G_{x}\right) \\
& =\operatorname{dim}\left(J^{-1}(\alpha)\right)-\operatorname{dim}\left(G_{\alpha}\right)+\operatorname{dim}(G)-\operatorname{dim}(M)+\operatorname{dim}\left(J^{-1}(\alpha)\right) \text { by (2) } \\
& =2 \operatorname{dim}\left(J^{-1}(\alpha)\right)+\operatorname{dim}\left(a_{\bar{J}}^{G}(\alpha)\right)-\operatorname{dim}(M) .
\end{aligned}
$$

(4) If $\alpha$ is a regular value of $J: M \rightarrow \mathfrak{g}^{*}$, the action of $G$ on $M$ is locally free in a neighborhood of every point $x \in J^{-1}(\alpha)$, by (34.3.5), i.e., the isotropy group $G_{x}$ is discrete.

This follows from $\operatorname{codim}_{M}\left(J^{-1}(\alpha)\right)=\operatorname{dim}(\mathfrak{g})-\operatorname{dim}(G)$. 
34.13. Theorem. Weakly regular symplectic reduction. Consider a momentum mapping $J: M \rightarrow \mathfrak{g}^{*}$ for a Hamiltonian right group action $r$ : $M \times G \rightarrow M$ on a connected symplectic manifold $(M, \omega)$ with group 1-cocycle $\bar{J}: G \rightarrow \mathfrak{g}^{*}$ and Lie algebra 2 -cocycle $\bar{\jmath}: \bigwedge^{2} \mathfrak{g} \rightarrow \mathbb{R}$. Let $\alpha \in J(M) \subset \mathfrak{g}^{*}$ be a weakly regular value of $J$.

Then the pullback 2-form $\omega^{J^{-1}(\alpha)} \in \Omega^{2}\left(J^{-1}(\alpha)\right)$ of $\omega$ is of constant rank, invariant under the action of $G_{\alpha}$, and the leaves of the foliation described by its kernel are the orbits of the action of the connected component $G_{\alpha}^{0}$ of the isotropy group $G_{\alpha}:=\left\{g \in G: a_{\bar{J}}^{g}(\alpha)=\alpha\right\}$ in $J^{-1}(\alpha)$.

If moreover the orbit space $M_{\alpha}:=J^{-1}(\alpha) / G_{\alpha}^{0}$ is a smooth manifold, then there exists a unique symplectic form $\omega^{\alpha}$ on it such that for the canonical projection $\pi: J^{-1}(\alpha) \rightarrow M_{\alpha}$ we have $\pi^{*} \omega^{\alpha}=\omega^{J^{-1}(\alpha)}$.

Let $h \in C^{\infty}(M)^{G}$ be a Hamiltonian function on $M$ which is G-invariant; then $h \mid J^{-1}(\alpha)$ factors to $\bar{h} \in C^{\infty}\left(M_{\alpha}\right)$ with $\bar{h} \circ \pi=h \mid J^{-1}(\alpha)$. The Hamiltonian vector field $\operatorname{grad}^{\omega}(h)=H_{h}$ is tangent to $J^{-1}(\alpha)$ and the vector fields $H_{h} \mid J^{-1}(\alpha)$ and $H_{\bar{h}}$ are $\pi$-related. Thus their trajectories are mapped onto each other:

$$
\pi\left(\mathrm{Fl}_{t}^{H_{h}}(x)\right)=\mathrm{Fl}_{t}^{H_{\bar{h}}}(\pi(x)) .
$$

In this case we call $\left(M_{\alpha}=J^{-1}(\alpha) / G_{\alpha}^{0}, \omega^{\alpha}\right)$ the reduced symplectic manifold.

Proof. By (34.12.3) the 2-form $\omega^{J^{-1}(\alpha)} \in \Omega^{2}\left(J^{-1}(\alpha)\right)$ is of constant rank and the foliation corresponding to its kernel is given by the orbits of the unit component $G_{\alpha}^{0}$ of the isotropy group $G_{\alpha}$. Let us now suppose that the orbit space $M_{\alpha}=J^{-1}(\alpha) / G_{\alpha}^{0}$ is a smooth manifold. Since the 2 -form $\omega^{J^{-1}(\alpha)}$ is $G_{\alpha}^{0}$-invariant and horizontal for the projection

$$
\pi: J^{-1}(\alpha) \rightarrow J^{-1}(\alpha) / G_{\alpha}^{0}=M_{\alpha}
$$

it factors to a smooth 2 -form $\omega^{\alpha} \in \Omega^{2}\left(M_{\alpha}\right)$ which is closed and nondegenerate since we just factored out its kernel. Thus $\left(M_{\alpha}, \omega^{\alpha}\right)$ is a symplectic manifold and $\pi^{*} \omega^{\alpha}=\omega^{J^{-1}(\alpha)}$ by construction.

Now let $h \in C^{\infty}(M)$ be a Hamiltonian function which is invariant under $G$. By E. Noether's theorem (34.3.8) the momentum mapping $J$ is constant along each trajectory of the Hamiltonian vector field $H_{h}$; thus $H_{h}$ is tangent to $J^{-1}(\alpha)$ and $G_{\alpha}$-invariant on $J^{-1}(\alpha)$. Let $\bar{h} \in C^{\infty}\left(M_{\alpha}\right)$ be the factored function with $\bar{h} \circ \pi=h$, and consider $H_{\bar{h}} \in \mathfrak{X}\left(M_{\alpha}, \omega^{\alpha}\right)$. Then for $x \in J^{-1}(\alpha)$ we have

$$
\left(T_{x} \pi\right)^{*}\left(i_{T_{x} \pi \cdot H_{h}(x)} \omega^{\alpha}\right)=i_{H_{h}(x)} \pi^{*} \omega^{\alpha}=d h(x)=\left(T_{x} \pi\right)^{*}(d \bar{h}(\pi(x))) .
$$

Since $\left(T_{x} \pi\right)^{*}: T_{\pi(x)}^{*} M_{\alpha} \rightarrow T_{x}\left(J^{-1}(\alpha)\right)$ is injective, we see that $i_{T_{x} \pi . H_{h}(x)} \omega^{\alpha}=$ $d \bar{h}(\pi(x))$ and hence $T_{x} \pi \cdot H_{h}(x)=H_{\bar{h}}(\pi(x))$. Thus $H_{h} \mid J^{-1}(\alpha)$ and $H_{\bar{h}}$ are $\pi$-related and the remaining assertions follow from (3.14). 
34.14. Proposition. Constant rank symplectic reduction. Consider a momentum mapping $J: M \rightarrow \mathfrak{g}^{*}$ for a Hamiltonian right group action $r: M \times G \rightarrow M$ on a connected symplectic manifold $(M, \omega)$ with group 1-cocycle $\bar{J}: G \rightarrow \mathfrak{g}^{*}$ and Lie algebra 2 -cocycle $\bar{\jmath}: \bigwedge^{2} \mathfrak{g} \rightarrow \mathbb{R}$. Let $G$ be connected. Let $\alpha \in J(M) \subset \mathfrak{g}^{*}$ be such that $J$ has constant rank in a neighborhood of $J^{-1}(\alpha)$. We consider the orbit $\alpha . G=a_{\bar{J}}^{G}(\alpha) \subset \mathfrak{g}^{*}$.

(1) $J^{-1}(\alpha . G) \subset M$ is an initial $G$-invariant submanifold.

(2) The smooth map $J^{-1}(\alpha) \times G \rightarrow J^{-1}(\alpha . G),(x, g) \mapsto x . g$ factors to a diffeomorphism $J^{-1}(\alpha) \times_{G_{\alpha}} G \cong J^{-1}(\alpha . G)$.

(3) Let $\iota: J^{-1}(\alpha . G) \rightarrow M$ be the inclusion. Then $\iota^{*} \omega-\iota^{*} J^{*} \omega^{\bar{J}}$ is closed, of constant rank and $G$-invariant. The leaves of the foliation described by its kernel are the orbits of the $G$-action restricted to $J^{-1}(\alpha . G)$. Here $\omega^{\bar{J}}$ is the symplectic structure on the affine orbit $\alpha . G$ from theorem $(34.9 .2)$.

(4) If $M_{\alpha . G}:=J^{-1}(\alpha . G) / G$ is a manifold, then $\iota^{*} \omega-\iota^{*} J^{*} \omega^{\bar{J}}$ factors to a symplectic form $\omega^{M_{\alpha . G}}$ on $M_{\alpha . G}$ which is thus characterized by

$$
\iota^{*} \omega=\pi^{*} \omega^{M_{\alpha . G}}+(J \circ \iota)^{*} \omega^{\bar{J}}
$$

where $\pi: J^{-1}(\alpha . G) \rightarrow M_{\alpha . G}$ is the projection.

(5) The orbit spaces $J^{-1}(\alpha) / G_{\alpha}$ and $M_{\alpha . G}$ are homeomorphic, and they are symplectomorphic if one of the orbit spaces is a manifold.

(6) Let $h \in C^{\infty}(M)^{G}$ be a $G$-invariant Hamiltonian function on $M$. Then $h \mid J^{-1}(\alpha . G)$ factors to $\bar{h} \in C^{\infty}\left(M_{\alpha}\right)$ with $\bar{h} \circ \pi=h \mid J^{-1}(\alpha . G)$. The Hamiltonian vector field $\operatorname{grad}^{\omega}(f)=H_{h}$ is tangent to $J^{-1}(\alpha . G)$ and the vector fields $H_{h} \mid J^{-1}(\alpha . G)$ and $H_{\bar{h}}$ are $\pi$-related. Thus their trajectories are mapped onto each other:

$$
\pi\left(\mathrm{Fl}_{t}^{H_{h}}(x)\right)=\mathrm{Fl}_{t}^{H_{\bar{h}}}(\pi(x)) .
$$

Proof. (11) Let $\alpha \in J(M) \subset \mathfrak{g}^{*}$ be such that $J$ is of constant rank on a neighborhood of $J^{-1}(\alpha)$. Let $\alpha \cdot G=a_{\bar{J}}^{G}(\alpha)$ be the orbit though $\alpha$ under the twisted coadjoint action. Then $J^{-1}(\alpha \cdot G)=J^{-1}(\alpha) \cdot G$ by the $G$-equivariance of $J$. Thus the dimension of the isotropy group $G_{x}$ of a point $x \in J^{-1}(\alpha . G)$ does not depend on $x$ and is given by (34.12.2). It remains to show that the inverse image $J^{-1}(\alpha . G)$ is an initial submanifold which is invariant under $G$.

If $\alpha$ is a regular value for $J$, then $J$ is a submersion on an open neighborhood of $J^{-1}(\alpha . G)$ and $J^{-1}(\alpha . G)$ is an initial submanifold by lemma (2.16).

Under the weaker assumption that $J$ is of constant rank on a neighborhood of $J^{-1}(\alpha)$, we will construct an initial submanifold chart as in (2.13.1) centered at each $x \in J^{-1}(\alpha . G)$. Using a suitable transformation in $G$, we may assume 
without loss that $x \in J^{-1}(\alpha)$. We shall use the method of the proof of theorem (3.25),

Let $m=\operatorname{dim}(M), n=\operatorname{dim}(\mathfrak{g}), r=\operatorname{rank}(d J(x)), p=m-r=\operatorname{dim}\left(J^{-1}(\alpha)\right)$ and $k=\operatorname{dim}(\alpha . G) \leq l=\operatorname{dim}(x . G)$. Using that $\mathfrak{g}_{x} \subseteq \mathfrak{g}_{\alpha}$, we choose a basis $X_{1}, \ldots, X_{n}$ of $\mathfrak{g}$ such that

- $\zeta_{X_{1}}^{\mathfrak{g}^{*}}(\alpha), \ldots, \zeta_{X_{k}}^{\mathfrak{g}^{*}}(\alpha)$ is a basis of $T_{\alpha}(\alpha . G)$ and $X_{k+1}, \ldots, X_{n}$ is a basis of the isotropy algebra $\mathfrak{g}_{\alpha}$,

- $\zeta_{X_{1}}^{M}(x), \ldots, \zeta_{X_{l}}^{M}(x)$ is a basis of $T_{x}(x . G)$ and $X_{l+1}, \ldots, X_{n}$ is a basis of the isotropy algebra $\mathfrak{g}_{x}$.

By the constant rank theorem (1.13) there exists a chart $(U, u)$ on $M$ centered at $x$ and a chart $(V, v)$ on $\mathfrak{g}^{*}$ centered at $\alpha$ such that

$$
v \circ J \circ u^{-1}: u(U) \rightarrow v(V)
$$

has the following form:

$$
\left(x^{1}, \ldots, x^{m}\right) \mapsto\left(x^{1}, \ldots, x^{k}, x^{k+1}, \ldots, x^{r}, 0, \ldots, 0\right),
$$

and we may also assume that

- $\zeta_{X_{1}}^{\mathfrak{g}^{*}}(\alpha), \ldots, \zeta_{X_{k}}^{\mathfrak{g}^{*}}(\alpha),\left.\frac{\partial}{\partial v^{k+1}}\right|_{\alpha}, \ldots,\left.\frac{\partial}{\partial v^{n}}\right|_{\alpha}$ is a basis of $T_{\alpha}\left(\mathfrak{g}^{*}\right)$,

- $\zeta_{X_{1}}^{M}(x), \ldots, \zeta_{X_{l}}^{M}(x),\left.\frac{\partial}{\partial u^{l+1}}\right|_{x}, \ldots,\left.\frac{\partial}{\partial u^{m}}\right|_{x}$ is a basis of $T_{x}(M)$.

Then the mapping

$$
f\left(y^{1}, \ldots, y^{n}\right)=\left(\mathrm{Fl}_{y^{1}}^{\zeta_{X_{1}}^{\mathfrak{g}^{*}}} \circ \cdots \circ \mathrm{Fl}_{y^{k}}^{\zeta_{X_{k}}^{\mathfrak{g}^{*}}} \circ v^{-1}\right)\left(0, \ldots, 0, y^{k+1}, \ldots, y^{n}\right)
$$

is a diffeomorphism from a neighborhood of 0 in $\mathbb{R}^{n}$ onto a neighborhood of $\alpha$ in $\mathfrak{g}^{*}$. Let $(\tilde{V}, \tilde{v})$ be the chart $f^{-1}$, suitably restricted. We have

$$
\beta \in \alpha \cdot G \Longleftrightarrow\left(\mathrm{Fl}_{y^{1}}^{\zeta_{X_{1}}^{\mathrm{g}^{*}}} \circ \ldots \circ \mathrm{Fl}_{y^{k}}^{\zeta_{X_{k}}^{\mathrm{g}^{*}}}\right)(\beta) \in \alpha \cdot G
$$

for all $\beta$ and all $y^{1}, \ldots, y^{k}$ for which both expressions make sense. So we have

$$
f\left(y^{1}, \ldots, y^{n}\right) \in \alpha . G \Longleftrightarrow f\left(0, \ldots, 0, y^{k+1}, \ldots, y^{n}\right) \in \alpha . G,
$$

and consequently $\alpha \cdot G \cap \tilde{V}$ is the disjoint union of countably many connected sets of the form $\left\{\beta \in \tilde{V}:\left(\tilde{v}^{k+1}(\beta), \ldots, \tilde{v}^{n}(\beta)\right)=\right.$ constant $\}$, since $\alpha . G$ is second countable.

Now let us consider the situation on $M$. Since $J^{-1}(\alpha)$ is $G_{\alpha}$-invariant, exactly the vectors $\zeta_{X_{k+1}}^{M}(x), \ldots, \zeta_{X_{l}}^{M}(x)$ are tangent to $x . G_{\alpha} \subseteq J^{-1}(\alpha)$. The mapping

$$
g\left(x^{1}, \ldots, x^{m}\right)=\left(\mathrm{Fl}_{x^{1}}^{\zeta_{X_{1}}^{M}} \circ \ldots \circ \mathrm{Fl}_{x^{k}}^{\zeta_{X_{k}}^{M}} \circ u^{-1}\right)\left(0, \ldots, 0, x^{k+1}, \ldots, x^{m}\right)
$$


is a diffeomorphisms from a neighborhood of 0 in $\mathbb{R}^{m}$ onto a neighborhood of $x$ in $M$. Let $(\tilde{U}, \tilde{u})$ be the chart $g^{-1}$, suitably restricted. By $G$-invariance of $J$ we have

$$
\begin{aligned}
(J \circ g) & \left(x^{1}, \ldots, x^{m}\right)=\left(J \circ \mathrm{Fl}_{x^{1}}^{\zeta_{X_{1}}^{M}} \circ \ldots \circ \mathrm{Fl}_{x^{k}}^{\zeta_{X_{k}}^{M}} \circ u^{-1}\right)\left(0, \ldots, 0, x^{k+1}, \ldots, x^{m}\right) \\
& =\left(\mathrm{Fl}_{x^{1}}^{\zeta_{X_{1}}^{\mathrm{g}^{*}}} \circ \ldots \circ \mathrm{Fl}_{x^{k}}^{\zeta_{X_{k}}^{\mathrm{g}^{*}}} \circ v^{-1} \circ v \circ J \circ u^{-1}\right)\left(0, \ldots, 0, x^{k+1}, \ldots, x^{m}\right) \\
& =\left(\mathrm{Fl}_{x^{1}}^{\zeta_{X_{1}}^{\mathrm{g}^{*}}} \circ \ldots \circ \mathrm{Fl}_{x^{k}}^{\zeta_{X_{k}}^{\mathrm{g}^{*}}} \circ v^{-1}\right)\left(0, \ldots, 0, x^{k+1}, \ldots, x^{r}, 0, \ldots, 0\right) \\
& =f\left(x^{1}, \ldots, x^{k}, x^{k+1}, \ldots, x^{r}, 0, \ldots, 0\right)
\end{aligned}
$$

and thus

$$
\begin{aligned}
& g\left(x^{1}, \ldots, x^{m}\right) \in J^{-1}(\alpha . G) \\
& \Longleftrightarrow(J \circ g)\left(x^{1}, \ldots, x^{m}\right)=f\left(x^{1}, \ldots, x^{k}, x^{k+1}, \ldots, x^{r}, 0, \ldots, 0\right) \in \alpha . G \\
& \Longleftrightarrow f\left(0_{\mathbb{R}^{k}}, x^{k+1}, \ldots, x^{r}, 0_{\mathbb{R}^{n-r}}\right) \in \alpha . G .
\end{aligned}
$$

Consequently, $\left(J^{-1}(\alpha . G)\right) \cap \tilde{U}$ is the disjoint union of countably many connected sets of the form $\left\{x \in \tilde{U}:\left(\tilde{u}^{k+1}(x), \ldots, \tilde{u}^{r}(x)\right)=\right.$ constant $\}$, since $\alpha . G$ is second countable. We have proved now that $J^{-1}(\alpha . G)$ is an initial submanifold or $M$.

(2) The induced map $J^{-1}(\alpha) \times_{G_{\alpha}} G \rightarrow J^{-1}(\alpha . G),[(x, g)] \mapsto x . g$ is a bijective submersion, and thus a diffeomorphism.

(31) Let $x \in J^{-1}(\alpha)$ and $X, Y \in \mathfrak{g}$. Then

$$
\begin{aligned}
\left(\iota^{*} \omega\right)_{x}\left(\zeta_{X}(x), \zeta_{Y}(x)\right) & =\omega_{x}\left(H_{j X}(x), H_{j Y}(x)\right) \\
& =-\{j X, j Y\}(x) \quad \text { by }(31.21) \\
& =-\left\{\operatorname{ev}_{X}, \operatorname{ev}_{Y}\right\}^{\bar{J}}(\alpha) \quad \text { by }(34.9 .2) \\
& =\omega_{\alpha}^{\bar{J}}\left(\zeta_{X}^{a_{\bar{J}}}(\alpha), \zeta_{Y}^{a_{\bar{J}}}(\alpha)\right) \quad \text { by }(34.9 .3)
\end{aligned}
$$

where $\omega^{\bar{J}}$ is the symplectic structure from (34.9.2) on the affine orbit $\alpha . G$. Let $\xi_{1}, \xi_{2} \in T_{x} J^{-1}(\alpha . G)$. By (2) we may (nonuniquely) decompose $\xi_{i}$ as $\xi_{i}=\eta_{i}+\zeta_{X_{i}}(x) \in T_{x} J^{-1}(\alpha)+T_{x}(x . G)$, where $i=1,2$. By (34.3.3) and (7) we have

$$
\left(\iota^{*} \omega\right)_{x}\left(\xi_{1}, \xi_{2}\right)=\omega_{x}^{J^{-1}(\alpha)}\left(\eta_{1}, \eta_{2}\right)+\left(\iota^{*} J^{*} \omega^{\bar{J}}\right)_{x}\left(\zeta_{X_{1}}(x), \zeta_{X_{2}}(x)\right)
$$

where we also use the notation from theorem (34.13). Thus

$$
\operatorname{ker}\left(\iota^{*} \omega-\iota^{*} J^{*} \omega^{\bar{J}}\right)_{x}=T_{x}(x \cdot G)+\operatorname{ker} \omega_{x}^{J^{-1}(\alpha)}=T_{x}(x \cdot G)
$$

by $(34.12 .3)$. Therefore, $\iota^{*} \omega-\iota^{*} J^{*} \omega^{\bar{J}}$ is closed, $G$-invariant and the leaves of the foliation described by its kernel coincide with the orbits of the $G$-action. By (34.12.2) this form is also of constant rank. 
(4) follows immediately from (3).

(51) By (2) the orbit spaces in question are homeomorphic and diffeomorphic if one of them is a manifold. In the latter case they are also symplectomorphic because of the formula in (4).

(6) Hamiltonian reduction follows similarly as in theorem (34.13).

34.15. Example: Coadjoint orbits. Let $G$ be a Lie group acting upon itself by inversion of left multiplication, i.e., $x . g=g^{-1} x$. Consider $T^{*} G$ with its canonical symplectic structure $\omega_{G}$ from (31.9). The cotangent lifted action by $G$ on $T^{*} G=G \times \mathfrak{g}^{*}$ (trivialized via left multiplication) is given by $(x, \alpha) \cdot g=\left(g^{-1} x, \alpha\right)$. According to (34.6.3) this action is strongly Hamiltonian with momentum mapping given by

$$
\langle J(x, \alpha), X\rangle=\left\langle\alpha, \zeta_{X}(x)\right\rangle=\left\langle-\operatorname{Ad}^{*}\left(x^{-1}\right) \cdot \alpha, X\right\rangle
$$

where $X \in \mathfrak{g}$. The $G$ action is free whence all points of $\mathfrak{g}^{*}$ are regular values for $J$. Let $O \subset \mathfrak{g}^{*}$ be a coadjoint orbit. Then $J^{-1}(O)=G \times(-O)$ and $\iota^{*} \omega_{G}-(J \circ \iota)^{*} \omega_{O}$ is basic with respect to the projection $J^{-1}(O) \rightarrow$ $J^{-1}(O) / G=-O$. (Here $\iota: J^{-1}(O) \rightarrow G \times \mathfrak{g}^{*}$ is the inclusion and $\omega_{O}$ is the coadjoint orbit symplectic form from (31.14).) The reduced symplectic space is thus given by $\left(-O,-\omega_{O}\right) \cong\left(O, \omega_{O}\right)$.

If we consider the action by $G$ on itself given by right multiplication, we see that $\left(O,-\omega_{O}\right)$ is the symplectic reduction of $\left(T^{*} G, \omega_{G}\right)$.

34.16. Example of a symplectic reduction: The space of Hermitian matrices. Let $G=S U(n)$ act on the space $H(n)$ of complex Hermitian $(n \times n)$-matrices by conjugation, where the inner product is given by the (always real) trace $\operatorname{Tr}(A B)$. We also consider the linear subspace $\Sigma \subset H(n)$ of all diagonal matrices; they have real entries. For each Hermitian matrix $A$ there exists a unitary matrix $g$ such that $g \mathrm{Ag}^{-1}$ is diagonal with eigenvalues decreasing in size. Thus a fundamental domain (we will call it a chamber) for the group action is here given by the quadrant $C \subset \Sigma$ consisting of all real diagonal matrices with eigenvalues $\lambda_{1} \geq \lambda_{2} \geq \cdots \geq \lambda_{n}$. There are no further identifications in the chamber; thus $H(n) / S U(n) \cong C$.

We are interested in the following problem: Consider a straight line $t \mapsto$ $A+t V$ of Hermitian matrices. We want to describe the corresponding curve of eigenvalues $t \mapsto \lambda(t)=\left(\lambda_{1}(t) \geq \cdots \geq \lambda_{n}(t)\right)$ of the Hermitian matrix $A+t V$ as precisely as possible. In particular, we want to find an ordinary differential equation describing the evolution of eigenvalues. We follow here the development in [4] which was inspired by [103].

(1) Hamiltonian description. Let us describe the curves of eigenvalues as trajectories of a Hamiltonian system on a reduced phase space. Let $T^{*} H(n)=$ $H(n) \times H(n)$ be the cotangent bundle where we identified $H(n)$ with its 
dual by the inner product, so the duality is given by $\langle\alpha, A\rangle=\operatorname{Tr}(A \alpha)$. Then the canonical 1-form is given by $\vartheta\left(A, \alpha, A^{\prime}, \alpha^{\prime}\right)=\operatorname{Tr}\left(\alpha A^{\prime}\right)$, the symplectic form is $\omega_{(A, \alpha)}\left(\left(A^{\prime}, \alpha^{\prime}\right),\left(A^{\prime \prime}, \alpha^{\prime \prime}\right)\right)=\operatorname{Tr}\left(A^{\prime} \alpha^{\prime \prime}-A^{\prime \prime} \alpha^{\prime}\right)$, and the Hamiltonian function for the straight lines $(A+t \alpha, \alpha)$ on $H(n)$ is $h(A, \alpha)=$ $\frac{1}{2} \operatorname{Tr}\left(\alpha^{2}\right)$. The action $S U(n) \ni g \mapsto\left(A \mapsto g A g^{-1}\right)$ lifts to the action $S U(n) \ni g \mapsto\left((A, \alpha) \mapsto\left(g A g^{-1}, g \alpha g^{-1}\right)\right)$ on $T^{*} H(n)$ with fundamental vector fields $\zeta_{X}(A, \alpha)=(A, \alpha,[X, A],[X, \alpha])$ for $X \in \mathfrak{s u}(n)$, and with generating functions $j_{X}(A, \alpha)=\vartheta\left(\zeta_{X}(A, \alpha)\right)=\operatorname{Tr}(\alpha[X, A])=\operatorname{Tr}([A, \alpha] X)$. Thus the momentum mapping $J: T^{*} H(n) \rightarrow \mathfrak{s u}(n)^{*}$ is given by $\langle X, J(A, \alpha)\rangle=$ $j_{X}(A, \alpha)=\operatorname{Tr}([A, \alpha] X)$. If we identify $\mathfrak{s u}(n)$ with its dual via the inner product $\operatorname{Tr}(X Y)$, the momentum mapping is $J(A, \alpha)=[A, \alpha]$. Along the line $t \mapsto A+t \alpha$ the momentum mapping is constant: $J(A+t \alpha, \alpha)=[A, \alpha]=Y \in$ $\mathfrak{s u}(n)$. Note that for $X \in \mathfrak{s u}(n)$ the evaluation on $X$ of $J(A+t \alpha, \alpha) \in \mathfrak{s u}(n)^{*}$ equals the inner product:

$$
\langle X, J(A+t \alpha, \alpha)\rangle=\operatorname{Tr}\left(\frac{d}{d t}(A+t \alpha), \zeta_{X}(A+t \alpha)\right),
$$

which is obviously constant in $t$; compare with the general result of Riemann transformation groups (30.1).

According to principles of symplectic reduction (34.12) we have to consider for a regular value $Y$ (and later for an arbitrary value) of the momentum mapping $J$ the submanifold $J^{-1}(Y) \subset T^{*} H(n)$. The null distribution of $\omega \mid J^{-1}(Y)$ is integrable (with constant dimensions since $Y$ is a regular value) and its leaves are exactly the orbits in $J^{-1}(Y)$ of the isotropy group $S U(n)_{Y}$ for the coadjoint action, by (34.13). So we have to consider the orbit space $J^{-1}(Y) / S U(n)_{Y}$. If $Y$ is not a regular value of $J$, the inverse image $J^{-1}(Y)$ is a subset which is described by polynomial equations since $J$ is polynomial (in fact quadratic), so $J^{-1}(Y)$ is stratified into submanifolds; symplectic reduction works also for this case; see [210, [16], or [185].

(2) The case of momentum $Y=0$ gives billiard of straight lines in $C$, reflected at the walls. If $Y=0$, then $S U(n)_{Y}=S U(n)$ and $J^{-1}(0)=\{(A, \alpha)$ : $[A, \alpha]=0\}$, so $A$ and $\alpha$ commute. If $A$ is regular (i.e., all eigenvalues are distinct), using a uniquely determined transformation $g \in S U(n)$, we move the point $A$ into the open chamber $C^{o} \subset H(n)$, so $A=\operatorname{diag}\left(a_{1}>a_{2}>\cdots>a_{n}\right)$ and since $\alpha$ commutes with $A$, it is also in diagonal form. The symplectic form $\omega$ restricts to the canonical symplectic form on $C^{o} \times \Sigma=C^{o} \times \Sigma^{*}=$ $T^{*}\left(C^{o}\right)$. Thus symplectic reduction gives $\left(J^{-1}(0) \cap\left(T^{*} H(n)\right)_{\mathrm{reg}}\right) / S U(n)=$ $T^{*}\left(C^{o}\right) \subset T^{*} H(n)$. By [210] we also use symplectic reduction for nonregular $A$ and we get (see in particular [122, 3.4]) $J^{-1}(0) / S U(n)=T^{*} C$, the stratified cotangent cone bundle of the chamber $C$ considered as stratified space. Namely, if one root $\varepsilon_{i}(A)=a_{i}-a_{i+1}$ vanishes on the diagonal matrix $A$, then the isotropy group $S U(n)_{A}$ contains a subgroup $S U(2)$ corresponding to these coordinates. Any matrix $\alpha$ with $[A, \alpha]=0$ contains an arbitrary 
Hermitian submatrix corresponding to the coordinates $i$ and $i+1$, which may be brought into diagonal form with the help of this $S U(2)$ so that $\varepsilon_{i}(\alpha)=\alpha_{i}-\alpha_{i+1} \geq 0$. Thus the tangent vector $\alpha$ with foot point in a wall is either tangent to the wall (if $\alpha_{i}=\alpha_{i+1}$ ) or points into the interior of the chamber $C$. The Hamiltonian $h$ restricts to $C^{o} \times \Sigma \ni(A, \alpha) \mapsto \frac{1}{2} \sum_{i} \alpha_{i}^{2}$, so the trajectories of the Hamiltonian system here are again straight lines which are reflected at the walls.

(3) The case of general momentum $Y$. If $Y \neq 0 \in \mathfrak{s u}(n)$ and if $S U(n)_{Y}$ is the isotropy group of $Y$ for the adjoint representation, then by the references at the end of (1) (concerning the singular version of (34.14) with stratified orbit space) we may pass from $Y$ to the coadjoint orbit $\mathcal{O}(Y)=\operatorname{Ad}^{*}(S U(n))(Y)$ and get

$$
J^{-1}(Y) / S U(n)_{Y}=J^{-1}(\mathcal{O}(Y)) / S U(n),
$$

where the (stratified) diffeomorphism is symplectic.

(4) The Calogero-Moser system. As the simplest case we assume that $Y^{\prime} \in$ $\mathfrak{s u}(n)$ is not zero but has maximal isotropy group, and we follow [103]. So we assume that $Y^{\prime}$ has complex rank 1 plus an imaginary multiple of the identity, $Y^{\prime}=\sqrt{-1}\left(c \mathbb{I}_{n}+v \otimes v^{*}\right)$ for $0 \neq v=\left(v^{i}\right)$ a column vector in $\mathbb{C}^{n}$. The coadjoint orbit is then $\mathcal{O}\left(Y^{\prime}\right)=\left\{\sqrt{-1}\left(c \mathbb{I}_{n}+w \otimes w^{*}\right): w \in\right.$ $\left.\mathbb{C}^{n},|w|=|v|\right\}$, isomorphic to $S^{2 n-1} / S^{1}=\mathbb{C} P^{n}$, of real dimension $2 n-2$. Consider $\left(A^{\prime}, \alpha^{\prime}\right)$ with $J\left(A^{\prime}, \alpha^{\prime}\right)=Y^{\prime}$, choose $g \in S U(n)$ such that $A=$ $g A^{\prime} g^{-1}=\operatorname{diag}\left(a_{1} \geq a_{2} \geq \cdots \geq a_{n}\right)$, and let $\alpha=g \alpha^{\prime} g^{-1}$. Then the entry of the commutator is $[A, \alpha]_{i j}=\alpha_{i j}\left(a_{i}-a_{j}\right)$. So $[A, \alpha]=g Y^{\prime} g^{-1}=: Y=$ $\sqrt{-1}\left(c \mathbb{I}_{n}+g v \otimes(g v)^{*}\right)=\sqrt{-1}\left(c \mathbb{I}_{n}+w \otimes w^{*}\right)$ has zero diagonal entries; thus $0<w^{i} \bar{w}^{i}=-c$ and $w^{i}=\exp \left(\sqrt{-1} \vartheta_{i}\right) \sqrt{-c}$ for some $\vartheta_{i}$. But then all offdiagonal entries $Y_{i j}=\sqrt{-1} w^{i} \bar{w}^{j}=-\sqrt{-1} c \exp \left(\sqrt{-1}\left(\vartheta_{i}-\vartheta_{j}\right)\right) \neq 0$, and $A$ has to be regular. We may use the remaining gauge freedom in the isotropy group $S U(n)_{A}=S\left(U(1)^{n}\right)$ to put $w^{i}=\exp (\sqrt{-1} \vartheta) \sqrt{-c}$ where $\vartheta=\sum \vartheta_{i}$. Then $Y_{i j}=-c \sqrt{-1}$ for $i \neq j$.

So the reduced space $\left(T^{*} H(n)\right)_{Y}$ is diffeomorphic to the submanifold of $T^{*} H(n)$ consisting of all $(A, \alpha) \in H(n) \times H(n)$ where $A=\operatorname{diag}\left(a_{1}>a_{2}>\right.$ $\left.\cdots>a_{n}\right)$ and where $\alpha$ has arbitrary diagonal entries $\alpha_{i}:=\alpha_{i i}$ and offdiagonal entries $\alpha_{i j}=Y_{i j} /\left(a_{i}-a_{j}\right)=-c \sqrt{-1} /\left(a_{i}-a_{j}\right)$. We can thus use $a_{1}, \ldots, a_{n}, \alpha_{1}, \ldots, \alpha_{n}$ as coordinates. The invariant symplectic form pulls back to $\omega_{(A, \alpha)}\left(\left(A^{\prime} \alpha^{\prime}\right),\left(A^{\prime \prime}, \alpha^{\prime \prime}\right)\right)=\operatorname{Tr}\left(A^{\prime} \alpha^{\prime \prime}-A^{\prime \prime} \alpha^{\prime}\right)=\sum\left(a_{i}^{\prime} \alpha_{i}^{\prime \prime}-a_{i}^{\prime \prime} \alpha_{i}^{\prime}\right)$. The invariant Hamiltonian $h$ restricts to the Hamiltonian

$$
h(A, \alpha)=\frac{1}{2} \operatorname{Tr}\left(\alpha^{2}\right)=\frac{1}{2} \sum_{i} \alpha_{i}^{2}+\frac{1}{2} \sum_{i \neq j} \frac{c^{2}}{\left(a_{i}-a_{j}\right)^{2}} .
$$

This is the famous Hamiltonian function of the Calogero-Moser completely integrable system; see [168], [180, [103], and [193, 3.1 and 3.3]. The 
corresponding Hamiltonian vector field and the differential equation for the eigenvalue curve are then

$$
\begin{gathered}
H_{h}=\sum_{i} \alpha_{i} \frac{\partial}{\partial a_{i}}+2 \sum_{i} \sum_{j: j \neq i} \frac{c^{2}}{\left(a_{i}-a_{j}\right)^{3}} \frac{\partial}{\partial \alpha_{i}}, \\
\ddot{a}_{i}=2 \sum_{j \neq i} \frac{c^{2}}{\left(a_{i}-a_{j}\right)^{3}}, \\
\left(a_{i}-a_{j}\right)^{\cdot \cdot}=2 \sum_{k: k \neq i} \frac{c^{2}}{\left(a_{i}-a_{k}\right)^{3}}-2 \sum_{k: k \neq j} \frac{c^{2}}{\left(a_{j}-a_{k}\right)^{3}} .
\end{gathered}
$$

Note that the curve of eigenvalues avoids the walls of the Weyl chamber $C$.

(5) Degenerate cases of nonzero momenta of minimal rank. Let us discuss now the case of nonregular diagonal $A$. Namely, if one root, say $\varepsilon_{12}(A)=$ $a_{1}-a_{2}$, vanishes on the diagonal matrix $A$, then the isotropy group $S U(n)_{A}$ contains a subgroup $S U(2)$ corresponding to these coordinates. Consider $\alpha$ with $[A, \alpha]=Y$; then $0=\alpha_{12}\left(a_{1}-a_{2}\right)=Y_{12}$. Thus $\alpha$ contains an arbitrary Hermitian submatrix corresponding to the first two coordinates, which may be brought into diagonal form with the help of this $S U(2) \subset S U(n)_{A}$ so that $\varepsilon_{12}(\alpha)=\alpha_{1}-\alpha_{2} \geq 0$. Thus the tangent vector $\alpha$ with foot point $A$ in a wall is either tangent to the wall (if $\alpha_{1}=\alpha_{2}$ ) or points into the interior of the chamber $C$ (if $\alpha_{1}>\alpha_{2}$ ). Note that then $Y_{11}=Y_{22}=Y_{12}=0$.

Let us now assume that the momentum $Y$ is of the form $Y=\sqrt{-1}\left(c \mathbb{I}_{n-2}+\right.$ $\left.v \otimes v^{*}\right)$ for some vector $0 \neq v \in \mathbb{C}^{n-2}$. We can repeat the analysis of (4) in the subspace $\mathbb{C}^{n-2}$ and get for the Hamiltonian function (where $I_{1,2}=$ $\{(i, j): i \neq j\} \backslash\{(1,2),(2,1)\})$

$$
\begin{gathered}
h(A, \alpha)=\frac{1}{2} \operatorname{Tr}\left(\alpha^{2}\right)=\frac{1}{2} \sum_{i=1}^{n} \alpha_{i}^{2}+\frac{1}{2} \sum_{(i, j) \in I_{1,2}} \frac{c^{2}}{\left(a_{i}-a_{j}\right)^{2}}, \\
H_{h}=\sum_{i=1}^{n} \alpha_{i} \frac{\partial}{\partial a_{i}}+2 \sum_{(i, j) \in I_{1,2}} \frac{c^{2}}{\left(a_{i}-a_{j}\right)^{3}} \frac{\partial}{\partial \alpha_{i}}, \\
\ddot{a}_{i}=2 \sum_{\left\{j:(i, j) \in I_{1,2}\right\}} \frac{c^{2}}{\left(a_{i}-a_{j}\right)^{3}} .
\end{gathered}
$$

(6) The case of general momentum $Y$ and regular $A$. Starting again with some regular $A^{\prime}$, consider $\left(A^{\prime}, \alpha^{\prime}\right)$ with $J\left(A^{\prime}, \alpha^{\prime}\right)=Y^{\prime}$, choose $g \in S U(n)$ such that $A=g A^{\prime} g^{-1}=\operatorname{diag}\left(a_{1}>a_{2}>\cdots>a_{n}\right)$, and let $\alpha=g \alpha^{\prime} g^{-1}$ and $Y=g Y^{\prime} g^{-1}=[A, \alpha]$. Then the entry of the commutator is $Y_{i j}=$ $[A, \alpha]_{i j}=\alpha_{i j}\left(a_{i}-a_{j}\right)$; thus $Y_{i i}=0$. We may pass to the coordinates $a_{i}$ and $\alpha_{i}:=\alpha_{i i}$ for $1 \leq i \leq n$ on the one hand, and $Y_{i j}$ for $i \neq j$ on the other hand, with the linear relation $Y_{j i}=-\overline{Y_{i j}}$ and with $n-1$ nonzero entries 
$Y_{i j}>0$ with $i>j$ (chosen in lexicographic order) by applying the remaining isotropy group $S U(n)_{A}=S\left(U(1)^{n}\right)=\left\{\operatorname{diag}\left(e^{\sqrt{-1} \vartheta_{1}}, \ldots, e^{\sqrt{-1} \vartheta_{n}}\right): \sum \vartheta_{i} \in\right.$ $2 \pi \mathbb{Z}\}$. This choice of coordinates $\left(a_{i}, \alpha_{i}, Y_{i j}\right)$ shows that the reduced phase space $J^{-1}(\mathcal{O}(Y)) / S U(n)$ is stratified symplectomorphic to $T^{*} C^{o} \times((\mathcal{O}(Y) \cap$ $\left.\left.\mathfrak{s u}(n)_{A}^{\perp}\right) / S U(n)_{A}\right)$; see [86, 85] and [87. In these coordinates, the Hamiltonian function is as follows:

$$
\begin{aligned}
h(A, \alpha) & =\frac{1}{2} \operatorname{Tr}\left(\alpha^{2}\right) \\
& =\frac{1}{2} \sum_{i} \alpha_{i}^{2}-\frac{1}{2} \sum_{i \neq j} \frac{Y_{i j} Y_{j i}}{\left(a_{i}-a_{j}\right)^{2}}, \\
d h & =\sum_{i} \alpha_{i} d \alpha_{i}+\sum_{i \neq j} \frac{Y_{i j} Y_{j i}}{\left(a_{i}-a_{j}\right)^{3}}\left(d a_{i}-d a_{j}\right)-\frac{1}{2} \sum_{i \neq j} \frac{d Y_{i j} \cdot Y_{j i}+Y_{i j} \cdot d Y_{j i}}{\left(a_{i}-a_{j}\right)^{2}} \\
(7) \quad & =\sum_{i} \alpha_{i} d \alpha_{i}+2 \sum_{i \neq j} \frac{Y_{i j} Y_{j i}}{\left(a_{i}-a_{j}\right)^{3}} d a_{i}-\sum_{i \neq j} \frac{Y_{j i}}{\left(a_{i}-a_{j}\right)^{2}} d Y_{i j} .
\end{aligned}
$$

The invariant symplectic form on $T H(n)$ pulls back, in these coordinates, to the symplectic form which is the product of the following two structures. The first one is $\omega_{(A, \alpha)}\left(\left(A^{\prime} \alpha^{\prime}\right),\left(A^{\prime \prime}, \alpha^{\prime \prime}\right)\right)=\operatorname{Tr}\left(A^{\prime} \alpha^{\prime \prime}-A^{\prime \prime} \alpha^{\prime}\right)=\sum\left(a_{i}^{\prime} \alpha_{i}^{\prime \prime}-a_{i}^{\prime \prime} \alpha_{i}^{\prime}\right)$ which equals $\sum_{i} d a_{i} \wedge d \alpha_{i}$. The second one comes by reduction from the Poisson structure on $\mathfrak{s u}(n)$ which is given by

$$
\begin{aligned}
P_{Y}(U, V) & =\operatorname{Tr}(Y[U, V])=\sum_{m, n, p}\left(Y_{m n} U_{n p} V_{p m}-Y_{m n} V_{n p} U_{p m}\right), \\
P_{Y} & =\sum_{i \neq j, k \neq l} P_{Y}\left(d Y_{i j}, d Y_{k l}\right) \partial_{Y_{i j}} \otimes \partial_{Y_{k l}} \\
& =\sum_{i \neq j, k \neq l} \sum_{m, n}\left(Y_{m n} \delta_{n i} \delta_{j k} \delta_{l m}-Y_{m n} \delta_{n k} \delta_{l i} \delta_{j m}\right) \partial_{Y_{i j}} \otimes \partial_{Y_{k l}} \\
& =\sum_{i \neq j, k \neq l}\left(Y_{l i} \delta_{j k}-Y_{j k} \delta_{l i}\right) \partial_{Y_{i j}} \otimes \partial_{Y_{k l}} .
\end{aligned}
$$

Since this Poisson 2-vector field is tangent to the orbit $\mathcal{O}(Y)$ and is $S U(n)$ invariant, we can push it down to the stratified orbit space

$$
\left(\mathcal{O}(Y) \cap \mathfrak{s u}(n)_{A}^{\perp}\right) / S U(n)_{A} .
$$

The latter space is the singular reduction of $\mathcal{O}(Y)$ with respect to the $S U(n)_{A}$-action. There it maps $d Y_{i j}$ to (remember that $Y_{i i}=0$ )

$$
\check{P}_{Y}\left(d Y_{i j}\right)=\sum_{k \neq l}\left(Y_{l i} \delta_{j k}-Y_{j k} \delta_{l i}\right) \partial_{Y_{k l}}=\sum_{k}\left(Y_{k i} \partial_{Y_{j k}}-Y_{j k} \partial_{Y_{k i}}\right) .
$$


So the Hamiltonian vector field is

$$
\begin{aligned}
H_{h}= & \sum_{i} \alpha_{i} \partial_{a_{i}}-2 \sum_{i \neq j} \frac{Y_{i j} Y_{j i}}{\left(a_{i}-a_{j}\right)^{3}} \partial_{\alpha_{i}} \\
& \quad-\sum_{i \neq j} \frac{Y_{j i}}{\left(a_{i}-a_{j}\right)^{2}} \sum_{k}\left(Y_{k i} \partial_{Y_{j k}}-Y_{j k} \partial_{Y_{k i}}\right) \\
= & \sum_{i} \alpha_{i} \partial_{a_{i}}-2 \sum_{i \neq j} \frac{Y_{i j} Y_{j i}}{\left(a_{i}-a_{j}\right)^{3}} \partial_{\alpha_{i}}+\sum_{i, j, k}\left(\frac{Y_{j i} Y_{j k}}{\left(a_{i}-a_{j}\right)^{2}}-\frac{Y_{i j} Y_{k j}}{\left(a_{j}-a_{k}\right)^{2}}\right) \partial_{Y_{k i}} .
\end{aligned}
$$

The differential equation thus becomes (remember that $Y_{j j}=0$ ):

$$
\begin{aligned}
\dot{a}_{i} & =\alpha_{i} \\
\dot{\alpha}_{i} & =-2 \sum_{j} \frac{Y_{i j} Y_{j i}}{\left(a_{i}-a_{j}\right)^{3}} \\
& =2 \sum_{j} \frac{\left|Y_{i j}\right|^{2}}{\left(a_{i}-a_{j}\right)^{3}}, \\
\dot{Y}_{k i} & =\sum_{j}\left(\frac{Y_{j i} Y_{j k}}{\left(a_{i}-a_{j}\right)^{2}}-\frac{Y_{i j} Y_{k j}}{\left(a_{j}-a_{k}\right)^{2}}\right) .
\end{aligned}
$$

Consider the matrix $Z$ with $Z_{i i}=0$ and $Z_{i j}=Y_{i j} /\left(a_{i}-a_{j}\right)^{2}$. Then the differential equations become:

$$
\begin{aligned}
& \ddot{a}_{i}=2 \sum_{j} \frac{\left|Y_{i j}\right|^{2}}{\left(a_{i}-a_{j}\right)^{3}}, \\
& \dot{Y}=\left[Z, Y^{*}\right] .
\end{aligned}
$$

This is the Calogero-Moser integrable system with spin; see [13], 14], and 86, 85.

(8) The case of general momentum $Y$ and singular $A$. Let us consider the situation of (6), when $A$ is not regular. Let us assume again that one root, say $\varepsilon_{12}(A)=a_{1}-a_{2}$, vanishes on the diagonal matrix $A$. Consider $\alpha$ with $[A, \alpha]=Y$. From $Y_{i j}=[A, \alpha]_{i j}=\alpha_{i j}\left(a_{i}-a_{j}\right)$ we conclude that $Y_{i i}=0$ for all $i$ and also $Y_{12}=0$. The isotropy group $S U(n)_{A}$ contains a subgroup $S U(2)$ corresponding to the first two coordinates and we may use this to move $\alpha$ into the form that $\alpha_{12}=0$ and $\varepsilon_{12}(\alpha) \geq 0$. Thus the tangent vector $\alpha$ with foot point $A$ in the wall $\left\{\varepsilon_{12}=0\right\}$ is either tangent to the wall when $\alpha_{1}=\alpha_{2}$ or points into the interior of the chamber $C$ when $\alpha_{1}>\alpha_{2}$. We can then use the same analysis as in (6) where we use now that $Y_{12}=0$. 
In the general case, when some roots vanish, we get for the Hamiltonian function, vector field, and differential equation:

$$
\begin{aligned}
& h(A, \alpha)=\frac{1}{2} \operatorname{Tr}\left(\alpha^{2}\right)=\frac{1}{2} \sum_{i} \alpha_{i}^{2}+\frac{1}{2} \sum_{\left\{(i, j): a_{i}(0) \neq a_{j}(0)\right\}} \frac{\left|Y_{i j}\right|^{2}}{\left(a_{i}-a_{j}\right)^{2}}, \\
& H_{h}=\sum_{i} \alpha_{i} \partial_{a_{i}}+2 \sum_{(i, j): a_{j}(0) \neq a_{i}(0)} \frac{\left|Y_{i j}\right|^{2}}{\left(a_{i}-a_{j}\right)^{3}} \partial_{\alpha_{i}} \\
& \quad+\sum_{(i, j): a_{j}(0) \neq a_{i}(0)} \sum_{k} \frac{Y_{j i} Y_{j k}}{\left(a_{i}-a_{j}\right)^{2}} \partial_{Y_{k i}}-\sum_{(j, k): a_{j}(0) \neq a_{k}(0)} \sum_{i} \frac{Y_{i j} Y_{k j}}{\left(a_{j}-a_{k}\right)^{2}} \partial_{Y_{k i}}, \\
& \ddot{a}_{i}=2 \sum_{j: a_{j}(0) \neq a_{i}(0)} \frac{\left|Y_{i j}\right|^{2}}{\left(a_{i}-a_{j}\right)^{3}}, \quad \dot{Y}=\left[Z, Y^{*}\right],
\end{aligned}
$$

where we use the same notation as above. It would be very interesting to investigate the reflection behavior of this curve at the walls.

34.17. Example: Symmetric matrices. We finally treat the action of $S O(n)=S O(n, \mathbb{R})$ on the space $S(n)$ of real symmetric matrices by conjugation. Following the method of (34.16.6) and (34.16.7), we get the following result. Let $t \mapsto A^{\prime}+t \alpha^{\prime}$ be a straight line in $S(n)$. Then the ordered set of eigenvalues $a_{1}(t), \ldots, a_{n}(t)$ of $A^{\prime}+t \alpha^{\prime}$ is part of the integral curve of the following vector field:

$$
\begin{aligned}
H_{h}= & \sum_{i} \alpha_{i} \partial_{a_{i}}+2 \sum_{(i, j): a_{j}(0) \neq a_{i}(0)} \frac{Y_{i j}^{2}}{\left(a_{i}-a_{j}\right)^{3}} \partial_{\alpha_{i}} \\
& -\sum_{(i, j): a_{i}(0) \neq a_{j}(0)} \sum_{k} \frac{Y_{i j} Y_{j k}}{\left(a_{i}-a_{j}\right)^{2}} \partial_{Y_{k i}}+\sum_{(j, k): a_{j}(0) \neq a_{k}(0)} \sum_{i} \frac{Y_{i j} Y_{j k}}{\left(a_{j}-a_{k}\right)^{2}} \partial_{Y_{k i}}, \\
\ddot{a}_{i}= & 2 \sum_{(i, j): a_{j}(0) \neq a_{i}(0)} \frac{Y_{i j}^{2}}{\left(a_{i}-a_{j}\right)^{3}}, \\
\dot{Y}= & {[Z, Y], \quad \text { where } Z_{i j}=-\frac{Y_{i j}}{\left(a_{i}-a_{j}\right)^{2}}, }
\end{aligned}
$$

where we also note that $Y_{i j}=Z_{i j}=0$ whenever $a_{i}(0)=a_{j}(0)$. 


\section{List of Symbols}

$(a, b) \quad$ open interval or pair

$[a, b]$ closed interval

$[X, Y] \quad$ Lie bracket, commutator, Frölicher-Nijehuis bracket

$\langle\alpha, X\rangle \quad$ usually a duality $V^{*} \times V \rightarrow \mathbb{R}$

$\operatorname{Ad}(g) \quad$ adjoint action of a Lie group on its Lie algebra

$\operatorname{ad}(X)=[X, \quad] \quad$ adjoint derivative of a Lie algebra

$\alpha: J^{r}(M, N) \rightarrow M \quad$ the source mapping of jets

$\beta: J^{r}(M, N) \rightarrow N$ the target mapping of jets

$B_{x}(r) \quad$ open ball with center $x$ and radius $r>0$

$\operatorname{conj}_{g}(h)=g h g^{-1} \quad$ conjugation in a Lie group

$\Gamma(E)$, also $\Gamma(E \rightarrow M)$ the space of smooth sections of a fiber bundle

$\mathbb{C}$ field of complex numbers

$C: T M \times_{M} T M \rightarrow T T M \quad$ connection or horizontal lift

$C^{\infty}(M, \mathbb{R})$ or $C^{\infty}(M)$ the space of smooth functions on a manifold $M$

$d$ usually the exterior derivative

$(E, p, M, S)$, also simply $E$ usually a fiber bundle with total space $E$, base $M$, and standard fiber $S$

exp exponential mapping from a Lie algebra to its Lie group

$\exp _{x}^{g} \quad$ geodesic exponential mapping centered at $x$

$\mathrm{Fl}_{t}^{X}$, also $\mathrm{Fl}(t, X)$ the flow of a vector field $X$

$K: T T M \rightarrow M$ the connector of a covariant derivative 
$G$ usually a general Lie group with multiplication $\mu: G \times G \rightarrow G$; we use $g h=\mu(g, h)=\mu_{g}(h)=\mu^{h}(g)$

$\mathfrak{g}=\operatorname{Lie}(G) \quad$ usually a Lie algebra for a Lie group $G$

$\mathbb{H}$ skew field of quaternions

$\mathbb{I}_{k}$ or $\mathbb{I}_{\mathbb{R}^{k}} \quad$ short for the $k \times k$-identity matrix $\operatorname{Id}_{\mathbb{R}^{k}}$.

$i_{X} \quad$ insertion operator of a vector field in a form

$J^{r}(E) \quad$ the bundle of $r$-jets of sections of a fiber bundle $E \rightarrow M$

$J^{r}(M, N)$ the bundle of $r$-jets of smooth functions from $M$ to $N$

$j^{r} f(x)$, also $j_{x}^{r} f \quad$ the $r$-jet of a mapping or function $f$

$\kappa_{M}: T T M \rightarrow T T M \quad$ the canonical flip mapping

$\ell: G \times S \rightarrow S$ usually a left action

$\mathcal{L}_{X} \quad$ Lie derivative along a vector field $X$

$M \quad$ usually a manifold

$\mu: G \times G \rightarrow G \quad$ usually the multiplication on a Lie group, $\mu(g, h)=g \cdot h=$ $\mu_{g}(h)=\mu^{h}(g)$, so $\mu_{g}$ is left translation by $g$ and $\mu^{h}$ is right translation by $h$

$\mathbb{N}$ natural numbers $>0$

$\mathbb{N}_{0} \quad$ nonnegative integers

$\nabla_{X}$, pronounced 'Nabla', covariant derivative along $X$

$\nu: G \rightarrow G, \nu(g)=g^{-1} \quad$ usually the inversion on a Lie group

$\operatorname{Pt}(c, t) \quad$ parallel transport along a curve $c$ from time 0 to time $t$

$p: P \rightarrow M$ or $(P, p, M, G) \quad$ a principal bundle with structure group $G$

$\pi_{l}^{r}: J^{r}(M, N) \rightarrow J^{l}(M, N) \quad$ projections of jets

$\mathbb{R}$ field of real numbers

$r: P \times G \rightarrow P \quad$ usually a right action, in particular the principal right action of a principal bundle

$T M$ the tangent bundle of a manifold $M$ with projection $\pi_{M}: T M \rightarrow M$

$T f: T M \rightarrow T N \quad$ tangent mapping of $f: M \rightarrow N$

$\mathbb{Z} \quad$ integers 


\section{Bibliography}

1. R. Abraham, Lectures of Smale on differential topology, Columbia University, New York, 1962, Lecture Notes.

2. R. Abraham and J. Marsden, Foundations of mechanics, Addison-Wesley, 1978.

3. A. Alekseev, A. Malkin, and E. Meinrenken, Lie group valued moment maps, J. Differ. Geom 48 (1998), 445-495.

4. D. Alekseevsky, A. Kriegl, M. Losik, and P.W. Michor, The Riemannian geometry of orbit spaces. The metric, geodesics, and integrable systems, Publicationes Mathematicae 62 (2003), 247-276.

5. D. Alekseevsky and P. Michor, Differential geometry of $\mathfrak{g}$-manifolds, Diff. Geom. Appl. 5 (1995), 371-403.

6. D. Alekseevsky, P.W. Michor, and W.A.F. Ruppert, Extensions of Lie algebras, math.DG/0005042, 2000.

7. __ Extensions of super Lie algebras, J. Lie Theory 15 (2005), 125-134, math.QA/0101190.

8. R. Almeida and P. Molino, Suites d'atiyah et feuilletages transversalement complets, C. R. Acad. Sci. Paris 300, Ser. 1 (1985), 13-15.

9. W. Ambrose and I.M. Singer, A theorem on holonomy, Trans. Amer. Math. Soc. 75 (1953), 428-443.

10. V.I. Arnold, Mathematical methods of classical mechanics, Springer-Verlag, New York, 1978.

11. M. Atiyah, R. Bott, and V.K. Patodi, On the heat equation and the index theorem, Inventiones Math. 19 (1973), 279-330.

12. J.A. Azcárraga and J.M. Izquierdo, Lie groups, Lie algebras, cohomology and some applications in physics, Cambridge University Press, Cambridge, UK, 1995, Cambridge Monographs on Mathematical Physics.

13. O. Babelon and M. Talon, The symplectic structure of the spin Calogero model, Phys. Lett. A 236, 5-6 (1997), 462-468, arXiv:q-alg/9707011.

14. _ The symplectic structure of rational Lax pair, Phys. Lett. A 257, 3-4 (1999), $139-144$ 
15. R. Baer, Erweiterungen von Gruppen und ihren Isomorphismen, Math. Zeit. 38 (1934), 375-416.

16. L. Bates and E. Lerman, Proper group actions and symplectic stratified spaces, Pacific J. Math. 181 (1997), 201-229.

17. A.L. Besse, Einstein manifolds, Springer-Verlag, Berlin, 1987, Ergebnisse der Mathematik, 3. Folge, Band 10.

18. E. Bierstone and P.D. Milman, Composite differentiable functions, Ann. of Math. 116 (1982), 541-558.

19. E. Bierstone, P.D. Milman, and W. Pawłucki, Composite differentiable functions, Duke Math. J. 83 (1996), 607-620.

20. J. Boman, Differentiability of a function and of its composition with a function of one variable, Math. Scand. 20 (1967), 249-268.

21. B. Boos and D.D. Bleeker, Topology and analysis, Springer-Verlag, New York, 1985 (1977)

22. R. Bott, Non-degenerate critical manifolds, Ann. Math. 60 (1954), 248-261.

23. R. Bott and H. Samelson, Applications of the theory of Morse to symmetric spaces, Amer. J. Math. 80 (1958), 964-1029.

24. J.-P. Bourguignon, Une stratification de l'espace des structures Riemanniens, Compositio Mathematica 30 (1975), 1-41.

25. G.E. Bredon, Introduction to compact transformation groups, Academic Press, New York, 1972.

26. T. Bröcker and K. Jänich, Einfürung in die Differentialtopologie, Springer-Verlag, Heidelberg, 1973.

27. K.S. Brown, Cohomology of groups, Springer-Verlag, New-York, 1982, Graduate Texts 87.

28. H. Bursztyn, M. Crainic, A. Weinstein, and C. Zhu, Integration of twisted Dirac brackets, Duke Math. J. 123 (2004), 549-607.

29. H. Bursztyn and O. Radko, Gauge equivalence of Dirac structures, Ann. Inst. Fourier 53 (2003), 309-337.

30. A. Cabras and A. M. Vinogradov, Extensions of the Poisson bracket to differential forms and multi-vector fields, J. Geom. Physics 9 (1992), 75-100.

31. E. Calabi, Sur les extensions des groupes topologiques, Brioschi Annali di Mat. Pura e Appl. Ser 432 (1951), 295-370.

32. A. Cap and J. Slovak, Parabolic geometries I: Background and general theory, AMS, Providence, 2009, Surveys and Monographs.

33. É. Cartan, Leçons sur la géométrie des espaces de riemann, Gauthiers-Villars, Paris, 1928.

34. R. Cohen, Immersions of manifolds, Proc. Nat. Acad. Sci. USA 79 (1982), 33903392.

35. L. Conlon, Variational completeness and K-transversal domains, J. Differential Geom. 5 (1971), 135-147.

36. A class of variationally complete representations, J. Differential Geom. 7 (1972), 149-160.

37. T. Courant, Dirac manifolds, Trans. AMS 319 (1990), 631-661.

38. J. Dadok, Polar coordinates induced by actions of compact Lie groups, TAMS 288 (1985), 125-137. 
39. J. Dadok and V. Kac, Polar representations, J. of Algebra 92 (1985), 504-524.

40. M. Davis, Smooth g-manifolds as collections of fiber bundles, Pac. J. Math. 77 (1978), $315-363$.

41. J. Dieudonne, Foundations of modern analysis I, Academic Press, 1960.

42. S. Donaldson, An application of gauge theory to the topology of 4-manifolds, J. Diff. Geo. 18 (1983), 269-316.

43. J.J. Duistermaat and J.A.C. Kolk, Lie groups, Springer-Verlag, 1999.

44. J.L. Dupont, Curvature and characteristic classes, Lecture Notes in Mathematics, vol. 640, Springer-Verlag, Berlin, 1978.

45. D. Ebin, The manifold of Riemannian metrics, Proc. Symp. Pure Math. AMS 15 (1970), 11-40.

46. D.G. Ebin and J.E. Marsden, Groups of diffeomorphisms and the motion of an incompressible fluid, Ann. Math. 92 (1970), 102-163.

47. D.J. Eck, Gauge-natural bundles and generalized gauge theories, Mem. Amer. Math. Soc. 247 (1981), vi +48.

48. _ Product preserving functors on smooth manifolds, J. Pure and Applied Algebra 42 (1986), 133-140.

49. J. Eells, A setting for global analysis, Bull AMS 72 (1966), 571-807.

50. J. Eells and L. Lemaire, A report on harmonic maps, Bull. London Math. Soc. 10 (1978), 1-68.

51. J Eells and L. Lemaire, Selected topics in harmonic mappings, vol. 50, Am. Math. Soc., Providence, 1983.

52. Another report on harmonic maps, Bull. London Math. Soc. 20 (1988), $385-524$.

53. C. Ehresmann, Les prolongements d'une variété différentiable I, II, III, C. R. Acad. Sci. Paris 233 (1951), I: 598-600, II: 777-779, III: 1081-1083.

54. __ Gattungen von Lokalen Strukturen, Jahresberichte der Deutschen Math. Ver. 60-2 (1957), 49-77.

55. S. Eilenberg, Foundations of fiber bundles, University of Chicago, Summer 1957.

56. S. Eilenberg and S. MacLane, Cohomology theory in abstract groups, II. Group extensions with non-abelian kernel, Ann. Math. (2) 48 (1947), 326-341.

57. T. Eisenkölbl, Personal communication, 2002.

58. C. Emmrich and H. Römer, Orbifolds as configuration spaces of systems with gaugesymmetries, Commun. Math. Phys. 129 (1990), 69-94.

59. D.B.A. Epstein, Natural tensors on Riemannian manifolds, J. Diff. Geom. 10, 631645 .

60. D.B.A. Epstein and W.P. Thurston, Transformation groups and natural bundles, Proc. London Math. Soc. 38 (1979), 219-236.

61. D.S. Freed and D. Groisser, The basic geometry of the manifold of Riemannian metrics and of its quotient by the diffeomorphism group, Michigan Math. J. 36 (1989), 323-344.

62. M.H. Freedman, The topology of four dimensional manifolds, J. Diff. Geo. 17 (1982), $357-454$.

63. M.H. Freedman and F. Luo, Selected applications of geometry to low-dimensional topology, University Lecture Series, vol. 1, Amer. Math. Soc., 1989. 
64. A. Frölicher and A. Kriegl, Linear spaces and differentiation theory, J. Wiley, Chichester, 1988, Pure and Applied Mathematics.

65. A. Frölicher and A. Nijenhuis, Theory of vector valued differential forms. Part I, Indagationes Math 18 (1956), 338-359.

66. P. Garcia Pérez, Gauge algebras, curvature, and symplectic structure, J. Diff. Geom. 12 (1977), 209-227.

67. I.M. Gelfand and I.Ya. Dorfman, Hamiltonian operators and the classical YangBaxter equation, Funct. Anal. Appl. 16 (1982), 241-248.

68. F. Gesztesy and H. Holden, Soliton equations and their algebro-geometric solutions, volume I: $(1+1)$-dimensional continuous models, Cambridge Studies in Advanced Mathematics, vol. 79, Cambridge University Press, Cambridge, 2003.

69. F. Gesztesy, H. Holden, J. Michor, and G. Teschl, Soliton equations and their algebrogeometric solutions, volume II: $(1+1)$-dimensional discrete models, Cambridge Studies in Advanced Mathematics, Cambridge University Press, Cambridge, 2008.

70. O. Gil-Medrano and P.W. Michor, The Riemannian manifold of all Riemannian metrics, Quarterly J. Math. Oxford 42 (1991), 183-202.

71. O. Gil-Medrano, P.W. Michor, and M. Neuwirther, Pseudoriemannian metrics on spaces of bilinear structures, Quarterly J. Math. Oxford 43 (1992), 201-221.

72. P.B. Gilkey, The spectral geometry of a Riemannian manifold, J. Diff. Geom. 10 (1975), 601-618.

73. _ Invariance theory, the heat equation and the index theorem, Publish or Perish, Wilmington, Del. (USA), 1984.

74. J. Giraud, Cohomologie non abélienne, Springer-Verlag, Berlin, 1971, Grundlehren 179.

75. G. Glaeser, Fonctions composées différentiables, Ann. of Math. 77 (1963), 193-209.

76. Yu. Gliklikh, Global analysis in mathematical physics. Geometric and stochastic methods, Springer-Verlag, New York, 1997, Aplied Mathematical Sciences.

77. R. Godement, Topologie algébrique et théorie des fausceaux, Hermann, Paris, 1958.

78. R. Gompf, Three exotic $\mathbb{R}^{4}$ 's and other anomalies, J. Diff. Geo. 18 (1983), 317-328.

79. J. Grabowski, Z-graded extensions of Poisson brackets, Rev. Math. Phys. 9 (1997), no. $1,1-27$.

80. W. Greub, S. Halperin, and R. Vanstone, Connections, curvature, and cohomology I, II, III, Academic Press, New York and London, 1972, 1973, 1976.

81. J. Hadamard, Les surfaces à courbures opposées et leurs lignes géodésiques, J. Math. Pures Appl. 4 (1898), 27-73.

82. S. Helgason, Differential geometry, lie groups, and symmetric spaces, Graduate Studies in Mathematics, vol. 34, AMS, Providence, 1978, 2001.

83. P.J. Hilton and U. Stammbach, A course in homological algebra, Graduate Texts in Mathematics, vol. 4, Springer-Verlag, New York, 1971, 1997.

84. M.W. Hirsch, Differential topology, Springer-Verlag, New York, 1976, GTM 33.

85. S. Hochgerner, Spinning particles in a Yang-Mills field, math.SG/0602062.

86. Singular cotangent bundle reduction 86 spin Calogero-Moser systems, Diff. Geom. Appl. (2008), math.SG/0411068.

87. S. Hochgerner and A. Rainer, Singular Poisson reduction of cotangent bundles, Rev. Mat. Complut. 19 (2006), 431-466, math.SG/0508455. 
88. G. Hochschild, Group extensions of Lie groups I, II, Ann Math. 54 (1951), 96-109; $537-551$.

89. Cohomology classes of finite type and finite dimensional kernels for Lie algebras, Am. J. Math. 76 (1954), 763-778.

90. _ The structure of Lie groups, Holden Day, San Francisco, 1965.

91. G.P. Hochschild and J.-P. Serre, Cohomology of group extensions, Trans. AMS 74 (1953), 110-134.

92. __ Cohomology of Lie algebras, Ann. Math. 57 (1953), 591-603.

93. O. Hölder, Die Gruppen der Ordnungen $p^{3}, p q^{2}, p q r, p^{4}$, Mathematische Annalen 43 (1893), 301-412.

94. L. Hörmander, The analysis of linear partial differential operators I, Springer-Verlag, Berlin, 1983, Grundlehren 256.

95. J.E. Humphreys, Reflection groups and Coxeter groups, Cambridge Studies in Advanced Mathematics, vol. 29, Cambridge University Press, Cambridge, 1990, 1992.

96. N. Jacobson, Lie algebras, J. Wiley-Interscience, 1962.

97. H Joris, Une $c^{\infty}$-application non-immersive qui possède la propriété universelle des immersions, Archiv Math. 39 (1982), 269-277.

98. H. Joris and E. Preissmann, Germes pseudo-immersifs de $\left(\mathbb{R}^{n}, 0\right)$ dans $\left(\mathbb{R}^{n}, 0\right), \mathrm{C} . \mathrm{R}$. Acad. Sci., Paris, Ser. I 305 (1987), 261-263.

99. _ Pseudo-immersions, Ann. Inst. Fourier 37 (1987), 195-221.

100. G. Kainz, A. Kriegl, and P.W. Michor, $c^{\infty}$-algebras from the functional analytic viewpoint, J. Pure Appl. Algebra 46 (1987), 89-107.

101. G. Kainz and P.W. Michor, Natural transformations in differential geometry, Czechoslovak Math. J. 37 (1987), 584-607.

102. F. Kamber and P.W. Michor, Completing Lie algebra actions to Lie group actions, Electron. Res. Announc. Amer. Math. Soc. 10 (2004), 1-10.

103. D. Kazhdan, B. Kostant, and S. Sternberg, Hamiltonian group actions and dynamical systems of Calogero type, Comm. Pure Appl. Math. 31 (1978), 481-501.

104. R. Kerner, Generalization of the Kaluza-Klein theory for an arbitrary non-Abelian group, Ann. Inst. Henri Poincare 9 (1968), 143-152.

105. A.A. Kirillov, Elements of the theory of representations, Springer-Verlag, Berlin, 1976.

106. S. Kobayashi, Riemannian manifolds without conjugate points, Ann. Mat. Pura Appl. 53 (1961), 149-155.

107. S. Kobayashi and K. Nomizu, Foundations of differential geometry. I, II, J. WileyInterscience, I: 1963, II: 1969.

108. I. Kolář, P.W. Michor, and J. Slovák, Natural operations in differential geometry, Springer-Verlag, Berlin, Heidelberg, New York, 1993.

109. Y. Kosmann-Schwarzbach, Derived brackets, Lett. Math. Phys. 69 (2004), 61-87.

110. B. Kostant, Orbits, symplectic structures, and representation theory, p. 71, Nippon Hyoronsha, Tokyo, 1966.

111. J.L. Koszul, Sur certains groupes de transformations de Lie, pp. 137-141, Centre National de la Recherche Scientifique, Paris, 1953.

112. __ Crochet de Schouten-Nijenhuis et cohomologie, Astérisque (1985), no. Numero Hors Serie, 257-271, The mathematical heritage of Élie Cartan (Lyon, 1984). 
113. A. Kriegl and P.W. Michor, The convenient setting for global analysis, Surveys and Monographs, vol. 53, AMS, Providence, 1997.

114. J. Kubarski, About Stefan's definition of a foliation with singularities: a reduction of the axioms, Bull. Soc. Math. France 118 (1990), 391-394.

115. M. Kummer, On the construction of the reduced phase space of a Hamiltonian system with symmetry, Univ. Math. J. 30 (1981), pp. 281-291.

116. H. Lawson and M.L. Michelsohn, Spin geometry, Princeton University Press, Princeton, 1989.

117. P. Lecomte, Sur la suite exacte canonique associée à un fibré principal, Bul. Soc. Math. France 13 (1985), 259-271.

118. _ On some sequence of graded Lie algebras asociated to manifolds, Ann. Global Analysis Geom. 12 (1994), 183-192.

119. P. Lecomte, P.W. Michor, and H. Schicketanz, The multigraded Nijenhuis-Richardson algebra, its universal property and application, J. Pure Appl. Algebra 77 (1992), 87102.

120. P. Lecomte and C. Roger, Sur les déformations des algèbres de courants de type réductif, C. R. Acad. Sci. Paris, I 303 (1986), 807-810.

121. John M. Lee, Introduction to smooth manifolds, second ed., Graduate Texts in Mathematics, vol. 218, Springer, New York, 2013. MR 2954043

122. E. Lerman, R. Montgomery, and R. Sjamaar, Examples of singular reduction, London Math. Soc. Lecture Note Ser., vol. 192, pp. 127-155, Cambridge Univ. Press, Cambridge, 1993.

123. P. Libermann and C.M. Marle, Symplectic geometry and analytical mechanics, D. Reidel, Dordrecht, 1987.

124. A. Lichnerowicz, Les variétés de Poisson et leur algébres de Lie associées, J. Diff. Geom. 12 (1977), 253-300.

125. S. Lie, Theorie der Transformationsgruppen. Zweiter Abschnitt, Teubner, Leipzig, 1890.

126. D. Luna, Fonctions différentiables invariantes sous l'operation d'un groupe réductif, Ann. Inst. Fourier 26 (1976), 33-49.

127. K. Mackenzie, Lie groupoids and Lie algebroids in differential geometry, London Math. Soc. Lecture Notes Ser., vol. 124, Cambridge Univ. Press, Cambridge, 1987.

128. G.W. Mackey, Induced representations of locally compact groups I, Ann. Math. 55 (1952), 101-139.

129. L Les ensembles boréliens et les extensions des groupes, J. Math. 36 (1957), $171-178$.

130. F. Magri and C. Morosi, A geometrical characterization of integrable Hamiltonian systems through the theory of Poisson-Nijenhuis manifolds, Quaderno, Univ. Milano 19 (1984).

131. B. Malgrange, Ideals of differentiable functions, Oxford Univ. Press, 1966.

132. L. Mangiarotti and M. Modugno, Graded Lie algebras and connections on a fibred space, Journ. Math. Pures et Appl. 83 (1984), 111-120.

133. G. Marmo, E. Saletan, A. Simoni, and B. Vitale, Dynamical systems. A differential geometric approach to symmetry and reduction, Wiley-Interscience, Chichester, 1985.

134. J. Marsden and M. Perlmutter, The orbit bundle picture of cotangent bundle reduction, C. R. Math. Acad. Sci. Soc. R. Can. 22 (2000), 35-54. 
135. J. Marsden and T. Ratiu, Introduction to mechanics and symmetry, Springer-Verlag, New York, 1999.

136. J. Marsden and A. Weinstein, Some comments on the history, theory, and applications of symplectic reduction, Quantization of singular symplectic quotients, Birkhäuser, 2001, Progress in Math. 198.

137. J.N. Mather, Notes on topological stability, Harvard, 1970, mimeographed notes.

138. _ Differentiable invariants, Topology 16 (1977), 145-155.

139. M. Mauhart, Iterierte Lie Ableitungen und Integrabilität, Master's thesis, Univ. Wien, 1990.

140. M. Mauhart and P.W. Michor, Commutators of flows and fields, Archivum Mathematicum (Brno) 28 (1992), 228-236.

141. F. Michor, Die Mathematik der Planetenbewegung, BRG Klosterneuburg, 2000, Fachbereichsarbeit aus Mathematik.

142. P.W. Michor, Manifolds of differentiable mappings, Shiva, Orpington, 1980.

143. _ Manifolds of smooth mappings IV: Theorem of de Rham, Cahiers Top. Geo. Diff. 24 (1983), 57-86.

144. _ A convenient setting for differential geometry and global analysis I, II, Cahiers Topol. Geo. Diff. 25 (1984), 63-109, 113-178.

145. _ A generalisation of Hamiltonian mechanics, J. Geometry and Physics 2 (1985), 67-82.

146. , Remarks on the Frölicher-Nijenhuis bracket, Proceedings of the Conference on Differential Geometry and its Applications, Brno 1986, D. Reidel, 1987, pp. $197-$ 220 .

147. _ Remarks on the Schouten-Nijenhuis bracket, Suppl. Rendiconti del Circolo Matematico di Palermo, Serie II, 16 (1987), 208-215.

148. _ Gauge theory for diffeomorphism groups, Proceedings of the Conference on Differential Geometric Methods in Theoretical Physics (Como, 1987) (K Bleuler and M Werner, eds.), Kluwer, 1988, pp. 345-371.

149. _ Graded derivations of the algebra of differential forms associated with a connection, Differential Geometry, Proceedings (Peniscola, 1988), Lecture Notes in Math., vol. 1410, Springer-Verlag, 1989, pp. 249-261.

150. Knit products of graded Lie algebras and groups, Suppl. Rendiconti Circolo Matematico di Palermo, Ser. II 22 (1989), 171-175.

151. _ The moment mapping for a unitary representation, J. Global Analysis and Geometry 8 (1990), 299-313.

152. G_ Gauge theory for fiber bundles, Monographs and Textbooks in Physical Sciences, Lecture Notes, vol. 19, Bibliopolis, Napoli, 1991.

153. _ The relation between systems and associated bundles, Ann. Mat. Pura Appl. 163 (1993), 385-399.

154. __ Basic differential forms for actions of Lie groups, Proc. AMS 124 (1996), 1633-1642.

155. _ _ The Jacobi flow, Rend. Sem. Mat. Univ. Pol. Torino 54 (1996), 365-372.

156. $\ldots$ Basic differential forms for actions of Lie groups II, Proc. AMS 125 (1997), 2175-2177.

157. J. Milnor, On manifolds homeomorphic to the 7-sphere, Annals of Math. 64 (1956), 399-405. 
158. J. Milnor and J. Stasheff, Characteristic classes, Annals of Math. Studies, vol. 76, Princeton University Press, Princeton, 1974.

159. I. Moerdijk and Reyes G.E., Rings of smooth funcions and their localizations I, J. Algebra 99 (1986), 324-336.

160. _ Rings of smooth funcions and their localizations II, Mathematical logic and theoretical computer science (College Park, Md., 1984-1985), Lecture Notes in Pure and Appl. Math., vol. 106, Dekker, New York, 1987, pp. 277-300.

161. J. Monterde, Generalized symplectomorphisms, Lecture Notes in Math, vol. 1410, pp. 262-271, Springer-Verlag, Berlin, Peñiscola, 1988, 1989.

162. J. Monterde and A. Montesinos, Integral curves of derivations, J. Global Analysis and Geometry 6 (1988), 177-189.

163. D. Montgomery and L. Zippin, Transformation groups, J. Wiley-Interscience, New York, 1955.

164. M. Mori, On the three-dimensional cohomology group of Lie algebras, J. Math. Soc. Japan 5 (1953), 171-183.

165. A. Morimoto, Prolongations of geometric structures, Math. Inst. Nagoya University, Chiknsa-Ku, Nagoya, Japan, 1969.

166. J. Morrow, The denseness of complete Riemannian metrics, J. Diff. Geo. 4 (1970), $225-226$.

167. M. Morse, The behavior of a function on its critical set, Ann. Math. 40 (1939), 345-396.

168. J. Moser, Three integrable Hamiltonian systems connected with isospectral deformations, Adv. Math. 16 (1975), 197-220.

169. J. Nagata, Modern dimension theory, North Holland, 1965.

170. M. Nagata, On the 14-th problem of Hilbert, Amer. J. Math. 81 (1959), 766-772.

171. K.-H. Neeb, A note on central extensions of Lie groups, J. Lie Theory 6 (1996), 207-213.

172. Holomorphy and convexity in Lie theory, de Gruyter, Berlin, 1999.

173. A. Newlander and L. Nirenberg, Complex analytic coordinates in almost complex manifolds, Ann. of Math. 65 (1957), 391-404.

174. A. Nijenhuis, On the holonomy groups of linear connections. IA. IB, Indagationes Math. 15 (1953), 233-240, 241-249.

175. On the holonomy groups of linear connections. II, Indagationes Math. 16 (1954), 17-25.

176. J Jacobi-type identities for bilinear differential concomitants of certain tensor fields I, Indagationes Math. 17 (1955), 390-403.

177. __ Natural bundles and their general properties, Differential Geometry in Honor of K. Yano, Kinokuniya, Tokio, 1972, pp. 317-334.

178. A. Nijenhuis and R. Richardson, Deformation of Lie algebra structures, J. Math. Mech. 17 (1967), 89-105.

179. K. Nomizu and H. Ozeki, The existence of complete Riemannian metrics, Proc. AMS 12 (1961), 889-891.

180. M. Olshanetskii and A. Perelomov, Geodesic flows on symmetric spaces of zero curvature and explicit solution of the generalized Calogero model for the classical case, Funct. Anal. Appl. 10, 3 (1977), 237-239. 
181. B. O'Neill, The fundamental equations of a submersion, Mich. Math. J. 13 (1966), 459-469.

182. A.L. Onishchik, Topology of transitive transformation groups, Johann Ambrosius Barth, Leipzig, Berlin, Heidelberg, 1994.

183. A.L. Onishchik and E. Vinberg, Lie groups and Lie algebras I, Encyclopedia of Math. Sciences, vol. 20, Springer-Verlag, Heidelberg, 1993.

184. J.-P. Ortega and T Ratiu, Singular reduction of Poisson manifolds, Lett. Math. Phys. 46 (1998), 359-372.

185. J.-P. Ortega and T. Ratiu, Momentum maps and Hamiltonian reduction, Progress in Mathematics, vol. 222, Birkhäuser, Boston, 2004.

186. R.S. Palais, A global formulation of the Lie theory of transformation groups, Mem. AMS 22 (1957).

187. of Math. 73 (1961), 295-323.

188. R.S. Palais and C.L. Terng, Natural bundles have finite order, Topology 16 (1977), 271-277.

189. __ A general theory of canonical forms, Trans. AMS 300 (1987), 771-789.

190. _ Critical point theory and submanifold geometry, Springer-Verlag, Berlin, 1988, Lecture Notes in Mathematics 1353.

191. S. Parameswaran, Skew-symmetric determinants, Amer. Math. Monthly 61 (1954), 116.

192. R. Penrose and W. Rindler, Spinors and space-time, vol. 1., vol. 2, Cambridge University Press, 1987, 1988.

193. A. Perelomov, Integrable systems of classical mechanics and Lie algebras, Birkhäuser, Basel, 1990.

194. H.V. Pitie, Characteristic classes of foliations, Research Notes in Mathematics, vol. 10, Pitman, London, 1976.

195. A. Pressley and G. Segal, Loop groups, Oxford Mathematical Monographs, Oxford University Press, 1986.

196. F. Quinn, Ends III, J. Diff. Geo. 17 (1982), 503-521.

197. A. Sard, The measure of the critical points of differentiable maps, Bull. AMS 48 (1942), 883-890.

198. G. Sartori, A theorem on orbit structures (strata) of compact linear Lie groups, J. Math. Phys. 24 (1983), 765-768.

199. H. Schicketanz, On derivations and cohomology of the Lie algebra of vector valued forms related to a smooth manifold, Bull. Soc. Roy. Sci. Liége 57 (1988), 599-617.

200. J.A. Schouten, Über Differentialkonkomitanten zweier kontravarianten Grössen, Indagationes Math. 2 (1940), 449-452.

201. O. Schreier, Über die Erweiterungen von Gruppen I, Monatsh. Math. Physik 34 (1926), 164-180.

202. __ Über die Erweiterungen von Gruppen II, Hamburger Abh. 4 (1926), 321-346.

203. I. Schur, Über eine Klasse von Mittelbildungen mit Anwendungen in der Determinantentheorie, Sitzungsberichte der Berliner Mathematischen Gesellschaft 22 (1923), 9-20.

204. G.W. Schwarz, Smooth functions invariant under the action of a compact Lie group, Topology 14 (1975), 63-68. 
205. R.T. Seeley, Extension of $c^{\infty}$-functions defined in a half space, Proc. AMS 15 (1964), 625-626.

206. J.-P. Serre, Cohomologie des groupes discrets, Séminaire Bourbaki 399 (1970/71).

207. _ Cohomologie des groupes discrets, Ann. of Math. Studies 70 (1971), 77-169, Princeton University Press.

208. P. Shanahan, The Atiyah-Singer index theorem, Lecture Notes in Mathematics, vol. 638, Springer-Verlag, Berlin, 1978.

209. U. Shukla, A cohomology for Lie algebras, J. Math. Soc. Japan 18 (1966), 275-289.

210. R. Sjamaar and E. Lerman, Stratified symplectic spaces and reduction, Ann. Math. 134 (1991), 375-422.

211. J. Slovák, Peetre theorem for nonlinear operators, Annals of Global Analysis and Geometry 6 (1988), 273-283.

212. L. Solomon, Invariants of finite reflection groups, Nagoya Math. J. 22 (1963), 57-64.

213. J.M. Souriau, Quantification géométrique, Comm. Math. Phys. 1 (1966), 374-398.

214. P. Stefan, Accessible sets, orbits, and foliations with singularities, Proc. London Math. Soc. 29 (1974), 699-713.

215. R. Stöcker and H. Zieschang, Algebraische Topologie, Teubner, Stuttgart, 1988.

216. J. Szenthe, A generalization of the Weyl group, Acta Math. Hungarica 41 (1983), $347-357$.

217. _ Orthogonally transversal submanifolds and the generalizations of the Weyl group, Period. Math. Hungarica 15 (1984), 281-299.

218. C.L. Terng, Natural vector bundles and natural differential operators, American J. of Math. 100 (1978), 775-828.

219. _ A convexity theorem for isoparametric submanifolds, Invent. Math. 85 (1986), 487-492.

220. _ Isoparametric submanifolds and their Coxeter groups, J. Diff. Geom. 1985 (21), 79-107.

221. J.C. Tougeron, Idéaux de fonctions différentiables, Springer-Verlag, 1972, Ergebnisse d. Math. 71.

222. W.M. Tulczyjew, The graded Lie algebra of multivector fields and the generalized Lie derivative of forms, Bull. Acad. Polon. Sci. 22, 9 (1974), 937-942.

223. I. Vaisman, Lectures on the geometry of Poisson manifolds, Progress in Mathematics, vol. 118, Birkhäuser Verlag, Basel, 1994.

224. V.S. Varadarajan, Lie groups, Lie algebras, and their representations, Prentice-Hall, Springer-Verlag, Englewood Cliffs, N.J., New York, 1974 1984, 2nd edition.

225. A. Weil, Théorie des points proches sur les variétés differentielles, Colloque de topologie et géométrie différentielle (Strasbourg), 1953, pp. 111-117.

226. A. Weinstein, A universal phase space for particles in a Yang-Mills field, Lett. Math. Phys. 2 (1978), 417-420.

227. H. Whitney, Analytic extensions of differentiable functions defined in closed sets, Trans. AMS 36 (1934), 63-89.

228. _ Differentiable even functions, Duke Math. J. 10 (1943), 159-166.

229. - The selfintersections of a smooth n-manifold in $2 n$-space, Annals of Math. 45 (1944), 220-293.

230. S. Wong, Field and particle equations for the classical Yang-Mills field and particles with isotopic spin, Il Nuovo Cimento 65A (1970), 689-694. 
231. H. Yamabe, On an arcwise connected subgroup of a Lie group, Osaka Math. J. 2 (1950), 13-14. 



\section{Index}

adapted orthonormal frame, 333

adjoint representation, 51

affine action, 66

affine manifold, 298

algebraic Bianchi identity, 302

algebraic bracket, 192

algebraic derivation, 192

almost complex structure, 199

$\bar{\alpha}$-pair, 180

angular momentum, 455

angular momentum of a planetary

movement, 428

anholonomic frame field, 22

associated bundle, 215

atlas, 1

base of a fibered manifold, 16

base of a vector bundle, 100

base space of a fiber bundle, 200

basic cohomology, 402

basic forms, 402

basic vector field, 337

basis of a fiber bundle, 200

Betti number, 129

Bianchi identity, 172302

Bianchi identity on a fiber bundle, 201

bundle, 200

$C^{k}$-atlas, 1

$C^{k}$-equivalent atlases, 1

Caley-Hamilton equation, 259

canonical flip, 107

canonical symplectic structure, 417

Cartan moving frame version of a

connection, 232

Čech cohomology set, 101 center of a Lie algebra, 64

center of a Lie group, 64

central extension of a Lie algebra, 170,463

centralizer in a Lie algebra, 64

centralizer in a Lie group, 64

characteristic class of the invariant

polynomial, 252

chart, 1 .

charts with boundary, 126

Chern character, 265

Chern classes, 264

Chern-Weil form, 251

Chern-Weil homomorphism, 252

Chevalley cohomology of the Lie algebra, 166

Christoffel forms, 203

Christoffel symbol, 276, 283

classical complex Lie groups, 44

classical second fundamental form, 332

classifying spaces, 220

closed form, 121

coadjoint representation, 421

cocurvature, 197

cocycle condition, 200

cocycle of transition functions, 100,200

Codazzi-Mainardi equation, $330 \lcm{332} \lcm{335}$

cohomological integral, 150

cohomologous, 101211

cohomology classes, 101

cohomology group, 132

coisotropic submanifold, 417

coisotropic subspace, 414

compatible, 99

compatible symplectic and complex

structures, 424

complete connection, 204 
complete Riemann manifold, 292

complete vector field, 25

completely integrable Hamiltonian system, 434

complex line bundles, 103

conformal diffeomorphism, 294

conformal Riemann metrics, 294

conjugate point, 353

conjugation, 51

connection, 172197

connection form, Lie algebra valued, 229

connection on a fiber bundle, 201

connector, 245,283

contact of order, 266

cotangent bundle, 113

covariant derivative, 245

covariant derivative of tensor fields, 287

covariant derivative on a manifold, 277

covariant derivative, compatible with the

pseudo-Riemann metric, 278

covariant exterior derivative, 234, 247

covariant exterior differential, 172

curl, 326

curvature, $171,197,246$

curvature form, Lie algebra valued, 230

curvature matrix, 311

curvature of the covariant derivative, 301

curve of local diffeomorphisms, 29

Darboux' theorem, 422

de Rham cohomology algebra, 129

de Rham cohomology algebra with compact supports, 139

degree of a mapping, 156

densities, 122

density or volume of the Riemann metric, 125

derivation, 5,191

diffeomorphic, 3

diffeomorphism, 3

differential form, 115

differential group of order $k, 269$

differential of a function, 9

Dirac structure, 450

distance increasing, 359

distinguished chart, 37

distinguished chart for a foliation, 33

distribution, 32 34

divergence, 325

double ratio, invariance of, 320

dual coframe, 313

effective action, 66

Ehresmann connection, 205

electric potential, 327

ellipsoid, 15

energy of a curve, 274 equivalent vector bundle atlases, 100

equivariant normal field, 373

Euler-Poincaré characteristic, 130

evolution operator, 39

exact form, 121

exponential mapping, 49

exponential mapping of a spray, 281

extension of Lie algebras, 170

exterior derivative, 119

$f$-dependent, 198

$f$-related, 26,198

1-form, 113

Fermi chart, 347,348

fiber, 100

fiber bundle, 200

fiber chart of a fiber bundle, 200

fiber integration, 142

fibered composition of jets, 268

fibered manifold, 16

first Chern class, 103

first nonvanishing derivative, 29

first Stiefel-Whitney class, 103

fixpoint group, 68

flow line, 23

flow prolongation, 111

focal points, 354

foliation, 35

foliation corresponding to an integrable vector subbundle, 33

Frölicher-Nijenhuis bracket, 193

fractional linear transformations, 318

frame field, 22, 103,310

free action, 66

fundamental vector field, 6768

$G$-atlas, 211

$G$-bundle, 211

$G$-bundle structure, 210

$G$-invariant, 402

$G$-invariant pseudo-Riemann metric, 366

$G$-manifold, 66

$G$-space, 66

gauge transformations, 224

Gauß's equation, 330

general linear group, 42

generalized Hamiltonian function, 452

geodesic, 277

geodesic distance, 291

geodesic spray, 279

geodesic structure on a manifold, 279

geodesic symmetry, 368

geometric objects, 272

germ of $f$ at $x, 5$

global flow, 25

graded derivation, 191

graded differential space, 132 
gradient, 325

Grassmann manifold, 213

group cohomology, 459

$\mathbb{H}$-linear, 45

Haar measure, 160

hairy ball theorem, 157

half-space, 126

Hamilton's equations, 411

Hamiltonian group action, 452

Hamiltonian mapping, 449

Hamiltonian system, 434

Hamiltonian vector field, 412,426

Hamiltonian vector field for a Poisson structure, 441

Hodge $*$-operator, 324

Hodge isomorphism, 325

holonomic frame field, 22,113

holonomy group, 206237

holonomy Lie algebra, 206

homogeneous space, 64 .

homomorphism of $G$-bundles, 218

homomorphism over $\Phi$ of principal bundles, 214

homotopy operator, 131,403

Hopf, Rinov, 292

horizontal, 402

horizontal bundle of a fiber bundle, 201

horizontal differential forms, 234

horizontal foliation, 202

horizontal lift, 172,282

horizontal lift of the vector field, 299

horizontal lift on a fiber bundle, 201

horizontal projection, 201

horizontal space, 197

horizontal subbundle, 336

horizontal vector field, 337

horizontal vectors of a fiber bundle, 201

hyperboloid, 15

ideal, 64

idealizer in a Lie algebra, 64

immersed submanifold, 17

immersion at, 17

index, 266

index of the metric, 273

induced connection, $1 7 2 \longdiv { 2 4 1 } 2 4 2$

induced representation, 223

infinitesimal automorphism, 36

infinitesimal gauge transformation, 224

infinitesimal strongly Hamiltonian action, 457

infinitesimally free action, 66

infinitesimally transitive action, 66

initial submanifold, 18

inner automorphism, 51

insertion operator, 117 integrable, 35

integrable subbundle of a tangent bundle, 32

integral curve, 23

integral manifold, 34

integral of a differential form, 126

integral of the density, 123

invariant of the Lie algebra, 251

invertible, 271

involution, 107

involutive distribution, 38

involutive set of local vector fields, 38

involutive subbundle of a tangent bundle, 32

irreducible principal connection, 238

isometric action, 67

isometry, 363

isoparametric submanifolds, 400

isotropic submanifold, 417

isotropic subspace, 414

isotropy representation, 372

isotropy subgroup, 68, 73

isotropy type, 73

Jacobi differential equation, 345

Jacobi fields, 345

Jacobi operator, 352

jet, 267

$k$-form, 115

$k$-th order frame bundle, 271

Killing fields, 307

Killing vector field, 363

Koszul bracket, 448

Lagrange-Grassmann manifold, 415

Lagrangian submanifold, 417

Lagrangian subspace, 414

leaf, 35

leaves of the foliation, 33

Lebesque measure 0, 12

left invariant differential form, 158

left action of a Lie group, 66

left invariant, 46

left invariant differential form, 163

left logarithmic derivative, 53

length of a curve, 274

Levi-Civita covariant derivative, 278

Lie algebra, 23

Lie algebra of infinitesimal automorphisms of the Poisson structure, 441

Lie algebra valued connection form, 229

Lie algebra valued curvature form, 230

Lie bracket of vector fields, 23

Lie derivative along a vector valued form, 193

Lie derivative of differential forms, 116 
Lie derivative of sections of a natural bundle, 109

Lie derivative of vector fields, 27

Lie group, 41

Lie subgroup, 60

linear action, 66

linear connection, $244,245,282$

linear frame bundle, 109,220

linear momentum, 455

Liouville form, 417

Liouville vector field, 418

Liouville volume, 416

local diffeomorphism, 3

local frame, 32310

local vector field, 21

locally Hamiltonian vector fields, 426

long exact cohomology sequence with

compact supports of the pair, 150

$m$-cube of width $w>0$ in $\mathbb{R}^{m}, 12$

magnetic potential, 327

manifold pair, 138, 150

manifold with boundary, 126

mapping degree for a proper mapping, 145

Maslov class, 416

Maurer-Cartan, 203

Maurer-Cartan form, 53

maximal integral manifold, 34

Maxwell equations, 326

Mayer-Vietoris sequence, 133

measure 0,12

Möbius transformations, 318

momentum, 411

momentum mapping, 453

multiplicity of a conjugate point, 353

multivector field, 440

natural bilinear mappings, 199

natural bundles, 272

natural lift, 111

natural transformation, 110

natural vector bundle, 108

Nijenhuis tensor, 199

Nijenhuis-Richardson bracket, 192

normal bundle, 371

normal slice, 372

normalizer in a Lie algebra, 64

normalizer in a Lie group, 64

$\omega$-respecting vector fields, 426

orbit of a Lie group, 66

orbit type, 73

orientable double cover, 151

orientable manifold, 125

orientations of a manifold, 125

oriented manifold, 125

orthogonal action, 66 orthogonal group, 42

orthonormal frame, 310

orthonormal frame bundle, 220 314

orthonormal frame field, 220

1-parameter subgroup, 48

1 -parameter variation through geodesics,

$$
345
$$

parallel transport, 299

parallel vector field, 298

parameterized by arc-length, 289

perihel, 430

Pfaffian, 262

Pfaffian class, 263

phase space, 432

physicists' version of a connection, 232

planetary orbit, 430

plaque, 3337

Poincaré duality, 146

Poincaré duality operator, 147

Poincaré polynomial, 130

Poisson bracket, 426

Poisson cohomology, 445

Poisson morphism, 443

Poisson structure, 439

polar action, 388

Pontryagin character, 260

Pontryagin classes, 255

Pontryagin numbers, 257

principal bundle, 211

principal bundle atlas, 211

principal connection, 229

principal fiber bundle, 211

principal fiber bundle homomorphism, 214

principal orbit, 74

principal right action, 211

product manifold, 11

projectable vector field, 337

projection of a fiber bundle, 200

projection of a vector bundle, 100

proper action, 77

proper homotopy, 141

proper mapping, 77

proper smooth mappings, 139

pseudo-Riemann locally symmetric space, 368

pseudo-Riemann metric, 273

pseudo-Riemann symmetric space, 368

pullback of a fiber bundle, 202

pullback vector bundle, 105

pure manifold, 1 ]

quasi-periodic flow, 438

quaternionic unitary group, 45

quaternionically linear, 45

quaternionically unitary, 45

quaternions, 58 
real line bundle, 102

reduction of the structure group, 215

reductive decomposition of a Lie algebra, 369

regular point, 74

regular point of a mapping, 13

regular value, 9

regular value of a mapping, 13

relative de Rham cohomology, 138

relative de Rham cohomology with

compact supports, 150

relative Poincaré lemma, 422

representation, 51

residual subset of a manifold, 12

restricted holonomy group, 206, 237

Riemann locally symmetric space, 368

Riemann metric, 220,273

Riemann normal coordinate system, 281

Riemann submersion, 336

Riemann symmetric space, 368

right action of a Lie group, 66

right invariant, 46

right logarithmic derivative, 52

right trivialized derivative, 160

saddle, 15

Schouten-Nijenhuis bracket, 440

second fundamental form, 327

section, 388

section of a vector bundle, 100

sectional curvature, 307

semidirect product, 71

semidirect product of Lie algebras, 170.

set of (Lebesque) measure 0 in a manifold, 12

shape form, 327

shape operator, 329

short exact sequence, 133

signature, 156

signature of the metric, 273

signed algebraic complements, 57

singular distribution, 34

singular foliation, 35

singular point, 74

singular point of a mapping, 13

singular value of a mapping, 13

skew-field, 58

slice, 75

slice representation, 372

smooth distribution, 34

smooth functor, 104

smooth partitions of unity, 4

soldering form, 314

source mapping, 267

source of a jet, 267

space of all covariant derivatives, 287

space of closed forms, 129 space of exact forms, 129

spanning subsets, 34

special linear group, 43

special orthogonal group, 42

special unitary, 45

sphere, 2

spray, 280

stable, 35

stably equivalent, 257

standard fiber, 99, 200

stereographic atlas, 2

Stiefel manifold, 214

strongly Hamiltonian group action, 457

structure, 1

submanifold, 9

submanifold charts, 9

submersion, 16

support of a section, 100

support of a smooth function, 4

support of a vector field, 25

symmetric connection, 282

symmetric covariant derivative, 277

symplectic action, 67

symplectic gradient, 426

symplectic group, 43

symplectic group action, 451

symplectic manifold, 416

symplectic orthogonal, 413

symplectic structure, 412

symplectic subspace, 414

symplectomorphisms, 412

tangent bundle, 8

tangent space of $M$ at $x, 6$

tangent vector, 5

target mapping, 267

target of a jet, 267

tensor field, 110113

theorema egregium, 330

theorema egregium proper, 333

time dependent vector field, 39]

Todd class, 265

topological manifold, 1

torsion form, 313

torsion of a covariant derivative, 285

torsion-free connection, 282

torsion-free covariant derivative, 277

torus, 15

total Chern class, 264

total Pontryagin class, 256

total space, 16

total space of a fiber bundle, 200

total space of a vector bundle, 100

totally geodesic immersion, 328

trace classes of a complex vector bundle, 264

trace coefficients, 257 
transformation formula for multiple integrals, 122

transition function, 99,200

transitive action, 66

transversal, 20

Trotter's formula, 55

truncated composition, 268

tubular neighborhood, 372

typical fiber, 99

unimodular Lie group, 159

unitary, 44

universal 1-form, 418

universal connection, 418

universal curvature, 418

variation, 274354

variational vector field, 274

vector bundle, 100

vector bundle atlas, 99

vector bundle chart, 99

vector bundle functor, 108

vector bundle homomorphism, 104

vector bundle isomorphism, 104

vector field, 21

vector product, 45

vector subbundle, 104

vector subbundle of a tangent bundle, 31

vector valued differential forms, 192

vertical bundle, 107

vertical bundle of a fiber bundle, 201, 228

vertical lift, 107

vertical projection, 107, 201

vertical space, 197

vertical subbundle, 336

vertical vector field, 337

volume, 125

volume bundle, 122

weakly regular value, 464

wedge product, 115

Weingarten equation, 332

Weingarten formula, 329

Weingarten mapping, 329

Weyl group, generalized, 394

zero section of a vector bundle, 100

zero set, 4 